CMS Physics Technical Design Report, Volume II: Physics Performance

This content has been downloaded from IOPscience. Please scroll down to see the full text. 2007 J. Phys. G: Nucl. Part. Phys. 34995

(http://iopscience.iop.org/0954-3899/34/6/S01)

View the table of contents for this issue, or go to the journal homepage for more

Download details:

IP Address: 193.140.249.2

This content was downloaded on 15/08/2016 at $12: 10$

Please note that terms and conditions apply. 


\title{
CMS Physics Technical Design Report, Volume II: Physics Performance
}

\author{
The CMS Collaboration
}

Received 3 January 2007

Published 20 April 2007

Online at stacks.iop.org/JPhysG/34/995

\begin{abstract}
CMS is a general purpose experiment, designed to study the physics of pp collisions at $14 \mathrm{TeV}$ at the Large Hadron Collider (LHC). It currently involves more than 2000 physicists from more than 150 institutes and 37 countries. The LHC will provide extraordinary opportunities for particle physics based on its unprecedented collision energy and luminosity when it begins operation in 2007.
\end{abstract}

The principal aim of this report is to present the strategy of CMS to explore the rich physics programme offered by the LHC. This volume demonstrates the physics capability of the CMS experiment. The prime goals of CMS are to explore physics at the $\mathrm{TeV}$ scale and to study the mechanism of electroweak symmetry breaking - through the discovery of the Higgs particle or otherwise. To carry out this task, CMS must be prepared to search for new particles, such as the Higgs boson or supersymmetric partners of the Standard Model particles, from the start-up of the LHC since new physics at the TeV scale may manifest itself with modest data samples of the order of a few $\mathrm{fb}^{-1}$ or less.

The analysis tools that have been developed are applied to study in great detail and with all the methodology of performing an analysis on CMS data specific benchmark processes upon which to gauge the performance of CMS. These processes cover several Higgs boson decay channels, the production and decay of new particles such as $Z^{\prime}$ and supersymmetric particles, $B_{s}$ production and processes in heavy ion collisions. The simulation of these benchmark processes includes subtle effects such as possible detector miscalibration and misalignment. Besides these benchmark processes, the physics reach of CMS is studied for a large number of signatures arising in the Standard Model and also in theories beyond the Standard Model for integrated luminosities ranging from $1 \mathrm{fb}^{-1}$ to $30 \mathrm{fb}^{-1}$. The Standard Model processes include QCD, $B$-physics, diffraction, detailed studies of the top quark properties, and electroweak physics topics such as the $W$ and $Z^{0}$ boson properties. The production and decay of the Higgs particle is studied for many observable decays, and the precision with which the Higgs boson properties can be derived is determined. About ten different supersymmetry benchmark points are analysed using full simulation. The CMS discovery reach is evaluated in the SUSY parameter space covering a large variety of decay signatures. 
Furthermore, the discovery reach for a plethora of alternative models for new physics is explored, notably extra dimensions, new vector boson high mass states, little Higgs models, technicolour and others. Methods to discriminate between models have been investigated.

This report is organized as follows. Chapter 1, the Introduction, describes the context of this document. Chapters 2-6 describe examples of full analyses, with photons, electrons, muons, jets, missing $E_{\mathrm{T}}$, B-mesons and $\tau$ 's, and for quarkonia in heavy ion collisions. Chapters 7-15 describe the physics reach for Standard Model processes, Higgs discovery and searches for new physics beyond the Standard Model.

\section{Acknowledgments}

This report is the result of several years of work on the preparation for physics analysis at the LHC with CMS. Subprojects in all areas were involved (Detector, PRS, Software, and Computing) in order to produce the large Monte Carlo simulation samples needed, to develop the software to analyse those samples, to perform the studies reported in this Report, and to write and review our findings.

We wish to thank, for the many useful discussions, our theory and phenomenology colleagues, in particular J Campbell, D Dominici, A Djouadi, S Heinemeyer, W Hollik, V Khoze, T Plehn, M Raidal, M Spira and G Weiglein for their contributions to this Report.

For their constructive comments and guidance, we would like to thank the CPT internal reviewers: J Alexander, J Branson, Y Karyotakis, M Kasemann and R Tenchini.

We would like to thank L Malgeri and R Tenchini for their efficient organisation of the CMS Notes.

For their patience in meeting sometimes impossible demands, we wish to thank the CMS Secretariat: K Aspola, M Azeglio, N Bogolioubova, D Denise, D Hudson, G Martin, and M C Pelloux.

We also would like to thank $\mathrm{G}$ Alverson and L Taylor for their invaluable technical assistance in the preparation of this manuscript.

Finally, we wish to thank the CMS management for their strong support and encouragement.

The CMS Collaboration

Trademark notice. All trademarks appearing in this Report are acknowledged as such. 


\section{The CMS Collaboration}

Yerevan Physics Institute, Yerevan, ARMENIA

G L Bayatian, S Chatrchyan, G Hmayakyan, A M Sirunyan

Institut für Hochenergiephysik der OeAW, Wien, AUSTRIA

W Adam, T Bergauer, M Dragicevic, J Erö, M Friedl, R Fruehwirth, V Ghete, P Glaser, J Hrubec, M Jeitler, M Krammer, I Magrans, I Mikulec, W Mitaroff, T Noebauer, M Pernicka, P Porth, H Rohringer, J Strauss, A Taurok, W Waltenberger, G Walzel, E Widl, C-E Wulz

Research Institute for Nuclear Problems, Minsk, BELARUS

A Fedorov, M Korzhik, O Missevitch, R Zuyeuski

National Centre for Particle and High Energy Physics, Minsk, BELARUS

V Chekhovsky, O Dvornikov, I Emeliantchik, A Litomin, V Mossolov, N Shumeiko, A Solin, R Stefanovitch, J Suarez Gonzalez, A Tikhonov

\section{Byelorussian State University, Minsk, BELARUS}

V Petrov

Vrije Universiteit Brussel, Brussel, BELGIUM

J D'Hondt, S De Weirdt, R Goorens, J Heyninck, S Lowette, S Tavernier, W Van Doninck ${ }^{1}$, L Van Lancker

Université Libre de Bruxelles, Bruxelles, BELGIUM

O Bouhali, B Clerbaux, G De Lentdecker, J P Dewulf, T Mahmoud, P E Marage, L Neukermans, V Sundararajan, C Vander Velde, P Vanlaer, J Wickens

Université Catholique de Louvain, Louvain-la-Neuve, BELGIUM

S Assouak, J L Bonnet, G Bruno, J Caudron, B De Callatay, J De Favereau De Jeneret, S De Visscher, C Delaere, P Demin, D Favart, E Feltrin, E Forton, G Grégoire, S Kalinin, D Kcira, T Keutgen, G Leibenguth, V Lemaitre, Y Liu, D Michotte, O Militaru, A Ninane, S Ovyn, T Pierzchala, K Piotrzkowski, V Roberfroid, X Rouby, D Teyssier, O Van der Aa, M Vander Donckt

Université de Mons-Hainaut, Mons, BELGIUM

E Daubie, P Herquet, A Mollet, A Romeyer

Universiteit Antwerpen, Wilrijk, BELGIUM

W Beaumont, M Cardaci, E De Langhe, E A De Wolf, L Rurua

Centro Brasileiro de Pesquisas Fisicas, Rio de Janeiro, RJ, BRAZIL

M H G Souza

Universidade do Estado do Rio de Janeiro, Rio de Janeiro, RJ, BRAZIL

V Oguri, A Santoro, A Sznajder

Instituto de Fisica, Universidade Federal do Rio de Janeiro, Rio de Janeiro, RJ, BRAZIL M Vaz

Instituto de Fisica Teorica, Universidade Estadual Paulista, Sao Paulo, SP, BRAZIL

E M Gregores, S F Novaes

\footnotetext{
${ }^{1}$ Also at CERN, European Organization for Nuclear Research, Geneva, Switzerland.
} 
Institute for Nuclear Research and Nuclear Energy, Sofia, BULGARIA

T Anguelov, G Antchev, I Atanasov, J Damgov, N Darmenov ${ }^{1}$, L Dimitrov, V Genchev ${ }^{1}$, P Iaydjiev, B Panev, S Piperov, S Stoykova, G Sultanov, I Vankov

University of Sofia, Sofia, BULGARIA

A Dimitrov, V Kozhuharov, L Litov, M Makariev, A Marinov, E Marinova, S Markov, M Mateev, B Pavlov, P Petkov, C Sabev, S Stoynev, Z Toteva ${ }^{1}$, V Verguilov

Institute of High Energy Physics, Beijing, CHINA

G M Chen, H S Chen, K L He, C H Jiang, W G Li, H M Liu, X Meng, X Y Shen, H S Sun, M Yang, W R Zhao, H L Zhuang

Peking University, Beijing, CHINA

Y Ban, J Cai, S Liu, S J Qian, Z C Yang, Y L Ye, J Ying

University for Science and Technology of China, Hefei, Anhui, CHINA

J Wu, Z P Zhang

Technical University of Split, Split, CROATIA

N Godinovic, I Puljak, I Soric

University of Split, Split, CROATIA

Z Antunovic, M Dzelalija, K Marasovic

Institute Rudjer Boskovic, Zagreb, CROATIA

V Brigljevic, D Ferencek, K Kadija, S Morovic, M Planinic ${ }^{2}$

University of Cyprus, Nicosia, CYPRUS

C Nicolaou, A Papadakis, P A Razis, D Tsiakkouri

National Institute of Chemical Physics and Biophysics, Tallinn, ESTONIA

A Hektor, M Kadastik, K Kannike, E Lippmaa, M Müntel, M Raidal

Laboratory of Advanced Energy Systems, Helsinki University of Technology, Espoo, FINLAND

P A Aarnio

Helsinki Institute of Physics, Helsinki, FINLAND

S Czellar, E Haeggstroem, A Heikkinen, J Härkönen, V Karimäki, R Kinnunen, T Lampén, K Lassila-Perini, S Lehti, T Lindén, P R Luukka, S Michal ${ }^{1}$, T Mäenpää, J Nysten, M Stettler ${ }^{1}$, E Tuominen, J Tuominiemi, L Wendland

Lappeenranta University of Technology, Lappeenranta, FINLAND

T Tuuva

Laboratoire d'Annecy-le-Vieux de Physique des Particules, IN2P3-CNRS, Annecy-le-Vieux, FRANCE

J P Guillaud, P Nedelec, D Sillou

DSM/DAPNIA, CEA/Saclay, Gif-sur-Yvette, FRANCE

M Anfreville, S Beauceron, E Bougamont, P Bredy, R Chipaux, M Dejardin, D Denegri, J Descamps, B Fabbro, J L Faure, S Ganjour, F X Gentit, A Givernaud, P Gras, G Hamel de Monchenault, P Jarry, F Kircher, M C Lemaire ${ }^{3}$, B Levesy ${ }^{1}$, E Locci, J P Lottin,

\footnotetext{
${ }^{2}$ Also at University of Zagreb, Zagreb, Croatia.

${ }^{3}$ Also at California Institute of Technology, Pasadena, USA.
} 
I Mandjavidze, M Mur, E Pasquetto, A Payn, J Rander, J M Reymond, F Rondeaux, A Rosowsky, Z H Sun, P Verrecchia

Laboratoire Leprince-Ringuet, Ecole Polytechnique, IN2P3-CNRS, Palaiseau, FRANCE S Baffioni, F Beaudette, M Bercher, U Berthon, S Bimbot, J Bourotte, P Busson, M Cerutti, D Chamont, C Charlot, C Collard, D Decotigny, E Delmeire, L Dobrzynski, A M Gaillac, Y Geerebaert, J Gilly, M Haguenauer, A Karar, A Mathieu, G Milleret, P Miné, P Paganini, T Romanteau, I Semeniouk, Y Sirois

Institut Pluridisciplinaire Hubert Curien, IN2P3-CNRS, ULP, UHA Mulhouse, Strasbourg, FRANCE

J D Berst, J M Brom, F Didierjean, F Drouhin ${ }^{1}$, J C Fontaine ${ }^{4}$, U Goerlach ${ }^{5}$, P Graehling, L Gross, L Houchu, P Juillot, A Lounis ${ }^{5}$, C Maazouzi, D Mangeol, C Olivetto, T Todorov ${ }^{1}$, P Van Hove, D Vintache

Institut de Physique Nucléaire, IN2P3-CNRS, Université Claude Bernard Lyon 1, Villeurbanne, FRANCE

M Ageron, J L Agram, G Baulieu, M Bedjidian, J Blaha, A Bonnevaux, G Boudoul ${ }^{1}$, E Chabanat, C Combaret, D Contardo ${ }^{1}$, R Della Negra, P Depasse, T Dupasquier, H El Mamouni, N Estre, J Fay, S Gascon, N Giraud, C Girerd, R Haroutunian, J C Ianigro, B Ille, M Lethuillier, N Lumb ${ }^{1}$, H Mathez, G Maurelli, L Mirabito ${ }^{1}$, S Perries, O Ravat

Institute of High Energy Physics and Informatization, Tbilisi State University, Tbilisi, GEORGIA

R Kvatadze

Institute of Physics Academy of Science, Tbilisi, GEORGIA

V Roinishvili

RWTH, I. Physikalisches Institut, Aachen, GERMANY

R Adolphi, R Brauer, W Braunschweig, H Esser, L Feld, A Heister, W Karpinski, K Klein, C Kukulies, J Olzem, A Ostapchuk, D Pandoulas, G Pierschel, F Raupach, S Schael, G Schwering, M Thomas, M Weber, B Wittmer, M Wlochal

RWTH, III. Physikalisches Institut A, Aachen, GERMANY

A Adolf, P Biallass, M Bontenackels, M Erdmann, H Fesefeldt, T Hebbeker, S Hermann, G Hilgers, K Hoepfner ${ }^{1}$, C Hof, S Kappler, M Kirsch, D Lanske, B Philipps, H Reithler, T Rommerskirchen, M Sowa, H Szczesny, M Tonutti, O Tsigenov

RWTH, III. Physikalisches Institut B, Aachen, GERMANY

F Beissel, M Davids, M Duda, G Flügge, T Franke, M Giffels, T Hermanns, D Heydhausen, S Kasselmann, G Kaussen, T Kress, A Linn, A Nowack, M Poettgens, O Pooth, A Stahl, D Tornier, M Weber

Deutsches Elektronen-Synchrotron, Hamburg, GERMANY

A Flossdorf, B Hegner, J Mnich, C Rosemann

University of Hamburg, Hamburg, GERMANY

G Flucke, U Holm, R Klanner, U Pein, N Schirm, P Schleper, G Steinbrück, M Stoye, R Van Staa, K Wick

\footnotetext{
${ }^{4}$ Also at Université de Haute-Alsace, Mulhouse, France.

${ }^{5}$ Also at Université Louis Pasteur, Strasbourg, France.
} 
Institut für Experimentelle Kernphysik, Karlsruhe, GERMANY

P Blüm, V Buege, W De Boer, G Dirkes ${ }^{1}$, M Fahrer, M Feindt, U Felzmann, J Fernandez Menendez $^{6}$, M Frey, A Furgeri, F Hartmann ${ }^{1}$, S Heier, C Jung, B Ledermann, Th. Müller, M Niegel, A Oehler, T Ortega Gomez, C Piasecki, G Quast, K Rabbertz, C Saout, A Scheurer, D Schieferdecker, A Schmidt, H J Simonis, A Theel, A Vest, T Weiler, C Weiser, J Weng ${ }^{1}$, V Zhukov $^{7}$

University of Athens, Athens, GREECE

G Karapostoli ${ }^{1}$, P Katsas, P Kreuzer, A Panagiotou, C Papadimitropoulos

Institute of Nuclear Physics "Demokritos", Attiki, GREECE

G Anagnostou, M Barone, T Geralis, C Kalfas, A Koimas, A Kyriakis, S Kyriazopoulou, D Loukas, A Markou, C Markou, C Mavrommatis, K Theofilatos, G Vermisoglou, A Zachariadou

University of Ioánnina, Ioánnina, GREECE

$\mathrm{X}$ Aslanoglou, I Evangelou, P Kokkas, N Manthos, I Papadopoulos, G Sidiropoulos, F A Triantis

KFKI Research Institute for Particle and Nuclear Physics, Budapest, HUNGARY

G Bencze ${ }^{1}$, L Boldizsar, C Hajdu ${ }^{1}$, D Horvath ${ }^{8}$, A Laszlo, G Odor, F Sikler, N Toth, G Vesztergombi, P Zalan

Institute of Nuclear Research ATOMKI, Debrecen, HUNGARY

J Molnar

University of Debrecen, Debrecen, HUNGARY

N Beni, A Kapusi, G Marian, P Raics, Z Szabo, Z Szillasi, G Zilizi

Panjab University, Chandigarh, INDIA

H S Bawa, S B Beri, V Bhandari, V Bhatnagar, M Kaur, R Kaur, J M Kohli, A Kumar, J B Singh

University of Delhi, Delhi, INDIA

A Bhardwaj, S Bhattacharya ${ }^{9}$, S Chatterji, S Chauhan, B C Choudhary, P Gupta, M Jha, K Ranjan, R K Shivpuri, A K Srivastava

Bhabha Atomic Research Centre, Mumbai, INDIA

S Borkar, M Dixit, M Ghodgaonkar, S K Kataria, S K Lalwani, V Mishra, A K Mohanty, A Topkar

Tata Institute of Fundamental Research - EHEP, Mumbai, INDIA

$\mathrm{T}$ Aziz, S Banerjee, S Bose, N Cheere, S Chendvankar, $\mathrm{P}$ V Deshpande, M Guchait ${ }^{10}$, A Gurtu, M Maity ${ }^{11}$, G Majumder, K Mazumdar, A Nayak, M R Patil, S Sharma, K Sudhakar, S C Tonwar

\footnotetext{
${ }^{6}$ Now at Instituto de Física de Cantabria (IFCA), CSIC-Universidad de Cantabria, Santander, Spain.

${ }^{7}$ Also at Moscow State University, Moscow, Russia.

${ }^{8}$ Also at Institute of Nuclear Research ATOMKI, Debrecen, Hungary.

${ }^{9}$ Also at University of California, San Diego, La Jolla, USA.

${ }^{10}$ Also at Tata Institute of Fundamental Research - HECR, Mumbai, India.

${ }^{11}$ Also at University of Visva-Bharati, Santiniketan, India.
} 
Tata Institute of Fundamental Research - HECR, Mumbai, INDIA

B S Acharya, S Banerjee, S Bheesette, S Dugad, S D Kalmani, V R Lakkireddi, N K Mondal, N Panyam, P Verma

Institute for Studies in Theoretical Physics \& Mathematics (IPM), Tehran, IRAN

M Arabgol, H Arfaei, M Hashemi, M Mohammadi, M Mohammadi Najafabadi, A Moshaii, S Paktinat Mehdiabadi

University College Dublin, Dublin, IRELAND

M Grunewald

Università di Bari, Politecnico di Bari e Sezione dell’ INFN, Bari, ITALY

M Abbrescia, L Barbone, A Colaleo ${ }^{1}$, D Creanza, N De Filippis, M De Palma, G Donvito, L Fiore, D Giordano, G Iaselli, F Loddo, G Maggi, M Maggi, N Manna, B Marangelli, M S Mennea, S My, S Natali, S Nuzzo, G Pugliese, V Radicci, A Ranieri, F Romano, G Selvaggi, L Silvestris, P Tempesta, R Trentadue, G Zito

Università di Bologna e Sezione dell' INFN, Bologna, ITALY

G Abbiendi, W Bacchi, A Benvenuti, D Bonacorsi, S Braibant-Giacomelli, P Capiluppi, F R Cavallo, C Ciocca, G Codispoti, I D’Antone, G M Dallavalle, F Fabbri, A Fanfani, P Giacomelli ${ }^{12}$, C Grandi, M Guerzoni, L Guiducci, S Marcellini, G Masetti, A Montanari, F Navarria, F Odorici, A Perrotta, A Rossi, T Rovelli, G Siroli, R Travaglini

Università di Catania e Sezione dell' INFN, Catania, ITALY

S Albergo, M Chiorboli, S Costa, M Galanti, G Gatto Rotondo, F Noto, R Potenza, G Russo, A Tricomi, C Tuve

Università di Firenze e Sezione dell' INFN, Firenze, ITALY

A Bocci, G Ciraolo, V Ciulli, C Civinini, R D’Alessandro, E Focardi, C Genta, P Lenzi, A Macchiolo, N Magini, F Manolescu, C Marchettini, L Masetti, S Mersi, M Meschini, S Paoletti, G Parrini, R Ranieri, M Sani

Università di Genova e Sezione dell' INFN, Genova, ITALY

P Fabbricatore, S Farinon, M Greco

Istituto Nazionale di Fisica Nucleare e Universita Degli Studi Milano-Bicocca, Milano, ITALY

G Cattaneo, A De Min, M Dominoni, F M Farina, F Ferri, A Ghezzi, P Govoni, R Leporini, S Magni, M Malberti, S Malvezzi, S Marelli, D Menasce, L Moroni, P Negri, M Paganoni, D Pedrini, A Pullia, S Ragazzi, N Redaelli, C Rovelli, M Rovere, L Sala, S Sala, R Salerno, T Tabarelli de Fatis, S Vigano'

Istituto Nazionale di Fisica Nucleare de Napoli (INFN), Napoli, ITALY

G Comunale, F Fabozzi, D Lomidze, S Mele, P Paolucci, D Piccolo, G Polese, C Sciacca

Università di Padova e Sezione dell' INFN, Padova, ITALY

P Azzi, N Bacchetta ${ }^{1}$, M Bellato, M Benettoni, D Bisello, E Borsato, A Candelori, P Checchia, E Conti, M De Mattia, T Dorigo, V Drollinger, F Fanzago, F Gasparini, U Gasparini, M Giarin, P Giubilato, F Gonella, A Kaminskiy, S Karaevskii, V Khomenkov, S Lacaprara, I Lippi, M Loreti, O Lytovchenko, M Mazzucato, A T Meneguzzo, M Michelotto, F Montecassiano', M Nigro, M Passaseo, M Pegoraro, G Rampazzo, P Ronchese, E Torassa, S Ventura, M Zanetti, P Zotto, G Zumerle

12 Also at University of California, Riverside, Riverside, USA. 
Università di Pavia e Sezione dell' INFN, Pavia, ITALY

G Belli, U Berzano, C De Vecchi, R Guida, M M Necchi, S P Ratti, C Riccardi, G Sani, P Torre, P Vitulo

Università di Perugia e Sezione dell' INFN, Perugia, ITALY

F Ambroglini, E Babucci, D Benedetti, M Biasini, G M Bilei ${ }^{1}$, B Caponeri, B Checcucci, L Fanò, P Lariccia, G Mantovani, D Passeri, M Pioppi, P Placidi, V Postolache, D Ricci ${ }^{1}$, A Santocchia, L Servoli, D Spiga

Università di Pisa, Scuola Normale Superiore e Sezione dell' INFN, Pisa, ITALY

P Azzurri, G Bagliesi, A Basti, L Benucci, J Bernardini, T Boccali, L Borrello, F Bosi, F Calzolari, R Castaldi, C Cerri, A S Cucoanes, M D'Alfonso, R Dell'Orso, S Dutta, L Foà, S Gennai ${ }^{13}$, A Giammanco, A Giassi, D Kartashov, F Ligabue, S Linari, T Lomtadze, G A Lungu, B Mangano, G Martinelli, M Massa, A Messineo, A Moggi, F Palla, F Palmonari, G Petrucciani, F Raffaelli, A Rizzi, G Sanguinetti, G Segneri, D Sentenac, A T Serban, G Sguazzoni, A Slav, P Spagnolo, R Tenchini, G Tonelli, A Venturi, P G Verdini, M Vos

Università di Roma I e Sezione dell' INFN, Roma, ITALY

S Baccaro ${ }^{14}$, L Barone, A Bartoloni, F Cavallari, S Costantini, I Dafinei, D Del Re ${ }^{9}$, M Diemoz, C Gargiulo, E Longo, P Meridiani, G Organtini, S Rahatlou

Università di Torino e Sezione dell' INFN, Torino, ITALY

E Accomando, M Arneodo ${ }^{15}$, A Ballestrero, R Bellan, C Biino, S Bolognesi, N Cartiglia, G Cerminara, M Cordero, M Costa, G Dellacasa, N Demaria, E Maina, C Mariotti, S Maselli, P Mereu, E Migliore, V Monaco, M Nervo, M M Obertino, N Pastrone, G Petrillo, A Romero, M Ruspa ${ }^{15}$, R Sacchi, A Staiano, P P Trapani

Università di Trieste e Sezione dell' INFN, Trieste, ITALY

S Belforte, F Cossutti, G Della Ricca, A Penzo

Kyungpook National University, Daegu, KOREA

K Cho, S W Ham, D Han, D H Kim, G N Kim, J C Kim, W Y Kim, M W Lee, S K Oh, W H Park, S R Ro, D C Son, J S Suh

Chonnam National University, Kwangju, KOREA

J Y Kim

Konkuk University, Seoul, KOREA

S Y Jung, J T Rhee

Korea University, Seoul, KOREA

B S Hong, S J Hong, K S Lee, I Park, S K Park, K S Sim, E Won

Seoul National University, Seoul, KOREA

S B Kim

Universidad Iberoamericana, Mexico City, MEXICO

S Carrillo Moreno

Centro de Investigacion y de Estudios Avanzados del IPN, Mexico City, MEXICO

H Castilla Valdez, A Sanchez Hernandez

\footnotetext{
13 Also at Centro Studi Enrico Fermi, Roma, Italy.

14 Also at ENEA - Casaccia Research Center, S. Maria di Galeria, Italy.

15 Now at Università del Piemonte Orientale, Novara, Italy.
} 
Benemerita Universidad Autonoma de Puebla, Puebla, MEXICO

H A Salazar Ibarguen

Universidad Autonoma de San Luis Potosi, San Luis Potosi, MEXICO

A Morelos Pineda

University of Auckland, Auckland, NEW ZEALAND

R N C Gray, D Krofcheck

University of Canterbury, Christchurch, NEW ZEALAND

N Bernardino Rodrigues, P H Butler, J C Williams

National Centre for Physics, Quaid-I-Azam University, Islamabad, PAKISTAN

Z Aftab, M Ahmad, U Ahmad, I Ahmed, J Alam Jan, M I Asghar, S Asghar, M Hafeez, H R Hoorani, M Ibrahim, M Iftikhar, M S Khan, N Qaiser, I Rehman, T Solaija, S Toor

Institute of Nuclear Physics, Polish Academy of Sciences, Cracow, POLAND

J Blocki, A Cyz, E Gladysz-Dziadus, S Mikocki, J Turnau, Z Wlodarczyk ${ }^{16}$, P Zychowski

Institute of Experimental Physics, Warsaw, POLAND

K Bunkowski, H Czyrkowski, R Dabrowski, W Dominik, K Doroba, A Kalinowski, M Konecki, J Krolikowski, I M Kudla, M Pietrusinski, K Pozniak ${ }^{17}$, W Zabolotny ${ }^{17}$, P Zych

Soltan Institute for Nuclear Studies, Warsaw, POLAND

M Bluj, R Gokieli, L Goscilo, M Górski, K Nawrocki, P Traczyk, G Wrochna, P Zalewski

Laboratório de Instrumentaçoãe Física Experimental de Partículas, Lisboa, PORTUGAL

R Alemany-Fernandez, C Almeida, N Almeida, A Araujo Trindade, P Bordalo, P Da Silva Rodrigues, M Husejko, A Jain, M Kazana, P Musella, S Ramos, J Rasteiro Da Silva, P Q Ribeiro, M Santos, J Semiao, P Silva, I Teixeira, J P Teixeira, J Varela ${ }^{1}$

Joint Institute for Nuclear Research, Dubna, RUSSIA

S Afanasiev, K Babich, I Belotelov, V Elsha, Y Ershov, I Filozova, A Golunov, I Golutvin, N Gorbounov, I Gramenitski, V Kalagin, A Kamenev, V Karjavin, S Khabarov, V Khabarov, Y Kiryushin, V Konoplyanikov, V Korenkov, G Kozlov, A Kurenkov, A Lanev, V Lysiakov, A Malakhov, I Melnitchenko, V V Mitsyn, K Moisenz, P Moisenz, S Movchan, E Nikonov, D Oleynik, V Palichik, V Perelygin, A Petrosyan, E Rogalev, V Samsonov, M Savina, R Semenov, S Shmatov, S Shulha, V Smirnov, D Smolin, A Tcheremoukhine, O Teryaev, E Tikhonenko, S Vassiliev, A Vishnevskiy, A Volodko, N Zamiatin, A Zarubin, P Zarubin, E Zubarev

\section{Petersburg Nuclear Physics Institute, Gatchina (St Petersburg), RUSSIA}

N Bondar, V Golovtsov, A Golyash, Y Ivanov, V Kim, V Kozlov, V Lebedev, G Makarenkov, E Orishchin, A Shevel, V Sknar, I Smirnov, V Sulimov, V Tarakanov, L Uvarov, G Velichko, S Volkov, A Vorobyev

\section{Institute for Nuclear Research, Moscow, RUSSIA}

Yu Andreev, A Anisimov, S Gninenko, N Golubev, D Gorbunov, M Kirsanov, A Kovzelev, N Krasnikov, V Matveev, A Pashenkov, V E Postoev, A Sadovski, A Solovey, A Solovey, D Soloviev, L Stepanova, A Toropin

\footnotetext{
16 Also at Institute of Physics, Swietokrzyska Academy, Kielce, Poland.

${ }^{17}$ Also at Warsaw University of Technology, Institute of Electronic Systems, Warsaw, Poland.
} 
Institute for Theoretical and Experimental Physics, Moscow, RUSSIA

V Gavrilov, N Ilina, V Kaftanov ${ }^{1}$, I Kiselevich, V Kolosov, M Kossov ${ }^{1}$, A Krokhotin, S Kuleshov, A Oulianov, G Safronov, S Semenov, V Stolin, E Vlasov', V Zaytsev

P N Lebedev Physical Institute, Moscow, RUSSIA

A M Fomenko, N Konovalova, V Kozlov, A I Lebedev, N Lvova, S V Rusakov, A Terkulov

Moscow State University, Moscow, RUSSIA

E Boos, M Dubinin ${ }^{3}$, L Dudko, A Ershov, A Gribushin, V Ilyin, V Klyukhin ${ }^{1}$, O Kodolova, I Lokhtin, S Petrushanko, L Sarycheva, V Savrin, A Sherstnev, A Snigirev, K Teplov, I Vardanyan

State Research Center of Russian Federation - Institute for High Energy Physics, Protvino, RUSSIA

V Abramov, I Azhguirei, S Bitioukov, K Datsko, A Filine, P Goncharov, V Grishin, A Inyakin, V Kachanov, A Khmelnikov, D Konstantinov, A Korablev, V Krychkine, A Levine, I Lobov, V Petrov, V Pikalov, R Ryutin, S Slabospitsky, A Sourkov ${ }^{1}$, A Sytine, L Tourtchanovitch, S Troshin, N Tyurin, A Uzunian, A Volkov, S Zelepoukine ${ }^{18}$

Vinca Institute of Nuclear Sciences, Belgrade, SERBIA

P Adzic, D Krpic ${ }^{19}$, D Maletic, P Milenovic, J Puzovic ${ }^{19}$, N Smiljkovic ${ }^{1}$, M Zupan

Centro de Investigaciones Energeticas Medioambientales y Tecnologicas, Madrid, SPAIN

M Aguilar-Benitez, J Alberdi, J Alcaraz Maestre, M Aldaya Martin, P Arce ${ }^{1}$, J M Barcala, C Burgos Lazaro, J Caballero Bejar, E Calvo, M Cardenas Montes, M Cerrada, M Chamizo Llatas, N Colino, M Daniel, B De La Cruz, C Fernandez Bedoya, A Ferrando, M C Fouz, P Garcia-Abia, J M Hernandez, M I Josa, J M Luque, J Marin, G Merino, A Molinero, J J Navarrete, J C Oller, E Perez Calle, L Romero, J Salicio, C Villanueva Munoz, C Willmott, C Yuste

Universidad Autónoma de Madrid, Madrid, SPAIN

C Albajar, J F de Trocóniz, M Fernandez, I Jimenez, R F Teixeira

Universidad de Oviedo, Oviedo, SPAIN

J Cuevas, J M Lopez, H Naves Sordo, J M Vizan Garcia

Instituto de Física de Cantabria (IFCA), CSIC-Universidad de Cantabria, Santander, SPAIN

A Calderon, D Cano Fernandez, I Diaz Merino, L A Garcia Moral, G Gomezo, I Gonzalez Cabellero, J Gonzalez Sanchez, A Lopez Virto, J Marco, R Marco, C Martinez Rivero, P Martinez Ruiz del Arbol, F Matorras, A Patino Revuelta ${ }^{1}$, T Rodrigo, D Rodriguez Gonzalez, A Ruiz Jimeno, M Sobron Sanudo, I Vila, R Vilar Cortabitarte

CERN, European Organization for Nuclear Research, Geneva, SWITZERLAND

D Abbaneo, S M Abbas, L Agostino, I Ahmed, S Akhtar, N Amapane, B Araujo Meleiro, S Argiro ${ }^{20}$, S Ashby, P Aspell, E Auffray, M Axer, A Ball, N Bangert, D Barney, C Bernet, W Bialas, C Bloch, P Bloch, S Bonacini, M Bosteels, V Boyer, A Branson, A M Brett,

\footnotetext{
18 Also at Institute for Particle Physics, ETH Zurich, Zurich, Switzerland.

19 Also at Faculty of Physics of University of Belgrade, Belgrade, Serbia.

${ }^{20}$ Also at INFN-CNAF, Bologna, Italy.
} 
H Breuker, R Bruneliere, O Buchmuller, D Campi, T Camporesi, E Cano, E Carrone, A Cattai, R Chierici, T Christiansen, S Cittolin, E Corrin, M Corvo, S Cucciarelli, B Curé, A De Roeck, D Delikaris, M Della Negra, D D'Enterria, A Dierlamm, A Elliott-Peisert, M Eppard, H Foeth, R Folch, S Fratianni, W Funk, A Gaddi, M Gastal, J C Gayde, H Gerwig, K Gill, A S Giolo-Nicollerat, F Glege, R Gomez-Reino Garrido, R Goudard, J Gutleber, M Hansen, J Hartert, A Hervé, H F Hoffmann, A Honma, M Huhtinen, G Iles, V Innocente, W Jank, P Janot, K Kloukinas, C Lasseur, M Lebeau, P Lecoq, C Leonidopoulos, M Letheren, C Ljuslin, R Loos, G Magazzu, L Malgeri, M Mannelli, A Marchioro, F Meijers, E Meschi, R Moser, M Mulders, J Nash, R A Ofierzynski, A Oh, P Olbrechts, A Onnela, L Orsini, I Pal, G Papotti, R Paramatti, G Passardi, B Perea Solano, G Perinic, P Petagna, A Petrilli, A Pfeiffer, M Pimiä, R Pintus, H Postema, R Principe, J Puerta Pelayo, A Racz, J Rehn, S Reynaud, M Risoldi, P Rodrigues Simoes Moreira, G Rolandi, P Rosinsky, P Rumerio, H Sakulin, D Samyn, F P Schilling, C Schwick, C Schäfer, I Segoni, A Sharma, P Siegrist, N Sinanis, P Sphicas ${ }^{21}$, M Spiropulu, F Szoncsó, O Teller, D Treille, J Troska, E Tsesmelis, D Tsirigkas, A Tsirou, D Ungaro, F Vasey, M Vazquez Acosta, L Veillet, P Vichoudis, P Wertelaers, A Wijnant, M Wilhelmsson, I M Willers

\section{Paul Scherrer Institut, Villigen, SWITZERLAND}

W Bertl, K Deiters, W Erdmann, K Gabathuler, S Heising, R Horisberger, Q Ingram, H C Kaestli, D Kotlinski, S König, D Renker, T Rohe, M Spira

\section{Institute for Particle Physics, ETH Zurich, Zurich, SWITZERLAND}

B Betev, G Davatz, G Dissertori, M Dittmar, L Djambazov, J Ehlers, R Eichler, G Faber, K Freudenreich, J F Fuchs ${ }^{1}$, C Grab, A Holzner, P Ingenito, U Langenegger, P Lecomte, G Leshev, A Lister ${ }^{22}$, P D Luckey, W Lustermann, J D Maillefaud ${ }^{1}$, F Moortgat, A Nardulli, F Nessi-Tedaldi, L Pape, F Pauss, H Rykaczewski ${ }^{23}$, U Röser, D Schinzel, A Starodumov ${ }^{24}$, F Stöckli, H Suter, L Tauscher, P Trüb ${ }^{25}, \mathrm{H}$ P von Gunten, M Wensveen ${ }^{1}$

Universität Zürich, Zürich, SWITZERLAND

E Alagoz, C Amsler, V Chiochia, C Hoermann, K Prokofiev, C Regenfus, P Robmann, T Speer, S Steiner, L Wilke

National Central University, Chung-Li, TAIWAN

S Blyth, Y H Chang, E A Chen, A Go, C C Hung, C M Kuo, W Lin

\section{National Taiwan University (NTU), Taipei, TAIWAN}

P Chang, Y Chao, K F Chen, Z Gao ${ }^{1}$, Y Hsiung, Y J Lei, J Schümann, J G Shiu, K Ueno, Y Velikzhanin, P Yeh

\section{Cukurova University, Adana, TURKEY}

S Aydin, M N Bakirci, S Cerci, I Dumanoglu, S Erturk, S Esen, E Eskut, A Kayis Topaksu, P Kurt, H Ozkurt, A Polatöz, K Sogut, H Topakli, M Vergili, T Yetkin, G Önengüt

\footnotetext{
${ }^{21}$ Also at University of Athens, Athens, Greece.

22 Now at University of California, Davis, Davis, USA.

${ }^{23}$ Now at ESO, Munich-Garching, Germany.

24 Also at Institute for Theoretical and Experimental Physics, Moscow, Russia.

25 Also at Paul Scherrer Institut, Villigen, Switzerland.
} 
Middle East Technical University, Physics Department, Ankara, TURKEY

H Gamsizkan, C Ozkan, S Sekmen, M Serin-Zeyrek, R Sever, E Yazgan, M Zeyrek

Bogaziçi University, Department of Physics, Istanbul, TURKEY

A Cakir ${ }^{26}$, K Cankocak ${ }^{27}, \mathrm{M}$ Deliomeroglu, D Demir ${ }^{26}$, K Dindar, E Gülmez, E Isiksal $^{28}$, M Kaya $^{29}$, O Kaya, S Ozkorucuklu ${ }^{30}, \mathrm{~N} \mathrm{Sonmez}^{31}$

Institute of Single Crystals of National Academy of Science, Kharkov, UKRAINE

B Grinev, V Lyubynskiy, V Senchyshyn

National Scientific Center, Kharkov Institute of Physics and Technology, Kharkov, UKRAINE

L Levchuk, P Sorokin

University of Bristol, Bristol, UNITED KINGDOM

D S Bailey, T Barrass, J J Brooke, R Croft, D Cussans, D Evans, R Frazier, N Grant, M Hansen, G P Heath, H F Heath, B Huckvale, C Lynch, C K Mackay, S Metson, D M Newbold ${ }^{32}$, V J Smith, R J Tapper

Rutherford Appleton Laboratory, Didcot, UNITED KINGDOM

S A Baird, K W Bell, R M Brown, D J A Cockerill, J A Coughlan, P S Flower, V B Francis, M French, J Greenhalgh, R Halsall, J Hill, L Jones, B W Kennedy, L Lintern, A B Lodge, J Maddox, Q Morrissey, P Murray, M Pearson, S Quinton, J Salisbury, A Shah, C Shepherd-Themistocleous, B Smith, M Sproston, R Stephenson, S Taghavirad, I R Tomalin, J H Williams

Imperial College, University of London, London, UNITED KINGDOM

F Arteche ${ }^{1}$, R Bainbridge, G Barber, P Barrillon, R Beuselinck, F Blekman, D Britton, D Colling, G Daskalakis, G Dewhirst, S Dris ${ }^{1}$, C Foudas, J Fulcher, S Greder, G Hall, J Jones, J Leaver, B C MacEvoy, O Maroney, A Nikitenko ${ }^{24}$, A Papageorgiou, D M Raymond, M J Ryan, C Seez, P Sharp ${ }^{1}$, M Takahashi, C Timlin, T Virdee ${ }^{1}$, S Wakefield, M Wingham, A Zabi, Y Zhang, O Zorba

Brunel University, Uxbridge, UNITED KINGDOM

C Da Via, I Goitom, P R Hobson, P Kyberd, C Munro, J Nebrensky, I Reid, O Sharif, R Taylor, L Teodorescu, S J Watts, I Yaselli

Boston University, Boston, Massachusetts, USA

E Hazen, A H Heering, D Lazic, E Machado, D Osborne, J Rohlf, L Sulak, F Varela Rodriguez, S Wu

Brown University, Providence, Rhode Island, USA

D Cutts, R Hooper, G Landsberg, R Partridge, S Vanini ${ }^{33}$

\footnotetext{
26 Also at Izmir Institute of Technology (IYTE), Izmir, Turkey.

27 Also at Mugla University, Mugla, Turkey.

28 Also at Marmara University, Istanbul, Turkey.

${ }^{29}$ Also at Kafkas University, Kars, Turkey.

${ }^{30}$ Also at Suleyman Demirel University, Isparta, Turkey.

31 Also at Ege University, Izmir, Turkey.

32 Also at Rutherford Appleton Laboratory, Didcot, United Kingdom.

33 Also at Università di Padova e Sezione dell' INFN, Padova, Italy.
} 
University of California, Davis, Davis, California, USA

R Breedon, M Case, M Chertok, J Conway, P T Cox, R Erbacher, J Gunion, B Holbrook, W Ko, R Lander, D Pellett, J Smith, A Soha, M Tripathi, R Vogt

University of California, Los Angeles, Los Angeles, California, USA

V Andreev, K Arisaka, D Cline, R Cousins, S Erhan ${ }^{1}$, M Felcini ${ }^{1}$, J Hauser, M Ignatenko, B Lisowski, D Matlock, C Matthey, B Mohr, J Mumford, S Otwinowski, G Rakness, P Schlein, Y Shi, J Tucker, V Valuev, R Wallny, H G Wang, X Yang, Y Zheng

University of California, Riverside, Riverside, California, USA

R Clare, D Fortin, D Futyan ${ }^{1}$, J W Gary, M Giunta ${ }^{1}$, G Hanson, G Y Jeng, S C Kao, H Liu, G Pasztor ${ }^{34}$, A Satpathy, B C Shen, R Stringer, V Sytnik, R Wilken, D Zer-Zion

University of California, San Diego, La Jolla, California, USA

J G Branson, E Dusinberre, J Letts, T Martin, M Mojaver, H P Paar, H Pi, M Pieri, A Rana, V Sharma, A White, F Würthwein

University of California, Santa Barbara, Santa Barbara, California, USA

A Affolder, C Campagnari, C Hill, J Incandela, S Kyre, J Lamb, J Richman, D Stuart, D White

California Institute of Technology, Pasadena, California, USA

J Albert, A Bornheim, J Bunn, J Chen, G Denis, P Galvez, M Gataullin, I Legrand, V Litvine, Y Ma, D Nae, H B Newman, S Ravot, S Shevchenko, S Singh, C Steenberg, X Su, M Thomas, V Timciuc, F van Lingen, J Veverka, B R Voicu ${ }^{1}$, A Weinstein, R Wilkinson, X Yang, Y Yang, L Y Zhang, K Zhu, R Y Zhu

Carnegie Mellon University, Pittsburgh, Pennsylvania, USA

T Ferguson, M Paulini, J Russ, N Terentyev, H Vogel, I Vorobiev

University of Colorado at Boulder, Boulder, Colorado, USA

J P Cumalat, W T Ford, D Johnson, U Nauenberg, K Stenson, S R Wagner

Cornell University, Ithaca, NY, USA

J Alexander, D Cassel, K Ecklund, B Heltsley, C D Jones, V Kuznetsov, J R Patterson, A Ryd, J Thom, P Wittich

Fairfield University, Fairfield, Connecticut, USA

C P Beetz, G Cirino, V Podrasky, C Sanzeni, D Winn

Fermi National Accelerator Laboratory, Batavia, Illinois, USA

S Abdullin' ${ }^{24}$, M A Afaq1, M Albrow, J Amundson, G Apollinari, M Atac, W Badgett, J A Bakken, B Baldin, L A T Bauerdick, A Baumbaugh, U Baur, P C Bhat, F Borcherding, K Burkett, J N Butler, H Cheung, I Churin, S Cihangir, M Demarteau, D P Eartly, J E Elias, V D Elvira, D Evans, I Fisk, J Freeman, P Gartung, F J M Geurts, D A Glenzinski, E Gottschalk, G Graham, D Green, G M Guglielmo, Y Guo, O Gutsche, A Hahn, J Hanlon, S Hansen, R M Harris, T Hesselroth, S L Holm, B Holzman, S Iqbal, E James, M Johnson, U Joshi, B Klima, J Kowalkowski, T Kramer, S Kwan, E La Vallie, M Larwill, S Los, L Lueking, G Lukhanin, S Lusin ${ }^{1}$, K Maeshima, P McBride, S J Murray, V O’Dell, M Paterno, J Patrick, D Petravick, R Pordes, O Prokofyev, V Rasmislovich, N Ratnikova, A Ronzhin, V Sekhri, E Sexton-Kennedy, T Shaw, D Skow, R P Smith, W J Spalding, L Spiegel, M Stavrianakou, G Stiehr, I Suzuki, P Tan, W Tanenbaum, S Tkaczyk, S Veseli, R Vidal, H Wenzel, J Whitmore, W J Womersley, W M Wu, Y Wu, A Yagil, J Yarba, J C Yun

${ }^{34}$ Also at KFKI Research Institute for Particle and Nuclear Physics, Budapest, Hungary. 
University of Florida, Gainesville, Florida, USA

D Acosta, P Avery, V Barashko, P Bartalini, D Bourilkov, R Cavanaugh, A Drozdetskiy, R D Field, Y Fu, L Gray, D Holmes, B J Kim, S Klimenko, J Konigsberg, A Korytov, K Kotov, P Levchenko, A Madorsky, K Matchev, G Mitselmakher, Y Pakhotin, C Prescott, P Ramond, J L Rodriguez, M Schmitt, B Scurlock, H Stoeck, J Yelton

Florida International University, Miami, Florida, USA

W Boeglin, V Gaultney, L Kramer, S Linn, P Markowitz, G Martinez, B Raue, J Reinhold

Florida State University, Tallahassee, Florida, USA

A Askew, M Bertoldi, W G D Dharmaratna, Y Gershtein, S Hagopian, V Hagopian, M Jenkins, K F Johnson, H Prosper, H Wahl

Florida Institute of Technology, Melbourne, Florida, USA

M Baarmand, L Baksay ${ }^{35}$, S Guragain, M Hohlmann, H Mermerkaya, R Ralich, I Vodopiyanov

University of Illinois at Chicago (UIC), Chicago, Illinois, USA

M R Adams, R R Betts, C E Gerber, E Shabalina, C Smith, T Ten

The University of Iowa, Iowa City, Iowa, USA

U Akgun, A S Ayan, A Cooper, P Debbins, F Duru, M Fountain, N George, E McCliment, J P Merlo, A Mestvirishvili, M J Miller, C R Newsom, E Norbeck, Y Onel, I Schmidt, S Wang

Iowa State University, Ames, Iowa, USA

E W Anderson, O Atramentov, J M Hauptman, J Lamsa

Johns Hopkins University, Baltimore, Maryland, USA

B A Barnett, B Blumenfeld, C Y Chien, D W Kim, P Maksimovic, S Spangler, M Swartz

The University of Kansas, Lawrence, Kansas, USA

P Baringer, A Bean, D Coppage, O Grachov, E J Kim, M Murray

Kansas State University, Manhattan, Kansas, USA

D Bandurin, T Bolton, A Khanov ${ }^{24}$, Y Maravin, D Onoprienko, F Rizatdinova, R Sidwell, N Stanton, E Von Toerne

University of Maryland, College Park, Maryland, USA

D Baden, R Bard, S C Eno, T Grassi, N J Hadley, R G Kellogg, S Kunori, F Ratnikov, A Skuja

Massachusetts Institute of Technology, Cambridge, Massachusetts, USA

R Arcidiacono, M Ballintijn, G Bauer, P Harris, I Kravchenko, C Loizides, S Nahn, C Paus, S Pavlon, C Roland, G Roland, K Sumorok, S Vaurynovich, G Veres, B Wyslouch

University of Minnesota, Minneapolis, Minnesota, USA

D Bailleux, S Corum, P Cushman, A De Benedetti, A Dolgopolov, R Egeland, G Franzoni, W J Gilbert, J Grahl, J Haupt, Y Kubota, J Mans, N Pearson, R Rusack, A Singovsky

University of Mississippi, University, Mississippi, USA

L M Cremaldi, R Godang, R Kroeger, D A Sanders, D Summers

University of Nebraska-Lincoln, Lincoln, Nebraska, USA

K Bloom, D R Claes, A Dominguez, M Eads, C Lundstedt, S Malik, G R Snow, A Sobol

35 Also at University of Debrecen, Debrecen, Hungary. 
State University of New York at Buffalo, Buffalo, New York, USA

I Iashvili, A Kharchilava

Northeastern University, Boston, Massachusetts, USA

G Alverson, E Barberis, O Boeriu, G Eulisse, Y Musienko ${ }^{36}$, S Muzaffar, I Osborne, S Reucroft, J Swain, L Taylor, L Tuura, D Wood

Northwestern University, Evanston, Illinois, USA

B Gobbi, M Kubantsev, H Schellman, M Schmitt, E Spencer, M Velasco

University of Notre Dame, Notre Dame, Indiana, USA

B Baumbaugh, N M Cason, M Hildreth, D J Karmgard, N Marinelli ${ }^{21}$, R Ruchti, J Warchol, M Wayne

The Ohio State University, Columbus, Ohio, USA

B Bylsma, L S Durkin, J Gilmore, J Gu, D Herman, P Killewald, K Knobbe, T Y Ling

Princeton University, Princeton, New Jersey, USA

P Elmer, D Marlow, P Piroué, D Stickland, C Tully, T Wildish, S Wynhoff, Z Xie

Purdue University, West Lafayette, Indiana, USA

A Apresyan, K Arndt, K Banicz, V E Barnes, G Bolla, D Bortoletto, A Bujak, A F Garfinkel,

O Gonzalez Lopez, L Gutay, N Ippolito, Y Kozhevnikov ${ }^{1}$, A T Laasanen, C Liu, V Maroussov, P Merkel, D H Miller, J Miyamoto, N Neumeister, C Rott, A Roy, A Sedov, I Shipsey

Purdue University Calumet, Hammond, Indiana, USA

N Parashar

Rice University, Houston, Texas, USA

G Eppley, S J Lee, J Liu, M Matveev, T Nussbaum, B P Padley, J Roberts, A Tumanov, $\mathrm{P}$ Yepes

University of Rochester, Rochester, New York, USA

A Bodek, H Budd, Y S Chung, P De Barbaro' ${ }^{1}$, R Demina, R Eusebi, G Ginther, Y Gotra, A Hocker, U Husemann, S Korjenevski, W Sakumoto, P Slattery, P Tipton, M Zielinski

Rutgers, the State University of New Jersey, Piscataway, New Jersey, USA

E Bartz, J Doroshenko, E Halkiadakis, P F Jacques, M S Kalelkar, D Khits, A Lath, A Macpherson ${ }^{1}$, L Perera, R Plano, K Rose, S Schnetzer, S Somalwar, R Stone, G Thomson, T L Watts

Texas Tech University, Lubbock, Texas, USA

N Akchurin, K W Carrell, K Gumus, C Jeong, H Kim, V Papadimitriou, A Sill, M Spezziga, E Washington, R Wigmans, L Zhang

Vanderbilt University, Nashville, Tennessee, USA

T Bapty, D Engh, W Johns, T Keskinpala, E Luiggi Lopez, S Neema, S Nordstrom, S Pathak, P Sheldon, E W Vaandering, M Webster

${ }^{36}$ Also at Institute for Nuclear Research, Moscow, Russia. 


\section{University of Virginia, Charlottesville, Virginia, USA}

M W Arenton, S Conetti, B Cox, R Hirosky, R Imlay, A Ledovskoy, D Phillips II, H Powell, M Ronquest, D Smith

\section{University of Wisconsin, Madison, Wisconsin, USA}

Y W Baek, J N Bellinger, D Bradley, D Carlsmith, I Crotty ${ }^{1}$, S Dasu, F Feyzi, T Gorski, M Grothe ${ }^{37}$, W Hogg, M Jaworski, P Klabbers, A Lanaro, R Loveless, M Magrans de Abril, D Reeder, W H Smith, D Wenman

Yale University, New Haven, Connecticut, USA

G S Atoyan ${ }^{36}$, S Dhawan, V Issakov, H Neal, A Poblaguev, M E Zeller

Institute of Nuclear Physics of the Uzbekistan Academy of Sciences, Ulugbek, Tashkent, UZBEKISTAN

B S Yuldashev

${ }^{37}$ Also at Università di Torino e Sezione dell' INFN, Torino, Italy. 


\section{Contents}

Acknowledgments

$\begin{array}{ll}\text { The CMS Collaboration } & 997\end{array}$

$\begin{array}{lll}\text { Chapter 1. Introduction } & 1022\end{array}$

$\begin{array}{ll}\text { 1.1. The full analyses } & 1024\end{array}$

$\begin{array}{lr}\text { 1.2. The physics reach } & 1025\end{array}$

1.3. Tools used in the studies for the PTDR 1026

1.3.1. Detector simulation and reconstruction 1026

$\begin{array}{ll}\text { 1.3.2. Pile-up treatment } & 1026\end{array}$

$\begin{array}{ll}\text { 1.3.3. Systematic effects on measurements } & 1027\end{array}$

$\begin{array}{ll}\text { 1.3.4. Event generators } & 1027\end{array}$

$\begin{array}{ll}\text { 1.3.5. Parton distributions and higher order corrections } & 1028\end{array}$

$\begin{array}{lr}\text { 1.4. Outlook } & 1028\end{array}$

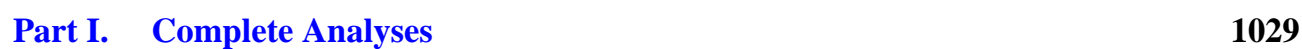

Chapter 2. Physics Studies with Photons and Electrons 1029

2.1. Benchmark Channel: $\mathbf{H} \rightarrow \gamma \gamma \quad 1029$

2.1.1. Higgs boson production and decay 1030

$\begin{array}{lr}\text { 2.1.2. Backgrounds } & 1030\end{array}$

2.1.3. Reconstruction, selection, and signal significance calculation 1032

2.1.4. Cut-based analysis 1034

2.1.5. Optimised analysis estimating $s / b$ for each event 1039

2.1.6. Measurement of the Higgs boson mass 1045

2.1.7. Summary 1046

2.2. Benchmark Channel: $\mathrm{H} \rightarrow \mathrm{ZZ}^{(*)} \rightarrow 4$ electrons $\quad 1046$

2.2.1. Datasets for signal and background processes 1047

$\begin{array}{ll}\text { 2.2.2. Data reduction } & 1049\end{array}$

2.2.3. Event selection and kinematic reconstruction 1051

$\begin{array}{ll}\text { 2.2.4. Systematics } & 1054\end{array}$

2.2.5. $\mathrm{H} \rightarrow 4 e$ Observability, mass and cross-section measurements 1059

$\begin{array}{lll}\text { Chapter 3. } & \text { Physics Studies with Muons } & 1063\end{array}$

3.1. Benchmark Channel: $\mathrm{H} \rightarrow \mathrm{ZZ}^{(*)} \rightarrow 4$ muons 1063

3.1.1. Physics processes and their simulation 1063

$\begin{array}{ll}\text { 3.1.2. Event selection } & 1064\end{array}$

3.1.3. Higgs boson search analysis 1066

3.1.4. Measurement of the Higgs boson properties at $\mathcal{L}=30 \mathrm{fb}^{-1} \quad 1073$

3.1.5. Conclusions 1076 
3.2. Benchmark Channel: $\mathrm{H} \rightarrow \mathrm{WW}^{(*)} \rightarrow 2$ muons

3.2.1. Introduction 1076

3.2.2. Physics processes 1077

$\begin{array}{ll}\text { 3.2.3. Event selection } & 1078\end{array}$

$\begin{array}{ll}3.2 .4 & \text { The trigger selection } \\ & 1078\end{array}$

3.2.5. Jet reconstruction and the jet veto 1080

3.2.6. Missing energy reconstruction and the MET cut 1081

3.2.7. The selection results 1082

3.2.8. Background estimation and systematics 1084

3.2.9. tì background normalisation $\quad 1085$

$\begin{array}{ll}\text { 3.2.10. WW background normalisation } & 1087\end{array}$

3.2.11. Other backgrounds normalisation $\quad 1089$

3.2.12. Detector misalignment systematics 1089

$\begin{array}{lr}\text { 3.2.13. Signal significance } & 1090\end{array}$

$\begin{array}{lr}\text { 3.2.14. Conclusions } & 1090\end{array}$

3.3. Benchmark Channel: $Z^{\prime} \rightarrow \mu \mu \quad 1091$

$\begin{array}{ll}\text { 3.3.1. Introduction } & 1091\end{array}$

$\begin{array}{ll}\text { 3.3.2. Signal and background processes } & 1091\end{array}$

$\begin{array}{ll}\text { 3.3.3. Event selection } & 1093\end{array}$

$\begin{array}{ll}\text { 3.3.4. Signal observability } & 1094\end{array}$

3.3.5. Distinguishing among $Z^{\prime}$ models 1100

3.3.6. Discriminating between different spin hypotheses 1102

$\begin{array}{lll}\text { Chapter 4. Physics Studies with Jets and } E_{\mathrm{T}}^{\text {miss }} & 1105\end{array}$

4.1. Benchmark Channel: new physics from dijets 1105

4.1.1. Dijet analysis 1105

4.1.2. Rates and efficiencies from jet triggers $\quad 1105$

4.1.3. Dijet mass distribution from QCD 1105

4.1.4. Searches using dijet mass 1106

4.1.5. Searches using dijet mass and angle 1108

$\begin{array}{ll}\text { 4.1.6. Systematic uncertainties } & 1108\end{array}$

4.2. Benchmark Channel: low mass supersymmetry 1110

4.2.1. Introduction $\quad 1110$

4.2.2. Jets and missing transverse energy at CMS 1111

4.2.3. Clean-up requirements 1111

4.2.4. Analysis path 1112

4.2.5. Missing transverse energy in QCD production 1112

$\begin{array}{ll}\text { 4.2.6. Indirect Lepton Veto } & 1114\end{array}$

4.2.7. The standard Z boson "candle" calibration 1115

$\begin{array}{ll}\text { 4.2.8. Analysis results } & 1117\end{array}$

4.2.9. Systematic uncertainties 1118

$\begin{array}{lr}\text { 4.2.10. Discussion } & 1120\end{array}$ 
5.1. Benchmark Channels: study of the decay $B_{s} \rightarrow J / \psi \phi$

5.1.1. Introduction

5.1.2. Event generation

5.1.3. Trigger selection

5.1.4. Offline selection and reconstruction $\quad 1125$

$\begin{array}{ll}\text { 5.1.5. The maximum likelihood analysis } & 1127\end{array}$

5.1.6. Result 1130

5.1.7. Systematics and detector effects 1132

5.1.8. Conclusion 1134

5.2. Associated production of MSSM heavy neutral Higgs bosons $\bar{b} \bar{b} H(A)$ with $\mathbf{H}(\mathbf{A}) \rightarrow \tau \tau$

5.2.1. Introduction

5.2.2. Event generation 1135

5.2.3. Level-1 and High Level trigger selections 1135

5.2.4. Off-line event selection 1136

5.2.5. Method of the Higgs boson mass reconstruction 1136

5.2.6. $\mathrm{H} \rightarrow \tau \tau \rightarrow 2 \mathrm{jet}$ analysis $\quad 1137$

5.2.7. $\mathrm{H} \rightarrow \tau \tau \rightarrow \mu+$ jet analysis $\quad 1142$

5.2.8. $\mathrm{H} \rightarrow \tau \tau \rightarrow e+$ jet analysis $\quad 1147$

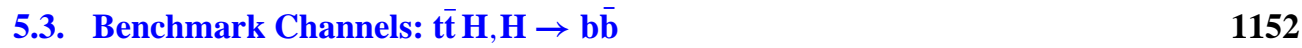

5.3.1. Introduction 1152

5.3.2. Event generation and simulation $\quad 1154$

5.3.3. Level-1 and high level trigger selections $\quad 1155$

5.3.4. Reconstruction 1156

$\begin{array}{ll}\text { 5.3.5. Event selection } & 1159\end{array}$

5.3.6. Discussion of systematic uncertainties 1164

5.3.7. Combined significance 1166

$\begin{array}{lll}\text { Chapter 6. } & \text { Physics Studies with Heavy Ions } & 1168\end{array}$

6.1. Benchmark Channel: $\mathrm{PbPb} \rightarrow \mathrm{Q} \overline{\mathbf{Q}}+\mathrm{X} \rightarrow \boldsymbol{\mu}^{+} \boldsymbol{\mu}^{-}+\mathrm{X} \quad 1168$

6.1.1. Simulation of physics and background processes 1168

$\begin{array}{ll}\text { 6.1.2. Reconstruction and analysis } & 1169\end{array}$

6.1.3. Results 1171

$\begin{array}{ll}\text { 6.1.4. Conclusions } & 1172\end{array}$

Part II. CMS Physics Reach $\quad 1174$

$\begin{array}{lll}\text { Chapter 7. Physics of Strong Interactions } & 1174\end{array}$

7.1. QCD and jet physics $\quad 1174$

$\begin{array}{ll}\text { 7.1.1. Introduction } & 1174\end{array}$

$\begin{array}{ll}\text { 7.1.2. Jet algorithms } & 1174\end{array}$

7.1.3. Trigger scheme, event selection and phase space 1176

$\begin{array}{ll}\text { 7.1.4. Input data } & 1176\end{array}$ 
7.1.5. Jet energy calibration 1177

7.1.6. NLO calculation 1177

7.1.7. Experimental and theoretical uncertainties 1177

7.1.8. Summary and outlook 1177

$\begin{array}{ll}\text { 7.2. } & \text { Underlying event studies }\end{array}$

7.2.1. Definition of the physics process and status of the art 1178

$\begin{array}{ll}\text { 7.2.2. Underlying event observables discussed for charged jet events } & 1179\end{array}$

$\begin{array}{ll}\text { 7.2.3. Feasibility studies } & 1181\end{array}$

7.2.4. Conclusions 1183

7.3. Physics of b-quarks and hadrons 1183

7.3.1. Inclusive b-quark production 1183

$\begin{array}{ll}\text { 7.3.2. Study of } B_{c} \text { hadrons } & 1189\end{array}$

$\begin{array}{ll}\text { 7.4. Diffraction and forward physics } & 1193\end{array}$

$\begin{array}{ll}\text { 7.4.1. Introduction } & 1193\end{array}$

7.4.2. The interest of diffractive interactions 1193

7.4.3. A survey of the accessible diffractive/forward processes 1194

7.5. Physics with heavy ions 1199

7.5.1. High-density QCD: heavy-ion physics 1199

7.5.2. Hard probes of QCD matter at LHC 1200

7.5.3. Gluon saturation and QGP colour screening via Quarkonia 1201

$\begin{array}{lll}\text { Chapter 8. Physics of Top Quarks } & 1202\end{array}$

8.1. Selection of $\mathrm{t} \overline{\mathrm{t}}$ events and measurement of the cross sections 1202

$\begin{array}{ll}\text { 8.1.1. Introduction } & 1202\end{array}$

8.1.2. Dileptonic channel 1202

8.1.3. Semi-leptonic channel 1206

8.1.4. Fully hadronic channel 1208

8.2. Measurement of the top quark mass 1212

$\begin{array}{ll}\text { 8.2.1. Dileptonic events } & 1212\end{array}$

8.2.2. Semi-leptonic events 1212

8.2.3. Fully hadronic events 1215

8.2.4. Top quark mass from $J / \psi$ final states 1218

8.2.5. Summary of top mass determinations 1222

8.3. Spin correlation in top-quark pair production 1223

8.3.1. Introduction 1223

8.3.2. Simulation of $\mathrm{t} \overline{\mathrm{t}}$ with spin correlation 1223

8.3.3. Online and offline event selection 1224

8.3.4. Estimation of correlation coefficient 1225

$\begin{array}{ll}\text { 8.4. Single top quark production } & 1227\end{array}$

$\begin{array}{ll}\text { 8.4.1. Introduction } & 1227\end{array}$

$\begin{array}{ll}\text { 8.4.2. Selection and cross section: t-channel } & 1229\end{array}$

8.4.3. Selection and cross section: tW-channel 1231 
8.4.4. Selection and cross section: s-channel 1234

$\begin{array}{ll}\text { 8.4.5. Conclusion } & 1237\end{array}$

8.5. Search for flavour changing neutral currents in top decays 1237

$\begin{array}{ll}\text { 8.5.1. Introduction } & 1237\end{array}$

$\begin{array}{ll}\text { 8.5.2. Signal and background generation } & 1238\end{array}$

$\begin{array}{ll}\text { 8.5.3. Selection strategies } & 1238\end{array}$

$\begin{array}{lr}\text { 8.5.4. Sensitivity estimation } & 1239\end{array}$

$\begin{array}{lll}\text { Chapter 9. } & \text { Electroweak Physics } & 1241\end{array}$

9.1. Production of $\mathrm{W}$ and $\mathrm{Z}$ bosons $\quad 1241$

$\begin{array}{ll}\text { 9.1.1. Introduction } & 1241\end{array}$

9.1.2. W/Z into electrons $\quad 1241$

$\begin{array}{ll}\text { 9.1.3. W/Z into muons } & 1244\end{array}$

9.1.4. Parton distribution functions and parton luminosities 1246

9.2. Muon pairs from the Drell-Yan process $\quad 1248$

$\begin{array}{ll}\text { 9.2.1. Introduction } & 1248\end{array}$

9.2.2. Cross section measurements $\quad 1249$

9.2.3. Prospects on the measurement of the forward-backward asymmetry $\quad 1251$

9.3. Determination of the W mass 1252

$\begin{array}{lr}\text { 9.3.1. Introduction } & 1252\end{array}$

9.3.2. Event selections 1253

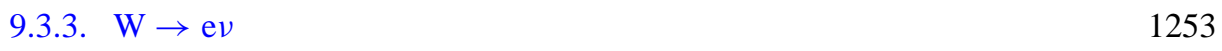

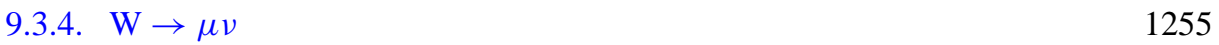

9.3.5. Expected precision and systematic uncertainties 1255

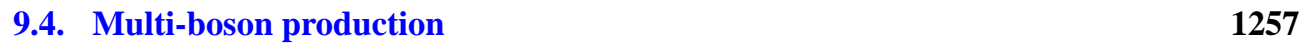

$\begin{array}{lr}\text { 9.4.1. Introduction } & 1257\end{array}$

$\begin{array}{ll}\text { 9.4.2. Signal definition and modelling } & 1258\end{array}$

$\begin{array}{lr}\text { 9.4.3. Background processes } & 1258\end{array}$

$\begin{array}{lr}\text { 9.4.4. } \mathrm{W}^{ \pm} Z^{0} \text { selection } & 1259\end{array}$

$\begin{array}{lr}\text { 9.4.5. } Z^{0} Z^{0} \text { selection } & 1259\end{array}$

9.4.6. Systematic uncertainties $\quad 1260$

$\begin{array}{lr}\text { 9.4.7. Results } & 1261\end{array}$

Chapter 10. Standard Model Higgs Bosons 1262

$\begin{array}{lr}\text { 10.1. Introduction } & 1262\end{array}$

10.2. Higgs boson channels $\quad 1266$

10.2.1. Inclusive Higgs boson production with $\mathrm{H} \rightarrow \mathrm{ZZ}^{(*)} \rightarrow \mathrm{e}+\mathrm{e}^{-} \mu^{+} \mu^{-} \quad 1266$

10.2.2. Inclusive Higgs boson production with $\mathrm{H} \rightarrow \mathrm{WW}^{*} \rightarrow 2 \ell 2 v \quad 1274$

10.2.3. The vector boson fusion production with $\mathrm{H} \rightarrow \tau \tau \rightarrow \ell+\tau$ jet $+E_{\mathrm{T}}^{\text {miss }} \quad 1279$

10.2.4. Searching for standard model Higgs via vector boson fusion in $\mathrm{H} \rightarrow \mathrm{W}^{+} \mathrm{W}^{-} \rightarrow \ell^{ \pm} v j j$ with $\mathrm{m}_{\mathrm{H}}$ from 120 to $250 \mathrm{GeV} / \mathrm{c}^{2} \quad 1283$

10.2.5. Vector boson fusion production with $\mathrm{H} \rightarrow \gamma \gamma$

10.2.6. Associated WH production with $\mathrm{H} \rightarrow \mathrm{WW}^{(*)} \rightarrow 2 \ell 2 v \quad 1291$ 
10.3.1. Accuracy of the Higgs boson mass measurement

10.3.2. Discovery reach for the Standard Model Higgs boson

10.3.3. Study of CP properties of the Higgs boson using angle correlation in the $\Phi \rightarrow \mathrm{ZZ} \rightarrow \mathrm{e}^{+} \mathrm{e}^{-} \mu^{+} \mu^{-}$process

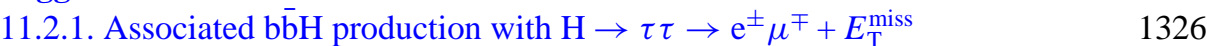

11.2.2. Associated $\mathrm{b} \overline{\mathrm{b}} \mathrm{H}$ production with $\mathrm{H} \rightarrow \mu^{+} \mu^{-}$

11.2.3. Associated $\mathrm{b} \overline{\mathrm{b}} \mathrm{H}$ production with $\mathrm{H} \rightarrow \mathrm{b} \overline{\mathrm{b}}$

11.2.4. Charged Higgs boson of $\mathrm{M}_{\mathrm{H}}<m_{\mathrm{t}}$ in $\mathrm{t} \overline{\mathrm{t}} \rightarrow \mathrm{H}^{ \pm} \mathrm{W}^{\mp} \mathrm{b} \overline{\mathrm{b}}$ production with $\mathrm{H}^{ \pm} \rightarrow \tau^{ \pm} \nu, \tau \rightarrow \nu+$ hadrons and $\mathrm{W}^{\mp} \rightarrow \ell^{\mp} \nu$

11.2.5. Charged Higgs boson of $\mathrm{M}_{\mathrm{H}}>m_{\mathrm{t}}$ in $\mathrm{gg} \rightarrow \mathrm{tbH}^{ \pm}$production with $\mathrm{H}^{ \pm} \rightarrow \tau^{ \pm} v, \tau \rightarrow$ hadrons $v$ and $\mathrm{W}^{\mp} \rightarrow j j$

11.2.6. Charged Higgs boson of $\mathrm{M}_{\mathrm{H}}>m_{\mathrm{t}}$ in $\mathrm{gg} \rightarrow \mathrm{tbH}^{ \pm}$production with $\mathrm{H}^{ \pm} \rightarrow$ tb

11.2.7. Search for the $\mathrm{A} \rightarrow \mathrm{Zh}$ decay with $\mathrm{Z} \rightarrow \ell^{+} \ell^{-}, \mathrm{h} \rightarrow \mathrm{b} \overline{\mathrm{b}}$

11.2.8. Search for $\mathrm{A}^{0} / \mathrm{H}^{0} \rightarrow \chi_{2}^{0} \chi_{2}^{0} \rightarrow 4 \ell+E_{\mathrm{T}}^{\text {miss }}$ channel in mSUGRA

11.3. Discovery reach and measurement of MSSM parameters

11.3.1. Benchmark scenarios for MSSM Higgs boson searches

11.3.2. Discovery reach in the $\mathrm{M}_{\mathrm{A}}-\tan \beta$ plane

\subsection{Introduction}

13.2.2. mSUGRA parameters and spectrum 
13.4. Hemisphere algorithm for separation of decay chains 1390

$\begin{array}{ll}\text { 13.4.1. Basic idea and goal } & 1390\end{array}$

$\begin{array}{ll}\text { 13.4.2. Seeding methods } & 1391\end{array}$

$\begin{array}{ll}\text { 13.4.3. Association methods } & 1391\end{array}$

$\begin{array}{ll}\text { 13.4.4. Results } & 1391\end{array}$

13.5. Inclusive analysis with missing transverse energy and jets 1393

13.5.1. Analysis path and results 1393

13.6. Inclusive muons with jets and missing transverse energy 1394

$\begin{array}{ll}\text { 13.6.1. Signal selection and backgrounds considered } & 1395\end{array}$

13.6.2. Results for $10 \mathrm{fb}^{-1}$ using full detector simulation and reconstruction $\quad 1396$

13.6.3. CMS Reach using inclusive muons with jets and missing energy 1397

13.7. Inclusive analyses with same sign dimuons $\quad 1398$

$\begin{array}{ll}\text { 13.7.1. Signal selection and backgrounds } & 1398\end{array}$

13.7.2. Results for full detector simulated mSUGRA samples 1399

$\begin{array}{lr}\text { 13.7.3. CMS inclusive reach } & 1399\end{array}$

13.8. Inclusive analyses with opposite sign dileptons $\quad \mathbf{1 4 0 0}$

$\begin{array}{lr}\text { 13.8.1. Signal selection and backgrounds } & 1400\end{array}$

$\begin{array}{ll}\text { 13.8.2. Results for point LM1 } & 1401\end{array}$

$\begin{array}{ll}\text { 13.8.3. CMS inclusive reach } & 1403\end{array}$

13.9. Inclusive analyses with ditaus $\quad \mathbf{1 4 0 4}$

$\begin{array}{ll}\text { 13.9.1. Event selection and background studies } & 1404\end{array}$

13.9.2. Discovery potential of mSUGRA with ditaus final states 1405

13.10. Inclusive analyses with Higgs $\quad 1406$

$\begin{array}{ll}\text { 13.10.1. Signal selection and backgrounds } & 1407\end{array}$

$\begin{array}{ll}\text { 13.10.2. Results at LM5 and systematics } & 1408\end{array}$

$\begin{array}{ll}\text { 13.10.3. CMS reach for inclusive Higgs production } & 1409\end{array}$

13.11. Inclusive SUSY search with $Z^{0} \quad 1410$

$\begin{array}{ll}\text { 13.11.1. Topology of the signal } & 1410\end{array}$

$\begin{array}{lr}\text { 13.11.2. Event selection } & 1410\end{array}$

13.11.3. Results and systematic uncertainties $\quad 1412$

13.11.4. CMS reach for inclusive $Z^{0}$ search $\quad 1412$

13.12. Inclusive analyses with top $\quad 1413$

13.12.1. Top quark and lepton reconstruction and identification 1413

$\begin{array}{ll}\text { 13.12.2. Signal selection and backgrounds } & 1414\end{array}$

$\begin{array}{ll}\text { 13.12.3. Results at point LM1 } & 1415\end{array}$

13.12.4. CMS reach for inclusive top search 1416

13.13. Mass determination in final states with ditaus $\quad 1416$

13.13.1. Extraction of mSUGRA mass spectra from the measurement of the end points of invariant mass distributions 
13.14. Direct $\chi_{2}^{0} \chi_{1}^{ \pm}$production in tri-leptons

13.14.2. Backgrounds and trigger path

13.14.3. Analysis path

13.14.4. Results at LM9 and systematics

13.14.5. CMS reach for the tri-lepton final state

13.15.1. Simulation details

13.15.2. Sleptons production and decays

13.15.3. Signature and backgrounds

13.15.4. Results

13.16. Lepton flavour violation in neutralino decay $\quad \mathbf{1 4 2 4}$

13.16.1. Signal selection and backgrounds

13.16.2. Results at CMS test points and reach

13.17. Summary of the reach with inclusive analyses $\quad 1427$

$\begin{array}{ll}\text { 13.17.1. Summary of the mSUGRA studies } & 1427\end{array}$

13.18. Look beyond mSUGRA 1429

13.18.1. Non-universal Higgs masses $\quad 1429$

Chapter 14. Extra Dimensions and New Vector Boson High Mass States 1435

14.1. Introduction 1435

14.1.1. Models with heavy vector bosons 1436

14.1.2. Arkani-Hamed-Dimopoulos-Dvali (ADD) models 1436

$\begin{array}{ll}\text { 14.1.3. Virtual graviton exchange } & 1439\end{array}$

14.1.4. Inverse TeV sized extra dimensions $\quad 1440$

14.1.5. Randall-Sundrum (RS) models 1441

14.2. High mass dielectron final states $\quad 1442$

$\begin{array}{ll}\text { 14.2.1. Event selection and correction } & 1443\end{array}$

$\begin{array}{ll}\text { 14.2.2. Mass peak distributions } & 1444\end{array}$

$\begin{array}{ll}\text { 14.2.3. Discovery potential of CMS } & 1444\end{array}$

$\begin{array}{ll}\text { 14.2.4. Systematic uncertainties } & 1447\end{array}$

$\begin{array}{ll}\text { 14.2.5. Identification of new particles } & 1447\end{array}$

14.3. High mass dimuon final states $\quad 1448$

14.3.1. The Randall-Sundrum model in the dimuon channel 1449

14.3.2. The ADD model in the dimuon channel 1451

14.4. High energy single lepton final states 1452

$\begin{array}{lr}\text { 14.4.1. Introduction } & 1452\end{array}$

14.4.2. Data samples $\quad 1453$

14.4.3. Event selection and analysis $\quad 1453$

14.4.4. Discovery and exclusion potential 1453

14.4.5. Systematic uncertainties $\quad 1454$

14.4.6. Summary 1455 
14.5.1. Dijet resonances and contact interactions 1455

14.5.2. Dijet resonance search 1456

14.6. High mass diphoton final states

14.6.1. Introduction $\quad 1459$

14.6.2. Event generation and kinematics pre-selection $\quad 1459$

14.6.3. Offline selection and analysis $\quad 1459$

$\begin{array}{ll}\text { 14.6.4. K-factors } & 1459\end{array}$

14.6.5. Results 1460

14.6.6. Systematic uncertainties for $30 \mathrm{fb}^{-1} \quad 1462$

14.7. Single $\gamma$ final state with $E_{\mathrm{T}}^{\text {miss }}$ from extra dimensions $\quad 1462$

14.7.1. Topology of single-photon final states $\quad 1462$

14.7.2. Backgrounds from the Standard Model 1463

14.7.3. Event selection 1464

14.7.4. Systematic uncertainties and discovery potential 1464

14.8. Black holes $\quad 1465$

14.8.1. Introduction to higher-dimensional black holes $\quad 1465$

14.8.2. Analysis selection path and results 1466

$\begin{array}{ll}\text { 14.9. Discussion } & 1467\end{array}$

$\begin{array}{ll}\text { Chapter 15. Alternative BSM Signatures } & 1469\end{array}$

15.1. Technicolour $\quad 1469$

15.1.1. The $\rho_{\mathrm{TC}} \rightarrow \mathrm{W}+\mathrm{Z}$ channel $\quad 1469$

15.2. Search for contact interactions with dimuons $\quad 1472$

$\begin{array}{ll}\text { 15.2.1. Analysis } & 1472\end{array}$

15.3. Search for contact interactions with dijets 1476

15.4. Heavy Majorana neutrinos and right-handed bosons $\quad 1477$

$\begin{array}{ll}\text { 15.4.1. Introduction } & 1477\end{array}$

15.4.2. Heavy Majorana neutrino production and decay 1478

$\begin{array}{ll}\text { 15.4.3. Analysis } & 1478\end{array}$

15.4.4. Results 1479

$\begin{array}{lr}\text { 15.5. Little Higgs models } & 1479\end{array}$

$\begin{array}{ll}\text { 15.5.1. Introduction } & 1479\end{array}$

$\begin{array}{ll}\text { 15.5.2. Analysis } & 1479\end{array}$

$\begin{array}{ll}\text { 15.6. Same sign top } & 1481\end{array}$

Appendix A. 95\% CL limits and $5 \sigma$ discoveries 1485

$\begin{array}{ll}\text { A.1. Estimators of significance } & 1485\end{array}$

A.2. On the true significance of a local excess of events 1487 
B.1. Theoretical uncertainties

B.1.1. Hard process description and parametric uncertainties 1490

B.1.2. Hard process scale 1491

$\begin{array}{ll}\text { B.1.3. PDF description } & 1492\end{array}$

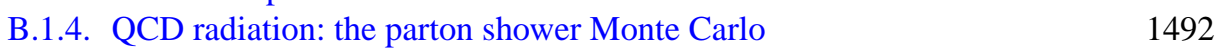

$\begin{array}{lr}\text { B.1.5. Fragmentation } & 1493\end{array}$

B.1.6. Minimum bias and underlying event 1495

B.1.7. Pile-up and LHC cross sections 1496

$\begin{array}{ll}\text { B.1.8. Decays } & 1497\end{array}$

B.1.9. LHAPDF and PDF uncertainties 1498

B.2. Experimental uncertainties $\quad 1500$

$\begin{array}{lr}\text { B.2.1. Luminosity uncertainty } & 1500\end{array}$

$\begin{array}{lr}\text { B.2.2. Track and vertex reconstruction uncertainties } & 1500\end{array}$

$\begin{array}{ll}\text { B.2.3. Muon reconstruction uncertainties } & 1500\end{array}$

B.2.4. Electromagnetic calibration and energy scale uncertainties 1501

$\begin{array}{ll}\text { B.2.5. Jet and missing transverse energy uncertainties } & 1501\end{array}$

$\begin{array}{lr}\text { B.2.6. Heavy-flavour tagging uncertainties } & 1503\end{array}$

Appendix C. Monte Carlo Models and Generators 1505

$\begin{array}{lr}\text { C.1. Introduction } & 1505\end{array}$

C.2. General scheme of generator usage in CMS 1506

$\begin{array}{lr}\text { C.3. CMkin } & 1507\end{array}$

$\begin{array}{lr}\text { C.4. Full event simulation generators } & \mathbf{1 5 0 8}\end{array}$

$\begin{array}{lr}\text { C.4.1. PYTHIA } & 1508\end{array}$

$\begin{array}{lr}\text { C.4.2. HERWIG } & 1509\end{array}$

$\begin{array}{lr}\text { C.4.3. ISAJET } & 1509\end{array}$

$\begin{array}{lr}\text { C.4.4. HIJING } & 1510\end{array}$

$\begin{array}{lr}\text { C.5. Tree level matrix element generators } & \mathbf{1 5 1 0}\end{array}$

$\begin{array}{lr}\text { C.5.1. ALPGEN } & 1510\end{array}$

C.5.2. COMPHEP 1510

C.5.3. MadGraph and MadeVent 1511

$\begin{array}{ll}\text { C.5.4. TOPREX } & 1511\end{array}$

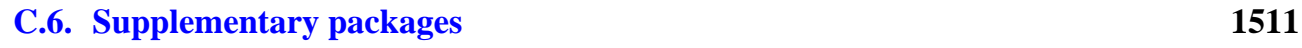

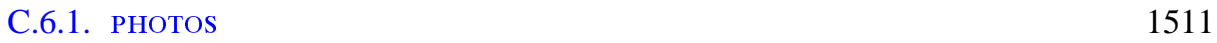

$\begin{array}{lr}\text { C.6.2. TAUOLA } & 1511\end{array}$

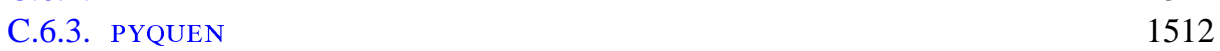

C.6.4. HYDJET 1512

$\begin{array}{ll}\text { C.7. K-factors for dilepton production } & 1512\end{array}$

Appendix D. GARCON: Genetic Algorithm for Rectangular Cuts OptimizatioN 1516 
E.2. Description of trigger tools

E.3. Triggering with forward detectors

E.3.2. Level-1 trigger rates for forward detectors trigger stream

E.3.3. Level-1 signal efficiencies

E.3.4. Effect of pile-up, beam-halo and beam-gas backgrounds

E.4.2. Evolution of DAQ-TDR triggers

E.4.3. New triggers

E.5.2. Level-1 trigger object corrections 


\section{Chapter 1. Introduction}

The Large Hadron Collider (LHC) [1], at the CERN Laboratory, the European Laboratory for Particle Physics, outside Geneva, Switzerland, will be completed in 2007. The LHC will be a unique tool for fundamental physics research and will be the highest energy accelerator in the world for many years following its completion. The LHC will provide two proton beams, circulating in opposite directions, at an energy of $7 \mathrm{TeV}$ each (centre-of-mass $\sqrt{s}=14 \mathrm{TeV}$ ). The CMS experiment [2,3] is a general purpose detector at the LHC to explore physics at an unprecedented physics energy scale, namely that at the TeV scale [4-6]. It is expected that the data produced at the LHC will elucidate the electroweak symmetry breaking mechanism (EWSB) and provide evidence of physics beyond the Standard Model. CMS will also be an instrument to perform precision measurements, e.g., of parameters of the Standard Model, mainly as a result of the very high event rates, as demonstrated for a few processes in Table 1.1 for a luminosity of $\mathcal{L}=2 \times 10^{33} \mathrm{~cm}^{-2} \mathrm{~s}^{-1}$. The $\mathrm{LHC}$ will be a $\mathrm{Z}$ factory, a $\mathrm{W}$ factory, a b quark factory, a top quark factory and even a Higgs or SUSY particle factory if these new particles have TeV scale masses.

The Physics Technical Design Report (PTDR) reports on detailed studies that have been performed with the CMS detector software and analysis tools. The CMS detector and its performance are described in detail in Volume 1 of the PTDR [7], while in the present Volume (Volume 2) the physics reach with the CMS detector is explored.

The CMS detector, shown in Fig. 1.1, measures roughly 22 metres in length, 15 metres in diameter, and 12,500 metric tons in weight. Its central feature is a huge, high field (4 tesla) solenoid, 13 metres in length, and 6 metres in diameter. Its "compact" design is large enough to contain the electromagnetic and hadron calorimetry surrounding a tracking system, and allows a superb muon detection system. All subsystems of CMS are bound by means of the data acquisition and trigger system.

In the CMS coordinate system the origin coincides with the nominal collision point at the geometrical center of the detector. The $z$ direction is given by the beam axis. The rest frame of the hard collision is generally boosted relative to the lab frame along the beam direction, $\theta$ is the polar angle with respect to the $z$ axis and $\phi$ the azimuthal angle with respect to the LHC plane. The detector solid angle segmentation is designed to be invariant under boosts along the $z$ direction. The pseudorapidity $\eta$, is related to the polar angle $\theta$ and defined as $\eta \equiv-\ln (\tan (\theta / 2))$. The transverse momentum component $z$-axis is given by $p_{\mathrm{T}}=p \sin \theta$ and similarly $E_{\mathrm{T}}=E \sin \theta$ is the transverse energy of a physics object.

The experiment comprises a tracker, a central calorimeter barrel part for $|\eta| \leqslant 1.5$, and endcaps on both sides, and muon detectors. The tracking system is made of several layers of silicon pixel and silicon strip detectors and covers the region $|\eta|<2.5$. The electromagnetic calorimeter consists of lead tungstate $\left(\mathrm{PbWO}_{4}\right)$ crystals covering $|\eta|<3$ (with trigger coverage $|\eta|<2.6)$. Its resolution at the initial luminosity $\left(\mathcal{L}=2 \times 10^{33} \mathrm{~cm}^{-2} \mathrm{~s}^{-1}\right)$ is $\Delta E / E=3 \% / \sqrt{E} \oplus 0.5 \%$. The surrounding hadronic calorimeter uses brass/scintillator tiles in the barrel and endcaps. Its resolution for jets, when combined with the electromagnetic calorimeter, is $\Delta E / E=100 \% / \sqrt{E} \oplus 5 \%$. The region $3<|\eta|<5$ is covered by forward calorimeters with a resolution of $\Delta E / E=180 \% / \sqrt{E} \oplus 10 \%$. Muons are measured in gas chambers in the iron return yoke. The muon momentum measurement using the muon chambers and the central tracker covers the range $|\eta|<2.4$ with a resolution of $\Delta p_{\mathrm{T}} / p_{\mathrm{T}}=5 \%$ at $p_{\mathrm{T}}=1 \mathrm{TeV}$ and $\Delta p_{\mathrm{T}} / p_{\mathrm{T}}=1 \%$ at $p_{\mathrm{T}}=100 \mathrm{GeV}$. The muon trigger extends over the pseudorapidity range $|\eta|<2.1$.

In total CMS has $\sim 10^{8}$ data channels that are checked each bunch crossing. The design data-size per event is about $1 \mathrm{MB}$. At start-up it is essential to allow for a larger event size, 
Table 1.1. Approximate event rates of some physics processes at the LHC for a luminosity of $\mathcal{L}=2 \times 10^{33} \mathrm{~cm}^{-2} \mathrm{~s}^{-1}$. For this table, one year is equivalent to $20 \mathrm{fb}^{-1}$.

\begin{tabular}{lcc}
\hline Process & Events/s & Events/year \\
\hline$W \rightarrow e v$ & 40 & $4 \times 10^{8}$ \\
$Z \rightarrow e e$ & 4 & $4 \times 10^{7}$ \\
$t \bar{t}$ & 1.6 & $1.6 \times 10^{7}$ \\
$b \bar{b}$ & $10^{6}$ & $10^{13}$ \\
$\tilde{g} \tilde{g}(m=1 \mathrm{TeV})$ & 0.002 & $2 \times 10^{4}$ \\
Higgs $(m=120 \mathrm{GeV})$ & 0.08 & $8 \times 10^{5}$ \\
Higgs $(m=120 \mathrm{GeV})$ & 0.08 & $8 \times 10^{5}$ \\
Higgs $(m=800 \mathrm{GeV})$ & 0.001 & $10^{4}$ \\
QCD jets $p_{\mathrm{T}}>200 \mathrm{GeV}$ & $10^{2}$ & $10^{9}$ \\
\hline
\end{tabular}

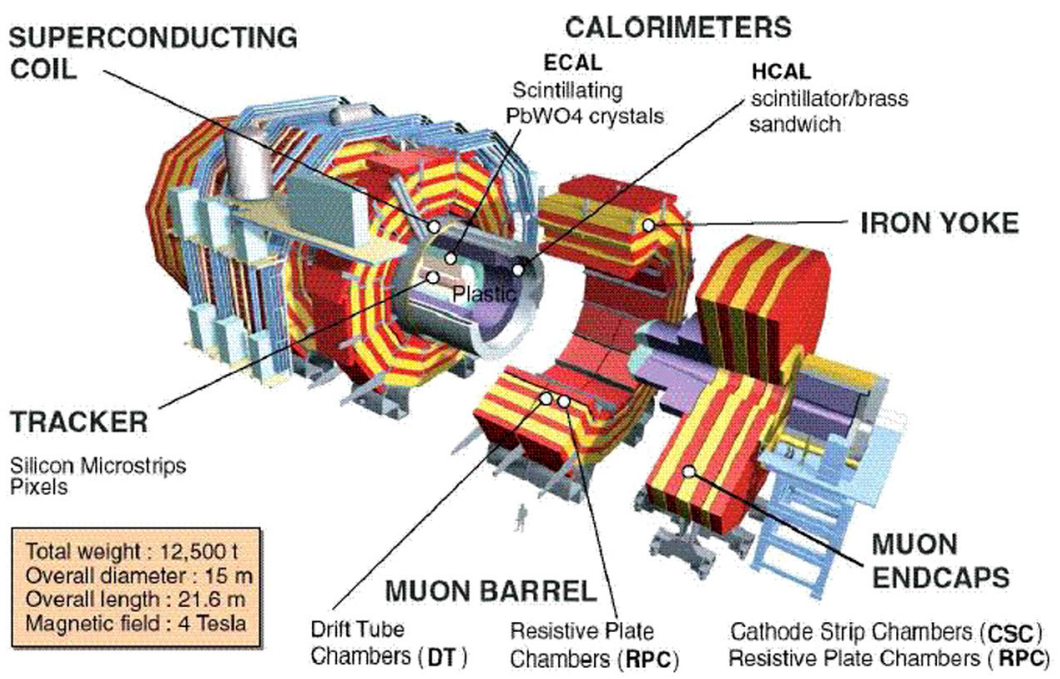

Figure 1.1. Three dimensional view of the CMS detector, and its detector components.

up to $1.5 \mathrm{MB}$ per event, in order to be able to thoroughly study and understand the detector performance.

This Volume is organised in two parts. In the first part a number of physics channels challenging for the detector are studied in detail. Each of these channels is associated with certain physics objects, such as electrons, photons, muons, jets, missing $E_{\mathrm{T}}$ and so on. The analyses are performed in a fully realistic environment as the one expected for real data. Methods on determining the backgrounds from the data as well as on evaluating the experimental systematic effects, e.g., due to miscalibration and misalignment, resolution and signal significance are developed. In short these analyses are performed imitating real data analyses to the maximum possible extent.

In the second part the physics reach is studied for a large number of physics processes, for data samples mostly with luminosities in the range of 1 to $30 \mathrm{fb}^{-1}$, expected to be collected during the first years of operation at the LHC. Standard model measurements of, e.g., W and top quark mass determinations are studied; many production and decay mechanisms for the SM and MSSM Higgs are studied, and several models beyond the Standard Model are explored. 


\subsection{The full analyses}

In total 11 analyses were studied in full detail. All the studies were performed with detailed Geant 4 based simulation of the CMS detector and reconstruction of the data, including event pile-up, and a detailed analysis of the systematics.

The $H \rightarrow \gamma \gamma$ analysis covers one of the most promising channels for a low mass Higgs discovery and for precision Higgs mass measurement at the LHC. This channel has been an important motivation for the design of the electromagnetic calorimeter (ECAL) of CMS. It is used here as a benchmark channel for identifying photons with high purity and efficiency, and as a driver for optimising the ECAL energy resolution and calibration of the analyses. Furthermore, new statistical techniques that make use of event kinematics and neural network event selection algorithms have been used to enhance the sensitivity in this channel.

The analysis $H \rightarrow Z Z \rightarrow 4$ electrons covers electron identification and selection optimisation. In particular, the classification of electron candidates according to quality criteria which depends on their passage through the material of the tracker was studied, and the impact on the Higgs search quantified.

The same process has been studied in the muon decay channel $H \rightarrow Z Z \rightarrow 4 \mu$. This process is an important benchmark for optimising the muon analysis tools. It is one of the cleanest discovery channels for a Standard Model Higgs with a mass up to $600 \mathrm{GeV} / \mathrm{c}^{2}$. Methods to minimise the systematics errors have been developed.

The channel $H \rightarrow W W \rightarrow 2 \mu 2 v$ is of particular importance if the mass of the Higgs is around $165 \mathrm{GeV} / \mathrm{c}^{2}$, and is again an interesting muon benchmark channel. The challenge is to establish with confidence a dimuon excess, since this channel does not allow reconstruction of the Higgs mass on an event by event basis. The event statistics after reconstruction and selection is large enough for an early discovery, even with about $1 \mathrm{fb}^{-1}$ of integrated luminosity, provided the systematic uncertainty on the background can be kept well under control.

The production of a new gauge boson with a mass in the $\mathrm{TeV}$ range is one of the possible early discoveries at the LHC. The clean final state for the decays into two high $p_{\mathrm{T}}$ leptons leads to a clearly detectable signal in CMS. The channel $Z^{\prime} \rightarrow \mu \mu$ was selected as a benchmark to study muons with $p_{\mathrm{T}}$ in the TeV/c range. Dedicated reconstruction techniques were developed for $\mathrm{TeV}$ muons and the experimental systematics e.g. due to misalignment effects were studied in detail.

Jets will be omnipresent in the LHC collisions. The analysis of dijets events and the dijet invariant mass has been studied in detail. A pre-scaling strategy of the jet threshold for the trigger, in order to allow a dijet mass measurement starting from approximately $300 \mathrm{GeV} / \mathrm{c}^{2}$ has been developed. Calibration procedures, and experimental and theoretical systematics on the dijet mass distribution have been evaluated in detail. The results were interpreted as sensitivities to new physics scenarios.

The determination of the missing transverse momentum in collisions at a hadron collider is in general a difficult measurement, since it is very susceptible to detector inefficiencies, mis-measurements, backgrounds such a halo muons or cosmic muons, and instrumental backgrounds. On the other hand, it is probably the most striking signature for new physics with escaping weakly interacting particles, such as the neutralinos in supersymmetry. A low mass mSUGRA SUSY benchmark point was selected to exercise a full analysis, including techniques to suppress spurious backgrounds as well as QCD residual contribution due to mis-measurements. Techniques to calibrate the $E_{\mathrm{T}}^{\text {miss }}$ with known Standard Model processes have been also developed. Such a low mass SUSY scenario could already be detected with $0.1 \mathrm{fb}^{-1}$ of data with a well understood detector and well controlled background. 
The decay $B_{s} \rightarrow J / \psi \phi$ is chosen as a benchmark channel since it is representative of exclusive $B$-physics studies. It allows to study the capability of CMS to identify, select and reconstruct a fully reconstructed decay of the $B_{s}$, which presents a significant challenge due to its relatively low momentum and high background. In addition, the measurement is performed of the width difference $\Delta \Gamma$ on a sample of untagged $B_{s} \rightarrow J / \psi \phi \rightarrow \mu^{+} \mu^{-} K^{+} K^{-}$candidates using a maximum likelihood fit of the time dependent angular distribution.

The detection of the $\tau$ particle will be very important at the LHC since, a clear excess of $\tau$ production is also a sign of new physics. The $\tau$ selection and analysis tools have been used to search for and measure the A/H heavy Higgs bosons in the MSSM. Various decay channels of the $\tau$ have been considered, and $\tau$ tagging tools have been deployed and refined. A $\tau$-trigger is very challenging but necessary for these physics studies, and has been studied in detail.

The process of associated production of a Higgs particle with top quarks, and with the Higgs decaying into b-quarks, is no doubt one of the most challenging channels studied in this part of the TDR. The physics interest is high since, this channel gives access to a measurement of the $H \rightarrow b b$ decay and thus, to the Yukawa coupling of the Higgs to the b quark. The inclusive $H \rightarrow b b$ production channel cannot be used due to a too large QCD $b \bar{b}$ background. This analysis uses techniques to tag $b$ quarks and calibration methods to reconstruct top quarks from multi-jet decays. Furthermore, the backgrounds such as $t \bar{t}$ jet-jet have been carefully examined. The results demonstrate that this will be a very challenging measurement even with the highest luminosity in the first phase of the LHC operation.

Finally, a benchmark channel for heavy ions collisions was studied. Quarkonia $(J / \psi, \Upsilon)$ were reconstructed and measured via the two muon decay modes. The particular challenge is an efficient track reconstruction in an environment of 2000 to perhaps even 5000 tracks produced per unit of rapidity. The analysis shows that the detection of the quarkonia is possible with reasonable efficiencies and leads to a good event statistics for detailed studies of the "melting" of these resonances in a hot dense region.

In general, these detailed studies in this first part of the PTDR have demonstrated that the CMS experiment is up and ready to meet the challenge, and can deliver measurements with the quality and precision as anticipated from its detector design.

\subsection{The physics reach}

The physics reach of the Report contains three main parts: Standard Model processes, Higgs searches and measurements and searches beyond the Standard Model.

The Standard Model sections contain a study of the strong interactions, top quark physics and electroweak physics. Jet production is revisited but this time to measure inclusive single jet $p_{\mathrm{T}}$ spectra, with emphasis placed on the experimental uncertainties related to such a measurement. The underlying event is still enigmatic, and procedures are outlined to get better insight with the first LHC data. B-hadrons will be copiously produced at the LHC and inclusive B production and $B_{c}$ production have been studied. At the LHC about one top quark pair is produced per second. Such a huge sample of top quarks allows for detailed measurements of the top quark properties such as cross sections and mass, spin properties, single top production, and searches for new physics in top decays. A detailed study on the mass measurement precision, limited by the systematics errors, is reported. In the electroweak part of this chapter, the production of $\mathrm{W}$ and $\mathrm{Z}$ bosons is discussed, as well as multi-boson production, and a precise measurement of the Drell-Yan process. The precision with which the mass of the $\mathrm{W}$ boson can be determined is analysed.

One of the main missions of the LHC is the discovery of the origin of the electroweak symmetry breaking mechanism. Therefore, the search for the Higgs particle is a major task 
for the experiments. The Higgs particle search is studied for the SM and MSSM Higgs(es) in the full mass range starting from the LEP exclusion limits. Detailed systematic studies were included in the estimates for the integrated luminosity needed for a $5 \sigma$ discovery. The methods used to calculate the $5 \sigma$ discovery limit are detailed in Appendix A. Over a large range of Higgs boson masses, a discovery is possible with a few $\mathrm{fb}^{-1}$, but for the interesting mass region below $130 \mathrm{GeV} / \mathrm{c}^{2}, 10 \mathrm{fb}^{-1}$ will be needed. MSSM Higgs discoveries are studied both for neutral and charged Higgs particles, and discovery regions are presented. Finally, the Higgs chapter also contains studies of other scalar particles such as the radion that emerges in models with warped extra dimensions, and a double charged Higgs that may be produced in Little Higgs scenarios.

The LHC will probe the TeV energy scale and is expected to break new ground. An important part of the CMS program will be to search for new physics. If low mass supersymmetry exists it will be within the reach of the LHC. The studies in this Report are mainly signature based, to test the discovery potential in as many channels as possible, using a number of chosen benchmark points covering a large part of different signatures. The discovery reach for scenarios with extra dimensions, and new vector bosons high mass states are analysed using several different experimental signals. The methods used to calculate the $5 \sigma$ discovery limit are detailed in Appendix A. Finally alternative signatures for new physics such as technicolour, contact interactions, heavy Majorana neutrinos, heavy top in Little Higgs models, and same sign top quarks have been analysed.

While many signals and processes have been studied, it was not the goal of this PTDR to study and to include all possible channels to give a full physics review. Besides, what is contained here in this Report, there are other ongoing analyses nearing completion on topics such as GMSB SUSY, UED extra dimensions, split SUSY scenarios, invisible Higgs production, TGC sensitivity of dibosons, strongly interacting vector boson scattering, and others. The channels included in this Report have however, been very instrumental to test and deploy the tools and techniques for performing physics studies with CMS at the LHC.

\subsection{Tools used in the studies for the PTDR}

\subsubsection{Detector simulation and reconstruction}

For the studies presented in this TDR, the CMS detector response was simulated using the package OSCAR [8]. It is an application of the Geant4 [9] toolkit for detector description and simulation. OSCAR is used to describe the detector geometry and materials. It also includes and uses information about the magnetic field. OSCAR reads the individual generated events and simulates the effects of energy loss, multiple scattering and showering in the detector materials with Geant4. The digitisation (simulation of the electronic response), the emulation of the Level-1 and High-Level Triggers (HLT), and the offline reconstruction of physics objects were performed with the CMS full-reconstruction ORCA package [10].

A number of analyses for the physics reach studies were performed with the fast parameterised simulation FAMOS [11]. FAMOS has been tuned to the detailed simulation and reconstruction and is roughly about a factor 1000 faster. FAMOS allows to perform, e.g., accurate sensitivity scans in a large parameter space of a model for new physics.

\subsubsection{Pile-up treatment}

The total inelastic cross section at the LHC is assumed to be $\sigma_{\mathrm{T}} \sim 80 \mathrm{mb}$. The LHC will operate at a bunch crossing rate of $40 \mathrm{MHz}$. Only $80 \%$ of the bunches will be filled, resulting in an effective bunch crossing rate of $32 \mathrm{MHz}$. The instantaneous luminosity in the first 
two years after start-up is expected to be $\mathcal{L}=2 \times 10^{33} \mathrm{~cm}^{-2} \mathrm{~s}^{-1}$ and subsequently upgraded to $\mathcal{L}=10^{34} \mathrm{~cm}^{-2} \mathrm{~s}^{-1}$ in a second phase. The average number of inelastic non-diffractive interactions per bunch crossing $\mu$ is $\mu=25$ at high and $\mu=5$ at low luminosity.

Both the detailed simulation and reconstruction chain OSCAR/ORCA and FAMOS allow the overlay of pile-up events, according to a Poisson distribution with average $\mu$, on top of real signal events, exactly as for real data. These events were sampled from a data base of $600 \mathrm{~K}$ minimum bias events, generated with parameters discussed in Appendix C.

All the studies reported in this TDR include the effects of pile-up on the signal. For all studies with luminosities up to $60 \mathrm{fb}^{-1} \mu=5$ was used. Several techniques have been developed to minimise the effect of pile-up, and have been used in the studies reported in this TDR. Both in-time and out-of-time pile-up has been included.

\subsubsection{Systematic effects on measurements}

The results of the PTDR Volume 1 were used to form the baseline for all systematic studies in this Volume. Systematic effects include energy scale uncertainties for the calorimeters, effects of misalignment, uncertainties in the background estimation either from theory or from techniques to estimate these backgrounds from data. Misalignments of the tracker and of the muon system expected at the initial and at the well-advanced stages of the data taking have been taken into account by using two misalignment scenarios developed in the framework of the CMS reconstruction.

A comprehensive review on the experimental and theoretical systematics used in this PTDR is presented in Appendix B.

\subsubsection{Event generators}

The studies for this physics TDR have been performed with a variety of event generators, suitably chosen for each processes studied. The main work-horse was PYTHIA, the general multi-purpose generator, and in some case checks have been performed with HERWIG. More specialised generators which include a more complete description of the relevant matrix elements, have been used for a number processes, as detailed in the analysis reports. A list of generators used in this TDR is given in Appendix C.

An important aspect for the LHC, is the QCD multi-jet production in various physics channels, and a correct and thorough understanding of Standard Model processes such as $\mathrm{W}+$ jets, $\mathrm{Z}+$ jets and $t \bar{t}+$ jet production will be paramount before discoveries can be claimed in channels such as jets $+E_{\mathrm{T}}^{\text {miss }}$ and jets + leptons. CMS will measure these Standard Model processes in an early phase of the experiment, to reduce the impact of inherent uncertainties in the Monte Carlo models on searches and discoveries, using methods demonstrated in this TDR. These will allow estimation of the expected backgrounds directly or will allow to tune the generators in order to use these with increased confidence in regions of phase space not directly accessible with measurements from the data.

Generators with multi-parton final states are available at Leading Order (LO) for most Standard Model processes. Recently, Next to Leading Order (NLO) generators have become available as well, be it for a more restricted number of processes. Sophisticated algorithms that match the hard jets generated by the matrix elements, with the softer parton jets, have become available. An example is the ALPGEN generator, which has been used for some studies and comparisons in this Report. For some of the detailed analyses, such as the $E_{\mathrm{T}}^{\text {miss }}$ low mass SUSY search, it was shown that the effect of using ALPGEN instead of PYTHIA did not lead to different result, while for other analyses, such as background to ttH production, the difference was important. 
Another difficulty in the estimation of the background to processes is the rate of QCD multi-jet events. Typically, samples of events of more than $10^{8}$ or $10^{9}$ events would be needed to cover possible tails. Detailed simulation of such background samples cannot be easily done, and therefore, other approaches were taken in this TDR. These include pre-selections at the generator level, fast simulation of large samples and factorising the efficiencies of independent selections cuts.

Hence, one has to keep in mind that the exact results presented in this TDR could depend on the generators. They should therefore, be taken as an indication albeit a good indication of what can be expected at the LHC.

\subsubsection{Parton distributions and higher order corrections}

One of the key differences between a hadron and an $e^{+} e^{-}$collider is that for hadrons the partons collide with a strongly varying incident energy, given by the distribution of the longitudinal momentum fraction $x$ of the parton in the proton. These parton densities are determined from data, in particular from deep inelastic scattering data and other measurements of hard scattering processes. Several groups have fitted parton distribution functions (PDFs) to these data, e.g., the CTEQ [12] and MRST [13] groups.

For the studies in this report, the simulated event samples were generated with CTEQ5L but CTEQ6 was used to normalise cross sections and to study the PDF uncertainties. CTEQ 6.1 has 40 different error PDFs, $20 \mathrm{PDFs}$ at positive error, and $20 \mathrm{PDFs}$ at negative error. We use the CTEQ6.1M eigenvector PDF sets [12] and the "master" equations as detailed in Appendix B to evaluate the uncertainties characterising current knowledge of the parton distributions.

The precise knowledge of the parton distributions will remain an extremely important subject for the physics at the LHC. Currently, a study group in the framework of the HERALHC workshop is tackling this topic in order to get as good knowledge as possible of the PDFs [14] and their uncertainties at the time of the startup of the LHC. Once the LHC starts data collection, several QCD process can be used to help to constrain the PDFs, as has been shown, e.g., using W production with studies at the HERA-LHC workshop.

\subsection{Outlook}

The work detailed in this Volume of the PTDR constitutes the pedestal for the physics studies that the experiment will pursue both at the start-up and the longer term running. In the process of carrying out these studies CMS has gained valuable experience in all aspects, both technical and strategic, in executing a high performance physics program. Of great value is also the identification of shortcomings and challenges that emerged in the context of completing these analyses.

As a follow-up of this work, CMS is planning an elaborate program for the start-up studies and physics commissioning from the combined magnet test effort (MTCC) as well as the experience of the upcoming computing, software and analysis challenge (CSA06) that incorporates the full calibration and alignment framework in combination with the full-trigger path exercise. The whole edifice for data collecting and analysis is expected to be complete and tested by the turn-on of the LHC in 2007. 


\section{Part I. Complete Analyses}

\section{Chapter 2. Physics Studies with Photons and Electrons}

\subsection{Benchmark Channel: $H \rightarrow \gamma \gamma$}

The $\mathrm{H} \rightarrow \gamma \gamma$ channel has been studied since the initial planning of the LHC and SSC as an important channel for the discovery of Higgs particles at masses beyond the upper reach of LEP and below about $150 \mathrm{GeV}[3,15,16]$. The signature sought in the inclusive analysis is two high $E_{\mathrm{T}}$ isolated photons. The challenge for discovery of a Higgs in this mode is the small branching fraction of about 0.002 , since in this mass range the dominant decay mode of the Higgs is $b \bar{b}$. The $\gamma \gamma$ decay mode can be well identified experimentally but the signal rate is small compared to the backgrounds coming both from two prompt photons (irreducible), and from those in which one or more of the photons are due to decay products or mis-identified particles in jets (reducible). It has long been understood that $\mathrm{H} \rightarrow \gamma \gamma$ can be detected as a narrow mass peak above a large background. The background magnitude can be determined from the region outside the peak. After event selection, for an integrated luminosity of $20 \mathrm{fb}^{-1}$ and for a Higgs boson mass of $120 \mathrm{GeV} / \mathrm{c}^{2}$, we expect approximately 350 signal events in a mass window of $2 \mathrm{GeV} / \mathrm{c}^{2}$ over 7000 background events. An example of a $p p \rightarrow \mathrm{H}+\mathrm{X}$ event with Higgs particle decay $\mathrm{H} \rightarrow \gamma \gamma$ is shown in colour plate CP1.

In this study we present two complementary inclusive analyses for the $\mathrm{H} \rightarrow \gamma \gamma$ channel: a standard cut based analysis and a high performance, discovery-oriented analysis, based on the method described in $[17,18]$. Both are carried out with our present knowledge of the expected background, estimated with full detector simulation. Further details can be found in [19]. The study concentrates on the first years of LHC operation and uses simulated events with pileup corresponding to a luminosity of $2 \times 10^{33} \mathrm{~cm}^{-2} \mathrm{~s}^{-1}$.

The idea of measuring the rate of background by using the mass regions adjoining the Higgs peak is extended to also measure the characteristics of the background, and using this information to help separate background from signal. The $\mathrm{H} \rightarrow \gamma \gamma$ channel is particularly well suited to this technique because the signal is relatively small and can be confined to a narrow mass region thanks to the excellent photon energy and position resolution of the CMS detector [7].

By using photon isolation and photon kinematic information, significant additional discrimination between signal and background can be achieved. The optimised analysis uses this information to discriminate between signal and background by comparing data in mass side-bands with signal Monte Carlo. Use is made of a neural network, but likelihood variables or other techniques may prove to be better in the future. The expected purity in terms of signal/background, corresponding to each event, can be estimated based on this information and each event then can be used optimally to evaluate the likelihood of a signal plus background hypothesis compared to a background-only hypothesis.

In the optimised analysis the expected signal to background ratio is calculated for each event. By dividing the cut-based analysis in various categories with different $s / b$ ratios results improve toward those that are obtained with the optimised analysis. If the maximum $s / b$ ratio in the optimised analysis is limited to the best category used in the cut-based analysis, the performances of the two analyses are nearly identical.

The optimised, discovery-oriented analysis is particularly appropriate to the $\mathrm{H} \rightarrow \gamma \gamma$ channel because the Higgs signal appears in a narrow mass peak allowing analysis of the large background in the mass side-bands. The analysis will not be limited by the poor simulation of the background once data will be available. 
Table 2.1. NLO cross sections for the different Higgs boson production processes and branching ratios.

\begin{tabular}{llllll}
\hline $\mathrm{M}_{\mathrm{H}}$ & $115 \mathrm{GeV} / \mathrm{c}^{2}$ & $120 \mathrm{GeV} / \mathrm{c}^{2}$ & $130 \mathrm{GeV} / \mathrm{c}^{2}$ & $140 \mathrm{GeV} / \mathrm{c}^{2}$ & $150 \mathrm{GeV} / \mathrm{c}^{2}$ \\
$\sigma$ (gg fusion) & $39.2 \mathrm{pb}$ & $36.4 \mathrm{pb}$ & $31.6 \mathrm{pb}$ & $27.7 \mathrm{pb}$ & $24.5 \mathrm{pb}$ \\
$\sigma(\mathrm{WVB}$ fusion) & $4.7 \mathrm{pb}$ & $4.5 \mathrm{pb}$ & $4.1 \mathrm{pb}$ & $3.8 \mathrm{pb}$ & $3.6 \mathrm{pb}$ \\
$\sigma(\mathrm{WH}, \mathrm{ZH}, t \bar{t} \mathrm{H})$ & $3.8 \mathrm{pb}$ & $3.3 \mathrm{pb}$ & $2.6 \mathrm{pb}$ & $2.1 \mathrm{pb}$ & $1.7 \mathrm{pb}$ \\
Total $\sigma$ & $47.6 \mathrm{pb}$ & $44.2 \mathrm{pb}$ & $38.3 \mathrm{pb}$ & $33.6 \mathrm{pb}$ & $29.7 \mathrm{pb}$ \\
$\mathrm{H} \rightarrow \gamma \gamma$ Branching ratio & 0.00208 & 0.00220 & 0.00224 & 0.00195 & 0.00140 \\
Inclusive $\sigma \times B . R$. & $99.3 \mathrm{fb}$ & $97.5 \mathrm{fb}$ & $86.0 \mathrm{fb}$ & $65.5 \mathrm{fb}$ & $41.5 \mathrm{fb}$ \\
\hline
\end{tabular}

The study described requires a comprehensive understanding and simulation of the CMS detector. The electromagnetic calorimeter is used to make the primary measurements of photon energy and position. The tracker is used to measure the position of the interaction vertex. The tracker, ECAL and HCAL are used to determine, if the photon candidate is well isolated. While background characteristics will be measured from data, the signal must be well simulated to perform the analysis described below. This requires a detailed understanding of the detector performance as well as its calibration.

\subsubsection{Higgs boson production and decay}

For this inclusive study the Higgs boson production mechanisms with the largest crosssections in the Standard Model have been simulated: gluon fusion, qqH production through Weak Vector Boson Fusion (WBF), associated Higgs production with $\mathrm{W}$ or $\mathrm{Z}$ bosons, and Higgs production associated with a $t \bar{t}$ pair. The cross sections for the different production processes [20] and the $\mathrm{H} \rightarrow \gamma \gamma$ branching ratios [21] are summarised in Table 2.1. The analysis described in this chapter has been limited to careful measurement of the inclusive diphoton channel, to address the main detector issues, and no use has been made of tagging leptons or jets. In the future, channel identification, based on additional leptons and jets. will improve the sensitivity. For the moment these 'tagged' channels are investigated individually in other studies [22, 23]. Figure 2.1 shows an event display of a $\mathrm{H} \rightarrow \gamma \gamma$ event with $\mathrm{M}_{\mathrm{H}}=120 \mathrm{GeV} / \mathrm{c}^{2}$.

\subsubsection{Backgrounds}

Backgrounds with two real prompt high $E_{\mathrm{T}}$ photons are called "irreducible", although they can be somewhat reduced due to kinematic differences from signal processes in which high mass particles are produced. Two photons can be produced from two gluons in the initial state through a "box diagram" or from initial quark and anti-quark annihilation.

Backgrounds in which at least one final state jet is interpreted as a photon are called "reducible" and are much harder to simulate since, jets are copiously produced at the LHC and Monte Carlo samples that correspond to $10 \mathrm{fb}^{-1}$ are much too large to fully simulate. Selections at generator level have been devised in order to be able to select multi-jet and $\gamma$ plus jets events that contribute to the background of the $\mathrm{H} \rightarrow \gamma \gamma$ channel and reject events that have negligible chance of producing background to the final analysis.

The $\gamma+$ jet sample can be viewed, from the selection point of view, as coming from two different sources: one where another photon is radiated during the fragmentation of the jet (two prompt photons), the other where there is only one prompt photon in the final state and the other photon candidate corresponds to a mis-identified jet or isolated $\pi^{0}$ (one prompt plus one fake photon). These two processes have been separated using generator level information, and are listed separately in the tables below. Also, different K-factors are applied. 


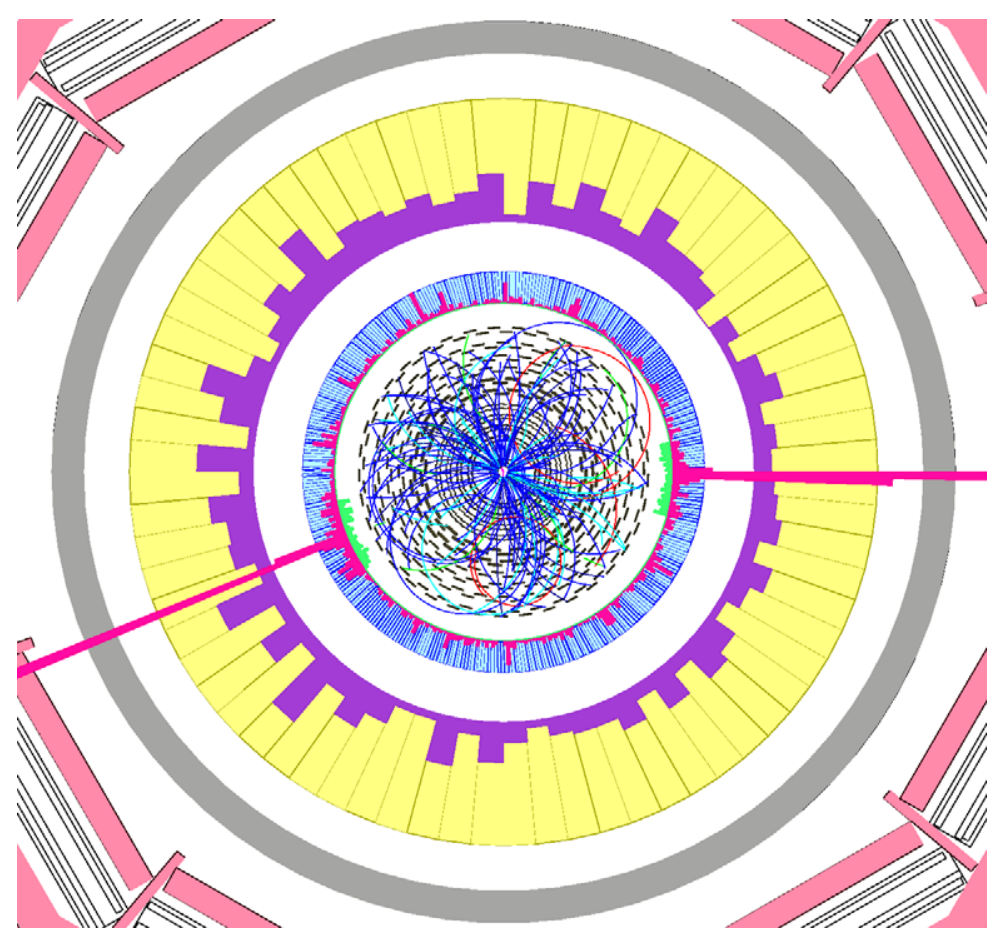

Figure 2.1. $\mathrm{H} \rightarrow \gamma \gamma$ event produced in gluon fusion with $\mathrm{M}_{\mathrm{H}}=120 \mathrm{GeV}$ observed in the CMS detector.

The generator level pre-selection of $\gamma+$ jet events that contribute to the $\mathrm{H} \rightarrow \gamma \gamma$ background is straightforward. For $\mathrm{pp} \rightarrow$ jets, a much tighter set of cuts at the particle generator level was carefully developed and studied. Groups of particles, protocandidates, which might form a photon candidate after event simulation are identified. Cuts are applied on the transverse energy of two protocandidates and on their invariant mass, and this involves an estimate on the lower and upper limits to the energy of the photon candidates that might be reconstructed from the protocandidates after the simulation. An estimate is also made on likely level of isolation of the resulting photon candidate.

With such selection a rejection of a factor of about 41000 can be obtained, with an estimated inefficiency of $14 \%$ for $\mathrm{pp} \rightarrow$ jets events generated with PYTHIA with $\hat{p}_{\perp}>30 \mathrm{GeV}$ (transverse momentum of the products of the hard interaction). The inefficiency after the final analysis selection was estimated by using a looser pre-selection similar to that used for the $\mathrm{pp} \rightarrow \gamma+$ jet simulation. Further details can be found in [19]. Events rejected by the preselection have rather low $E_{\mathrm{T}}$ photons and are not very important for the final analysis.

The Monte Carlo samples used are summarised in Table 2.2. All events were generated with PYTHIA [24], simulated with the GEANT-based [9] CMSIM [25] or OSCAR [8], and reconstructed with ORCA version 8.7.3 [10]. Pile-up events from minimum bias interactions were added to the hard interaction, assuming a luminosity of $\mathcal{L}=2 \times 10^{33} \mathrm{~cm}^{-2} \mathrm{~s}^{-1}$.

$\mathrm{K}$-factors are applied to take into account the expected differences between the lowest order cross sections given by PYTHIA and the NLO cross sections of the different background processes [26-30]. The K-factors used for each background are summarised in Table 2.3 and are estimated to have an uncertainty of $20-30 \%$. 
Table 2.2. Monte Carlo samples used in the $\mathrm{H} \rightarrow \gamma \gamma$ analysis with LO cross section from PYTHIA and total corresponding integrated luminosities of the analysed samples.

\begin{tabular}{|c|c|c|c|c|c|c|}
\hline Process & $\begin{array}{c}\hat{p}_{\perp} \\
(\mathrm{GeV} / \mathrm{c})\end{array}$ & $\begin{array}{c}\mathrm{M}_{\mathrm{H}} \\
\left(\mathrm{GeV} / \mathrm{c}^{2}\right)\end{array}$ & $\sigma(\mathrm{pb})$ & $\begin{array}{c}\text { Pre-sel. } \\
\sigma(\mathrm{pb})\end{array}$ & $\begin{array}{c}\text { Events } \\
\text { Analysed }\end{array}$ & $\begin{array}{c}\text { Int Lum. } \\
\left(\mathrm{fb}^{-1}\right)\end{array}$ \\
\hline $\mathrm{H} \rightarrow \gamma \gamma$ (gg fusion) & - & 120 & - & - & $181 \mathrm{~K}$ & - \\
\hline $\mathrm{H} \rightarrow \gamma \gamma$ (WB fusion) & - & 120 & - & - & $193 \mathrm{~K}$ & - \\
\hline $\mathrm{H} \rightarrow \gamma \gamma$ (gg fusion) & - & $115-150$ & - & - & $20 \mathrm{~K}$ & - \\
\hline $\mathrm{H} \rightarrow \gamma \gamma$ (WB fusion) & - & $115-150$ & - & - & $20 \mathrm{~K}$ & - \\
\hline $\mathrm{H} \rightarrow \gamma \gamma(\mathrm{WH}, \mathrm{ZH}, \mathrm{ttH})$ & - & $115-150$ & - & - & $20 \mathrm{~K}$ & - \\
\hline $\mathrm{pp} \rightarrow \gamma \gamma($ born $)$ & $>25$ & - & 82 & 44 & $920 \mathrm{~K}$ & 30 \\
\hline $\mathrm{pp} \rightarrow \gamma \gamma(\mathrm{box})$ & $>25$ & - & 82 & 31 & $668 \mathrm{~K}$ & 20 \\
\hline $\mathrm{pp} \rightarrow \gamma+$ jet & $>30$ & - & $5 \times 10^{4}$ & $2.5 \times 10^{3}$ & $5.5 \mathrm{M}$ & 2.2 \\
\hline $\mathrm{pp} \rightarrow$ jets & $>50$ & - & $2.8 \times 10^{7}$ & $4.7 \times 10^{3}$ & $4.5 \mathrm{M}$ & 1.0 \\
\hline Drell-Yan ee & - & - & $4 \times 10^{3}$ & $4 \times 10^{3}$ & $460 \mathrm{~K}$ & 0.1 \\
\hline
\end{tabular}

Table 2.3. Background K-factors applied to PYTHIA cross sections.

\begin{tabular}{ll}
\hline $\mathrm{pp} \rightarrow \gamma \gamma$ (Born) & 1.5 \\
$\mathrm{pp} \rightarrow \gamma \gamma$ (Box) & 1.2 \\
$\mathrm{pp} \rightarrow \gamma+$ jet $(2$ prompt $)$ & 1.72 \\
$\mathrm{pp} \rightarrow \gamma+$ jet (1 prompt +1 fake) & 1 \\
$\mathrm{pp} \rightarrow$ jets & 1 \\
\hline
\end{tabular}

\subsubsection{Reconstruction, selection, and signal significance calculation}

2.1.3.1. Trigger. $\quad \mathrm{H} \rightarrow \gamma \gamma$ events are selected with extremely high efficiency both by the Level-1 and High Level triggers that are described in details in Ref. [31]. Since in the analysis selection tighter $E_{\mathrm{T}}$ and isolation cuts are applied, the inefficiency due to the trigger is negligible.

2.1.3.2. Photon reconstruction. Photons are reconstructed with the standard ECAL algorithms [7, 32]. At this level the photon reconstruction efficiency is over $99.5 \%$ for photons in the region covered by the ECAL.

The energy resolution of reconstructed photons is excellent for photons that do not convert or that convert late in the tracker. Energy resolution deteriorates somewhat for photons that convert early in the tracker. Nevertheless, the photon energy resolution is substantially less affected by tracker material than is electron energy resolution and the Higgs reconstruction in the calorimeter is quite reliable even for converted photons.

For signal events, where this effect is relevant, the energy response of the individual crystals of the ECAL has been smeared using a miscalibration file randomly generated to correspond to the intercalibration precision expected after calibration with $\mathrm{W} \rightarrow \mathrm{e} v$ events obtained with an integrated luminosity of $10 \mathrm{fb}^{-1}$, as described in [7]. The precision is $0.3 \%$ in the central part on the barrel, growing up to $1.0 \%$ at the edge of the barrel and in the endcaps.

The tools that have been developed to identify and reconstruct photon conversions in the tracker [33], and $\pi^{0}$ rejection tools developed for the endcap silicon preshower detector and the barrel crystals, have not yet been included in the analysis.

2.1.3.3. Primary vertex identification. The bunch length at $\mathrm{LHC}$ has an $\mathrm{rms}$ width of $75 \mathrm{~mm}$ resulting in a longitudinal spread of interaction vertices of $53 \mathrm{~mm}$. If the mean longitudinal 
position is used (nominal vertex), the invariant mass of a two-photon state, such as the $\mathrm{H} \rightarrow \gamma \gamma$, is smeared by about $1.5 \mathrm{GeV} / \mathrm{c}^{2}$, due to the mis-measurement of the angle between the two photons related to the uncertainty of the photon directions.

The two high $E_{\mathrm{T}}$ photons coming from the Higgs boson decay are produced in association with other tracks that may come from the underlying event and initial state gluon radiation or from the other particles produced with the Higgs boson in the case of WBF fusion, WH or ZH production and $t \bar{t} \mathrm{H}$ production.

The charged tracks associated to the Higgs production vertex are typically harder than those coming from minimum bias interactions. Therefore, the vertex can be identified by reconstructing the primary vertices in the event and selecting the one that most likely corresponds to the Higgs boson production, based on charged tracks.

At low luminosity $\left(2 \times 10^{33} \mathrm{~cm}^{-2} \mathrm{~s}^{-1}\right)$ we are able to identify the correct vertex, defined as being within $5 \mathrm{~mm}$ of the actual vertex, in about $81 \%$ of the signal events passing the selection described in Section 2.1.4.1. Clearly, these results will be affected by any significant variation of the characteristics of the pileup events from what is simulated in our pileup samples.

2.1.3.4. Photon isolation. Detailed studies have been made of photon isolation and its optimisation [34, 35]. Fake photon signals due to jets can be rejected by looking for additional energetic particles accompanying the photon candidate. Charged pions and kaons can be detected in the tracker or in the calorimeters. Neutral pions and other particles decaying to photons can be detected in the ECAL. The hadron calorimeter may be important for detecting charged particles not efficiently reconstructed in the tracker, particularly at high $\eta$, or other particles like neutrons or $\mathrm{K}_{\text {long }}^{0}$.

2.1.3.5. Separation into categories based on lateral shower shape and pseudorapidity. The shower shape variable $\mathrm{R}_{9}$, defined as the fraction of the super-cluster energy found inside the $3 \times 3$ array of crystals centred around the highest energy crystal, is effective in distinguishing photon conversions in the material of the tracker. Photon candidates with large values of $\mathrm{R}_{9}$ either did not convert or converted late in the tracker and have good energy resolution. Photons converting early have lower values of $\mathrm{R}_{9}$ and worse energy resolution.

The variable $R_{9}$ has been shown to be very useful also in discriminating between photons and jets. This occurs both because of the conversion discrimination - either of the photons from a $\pi^{0}$ can convert - and because, looking in a small $3 \times 3$ crystal area inside the supercluster, the $\mathrm{R}_{9}$ variable can provide very local isolation information about narrow jets.

In the multi-category analysis, the events are separated into categories based on $\mathrm{R}_{9}$ so as to take advantage of better mass resolution where it is expected (the unconverted photons), and yet still use all the events (since the mass resolution varies by at most a factor of 2). This separation also tends to put background events involving jets into categories with lower $\mathrm{R}_{9}$.

We also find that photons detected in the endcaps have worse energy resolution and higher background than photons detected in the barrel so that it is useful to separate events with one or more photons in the endcaps from those with both photons in the barrel.

2.1.3.6. Calculation of confidence levels. Confidence levels are computed by using the Log Likelihood Ratio frequentist method, as described in [36]. Given the expected signal and background distributions in the final variable (the mass distribution for the cut-based analysis), we simulate many possible outcomes of the experiment by means of Monte Carlo. This is done both in the hypothesis that the signal exists and that it does not exist. To compute a confidence level, we order our trials according to an estimator. This is a single number 
that is useful to order random trials from most background-only-like to most signal-plusbackground-like. The simplest and probably best estimator is the Log Likelihood Ratio (LLR) which compares the likelihood of the data to come from a background-only distribution to the likelihood to come from a signal-plus-background distribution. Each likelihood is the product of probabilities from all the bins. The median confidence level is computed both for discovery and for exclusion.

2.1.3.7. Effect of systematic errors. To include systematic errors the background and signal expectation are randomised by the systematic error during the generation of the random trials, while keeping their expectations at the nominal value. If necessary, the correlations between the errors on the different analysis bins is included. It is observed that the signal systematic error has no effect on the median LLR of signal-plus-background experiments, nor on that of background-only experiments. Of course, the distribution corresponding to the signal-plus-background experiments is enlarged by the systematic error on the signal and this makes exclusion more difficult. On the other hand the effect of the systematic error on the background is very large, because of the small signal over background ratio. The mean of the distributions is still unchanged but the widths are enlarged both for background-only experiments and for signal-plus-background experiments. This decreases both the discovery and exclusion sensitivities.

\subsubsection{Cut-based analysis}

2.1.4.1. Selection. Two photon candidates are required with pseudo-rapidity $|\eta|<2.5$, with transverse energies larger than $40 \mathrm{GeV}$ and $35 \mathrm{GeV}$ respectively, and satisfying the following isolation requirements:

- No tracks with $p_{\mathrm{T}}$ larger than $1.5 \mathrm{GeV} / \mathrm{c}$ must be present inside a cone with $\Delta \mathrm{R}<0.3$ around the photon candidate. We only consider tracks with hits in at least two layers of the silicon pixel detector, therefore converted photons are likely to be rejected only if they convert before the second pixel layer.

- The total $E_{\mathrm{T}}$ of all ECAL island basic clusters with $0.06<\Delta \mathrm{R}<0.35$ around the direction of the photon candidate, regardless of whether they belong to the super-cluster or not must be less than $6 \mathrm{GeV}$ in the barrel and $3 \mathrm{GeV}$ in the endcaps.

- The total transverse energies of HCAL towers within $\Delta \mathrm{R}<0.3$ around the photon candidate must be less than $6 \mathrm{GeV}$ in the barrel and $5 \mathrm{GeV}$ in the endcaps.

In order to further reduce the background that is higher when at least one of the photons is detected in the electromagnetic calorimeter endcaps and to increase the performance of the analysis in the forward region additional isolation requirements are applied for events where one, or more, of the candidates has $|\eta|>1.4442$. For these events, the candidate in the barrel is required to satisfy the tighter isolation selection that is applied to photons in the endcaps: ECAL isolation less than $3 \mathrm{GeV}$ and HCAL isolation less than $5 \mathrm{GeV}$.

Figure 2.2 shows the mass distribution after the selection. The efficiency for a $120 \mathrm{GeV} / \mathrm{c}^{2}$ Higgs boson is $30 \%$ and the total expected background is $178 \mathrm{fb} / \mathrm{GeV}$. The number of expected background events for the different types of background is shown in Table 2.4 while the Higgs efficiency in different mass windows is shown in Table 2.5. The efficiency is computed using all generated signal events. The signal contribution to the total number of events is very small, particularly outside the mass region under study. The background can be estimated by a fit to the data mass distribution. 


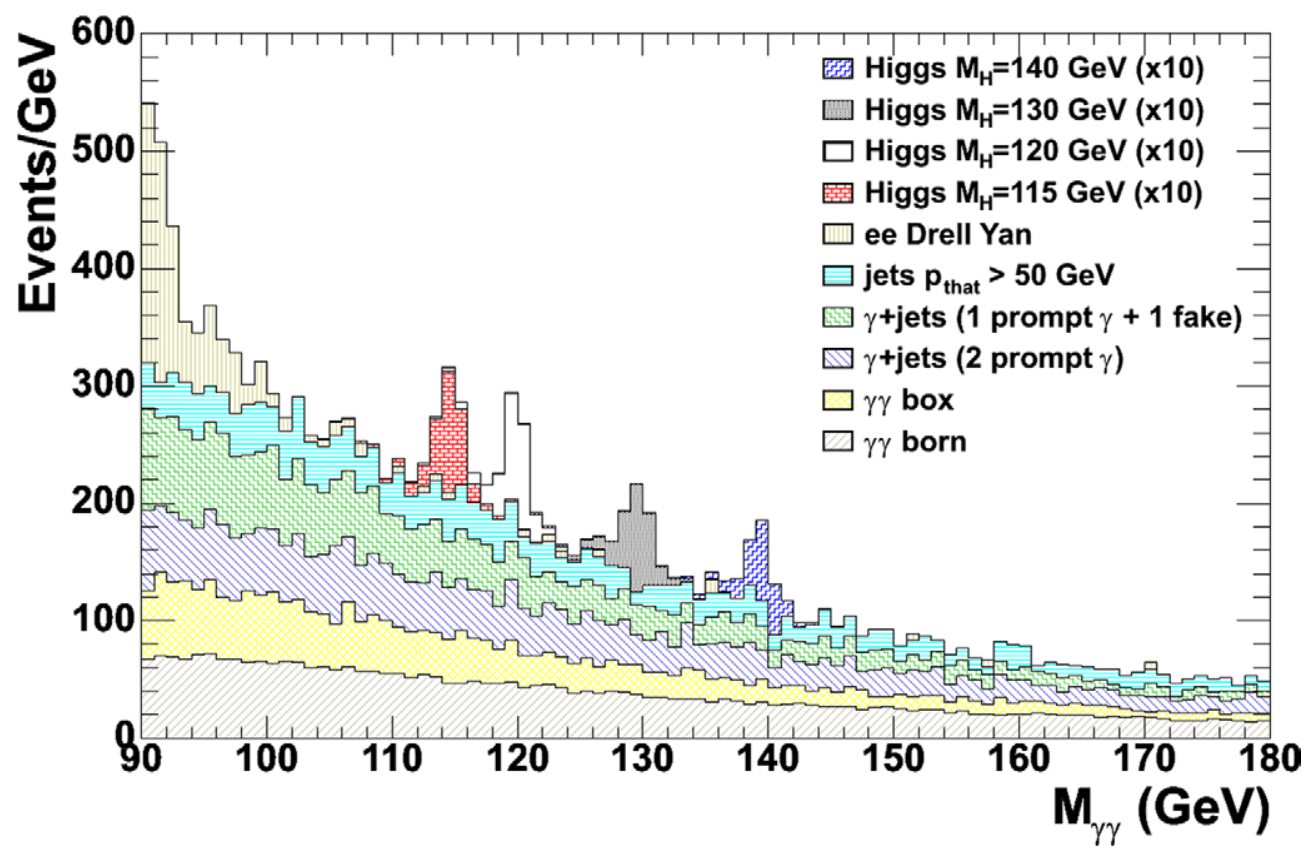

Figure 2.2. Diphoton invariant mass spectrum after the selection for the cut-based analysis. Events are normalised to an integrated luminosity of $1 \mathrm{fb}^{-1}$ and the Higgs signal, shown for different masses, is scaled by a factor 10 .

Table 2.4. Expected background after the selection for Higgs boson masses between 115 and $150 \mathrm{GeV} / \mathrm{c}^{2}$, expressed in $\mathrm{fb} / \mathrm{GeV}$.

\begin{tabular}{lccccc}
\hline Process & $115 \mathrm{GeV} / \mathrm{c}^{2}$ & $120 \mathrm{GeV} / \mathrm{c}^{2}$ & $130 \mathrm{GeV} / \mathrm{c}^{2}$ & $140 \mathrm{GeV} / \mathrm{c}^{2}$ & $150 \mathrm{GeV} / \mathrm{c}^{2}$ \\
\hline $\mathrm{pp} \rightarrow \gamma \gamma$ (Born) & 48 & 44 & 36 & 29 & 24 \\
$\mathrm{pp} \rightarrow \gamma \gamma$ (Box) & 36 & 31 & 23 & 16 & 12 \\
$\mathrm{pp} \rightarrow \gamma+$ jet (2 prompt) & 43 & 40 & 32 & 26 & 22 \\
$\mathrm{pp} \rightarrow \gamma+$ jet (prompt + fake) & 40 & 34 & 22 & 19 & 14 \\
$\mathrm{pp} \rightarrow$ jets & 29 & 27 & 20 & 18 & 14 \\
Drell-Yan ee & 2 & 2 & 1 & 1 & 1 \\
\hline Total background & 203 & 178 & 134 & 109 & 86 \\
\hline
\end{tabular}

Table 2.5. Selection efficiency for the Higgs signal in different mass windows.

\begin{tabular}{lccccc}
\hline $\begin{array}{l}\mathrm{M}_{\mathrm{H}} \\
\left(\mathrm{GeV} / \mathrm{c}^{2}\right)\end{array}$ & $\begin{array}{c}\text { Window } \\
\pm 1 \mathrm{GeV} / \mathrm{c}^{2}\end{array}$ & $\begin{array}{c}\text { Window } \\
\pm 1.5 \mathrm{GeV} / \mathrm{c}^{2}\end{array}$ & $\begin{array}{c}\text { Window } \\
\pm 2.5 \mathrm{GeV} / \mathrm{c}^{2}\end{array}$ & $\begin{array}{c}\text { Window } \\
\pm 5 \mathrm{GeV} / \mathrm{c}^{2}\end{array}$ & $\begin{array}{c}\text { Window } \\
\text { Total }\end{array}$ \\
\hline 115 & $17 \%$ & $21 \%$ & $25 \%$ & $28 \%$ & $29 \%$ \\
120 & $18 \%$ & $22 \%$ & $26 \%$ & $29 \%$ & $30 \%$ \\
130 & $18 \%$ & $22 \%$ & $27 \%$ & $31 \%$ & $32 \%$ \\
140 & $18 \%$ & $23 \%$ & $28 \%$ & $32 \%$ & $34 \%$ \\
150 & $28 \%$ & $24 \%$ & $29 \%$ & $33 \%$ & $36 \%$ \\
\hline
\end{tabular}


The error on the background estimation comes from two sources:

- the statistical precision which decreases with the size of the mass range that is used to perform the fit;

- the systematic error related to the shape of the function that is used to fit the distribution.

It is not possible to know the exact functional form of the background shape and the error must be estimated by assuming a function, simulating a distribution and then using a different function to fit the data. Clearly, this error grows with the size of the mass range used. For a reasonable mass range of $\pm 10-20 \mathrm{GeV} / \mathrm{c}^{2}$ excluding +3 and $-5 \mathrm{GeV} / \mathrm{c}^{2}$ from the Higgs boson mass under study and for an integrated luminosity of $20 \mathrm{fb}^{-1}$ the statistical and systematic errors are estimated to be $0.4 \%$ and $0.5 \%$ respectively. The statistical error decreases with the integrated luminosity while the systematic error is constant.

2.1.4.2. Splitting into categories. Changing the cuts or adding new discriminating variables to this analysis does not give large improvements in the sensitivity. This can be seen, for example, from the fact that it is not possible to use the very powerful variable, $R_{9}$, to reject events without loosing performance. This is because, the increase in $s / b$ ratio does not compensate the loss in efficiency.

The way to improve the sensitivity of the analysis is to keep all selected events but to split the sample into categories with different $s / b$ ratios.

The following 3 possibilities are considered:

- 1 single category;

- 4 categories from $2 \mathrm{R}_{9}^{\mathrm{min}}$ ranges $\left(\mathrm{R}_{9}^{\mathrm{min}}\right.$ larger or smaller than 0.93 ) times 2 pseudo-rapidity regions $|\eta|^{\max }$ in barrel or endcaps;

- 12 categories from $3 \mathrm{R}_{9}^{\min }$ ranges $\left(\mathrm{R}_{9}^{\min }>0.948,0.9<\mathrm{R}_{9}^{\min }<0.948\right.$ and $\left.\mathrm{R}_{9}^{\min }<0.9\right)$ times 4 pseudo-rapidity regions $\left(|\eta|^{\max }<0.9,0.9<|\eta|^{\max }<1.4442,1.4442<|\eta|^{\max }<2.1\right.$ and $|\eta|^{\max }>2.1$ ).

Figure 2.3 shows the mass spectrum after splitting into four categories. The signal over background ratio is much larger in the best category and the composition of the background varies between the different samples: irreducible backgrounds dominate for large $R_{9}$ and reducible backgrounds are larger for small $\mathrm{R}_{9}$.

Table 2.6 shows, for the 12 category analysis, the fraction of events along with the maximum $s / b$ ratio in each category.

2.1.4.3. Systematic errors. The total error on the background is approximately $0.65 \%$ and is due to the uncertainty of the function fit to the side-bands of the mass distribution, estimated to be $0.5 \%$, plus the statistical error on the fit that is approximately $0.4 \%$ for an integrated luminosity of $20 \mathrm{fb}^{-1}$.

An error of $0.65 \%$ has a very large effect on the discovery CL when only one category is used. The reason is that a large fraction of signal events corresponds to a very low $s / b$, of the order of a percent. The effect can be reduced by applying a cut on the signal over background $s / b$. This corresponds to using events in a mass window around the analysed mass, until $s / b$ becomes smaller than the chosen cut. The optimal cut for this analysis is 0.02 .

When the events are split into categories the number of background events in each category is reduced on average by $1 / \mathrm{N}_{\text {cat }}$ and this increases the statistical error on the background estimation by approximately a factor $\sqrt{\mathrm{N}_{\text {cat }}}$, but this error is completely uncorrelated between the different categories. The error related to the uncertainty of the fit function remains constant and it is also uncorrelated between the different categories because, due to the different cuts the background shapes are different and described by different 

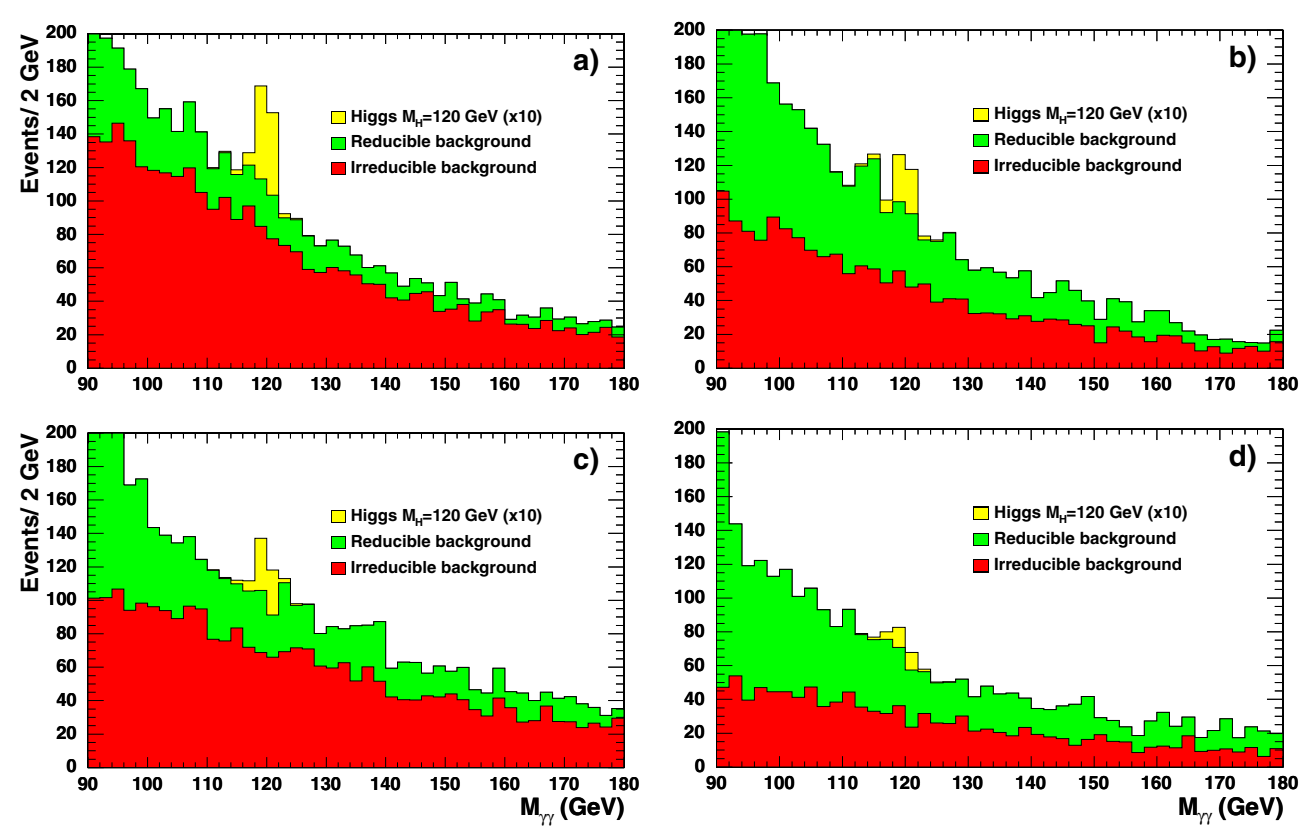

Figure 2.3. Invariant mass spectrum after the selection relative to the cut-based analysis with four categories defined in the text: barrel with large $R_{9}(a)$, barrel with small $R_{9}(b)$, endcaps with large $\mathrm{R}_{9}$ (c) and endcaps with small $\mathrm{R}_{9}(\mathrm{~d})$, Events are normalised to an integrated luminosity of $1 \mathrm{fb}^{-1}$ and the Higgs signal, shown for different masses, is scaled by a factor 10 .

Table 2.6. Fractions of events in each of the 12 categories and maximum $s / b$ in the mass region of $120 \mathrm{GeV} / \mathrm{c}^{2}$

\begin{tabular}{|c|c|c|c|c|c|c|c|c|}
\hline & \multicolumn{2}{|c|}{$|\eta|^{\max } \mid<0.9$} & \multicolumn{2}{|c|}{$0.9<|\eta|^{\max } \mid<1.4442$} & \multicolumn{2}{|c|}{$1.4442<|\eta|^{\max } \mid<2.1$} & \multicolumn{2}{|c|}{$|\eta|^{\max } \mid>2.1$} \\
\hline & frac. & $s / b$ & frac. & $s / b$ & frac. & $s / b$ & frac. & $s / b$ \\
\hline $\mathrm{R}_{9}^{\min }>0.948$ & $15.5 \%$ & $14.7 \%$ & $13.1 \%$ & $9.0 \%$ & $10.8 \%$ & $6.1 \%$ & $8.5 \%$ & $4.5 \%$ \\
\hline $0.9<\mathrm{R}_{9}^{\min }<0.948$ & $9.4 \%$ & $12.2 \%$ & $6.8 \%$ & $6.8 \%$ & $6.7 \%$ & $4.8 \%$ & $2.7 \%$ & $2.8 \%$ \\
\hline $\mathrm{R}_{9}^{\min }<0.9$ & $8.3 \%$ & $7.6 \%$ & $11.1 \%$ & $4.3 \%$ & $5.4 \%$ & $3.2 \%$ & $1.7 \%$ & $2.2 \%$ \\
\hline
\end{tabular}

functions. The total error is then less than the total error reduced by $1 / \mathrm{N}_{\text {cat }}$. This reduces the effect of the systematic error on the discovery.

The effect of the systematic error on the background estimation is also related to the signal over background of the analysis. A more sensitive analysis, for which a larger part of the signal has a higher $s / b$ ratio, is less affected by the same relative uncertainty on the background.

Clearly the current understanding of the background is affected by larger uncertainties such as: cross section, diphoton kinematic distributions and efficiency of the selection (mainly affected by jet fragmentation, pile-up and by the structure of the underlying events).

The systematic error on the signal, that as has been mentioned has no effect on the discovery $\mathrm{CL}$, has contributions from the theoretical uncertainty of the cross section $(+15-12 \%$ from the scale variation and $+4-5 \%)$, from the measurement of the integrated luminosity $(\sim 5 \%)$, from the trigger $(\sim 1 \%)$, from the analysis selection (that will be measured for example with $\mathrm{Z} \rightarrow \mu \mu \gamma)$ and from the uncertainties on the photon energy resolution. 
Table 2.7. Integrated luminosity needed to discover or exclude the Higgs boson with mass $120 \mathrm{GeV} / \mathrm{c}^{2}$ with or without taking into account the systematic errors $\left(\mathrm{fb}^{-1}\right)$.

\begin{tabular}{lcccccc}
\hline Analysis & $\begin{array}{c}5 \sigma \text { discovery } \\
\text { no syst }\end{array}$ & $\begin{array}{c}5 \sigma \text { discovery } \\
\text { syst }\end{array}$ & $\begin{array}{c}3 \sigma \text { evidence } \\
\text { no syst }\end{array}$ & $\begin{array}{c}3 \sigma \text { evidence } \\
\text { syst }\end{array}$ & $\begin{array}{c}95 \% \text { exclusion } \\
\text { no syst }\end{array}$ & \multicolumn{2}{c}{$\begin{array}{c}95 \% \text { exclusion } \\
\text { syst }\end{array}$} \\
\hline counting exp. & 27.4 & 48.7 & 10.0 & 13.2 & 4.5 & 6.5 \\
1 category & 24.5 & 39.5 & 8.9 & 11.5 & 4.1 & 5.8 \\
4 categories & 21.3 & 26.0 & 7.5 & 9.1 & 3.5 & 4.8 \\
12 categories & 19.3 & 22.8 & 7.0 & 8.1 & 3.2 & 4.4 \\
\hline
\end{tabular}

Other effects that could modify the ability to discover the Higgs boson are: uncertainties on the structure of the underlying events, that could change the efficiency of the primary vertex determination and the amount of material in the tracker before the electromagnetic calorimeter.

The effect on the performances of the analysis of an increase of $20 \%$ of the tracker material has been evaluated. The main effects on such change on the analysis would be:

- increase of the inefficiency of the track isolation requirements for early photon conversions, before or inside the second layer of the pixel detector.

- increase of the inefficiency of ECAL isolation cut;

- decrease of the value of $\mathrm{R}_{9}$ for all photons that would cause a migration of events from more sensitive categories to less sensitive categories.

It was estimated that such change would increase the luminosity needed to achieve a given discovery CL of approximately $6 \%$. Given that the amount of tracker material will be known with a precision of $\sim 2 \%$ the related systematic error is less than $1 \%$.

In what follows a conservative $20 \%$ systematic error on the signal is assumed. It affects exclusion of a signal, not discovery, since the signal rate is directly measured from data in case of discovery.

2.1.4.4. Results of the cut-based analysis. Table 2.7 shows the integrated luminosity needed to obtain $5 \sigma$ discovery or $95 \% \mathrm{CL}$ exclusion for a $120 \mathrm{GeV} / \mathrm{c}^{2}$ mass Higgs boson with the different splittings. The effect of the systematic errors is also shown. We can observe how the performance increases and the effect of the error on the background estimation decreases with the number of categories. In the three cases (1, 4 and 12 categories) the event selection is the same and that the differences in performance come from the splitting of the total sample in different sub-samples with different sensitivities $(s / b)$. In the split category analyses the computation of the log-likelihood ratio estimator is made separately for each $1 \mathrm{GeV} / \mathrm{c}^{2}$ bin in mass, whereas in the "counting experiment" only a single (optimum) mass window is evaluated.

The integrated luminosity needed for discovery and exclusion, using the 12-category analysis, for the mass range studied between 115 and $150 \mathrm{GeV} / \mathrm{c}^{2}$ are shown in the plots at the end of the section (Fig. 2.10). The Higgs boson can be discovered with mass between 115 and $140 \mathrm{GeV} / \mathrm{c}^{2}$ with less than $30 \mathrm{fb}^{-1}$ and excluded in the same mass range, at $95 \% \mathrm{CL}$, with less than $5 \mathrm{fb}^{-1}$.

As mentioned before, all these results have been obtained assuming an intercalibration of the ECAL, after having collected an integrated luminosity of $10 \mathrm{fb}^{-1}$. With the whole ECAL intercalibrated to a precision better than $0.5 \%$ over all the solid angle, the results improve such that approximately $10 \%$ less integrated luminosity is needed for discovery. 


\subsubsection{Optimised analysis estimating $s / b$ for each event}

In the optimised analysis 6 categories are used, 3 in which both photons are in the barrel and 3 in which at least 1 photon is in an endcap. The 3 categories are defined, as for the cut-based analysis, to have the lowest $R_{9}$ photon candidate with $R_{9}>0.948,0.948>R_{9}>0.90$ and $\mathrm{R}_{9}<0.90$ respectively. The categories are labelled with numbers from 0 to 5: first, the 3 barrel categories with decreasing values of $\mathrm{R}_{9}$ then the 3 endcap categories again with decreasing values of $R_{9}$.

2.1.5.1. Mass distributions in categories. The diphoton mass distributions enable the separation of signal from background. Signal peaks sharply at the Higgs mass while the backgrounds are quite smooth. This allows good estimation of the magnitude of the background under the peak.

The best mass resolution and the best $s / b$ ratio in the peak is found in category 0 , with high $\mathrm{R}_{9}$ in the barrel.

2.1.5.2. Loose selection of events for optimised analysis. Isolation requirements are applied to photon candidates prior to the computation of the neural network isolation variables $\mathrm{NN}_{\text {isol }}$ :

- the transverse $E_{\mathrm{T}}$ of the photon candidates must be larger than $40 \mathrm{GeV}$ and the absolute value of their pseudo-rapidity less than 2.5 ;

- no tracks with $p_{\mathrm{T}}$ larger than $1.5 \mathrm{GeV} / \mathrm{c}$ must be present inside a cone with $\Delta \mathrm{R}<0.1$ around the photon candidate;

- the total $E_{\mathrm{T}}$ of all ECAL island basic clusters with $\Delta \mathrm{R}<0.3$ around the photon candidate, excluding those belonging to the super-cluster itself must be less than $5 \mathrm{GeV}$;

- the total transverse energies of HCAL towers within $\Delta \mathrm{R}<0.35$ around the photon candidate must be less than $35 \mathrm{GeV}$;

- the sum of the transverse momenta of charged tracks within $\Delta \mathrm{R}<0.2$ around the photon candidate must be less than $100 \mathrm{GeV} / \mathrm{c}$.

Before optimising the final analysis, some additional cuts are applied. These both simplify the neural network training and slightly improve the performance. It is required that:

- the events pass the double photon High Level Trigger;

- the isolation neural net output is greater than 0.25 for both photons.

2.1.5.3. Optimised use of kinematic variables to separate signal and background. In addition to the mass, there are kinematic differences between signal and background. In particular the signal has a harder photon $E_{\mathrm{T}}$ distribution than the background - the background can have a high mass by having a large $\eta$ difference between the photon candidates. Weak Boson Fusion and associated production of a Higgs with other massive particles enhance these differences between signal and background. The large, reducible backgrounds often have photon candidates that are not well isolated.

As with the Higgs searches performed at LEP, higher performance can be achieved if the expected signal over background, $s / b$, is estimated for each event. This is particularly effective if, the $s / b$ varies significantly from event to event. This is the case here due to wide variations in photon isolation and photon $E_{\mathrm{T}}$. There is also significant dependence of the $s / b$ on photon conversion and on location in the detector.

One photon isolation variable $\mathrm{NN}_{\text {isol }}$ for each photon, is combined with kinematic variables to help separate signal and background. A neural net is trained to distinguish background events, taken from the mass side-bands, from signal Monte Carlo events. There is 


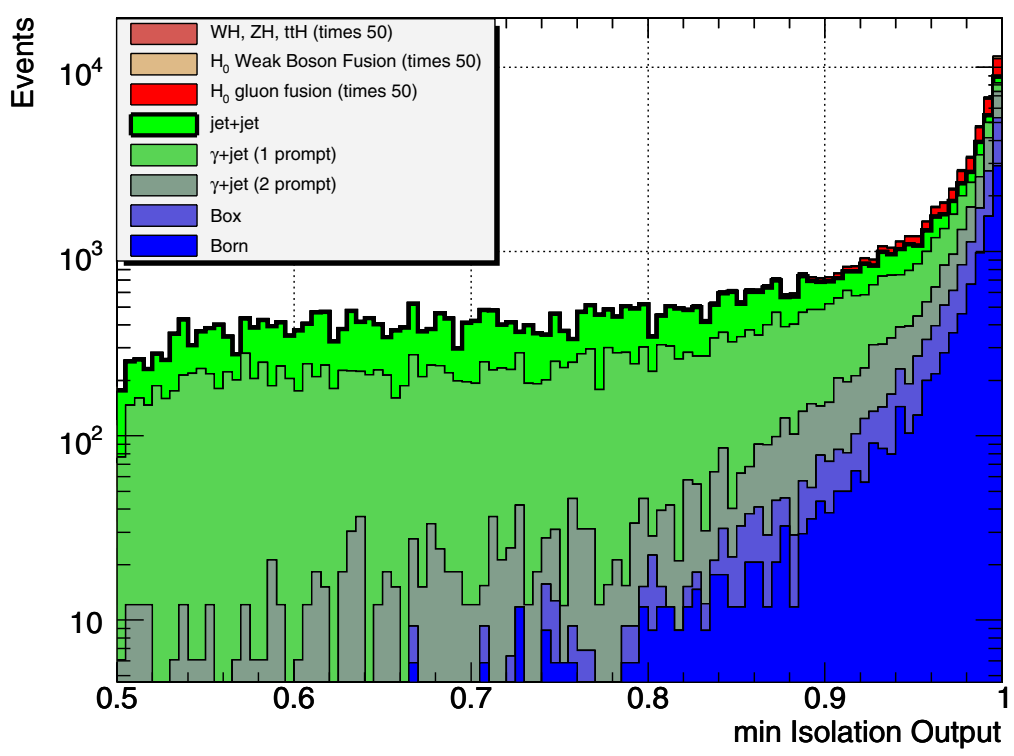

Figure 2.4. Distribution of the minimum value of the $\mathrm{NN}_{\mathrm{isol}}$ variables of the two photon candidates. Events are normalised to an integrated luminosity of $7.7 \mathrm{fb}^{-1}$ and the signal $\left(\mathrm{M}_{\mathrm{H}}=120 \mathrm{GeV} / \mathrm{c}^{2}\right)$ is scaled by a factor 50 .

no danger of over-training since background events from the signal mass region are not used and independent samples are used for the signal Monte Carlo. The input variables are devised to be insensitive to the diphoton mass so that the background rejection due to the kinematics and isolation is independent of the background rejection from the mass distribution.

Six variables are used as inputs to a neural net. They are the isolation $\mathrm{NN}$ outputs $\mathrm{NN}_{\text {isol }}$ for the 2 photons, the transverse energies of the 2 photons, normalised to the diphoton mass, the absolute value of the rapidity difference between the 2 photons, and the longitudinal momentum of the photon pair.

The distributions of the input variables are shown for signal and background in Figs. 2.4 and 2.5. Kinematic information that are likely to be highly sensitive to higher order corrections to the background simulation has not been used. Such information, like the $E_{\mathrm{T}}$ of the Higgs boson candidate, the $E_{\mathrm{T}}$ transverse to the photon direction, and information about additional jets will ultimately be useful but may not be reliable until better simulations or actual data are available to train on.

The neural net is trained in each of the 6 categories independently. The net has 6 input nodes, 12 intermediate nodes in a single layer, and 1 output node. The error function has been modified from the standard to improve training toward a high signal over background region. A minimum neural net output cut is applied that eliminates $1 \%$ of the signal in each category and a function is fit to the distribution above that cut. These functions are used to bin the data and to smooth the background in a limited region.

It is useful to examine the neural net output distribution for events from different sources (Fig. 2.6). Low NN outputs are dominated by photon candidates from jets which are not well isolated. The large peak at 0.85 represents both signal and background where the photon is relatively well isolated and the photon $E_{\mathrm{T}}$ is $\mathrm{M}_{\mathrm{H}} / 2$, corresponding to events with a large value of $\mathrm{NN}_{\text {isol }}$. Higher photon $E_{\mathrm{T}}$ events are found in the peak near 1 . There is an enhancement of the signal, particularly for the WBF and associated production processes. The background there is dominated by events with at least one jet interpreted as a photon. 

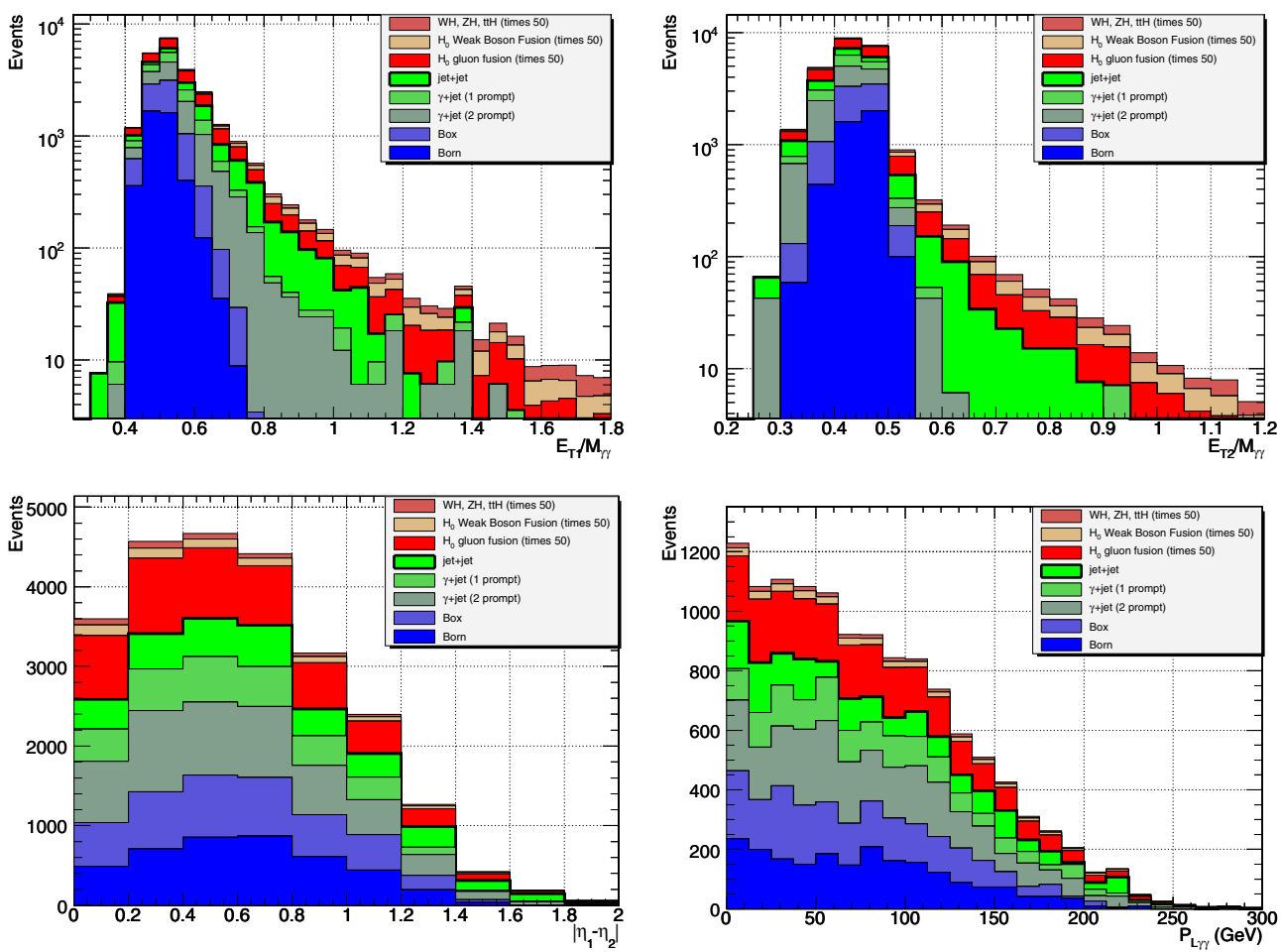

Figure 2.5. Distribution of the kinematic inputs to the neural network for signal and background sources. A value of the neural net output is required to be greater than 0.85 . Events are normalised to an integrated luminosity of $7.7 \mathrm{fb}^{-1}$ and the signal $\left(\mathrm{M}_{\mathrm{H}}=120 \mathrm{GeV} / \mathrm{c}^{2}\right)$ is scaled by a factor 50 .

2.1.5.4. Estimation of signal to background ratio for each event. In order to get the most information out of each event, the signal over background is estimated for each event. In the simplest analyses, cuts are applied to select only high signal over background events and those are counted. Such a simple analysis looses information because, some of the events that are cut could contribute to the measurement and because, some of the events that are accepted are not used optimally.

Events in the mass peak for the Higgs mass hypothesis under consideration have high signal over background expectation while events outside the peak have lower expected $s / b$. Similarly, events at high $\mathrm{NN}_{\text {kin }}$ output have higher $s / b$ expectation. The kinematics and isolation information in $\mathrm{NN}_{\text {kin }}$ has been made independent of mass information so the two $s / b$ ratios can be multiplied to get a good estimate of the $s / b$ expectation for the event:

$$
\left(\frac{s}{b}\right)_{\mathrm{est}}=\left(\frac{s}{b}\right)_{\text {mass }} \times\left(\frac{s}{b}\right)_{\text {kin }} \text {. }
$$

This is an estimate that is to bin signal and background events. If the estimate is bad, the performance of the analysis suffers because good $s / b$ events are not well separated from bad ones. It is not possible for a bad estimate to make the analysis appear to perform too well. The $s / b$ estimate need not be normalised correctly, since it is a relative number used to bin events.

The events are binned according to the $s / b$ estimate. Histograms are made in each of the six categories. The actual signal to background ratio is computed for the binned events and 


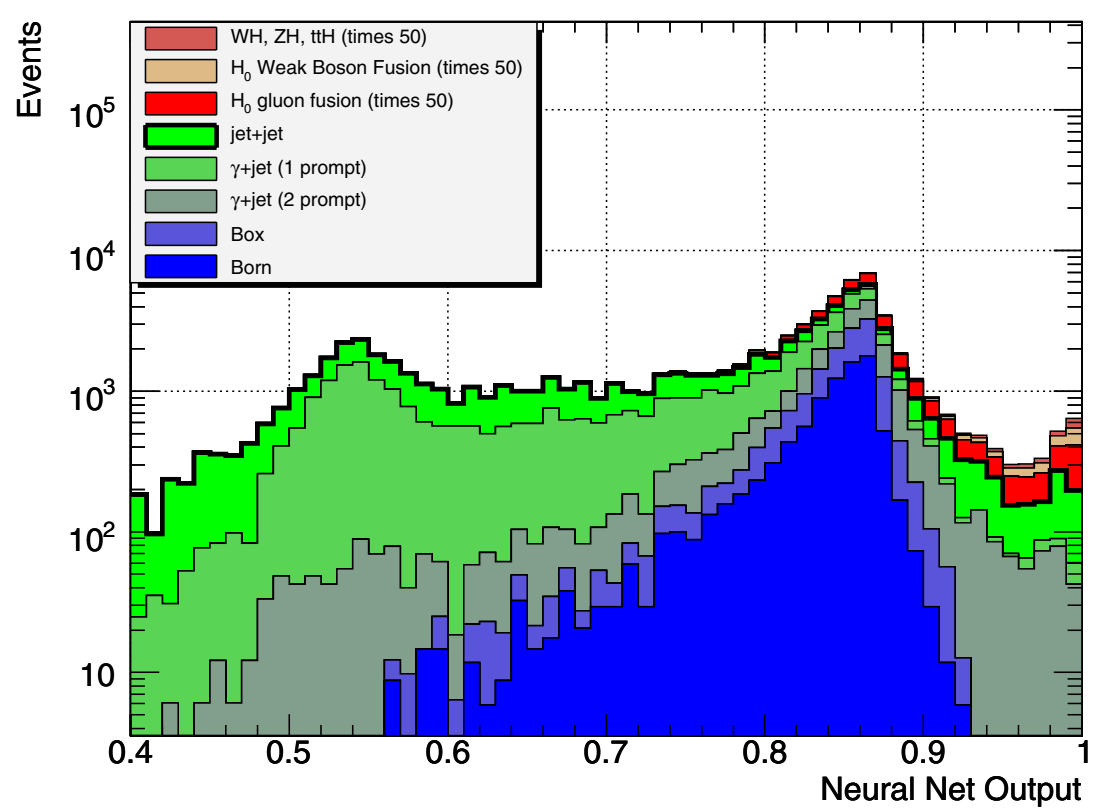

Figure 2.6. The neural net output for events in the barrel for each signal $\left(\mathrm{M}_{\mathrm{H}}=120 \mathrm{GeV} / \mathrm{c}^{2}\right)$ and background source. Events are normalised to an integrated luminosity of $7.7 \mathrm{fb}^{-1}$ and the Higgs signal is scaled by a factor 50 .

used to calculate confidence levels that data are consistent with a background-only hypothesis or with a signal-plus-background hypothesis.

2.1.5.5. Smoothing the background. The $\mathrm{H} \rightarrow \gamma \gamma$ channel has the good feature that the mass is essentially independent of isolation and suitably chosen kinematic variables. With this factorisation assumption, background can be smoothed well even in regions with low statistics.

The background expectation in a bin must be reliably estimated in order to correctly calculate confidence levels. Downward fluctuations in the background estimation can have a significant impact on the CL. The number of simulated events for the irreducible (jet) backgrounds is about one seventh of the number that will be available in the data at the time it would be expected to discover the Higgs. Therefore, problems with background estimation are even more difficult now than they will be when we have data.

The background distributions are very smooth in the mass variable, so the distribution in mass can be reliably smoothed. This is done by spreading each event over a $\pm 5 \mathrm{GeV} / \mathrm{c}^{2}$ region according to the functions fit to the mass distribution. A wider mass region could be used but this would interfere with the training of the analysis on an independent sample in the mass side-bands.

The background distribution in the neural net output is also smoothed over a region of \pm 0.05 using the fit functions. It is therefore, quite important that the background fit functions accurately represent the neural net distribution. In the smoothing process, the normalisation of the background is carefully maintained to high accuracy.

With this two-dimensional smoothing accurate background expectations are obtained except in the regions with extremely small amounts of background. In such regions, bins must be combined until sufficient background events are available. If a $s / b$ bin has too few MC 

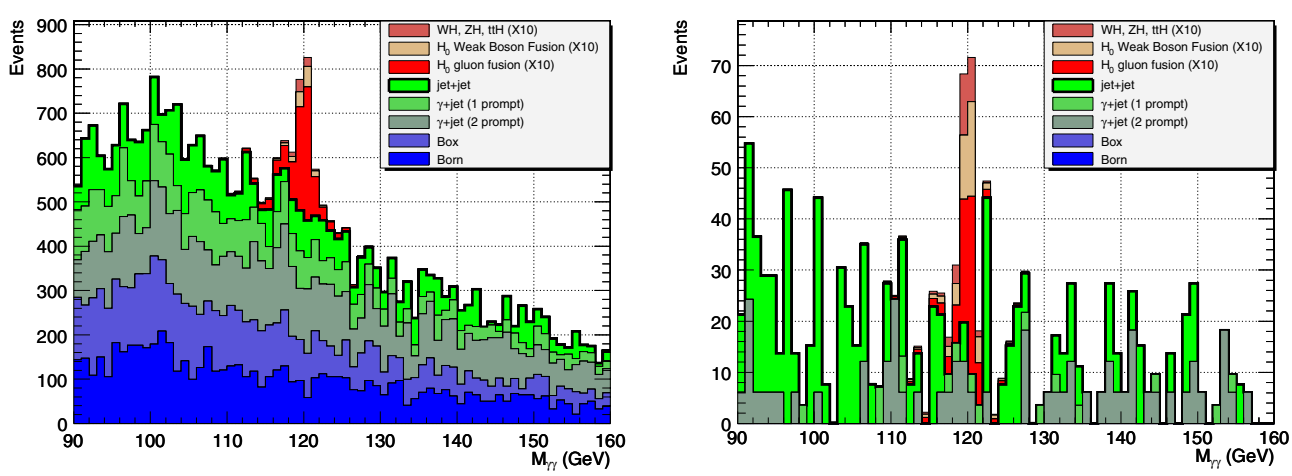

Figure 2.7. The diphoton mass distribution for each source for barrel events with kinematic neural net output greater than 0.85 (left) and 0.97 (right). Events are normalised to an integrated luminosity of $7.7 \mathrm{fb}^{-1}$ and the Higgs signal $\left(\mathrm{M}_{\mathrm{H}}=120 \mathrm{GeV} / \mathrm{c}^{2}\right)$ is scaled by a factor 10 .

background events contributing to it, it is combined with the nearest (lower $s / b$ ) bin. This is continued until there are sufficient events. This combination clearly reduces the sensitivity of the analysis but cannot be avoided without a more detailed understanding of the background, which is a goal for the future. At present, at least 20 Monte Carlo background events are required in a bin. Since the current MC samples contain about seven times less events than expected in the data, significant improvements are possible, allowing higher $s / b$ bins to be used, resulting in better performance.

Figure 2.7 shows the mass distributions for barrel events with two different cuts on the neural net output. The looser cut simply excludes most of the obviously non-isolated candidates. It can be seen that all of the backgrounds are important at this level. The tighter cut highly enhances the $s / b$ ratio and emphasises the importance of smoothing, which has not been applied to the background in this distribution.

Figure 2.8 shows the mass distribution for neural net output greater than 0.97 in category 0 . Again it is clear that smoothing in two dimensions is needed to get a reasonable estimate of the background. It is useful to note that even in this very high $s / b$ region, the largest contribution to the signal is from gluon fusion, although the relative contributions of the other production processes has increased.

2.1.5.6. Combination of categories into final $s / b$ distribution. At this point the signal and background is binned in $s / b$ in six categories. These could be used to calculate the confidence level, however, it seems most useful, in the light of future plans to analyse separate channels, to combine the categories into one $s / b$ plot in a similar way as may be used to recombine channels. The six histograms are combined into one which can be used calculate confidence levels. The combination is based on the actual signal to background in each bin. In principle, this is the same as combining results from different channels or even from different experiments in a way that makes optimal use of all channels and does not pollute high quality channels with data of lesser purity.

The final binning of data into $s / b$ bins is shown in Fig. 2.9. The plot extends from very low signal to background to a small number of events with $s / b>1$.

The relative contribution of barrel and endcap categories can be estimated from the total LLR computed and LLRs computed excluding each category. The six categories have rather widely varying contributions to the Log Likelihood Ratio and hence to the performance of the analysis. Table 2.8 shows the fraction of signal and the fraction of the LLR for each category. 


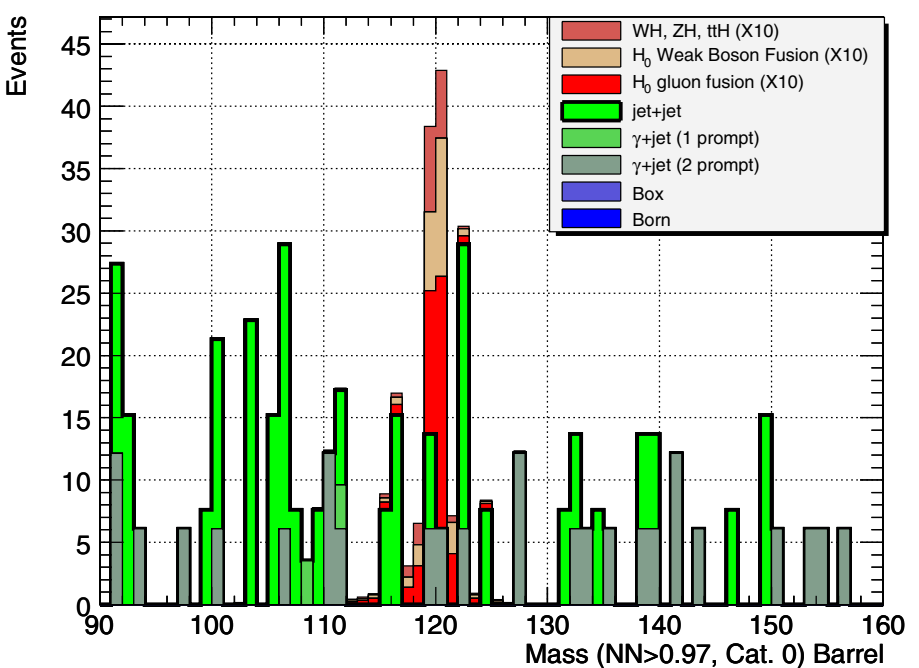

Figure 2.8. The diphoton mass distribution for each source for barrel events with kinematic neural net output greater than 0.97 and $\mathrm{R}_{9}>0.948$. Events are normalised to an integrated luminosity of $7.7 \mathrm{fb}^{-1}$ and the Higgs signal $\left(\mathrm{M}_{\mathrm{H}}=120 \mathrm{GeV} / \mathrm{c}^{2}\right)$ is scaled by a factor 10 .

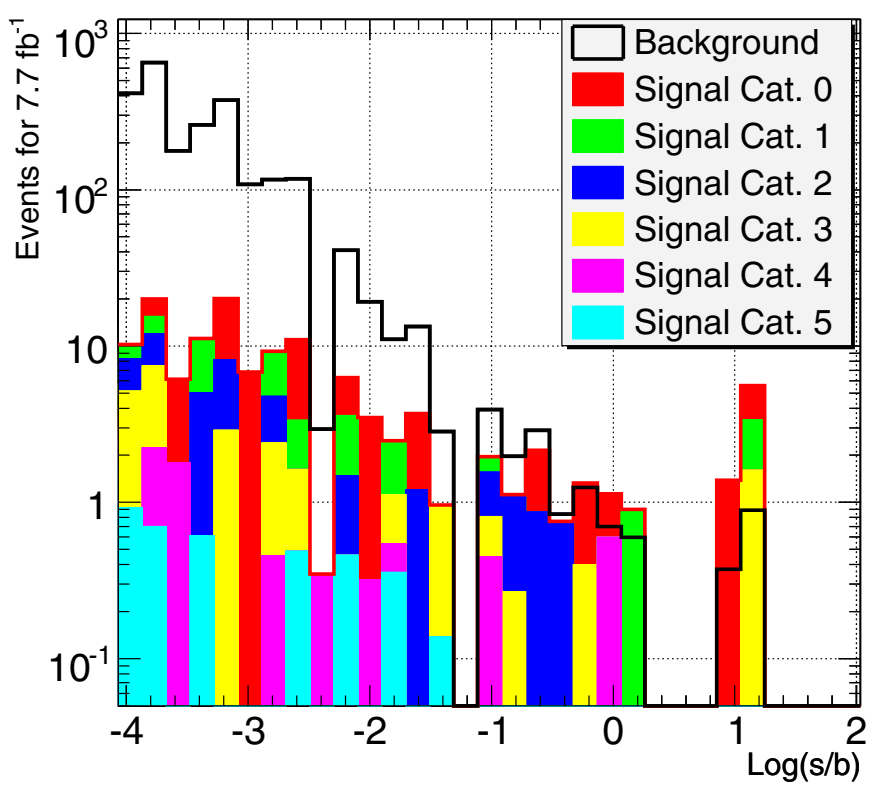

Figure 2.9. The final distribution of binned signal $\left(\mathrm{M}_{\mathrm{H}}=120 \mathrm{GeV} / \mathrm{c}^{2}\right)$ and background in $\log (s / b)$ for an integrated luminosity of $7.7 \mathrm{fb}^{-1}$. Here the Higgs signal is normalised to the integrated luminosity and the statistics benefits of the smoothing of the background. Signal and background events are added independently.

Some of the categories have a fairly small effect on the final result. This remains true after the application of systematic normalisation uncertainties described below. It is clear that photon conversions result in a significant deterioration of the performance. It is hoped to mitigate this somewhat by using the conversion track reconstruction in the future, but the poorer mass resolution cannot be recovered and a big effect is not expected. 
Table 2.8. Performance in the six categories for $\mathrm{M}_{\mathrm{H}}=120 \mathrm{GeV} / \mathrm{c}^{2}$.

\begin{tabular}{lcr}
\hline Category & Signal\% & LLR \% \\
\hline 0 & 27.8 & 48.0 \\
1 & 16.1 & 24.8 \\
2 & 21.7 & 11.9 \\
3 & 16.6 & 9.7 \\
4 & 9.0 & 4.1 \\
5 & 8.8 & 1.5 \\
\hline
\end{tabular}
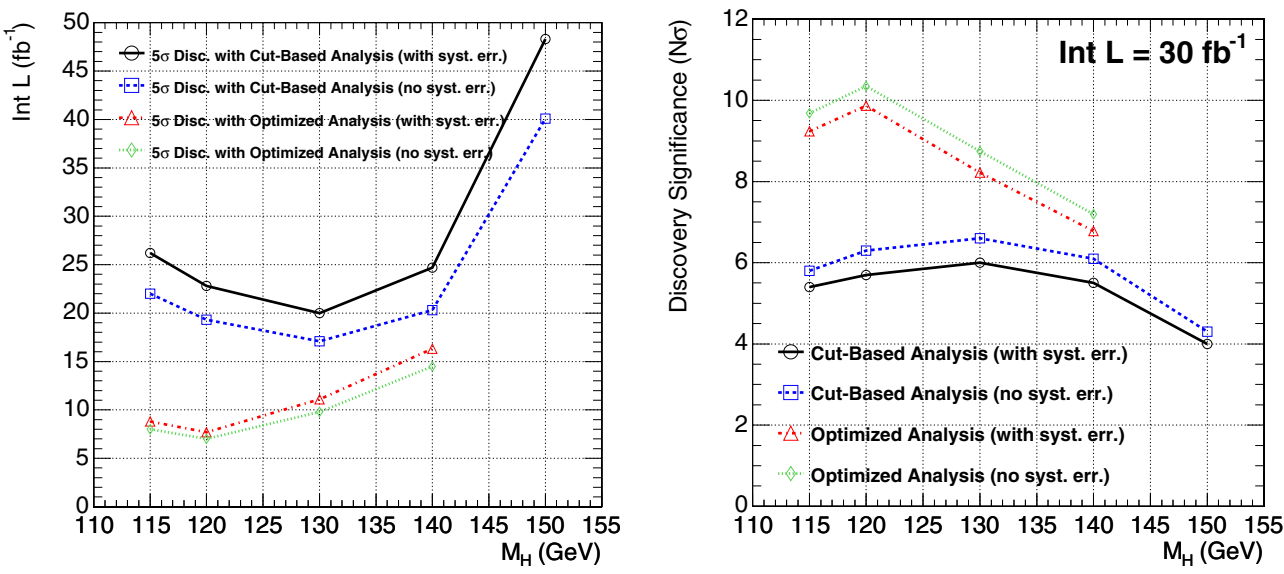

Figure 2.10. Integrated luminosity needed for a $5 \sigma$ discovery (left) and discovery sensitivity with an integrated luminosity of $30 \mathrm{fb}^{-1}$ (right) with the optimised analysis. The results from the cut-based analysis in 12 categories are also shown for comparison.

2.1.5.7. Results of the optimised analysis. The same estimates of systematic error are used to obtain the results in the optimised analysis as are used in the cut-based analysis. Most of the development and studies have been made for a Higgs mass of $120 \mathrm{GeV} / \mathrm{c}^{2}$. For this mass, a $5 \sigma$ discovery can be made with about $7 \mathrm{fb}^{-1}$ luminosity. A $1 \%$ background normalisation uncertainty corresponds to an increase of the luminosity needed for a $5 \sigma$ discovery from $7 \mathrm{fb}^{-1}$ to $7.7 \mathrm{fb}^{-1}$.

There is a great deal of uncertainty in this benchmark estimate of luminosity due to our poor understanding of the backgrounds we will contend with when the LHC starts running, however, this is not considered here as a systematic error on a discovery since, it is proposed to measure the background from the data. Figure 2.10 shows the luminosity needed for a $5 \sigma$ discovery and the discovery sensitivity with an integrated luminosity of $30 \mathrm{fb}^{-1}$ for several Higgs masses, both for the fully optimised analysis and for the cut-based analysis using 12 categories described in Section 2.1.4.4. It seems possible to discover, or at least have strong evidence for a low mass Higgs in the first good year of running.

\subsubsection{Measurement of the Higgs boson mass}

If the Higgs boson will be discovered in the $\mathrm{H} \rightarrow \gamma \gamma$ channel then we will be able to measure its mass. We have studied the mass measurements with the cut based analysis with two different methods: 
Table 2.9. Expected statistical errors on the Higgs boson mass measurement for $30 \mathrm{fb}^{-1}$.

\begin{tabular}{lccccc}
\hline $\mathrm{M}_{\mathrm{H}}$ & $115 \mathrm{GeV} / \mathrm{c}^{2}$ & $120 \mathrm{GeV} / \mathrm{c}^{2}$ & $130 \mathrm{GeV} / \mathrm{c}^{2}$ & $140 \mathrm{GeV} / \mathrm{c}^{2}$ & $150 \mathrm{GeV} / \mathrm{c}^{2}$ \\
\hline All events & $184 \mathrm{MeV} / \mathrm{c}^{2}$ & $184 \mathrm{MeV} / \mathrm{c}^{2}$ & $201 \mathrm{MeV} / \mathrm{c}^{2}$ & $222 \mathrm{MeV} / \mathrm{c}^{2}$ & $298 \mathrm{MeV} / \mathrm{c}^{2}$ \\
& $0.16 \%$ & $0.15 \%$ & $0.15 \%$ & $0.16 \%$ & $0.20 \%$ \\
\hline 12 & $127 \mathrm{MeV} / \mathrm{c}^{2}$ & $139 \mathrm{MeV} / \mathrm{c}^{2}$ & $129 \mathrm{MeV} / \mathrm{c}^{2}$ & $156 \mathrm{MeV} / \mathrm{c}^{2}$ & $204 \mathrm{MeV} / \mathrm{c}^{2}$ \\
categories & $0.11 \%$ & $0.12 \%$ & $0.10 \%$ & $0.11 \%$ & $0.14 \%$ \\
\hline
\end{tabular}

- measurement from the $\Delta \log ($ likelihood) using all events;

- measurement from the $\Delta \log ($ likelihood) using the cut-based analysis split in 12 categories.

The expected statistical errors are shown in Table 2.9 for an integrated luminosity of $30 \mathrm{fb}^{-1}$. The statistical errors simply scale with $1 / \sqrt{\text { Int L }}$. The errors are slightly asymmetric, due to the tail of the reconstructed Higgs mass distribution at lower masses, the positive error being approximately $10 \%$ smaller than the negative. The table shows the average between the two.

As we can see the statistical error will be 0.1 to $0.2 \%$ already with $30 \mathrm{fb}^{-1}$, when the significance of the discovery would be 5 to $6 \sigma$ with the cut based analysis. Of course, this measurement will be affected by the uncertainty of the absolute scale of the photon energy measurement that will be derived for example by the measurement of the $\mathrm{Z}$ mass in the radiative $\mathrm{Z}$ decays $\mathrm{Z} \rightarrow \mu \mu \gamma$.

\subsubsection{Summary}

A standard cut-based analysis can discover the Higgs boson with $5 \sigma$ significance between the LEP lower limit and $140 \mathrm{GeV} / \mathrm{c}^{2}$ with less than $30 \mathrm{fb}^{-1}$ of integrated luminosity. Approximately $5 \mathrm{fb}^{-1}$ are needed to exclude its existence in the same mass range.

It has been shown that the $\mathrm{H} \rightarrow \gamma \gamma$ channel can be used to discover a low mass Higgs with an integrated luminosity not too different from that needed for higher mass Higgs, $7.7 \mathrm{fb}^{-1}$ at $120 \mathrm{GeV} / \mathrm{c}^{2}$ with an analysis using an event by event estimation of the $s / b$ ratio. Because of the excellent mass resolution expected in the diphoton channel, the background rate and characteristics from the data can be determined from diphoton events at masses away from the Higgs mass hypothesis.

An inclusive analysis has been presented. In future the various signal channels will be identified by looking for additional jets, leptons, or missing energy. This will clearly improve the sensitivity of the analysis.

\subsection{Benchmark Channel: $H \rightarrow Z Z^{(*)} \rightarrow 4$ electrons}

One of the most promising road towards a discovery at the LHC of the Higgs boson postulated in the SM is via single production followed by a cascade decay into charged leptons, $\mathrm{H} \rightarrow \mathrm{ZZ}^{(*)} \rightarrow l^{+} l^{-} l^{+} l^{-}$.

The single Higgs boson production benefits from a high cross-section, with values of about $40 \times 10^{3} \mathrm{fb}$ at $m_{\mathrm{H}}=130 \mathrm{GeV} / \mathrm{c}^{2}$ and decreasing monotonically to about $10 \times 10^{3} \mathrm{fb}$ around $m_{\mathrm{H}}=300 \mathrm{GeV} / \mathrm{c}^{2}$. The production cross-section is dominated ( $\left.\gtrsim 80 \%\right)$ over this mass range by gluon-gluon fusion processes via triangular loops involving heavy quark (mostly the top quark) flavours. The branching ratio for the $\mathrm{H} \rightarrow \mathrm{ZZ}^{(*)}$ decay in the SM is sizeable for any $m_{\mathrm{H}}$ value above $130 \mathrm{GeV} / \mathrm{c}^{2}$. It remains above $2 \%$ for $m_{\mathrm{H}} \leqslant 2 \times M_{W}$ with a peak above $8 \%$ around $m_{\mathrm{H}} \simeq 150 \mathrm{GeV} / \mathrm{c}^{2}$, and rises to values of 20 to $30 \%$ for $m_{\mathrm{H}} \geqslant 2 \times m_{\mathrm{Z}}$. The 
$\mathrm{Z}$ bosons have a $10 \%$ probability to yield a pair of charged leptons. Thus, the decay chain $\mathrm{H} \rightarrow \mathrm{ZZ}^{(*)} \rightarrow l^{+} l^{-} l^{+} l^{-}$(in short $\mathrm{H} \rightarrow 4 l$ ) offers a possibly significant and very clean and simple multi-lepton final state signature for the SM Higgs boson at the LHC. An example of an event candidate in the CMS detector for the Higgs boson decay channel $\mathrm{H} \rightarrow \mathrm{ZZ}^{*} \rightarrow 4 \mathrm{e}$ is shown in colour plate $\mathrm{CP} 2$.

Ultimately, the channel can provide a precision determination of the Higgs boson mass and production cross-section. The anti-correlation of the $\mathrm{Z}$ spin projections in the $\mathrm{H} \rightarrow \mathrm{ZZ}$ decay and the polarisation of each $Z$ boson can be used to constrain, and eventually determine, the spin and $\mathrm{CP}$ quantum numbers of the Higgs resonance. Furthermore, the $\mathrm{ZZ}^{(*)}$ and $\mathrm{WW}^{(*)}$ decay modes are related via $S U(2)$ and the combination of channels could allow for cancellation of some systematic uncertainties in a determination of the Higgs coupling. But first and foremost is the necessity to be best prepared for a discovery at the LHC.

In this section, the discovery potential of the CMS experiment for the SM Higgs boson is discussed in the mass range of $120 \leqslant m_{\mathrm{H}} \leqslant 300 \mathrm{GeV} / \mathrm{c}^{2}$, focusing on the $4 e$ channel. The analysis [37] relies on a detailed simulation of the detector response in the experimental conditions of the first years of low luminosity LHC running. The signal and background Monte Carlo datasets used for this prospective are described in Section 2.2.1. The detailed High Level Trigger (HLT) and reconstruction algorithms used at each step of this analysis have been presented in [7]. Basic, and in part compulsory, triggering and pre-selection steps for data reduction are described in Section 2.2.2. Simple observables from the electron reconstruction are used to characterise the event signature for this pre-selection step. The final event selection relies on more involved requirements for primary electrons coupled with basic event kinematics and is presented in Section 2.2.3. The selection is optimised to preserve a best signal detection efficiency and highest significance for a discovery. Emphasis is put on realistic strategies for the control of experimental errors and the estimation of systematic uncertainties on physics background rates. These are described in Section 2.2.4. Results on the expected discovery reach of the SM Higgs boson in CMS in the $\mathrm{H} \rightarrow 4 e$ channel and for the measurement of its mass, width and cross-section are finally presented in Section 2.2.5.

\subsubsection{Datasets for signal and background processes}

Monte Carlo data samples for the signal from single SM Higgs boson production as well as for $\mathrm{SM}$ background from $\mathrm{ZZ}^{(*)}$ pair production, $\mathrm{tt}$ pair production and $\mathrm{Zb} \overline{\mathrm{b}}$ associated production are used. The signal and background processes are generated for pp collisions at the LHC at a centre-of-mass energy $\sqrt{\mathrm{pp}}=14 \mathrm{TeV}$, with pile-up conditions from multiple collisions as expected in a collider machine configuration providing an instantaneous luminosity of $2 \times 10^{33} \mathrm{~cm}^{-2} \mathrm{~s}^{-1}$ (of $\mathcal{O}(10) \mathrm{fb}^{-1} /$ year). All cross-sections are normalised within acceptance to Next to Leading Order (NLO) calculations. The event generators are interfaced with PHотоs $[38,39]$ for the simulation of QED final state radiations. The non-perturbative parton density functions (PDFs) in the proton are taken to be the CTEQ6 distributions [12].

The Higgs boson is produced via either gluon fusion and weak boson fusion processes. The $4 e$ signal samples are generated at various $m_{\mathrm{H}}$ with PYTHIA [24]. The Higgs boson is forced to decay into a $\mathrm{Z}$ boson pair. The $\mathrm{Z}$ bosons are subsequently forced to undergo a decay in electron-positron pair. The signal is normalised to the value of total cross-section at NLO calculated including all Higgs boson production processes via HIGLU [40], with branching ratios $B R\left(\mathrm{H} \rightarrow \mathrm{ZZ}^{(*)}\right)$ calculated via HDECAY [41].

In the $4 e$ channel (and similarly for the $4 \mu$ channel), an additional enhancement of the signal is considered which is due to the constructive final state interference between like-sign electrons originating from different $Z^{(*)}$ bosons [42]. This enhancement has been 
Table 2.10. Total cross-sections at NLO (pb), cross-section in the $4 e$ channel within acceptance $(\mathrm{fb})$, and number of accepted events in data samples available for analysis.

\begin{tabular}{lccr}
\hline$m_{\mathrm{H}}\left(\mathrm{GeV} / \mathrm{c}^{2}\right)$ & $\sigma_{N L O}(\mathrm{pb})$ & $\sigma_{N L O} \times B R \times A c c .(\mathrm{fb})$ & $N_{\text {simul. }}$ \\
\hline 115 & 47.73 & 0.27 & 10000 \\
120 & 44.30 & 0.48 & 10000 \\
130 & 38.44 & 1.11 & 10000 \\
140 & 33.69 & 1.78 & 10000 \\
150 & 29.81 & 1.94 & 10000 \\
160 & 26.56 & 0.92 & 10000 \\
170 & 23.89 & 0.43 & 10000 \\
180 & 21.59 & 0.98 & 10000 \\
190 & 19.67 & 3.58 & 10000 \\
200 & 17.96 & 3.94 & 10000 \\
250 & 12.37 & 3.07 & 10000 \\
300 & 9.58 & 2.60 & 10000 \\
\hline $\mathrm{ZZ} \mathrm{Z}^{(*)}$ & 29.0 & 20.2 & 150000 \\
$\mathrm{Zb} \bar{b}$ & 276.3 & 120.4 & 87000 \\
$\overline{\mathrm{t}}$ & 840 & 194.0 & 500000 \\
\hline
\end{tabular}

re-evaluated with COMPHEP [43] and amounts to a factor $1.130 \pm 0.006$ at $m_{\mathrm{H}}=$ $115 \mathrm{GeV} / \mathrm{c}^{2}$, slowly decreasing to a negligible value when approaching $m_{\mathrm{H}} \approx 2 m_{\mathrm{Z}}$.

The $\mathrm{ZZ}^{(*)} \mathrm{SM}$ background continuum is generated using PYTHIA [24]. This includes only the $t$-channel contribution with $q \bar{q}$ in the initial state. The missing $s$-channel might contribute up to $10 \%$ for low Higgs boson masses and can be neglected for higher masses. The differential cross-section is re-weighted using $m_{4 e}$ dependent NLO $K$-factors obtained with MCFM 4.1, with an average $K$-factor of $\left\langle K_{N L O}\right\rangle=1.35$. Both $\mathrm{Z}$ bosons are constrained within the mass range $5-150 \mathrm{GeV} / \mathrm{c}^{2}$ and are forced to decay into charged lepton pairs, with the $\tau$ leptons subsequently forced to undergo leptonic decays via $\tau \rightarrow \mu \nu$ or $\tau \rightarrow e \nu$. The missing $g g$ contribution is estimated to be of order $20 \%$ at LO [42], with $\pm 8 \%$ uncertainties and with unknown NLO $K$-factors. Recent calculations with TopReX [44] of the gluon fusion production process of two real $\mathrm{Z}$ confirm the above assumptions, and this contribution has been shown to remain stable after kinematic cuts for a $\mathrm{H} \rightarrow 4 l$ analysis. The cross-section here is simply increased by the mean expected contribution.

The $\bar{t} \bar{t}$ background sample is also generated with PYTHIA [24], with W bosons and $\tau$ leptons forced to leptonic decays, but with $b$ quarks left to decay freely. Both gluon fusion and quark annihilation initial states are simulated and the cross-section is normalised to the NLO value of $840 \pm 5 \%$ (scale) $\pm 3 \%$ (PDF) pb [45].

The $\mathrm{Zb} \overline{\mathrm{b}}$ background is generated using all lowest order $g g \rightarrow e^{+} e^{-} \mathrm{b} \overline{\mathrm{b}}$ and $q q^{\prime} \rightarrow e^{+} e^{-} \mathrm{b} \overline{\mathrm{b}}$ diagrams (excluding diagrams involving the SM Higgs boson) calculated with COMPHEP [43] and interfaced with PYTHIA [24] for showering and hadronisation. All possible combinations of quarks are considered in the initial state. The total LO cross-section for $m_{e e}>5 \mathrm{GeV} / \mathrm{c}^{2}$ is $115 \mathrm{pb}$ of which about $89 \%$ originates from $g g$ processes, $7.7 \%$ involve u-like quarks and $3.2 \%$ involve d-like quarks in the initial state. The hadronisation and decay of the b quarks are left free. A NLO $K$-factor of $2.4 \pm 0.3$ is applied. Signal and background events are filtered at generator level for further analysis if satisfying the following acceptance requirements: $\geqslant 2 e^{+}$ and $\geqslant 2 e^{-}$with $p_{\mathrm{T}}^{e}>5 \mathrm{GeV} / \mathrm{c}$ in $|\eta|<2.7$. In addition for the $\mathrm{Zb} \overline{\mathrm{b}}$ background, at least two $e^{+} e^{-}$pairs with invariant mass in the range $5-400 \mathrm{GeV} / \mathrm{c}^{2}$ are required. In Table 2.10 crosssections at NLO and after pre-selection, as well as number of events in data samples available for analysis after pre-selection are given. 
Detailed simulation of the CMS detector is performed using the official CMS simulation OSCAR. Reconstruction of physics objects is performed in ORCA.

\subsubsection{Data reduction}

The events of interest for the Higgs boson search in the $\mathrm{H} \rightarrow 4 e$ channel must satisfy a minimal set of requirements.

A first and compulsory condition for the events is to satisfy the CMS Level 1 (hardware) trigger conditions and the filtering of the (software) HLT. This triggering step is described in Section 2.2.2.1. The basic electron triggers are expected to be saturated by SM processes such as the single $\mathrm{Z}$ and $\mathrm{W}$ production. Further filtering is obtained with a minimal set of additional electron requirements as described in Section 2.2.2.2.

The pre-selection must preserve the signal acceptance, and especially the electron reconstruction efficiency, until later stages where the analysis can best profit from more involved algorithms applied to reduced event samples.

2.2.2.1. Triggering. The events must have satisfied the single $e$, double $e$ or double relaxed $e$ requirements at $\mathrm{L} 1 / \mathrm{HLT}$ level. The single e trigger requires one isolated (charged) "electromagnetic" object with a threshold set at a reconstructed transverse energy in the electromagnetic calorimeter (ECAL) of $E_{\mathrm{T}}=26 \mathrm{GeV}$. The double $e$ trigger requires two isolated (charged) "electromagnetic" objects, each above a threshold of $E_{\mathrm{T}}=14.5 \mathrm{GeV}$. In contrast, the double relaxed $e$ trigger does not imposed isolation for the (charged) "electromagnetic" objects and the increased rate is compensated by a higher threshold of $E_{\mathrm{T}}=21.8 \mathrm{GeV}$.

The trigger efficiency for the Higgs boson signal, normalised to the cross-section within acceptance as defined in Section 2.2.1, is above 95\% for masses $m_{\mathrm{H}}>130 \mathrm{GeV} / \mathrm{c}^{2}$.

2.2.2.2. Pre-selection of four electron candidates. Following the Level-1 and HLT filtering steps, the event candidates must further satisfy basic electron pre-selection requirements. These requirements are designed to reduce possible background sources involving "fake" electron contamination from QCD jets.

For Higgs bosons with a mass $m_{\mathrm{H}}$ below $300 \mathrm{GeV} / \mathrm{c}^{2}$, the $4 e$ final state always involves at least one (or few) low $p_{\mathrm{T}}^{e}$ electron(s). In the range of $m_{\mathrm{H}}$ values below the $\mathrm{Z}$ pair production threshold, where the $\mathrm{Z}$ and $\mathrm{Z}^{*}$ bosons themselves receive in general only small transverse momentum, the mean $p_{\mathrm{T}}^{e}$ of the softest electron falls in a range where a full combination of tracking and calorimetry information becomes important. The $p_{\mathrm{T}}^{e}$ spectra for signal events at $m_{\mathrm{H}}=150 \mathrm{GeV} / \mathrm{c}^{2}$ is shown in Fig. 2.11a. The softest electron, which generally couples to the off-shell $\mathrm{Z}^{(*)}$, has a most probable $p_{\mathrm{T}}^{e}$ value below $10 \mathrm{GeV} / \mathrm{c}$ for masses $m_{\mathrm{H}} \lesssim 140 \mathrm{GeV} / \mathrm{c}^{2}$. Hence, an excellent electron reconstruction is essential down to very low $p_{\mathrm{T}}^{e}$ values, well below the range of $p_{\mathrm{T}}^{e} \simeq 40-45 \mathrm{GeV} / \mathrm{c}$ for which the reconstruction will be best constrained in CMS via measurements with SM single $\mathrm{Z}$ and single $\mathrm{W}$ production. The control of systematic uncertainties from experimental data is a major issue for such low $p_{\mathrm{T}}^{e}$ electrons and this will be discussed in detail in Section 2.2.4.

This analysis makes use of the elaborate reconstruction procedures which have been introduced very recently in CMS and have been described in detail in Ref. [46]. The electron identification and momentum measurements are somewhat distorted by the amount of tracker material which is distributed in front of the ECAL, and by the presence of a strong magnetic field aligned with the collider beam $z$ axis. The procedures introduced in Ref. [46] provide new useful observables that allow to better deal with these detector effects, combining information from the pixel detector, the silicon strip tracker and the ECAL. 

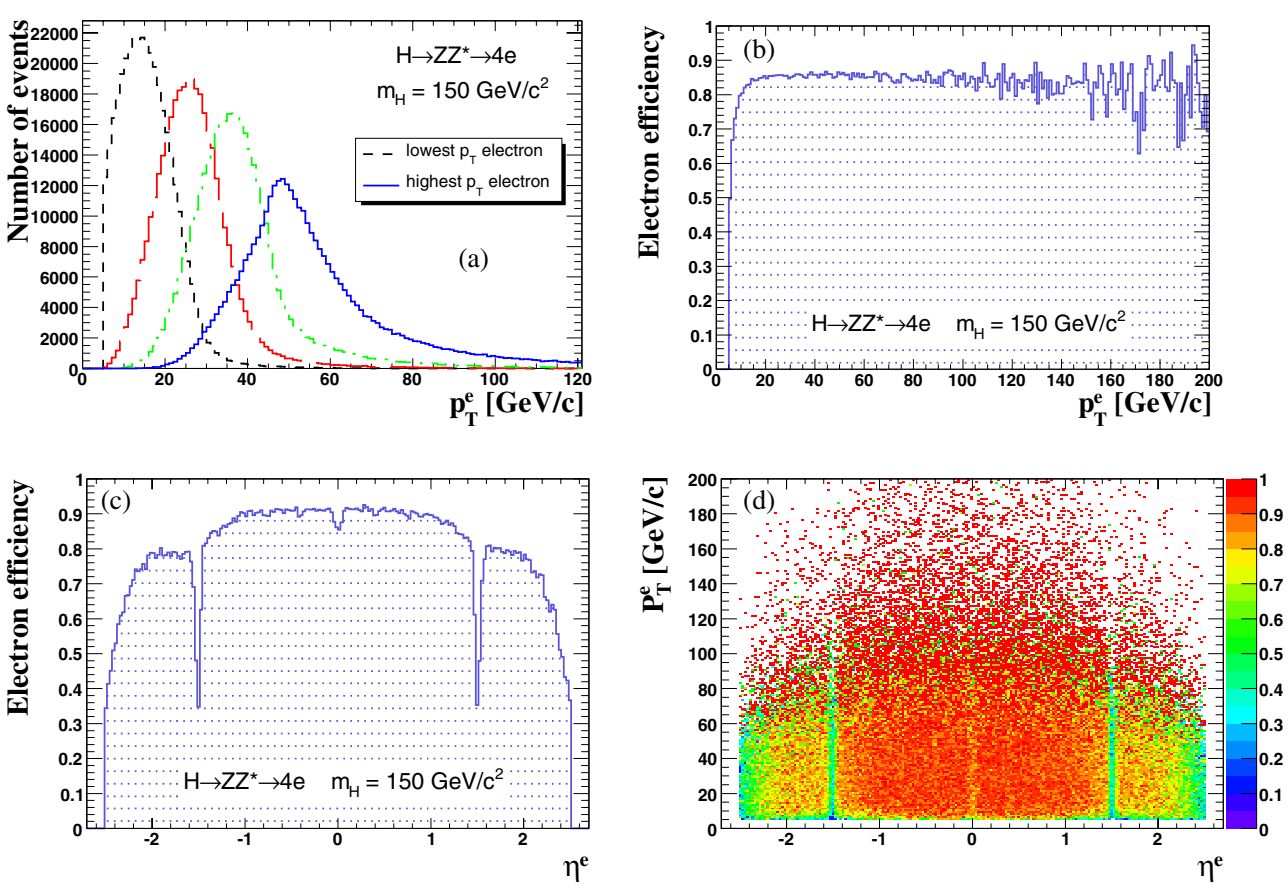

Figure 2.11. Electrons in SM Higgs boson $4 e$ decay channel for $m_{\mathrm{H}}=150 \mathrm{GeV} / \mathrm{c}^{2}$ : (a) transverse momentum of each of the four final state electrons; (b) efficiency at pre-selection as a function of $p_{\mathrm{T}}^{e}$; (c) efficiency at pre-selection as a function of $\eta_{e}$; (d) efficiency in the $p_{\mathrm{T}}^{e}$ versus $\eta_{e}$ plane.

The pre-selection of the signal event candidates relies on the presence of at least $2 e^{+}$and $2 e^{-}$candidates within the acceptance $|\eta|<2.5$ and each with $p_{\mathrm{T}}>5 \mathrm{GeV} / \mathrm{c}$, verifying the following characteristics:

- $E_{\mathrm{sc}} / p_{\text {in }}<3$, where $E_{\mathrm{sc}}$ is the supercluster energy and $p_{\text {in }}$ the track momentum at the interaction vertex;

- $\left|\Delta \phi_{\text {in }}\right|=\left|\phi_{\text {sc }}-\phi_{\text {in }}^{\text {extrap }}\right|<0.1$, where $\phi_{\text {sc }}$ is the energy weighted $\phi$ position of the supercluster and $\phi_{\text {in }}^{\text {extrap }}$ is the $\phi$ of the track at vertex, extrapolated to the ECAL assuming a perfect helix;

- $\left|\Delta \eta_{\text {in }}\right|=\left|\eta_{\text {sc }}-\eta_{\text {in }}^{\text {extrap }}\right|<0.02$, with notations as above;

- $H / E<0.2$, where $H$ is the energy deposited in the HCAL tower just behind the electromagnetic seed cluster and $E$ the energy of the electromagnetic seed cluster;

- $\sum_{\text {cone }} p_{\mathrm{T}}^{\text {tracks }} / p_{\mathrm{T}}^{e}<0.5$, a loose track isolation requirement, whose calculation will be described in Section 2.2.3.1.

The electron pre-selection efficiency is shown in Fig. 2.11b and Fig. 2.11c as a function of $p_{\mathrm{T}}^{e}$ and $\eta^{e}$ for the electrons from Higgs boson events at $m_{\mathrm{H}}=150 \mathrm{GeV} / \mathrm{c}^{2}$. The efficiency steeply rises and reaches a plateau around $86 \%$ for $p_{\mathrm{T}}^{e} \gtrsim 20 \mathrm{GeV} / \mathrm{c}$. The efficiency is above $90 \%$ for $|\eta| \lesssim 1.1$ and decreases towards the edge of the tracker acceptance when approaching $|\eta| \simeq 2.5$. The pre-selection efficiency for electrons from the same sample is represented in Fig. $2.11 \mathrm{~d}$ as a two-dimensional map in the $p_{\mathrm{T}}$ versus $\eta$ plane.

The absolute efficiencies for the Higgs boson signal at different $m_{\mathrm{H}}$ values and for the backgrounds are shown in Fig. 2.12a after triggering and the multi-electron pre-selection step. The acceptance for the Higgs boson signal is maintained above $50 \%$ in the full relevant mass range. 

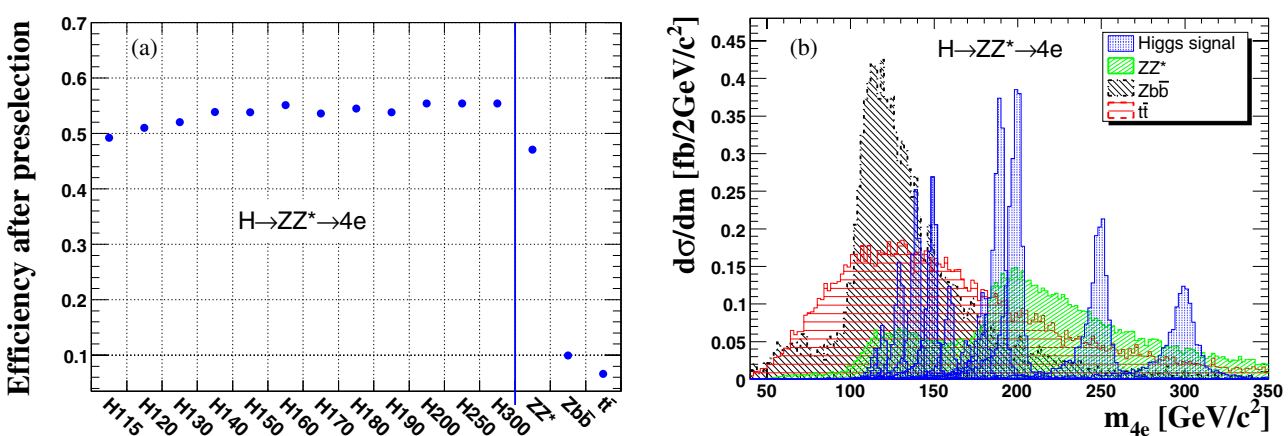

Figure 2.12. Higgs boson signal and dominant background sources after pre-selection step: (a) overall pre-selection efficiency for $m_{\mathrm{H}}$ in the range from 115 to $300 \mathrm{GeV} / \mathrm{c}^{2}$ and the background from $\mathrm{ZZ}^{(*)}$ continuum, from $\mathrm{Zb} \overline{\mathrm{b}}$ and $\overline{\mathrm{t}}$; (b) separate signal and background contributions to the spectrum of reconstructed invariant mass $m_{4 e}$.

The signal and background events fulfilling the triggering and pre-selection steps are represented in the reconstructed invariant mass $m_{4 e}$ spectrum in Fig. 2.12b. The Higgs boson signal is seen to emerge above the background for masses around $150 \mathrm{GeV} / \mathrm{c}^{2}$ and above $\simeq 2 m_{\mathrm{Z}}$. More background suppression is required elsewhere.

\subsubsection{Event selection and kinematic reconstruction}

The further steps of the event selection rely on a more detailed characterisation of the electron candidates and simple kinematic expectations. The electrons from the Higgs boson, in contrast to at least one $e^{+} e^{-}$pair from the $\overline{\mathrm{t}}$ and $\mathrm{Zb} \overline{\mathrm{b}}$ backgrounds, are isolated and originate from a common primary vertex. The corresponding analysis requirements are discussed in Section 2.2.3.1. Profiting from the expectation of a narrow resonance in the $m_{4 e}$ spectrum, and of the likely presence of a real $\mathrm{Z}$ boson in the final state, the kinematics and its simple evolution with $m_{\mathrm{H}}$ can be further exploited. The electrons of the $e^{+} e^{-}$pair at lowest $m_{e e}$ have on average a much harder $p_{\mathrm{T}}^{e}$ spectrum for the Higgs boson signal than for the $t \bar{t}$ and $t \bar{t}$ backgrounds. Moreover, the combination of the $\mathrm{Z}$ and $\mathrm{Z}^{(*)}$ mass spectra distinguishes the Higgs boson signal from the $\mathrm{ZZ}^{(*)} \mathrm{SM}$ background continuum. These kinematic requirements are discussed in Section 2.2.3.2.

2.2.3.1. Isolated primary electrons. A loose vertex constraint is first imposed on the longitudinal impact parameter for the four electron candidates in each event. All electrons should verify $I P_{L} / \sigma_{L}<13$, where $\sigma_{L}$ is the error on the longitudinal impact parameter $I P_{L}$. The main vertex constraint is imposed on the transverse impact parameter of the electrons to suppress secondary vertices. Secondary electrons appear for instance in semi-leptonic decays in the hadronisation of the $\mathrm{b}$ quark jets in $\mathrm{Zb} \bar{b}$ and $t \bar{t}$ background events. The sum of the transverse impact parameter significance $\left(I P_{\mathrm{T}} / \sigma_{\mathrm{T}}\right)$, i.e. the ratio of the transverse impact parameter $I P_{\mathrm{T}}$ over its error $\sigma_{\mathrm{T}}$, is shown in Fig. 2.13a (Fig. 2.13b) for the $e^{+} e^{-}$pair with invariant mass $m_{e e}$ closest (next-to-closest) to the nominal $\mathrm{Z}$ boson mass $m_{\mathrm{Z}}$. For both of these background sources, the displaced vertices are most likely to appear in the softest pair of reconstructed electrons. A best rejection power is obtained by imposing $\sum I P_{\mathrm{T}} / \sigma_{\mathrm{T}}<30$ for the pair with $m_{e e} \simeq m_{\mathrm{Z}}$ and a more stringent cut of $\sum I P_{\mathrm{T}} / \sigma_{\mathrm{T}}<15$ for the other pair. 

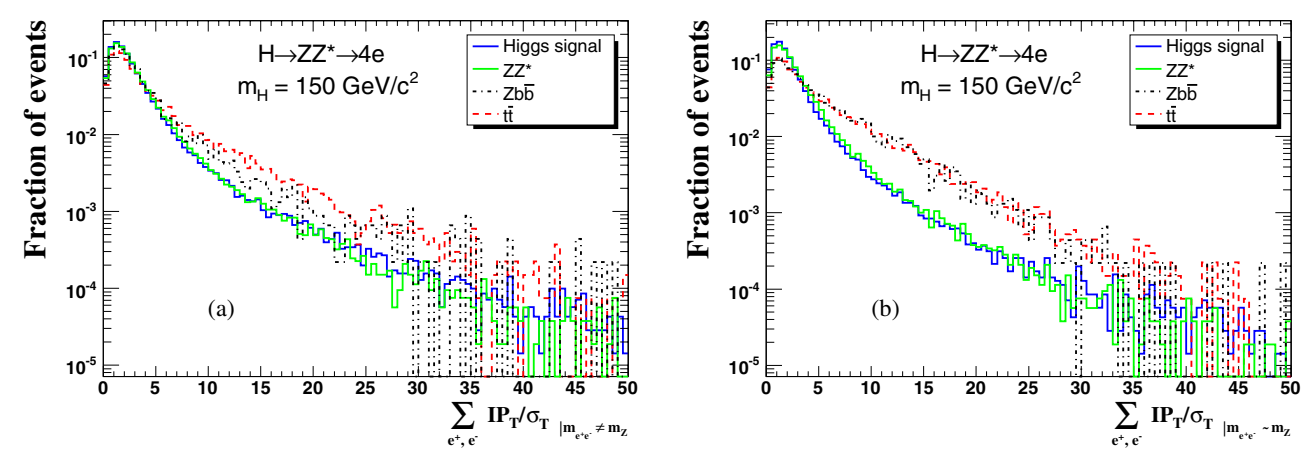

Figure 2.13. Sum of the transverse impact parameter significance $\left(I P_{\mathrm{T}} / \sigma_{\mathrm{T}}\right)$ of $e^{+} e^{-}$pairs for a Higgs boson at $m_{\mathrm{H}}=150 \mathrm{GeV} / \mathrm{c}^{2}$, for the $\mathrm{ZZ}^{(*)}$ continuum, and for $\mathrm{Zb} \overline{\mathrm{b}}$ and $\overline{\mathrm{t}}$ : (a) $\sum I P_{\mathrm{T}} / \sigma_{\mathrm{T}}$ from the electrons of the $e^{+} e^{-}$pair with a reconstructed mass $m_{e e}$ best matching the $\mathrm{Z}$ boson mass; (b) $\sum I P_{\mathrm{T}} / \sigma_{\mathrm{T}}$ from the second $e^{+} e^{-}$pair.

Another powerful discriminant against secondary electrons in $\mathrm{b}$ jets or in general against fake electrons in QCD jets, is provided by isolation requirements. The electrons coupled to the $\mathrm{Z}$ or $\mathrm{Z}^{(*)}$ in the $\mathrm{H} \rightarrow 4 e$ channel are expected to be on average well isolated from hadronic activity. Hadronic activity in single Higgs boson production appears in NLO processes, in the recoil against the Higgs boson. The Higgs boson itself generally receives a significant longitudinal boost in the laboratory reference frame but, as a scalar, decays uniformly in its centre-of-mass reference frame. In contrast, the electrons in the $\mathrm{b}$ jets from $\bar{t} \overline{\mathrm{t}}$ or $\mathrm{Zb} \overline{\mathrm{b}}$ are accompanied by significant hadronic activity.

Two partly complementary observables can be best used for the isolation of low $p_{\mathrm{T}}^{e}$ electrons. These rely either on measurements of primary tracks or on the energy flow in the hadronic calorimeter (HCAL). Both observables are insensitive to the eventual electroninduced electromagnetic showering in the tracker material. For the "track isolation", an isolation cone of size $\Delta R=\sqrt{\Delta \eta^{2}+\Delta \phi^{2}}=0.2$ is defined around the electron direction, and tracks with $p_{\mathrm{T}}>1.5 \mathrm{GeV} / \mathrm{c}$ originating from the same primary vertex within $\left|\Delta I P_{L}\right|<0.1$ $\mathrm{cm}$ are considered. To avoid suppressing signal events, tracks attached to an electron candidate of opposite charge, and giving $m_{e^{+} e^{-}}>10 \mathrm{GeV} / \mathrm{c}^{2}$, are discarded. All the 4 electrons from the Higgs boson candidate events must satisfy $\sum_{\text {cone }} p_{\mathrm{T}}^{\text {tracks }} / p_{\mathrm{T}}^{e}<0.1$. Distributions of this track isolation observable are shown in Fig. 2.14a. For the "hadronic isolation", all HCAL towers in an isolation cone size as above, and contributing with $E_{\mathrm{T}}>0.5 \mathrm{GeV}$ are considered in the ratio $\sum_{\text {cone }} E_{\mathrm{T}}^{H C A L} / p_{\mathrm{T}}^{e}$. This ratio is required to be below 0.05 for at least three electrons. The cut is relaxed to 0.2 for the fourth electron. Distributions of this hadronic isolation observable are shown in Fig. 2.14b.

Further electron identification requirements must be imposed to suppress the possible background, involving "fake" electrons, from Drell-Yan processes at NLO where a $Z^{(*)}$ recoils against jet(s). Different electron identification cuts are used depending on the distinct classes of track-supercluster electron patterns [46] in order to preserve the electron detection efficiency at all $\eta^{e}$. More details can be found in Ref. [37]. This tightening of the electron identification entails an absolute efficiency loss for the Higgs boson signal below $5 \%$.

2.2.3.2. Kinematics. The cascade $\mathrm{H} \rightarrow \mathrm{ZZ}^{(*)} \rightarrow 4 e$ for a Higgs boson, mostly produced at small transverse momentum, leads to very distinctly ordered $p_{\mathrm{T}}^{e}$ spectra for the four final state electrons. Moreover, the $p_{\mathrm{T}}^{e}$ spectra of the softest electrons for the Higgs boson signal is on average harder than the one expected from secondary electrons from the $\mathrm{Zb} \overline{\mathrm{b}}$ or $\overline{\mathrm{t}}$ backgrounds. Thus, it is advantageous to profit from the knowledge of the expected $p_{\mathrm{T}}^{e}$ 

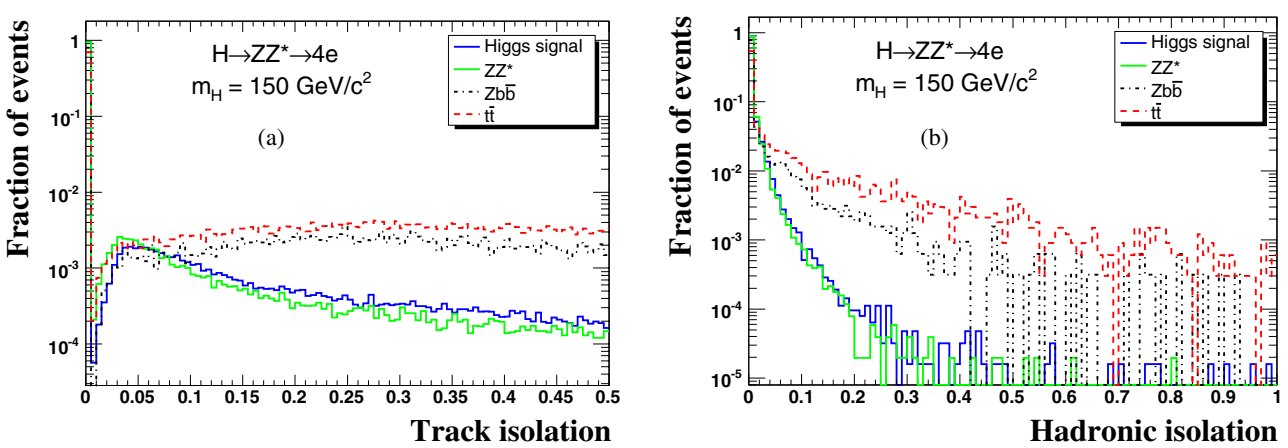

Figure 2.14. Electron isolation observables for the signal at $m_{\mathrm{H}}=150 \mathrm{GeV} / \mathrm{c}^{2}$ and the SM backgrounds: (a) track isolation, $\sum_{\text {cone }} p_{\mathrm{T}}^{\text {tracks }} / p_{\mathrm{T}}^{e}$; (b) hadronic isolation, $\sum_{\text {cone }} E_{\mathrm{T}}^{H C A L} / p_{\mathrm{T}}^{e}$, for the second least isolated electrons.

Table 2.11. Electron $p_{\mathrm{T}}$ cuts, from the lowest to the highest $p_{\mathrm{T}}$ electron and reconstructed $\mathrm{Z}_{1}$ and $\mathrm{Z}_{2}$ invariant mass cuts.

\begin{tabular}{|c|c|c|c|c|c|c|c|c|}
\hline $\begin{array}{l}m_{\mathrm{H}} \\
\left(\mathrm{GeV} / \mathrm{c}^{2}\right)\end{array}$ & $p_{\mathrm{T}}^{1}$ & $\begin{array}{c}p_{\mathrm{T}}^{2} \\
(\mathrm{G}\end{array}$ & $\begin{array}{l}p_{\mathrm{T}}^{3} \\
/ / \mathrm{c}\end{array}$ & $p_{\mathrm{T}}^{4}$ & $m_{\mathrm{Z}_{1}}^{\min }$ & $\begin{array}{r}m_{\mathrm{Z}_{1}}^{\max } \\
(\mathrm{Ge}\end{array}$ & $\begin{array}{l}m_{\mathrm{Z}_{2}}^{\min } \\
\left.{ }^{2}\right)\end{array}$ & $m_{\mathrm{Z}_{2}}^{\max }$ \\
\hline 115 & 7 & 10 & 10 & 15 & 51 & 101 & 10 & 50 \\
\hline 120 & 7 & 12 & 15 & 15 & 51 & 101 & 10 & 50 \\
\hline 130 & 7 & 12 & 15 & 15 & 61 & 101 & 10 & 60 \\
\hline 140 & 7 & 12 & 15 & 15 & 71 & 101 & 10 & 65 \\
\hline 150 & 7 & 12 & 15 & 15 & 71 & 101 & 15 & 65 \\
\hline 160 & 7 & 15 & 15 & 15 & 71 & 101 & 15 & 70 \\
\hline 170 & 7 & 15 & 15 & 20 & 81 & 101 & 20 & 80 \\
\hline 180 & 7 & 15 & 15 & 20 & 81 & 101 & 30 & 90 \\
\hline 190 & 7 & 15 & 20 & 30 & 81 & 101 & 40 & 100 \\
\hline 200 & 7 & 15 & 25 & 30 & 81 & 101 & 40 & 110 \\
\hline 250 & 7 & 20 & 40 & 50 & 51 & 131 & 20 & 200 \\
\hline 300 & 7 & 30 & 40 & 60 & 51 & 131 & 15 & 300 \\
\hline
\end{tabular}

distributions for the Higgs boson signal. A best set of $p_{\mathrm{T}}^{e}$ cuts as a function of $m_{\mathrm{H}}$ is given in Table 2.11.

The cut on the softest electron is maintained to a lowest value for simplicity and to preserve the signal efficiency at low $m_{\mathrm{H}}$. Otherwise the $p_{\mathrm{T}}^{e}$ cuts are seen to slowly evolve for as long as $m_{\mathrm{H}}<2 m_{\mathrm{Z}}$ and then rise faster above the $\mathrm{Z}$ pair production threshold. The $p_{\mathrm{T}}^{e}$ cuts lead for example [37] to a reduction by a factor of 5 to 10 of the $\mathrm{Zb} \overline{\mathrm{b}}$ background, and a factor of 3 to 5 of the $\overline{\mathrm{t}}$ background for $m_{4 e}<2 m_{\mathrm{Z}}$. Both backgrounds are also heavily suppressed above $2 m_{\mathrm{Z}}$.

Labelling $\mathrm{Z}_{1}$ the boson reconstructed with an $m_{e e}$ closest to the nominal $\mathrm{Z}$ mass and $\mathrm{Z}_{2}$ the one reconstructed from the second $e^{+} e^{-}$pair, one expects for $m_{4 e}<2 m_{\mathrm{Z}}$ in the case of the Higgs boson signal that $m_{4 e} \simeq m_{\mathrm{Z}_{1}}+m_{\mathrm{Z}_{2}}$ with most often the presence of a $\mathrm{Z}$ boson on its mass shell, $m_{\mathrm{Z}_{1}} \simeq m_{\mathrm{Z}}$. The $\mathrm{Z}$ boson masses saturate the phase space and are dominantly produced with small velocity in the Higgs boson rest frame. The requirement of one real $\mathrm{Z}$ boson suppresses further the $\bar{t} \bar{t}$ backgrounds for low $m_{4 e}$. The cut on $\mathrm{Z}_{2}$ is powerful against the $\mathrm{ZZ}^{(*)}$ continuum and further suppresses the $\mathrm{Zb} \overline{\mathrm{b}}$ and $\overline{\mathrm{t}} \overline{\mathrm{t}}$ backgrounds. A set of optimal $\mathrm{Z}_{1}$ and $\mathrm{Z}_{2}$ cuts is given in Table 2.11 as a function of $m_{\mathrm{H}}$. The cuts lead for example [37] 

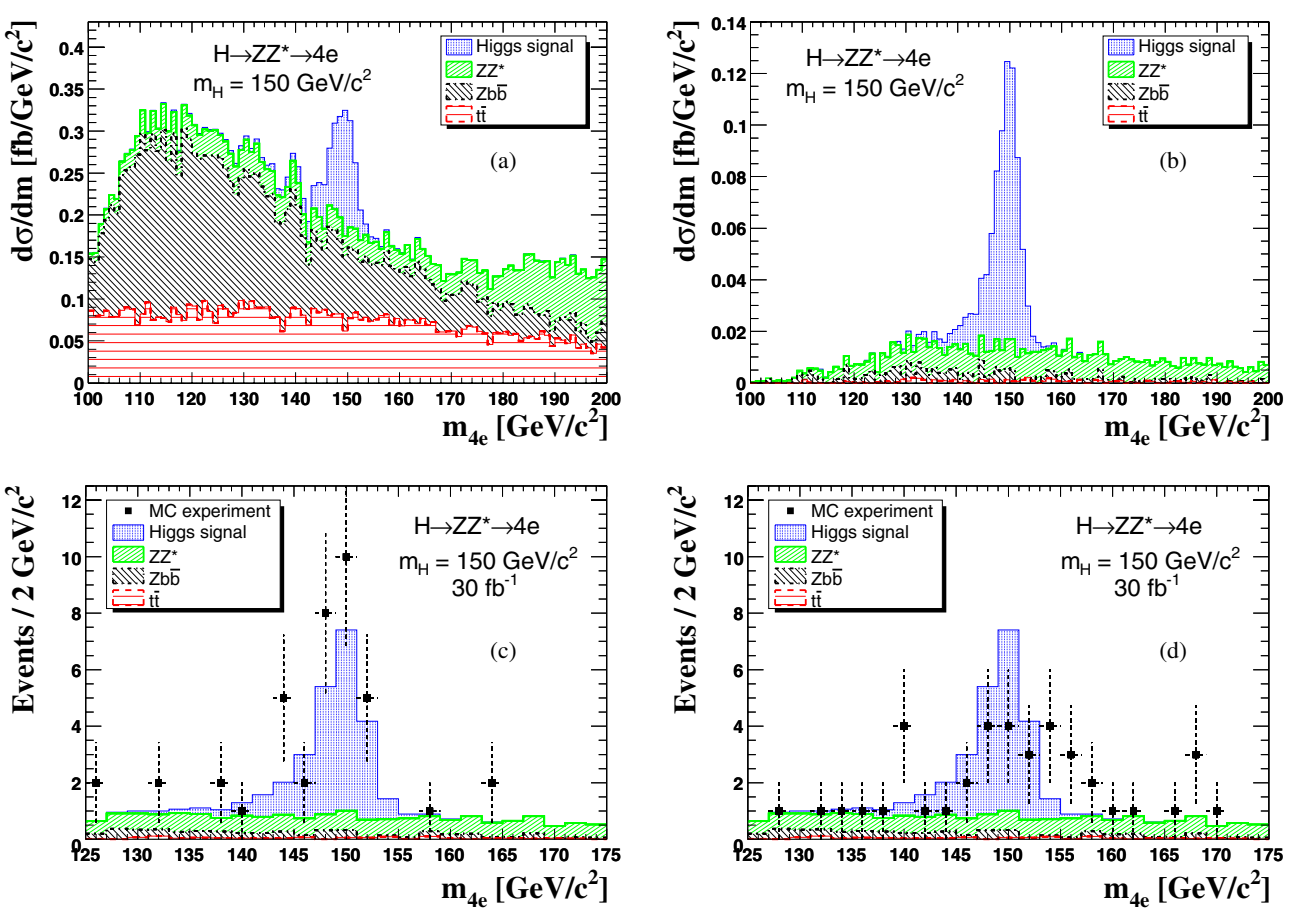

Figure 2.15. Distributions of the reconstructed invariant mass $m_{4 e}$ for the SM Higgs bosons signal at $m_{\mathrm{H}}=150 \mathrm{GeV} / \mathrm{c}^{2}$ and for the SM backgrounds after (a) pre-selection step and (b) after all cuts. The number of events are normalised in cross-section. Single Monte Carlo experiments corresponding to an integrated luminosity of $30 \mathrm{fb}^{-1}$ for (c) a favourable case and (d) a less favourable case.

for $m_{4 e} \simeq 150 \mathrm{GeV} / \mathrm{c}^{2}$ to a reduction of the $\mathrm{ZZ}^{(*)}$ continuum by a factor of about 6.5 and a reduction of the $\bar{t} \bar{t}$ background by a factor of about 2.5 .

Figure $2.15 \mathrm{a}$ shows as an illustration the expected $m_{4 e}$ invariant mass distributions for the signal at $m_{\mathrm{H}}=150 \mathrm{GeV} / \mathrm{c}^{2}$ and for backgrounds after triggering and pre-selection. The further background suppression from the isolated primary electron requirement, the $p_{\mathrm{T}}^{e}$ and $\mathrm{Z}$ mass cuts is seen by comparison in Fig. 2.15b. The global selection efficiency (normalised to the acceptance defined at the generation level) is given in Table 2.12 for the signal and backgrounds. Figures $2.15 \mathrm{c}$ and $2.15 \mathrm{~d}$ show for illustration the possible outcome of two random Monte Carlo experiments corresponding to favourable and less favourable fluctuations of the Higgs boson signal for an integrated luminosity of $30 \mathrm{fb}^{-1}$. The Poissonian probability to have equal or more favourable (respectively equal or less favourable) fluctuations is of about $5 \%$ for the example cases shown.

\subsubsection{Systematics}

In this section the systematic errors are discussed in the context of a discovery via a simple event counting method. The "theoretical" and "experimental" sources of errors are distinguished. The theoretical uncertainties concern the estimation of the background rates within the cuts defining the acceptance of the Higgs boson signal and are discussed in Section 2.2.4.1. The experimental uncertainties take into account the limited knowledge of 
Table 2.12. Summary of selection efficiencies normalised to the generation pre-selection efficiency.

\begin{tabular}{lcccccccccccc}
\hline$m_{\mathrm{H}}$ & 115 & 120 & 130 & 140 & 150 & $\begin{array}{c}160 \\
\left(\mathrm{GeV} / \mathrm{c}^{2}\right)\end{array}$ \\
\hline Signal & 24.3 & 26.0 & 31.2 & 35.2 & 36.0 & 37.4 & 38.0 & 39.9 & 40.9 & 42.5 & 41.2 & 38.6 \\
$\mathrm{ZZ}^{(*)}$ & 5.24 & 4.94 & 5.68 & 5.95 & 5.14 & 5.23 & 6.87 & 17.8 & 25.1 & 26.2 & 22.3 & 13.9 \\
$\mathrm{Zb} \overline{\mathrm{b}}$ & 0.22 & 0.16 & 0.17 & 0.17 & 0.16 & 0.10 & 0.097 & 0.068 & 0.037 & 0.031 & 0.013 & 0.001 \\
$\overline{\mathrm{tt}}$ & 0.054 & 0.044 & 0.043 & 0.033 & 0.032 & 0.022 & 0.021 & 0.011 & 0.008 & 0.008 & 0.013 & 0.006 \\
\hline
\end{tabular}

the detector responses and efficiencies, and of the corresponding Monte Carlo modelling. These are discussed in Section 2.2.4.2. A comparison of different methods for the control of background systematics is presented in Section 2.2.4.3.

2.2.4.1. Theoretical errors. The theoretical uncertainty on the number of background events in the signal region from PDFs and QCD scales variations has been estimated by the MCFM program [47]. CTEQ6M PDF are used and 20 eigenvector parameters have been varied by $\pm 1 \sigma$. Both QCD normalisation and factorisation scales have been varied independently up and down for a factor two from their nominal values of $2 m_{Z}$. The resulting uncertainties from PDF and QCD scale are of the order of $6 \%$ for direct estimation of ZZ background, from 2 to $8 \%$ for normalisation to single $Z \rightarrow 2 e$, and from 0.5 to $4 \%$ for the normalisation to sidebands (discussed further in Section 2.2.4.3). The gluon fusion cross-section uncertainties in the ZZ background of $8 \%$ is also considered as a part of theoretical uncertainties.

The uncertainty on the normalisation of the measurements to the $p p$ luminosity of the LHC collider is estimated to be of the order of $3 \%$ for an integrated luminosity above $10 \mathrm{fb}^{-1}$.

2.2.4.2. Experimental errors. The main remaining sources of experimental systematics expected in the CMS experiment after having collected of $\mathcal{O}(10) \mathrm{fb}^{-1}$, and relevant for the $\mathrm{H} \rightarrow 4 e$ channel, originate from uncertainties on knowledge of the amount of tracker material in front of the ECAL, from the precision of the (pattern dependent) energy calibration of electron objects, and from the control of electron efficiencies. The strategy adopted consists of relying on experimental data, and in particular on single $\mathrm{Z}$ and $\mathrm{W}$ production, to minimise these systematic errors. The electrons from $\mathrm{W} \rightarrow e v$ and $\mathrm{Z} \rightarrow e e$ decays are used to control the energy measurements and reconstruction efficiencies.

A change of the integral amount of tracker material traversed by electrons before reaching the ECAL is susceptible of affecting the electron selection and identification efficiencies, as well as energy measurement scales and resolution. The uncertainty on the material budget will limit the precision of the acceptance calculations, when using the Monte Carlo model to extrapolate away from the kinematic domain best constrained via single $\mathrm{Z}$ and $\mathrm{W}$ measurements.

There are many observables that are directly or indirectly sensitive to the amount of tracker material, and that have been used in collider experiments. Examples are the distribution of converted photon vertices, or the shape of the $E / p$ comparing tracker momentum measurement $p$ to the energy $E$ measured in the calorimeter in finite cluster volume, or a comparison of data and Monte Carlo for the $\mathrm{Z}$ mass resolution, etc. A new technique is used which is based on the electron GSF tracking introduced recently in Ref. [46]. The difference between the momentum magnitude at vertex and at the last hit, $p_{\text {in }}-p_{\text {out }}$, is a measure of the integral amount of bremsstrahlung. The mean fraction $f_{\text {brem }}$ of the energy radiated along the complete trajectory is roughly proportional to the integral amount 

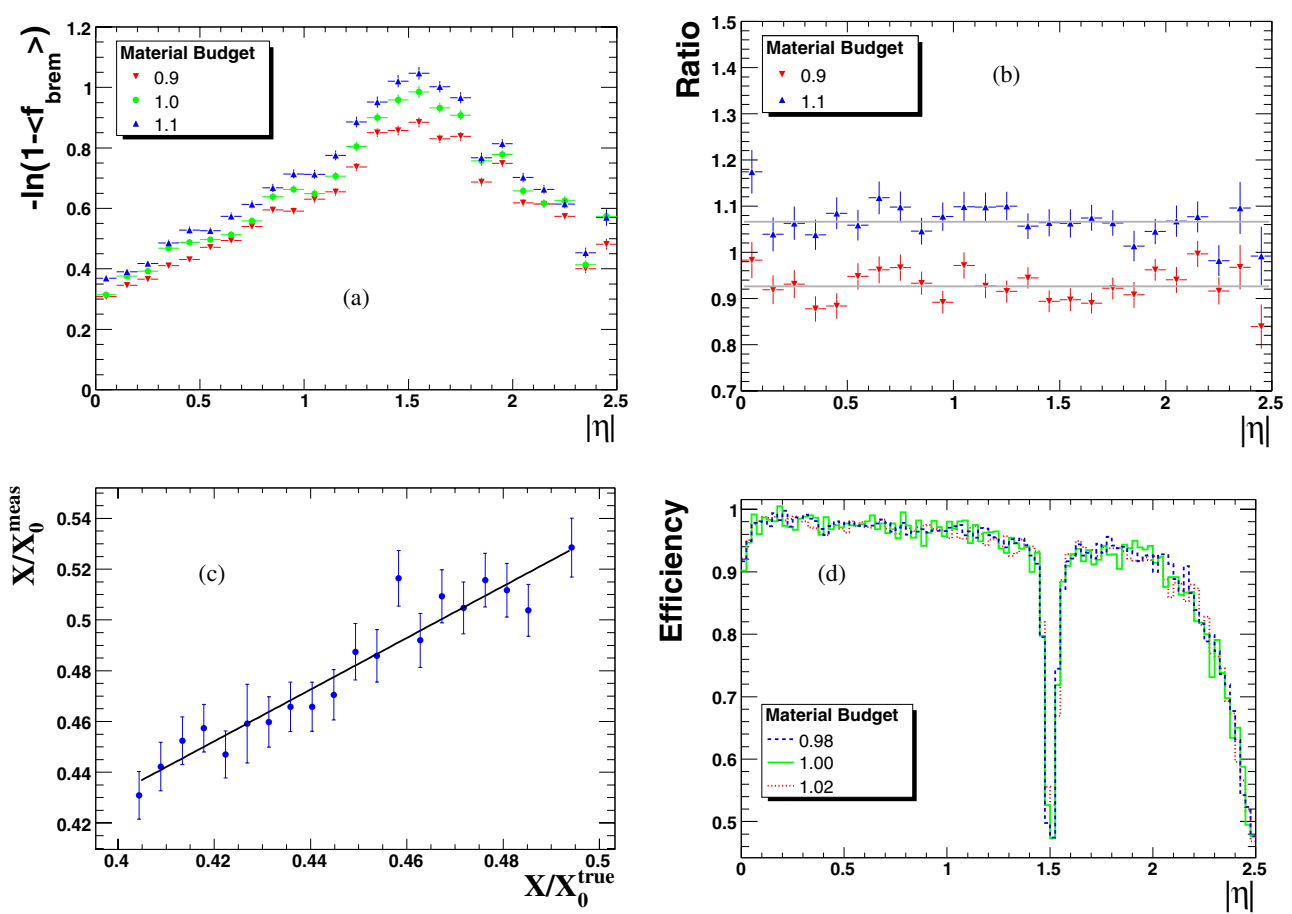

Figure 2.16. Sensitivity to variations of the tracker material budget from electron measurements based on GSF tracks: (a) measured amount of material as a function of $|\eta|$ for the nominal tracker configuration and for an integral material budget changed by $\pm 10 \%$; (b) ratio of the measured mount of material as a function of $|\eta|$; (c) measured versus true thickness in $X_{0}$ of the tracker material; (d) effect of a change of $2 \%$ of the material budget on the electron reconstruction efficiency.

of material traversed. Hence, one can relate $f_{\text {brem }}$ to the material thickness $X / X_{0}$ where $X_{0}$ is the characteristic radiation length via the formula $\langle X\rangle / X_{0} \simeq-\ln \left(1-f_{\text {brem }}\right)$, where $f_{\text {brem }}=\left(p_{\text {in }}-p_{\text {out }}\right) / p_{\text {in }}$.

The amount of tracker material measured in this way for single electron data is shown in Fig. 2.16a. The results obtained in the configuration corresponding to the nominal tracker material coincide very well with the known material distribution as given in Ref. [7]. Figure $2.16 \mathrm{~b}$ shows the ratio of the measured material thickness obtained in configurations where the amount of material was changed by $\pm 10 \%$, normalised to the measurement results in the nominal case. The ratio is found to be remarkably stable as a function of $\eta$, despite the fact that the integral amount of material has a strong $\eta$ dependence. Thus, single electrons can be used in CMS to tune the Monte Carlo model of the tracker material per $\eta$ slice. Figure 2.16c shows that in a given $\eta$ slice the measured material thickness is linearly correlated to a change (at least within a range of $\pm 10 \%$ ) of the true material thickness. Similar results are obtained when considering various restricted range of $p_{\mathrm{T}}^{e}$ within a sample of uniformly distributed electrons in the $p_{\mathrm{T}}^{e}$ range from 5 to $100 \mathrm{GeV} / \mathrm{c}$. With the electron statistics expected from single $\mathrm{Z}$ production for an integrated LHC luminosity of $\mathcal{O}(10) \mathrm{fb}^{-1}$, it should be possible to determine the tracker material thickness to a precision better than $2 \%$ over the full acceptance in $\eta$. Figure $2.16 \mathrm{~d}$ shows that such a $2 \%$ uncertainty on the material budget will have almost no effect on electron reconstruction efficiency. 

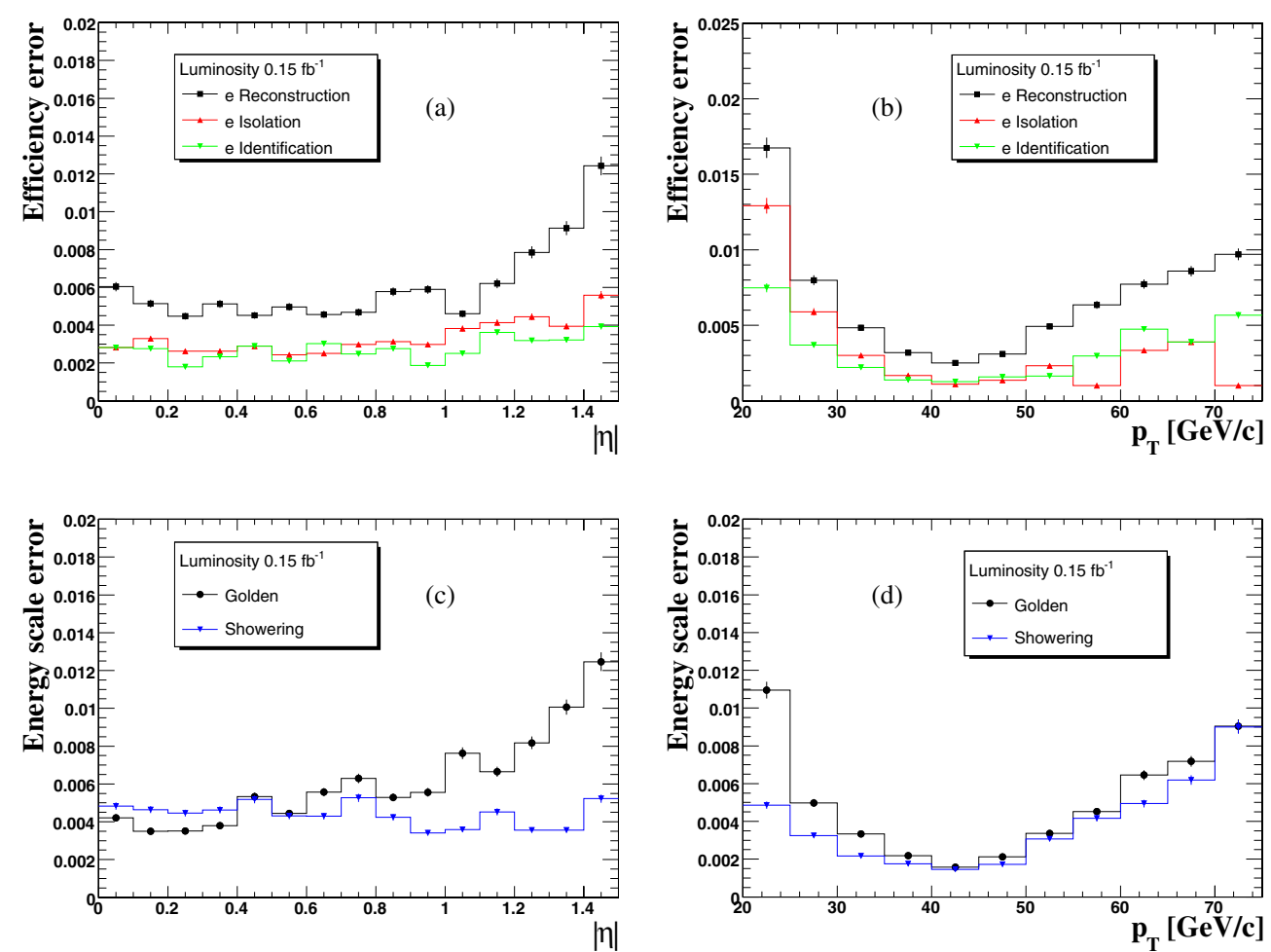

Figure 2.17. Control of experimental uncertainties using SM data; uncertainties on measurements of electron reconstruction, isolation and identification as a function of (a) $\eta$ and (b) $p_{\mathrm{T}}$; uncertainties on measuring the energy scale for golden and showering electrons as a function of (c) $\eta$ and (d) $p_{\mathrm{T}}$.

Electron reconstruction efficiencies and energy scales will be controlled by electrons from $\mathrm{W} \rightarrow e v$ and $\mathrm{Z} \rightarrow e e$ decay. Huge cross-sections of these two processes will allow for a significant reduction of reconstruction uncertainties already after few $\mathrm{fb}^{-1}$. Electrons from $\mathrm{Z} \rightarrow e e$ are produced centrally with a characteristic Jacobian $p_{\mathrm{T}}$ distributions around $45 \mathrm{GeV} / \mathrm{c}$. It is therefore, expected that the best control of experimental systematics is obtained in the central part of the detector and for electrons around the Jacobian peak.

Electron reconstruction uncertainties as a function of $\eta$ and $p_{\mathrm{T}}$ are given in Fig. 2.17a and Fig. 2.17b respectively, for an integrated luminosity of $0.15 \mathrm{fb}^{-1}$. The expected behaviour of increased uncertainties when moving away from the Jacobian peak or from the central $\eta$ region can be clearly seen. From the expected reconstruction errors evolution with the luminosity, all reconstruction efficiency uncertainties can be safely absorbed in a single factor of $1 \%$ per electron, for integrated luminosities larger than $10 \mathrm{fb}^{-1}$.

The second important systematic effect is the uncertainty on the energy scale determination. Using single $\mathrm{Z}$ production, it has been shown in Ref. [48] that the absolute energy scale for electrons can in principle be controlled with great precision with average uncertainties reaching values below $0.1 \%$. The systematic uncertainty has to be studied as a function of $p_{\mathrm{T}}^{e}$ and $\eta^{e}$ given the different electron spectrum in $\mathrm{H} \rightarrow \mathrm{ZZ}^{(*)} \rightarrow 4 e$ and $\mathrm{Z} \rightarrow e e$ decays. The reachable precision depends on the amount of integrated LHC luminosity. In this analysis, the second leg of a $\mathrm{Z}$ boson decay, tagged as an electron by imposing stringent 
electron identification requirements on the first leg combined with a kinematic constraint to the $\mathrm{Z}$ boson mass, is used as a probe to estimate systematics on the energy scale.

Uncertainties versus $\eta$ and $p_{\mathrm{T}}$ for golden and showering electrons are shown in Fig. 2.17c and Fig. $2.17 \mathrm{~d}$, for the integrated luminosity of $0.15 \mathrm{fb}^{-1}$. With expected evolution of these uncertainties with the luminosity, it is found that an uncertainty in energy scale of $0.5 \%$ in the barrel region, and $1 \%$ in the endcaps, for integrated luminosities larger than $10 \mathrm{fb}^{-1}$, can be safely considered.

2.2.4.3. Control of background rates. Following the primary and isolated electron selection and the application of basic kinematic requirements, only the $\mathrm{ZZ}^{(*)}$ continuum remains as the dominant or sole background over the full mass range in consideration for the SM Higgs boson search. Thus, the determination of the mean expected number of SM ZZ ${ }^{(*)}$ background events in the signal region, defined e.g. by a simple sliding window in the $m_{4 e}$ spectrum, remains as a key issue.

The three main methods for the estimation of $\mathrm{ZZ}^{(*)}$ continuum contribution to the background in the signal region are:

- direct simulation of the $\mathrm{ZZ}^{(*)} \rightarrow 4 e$ process,

- normalisation to the $\mathrm{Z} \rightarrow 2 e$ data,

- normalisation to the sidebands.

The first method entirely relies on existing SM constraints and the theoretical knowledge, with uncertainties coming from the PDFs used to describe the colliding protons and from QCD scale variations. It furthermore is reliant on the LHC luminosity uncertainties, and on the Monte Carlo modelling of the acceptance and detector response for the uncertainties arising from electron reconstruction and selection. Otherwise, the method potentially benefits from the fact that the statistical precision on the mean background expectation is only limited by the Monte Carlo statistics, and can therefore be assumed negligible in the context of a prospective for an analysis to be performed in a future CMS experiment.

The second method aims at profiting from the fact that the SM single $\mathrm{Z}$ production cross-sections is measured with great precision in an experiment which will have integrated a luminosity of $\mathcal{O}(10) \mathrm{fb}^{-1}$ at the LHC. Using the ratio of $\mathrm{ZZ} \rightarrow 4 e$ to $\mathrm{Z} \rightarrow 2 e$ rates allows to profit from a full cancellation of $p p$ luminosity uncertainties, while providing a partial cancellation of PDF and QCD scale variations uncertainties (due to their correlations in a part of the initial state phase space) and a partial cancellation of experimental uncertainties.

In the method of the normalisation from sidebands, the number of background events inside the acceptance of the signal region is determined from the number of background events measured outside the signal region, by multiplying the latter with the ratio $\alpha_{\mathrm{MC}}$ between inside and outside expectations as determined using Monte Carlo simulation. Using the sidebands one also expects to fully cancel luminosity uncertainties, to reduce PDF and QCD scale variation uncertainties and substantially reduce experimental uncertainties too. Statistical errors with sidebands normalisation come from the statistics of the background rate outside the signal region and can be a limiting factor for the method. By relaxing some of late analysis cuts, such as invariant $\mathrm{Z}$ mass, the background events rate outside the signal region increases, reducing therefore statistical errors for this method. The price to pay is an increased background rate in the signal region too and, therefore, some balancing is needed.

Using results from previous sections, both theoretical and experimental uncertainties are evaluated for two methods: normalisation to the $\mathrm{Z} \rightarrow 2 e$ measurements and normalisation to the sidebands. For the normalisation to single $\mathrm{Z} \rightarrow 2 e$ measurements results are shown in Fig. 2.18a. The overall systematic uncertainty with this method is of about 5\%. Experimental 

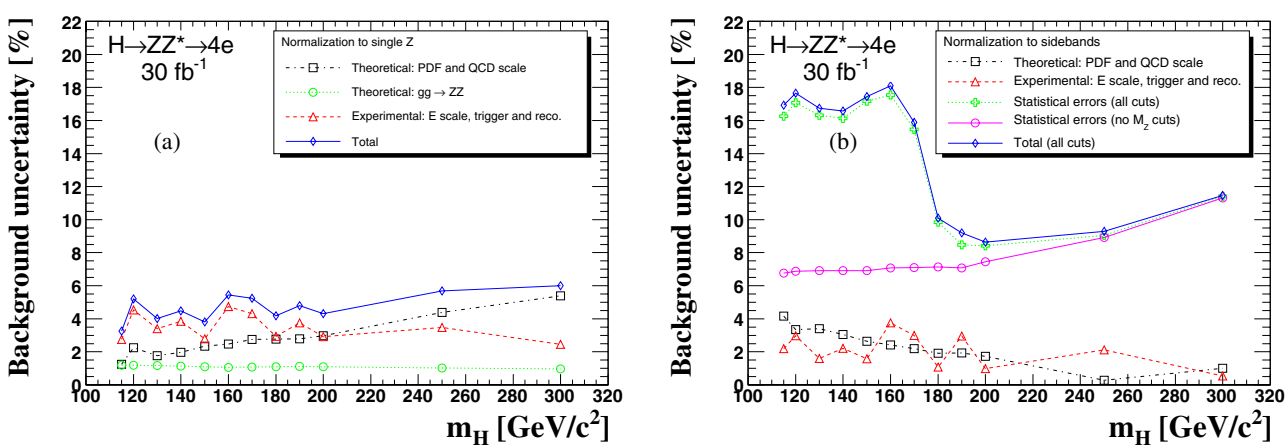

Figure 2.18. Theoretical and experimental uncertainty estimations for both methods for evaluation of background from data: (a) normalisation to the single $\mathrm{Z} \rightarrow 2 e$ measurements and (b) normalisation to the sidebands. Expected statistical errors for sidebands are also shown, for integrated luminosity of $30 \mathrm{fb}^{-1}$.

Table 2.13. Expected number of Higgs boson signal $\left(N_{S}\right)$ and SM background $\left(N_{B}\right)$ events for an integrated luminosity of $30 \mathrm{fb}^{-1}$, in the optimised window for the reconstructed invariant mass $m_{4 e}$. The uncertainties $\left(\delta N_{B}\right)$ are given for systematics from experimental (exp.) and theoretical (theo.) sources, for an analysis where the $\mathrm{ZZ}^{(*)}$ continuum has been normalised to the measurement of single $\mathrm{Z}$ production.

\begin{tabular}{|c|c|c|c|c|c|c|c|c|c|c|c|c|}
\hline$m_{\mathrm{H}}$ & 115 & \multicolumn{10}{|c|}{$\left(\mathrm{GeV} / \mathrm{c}^{2}\right)$} & 300 \\
\hline$N_{S}$ & 1.52 & 2.97 & 8.18 & 15.80 & 17.19 & 8.38 & 3.76 & 9.95 & 34.05 & 38.20 & 27.68 & 21.69 \\
\hline$N_{B}$ & 2.26 & 1.94 & 3.71 & 4.31 & 3.68 & 3.10 & 3.37 & 6.42 & 14.62 & 17.29 & 13.40 & 7.63 \\
\hline$\delta N_{B}$ & & & & & & & & & & & & \\
\hline exp. & 0.063 & 0.089 & 0.126 & 0.167 & 0.105 & 0.148 & 0.145 & 0.187 & 0.551 & 0.505 & 0.466 & 0.187 \\
\hline theo. & 0.039 & 0.049 & 0.079 & 0.098 & 0.095 & 0.084 & 0.100 & 0.191 & 0.440 & 0.549 & 0.602 & 0.417 \\
\hline
\end{tabular}

uncertainties are seen to dominate for $m_{\mathrm{H}} \simeq 2 m_{\mathrm{Z}}$ while theoretical errors take over above the pair production threshold. Uncertainties for the sidebands normalisation are shown in Fig. 2.18b. Statistical uncertainties scale as the square root of the number of background events outside the signal region and are shown for an integrated luminosity of $30 \mathrm{fb}^{-1}$ and for two analysis scenarios: after all analysis cuts and without cuts on the mass of both $\mathrm{Z}$ bosons. A trade-off in the second method is in a somewhat lower nominal significance (for about $8 \%$ ) while statistical errors decrease by a factor of about 2.5. Full significance calculations with and without systematics and statistical uncertainties are presented in the following section.

\subsection{5. $H \rightarrow 4 e$ Observability, mass and cross-section measurements}

2.2.5.1. Discovery reach. A simple counting experiment is used here to quantify the sensitivity of the experiment to the presence of a Higgs boson signal. The expected number of signal $\left(N_{S}\right)$ and background $\left(N_{B}\right)$ events are evaluated in a sliding window whose central position $m_{4 e}$ varies between 100 and $320 \mathrm{GeV} / \mathrm{c}^{2}$. The size of the optimal window increases progressively from $6 \mathrm{GeV} / \mathrm{c}^{2}$ at $m_{4 e}=115 \mathrm{GeV} / \mathrm{c}^{2}$ to $24 \mathrm{GeV} / \mathrm{c}^{2}$ at $m_{4 e}=300 \mathrm{GeV} / \mathrm{c}^{2}$. The Table 2.13 presents for each Higgs boson mass hypothesis the mean expected number of signal and background events, and associated uncertainties. 

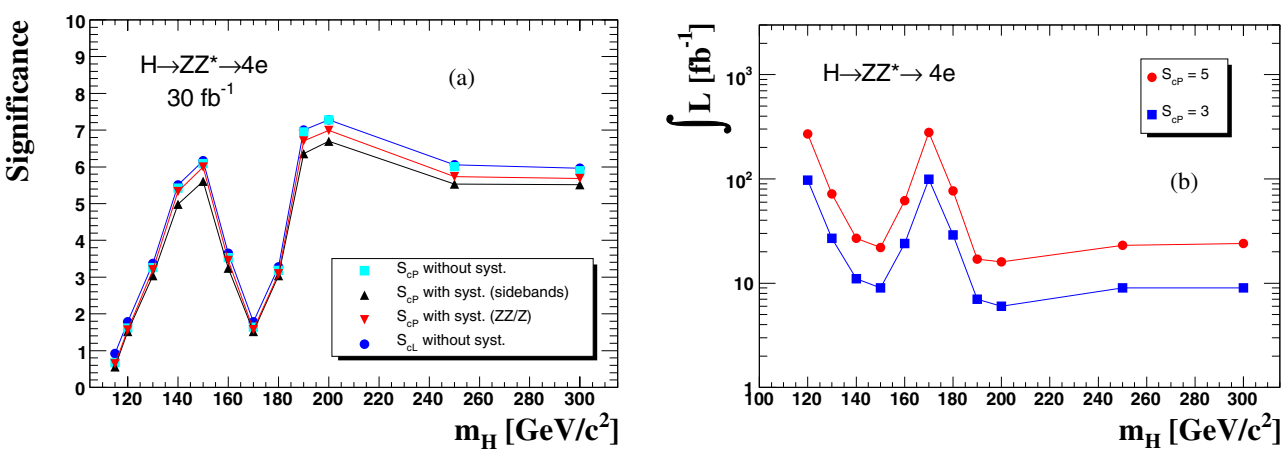

Figure 2.19. (a) Significance $S_{C P}$ for an integrated luminosity of $30 \mathrm{fb}^{-1}$ as a function of the Higgs boson mass without and with systematics included in both options of $\mathrm{ZZ}^{(*)}$ normalisation to the measured sidebands or the measured single $\mathrm{Z}$ production cross-section. The significance $S_{c L}$ is also shown. (b) Luminosity needed for a $3 \sigma$ observation and $5 \sigma$ discovery with the systematics included using $\mathrm{ZZ}^{(*)}$ normalisation to the $\mathrm{Z}$ cross-section.

The significance of the $\mathrm{H} \rightarrow 4 e$ signal observation is shown as a function of $m_{\mathrm{H}}$ in Fig. 2.19a as expected for an integrated luminosity of $30 \mathrm{fb}^{-1}$. The results are given for both the $S_{c P}$ and the $S_{C L}$ significance estimators. The $S_{C P}$ is defined as the probability for a Poisson distribution with mean $N_{B}$ to observe a number of events equal or greater than $N_{S}+N_{B}$, converted in the equivalent number of standard deviations of a Gaussian distribution. The $S_{c L}$ corresponds to the widely used log-likelihood ratio significance [49] and is given for comparison. The effect of including experimental and theoretical systematics, described in section 2.2.4 and listed in Table 2.13, on the significance $S_{c P}$ [50] is also shown, for two different methods of controlling the background uncertainties. A signal observation with a significance above 3 standard deviations is expected in the $\mathrm{H} \rightarrow 4 e$ channel alone for $m_{\mathrm{H}}$ in the range from 130 to $160 \mathrm{GeV} / \mathrm{c}^{2}$, and above $180 \mathrm{GeV} / \mathrm{c}^{2}$. The integrated luminosity needed for a 5 standard deviations discovery of the SM Higgs boson in the $\mathrm{H} \rightarrow 4 e$ channel alone is also shown as a function of $m_{\mathrm{H}}$ in Fig. 2.19b. Systematic errors from normalisation to the $\mathrm{Z}$ cross-section have been included.

2.2.5.2. Mass, width and cross-section measurements. At an early stage of the Higgs boson search and discovery in the $\mathrm{H} \rightarrow 4 e$ channel, given very low statistics, a robust and simple estimation of $m_{\mathrm{H}}$ can be obtained by a simple mean (or weighted mean) of the $m_{4 e}$ values measured for individual events. The events falling in the pre-defined optimal mass window introduced in the above Section 2.2.5.1 and used to establish the signal significance, can be used for such purposes. For higher statistics, a fit of the $m_{4 e}$ mass distribution to a signal plus background shape can be used to extract simultaneously the mass and the cross-section $\times$ branching ratio of a Higgs boson signal. Detector effects dominate the Higgs boson mass resolution below the $\mathrm{Z}$ pair production threshold and a sensitivity to the Higgs boson intrinsic width is expected only for masses well above $2 m_{\mathrm{Z}}$.

The precision on the parameter measurements for the Higgs boson depend on the quality of the reconstructed electrons and can, in general, be improved using event-by-event errors on the electron momentum estimation [46]. Example cases for two different sub-samples of Higgs boson events differing by the pattern of the four reconstructed electrons are presented in Fig. 2.20. Clearly, event candidates built from four non-showering electrons in the barrel part of the ECAL, a subset representing only about $1.76 \%$ of all signal events, allow for 

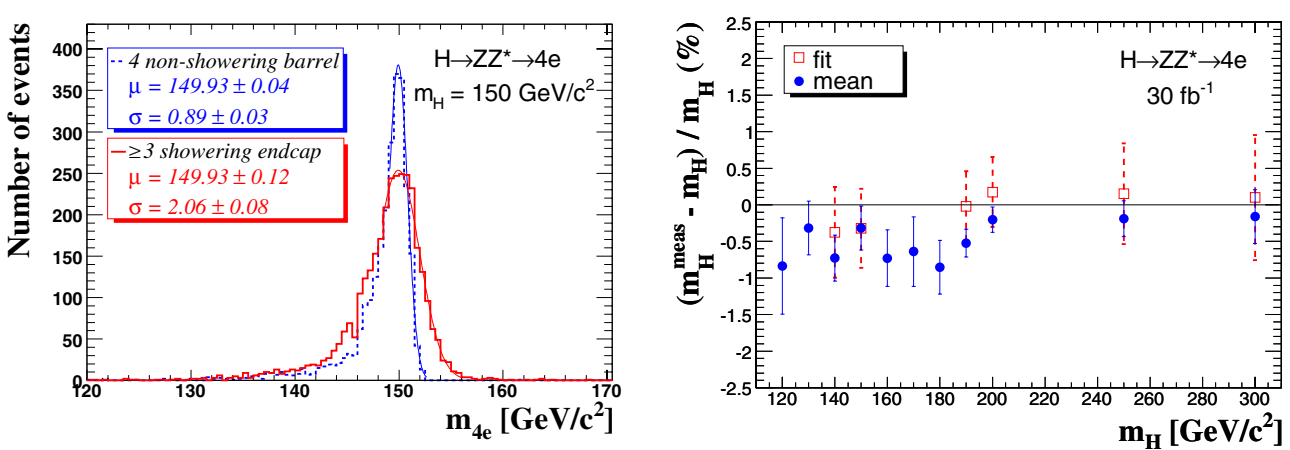

Figure 2.20. Mass measurements: (a) example case for two different event sub-samples differing by the pattern of the four reconstructed electrons; (b) relative errors as a function of the Higgs boson mass using the mean mass and the fitted mass as obtained for an integrated luminosity of $30 \mathrm{fb}^{-1}$.

a much better $m_{\mathrm{H}}$ measurement (smallest errors on average and least dispersion of the mass measurement errors) than candidates built mainly from e.g. showering electrons in the endcaps part of the ECAL. About $36.7 \%$ of the signal event candidates contain three or more showering electrons. A weighted mean of the events of the $m_{4 e}$ distribution falling in the signal window has been considered for the estimation of the Higgs boson mass in Ref. [37]. A simple mean can be also used for simplicity.

The reconstructed Higgs boson mass and its error obtained from the mean value for events falling in the expected signal window is presented in Fig. 2.20b. The error is obtained from the dispersion of the mean values obtained from large number of Monte Carlo experiments at an integrated luminosity of $30 \mathrm{fb}^{-1}$. The results are shown as a function of the Higgs boson mass. The systematic bias on the mass estimate for the low $m_{\mathrm{H}}$ cases for this simple mean approach is due to the asymmetric shape of the reconstructed signal and can be modelled. In the mass ranges where the Higgs boson signal significance exceeds 3 standard deviations, the uncertainty on the mass determination is found to be everywhere below $0.4 \%$. It reaches values below $0.2 \%$ for $m_{\mathrm{H}} \simeq 200 \mathrm{GeV} / \mathrm{c}^{2}$. For comparison, results obtained by fitting the $m_{4 e}$ distribution are also shown. The fit method requires a significant number of events (typically $\gtrsim \mathcal{O}(10))$ to converge and provide reasonably stable results. The $m_{4 e}$ distribution is fitted by a signal plus background shape. The signal contribution is modelled with two Gaussians, describing respectively the core and the low $m_{4 e}$ tail of the signal distribution. The tail parameters (fraction, mean and dispersion) are fixed by fitting the "signal only" expectation. The background is modelled using a flat distribution up to about $m_{4 e} \approx 2 m_{\mathrm{Z}}$ and a linear function (non-zero slope) for higher Higgs boson masses. This has been found to provide a sufficiently good model of the observation in a restricted mass range around the signal region. A likelihood fit is then performed on each Monte Carlo experiments and the reconstructed mass and precision are extracted from the distribution of the fitted values of the peak of the Gaussian core. Where the fit can be performed, Fig. 2.20b shows that an unbiased estimation of $m_{\mathrm{H}}$ is obtained within errors.

The fitted number of signal events is used to estimate the production cross-section by correcting for the global acceptance efficiency. The statistical precision on this measurement is here also obtained from the width of the distribution of the fitted parameters in Monte Carlo experiments. An unbiased measurement of the cross-section is obtained over the full mass range considered here, with a precision of the cross-section measurement between 
20 and $30 \%$. With such a precision, the influence of the detector systematics (about 5\%) and of the uncertainty on the luminosity measurement (less than $3 \%$ for $30 \mathrm{fb}^{-1}$ ) is marginal. For an integrated luminosity of $60 \mathrm{fb}^{-1}$, the precision on the cross-section measurement improves to about $15 \%$.

A measurement of the width is possible only for Higgs boson masses above $\gtrsim 2 m_{Z}$ where at the same time the Higgs natural width is becoming large and the detector resolution is improving. A Gaussian width with central values of about $2.3 \mathrm{GeV} / \mathrm{c}^{2}$ for $m_{\mathrm{H}}=200 \mathrm{GeV} / \mathrm{c}^{2}$ and $4.2 \mathrm{GeV} / \mathrm{c}^{2}$ for $m_{\mathrm{H}}=300 \mathrm{GeV} / \mathrm{c}^{2}$ is obtained from the fit, but with a rather large uncertainty of about $50 \%$. 


\section{Chapter 3. Physics Studies with Muons}

\subsection{Benchmark Channel: $H \rightarrow Z Z^{(*)} \rightarrow 4$ muons}

The $H \rightarrow Z Z^{(*)} \rightarrow 4 \mu$ process is one of the cleanest channels for discovering the Standard Model Higgs boson at LHC. This section presents the CMS potential for discovering the Higgs boson in this decay mode and measuring its mass, width, and production cross section, in the range of Higgs boson masses from $115 \mathrm{GeV} / \mathrm{c}^{2}$ to $600 \mathrm{GeV} / \mathrm{c}^{2}$. Both signal and background event samples are generated at the Leading Order (LO) approximation, and Next to Leading Order (NLO) production cross sections, computed using different methods, are used for their normalisation. To simulate the detector response and reconstruct physics objects, the full CMS detector simulation and reconstruction software was used. A full treatment of the most important theoretical and instrumental systematic uncertainties are presented, together with their effect on the evaluation of the significance of the Higgs boson observation and on the measurement of its parameters. To minimise systematic uncertainties, new methods of reconstructing the most important corrections directly from data were developed.

\subsubsection{Physics processes and their simulation}

The Higgs boson event samples for 18 Higgs boson mass points and the three main background processes, $t \bar{t},\left(Z^{(*)} / \gamma^{*}\right) b \bar{b}$ and $\left(Z^{(*)} / \gamma^{*}\right)\left(Z^{(*)} / \gamma^{*}\right)$ were simulated using the CMS simulation [8] and reconstruction [10] software. These three backgrounds will be hereafter referred to as $t \bar{t}, \mathrm{Zb} \overline{\mathrm{b}}$ and $\mathrm{ZZ}$, respectively. Details on the generator-level simulation conditions, cross sections and K-factors can be found in [51]. Many other plausible

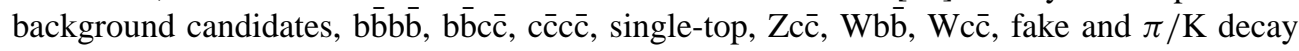
muons in QCD, were considered and found to be negligible. An example of an $\mathrm{H} \rightarrow \mathrm{ZZ} \rightarrow 4 \mu$ event is shown in colour plate $\mathrm{CP} 3$.

Only events with at least $2 \mu^{+}$and $2 \mu^{-}$in pseudorapidity range $|\eta|<2.4$ and with $p_{\mathrm{T}}>3 \mathrm{GeV} / \mathrm{c}$ were retained for further analysis. Muons outside these kinematic limits could not be reconstructed in the CMS detector. Additional cuts were applied on dimuon invariant masses for the Higgs boson samples $\left(m_{\mathrm{Z}}>5 \mathrm{GeV} / \mathrm{c}^{2}\right)$ and for $\mathrm{ZZ}$ and $\mathrm{Zb} \overline{\mathrm{b}}$ samples $\left(m \mu^{+} \mu^{-}>5 \mathrm{GeV} / \mathrm{c}^{2}\right)$. The first $\mu^{+} \mu^{-}$pair in the $\mathrm{ZZ}$ and $\mathrm{Zb} \overline{\mathrm{b}}$ samples was defined as the one with its invariant mass closest to $m_{\mathrm{Z}}$, while the second $\mu^{+} \mu^{-}$pair was made out of the two remaining highest $p_{\mathrm{T}}$ muons of opposite charge. These cuts do not bias the Monte Carlo samples since all the analysis cuts, described below, are tighter.

The Higgs boson samples were generated with PYTHIA 6.225 [24] (LO gluon and weak boson fusion, $\mathrm{gg} \rightarrow \mathrm{H}$ and $\mathrm{q} \overline{\mathrm{q}} \rightarrow \mathrm{q} \overline{\mathrm{q}} \mathrm{H})$ interfaced via CMKIN [52]. Events were re-weighted to correspond to the total NLO cross section $\sigma(\mathrm{pp} \rightarrow \mathrm{H}) \cdot B R(\mathrm{H} \rightarrow \mathrm{ZZ}) \cdot B R(\mathrm{Z} \rightarrow 2 \ell)^{2}$ (Fig. 3.1). The cross section $\sigma(\mathrm{pp} \rightarrow \mathrm{H})$ and the branching ratio $B R(\mathrm{H} \rightarrow \mathrm{ZZ})$ were taken from [53]; $B R(\mathrm{Z} \rightarrow 2 \ell)=0.101$ [54]. Interference of permutations of identical leptons originating from different $\mathrm{Z}$ bosons results in an enhancement to the cross section for $\mathrm{H} \rightarrow \mathrm{ZZ}^{(*)} \rightarrow 4 \ell$ ) processes with identical leptons [51], which is about $15 \%$ for $m_{\mathrm{H}}=$ $115 \mathrm{GeV} / \mathrm{c}^{2}$ and steadily goes to zero for $m_{\mathrm{H}}=180 \mathrm{GeV} / \mathrm{c}^{2}$. This correction was calculated with COMPHEP.

The $t \bar{t}$ sample was generated with PYTHIA $6.225(\mathrm{LO} \mathrm{gg} \rightarrow t \bar{t}$ and $\mathrm{q} \bar{q} \rightarrow t \bar{t})$. Events were re-weighted to correspond to the total NLO cross section $\sigma(\mathrm{pp} \rightarrow t \bar{t}) \cdot B R(\mathrm{~W} \rightarrow \ell \nu)^{2}$. The NLO cross section $\sigma(\mathrm{pp} \rightarrow \mathrm{t} \overline{\mathrm{t}})=840 \mathrm{pb}$ was taken from [55] and the branching ratio $B R(\mathrm{~W} \rightarrow \ell v)=0.320$ from [54].

The $\mathrm{Zb} \overline{\mathrm{b}} \rightarrow \mu^{+} \mu^{-} \mathrm{b} \overline{\mathrm{b}}$ sample was generated with the COMPHEP 4.2p1 [43] matrix element generator, interfaced to PYтнін 6.225 for showering and hadronisation. Included 


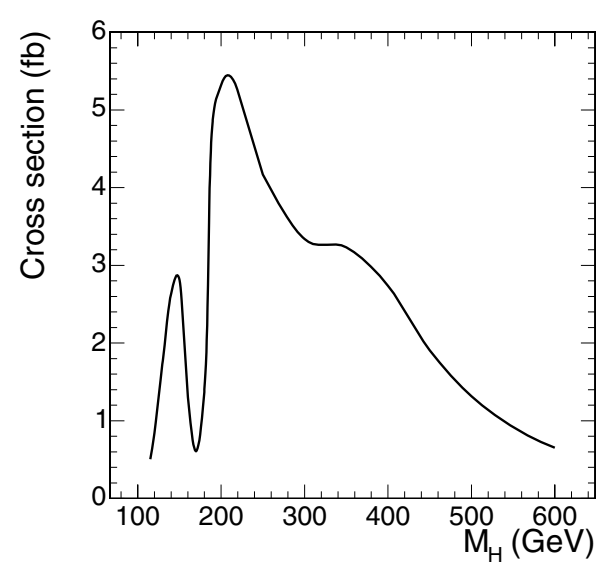

Figure 3.1. Standard Model NLO cross section for the process $\left(Z^{(*)} \rightarrow 4 \mu v\right.$ s. Higgs boson mass.

sub-processes were $\mathrm{q} \overline{\mathrm{q}} / \mathrm{gg} \rightarrow\left(\mathrm{Z} / \gamma^{*}\right) \mathrm{b} \overline{\mathrm{b}} \rightarrow \mu^{+} \mu^{-} \mathrm{b} \overline{\mathrm{b}}$. The corresponding CompHEP LO cross section was found to be $116 \mathrm{pb}$. To obtain the NLO cross section a NLO K-factor $K_{N L O}=$ $2.4 \pm 0.3$, computed with MCFM [56], was used.

The $\mathrm{q} \overline{\mathrm{q}} \rightarrow \mathrm{ZZ} \rightarrow 4 \mu$ and $\mathrm{q} \overline{\mathrm{q}} \rightarrow \mathrm{ZZ} \rightarrow 2 \mu 2 \tau$ event samples were generated with CoMPHEP, including both the t- and s-channel diagrams [57]. The CoMPHEP events were further interfaced to PYTHIA 6.225 for showering and hadronisation. The CoMPHEP LO cross sections for the two sub-processes were $113 \mathrm{fb}$ and $157 \mathrm{fb}$. To account for contributions due to all the NLO diagrams and due to the NNLO gluon fusion ( $g g \rightarrow \mathrm{ZZ}$, known to contribute $\sim 20 \%$ with respect to the LO [42] cross section), events are reweighted with the $\mathrm{m}_{4 \mu}$-dependent $\mathrm{K}$-factor $K\left(\mathrm{~m}_{4 \mu}\right)=K_{N L O}\left(\mathrm{~m}_{4 \mu}\right)+0.2$. The NLO K-factor $K_{N L O}\left(\mathrm{~m}_{4 \mu}\right)$ was obtained with MCFM. The details on the dynamic differences between NLO and LO are summarised elsewhere [58].

The $\mathrm{m}_{4 \mu}$ distributions for a Higgs boson signal of $m_{\mathrm{H}}=140 \mathrm{GeV} / \mathrm{c}^{2}$ and the main backgrounds are shown in Fig. 3.2 after the pre-selection cuts described above.

\subsubsection{Event selection}

3.1.2.1. Trigger and offline muon selection. CMS has been designed and optimised to detect and reconstruct muons. These particles provide a very clean signature and thus a very high trigger efficiency, with an average of $98 \%$ for the Level-1 Global Muon Trigger [7]. The inclusive muon triggers based on the selection of a single muon with $p_{\mathrm{T}}>19 \mathrm{GeV} / \mathrm{c}$ or dimuons with $p_{\mathrm{T}}>7 \mathrm{GeV} / \mathrm{c}$ assures an efficiency of practically $100 \%$ for collecting events with four high- $p_{\mathrm{T}}$ muons.

In order to minimise muon reconstruction systematic uncertainties, we select only those reconstructed muons that have transverse momentum $p_{\mathrm{T}}>7 \mathrm{GeV} / \mathrm{c}$, if they are in the central pseudo-rapidity region $(|\eta|<1.1)$, or with momentum $p>13 /, \mathrm{GeV} / \mathrm{c}$, if they are in the endcaps $(|\eta|>1.1)$ [59]. These cuts do not affect the number of accepted signal events significantly.

Also, we require that all four possible combinations of the reconstructed dimuon masses be above $12 \mathrm{GeV} / \mathrm{c}^{2}, m_{\mu^{+} \mu^{-}}>12 \mathrm{GeV} / \mathrm{c}^{2}$. As in the previous case, this cut has a very little effect on the Higgs boson events and is primarily intended to suppress poorly simulated hadron background contributions originating from charmonium and bottomium dimuon decays. 


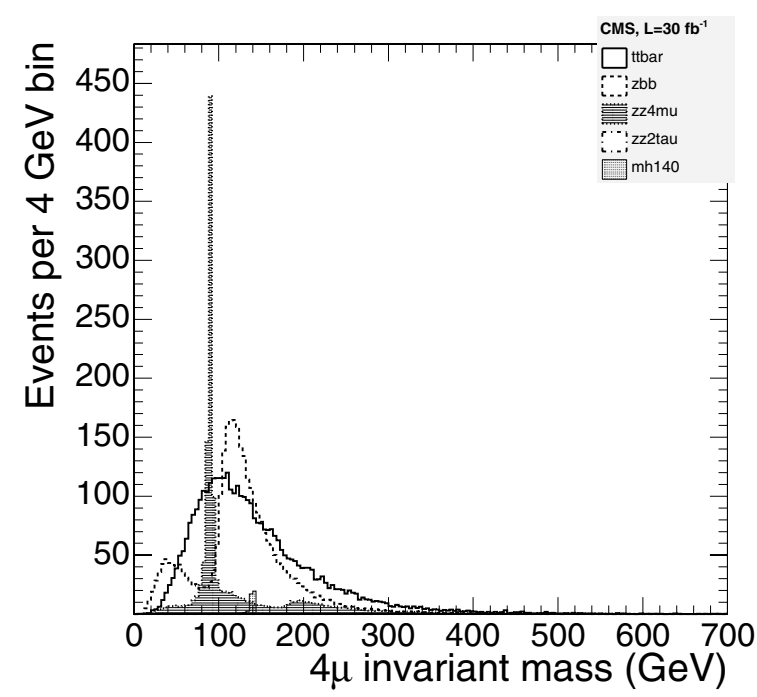

Figure 3.2. Distributions of $\mathrm{m}_{4 \mu}$ after pre-selection cuts for $t \bar{t}, \mathrm{Zb} \overline{\mathrm{b}}, \mathrm{ZZ}$ and a Higgs boson signal of $m_{\mathrm{H}}=140 \mathrm{GeV} / \mathrm{c}^{2}$.

3.1.2.2. Discriminating variables. The $\mathrm{H} \rightarrow \mathrm{ZZ}^{(*)} \rightarrow 4 \mu$ signal presents a characteristic topology, which consists of two opposite charge muon-pairs in the final state. All four muons are isolated, have a high transverse momentum and point to the same Z-boson mass, depending on the restrictions in the phase space introduced by the Higgs boson mass itself. The four-muon invariant mass peaks at the Higgs boson mass, within the detector resolution. The width of the resonant peak accounts for the natural Higgs boson width and the detector resolution.

In $\mathrm{Zb} \overline{\mathrm{b}}$ and $t \bar{t}$ background events, two of the muons come from b-quark decays and are usually found within a jet (i.e., non-isolated), have lower transverse momenta and often exhibit detectable displaced vertices. The isolation is defined as the amount of transverse energy in the calorimeter (calorimeter isolation), or the sum of the transverse momentum of the tracks reconstructed in the tracker (tracker isolation), inside a cone in $\eta$ - $\phi$ space with a radius $R \equiv \sqrt{(\Delta \eta)^{2}+(\Delta \phi)^{2}}$ around each muon. Figure 3.3 (left) shows the distribution of the calorimeter isolation variable for the least isolated muon, for two potential Higgs boson signals, $150 \mathrm{GeV} / \mathrm{c}^{2}$ and $300 \mathrm{GeV} / \mathrm{c}^{2}$, and for the background. Requiring a maximum isolation in all four muons drastically suppresses $t \bar{t}$ and $\mathrm{Zb} \overline{\mathrm{b}}$ contamination.

Further restrictions on the $p_{\mathrm{T}}$ spectrum of the 2 lowest $p_{\mathrm{T}}$ muons in the event (see Fig. 3.3 (right), for the 2 nd lowest $p_{\mathrm{T}}$ muon) reduces even more the $t \bar{t}$ and $\mathrm{Zb} \overline{\mathrm{b}}$ contamination. In this way, the $\mathrm{ZZ}$ background, which presents a topology very similar to that of the signal, becomes the dominant and irreducible background. Only the four-muon mass distribution, the main discriminant, allows the resonant Higgs signal to be identified over the continuum $\mathrm{ZZ}$ production.

Distinction on the basis of dimuon invariant mass or displaced vertices does not increase the Higgs boson signal over the ZZ background. However, they may play an important role in eliminating other possible unaccounted for backgrounds, arising from the primary interactions, accelerator beam halo, detector mis-performance, etc.

Additional variables that may help discriminating $\mathrm{H}$ from the dominant $\mathrm{ZZ}$ background have been studied: $p_{\mathrm{T}}(4 \mu)$, number of jets and their $E_{\mathrm{T}}$, etc. However, these variables are driven by the NLO production processes, while our samples were generated at the Leading 

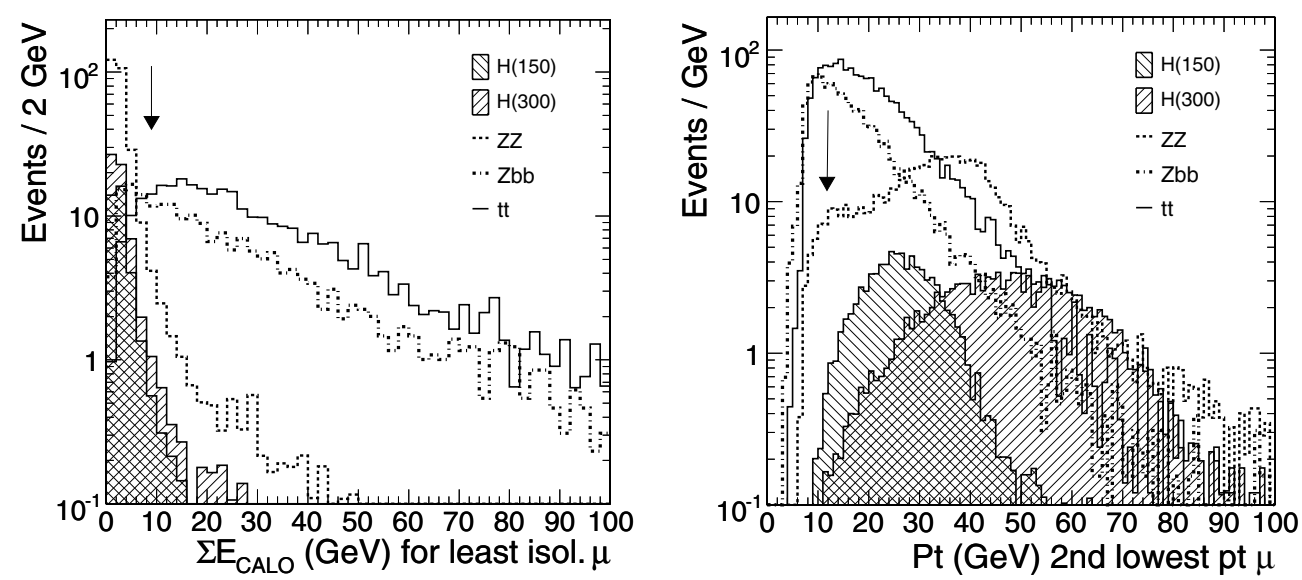

Figure 3.3. Examples of discriminating variables: (left) muon calorimeter-based isolation $\sum E_{\mathrm{T}}$ for the least isolated muon and (right) transverse momentum of the $2^{\text {nd }}$ lowest $p_{\mathrm{T}}$ muon. The hatched histograms represent the Higgs boson signals of masses $150 \mathrm{GeV} / \mathrm{c}^{2}$ and $300 \mathrm{GeV} / \mathrm{c}^{2}$, while the solid, dashed and dash-dotted lines indicate the contribution from the $t \bar{t}, \mathrm{ZZ}$ and $\mathrm{Zb} \overline{\mathrm{b}}$ backgrounds, respectively. The arrows indicate the positions of the cuts.

Order by PYTHIA and COMPHEP. Therefore, any conclusions that we might derive from these samples would not be reliable. Some muon angular distributions also have some differences originating from the underlying spin structures, but they are not sufficiently discriminating to be used and may be strongly affected by the NLO diagrams.

\subsubsection{Higgs boson search analysis}

3.1.3.1. Search using $\mathrm{m}_{4 \mu}$-independent cuts. Given the clear signature of the Higgs boson events, the signal extraction has been performed with a unique set of cuts, independent the Higgs boson mass, the details can be found in [60]. A Higgs mass-independent analysis is expected to minimise the dependence on the simulation of the discriminating variables in the Monte Carlo and the sensitivity to systematic errors. It is also readily applicable to real data and robust under variations of the detector conditions (calibrations, resolutions, efficiencies). Moreover, in our case, a mass-dependent selection does not significantly increase the significance of observing a signal.

A unique set of selection cuts has been designed to make the analysis robust when applied to real data. As explained below, some of the cuts (dimuon invariant mass, $p_{\mathrm{T}}$ cuts on the two hardest muons and isolation cuts on the two most isolated muons) slightly decrease the signal significance but make the selection more robust under imperfect conditions in the detector.

A loose requirement on the invariant mass of the pair of unlike-sign muons in the event which is closer to the nominal Z-boson mass, namely, $70 \mathrm{GeV} / \mathrm{c}^{2}<m_{\mu^{+} \mu^{-}}<100 \mathrm{GeV} / \mathrm{c}^{2}$, leaves more than $90 \%$ of the signal, while eliminating around $50 \%$ of the $t \bar{t}$ contamination. The loss in the signal is due to the internal bremsstrahlung and $\mathrm{Z} \rightarrow 2 \tau \rightarrow 2 \mu 4 \nu$ decays.

Cuts of $12 \mathrm{GeV} / \mathrm{c}$ and $8 \mathrm{GeV} / \mathrm{c}$ are set on the $p_{\mathrm{T}}$ of the two lowest- $p_{\mathrm{T}}$ muons. The $p_{\mathrm{T}}$ of the two highest- $p_{\mathrm{T}}$ muons must be larger than $15 \mathrm{GeV} / \mathrm{c}$. The latter cut affects neither the signal nor the background, but is considered useful for eliminating unexpected background in real data. The efficiency of the $p_{\mathrm{T}}$ cuts in the signal is close to $90 \%$ while it suppresses around $50 \%$ of the remaining $\mathrm{Zb} \overline{\mathrm{b}}$ events, $40 \%$ of the $t \bar{t}$ events and about $20 \%$ of the $\mathrm{ZZ}$ background. 

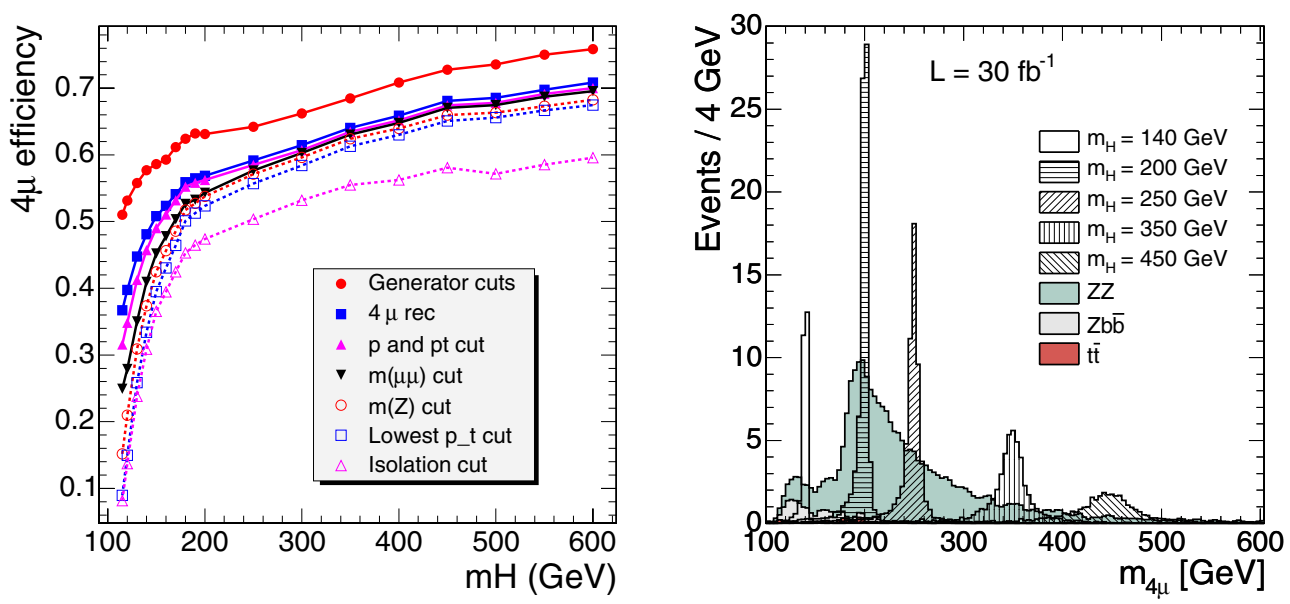

Figure 3.4. (Left) $\mathrm{H} \rightarrow \mathrm{ZZ}^{(*)} \rightarrow 4 \mu$ efficiency $v s$. $m_{\mathrm{H}}$ after different cuts are applied. (Right) Reconstructed four-muon invariant mass distribution, for an integrated luminosity of $30 \mathrm{fb}^{-1}$, for background (shaded histograms) and several Higgs signals (hatched), after the selection criteria are applied.

For the purposes of the isolation cut optimisation, different cone radii and several energy and transverse momentum thresholds have been studied. Those yielding the maximum signal significance are, for calorimeter isolation, a cone radius of 0.24 and energy thresholds of $5 \mathrm{GeV}$ and $9 \mathrm{GeV}$, while for tracker isolation a cone radius of 0.20 and $p_{\mathrm{T}}$ thresholds of $2.5 \mathrm{GeV} / \mathrm{c}$ and $4 \mathrm{GeV} / \mathrm{c}$. The numbers are given for the two least isolated muons. Although a requirement on the isolation of the two most isolated muons does not increase the signal significance, following the same argument as in the case of the $p_{\mathrm{T}}$ cuts, a cut of $3.5 \mathrm{GeV} / \mathrm{c}$ and $5 \mathrm{GeV} / \mathrm{c}$ for the calorimeter isolation and $2 \mathrm{GeV} / \mathrm{c}$ and $2.5 \mathrm{GeV} / \mathrm{c}$ for the tracker isolation is set for the two most isolated muons.

After these cuts, $\mathrm{Zb} \bar{b}$ and $t \bar{t}$ events are suppressed to a negligible level in comparison to the remaining $\mathrm{ZZ}$ background. The efficiencies of each selection cut over the signal, for the 18 Higgs mass points studied, are shown in Fig. 3.4 (left). The four-muon mass distributions for signal and background events that survive the selection cuts are displayed in Fig. 3.4 (right).

In order to estimate the statistical significance of the signal, the log-likelihood ratio (LLR) statistical method $[61,62]$ is used. The distribution to discriminate signal and background is the four-muon invariant mass (Fig. 3.4 (right)). This distribution, for each Higgs boson mass hypothesis and for the background, is used to calculate the $\log$ likelihood ratio, $-2 \ln Q$, which is then used to evaluate the compatibility of the data with either the signal plus background or the background-only hypothesis [60]. The $-2 \ln Q$ estimator is sensitive both to the normalisation and the shape of the discriminant. Each event in the sum has a weight $\ln (1+s / b)$ which depends on the signal-to-background ratio, $s / b$, in the bin where it is found, which in turn depends on the $m_{\mathrm{H}}$ hypothesis. The whole spectrum of the discriminant variable enters the LLR calculation. This avoids any ambiguity in the definition of a signal region for determining the signal significance, present in counting methods.

Figure 3.5 (left) shows the statistical significance, $S_{L} \equiv \sqrt{<2 \ln Q>}$, for an integrated luminosity $30 \mathrm{fb}^{-1}$ at different $\mathrm{m}_{4 \mu}$ invariant masses, should the Higgs boson exist at one of these masses. Based on this distribution, the plot on the right depicts the integrated luminosity required to reach a statistical significance of the signal of $3 \sigma$ and $5 \sigma$, as function of $m_{\mathrm{H}}$. The expected integrated luminosity required to exclude the signal at the $95 \%$ confidence level 

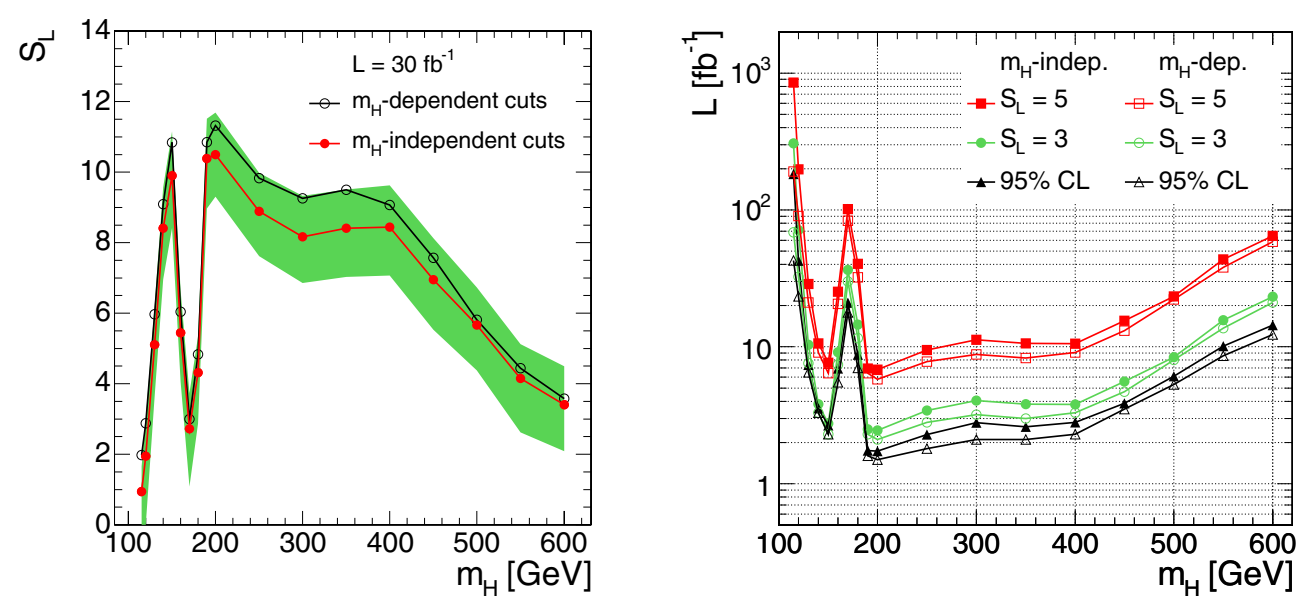

Figure 3.5. (Left) Statistical significance of the signal, $S_{L}$, as function of the Higgs boson mass for an integrated luminosity of $30 \mathrm{fb}^{-1}$, for mass-independent cuts (filled circles) and mass-dependent cuts (empty circles). The shaded band represents the statistical uncertainty on $S_{L}$. (Right) Integrated luminosity, for mass-independent (lines with filled squares, circles, and triangles) and mass-dependent cuts (lines with empty pointers), required to achieve a statistical significance of three (middle pair of curves) and five (upper pair of curves) standard deviations, as a function of the Higgs mass. The integrated luminosity required for excluding a Higgs boson signal at the $95 \%$ C.L. in a background-only experiment is also displayed (lower pair of curves).

in a background-only experiment is also shown as function of $m_{\mathrm{H}}$. The effect of including systematic uncertainties (subsection 3.1.3.3) in the calculation of $S_{L}$ is at the level of $15 \%$ $20 \%$ of the statistical accuracy of the expected significance, supporting that this analysis is not dominated by systematic uncertainties.

In order to more accurately quantify the degree of compatibility of the observed data with any of the two hypotheses, the confidence levels $\mathrm{CL}_{b}$ and $\mathrm{CL}_{s}$ are defined using the $-2 \ln Q$ probability density functions, pdf, for both the background-only and the signal-plusbackground hypotheses (details can be found in Refs. [60, 61]).

The presence of a signal can be inferred from the behaviour of $1-\mathrm{CL}_{b}$ for the background-only hypothesis, which is the probability of observing in a sample of simulated background-only experiments a more signal-like value of $-2 \ln Q$. The observation of the value $1-\mathrm{CL}_{b}=2.85 \times 10^{-7}$ indicates a $5 \sigma$ excess in the data with respect to the background expectation. While $\mathrm{CL}_{b}$ quantifies the lack of compatibility of an excess of observed events with the background-only hypothesis, $\mathrm{CL}_{s}$ gives information about how compatible it is with an actual signal (Fig. 3.6).

3.1.3.2. Search using $\mathrm{m}_{4 \mu}$-dependent cuts. One can take advantage of the fact that the Higgs boson resonance $\mathrm{H} \rightarrow \mathrm{ZZ}^{(*)} \rightarrow 4 \mu$ is relatively narrow and use $\mathrm{m}_{4 \mu}$-dependent cuts for its search. All details of such search strategy can be found in [51]. The analysis steps in this case would be as follows:

- First, events with 4 muons $\left(2 \mu^{+} 2 \mu^{-}\right)$satisfying $p_{\mathrm{T}}, p$, and $m_{\mu^{+} \mu^{-}}$quality cuts as described in Section 3.1.2.1 are selected. This ensures that muons are reliably reconstructed and removes a "contamination" originating from heavy quarkonia decays.

- Second, after reconstructing a four-muon invariant mass, the $\mathrm{m}_{4 \mu}$-dependent cuts are applied. The cuts, being smooth functions of $\mathrm{m}_{4 \mu}$, are optimised in such a way that they maximise the significance of the Higgs signal excess at all Higgs boson mass points. 

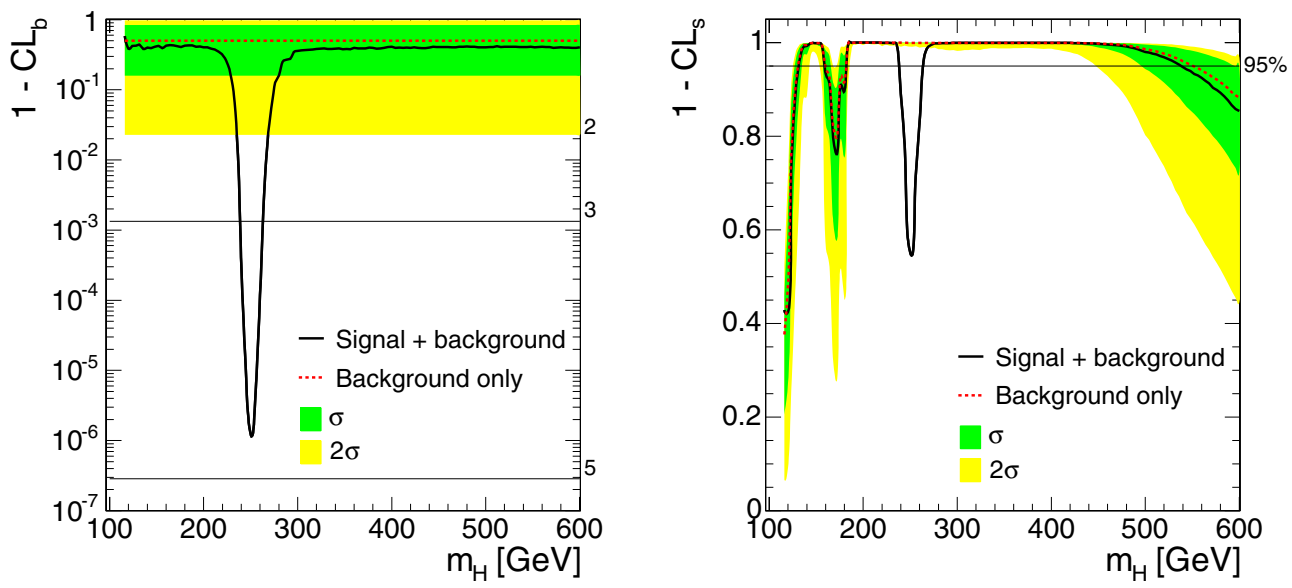

Figure 3.6. Mean values for $1-\mathrm{CL}_{b}$ (left) and $1-\mathrm{CL}_{s}$ (right) as a function of the Higgs boson mass hypothesis, assuming existence of Higgs boson at $250 \mathrm{GeV} / \mathrm{c}^{2}$ mass and for an integrated luminosity of $10 \mathrm{fb}^{-1}$. The observation of the Higgs is just a little bit shy of the $5 \sigma$ discovery (left). The mass points for which the curve $1-\mathrm{CL}_{s}$ is above 0.95 are excluded at $95 \% \mathrm{CL}$ (right). The $1 \sigma$ and $2 \sigma$ bands on $1-\mathrm{CL}_{b}$ and $1-\mathrm{CL}_{s}$, originating from the Poisson statistical fluctuations of the number of background events in each bin of the discriminant distribution, are also shown.

- And finally, the resulting $\mathrm{m}_{4 \mu}$ distribution is analysed for the presence of a Higgs boson resonance. The search can be done using either the LLR significance $S_{L}$ estimator built for the whole spectrum or the LLR $S_{c L}$ estimator built for a single-bin, or signal window (counting experiment). The direct comparison of the results can be found in [51].

To perform the desired $\mathrm{m}_{4 \mu}$-dependent cut optimisation, we used a recently developed program GARCON [63]. The counting experiment significance estimator $S_{c L}$ is the natural tool for such optimisation. The first half of the available Monte Carlo statistics was used for the cut optimisation. The results for the 18 Higgs mass points were then fit to obtain smooth $\mathrm{m}_{4 \mu^{-}}$ dependent cuts. It was found that, given the level of the expected dominant backgrounds $(t \bar{t}$, $\mathrm{Zb} \bar{b}, \mathrm{ZZ}$ ), there are only three critical discriminating cuts (details are given in Ref. [51]):

- The muon isolation cut, both tracker- and calorimeter-based, on the worst isolated muon, or equivalently one common cut on all four muons. This cut strongly suppresses $t \bar{t}$ and $\mathrm{Zb} \overline{\mathrm{b}}$ backgrounds. The cuts gets tighter and tighter as $\mathrm{m}_{4 \mu}$ gets smaller since $\mathrm{Zb} \overline{\mathrm{b}}$ and $t \bar{t}$ increase (Fig. 3.2).

- The $p_{\mathrm{T}}$ on the second lowest $p_{\mathrm{T}}$ muon, or equivalently one common cut on the three highest $p_{\mathrm{T}}$ muons. This cut helps to further suppress $\mathrm{Zb} \overline{\mathrm{b}}$ background to the level well below $\mathrm{ZZ}$ and reduces the $\mathrm{ZZ}$ background at high four-muon invariant masses. This cut becomes more stringent with increasing $\mathrm{m}_{4 \mu}$.

- The $\mathrm{m}_{4 \mu}$ window being used for scanning over the background. It roughly corresponds to the $\pm 2 \sigma$ width, where $\sigma$ is the Higgs boson peak width that includes the detector resolution and the Standard Model Higgs boson width.

The final results are obtained by applying these cuts to the second half of the available Monte Carlo statistics. The observed stability of the results ensures that the cut optimisation did not pick peculiar phase space corners corresponding to statistical flukes. After applying the cuts, the $t \bar{t}$ and $\mathrm{Zb} \overline{\mathrm{b}}$ backgrounds are now suppressed well below the irreducible $\mathrm{ZZ}$ background. 
Figure 3.5 shows, for different Higgs boson masses, the expected significance $S_{L}$

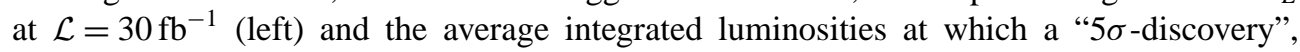
"3 $\sigma$-evidence", and exclusion at $95 \% \mathrm{CL}$ are expected (right). The gain in significance with respect to the flat, $\mathrm{m}_{4 \mu}$-independent, cuts can be easily translated into probabilistic terms. For example, the Higgs boson with $m_{\mathrm{H}}=130 \mathrm{GeV} / \mathrm{c}^{2}$ is right at the " $5 \sigma$-discovery" threshold for an integrated luminosity $\mathcal{L}=30 \mathrm{fb}^{-1}$. The difference in the average expected significance, 5.1 and 6.0, means in this case that the chances of observing significance in excess of 5 for $m_{\mathrm{H}}=130 \mathrm{GeV} / \mathrm{c}^{2}$ at $\mathcal{L}=30 \mathrm{fb}^{-1}$ are $55 \%$ for the flat cuts and $80 \%$ for the $\mathrm{m}_{4 \mu}$-dependent cuts.

3.1.3.3. Systematic errors. The analysis of the systematic errors can be sub-divided into two distinct stages. First, one needs to understand the level of uncertainties in predicting the level of background in the vicinity of a particular $\mathrm{m}_{4 \mu}$ point being investigated for a possible event excess. Second, these uncertainties in the background need to be included in the evaluation of the significance of an excess of events, should it be observed.

Uncertainties in the signal are not very important for establishing an excess of events over the background. It is the uncertainties in the background that are of main concern. After applying the analysis cuts as described earlier, the $\mathrm{ZZ}$ production is the dominant irreducible background with all other processes giving much smaller contributions. This reduces the analysis of systematic errors to those of the $\mathrm{ZZ} \rightarrow 4 \mu$ process.

One can try to evaluate the theoretical and detector performance related uncertainties starting from the first principles. However, especially during the earlier stages of the detector operation when the changes in the system are frequent and hard to monitor and timely incorporate into the detector Monte Carlo simulation, these estimations have limited predictability. Therefore, we developed methods evaluating various corrections, such as muon reconstruction efficiency, muon isolation cut efficiency, directly from data in order to minimise reliance on the Monte Carlo simulation, and, thus, significantly reducing the associated systematic errors. Also, throughout this analysis, we estimate the background around a particular $\mathrm{m}_{4 \mu}$ with reference to a measured control sample. Note that this completely eliminates uncertainties associated with measuring the luminosity and reduces the sensitivity to PDF and QCD-scales. For the control sample, we use either the inclusive $\mathrm{Z} \rightarrow 2 \mu$ process or sidebands of the $\mathrm{m}_{4 \mu}$ spectrum itself.

The main uncertainties can be grouped as follows:

1. Uncertainties associated with the background production rates, i.e. not directly related to CMS Detector performance itself:

- ZZ: PDF and QCD scale uncertainties described in details in Ref. [47].

- ZZ: NLO and NNLO contributions vs LO described in details in Ref. [58] plus some related issues are discussed in Ref. [42]. These possible uncertainties are not taken into account in the results shown below, for details see Ref. [51].

- LHC luminosity: when we estimate the ZZ background events in the signal region via the measured number of events in the control samples, the luminosity uncertainties largely cancel out.

2. Uncertainties associated with the CMS detector performance (hardware/software) and our analysis-specific cuts:

- ZZ: Trigger efficiency, being very close to $100 \%$ due to presence of four muons, does not have substantial systematic errors. 

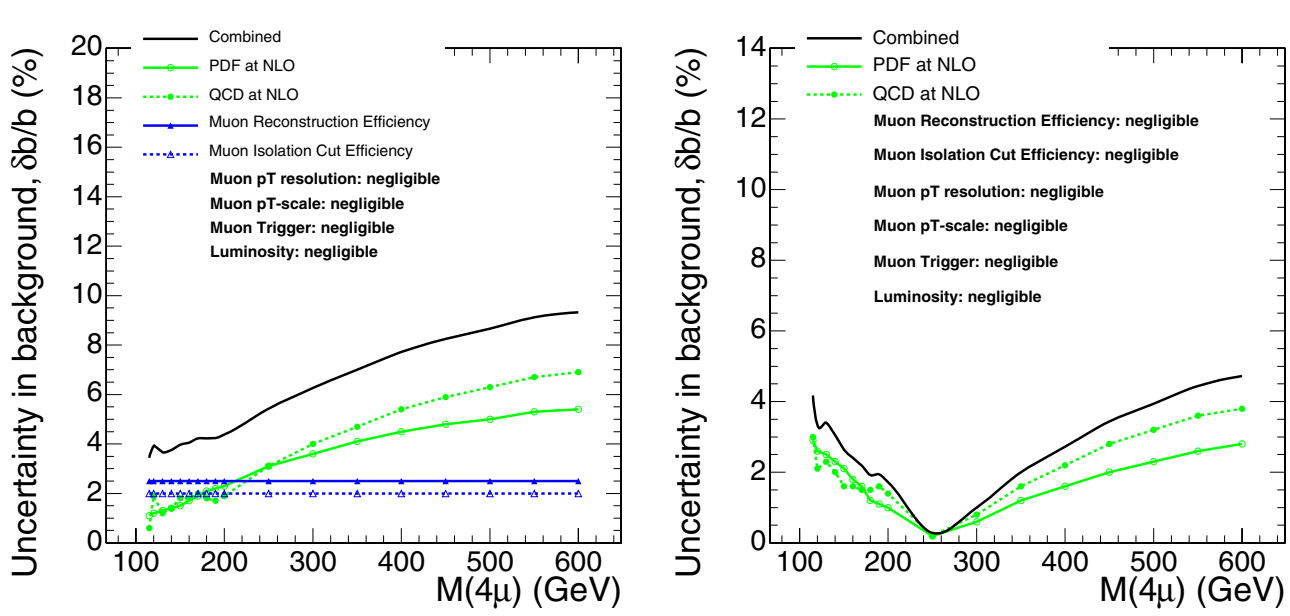

Figure 3.7. Uncertainties in the count of the $\mathrm{ZZ} \rightarrow 4 \mu$ background events in the signal region window at different $\mathrm{m}_{4 \mu}$. The window size is $\pm 2 \sigma$ of the expected experimental Higgs resonance width. (Left) The background event count in the signal region is derived from the measured number of $\mathrm{Z} \rightarrow 2 \mu$ events. (Right) The background event count in the signal region, $b$, is calculated from the number of $\mathrm{ZZ} \rightarrow 4 \mu$ events $B$ in the range $100 \mathrm{GeV} / \mathrm{c}^{2}-700 \mathrm{GeV} / \mathrm{c}^{2}$ (excluding the signal region window), i.e. $b=\rho \cdot B$.

- ZZ: The muon reconstruction efficiency is determined directly from data [59]. The associated systematic error is less than $1 \%$ per muon. Using normalisation to the measured $\mathrm{Z} \rightarrow 2 \mu$ process, this leaves us with $2 \%$ uncertainty per event for the $\mathrm{ZZ} \rightarrow 4 \mu$ background production.

- ZZ: The muon isolation cut efficiency is also determined directly from data [64] with about $2 \%$ uncertainty per event.

- Higgs: $\mathrm{m}_{4 \mu}$ resolution is affected by muon $p_{\mathrm{T}}$ resolution. This almost does not affect the background distribution. In [51], we show that even making a mistake in the $\mathrm{m}_{4 \mu}$ distribution width by as much as $25 \%$ has only a tiny effect on evaluating a significance of an excess of events. The muon $p_{\mathrm{T}}$ resolution is fairly easy to measure from data using the measured $J / \psi$ and $Z$ peak widths with the precision much better than needed.

- ZZ: $\mathrm{m}_{4 \mu}$ scale. The effect of these uncertainties on the number of background events in a signal window appears only on steep slopes of the $m_{4 \mu}$ distribution. For the steepest part of the $\mathrm{m}_{4 \mu}$ distribution in the $180 \mathrm{GeV} / \mathrm{c}^{2}-200 \mathrm{GeV} / \mathrm{c}^{2}$ range, we obtain $\delta b / b \sim 0.1 \delta \mathrm{m}_{4 \mu}$, where $\mathrm{m}_{4 \mu}$ is in $\mathrm{GeV} / \mathrm{c}^{2}$ and $b$ is the number of background events. This implies that to be able to neglect this effect, one needs to know the momentum scale with precision of $0.1 \mathrm{GeV}$ at $p_{\mathrm{T}} \sim 50 \mathrm{GeV} / \mathrm{c}$. This can be easily achieved with just a few hundreds of $\mathrm{Z} \rightarrow 2 \mu$ events.

Fig. 3.7 summaries all systematic errors on the expected number of events in the $Z \rightarrow 4 \mu$ background for the two methods: via referencing to the total measured $\mathrm{Z} \rightarrow 2 \mu$ cross section and via referencing to the event count in the sidebands of the $\mathrm{m}_{4 \mu}$ spectrum itself.

\section{Significance with the background uncertainties included}

For the Gaussian-like signal over relatively flat background, the $S_{L}$ and $S_{C L}$ estimators are strongly correlated, with the typical difference of 5\%-10\% [51]. This stems from the fact that the signal peak is very localised and the background is relatively flat. This allows us to study 


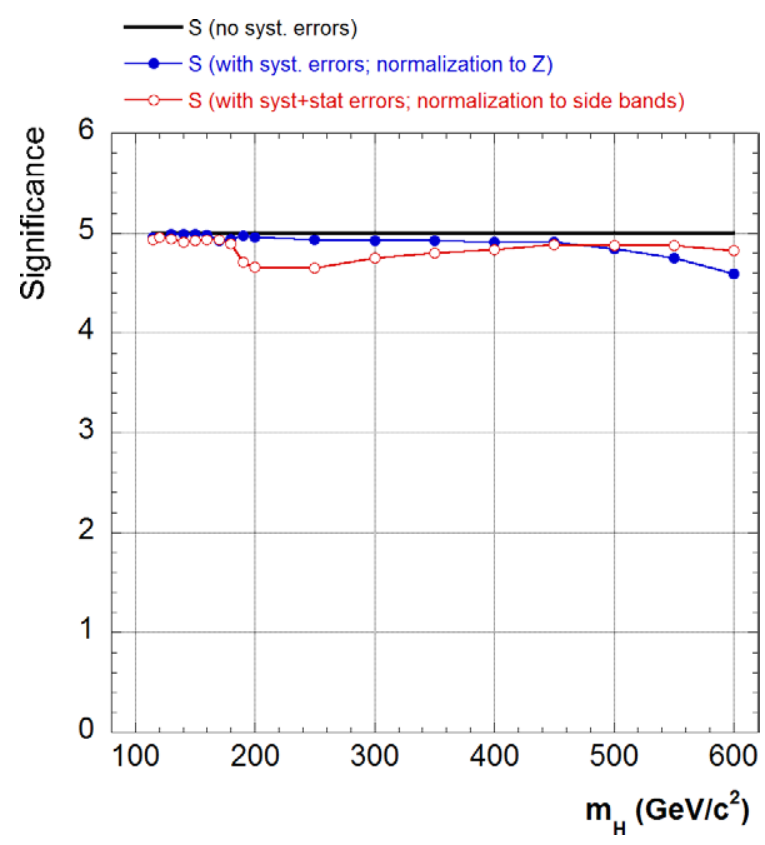

Figure 3.8. Effect of including systematic errors into evaluation of significance at the time of measurements. The reference luminosities, dependent on the Higgs boson mass, are chosen to correspond to an observation of significance $S=5$ without systematic errors. Solid circles show degrading of significance for the case of systematic errors when the background is evaluated from the measured $\mathrm{Z} \rightarrow 2 \mu$ cross section. Open circles show the effect for the case when the background in signal region is normalised to the sidebands.

the effect of systematic errors on the evaluation of significance at the time of measurements using the counting experiment approach, for which everything can be done analytically. All details on the method we use can be found in Ref. [51]. The method allows to account for the theoretical and instrumental systematic errors as well as for statistical errors when a control sample with a limited event count is used.

The final result of these studies is presented in Fig. 3.8. Starting from an integrated luminosity at which the statistical significance of a Higgs boson observation would be equal to 5 (if the level of background without any errors was known), the figure shows how this significance must be de-rated due to the systematic errors at the time of the measurements as described in the previous sub-section. The effect of systematic errors at low or high luminosities is not as important: at lower luminosity the significance is not sufficient to make serious claims, anyway; while after surpassing the significance of 5 , the existence of the Higgs boson can be considered established and the focus must be switched to measuring its parameters.

The two curves with full and open circles show the difference of the two methods for evaluating the background in the signal region: via normalisation to the measured $\mathrm{Z} \rightarrow$ $2 \mu$ cross section, and via normalisation to the event count in sidebands $\left(100 \mathrm{GeV} / \mathrm{c}^{2}\right.$ to $700 \mathrm{GeV} / \mathrm{c}^{2}$, excluding the signal region). The effect of systematic errors at lower luminosities becomes smaller for the former method and quickly diverges for the latter. As the luminosity increases, the trends obviously reverse. Around the threshold of $S=5$, the difference between the two methods is not very dramatic; the true benefit of using two approaches to estimating background from data is in their complementarity. 


\section{Local significance and overall statistical fluctuation probability}

In a search for a relatively narrow $4 \mu$ invariant mass peak over a broad background spectrum, one must take into account that the probability of observing a background fluctuation giving an excess of events consistent with a Higgs hypothesis of some particular mass might be considerably higher that the local significance calculated for a given mass might imply. This over-estimation of significance strongly depends on how the analysis is set and what constraints/priors on the "phase space" of parameters are used. For example, in a search specifically tailored for the Standard Model Higgs, the only free parameter is the Higgs boson mass; its width, production cross section, and decay branching ratios are dependent on the mass. To make the search even more constrained, one can use a prior on the Higgs mass as it comes out from the precision electroweak measurements. A specific case study showing the potential scope of the effect, which may be comparable or even larger than the effect of the systematic errors discussed above, is given in Appendix A.

\subsubsection{Measurement of the Higgs boson properties at $\mathcal{L}=30 \mathrm{fb}^{-1}$}

The capabilities of the CMS detector to measure the mass, cross section and width of the Higgs boson are determined for an integrated luminosity of $30 \mathrm{fb}^{-1}$ [65]. These parameters are measured using a binned maximum likelihood fit to the reconstructed four-muon invariant mass, which includes the signal and background contributions after all the selection cuts have been applied (Fig. 3.4 (right)). The 'observed' distribution, $f_{s b}$, is expressed in terms of the signal, $p_{s}$, and background, $p_{b}$, probability density functions (pdf) as:

$$
f_{s b}\left(\mathrm{~m}_{4 \mu} ; m_{f i t}, \Gamma, N_{s}, N_{b}\right)=N_{s} \cdot p_{s}\left(\mathrm{~m}_{4 \mu} ; m_{f i t}, \Gamma\right)+N_{b} \cdot p_{b}\left(\mathrm{~m}_{4 \mu}\right)
$$

$N_{s}$ is the number of signal events, $N_{b}$ the number of background events, $m_{f i t}$ the position of the mass peak and $\Gamma$ the intrinsic width of the Higgs boson. The signal pdf is the sum of two contributions: a convolution of a Breit-Wigner signal shape with a Gaussian distribution that accounts for detector resolution, $p_{\text {core }}$, and a function that reproduces the radiative tail due to internal bremsstrahlung, $p_{\text {tail }}$ :

$$
p_{s}=\beta \cdot p_{\text {core }}\left(\mathrm{m}_{4 \mu} ; m_{f i t}, \Gamma, \sigma\right)+(1-\beta) \cdot p_{\text {tail }}\left(\mathrm{m}_{4 \mu} ; m_{\text {fit }}, \tau\right)
$$

where $1-\beta$ is the fraction of signal events in the radiative tail. The tail shape is parameterised ad hoc as

$$
p_{\text {tail }}=\frac{\left(\mathrm{m}_{4 \mu}-m_{f i t}\right)^{2}}{2 \tau^{3}} \exp \left(\frac{\mathrm{m}_{4 \mu}-m_{f i t}}{\tau}\right)
$$

if $\mathrm{m}_{4 \mu}<m_{f i t}$ and is zero otherwise [66]. Figure 3.9 (left) illustrates the different contributions to $f_{s b}$. The $p_{s}$ function is fitted to the signal-only distributions to obtain the parameters of the radiative tail, which remain fixed in the fit to the signal plus background spectra.

For Higgs boson masses below $190 \mathrm{GeV} / \mathrm{c}^{2}$, the intrinsic width is negligibly small compared to the mass spread introduced by the experimental resolution and the signal is thus approximated by a Gaussian shape. For masses above $400 \mathrm{GeV} / \mathrm{c}^{2}$, the natural width of the Higgs is much larger than the experimental resolution, hence the description using a pure Breit-Wigner function yields similar parameters as those obtained from the convolution.

The detector resolution is extracted from the $\mathrm{m}_{4 \mu}$ distribution of $\mathrm{ZZ}$ events with a fourmuon mass above $2 m_{\mathrm{Z}}$, for which the kinematics is similar to that of the signal. For masses below $2 m_{\mathrm{Z}}$, the intrinsic Higgs boson width is negligible, therefore the resolution is measured directly from the width of the $\mathrm{m}_{4 \mu}$ distribution. This width has been found to be consistent with the extrapolation of the resolution determined using $\mathrm{ZZ}$ events. 

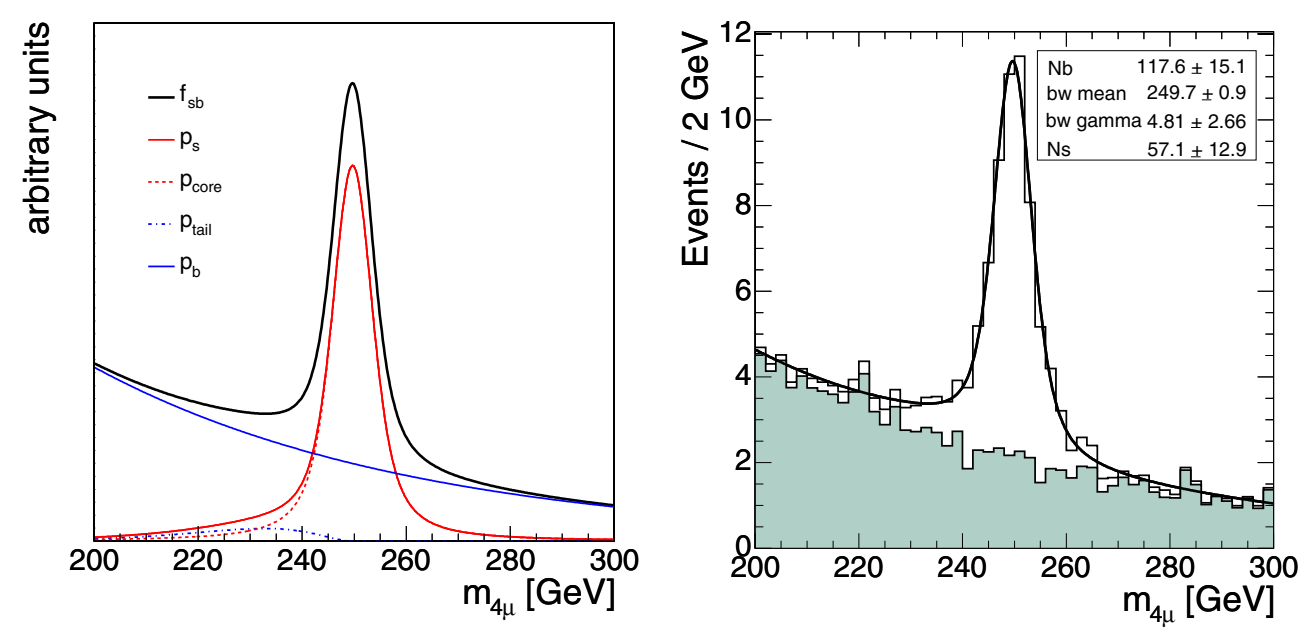

Figure 3.9. (Left) Example of the shapes of the different contributions to $f_{s b}$. (Right) Data-like distribution expected for a Higgs boson signals of $m_{\mathrm{H}}=250 \mathrm{GeV} / \mathrm{c}^{2}$, for an integrated luminosity of $30 \mathrm{fb}^{-1}$, together with the result of the fit (solid line) and the expected background (shaded area). This pseudo-experiment is selected randomly.
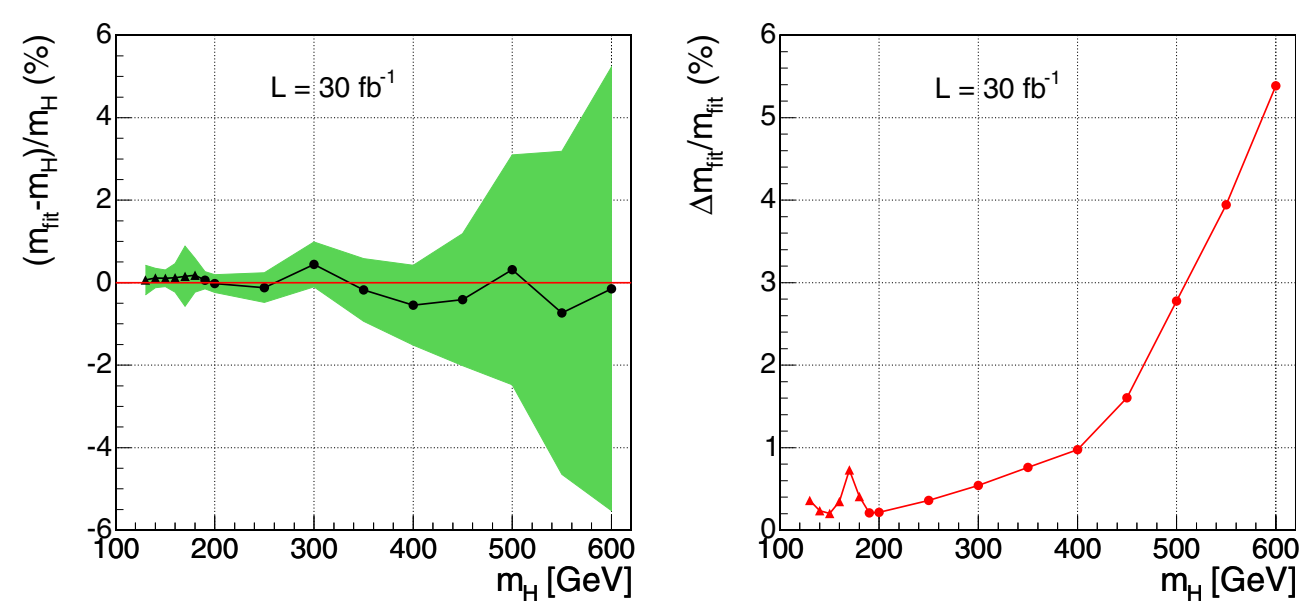

Figure 3.10. (Left) Relative shift of the fitted value of the Higgs boson mass with respect to the input $m_{\mathrm{H}}$ value, as function of $m_{\mathrm{H}}$. The shaded area is the error in the determination of the peak value from the fit, also shown as function of the Higgs boson mass (right). The dots correspond to the result of the convolution and the triangles to the Gaussian approximation.

The background pdf, $p_{b}$, is approximated by either a polynomial or an exponential function, depending on the mass region under study. The parameters are determined performing a binned maximum likelihood fit to the background sample. The parameters defining the shape of the background are fixed in the global fit to signal plus background, but not its normalisation.

The values of the parameters, together with their errors, are obtained directly from the fit. The result of the fit to the signal plus background distribution is shown in Fig. 3.9 (right) for a Higgs boson signal of $m_{\mathrm{H}}=250 \mathrm{GeV} / \mathrm{c}^{2}$. Figure 3.10 (left) depicts the relative shift of the fitted Higgs boson mass with respect to the true mass, together with its statistical error. These values are compatible with zero in the full range of masses, which means that the true mass 

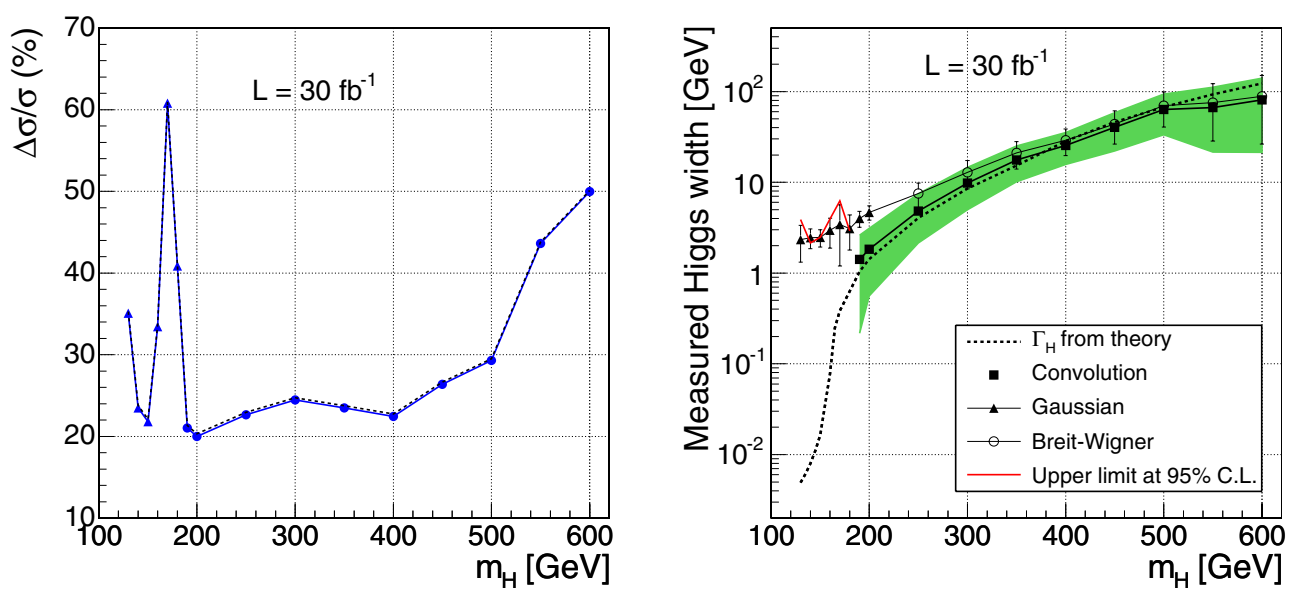

Figure 3.11. (Left) Relative error in the cross-section measurement, $\Delta N_{s} / N_{s}$, as a function of the $m_{\mathrm{H}} . \Delta N_{s}$ is the statistical error of $N_{s}$ obtained from the fit. The dots correspond to the result of the convolution and the triangles to the Gaussian approximation. The dashed line indicates the impact of the systematic uncertainties. (Right) Measured Higgs boson width (squares), its statistical error (green band) and the theoretical calculation of $\Gamma_{\mathrm{H}}$ (dashed line). Upper limits to the width at 95\% C. L. are shown (red line) for $m_{\mathrm{H}}<190 \mathrm{GeV} / \mathrm{c}^{2}$. The result of Gaussian (triangles) and BreitWigner (dots) fits are also shown for comparison.

is accurately recovered after applying the fitting method to the reconstructed sample. The evolution of the relative error as a function of the true mass is displayed in Fig. 3.10 (right), showing that the mass can be measured with precisions from $0.1 \%$ to $5.4 \%$. The increase in this error around $170 \mathrm{GeV} / \mathrm{c}^{2}$ is due to the smaller signal statistics caused by the suppression of the $\mathrm{H} \rightarrow \mathrm{ZZ}^{(*)}$ decay at this mass. The increasing uncertainty at higher masses is due to the smaller production cross sections, the larger intrinsic width of the Higgs boson and, to a lesser extent, the worse resolution for high $p_{\mathrm{T}}$ muons.

The number of signal and background events is obtained from the fit. The relative error in the cross-section measurement is determined from the number of signal events $\left(N_{s}\right)$ and its statistical uncertainty $\left(\Delta N_{s}\right)$ as $\Delta N_{s} / N_{s}$, shown in Fig. 3.11 (left) as function of the Higgs boson mass. The contribution of the background is properly taken into account, as its normalisation is a free parameter in the fit. The cross section can be determined with a precision between $20 \%$ and $45 \%$, except for masses below $130 \mathrm{GeV} / \mathrm{c}^{2}$, where the statistics is low.

The measured width, together with its statistical error, is presented in Fig. 3.11 (right) as function of the true mass. The width can be determined with an error between $35 \%$ and $45 \%$ above $190 \mathrm{GeV} / \mathrm{c}^{2}$. Below this mass there is no sensitivity to the Higgs boson width and upper limits at $95 \%$ confidence level (C.L.) are set. For the sake of comparison, the width obtained by fitting only a Gaussian for masses below $200 \mathrm{GeV} / \mathrm{c}^{2}$ and only a Breit-Wigner for masses above $200 \mathrm{GeV} / \mathrm{c}^{2}$ is also shown, together with the statistical uncertainty. The Breit-Wigneronly fits do not take into account the detector resolution, and therefore the intrinsic theoretical values are not recovered.

The measurement of the parameters is affected by systematic uncertainties in the muon momentum resolution (determined from data), in the muon reconstruction efficiency (around 2\%) and those associated to the selection cuts (close to 1\%) [60]. These systematic uncertainties are mostly uncorrelated. The impact in the measured mass and width is small. The cross-section measurement is also affected by the uncertainty in the luminosity determination, which is around 3\% (Fig. 3.11 (left)). 
The results obtained for Higgs boson masses around $170 \mathrm{GeV} / \mathrm{c}^{2}$ and above $500 \mathrm{GeV} / \mathrm{c}^{2}$, for which the expected number of events is somewhat low for $\mathcal{L}=30 \mathrm{fb}^{-1}$, have to be taken as representative results for the typical expected distributions. The higher errors of the parameters for those $m_{\mathrm{H}}$ values are consistent with statistics. For extending the measurement of the Higgs boson parameters to smaller masses or to lower luminosities, it should be more appropriate to extract the parameters from a large set of randomly chosen four-muon mass distributions with the correct statistics.

\subsubsection{Conclusions}

Discovery of the Standard Model Higgs boson and measurement of its mass, production cross section and width in the "golden" decay mode $\mathrm{H} \rightarrow \mathrm{ZZ}^{(*)} \rightarrow 4 \mu$ were analysed with the CMS Detector. The explored range of Higgs boson masses was $115 \mathrm{GeV} / \mathrm{c}^{2}-600 \mathrm{GeV} / \mathrm{c}^{2}$. The Monte Carlo samples were normalised to represent the NLO cross sections, including $\mathrm{m}_{4 \mu}$-dependent K-factors. To simulate the detector response and reconstruct physics objects, the full CMS Detector simulation and reconstruction software was used. The Higgs boson discovery potential was explored for different analysis variations, including the use of $\mathrm{m}_{4 \mu}$-dependent and flat cuts, log-likelihood ratio based on the full $\mathrm{m}_{4 \mu}$ spectrum and a straightforward counting experiment approach. A full treatment of the most important theoretical and instrumental systematic errors and their effect on evaluation of significance of the Higgs boson observation as well as measuring its parameters were presented. To minimise systematic errors, a number of methods of reconstructing the necessary corrections directly from data were developed.

It was shown that at $\sim 2 \mathrm{fb}^{-1}$ of integrated luminosity, CMS would be able to start excluding the Standard Model Higgs boson at $95 \% \mathrm{CL}$ for $m_{\mathrm{H}}$ in vicinity of $200 \mathrm{GeV} / \mathrm{c}^{2}$. By the time CMS reaches $\sim 30 \mathrm{fb}^{-1}$, it would exclude the Standard Model Higgs boson in its four-muon decay mode in the mass range $m_{\mathrm{H}}=120 \mathrm{GeV} / \mathrm{c}^{2}-600 \mathrm{GeV} / \mathrm{c}^{2}$, if indeed it does not exist.

The discoveries at the level of " $5 \sigma$ " local significance could be already possible at $\sim 10 \mathrm{fb}^{-1}$ for $m_{\mathrm{H}}$ in the range $140 \mathrm{GeV} / \mathrm{c}^{2}-150 \mathrm{GeV} / \mathrm{c}^{2}$ and $190 \mathrm{GeV} / \mathrm{c}^{2}-400 \mathrm{GeV} / \mathrm{c}^{2}$. By the time $\sim 30 \mathrm{fb}^{-1}$ are collected, the discovery range would open up to $130 \mathrm{GeV} / \mathrm{c}^{2}-160 \mathrm{GeV} / \mathrm{c}^{2}$ and $180 \mathrm{GeV} / \mathrm{c}^{2}-500 \mathrm{GeV} / \mathrm{c}^{2}$. An observation of the Higgs boson with the mass $m_{\mathrm{H}} \sim$ $170 \mathrm{GeV} / \mathrm{c}^{2}$ or $\sim 600 \mathrm{GeV} / \mathrm{c}^{2}$ in the $\mathrm{H} \rightarrow \mathrm{ZZ}^{(*)} \rightarrow 4 \mu$ decay channel would require an integrated luminosity of the order of $100 \mathrm{fb}^{-1}$.

At the integrated luminosity of $\sim 30 \mathrm{fb}^{-1}$, the Higgs boson mass could be measured with a precision between $0.1 \%$ and $5.4 \%$, depending on its mass. The intrinsic width could be measured only for the Higgs boson heavier than $190 \mathrm{GeV} / \mathrm{c}^{2}$, with a precision $\sim 35 \%$. For lower masses, the Higgs boson width becomes much smaller than the detector resolution and only upper limits of the order of a few $\mathrm{GeV}$ could be set. The production cross section would be determined with a precision $\sim 30 \%$.

\subsection{Benchmark Channel: $H \rightarrow W W^{(*)} \rightarrow 2$ muons}

\subsubsection{Introduction}

Previous studies $[67,68]$ demonstrated the relevance of the $\mathrm{H} \rightarrow \mathrm{ZZ}^{(*)} \rightarrow 2 / 2 v$ channel for the Higgs discovery with an integrated luminosity of less than $5 \mathrm{fb}^{-1}$. The physics study was performed on the data produced at the end of the full simulation, trigger and off-line detector 
reconstruction chain, including realistic assumptions for the sub-detectors misalignments. The goal of this study is to provide the discovery potential as a function of the Higgs mass using detailed simulation reconstruction code, considering all the relevant background contributions and providing an as much as possible complete estimation of the systematic errors. The muon reconstruction has an average efficiency in the detector geometrical acceptance $(\eta<2.4)$ of $95-99 \%$ for the transverse momentum ranging from $5 \mathrm{GeV} / \mathrm{c}$ up to $P_{\mathrm{T}}=1 \mathrm{TeV} / \mathrm{c}$, as extensively discussed in [7], while the fraction of mis-assigned charge for muons with $P_{\mathrm{T}}=100 \mathrm{GeV} / \mathrm{c}$ is less than $0.1 \%$.

\subsubsection{Physics processes}

3.2.2.1. Signal processes. The signal was studied in the range between 130 to $180 \mathrm{GeV}$ using 7 samples of datasets (Table 3.1). The generation was done using the PYTHIA program [69], considering the most relevant signal sources:

$$
\begin{aligned}
& g g \rightarrow H \rightarrow W W^{(*)} \rightarrow 2 \mu 2 v \\
& q \bar{q} \rightarrow V V q^{\prime} \bar{q}^{\prime} \rightarrow H q^{\prime} \bar{q}^{\prime} ; H \rightarrow W W^{(*)} \rightarrow 2 \mu 2 v
\end{aligned}
$$

In the simulation, digitisation and reconstruction the effect of the event pile up expected at the machine luminosity $2 \times 10^{33} \mathrm{~cm}^{-1} \mathrm{~s}^{-2}$ was included. An example of a pp $\rightarrow \mathrm{H}+\mathrm{X}$ event with $\mathrm{H} \rightarrow \mathrm{WW} \rightarrow \mu \nu \mu \nu$ is shown in colour plate $\mathrm{CP} 4$.

3.2.2.2. Background processes. The dominant background giving the largest contribution at the end of the complete selection chain, is the irreducible one from the continuum production of $\mathrm{W}$ pairs decaying into muons and neutrinos. Other significant or critical sources of backgrounds are the production of top quarks and the Drell-Yan muon pairs. The most important backgrounds are thus the processes:

$$
\begin{aligned}
& q \bar{q} \rightarrow W^{+} W^{-} \rightarrow 2 \mu 2 v \\
& g g \rightarrow t \bar{t} \rightarrow 2 \mu 2 v \\
& q \bar{q} \rightarrow \gamma^{*}, Z \rightarrow 2 \mu
\end{aligned}
$$

Further contributions from $b \bar{b}, g g W W, W Z, Z Z$, and $W t$ production processes were also considered. A part from $W t$ and $g g \rightarrow W W$, all the processes have been generated with PYTHIA. For the former process, the TopReX Monte Carlo [44] has been used which correctly takes into account the top mass and the spin correlations throughout the decay chain. The latter dataset has been simulated starting from a Monte Carlo sample produced by N. Kauer et al. [70]. The full list of dataset samples used for the background study is given in Table 3.2.

3.2.2.3. Cross sections at NLO. All the processes considered in this study have been simulated with LO accuracy. In order to approximate the NLO predictions for the signal and the W-pair background, phase space depended reweighting K-factors has been applied [71]. These factors have been obtained by matching respectively the $p_{\mathrm{T}}$ distribution of the Higgs and of the $W^{+} W^{-}$system provided by PYTHIA to the one predicted by MC@ NLO $[72]^{38}$. The $\mathrm{K}\left(p_{\mathrm{T}}\right)$ factors used for each $p_{\mathrm{T}}$ intervals are given in Appendix of [73]. The absolute cross sections for Higgs production through gluon-gluon fusion and vector boson fusion have been calculated [20] and are listed in Table 3.1.

\footnotetext{
${ }^{38}$ For the signal, only the Higgs production through the gluon-gluon mechanism has been reweighted with $\mathrm{K}\left(p_{\mathrm{T}}\right)$ factors accordingly to NLO description.
} 
Table 3.1. The cross section at the next-to-leading order for Higgs production through gluon fusion and vector boson fusion (VFB) processes and the number of generated events are reported.

\begin{tabular}{lccc}
\hline $\begin{array}{l}\text { Higgs mass } \\
\left(\mathrm{GeV} / \mathrm{c}^{2}\right)\end{array}$ & $\begin{array}{c}\sigma^{N L O} \times B R(2 l) \\
\text { Gluon Fusion }(\mathrm{pb})\end{array}$ & $\begin{array}{c}\sigma^{N L O} \times B R(2 l) \\
\mathrm{VBF}(\mathrm{pb})\end{array}$ & $\begin{array}{c}\sigma^{N L O} \times B R(2 l) \\
\text { num. of events }\end{array}$ \\
\hline 130 & 0.94 & 0.12 & 20000 \\
140 & 1.39 & 0.19 & 20000 \\
150 & 1.73 & 0.25 & 17000 \\
160 & 2.03 & 0.31 & 44000 \\
165 & 2.04 & 0.32 & 49000 \\
170 & 1.95 & 0.31 & 40000 \\
180 & 1.71 & 0.28 & 20000 \\
\hline
\end{tabular}

Table 3.2. The cross section at the next-to-leading order for the background processes. The $g g \rightarrow$ $W W$ process is generated using a matrix element program linked to PYTHIA for the showering [70]. This process is only known at LO. (*) For $b \bar{b} \rightarrow 2 \mu$ the pre-selection $p_{\mathrm{T}}>20,10 \mathrm{GeV} / \mathrm{c}$ was applied.

\begin{tabular}{lcr}
\hline Channel & $\sigma^{N L O} \times B R(p b)$ & num. of ev. \\
\hline$q q \rightarrow W W \rightarrow 2 l$ & 11.7 & 164000 \\
$t \bar{t}$ & 840 & 548000 \\
$g g \rightarrow W W \rightarrow 2 l$ & $0.54(\mathrm{LO})$ & 50000 \\
$\gamma^{*}, Z$ & 145000 & 2700000 \\
$b \bar{b} \rightarrow 2 \mu$ & $710(\mathrm{LO})\left(^{*}\right)$ & 640000 \\
$Z W \rightarrow 3 l$ & 1.63 & 72000 \\
$t W b \rightarrow 2 l$ (TopREX) & 3.4 & 191000 \\
$Z Z \rightarrow 2 l$ & 1.52 & 99000 \\
\hline
\end{tabular}

No reweighting has been applied to the other processes, whose total cross sections have been simply rescaled accordingly the NLO calculation performed sing the MCFM Monte Carlo program $[55,74,75]$. These cross sections are reported in Table 3.2.

\subsubsection{Event selection}

The signal selection requires the identification of two high $p_{\mathrm{T}}$ isolated muons. The background reduction is obtained applying suitable kinematic cuts to the reconstructed muons, a veto on the presence of central jets and a high missing $E_{\mathrm{T}}$ (MET) in the event. As discussed in the following sections, separate optimisations were performed independently on the muon isolation variables, jet and missing energy thresholds and on the muons kinematical variables.

\subsubsection{The trigger selection}

Events passing the global Level-1 trigger must be reduced with a more restricted trigger requirement to limit the recorded event rate. Two trigger streams were considered in this analysis:

1. the HLT double muon stream;

2. the OR of the HLT single muon and double muon stream. 
Before any selection the single or double muon HLT trigger efficiency is $92 \%$, while the double muon HLT trigger efficiency is 80\% [76]. After the off-line cuts for the Higgs selection, which will be described in detail in the following section, the overall efficiency of the first stream relative to the second one is found to be $(97 \pm 1) \%$, for $m_{H}=165 \mathrm{GeV} / \mathrm{c}^{2}$. In the following, the trigger selection used was the HLT double muon stream, for which the trigger rate is predicted to be a factor $\sim 7$ smaller than the single muon one [76].

3.2.4.1. The muon identification and isolation. A first event selection based on the identification of two prompt muons required:

- Level-1 and HLT dimuon trigger bits found;

- two oppositely charged muons reconstructed by the Global Muon reconstructor algorithm developed in ORCA, as described in [7].

The first requirement assures the events to be found in the CMS dimuon data stream, which currently foresees a symmetric threshold of $7 \mathrm{GeV} / \mathrm{c}$ on the $p_{\mathrm{T}}$ of both muons as reconstructed by the High Level Trigger algorithm, for operations at a machine luminosity of $2 \times 10^{33} \mathrm{~cm}^{-2} \mathrm{~s}^{-1}$; in addition, at least one of the muons must fulfill the HLT isolation criteria [76]. As discussed in Ref. [76], the trigger rate for this datastream is predicted to be about $4 \mathrm{~Hz}$.

At the off-line reconstruction and selection stage, two cones were considered for the isolation around each reconstructed muon tracks. The $\sum P_{\mathrm{T}}$ summed over all the charged track candidates found in the Tracker detector was accounted inside the first cone. The $\sum E t$ over the energy deposits in the ECAL and HCAL towers was accounted in the second cone. The size of a cone around a muon track is defined as $\Delta R=\sqrt{\Delta \eta^{2}+\Delta \phi^{2}}$. A muon is considered to be isolated if the $\sum P t\left(\sum E t\right)$ inside the considered cones of size $\Delta R_{\text {Tracker }}$ $\left(\Delta R_{\text {Calo }}\right)$ is below the threshold $P_{\mathrm{T}}(\max )\left(E_{\mathrm{T}}(\max )\right)$. An optimisation study was performed to find the four parameters:

$$
\begin{array}{llll}
\text { (1) } \Delta R_{\text {Tracker }} & \text { (2) } P_{\mathrm{T}}(\max ) & \text { (3) } \Delta R_{\text {Calo }} & \text { (4) } E_{\mathrm{T}}(\max )
\end{array}
$$

searching for the highest signal over background ratio. The optimisation was performed using the signal dataset with $m_{H}=165 \mathrm{GeV} / \mathrm{c}^{2}$ and the $b \bar{b}$ background dataset, which is the most sensitive to the isolation cut. At this first stage of the selection, the background reduction was not requested to be very large, thus keeping the signal reduction relatively small; for each combination of the cones:

$$
\Delta R_{\text {Tracker }}=0.25,0.3,0.35,0.4 \quad \Delta R_{\text {Calo }}=0.25,0.3,0.35,0.4
$$

the cut efficiency of $85 \%$ for the signal was requested. With two free parameters, $E_{\mathrm{T}}(\max )$ and $P_{\mathrm{T}}$ (max), several solutions are possible. A reasonable choice is to give the same weight to the Tracker and Calorimeter isolation cuts. The mean and the r.m.s. values of the $p_{\mathrm{T}}$ and energy deposition for the signal dataset within different cones are reported in [77]. For each set of isolation cones $\left(\Delta R_{\text {Tracker }}, \Delta R_{\text {Calo }}\right)$ the $E_{\mathrm{T}}$ and $P_{\mathrm{T}}$ thresholds were chosen as follows:

$$
\begin{aligned}
& E_{\mathrm{T}}^{\text {thresh }}=<E_{\mathrm{T}}>+x \cdot \sigma\left(E_{\mathrm{T}}\right) \\
& P_{\mathrm{T}}^{\text {thresh }}=<P_{\mathrm{T}}>+x \cdot \sigma\left(P_{\mathrm{T}}\right)
\end{aligned}
$$

where the parameter $x$ was set to the value giving the required $85 \%$ efficiency for the signal. Figure 3.12 shows the resulting background selection efficiency. 


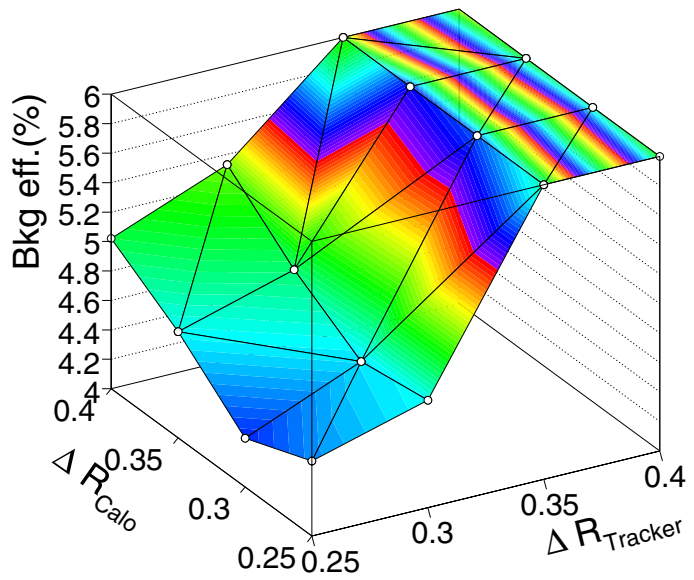

Figure 3.12. $b \bar{b}$ background efficiencies for the 16 combinations of cones considered for the muon isolation selection cut.

The best selection is obtained with:

$$
\Delta R_{\text {Tracker }}=0.25 \quad P_{\mathrm{T}}<2.0 \mathrm{GeV} / \mathrm{c} \quad \Delta R_{\text {Calo }}=0.3 \quad E_{\mathrm{T}}<4.7 \mathrm{GeV}
$$

corresponding to $x=1.8$ for the energy deposition and $P_{\mathrm{T}}$ cut. The isolation cuts used in the analysis were:

$$
\Delta R_{\text {Tracker }}=0.25 \quad P_{\mathrm{T}}<2.0 \mathrm{GeV} / \mathrm{c} \quad \Delta R_{\text {Calo }}=0.3 \quad E_{\mathrm{T}}<5.0 \mathrm{GeV} .
$$

\subsubsection{Jet reconstruction and the jet veto}

The reconstruction of jets is needed to obtain a strong $t \bar{t}$ background reduction by applying a jet veto. The jet reconstruction algorithms can use the raw energy sum of the ECAL and HCAL towers, either with a fixed energy threshold or with $\eta$-dependent thresholds. The $\eta$-dependent threshold does not improve the $t \bar{t}$ background rejection with respect to a fixed combined $E_{\mathrm{T}}$ and $E$ thresholds [73]. The jets reconstructed from raw energies with fixed $E_{\mathrm{T}}$ and $E$ thresholds were finally chosen to be used for the JET veto. A strong $E_{\mathrm{T}}$ cut helps in the background reduction. However, below $E_{\mathrm{T}}=25 \mathrm{GeV}$ the fraction of jets matching with a generated jet starts to decrease, because of ghost jet candidates mainly due to pileup events. The matching was defined within a cone around the reconstructed jet candidate $\Delta R_{\text {rec-gen jet }}<0.3$. In order to reduce the number of fake jets, a quality parameter was introduced:

$$
\alpha=\sum_{\text {selected tracks }} P_{\mathrm{T}} / E_{\mathrm{T}}(j e t)
$$

where the selected tracks are those inside the jet $\left(\Delta R_{t r k-j e t}<0.5\right)$ with more than 5 associated hits, pointing to the primary interaction vertex $\left(\left|z_{t r k}-z_{v t x}\right|<0.4 \mathrm{~cm}\right)$. The mean value of $\alpha$ is 0.66 (two third of the jet energy on average is due to charged particles). A reconstructed jet candidate with $E_{\mathrm{T}}$ in the low energy region $(<20 \mathrm{GeV})$ was considered only if $\alpha>0.2$. It has been shown [73] that this selection significantly reduces the number of fake jets (the fraction of matched jets being greater than $90 \%$ for $E_{\mathrm{T}}>15 \mathrm{GeV}$ ) with negligible loss of reconstruction efficiency for true jets. Different jet reconstruction algorithms were tested. The best signal $\left(m_{H}=165 \mathrm{GeV} / \mathrm{c}^{2}\right) /$ background $(t \bar{t})$ ratio was obtained using an iterative 

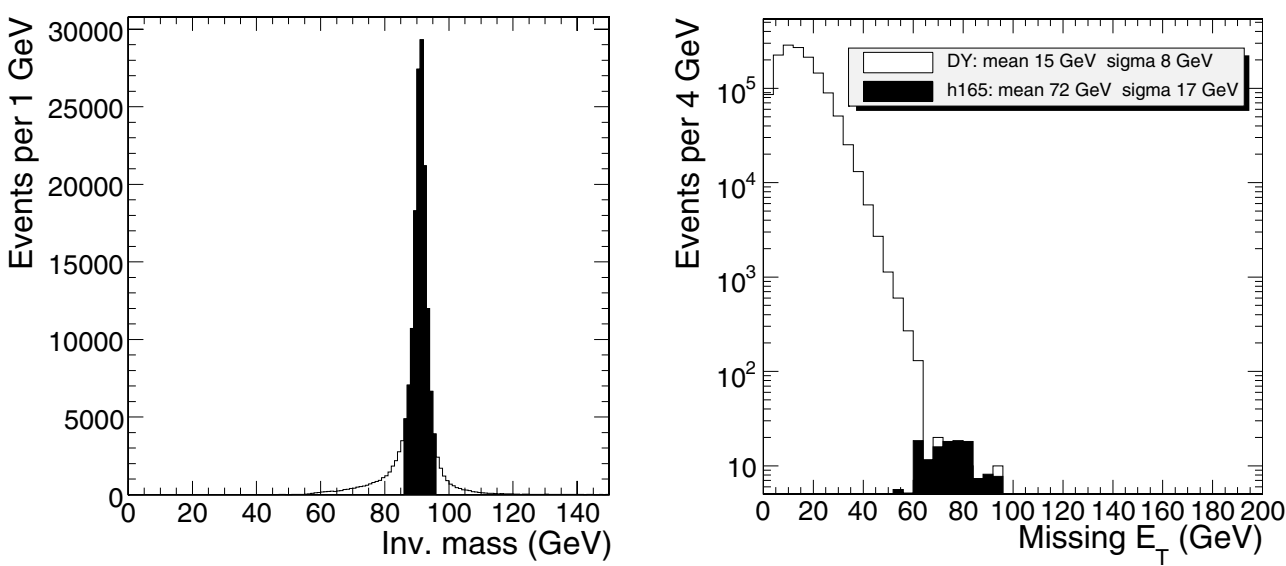

Figure 3.13. Reconstructed dimuon invariant mass for Drell-Yan events selected inside the Z mass region (left, black area); MET distributions for the selected Drell-Yan events and for signal events scaled at the integrated luminosity $\mathcal{L}=10 \mathrm{fb}^{-1}$ (right).

cone algorithm [78] with a cone size $\mathrm{R}=0.5$ and calorimeter towers having raw energies $E_{\mathrm{T}}^{\text {tower }}>0.5 \mathrm{GeV}$ and $E^{\text {tower }}>0.8$. To summarise, the jet veto is applied if:

$$
E_{\mathrm{T}}>15 \mathrm{GeV} \quad\left|\eta_{j e t}\right|<2.5
$$

and the $\alpha$ cut is required in the jet energy range $15 \mathrm{GeV}<E_{\mathrm{T}}<20 \mathrm{GeV}$.

\subsubsection{Missing energy reconstruction and the MET cut}

The transverse missing energy is reconstructed with the sum of the ECAL and HCAL tower raw energies, corrected for the muons energy contribution. The most sensitive background to the MET cut is the dimuon production from Drell-Yan (DY) process. The right plot in Fig. 3.13 shows the MET distributions for DY events having a reconstructed dimuon invariant mass inside the $\mathrm{Z}$ mass region (shown by the black area in the left plot), and for signal events with $m_{H}=165 \mathrm{GeV} / \mathrm{c}^{2}$. The signal and background distribution were normalised to an integrated luminosity $\mathcal{L}=10 \mathrm{fb}^{-1}$.

A MET threshold of $47 \mathrm{GeV}$ is $4 \sigma$ over the mean value for the background and $1.5 \sigma$ under the mean value for the signal. Drell-Yan events are thus strongly suppressed by applying a MET threshold. The cut used in this analysis was MET $>50 \mathrm{GeV}$.

3.2.6.1. The kinematic cuts. The kinematic of the two muons is different for signal and background:

- signal events from gluon-gluon scattering are more central than the $W^{+} W^{-}$background from $q \bar{q}$ scattering, thus resulting in a slightly more central rapidity distribution for the decay muons;

- due to the scalar nature of the Higgs boson and of the V-A structure of the weak interaction, for Higgs masses close to $2 M_{W}$, the $W^{+} W^{-}$spin correlation plays in favour of small opening angles between the two muons;

- signal events have a lepton $P_{\mathrm{T}}$ spectra peak close to $M_{W} / 2$;

- DY background has a two muons invariant mass peak at $M_{Z}$. 
In addition, the muons from $\mathrm{b}$ quarks (as in the case of the $b \bar{b}$ background and eventually from $t \bar{t}$ ) have large impact parameters. The following cuts were applied before the optimisation of the kinematical cuts:

1. $\left.\mid\left(\mu_{1}\right)\right)||,\left(\mu_{2}\right) \mid<2.0$ (pseudorapidity of the two muons);

2. I $P\left(\mu_{1}\right), I P\left(\mu_{2}\right)<3 \sigma$ (impact parameter of the two muons);

3. $P_{\mathrm{T}}\left(\mu_{\max }\right)<55 \mathrm{GeV} / \mathrm{c}$ (transverse momentum of the two muons);

4. $m_{\mu_{1} \mu_{2}}>12 \mathrm{GeV} / \mathrm{c}_{2}$ (invariant mass of the two muons);

5. $\Delta \phi_{\mu_{1} \mu_{2}}<0.8$ (opening angle between the two muons).

Cut 1 is useful for the WW background reduction, as well as cuts 3 and 5 . Cut 2 reduces the $b \bar{b}$ events, while cut 4 rejects potential background from b-resonances. After the requirement of the muon isolation described before, the overall signal efficiency for cuts 1 to 4 is about $90 \%$. The distribution of the variable $\Delta \phi \mu 1 \mu_{2}$ will be used to search for the Higgs signal.

The optimisation study was performed by varying the following cuts:

$$
\begin{aligned}
& P_{\mathrm{T}}\left(\mu_{\text {max }}\right)>25,30,35,40 \mathrm{GeV} / \mathrm{c} P_{\mathrm{T}}\left(\mu_{\text {min }}\right)>15,20,25,30 \mathrm{GeV} / \mathrm{c}^{2} \\
& m_{\mu_{1} \mu_{2}}<35,40,45,50,55,60 \mathrm{GeV} / \mathrm{c}^{2}
\end{aligned}
$$

to find the set of cuts giving the best significance. The estimator $S_{c P}$ was used, which gives the significance using the Poisson distribution [79]. The input of the estimator are the number of signal and background events, the statistical uncertainties and the theoretical systematics in the background. The optimisation was performed using as before the signal dataset with $M_{H}=165 \mathrm{GeV} / \mathrm{c}^{2}$, and using all the background contributions, properly normalised considering their production cross sections.

The optimisation result could depend on the statistics of the event data samples and on the estimated systematic errors. We searched for the maximum significance in four different conditions:

$$
\mathcal{L}=1 \mathrm{fb}^{-1} \quad \mathcal{L}=2 \mathrm{fb}^{-1} \quad \text { syst. err. }=10 \% \quad \text { syst. err. }=15 \%
$$

Figure 3.14 shows, as an example, the significance expected as a function of $p_{\mathrm{T}}\left(\mu_{\max }\right)$ and $p_{\mathrm{T}}\left(\mu_{\text {min }}\right)$ cuts for two different values of the dimuon invariant mass cut, for the case of an integrated luminosity $L=1 \mathrm{fb}^{-1}$ and an overall $10 \%$ systematic error.

The following cuts:

$$
P_{\mathrm{T}}\left(\mu_{\text {max }}\right)>35 \mathrm{GeV} / \mathrm{c} P_{\mathrm{T}}\left(\mu_{\text {min }}\right)>25 \mathrm{GeV} / \mathrm{c} m_{\mu_{1} \mu_{2}}<50 \mathrm{GeV} / \mathrm{c}^{2}
$$

give the maximum significance (about 3.0 for $\mathcal{L}=1 \mathrm{fb}^{-1}$ and an assumed syst. err. $=10 \%$ ) in all the four conditions.

\subsubsection{The selection results}

The optimised selection cuts discussed above were applied to the background and signal samples. The list of cuts is described in Table 3.3. The expected number of events for a luminosity of $1 \mathrm{fb}^{-1}$ are given in Table 3.4 for the signals and the backgrounds.

Figure 3.15 shows the distributions of the MET, $P_{\mathrm{T}}\left(\mu_{\text {max }}\right), P_{\mathrm{T}}\left(\mu_{\text {min }}\right)$ and $m_{\mu 1 \mu 2}$ variables for the signal and the three most important backgrounds after the jet-veto and the following selection cuts applied in the order reported in the Table 3.3.

Figure 3.16 shows the final distribution obtained for the azimuth angle difference between the muons, expected for an integrated luminosity $\mathcal{L}=10 \mathrm{fb}^{-1}$ and for the Higgs signal of mass $m_{H}=165 \mathrm{GeV} / \mathrm{c}^{2}$. 

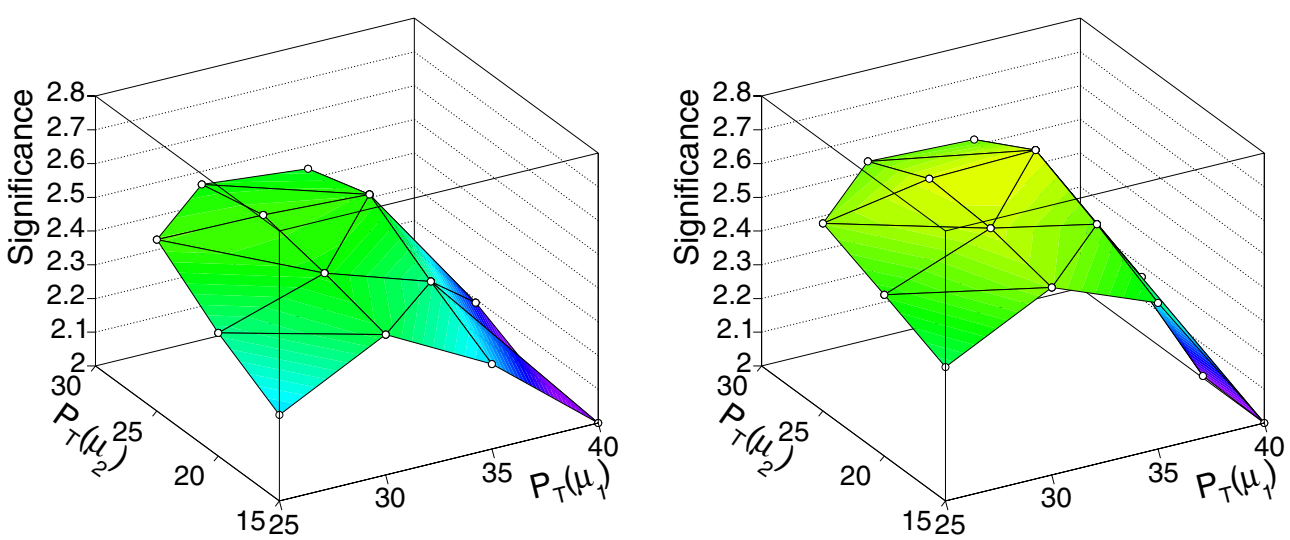

Figure 3.14. Significance as a function of $P_{\mathrm{T}}$ cuts for $m_{\mu_{1} \mu_{2}}<40 \mathrm{GeV} / \mathrm{c}^{2}$ (left) and for $m_{\mu_{1} \mu_{2}}<50 \mathrm{GeV} / \mathrm{c}^{2}$ (right) with $\mathcal{L}=1 \mathrm{fb}^{-1}$ and syst. err. $=10 \%$

Table 3.3. The list of cuts applied to the signal and background samples.

\begin{tabular}{llll}
\hline 1 & L1+HLT dimuon & 6 & MET $>50 \mathrm{GeV}$ \\
2 & $2 \mu$ opposite charge & 7 & $35 \mathrm{GeV} / \mathrm{c}<P_{\mathrm{T}}\left(\mu_{\text {max }}\right)<55 \mathrm{GeV} / \mathrm{c}$ \\
3 & Isolation & 8 & $25 \mathrm{GeV} / \mathrm{c}<P_{\mathrm{T}}\left(\mu_{\text {min }}\right)$ \\
4 & $\eta<2.0$ IP $<3 \sigma$ & 9 & $m_{\mu_{1} \mu_{2}}<50 \mathrm{GeV} / \mathrm{c}^{2}$ \\
5 & Jet Veto & 10 & $\Delta \phi_{\mu_{1} \mu_{2}}<0.8$ \\
\hline
\end{tabular}

Table 3.4. The expected number of events for a luminosity of $1 \mathrm{fb}^{-1}$ for the signal with Higgs masses between 130 and $180 \mathrm{GeV} / \mathrm{c}^{2}$ and for the backgrounds.

\begin{tabular}{lccc}
\hline & L1+HLT dimuon & All cuts & $\varepsilon_{\text {tot }}$ \\
\hline$m_{H}=130 \mathrm{GeV} / \mathrm{c}^{2}$ & 112 & $0.68 \pm 0.19$ & $(0.07 \pm 0.02) \%$ \\
$m_{H}=140 \mathrm{GeV} / \mathrm{c}^{2}$ & 162 & $1.7 \pm 0.4$ & $(0.12 \pm 0.03) \%$ \\
$m_{H}=150 \mathrm{GeV} / \mathrm{c}^{2}$ & 228 & $5.3 \pm 0.8$ & $(0.26 \pm 0.04) \%$ \\
$m_{H}=160 \mathrm{GeV} / \mathrm{c}^{2}$ & 256 & $12.6 \pm 0.7$ & $(0.58 \pm 0.04) \%$ \\
$m_{H}=165 \mathrm{GeV} / \mathrm{c}^{2}$ & 264 & $14.3 \pm 0.8$ & $(0.64 \pm 0.04) \%$ \\
$m_{H}=170 \mathrm{GeV} / \mathrm{c}^{2}$ & 259 & $11.0 \pm 0.7$ & $(0.53 \pm 0.03) \%$ \\
$m_{H}=180 \mathrm{GeV} / \mathrm{c}^{2}$ & 233 & $5.9 \pm 0.8$ & $(0.30 \pm 0.04) \%$ \\
$q q \rightarrow W W$ & 1040 & $4.1 \pm 0.5$ & $(0.036 \pm 0.005) \%$ \\
$t \bar{t} \rightarrow 2 \mu 2 v$ & 17007 & $2.6 \pm 0.3$ & $(0.012 \pm 0.001) \%$ \\
$g g \rightarrow W W$ & 58 & $1.0 \pm 0.1$ & $(0.18 \pm 0.02) \%$ \\
$\gamma^{*}, Z \rightarrow 2 \mu$ & 720653 & $0.3 \pm 0.3$ & $(4 \pm 4) 10-5 \%$ \\
$b \bar{b} \rightarrow 2 \mu 2 v$ & 69374 & 0 & $0 \%$ \\
$W t$ & 615 & $0.57 \pm 0.10$ & $(0.017 \pm 0.003) \%$ \\
$Z Z$ & 218 & $0.18 \pm 0.05$ & $(0.012 \pm 0.003) \%$ \\
$Z W$ & 384 & $0.13 \pm 0.05$ & $(0.008 \pm 0.003) \%$ \\
\hline
\end{tabular}

As stated above, all the numbers at the various selection steps refer to the analysis applied to the HLT dimuon stream. For comparison, the event numbers after all the selection cuts were also studied for the case in which the analysis were performed on the data including the single muon trigger data stream. The inclusion of this datastream, which is foreseen to have a rate about 7 times larger than the dimuon stream [76], would result in a $(3 \pm 1) \%$ increase of 

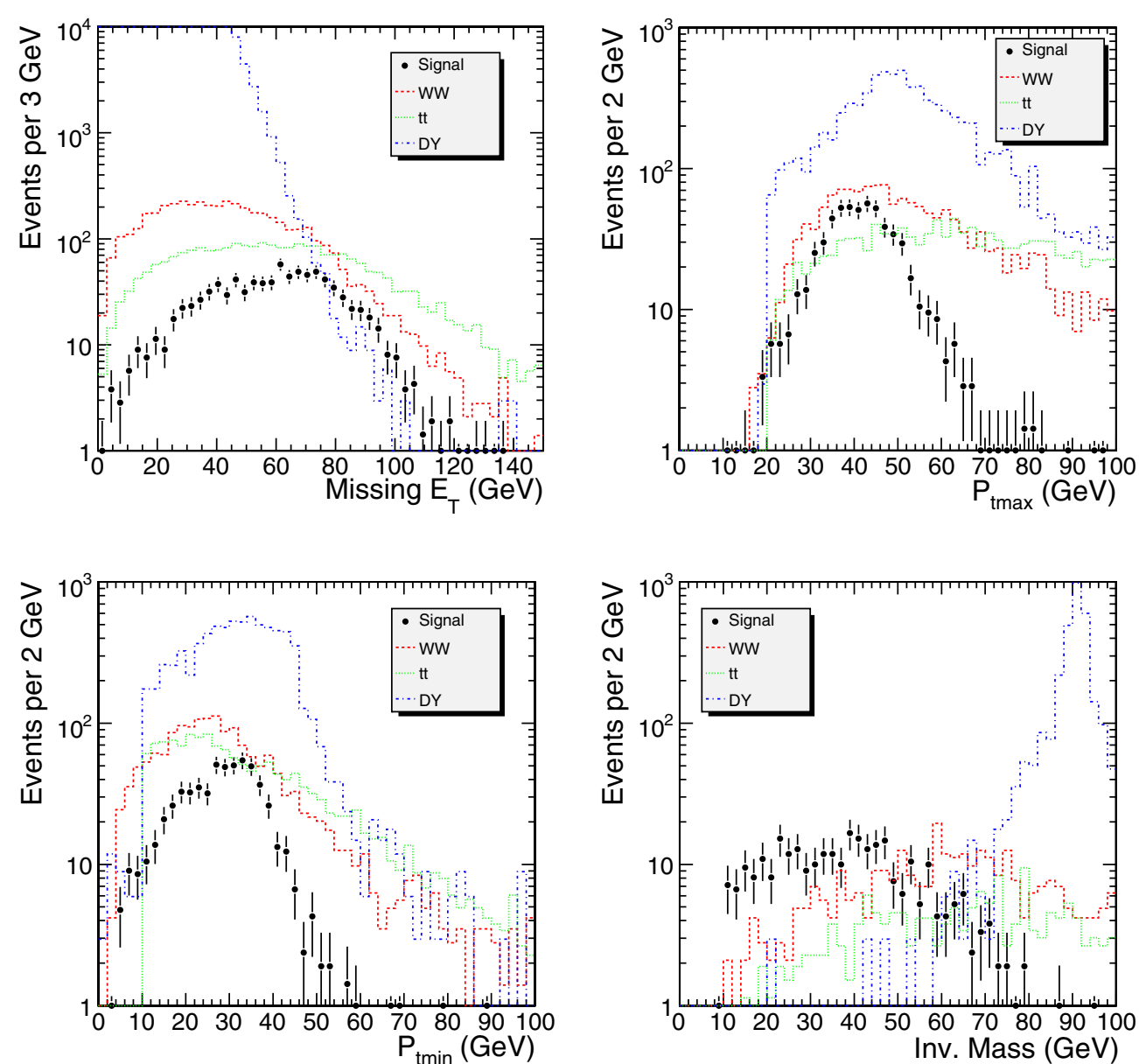

Figure 3.15. Distributions of the missing energy, transverse momentum and invariant mass for a luminosity of $10 \mathrm{fb}^{-1}$ following the cut list order.

the overall signal selection efficiency. The Higgs search with mass appreciably different than $165 \mathrm{GeV} / \mathrm{c}^{2}$ can take advantage from a dedicated cut optimisation, such as the one reported in [77].

\subsubsection{Background estimation and systematics}

The precise understanding of the backgrounds is the most critical issue concerning this Higgs discovery channel. The direct use of the Monte Carlo predictions, i.e. $N_{b k g, M C}=\sigma_{b k g, M C} \cdot \varepsilon_{f f}$, leads to high systematic uncertainties due either to theoretical calculation and to experimental systematics. The most reliable approach to address this problem is to measure the different sources of background directly from the data. The commonly used method to extrapolate the background contribution directly from the data consists of selecting a signal-free phase space region (control region) where a given background process is enhanced. The normalisation from data for the two most relevant background, i.e. $t \bar{t}$ and $W W$ has been addressed. For both backgrounds, a dedicated control region was defined. The number of background events in 


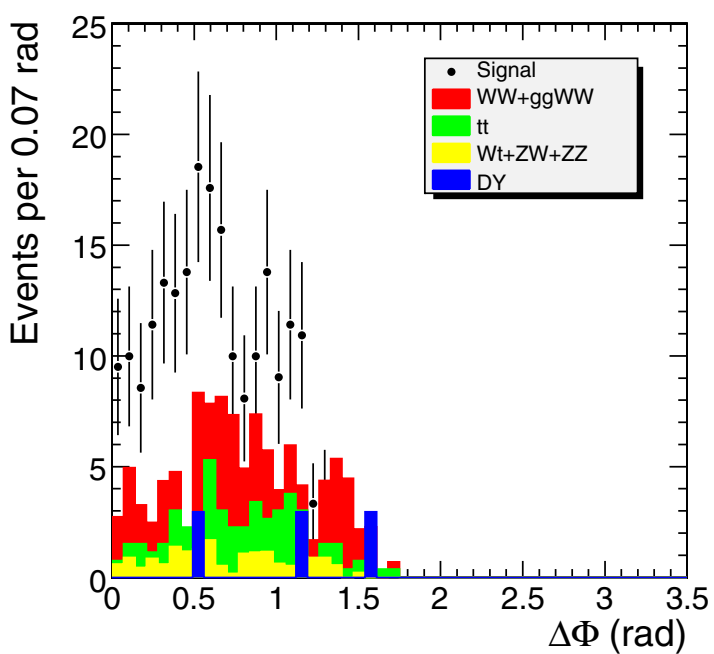

Figure 3.16. Distribution of the angle between the two muons for a luminosity of $10 \mathrm{fb}^{-1}$ at the end of the selection.

the signal region can then be estimated through:

$$
N_{\text {signal_reg }}=\frac{N_{\text {signal_reg }}^{\text {MonteCarlo }}}{N_{\text {control_reg }}^{\text {MonteCarlo }}} N_{\text {control_reg }}
$$

where $N_{\text {signal_reg }}^{\text {MonteCarlo }}$ and $N_{\text {control_reg }}^{\text {MonteCarlo }}$ are the numbers of events predicted by the Monte Carlo simulation in the signal and control region. The error on the ratio $N_{\text {signal_reg }}^{\text {MonteCarlo }} / N_{\text {control_reg }}^{\text {MonteCarlo }}$ accounts for a theoretical contribution (scale variation, PDF uncertainty) and detector systematics effects. The precision with which the number of $N_{\text {signal_reg }}$ can be predicted depends also on the statistical error on $N_{\text {control_reg. }}$.

\subsection{9. $t \bar{t}$ background normalisation}

Since the presence of two b-tagged jets is a striking evidence for $t \bar{t}$ events, the most natural control region for this process is then defined by applying the same selection cuts as for the signal region but the jet veto, with the additional request of two b-tagged jets in the detector acceptance ${ }^{39}$. The $t \bar{t}$ evaluation from the data for the $H \rightarrow W W^{(*)}$ channel has been studied in Ref. [80] to which we refer for further details. In this study, a jet is tagged as a b-jet if its measured $E_{\mathrm{T}}$ is greater then $20 \mathrm{GeV}$ and if there are at least two tracks belonging to the jet (i.e. within a cone of 0.5 around the jet axis) whose $\sigma_{\mathrm{IP}}$ is higher than 2 . With such settings the double b-tagging efficiency for $t \bar{t}$ events is $\mathcal{O}(30 \%$; ). The mis-tagging rate has been calculated from the ratio between the number of b-tagged jets and the total number of jet with $E_{\mathrm{T}}>20 \mathrm{GeV}$ in the fully simulated DY sample and it resulted to be $\mathcal{O}(3 \%$; ).

In the following, we consider the background processes in the $t \bar{t}$ control region. For $1 \mathrm{fb}^{-1}$ the number of $t \bar{t}$ events in the control region just defined is foreseen to be 17 , whereas the contribution from the signal and $W t$ is completely negligible (in both cases smaller than 0.1 events).

\footnotetext{
${ }^{39}$ In Ref. [80] an additional control region for $t \bar{t}$ events defined by requiring two high $E_{\mathrm{T}}$ jets instead of two b-tagged jets has been proposed. However, it has been shown there, that due to the high contamination from Drell-Yan events, this control region is less indicate for same flavour lepton final states.
} 
Table 3.5. Sources of uncertainty for the $t \bar{t}$ background normalisation procedure. Results are shown for 1,5 and $10 \mathrm{fb}^{-1}$.

\begin{tabular}{lcccccc}
\hline $\begin{array}{l}\text { Luminosity } \\
\left(\mathrm{fb}^{-1}\right)\end{array}$ & $\begin{array}{c}\text { Theoretical } \\
\text { error }\end{array}$ & \multicolumn{3}{c}{ Detector systematics } & $\begin{array}{c}\text { Statistical } \\
\text { error }\end{array}$ & $\begin{array}{c}\text { Total } \\
\text { error }\end{array}$ \\
\cline { 3 - 6 } 1 & $10 \%$ & $10 \%$ & $4 \%$ & $11 \%$ & $24 \%$ & $30 \%$ \\
5 & $10 \%$ & $6 \%$ & $4 \%$ & $9 \%$ & $11 \%$ & $19 \%$ \\
10 & $10 \%$ & $6 \%$ & $4 \%$ & $7 \%$ & $8 \%$ & $16 \%$ \\
\hline
\end{tabular}

Not all the processes with $2 \mu+2 b+E_{t}^{\text {miss }}$ as final state have been fully simulated for this analysis, nevertheless general considerations and fast Monte Carlo generator level cross checks lead to exclude other sources of backgrounds, as briefly outlined in the following.

The more natural concurrent process is the non-resonant $W^{+} W^{-} \rightarrow 2 \mu+b \bar{b}$ which is suppressed with respect to $t \bar{t}$. Its cross section is indeed expected to be smaller than $0.3 \mathrm{pb}$. Assuming the same efficiency for the kinematic selections as for the $W^{+} W^{-} \rightarrow 2 \mu(\sim 0.07 \%)$ and including the double-b tagging efficiency, less than 0.1 events are expected for $1 \mathrm{fb}^{-1}$ in the control region.

In the fully simulated Drell-Yan sample used in this analysis, the eventual additional $b \bar{b}$ pair comes only from a gluon splitting; the main mechanism of $\gamma^{*} / Z^{*}+2 b$ is not included. For an estimation of the contamination of the $t \bar{t}$ control region due to this process we thus used a parton level sample generated with a matrix element Monte Carlo (MADGRAPH [81]). Applying the signal kinematic selections, but the $E_{\mathrm{T}}$ cut on the latter sample, $\sim 10$ events are expected for $1 \mathrm{fb}^{-1}$. The rejection due to $E_{\mathrm{T}}$ cut has been calculated from the fully simulated sample where actually two b-quarks were present in the final state and it turned to be smaller than $1 \%$. Considering also the efficiency for the double b-tagging, we can safely exclude this as a dangerous background.

In the following the various contribution of uncertainty in the $t \bar{t}$ normalization procedure are listed and described. The results are summarised in Table 3.5 for 1,5 and $10 \mathrm{fb}^{-1}$.

- Theoretical uncertainty. The theoretical uncertainty of the $t \bar{t}$ cross section ratio $\sigma_{\text {signal_reg }} / \sigma_{\text {control_reg }}$ has been studied in [82] at parton level with LO precision by varying the reorganisation and factorisation scale. The error has been estimated to range between $3 \%$ to $10 \%$ mostly due to the choice of PDF. Some studies were done also at NLO: $E_{\mathrm{T}}$ spectra and multiplicity of jets are not affected by higher order contributions but the estimate of the theoretical error at NLO is not available. In the following we will, assume the theoretical uncertainty on the $t \bar{t}$ normalisation procedure to be $10 \%$.

- Jet Energy Scale (JES) uncertainty. In the background normalisation procedures we proposed, the JES uncertainty is particularly important since it affects in an opposite sense the signal region, defined by vetoing the jets, and the control region where the presence of two jets is required. To take into account this sort of anti-correlation of $\epsilon_{\text {signal_reg }}$ and $\epsilon_{\text {control_reg, }}$, we estimate the effect of the JES uncertainty directly on their ratio by rescaling the measured jet four momentum by a fractional uncertainty (i.e. $\mathrm{P}_{\text {jet }}^{\mu}=(1+\lambda) P_{\text {jet }}^{\mu}$ ). The

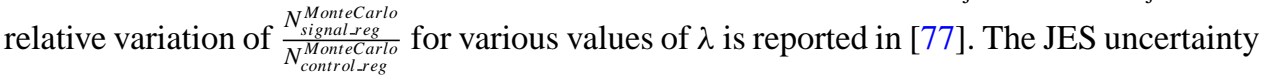
foreseen at CMS is $\mathcal{O}(5 \%)$ for $1 \mathrm{fb}^{-1}$ and it is expected to decrease down to $\sim 3 \%$ for $5 \mathrm{fb}^{-1}$ (thanks to the calibration on the W mass) [7]. The effect of the JES uncertainty is $10 \%$ for $1 \mathrm{fb}^{-1}$ and $6 \%$ for $5 \mathrm{fb}^{-1}$. 


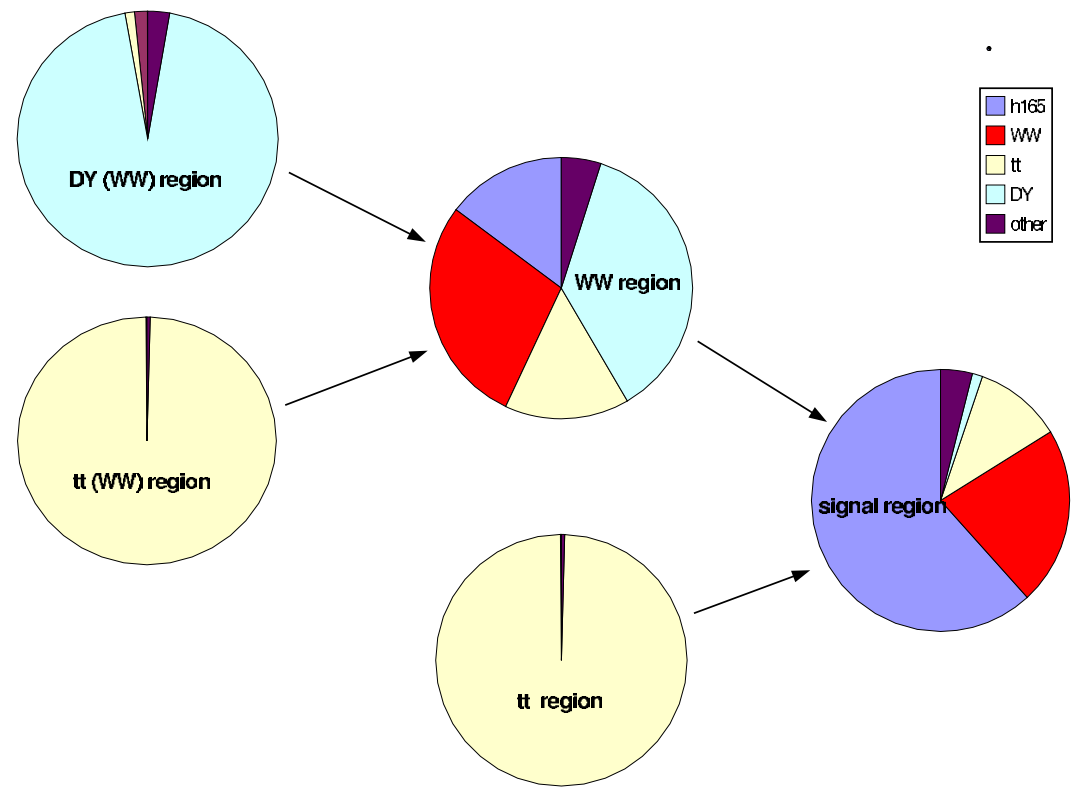

Figure 3.17. Scheme for background normalisation from the data in different phase space regions: the signal region, the $t \bar{t}$ region, the WW region, the DY (WW) region, and the $t \bar{t}(\mathrm{WW})$ region. The arrows indicate the extrapolation of the number of events determined in the corresponding "control region" into the corresponding "target region". Each region is represented by a pie chart that shows the fractions of certain types of events: h165 is the Higgs signal with $m_{h}=165 \mathrm{GeV} / \mathrm{c}^{2}$, WW is the sum of WW backgrounds, $t \bar{t}$ is the $t \bar{t}$ background, DY is the Drell-Yan background, and other is the sum of the $\mathrm{Wt}, \mathrm{ZW}$ and $\mathrm{ZZ}$ backgrounds. The number of expected events in each region is reported in Table 3.6.

- $\alpha$ criterion uncertainty. To estimate the systematic uncertainty due to $\alpha$ criterion, the value of the cut has been varied from 0.15 to 0.25 . Moreover, different values of the minimum $p_{\mathrm{T}}$ for a track to be included in the sum have been tried, from 2 to $3 \mathrm{GeV} / \mathrm{c}$. The consequent variation of the jet veto efficiency (affecting only $N_{\text {signal_reg }}^{\text {MonteCarlo }}$ ) is relatively small, i.e. of the order of $4 \%$.

- b-Tagging uncertainty. The uncertainty on the b-tagging efficiency will be estimated exploiting $t \bar{t}$ events as calibration samples. The precision with which the b-tagging efficiency will be known is expected to be $\pm 11 \%$ for $1 \mathrm{fb}^{-1}$ integrated luminosity and it is foreseen to improve to $\pm 7 \%$ with $10 \mathrm{fb}^{-1}$ [83].

- Uncertainties on the composition of the control region. As it has been shown in the previous section, $t \bar{t}$ is the dominant process in the chosen control region, other processes contributing less than $1 \%$. It is then safe to simply neglect this source of systematic error.

- Statistical uncertainty on $\boldsymbol{N}_{\text {control_reg }}$. Assuming a Poissonian behaviour, the statistical uncertainty scales with the integrated luminosity as the square root of the number of $t \bar{t}$ events in the control region.

\subsubsection{WW background normalisation}

In contrast to the $t \bar{t}$ background normalisation, which can be performed using an almost completely pure $t \bar{t}$ control sample, it is impossible to isolate the WW background in a 
clean way, which means that contributions of other processes have to be subtracted and their systematic uncertainties have to be taken into account during the normalisation procedure of the WW background, including gg $\rightarrow \mathrm{W}^{+} \mathrm{W}^{-}$events. In Fig. 3.17 the overall background normalisation strategy is illustrated. There are four phase space regions involved in the WW background normalisation. Each region is defined with a certain set of cuts:

- signal region: the selection of events in the signal region as described above.

- WW region: same as in the signal region, but $\Delta \phi_{\mu_{1} \mu_{2}}=2>0.8$ and $50 \mathrm{GeV} / \mathrm{c}^{2}<m_{\mu_{1} \mu_{2}}<$ $80 \mathrm{GeV} / \mathrm{c}^{2}$.

- DY (WW) region: same as in the WW region, but $80 \mathrm{GeV} / \mathrm{c}^{2}<m_{\mu_{1} \mu_{2}}<100 \mathrm{GeV} / \mathrm{c}^{2}$.

- $t \bar{t}$ (WW) region: same as in the WW region, but the jet veto is replaced with the requirement of two b-tagged jets $\left(E_{t}>20 \mathrm{GeV}\right.$ and two tracks with $\left.\sigma_{I P}>2\right)$.

In all cases, the selection is independent of the Higgs mass hypothesis. The total number of events in each region is given in Table 3.6, and the contributions of individual processes are represented in form of pie charts in Fig. 3.17. The main contamination of the WW region is due to Drell-Yan, $t \bar{t}$ and the Higgs signal. The number of Drell-Yan and $t \bar{t}$ is determined by extrapolating the corresponding numbers from relatively clean control regions and are subtracted from the WW region. Additional small contributions from other backgrounds in the WW region are determined from Monte Carlos and then subtracted. So far, no concrete method has been established to subtract Higgs events from the WW control region. Therefore, we choose the conservative approach to treat these Higgs events as an additional background in the WW region.

- Theoretical uncertainties. The theoretical uncertainties of $\mathrm{W}$ pair production with subsequent decay to leptons have been studied in detail in Ref. [84], and the main sources of potential uncertainties of the shapes of kinematic variables turn out to be spin correlations, underlying event, and scale dependence. The effect of spin correlations can be taken into account properly with the correct choice of an event generator, and the underlying event is expected to be measured from the data with sufficient precision. The shape dependence on the choice of the reorganisation and factorisation scales is sizable in case of the contribution from the $\mathrm{gg} \rightarrow \mathrm{W}^{+} \mathrm{W}^{-}$subprocess, because the higher order corrections are unknown in this case. For the cuts, described below, this uncertainty is about $9 \%$ and is taken into account in the following.

- Statistical error and uncertainties on the composition of the control region. All background normalisation uncertainties are calculated in the following way:

$$
\delta_{\text {extrapolation }}=\sum_{i} \sqrt{n_{\text {total }}+\left(n_{i} \times \delta_{i}\right)^{2}} \times \varepsilon_{\text {control } \rightarrow \text { target }}
$$

where $n_{\text {total }}$ is the total number of events ${ }^{40}$ in the corresponding control region, $n_{i} \times \delta_{i}$ is the product of the number of events and the systematic uncertainty of an individual process in the control region, and $\varepsilon_{\text {control } \rightarrow \text { target }}$ is the extrapolation efficiency from the control region to the target region, e.g. the signal region.

The WW background normalisation requires three extrapolations from control regions to target regions:

- DY (WW) region $\Rightarrow$ WW region: with an extrapolation uncertainty of 5\% [85] the extrapolated number of events and the uncertainty from Eq. 3.18 is $15.86 \pm 1.23$ events $\left(79.29 \pm 4.49\right.$ events) for $1 \mathrm{fb}^{-1}\left(5 \mathrm{fb}^{-1}\right)$ of integrated luminosity.

40 This term takes into account the statistical fluctuations of the control sample. 
Table 3.6. Number of expected events in all the regions with an integrated luminosity of $1 \mathrm{fb}^{-1}$. The signal region numbers are referred to $m_{H}=165 \mathrm{GeV} / \mathrm{c}^{2}$.

\begin{tabular}{lccccc}
\hline Channel & Signal region & $t \bar{t}$ region & WW region & $t \bar{t}(\mathrm{WW})$ region & DY $(\mathrm{WW})$ region \\
\hline Signal & $\mathbf{1 4 . 3}$ & 0.0 & 6.0 & 0.0 & 0.1 \\
$t \bar{t}$ & 2.6 & $\mathbf{1 7 . 0}$ & 6.2 & 24.7 & 3.2 \\
WW & 5.1 & 0.0 & $\mathbf{1 1 . 5}$ & 0.0 & 4.4 \\
DY & 0.3 & 0.0 & 15.0 & 0.0 & 267 \\
Wt,ZZ,WZ & 0.8 & 0.1 & 1.9 & 0.1 & 7.3 \\
all & 23.1 & 17.1 & 40.6 & 24.8 & 282 \\
\hline
\end{tabular}

- $t \bar{t}(\mathrm{WW})$ region $\Rightarrow \mathrm{WW}$ region: with an extrapolation uncertainty of $20 \%(15 \%)$ [80] the extrapolated number of events and the uncertainty from Eq. 3.18 is $6.19 \pm 1.75$ events $(30.93 \pm 5.41$ events $)$ for $1 \mathrm{fb}^{-1}\left(5 \mathrm{fb}^{-1}\right)$ of integrated luminosity.

- WW region $\Rightarrow$ signal region: as illustrated in Fig. 3.17, the first two items are inputs to this extrapolation, which means that the obtained numbers of Drell-Yan and $t \bar{t}$ events are subtracted in the WW region and the corresponding uncertainties are propagated. The extrapolation uncertainty of WW events, which is mainly due to the unknown higher order correction of the $\mathrm{gg} \rightarrow \mathrm{W}^{+} \mathrm{W}^{-}$contribution [84], amounts to $9 \%$ for the cuts used in this analysis. In addition, the remaining backgrounds are estimated and subtracted with the following uncertainties: $\delta_{\mathrm{Wt}}=40 \%, \delta_{\mathrm{ZW}}=20 \%$ and $\delta_{\mathrm{ZZ}}=20 \%$. According to Eq. 3.18 we obtain $7.35 \pm 3.04$ events $(36.77 \pm 7.85$ events $)$ for $1 \mathrm{fb}^{-1}\left(5 \mathrm{fb}^{-1}\right)$ of integrated luminosity.

The results of the last item are used for the calculation of the Higgs discovery potential with $m_{h}=165 \mathrm{GeV} / \mathrm{c}^{2}$, and an integrated luminosity of either $1 \mathrm{fb}^{-1}$ or $5 \mathrm{fb}^{-1}$.

Furthermore, it should be pointed out that the entire background normalisation procedure is performed using only the dimuon data set and therefore no additional data sets are needed. In this way, potential uncertainties due to different trigger efficiencies and different integrated luminosities of other data sets do not play a role.

\subsubsection{Other backgrounds normalisation}

The Drell-Yan background has been normalised to estimate the contamination in the WW region. The same results can be achieved in the signal region. Figure 3.15 demonstrates that the invariant mass cut $80 \mathrm{GeV} / \mathrm{c}^{2}$ to $100 \mathrm{GeV} / \mathrm{c}^{2}$ defines a clean control region. $\mathrm{ZW}$ background can be normalised by requiring one additional lepton in the final state and removing the $\Delta \phi$ and the invariant mass cuts. ZZ background can be normalised by requiring two additional leptons in the final state and removing the $\Delta \phi$ and the invariant mass cuts. They are expected to contribute to the total background by only $3 \%$ (DY), $1 \%$ (ZW) and $1 \%$ (ZZ). For the Wt background, it is not easy to define a normalisation region. As this process is expected not to represent a sizable fraction of the total background $(\sim 6 \%)$, the Monte Carlo prediction will be then directly used, the cross section theoretical uncertainty is estimated to be about $30 \%$ at LO and $10 \%$ at NLO [75].

\subsubsection{Detector misalignment systematics}

A study for the misalignment impact on the track reconstruction has been done [86]. In the fist data scenario $\left(100 \mathrm{pb}^{-1}-1 \mathrm{fb}^{-1}\right)$ the muon chamber position uncertainty is expected to be $1 \mathrm{~mm}$ and the orientation uncertainty about $0.2 \mathrm{mrad}$. The tracker position uncertainty is expected to be about $5 \mu \mathrm{m}$ for TPE, $10 \mu \mathrm{m}$ for TPB, $50 \mu \mathrm{m}$ for TEC and TOB, $100 \mu \mathrm{m}$ 
Table 3.7. Total background and error for integrated luminosity of 1 and $5 \mathrm{fb}^{-1}$. The two options for the signal contamination in the WW control region were considered.

\begin{tabular}{lccc}
\hline Option & Luminosity & Total background & Total error \\
\hline 1. & $1 \mathrm{fb}^{-1}$ & 8.8 & $3.2(36 \%)$ \\
& $5 \mathrm{fb}^{-1}$ & 44.0 & $8.3(19 \%)$ \\
2. & $1 \mathrm{fb}^{-1}$ & 11.0 & $3.2(29 \%)$ \\
& $5 \mathrm{fb}^{-1}$ & 55.3 & $8.3(15 \%)$ \\
\hline
\end{tabular}

for TIB and $400 \mu \mathrm{m}$ for TID. The results from simulation show the muon reconstruction efficiency will be unaffected, while the momentum resolution (for $100 \mathrm{GeV} / \mathrm{c}$ tracks) will be reduced from 1-2 \% to 4-5\%. Under these circumstances, the systematic contribution to the signal and background selection is expected to be negligible with respect to the background normalisation systematics.

\subsubsection{Signal significance}

The signal significance can be obtained using counting or Likelihood methods. Here, the counting $S_{c P}$ method (See Appendix A) was used. $S_{c P}$ is the probability, converted in equivalent number of sigmas, to observe at least $N_{s}+N_{b}$ events from Poisson distribution with mean $N_{b}$. The presence of systematic errors influences the significance calculations. The hypothesis is to find the same number of signal and background events predicted by the Monte Carlo. The systematic errors due to the $t \bar{t}$ and WW background normalisation methods were included. Two options were considered:

1. the signal contamination in the WW control region can be subtracted;

2. the signal contamination in the WW control region must be considered as additional background.

The option 1 was considered to have a comparison with the $H \rightarrow W W \rightarrow 2 l 2 v$ analysis [73]. Table 3.7 summaries the total backgrounds and errors for different integrated luminosities. The systematics and statistical errors due to the limited Monte Carlo statistics are included.

The signal to background ratio as a function of different Higgs masses and the signal significance are shown in Fig. 3.18.

\subsubsection{Conclusions}

The possibility to discover the Higgs boson particle through its decay channel into $\left(W W^{(*)} \rightarrow\right.$ $2 \mu 2 v$ was studied in detail. Particular attention was given to the event selection optimisation, in the determination of the number of background events from the data and the evaluation of the experimental and theoretical systematical uncertainties. Taking all these effects into account, it was shown that in the Higgs mass range $155-175 \mathrm{GeV} / \mathrm{c}^{2}$ a signal significance bigger than 3 standard deviations can be achieved with $5 \mathrm{fb}^{-1}$ integrated luminosity. On the other hand, with $1 \mathrm{fb}^{-1}$ luminosity only a 2 sigma significance can be achieved even in the most favourable case $m_{H} \sim 2 m_{W}$, when this final state topology alone is used for the Higgs search. 

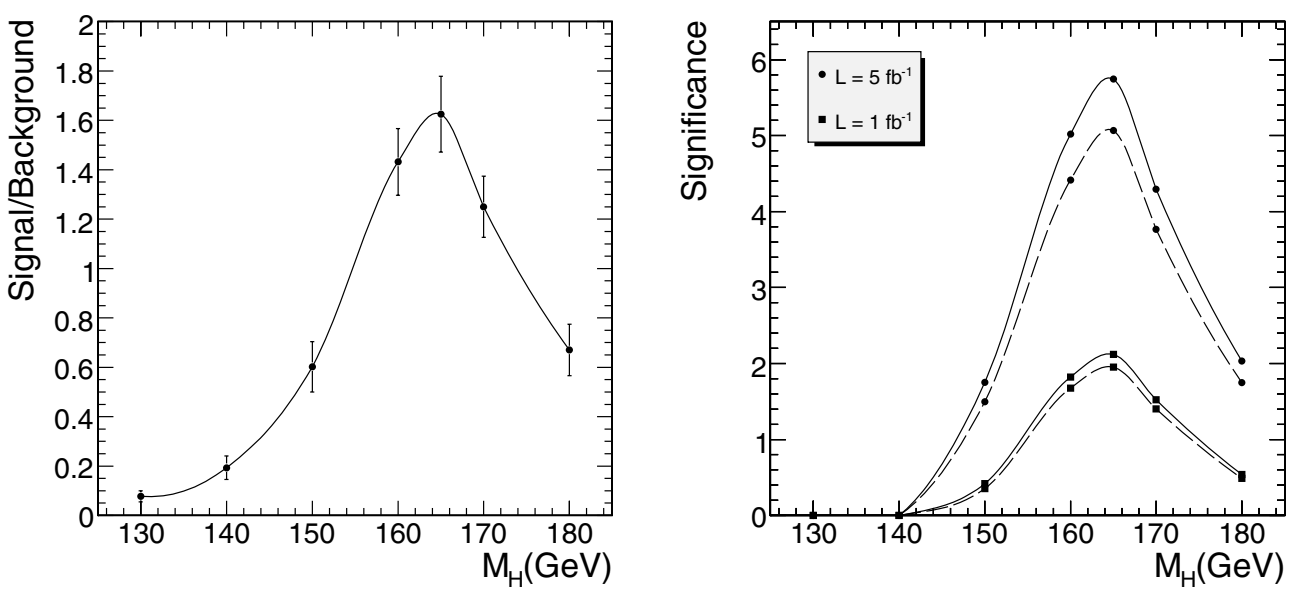

Figure 3.18. Signal to background ratio for the option 1 as a function of different Higgs masses. Error bars are the statistical contribution due to the limited Monte Carlo statistics (left). Significance as a function of different Higgs masses with a luminosity of 1 and $5 \mathrm{fb}^{-1}$, solid line for the option 1 , dashed line for the option 2 (right).

\subsection{Benchmark Channel: $Z^{\prime} \rightarrow \mu \mu$}

\subsubsection{Introduction}

Additional heavy neutral gauge bosons $\left(\mathrm{Z}^{\prime}\right)$ are predicted in many superstring-inspired [87, 88] and grand unified theories (GUTs) [89], as well as in dynamical symmetry breaking [90] and "little Higgs" [91] models. There are no reliable theoretical predictions, however, of the $\mathrm{Z}^{\prime}$ mass scale. Current lower limits on the $\mathrm{Z}^{\prime}$ mass are (depending on the model) of the order of $600-900 \mathrm{GeV} / \mathrm{c}^{2}$ [54]. The mass region up to about $1 \mathrm{TeV} / \mathrm{c}^{2}$ is expected to be explored at Run II at the Tevatron $[92,93]$. The LHC offers the opportunity to search for $Z^{\prime}$ bosons in a mass range significantly larger than $1 \mathrm{TeV} / \mathrm{c}^{2}$.

Observability of the $\mathrm{Z}^{\prime} \rightarrow \mu^{+} \mu^{-}$channel in CMS is discussed in Sections 3.3.2-3.3.4. Since narrow graviton resonances such as those in Randall-Sundrum models [94] can also decay to lepton pairs (Section 14.3.1), much of the discussion in these sections is also applicable to them. If a new resonance is discovered, the characterisation of its spin and couplings will proceed via the traditional methods of measuring production and decay probabilities and distributions. For example, the two-photon decay should be observable for a graviton and not for a $Z^{\prime}$, as discussed in Section 14.6. The measurement of forward-backward asymmetries of leptonic decay products, both at the resonance peak and off the peak, yields information on parity-violating couplings and hence can help distinguish among different $Z^{\prime}$ models (Section 3.3.5). Angular distributions of the decay products can also be used for spin discrimination (Section 3.3.6). A simulated event of a dimuon decay of $3 \mathrm{TeV} / \mathrm{c}^{2} \mathrm{Z}^{\prime}$ is shown in colour plate CP5.

\subsubsection{Signal and background processes}

3.3.2.1. Signal $\mathrm{Z}^{\prime} \rightarrow \mu^{+} \mu^{-}$. Signal and background samples were generated with PYTHIA [69] version 6.227 (with photon emission off incoming or outgoing quarks and leptons switched on) and the CTEQ6L set of parton distribution functions [12] from LHAPDF [95] version 4.1.1. 
Table 3.8. Summary of expected properties of $\mathrm{Z}^{\prime}$ bosons for six studied models. For each model, the first column shows the ratio of the total $\mathrm{Z}^{\prime}$ decay width $\Gamma$ to its mass $M$, the second column shows the dimuon branching ratio $\mathrm{Br}$. The three middle columns, labelled $\sigma^{\mathrm{LO}} \cdot \mathrm{Br}$, give the product of the pure- $\mathrm{Z}^{\prime}$ leading-order production cross section and the branching ratio for three studied $Z^{\prime}$ masses; the last three columns give $\sigma^{\mathrm{LO}} \cdot \mathrm{Br}$ obtained when the full $\gamma^{*} / \mathrm{Z}^{0} / \mathrm{Z}^{\prime}$ interference structure is included. The numbers quoted are for the mass intervals above $400 \mathrm{GeV} / \mathrm{c}^{2}$ for $M=1 \mathrm{TeV} / \mathrm{c}^{2}$, above $1.5 \mathrm{TeV} / \mathrm{c}^{2}$ for $M=3 \mathrm{TeV} / \mathrm{c}^{2}$, and above $3 \mathrm{TeV} / \mathrm{c}^{2}$ for $M=5 \mathrm{TeV} / \mathrm{c}^{2}$. The values of $\sigma \cdot \mathrm{Br}$ in the three middle columns correspond to $\mathrm{Z}^{\prime}$-only samples not used in our study; the values in the last three columns refer to the full-interference samples that we did use.

\begin{tabular}{|c|c|c|c|c|c|c|c|c|}
\hline \multirow[t]{2}{*}{ Model } & \multirow[t]{2}{*}{$\Gamma / M \%$} & \multirow[t]{2}{*}{$\begin{array}{c}\mathrm{Z}^{\prime} \rightarrow \mu^{+} \mu^{-} \\
\text {BR in } \%\end{array}$} & \multicolumn{3}{|c|}{$\begin{array}{c}\sigma^{\mathrm{LO}} \cdot \mathrm{Br}, \mathrm{fb} \\
(\text { (PYTHIA) }\end{array}$} & \multicolumn{3}{|c|}{$\begin{array}{c}\sigma^{\mathrm{LO}} \cdot \mathrm{Br}, \text { full interference, } \\
\text { fb (РYTHIA) }\end{array}$} \\
\hline & & & $1 \mathrm{TeV} / \mathrm{c}^{2}$ & $3 \mathrm{TeV} / \mathrm{c}^{2}$ & $5 \mathrm{TeV} / \mathrm{c}^{2}$ & $1 \mathrm{TeV} / \mathrm{c}^{2}$ & $3 \mathrm{TeV} / \mathrm{c}^{2}$ & $5 \mathrm{TeV} / \mathrm{c}^{2}$ \\
\hline $\mathrm{Z}_{\mathrm{SSM}}$ & 3.1 & 3.0 & 480 & 1.9 & 0.034 & 610 & 2.8 & 0.050 \\
\hline $\mathrm{Z}_{\psi}$ & 0.6 & 4.0 & 130 & 0.5 & 0.009 & 340 & 1.7 & 0.032 \\
\hline $\mathrm{Z}_{\eta}$ & 0.7 & 3.4 & 150 & 0.6 & 0.011 & 370 & 1.8 & 0.035 \\
\hline $\mathrm{Z}_{\chi}$ & 1.3 & 5.7 & 280 & 1.0 & 0.014 & 500 & 2.2 & 0.038 \\
\hline $\mathrm{Z}_{\mathrm{LRM}}$ & 2.2 & 2.3 & 310 & 1.2 & 0.020 & 500 & 2.3 & 0.040 \\
\hline $\mathrm{Z}_{\text {ALRM }}$ & 1.6 & 8.6 & 580 & 2.6 & 0.051 & 740 & 3.7 & 0.077 \\
\hline
\end{tabular}

From a large variety of $Z^{\prime}$ bosons described in the literature, we consider six which are frequently discussed, and whose properties are representative of a broad class of extra gauge bosons:

- $\mathrm{Z}_{\mathrm{SSM}}$ within the Sequential Standard Model (SSM), which has the same couplings as the Standard Model $Z^{0}$; it is available in PYTHIa [24].

- $\mathrm{Z}_{\psi}, \mathrm{Z}_{\eta}$ and $\mathrm{Z}_{\chi}$, arising in $\mathrm{E}_{6}$ and $\mathrm{SO}(10)$ GUT groups. Couplings to quarks and leptons were obtained from Refs. [96, 97].

- $\mathrm{Z}_{\mathrm{LRM}}$ and $\mathrm{Z}_{\mathrm{ALRM}}$, arising in the framework of the so-called "left-right" [98] and "alternative left-right" $[92,93]$ models. Their couplings were obtained from Ref. [92, 93], with the choice of $g_{R}=g_{L}$.

The generation of signal events with PYTHIA includes the full $\gamma^{*} / Z^{0} / Z^{\prime}$ interference structure. We assume that $Z^{\prime}$ bosons decay only to three ordinary families of quarks and leptons and that no exotic decay channels are open. Properties for these models are in Table 3.8. The cross sections are shown at leading order (LO), as predicted by PYTHIA. We scale them by a constant $K$ factor of 1.35 , see Appendix C, in order to take into account the next-to-next-to-leading order (NNLO) QCD corrections. Electroweak higherorder corrections are not yet accounted for (see discussion in Section 3.3.4.4.1).

3.3.2.2. Background from Drell-Yan production and other processes. The dominant (and irreducible) background to $\mathrm{pp} \rightarrow \mathrm{Z}^{\prime} \rightarrow \mu^{+} \mu^{-}$is the Drell-Yan production of muon pairs, $\mathrm{pp} \rightarrow \gamma / Z^{0} \rightarrow \mu^{+} \mu^{-}$. The Drell-Yan cross section in PYTHIA was scaled by the same $K$ factor of 1.35, see Appendix C, to get an agreement with the NNLO QCD calculations.

The overall contribution from ZZ, ZW, WW, and $t \bar{t}$ was found to be at the level of only a few percent of the Drell-Yan background and can be further suppressed by signal-selection criteria with almost no reduction in signal efficiency; we neglect this contribution. A few other potential background sources (like cosmics, jet-jet, $\mathrm{W}$-jet, $b \bar{b}$, hadron punchthroughs, and poorly measured $\mathrm{Z}^{0} \rightarrow \mu^{+} \mu^{-}$events) have not been studied yet, but their contribution is expected to be small. 
3.3.2.3. Simulation and reconstruction. The detector response was simulated with the detailed CMS detector simulation and reconstruction software, including pile-up events. Misalignments of the tracker and of the muon system expected at the initial and at the welladvanced stages of the data taking have been taken into account by using two misalignment scenarios developed in the framework of the CMS reconstruction, referred to as the "first data" and the "long term" scenarios [86]:

- The "first data" scenario gives an estimate of the alignment achieved with an integrated luminosity of about $0.1 \mathrm{fb}^{-1}$ and corresponds to the situation when the pixel detector is aligned with tracks and the first information from the Laser Alignment System (LAS) is available for the muon detectors.

- The "long term" scenario describes the expected residual alignment uncertainties. Once the performance of the LAS reaches its design level and the alignment with tracks is done in all tracking detectors. The current estimate is that, this can be achieved with an integrated luminosity of about $1 \mathrm{fb}^{-1}$.

As a result, for each of the $\mathrm{Z}^{\prime}$ models above, several sets of simulated samples corresponding to different possible combinations of luminosities and misalignment scenarios were produced at each of three mass values of 1,3 , and $5 \mathrm{TeV} / \mathrm{c}^{2}$. Since the Drell-Yan cross section falls rapidly with the mass of the muon pair, Drell-Yan background was generated in six mass intervals (with lower mass bounds of $0.2,0.4,1,1.5,2$, and $3 \mathrm{TeV} / \mathrm{c}^{2}$ ), again for different combinations of luminosities and misalignment scenarios.

\subsubsection{Event selection}

For $\mu^{+} \mu^{-}$invariant mass between $1 \mathrm{TeV} / \mathrm{c}^{2}$ and $5 \mathrm{TeV} / \mathrm{c}^{2}$, the fraction of Drell-Yan events with both muons within the full geometrical acceptance of the muon system $(|\eta|<2.4)$ increases from about $80 \%$ at $1 \mathrm{TeV} / \mathrm{c}^{2}$ to almost $95 \%$ at very high masses. The acceptance of $\mathrm{Z}^{\prime} \rightarrow \mu^{+} \mu^{-}$events is very similar.

We require that the event pass the logical OR of single-muon and dimuon triggers, both Level-1 and HLT. We use the default ORCA implementations of low-luminosity and highluminosity muon trigger algorithms described in Refs. [7, 76], with the exception of the HLT calorimeter isolation criterion requiring that the weighted sum of energy deposits in ECAL and HCAL in a cone around the muon direction be below a pre-defined threshold. Its current implementation leads to significant efficiency losses for isolated high- $p_{\mathrm{T}}$ muons (since they are often accompanied by electromagnetic showers); we do not apply HLT calorimeter isolation in this study (tracker isolation is applied). An increase in the trigger rate in the absence of calorimeter isolation should be mitigated by higher $p_{\mathrm{T}}$ thresholds; we have checked that raising the $p_{\mathrm{T}}$ thresholds of the single-muon HLT by $10-20 \mathrm{GeV}$ with respect to their nominal values changes trigger efficiency for our signals by a negligible amount. For the $\mathrm{Z}^{\prime}$ models that we study (as well as for the Drell-Yan background), the combined Level-1/HLT trigger efficiency is about $98 \%$ at $1 \mathrm{TeV} / \mathrm{c}^{2}$ and decreases with the $\mathrm{Z}^{\prime}$ mass down to about $95 \%$ at $5 \mathrm{TeV} / \mathrm{c}^{2}$. At high luminosity, the trigger efficiency is $95 \%$ at $1 \mathrm{TeV} / \mathrm{c}^{2}$ and $93 \%$ at $5 \mathrm{TeV} / \mathrm{c}^{2}$. These efficiencies are relative to having at least one muon inside the geometrical acceptance of the muon trigger $(|\eta|<2.1)$ and both muons from the $\mathrm{Z}^{\prime}$ decay inside the full acceptance of the muon system. No dependence of trigger efficiency on tracker and muon misalignment has been observed, in agreement with the results reported in Ref. [99].

We require that at least two muons of opposite sign charge be reconstructed offline. Detailed description of offline muon reconstruction can be found in Ref. [7]. For each muon candidate, we examine the results of fits to two subsets of hits associated to this 
candidate: (1) excluding all muon hits except for those in the innermost muon station, and (2) excluding hits in muon chambers appearing to contain electromagnetic showers. Optimal performance for high- $p_{\mathrm{T}}$ muons is achieved by choosing the best fit on a track-by-track basis using goodness-of-fit variables. The fraction of $Z^{\prime}$ events with an opposite-sign dimuon reconstructed offline is about $97 \%$ at $1 \mathrm{TeV} / \mathrm{c}^{2}$ for both the "first data" and the "long term" misalignment scenarios, and decreases slightly with the $Z^{\prime}$ mass, to about $95 \%$ at $5 \mathrm{TeV} / \mathrm{c}^{2}$ for the "long term" misalignment scenario. The efficiencies quoted are calculated relative to the number of events accepted by the trigger and with both muons from the $\mathrm{Z}^{\prime}$ decay within the full geometrical acceptance of the muon system.

The overall efficiency - including acceptance, trigger and offline reconstruction - for $\mathrm{Z}^{\prime} \rightarrow \mu^{+} \mu^{-}$events with a mass between 1 and $5 \mathrm{TeV} / \mathrm{c}^{2}$ lies in the range of $77-85 \%$ at low luminosity, and of $75-83 \%$ at high luminosity.

\subsubsection{Signal observability}

The search for a new resonance is performed with an unbinned maximum likelihood fit to the $\mu^{+} \mu^{-}$invariant mass spectrum over a range which includes Drell-Yan continuum as well as a possible peak. The fit takes as input the presumed signal and background shapes, and determines the best-fit background normalisation. More details are given in Refs. [100, 101].

3.3.4.1. Mass spectra and fitting procedure. Prior to the calculation of the invariant mass of an opposite-sign muon pair, $\sqrt{s}$, a search for photon candidates in a cone with a radius of $\Delta R=\sqrt{(\Delta \phi)^{2}+(\Delta \eta)^{2}}<0.1$ around the trajectory of each muon is performed, and the 4-momentum of the photon candidate with the smallest $\Delta R$ in the cone is added to the 4-momentum of the muon. This procedure recovers some of the energy lost by the muon via final state radiation and radiative processes in the detector, thus improving the invariant mass resolution.

The resolution for $\sqrt{s}$ depends strongly on the misalignment scenario, and weakly on the amount of pile-up. If the "long term" misalignment scenario for the tracker and the muon chambers is considered, the sigma of the Gaussian fit to the mass resolution curves varies from $4.2 \%$ at $1 \mathrm{TeV} / \mathrm{c}^{2}$ to $9.0 \%$ at $5 \mathrm{TeV} / \mathrm{c}^{2}$; the RMS truncated at $\pm 30 \%$ is $\sim 6 \%$ at $1 \mathrm{TeV} / \mathrm{c}^{2}$ and $\sim 10 \%$ at $5 \mathrm{TeV} / \mathrm{c}^{2}$. The corresponding numbers for the "first data" misalignment scenario at $1 \mathrm{TeV} / \mathrm{c}^{2}$ are $\sigma=12.5 \%$ and RMS $\sim 12 \%$. The bias in the mass resolution does not exceed $1 \%$ for the "long term" scenario at all masses considered and for the "first data" scenario at $1 \mathrm{TeV} / \mathrm{c}^{2}$.

An example of the $\sqrt{s}$ spectra showing $1 \mathrm{TeV} / \mathrm{c}^{2} \mathrm{Z}_{\eta}$ signal and Drell-Yan background is in Fig. 3.19. The left-hand plot shows generated mass spectra (100\% efficiency with no detector- and reconstruction-related effects); it can be compared to the right-hand plot for fully-recon structed events using the "first data" misalignment scenario. Signal peak is clearly visible in spite of the poor mass resolution.

The mass spectra in Fig. 3.19 are obtained by re-scaling the simulated spectra with large statistics down to a modest number of events characteristic for the regime close to the discovery limit; the statistical fluctuations are thus not to scale. In what follows, we use ensembles of Monte Carlo pseudo-experiments selected from available large-statistics samples. The number of events in each experiment, $N_{\text {evt }}$, fluctuates according to a Poisson distribution with a mean of $\sigma \cdot \operatorname{Br} \cdot \int \mathcal{L} d t \cdot \varepsilon$, where $\int \mathcal{L} d t$ is the integrated luminosity and $\varepsilon$ is the combined trigger and reconstruction efficiency.

In order to test for the existence of a resonance and to measure its parameters if it is found to exist, an unbinned maximum likelihood fit of the $\sqrt{s}$ values in each MC experiment 

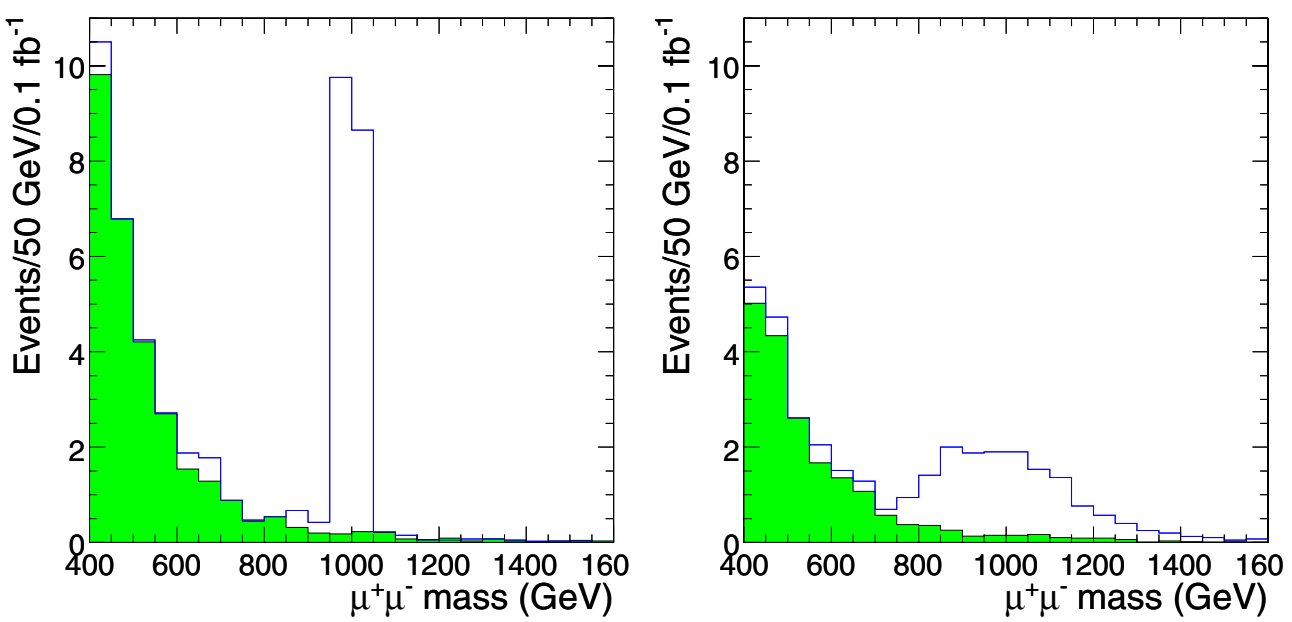

Figure 3.19. Histograms of the $\mu^{+} \mu^{-}$invariant mass for $1 \mathrm{TeV} / \mathrm{c}^{2} \mathrm{Z}_{\eta}$ plus background (open histogram) and for background only (shaded histogram), at the event-generator level (left) and for events selected by the Level-1/HLT triggers and reconstructed assuming the "first data" misalignment scenario (right). The number of events per bin is normalised to an integrated luminosity of $0.1 \mathrm{fb}^{-1}$.

is appropriate. One can imagine that, in the initial data analysis, one is confident about the background shape but not the absolute normalisation. In this case, data can be fit with a sum of signal and background shapes, presumed known, with the signal fraction as a free parameter. In the presence of a signal, one can fix or let vary the mass and the width as well. Thus, as a model of the probability density function (pdf), $p$, of the parent population of the observed mass spectra, we use

$$
p\left(\sqrt{s} ; f_{s}, m_{0}, \Gamma\right)=f_{s} \cdot p_{s}\left(\sqrt{s} ; m_{0}, \Gamma\right)+\left(1-f_{s}\right) \cdot p_{b}(\sqrt{s}) .
$$

Here:

- $p_{s}$, the pdf of the signal, is a convolution of a Breit-Wigner signal shape with a Gaussian accounting for mass resolution smearing. The convolution includes the dependence of the mass resolution on $\sqrt{s}$, but the radiative tail of the signal is not yet accounted for.

- $p_{b}$, the pdf of the background, is modelled as an exponential, $\exp \left(-k \cdot \sqrt{s}^{0.3}\right)$, with the parameter $k$ determined from fits to Drell-Yan events. This pdf, with the value of $k$ of 2.0, gives a good description of the background shape in the whole mass region between 400 and $5000 \mathrm{GeV} / \mathrm{c}^{2}$.

There are three free parameters in the fit: the signal fraction $f_{s}=N_{s} /\left(N_{s}+N_{b}\right)$, the position of the mass peak $m_{0}$, and the full width at half maximum (FWHM), $\Gamma$, of the signal. The shape of the background distribution is fixed, while its level is determined by the fit: $f_{s}$ is a free parameter. Therefore, the fit explores the difference in shape between the signal and the background, and is not sensitive to uncertainties in the expected signal and background levels.

The background shape is currently determined from fits to large-statistics backgroundonly simulated distributions in the full mass region of interest, including the region under the signal peak. In the real experiment, the shape will likely have to be extracted from the data in signal-free regions. The accuracy of predicting the background shape is an important contribution to the systematic uncertainty of the analysis and is discussed in Section 3.3.4.4.3. 
Table 3.9. Average values of the likelihood-ratio significance estimator $S_{\mathcal{L}}$ for six different $\mathrm{Z}^{\prime}$ models, at three signal mass points and for a few representative values of an integrated luminosity. The uncertainties shown are statistical only.

\begin{tabular}{lrrr}
\hline Mass & $1 \mathrm{TeV} / \mathrm{c}^{2}$ & $3 \mathrm{TeV} / \mathrm{c}^{2}$ & $5 \mathrm{TeV} / \mathrm{c}^{2}$ \\
\hline $\int \mathcal{L} d t$ & $0.1 \mathrm{fb}^{-1}$ & $10 \mathrm{fb}^{-1}$ & $300 \mathrm{fb}^{-1}$ \\
$\mathrm{Z}_{\mathrm{SSM}}$ & $12.4 \pm 0.2$ & $10.1 \pm 0.2$ & $5.8 \pm 0.1$ \\
$\mathrm{Z}_{\psi}$ & $5.1 \pm 0.2$ & $4.4 \pm 0.1$ & $2.4 \pm 0.2$ \\
$\mathrm{Z}_{\eta}$ & $5.5 \pm 0.2$ & $5.1 \pm 0.1$ & $2.9 \pm 0.1$ \\
$\mathrm{Z}_{\chi}$ & $9.1 \pm 0.2$ & $6.7 \pm 0.2$ & $3.2 \pm 0.1$ \\
$\mathrm{Z}_{\text {LRM }}$ & $9.0 \pm 0.2$ & $7.4 \pm 0.2$ & $4.1 \pm 0.1$ \\
$\mathrm{Z}_{\text {ALRM }}$ & $13.3 \pm 0.3$ & $11.8 \pm 0.2$ & $7.7 \pm 0.2$ \\
\hline
\end{tabular}

Ref. [100] contains examples of results of fits to Monte Carlo small-event samples. With even the small number of events needed to give evidence of a resonance, the mass is determined fairly well, with a precision of $4-8 \%$ depending on the resonance mass and alignment uncertainties. However, for the narrow resonances under study, typically little information can be obtained about the width.

3.3.4.2. Significance estimator. We follow closely the approach of Ref. [102], which is based on the theorem of Wilks [103]. The test statistic is the likelihood-ratio estimator $S_{\mathcal{L}}$ :

$$
S_{\mathcal{L}}=\sqrt{2 \ln \left(\mathcal{L}_{s+b} / \mathcal{L}_{b}\right)}
$$

where $\mathcal{L}_{s+b}$ is the maximum likelihood value obtained in the full signal-plus-background fit, and $\mathcal{L}_{b}$ is the maximum likelihood from the background-only fit. Studies show [100] that in the small-statistics low-background regime characteristic of a $Z^{\prime}$ search, the asymptotic conditions of Wilks's theorem [103] are satisfied well enough and $S_{\mathcal{L}}$ is the number of Gaussian-equivalent standard deviations a measurement lies from the value predicted by a background-only (null) hypothesis. This requires fixing both $m_{0}$ and $\Gamma$ in the fits using the pdf of Eq. (3.19).

We follow a common convention in using the (arbitrary, but useful for comparison) specification that $S>5$ is necessary to establish a discovery. This $S$ refers to the local excess without accounting for the degree of freedom due to the unknown mass; how one might derate $S$ in a time-dependent way in this context as data comes in will be the subject of a future study.

3.3.4.3. Discovery potential in $\mathrm{Z}^{\prime} \rightarrow \mu^{+} \mu^{-}$channel. Table 3.9 gives a summary of the signal significance expected for different $Z^{\prime}$ models, masses and integrated luminosities. The numbers shown are for the "first data" misalignment scenario and low luminosity parameters for $\int \mathcal{L} d t=0.1 \mathrm{fb}^{-1}$, the "long term" misalignment scenario and low luminosity parameters for $10 \mathrm{fb}^{-1}$, and the "long term" misalignment scenario and high luminosity parameters for $300 \mathrm{fb}^{-1} . S_{\mathcal{L}}$ scales as expected with the square root of $\int \mathcal{L} d t$.

We use the same combinations of luminosities and misalignment scenarios to calculate the integrated luminosity needed to reach $5 \sigma$ significance. The results for various $\mathrm{Z}^{\prime}$ models are shown in Fig. 3.20 as a function of $Z^{\prime}$ mass. One can see that:

- A very low integrated luminosity, less than $0.1 \mathrm{fb}^{-1}$, and non-optimal alignment of the tracker and the muon detectors should be sufficient to discover $Z^{\prime}$ bosons at $1 \mathrm{TeV} / \mathrm{c}^{2}$, a mass value which will likely be above the Tevatron reach. One would need about $50 \%$ less data to reach the same signal significance if, the optimal alignment is achieved. 


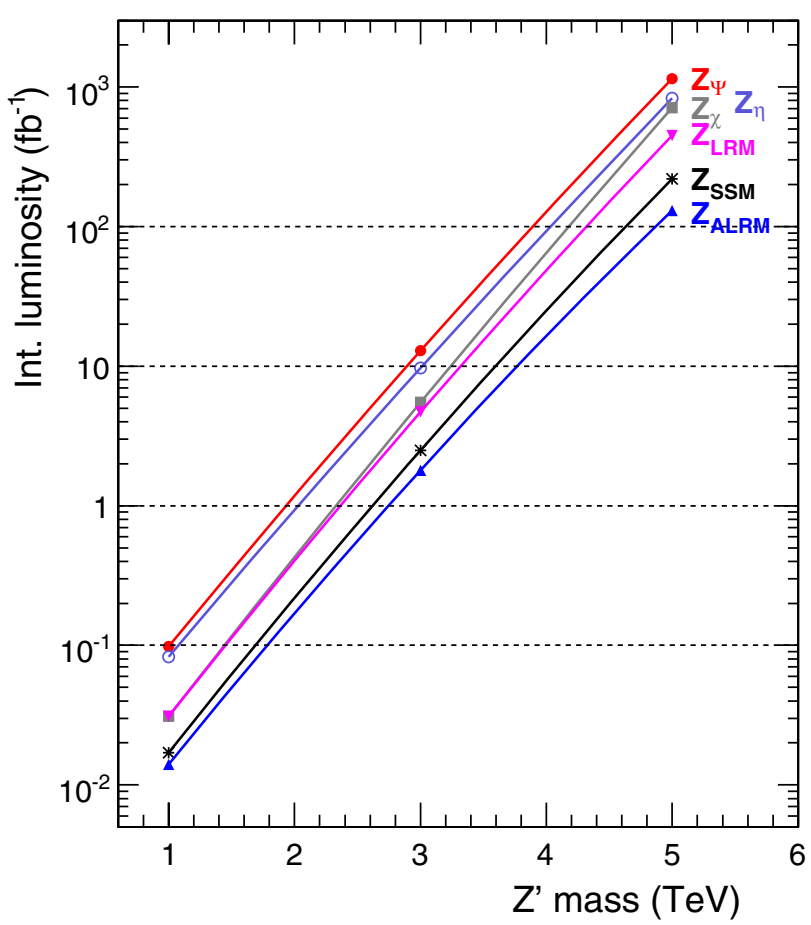

Figure 3.20. Integrated luminosity needed to reach $5 \sigma$ significanc $\left(S_{\mathcal{L}}=5\right)$ as a function of $Z^{\prime}$ mass for (top to bottom) $\mathrm{Z}_{\psi}, \mathrm{Z}_{\eta}, \mathrm{Z}_{\chi}, \mathrm{Z}_{\mathrm{LRM}}, \mathrm{Z}_{\mathrm{SSM}}$ and $\mathrm{Z}_{\mathrm{ALRM}}$. Symbols indicate fully-simulated mass-luminosity points, lines are the results of interpolations between the points.

- An integrated luminosity of $10 \mathrm{fb}^{-1}$ is sufficient to reach $5 \sigma$ significance at $3 \mathrm{TeV} / \mathrm{c}^{2}$ for most (but not all) of the $\mathrm{Z}^{\prime}$ models considered if the optimal alignment is available: depending on the model, the mass reach is in the range between 2.9 and $3.8 \mathrm{TeV} / \mathrm{c}^{2}$.

- An integrated luminosity of $100 \mathrm{fb}^{-1}$ does not allow one to obtain $5 \sigma$ significance at $5 \mathrm{TeV} / \mathrm{c}^{2}$ with only the $\mathrm{Z}^{\prime} \rightarrow \mu^{+} \mu^{-}$channel for any of the models considered: the corresponding mass reach lies in the region between 3.9 and $4.9 \mathrm{TeV} / \mathrm{c}^{2}$.

These estimates of signal significance do not incorporate systematic uncertainties, which we discuss in the next section.

3.3.4.4. Systematic uncertainties. The main sources of systematic uncertainties are expected to be (a) theoretical uncertainties (parton distributions, higher-order corrections, etc.), (b) uncertainties arising from an imperfect knowledge of the detector (alignment, calibration, magnetic field), and (c) uncertainties in the fitting procedure (background shape, functional forms of pdf's, mass resolution, etc.).

3.3.4.4.1. Theoretical uncertainties. Our current estimates of the $\mathrm{Z}^{\prime}$ mass reach depend on the accuracy of the modelling of the Standard Model processes and of the $Z^{\prime}$ boson production. The following sources of theoretical uncertainties have been studied.

- Higher-order QCD corrections. We use a constant $K_{\mathrm{QCD}}^{\mathrm{NNLO}}$ factor of 1.35 to rescale PYTHIA cross sections for Drell-Yan and $Z^{\prime}$ bosons to NNLO QCD predictions. This is an approximation, since such a reweight does not take into account variations of the ratio of NNLO and LO cross sections with the invariant mass and other observables, such as rapidity and $p_{\mathrm{T}}$. It is shown in Appendix $\mathrm{C}$ that the variations of the $K_{\mathrm{QCD}}^{\mathrm{NNLO}}$ factor with the mass in 
the mass interval between $500 \mathrm{GeV} / \mathrm{c}^{2}$ and $5 \mathrm{TeV} / \mathrm{c}^{2}$ is in the range of $\Delta K_{\mathrm{QCD}}= \pm 0.05$; the dependence on other observables and the ensuing impact on acceptance, efficiency, etc. remains to be studied. Since $K$ is expected to be nearly identical for the signal and dominant background, the effect of changes in $K$ from the nominal value $K_{0}=1.35$ is to scale the expected significance by $\sqrt{K / K_{0}}$.

- Higher-order electroweak corrections. Only preliminary estimates of electroweak nextto-leading order corrections exist for the LHC and $\sqrt{s}>1 \mathrm{TeV} / \mathrm{c}^{2}[104,105]$. Currently, we use $K_{\mathrm{EW}}=1$ for the central values of signal and background cross-sections, and assign an uncertainty of $\Delta K_{\mathrm{EW}}= \pm 0.10$ based on discussions in Refs. [104, 105].

- Parton distribution functions (PDFs). We use the CTEQ6.1M eigenvector PDF sets [12] and the "master" equations in Ref. [106] to evaluate the uncertainties characterising current knowledge of the parton distributions. The effect on the total cross section $\sigma$ was found to be similar for the Drell-Yan background and for the studied $\mathrm{Z}^{\prime}$ models at any given mass, with uncertainties lying in the range of $\frac{\Delta \sigma}{\sigma}={ }_{+4 \%}^{-7 \%}$ at $\sqrt{s}=1 \mathrm{TeV} / \mathrm{c}^{2}$, rising to ${ }_{+12 \%}^{-10 \%}$ at $\sqrt{s}=3 \mathrm{TeV} / \mathrm{c}^{2}$, and reaching as much as ${ }_{+30 \%}^{-20 \%}$ at $\sqrt{s}=5 \mathrm{TeV} / \mathrm{c}^{2}$. The effect on other observables and on the acceptance has not been studied yet, but is expected to be small.

- Hard process scale. The dependence of the observables on the choice for renormalisation and factorisation $Q^{2}$ scales, $\mu_{R}$ and $\mu_{F}$, is unphysical and is commonly taken as a rough estimate of the uncertainty due to unaccounted higher orders in QCD calculations. The study of the sensitivity of the Drell-Yan cross section to the choice for the QCD scale is described in Appendix C. Both $\mu_{F}$ and $\mu_{R}$ were varied in the range of $\sqrt{s} / 2<\mu<2$ $\sqrt{s}$ around the default choice of $\mu=\sqrt{s}$, and the mass-dependent variations of the cross section obtained. At NNLO, they are smaller than $\pm 1 \%$ at $1 \mathrm{TeV} / \mathrm{c}^{2}$, but as large as $-25 \%$ (for $\mu=2 \sqrt{s}$ ) and $+5 \%$ (for $\mu=2 \sqrt{s}$ ) at $5 \mathrm{TeV} / \mathrm{c}^{2}$. We use the NNLO estimates given in Appendix $\mathrm{C}$ for both the Drell-Yan and the $\mathrm{Z}^{\prime}$ bosons.

Since our analysis relies only on the background shape and not on any assumptions about background normalisation, the uncertainties in signal and background cross sections described in this section will not have any direct impact on the calculation of significance once a data set is in hand. They do effect, however, estimates of the $Z^{\prime}$ mass reach based on Monte Carlo predictions for the signal and the background. We combine them in quadrature, and use the obtained mass-dependent band as $1 \sigma$ uncertainty in the expected number of signal and background events. This band is then translated into $1 \sigma$ uncertainty in the prediction of the mean integrated luminosity needed to reach $5 \sigma$ significance for any given $\mathrm{Z}^{\prime}$ model. This uncertainty, and the best estimates of the luminosity, is shown in Fig. 3.21 for the models with the smallest and the largest values of $\sigma \cdot \mathrm{Br}$ among the models studied, $\mathrm{Z}_{\psi}$ and $\mathrm{Z}_{\mathrm{ALRM}}$.

3.3.4.4.2. Uncertainties in the detector performance. The key element in the performance of high- $p_{\mathrm{T}}$ muon reconstruction and, therefore, for the $\mathrm{Z}^{\prime}$ mass reach is the alignment of the tracker and the muon system. Unlike the muons in the region of low and moderate $p_{\mathrm{T}}$ values, where the influence of the tracker alignment is predominant, both the tracker alignment and the muon system alignment play an important role for the muons at TeV scale. We take them into account by using the two realistic misalignment scenarios developed in the CMS reconstruction, the "first data" and the "long term". These scenarios, however, are only based on the current best estimates (and sometimes guesses) of expected alignment uncertainties and will be refined as better estimates from alignment studies become available. Therefore, they have intrinsic uncertainties, which at the moment cannot be evaluated. As discussed above and in Ref. [99], neither the trigger efficiency nor the offline reconstruction efficiency for high- $p_{\mathrm{T}}$ muons is affected by the misalignment even in the worst-case scenario once the alignment 


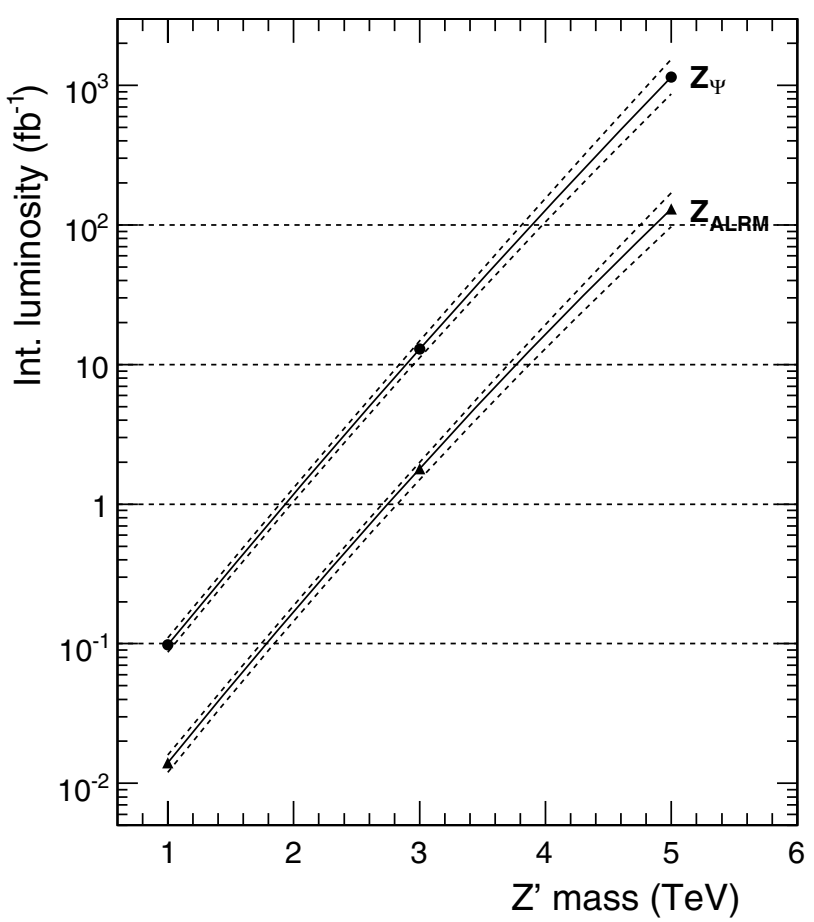

Figure 3.21. Integrated luminosity needed to reach $5 \sigma$ significance $\left(S_{\mathcal{L}}=5\right)$ as a function of $\mathrm{Z}^{\prime}$ mass for $\mathrm{Z}_{\psi}$ and $\mathrm{Z}_{\mathrm{ALRM}}$ models. Solid lines show the best estimates, dashed lines indicate boundaries of the band corresponding to the predictions with $\pm 1 \sigma$ theoretical uncertainty.

position uncertainties are used in reconstruction algorithms [86]. So uncertainties in alignment translate mainly into uncertainties in the invariant mass resolution. We show below that even sizable variations in the width of the mass resolution have only a small impact on the $\mathrm{Z}^{\prime}$ mass reach.

Another potentially important source of systematic uncertainties is the uncertainty in the calibration precision of the muon chambers. The impact of uncertainties in the calibration of the Drift Tube chambers on the $Z^{\prime}$ mass reach has been studied by (1) changing the $t_{0}$ offsets for all chambers by $\pm 2 \mathrm{~ns}$, and (2) scaling drift velocity (changing time-to-distance relationship) by $\pm 3 \%$. These variations represent conservative upper bounds on corresponding effects [107]. The effect of changing $t_{0}$ offset was found to be negligible for $Z^{\prime}$ samples at all studied mass values and for both misalignment scenarios considered. The scaling of drift velocity has a negligible impact for the "first data" misalignment scenario with its rather poor mass resolution, but results in an increase of 5-10\% in the width of the mass resolution for the "long term" scenario (no change in trigger and dimuon reconstruction efficiencies). This translates into a negligible effect in the $Z^{\prime}$ mass reach. Uncertainties in the calibration of the Cathode Strip Chambers are less critical and hence are expected to have a negligible impact on the $\mathrm{Z}^{\prime}$ detection as well.

The effect of uncertainties in the knowledge of the magnetic field remains to be studied.

3.3.4.4.3. Uncertainties in background shape and mass resolution. Many experimental uncertainties have a negligible or small impact on the results of our studies because, the proposed analysis method is not sensitive to uncertainties in the predicted levels of signal and background processes. For example, only the mass dependence of the uncertainty in the 
muon reconstruction efficiency needs to be taken into account, not the absolute uncertainty. The same is true for the trigger efficiency and for the uncertainty in the $\sqrt{s}$ scale. Among those uncertainties that do not cancel out, two seem to be particularly important: the uncertainty in the background shape, and the uncertainty in the mass resolution.

As described above, the background shape is currently determined from fits to background distributions predicted by the Monte Carlo simulation. In the analysis of real data, this MC-based shape will be compared with (and perhaps tuned to) the background shape in the region of low masses where one has high statistics of background events. The issue is then the reliability of the extrapolation from the steeply falling spectrum into the candidate signal region. This will have to be studied in detail, once the real data starts to be available. What is interesting to explore at this stage of analysis is how rapidly the significance deteriorates as the ratio of background events in the highstatistics normalisation region to background events in the candidate signal region is wrongly predicted by the MC-motivated background shape. To study this, we multiply our background pdf ( $p_{b}$ in Eq. (3.19)) by a function which is unity in the high-statistics background-only region and smoothly transitions to a tunable value, $f$, under the candidate mass peak. Values of integrated luminosity were chosen to correspond to $5 \sigma$ significance for each model at $f=1$. For $f=2$ (assuming twice as much background in the signal region as there really is), $5 \sigma$ becomes $4.2 \sigma$ for $\mathrm{Z}_{\mathrm{ALRM}}$ and is about $3.7 \sigma$ for $\mathrm{Z}_{\psi}$. For $f$ around 1.1 or 1.2 , the change in $S$ is of the order of a few per cent.

Sensitivity of the $Z^{\prime}$ mass reach to uncertainties in the invariant mass resolution has been studied by applying extra Gaussian smearing to the reconstructed values of $\sqrt{s}$ of both the signal and background events and comparing the signal significance obtained with modified $\sqrt{s}$ values to that calculated with the nominal $\sqrt{s}$ values. We found that an increase of $10 \%$ in the mass resolution width, $\sigma_{M}$, reduces the signal significance by less than $2 \%$ at the values of $S_{\mathcal{L}}$ close to $5 ; 20 \%$ worse resolution gives $5 \%$ or less smaller $S_{\mathcal{L}}$. The effect is not very big, indicating that an approximate knowledge of $\sigma_{M}$ should suffice. (This exercise does not check, however, the effect of extreme tails of the mass resolution being bigger than expected, which could lead to a background shape (and amount) different from that obtained from the simulation.) The knowledge of $\sigma_{M}$ as a function of $\sqrt{s}$ is also used in the pdf of the signal in Eq. (3.19), where it defines the width of a Gaussian accounting for resolution smearing of the signal shape. This does not need to be very precise either: assuming resolution $20 \%$ better that it really is reduces $S_{\mathcal{L}}$ by less than $1 \%$.

\subsubsection{Distinguishing among $\mathrm{Z}^{\prime}$ models}

The forward-backward asymmetry, $A_{\mathrm{FB}}$, of the leptonic decay products provides information on parity-violating couplings, on and off resonance, as discussed for example in Refs. [96, 108].

The forward-backward asymmetry for $q \bar{q} \rightarrow \mu^{+} \mu^{-}$interactions is defined as (e.g., Refs. [109, 110])

$$
A_{\mathrm{FB}}=\frac{\sigma_{F}-\sigma_{B}}{\sigma_{F}+\sigma_{B}}
$$

where

$\sigma_{F} \equiv \int_{0}^{1} \frac{d \sigma\left(q \bar{q} \rightarrow \mu^{+} \mu^{-}\right)}{d \cos \theta^{*}} d \cos \theta^{*}, \quad \sigma_{B} \equiv \int_{-1}^{0} \frac{d \sigma\left(q \bar{q} \rightarrow \mu^{+} \mu^{-}\right)}{d \cos \theta^{*}} d \cos \theta^{*}$, 
and where $\theta^{*}$ is the angle in the dimuon centre-of-mass $(\mathrm{CM})$ reference frame between the negative muon and the incident quark. For spin- $1 \gamma^{*} / Z^{0} / Z^{\prime}$ propagators, the probability density function $P\left(\cos \theta^{*}\right)$ is most generally of the form

$$
P\left(\cos \theta^{*} ; A_{\mathrm{FB}}, b\right)=\frac{3}{2(3+b)}\left(1+b \cos ^{2} \theta^{*}\right)+A_{\mathrm{FB}} \cos \theta^{*} .
$$

Although $b=1$ from general considerations, in the fits described here $b$ is typically left as a free parameter. In Ref. [97], Rosner expresses $A_{\mathrm{FB}}$ for $f \bar{f} \rightarrow \gamma^{*} / \mathbf{Z}^{0} / \mathbf{Z}^{\prime} \rightarrow \mu^{+} \mu^{-}$events in terms of the left- and right-handed couplings of the photon, $Z^{0}$, and $Z^{\prime}$ to $u$ quarks, $d$ quarks, and charged leptons. More details, including the couplings for the models studied, are given in Ref. [111].

For CM energies well above the $Z^{0}$ peak, the Drell-Yan background has a characteristic $A_{\mathrm{FB}}$ of about 0.6 [109], and provides a useful starting point.

3.3.5.1. Uncertainty in the sign of $\cos \theta^{*}$ in $p p$ collisions. In proton-proton interactions, the quark direction is ambiguous experimentally since a quark can originate with equal probability from either proton, and the sign of $\cos \theta^{*}$ is not directly measurable. We follow Ref. [112] and infer the sign of $\cos \theta^{*}$ by assuming that the longitudinal motion of the dimuon system is in the direction of the proton contributing the annihilating quark, since a quark in a proton typically carries a larger momentum fraction $x$ than does an anti-quark. We refer to the inference of the wrong sign of $\cos \theta^{*}$ as "mistagging" the sign. If not accounted for, the mistagged events, particularly at low $y$, reduce ("dilute") the apparent value of $A_{\mathrm{FB}}$. Some authors deal with this problem by removing events below a chosen $y$ threshold [112], or by examining $A_{\mathrm{FB}}$ in bins of $y$ [113]; in Ref. [111], an approached is described which assigns the probability of a mistag on an event-by-event basis, thus using all events in a given sample. As knowledge of the mistagging probability depends on the Parton Distribution Functions, the effect of uncertainties in PDFs must be evaluated, and will be the subject of future work.

3.3.5.2. Other uncertainties. The transverse momentum $p_{\mathrm{T}}$ of the annihilating quark and/or anti-quark provides another source of uncertainty in the measurement of $\cos \theta^{*}$, since the observable quantity is the vector sum of these transverse momenta. We use the Collins-Soper reference frame [114], in which angles are measured with respect to the axis that bisects the target and beam axes in the dimuon CM frame, to minimise the effect of $p_{\mathrm{T}}$ on the measurement of $\cos \theta^{*}$, and let $\theta_{\mathrm{CS}}^{*}$ denote the polar angle of the $\mu^{-}$in this frame.

As described in Ref. [111], the effect of detector acceptance, combined with high mistag probability for events near $y=0$, means that events lying near the edges of acceptance carry the largest information for the $A_{\mathrm{FB}}$ measurement. Hence, in addition to trying to obtain maximum acceptance, it is particularly important to understand the effect of any asymmetries in the acceptance which may arise as a result of the real detector efficiencies not being perfectly symmetric or of the beam crossing not being perfectly centred.

3.3.5.3. Likelihood function and fitting procedure. Since a $Z^{\prime}$ can be discovered with a small number of events (Section 3.3.4), and since the search for anomalous $A_{\mathrm{FB}}$ in the highest mass continuum Drell-Yan events at any given luminosity will use a restricted sample of events, we consider an unbinned likelihood fit. The procedure and results with statistical errors only are described in Ref. [111]. The results of numerous fits can be summarised simply with a nominal statistical uncertainty in $A_{\mathrm{FB}}$ of 0.09 in a fit with 400 events for $1 \mathrm{TeV} / \mathrm{c}^{2} \mathrm{Z}^{\prime}$ samples, and of 0.08 with 400 events for $3 \mathrm{TeV} / \mathrm{c}^{2}$ samples. Ref. [111] also reviews an appropriate hypothesis-testing methodology for distinguishing between $\mathrm{Z}^{\prime}$ models. 
Table 3.10. Angular distributions for the decay products of spin-1 and spin-2 resonances, considering only even terms in $\cos \theta^{*}$.

\begin{tabular}{lll}
\hline Channel & \multicolumn{1}{c}{$d$-functions } & Normalised density for $\cos \theta^{*}$ \\
\hline$q \bar{q} \rightarrow G^{*} \rightarrow f \bar{f}$ & $\left|d_{1,1}^{2}\right|^{2}+\left|d_{1,-1}^{2}\right|^{2}$ & $P_{q}=\frac{5}{8}\left(1-3 \cos ^{2} \theta^{*}+4 \cos ^{4} \theta^{*}\right)$ \\
$g g \rightarrow G^{*} \rightarrow f \bar{f}$ & $\left|d_{2,1}^{2}\right|^{2}+\left|d_{2,-1}^{2}\right|^{2}$ & $P_{g}=\frac{5}{8}\left(1-\cos ^{4} \theta^{*}\right)$ \\
$q \bar{q} \rightarrow \gamma^{*} / \mathrm{Z}^{0} / \mathrm{Z}^{\prime} \rightarrow f \bar{f}$ & $\left|d_{1,1}^{1}\right|^{2}+\left|d_{1,-1}^{1}\right|^{2}$ & $P_{1}=\frac{3}{8}\left(1+\cos ^{2} \theta^{*}\right)$ \\
\hline
\end{tabular}

\subsubsection{Discriminating between different spin hypotheses}

In order to distinguish the spins of a spin-1 Z' bosons and a spin-2 gravitons in a dilepton decay mode, Ref. [115] considers an unbinned likelihood ratio statistic incorporating the angles of the decay products. The statistical interpretation of this statistic is discussed in detail in Ref. [116], also considering the possibility of spin 0 .

To leading order, the sub-diagram for $\mathrm{Z}^{\prime}$ formation is quark-anti-quark $(q \bar{q})$ annihilation, while for a graviton there exist both $q \bar{q}$ annihilation and gluon-gluon ( $g g$ ) fusion. One defines $\theta^{*}$ as the angle in the dilepton centre-of-mass reference frame between the negative lepton $\ell^{-}$ and the incident quark or gluon. In this section, we consider only the parity-conserving terms; inference from these terms can be combined with that of the parity-violating terms giving rise to $A_{\mathrm{FB}}$.

For light lepton decay products, the angular probability density functions in the absence of interference are in Table 3.10. These are determined from angular momentum considerations and do not depend on the couplings. For the spin-2 graviton, only the relative fractions of $q \bar{q}$ annihilation, gluon fusion, and background (predominantly from the DrellYan continuum) events are needed to arrive at a parameter-free form for the expected distribution. (For spin 1, the resonance and the Drell-Yan background have the same form.)

The fractions of generated events arising from these processes are denoted by $\epsilon_{q}, \epsilon_{g}$, and $\epsilon_{1}$, respectively, with $\epsilon_{q}+\epsilon_{g}+\epsilon_{1}=1$. Then the form of the probability density $P\left(\cos \theta^{*}\right)$ is

$$
P\left(\cos \theta^{*}\right)=\epsilon_{q} P_{q}+\epsilon_{g} P_{g}+\epsilon_{1} P_{1} .
$$

As in the $A_{\mathrm{FB}}$ measurements, we let $\theta_{\mathrm{CS}}^{*}$ denote the polar angle of the $\ell^{-}$in the CollinsSoper frame. Experimentally, one will obtain a set of events with $\theta_{\mathrm{CS}}^{*}$ measured along with other quantities such as dilepton transverse momentum $p_{\mathrm{T}}^{\text {dil }}$ and rapidity $y^{\text {dil }}$. From these, one can construct the probability density $P_{\mathrm{acc}}\left(\cos \theta_{\mathrm{CS}}^{*}\right)$ for events accepted (observed) in an experiment for each hypothesis $H_{i}$, where $i$ labels the model such as $\mathrm{Z}^{\prime}$ or $\mathrm{G}^{*}$. In this study, we consider only the angular information and integrate over $p_{\mathrm{T}}^{\mathrm{dil}}, y^{\mathrm{dil}}$, and any other relevant quantities; if one has confidence that these quantities are well described by the event generators, more variables can be added to $P_{\text {acc }}$. Since we do not add this information, $P_{\text {acc }}$ for accepted events approximately factorises:

$$
P_{\mathrm{acc}}\left(\cos \theta_{\mathrm{CS}}^{*} \mid H_{i}\right)=P\left(\cos \theta_{\mathrm{CS}}^{*} \mid H_{i}\right) \bar{\Omega}\left(\cos \theta_{\mathrm{CS}}^{*}\right),
$$

where $P\left(\cos \theta_{\mathrm{CS}}^{*} \mid H_{i}\right)$ is from Eq. (3.24) with the $\varepsilon_{j}$ set appropriately for the model considered (e.g. for the spin-1 hypothesis, we set $\epsilon_{1}=1$ and $\epsilon_{q}=\epsilon_{g}=0$ ), and $\bar{\Omega}$ is the acceptance averaged over $p_{\mathrm{T}}, y$, etc.

Eq. (3.25) has no free parameters, if the fractions $\varepsilon_{q}, \varepsilon_{g}$, and $\varepsilon_{1}$ are considered to be fixed. For each observed event, one evaluates $P_{\mathrm{acc}}\left(\cos \theta_{\mathrm{CS}}^{*} \mid H_{i}\right)$ at the observed $\cos \theta_{\mathrm{CS}}^{*}$ to obtain the likelihood $\mathcal{L}\left(H_{i}\right)$ of that event under the given hypothesis. The combined likelihood of the data set under a hypothesis is then the product of the events' likelihoods; henceforth in this paper, 
Table 3.11. Integrated luminosity and numbers of signal and background events $N_{s}$ and $N_{b}$ required to discriminate spin-1 and spin-2 hypotheses with $\alpha=\beta$ corresponding to $2 \sigma$ (onetailed). The first column indicates the mass of the resonance; the second column shows the values of the RS ratio $c=k / \bar{M}_{\mathrm{Pl}}$; the third column specifies the integrated luminosity needed for $2 \sigma$ discrimination; the last two columns show the corresponding numbers of signal and background events.

\begin{tabular}{lcccc}
\hline$\sqrt{s}, \mathrm{TeV}$ & $c$ & $\int \mathcal{L} d t, \mathrm{fb}^{-1}$ & $N_{s}$ & $N_{b}$ \\
\hline 1.0 & 0.01 & 50 & 200 & 87 \\
1.0 & 0.02 & 10 & 146 & 16 \\
1.5 & 0.02 & 90 & 174 & 41 \\
3.0 & 0.05 & 1200 & 154 & 22 \\
3.0 & 0.10 & 290 & 148 & 6 \\
\hline
\end{tabular}

$\mathcal{L}\left(H_{i}\right)$ refers to this product unless otherwise stated. As Ref. [116] discusses, the absence of free parameters means that the Neyman-Pearson hypothesis testing for simple hypothesis testing is applicable.

For testing a simple null hypothesis $H_{A}$ of one spin against another simple alternative spin hypothesis $H_{B}$, we use the likelihood ratio $\lambda=\mathcal{L}\left(H_{A}\right) / \mathcal{L}\left(H_{B}\right)$, with critical region again chosen such that $\alpha=\beta$. For investigating and summarising which values of $\lambda^{\text {cut }}$ correspond to which values of $\alpha$ and $\beta$, the quantity $-2 \ln \lambda=2 \ln \mathcal{L}\left(H_{B}\right)-2 \ln \mathcal{L}\left(H_{A}\right)$ is particularly useful. For simple hypotheses $H_{A}$ and $H_{B}$, the central limit theorem implies that $-2 \ln \lambda$ tends to a Gaussian.

3.3.6.1. Testing spin 1 versus spin 2. A detailed discussion of the intermediate steps in applying the above method for discriminating spin 1 from spin 2 is in Ref. [116], using large samples of $Z^{\prime}$ and $G^{*}$ events (from the Randall-Sundrum (RS) model [94]) generated with HERWIG. (Generator-level results using PYTHIA are completely compatible.) The ratio $\lambda$ of the likelihoods of the hypotheses is calculated for each event, assigning spin 1 as the null hypothesis $H_{A}$ and spin 2 as the alternative hypothesis $H_{B}$. In taking the ratio, the average acceptance cancels to good approximation and one essentially recovers the ratios of the angular forms. Histograms of $-2 \ln \lambda$ for these events are highly asymmetric and strongly peaked at one side [116]. In view of the asymmetries in the underlying event histograms, the convergence of the sums of $-2 \ln \lambda$ values for $N$ selected events toward Gaussians is quite striking. The means and rms deviations of the sums are in excellent agreement with the means and rms deviations of the respective event histograms scaled by $N$ and $\sqrt{N}$, respectively, as expected from the central limit theorem.

The statistical technique of Ref. [116] has been applied to fully-reconstructed $Z^{\prime}$ and $G^{*}$ events [117]. Details of simulation, trigger and reconstruction are described in Sections 3.3.2, 3.3.3 and 14.3.1. From ensembles of pseudo-experiments, we determine the number $N$ of events per experiment corresponding to various values of $\alpha=\beta$, expressed in equivalent number of Gaussian standard deviations " $\sigma$ " for one-tailed tests, e.g., for $\alpha=0.159$, we report $\alpha=1 \sigma$, and so on. The values of $\alpha$ so obtained scale as expected as $\sqrt{N}$.

Table 3.11 contains, for different studied masses and values of the Randall-Sundrum ratio $c=k / \bar{M}_{\mathrm{Pl}}$, the integrated luminosity needed for a $2 \sigma$ significance, and the corresponding numbers of signal and background events. All numbers are for the "long term" misalignment scenario; the cross section for $\mathrm{Z}^{\prime}$ production is assumed to be equal to that of $\mathrm{G}^{*}$ with the given $c$ value. Of course, because the production cross section falls rather steeply with mass, the integrated luminosity needed for spin discrimination increases with mass. For RS gravitons, the production cross section scales as $c^{2}$; therefore, the integrated luminosity required for spin 


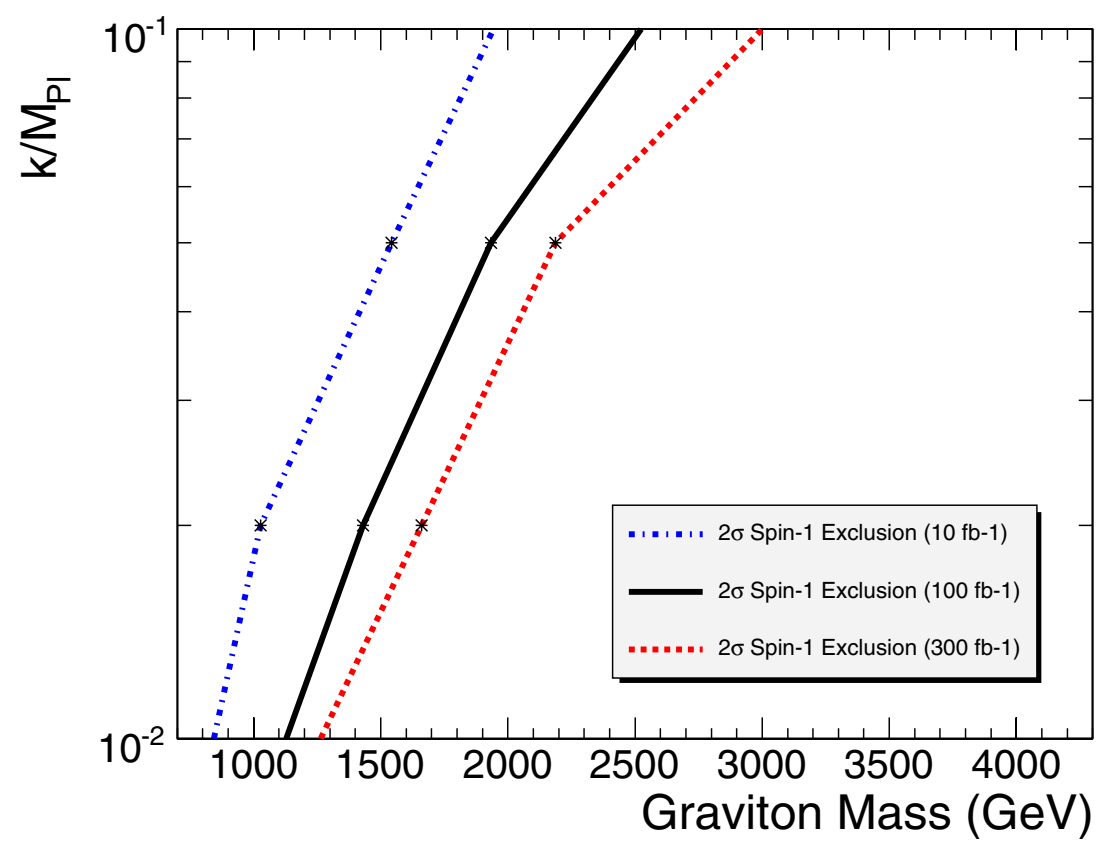

Figure 3.22. Region in the plane of $M_{\mathrm{G}^{*}-c}$ in which Randall-Sundrum $\mathrm{G}^{*}$ can be distinguished from $\mathrm{Z}^{\prime}$ having an equal cross section with $2 \sigma$ significance if one treats two spin hypotheses symmetrically, for a few representative values of the integrated luminosity. The region which can be probed lies to the left of the lines.

discrimination quickly increases as $c$ gets smaller, and so does the number of signal events, because of a larger background contamination. The region in the plane of $M_{\mathrm{G}^{*}}-c$ in which Randall-Sundrum $\mathrm{G}^{*}$ can be distinguished from $\mathrm{Z}^{\prime}$ with $2 \sigma$ significance if one treats two spin hypotheses symmetrically is shown in Fig. 3.22 for a few representative values of the integrated luminosity.

Alternatives to the $\alpha=\beta$ criterion, in particular tests in which $\alpha$ is minimised for one hypothesis at the cost of increase in $\beta$, are discussed in Ref. [116].

3.3.6.2. Discrimination from spin 0 . While the motivation of discriminating $\mathrm{Z}^{\prime}$ from $\mathrm{G}^{*}$ has focused studies on discriminating spin 1 from spin 2, another possibility to be considered is spin 0 resonance (which is uniform in $\cos \theta^{*}$ ). For accepted spin- 0 events, the probability density for $\cos \theta_{\mathrm{CS}}^{*}$ is somewhat in between the mostly concave-upward function for spin 1 and the predominantly concave-downward function for spin 2.

As discussed in Ref. [116], discriminating either spin 1 or spin 2 from spin 0 requires significantly more events than discriminating spin 2 from spin 1. 


\section{Chapter 4. Physics Studies with Jets and $E_{\mathrm{T}}^{\text {miss }}$}

\subsection{Benchmark Channel: new physics from dijets}

Inclusive dijet production $(p p \rightarrow 2$ jets $+X)$ is the dominant LHC hard scattering process. Simple to observe, and rich in potential signals of new physics, dijets are expected to be one of the earliest CMS measurements. In this section we discuss the measured distributions and their systematic uncertainties [118]. In section 14.5.2 and 15.3 we use these distributions to estimate our sensitivity to specific models of new physics.

\subsubsection{Dijet analysis}

We use samples generated using PYTHIA dijet processes mixed with pileup of minimum bias interactions for an assumed luminosity of $2 \times 10^{33} \mathrm{~cm}^{-2} \mathrm{~s}^{-1}$, simulated with OSCAR and reconstructed with ORCA. Jets are reconstructed as localised energy depositions in the CMS calorimeters arranged in a projective tower geometry. The jet energy $E$ is defined as the scalar sum of the calorimeter tower energies inside a cone of radius $R=\sqrt{(\Delta \eta)^{2}+(\Delta \phi)^{2}}=0.5$, centred on the jet direction. The jet momentum $\vec{P}$ is the corresponding vector sum of energies, with the vector pointing in the tower direction. Both the jet energy and momentum are corrected back to the particles in the jet cone originating from the hard interaction excluding pileup [119]. We define the dijet system as the two jets with the highest $p_{\mathrm{T}}$ in an event (leading jets) and define the dijet mass $m=\sqrt{\left(E_{1}+E_{2}\right)^{2}-\left(\vec{P}_{1}+\vec{P}_{2}\right)^{2}}$. We select events in which the leading jets each have $|\eta|<1$. This cut enhances our sensitivity to new physics, produced at low $|\eta|$, compared to the predominantly $t$-channel processes from the QCD background. In all plots that are a function of dijet mass, we plot in bins of width equal to the Gaussian resolution measured in section 4.1.4.1.

\subsubsection{Rates and efficiencies from jet triggers}

We use simulated data from the single jet triggers discussed in Appendix E.4.3.2. From the three trigger tables for luminosities of $\mathcal{L}=10^{32}, 10^{33}, 10^{34} \mathrm{~cm}^{-2} \mathrm{~s}^{-1}$ we expect initial samples of size at least $100 \mathrm{pb}^{-1}, 1 \mathrm{fb}^{-1}$, and $10 \mathrm{fb}^{-1}$ respectively. This is from $10^{6}$ seconds of collisions, equivalent to one month of continuous operation at $40 \%$ efficiency. In Fig. 4.1 we show the rate expected from these triggers as a function of dijet mass. By construction there are comparable events in each trigger, and a high statistics overlap between triggers for a given table. We see that the highest mass dijet is expected to be 5, 6 and $7 \mathrm{TeV}$ for samples of size $100 \mathrm{pb}^{-1}, 1 \mathrm{fb}^{-1}$, and $10 \mathrm{fb}^{-1}$ respectively. In Fig. 4.2 we show the trigger efficiency vs. dijet mass, measured for each trigger using the neighbouring trigger with a lower $p_{\mathrm{T}}$ threshold, and explicitly show the mass cuts that are fully efficient. In Fig. 4.3 we show the data we will use to measure the cross section. We use each trigger where it is fully efficient and stop using the trigger where the next trigger is fully efficient. Fig. 4.3 shows there are adequate numbers of fully efficient events for analysis.

\subsubsection{Dijet mass distribution from QCD}

In Fig. 4.4 we combine the triggers to produce a cross section across the full mass spectrum. The prescaled triggers allow us to measure mass down to $300 \mathrm{GeV} / \mathrm{c}^{2}$, or even smaller if we can understand the efficiency of the lowest threshold trigger. The mass measured with the prescaled triggers will allow us to connect to dijet masses measured at the Tevatron. 

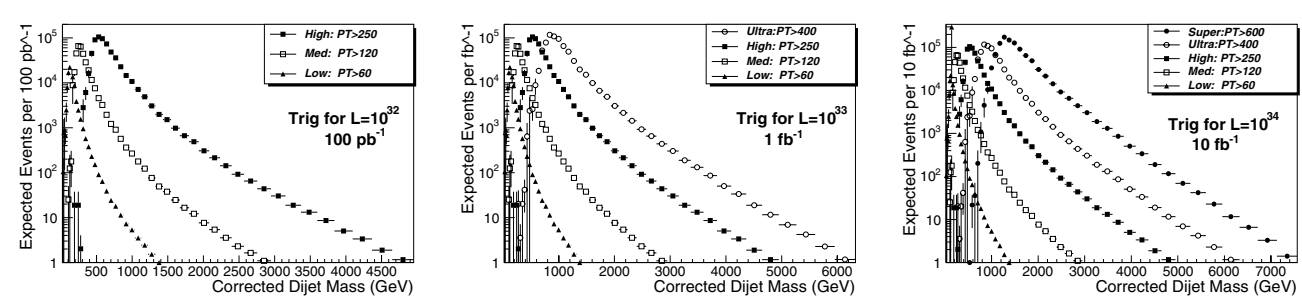

Figure 4.1. Rate of jet trigger as a function of dijet mass. The 3 plots correspond to 3 trigger tables, and each plot shows multiple triggers with various $p_{\mathrm{T}}$ thresholds and prescales.

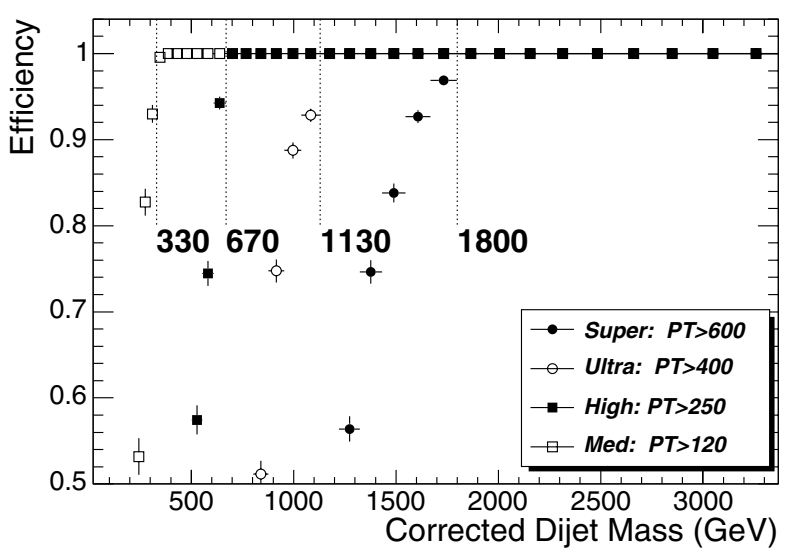

Figure 4.2. Jet trigger efficiency (points) and fully efficient dijet mass cuts (lines).
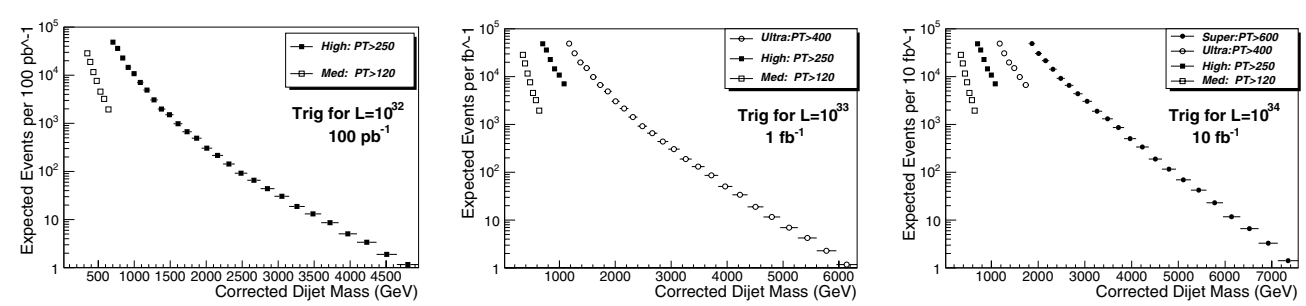

Figure 4.3. Rate of jet trigger for cross section measurement. Same triggers as Fig. 4.1.

In Fig. 4.5 we show the fractional statistical error on the cross section, the simplest measure of our sensitivity to new physics. Figure 4.5 shows that our prescaled triggers will allow a measurement of QCD with 1-3\% statistical accuracy. The unprescaled triggers will have $1 \%$ error at threshold and the first unprescaled sample begins at a mass of $670 \mathrm{GeV} / \mathrm{c}^{2}$, giving us full sensitivity to new physics in a region that overlaps with previous dijet mass measurements at the Tevatron.

\subsubsection{Searches using dijet mass}

Here we will discuss the signal and background distributions that are needed for a dijet resonance search using the mass distribution. In section 14.5.2 we use these techniques to estimate our sensitivity to seven models of narrow dijet resonances. 


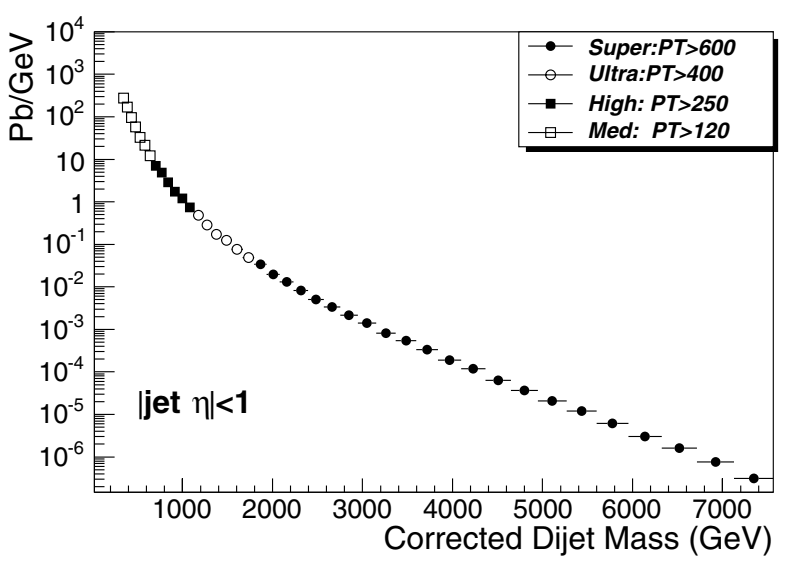

Figure 4.4. Cross section vs. dijet mass and the contributing jet triggers.
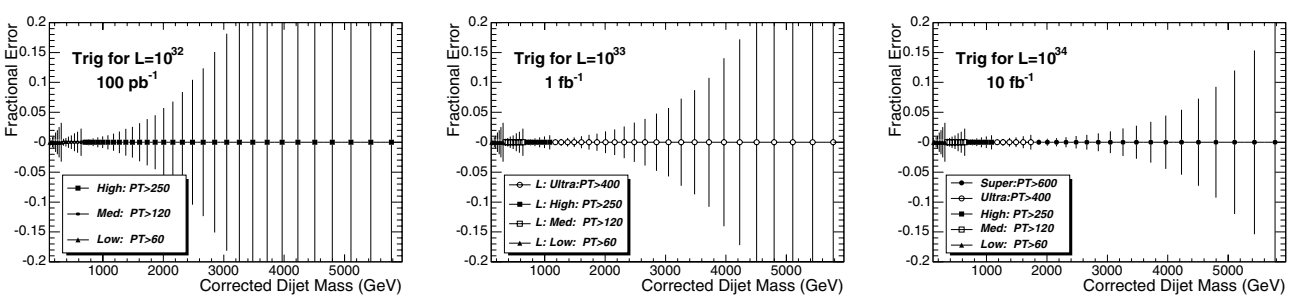

Figure 4.5. Fractional statistical error on the jet cross section for the samples in Fig. 4.5.

4.1.4.1. Narrow dijet resonance shapes. The simulated shape of a narrow dijet resonance in CMS is shown in Figure 4.6. The shape is composed of a Gaussian distribution from jet energy resolution and a long tail to low mass. The measured RMS of the Gaussian component is $\sigma / M=0.045+1.3 / \sqrt{M}$. The long tail to low mass comes predominately from final state QCD radiation (extra jets) which reduce the reconstructed mass. All resonances with a natural width significantly less than our resolution should look similar to this in the CMS detector. The model used in Figure 4.6 was a $Z^{\prime}$ from PYTHIA.

4.1.4.2. QCD background to dijet resonances. Figure 4.6 compares a $Z^{\prime}$ signal cross section to the QCD background found in section 4.1.3. The differential cross section for the QCD background is well fit by a simple parametrisation of the form

$$
\frac{d \sigma}{d m}=\frac{p_{0}(1-m / \sqrt{s})^{p_{1}}}{m^{p_{2}}}
$$

where $m$ is the dijet mass, $\sqrt{s}=14000 \mathrm{GeV} / \mathrm{c}^{2}$ is the collision energy, and $p_{0}, p_{1}, p_{2}$ are arbitrary parameters. The resonance sensitivity estimates in section 14.5 .2 use this parametrisation to smooth away background fluctuations in our simulation sample. In a search with real data, a similar parametrisation could be used to simply model the measured background, as was done by CDF [120], or a full NLO QCD calculation smeared with the jet resolution could be used to model the background, as was done by D0 [121]. 

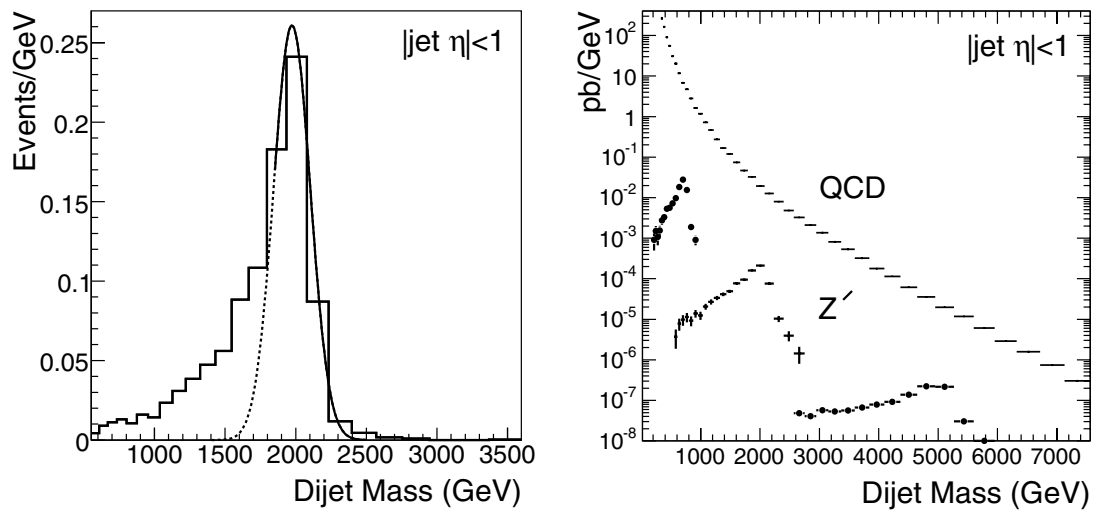

Figure 4.6. (Left) The dijet mass distribution from a $2 \mathrm{TeV} / \mathrm{c}^{2} Z^{\prime}$ (histogram) is fit with a Gaussian (solid curve) from the peak region to high mass and the Gaussian is extended to lower mass (dashed curve). (Right) The differential cross section as a function of dijet mass for the QCD background and three $Z^{\prime}$ signals with a mass of $0.7,2$, and $5 \mathrm{TeV} / \mathrm{c}^{2}$.

\subsubsection{Searches using dijet mass and angle}

Here we will discuss the signal and background distributions that are used for searches for new physics in the dijet mass and angular distribution simultaneously. This technique can be used to confirm resonances observed in the dijet mass distribution, and measure their spin, or to discover other new physics that could affect the dijet angular distribution. In section 15.3 we use these techniques to estimate our sensitivity to a model of quark contact interactions.

4.1.5.1. Dijet ratio: $N(|\eta|<0.5) / N(0.5<|\eta|<1.0)$. The ratio of the number of dijets in which both jets have $|\eta|<0.5$ to the number of dijets in which both jets have $0.5<|\eta|<1.0$ was first introduced by D0 to search for contact interactions as a function of dijet mass [122]. It is the simplest measure of the most sensitive part of the angular distribution, providing a single number we can measure as a function of dijet mass. In Figure 4.7 we show our lowest order calculation of the dijet ratio from QCD compared with a left-handed contact interaction among quarks $[123,124]$ at three different values of the contact interaction scale. For this calculation we used the same code as [125] with modern parton distributions [12]. Lowest order QCD gives a fairly flat dijet ratio around 0.6 while the contact interactions produce an increase in the dijet ratio at high mass. Figure 4.7 also shows that a full CMS detector simulation of the dijet ratio from QCD, using the samples discussed in section 4.1.3, is indistinguishable from a flat ratio of 0.6 within the simulation statistical uncertainty.

\subsubsection{Systematic uncertainties}

In figure 4.8 we present estimates of systematic uncertainties on both the dijet cross section and the dijet ratio. The systematics discussed below have a large effect on the cross section and little effect on the dijet ratio.

4.1.6.1. Absolute jet energy scale. We have concluded that an overall uncertainty on the jet energy scale in the barrel of $\pm 5 \%$ is achievable [126]. We have propagated this energy scale error to the dijet mass cross section by measuring the effect of a $\pm 5 \%$ change in mass on a smooth fit to the dijet mass cross section. As shown in figure 4.8, the resulting upper uncertainty on the cross section varies from $30 \%$ at a dijet mass of $0.3 \mathrm{TeV} / \mathrm{c}^{2}$ to $80 \%$ at 

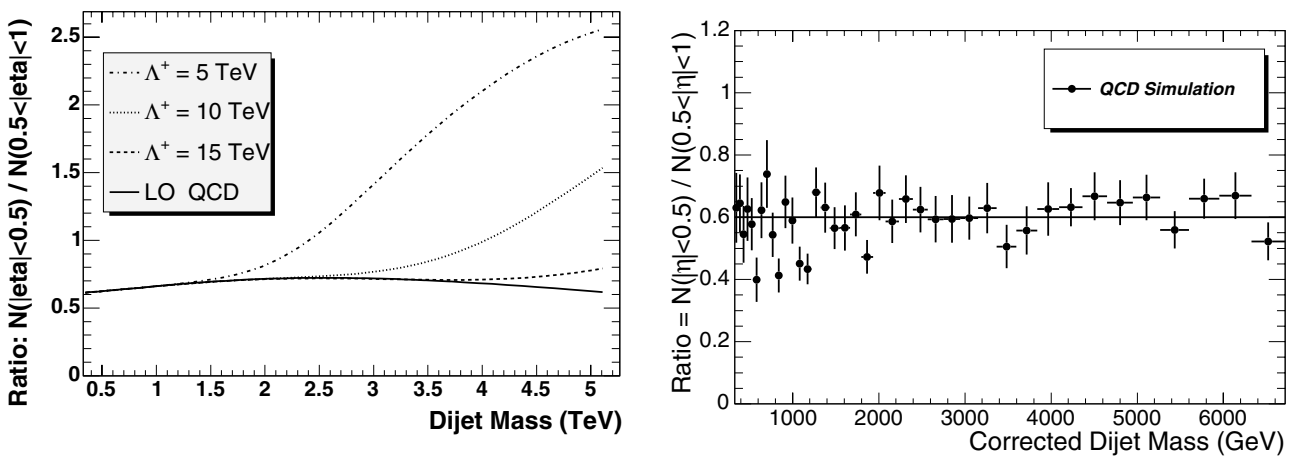

Figure 4.7. (Left) A lowest order calculation of the dijet ratio from QCD (solid curve) is compared with QCD plus a quark contact interaction at a scale $\Lambda^{+}$of $15 \mathrm{TeV}$ (dashed), $10 \mathrm{TeV}$ (dotted) and $5 \mathrm{TeV}$ (dot-dashed). (Right) The dijet ratio in the CMS simulation from QCD (points) is compared to the value 0.6 (line).
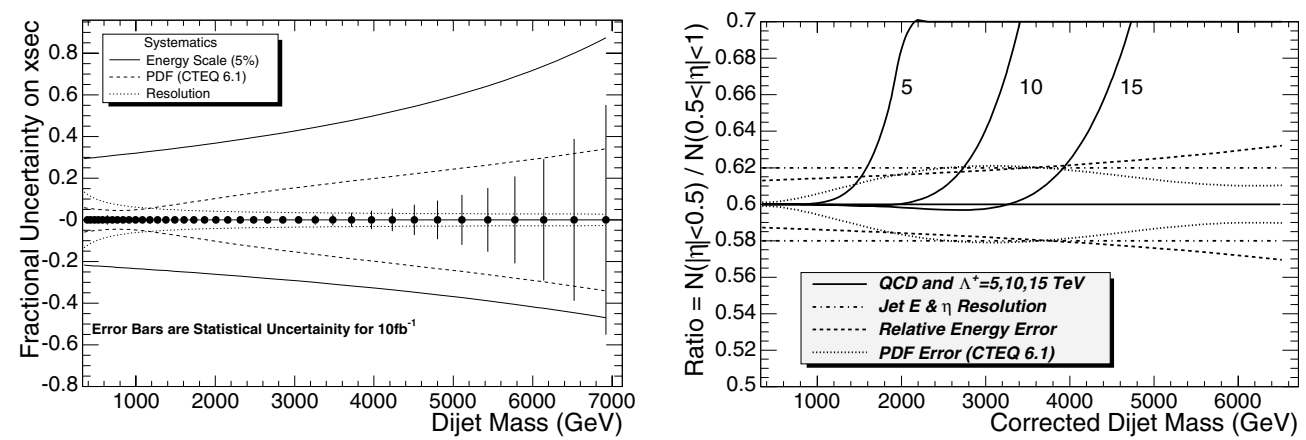

Figure 4.8. (Left) Systematic uncertainty on the dijet cross section due to jet energy scale (solid curve), parton distributions (dashed curve), and calorimeter energy and $\eta$ resolution (dotted curve) are compared to the statistical uncertainties for $10 \mathrm{fb}^{-1}$ (error bars). (Right) Systematic bounds on the dijet ratio from uncertainties in the relative jet energy scale (dashed curve), parton distributions (dotted curve), and calorimeter energy and $\eta$ resolution (dot dash curve), are compared to the expectations of QCD and three contact interaction scales (solid line and curves).

a dijet mass of $6.5 \mathrm{TeV} / \mathrm{c}^{2}$. This large systematic uncertainty, increasing with dijet mass, is the primary reason we do not use the dijet mass distribution to search for quark contact interactions. For the dijet ratio the absolute jet energy scale uncertainty has no effect, because the dijet ratio is flat versus dijet mass. The uncertainty cancels out in the ratio.

4.1.6.2. Relative jet energy scale. We have shown that by using dijet balance an uncertainty of $\pm 0.5 \%$ is achievable [127] for the relative jet energy scale as a function of $\eta$ within the barrel, in 0.1 steps in $\eta$. Here we assume that the relative jet energy scale, defined in this analysis as the uniformity in energy scale in the region $0.5<|\eta|<1.0$ compared to $|\eta|<0.5$, can be determined to $\pm 0.5 \%$. For the cross section as a function of mass this uncertainty is negligible compare to the $\pm 5 \%$ error in the absolute energy scale. We have propagated this error to the dijet ratio by measuring the effect of a $\pm 0.5 \%$ change in dijet mass for the measurement of $N(0.5<|\eta|<1)$ while keeping $N(|\eta|<0.5)$ unchanged. As shown in figure 4.8 , the resulting upper uncertainty in the ratio varies from $0.013(2 \%)$ at a mass of $0.3 \mathrm{TeV} / \mathrm{c}^{2}$ to $0.032(5 \%)$ at a mass of $6.5 \mathrm{TeV} / \mathrm{c}^{2}$. 
4.1.6.3. Resolution. The effect of calorimeter resolution is the difference between the measurement with jets constructed from MC particles (Gen Jets) and the measurement with jets constructed from calorimeter depositions and corrected (Rec Jets). This difference, often called the smearing due to calorimeter resolution, is taken as a bound on the size of the systematic uncertainty due to resolution. For the cross section, the difference between Rec Jets and Gen Jets is small. This smearing varies from $15 \%$ at $0.3 \mathrm{TeV}$ to $3 \%$ at $6.5 \mathrm{TeV}$, as shown in Figure 4.8. For the ratio, there is no change between Gen Jets and corrected Rec Jets within the Monte Carlo statistics presented in Fig. 4.7, and the statistical error on the simulation gives a bound on the systematic of $0.02(3 \%)$ in the ratio, which is shown in Figure 4.8.

4.1.6.4. Parton distributions. We have used these 40 PDFs of CTEQ6.1 and the recommended procedure [12] to calculate the PDF uncertainties on both the cross section and the dijet ratio using our lowest order QCD calculation. As shown in figure 4.8, the resulting upper uncertainty in the cross section varies from $5 \%$ at a dijet mass of $0.3 \mathrm{TeV} / \mathrm{c}^{2}$ to $32 \%$ at a dijet mass of $6.5 \mathrm{TeV} / \mathrm{c}^{2}$. As shown in figure 4.8 , the resulting uncertainty in the dijet ratio peaks at a value of $0.02(3 \%)$ in the ratio at a mass of around $3.5 \mathrm{TeV} / \mathrm{c}^{2}$, and declines at both lower and higher masses.

4.1.6.5. Luminosity, efficiency and acceptance. The luminosity uncertainty on the cross section is around $10 \%$, small compared to other uncertainties, and has no affect on the dijet ratio. For the masses we consider in this analysis there is full efficiency for finding a dijet in the event with negligible uncertainty. The acceptance for jets is defined by the cut in $\eta$, and any measured jet distributions must be compared to calculations using the same $\eta$ cuts, with negligible uncertainty in the comparison of measured and calculated jet $\eta$.

\subsection{Benchmark Channel: low mass supersymmetry}

\subsubsection{Introduction}

$R$-parity conserving SUSY leads to characteristic signatures with missing transverse energy in the final state due to the stable lightest supersymmetric particle (LSP). In the search described below for the bosonic partners of quarks (squarks) and the fermionic partners of gluons (gluinos) it is assumed that the LSP is weakly interacting, as is the case for most of the MSSM parameter space.

This analysis focuses on gluino and squark production within the minimal supergravity model (mSUGRA). In this model the entire SUSY mass spectrum is essentially determined by only five unknown parameters: the common scalar mass at the GUT scale, $M_{0}$; the common gaugino mass at the GUT scale, $M_{1 / 2}$; the common trilinear coupling at the GUT scale, $A_{0}$; the sign of the Higgsino mixing parameter, $\operatorname{sign}(\mu)$; and the ratio of the Higgs vacuum expectation values, $\tan \beta$.

We investigate whether the production and decay of gluinos and scalar quarks is observable in the rate of $\geqslant 3$-jet events with large missing transverse energy. The large missing energy originates from the two LSPs in the final states of the squark and gluino decays. The three or more hadronic jets result from the hadronic decays of the squarks and/or gluinos. We use the ISAJET (7.69) Monte Carlo program interfaced with PYTHIA (6.225) which provides parton shower and an underlying event model to generate squark and gluino production with parameters $M_{0}=60 \mathrm{GeV} / \mathrm{c}^{2}, M_{1 / 2}=250 \mathrm{GeV} / \mathrm{c}^{2}, A_{0}=0, \mu>0$ and $\tan \beta=10$ (LM1 test point). For this set of parameters $m(\tilde{g}) \sim 600 \mathrm{GeV} / \mathrm{c}^{2}, m(\tilde{q}) \sim 550 \mathrm{GeV} / \mathrm{c}^{2},(m(\tilde{g})>m(\tilde{q}))$ and production of $\tilde{g} \tilde{q}$ is $53 \%, \tilde{q} \tilde{q} 28 \%$ and $\tilde{g} \tilde{g} 12 \%$. The decay $\tilde{g} \rightarrow \tilde{q}_{L, R}+q$ is dominant. 
Specifically the gluino and squark decays proceed as follows:

$$
\begin{aligned}
& \tilde{g} \rightarrow q \overline{\tilde{q}}_{L, R}, \quad \text { or } \quad \tilde{g} \rightarrow \bar{q} \tilde{q}_{L, R} \\
& \tilde{q_{R}} \rightarrow q \tilde{\chi}_{1}^{0},(100 \%) \\
& \tilde{q}_{L} \rightarrow q+\tilde{\chi}_{2}^{0},(30 \%) \\
& \tilde{q}_{L} \rightarrow q+\tilde{\chi}_{1}^{+},(70 \%)
\end{aligned}
$$

while the charginos and neutralinos decay as follows:

$$
\begin{aligned}
& \tilde{\chi}_{2}^{0} \longrightarrow \tilde{\ell}_{R} \ell,(11.2 \%) \\
& \tilde{\chi}_{2}^{0} \longrightarrow \tilde{\tau}_{1} \tau,(46 \%) \\
& \tilde{\chi}_{1}^{+} \longrightarrow \tilde{v}_{L} \ell,(36 \%) .
\end{aligned}
$$

The total LO production cross section for squarks and gluinos at this point of the mSUGRA parameter space is $49 \mathrm{pb}$. An example of a SUSY candidate is shown in colour plate CP6. The major Standard Model background components for a multi-jet plus large missing transverse energy search include production of $Z+$ jets with the $Z$ decaying invisibly, $W+$ jets, top-antitop pairs, dibosons, single top and QCD jets.

\subsubsection{Jets and missing transverse energy at CMS}

Jets are defined as localised energy depositions in the calorimeters and are reconstructed using an iterative clustering algorithm with a fixed cone of radius $\Delta R \equiv \sqrt{\Delta \eta^{2}+\Delta \phi^{2}}=0.5$ in $\eta-\phi$ space [7]. Jets are ordered in transverse energy, $E_{\mathrm{T}}=E \sin \theta$, where $E$ is the scalar sum of energy deposited in the calorimeter towers within the cone, and $\theta$ is the angle formed by the beam-line, the event vertex, and the cone centre. Jets with uncorrected $E_{\mathrm{T}}>30 \mathrm{GeV}$ and with $|\eta|<3$ are used throughout this analysis.

The offline missing transverse energy is defined as the negative vector sum of the transverse energy in the electromagnetic and hadronic calorimeter towers, $E_{\mathrm{T}}^{\text {miss }}=$ $-\sum_{i}\left(E_{i} \sin \theta_{i}\right) \hat{n}_{i}$, where $E_{i}$ is the energy of the $i$-th tower, $\hat{n}_{i}$ is a transverse unit vector pointing to the centre of each tower, and $\theta_{i}$ is the polar angle of the tower; the sum extends to $|\eta|<5$. The data sample is selected with a hardware trigger which requires $E_{\mathrm{T}}^{\text {miss, } L 1}>46 \mathrm{GeV}$ $\left(|\eta|<5\right.$ coverage) and a central jet of $E_{\mathrm{T}}>88 \mathrm{GeV}$. A parametrisation of the Level-1 trigger efficiency as measured in a dijet sample is applied to all data analysed. For the confirmation of the High Level Trigger (HLT) the $E_{\mathrm{T}}^{\text {miss }}$ is required to be above $200 \mathrm{GeV}$ where the HLT trigger is fully efficient. In the following sections we detail the methodology and analysis strategies towards a search for SUSY using a dataset of events collected according to the missing transverse energy plus jet Level-1 and HLT trigger path.

\subsubsection{Clean-up requirements}

In anticipation of real data a pre-selection is used to reject accelerator- and detector-related backgrounds (such as beam halo and noise), and cosmic ray events. At least one primary vertex is required in the event and the pre-selection uses the event electromagnetic fraction, $F_{e m}$ (defined as the $E_{\mathrm{T}}$-weighted jet electromagnetic fraction sum over the electromagnetic calorimeter acceptance, $\left|\eta_{d}\right| \leqslant 3.0$ ) and event charged fraction, $F_{c h}$ (defined as the average over the jets ratio of the sum of the $P_{\mathrm{T}}$ of the associated to the jet tracks for jets within $|\eta|<1.7$, over the calorimetric jet transverse energy) to distinguish between real and fake jet events. The pre-selection requirements and their efficiency on the signal are shown in 
Table 4.1. Cleanup pre-selection efficiency.

\begin{tabular}{lccc}
\hline Sample/Requirement & $F_{\text {em }}>0.1$ & $F_{c h}>0.175$ & Both (\%) \\
\hline LM1 & $99.88 \%$ & $91.32 \%$ & $91.24 \%$ \\
\hline
\end{tabular}

Table 4.2. The $E_{\mathrm{T}}^{\text {miss }}+$ multi - jet SUSY search analysis path.

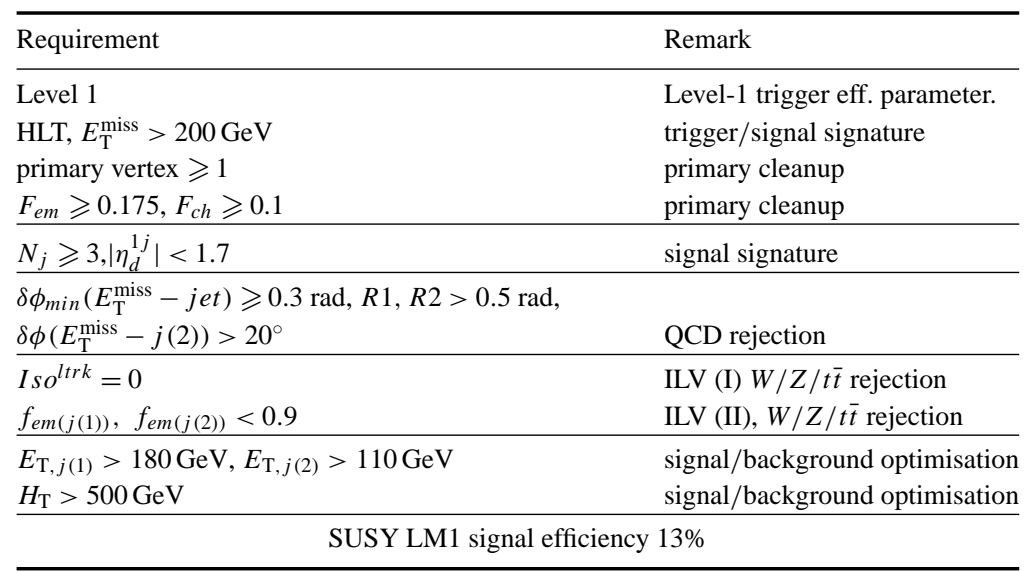

Table 4.1. The values of the requirements are chosen based on the Tevatron data where similar requirements have been used to clean the high $p_{\mathrm{T}}$ multi-jet plus large missing transverse energy datasets from a number of spurious and instrumental backgrounds that tend to appear as spikes in the low end of the event electromagnetic and charge fraction distributions.

\subsubsection{Analysis path}

Events that are accepted by the pre-selection requirements, proceed through the analysis path if they have missing transverse energy $E_{\mathrm{T}}^{\text {miss }}>200 \mathrm{GeV}$ and at least three jets with $E_{\mathrm{T}} \geqslant 30 \mathrm{GeV}$ within $|\eta|<3$. In addition the leading jet is required to be within the central tracker fiducial volume i.e. $|\eta|<1$.7. These requirements directly define the SUSY signal signature. The rest of the analysis path is designed based on elimination of the major classes of backgrounds: the QCD production, top-anti-top pairs and the $W / Z$-QCD associated production. In Table 4.2 the path is shown with a remark indicating the reason and aim of each selection step.

In the following sections the motivation and details of the analysis path are discussed.

\subsubsection{Missing transverse energy in QCD production}

Due the very high QCD production cross section the Standard Model background to a large missing transverse energy plus jets data-sample is dominated by QCD events. The observed missing transverse energy in QCD jet production is largely a result of jet mis-measurements and detector resolution. In Figure 4.9 the missing transverse energy full spectrum is shown for QCD 3-jet events in the $\hat{p_{\mathrm{T}}}$ region between $120 \mathrm{GeV} / \mathrm{c}$ and $1.8 \mathrm{TeV} / \mathrm{c}$.

It is to be noted that due to finite computing resources and the large production cross section it is unrealistic to fully simulate and reconstruct samples with adequate Monte Carlo statistics. It is also unrealistic due to the trigger and data acquisition bandwidth constraints and the large QCD production cross section to collect QCD datasets with low $E_{\mathrm{T}}$ thresholds during data-taking. However the CMS trigger table includes a large number of prescaled 


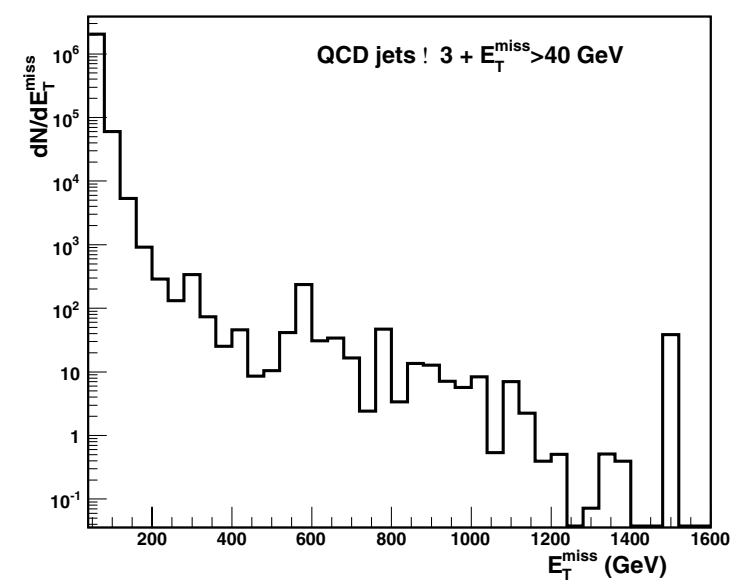

Figure 4.9. $E_{\mathrm{T}}^{\text {miss }}$ distribution in QCD 3 -jet events.
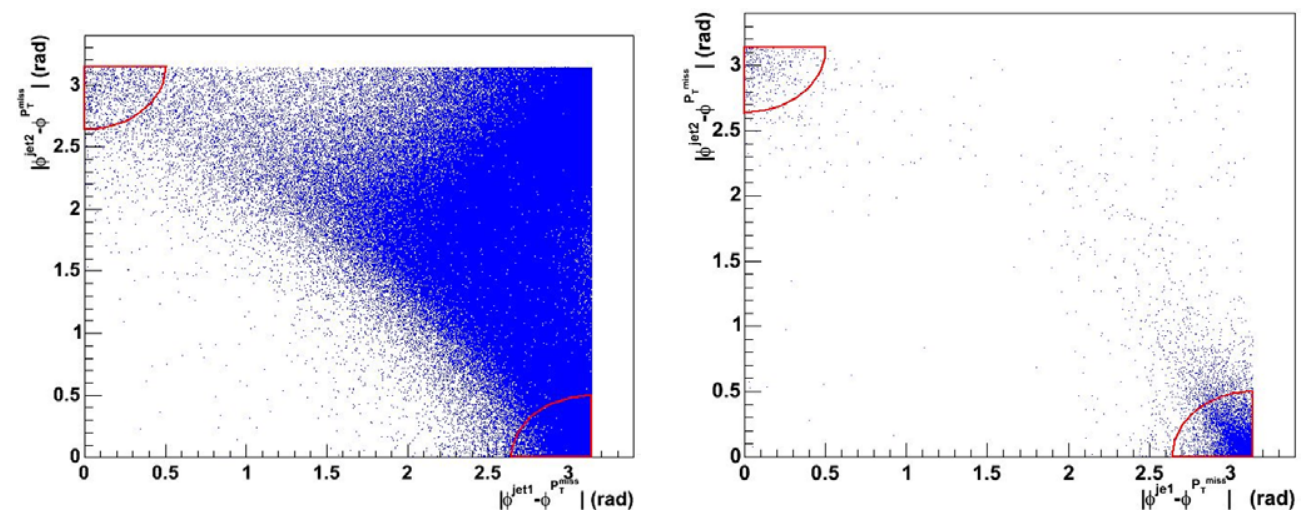

Figure 4.10. $\delta \phi_{1}$ versus $\delta \phi_{2}$ for (left) SUSY signal and (right) QCD dijet events.

QCD trigger paths that will be used to extract the shape of the missing transverse energy and the direct normalisation for the QCD background component in all-hadronic events with large missing energy. In addition, topological requirements are designed to eliminate as much as possible the QCD contribution. Well measured QCD dijet events with back-toback in $\phi$ jet topology are used for obtaining jet corrections. These are well balanced events with low missing transverse energy. Large missing energy in QCD events originates from jet mis-measurements. In such events the highest $E_{\mathrm{T}}$ jet is typically the most accurately measured. When any jet in the event is mis-measured, usually the second or third jet, the $E_{\mathrm{T}}^{\mathrm{miss}}$ direction is pulled close in $\phi$ to the mis-measured jet direction. We eliminate such residual QCD component by using the correlation in the $\delta \phi_{1}=\left|\phi_{\mathrm{j}(1)}-\phi\left(E_{\mathrm{T}}^{\mathrm{miss}}\right)\right|$ versus $\delta \phi_{2}=$ $\left|\phi_{\mathrm{j}(2)}-\phi\left(E_{\mathrm{T}}^{\mathrm{miss}}\right)\right|$ plane, as shown is Figure 4.10. Events with $R_{1}>0.5 \mathrm{rad}$ and $R_{2}>0.5$ $\mathrm{rad}$, where $R_{1}=\sqrt{\delta \phi_{2}^{2}+\left(\pi-\delta \phi_{1}\right)^{2}}$ and $R_{2}=\sqrt{\delta \phi_{1}^{2}+\left(\pi-\delta \phi_{2}\right)^{2}}$, are accepted. In addition we require that no jet in the event be closer than 0.3 rad to the missing energy direction and that the second jet be further than $20^{\circ}$ from it (Figure 4.11).

After a baseline selection of $N_{j} \geqslant 2$ and $E_{\mathrm{T}}^{\text {miss }}>93 \mathrm{GeV}$ the cumulative efficiency of the angular requirements is $\sim 90 \%$ for the SUSY signal. They reject $\sim 85 \%$ of all QCD events. 

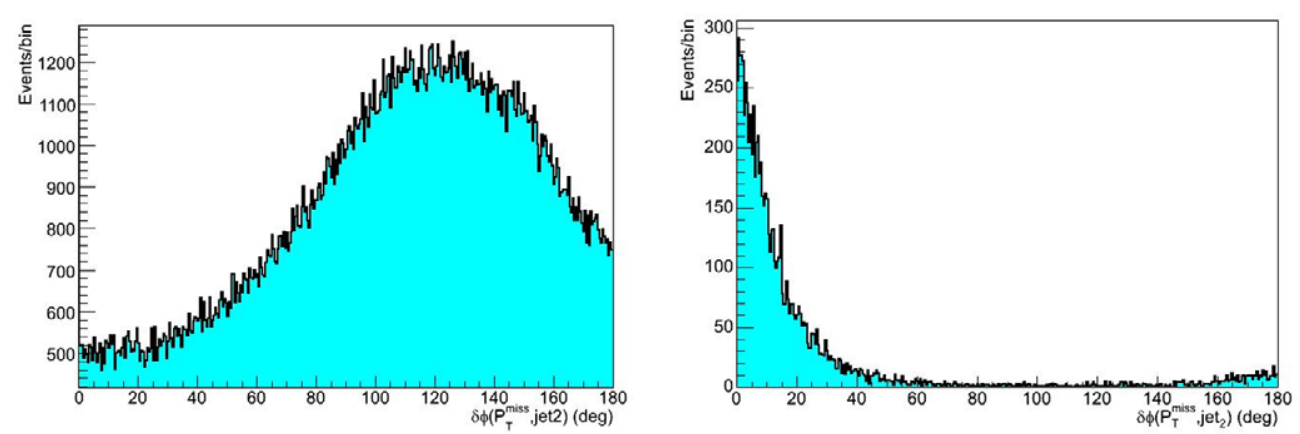

Figure 4.11. $\delta \phi_{2}=\left|\phi_{\mathrm{j}(2)}-\phi\left(E_{\mathrm{T}}^{\mathrm{miss}}\right)\right|$ for (left) SUSY signal and (right) QCD dijet events.

\subsubsection{Indirect Lepton Veto}

$W$ and $Z+$ jet events with large boson $P_{\mathrm{T}}$ and leptonic decays of the boson are backgrounds to a large missing transverse energy plus multijet search. Similarly semileptonic $t \bar{t}$ events where the $W$ boson decays leptonically constitute a background. In the $W$ leptonic decays there is real missing energy due to the neutrino while in the $Z$ decays the missing energy is mostly due to $\tau$ decays or missed leptons. Residual background when the bosons decaying hadronically (with missing energy due to jet mis-measurements) are accounted for using the real multi-jet data triggers.

In this analysis there is no explicit lepton identification. Leptons in the signal SUSY events result from cascade decays of squarks and gluinos through charginos and neutralinos. To reduce the large background contribution mainly from $W(\rightarrow \ell v)+j e t s$ and $t \bar{t}$ production and decays, an indirect lepton veto (ILV) scheme is designed. The aim of the indirect lepton veto is twofold: (a) to retain large signal efficiency and (b) to achieve large rejection of the $W, Z, t \bar{t}$ backgrounds (independent of the MC used, namely parton shower only versus complete matrix element in particular for the higher jet multiplicity bins).

Given that electrons are also clustered as jets, the jet electromagnetic fraction, $f_{e m}$, which is close to 1 for electrons, is efficient in rejecting backgrounds events containing electrons while retaining good efficiency in the LM1 SUSY inclusive signal. Events are selected if the two highest $E_{\mathrm{T}}$ jets are not purely electromagnetic, i.e. $f_{e m, j(1)}<0.9$ and $f_{e m, j(2)}<0.9$. The leading and second jet electromagnetic fraction distributions for $W \rightarrow e v+\geqslant 2$ jets are shown in Figure 4.12. The corresponding distributions for the SUSY LM1 signal are shown in Figure 4.13. The signal efficiency is $\sim 87 \%$ while $90 \%$ of the $W \rightarrow e v+\geqslant 2$ jets are rejected. A systematic uncertainty of $5 \%$ on the background rejection efficiency is assigned due to a variation between PYTHIA and ALPGEN + PYTHIA samples.

To further reject electrons, muons and taus from $W$ and $Z$ decays while retaining the SUSY signal efficiency a tracking isolation strategy is employed as follows: if the leading track in the event has $p_{\mathrm{T}} \geqslant 15 \mathrm{GeV} / \mathrm{c}$ and the ratio of the sum of the $P_{\mathrm{T}}$ of all tracks around it in a cone of $\Delta R=0.35$ over the $p_{\mathrm{T}}$ of the track is less than $10 \%$ the event is dropped. The requirement of accepting events with a non-isolated leading track is noted in Table 4.2 as Iso ${ }^{\text {ltrk }}=0$.

The leading isolated track veto has $\sim 92 \%$ signal efficiency while it rejects $\sim 50 \%$ of the $W / Z+$ jets events (in PYTHIA as well as ALPGEN generated samples). The cumulative $W / Z+$ jets rejection efficiency when both requirements of the indirect lepton veto are applied is between $50 \%$ and $90 \%$ depending on the lepton flavour, with lower rejection as expected when the boson decay product includes a $\tau$ lepton. When applied in the full analyses path it rejects $40 \%$ of $t \bar{t}$ inclusive events. The cumulative SUSY signal efficiency is $\sim 80 \%$. 

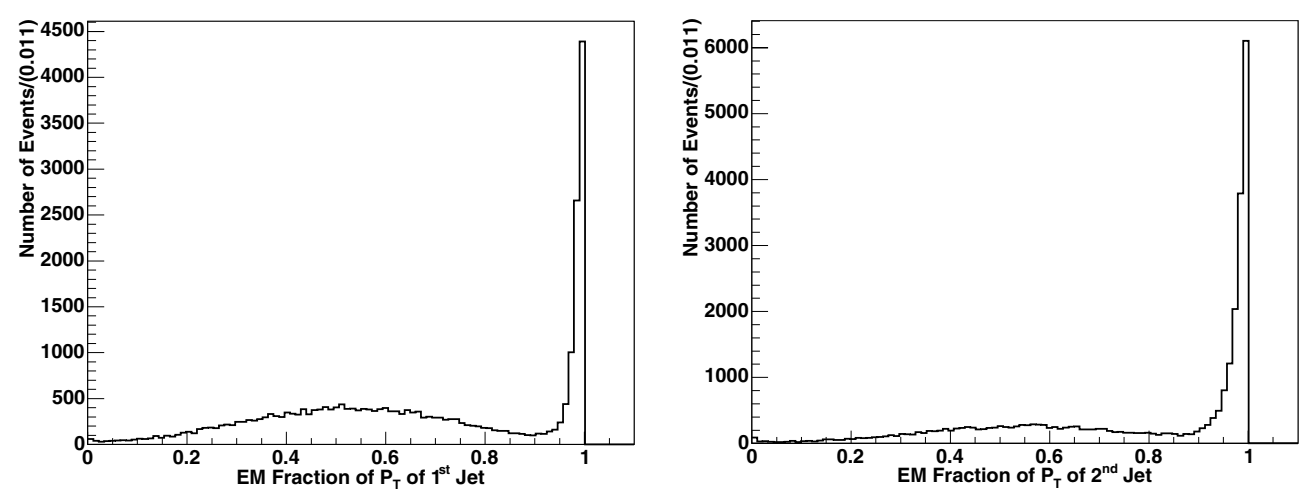

Figure 4.12. Electromagnetic fraction of (left) leading and (right) second jet in $W \rightarrow e \nu+\geqslant 2$ jets events.
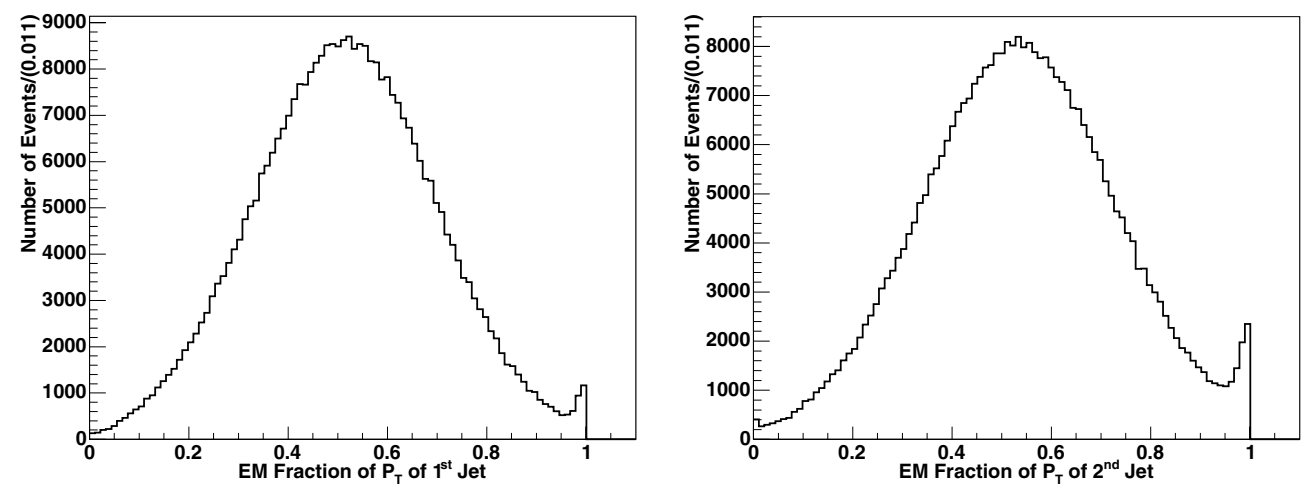

Figure 4.13. Electromagnetic fraction of (left) leading and (right) second jet in SUSY LM1 events.

\subsubsection{The standard $Z$ boson "candle" calibration}

Events with large missing transverse energy and $\geqslant 3$ jets in the final state are expected from $Z(\rightarrow v \bar{v})+\geqslant 3$ jets and $W(\rightarrow \tau v)+\geqslant 2$ jets (the third jet originating from the hadronic $\tau$ decay) processes. Additional residual contribution is expected also from $W(\rightarrow \mu \nu), e v+\geqslant$ 3 jets. In what follows a comprehensive normalisation program is described that relies on the $Z$ + multi-jet data to accurately estimate the $W$ and $Z+$ multi-jet background contribution in a large $E_{\mathrm{T}}^{\text {miss }}$ plus multi-jet search.

The $Z+N$ jets cross section is proportional to $a_{s}^{N}$ : for each additional jet in the $Z$ event the cross section falls by a factor proportional to $a_{s}$. The ratio of the number of events in adjacent jet multiplicity bins should remain constant and be proportional to the strong coupling constant. The multiplicity breakdown will be measured in the data and the slope returned by the exponential fit will be $R=\frac{d N_{\text {events }}}{d N_{\text {jets }}}=\frac{\mathcal{L} d \sigma}{d N_{\text {jets }}}$. This ratio measured as the two to three jet ratio in PYTHIA $W+$ jets and $Z+$ jets is $\sim 2.3$. An illustration of the result of the measurement that will be performed with the real data is shown in Figure 4.14 using the ALPGEN Monte Carlo cross section after parton shower matching.

The Monte Carlo predictions for events with $\geqslant 3$ jets and $Z$ boson $P_{\mathrm{T}}>200 \mathrm{GeV} / \mathrm{c}$ will be normalised to the observed $Z(\rightarrow \mu \mu)+2$ jets data sample (where $Z$ boson 


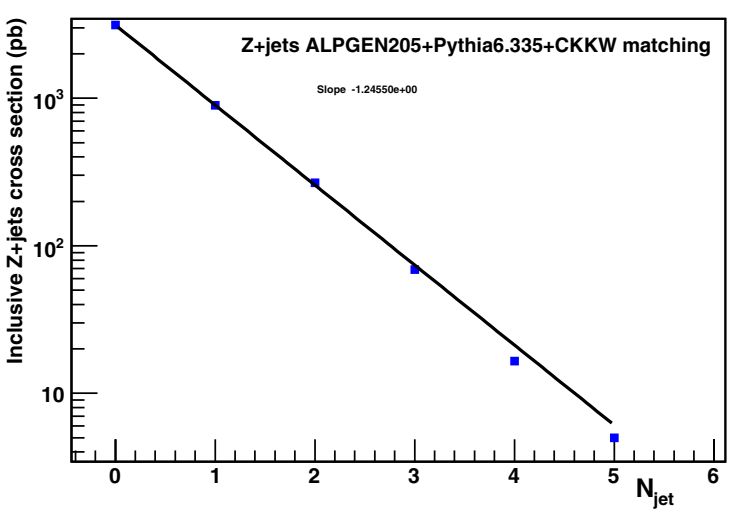

Figure 4.14. Illustration of the measurement of the $R=\frac{d N_{\text {events }}}{d N_{\text {jets }}}$ ratio in the $Z+$ jets data. Here the ALPGEN Monte Carlo cross section is used after parton shower matching and the theoretical returned ratio is 3.8. No $Z$ boson $P_{\mathrm{T}}$ requirement is used for these estimates. Slope $=-1.24550$

$\left.P_{\mathrm{T}}>200 \mathrm{GeV} / \mathrm{c}\right)$ via the measured $R=\frac{d N_{\text {events }}}{d N_{\text {jets }}}$ ratio, where $d N_{\text {events }}$ is the number of events accumulated with $\sim 1 \mathrm{fb}^{-1}$ of data.

The ratio $\rho \equiv \frac{\sigma(p p \rightarrow W(\rightarrow \mu v)+j e t s)}{\sigma\left(p p \rightarrow Z\left(\rightarrow \mu^{+} \mu^{-}\right)+j e t s\right)}$ will be used to normalise the $W+$ jets Monte Carlo predictions. Assuming lepton universality, the predictions for the number of events with $\geqslant 2$ jets and $\geqslant 3$ jets from $W$ and $Z$ production and decays to all flavours will be normalised to the $Z\left(\rightarrow \mu^{+} \mu^{-}\right)+\geqslant 2$ jets data. By normalising the MC predictions to data large systematic effects are avoided that are due to the renormalisation scale, the choice of parton density functions, initial- and final-state radiation, and the jet energy scale. The total uncertainty $(\sim 5 \%)$ is then dominated by the uncertainty on the luminosity measurement, the uncertainty on the measured ratio $R=\frac{d N_{\text {events }}}{d N_{\text {jets }}}$ (to be measured with the data), and the uncertainty on the ratio $\rho$ as a function of the jet multiplicity, $N_{\text {jet }}$.

The method will be used to absolutely normalise the Monte Carlo predictions for $Z(\rightarrow v \bar{v})+\geqslant 3$ jets assuming that after detector simulation they will be tuned to reproduce the kinematic distributions observed in the "candle" data sample and the ratios discussed above. Note that the actual data "candle" sample can be used stand-alone to predict the rate and event kinematics of the $Z(\rightarrow v \bar{v})+\geqslant 3$ jets process.

In this study the $Z \rightarrow \mu \mu+\geqslant 2$ jets with $Z_{p T}>200 \mathrm{GeV} / \mathrm{c}$ is the "candle" data sample. Both the muon and electron decays of the $Z$ will be used as the standardisable candle, but for the purposes of demonstrating the method, the $Z$ muon decays are chosen. The additional advantage of the muon channel is the efficient CMS muon detection due to the tracking and muon systems. Since the completely raw missing transverse energy is used (as is expected to be the case at the start-up of the experiment), the shape of the $E_{\mathrm{T}}^{\text {miss }}$ distribution of the measured the $Z \rightarrow \mu \mu+\geqslant 2$ jet events will be very close to the shape of the invisible $Z \rightarrow \nu \nu+\geqslant 2$ jet events as shown in Figure 4.15. The muon decays of the $Z$ are selected from an inclusive sample using the following requirements as baseline selection: (a) at least one primary vertex, (b) at least 2 jets with $E_{\mathrm{T}} \geqslant 30 \mathrm{GeV}$, and $\left|\eta_{d}\right| \leqslant 3$, (c) $E_{\mathrm{T}}^{\text {miss }}>200 \mathrm{GeV}$ and (d) for the $Z$ boson identification two reconstructed muons with invariant mass closest to the measured $Z$ boson mass $\left(91.2 \mathrm{GeV} / \mathrm{c}^{2}\right)$ and within $20 \mathrm{GeV} / \mathrm{c}^{2}$. The " $Z$-mass" tag requirement is $90 \%$ efficient. The selected candle sample dimuon invariant mass is shown in Figure 4.16 overlaid with the one using the Monte Carlo truth. Considering both the electron and muon decays of the $Z$ boson, a statistically adequate (5\% precision) "candle" sample to normalise the $Z \rightarrow v v+\geqslant 2$ jet predictions for $E_{\mathrm{T}}^{\mathrm{miss}}>200 \mathrm{GeV}$ will be obtained with $\sim 1.5 \mathrm{fb}^{-1}$. 


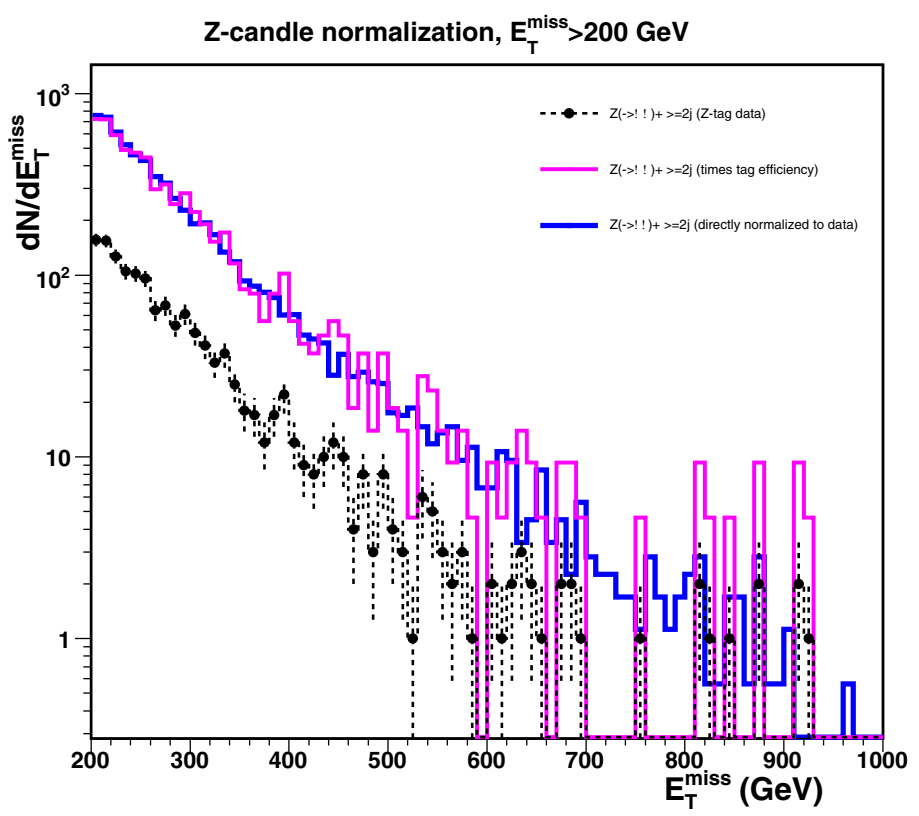

Figure 4.15. $E_{\mathrm{T}}^{\text {miss }}$ in $Z \rightarrow \mu \mu+\geqslant 2$ jets candle sample and normalised $E_{\mathrm{T}}^{\text {miss }}$ in $Z \rightarrow \nu \bar{\nu}+\geqslant$ 2 jets sample.

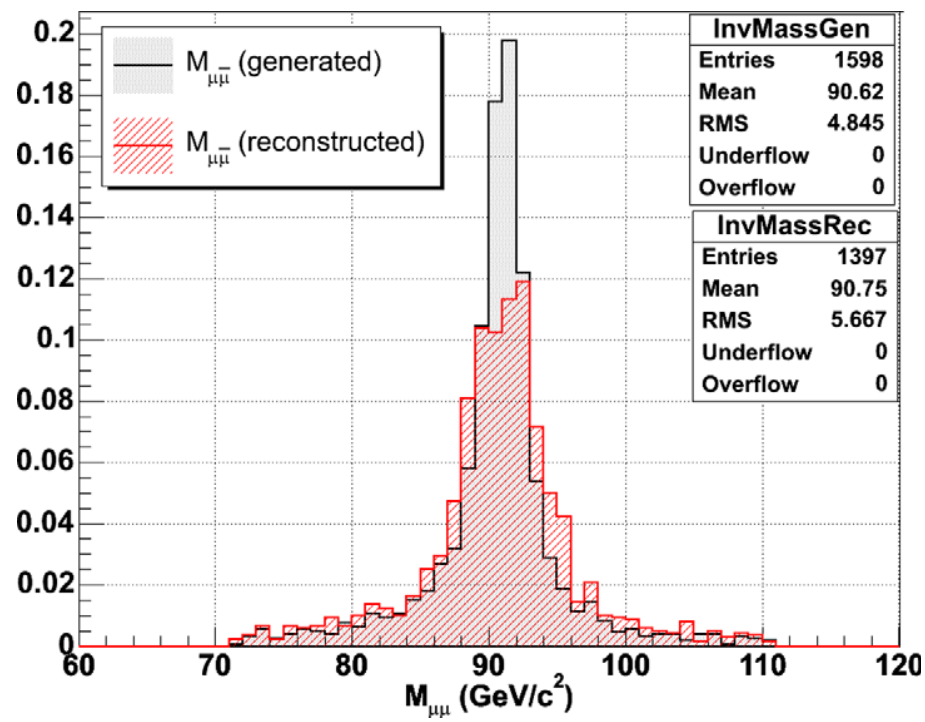

Figure 4.16. Reconstructed and generator level $Z$ dimuon invariant mass for $Z \rightarrow \mu \mu+\geqslant 2$ jets and $E_{\mathrm{T}}^{\mathrm{miss}}>200 \mathrm{GeV}$.

\subsubsection{Analysis results}

The signal to background ratio is further enhanced in the final steps of the analysis (shown in Table 4.2) by requiring the two leading jets $E_{\mathrm{T}}$ be above 180 and $110 \mathrm{GeV}$ respectively. Furthermore the $H_{\mathrm{T}}$ in the event is required to be $H_{\mathrm{T}} \equiv E_{\mathrm{T}(2)}+E_{\mathrm{T}(3)}+E_{\mathrm{T}(4)}+E_{\mathrm{T}}^{\text {miss }}>500 \mathrm{GeV}$. 
Table 4.3. Selected SUSY and Standard Model background events for $1 \mathrm{fb}^{-1}$.

\begin{tabular}{cccccc}
\hline Signal & $t \bar{t}$ & single $t$ & $Z(\rightarrow v \bar{v})+$ jets & $(W / Z, W W / Z Z / Z W)+$ jets & QCD \\
\hline 6319 & 53.9 & 2.6 & 48 & 33 & 107 \\
\hline
\end{tabular}

The global signal efficiency for the analysis is $13 \%$ while the signal to background ratio is $\sim 26$. The results are shown in Table 4.3.

Due to the QCD Monte Carlo limited statistics to derive the QCD background component the analysis path is followed without the topological QCD clean-up requirements and ILV requirements. The estimate is conservative and is based on factorising the clean-up and ILV efficiency and assuming them uncorrelated with the rest of the analysis requirements. A parametrisation of the QCD topological clean-up requirements efficiency as a function of the $E_{\mathrm{T}}^{\mathrm{miss}}$ is used for $E_{\mathrm{T}}^{\mathrm{miss}}>700 \mathrm{GeV}$.

\subsubsection{Systematic uncertainties}

4.2.9.1. $E_{\mathrm{T}}^{\mathrm{miss}}$ shape systematic uncertainty due to tails in the jet resolution. A bootstraplike study is performed to estimate the systematic uncertainty of the $E_{\mathrm{T}}^{\text {miss }}$ due to the non Gaussian tails in the jet resolution. The study uses the inclusive $t \bar{t}$ sample. The events are re-weighed according to a grading of the mis-measured jets, and on a jet-by-jet basis. The grading of a jet being considered mis-measured is derived from the jet resolution shape of jets in three $E_{\mathrm{T}}$ bins. Jets are considered mis-measured when they fall in the non-Gaussian tails of the jet resolution. The event weight is derived using each jet's weight and for three different scenarios that involve one, two or three jets being simultaneously mis-measured and positively contributing to the enhancement of the $E_{\mathrm{T}}^{\mathrm{miss}}$ tail. As an example when one jet is assumed to be undermeasured, $15 \%$ of the events that include the undermeasured jet (as determined by the corresponding resolution curves) are weighted up by up to $15 \%$. A larger weight is assigned to the events with a jet lying on the downward going tail (and depending on the $E_{\mathrm{T}}$ of the jet) thus exaggerating the non-Gaussian jet resolution tail. The further the jet in the event is out on the tail the larger is the weight assigned to it.

The ratio of the $E_{\mathrm{T}}^{\text {miss }}$ distribution resulting from the one, two and three under-measured jets scenarios study over the nominal $E_{\mathrm{T}}^{\mathrm{miss}}$ is shown in Figure 4.17 and it shows graphically the positive systematic uncertainty band as a function of the $E_{\mathrm{T}}^{\mathrm{miss}}$ due to jet tails in the resolution.

The positive systematic uncertainty due to one mis-measured jet in the high $E_{\mathrm{T}}^{\text {miss }}$ tails is estimated over the bins where in the nominal distribution we have enough statistics, namely between 180 and $240 \mathrm{GeV}$ (statistical uncertainty $<5 \%$ ). The result is $8.5 \%$. For the scenario with the two undermeasured jets, and assuming that $50 \%$ of the times the simultaneous undermeasurement results in the overestimate of the $E_{\mathrm{T}}^{\mathrm{miss}}$ the result is $6 \%$ and for the case of the three under-measured jets it is also $6 \%$. We take the weighted average of these three scenarios, namely $7 \%$, as an index of the positive systematic uncertainty due to the tails of the jet resolution in the tails of the $E_{\mathrm{T}}^{\text {miss }}$ above $180 \mathrm{GeV}$. The result in the method presented is bound to overestimate the increase in the tails, since by design positive interference of all under-measured jets in the event is considered (in reality there is some combinatorial compensation in the $E_{\mathrm{T}}^{\text {miss }}$ vector given the jet topology). The ultimate measurement of the shape of the high $E_{\mathrm{T}}^{\text {miss }}$ tails and its systematic should be done using Standard Model candle physics processes in the real data such as the $Z+$ jets and the $t \bar{t}$ data sample. 


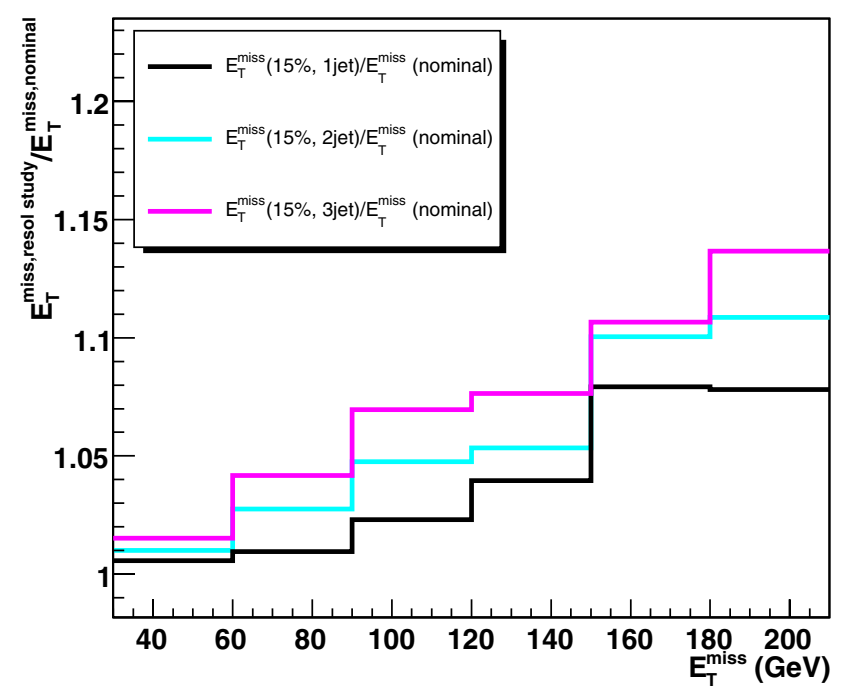

Figure 4.17. Ratio of $E_{\mathrm{T}}^{\mathrm{miss}}$ weighted distribution for one, two and three under-measured jets (described in the text) over the corresponding nominal $E_{\mathrm{T}}^{\mathrm{miss}}$ distribution.

4.2.9.2. Jet energy scale. The jet energy scale (JES) uncertainty in all hadronic analyses is playing an important role since the jet energy spectrum is steeply falling. To determine the effect of the JES uncertainty each jet four-vector is scaled with the uncertainty value $\alpha$ as follow:

$$
\begin{aligned}
p_{\text {scaled }}^{\mu, j e t} & =(1 \pm \alpha) \cdot p_{\text {meas }}^{\mu, \text { jet }} \\
& =(1 \pm \alpha) \cdot\left(p_{x}, p_{y}, p_{z}, E\right)
\end{aligned}
$$

The JES uncertainty for the high $E_{\mathrm{T}}$ jets that enter this analysis is taken to be about $7 \%$ for $1 \mathrm{fb}^{-1}$. The resulting uncertainty in the overall analysis acceptance times efficiency in $t \bar{t}$ and QCD events is $22 \%$.

4.2.9.3. Luminosity uncertainty. Since the $W / Z+$ jets background is taken to be normalised with real data, the estimate carries the luminosity uncertainty on it. Hence a $\pm 5 \%$ uncertainty is taken on the background estimates due to the luminosity measurement.

4.2.9.4. ALPGEN-PYTHIA $I L V$. As discussed in section 4.2 .6 a $5 \%$ positive systematic on the background estimate is taken due to the variation in efficiency of the ILV requirement between ALPGEN and PYTHIA.

4.2.9.5. Total background systematic. In summary for the major background components the uncertainties are as follows:

- $t \bar{t}$ uncertainties: $7 \% E_{\mathrm{T}}^{\text {miss }}$ shape, $22 \% \mathrm{JES}, 13 \%$ statistical.

- $Z \rightarrow v \bar{v}+$ jets, $W / Z+$ jets: $5 \%$ Luminosity (direct candle normalisation to the data).

- QCD: $E_{\mathrm{T}}^{\mathrm{miss}} 7 \%$ shape, $22 \%$ JES, $10 \%$ statistical.

The number of backgrounds events per background component and their uncertainties are tabulated in Table 4.4 . 
Table 4.4. Standard Model background components and uncertainties for $1 \mathrm{fb}^{-1}$

\begin{tabular}{lccc}
\hline$t \bar{t}$, single top & $Z(\rightarrow v \bar{v})+$ jets & $(W / Z, W W / Z Z / Z W)+$ jets & QCD \\
\hline $56 \pm 11($ sys $) \pm 7.5($ stat $)$ & $48 \pm 3.5$ (all) & $33 \pm 2.5$ (all) & $107 \pm 25($ sys $) \pm 10($ stat $)$ \\
\hline
\end{tabular}
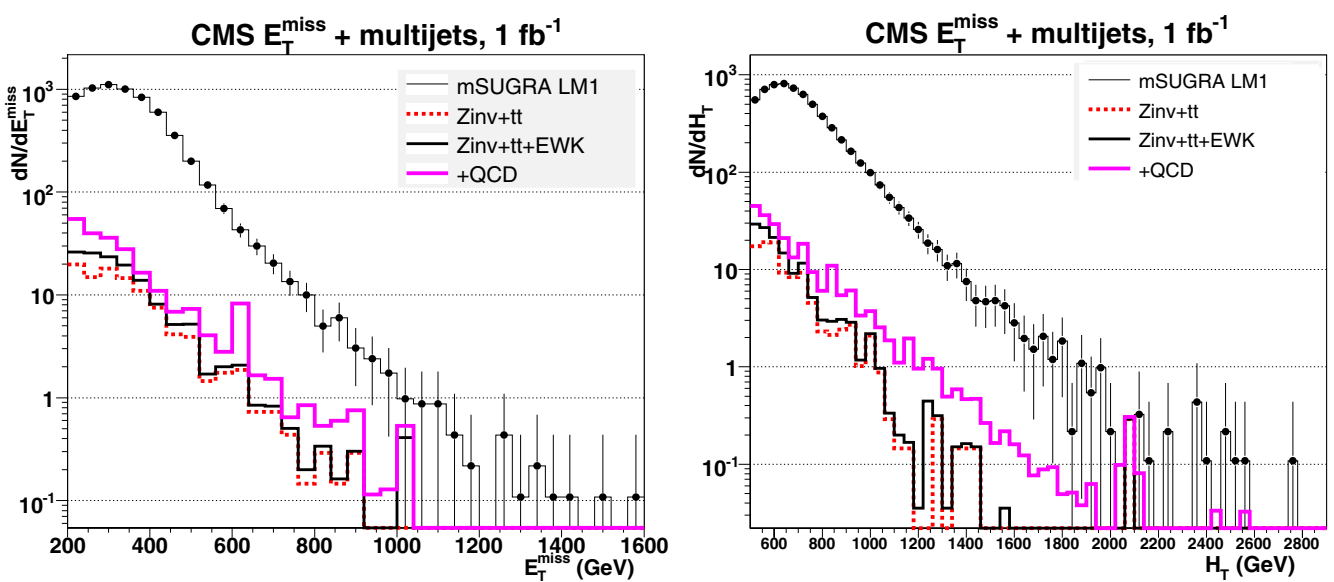

Figure 4.18. LM1 signal and Standard Model background distributions for $E_{\mathrm{T}}^{\text {miss }}$ (left) and $H_{\mathrm{T}}$ (right).

\subsubsection{Discussion}

In conclusion, based on the Standard Model background estimates and their uncertainties, a $5 \sigma$ observation of low mass SUSY at LM1 (gluino mass $600 \mathrm{GeV} / \mathrm{c}^{2}$ ) is in principle achievable with $\sim 6 / \mathrm{pb}$ in events with large missing energy plus multi-jets. It is found that with $\sim 1.5 \mathrm{fb}^{-1}$ the $W / Z+$ jets background including the invisible decays of the $Z$ boson which constitutes a large irreducible background component can be reliably normalised using the $Z \rightarrow \mu \mu$ and $Z \rightarrow e e+$ multi-jet data candle. With adequate data-based strategies of controlling and estimating the Standard Model backgrounds and their uncertainties, low mass SUSY will be discovered with $0.1-1 \mathrm{fb}^{-1}$. Furthermore the global raw $E_{\mathrm{T}}^{\text {miss }}$ measurement from the calorimeter towers can be calibrated for multi-jet topologies using the tracking and muons systems and the $Z \rightarrow \mu \mu+$ multi-jet candle data sample. This analysis demonstrates that the $E_{\mathrm{T}}^{\text {miss }}$ measurement from the calorimeter towers can be used as such at the startup of the experiment provided that adequate strategies are in place to discard spurious instrumental backgrounds. It is also found that an indirect lepton veto makes possible the $t \bar{t}$ and $W / Z+$ jets background rejection, without compromising the inclusive nature of the search. In anticipation of data, there is no accurate way of accurately predicting the contribution of the QCD background tails; although the full matrix element Monte Carlo predictions (such as ALPGEN) are to date far more complete, the experiment has in place proper prescaled QCD triggers in order to estimate this background component using directly the data.

Finally the comparison of the signal, total background estimated and its components for the $E_{\mathrm{T}}^{\text {miss }}, H_{\mathrm{T}}, N_{j e t}$ and $M_{\text {eff }} \equiv E_{\mathrm{T}(1)}+E_{\mathrm{T}(2)}+E_{\mathrm{T}(3)}+E_{\mathrm{T}(4)}+E_{\mathrm{T}}^{\text {miss }}$ are shown in Figure 4.18. It is to be underlined that the slopes of the tails of the missing energy, $H_{\mathrm{T}}$, and $M_{e f f}$ distributions are very similar between the Standard Model background and the low mass SUSY signal. 

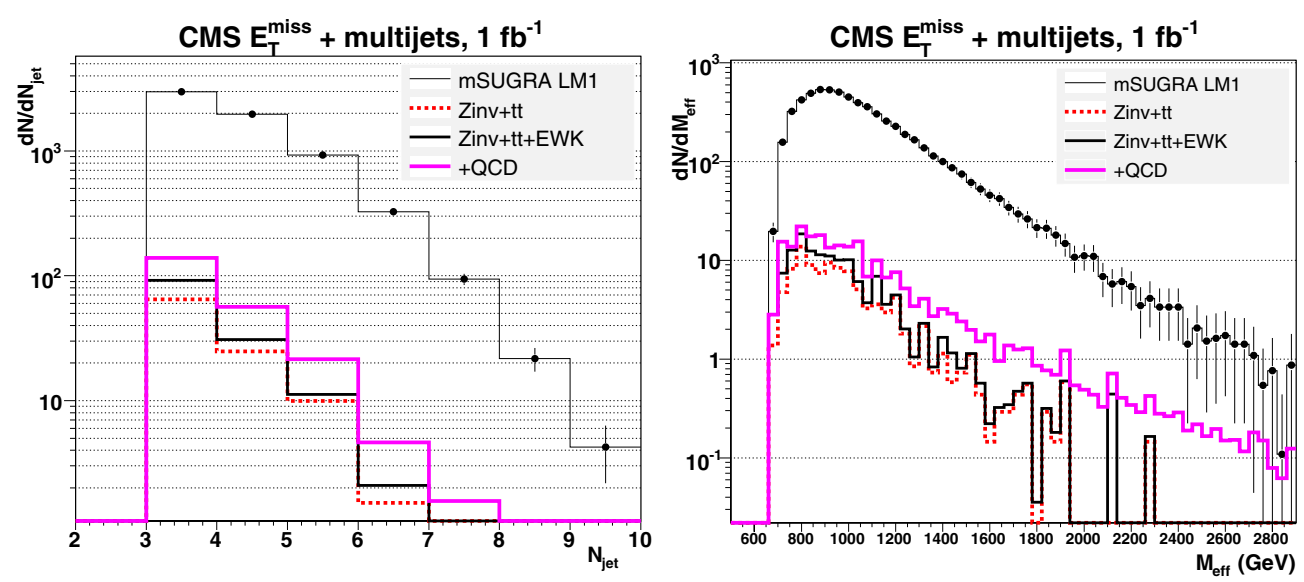

Figure 4.19. LM1 signal and Standard Model background distributions for Jet Multiplicity (left) and $M_{\text {eff }}$ (right).
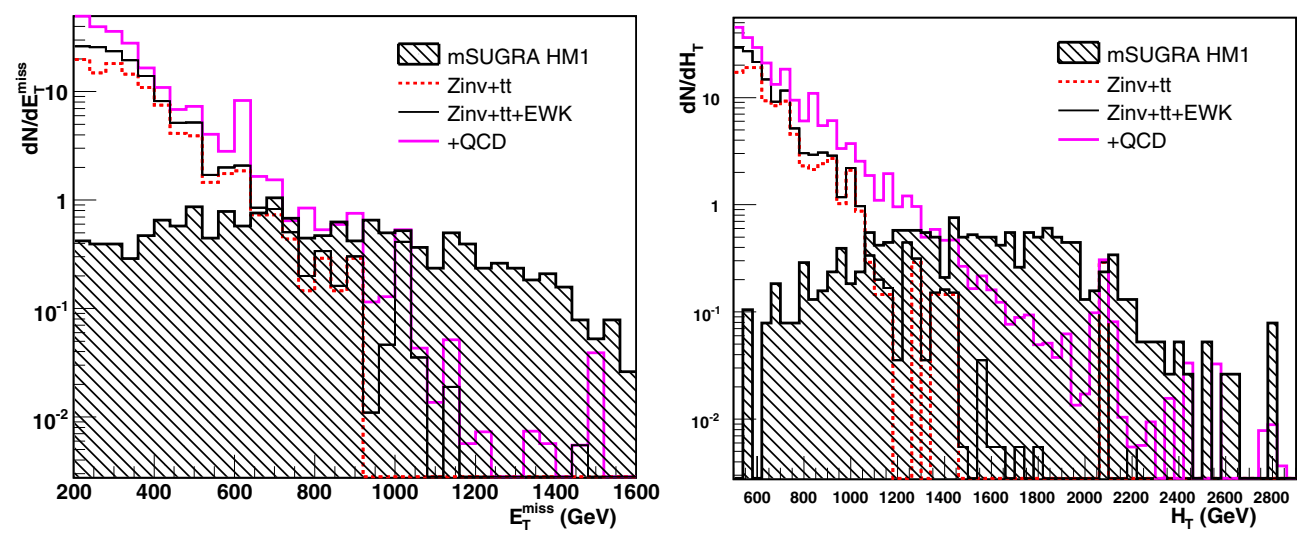

Figure 4.20. HM1 signal and Standard Model background distributions $\left(1 \mathrm{fb}^{-1}\right)$ for $E_{\mathrm{T}}^{\text {miss }}$ (left) and $H_{\mathrm{T}}$ (right).

Applying the analysis in the high mass SUSY test point HM1 (with parameters $M_{0}=180 \mathrm{GeV} / \mathrm{c}^{2}, M_{1 / 2}=850 \mathrm{GeV} / \mathrm{c}^{2}, A_{0}=0, \mu>0$ and $\tan \beta=10$ ) where $m(\tilde{g}) \sim 1890$ $\mathrm{GeV} / \mathrm{c}^{2}, m(\tilde{q}) \sim 1700 \mathrm{GeV} / \mathrm{c}^{2}$ the signal efficiency is $28 \%$. The $E_{\mathrm{T}}^{\text {miss }}$ and $H_{\mathrm{T}}$ distributions comparison between the HM1 SUSY signal and Standard Model backgrounds are shown in Figure 4.20. To perform a SUSY reach scan over the mSUGRA parameter space the optimised analysis requirements for high mass SUSY are used with $E_{\mathrm{T}}^{\text {miss }}>600 \mathrm{GeV}$ and $H_{\mathrm{T}}>1500 \mathrm{GeV}$ (cf. section 13.5). 


\section{Chapter 5. Physics Studies with Tracks, $B$ mesons, and taus}

\subsection{Benchmark Channels: study of the decay $B_{s} \rightarrow J / \psi \phi$}

\subsubsection{Introduction}

The decay $B_{s}^{0} \rightarrow J / \psi \phi \rightarrow \mu^{+} \mu^{-} K^{+} K^{-}$is of particular interest, since it allows to study many properties of the $B_{s}^{0}$ system, such as the differences between the widths and the masses of the two weak eigenstates, $B_{s}^{H}$ and $B_{s}^{L}$. Contrary to the $B^{0}$ system, the difference between the widths $\Delta \Gamma_{s}$ of the two weak eigenstates is expected to be large, with a relative difference $\Delta \Gamma_{s} / \bar{\Gamma}_{s}$ predicted to be in the order of $10 \%$ in the Standard Model. The first measurement from $\operatorname{CDF}\left(\Delta \Gamma_{s} / \bar{\Gamma}_{s}=\left(65_{-33}^{+25} \pm 1\right) \%\right.$ [128] $)$ and the new preliminary result from $\mathrm{D} \emptyset\left(\Delta \Gamma_{s} / \bar{\Gamma}_{s}=\left(15 \pm 10_{-4}^{+3}\right) \%\right.$ [129] $)$ have discrepancies between the two measured values themselves and with the Standard Model prediction. It is only very recently that a first measurement of the mass difference, $\Delta m_{s}$, has been performed at CDF. Time-integrated measurements are not possible, as the time-integrated mixing probability $\chi$ saturates at a value of 0.5 for large mass differences, and in time-dependent measurements, the high mass difference generates very rapid oscillations. As in the $B_{s}^{0}$ system the ratio $\Delta m_{s} / \Delta \Gamma_{s}$ depends on the ratio $\left|V_{c b} V_{c s}\right| /\left|V_{t b} V_{t s}\right|$, which is quite well known, and on QCD corrections, a measurement of $\Delta \Gamma_{s}$ would therefore yield an independent measurement of $\Delta m_{s}$. With the measurement already performed in the $B^{0}$ system, the ratio between the mixing parameters of the $B^{0}$ and $B_{s}^{0}$ could provide a measurement of the ratio $\left|V_{t s}\right| /\left|V_{t d}\right|$.

Furthermore, this decay provides one of the best ways to determine the height of the Unitarity Triangle, $\eta$ in the Wolfenstein parametrisation. At first order of the Wolfenstein parametrisation, the CP-violating weak phase $\phi_{C K M}=\left[\arg \left(V_{c s}^{*} V_{c b}\right)-\arg \left(V_{t s}^{*} V_{t b}\right)\right]$, measured in the rate asymmetry, cancels, and higher order terms have to be taken, yielding a weak phase $\phi_{C K M}=2 \lambda^{2} \eta$. The weak phase is therefore expected to be very small, of the order of 0.03 . The measurement of a significantly larger phase would indicate contributions from non-Standard Model processes.

Because of the relative orbital angular momentum between the decay products, the $J / \psi \phi$ final state is an admixture of CP-even and CP-odd states, and the total rate asymmetry suffers from a partial cancellation. As the $\mathrm{CP}$-even and CP-odd components have different angular dependences, an analysis of the angular correlation of the decay will allow to separate the two states, thereby permitting to access the different parameters.

With a total $B$ production cross section at $\sqrt{s}=14 \mathrm{TeV}$ expected to be as high as $500 \mu \mathrm{b}$, a substantial number of fully reconstructed $B_{s}^{0}$ candidates can be expected. Nevertheless, a high background has to be dealt with. The main sources of backgrounds identified are those containing a $J / \psi$ decaying to two muons susceptible to satisfy the Level-1 trigger requirements.

The decay $B_{s}^{0} \rightarrow J / \psi \phi$ is chosen as a benchmark channel since it is representative of exclusive $B$ physics studies. It allows to study the capability of CMS to identify, select and fully reconstruct the decay of the $B_{s}^{0}$, which presents a significant challenge due to its relatively low momentum and high background. In addition, the measurement of the width difference $\Delta \Gamma_{s}$ on a sample of untagged $B_{s}^{0} \rightarrow J / \psi \phi \rightarrow \mu^{+} \mu^{-} K^{+} K^{-}$candidates using a maximum likelihood fit of the time dependent angular distribution can be attempted. An example of a pp $\rightarrow B_{s}+X$ event with $B_{s} \rightarrow J \psi \phi$ is shown in colour plate CP7.

\subsubsection{Event generation}

In addition to the signal itself, the main backgrounds identified have been simulated with low luminosity pile-up $\left(\mathcal{L}=2 \times 10^{33} \mathrm{~cm}^{-2} \mathrm{~s}^{-1}\right)$. Kinematic requirements were applied in 
order to ensure that a significant fraction of the generated events would fulfil the Level-1 trigger requirements and that the final state particles are within the acceptance of the tracker $(|\eta|<2.5)$. The transverse momentum of the muons is thus required to be above $3 \mathrm{GeV} / \mathrm{c}$ for muons in the barrel $(|\eta|<1.2)$ and $2 \mathrm{GeV} / \mathrm{c}$ elsewhere. For the signal, the momenta of the kaons are required to be above $0.8 \mathrm{GeV} / \mathrm{c}$.

For the samples composed of events with decays of $B$ hadrons, $b \bar{b}$ pairs were generated with PYTHIA 6.215 . The MSEL $=1$ card was used in order to correctly reproduce the three different contributions to the total cross section (parton fusion, flavour excitation, and gluon splitting). The fragmentation of the $b$ quark is performed by PYTHIA and the subsequent decay of the $B$ hadron is performed using the SIMUB generator [130], a dedicated $B$ physics event generator. The decay $B_{s}^{0} \rightarrow J / \psi \phi$ has to be performed with sIMUB, since PYTHIA does not take into account the angular distributions of the final decay products.

One of the $b$ quarks in the event is forced to hadronise to a $B_{s}^{0}$ or $\bar{B}_{s}^{0}$ meson and to decay through the complete decay chain. With the kinematic requirements, using the worldaverage branching ratios for the decays of the $B_{s}^{0}, J / \psi$ and $\phi$ mesons [54], the cross section is predicted to be $\sigma\left(B_{s}^{0} \rightarrow J / \psi \phi \rightarrow \mu^{+} \mu^{-} K^{+} K^{-}\right)=74 \pm 27 \mathrm{pb}$.

The inclusive decays of $B$ hadrons to final states with a $J / \psi$ resonance are expected to be the most important background for the measurement. These were simulated using PYTHIA, since no detailed simulation of angular distributions of the final decay products is needed. In order to increase the number of events similar to the signal events, a pair of oppositely charged particles with $p_{\mathrm{T}}>0.5 \mathrm{GeV} / \mathrm{c}$ and $|\eta|<2.5$ forming a fake $\phi$ candidate is required in a region $(|\Delta \eta|<1.5,|\Delta \varphi|<1.5)$ around the $J / \psi$ direction and with an invariant mass within $30 \mathrm{MeV} / \mathrm{c}^{2}$ of the world-average $\phi$ mass. In addition, this fake $\phi$ candidate is required to form a fake $B_{s}^{0}$ candidate with an invariant mass within $300 \mathrm{MeV} / \mathrm{c}^{2}$ of the world-average $B_{s}^{0}$ mass. The cross section, including the kinematic requirements and branching-fractions, is estimated to be $\sigma(b \rightarrow J / \psi X)=3.20 \pm 0.3 \mathrm{nb}$.

Furthermore, a sample of $B^{0} \rightarrow J / \psi K^{* 0} \rightarrow \mu^{+} \mu^{-} K^{+} \pi^{-}$events were simulated, since this final state can be misidentified as a $B_{s}^{0} \rightarrow J / \psi \phi$ decay. In addition, this decay has a similar differential decay rate $[131,132]$ to the studied $B_{s}^{0}$ decay. The $B^{0}$ decay is simulated with SIMUB, where one of the $b$ quarks in the event is forced to hadronise to a $B^{0}$ or $\bar{B}^{0}$ meson, and to decay through the complete decay chain. With the kinematic requirements, and using the world-average branching ratios, the cross section is predicted to be $\sigma\left(B^{0} \rightarrow J / \psi K^{* 0} \rightarrow\right.$ $\left.\mu^{+} \mu^{-} K^{+} \pi^{-}\right)=366 \pm 22 \mathrm{pb}$.

The uncertainties quoted on the estimates above do not include the uncertainties on the total $b \bar{b}$ cross section at LHC energies, the $b$ fragmentation functions, the transverse momentum distribution of $b$ quarks, and the uncertainties introduced by using the model of $b \rightarrow J / \psi X$ decays in PYTHIA. However, since both the signal and background are proportional to the same $b \bar{b}$ cross section, the signal-to-background ratio is unaffected by the corresponding uncertainty. The parameters used in the simulation of the $B_{s}^{0} \rightarrow J / \psi \phi$ and $B^{0} \rightarrow J / \psi K^{* 0}$ decays are given in Table 5.1.

The direct production of $J / \psi$ mesons is an important background at trigger level. Measurements at the Tevatron [133] have shown that predictions of the colour-singlet model, which is presently the one implemented in the PYTHIA generator, underestimate the measurements by several orders of magnitude. Perturbative QCD is used in this model to generate $c \bar{c}$ pairs, which then hadronise to a charmonium state in a non-perturbative way.

The observed discrepancy has led to a different approach [134], which has been implemented in a modified version of PYTHIA 6.225, tuned on Tevatron data. A $c \bar{c}$ pair is first formed taking into account all perturbative QCD diagrams, regardless of the final colour 
Table 5.1. Values used for the mixing parameters, decay amplitudes, strong and weak phases in the simulation of the $B_{s}^{0} \rightarrow J / \psi \phi$ and $B^{0} \rightarrow J / \psi K^{* 0}$ Monte Carlo sample.

\begin{tabular}{lll}
\hline Parameter & $B_{s}^{0} \rightarrow J / \psi \phi$ & $B^{0} \rightarrow J / \psi K^{* 0}$ \\
\hline$\tau=1 / \bar{\Gamma}$ & $1.405 \times 10^{-12} \mathrm{~s}$ & $1.528 \times 10^{-12} \mathrm{~s}$ \\
$\Delta \Gamma / \bar{\Gamma}$ & -0.2 & 0 \\
$\Delta m$ & $17.8 \mathrm{ps}^{-1}$ & $0.509 \mathrm{ps}^{-1}$ \\
$\left|A_{0}(0)\right|^{2} / \Gamma$ & 0.570 & 0.570 \\
$\left|A_{\|}(0)\right|^{2} / \Gamma$ & 0.217 & 0.217 \\
$\left|A_{\perp}(0)\right|^{2} / \Gamma$ & 0.213 & 0.213 \\
$\delta_{1}$ & $\pi$ & $\pi$ \\
$\delta_{2}$ & 0 & 0 \\
$\phi$ & -0.04 & 0 \\
\hline
\end{tabular}

state. The $c \bar{c}$ state is then transformed into a colour-singlet by non-perturbative processes, such as the emission of a soft gluon.

This version of PYTHIA has been used to simulate a sample of $J / \psi$ decaying to two muons for background studies. The $J / \psi$ production cross section is calculated to be $141 \mu \mathrm{b}$. Taking the $J / \psi \rightarrow \mu^{+} \mu^{-}$branching ratio and the kinematic requirements into account, a cross section of $310 \pm 5 \mathrm{nb}$ is expected. Only the statistical uncertainty is quoted and used; the large uncertainties on the total cross section for $J / \psi$ production and on the $p_{\mathrm{T}}$ distribution are not included.

\subsubsection{Trigger selection}

5.1.3.1. The Level-1 Trigger. The $B_{s}^{0}$ decay chain is selected at Level-1 by the dimuon trigger stream. At low luminosity it is foreseen [76] to use an identical threshold of $3 \mathrm{GeV} / \mathrm{c}$ on the transverse momentum of each muon, still keeping a low bandwidth occupancy of $0.9 \mathrm{kHz}$. Such a low $p_{\mathrm{T}}$ threshold ensures a very high selection efficiency on this channel, with a rate low-enough to allow the use of lower quality muon candidates in the endcap region, recovering full geometrical acceptance of the muon detector up to $|\eta|<2.4$. For this decay, two of the identified muons are required to have opposite charge.

5.1.3.2. The High-Level Trigger. In the HLT, the signal events are identified by doing a full reconstruction of the $B_{s}^{0}$ decay, imposing invariant mass and vertex constraints. Indeed, at this stage, tracks can be reconstructed in the tracker in restricted $(\eta, \phi)$ regions via a partial reconstruction algorithm, where only the first 5 hits are used [7, Section 6.4.3.2]. To define the tracking regions, the primary (interaction) vertex is first identified and reconstructed using only hits in the Pixel detector, with the "Divisive Method" described in reference [135]. Since the primary vertex of $b \bar{b}$ events involves low momentum tracks, the three vertex candidates with the highest sum of the $p_{\mathrm{T}}^{2}$ of the tracks, which is the default selection criterion, have to be retained in order to achieve a good efficiency.

For the muons, the tracking regions are chosen around the direction of the muons identified at Level-1. Since no link to the muon detectors can be done at this stage, all track pairs of opposite charge for which the invariant mass is within $150 \mathrm{MeV} / \mathrm{c}^{2}$ of the worldaverage $J / \psi$ mass are retained. The resolution on the invariant mass of the $J / \psi$ meson is found to be $51 \mathrm{MeV} / \mathrm{c}^{2}$. In addition, the $p_{\mathrm{T}}$ of each muon is required to be above $2.5 \mathrm{GeV} / \mathrm{c}$ in $|\eta|<1.2$ or $2 \mathrm{GeV} / \mathrm{c}$ in $|\eta| \geqslant 1.2$, and the $p_{\mathrm{T}}$ of the $J / \psi$ candidate above $4 \mathrm{GeV} / \mathrm{c}$. To remove the prompt $J / \psi$ background, the two muon candidates are then fitted to a common 

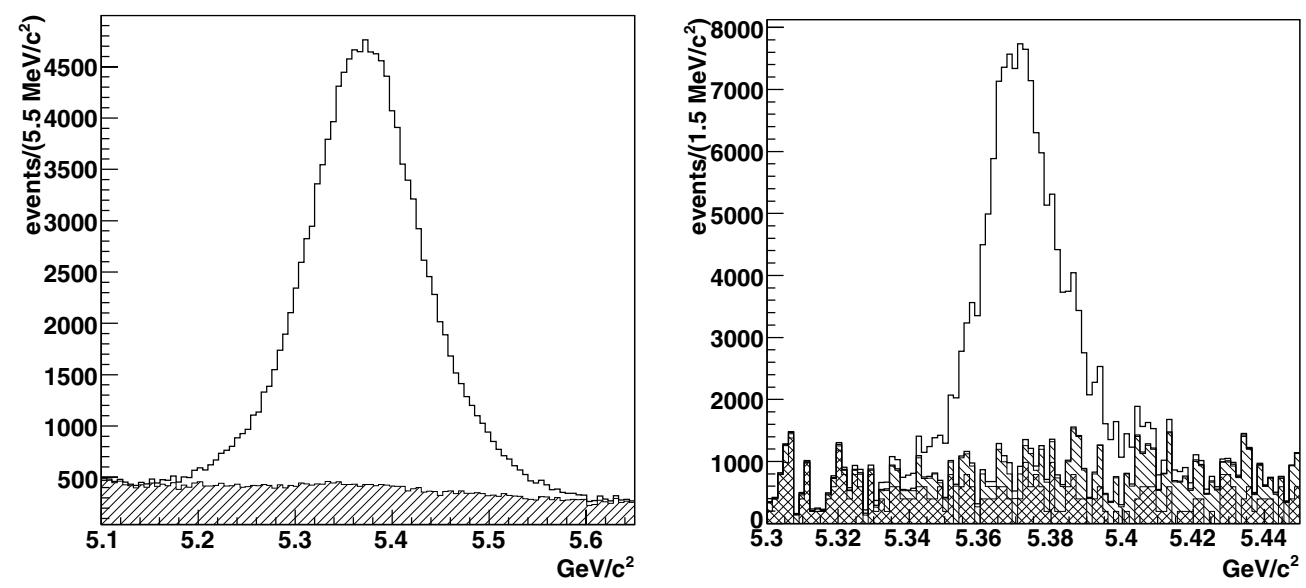

Figure 5.1. Four-track invariant mass distribution after the HLT (left) and offline (right) requirements. The right distribution includes only combinatorial background and the left distribution the expected inclusive $b \rightarrow J / \psi X$ and $B^{0} \rightarrow J / \psi K^{* 0}$ background.

decay vertex. The $\chi^{2}$ of the fit is required to be below 10 and the significance of the transverse decay length is required to be above 3. Furthermore, the transverse momentum of the $J / \psi$ candidate is required to be nearly parallel to its flight path in the transverse plane, since the $J / \psi$ mesons produced in the decays of $B_{s}^{0}$ mesons are collimated around the direction of the $B_{s}^{0}$ meson by the relativistic boost. The cosine of the angle between the reconstructed momentum vector and the vector pointing from the production to the decay vertex is thus required to be larger than 0.9 .

To reconstruct the kaons, a tracking region is chosen around the direction of each $J / \psi$ candidate. Assigning the kaon mass to the reconstructed tracks, all oppositely charged track pairs for which the invariant mass is within $20 \mathrm{MeV} / \mathrm{c}^{2}$ of the world-average mass of the $\phi$ meson are retained, for a resolution on the invariant mass of the $\phi$ meson of $4.5 \mathrm{MeV} / \mathrm{c}^{2}$. The $p_{\mathrm{T}}$ of each of the kaon tracks is required to be above $0.7 \mathrm{GeV} / \mathrm{c}$, the $p_{\mathrm{T}}$ of the $\phi$ candidate above $1 \mathrm{GeV} / \mathrm{c}$ and the $p_{\mathrm{T}}$ of the $B_{s}^{0}$ candidate above $5 \mathrm{GeV} / \mathrm{c}$. With the two muon candidates, the four-track invariant mass is required to be within $200 \mathrm{MeV} / \mathrm{c}^{2}$ of the worldaverage mass of the $B_{s}^{0}$ meson. The resolution on the invariant mass of the $B_{s}^{0}$ meson is found to be $65 \mathrm{MeV} / \mathrm{c}^{2}$. Here as well, a vertex fit of the four tracks is performed, imposing similar requirements as above.

The distribution of the invariant mass of the candidates after the HLT requirements is shown in Figure 5.1 (left). The efficiencies for the different criteria, which include the respective reconstruction efficiencies, are given in Table 5.2 for the signal and the different background samples, together with the estimated rate. The total rate for this selection is well below $1 \mathrm{~Hz}$, and a yield of approximately 456000 signal events can be expected within $30 \mathrm{fb}^{-1}$ of data.

\subsubsection{Offline selection and reconstruction}

The first step in the offline selection is similar to the HLT selection, with the difference that the complete information from the detector is available. Candidates are reconstructed by combining two muons of opposite charge with two further tracks of opposite charge. As CMS does not possess a particle identification system suitable for this measurement, all 
Table 5.2. Trigger selection efficiencies for the signal and background (defined with respect to the number of generated events) after each requirement, and estimated HLT rate.

\begin{tabular}{lcccc}
\hline Requirement & Signal & \multicolumn{3}{c}{ Background } \\
\cline { 3 - 5 } & $B_{s}^{0} \rightarrow J / \psi \phi$ & Inclusive $b \rightarrow J / \psi X$ & $B^{0} \rightarrow J / \psi K^{* 0}$ & Prompt $J / \psi$ \\
\hline Level-1 & $45.76(6) \%$ & $38.25(13) \%$ & $46.91(13) \%$ & $36.91(12) \%$ \\
HLT- $J / \psi$ selection & $28.69(7) \%$ & $21.91(11) \%$ & $30.28(12) \%$ & $0.65(2) \%$ \\
HLT- $\phi$ selection & $20.50(6) \%$ & $1.23(3) \%$ & $0.961(26) \%$ & $0.0007(7) \%$ \\
HLT rate $(\mathrm{Hz})$ & $0.03034(8)$ & $0.0792(18)$ & $0.0077(2)$ & $0.002(2)$ \\
\hline
\end{tabular}

measured tracks have to be considered as possible kaon candidates, which adds a substantial combinatorial background. At this stage, only loose requirements are applied, which are tightened after a kinematic fit.

First, all muons in the event are reconstructed using the global muon reconstruction algorithm [7, Section 9.1.3]. This algorithm is not fully efficient for low- $p_{\mathrm{T}}$ muons from $J / \psi$ decays, being more suited to the reconstruction of high- $p_{\mathrm{T}}$ muons. Therefore, all tracks are reconstructed with the standard track reconstruction algorithm [7, Section 6.5]. Trackpairs of opposite charge for which the invariant mass is within $120 \mathrm{MeV} / \mathrm{c}^{2}$ of the worldaverage $J / \psi$ mass are retained as a $J / \psi$ candidate. The $p_{\mathrm{T}}$ of each muon is required to be above $3 \mathrm{GeV} / \mathrm{c}$ in $|\eta|<1.2$ or $2 \mathrm{GeV} / \mathrm{c}$ in $|\eta| \geqslant 1.2$, and the $p_{\mathrm{T}}$ of the $J / \psi$ candidate above $4 \mathrm{GeV} / \mathrm{c}$. The muon identification algorithm which uses information from the muon detector [7, Section 9.2.1.2], is applied to both tracks forming the $J / \psi$ candidate. A $J / \psi$ candidate is confirmed if both tracks share more than half of their hits in the silicon tracker with the muon tracks reconstructed by the global muon reconstructor, or if their compatibility score returned by the muon identification algorithm is greater than 0.1 .

To reconstruct the $\phi$ meson, all tracks reconstructed with the standard track reconstruction algorithm are used. Requiring the $p_{\mathrm{T}}$ of each track to be above $0.8 \mathrm{GeV} / \mathrm{c}$ and assigning a kaon mass to the thus reconstructed tracks, all oppositely charged track pairs for which the invariant mass is within $20 \mathrm{MeV} / \mathrm{c}^{2}$ of the world-average mass of the $\phi$ meson are retained. The $p_{\mathrm{T}}$ of the $\phi$ candidate is required to be above $1 \mathrm{GeV} / \mathrm{c}$, and the $p_{\mathrm{T}}$ of the $B_{s}^{0}$ candidate above $5 \mathrm{GeV} / \mathrm{c}$

A kinematic fit [136] is then made, where the four tracks are constrained to come from a common vertex and the invariant mass of the two muons is constrained to be equal to the mass of the $J / \psi$. Since the natural width of the $\phi$ meson is of the same order as the resolution due to the reconstruction, no mass constraint is applied to the two kaon tracks. With this fit, a resolution on the invariant mass of the $B_{s}^{0}$ meson of $14 \mathrm{MeV} / \mathrm{c}^{2}$ is found. The confidence level of the fit is required to be greater than $1 \times 10^{-3}$ (seven degrees of freedom). The invariant mass of the two kaons is required to be within $8 \mathrm{MeV} / \mathrm{c}^{2}$ of the world-average mass of the $\phi$ meson. Finally, the cosine of the angle between the reconstructed momentum vector of the $B_{s}^{0}$ candidate and the vector pointing from the production to the decay vertex is required to be larger than 0.95 . The distribution of the invariant mass of the candidates after all selection requirements is shown in Figure 5.1 (right).

The primary vertex is not used at this stage, since the efficiency of the standard primary vertex finder [7, Section 6.6.4], which uses all fully reconstructed tracks, is $92 \%$, and drops to $83 \%$ if the vertex is required to be within $500 \mu \mathrm{m}$ from the simulated vertex. In order to prevent this unnecessary loss of efficiency, no use is made of the primary vertex, and all quantities of interest are evaluated in the transverse plane. 
Table 5.3. Offline selection efficiencies for the signal and background (defined with respect to the number of generated events) after each requirement.

\begin{tabular}{lllll}
\hline Requirement & Signal & \multicolumn{3}{c}{ Background } \\
\cline { 3 - 5 } & $B_{s}^{0} \rightarrow J / \psi \phi$ & $b \rightarrow J / \psi X$ & $B^{0} \rightarrow J / \psi K^{* 0}$ & Prompt $J / \psi$ \\
\hline HLT selection & $20.50(6) \%$ & $1.23(3) \%$ & $0.937(14) \%$ & $0.0007(7) \%$ \\
Reconstruction + Basic $p_{\mathrm{T}}$ req. & $18.15(5) \%$ & $0.63(2) \%$ & $0.675(12) \%$ & $0.0007(7) \%$ \\
Muon Identification & $17.89(5) \%$ & $0.585(19) \%$ & $0.636(11) \%$ & $0.0007(7) \%$ \\
Kinematic fit $\chi^{2}$ req. & $16.58(5) \%$ & $0.282(14) \%$ & $0.503(10) \%$ & $0.0007(7) \%$ \\
Pointing constraint & $16.48(5) \%$ & $0.258(13) \%$ & $0.497(10) \%$ & - \\
$\phi$ mass req. & $14.65(5) \%$ & $0.113(13) \%$ & $0.202(10) \%$ & - \\
\hline
\end{tabular}

Table 5.4. Expected cross sections for the signal and background, after each requirement, with number of expected events.

\begin{tabular}{lllll}
\hline & Signal & \multicolumn{3}{c}{ Background } \\
\cline { 3 - 5 } & $B_{s}^{0} \rightarrow J / \psi \phi$ & Inclusive $b \rightarrow J / \psi X$ & $B^{0} \rightarrow J / \psi K^{*} 0$ & Prompt $J \psi$ \\
\hline$\sigma \times$ BR & $2.87 \pm 1.07 \mathrm{nb}$ & $682 \pm 64 \mathrm{nb}$ & $20.4 \pm 1.7 \mathrm{nb}$ & $141 \mu \mathrm{b}$ \\
Kin. preselection & $74 \pm 27 \mathrm{pb}$ & $3.20 \pm 0.3 \mathrm{nb}$ & $366 \pm 22 \mathrm{pb}$ & $176 \pm 2 \mathrm{nb}$ \\
Level-1 & $34 \pm 12 \mathrm{pb}$ & $1.22 \pm 0.11 \mathrm{nb}$ & $172 \pm 10 \mathrm{pb}$ & $65 \pm 1 \mathrm{nb}$ \\
HLT & $15.2 \pm 5.5 \mathrm{pb}$ & $39.4 \pm 3.8 \mathrm{pb}$ & $3.52 \pm 0.21 \mathrm{pb}$ & $1.2 \pm 1.2 \mathrm{pb}$ \\
Offline & $10.9 \pm 4.0 \mathrm{pb}$ & $3.62 \pm 0.54 \mathrm{pb}$ & $0.74 \pm 0.06 \mathrm{pb}$ & - \\
Events per $30 \mathrm{fb}^{-1}$ & 327000 & 108500 & 22200 & - \\
\hline
\end{tabular}

With this selection, a yield of approximately 327000 signal events can be expected within $30 \mathrm{fb}^{-1}$ of data, with a background of 108500 events. The efficiencies for the different criteria, which include the respective reconstruction efficiencies, are given in Table 5.3 for the signal and the different background samples, and the expected cross sections are given in Table 5.4. These do not include a requirement on the four-track invariant mass of the candidates, since the sidebands will be used later in the analysis. However, only a small fraction of these events are directly under the $B_{s}^{0}$ peak, and even a simple cut will reduce the number of background events by a significant factor.

\subsubsection{The maximum likelihood analysis}

The final state of the decay of a pseudo-scalar $B$ meson into two vector mesons $B \rightarrow V_{1} V_{2}$ is an admixture of CP-even and CP-odd states [131,132,137]. The CP-odd states correspond to transitions in which the relative orbital momentum $L$ between the two vector mesons is 1 and the CP-even states to transitions in which $L$ is either 0 or 2 . The amplitude of the decay can be decomposed in three independent decay amplitudes which correspond to the linear polarisation states of the two mesons. The first, $A_{0}$, describes states in which the linear polarisation vectors are longitudinal and is CP-even. The other two describe states in which the linear polarisation vectors are transverse, either parallel $\left(A_{\|}-\mathrm{CP}\right.$-even) or perpendicular $\left(A_{\perp}-\mathrm{CP}\right.$-odd) to each other.

The differential decay rate can be written as:

$$
\frac{\mathrm{d}^{4} \Gamma\left(B_{s}(t)\right)}{\mathrm{d} \Theta \mathrm{d} t}=f(\Theta, \alpha, t)=\sum_{i=1}^{6} O_{i}(\alpha, t) \cdot g_{i}(\Theta),
$$

where $O_{i}$ are the kinematics-independent observables and $g_{i}$ the angular distributions. The set of physical parameters are represented by $\alpha$ and the angles which define the kinematics are 
generically denoted $\Theta$. The time evolution of the different observables is given by bilinear combinations of the polarisation amplitudes, $\left|A_{0}(t)\right|^{2},\left|A_{\|}(t)\right|^{2},\left|A_{\perp}(t)\right|^{2}, \Im\left(A_{\|}^{*}(t) A_{\perp}(t)\right)$, $R\left(A_{0}^{*}(t) A_{\|}(t)\right)$ and $\Im\left(A_{0}^{*}(t) A_{\perp}(t)\right)$. These are functions of the widths of the two light and heavy eigenstates, $\Gamma_{L}$ and $\Gamma_{H}$, the weak phase $\phi_{C K M}$, the magnitudes of the amplitudes at $t=0\left(A_{0}(0), A_{\|}(0)\right.$ and $\left.A_{\perp}(0)\right)$ which describe all hadronisation effects, and, for a flavour-tagged sample, the mass difference $\Delta m_{s}=m_{H}-m_{L}$. Since the overall phase of the polarisation states is not observable, two strong phases are defined as $\delta_{1} \equiv \arg \left|A_{\|}{ }^{*} A_{\perp}\right|$ and $\delta_{2} \equiv \arg \left|A_{0}^{*} A_{\perp}\right|$. These are CP conserving, and are expected to be $0(\bmod \pi)$ in the absence of final-state interactions. Assuming $S U$ (3) flavour-symmetry, the magnitudes and the two strong phases are equal for the decays $B_{s}^{0} \rightarrow J / \psi \phi$ and $B^{0} \rightarrow J / \psi K^{* 0}$ in unmixed samples. The measurement of these parameters is of interest to study and improve the phenomenological models used to calculate all hadronic effects.

In such decays, the kinematics are uniquely defined by a set of three angles. The transversity base is used in this analysis, in which the set of variables is $\Theta=(\cos \theta, \phi, \cos \varphi)$. In this base, $(\theta, \varphi)$ are the polar and azimuthal angles of the momentum of the $\mu^{+}$in the $J / \psi$ rest frame. This coordinate system is defined such that the $\phi$ moves in the positive $x$ direction and the $z$ axis is perpendicular to the decay plane of the decay $\phi \rightarrow K^{+} K^{-}$. The angle $\psi$ is defined in the rest frame of the $\phi$ as the negative cosine of the angle between the $K^{+}$direction and the $J / \psi$ direction.

In order to measure the values of the different parameters, an unbinned maximum likelihood fit is performed on the observed time evolution of the angular distribution. In the absence of background and without distortion, the p.d.f. describing the data would be the original differential decay rate $f(\Theta, \alpha, t)$ (Equation (5.1)). The distortion of this distribution by the detector acceptance, trigger efficiency and the different selection criteria is taken into account by an efficiency term $\varepsilon(t, \Theta)$. In addition, a term describing the background has to be added.

It is assumed that the efficiency can be factorised in two functions, the first modelling the effects of the decay length requirements and the second the distortion of the angular distribution,

$$
\epsilon(t, \Theta)=\epsilon(t) \cdot \epsilon(\Theta) \text {. }
$$

The angular efficiency is described by an expansion of products of spherical harmonics [138]:

$$
\begin{aligned}
& \epsilon(\Theta)=\sum_{L R M} T_{L R M}^{\epsilon} \cdot \mathcal{Y}_{L R M}(\Theta), \\
& \text { with } \mathcal{Y}_{L R M}(\Theta)=\sqrt{2 \pi} \cdot Y_{L M}(\theta, \varphi) \cdot Y_{R M}(\psi, 0),
\end{aligned}
$$

where $\mathcal{Y}_{L R M}$ are orthonormal basis functions and $Y_{L M}, Y_{R M}$ are spherical harmonic functions. In principle, $L$ and $R$ run from 0 to infinity and the sum over $M$ from $-\min (L ; R)$ to $+\min (L ; R)$, but it has been found that the expansion can be limited to $L, R \leqslant 8$. These $\mathcal{Y}_{L R M}$ functions describe the partial waves involved in a scalar $\rightarrow$ vector decay [139]. The moments of the efficiency are determined from a Monte Carlo simulation with full detector simulation:

$$
\begin{aligned}
T_{L R M}^{\epsilon} & =\int \epsilon(\Theta) \cdot \mathcal{Y}_{L R M}^{*}(\Theta) \mathrm{d} \Theta \\
& \approx \frac{1}{N_{\text {gen }}} \sum_{i=1}^{N_{\text {obs }}} \frac{1}{f\left(\Theta_{i}\right)} \mathcal{Y}_{L R M}^{*}\left(\Theta_{i}\right),
\end{aligned}
$$

where $f\left(\Theta_{i}\right)$ is the expected time-integrated angular distribution (Equation (5.1)). 
The time-dependent efficiency describes mainly the effects of the requirements on the proper decay length distribution. After the initial turn-on and a stable plateau, a deficit of events can be observed. Initial studies attribute this decrease in efficiency to the restrictions imposed on the seeds by the tracking regions in the HLT, which cause an additional track reconstruction inefficiency for displaced tracks such as those originating from $B$ decays. The tolerance on the transverse and the longitudinal direction imposed on the tracking regions in the HLT results in an implicit cut on the impact parameters. Further studies are needed to find solutions to alleviate this inefficiency. Without corrections, the main effect of this inefficiency would be to lower the estimated lifetime of the longer-lived eigenstate $B_{s}^{H}$.

The different features in this distribution cannot easily be described by a simple function. Two sigmoidal functions combined with a quadratic function are used to describe the efficiency:

$$
\epsilon(t)= \begin{cases}c \cdot\left(1+\tanh \left(\frac{t-t_{0}}{\Delta t_{1}}\right)\right) & t<t_{0} \\ \left(a \cdot t^{2}+b \cdot t+c\right) \cdot\left(1+\tanh \left(\frac{t-t_{0}}{\Delta t_{2}}\right)\right) & t>t_{0} .\end{cases}
$$

The parameters are found by fitting this function to the distribution obtained by the full Monte Carlo simulation.

The best way to gauge our ability to account for all effects and our capacity to correct them through this time-dependent efficiency curve is by comparing the proper time distributions foreseen by the simulation and observed in the data for the different $B$ mesons. The first obvious choice is again the decay $B^{0} \rightarrow J / \psi K^{* 0}$, which is very similar to the studied $B_{s}^{0}$ decay, and for which the lifetime has been measured with a high precision. Any discrepancy between the efficiency determined by Monte Carlo and the data will be reflected in a mismeasurement of the $B^{0}$ lifetime. Further studies would be needed to determine the sensitivity of the efficiency on the lifetime of the selected $B$ meson. It is dubious whether the number of $B_{s}^{0}$ events recovered in other trigger streams such as the dimuon stream, which has no decay length requirement, would be enough to estimate the time-dependent efficiency.

The background can be divided in two different types of distributions. The first type arises from misidentified $B^{0} \rightarrow J / \psi K^{* 0} \rightarrow \mu^{+} \mu^{-} K^{+} \pi^{-}$events, which has a similar differential decay rate $[131,132]$ to the decay of interest. The width difference of the two eigenstates of the $B^{0}$ are assumed to be negligible, and no CP violation is present since the final state is flavour specific. To describe this background in the dataset, it is not possible to use its time dependent angular distribution, which is in principle well known, since all variables are mismeasured because of the misidentification of the $\pi$. In addition, the distortion of the distribution due to the various requirements is much more severe than in the case of the $B_{s}^{0}$. Indeed, due to its lower mass, the momentum of the $\pi$ in the laboratory frame is lower than that of the corresponding $K$ when the $\pi$ is emitted in the direction opposite to the momentum of the $K^{* 0}$.

The same set of functions $\mathcal{Y}_{L R M}(\Theta)$ (Equation (5.4)) is used to model the angular distribution $f_{d}(\Theta)$ of this background, with the moments computed in the following way:

$$
\begin{aligned}
T_{L R M}^{b} & =\int b(\Theta) \cdot \mathcal{Y}_{L R M}^{*}(\Theta) \mathrm{d} \Theta \\
& \approx \frac{1}{N_{b}} \sum_{i=1}^{N_{b}} \mathcal{Y}_{L R M}^{*}\left(\Theta_{i}\right) .
\end{aligned}
$$

Here as well, the expansion is done up to $L, R \leqslant 8$. The functions are obtained by a Monte Carlo simulation and can be cross-checked by a fully reconstructed sample of well-identified $B^{0} \rightarrow J / \psi K^{* 0}$ decays misreconstructed as $B_{s}^{0}$ candidates. 
The time dependence of this background is modelled as a single exponential decay, again with a time-dependent efficiency. The lifetime $\tau_{d}$ is left as a free parameter, since the mismeasurement of the proper decay length precludes using the well-measured lifetime of the $B^{0}$.

The other sources of background are assumed to have no angular dependence. The distribution of their proper decay time is modelled by two exponential decays, the first describing the short-lived prompt background and the second misidentified long-lived heavy-flavour hadrons.

A better separation of the signal and background is obtained by using the events in a wider invariant mass region between 5.219 and $5.559 \mathrm{GeV} / \mathrm{c}^{2}$, and including in the fit the distribution of the invariant mass of the candidates. The distribution of the $B_{s}^{0}$ candidates is modelled by a Gaussian $G_{s}\left(m ; m_{s}, \sigma_{s}\right)$, where $m_{s}$ is the mass of the $B_{s}^{0}$ meson and $\sigma_{s}$ the variance due to the reconstruction. The distribution of the misidentified $B^{0} \rightarrow J / \psi K^{* 0}$ decays can reasonably well be modelled in the chosen region by a Gaussian $G_{d}\left(m ; m_{d}, \sigma_{d}\right)$. Because of the misidentification of the pion, $m_{d}$ will not correspond to the true mass of the $B^{0}$ meson, and will be left as a free parameter in the fit. The other sources of background are assumed to have a flat mass distribution and will be modelled by a linear function $L(m)$.

The total p.d.f. to be fit is thus given by

$$
\begin{aligned}
\mathcal{P}= & \left(1-b_{d}-b_{c}\right) \cdot \epsilon(t, \Theta) \cdot f(\Theta, \alpha, t) \cdot G_{s}\left(m ; m_{s}, \sigma_{s}\right) \\
& +b_{d} \cdot f_{d}(\Theta) \cdot \epsilon(t) \cdot \frac{1}{\tau_{d}} e^{-t / \tau_{d}} \cdot G_{d}\left(m ; m_{d}, \sigma_{d}\right) \\
& +b_{c} \cdot \epsilon(t) \cdot\left(\frac{1}{\tau_{c l}} e^{-t / \tau_{c s}}+\frac{1}{\tau_{c l}} e^{-t / \tau_{c l}}\right) \cdot L(m),
\end{aligned}
$$

where $b_{d}$, respectively $b_{c}$, are the fraction of misidentified $B^{0}$ background, respectively combinatorial background, in the sample. These parameters are left free in the fit. The resolution of the proper decay length is taken into account by convolving the p.d.f. with a Gaussian resolution function. The standard deviation of the Gaussian is taken as the uncertainty of each candidate's proper decay length measurement multiplied by a scale factor, which is left free in the fit. Since the uncertainties of the measured angles are found to be small, these are not taken into account in the fit. A contribution is added to the systematic uncertainty to reflect this omission.

\subsubsection{Result}

Due to the high production cross sections of the identified backgrounds, only limited samples could be generated and analysed, which do not permit to have a final dataset with the foreseen signal-to-background ratio. Indeed, the signal sample corresponds to an integrated luminosity of $6.8 \mathrm{fb}^{-1}$, while the inclusive background corresponds to an integrated luminosity of barely $48 \mathrm{pb}^{-1}$. The situation is somewhat better for the decay $B^{0} \rightarrow J / \psi K^{* 0}$, for which the sample corresponds to an integrated luminosity of $1.3 \mathrm{fb}^{-1}$.

First, a fit was performed on the complete set of selected and associated $B_{s}^{0}$ candidates only, using the efficiency functions determined in the previous section. The relative width difference $\Delta \Gamma_{s} / \bar{\Gamma}_{s}$ can be determined with an uncertainty of 0.016 (Table 5.5), but no sensitivity on the weak phase and the strong phases is obtained.

Then, a sample corresponding to an integrated luminosity of $1.3 \mathrm{fb}^{-1}$ is considered, which allows to have a realistic ratio of $B^{0} \rightarrow J / \psi K^{* 0}$ and signal events. With the low number of background events which remain after all selection requirements, an accurate model through the described p.d.f. is not possible. In addition, the low number of $B^{0} \rightarrow J / \psi K^{* 0}$ events 
Table 5.5. Results of the maximum likelihood fit for 73813 signal events.

\begin{tabular}{lclll}
\hline Parameter & Input value & Result & Stat. error & Rel. error \\
\hline$\left|A_{0}(0)\right|^{2}$ & 0.57 & 0.57398 & 0.00267 & $0.4 \%$ \\
$\left|A_{\|}(0)\right|^{2}$ & 0.217 & 0.21808 & 0.00473 & $2.1 \%$ \\
$\left|A_{\perp}(0)\right|^{2}$ & 0.213 & 0.20794 & 0.00396 & $1.9 \%$ \\
$\bar{\Gamma}_{s}$ & $0.712 \mathrm{ps}^{-1}$ & $0.712358 \mathrm{ps}^{-1}$ & $0.00350643 \mathrm{ps}^{-1}$ & $0.5 \%$ \\
$\Delta \Gamma_{s}$ & $0.142 \mathrm{ps}^{-1}$ & $0.134645 \mathrm{ps}^{-1}$ & $0.0108247 \mathrm{ps}^{-1}$ & $8.0 \%$ \\
$\Delta \Gamma_{s} / \bar{\Gamma}_{s}$ & 0.2 & 0.189013 & 0.0157993 & $8.4 \%$ \\
$\delta_{1}$ & $\pi$ & 2.94405 & 0.632682 & \\
$\delta_{2}$ & 0 & -0.109493 & 0.639713 & \\
$\phi_{C K M}$ & -0.04 & -0.0297427 & 0.0758856 & \\
\hline
\end{tabular}

Table 5.6. Results of the maximum likelihood fit for an integrated luminosity of $1.3 \mathrm{fb}^{-1}$ (signal only).

\begin{tabular}{lllll}
\hline Parameter & Input value & Result & Stat. error & Rel. error \\
\hline$\left|A_{0}(0)\right|^{2}$ & 0.57 & 0.5859 & 0.0062 & $1.1 \%$ \\
$\left|A_{\|}(0)\right|^{2}$ & 0.217 & 0.2141 & 0.0078 & $3.6 \%$ \\
$\left|A_{\perp}(0)\right|^{2}$ & 0.213 & 0.2002 & 0.0064 & $3.2 \%$ \\
$\bar{\Gamma}_{s}$ & $0.712 \mathrm{ps}^{-1}$ & $0.7018 \mathrm{ps}^{-1}$ & $0.0081 \mathrm{ps}^{-1}$ & $1.2 \%$ \\
$\Delta \Gamma_{s}$ & $0.142 \mathrm{ps}^{-1}$ & $0.1470 \mathrm{ps}^{-1}$ & $0.0256 \mathrm{ps}^{-1}$ & $17.4 \%$ \\
$\Delta \Gamma_{s} / \bar{\Gamma}_{s}$ & 0.2 & 0.2095 & 0.0371 & $18.1 \%$ \\
\hline
\end{tabular}

Table 5.7. Results of the maximum likelihood fit for an integrated luminosity of $1.3 \mathrm{fb}^{-1}$ (signal and background).

\begin{tabular}{lllll}
\hline Parameter & Input value & Result & Stat. error & Rel. error \\
\hline$\left|A_{0}(0)\right|^{2}$ & 0.57 & 0.5823 & 0.0061 & $1.1 \%$ \\
$\left|A_{\|}(0)\right|^{2}$ & 0.217 & 0.2130 & 0.0077 & $3.6 \%$ \\
$\left|A_{\perp}(0)\right|^{2}$ & 0.213 & 0.2047 & 0.0065 & $3.2 \%$ \\
$\bar{\Gamma}_{s}$ & $0.712 \mathrm{ps}^{-1}$ & $0.7060 \mathrm{ps}^{-1}$ & $0.0080 \mathrm{ps}^{-1}$ & $1.1 \%$ \\
$\Delta \Gamma_{s}$ & $0.142 \mathrm{ps}^{-1}$ & $0.1437 \mathrm{ps}^{-1}$ & $0.0255 \mathrm{ps}^{-1}$ & $17.7 \%$ \\
$\Delta \Gamma_{s} / \bar{\Gamma}_{s}$ & 0.2 & 0.2036 & 0.0374 & $18.4 \%$ \\
\hline
\end{tabular}

does not permit an accurate estimate of either the angular distribution or of its time-dependent efficiency. As such, the background events are simply added to the dataset and their expected distribution is not included in the p.d.f. used in the fit. The p.d.f. would thus simply describe the $B_{s}^{0}$ distribution:

$$
\mathcal{P}=\epsilon(t, \Theta) \cdot f(\Theta, \alpha, t) .
$$

With such a fit in which the invariant mass of the candidates is not taken into account, a requirement on the invariant mass of the candidates would obviously be made, choosing a window of $\pm 36 \mathrm{MeV} / \mathrm{c}^{2}$ around the world-average $B_{s}^{0}$ mass. This reduces the number of $B^{0}$ background events by a further $59 \%$, while reducing the number of signal candidates by $2.9 \%$. The results of the fit without background is given in Table 5.6 and with background in Table 5.7. With the lower number of $B_{s}^{0}$ candidates, the statistical uncertainty of the measurement is, as expected, markedly worse. As can be seen, the influence of the background is very small, with only a slight degradation of the width difference. The distribution of the proper decay length of the selected events with the fit projection is shown in Figure 5.2. 


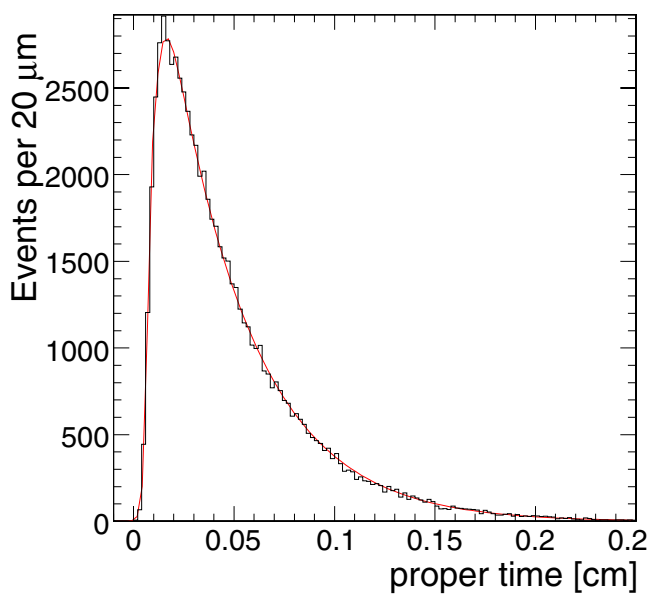

Figure 5.2. Distributions of the proper decay length of the selected signal and background events with fit projection.

Table 5.8. List of systematic uncertainties with effect on the predictions of the rates.

\begin{tabular}{llll}
\hline Source & HLT uncert. & Offline uncert. & Common uncert. \\
\hline Branching ratio $B_{s}^{0}$ & & & $36.4 \%$ \\
Branching ratio $B^{0}$ & & & $6 \%$ \\
Branching ratio $b \rightarrow J / \psi X$ & & $2 \%$ & $9 \%$ \\
Tracking inefficiency & $2 \%$ & $1.4 \%$ & \\
Muon reconstruction & - & - & \\
Misalignment & $17 \%$ & & \\
\hline
\end{tabular}

Table 5.9. List of systematic uncertainties with effect on the measurements.

\begin{tabular}{llllll}
\hline Source & $\left|A_{0}(0)\right|^{2}$ & $\left|A_{\|}(0)\right|^{2}$ & $\left|A_{\perp}(0)\right|^{2}$ & $\bar{\Gamma}_{s}$ & $\Delta \Gamma_{s} / \bar{\Gamma}_{s}$ \\
\hline Bckg. distrib. & 0.0034 & 0.0011 & 0.0045 & 0.0043 & 0.0059 \\
S/B ratio & 0.0037 & 0.0001 & 0.0024 & 0.0025 & 0.0055 \\
Resolution & - & - & - & 0.00060 & 0.0045 \\
Ang. distortion & 0.0143 & 0.0061 & 0.0082 & 0.00083 & 0.0010 \\
$c \tau$ distortion & 0.0016 & 0.00073 & 0.0023 & 0.0221 & 0.0146 \\
Alignment & 0.00012 & 0.00042 & 0.00055 & 0.00040 & 0.0014 \\
Total & 0.0152 & 0.0063 & 0.0099 & 0.0227 & 0.0173 \\
\hline
\end{tabular}

\subsubsection{Systematics and detector effects}

The list of systematic uncertainties which were considered are summarised in two tables. The first, Table 5.8, summarises the uncertainties which affect the HLT rate and the number of foreseen events after all selection requirements. The second, Table 5.9, summarises the uncertainties which affect the measurement of the various parameters.

- Signal and background statistics. Among the various uncertainties listed in Section 5.1.2, the largest single source of uncertainty in the estimate of the number of events is obviously 
the poor knowledge of the $B_{s}^{0} \rightarrow J / \psi \phi$ branching ratio. The uncertainties quoted on the estimates above do not include the uncertainties on the total $b \bar{b}$ cross section at LHC energies, the $b \rightarrow B^{0}$ fragmentation functions, the transverse momentum distribution of $b$ quarks. However, since both the signal and background are proportional to the same $b \bar{b}$ cross section, the signal-to-background ratio is unaffected by the corresponding uncertainty.

- Track reconstruction efficiency. A $1 \%$ uncertainty per track on the track reconstruction efficiency is assumed for all tracks.

- Muon reconstruction. The selection relies heavily on the correct identification of muons. A $1 \%$ uncertainty per track on the combined muon identification procedure is assumed.

- Tracker and muon detector misalignment. The study has been conducted with a perfectly aligned detector. To gauge the sensitivity of the analysis with respect to the alignment the analysis has been repeated on a detector with the short-term alignment scenario. This scenario is expected to be representative of the relative misalignment of the detector components during the initial data taking period [86]. The effects of misalignment of the tracker on various aspects of track and vertex reconstruction have been extensively studied and reported in $[140,141]$. The degradation affect both the selection, mostly through the requirement on the significance of the transverse decay length of the $J / \psi$ in the HLT, and the analysis, through the degradation of the measurement of the proper decay length. The resolution of the latter is degraded from $24 \mu \mathrm{m}$ for a perfectly aligned detector to $32 \mu \mathrm{m}$ with the short-term alignment. The HLT efficiency is degraded by some $17 \%$ with respect to a perfectly aligned detector.

- Background distributions. To gauge the influence of the background on the fit, the variation observed between the fits performed on the reduced $1.3 \mathrm{fb}^{-1}$ dataset with and without these events is added to the systematic uncertainty ("Bckg. distrib." in the table).

Since the signal-to-background ratio has a significant uncertainty, the fit performed on the reduced $1.3 \mathrm{fb}^{-1}$ sample is repeated varying the number of $B_{s}^{0}$ signal events to match the uncertainty in the signal-to-background ratio. For this estimate, a different uncertainty for the $B_{s}^{0}$ branching fraction has been chosen, since it is believed that it will be measured again in the current run of the Tevatron. Two main uncertainties plagued the measurement done at CDF in Run I, the low number of observed $B_{s}^{0}$ candidates and the uncertainty on the fragmentation. Based on recent publications, it is estimated that approximately 30 times more $B_{s}^{0} \rightarrow J / \psi \phi$ decays than in Run 1 should already be collected in the current dataset of $1 \mathrm{fb}^{-1}$. The uncertainty of the branching fraction is therefore reduced to $20 \%$. For the other uncertainties, the numbers listed in Table 5.8 are used. The variation observed on the fit is listed under the heading "S/B ratio." In a larger dataset, where the full p.d.f. (Eq. 5.11) is used, the influence of the uncertainty on the signal-to-background ratio should be much smaller, since the fractions of background events in the dataset are free parameters in the fit.

- Distortion of the proper-time distribution (“c $\tau$ distortion”). Other fits were then performed where the parameters of the time dependent efficiency function are varied by one standard deviation. The mean variation of the fitted parameters was added to the systematic uncertainty. As already mentioned, the decay $B^{0} \rightarrow J / \psi K^{* 0}$ can be used to compare the accuracy of this model by comparing the Monte Carlo prediction with the efficiency function observed in the data.

- Distortion of the angular distributions ("Ang. distortion"). The expansion used to model the distortion of the angular distributions (Equation (5.3)) is limited to $L, R \leqslant 8$. When 
Table 5.10. Results of the maximum likelihood fit for an integrated luminosity of $1.3 \mathrm{fb}^{-1}$ (signal and background).

\begin{tabular}{lllllll}
\hline Parameter & Input value & Result & Stat. error & Sys. error & Total error & Rel. error \\
\hline$\left|A_{0}(0)\right|^{2}$ & 0.57 & 0.5823 & 0.0061 & 0.0152 & 0.0163 & $2.8 \%$ \\
$\left|A_{\|}(0)\right|^{2}$ & 0.217 & 0.2130 & 0.0077 & 0.0063 & 0.0099 & $4.6 \%$ \\
$\left|A_{\perp}(0)\right|^{2}$ & 0.213 & 0.2047 & 0.0065 & 0.0099 & 0.0118 & $5.8 \%$ \\
$\bar{\Gamma}_{s}$ & $0.712 \mathrm{ps}^{-1}$ & $0.7060 \mathrm{ps}^{-1}$ & $0.0080 \mathrm{ps}^{-1}$ & $0.0227 \mathrm{ps}^{-1}$ & $0.0240 \mathrm{ps}^{-1}$ & $3.4 \%$ \\
$\Delta \Gamma_{s}$ & $0.142 \mathrm{ps}^{-1}$ & $0.1437 \mathrm{ps}^{-1}$ & $0.0255 \mathrm{ps}^{-1}$ & $0.0113 \mathrm{ps}^{-1}$ & $0.0279 \mathrm{ps}^{-1} \mathrm{ps}^{-1}$ & $19 \%$ \\
$\Delta \Gamma_{s} / \bar{\Gamma}_{s}$ & 0.2 & 0.2036 & 0.0374 & 0.0173 & 0.0412 & $20 \%$ \\
\hline
\end{tabular}

limiting the expansion to $L, R \leqslant 6$ or $L, R \leqslant 10$, the result of the fit shows negligible differences. In addition, to account for the possibility that the efficiencies do not factorise and that the angular efficiency is grossly miscalculated, the fit is also repeated without the angular efficiency, i.e. without correction of the distortion. While this has little influence on the estimated lifetimes, a large variation is found for the amplitudes. This variation is used as systematic uncertainty.

- Resolution on the angular variables ("Resolution"). In order to estimate the influence of the uncertainties of the angles and the proper decay length on the fit, a fully controlled toy Monte Carlo was used, in which only the proper time and angles were generated according to the expected p.d.f. and smeared with Gaussian resolution functions. The default standard deviations are taken to be equal to those measured in the Monte Carlo with full detector simulation. The simulation was then repeated without smearing and with a substantial smearing, where the resolution is taken to be two times larger than in the default simulation. The value of parameters found in both cases were very close to the values found with the default smearing, and the observed variation is added to the systematic uncertainty.

\subsubsection{Conclusion}

The present section describes a study on the selection of the $B_{s}^{0} \rightarrow J / \psi \phi$ decay and the measurement of the width difference $\Delta \Gamma_{s}$ in absence of flavour tagging. An example of a trigger algorithm is presented which would be efficient for this decay and would reject a large fraction of the background. It is based on the identification of $J / \psi$ and $B_{s}^{0}$ candidates with a displaced decay vertex. Nevertheless, this trigger precludes the selection of other decays of the $B$ meson, and should certainly evolve as a true precursor to a $B$ physics trigger. Indeed, the strategy proposed for the Level-2 would select inclusive $b \rightarrow J / \psi$ decays with high efficiency and good purity with respect to the prompt $J / \psi$ background. Large uncertainties nevertheless plague the estimates of rates, since large uncertainties remain on the $b$-quark and prompt $J / \psi$ production cross sections, on their momentum distributions, and on the $b \rightarrow B_{s}^{0}$ fragmentation function.

A first measurement of one of the main parameters of the $B_{s}^{0}$ system, the relative difference of the widths of the weak eigenstates could be determined with a statistical uncertainty of 0.011 in a sample corresponding to an integrated luminosity of $10 \mathrm{fb}^{-1}$. A first measurement undertaken on approximately $1.3 \mathrm{fb}^{-1}$ of data could already yield a measurement with an uncertainty of $20 \%$ (Table 5.10). A natural extension of this study should be a tagged analysis, for which flavour tagging algorithms need to be developed. 


\subsection{Associated production of MSSM heavy neutral Higgs bosons $\bar{b} \bar{b} H(A)$} with $H(A) \rightarrow \tau \tau$

\subsubsection{Introduction}

The observation of a heavy neutral scalar accompanied by b-jets and decaying into two $\tau$ leptons would be an important sign of a MSSM Higgs sector. In the MSSM the associated Higgs boson production $\mathrm{gg} \rightarrow \mathrm{b} \overline{\mathrm{b}} \mathrm{H}(\mathrm{A})$ is dominant at large values of $\tan \beta$. The cross section of the $\mathrm{gg} \rightarrow \mathrm{b} \overline{\mathrm{b}} \mathrm{H}(\mathrm{A}), \mathrm{H}(\mathrm{A}) \rightarrow \tau \tau$ process is proportional to $\tan ^{2} \beta_{\text {eff }}$ and will be used in a global fit together with other relevant measurements to determine the SUSY parameters simultaneously. An example of a pp $\rightarrow \mathrm{H}+\mathrm{X}$ event with $\mathrm{H} \rightarrow \tau \nu \tau \nu$ is shown in colour plate CP8.

This channel is an excellent benchmark for the $\mathrm{b}$ - and $\tau$-tagging, jet and missing $\mathrm{E}_{\mathrm{T}}$ reconstruction. The final state with two $\tau$-jets requires $\tau$ tagging both at Level-1 and High Level Trigger. Along with reconstruction and tagging issues, a large number of various Standard Model backgrounds including QCD multi-jet production must be well understood from the real data to be able to establish a discovery.

\subsubsection{Event generation}

The signal events were generated by PYTHIA using processes the $181(\mathrm{gg} \rightarrow \mathrm{b} \overline{\mathrm{b}} \mathrm{H})$ and $152(\mathrm{gg} \rightarrow \mathrm{H})$ for three values of the Higgs boson mass: 200,500 and $800 \mathrm{GeV} / \mathrm{c}^{2}$. The backgrounds considered were QCD multi-jet events (for $\tau \tau \rightarrow \mathrm{jj}$ mode), $\mathrm{tt}$, b $\bar{b}$, Drell-Yan production of $\mathrm{Z} / \gamma^{*}, \mathrm{~W}+\mathrm{jet}, \mathrm{Wt}$ and $\tau \tau \mathrm{b} \overline{\mathrm{b}}$. All background processes except $\tau \tau \mathrm{b} \overline{\mathrm{b}}$ were generated with PYTHIA. The $\tau \tau \mathrm{b} \bar{b}$ process was generated by COMPHEP.

In order to reduce CPU time for full detector simulation and event reconstruction loose pre-selections were applied for some of the backgrounds at the generation level. The description of the pre-selections for each final state can be found in the following sections.

The cross sections for the associated Higgs boson production $g g \rightarrow b \bar{b} H(A)$ and the branching ratio $\mathrm{H}(\mathrm{A}) \rightarrow \tau \tau$ were calculated using FeynHiggs $2.3 .2[142-144]^{41}$ in the $\mathrm{m}_{\mathrm{h}}^{\max }$ scenario with $\mu=200 \mathrm{GeV} / \mathrm{c}^{2}$ (see Section 11.3.1).

The uncertainty of the measured cross section of the $b(\bar{b}) A, A \rightarrow \tau \tau$ process will include the uncertainty of the Monte Carlo generation. The verification of the Monte Carlo generation for the Higgs boson production with the associated $b$-jets will be done with the real data using $\mathrm{b} \overline{\mathrm{b} Z}(\mathrm{Z} \rightarrow \ell \ell)$ events [145].

\subsubsection{Level-1 and High Level trigger selections}

The $\tau \tau \rightarrow \mathrm{jj}$ final state is triggered by Level-1 single or double tau triggers with thresholds of $93 \mathrm{GeV}$ for the single and $66 \mathrm{GeV}$ for the double tau trigger. It is followed by the double $\tau$-jet tagging at High Level Trigger. Currently there are two selection strategies at HLT under consideration [146]. In the first strategy the calorimeter isolation using the electromagnetic calorimeter is applied to the first $\tau$-jet in order to reduce the Level-1 output rate by a factor of 3 . The tracker isolation is then applied on both jets using the tracks reconstructed with the pixel detector only. The second strategy performs tracker isolation right after the Level-1 trigger decision and uses the full tracker with regional track finding and a restricted number of hits to reconstruct tracks. In this analysis the first method is exploited.

The $\tau \tau \rightarrow \mu \mathrm{j}$ final state uses the single muon trigger at Level- 1 with a threshold of $14 \mathrm{GeV}$. At the High Level the combined muon-plus- $\tau$-jet trigger is used with thresholds of $15 \mathrm{GeV}$ for the muon and of $40 \mathrm{GeV}$ for the $\tau$-jet.

\footnotetext{
${ }^{41}$ The code can be obtained from http://www.feynhiggs.de
} 
The $\tau \tau \rightarrow$ ej final state uses the Level-1 single electron trigger with a threshold of $23 \mathrm{GeV}$ together with the combined electron-plus- $\tau$-jet trigger with thresholds of $14 \mathrm{GeV}$ for the electron and $52 \mathrm{GeV}$ for the $\tau$-jet. At High Level again the single electron trigger with a threshold of $26 \mathrm{GeV}$ and the combined electron-plus- $\tau$-jet trigger with a threshold of $16 \mathrm{GeV}$ for the electron is used. No threshold is applied for the $\tau$-jet candidate.

At High Level Trigger, for both the $\tau \tau \rightarrow \mu \mathrm{j}$ and the $\tau \tau \rightarrow$ ej final states, the ECAL and pixel track isolation is applied on the $\tau$-jet candidate similar to what is used in the double $\tau$-jet trigger. For the lepton (e and $\mu$ ) the same selections are used as for the single electron and muon High Level triggers. The lepton and $\tau$-jet are required to stem from the same vertex found with the pixel detector. Only the tracks from this vertex are used in the tracker isolation.

The search strategy for $\tau$-jet candidates at High Level Trigger for the combined muonplus- $\tau$-jet and electron-plus- $\tau$-jet triggers is the following: Two calorimeter jets are always reconstructed with the regional jet finder in the regions given by the two highest $\mathrm{E}_{\mathrm{T}}$ Level-1 $\tau$-jets. For the muon-plus- $\tau$-jet trigger the first (highest $\mathrm{E}_{\mathrm{T}}$ ) jet is taken as $\tau$-jet candidate. For the electron-plus- $\tau$-jet trigger the requirement of non collinearity of the jet and the HLT electron candidate, $\Delta \mathrm{R}(\mathrm{e}-\mathrm{jet})>0.3$, is checked for each jet, where $\Delta \mathrm{R}(\mathrm{e}-\mathrm{jet})$ is the distance in $\eta-\varphi$ space between the electron and the jet. The first non collinear jet is taken as the $\tau$-jet candidate.

\subsubsection{Off-line event selection}

The first step in the off-line analysis is the $\tau$-jet identification. The calorimeter jet is reconstructed in the $\eta-\varphi$ region of the High Level Trigger $\tau$-jet candidate with the iterative cone algorithm using a cone size of 0.4 . A number of requirements for $\tau$-jet identification [146] is applied in addition to the tracker isolation which is tighter off-line than at the HLT and uses the tracks reconstructed with the full tracker. The additional $\tau$-jet identification criteria include requirements to have one or three tracks in the signal cone and opposite charge of the two $\tau$-jets for the $\tau \tau \rightarrow \mathrm{jj}$ mode or the lepton and the $\tau$-jet for the $\tau \tau \rightarrow \ell \mathrm{j}$ modes and cuts on the transverse impact parameter and on the $p_{\mathrm{T}}$ of the leading track in the signal cone. Finally an electron rejection criterion was applied for the jets. The $\tau$-jet tagging reduces the QCD multi-jet (including $b \bar{b}$ ) and the $\mathrm{W}+$ jet backgrounds.

The associated $\mathrm{b} \bar{b} \mathrm{H}(\mathrm{A})$ production dominates at high values of $\tan \beta$, thus it is natural to apply b-jet tagging which must suppress Drell-Yan $\tau \tau$ production and eliminate further the QCD multi-jet and the $\mathrm{W}+$ jet backgrounds. Since the $b$-jets in the signal are very soft in $\mathrm{E}_{\mathrm{T}}$ and have flat distribution in pseudorapidity only single $b$ tagging is applied. Furthermore, it is possible to veto events with additional jets to reduce $\mathrm{t} \overline{\mathrm{t}}$ background. The $\tau$-jets found in the first step are not considered for $\mathrm{b}$ tagging. Non $\tau$-jet candidates are reconstructed with the iterative cone algorithm using a cone size of 0.5 .

The energy of the $\tau$-jet is corrected with a dedicated calibration obtained from MonteCarlo sample of single $\tau$-jets at low luminosity. The energy of other jets in the event is corrected applying Monte Carlo calibration evaluated from the QCD multi-jet events at low luminosity.

\subsubsection{Method of the Higgs boson mass reconstruction}

Despite the escaping neutrinos, the Higgs boson mass can be reconstructed in the $\mathrm{H} \rightarrow \tau \tau$ channels from the visible $\tau$ momenta (leptons or $\tau$-jets) and the missing transverse energy $\left(\mathrm{E}_{\mathrm{T}}^{\mathrm{miss}}\right)$ with the collinearity approximation for the neutrinos from highly boosted $\tau$ 's. The mass resolution depends on the angle $\Delta \varphi$ between the visible $\tau$ momenta as $1 / \sin (\Delta \varphi)$ and is sensitive to the $\mathrm{E}_{\mathrm{T}}^{\text {miss }}$ measurement, both in magnitude and particularly in direction. The measurement of $\mathrm{E}_{\mathrm{T}}^{\mathrm{miss}}$ is affected by the non-linear calorimeter response. A method to improve 
the $E_{T}^{\text {miss }}$ scale based on the jet energy corrections was used [147, 148]. The correction of the missing $\mathrm{E}_{\mathrm{T}}$ scale improves the reconstruction efficiency by reducing the number of events with negative reconstructed $\tau$ lepton and neutrino energies. In particular, for the case of the $\tau \tau \rightarrow \mathrm{jj}$ final state the efficiency is improved by factor of $\simeq 1$.6. The $\tau \tau$ mass reconstruction method will be verified with the real data using $\mathrm{Z} \rightarrow \tau \tau \rightarrow \mathrm{e}(\mu)+$ jet and $\mathrm{Z} \rightarrow \tau \tau \rightarrow \mathrm{e}+\mu$ channels [145, 149].

\subsection{6. $\mathrm{H} \rightarrow \tau \tau \rightarrow$ 2jet analysis}

A detailed description of the analysis can be found in [150].

5.2.6.1. Event generation and pre-selections. The t⿱t, Drell-Yan production of $\mathrm{Z} / \gamma^{*}, \mathrm{~W}+\mathrm{jet}$ and $\mathrm{Wt}$ backgrounds were generated with PYTHIA, forcing $\mathrm{W} \rightarrow \tau \nu$ and $\mathrm{Z} / \gamma^{*} \rightarrow \tau \tau$ decays. The TAUOLA package was used for $\tau$-lepton decays into all possible decay modes.

The $\mathrm{Z} / \gamma^{*}$ generation was split into three bins of generated di $\tau$-lepton mass $\mathrm{m}_{\tau \tau}$ : $80-130 \mathrm{GeV} / \mathrm{c}^{2}, 130-300 \mathrm{GeV} / \mathrm{c}^{2}$ and $>300 \mathrm{GeV} / \mathrm{c}^{2}$. The $\tau \tau \mathrm{b} \bar{b}$ generation was divided into two bins of generated di $\tau$-lepton mass $\mathrm{m}_{\tau \tau}: 60-100 \mathrm{GeV} / \mathrm{c}^{2}$ and $>100 \mathrm{GeV} / \mathrm{c}^{2}$. The $\tau \tau \mathrm{b} \bar{b}$ background, generated with COMPHEP, was propagated to PYTHIA for showering, hadronisation and $\tau$ lepton decays into all possible modes.

The $\mathrm{W}+$ jet background was generated using PYTHIA processes 16 and 31 and with $\hat{\mathrm{p}}_{\mathrm{T}}>65 / \mathrm{GeV} / \mathrm{c}$. The QCD multi-jet background generation was done for four bins in $\hat{\mathrm{p}}_{\mathrm{T}}$ : $50-80,80-120,120-170$ and $>170 \mathrm{GeV} / \mathrm{c}$.

The loose pre-selections at the level of generation were applied for all backgrounds (except $\tau \tau \mathrm{b} \bar{b}$ ): the event was required to have at least two " $\tau$-like" jets. The jets were reconstructed with the PYTHIA PYCELL routine using a cone size of 0.5 . A jet is selected as " $\tau$-like" if it has $\mathrm{E}_{\mathrm{T}}^{\mathrm{MC}}>50 \mathrm{GeV},\left|\eta^{\mathrm{MC}}\right|<2.4$ and a transverse momentum of the leading stable charged particle in the jet, $\mathrm{p}_{\mathrm{T}}^{\mathrm{MC}}>30 \mathrm{GeV} / \mathrm{c}$. These cuts are looser than the ones applied at the trigger and off-line $\tau$-jet selections. For $\mathrm{Z} / \gamma^{*}$ background no cut was applied on $\mathrm{p}_{\mathrm{T}}^{\mathrm{MC}}$.

For the signal events the Higgs boson was forced to decay into two $\tau$ leptons and the $\tau$ lepton was decayed hadronically using TAUOLA. No pre-selections were applied for the signal events.

5.2.6.2. Event selections. The calorimeter $\tau$-jet jet candidates are reconstructed in the $\eta-\varphi$ regions of the High Level Trigger $\tau$-jet candidates, thus no "volunteers" are searched for. This is motivated by the high $(\simeq 100 \%)$ purity of the HLT $\tau$-jet candidates (fraction of true $\tau$-jets matched with $\tau$-jet candidates).

A cut on the uncalibrated transverse jet energy for each of the two $\tau$-jet candidates was required. It was $\mathrm{E}_{\mathrm{T}}>50 \mathrm{GeV}$ for $\mathrm{M}_{\mathrm{A}}=200 \mathrm{GeV} / \mathrm{c}^{2}$. For higher Higgs boson masses asymmetrical cuts were used: $100,50 \mathrm{GeV}$ for $\mathrm{M}_{\mathrm{A}}=500 \mathrm{GeV} / \mathrm{c}^{2}$ and $150,50 \mathrm{GeV}$ for $\mathrm{M}_{\mathrm{A}}=800 \mathrm{GeV} / \mathrm{c}^{2}$. It allows more effective rejection of the QCD multi-jet background. The following $\tau$-jet identification criteria were then used:

- tracker isolation with parameters: $\mathrm{R}_{\mathrm{m}}=0.1, \mathrm{R}_{\mathrm{S}}=0.04, \mathrm{R}_{\mathrm{i}}=0.5, \mathrm{p}_{\mathrm{T}}^{\mathrm{i}}=1 \mathrm{GeV} / \mathrm{c}$;

- transverse momentum of the leading track $>35 \mathrm{GeV} / \mathrm{c}$;

- one or three tracks in the signal cone $\mathrm{N}_{\mathrm{tr}}^{\mathrm{S}}$ for $\mathrm{M}_{\mathrm{A}}=200 \mathrm{GeV} / \mathrm{c}^{2}$. For higher Higgs boson masses an effective background rejection is only possible by requiring only one track in the signal cone.

Finally, the two $\tau$-jet candidates were required to have opposite charge. The charge was calculated as the sum of charges of the tracks in the signal cone.

After identification of two $\tau$-jets the other jets in the event were considered. It was required to have only one additional jet with uncalibrated energy $\mathrm{E}_{\mathrm{T}}^{\text {raw }}>20 \mathrm{GeV}$ and $|\eta|<2.4$. It had to be tagged as $b$-jet. The $b$-jet identification was performed using the impact parameter 
Table 5.11. The summary table of the selections for signals of $M_{A}=200,500$ and $800 \mathrm{GeV} / \mathrm{c}^{2}$.

\begin{tabular}{|c|c|c|c|}
\hline & $\begin{array}{l}\mathrm{m}_{\mathrm{A}}=200 \mathrm{GeV} / \mathrm{c}^{2} \\
\tan \beta=20\end{array}$ & $\begin{array}{l}\mathrm{m}_{\mathrm{A}}=500 \mathrm{GeV} / \mathrm{c}^{2} \\
\tan \beta=30\end{array}$ & $\begin{array}{l}\mathrm{m}_{\mathrm{A}}=800 \mathrm{GeV} / \mathrm{c}^{2} \\
\tan \beta=40\end{array}$ \\
\hline \multicolumn{4}{|c|}{ Cross sections and branching ratios } \\
\hline$\sigma(\mathrm{gg} \rightarrow \mathrm{b} \bar{b}(\mathrm{~A}+\mathrm{H}))(\mathrm{fb})$ & $45795+44888$ & $2741+2744$ & $677+677$ \\
\hline $\mathrm{BR}(\mathrm{H} / \mathrm{A} \rightarrow \tau \tau)$ & 0.1 & 0.082 & 0.087 \\
\hline $\mathrm{BR}(\tau \rightarrow \text { hadrons })^{2}$ & & $0.65 \times 0.65$ & \\
\hline$\sigma \times \mathrm{BR}(\mathrm{fb})$ & 3831 & 190 & 49.8 \\
\hline \multicolumn{4}{|c|}{ Experimental selection efficiencies } \\
\hline Level-1 Trigger & 0.506 & 0.854 & 0.896 \\
\hline HLT & 0.289 & 0.319 & 0.314 \\
\hline two off-line calo $\tau$ jets & 0.997 & 0.999 & 0.999 \\
\hline cuts on $\mathrm{E}_{\mathrm{T}} \tau$ jets & 0.430 & 0.755 & 0.780 \\
\hline two off-line $\tau$ candidates & 0.674 & 0.716 & 0.675 \\
\hline $\mathrm{p}_{\mathrm{T}}^{\mathrm{ltr}}>35 \mathrm{GeV} / \mathrm{c}$ & 0.326 & 0.616 & 0.713 \\
\hline tracker isolation & 0.859 & 0.950 & 0.954 \\
\hline $\mathrm{N}_{\text {tracks }}$ in signal cone & 0.81 & 0.67 & 0.78 \\
\hline $\mathrm{Q}_{\tau 1} \times \mathrm{Q}_{\tau 2}=-1$ & 0.98 & 0.94 & 0.94 \\
\hline $\begin{array}{l}\geqslant 1 \text { extra jet, } \\
\mathrm{E}_{\mathrm{T}}^{\text {raw }}>20 \mathrm{GeV},|\eta|<2.4\end{array}$ & 0.21 & 0.27 & 0.31 \\
\hline $\begin{array}{l}\text { only } 1 \text { extra jet, } \\
\mathrm{E}_{\mathrm{T}}^{\text {raw }}>20 \mathrm{GeV},|\eta|<2.4\end{array}$ & 0.83 & 0.82 & 0.78 \\
\hline \multicolumn{4}{|l|}{$\mathbf{M}_{\tau \tau}$ reconstruction efficiency } \\
\hline $\mathrm{E}_{\tau 1, \tau 2}>0$ & 0.93 & 0.93 & 0.92 \\
\hline $\mathrm{E}_{\nu 1, v 2}>0$ & 0.56 & 0.67 & 0.67 \\
\hline total mass reconstruction & 0.52 & 0.62 & 0.62 \\
\hline b tagging of the extra jet & 0.36 & 0.44 & 0.41 \\
\hline $\mathbf{M}_{\tau \tau}$ mass window & $150-300 \mathrm{GeV} / \mathrm{c}^{2}$ & $400-700 \mathrm{GeV} / \mathrm{c}^{2}$ & $600-1100 \mathrm{GeV} / \mathrm{c}^{2}$ \\
\hline mass window efficiency & 0.81 & 0.73 & 0.81 \\
\hline total efficiency & $2.5 \times 10^{-4}$ & $2.4 \times 10^{-3}$ & $3.6 \times 10^{-3}$ \\
\hline$\sigma$ after selections $(\mathrm{fb})$ & 0.96 & 0.46 & 0.19 \\
\hline number of events for $60 \mathrm{fb}^{-1}$ & 58.0 & 27.0 & 11.0 \\
\hline
\end{tabular}

tagging in 3D space [151]. The jet had to have at least three tracks with an impact parameter significance $>2$. The purity of the b-tagged jet for the signal is very high $(>95 \%)$.

The di $\tau$-jet mass reconstruction efficiency is affected by the requirements to have a positive reconstructed energy of both neutrinos, $\mathrm{E}_{\mathrm{T}}^{\nu_{1}, \nu_{2}}>0$. In the missing $\mathrm{E}_{\mathrm{T}}$ corrections jets with raw energy $\mathrm{E}_{\mathrm{T}}^{\mathrm{raw}}>25$ were used.

5.2.6.3. Expected number of selected events. This section summarises the event selections, the corresponding cross sections and expected number of events for the signal and the background processes after the selections. The efficiency of all selections shown in the tables of this section was evaluated relative to the previous selection.

Signal. Table 5.11 summarises the expectations for a signal of $\mathrm{M}_{\mathrm{A}}=200,500$ and $800 \mathrm{GeV} / \mathrm{c}^{2}$. The signal cross sections and the branching ratios were obtained for the $\mathrm{m}_{\mathrm{h}}^{\max }$ scenario with $\mu=200 \mathrm{GeV} / \mathrm{c}^{2}$ (see Section 11.3.1).

QCD multi-jet background. Despite the huge amount of generated events (more than one million) and generation pre-selections, the statistics of the QCD multi-jet background events is not enough to ensure a large number of Monte Carlo events passing all the selections. In order to decrease the statistical uncertainties a factorisation of the selections was applied. All 
Table 5.12. The summary table of the selections for the QCD multi-jet background. The selections are factorised as explained in the text. The requirement to have opposite charge $\tau$-jet candidates $\left(\mathrm{Q}_{1} \times \mathrm{Q}_{2}=-1\right)$ is not included.

\begin{tabular}{|c|c|c|c|c|}
\hline & \multicolumn{4}{|c|}{ QCD dijet background in bins of generated $\hat{\mathrm{p}}_{\mathrm{T}}$} \\
\hline & $>170 \mathrm{GeV} / \mathrm{c}$ & $120-170 \mathrm{GeV} / \mathrm{c}$ & $80-120 \mathrm{GeV} / \mathrm{c}$ & $50-80 \mathrm{GeV} / \mathrm{c}$ \\
\hline$\sigma(\mathrm{fb})$ & $1.33 \times 10^{8}$ & $5.03 \times 10^{8}$ & $2.94 \times 10^{9}$ & $2.08 \times 10^{10}$ \\
\hline$\varepsilon_{\text {kine pres. }}$ & $2.12 \times 10^{-1}$ & $4.19 \times 10^{-2}$ & $5.77 \times 10^{-3}$ & $2.44 \times 10^{-4}$ \\
\hline \multicolumn{5}{|c|}{ Group 1 cuts: Level-1 trigger $+\mathrm{L} 2$ and offline calo reco $+\mathrm{E}_{\mathrm{T}}$ cut } \\
\hline Level-1 trigger & 0.562 & 0.726 & 0.715 & 0.461 \\
\hline Two Level 2 calo jets with $\Delta \mathrm{R}_{\mathrm{J}} \mathrm{J}>1.0$ & 0.927 & 0.959 & 0.982 & 0.987 \\
\hline two off-line calo $\tau$ jets & 0.975 & 0.975 & 0.982 & 0.994 \\
\hline cuts on $\mathrm{E}_{\mathrm{T}} \tau$ jets & 0.753 & 0.804 & 0.774 & 0.343 \\
\hline$\varepsilon_{\text {Group } 1}$ & 0.383 & 0.547 & 0.534 & 0.155 \\
\hline \multicolumn{5}{|c|}{ Group2 cuts: $\tau$-jet identification at HLT and off-line } \\
\hline HLT Calo+Pxl $\tau$ trigger & $7.15 \times 10^{-4}$ & $1.81 \times 10^{-3}$ & $4.44 \times 10^{-3}$ & $1.12 \times 10^{-2}$ \\
\hline Two off-line $\tau$ candidates & 0.86 & 0.84 & 0.825 & 0.84 \\
\hline $\mathrm{p}_{\mathrm{T}}^{\mathrm{ltr}}>35 \mathrm{GeV} / \mathrm{c}$ & 0.47 & 0.41 & 0.42 & 0.38 \\
\hline Tracker isolation & 0.24 & 0.21 & 0.25 & 0.35 \\
\hline \multicolumn{5}{|c|}{ Factorised inside group 2} \\
\hline 1 or 3 prongs in ${ }^{1 \mathrm{st}} \tau$ jet & 0.66 & 0.92 & 0.63 & 0.72 \\
\hline 1 or 3 prongs in ${ }^{2 \text { nd }} \tau$ jet & 0.48 & 0.54 & 0.65 & 0.72 \\
\hline$\varepsilon_{\text {Group } 2 / \varepsilon_{\text {Group } 1}}$ & $2.30 \times 10^{-5}$ & $6.33 \times 10^{-5}$ & $1.63 \times 10^{-4}$ & $6.54 \times 10^{-4}$ \\
\hline \multicolumn{5}{|c|}{ Group3 cuts: extra jet reco and $b$ tagging plus $M_{\tau \tau}$ reco and mass window } \\
\hline $\begin{array}{l}\geqslant 1 \text { extra jet, } \\
\mathrm{E}_{\mathrm{T}}^{\text {raw }}>20 \mathrm{GeV},|\eta|<2.4\end{array}$ & 0.463 & 0.235 & 0.127 & 0.090 \\
\hline $\begin{array}{l}\text { Only } 1 \text { extra jet, } \\
\mathrm{E}_{\mathrm{T}}^{\text {raw }}>20 \mathrm{GeV},|\eta|<2.4\end{array}$ & 0.661 & 0.817 & 0.863 & 0.855 \\
\hline \multicolumn{5}{|c|}{ Factorised inside group $3: M_{\tau \tau}$ and $b$ tagging } \\
\hline $\mathrm{E}_{\tau 1, \tau 2}>0$ & 0.921 & 0.898 & 0.882 & 0.834 \\
\hline $\mathrm{E}_{v 1, v 2}>0$ & 0.701 & 0.683 & 0.657 & 0.625 \\
\hline Total mass reconstruction & 0.646 & 0.613 & 0.579 & 0.522 \\
\hline$b$ tagging of the extra jet & 0.098 & 0.050 & 0.033 & 0.016 \\
\hline $\mathrm{M}_{\tau \tau}$ window: $150-300 \mathrm{GeV} / \mathrm{c}^{2}$ & 0.142 & 0.295 & 0.433 & 0.430 \\
\hline$\varepsilon_{\text {Group } 3} / \varepsilon_{\text {Group } 1}$ & $2.77 \times 10^{-3}$ & $1.75 \times 10^{-3}$ & $9.15 \times 10^{-4}$ & $2.28 \times 10^{-4}$ \\
\hline$\varepsilon_{\text {Group } 1} \times \varepsilon_{\text {Group } 2} \times \varepsilon_{\text {Group } 3}$ & $2.44 \times 10^{-8}$ & $6.07 \times 10^{-8}$ & $7.98 \times 10^{-8}$ & $2.84 \times 10^{-8}$ \\
\hline$\sigma$ after selections $(\mathrm{fb})$ & 0.69 & 1.28 & 1.35 & 0.144 \\
\hline Number of events for $60 \mathrm{fb}^{-1}$ & 41.4 & 76.7 & 81.2 & 8.7 \\
\hline
\end{tabular}

selections were combined in three groups as shown in Table 5.12. Group1 includes the Level1 trigger and the calorimetric reconstruction of the $\tau$-jets (at HLT and offline). It includes also the cut on the transverse energy of the jets. After the event passed the Group1 selections the two other selection groups (Group2 and Group3) were applied independently. Group2 is essentially the $\tau$-jet identification part of the analysis, i.e. the tracker isolation (at HLT and offline), the cut on the $\mathrm{p}_{\mathrm{T}}$ of the leading track and the selection on the number of tracks inside the signal cone. Group 3 describes the selections on the one extra jet in the event, the $b$ tagging and the di $\tau$-jet mass reconstruction. The choice of the second and third selection groups was made minimising the correlation among them. A further factorisation was done for some selections inside the groups. Table 5.12 summarises the selections and the QCD multi-jet background estimates for the signal of $\mathrm{M}_{\mathrm{A}}=200 \mathrm{GeV} / \mathrm{c}^{2}$. The requirement to have opposite charge $\tau$-jet candidates $\left(\mathrm{Q}_{1} \times \mathrm{Q}_{2}=-1\right)$ is not included in Table 5.12. It reduces the QCD multi-jet 
Table 5.13. The number of expected events with $60 \mathrm{fb}^{-1}$ and efficiencies of some of the selections for the irreducible backgrounds.

\begin{tabular}{|c|c|c|c|c|c|}
\hline process & $\begin{array}{l}N_{\text {exp. }} \text { at } \\
60 \mathrm{fb}^{-1}\end{array}$ & $\begin{array}{l}\mathrm{Q}_{\tau 1} \times \mathrm{Q}_{\tau 2} \\
=-1\end{array}$ & $\begin{array}{l}\text { only one } \\
\text { extra jet }\end{array}$ & $\begin{array}{l}\text { b tag. } \\
\text { jet }\end{array}$ & $\begin{array}{l}\mathbf{M}_{\tau \tau} \\
\text { window }\end{array}$ \\
\hline$t \bar{t}$ & 0.64 & 0.96 & 0.36 & 0.42 & 0.11 \\
\hline $\mathrm{W}+\mathrm{j}$ & 0.33 & 0.81 & 0.15 & 0.06 & 0.12 \\
\hline Wt & 0.26 & 0.96 & 0.49 & 0.44 & 0.23 \\
\hline \multicolumn{6}{|c|}{$\mathrm{Z} / \gamma^{*} \rightarrow \tau \tau$ in bins of generated $\mathrm{m}_{\tau \tau}$} \\
\hline $130<\mathrm{m}_{\tau \tau}<300 \mathrm{GeV} / \mathrm{c}^{2}$ & 3.80 & 0.96 & 0.23 & 0.06 & 0.61 \\
\hline $\mathrm{m}_{\tau \tau}>300 \mathrm{GeV} / \mathrm{c}^{2}$ & 0.18 & 0.95 & 0.27 & 0.05 & 0.04 \\
\hline$\tau \tau \mathrm{b} \overline{\mathrm{b}}, \mathrm{m}_{\tau \tau}>100 \mathrm{GeV} / \mathrm{c}^{2}$ & 0.86 & 0.98 & 0.39 & 0.44 & 0.38 \\
\hline
\end{tabular}

background by another factor of two, leading to 104 events of the QCD multi-jet background expected with $60 \mathrm{fb}^{-1}$. With the selections applied to search for signals of $\mathrm{M}_{\mathrm{A}}=500 \mathrm{GeV} / \mathrm{c}^{2}$ and $\mathrm{M}_{\mathrm{A}}=800 \mathrm{GeV} / \mathrm{c}^{2}$ the expected numbers of the QCD multi-jet background with $60 \mathrm{fb}^{-1}$ are 25.0 and 4.0, respectively.

Irreducible background. The irreducible background which remains after all selections were applied is the small part of the total background dominated by the QCD multi-jet events. Table 5.13 summarises the expected number of events from the irreducible background with $60 \mathrm{fb}^{-1}$ for the selections used to search for a signal of $\mathrm{M}_{\mathrm{A}}=200 \mathrm{GeV} / \mathrm{c}^{2}$. In total, 6.0 events are expected. The efficiencies of some of the selections are also shown in the table. With the selections applied to search for signals of $\mathrm{M}_{\mathrm{A}}=500 \mathrm{GeV} / \mathrm{c}^{2}$ and $\mathrm{M}_{\mathrm{A}}=800 \mathrm{GeV} / \mathrm{c}^{2}$ the expected numbers of the irreducible background with $60 \mathrm{fb}^{-1}$ are 4.0 and 1.0 , respectively.

5.2.6.4. Detector effects, experimental systematics and evaluation of the background from data.

$\mathbf{E}_{\mathbf{T}}^{\text {miss }}$ and jet energy scale uncertainties. The effect of the $E_{\mathrm{T}}^{\text {miss }}$ and the jet energy scale uncertainty on the Higgs boson mass reconstruction efficiency was estimated. The $\mathrm{E}_{\mathrm{T}}^{\text {miss }}$ is reconstructed with the Type 1 corrections in the following form:

$$
\mathrm{E}_{\mathrm{Tx}(\mathrm{y})}^{\mathrm{miss}}=-\left(\mathrm{E}_{\mathrm{Tx}(\mathrm{y})}^{\mathrm{raw}}+\sum_{\text {jets }}\left(\mathrm{E}_{\mathrm{Tx}(\mathrm{y})}^{\text {corr.jet }}-\mathrm{E}_{\mathrm{Tx}(\mathrm{y})}^{\mathrm{rawjet}}\right)\right)
$$

where $E_{\mathrm{T} x(y)}^{\text {raw }}$ is the sum over the raw calorimeter tower energies from calorimeter towers and the jet sum in the equation is over jets with a reconstructed $\mathrm{E}_{\mathrm{T}}^{\mathrm{raw}}>25 \mathrm{GeV}$. The formula can be rewritten in the form:

$$
\mathrm{E}_{\mathrm{T} x(y)}^{\text {miss }}=-\left(\left[\mathrm{E}_{\mathrm{Tx}(\mathrm{y})}^{\mathrm{raw}}-\sum_{\text {jets }} \mathrm{E}_{\mathrm{Tx}(\mathrm{y})}^{\text {rawjet }}\right]_{\text {low } \mathrm{E}_{\mathrm{T}}}+\left[\sum_{\text {jets }} \mathrm{E}_{\mathrm{Tx}(\mathrm{y})}^{\text {corr.jet }}\right]_{\mathrm{high} E_{\mathrm{T}}}\right)
$$

representing of low and high $\mathrm{E}_{\mathrm{T}}$ parts. For the low $\mathrm{E}_{\mathrm{T}}$ part a scale uncertainty of $10 \%$ was applied, while for the high $\mathrm{E}_{\mathrm{T}}$ part $3 \%$ uncertainty was used. The variation of the scale is applied independently for the two parts to obtain the maximal upper and lower deviations from the case with no uncertainty. It was found that the $\mathrm{E}_{\mathrm{T}}^{\mathrm{miss}}$ scale uncertainty brings the largest contribution to the uncertainty of the Higgs boson mass reconstruction efficiency. In the worst case the uncertainty reaches $3 \%$. The mean fitted value of the $\mathbf{M}_{\tau \tau}$ distribution for a 

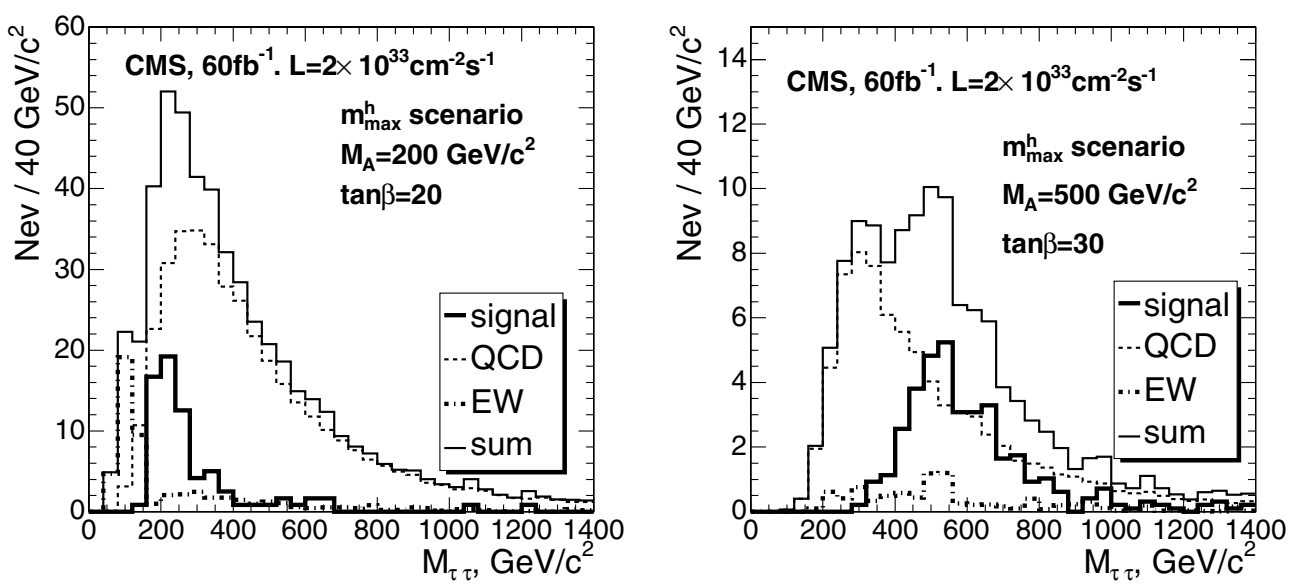

Figure 5.3. The expected $M_{\tau \tau}$ distributions for the signal of $M_{A}=200 \mathrm{GeV} / \mathrm{c}^{2}, \tan \beta=20$ (left plot) and $\mathrm{M}_{\mathrm{A}}=500 \mathrm{GeV} / \mathrm{c}^{2}, \tan \beta=30$ (right plot) and the background with $60 \mathrm{fb}^{-1}$. Thick solid histogram - signal in the $\mathrm{m}_{\mathrm{h}}^{\max }$ scenario; dashed histogram - the QCD multi-jet background; thick dashed-dotted histogram - the irreducible background; normal solid histogram - signal plus background.

signal of $\mathrm{M}_{\mathrm{A}}=500 \mathrm{GeV} / \mathrm{c}^{2}$ is varied from $-10 \mathrm{GeV} / \mathrm{c}^{2}$ to $+16 \mathrm{GeV} / \mathrm{c}^{2}$ relative to the mean value evaluated without the scale uncertainty taken into account.

Tracker misalignment. The effect of the tracker misalignment on the rate of fake $\tau$-jets from the QCD multi-jet background was studied for the first data taking scenario (Scenario 1) and the long term data taking scenario (Scenario 2). The tracker isolation efficiency and the efficiency of the track counting in the signal cone (one or three tracks requirement) was compared with the performance of the perfect tracker alignment (Scenario 0).

It was found that in the Scenario 2 the QCD multi-jet background can be increased by $\simeq 11 \%$ due to the change of the tracker isolation efficiency. The efficiency of the requirement to have one track in the signal cone is increased by $\simeq 10 \%$ in the Scenario 2 relative to the perfect alignment.

The measurement of the QCD multi-jet background from the data. Figure 5.3 (left plot) shows the expected $\mathrm{M}_{\tau \tau}$ distribution for two signal samples and the background. The QCD multi-jet background is the biggest background in this analysis. The following way to evaluate this background from the data is proposed: A control sample must be used where all signal selections are applied except the mass window and the requirement to have an opposite charge of the two $\tau$-jet candidates. It is proposed to select, instead, the sample with the same charge of the two $\tau$-jet candidates (SS sample). The contamination of the signal events and irreducible background is negligible in the SS sample, thus giving the possibility to predict from the data the QCD multi-jet background in a given mass window from the number of event and the measured shape of the di $\tau$-jet mass in SS sample. The expected number of QCD multi-jet SS events after all selections, but the mass window, used for the signal of $M_{A}=200 \mathrm{GeV} / \mathrm{c}^{2}$ is 380 with $60 \mathrm{fb}^{-1}$. Neglecting the uncertainty of the measured shape of the di $\tau$-jet mass leads to $5 \%$ statistical uncertainty of the QCD multi-jet background estimates under the signal mass window. For the $\mathrm{M}_{\mathrm{A}}=500$ (800) $\mathrm{GeV} / \mathrm{c}^{2}$ selections about 80 (28) SS QCD multi-jet events are expected, thus giving $\simeq 10$ (20) $\%$ statistical uncertainty. 
Table 5.14. The lower limit of $\tan \beta$ where a $5 \sigma$ discovery is possible with $60 \mathrm{fb}^{-1}$.

\begin{tabular}{llll}
\hline $\begin{array}{l}\text { Low } \tan \beta \text { limit } \\
\text { for } 5 \sigma \text { discovery }\end{array}$ & $\begin{array}{l}\text { Higgs boson mass } \\
\mathrm{m}_{\mathrm{A}}=200 \mathrm{GeV} / \mathrm{c}^{2}\end{array}$ & $\mathrm{~m}_{\mathrm{A}}=500 \mathrm{GeV} / \mathrm{c}^{2}$ & $\mathrm{~m}_{\mathrm{A}}=800 \mathrm{GeV} / \mathrm{c}^{2}$ \\
\hline no systematics & 20 & 32 & 46 \\
with systematics & 21 & 34 & 49 \\
\hline
\end{tabular}

5.2.6.5. Discovery reach in the $\mathrm{M}_{\mathrm{A}}-\tan \beta$ plane. Table 5.14 shows the lowest value of $\tan \beta$ for the three Higgs boson masses considered in the analysis, where the $5 \sigma$ discovery is possible with $60 \mathrm{fb}^{-1}$. It is shown with and without QCD multi-jet background systematic uncertainty taken into account. The significance of the discovery is calculated with the $\mathrm{S}_{\mathrm{cP}}$ method.

The extension of the discovery reach to lower values of $\tan \beta$ would be possible with a lower threshold on the energy of the additional jet in the event, provided that the fake jets will be then suppressed with the jet-tracks matching criteria. Another improvement is expected from the increase of the Higgs boson mass reconstruction efficiency using the improved missing $\mathrm{E}_{\mathrm{T}}$ measurement from energy-flow like algorithms. Finally, improved b-jet tagging performance is expected to extend the discovery reach to lower values of $\tan \beta$.

\subsection{7. $\mathrm{H} \rightarrow \tau \tau \rightarrow \mu+$ jet analysis}

A detailed description of the analysis can be found in [152].

5.2.7.1. Event generation and pre-selections. For the irreducible Drell-Yan (DY) $\tau \tau$ background the $\tau_{1(2)} \rightarrow \mu \nu \nu, \tau_{2(1)} \rightarrow$ hadrons $+\nu$ decays were forced in PYTHIA. The events containing $\mathrm{b}$ quarks were rejected to avoid the double counting with the $\tau \tau \mathrm{b} \overline{\mathrm{b}}$ background. For the other background processes, $\overline{\mathrm{t}}, \mathrm{Wt}, \mathrm{W}+\mathrm{jet}$ and $\mathrm{b} \overline{\mathrm{b}}$ no specific decay mode was forced.

The DY $\tau \tau$ background was produced in two ranges of the $\tau \tau$ invariant mass: $40<\mathrm{m}_{\tau \tau}<120 \mathrm{GeV} / \mathrm{c}^{2}$ and $\mathrm{m}_{\tau \tau}>120 \mathrm{GeV} / \mathrm{c}^{2}$. For $\tau \tau \mathrm{b} b$ the following mass bins were used: $60<\mathrm{m}_{\tau \tau}<100 \mathrm{GeV} / \mathrm{c}^{2}$ and $\mathrm{m}_{\tau \tau}>100 \mathrm{GeV} / \mathrm{c}^{2}$. The $\mathrm{W}+$ jet background was generated with $\hat{P}_{\mathrm{T}}>20 \mathrm{GeV} / \mathrm{c}^{2}$.

The SUSY background has been estimated using the events for the LM2 mSUGRA test point (see Section 13.3.2) with the total NLO SUSY cross section of $9.4 \mathrm{pb}$. For this point $\tan \beta=35$, which makes the stau and tau production rate potentially dangerous. The number of events after all selection has been estimated to be less than one, therefore the SUSY background has been considered negligible, and was not studied in detail.

For the signal generation the Higgs boson was forced to decay into a $\tau$ pair. The $\tau$ leptons were decayed using TAUOLA and events with $\tau_{1(2)} \rightarrow \mu \nu \nu, \tau_{2(1)} \rightarrow$ hadrons $+\nu$ decays were selected.

The pre-selections at generation level were chosen in a way that selected events are likely to pass the trigger selection. The requirements were: The isolation of the muon was defined as absence of charged particles with $\mathrm{p}_{\mathrm{T}}>1 \mathrm{GeV} / \mathrm{c}$ within a cone of radius 0.2 in the $\eta-\varphi$ space around the muon momentum direction. Isolation for the $\tau$-like jet allowed for at most one charged particle with $\mathrm{p}_{\mathrm{T}}>1 \mathrm{GeV} / \mathrm{c}$ in the ring with an inner radius of 0.1 and an outer radius of 0.4 around the highest $p_{\mathrm{T}}$ charged particle in the jet. The leading track was required to have $p_{\mathrm{T}}>3 \mathrm{GeV} / \mathrm{c}$. The $\tau \tau \mathrm{b} \overline{\mathrm{b}}$ events were generated without the pre-selection requirements.

Details on $b \bar{b}$ generation are explained in [153]. 
5.2.7.2. Event selection. The off-line $\tau$-jet identification uses the parameters of the pixel HLT $\tau$ isolation, but with fully reconstructed tracks instead of pixel tracks. Additionally one or three tracks are required in the signal cone. For the $\tau$-jet direction, the sum of the momenta of the signal tracks was used, improving the direction resolution. The leading $\tau$-jet track is required to have $p_{\mathrm{T}}>10 \mathrm{GeV} / \mathrm{c}$ in case of one track in the signal cone, and $p_{\mathrm{T}}>20 \mathrm{GeV} / \mathrm{c}$ for three tracks, in order to suppress the $b \bar{b}$ and DY $\tau$ backgrounds.

To select events with associated $\mathrm{b} \overline{\mathrm{b}} H(\mathrm{~A})$ production, one b-tagged jet with calibrated $E_{\mathrm{T}}>20 \mathrm{GeV}$ was required. For the b tagging, the track counting method was used [151]: the jet is $b$ tagged if it has at least two tracks with a $2 \mathrm{D}$ transverse impact parameter significance greater than two. The $\mathrm{b}$ tagging efficiency, including the jet finding, for the signal is $17 \%$ for $\mathrm{M}_{\mathrm{A}}=200 \mathrm{GeV} / \mathrm{c}^{2}$ and $27 \%$ for $\mathrm{M}_{\mathrm{A}}=500 \mathrm{GeV} / \mathrm{c}^{2}$. For the backgrounds with a real b-jet it is $67 \%$ for $\mathrm{t} \overline{\mathrm{t}}$ and $46 \%$ for $\mathrm{Wt}$ processes. For the backgrounds without a real b-jet the mistagging efficiency is $1 \%$ for the $\mathrm{W}+\mathrm{jet}$ and $3 \%$ for the DY $\tau \tau$ processes. The $\mathrm{b}$ tagging purity for the signal and the t⿱t background is $95 \%$; it is $90 \%$ for the $\mathrm{Wb}$ and the $\tau \tau \mathrm{b} \bar{b}$ processes.

Events containing $\mathrm{W}$ bosons decaying into $\mu+v_{\mu}$ are suppressed using a cut on the transverse mass of the muon and the missing transverse energy: $m_{\mathrm{T}}=$ $\sqrt{2 \cdot p_{\mathrm{T}}^{\mu} \cdot \mathrm{E}_{\mathrm{T}}\left(1-\cos \left(\overrightarrow{\mathrm{p}}_{\mathrm{T}}^{\mu}, \overrightarrow{\mathrm{E}}_{\mathrm{T}}\right)\right)}$, where $\overrightarrow{\mathrm{E}}_{\mathrm{T}}$ is the missing transverse energy. The distribution of $m_{\mathrm{T}}$ has a Jacobian peak near the $\mathrm{W}$ mass. Rejecting events with $\mathrm{m}_{\mathrm{T}}>60 \mathrm{GeV}$ largely reduces the $\mathrm{t}, \mathrm{Wt}$ and $\mathrm{W}+$ jet backgrounds while retaining a good fraction of the signal events.

The additional selection against the $\bar{t}$ background is the central jet veto. All events containing an additional jet (to the $\tau$ jet and the b-tagged jet) in the central region, $|\eta|<2.5$, and with a calibrated $\mathrm{E}_{\mathrm{T}}>20 \mathrm{GeV}$ were rejected.

The electrons from the $\mathrm{W}$ boson decays in the $\mathrm{t} \overline{\mathrm{t}}$ and $\mathrm{Wt}$ backgrounds can be misidentified as $\tau$-jets. For the electron rejection a cut on the ratio of the $\tau$-jet energy measured in the HCAL $\left(\mathrm{E}^{\mathrm{HCAL}}\right)$ to the leading track momentum $\left(\mathrm{p}^{\mathrm{ltr}}\right), \mathrm{f}=\mathrm{E}^{\mathrm{HCAL}} / p^{\text {trt }}$, was used for the events with one track in the signal cone. The cut $f>0.2$ retains $90 \%$ of the signal events, while it rejects $95 \%$ of the events with the real electrons. The cut on the upper value of the ratio is efficient against jets with a large fraction of neutral hadrons. The requirement $\mathrm{f}<1.1$ rejects $50 \%$ of $\mathrm{W}+\mathrm{j}$ and $b \bar{b}$ events and only $20 \%$ of signal events. Figure 5.4 shows the integrated distribution of the parameter $\mathrm{f}$ for the signal and the background events selected by the High Level trigger. The labels on the right part of the figure are ordered by decreasing selection efficiency in the acceptance region of $0.2<\mathrm{f}<1.1$, marked by the arrows.

The Higgs boson mass reconstruction requires the rejection of events with a $\mu$ and a $\tau$ jet in a back-to-back topology, therefore the cut $\cos \left(\Delta \varphi\left(\overrightarrow{\mathrm{p}}_{\mathrm{T}}, \overrightarrow{\mathrm{E}}_{\mathrm{T}}^{\text {jet }}\right)\right)>-0.9962$ was used. In addition, an upper cut on $\cos \left(\Delta \varphi\left(\overrightarrow{\mathrm{p}}_{\mathrm{T}}, \overrightarrow{\mathrm{E}}_{\mathrm{T}}^{\text {jet }}\right)\right)<-0.5$ was used, retaining most of the signal events, while visibly reducing a fraction of the background events. Finally, the events with a negative reconstructed neutrino energy were rejected.

5.2.7.3. Expected number of selected events. Table 5.15 presents the production cross sections in $\mathrm{fb}$ and the individual selection efficiencies for signals of $\mathrm{M}_{\mathrm{A}}=200$ and $500 \mathrm{GeV} / \mathrm{c}^{2}$. The signal cross sections and the branching ratios were obtained for the $\mathrm{m}_{\mathrm{h}}^{\max }$ scenario with $\mu=200 \mathrm{GeV}$ (see Section 11.3.1). Tables 5.16-5.18 summarise the cross sections and the individual selection efficiencies for the background processes. The total efficiency of all selections and the cross sections after all selections are also presented at the end of the tables. The events were counted in the $\mathbf{M}_{\tau \tau}$ mass windows with the width taken to be $\pm \sigma$, where $\sigma$ is given by the standard deviation of a Gaussian fit of the signal $\mathrm{M}_{\tau \tau}$ distributions. The value of $\sigma$ is $41 \mathrm{GeV} / \mathrm{c}^{2}$ for $\mathrm{M}_{\mathrm{A}}=200 \mathrm{GeV} / \mathrm{c}^{2}$, whereas it is $83 \mathrm{GeV} / \mathrm{c}^{2}$ for $\mathrm{m}_{\mathrm{A}}=500 \mathrm{GeV} / \mathrm{c}^{2}$. With an integrated luminosity of $20 \mathrm{fb}^{-1}$ the expected number of signal (background) events is 


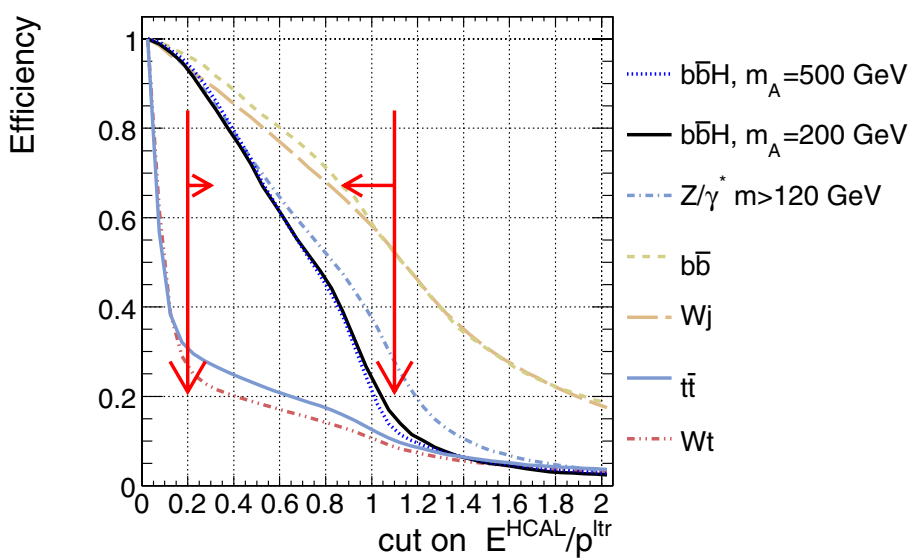

Figure 5.4. The integrated distribution of the parameter $\mathrm{f}=\mathrm{E}^{\mathrm{HCAL}} / \mathrm{p}_{\mathrm{T}}^{\mathrm{ltr}}$. The acceptance region of $0.2<\mathrm{f}<1.1$ is marked by the arrows.

Table 5.15. The production cross sections, in $\mathrm{fb}$, and the individual selection efficiencies for the signal.

\begin{tabular}{lll}
\hline & \multicolumn{1}{c}{$g g \rightarrow b \bar{b}(A+H), A, H \rightarrow \tau \tau$} \\
\cline { 2 - 3 } & $\begin{array}{l}\mathrm{M}_{\mathrm{A}}=200 \mathrm{GeV} / \mathrm{c}^{2} \\
\tan (\beta)=20\end{array}$ & $\begin{array}{l}\mathrm{M}_{\mathrm{A}}=500 \mathrm{GeV} / \mathrm{c}^{2} \\
\tan (\beta)=30\end{array}$ \\
\hline$\sigma \times \mathrm{BR}[\mathrm{fb}]$ & $9.12 \cdot 10^{3}$ & $4.51 \cdot 10^{2}$ \\
kine pre-selection & $9.47 \cdot 10^{-2}$ & $1.65 \cdot 10^{-1}$ \\
Level-1 trigger & $8.99 \cdot 10^{-1}$ & $9.09 \cdot 10^{-1}$ \\
HLT & $4.17 \cdot 10^{-1}$ & $4.99 \cdot 10^{-1}$ \\
offline $\tau$-jet isolation & $9.54 \cdot 10^{-1}$ & $9.60 \cdot 10^{-1}$ \\
1 or 3 tk. in $\tau$-jet signal cone & $9.12 \cdot 10^{-1}$ & $9.19 \cdot 10^{-1}$ \\
$\mathrm{p}_{\mathrm{T}}^{\text {ltr }}>10 \mathrm{GeV} / \mathrm{c}$ & $9.05 \cdot 10^{-1}$ & $9.55 \cdot 10^{-1}$ \\
$Q_{\mu} \cdot Q_{j e t}=-1$ & $9.61 \cdot 10^{-1}$ & $9.60 \cdot 10^{-1}$ \\
single b tagging & $1.73 \cdot 10^{-1}$ & $2.56 \cdot 10^{-1}$ \\
no jet with $E_{\mathrm{T}}>20,|\eta|<2.5$ & $8.53 \cdot 10^{-1}$ & $7.72 \cdot 10^{-1}$ \\
$m_{\mathrm{T}}(l, M E T)<60 \mathrm{GeV}$ & $8.33 \cdot 10^{-1}$ & $7.01 \cdot 10^{-1}$ \\
$-0.996<\cos (\Delta \varphi)<-0.5$ & $8.05 \cdot 10^{-1}$ & $7.51 \cdot 10^{-1}$ \\
electron veto: $0.2<f<1.1$ & $8.22 \cdot 10^{-1}$ & $8.54 \cdot 10^{-1}$ \\
$E_{v 1}>0, E_{v 2}>0$ & $6.84 \cdot 10^{-1}$ & $7.68 \cdot 10^{-1}$ \\
total efficiency: & $1.66 \cdot 10^{-3}$ & $4.53 \cdot 10^{-3}$ \\
$\sigma$ after selections $[\mathrm{fb}]:$ & $1.52 \cdot 10^{1}$ & 2.05 \\
\hline & & \\
\hline & &
\end{tabular}

146 (127) for $\mathrm{m}_{\mathrm{A}}=200 \mathrm{GeV} / \mathrm{c}^{2}, \tan \beta=20$, and 21 (61) for $\mathrm{m}_{\mathrm{A}}=500 \mathrm{GeV} / \mathrm{c}^{2}, \tan \beta=30$. Figure 5.5 shows the expected $\tau \tau$ mass distribution for the total background and for the signal plus background for $\mathrm{M}_{\mathrm{A}}=200 \mathrm{GeV} / \mathrm{c}^{2}, \tan \beta=20$ and $\mathrm{M}_{\mathrm{A}}=500 \mathrm{GeV} / \mathrm{c}^{2}, \tan \beta=30$.

5.2.7.4. Background estimates and uncertainty. After all off-line selections the main background is represented by the $\tau \tau \mathrm{b} \bar{b}$, DY $\tau \tau$ and the $\bar{t} \bar{t}$ production processes. The contribution of the non $\mathrm{Z} / \gamma^{*}$ background, mainly the $\overline{\mathrm{t}}$ events, can be estimated applying the inversion of the electron veto: $\mathrm{f}<0.1$ instead of $0.2<\mathrm{f}<1.1$. All other cuts must be the same, including the $\mathrm{M}_{\tau \tau}$ mass window. A relatively pure sample of $\bar{t} \bar{t}$ can be selected, since 
Table 5.16. The production cross sections, in $\mathrm{fb}$, and the individual selection efficiencies for the reducible background processes.

\begin{tabular}{lllll}
\hline & $t \bar{t}$ & $W+j e t$ & $W t$ & $b \bar{b}$ \\
\hline$\sigma[\mathrm{fb}]$ & $8.40 \cdot 10^{5}$ & $4.15 \cdot 10^{7}$ & $6.20 \cdot 10^{4}$ & $2.29 \cdot 10^{10}$ \\
kine preselection & $9.01 \cdot 10^{-2}$ & $1.44 \cdot 10^{-2}$ & $6.58 \cdot 10^{-2}$ & $7.56 \cdot 10^{-4}$ \\
Level-1 trigger & $9.06 \cdot 10^{-1}$ & $8.40 \cdot 10^{-1}$ & $8.91 \cdot 10^{-1}$ & $2.26 \cdot 10^{-2}$ \\
$\mathrm{H} \mathrm{LT}$ & $9.61 \cdot 10^{-2}$ & $4.16 \cdot 10^{-2}$ & $1.05 \cdot 10^{-1}$ & $2.36 \cdot 10^{-4}$ \\
offline $\tau$-jet isolation & $8.51 \cdot 10^{-1}$ & $6.70 \cdot 10^{-1}$ & $8.79 \cdot 10^{-1}$ & $8.69 \cdot 10^{-1}$ \\
1 or 3 tk. in $\tau$-jet signal cone & $8.92 \cdot 10^{-1}$ & $6.30 \cdot 10^{-1}$ & $9.07 \cdot 10^{-1}$ & $7.19 \cdot 10^{-1}$ \\
$\mathrm{p}_{\mathrm{T}}^{\text {ltr }}>10 \mathrm{GeV} / \mathrm{c}$ & $9.42 \cdot 10^{-1}$ & $8.58 \cdot 10^{-1}$ & $9.37 \cdot 10^{-1}$ & $7.17 \cdot 10^{-1}$ \\
$Q_{\mu} \cdot Q_{\text {jet }}=-1$ & $9.18 \cdot 10^{-1}$ & $7.31 \cdot 10^{-1}$ & $9.52 \cdot 10^{-1}$ & $5.45 \cdot 10^{-1}$ \\
Single b tagging & $6.73 \cdot 10^{-1}$ & $1.09 \cdot 10^{-2}$ & $4.56 \cdot 10^{-1}$ & $9.42 \cdot 10^{-2}$ \\
no jet with $E_{\mathrm{T}}>20,|\eta|<2.5$ & $3.43 \cdot 10^{-1}$ & $8.17 \cdot 10^{-1}$ & $8.60 \cdot 10^{-1}$ & $4.30 \cdot 10^{-1}$ \\
$m_{\mathrm{T}}(l, M E T)<60 \mathrm{GeV} / \mathrm{c}^{2}$ & $3.53 \cdot 10^{-1}$ & $3.76 \cdot 10^{-1}$ & $3.62 \cdot 10^{-1}$ & 1.00 \\
$-0.996<\cos (\Delta \varphi)<-0.5$ & $4.95 \cdot 10^{-1}$ & $6.56 \cdot 10^{-1}$ & $4.51 \cdot 10^{-1}$ & $4.16 \cdot 10^{-1}$ \\
electron veto: $<0.2<f<1.1$ & $1.65 \cdot 10^{-1}$ & $4.76 \cdot 10^{-1}$ & $1.27 \cdot 10^{-1}$ & $2.98 \cdot 10^{-1}$ \\
$E_{\nu 1}>0, E_{\nu 2}>0$ & $4.08 \cdot 10^{-1}$ & $2.00 \cdot 10^{-1}$ & $4.15 \cdot 10^{-1}$ & $3.60 \cdot 10^{-1}$ \\
total efficiency: & $1.54 \cdot 10^{-5}$ & $3.31 \cdot 10^{-8}$ & $1.66 \cdot 10^{-5}$ & $7.86 \cdot 10^{-11}$ \\
$\sigma$ after selections $[\mathrm{fb}]:$ & $1.30 \cdot 10^{1}$ & 1.37 & 1.03 & 1.80 \\
\hline
\end{tabular}

Table 5.17. The production cross sections, in $\mathrm{fb}$, and the individual selection efficiencies for the irreducible background processes.

\begin{tabular}{lll}
\hline & \multicolumn{2}{c}{$Z / \gamma^{*} \rightarrow \tau \tau \rightarrow \mu+$ jet } \\
\cline { 2 - 3 } & $40<m_{\tau \tau}<120 \mathrm{GeV} / \mathrm{c}^{2}$ & $m_{\tau \tau}>120 \mathrm{GeV} / \mathrm{c}^{2}$ \\
\hline$\sigma \times \mathrm{BR}[\mathrm{fb}]$ & $4.63 \cdot 10^{5}$ & $4.88 \cdot 10^{3}$ \\
kine preselection & $6.56 \cdot 10^{-2}$ & $2.14 \cdot 10^{-1}$ \\
Level-1 trigger & $8.00 \cdot 10^{-1}$ & $8.28 \cdot 10^{-1}$ \\
HLT & $1.03 \cdot 10^{-1}$ & $2.77 \cdot 10^{-1}$ \\
offline $\tau$-jet isolation & $9.12 \cdot 10^{-1}$ & $9.40 \cdot 10^{-1}$ \\
1 or 3 tk. in $\tau$-jet signal cone & $9.03 \cdot 10^{-1}$ & $8.93 \cdot 10^{-1}$ \\
$\mathrm{p}_{\mathrm{T}}^{\text {ltr }}>10 \mathrm{GeV} / \mathrm{c}$ & $8.12 \cdot 10^{-1}$ & $9.00 \cdot 10^{-1}$ \\
$Q_{\mu} \cdot Q_{j e t}=-1$ & $9.47 \cdot 10^{-1}$ & $9.33 \cdot 10^{-1}$ \\
single b tagging & $2.68 \cdot 10^{-2}$ & $2.51 \cdot 10^{-2}$ \\
no jet with $E_{\mathrm{T}}>20,|\eta|<2.5$ & $7.77 \cdot 10^{-1}$ & $6.98 \cdot 10^{-1}$ \\
$m_{\mathrm{T}}(l, M E T)<60 \mathrm{GeV} / \mathrm{c}^{2}$ & $9.41 \cdot 10^{-1}$ & $7.74 \cdot 10^{-1}$ \\
$-0.996<\cos (\Delta \varphi)<-0.5$ & $3.75 \cdot 10^{-1}$ & $6.57 \cdot 10^{-1}$ \\
electron veto: $0.2<f<1.1$ & $6.46 \cdot 10^{-1}$ & $7.29 \cdot 10^{-1}$ \\
$E_{\nu 1}>0, E_{\nu 2}>0$ & $6.45 \cdot 10^{-1}$ & $6.46 \cdot 10^{-1}$ \\
total efficiency: & $1.31 \cdot 10^{-5}$ & $1.75 \cdot 10^{-4}$ \\
$\sigma$ after selections $[\mathrm{fb}]:$ & 6.08 & $8.53 \cdot 10^{-1}$ \\
\hline & &
\end{tabular}

the requirement $\mathrm{f}<0.1$ rejects more than $95 \%$ of all processes except the $\overline{\mathrm{tt}}$ and $\mathrm{Wt}$ as shown in Figure 5.4. The number of the non $\mathrm{Z} / \gamma^{*}$ background events in the signal region can be then predicted using the ratio of the $\mathrm{tt}_{\mathrm{t}}$ events in the signal region of $0.2<\mathrm{f}<1.1$ and in the region of $\mathrm{f}<0.1$. This ratio can be obtained from Monte-Carlo simulation or from real $\bar{t} \overline{t a t a}$. The systematic uncertainty on the number of the non $\mathrm{Z} / \gamma^{*}$ background events predicted using this method has two contributions:

- The uncertainty of the HCAL energy scale, since the variable $f=E^{\mathrm{HCAL}} / \mathrm{p}^{\mathrm{ltr}}$ includes the HCAL part of the $\tau$-jet candidate energy measured by the calorimeter. It is taken as $3 \%$. 
Table 5.18. The production cross sections, in $\mathrm{fb}$, and the individual selection efficiencies for the irreducible background processes.

\begin{tabular}{lll}
\hline & \multicolumn{2}{c}{$b b(Z \rightarrow \tau \tau)$} \\
\cline { 2 - 3 } & $60<m_{\tau \tau}<100 \mathrm{GeV} / \mathrm{c}^{2}$ & $m_{\tau \tau}>100 \mathrm{GeV} / \mathrm{c}^{2}$ \\
\hline$\sigma \times$ BR [fb] & $2.61 \cdot 10^{4}$ & $1.05 \cdot 10^{3}$ \\
kine preselection & 1.00 & 1.00 \\
Level-1 trigger & $1.41 \cdot 10^{-1}$ & $1.64 \cdot 10^{-1}$ \\
HLT & $4.10 \cdot 10^{-3}$ & $1.21 \cdot 10^{-2}$ \\
offline $\tau$-jet isolation & $9.05 \cdot 10^{-1}$ & $9.34 \cdot 10^{-1}$ \\
1 or 3 tk. in $\tau$-jet signal cone & $9.12 \cdot 10^{-1}$ & $9.17 \cdot 10^{-1}$ \\
p $\mathrm{p}_{\mathrm{T}}^{\text {tr }}>10 \mathrm{GeV} / \mathrm{c}$ & $8.60 \cdot 10^{-1}$ & $8.98 \cdot 10^{-1}$ \\
$Q_{\mu} \cdot Q_{j e t}=-1$ & $9.41 \cdot 10^{-1}$ & $9.48 \cdot 10^{-1}$ \\
single b tagging & $2.73 \cdot 10^{-1}$ & $2.75 \cdot 10^{-1}$ \\
no jet with $E_{\mathrm{T}}>20,|\eta|<2.5$ & $7.20 \cdot 10^{-1}$ & $7.72 \cdot 10^{-1}$ \\
$m_{\mathrm{T}}(l, M E T)<60 \mathrm{GeV}$ & $9.68 \cdot 10^{-1}$ & $8.80 \cdot 10^{-1}$ \\
$-0.996<\cos (\Delta \varphi)<-0.5$ & $4.23 \cdot 10^{-1}$ & $5.84 \cdot 10^{-1}$ \\
electron veto: $0.2<f<1.1$ & $6.98 \cdot 10^{-1}$ & $5.11 \cdot 10^{-1}$ \\
$E_{\nu 1}>0, E_{v 2}>0$ & $4.32 \cdot 10^{-1}$ & $5.62 \cdot 10^{-1}$ \\
total efficiency: & $6.64 \cdot 10^{-5}$ & $2.76 \cdot 10^{-4}$ \\
$\sigma$ after selections $[\mathrm{fb}]:$ & 1.74 & $2.89 \cdot 10^{-1}$ \\
\hline
\end{tabular}

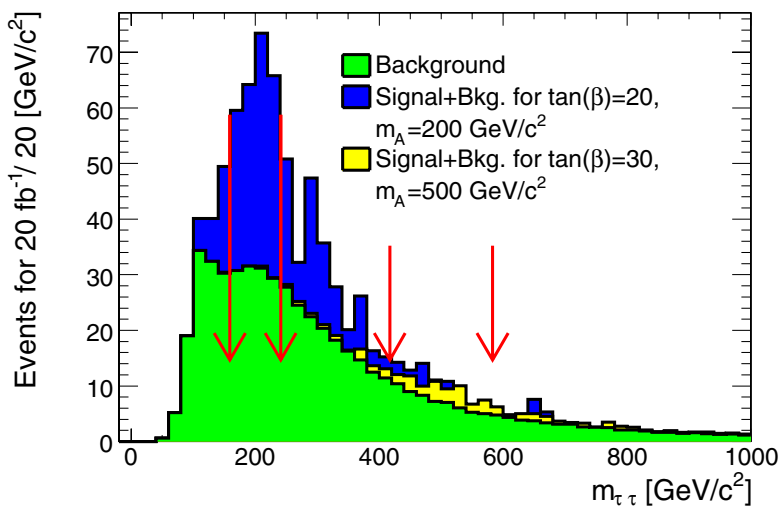

Figure 5.5. The reconstructed $\tau \tau$ mass distribution. The signal and the background contributions are shown with $20 \mathrm{fb}^{-1}$. The mass windows in which the events are counted for the significance calculations are shown.

- The uncertainty of the shape of the distribution of $\mathrm{f}$. The shape is obtained from $\mathrm{t} \overline{\mathrm{t}}$ events only, however a small fraction of events from the other processes is present in the "normalisation" region of $\mathrm{f}<0.1$. It leads to an uncertainty of $\simeq 12 \%$.

The contribution from the other systematic uncertainties, e.g. b tagging is expected to be small, due to the cancellation in the efficiency ratio. The total uncertainty on the number of the non $\mathrm{Z} / \gamma^{*}$ background events is thus $12.4 \%$.

The $\mathrm{Z} / \gamma^{*}$ background consists of two parts: the $\tau \tau \mathrm{b} \overline{\mathrm{b}}$ process and the DY $\tau \tau$ process without genuine $\mathrm{b}$ quarks in the event. The DY $\tau \tau$ background can be predicted using the DY $\ell \ell(\ell=\mathrm{e}, \mu)$ cross section, to be measured with high precision at LHC, and the selection 


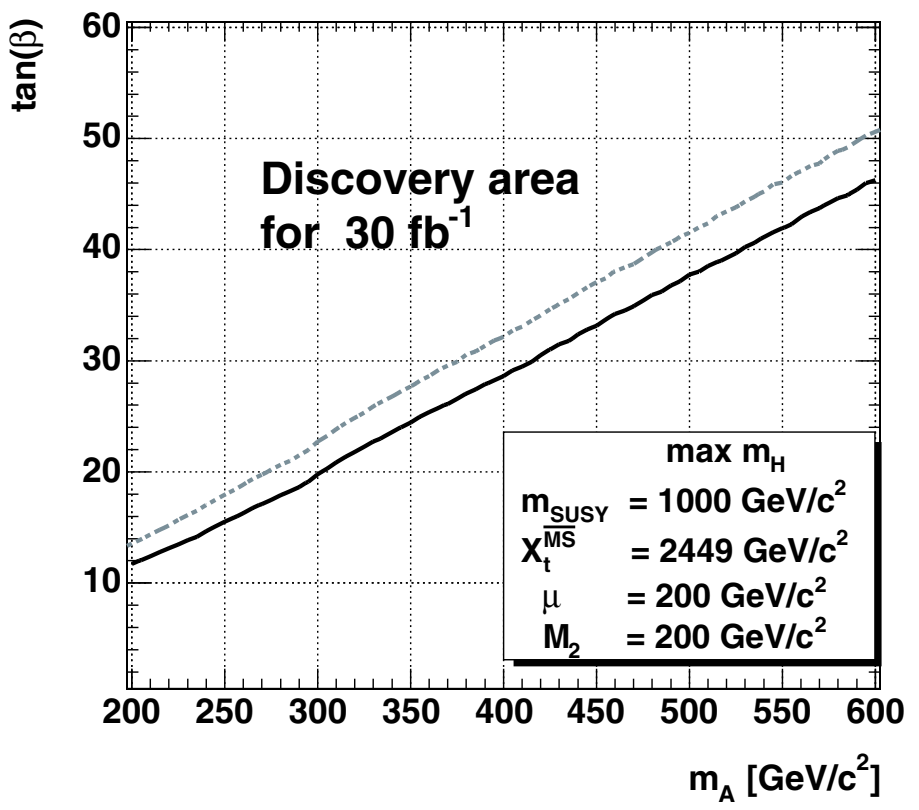

Figure 5.6. The $5 \sigma$ discovery region in the $\mathrm{M}_{\mathrm{A}}-\tan \beta$ plane with $30 \mathrm{fb}^{-1}$ of the integrated luminosity for the $\mathrm{m}_{\mathrm{h}}^{\max }$ MSSM scenario. The regions are shown without (lower curve) and with (upper curve) the uncertainty on the background taken into account.

efficiency obtained from the Monte-Carlo. The systematic uncertainty on the number of DY $\tau \tau$ events has two main contributions due to:

- The jet scale uncertainty. The number of the events in the $\mathbf{M}_{\tau \tau}$ signal window varies by $\pm 6 \%$ for jet scale variations of $\pm 3 \%$ and missing transverse energy scale variations of $\pm 5 \%$.

- The b-mistagging uncertainty. A conservative estimate of $5 \%$ is taken.

The total uncertainty on the number of the DY $\tau \tau$ events with the jet mistagged as a b-jet is therefore $8 \%$.

For the $\tau \tau \mathrm{b} \overline{\mathrm{b}}$ background estimates the systematic uncertainty has the following main contributions:

- The uncertainty of the $\mu \mu \mathrm{b} \bar{b}$ cross section measurement (without the luminosity uncertainty) is $14 \%$ [145].

- The jet scale uncertainty. It is assumed to be the same as for the DY $\tau \tau$ events.

The total uncertainty on the number of the $\tau \tau \mathrm{b} \bar{b}$ events is $15 \%$.

5.2.7.5. Discovery reach in the $\mathrm{M}_{\mathrm{A}}-\tan \beta$ plane. The CMS discovery reach in the $\mathrm{M}_{\mathrm{A}}-$ $\tan \beta$ plane with $30 \mathrm{fb}^{-1}$ in the $\mathrm{m}_{\mathrm{h}}^{\max }$ scenario is shown in Figure 5.6. The $5 \sigma$ discovery curves are shown without (lower curve) and with (upper curve) the uncertainty on the background taken into account.

\subsection{8. $\mathrm{H} \rightarrow \tau \tau \rightarrow e+$ jet analysis}

A detailed description of the analysis can be found in [154]. 
5.2.8.1. Event generation. The signal process $\mathrm{gg} \rightarrow \mathrm{bbH} / \mathrm{A}, \mathrm{H} / \mathrm{A} \rightarrow \tau \tau, \tau_{1} \rightarrow \mathrm{e} v_{\mathrm{e}} \nu_{\tau}, \tau_{2} \rightarrow$ $\tau$ jet $+v_{\tau}$ leads to a final state of one isolated electron, an isolated $\tau$ jet and one or two detectable $\mathrm{b}$ jets. The background with genuine $\tau$ 's is due to two types of events, $\mathrm{Z} / \gamma^{*}$ events decaying into $\tau \tau$, and the $\bar{t}$ events, where the $\mathrm{e}+\tau$ jet final state can come from direct $\mathrm{W}$ decays to an electron and a $\tau$ or through $\mathrm{W} \rightarrow \tau \nu_{\tau} \rightarrow \mathrm{e} \nu_{\mathrm{e}} \nu_{\tau} \nu_{\tau}$ decays:

- $\mathrm{Z} / \gamma^{*} \rightarrow \tau \tau \rightarrow \mathrm{e}+\tau \mathrm{jet}+\mathrm{X}$

- $\mathrm{b} \overline{\mathrm{b}} Z / \gamma^{*}, \mathrm{Z} / \gamma^{*} \rightarrow \tau \tau \rightarrow \mathrm{e}+\tau \mathrm{jet}+\mathrm{X}$

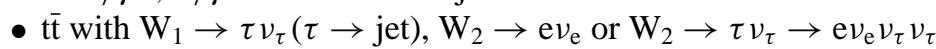

- Wt, with $\mathrm{W}_{1} \rightarrow \tau \nu_{\tau}(\tau \rightarrow$ jet $), \mathrm{W}_{2} \rightarrow \mathrm{ev}_{\mathrm{e}}$ or $\mathrm{W}_{2} \rightarrow \tau \nu_{\tau} \rightarrow \mathrm{ev}_{\mathrm{e}} \nu_{\tau} \nu_{\tau}$.

Background can arise also from the processes where a hadronic jet or an electron leads to a fake $\tau$ :

- $\mathrm{W}+$ jet, with $\mathrm{W} \rightarrow \mathrm{e} v_{\mathrm{e}}$

- $\mathrm{Z} / \gamma^{*} \rightarrow \mathrm{e}^{+} \mathrm{e}^{-}$

- $\mathrm{b} \overline{\mathrm{b} Z} / \gamma^{*}, \mathrm{Z} / \gamma^{*} \rightarrow \mathrm{e}^{+} \mathrm{e}^{-}$

- $\mathrm{t} \overline{\mathrm{t}}$ with $\mathrm{W} \rightarrow$ jj or $\mathrm{W} \rightarrow \mathrm{e} v_{\mathrm{e}}$.

The QCD multi-jet production is a large potential background through hadronic jets faking both the electron and the $\tau$ jet.

For the inclusive $\mathrm{Z} / \gamma^{*}$ production the events containing $\mathrm{b}$ quarks in the final state were removed to avoid double counting with the $\tau \tau \mathrm{b} \bar{b}$ background. The single top (Wt) events were generated with TopREX [44]. The $\tau$ decays in the signal were performed with the TAUOLA package [155].

5.2.8.2. Event selection. In the offline reconstruction an isolated electron from the decay of one of the $\tau$ 's was first searched for. On the average $\sim 1.3$ reconstructed electron candidates were found in the signal events. The reconstructed electrons were first required to be isolated in the tracker demanding that no track with $\mathrm{p}_{\mathrm{T}}>1 \mathrm{GeV} / \mathrm{c}$ was found in a cone of $\Delta \mathrm{R}=0$. 4 around the electron candidate direction. The further electron identification was performed following the algorithm of Ref. [156]. The largest contribution to the identification efficiency and purity was obtained from the ratio of hadronic cluster energy to the electromagnetic energy of the cluster $\left(E^{\text {hadronic }} / E^{\text {elm }}<0.2\right)$ and from the ratio of the supercluster energy to the track momentum $\left(E^{\text {super cluster }} / \mathrm{p}^{\text {track }}>0.8\right)$. The identification efficiency, including the tracker isolation, was found to be $64.2 \%$. A good purity of $97.5 \%$ was obtained for the selected electrons.

The off-line $\tau$-jet identification was applied to the jets with $\mathrm{E}_{\mathrm{T}}^{\mathrm{jet}}>40 \mathrm{GeV}$ reconstructed in the calorimeter with the cone of 0.4 . The leading track with $p_{\mathrm{T}}^{\mathrm{ltr}}>10 \mathrm{GeV} / \mathrm{c}$ was searched for in a cone of $\mathrm{R}_{\mathrm{m}}=0.1$ around the $\tau$-jet direction. For an efficient isolation against the hadronic jets in the $\mathrm{W}+$ jet and QCD multi-jet backgrounds, a small signal cone, $\mathrm{R}_{\mathrm{S}}=0.04$, around the leading track was used. About $83 \%$ of the $\tau^{ \pm} \rightarrow$ hadron $^{ \pm}+n \pi^{0}+v_{\tau}$ decays were found to be reconstructed as one prong $\tau$ 's. Due to the small signal cone selected, $50 \%$ of the $\tau^{ \pm} \rightarrow 3$ hadrons $^{ \pm}+\mathrm{n} \pi^{0}+v_{\tau}$ decays were reconstructed as one or two prong $\tau$-jets. The cut $\mathrm{p}_{\mathrm{T}}^{\mathrm{ltr}}>20 \mathrm{GeV} / \mathrm{c}$ was found to be optimal for the suppression of the hadronic jets, in the presence of the QCD multi-jet background. The isolation was performed counting tracks with $\mathrm{p}_{\mathrm{T}}^{\mathrm{i}}>1 \mathrm{GeV} / \mathrm{c}$ in the area between the signal cone and the isolation cone, which was taken to be then same as the jet reconstruction cone, $\mathrm{R}_{\mathrm{i}}=0.4$. Following the method described in [146], at least eight hits were required in the full silicon tracker and an upper bound of $0.3 \mathrm{~mm}$ on the transverse impact parameter was set on the leading track in order to suppress the background from the fake tracks. 
Table 5.19. Production cross sections times branching fraction, efficiencies $(\%)$ for the selection cuts and numbers of events for $30 \mathrm{fb}^{-1}$ for the signal with $\tan \beta=20$ and for $\mathrm{M}_{\mathrm{A}}=130,200,300$ and $500 \mathrm{GeV} / \mathrm{c}^{2}$.

\begin{tabular}{lllll}
\hline $\mathrm{M}_{\mathrm{A}}\left(\mathrm{GeV} / \mathrm{c}^{2}\right)$ & 130 & 200 & 300 & 500 \\
$\sigma \times \mathrm{BR}(\mathrm{pb})$ & 18.2 & 4.15 & 0.85 & 0.071 \\
Level-1 and HLT & $1.53(8.4)$ & $0.64(15.4)$ & $0.18(21.6)$ & $2.0 \times 10^{-2}(28.7)$ \\
primary vertex & $1.44(94.1)$ & $0.60(94.2)$ & $0.18(97.2)$ & $1.9 \times 10^{-2}(93.6)$ \\
electron identification & $1.11(77.8)$ & $0.48(80.8)$ & $0.14(73.7)$ & $1.4 \times 10^{-2}(73.8)$ \\
one identified $\tau$ jet & $0.127(11.4)$ & $0.11(23.4)$ & $4.5 \times 10^{-2}(32.9)$ & $5.9 \times 10^{-3}(41.7)$ \\
$\mathrm{Q}^{\tau}$ jet $\times \mathrm{Q}^{\mathrm{e}}=-1$ & $0.127(100.0)$ & $0.11(99.1)$ & $4.5 \times 10^{-2}(99.3)$ & $5.8 \times 10^{-3}(99.0)$ \\
$\mathrm{m}_{\mathrm{T}}<40 \mathrm{GeV} / \mathrm{c}^{2}$ & $9.9 \times 10^{-2}(77.6)$ & $3.8 \times 10^{-2}(73.7)$ & $3.1 \times 10^{-2}(69.3)$ & $3.9 \times 10^{-3}(66.7)$ \\
$\geqslant 1$ jet, $\mathrm{E}_{\mathrm{T}}>20 \mathrm{GeV}$ & $4.5 \times 10^{-2}(45.9)$ & $3.8 \times 10^{-2}(46.6)$ & $1.5 \times 10^{-2}(48.6)$ & $2.1 \times 10^{-3}(53.5)$ \\
$\mathrm{b}$ tagging & $1.3 \times 10^{-2}(29.7)$ & $1.2 \times 10^{-2}(32.2)$ & $5.0 \times 10^{-3}(32.9)$ & $7.6 \times 10^{-4}(36.5)$ \\
jet veto & $8.1 \times 10^{-3}(60.2)$ & $7.2 \times 10^{-2}(62.5)$ & $3.1 \times 10^{-3}(63.2)$ & $4.6 \times 10^{-4}(61.0)$ \\
$\Delta \varphi\left(\tau_{1}, \tau_{2}\right)<175^{\circ}$ & $7.6 \times 10^{-3}(94.8)$ & $6.8 \times 10^{-3}(93.9)$ & $2.7 \times 10^{-3}(85.7)$ & $3.4 \times 10^{-4}(74.5)$ \\
$\mathrm{E}_{v_{1}, \nu_{2}}>0$ & $4.1 \times 10^{-3}(54.1)$ & $4.2 \times 10^{-3}(61.7)$ & $1.7 \times 10^{-3}(64.3)$ & $2.4 \times 10^{-4}(70.6)$ \\
$\mathrm{N}_{\mathrm{ev}}$ at $30 \mathrm{fb}^{-1}$ & 123.9 & 126.0 & 51.9 & 7.3 \\
\hline
\end{tabular}

The $\mathrm{Z} / \gamma^{*} \rightarrow \mathrm{e}^{+} \mathrm{e}^{-}$and $\mathrm{b} \overline{\mathrm{b}} \mathrm{Z} / \gamma^{*}, \mathrm{Z} / \gamma^{*} \rightarrow \mathrm{e}^{+} \mathrm{e}^{-}$backgrounds contain an isolated genuine electron to pass the electron cuts and are not significantly suppressed with the $\tau$-selection cuts. These electronic $\tau$ candidates were suppressed requiring a large energy deposition in the hadron calorimeter. A cut in the $\mathrm{E}_{\mathrm{T}}$ of the most energetic HCAL cell in the $\tau$ jet, $\mathrm{E}_{\mathrm{T}}($ max HCAL cell $)>2 \mathrm{GeV}$, was found to suppress the electrons with a factor of $\sim 7$. A further reduction was obtained comparing the HCAL energy and the leading track momentum of the $\tau$ jet. The cut $\mathrm{E}^{\mathrm{HCAL}} / \mathrm{p}^{\text {trr }}>0.35$, applied on the one-prong $\tau$ candidates only, was found to suppress further the electronic $\tau$ candidates by a factor of $\sim 1.8$. The $\mathrm{W}+$ jet events show a tail at large values of $\mathrm{E}^{\mathrm{HCAL}} / \mathrm{p}^{\text {ltr }}$ due to the neutral hadron component of the hadronic jets and were suppressed with the cut $\mathrm{E}^{\mathrm{HCAL}} / \mathrm{p}^{\text {ltr }}<1.5$.

Efficiencies of the $\tau$-jet selections are shown in Tables 5.19, 5.20 and 5.21. The purity of $\sim 97 \%$ is obtained for the signal events. A rejection factor of $\sim 400$ was obtained for the QCD multi-jet events generated with $50<\hat{\mathrm{p}}_{\mathrm{T}}<80 \mathrm{GeV} / \mathrm{c}$ when the $\tau$-jet selections described above were applied.

Finally, the charges of the electron and $\tau$ jet were required to be opposite. The charge of the $\tau$ jet was calculated as the sum of charges of the tracks in the signal cone.

The missing transverse energy measurement can be exploited to suppress the $t \bar{t}$ background with an upper bound on the transverse mass $\mathrm{m}_{\mathrm{T}}\left(\mathrm{e}, \mathrm{E}_{\mathrm{T}}^{\mathrm{miss}}\right)$ reconstructed from the electron and the missing transverse energy. Figure 5.7 shows the $\mathrm{m}_{\mathrm{T}}\left(\mathrm{e}, \mathrm{E}_{\mathrm{T}}^{\mathrm{miss}}\right)$ distribution for the signal events with $\mathrm{M}_{\mathrm{A}}=200 \mathrm{GeV} / \mathrm{c}^{2}$ and for the $\overline{\mathrm{t}}$ and $\mathrm{Z} / \gamma^{*} \rightarrow \mathrm{e}^{+} \mathrm{e}^{-}$backgrounds with the electron and $\tau$-jet selections. The selected upper bound $\mathrm{m}_{\mathrm{T}}\left(\mathrm{e}, \mathrm{E}_{\mathrm{T}}^{\text {miss }}\right)<40 \mathrm{GeV} / \mathrm{c}^{2}$ reduces the $t \bar{t}$ background with a factor of $\sim 4$.

The events were further selected when at least one jet (in addition to the $\tau$ jet) with calibrated $\mathrm{E}_{\mathrm{T}}^{\mathrm{jet}}>20 \mathrm{GeV}$ and $|\eta|<2.5$ was found and tagged as the $\mathrm{b}$ jet. A probabilistic secondary vertex algorithm with a discriminator cut from Ref. [157] was used for $b$ tagging. The cut in the discriminator was set to 0.8 , which suppresses efficiently the $\mathrm{Z} / \gamma^{*}$, $\mathrm{W}+\mathrm{jet}$ and the potential multi-jet background. The efficiency to tag at least one jet, including the jet finding efficiency, was found to be between 13 and $19 \%$ for the signal, below $1 \%$ for the $\mathrm{Z} / \gamma^{*}$ backgrounds and $1.3 \%$ for the $\mathrm{W}+\mathrm{jet}$ background. For the signal events the purity of the b-tagged jets is very high (99\%). 
Table 5.20. Background production cross sections times branching fraction, cross sections and efficiencies $(\%)$ for the selection cuts and number of events for $30 \mathrm{fb}^{-1}$

\begin{tabular}{lllll}
\hline & $\mathrm{Z} / \gamma^{*} \rightarrow \tau \tau$ & $\mathrm{b} \overline{\mathrm{b} Z} / \gamma^{*} \rightarrow \tau \tau$ & $\mathrm{Z} / \gamma^{*} \rightarrow \mathrm{e}^{+} \mathrm{e}^{-}$ & $\mathrm{b} \overline{\mathrm{b} Z} / \gamma^{*} \mathrm{e}^{+} \mathrm{e}^{-}$ \\
\hline$\sigma \times \mathrm{BR}(\mathrm{pb})$ & 331.8 & 27.0 & 1890 & 26.3 \\
pre-selection & $173.5(41.4)$ & & $811.2(42.9)$ & \\
Level-1 and HLT & $17.3(10.0)$ & $0.818(3.1)$ & $617.4(76.1)$ & $18.2(67.2)$ \\
primary vertex & $16.5(95.4)$ & $0.796(97.3)$ & $591.9(95.9)$ & $17.7(97.3)$ \\
no b's in DY Z/ $\gamma^{*}$ & $15.6(94.6)$ & & $561.8(94.9)$ & \\
electron identification & $11.6(74.4)$ & $0.585(80.2)$ & $278.1(50.1)$ & $9.31(52.6)$ \\
one identified $\tau$ jet & $0.13(1.2)$ & $1.0 \times 10^{-2}(1.8)$ & $3.40(1.2)$ & $9.0 \times 10^{-2}(1.0)$ \\
$\mathrm{Q}^{\tau \text { jet }} \times \mathrm{Q}^{\mathrm{e}}=-1$ & $0.13(96.3)$ & $1.0 \times 10^{-2}(100)$ & $3.31(97.4)$ & $8.8 \times 10^{-2}(97.8)$ \\
$\mathrm{m}_{\mathrm{T}}<40 \mathrm{GeV} / \mathrm{c}^{2}$ & $9.8 \times 10^{-2}(76.3)$ & $8.0 \times 10^{-3}(80.0)$ & $2.26(68.3)$ & $5.5 \times 10^{-2}(62.5)$ \\
$\geqslant 1$ jet, $\mathrm{E}_{\mathrm{T}}>20 \mathrm{GeV}$ & $4.0 \times 10^{-2}(40.6)$ & $5.6 \times 10^{-3}(70.0)$ & $0.85(37.6)$ & $3.0 \times 10^{-2}(54.2)$ \\
$\mathrm{b}$ tagging & $8.0 \times 10^{-4}(2.0)$ & $2.6 \times 10^{-3}(46.4)$ & $1.5 \times 10^{-2}(1.8)$ & $9.6 \times 10^{-3}(32.2)$ \\
jet veto & $5.2 \times 10^{-4}(65.0)$ & $1.5 \times 10^{-3}(57.7)$ & $6.0 \times 10^{-3}(41.4)$ & $5.9 \times 10^{-3}(67.4)$ \\
$\Delta \varphi\left(\tau_{1}, \tau_{2}\right)<175^{\circ}$ & $4.9 \times 10^{-4}(94.2)$ & $1.4 \times 10^{-3}(90.7)$ & $4.8 \times 10^{-3}(80.0)$ & $5.1 \times 10^{-3}(85.7)$ \\
$\mathrm{E}_{v_{1}, \nu_{2}}>0$ & $2.0 \times 10^{-4}(40.2)$ & $7.6 \times 10^{-4}(55.9)$ & $1.7 \times 10^{-3}(35.4)$ & $1.9 \times 10^{-3}(50.0)$ \\
$\mathrm{N}_{\mathrm{ev}}$ at $30 \mathrm{fb}^{-1}$ & 5.9 & 22.8 & 51.3 & 57.9 \\
\hline
\end{tabular}

Table 5.21. Background production cross sections times branching fraction ( $\mathrm{pb}$ ), cross sections and efficiencies $(\%)$ for the selection cuts and number of events for $30 \mathrm{fb}^{-1}$.

\begin{tabular}{llll}
\hline & $\mathrm{t} \overline{\mathrm{t}}$ & $\mathrm{Wt}$ & $\mathrm{W}+$ jet \\
\hline$\sigma \times \mathrm{BR}(\mathrm{pb})$ & 840 & 6.16 & 673.2 \\
pre-selection & & & $315.0(46.8)$ \\
Level-1 and HLT & $94.4(11.3)$ & $2.00(32.5)$ & $145.6(46.2)$ \\
primary vertex & $93.9(99.5)$ & $1.97(98.5)$ & $143.9(98.8)$ \\
electron identification & $66.7(71.0)$ & $1.43(72.6)$ & $114.2(79.4)$ \\
one id. $\tau$ jet & $0.66(0.95)$ & $4.10 \times 10^{-2}(2.87)$ & $0.57(0.5)$ \\
$\mathrm{Q}^{\tau}$ jet $\times \mathrm{Q}^{\mathrm{e}}=-1$ & $0.57(89.8)$ & $4.00 \times 10^{-2}(97.6)$ & $0.47(82.7)$ \\
$\mathrm{m}_{\mathrm{T}}\left(\mathrm{e}, \mathrm{E}_{\mathrm{T}}^{\mathrm{miss}}\right)<40 \mathrm{GeV} / \mathrm{c}^{2}$ & $0.14(24.3)$ & $8.0 \times 10^{-3}(20.0)$ & $0.12(25.2)$ \\
$\geqslant 1$ jet, $\mathrm{E}_{\mathrm{T}}>20 \mathrm{GeV}$ & $0.14(98.6)$ & $6.9 \times 10^{-3}(86.3)$ & $5.5 \times 10^{-2}(46.2)$ \\
$\mathrm{b}$ tagging & $9.4 \times 10^{-2}(68.6)$ & $4.1 \times 10^{-3}(59.4)$ & $1.6 \times 10^{-3}(2.9)$ \\
jet veto & $5.1 \times 10^{-3}(5.4)$ & $2.38 \times 10^{-3}(58.1)$ & $6.6 \times 10^{-4}(41.9)$ \\
$\Delta \varphi\left(\tau_{1}, \tau_{2}\right)<175^{\circ}$ & $4.9 \times 10^{-3}(96.4)$ & $2.33 \times 10^{-3}(98.0)$ & $5.6 \times 10^{-4}(83.9)$ \\
$\mathrm{E}_{v_{1}, v}>0$ & $2.0 \times 10^{-3}(40.9)$ & $9.60 \times 10^{-4}(41.2)$ & $2.1 \times 10^{-4}(38.5)$ \\
$\mathrm{N}_{\mathrm{ev}}$ at $30 \mathrm{fb}^{-1}$ & 60.3 & 28.8 & 6.4 \\
\hline
\end{tabular}

The $\bar{t} \bar{t}$ background, with a genuine electron, $\tau$ and $b$ jets, cannot be significantly suppressed with the cuts described above. This background, however, was suppressed applying the jet veto: the event must contain only the b-tagged jet with calibrated $\mathrm{E}_{\mathrm{T}}^{\mathrm{jet}}>20 \mathrm{GeV}$ and $\left|\eta^{\mathrm{jet}}\right|<2.5$. The fake jets, which generally do not contain tracks from the signal vertex, were suppressed with a cut in the fraction of the track $\mathrm{p}_{\mathrm{T}}$ sum to the jet $\mathrm{E}_{\mathrm{T}}, \alpha=\Sigma \mathrm{p}_{\mathrm{T}}^{\mathrm{t} \text { track }} / \mathrm{E}_{\mathrm{T}}^{\mathrm{jet}}$. The cut $\alpha>0.1$ was found to improve the veto efficiency for the signal by about $10 \%$. The jet veto efficiency is around $60 \%$ for the signal and $\sim 5 \%$ for the $\bar{t}$ background.

For the reconstruction of the $\tau \tau$ mass the events with back-to-back configurations between the electron and the $\tau$ jet were removed with an upper bound on the angle between the $\tau$ jet and the electron in the transverse plane $(\Delta \varphi(\mathrm{e}, \tau$ jet $))$. The reconstructed neutrino energies were required to be positive $\left(E_{v_{1}}>0\right.$ and $\left.E_{v_{2}}>0\right)$, which leads to a reduction 


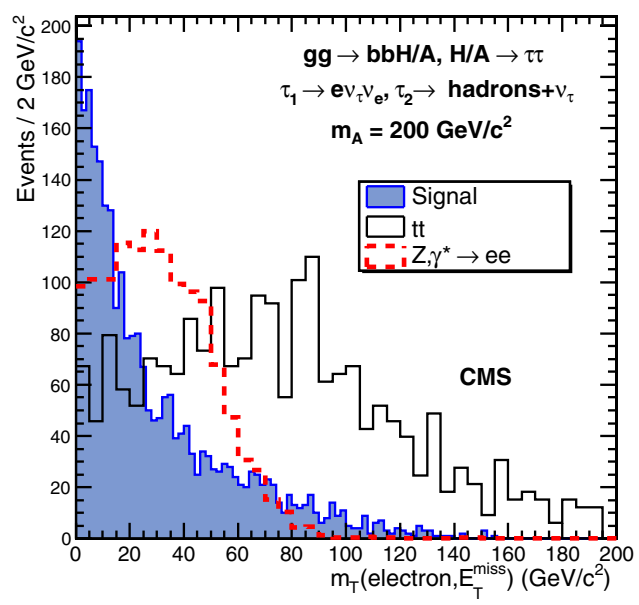

Figure 5.7. Distribution of transverse mass reconstructed from the electron and the missing transverse energy for the signal of $\mathrm{M}_{\mathrm{A}}=200 \mathrm{GeV} / \mathrm{c}^{2}$ and $\tan \beta=20$ (filled histogram), for the $\overline{\mathrm{t}}$ (solid line) and for the $\mathrm{Z} / \gamma^{*} \rightarrow \mathrm{e}^{+} \mathrm{e}^{-}$(dashed line) background. Histogram normalisation is arbitrary.

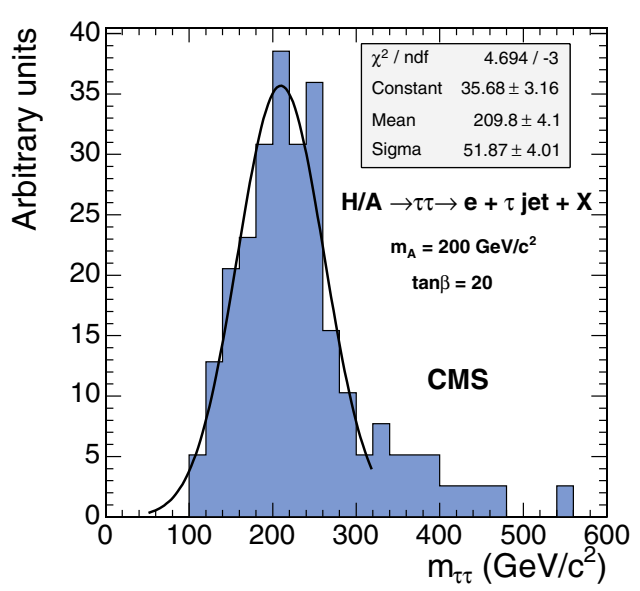

Figure 5.8. Reconstructed Higgs boson mass for $\mathrm{M}_{\mathrm{A}}=$ $200 \mathrm{GeV} / \mathrm{c}^{2}$ and $\tan \beta=20$.

of $\simeq 40 \%$ of the signal events, but rejects $\simeq 60 \%$ of the $\bar{t}, \mathrm{tW}$ and $\mathrm{W}+$ jet backgrounds. Figure 5.8 shows the reconstructed Higgs boson mass for the signal events with $\mathrm{M}_{\mathrm{A}}=$ $200 \mathrm{GeV} / \mathrm{c}^{2}$. The Gaussian fit yields a mass resolution of $25 \%$.

Table 5.19 shows the numbers of signal events with $\mathrm{M}_{\mathrm{A}}=130-500 \mathrm{GeV} / \mathrm{c}^{2}$ and $\tan \beta=20$ for $30 \mathrm{fb}^{-1}$ and the efficiency for all the event selection cuts described above. For $\mathrm{M}_{\mathrm{A}}=130$ and $140 \mathrm{GeV} / \mathrm{c}^{2}$, the mass of the lighter scalar Higgs boson $\mathrm{h}$ is only 4.4 and $11.2 \mathrm{GeV} / \mathrm{c}^{2}$ smaller than $\mathrm{M}_{\mathrm{A}}$. With the mass resolution, which can be reached in the $\mathrm{H} \rightarrow \tau \tau$ decay channels, the lighter scalar contributes to the signal and is added in the cross sections for $\mathrm{M}_{\mathrm{A}}=130$ and $140 \mathrm{GeV} / \mathrm{c}^{2}$. The contribution is 31 and $11 \%$ of the total production rate, respectively. Table 5.20 shows the number of events and efficiencies for the backgrounds originating from $\mathrm{Z} / \gamma^{*} \rightarrow \tau \tau$ and $\mathrm{Z} / \gamma^{*} \rightarrow \mathrm{e}^{+} \mathrm{e}^{-}$decays in the inclusive and in the associated $\mathrm{b} \overline{\mathrm{b} Z} / \gamma^{*}$ production. The efficiency of removing the $\mathrm{b} \overline{\mathrm{b} Z} / \gamma^{*}$ component from the inclusive $\mathrm{Z} / \gamma^{*}$ samples is also shown. Table 5.21 shows the same for the backgrounds involving W's from $\mathrm{t} \overline{\mathrm{t}}, \mathrm{Wt}$ and $\mathrm{W}+$ jet events. The cross section times branching fraction, trigger efficiency and the efficiency of the primary vertex reconstruction are also shown in the tables. The QCD multi-jet background after all selections was estimated to be 8.4 events for $30 \mathrm{fb}^{-1}$ in the mass window around $\mathrm{M}_{\mathrm{A}}=200 \mathrm{GeV} / \mathrm{c}^{2}$, which is $\simeq 10 \%$ of all other backgrounds.

Figures 5.9 and 5.10 show the reconstructed Higgs boson mass distributions of the $\mathrm{H} / \mathrm{A} \rightarrow \tau^{+} \tau^{-} \rightarrow$ electron + jet $+\mathrm{X}$ signal and the total background for $30 \mathrm{fb}^{-1}$ for $\mathrm{M}_{\mathrm{A}}=$ $200 \mathrm{GeV} / \mathrm{c}^{2}, \tan \beta=20$ and for $\mathrm{M}_{\mathrm{A}}=300 \mathrm{GeV} / \mathrm{c}^{2}, \tan \beta=25$. The sum of the $\mathrm{Z} / \gamma^{*} \rightarrow \mathrm{e}^{+} e^{-}$ and $\mathrm{b} \overline{\mathrm{b}} \mathrm{Z} / \gamma^{*} \rightarrow \mathrm{e}^{+} \mathrm{e}^{-}$backgrounds is shown separately in the figures.

5.2.8.3. Systematic uncertainties for the background determination. The background uncertainty was evaluated using the cross-section uncertainties (measured or predicted from the theory) and the experimental uncertainties for the event selections.

The uncertainty of the event selection efficiency is related to the uncertainty of the electron and $\tau$ identification, the absolute calorimeter scale and the b-tagging efficiency. 


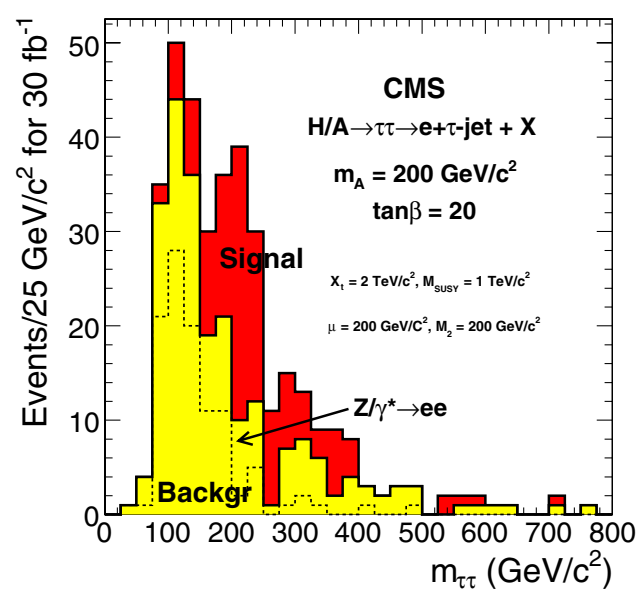

Figure 5.9. Reconstructed Higgs boson mass for the signal of $\mathrm{M}_{\mathrm{A}}=200 \mathrm{GeV} / \mathrm{c}^{2}, \tan \beta=20$ and for the total background for an integrated luminosity of $30 \mathrm{fb}^{-1}$. The dashed line shows the sum of the $\mathrm{Z} / \gamma^{*} \rightarrow \mathrm{e}^{+} e^{-}$and $\mathrm{b} \overline{\mathrm{b}} \mathrm{Z} / \gamma^{*} \mathrm{e}^{+} \mathrm{e}^{-}$backgrounds.

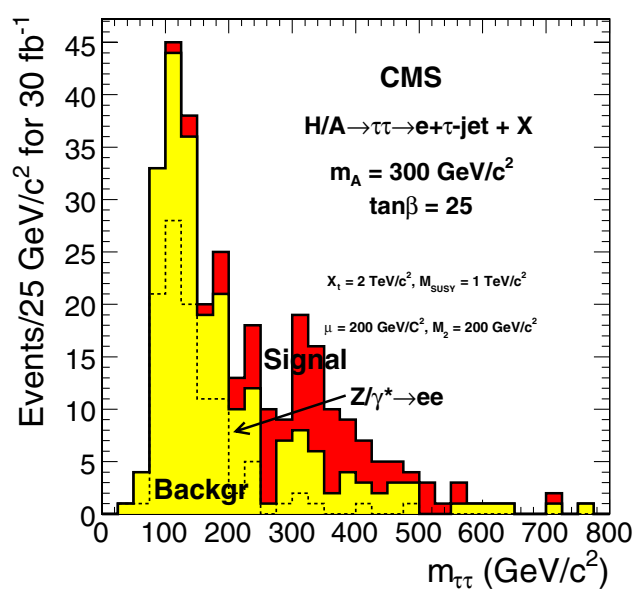

Figure 5.10. Reconstructed Higgs boson mass for the signal with $\mathrm{M}_{\mathrm{A}}=300 \mathrm{GeV} / \mathrm{c}^{2}, \tan \beta=25$ and for the total background for an integrated luminosity of $30 \mathrm{fb}^{-1}$. The dashed line shows the sum of the $\mathrm{Z} / \gamma^{*} \rightarrow \mathrm{e}^{+} e^{-}$and $\mathrm{b} \overline{\mathrm{b}} \mathrm{Z} / \gamma^{*} \mathrm{e}^{+} \mathrm{e}^{-}$backgrounds.

The systematic uncertainty due to the energy scale was estimated varying the jet energy and the $\mathrm{E}_{\mathrm{T}}^{\mathrm{miss}}$ values with the expected energy scale uncertainties yielding an average $5.1 \%$ uncertainty on the number of $\mathrm{Z} / \gamma^{*}$ events, $3.8 \%$ uncertainty on the number of $\mathrm{b} \overline{\mathrm{b}} \mathrm{Z} / \gamma^{*}$ events, $7.3 \%$ uncertainty on the number of $\bar{t} \bar{t}$ events, $11.3 \%$ uncertainty on the number of $\mathrm{tW}$ events and $11.8 \%$ uncertainty on the number of $\mathrm{W}+$ jet events passing the event selection cuts. A $5 \%$ uncertainty on the $\mathrm{b}$ tagging and mistagging efficiencies and a $2 \%$ uncertainty on the electron reconstruction and identification were used.

The uncertainty of the $\mathrm{Z} / \gamma^{*}$ cross section at the LHC is of the order of $1 \%$ [158]. For the $\overline{\mathrm{tt}}$ background the theoretical NLO cross section uncertainty derives from the scale uncertainty, taken to be 5\% according to Ref. [159], and the PDF uncertainty, about $2.5 \%$, yielding $5.6 \%$ for the total uncertainty. The same uncertainty is used for the cross sections of the $\mathrm{Wt}$ and $\mathrm{W}+\mathrm{jet}$ processes. The uncertainty of the $\mathrm{b} \overline{\mathrm{b}} Z / \gamma^{*}$ cross section measurement is estimated to be $14.2 \%$ in [145]. With these estimates, the total systematic uncertainty, including the luminosity uncertainty of $3 \%$ [7], was found to be $8.1 \%, 15.9 \%, 11.1 \%, 14.0 \%$ and $14.5 \%$ for the $\mathrm{Z} / \gamma^{*}, \mathrm{~b} \overline{\mathrm{b}} Z / \gamma^{*}, \mathrm{t} \overline{\mathrm{t}}, \mathrm{Wt}$ and $\mathrm{W}+$ jet backgrounds, respectively.

5.2.8.4. Discovery reach in the $\mathrm{M}_{\mathrm{A}}-\tan (\beta)$ plane. Table 5.22 shows the number of signal plus background events and the number of background events for $30 \mathrm{fb}^{-1}$ in the selected mass windows and the signal significance calculated according to Poisson statistics, with and without the background systematics taken into account. The mass windows were selected to optimise the significance. The $\mathrm{m}_{\mathrm{h}}^{\max }$ scenario was used.

Figure 5.11 shows the $5 \sigma$ discovery region in the $\mathrm{M}_{\mathrm{A}}-\tan \beta$ plane for $30 \mathrm{fb}^{-1}$ in the $\mathrm{m}_{\mathrm{h}}^{\max }$ scenario, evaluated with and without background systematics.

\subsection{Benchmark Channels: $t \bar{t} H, H \rightarrow b \bar{b}$}

\subsubsection{Introduction}

The Higgs boson decay to $\mathrm{b} \overline{\mathrm{b}}$ is the dominant mode for the Higgs mass range up to $m_{H} \sim 135 \mathrm{GeV} / \mathrm{c}^{2}$. Direct Higgs production is almost impossible to detect via this decay 
Table 5.22. Number of signal-plus-background events and the number of background events in the selected mass windows for $30 \mathrm{fb}^{-1}$ and the signal significance without $\left(\mathrm{S}_{\text {no syst. }}\right)$ and with $\left(\mathrm{S}_{\mathrm{syst}}\right)$ the background systematics taken into account.

\begin{tabular}{llllll}
\hline & $\Delta \mathrm{m}_{\tau^{+} \tau^{-}}$ & $\mathrm{N}_{\mathrm{S}}+\mathrm{N}_{\mathrm{B}}$ & $\mathrm{N}_{\mathrm{B}}$ & $\mathrm{S}_{\text {no } \text { syst. }}$ & $\mathrm{S}_{\text {syst. }}$ \\
\hline $\mathrm{M}_{\mathrm{A}}=130 \mathrm{GeV} / \mathrm{c}^{2}, \tan \beta=20$ & $120-200 \mathrm{GeV} / \mathrm{c}^{2}$ & 176 & 83 & 8.9 & 6.4 \\
$\mathrm{M}_{\mathrm{A}}=140 \mathrm{GeV} / \mathrm{c}^{2}, \tan \beta=15$ & $130-220 \mathrm{GeV} / \mathrm{c}^{2}$ & 136 & 76 & 9.1 & 6.7 \\
$\mathrm{M}_{\mathrm{A}}=200 \mathrm{GeV} / \mathrm{c}^{2}, \tan \beta=20$ & $140-280 \mathrm{GeV} / \mathrm{c}^{2}$ & 175 & 83 & 8.8 & 6.3 \\
$\mathrm{M}_{\mathrm{A}}=300 \mathrm{GeV} / \mathrm{c}^{2}, \tan \beta=20$ & $240-480 \mathrm{GeV} / \mathrm{c}^{2}$ & 78 & 39 & 5.4 & 4.3 \\
$\mathrm{M}_{\mathrm{A}}=500 \mathrm{GeV} / \mathrm{c}^{2}, \tan \beta=50$ & $360-780 \mathrm{GeV} / \mathrm{c}^{2}$ & 57 & 22 & 6.2 & 5.3 \\
\hline
\end{tabular}

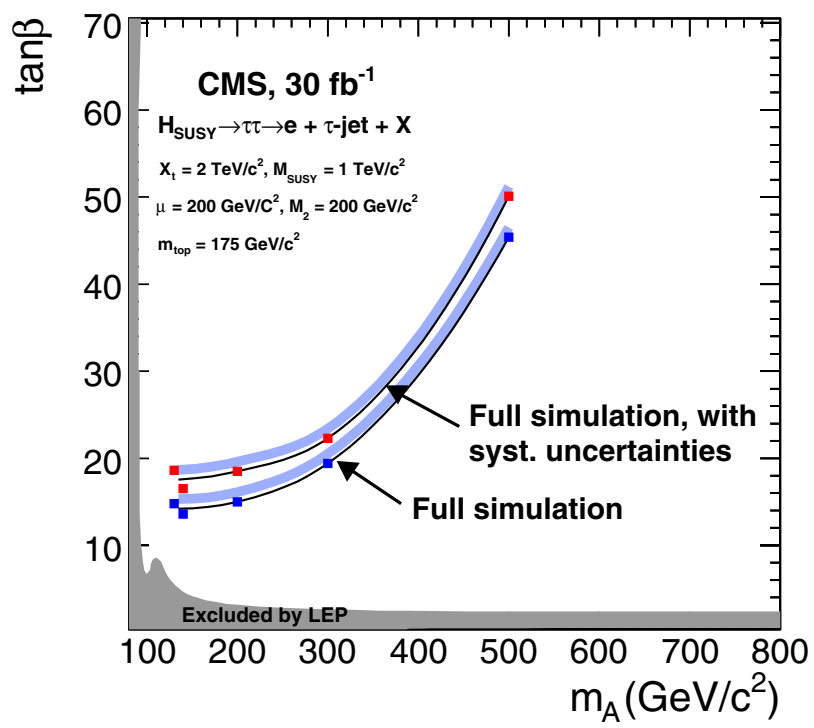

Figure 5.11. The $5 \sigma$ discovery region in the $\mathrm{M}_{\mathrm{A}}-\tan (\beta)$ plane for an integrated luminosity of $30 \mathrm{fb}^{-1}$ in the $\mathrm{m}_{\mathrm{b}}^{\max }$ scenario. The lower (upper) curve was evaluated without (with) the effect of background systematics taken into account.

as a result of the combination of an overwhelming QCD cross section for $\mathrm{b} \overline{\mathrm{b}}$ production and the inability to reconstruct the Higgs mass very precisely. While the latter is still true in the case of Higgs production in association with a $\bar{t} \bar{t}$ or $b \bar{b}$ pair, these channels hold promise because they entail substantially lower backgrounds. The separation of these events into 3 salient topologies follows as a result of the ways in which the two $\mathrm{W}$ bosons in the event decay. Thus, in addition to the four b jets, roughly $49 \%$ of these events also contain four hadronic jets (the all-hadron channel), while some $28 \%$ have two hadronic jets together with an isolated electron or muon and missing $E_{t}$ (the semi-leptonic channel), with a further $5 \%$ of events containing two oppositely-charged leptons (either of which can be an electron or muon) and missing $E_{t}$ (the dilepton channel). The remaining $14 \%$ of events correspond to those cases where one or both of the $\mathrm{W}$ bosons decay to a tau lepton and neutrino and are not easily distinguishable as such, as a result of the rich decay repertoire of the tau meson. In fact, these events do make a small contribution to the three other classes of events in the actual analyses. Additional hadronic jets can appear in these events and originate from initial and final state QCD radiation (IFSR). 
Table 5.23. NLO signal cross-sections and $\mathrm{H} \rightarrow \mathrm{b} \overline{\mathrm{b}}$ branching ratios for different Higgs mass hypotheses

\begin{tabular}{llll}
\hline$m_{\mathrm{H}}$ & $115 \mathrm{GeV} / \mathrm{c}^{2}$ & $120 \mathrm{GeV} / \mathrm{c}^{2}$ & $130 \mathrm{GeV} / \mathrm{c}^{2}$ \\
\hline$\sigma_{N L O}(\mathrm{pb})$ & 0.747 & 0.664 & 0.532 \\
$B R(\mathrm{H} \rightarrow \mathrm{b} \overline{\mathrm{b}})$ & 0.731 & 0.677 & 0.525 \\
\hline
\end{tabular}

A detailed description of the $\bar{t} \mathrm{H}$ analysis strategies and the results can be found in Reference [160]. All the results presented here are for an integrated luminosity of $60 \mathrm{fb}^{-1}$.

\subsubsection{Event generation and simulation}

As the identification of the signal relies upon the presence of top quark decay products, it comes as no surprise that the most significant backgrounds are those associated with $t \bar{t}$ events themselves. The main backgrounds are: $\mathrm{t} \overline{\mathrm{t} j \mathrm{j}}$, t⿱t $\mathrm{t} b \overline{\mathrm{b}}$ and $\mathrm{t} \overline{\mathrm{Z}} \mathrm{Z}$ with $\mathrm{Z} \rightarrow \mathrm{b} \overline{\mathrm{b}}$.

These processes are studied in detail and are presented here. Secondary background sources include pure QCD multi-jet events in the case of the all-hadron channel, and W/Z plus jets or dibosons plus jets events in the case of the semi-leptonic and dilepton channels. With the exception of QCD multi-jets, the latter have substantially lower production crosssections than $\bar{t} \bar{t}$ events but very similar topologies. They are therefore not studied in detail.

Details about the primary Monte Carlo data samples used in this analysis are available in Reference [160]. The semi-leptonic and all-hadron tt̄H signal samples were generated using COMPHEP (version 41.10) and PYTHIA (version 6.215), while the dilepton samples used PYTHIA only. Though a leading order Monte Carlo, PYTHIA is known to do a very good job of reproducing IFSR as well as parton shower effects. This is adequate for the signal samples. For the t⿱t plus jets backgrounds, greater care must be exercised. In particular, PYTHIA alone cannot be expected to do a realistic job since the relevant processes are not leading order. On the other hand, there is not currently a full next-to-leading order (NLO) MC for tt plus jets production. As a result, higher order matrix elements are used including additional radiated partons in conjunction with the parton showering of PYTHIA to produce the appropriate event topologies.

ALPGEN and PYTHIA are used for the matrix elements and parton showering, respectively, for the t⿱t plus $n$ jets background samples. The matching of the two generators is done in ALPGEN as discussed in Ref. [161]. In particular, all of the matrix elements for t⿱t plus $n$ additional hard partons are included and properly combined at each order taking into account the interference between amplitudes.

QCD events were generated with PYTHIA (version 6.215) in the $\hat{p}_{t}$ ranges from 120 to $170 \mathrm{GeV} / \mathrm{c}$ and greater than $170 \mathrm{GeV} / \mathrm{c}$.

For the simulation of the interaction with the detector, the CMS tools, providing GEANT3 and GEANT4 based simulation of the CMS detector have been used.

The NLO signal cross-sections for different Higgs mass hypotheses are given in Table 5.23 together with the branching ratios for $\mathrm{H} \rightarrow \mathrm{b}$ [162].

The leading order COMPHEP cross-sections for the different background processes together with the effective cross-sections after the application of the generator filters are listed in Table 5.24. The ALPGEN cross sections for the different jet multiplicity processes are listed in Table 5.25. A detailed comparison of ALPGEN versus COMPHEP for the tijj background is available in [160]. All the results that are presented here for the t⿱t $\mathrm{Nj}$ backgrounds are based on the ALPGEN samples, where available. 
Table 5.24. LO COMPHEP cross-sections and effective cross-sections after the generator filters of the considered background processes.

\begin{tabular}{lllll}
\hline & $\mathrm{QCD} \hat{p}_{t}=120-170 \mathrm{GeV} / \mathrm{c}$ & $\mathrm{QCD} \hat{p}_{t}>170 \mathrm{GeV} / \mathrm{c}$ & $\mathrm{tt} b \overline{\mathrm{b}}$ & $\mathrm{tt} Z$ \\
\hline$\sigma_{L O}(\mathrm{pb})$ & $3.82 \cdot 10^{5}$ & $1.05 \cdot 10^{5}$ & 3.28 & 0.65 \\
$\sigma_{L O} \times \varepsilon(\mathrm{pb})$ & 76.4 & 336.0 & 2.82 & 0.565 \\
\hline
\end{tabular}

Table 5.25. LO ALPGEN cross-sections for the different jet multiplicity samples.

\begin{tabular}{lllll}
\hline & exclusive $\overline{\mathrm{t}}+1 \mathrm{j}$ & exclusive $\overline{\mathrm{t}}+2 \mathrm{j}$ & exclusive $\overline{\mathrm{t}}+3 \mathrm{j}$ & inclusive $\overline{\mathrm{t}}+4 \mathrm{j}$ \\
\hline$\sigma_{L O}(\mathrm{pb})$ & 170 & 100 & 40 & 61 \\
\hline
\end{tabular}

Table 5.26. Signal and background efficiencies of the Level 1 and High Level Triggers.

\begin{tabular}{lllll}
\hline & & \multicolumn{3}{l}{ Single $e$} \\
& Single $\mu$ & Single $e$ & OR $\mu$ OR $\tau$ & Jets \\
\hline $\mathrm{H} \rightarrow \mathrm{b} \overline{\mathrm{b}}(\%)$ with $m_{H}=120 \mathrm{GeV} / \mathrm{c}^{2}$ & 63.5 & 52.4 & 76.7 & 24.9 \\
$\overline{\mathrm{tt}} \overline{\mathrm{b}}(\%)$ & 19.0 & 16.1 & 83.6 & 18.3 \\
$\overline{\mathrm{tt}} 1 \mathrm{j}(\%)$ & 13.9 & 11.3 & 53.0 & 2.9 \\
$\overline{\mathrm{t}} 2 \mathrm{j}(\%)$ & 14.0 & 11.1 & 59.8 & 6.2 \\
$\overline{\mathrm{tt}} \mathrm{j} \mathrm{j}(\%)$ & 14.0 & 11.1 & 68.5 & 11.4 \\
$\overline{\mathrm{tt}} 4 \mathrm{j}(\%)$ & 13.4 & 11.1 & 78.6 & 31.4 \\
$\overline{\mathrm{t}} \mathrm{Z}(\%)$ & 20.4 & 18.8 & 84.4 & 25.3 \\
$\mathrm{QCD} 120-170 \mathrm{GeV} / \mathrm{c}(\%)$ & 0.08 & 0.8 & 4.3 & 1.7 \\
$\mathrm{QCD}>170 \mathrm{GeV} / \mathrm{c}(\%)$ & 0.07 & 2.1 & 4.4 & 10.3 \\
\hline
\end{tabular}

\subsubsection{Level-1 and high level trigger selections}

A dedicated $\bar{t} \mathrm{H}$ trigger was not available and therefore was not implemented in the analysis. As a result, it is assumed in what follows that the signal is recorded by the CMS Level 1 (L1) and High Level Triggers (HLT) as described in [76]. Wherever possible, the cleaner signature of at least one isolated lepton in the final state is exploited. The semi-leptonic channels thus use the single muon (stream \#43) or single electron (stream \#2) triggers.

A logical "OR" of the single muon, single electron and single tau streams is used for the dilepton channel. The same trigger setups as for streams \#43 and \#2 were used, except that the $p_{\mathrm{T}}$ threshold was lowered to $15 \mathrm{GeV} / \mathrm{c}$ to permit selection of $20 \mathrm{GeV} / \mathrm{c}$ leptons later in the analysis. The tau trigger is the official stream (bit \#91). Jet triggers are used to select all-hadron events. In particular, the single-jet, 3-jet and 4-jet triggers with low luminosity thresholds [76][163] are combined (stream \#120 or \#122 or \#123).

Efficiencies for the various HLT and Level-1 triggers that were used are presented in Table 5.26. The efficiencies quoted are determined by counting the numbers of accepted events relative to the total numbers of events in each sample. In order to streamline the various studies that were performed, the analyses used different MC samples, produced with different final state constraints. Thus, efficiencies for single muon, single electron and fully hadronic final states were defined with respect to exclusive signal samples and inclusive background samples, as described in the preceding section. The dilepton channel efficiency on the other hand, was defined with respect to samples containing at least one leptonic top decay for the signal and inclusive samples for the backgrounds. 

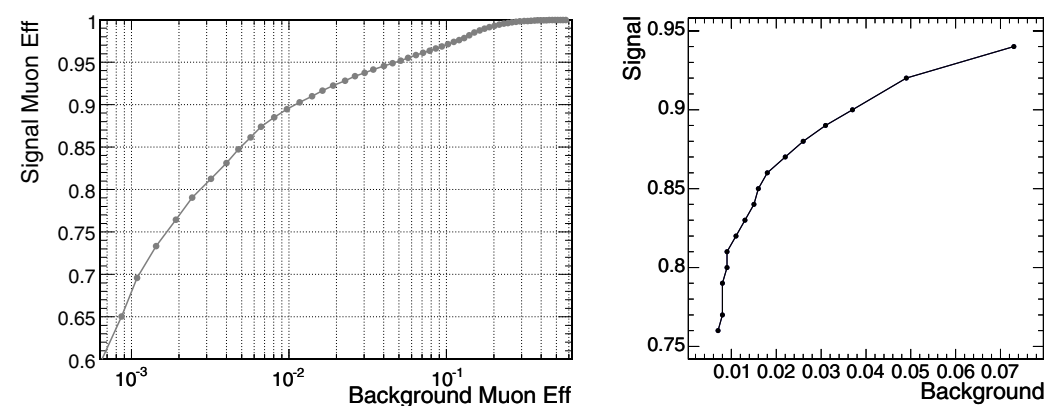

Figure 5.12. (Left) Performance of the muon likelihood discriminator for the semi-leptonic muon $\bar{t} \overline{\mathrm{t}} \mathrm{H}$ channel. (Right) Signal versus background electron efficiencies for likelihood values ranging from 0.006 (the upper point) with a step size of 0.006 , (i.e. approximately in the range $\left.1.0<-\log \left(L_{e}\right)<2.0\right)$.

\subsubsection{Reconstruction}

5.3.4.1. Muon reconstruction. The process of muon reconstruction begins in the Muon Chambers and is then extended to the tracking system, as described in Ref. [164]. For the studies presented here it is important to identify muons coming from $\mathrm{W}$ decays. To this end, additional selection criteria are applied to distinguish these muons, which will be referred to as signal muons, from the muons coming from other sources such as b decays. The latter will be referred to as background muons, even though they arise in signal events as well as background events. The desired discrimination between signal and background muons is achieved by constructing a discriminator that is based upon probability density functions (PDF) for the following observables associated with muon candidates:

- Transverse momentum, $p_{t}$.

- Track isolation, IsoTk.

- Calorimeter isolation, IsoCalo.

- Significance of track impact parameter, $S_{i p}=d / \sigma_{d}$.

The PDF's associated with these variables for signal and background muons are obtained by matching to generator-level muons.

The PDF's are combined into the following likelihood ratio:

$$
L=\Pi_{i} \frac{P_{i}^{s i g}\left(x_{i}\right)}{P_{i}^{s i g}\left(x_{i}\right)+P_{i}^{b k g}\left(x_{i}\right)}
$$

where $P_{i}^{s i g}$ and $P_{i}^{b k g}$ are the PDF's of an observable $x_{i}$ for signal and background muons, respectively.

The performance for signal and background muon discrimination are shown in Figure 5.12. For a signal muon efficiency of $90 \%$, only $1 \%$ of background muons are selected. The PDF's are constructed using a sample of tt $\mathrm{H}$ events with $\mathrm{m}_{\mathrm{H}}=120 \mathrm{GeV} / \mathrm{c}^{2}$ in which one and only one of the $\mathrm{W}$ bosons decays to a muon and neutrino, while the other one decays hadronically.

If the likelihood selection is used after the HLT, a dramatic improvement in QCD $\left(\hat{p}_{t}>170 \mathrm{GeV} / \mathrm{c}\right)$ rejection is possible with little or no loss in signal efficiency. For example, a small drop in signal efficiency from $63 \%$ to $60 \%$ reduces the QCD efficiency by more than a factor of 3 (i.e. from $0.07 \%$ to $0.02 \%$ ). 
5.3.4.2. Electron reconstruction. A full description of the electron reconstruction in CMS can be found in Ref. [46]. Electrons coming from W boson decays are typically characterised by isolated high transverse energy clusters. These electrons are thus efficiently identified by means of an isolation requirement applied to the electron candidate with respect to other reconstructed tracks in the event.

In analogy to the muon reconstruction and equation 5.13, a likelihood method is used to identify the signal electrons, making use of the following observables:

- the $p_{t}$ sum of tracks inside an isolation cone of radius $\Delta R=0.3$ around the candidate electron direction.

- the $\Delta R$ distance between the electron candidate and the closest track.

- the transverse momentum of the electron candidate, $p_{t}$.

- the ratio between the cluster energy and the track momentum, $E / p$.

- the ratio between the hadronic and electromagnetic energies of the cluster, $H / E$.

An appropriate choice of likelihood cut value has been studied by comparing signal versus background electron efficiencies as shown in Figure 5.12.

For a $-\log \left(L_{e}\right)$ cut value of 1.27 , signal electrons are selected with an efficiency of $84 \%$ and background electrons with an efficiency of $1.5 \%$. This value was chosen for the analyses described in subsequent sections.

Concerning the efficiency of the likelihood cut with respect to background rejection in ttijj events in which there were no isolated electrons coming from $\mathrm{W}$ decays, only $6 \%$ of these events were accepted for a likelihood cut of 1.27.

As in the case of the muon selection, the likelihood approach can be used to augment the HLT selection efficiency. Maintaining a roughly constant signal efficiency, the likelihood cut in combination with the HLT trigger yields an order of magnitude reduction in the QCD background selection efficiency.

5.3.4.3. Jet and missing $E_{\mathrm{T}}$ reconstruction. Jets are reconstructed using the iterative cone algorithm. A cone with $\Delta R=0.5$ is used when at least one $\mathrm{W}$ boson decays into leptons, while a smaller cone size was found to be more suitable for the more dense jet environments associated with the all-hadron channel (see below).

A calorimetric-tower energy threshold of $0.8 \mathrm{GeV}$ and a transverse-energy threshold of $0.5 \mathrm{GeV}$ are used. Calorimeter towers that exceed $1 \mathrm{GeV}$ are considered as jet seeds. For the leptonic channels, the jet energy is calibrated using MC calibrations [165] provided by the JetMET group for the corresponding set of reconstruction parameters.

The single lepton analyses, as described in more detail below, make use of an event likelihood to help select and properly reconstruct events and decay chains. This is facilitated, in part, by making use of the various invariant mass constraints associated with the top quark decays. The corresponding likelihoods thus rely upon the resolutions that are obtained for the invariant masses of the hadronically decaying $\mathrm{W}$ boson and the two top quarks. The "bestcase" invariant mass distribution for the hadronically decaying top quark is reconstructed by matching to generator-level parton information and shown in Figure 5.13. The distributions for the leptonically decaying top quark and the hadronically decaying W boson (Ref. [160]) have similar shapes but different RMS $\left(25.7 \mathrm{GeV} / \mathrm{c}^{2}\right.$ and $15.7 \mathrm{GeV} / \mathrm{c}^{2}$, respectively) since the longitudinal momentum of the leptonically decaying top quark has to be calculated from missing $E_{t}$. A reconstructed jet is considered as matched to the corresponding parton if their separation, $\Delta R_{j-p}$, is less than 0.3 . 

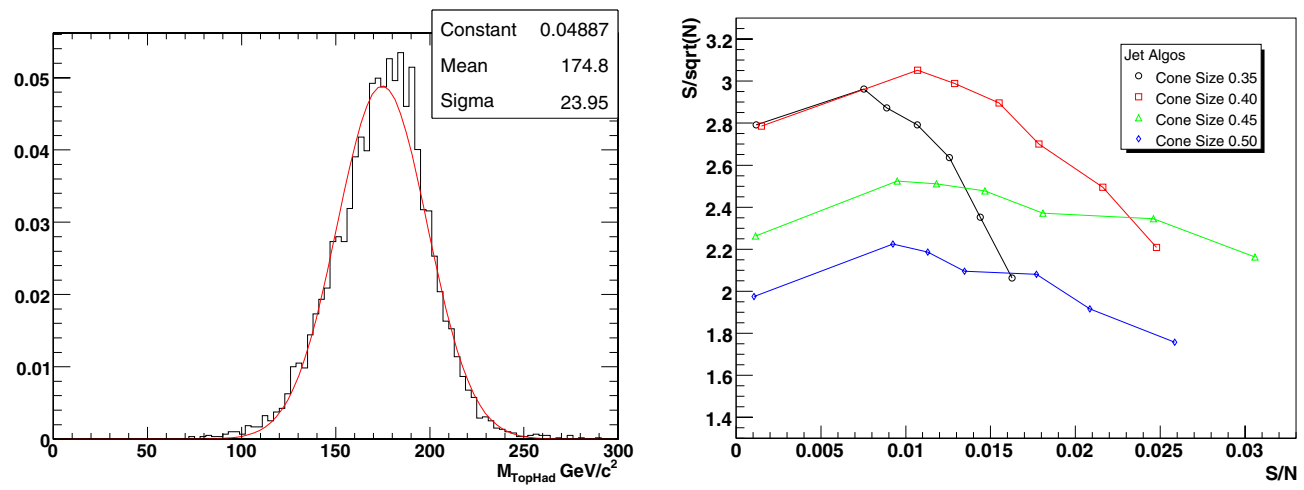

Figure 5.13. (Left) Invariant mass of the hadronically decaying Top quark using jet-parton matching with $\Delta R_{j-p}<0.3$. (Right) Change in significance and $S / N$ resulting from variations in the b-tagging discriminator for the various cone sizes indicated in the legend.

The missing transverse energy of the event $E_{\mathrm{t}}^{\text {miss }}$ is computed as

$$
E_{\mathrm{t}}^{\mathrm{miss}}=\sum_{i} E_{\mathrm{t}}^{\mathrm{tower}}-\left(\sum_{j} E_{\mathrm{t}}^{\mathrm{RawJet}}-\sum_{k} E_{\mathrm{t}}^{\text {CaliJet }}\right)+\sum_{m} E_{\mathrm{t}}^{\text {Muon }}
$$

where the sum with index $i$ runs over calorimeter towers, that with index $j$ runs over raw jets, $k$ runs over calibrated jets, and $m$ runs over the reconstructed muons of the event. Equation 5.14 thus takes into account the corrections due to jet calibration and the contributions of muons that are not measured in the calorimeter.

The choice of the jet reconstruction algorithm is an important step in the event selection optimisation for the all-hadron $\overline{\mathrm{t}} \mathrm{H}$ channel, where at least 8 jets are expected in the final state. For this reason, an optimisation is obtained by means of a simple "proto" analysis as described in Reference [160].

A dedicated $\mathrm{t} \overline{\mathrm{H}} \mathrm{H}$ calibration [166] is applied to help recover the original transverse energy of the associated parton. Reconstructed jets with a b-tagging discriminator value higher than 0.4 are calibrated using a separate b-jet calibration procedure.

Figure 5.13 shows the significance with respect to the $S / N$ ratio for a range of b-tag discriminator values for each of the several cone sizes indicated. Lower discriminator values yield higher significance but only at the cost of low $S / N$ while, on the contrary, higher discriminator values give lower significance but higher $S / N$. A good compromise is in the middle range of each of the curves where neither $S / N$ nor significance are unreasonably low. With this in mind, the best choice for the jet cone is seen to be $\Delta R=0.40$.

5.3.4.4. b-Tagging. The identification of jets from b-quarks is done with the Combined Secondary Vertex algorithm. This algorithm exploits secondary vertex and track properties to calculate a discriminator value which separates b-jets from non b-jets. A detailed description is published in Ref. [157] which also presents results of detailed studies of the performance of the b-tagging algorithm as applied to Monte Carlo $\bar{t}$ and QCD samples.

In the $\bar{t} \mathrm{t} H$ analyses, a fixed cut value for the b-tagging discriminator is applied, and four jets are required to pass this cut in the semi-leptonic and all-hadron channels, while only 3 jets are required to be tagged in the dilepton analysis. The misidentification rate of charm and light flavour jets as a function of the b-tagging efficiency is shown in Fig. 5.14 for the $\bar{t} \mathrm{H}$ and the $\bar{t}_{\mathrm{tj}} \mathrm{samples}$, respectively. It can be seen that the efficiencies are similar in these samples. 

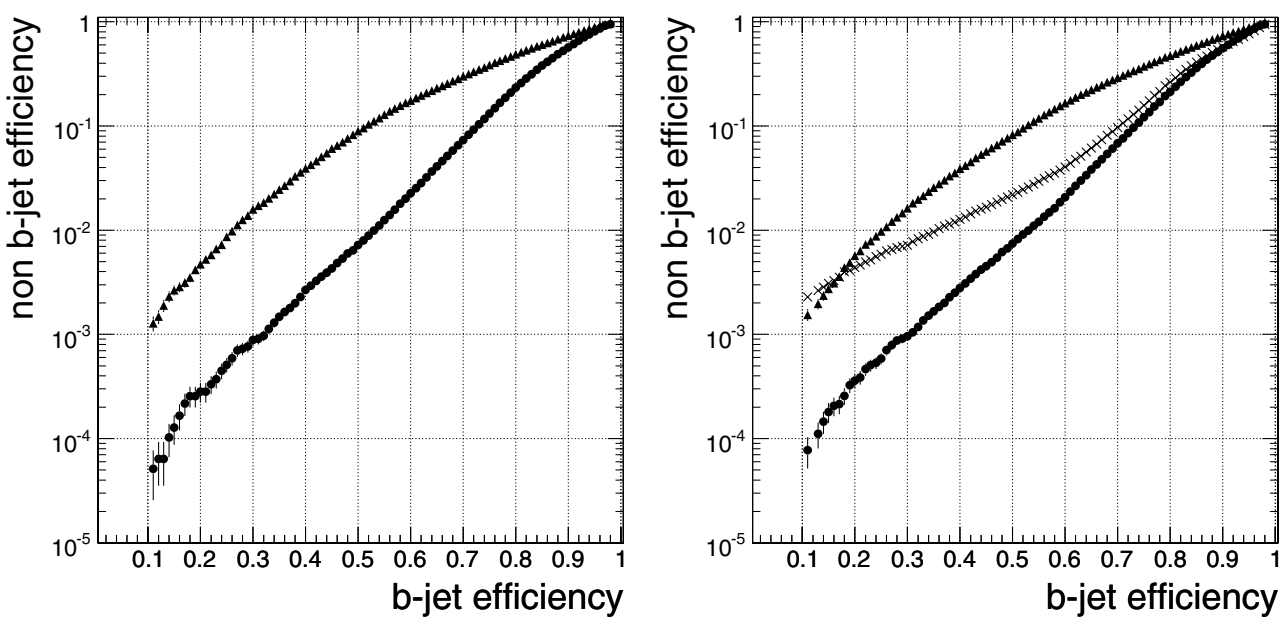

Figure 5.14. On the left: Non-b jet mistagging efficiency versus b-jet tagging efficiency for c-jets (triangles), and uds-jets (stars) for the $\mathrm{t} \overline{\mathrm{t}} \mathrm{H}$ sample with $m_{H}=120 \mathrm{GeV} / c^{2}$ and jets with a minimum transverse momentum of $20 \mathrm{GeV} / \mathrm{c}$. For this plot the "physics definition" of the original jet flavour has been used. In this definition there are no original gluon jets in the $\bar{t} \mathrm{H}$ sample. On the right: The corresponding plot for the tījj sample, where gluon jets are represented by crosses.

This fixed-cut b-tagging approach gives reasonable results, but is not necessarily optimal. Some potential improvements are possible such as the combination with a soft lepton tag or a discriminator cut which depends on $p_{t}$ and $\eta$ of the jets. Studies have shown that they have the potential to improve the results at the order of some percent. These improvements were not used in the current analyses.

\subsubsection{Event selection}

In this section the event selection for the different channels under consideration is described. In order to be able to combine the results from all the $\bar{t} \bar{H}$ search channels, the different channels use mutually exclusive event samples. This is most easily facilitated by coordinating how high $p_{t}$ electrons and muons from the $\mathrm{W}$ decays (previously referred to as signal leptons) are either selected or vetoed by the different analyses.

For the analyses reported here, the different data samples used were separated using selection and/or veto criteria based on the lepton likelihood value, as described in Ref. [160].

5.3.5.1. Semi-leptonic Channel: $\overline{\mathrm{t}} \mathrm{H} \rightarrow \mathrm{b} \overline{\mathrm{b}} \overline{\mathrm{b}} \mathrm{qq} \mathrm{q}^{\prime} \mu v_{\mu}$ and $\mathrm{b} \overline{\mathrm{b}} \mathrm{b} \overline{\mathrm{b}} \mathrm{qq} \mathrm{q}^{\prime} \mathrm{e} v_{e}$. The strategy for selecting $\bar{t} \bar{H}$ events with one isolated muon or electron in the final state can be summarised in the following three steps: pre-selection, choice of jet pairing and finally, selection. The preselection requires the HLT stream for a single muon or a single electron, one isolated lepton using the likelihood information as described in section 5.3.4.1 and 5.3.4.2, and 6 or 7 jets in the pseudorapidity region $|\eta|<3.0$ with a calibrated transverse energy larger than $20 \mathrm{GeV}$. In order to recover some efficiency, jets with $10 \mathrm{GeV}<E_{t}<20 \mathrm{GeV}$ are also accepted if they have at least two associated tracks pointing to the signal primary verte ${ }^{42}$ within a distance along the beam $(z)$ axis of $\left(\left|z_{P V}-z_{\text {track }}\right|<1 \mathrm{~mm}\right)$. The latter condition is required to reject low transverse energy fake jets, (i.e. jets that are not associated with any of the signature

\footnotetext{
42 The signal interaction is generally the one which allows the event to be triggered.
} 
partons in the signal event). For the single electron channel, the misidentification of the jet with the isolated electron has been excluded by imposing a veto on the jet if the electron lies inside a jet cone radius of 0.1 .

At least 4 jets are required to be tagged as b-jets with a minimal discriminator value corresponding to a b-efficiency of about $70 \%$.

To decrease the contamination from the dilepton channel, a double muon, double electron and muon-electron veto is applied, in which events with the second lowest $-\log \left(L_{\mu}\right)<1.4$ and events with $-\log \left(L_{e}\right)<1.2$ are rejected from the analysis. In the case of the semileptonic electron channel the previous cuts are applied respectively to the first muon likelihood candidate and to the second electron likelihood candidate. The application of these vetoes results in a lowering of the signal efficiency by about $2 \%$, while the total background rejection is increased by $13 \%$.

In order to perform a complete reconstruction of the event, the longitudinal momentum of the neutrino has to be computed from four-momentum conservation for the $\mathrm{W}$ boson: $m_{W}^{2}=\left(E^{\mu}+E^{\nu}\right)^{2}-\left(\vec{p}^{\mu}+\vec{p}^{v}\right)^{2}$. This equation gives 2 real solutions for $p_{z}^{v}$ in $66 \%$ of the cases, while in the remaining $34 \%$, the neutrino is assumed to be collinear with the lepton: $p_{z}^{v}=p_{z}^{l}$. This leads to a small degradation in the longitudinal momentum resolution, but the reconstruction efficiency of the leptonic $\mathrm{W}$ boson decay is increased to $100 \%$.

In order to choose the jet combination that does the best job of reconstructing the two top quarks, a likelihood, $L_{\text {Event }}$, is defined using masses, b-tagging and kinematic information from the whole event:

$$
L_{\text {Event }}=L_{\text {Mass }} \times L_{\text {bTag }} \times L_{\text {Kine }} .
$$

The mass information considered in the likelihood $L_{\text {Mass }}$ is the probability returned by the kinematic fit with invariant mass constraints (top quarks and hadronic $\mathrm{W}$ ) that is described in Reference [167].

The b-tagging function $L_{b T a g}$ is defined as the product of the b-tag discriminators: $L_{b T a g}=D_{T_{o p_{H a d}}} \times D_{T_{o p_{L e p}}} \times D_{H_{1}} \times D_{H_{1}} \times\left(1-D_{W 1}\right) \times\left(1-D_{W 2}\right)$; where Top Had $_{\text {Had }}$ and $T o p_{L e p}$ are expected to be the two b jets from the hadronic and leptonic top, respectively, while $H_{1}$ and $H_{2}$ are expected to be the two b jets coming from Higgs and $W_{1}$ and $W_{2}$ are the two jets from the hadronically-decaying $\mathrm{W}$ boson.

The kinematic function takes into account the observation that the b-jets coming from top quarks tend to be slightly more energetic than b-jets coming from the Higgs boson (see [160] for a definition).

Among all possible combinations of jet-parton assignments, the one with the highest value of $L_{\text {Event }}$ is chosen for use in the final reconstruction of the top quarks and the two remaining jets with highest b-tagging discriminator values are used to reconstruct the Higgs mass.

After the jet assignment is complete, additional criteria are applied to improve background rejection. In particular, a stronger b-tag requirement is applied on the event variable $L_{b \text { Sele }}=D_{T_{\text {op }} \text { Had }} \times D_{T_{\text {op }} \text { Lep }} \times D_{H_{1}} \times D_{H_{1}}$.

The signal significance as a function of the selection cut $L_{b S e l e}$ is shown in Figure 5.15.

The distributions of reconstructed Higgs mass for the final selected events are shown in Figure 5.16 for signal only (left) and for the combination of the different backgrounds (right) for the muon channel only (similar results for the electron channel can be found in [160]). The fraction of signal events where the two b-jets are correctly assigned to the Higgs boson (i.e. the pairing efficiency) is roughly $31 \%$ in the muon channel and about $29 \%$ for the electron channel. 

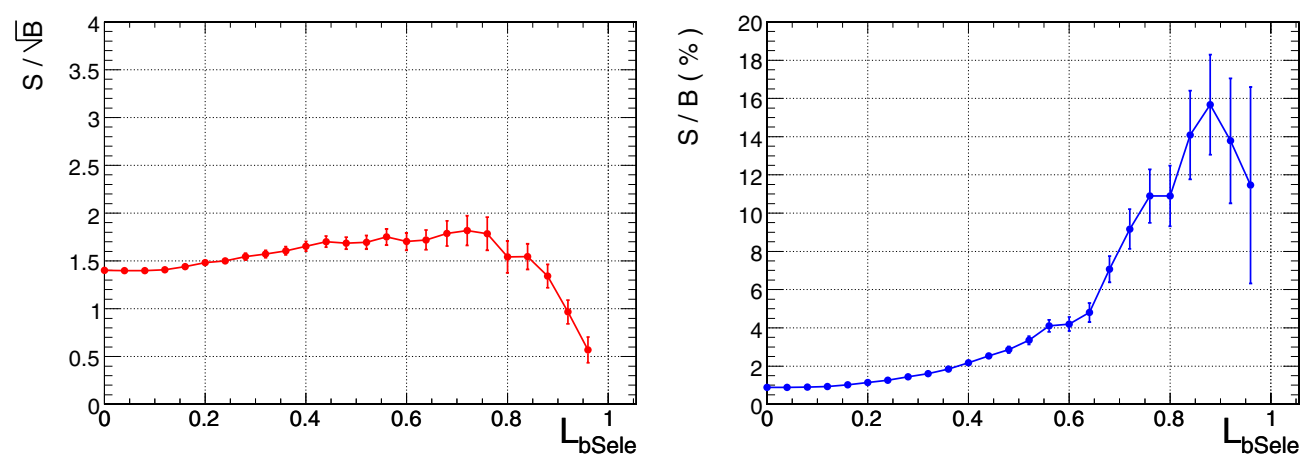

Figure 5.15. $\overline{\mathrm{t}} \mathrm{H}\left(W \rightarrow q q^{\prime}, W \rightarrow \mu \nu\right)$ : Signal Significance (left) and Signal to Background ratio (right) as function of the cut on $L_{b \text { Sele }}$.
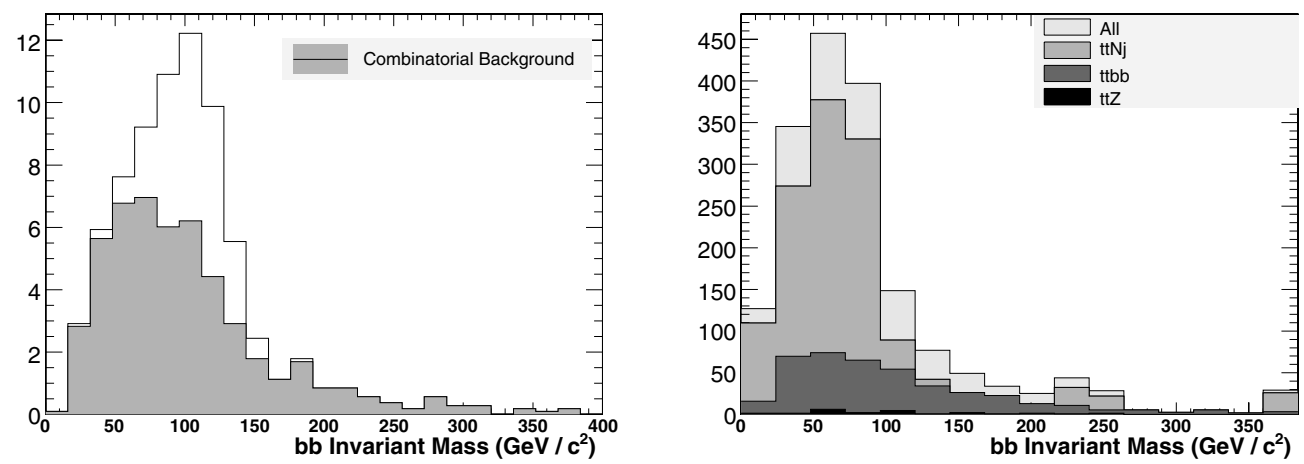

Figure 5.16. $\overline{\mathrm{t}} \mathrm{H}\left(W \rightarrow q q^{\prime}, W \rightarrow \mu \nu\right)$. (Left) Invariant $b \bar{b}$ mass for signal only (combinatorial background is shaded grey). (Right) The sum of the reconstructed $m_{b \bar{b}}$ spectra for backgrounds with a value of $L_{b S e l e}>0.55$. The distributions are normalised to an integrated luminosity of $60 \mathrm{fb}^{-1}$.

5.3.5.2. Results. The selection efficiencies with the corresponding numbers of expected events and signal significances are reported in Table 5.27 for the channels with a muon or an electron in the final state. The number of expected events is computed for an integrated luminosity of $60 \mathrm{fb}^{-1}$ in the Standard Model Higgs mass range from 115 to $130 \mathrm{GeV} / \mathrm{c}^{2}$.

5.3.5.3. Dilepton channel: $\mathrm{t} \overline{\mathrm{t}} \mathrm{H} \rightarrow \mathrm{b} \overline{\mathrm{b}} \mathrm{b} \overline{\mathrm{b}} \ell^{\prime} v^{\prime} \ell v$. Dilepton ttH events are selected by requiring two reconstructed leptons $(\mathrm{e}, \mu)$ accompanied by significant missing transverse energy and at least four but no more than seven jets, at least three of which have been b-tagged according to the Combined Secondary Vertex b-tagging algorithm.

Lepton identification is performed using the electron and muon likelihoods described in Section 5.3.4. In the semi-leptonic analyses, events with more than one identified lepton are vetoed, but in the dilepton analysis those events are retained. The likelihood acceptance cuts used for leptons in the dilepton channel are therefore chosen to be the same as the secondlepton veto cuts for both semi-leptonic channels. In this way, the sample of events for the dilepton $\overline{\mathrm{tt}} \mathrm{H}$ analysis is by construction strictly complementary to those used in the semileptonic channels. 
Table 5.27. Selection efficiency for $L_{b \text { Sele }}>0.55\left(\varepsilon_{\text {loose }}\right)$ and for $L_{b \text { Sele }}>0.75\left(\varepsilon_{\text {tight }}\right)$, number of expected events and signal significance in $60 \mathrm{fb}^{-1}$ for the muon and electron $\overline{\mathrm{tt}} \mathrm{H}$ channel. The numbers refer to the complete Higgs mass range.

\begin{tabular}{|c|c|c|c|c|c|}
\hline & Analysed Ev. & $\varepsilon_{\text {loose }}(\%)$ & $N_{\text {loose }}^{e v} 60 \mathrm{fb}^{-1}$ & $\varepsilon_{\text {tight }}(\%)$ & $N_{\text {tight }}^{e v} 60 \mathrm{fb}^{-1}$ \\
\hline \multicolumn{6}{|l|}{ muon channel } \\
\hline$\overline{\mathrm{tt}} \mathrm{H}(115)$ & 27768 & $2.00 \pm 0.08$ & $96 \pm 4$ & $0.80 \pm 0.05$ & $38 \pm 3$ \\
\hline$\overline{\mathrm{tt}} \mathrm{H}(120)$ & 41929 & $1.90 \pm 0.07$ & $75 \pm 3$ & $0.74 \pm 0.04$ & $29 \pm 2$ \\
\hline$\overline{\mathrm{tt}} \mathrm{H}(130)$ & 19466 & $2.23 \pm 0.11$ & $55 \pm 3$ & $0.84 \pm 0.07$ & $21 \pm 2$ \\
\hline$\overline{\mathrm{ttb}} \overline{\mathrm{b}}$ & 372737 & $0.247 \pm 0.008$ & $419 \pm 14$ & $0.0877 \pm 0.0048$ & $148 \pm 8$ \\
\hline$\overline{\mathrm{t}} 1 \mathrm{j}$ & 393000 & $0.0051 \pm 0.0011$ & $520 \pm 120$ & $0.00076 \pm 0.00044$ & $78 \pm 45$ \\
\hline$\overline{\mathrm{tt}} 2 \mathrm{j}$ & 568999 & $0.0105 \pm 0.0014$ & $633 \pm 82$ & $0.00070 \pm 0.00035$ & $42 \pm 21$ \\
\hline $\mathrm{tt} 3 \mathrm{j}$ & 101000 & $0.0050 \pm 0.0022$ & $119 \pm 53$ & 0 & $<27(68 \%$ C.L $)$ \\
\hline$\overline{\mathrm{tt}} 4 \mathrm{j}$ & 86697 & $0.0035 \pm 0.0020$ & $126 \pm 73$ & 0 & $<48(68 \%$ C.L. $)$ \\
\hline $\mathrm{Zt \overline {t }}$ & 50000 & $0.068 \pm 0.012$ & $23 \pm 4$ & $0.026 \pm 0.007$ & $9 \pm 2$ \\
\hline Total Background & & & 1840 & & $<352$ \\
\hline$S / \sqrt{B}$ & & & 2.2 & & 2.0 \\
\hline$S / B(115)$ & & & $5.1 \%$ & & $10.8 \%$ \\
\hline$S / \sqrt{B}(120)$ & & & 1.8 & & 1.6 \\
\hline$S / B(120)$ & & & $4.1 \%$ & & $8.2 \%$ \\
\hline$S / \sqrt{B}(130)$ & & & 1.3 & & 1.1 \\
\hline$S / B(130)$ & & & $3.0 \%$ & & $6.0 \%$ \\
\hline \multicolumn{6}{|l|}{ electron channel } \\
\hline$\overline{\mathrm{t}} \mathrm{H}(115)$ & 27692 & $1.39 \pm 0.07$ & $66 \pm 3$ & $0.52 \pm 0.04$ & $25 \pm 2$ \\
\hline$\overline{\mathrm{tt}} \mathrm{H}(120)$ & 42228 & $1.42 \pm 0.06$ & $56 \pm 2$ & $0.53 \pm 0.04$ & $21 \pm 1$ \\
\hline$\overline{\mathrm{tt}} \mathrm{H}(130)$ & 19127 & $1.57 \pm 0.09$ & $39 \pm 2$ & $0.61 \pm 0.06$ & $15 \pm 1$ \\
\hline $\mathrm{t} \overline{\mathrm{t}} \mathrm{b} \overline{\mathrm{b}}$ & 372737 & $0.176 \pm 0.007$ & $297 \pm 12$ & $0.0641 \pm 0.0041$ & $109 \pm 7$ \\
\hline$\overline{\mathrm{t}} 1 \mathrm{j}$ & 393000 & $0.0038 \pm 0.0010$ & $390 \pm 100$ & $0.00025 \pm 0.00025$ & $26 \pm 26$ \\
\hline $\mathrm{tt} 2 \mathrm{j}$ & 568999 & $0.0067 \pm 0.0011$ & $401 \pm 65$ & $0.00123 \pm 0.00046$ & $74 \pm 28$ \\
\hline $\mathrm{tt} 3 \mathrm{j}$ & 101000 & $0.0040 \pm 0.0020$ & $95 \pm 48$ & 0 & $<27(68 \%$ C.L $)$ \\
\hline$\overline{\mathrm{tt}} 4 \mathrm{j}$ & 86697 & $0.0023 \pm 0.0016$ & $84 \pm 60$ & 0 & $<48(68 \%$ C.L. $)$ \\
\hline $\mathrm{Zt \overline {t }}$ & 50000 & $0.064 \pm 0.011$ & $22 \pm 4$ & $0.022 \pm 0.007$ & $7 \pm 2$ \\
\hline Total Background & & & 1289 & & $<291$ \\
\hline$S / \sqrt{B}(115)$ & & & 1.8 & & 1.5 \\
\hline$S / B(115)$ & & & $5.1 \%$ & & $8.6 \%$ \\
\hline$S / \sqrt{B}(120)$ & & & 1.6 & & 1.2 \\
\hline$S / B(120)$ & & & $4.4 \%$ & & $7.2 \%$ \\
\hline$S / \sqrt{B}(130)$ & & & 1.1 & & 0.9 \\
\hline$S / B(130)$ & & & $3.0 \%$ & & $5.2 \%$ \\
\hline
\end{tabular}

The details of the dilepton $\overline{\mathrm{tt}} \mathrm{H}$ selection are summarised below:

- 2 oppositely-charged leptons (e, $\mu$ ) passing identification criteria $-\log \left(L_{\mu}\right)<1.4$ for muons, $-\log \left(L_{e}\right)<1.2$ for electrons).

- corrected $E_{\mathrm{T}}^{\mathrm{miss}}>40 \mathrm{GeV}$.

- 4 to 7 jets with calibrated $E_{\mathrm{T}}>20 \mathrm{GeV}$ and $|\eta|<2.5$.

- $\geqslant 3$ selected jets b-tagged with discriminator $D>0.7$.

The above is termed the "loose" working point because there is evidence that it is possible to increase the purity $(S / B)$ of the selection, by way of more stringent criteria:

- 4 to 6 jets with calibrated $E_{\mathrm{T}}>20 \mathrm{GeV}$ and $|\eta|<2.5$.

$\bullet \geqslant 4$ selected jets b-tagged with discriminator $D>0.7$. 
Table 5.28. Selection efficiency $\varepsilon_{\text {loose }}$ (including branching fraction where applicable) and resulting number of expected events $N_{\text {loose }}$ in $60 \mathrm{fb}^{-1}$, for the dilepton $\mathrm{t} \overline{\mathrm{t}} \mathrm{H}$ channel. For a glimpse of possible improvements, the same for a tighter set of cuts is provided $\left(\varepsilon_{t i g h t}, N_{t i g h t}\right)$. Also quoted are binomial errors arising from the finite sizes of processed datasets. The $\bar{t} \bar{t} H$ datasets are labelled by the generated Higgs mass in $\mathrm{GeV} / \mathrm{c}^{2}$ (parentheses).

\begin{tabular}{llllll}
\hline & \# analysed & $\varepsilon_{\text {loose }}(\%)$ & $N_{\text {loose }}^{e v}$ & $\varepsilon_{\text {tight }}(\%)$ & $N_{\text {tight }}^{\text {ev }}$ \\
\hline $\mathrm{t} \overline{\mathrm{H}}(115)$ & 27900 & $0.511 \pm 0.025$ & $168 \pm 8$ & $0.088 \pm 0.010$ & $29 \pm 3$ \\
$\mathrm{t} \overline{\mathrm{H}}(120)$ & 26141 & $0.490 \pm 0.025$ & $132 \pm 7$ & $0.070 \pm 0.009$ & $19 \pm 3$ \\
$\mathrm{t} \overline{\mathrm{H}}(130)$ & 25911 & $0.490 \pm 0.025$ & $82 \pm 4$ & $0.072 \pm 0.010$ & $12 \pm 2$ \\
$\overline{\mathrm{t}} \mathrm{b} \overline{\mathrm{b}}$ & 313894 & $0.637 \pm 0.014$ & $1080 \pm 24$ & $0.094 \pm 0.007$ & $159 \pm 12$ \\
$\mathrm{t} \overline{1} \mathrm{j}$ & 280385 & $0.0125 \pm 0.0021$ & $1270 \pm 220$ & 0 & $<42(68 \%$ C.L. $)$ \\
$\mathrm{t} \overline{\mathrm{t}} \mathrm{j}$ & 276917 & $0.0448 \pm 0.0040$ & $2690 \pm 240$ & $0.00144 \pm 0.00072$ & $87 \pm 43$ \\
$\mathrm{t} \overline{\mathrm{t}} \mathrm{j}$ & 90367 & $0.0553 \pm 0.0078$ & $1330 \pm 190$ & 0 & $<31(68 \%$ C.L. $)$ \\
$\mathrm{t} \mathrm{t} 4 \mathrm{j}$ & $0.0716 \pm 0.0077$ & $2620 \pm 280$ & $0.0025 \pm 0.0014$ & $92 \pm 53$ \\
$\mathrm{t} \overline{\mathrm{Z}}$ & 12281 & $0.304 \pm 0.017$ & $103 \pm 6$ & $0.0363 \pm 0.0057$ & $12 \pm 2$ \\
$\mathrm{all}$ backgrounds & 110156 & & 9090 & & $<422$ \\
$S / \sqrt{B}(115)$ & & 1.8 & & 1.4 \\
$S / B(115)$ & & $1.8(\%)$ & & $6.9(\%)$ \\
$S / \sqrt{B}(120)$ & & 1.4 & & 0.9 \\
$S / B(120)$ & & $1.5(\%)$ & & $4.5(\%)$ \\
$S / \sqrt{B}(130)$ & & 0.9 & & 0.6 \\
$S / B(130)$ & & $0.9(\%)$ & & $2.9(\%)$ \\
\hline
\end{tabular}

The generated $W^{-}$was forced to decay leptonically $(e, \mu, \tau)$, but the $W^{+}$was allowed to decay freely. This "non-exclusive" dataset incurs a branching ratio of $1 / 3$, which has been factored into the selection efficiencies reported in Table 5.28. This choice allows us to obtain a good estimate of the overlap of the contribution to the dilepton sample arising from semileptonic top decays which are mis-reconstructed as dilepton events; the same applies to tau decays which are mis-reconstructed as $e, \mu$.

The background events have small efficiency to pass the selection criteria, so very large samples must be analysed. To make these samples more manageable, a loose pre-selection requiring at least $3 \mathrm{~b}$-tags with discriminator $D>0.7$ is applied before analysis.

5.3.5.4. Results. The selection efficiencies for the two working points, with the corresponding number of expected events and the signal significance, are reported in Table 5.28. The number of expected events is computed for an integrated luminosity of $\mathrm{fb}^{-1}$.

Since the event selection is quite simple for the dilepton channel, it is possible to formulate simple equations predicting the selection efficiencies. This is detailed in Ref. [160], where some back-of-the-envelope calculations to estimate these efficiencies for both signal and backgrounds are presented, including some of the backgrounds that were not taken into account in this analysis.

5.3.5.5. All-hadron channel: $\overline{\mathrm{t}} \mathrm{H} \rightarrow \mathrm{b} \overline{\mathrm{b}} \mathrm{b} \overline{\mathrm{b}} \mathrm{qq}^{\prime} \mathrm{q}^{\prime \prime} \mathrm{q}^{\prime \prime \prime}$. A number of kinematic variables, together with the b-tagging discriminator, have been studied to optimise the signal selection with respect to background rejection. Moreover, in order to combine the results from the 4 different decay sub-channels, a veto on leptons has been applied using the complementary cut developed within the semi and fully leptonic decays analyses: events are discarded if $-\log \left(L_{\mu}\right)<1.4$ or $-\log \left(L_{e}\right)<1.2$. 
The final set of variables that are used in this analysis is the following:

- Jet Transverse Energy of the 8 most energetic jets in the tracker acceptance.

- Combined b-Tag discriminator variable for each jet.

- Centrality of the event defined as $\sum_{i=0}^{8} E_{\mathrm{T}}^{i} / E^{i}$.

- Centrality of the Higgs defined similarly, with the sum restricted to the 2 jets paired to the Higgs.

The jet-to-parton matching is performed using a $\chi^{2}$ method as defined in [160].

Two working points have been chosen: the first uses loose cuts on the b-tagging discriminator to get higher statistical significance (but lower $S / B$ ), while the second uses a tighter cut on the b-tagging discriminator to obtain a higher $S / B$ (but lower significance). For the first working point an event is selected if the following conditions are satisfied:

- $E_{\mathrm{T}}^{7 t h}>30 \mathrm{GeV}$ and $E_{\mathrm{T}}^{8 t h}>20 \mathrm{GeV}$ for the $E_{\mathrm{T}}$ ordered jets.

- the $\chi^{2}$ for each of the $2 W$ bosons and $2 t$ quarks are within 3 sigma of their expected values.

- the 3 highest combined b-tagging discriminators for the 4 jets associated to the $b$-partons must satisfy $D_{3}>0.80$.

- Higgs centrality higher than 0.55 and no cut on Event Centrality.

For the tight working point, the b-tagging discriminator for the third highest jet is required to satisfy $D_{3}>0.85$ and the fourth one $D_{4}>0.70$, while the event and Higgs centrality are required to exceed 0.55 and 0.80 , respectively.

All the applied cuts have been optimised to obtain the highest significance while keeping the $S / B$ ratio as high as possible. All values chosen for $E_{\mathrm{T}}^{7 t h}, E_{\mathrm{T}}^{8 t h}, D_{3}, D_{4}$, Event and Higgs centrality have been varied simultaneously, thereby mapping out the complete set of combinations within the following limits:

- $20 \mathrm{GeV}<E_{\mathrm{T}}^{8 \text { th }}<40 \mathrm{GeV}$.

- $E_{\mathrm{T}}^{8 t h}<E_{\mathrm{T}}^{7 t h}<E_{\mathrm{T}}^{8 t h}+40 \mathrm{GeV}$.

- $0.5<D_{3}$ and $D_{4}<0.95$.

- Event and Higgs Centrality in the range [0.50-0.95].

Variation of more than one cut has also been tested and the final implemented set of cut values is that for which significance and $S / B$ are optimal.

5.3.5.6. Results. The number of analysed events, selection efficiencies with the corresponding number of expected events and the signal significance are reported in Tables 5.29 for the all-hadron decay channel. Both working points are considered.

\subsubsection{Discussion of systematic uncertainties}

5.3.6.1. Estimation of "standard" CMS systematics. The uncertainties in various quantities, given the knowledge of the CMS experiment at the time of writing this note, are considered first. These differ from what they are expected to be after CMS has collected $60 \mathrm{fb}^{-1}$ of data.

In keeping with other CMS analyses, the following "standard" sources of systematic error are considered:

- Jet energy scale (JES) (3\% to $10 \%$ depending on $p_{t}$ ).

- Jet resolution (10\%).

- b-jet and c-jet tagging efficiencies (4\%).

- uds-jet tagging efficiencies $(10 \%)$.

- Luminosity (3\%). 
Table 5.29. Analysed events, selection efficiency, number of expected events and signal significance in $60 \mathrm{fb}^{-1}$ for the all-hadron $\mathrm{t} \overline{\mathrm{t}} \mathrm{H}$ channel for 2 different working points: $\varepsilon_{\text {loose }}$ and $\varepsilon_{\text {tight }}$. The numbers refer to the full mass range.

\begin{tabular}{llllll}
\hline & \# analysed & $\varepsilon_{\text {loose }}(\%)$ & $N_{\text {loose }}^{\text {ev }} 60 \mathrm{fb}^{-1}$ & $\varepsilon_{\text {tight }}(\%)$ & $N_{\text {tight }}^{\text {ev }} 60 \mathrm{fb}^{-1}$ \\
\hline$\overline{\mathrm{t}} \mathrm{H}(115)$ & 49636 & $2.32 \pm 0.07$ & $347 \pm 10$ & $0.294 \pm 0.015$ & $44 \pm 4$ \\
$\overline{\mathrm{t}} \mathrm{H}(120)$ & 163494 & $2.55 \pm 0.04$ & $314 \pm 5$ & $0.366 \pm 0.024$ & $45 \pm 2$ \\
$\overline{\mathrm{t}} \mathrm{H}(130)$ & 43254 & $2.80 \pm 0.08$ & $214 \pm 6$ & $0.358 \pm 0.029$ & $27 \pm 2$ \\
$\overline{\mathrm{t}} \overline{\mathrm{b}}$ & 203135 & $0.702 \pm 0.019$ & $1190 \pm 31$ & $0.0645 \pm 0.0056$ & $109 \pm 9$ \\
$\overline{\mathrm{t}} 1 \mathrm{j}$ & 1031551 & $0.0084 \pm 0.0009$ & $860 \pm 92$ & $0.0005 \pm 0.0002$ & $49 \pm 22$ \\
$\overline{\mathrm{t}} 2 \mathrm{j}$ & 559111 & $0.0333 \pm 0.0024$ & $2000 \pm 150$ & $0.0009 \pm 0.0004$ & $54 \pm 24$ \\
$\overline{\mathrm{t}} 3 \mathrm{j}$ & 68015 & $0.079 \pm 0.011$ & $1910 \pm 260$ & $0.0015 \pm 0.0015$ & $35 \pm 35$ \\
$\overline{\mathrm{t}} 4 \mathrm{j}$ & $0.182 \pm 0.014$ & $6660 \pm 500$ & $0.0021 \pm 0.0015$ & $75 \pm 53$ \\
$\mathrm{Z} \overline{\mathrm{t}}$ & 97334 & $0.358 \pm 0.021$ & $121 \pm 7$ & $0.0312 \pm 0.0062$ & $11 \pm 2$ \\
$\mathrm{qcd} 170$ & 80226 & $0.0238 \pm 0.0030$ & $4810 \pm 610$ & $0.0004 \pm 0.0004$ & $76 \pm 76$ \\
$\mathrm{qcd} 120$ & 264310 & $0.0018 \pm 0.0018$ & $83 \pm 83$ & $0 \pm 0$ & $<95(68 \% C . L)$. \\
$\mathrm{Total}$ Backgr. & 55128 & & 17600 & & $<505$ \\
$S / \sqrt{B}(115)$ & & & 2.6 & & 2.0 \\
$S / B(115)$ & & & $2.0 \%$ & & $8.7 \%$ \\
$S / \sqrt{B}(120)$ & & & 2.4 & & 2.0 \\
$S / B(120)$ & & & $1.8 \%$ & & $8.9 \%$ \\
$S / \sqrt{B}(130)$ & & & $1.6 \%$ & & 1.2 \\
$S / B(130)$ & & & & & $5.4 \%$ \\
\hline & & & & & \\
\end{tabular}

It is assumed that the systematics listed above are uncorrelated. Each source is varied independently which produces a change in the selection efficiency $\Delta \varepsilon$ and the corresponding change in expected event yields $\Delta N_{X}(X=\mathrm{ttH}, \mathrm{tt} 1 \mathrm{j}, \ldots)$ for the signal and background.

A very detailed breakdown of the various sources of systematic uncertainties and the methods of how they are computed for all the background and signal samples is available in Reference [160]. In Table 5.30, the systematic uncertainties are propagated to the expected signal significance for "tight" and "loose" working points.

5.3.6.2. Background rates from data. There are relatively large theoretical uncertainties in the cross-sections used to normalise the signal yields [162], and even larger theoretical uncertainties in those used for the $t \bar{t}+$ jets backgrounds [168]. These have not been included as part of the systematic errors considered above, because when the CMS experiment reaches maturity, estimating the $\bar{t}+$ jets background directly from data ought to be possible. In this way, the uncertainty associated with Monte Carlo derived tagging rates are avoided entirely. For example, the number of mis-tagged $\bar{t}+$ jets which can be factorised as follows:

$$
N_{t \bar{t} j j}^{m i s t a g}=N_{t \bar{t} j j}^{n o-t a g} \times \operatorname{Pr}\left(u d s \rightarrow b ; E_{\mathrm{T}}, \eta, \ldots\right)
$$

where $N_{t \bar{j} j j}^{n o-t a g}$ is a high purity (e.g. fully reconstructed with a mass window) top sample that has been obtained without requiring b-tagging and $\operatorname{Pr}\left(u d s \rightarrow b ; E_{\mathrm{T}}, \eta, \ldots\right)$ is a parameterised "fake matrix" that is derived from some independent dataset (e.g. dijet data) which yields the probability for a light quark jet to fake a secondary vertex. It may also be possible to derive this fake matrix from the top sample itself. If a high-purity (e.g. double-tagged and fully reconstructed) semi-leptonic top sample were selected, the jets belonging to the hadronic $W$ would provide a source of both light quark and charm jets. From these data, a measurement of the corresponding uds-tag and c-tag rates at the relevant energy could be directly obtained. 
Table 5.30. Significance before and after taking into account the uncertainty $d B$ in the total number of background events due to systematics.

\begin{tabular}{|c|c|c|c|c|c|c|c|}
\hline muon & $S / B$ & $S / \sqrt{B}$ & $S / \sqrt{B+d B^{2}}$ & dilepton & $S / B$ & $S / \sqrt{B}$ & $S / \sqrt{B+d B^{2}}$ \\
\hline \multicolumn{4}{|c|}{$L_{b \text { Sele }}>0.55\left(\varepsilon_{\text {loose }}\right)$} & \multicolumn{4}{|c|}{ 4-7 jets, 3-4 b-tagged ( $\left.\varepsilon_{\text {loose }}\right)$} \\
\hline $\mathrm{t} \overline{\mathrm{t}} \mathrm{H}(115)$ & 0.052 & 2.2 & 0.20 & $\mathrm{t} \overline{\mathrm{t}} \mathrm{H}(115)$ & 0.018 & 1.8 & 0.10 \\
\hline $\mathrm{t} \overline{\mathrm{t}} \mathrm{H}(120)$ & 0.041 & 1.8 & 0.15 & $\mathrm{t} \overline{\mathrm{t}} \mathrm{H}(120)$ & 0.015 & 1.4 & 0.08 \\
\hline $\mathrm{t} \overline{\mathrm{t}} \mathrm{H}(130)$ & 0.030 & 1.3 & 0.11 & $\mathrm{t} \overline{\mathrm{t}} \mathrm{H}(130)$ & 0.009 & 0.9 & 0.05 \\
\hline \multicolumn{4}{|c|}{$L_{b S e l e}>0.75\left(\varepsilon_{\text {tight }}\right)$} & \multicolumn{4}{|c|}{$4-6$ jets, $4-6$ b-tagged $\left(\varepsilon_{t i g h t}\right)$} \\
\hline $\mathrm{t} \overline{\mathrm{t}} \mathrm{H}(115)$ & 0.108 & 2.0 & 0.44 & $\mathrm{t} \overline{\mathrm{t}} \mathrm{H}(115)$ & 0.069 & 1.4 & 0.42 \\
\hline $\mathrm{t} \overline{\mathrm{t}} \mathrm{H}(120)$ & 0.082 & 1.6 & 0.34 & $\mathrm{t} \overline{\mathrm{t}} \mathrm{H}(120)$ & 0.045 & 0.9 & 0.27 \\
\hline $\mathrm{t} \overline{\mathrm{t}} \mathrm{H}(130)$ & 0.060 & 1.1 & 0.24 & $\mathrm{t} \overline{\mathrm{t}} \mathrm{H}(130)$ & 0.029 & 0.6 & 0.18 \\
\hline electron & $S / B$ & $S / \sqrt{B}$ & $S / \sqrt{B+d B^{2}}$ & hadron & $S / B$ & $S / \sqrt{B}$ & $S / \sqrt{B+d B^{2}}$ \\
\hline \multicolumn{4}{|c|}{$L_{b \text { Sele }}>0.55\left(\varepsilon_{\text {loose }}\right)$} & \multicolumn{4}{|c|}{ Working Point $\varepsilon_{\text {loose }}$} \\
\hline $\mathrm{t} \overline{\mathrm{t}} \mathrm{H}(115)$ & 0.051 & 1.8 & 0.20 & $\mathrm{t} \overline{\mathrm{t}} \mathrm{H}(115)$ & 0.020 & 2.6 & 0.07 \\
\hline $\mathrm{t} \overline{\mathrm{t}} \mathrm{H}(120)$ & 0.044 & 1.6 & 0.17 & $\mathrm{t} \overline{\mathrm{t}} \mathrm{H}(120)$ & 0.018 & 2.4 & 0.07 \\
\hline $\mathrm{t} \overline{\mathrm{t}} \mathrm{H}(130)$ & 0.030 & 1.1 & 0.12 & $\mathrm{t} \overline{\mathrm{t}} \mathrm{H}(130)$ & 0.012 & 1.6 & 0.05 \\
\hline \multicolumn{4}{|c|}{$L_{\text {bSele }}>0.75\left(\varepsilon_{\text {tight }}\right)$} & \multicolumn{4}{|c|}{ Working Point $\varepsilon_{t i g h t}$} \\
\hline $\mathrm{t} \overline{\mathrm{t}} \mathrm{H}(115)$ & 0.086 & 1.5 & 0.37 & $\mathrm{t} \overline{\mathrm{t}} \mathrm{H}(115)$ & 0.087 & 2.0 & 0.22 \\
\hline $\mathrm{t} \overline{\mathrm{t}} \mathrm{H}(120)$ & 0.072 & 1.2 & 0.31 & $\mathrm{t} \overline{\mathrm{t}} \mathrm{H}(120)$ & 0.089 & 2.0 & 0.22 \\
\hline $\mathrm{t} \overline{\mathrm{t}} \mathrm{H}(130)$ & 0.052 & 0.9 & 0.22 & $\mathrm{t} \overline{\mathrm{t}} \mathrm{H}(130)$ & 0.054 & 1.2 & 0.13 \\
\hline
\end{tabular}

\subsubsection{Combined significance}

Since the event samples for the channels studied in this note are strictly disjoint, the results can be combined by simply adding the individual signal yields (background yields) to obtain a summed $S(B)$.

For each of the considered systematics, the resultant error in background yields are added for all four channels, since they are by definition fully correlated. The summed errors are then added by quadratures to get a combined systematic uncertainty $d B$. One then calculates the significance, inclusive of systematic uncertainties in the background yield, according to the formula $S / \sqrt{B+d B^{2}}$.

It is of interest to see how much better the results have the potential to be at tighter working points for the various analyses. Since the systematic uncertainties are not well quantified at these "tight" working points, because of a lack in Monte Carlo Statistics, the same uncertainties as for the "loose" working points are used to reduce spurious statistical effects. This procedure can be justified by the observation that the impact of the b-tagging and uds-mistagging uncertainty is smaller at the "tight" working points and the JES uncertainty becomes dominant. Since the "tight" working points are defined by stronger b-tagging cuts, while keeping the $E_{\mathrm{T}}$ cuts constant, no major change in the relative systematic uncertainty is expected. A more detailed study of the systematic error at the "tight" working points for samples with enough Monte Carlo Statistics is available in Ref. [160].

It is difficult to predict at this time exactly what will be the level to which the backgrounds can be understood, because the tools required are not yet in existence and because this understanding requires real data. In view of this, it is interesting to consider how the combined significance of the measurements presented in this note would vary as a function of the fractional uncertainty in background cross-sections, i.e. as $d B_{x s e c} / B$.

The solid central line in Figure 5.17 shows how the combined significance $S / \sqrt{B+\left(d B_{s y s}+d B_{x s e c}\right)^{2}}$ degrades as a function of $d B_{x s e c} / B$. The signal and background yields for the tightest working points $\left(N_{\text {tight }}^{e v}\right.$ in Table 5.27, Table 5.28 and Table 5.29) 

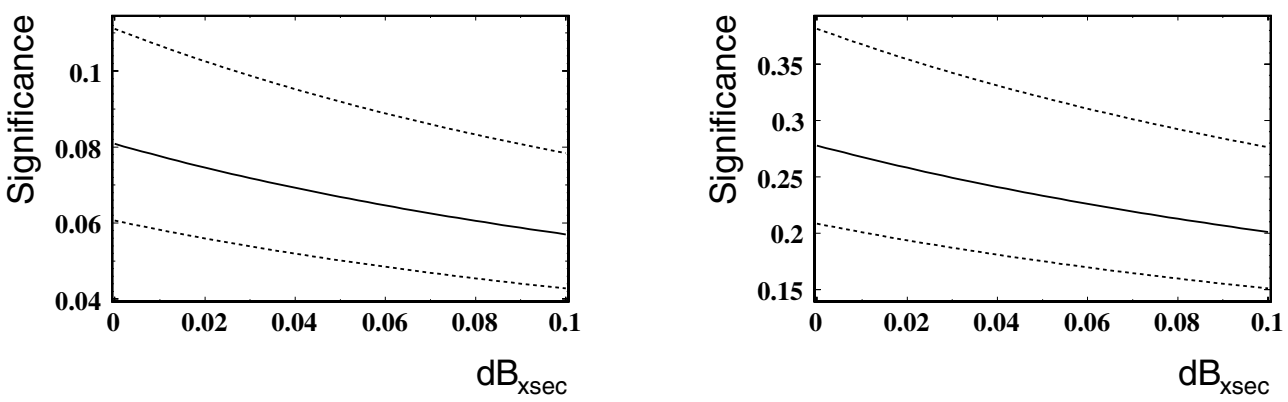

Figure 5.17. Expected range of combined significance (dilepton + semi-leptonic + all-hadron, and includes the systematic uncertainties estimated in Section 5.3.6.1) versus an additional systematic uncertainty on the background cross-section as a fraction of total background. (Left) Results for the "loose" working points. (Right) Results for the "tight" working points.
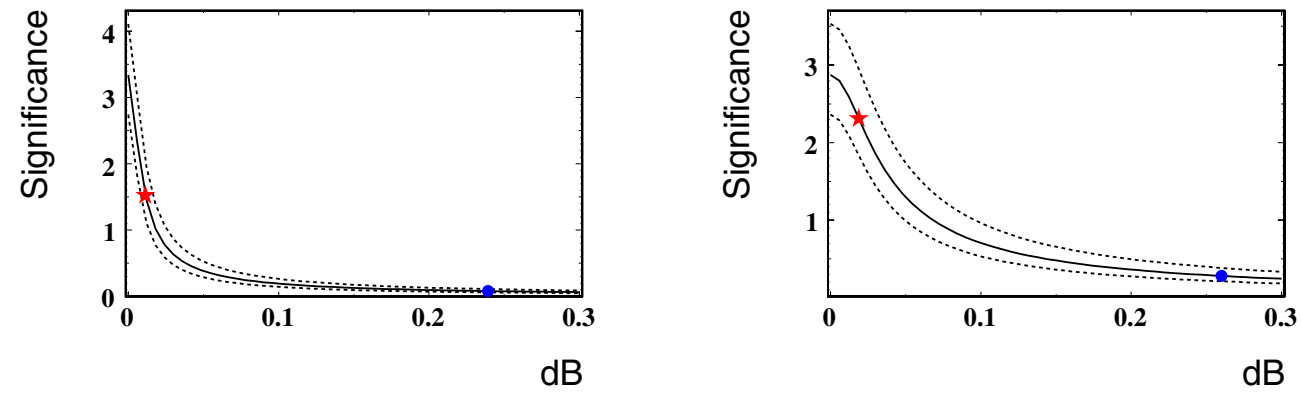

Figure 5.18. Expected range of combined significance (dilepton + semi-leptonic + allhadron) versus the total systematic uncertainty in background as a fraction of total background. (Left) Results for the "loose" working points. (Right) Results for the "tight" working points.

are used in the right side of Figure 5.17, because these give the best results after inclusion of systematics.

Other than this "fundamental" cross-section uncertainty, there is also the "correctible" errors in the cross-sections used at the time of writing, which can be compensated for once data has been collected. The upper and lower dashed curves in Figure 5.17 show the maximum and minimum allowed excursions, should the signal and background cross-sections be off by $10 \%$ and $20 \%$ respectively. Thus the upper (lower) dashed line corresponds to the signal cross-section scaled up (down) by $10 \%$ while at the same time the background cross-section is scaled down (up) by $20 \%$.

It is also of interest to see how much better the analyses could do if the total systematic uncertainty can be reduced (i.e. the region left of zero in Figure 5.17). Hence, Figure 5.18 shows the full range of obtainable significances, with the dot marking the currently estimated value with no cross-section uncertainty $\left(d B=d B_{s y s}\right)$. The star corresponds to what one would obtain for $1 \%$ and $4 \%$ uncertainties on the $\bar{t} \mathrm{t} N j$ and $\bar{t} \bar{b} \bar{b}$ backgrounds, respectively, an arbitrarily chosen reference. It is interesting to note that it does not quite yield a substantial significance, even though background uncertainties of $1 \%$ and $4 \%$ for $\mathrm{tt}_{\mathrm{tj}} \mathrm{and} \mathrm{t} \mathrm{t} \overline{\mathrm{b}}$ are probably substantially better than what will be accessible in reality. This highlights the challenge that is faced in observing $\mathrm{t} \overline{\mathrm{t}} \mathrm{H}$. 


\section{Chapter 6. Physics Studies with Heavy Ions}

\subsection{Benchmark Channel: $P b P b \rightarrow Q \bar{Q}+X \rightarrow \mu^{+} \mu^{-}+X$}

The measurement of the charmonium $\left(J / \psi, \psi^{\prime}\right)$ and bottomonium $\left(\Upsilon, \Upsilon^{\prime}, \Upsilon^{\prime \prime}\right)$ resonances in $\mathrm{PbPb}$ collisions at $\sqrt{s_{N N}}=5.5 \mathrm{TeV}$ provides crucial information on the many-body dynamics of high-density QCD matter. First, the step-wise suppression of heavy quarkonia production is generally agreed to be one of the most direct probes of Quark-Gluon-Plasma formation. Lattice QCD calculations of the heavy-quark potential indicate that colour screening dissolves the ground-state charmonium and bottomonium states, $J / \psi$ and $\Upsilon$, at $T_{\text {diss }} \approx 2 \cdot T_{\text {crit }}$ and 4 . $T_{\text {crit }}$, respectively. While the interest of charmonia production studies in heavy-ion collisions is well established from measurements done at the SPS and at RHIC, the clarification of some important remaining questions requires equivalent studies of the $\Upsilon$ family, only possible at the LHC energies. Second, the production of heavy-quarks proceeds mainly via gluongluon fusion processes and, as such, is sensitive to saturation of the gluon density at low- $x$ in the nucleus ("Colour Glass Condensate"). Measured departures from the expected "vacuum" (proton-proton) quarkonia cross-sections in $\mathrm{PbPb}$ collisions at $\mathrm{LHC}$ will thus provide valuable information not only on the thermodynamical state of the produced partonic medium, but also on the initial-state modifications of the nuclear parton (especially, gluon) distribution functions.

This first CMS heavy-ion physics analysis focuses on the measurement of the heavyquarkonia cross-sections in $\mathrm{PbPb}$ collisions at $\sqrt{s_{N N}}=5.5 \mathrm{TeV}$, via their dimuon decay channel. The generation of realistic signals and backgrounds, the dimuon reconstruction algorithm and the trigger, acceptance and efficiency corrections are discussed. The obtained dimuon mass resolutions, the signal over background as well as the expected yields in onemonth $\mathrm{PbPb}$ running are presented. An example of a $\Upsilon \rightarrow \mu^{+} \mu^{-}$event embedded in a $\mathrm{PbPb}$ collision is shown in colour plate CP9.

\subsubsection{Simulation of physics and background processes}

The relatively low $\Upsilon$ production rates $\left(\sim 10^{-4}\right.$ per $\mathrm{PbPb}$ event $)$ and the large number of particles to track in heavy-ion collisions make it very expensive computationally to use a full nucleus-nucleus event generator (such as e.g. HIJING [169]) with detailed detector simulation and reconstruction to obtain a statistically significant sample of signal events. Instead, a combination of fast and slow simulations are used in this analysis. The input signal and backgrounds are obtained from realistic distributions: NLO pQCD for heavyquark production processes, and HIJING for the soft background, constrained by extrapolations from lower energy heavy-ion data. A full detector and trigger simulation plus reconstruction are carried out for a few $10^{7}$ events with single and pair particles of the different types and the corresponding response functions (acceptances, resolutions, efficiencies, etc) are parameterised in a fast MC, used to obtain the final fully corrected yields. The response functions are cross-checked by comparing the final dimuon spectra obtained with the fast MC against $5 \times 10^{5} \mathrm{PbPb}$ HIJING events fully simulated and reconstructed in the detector.

The quarkonium production cross sections in $\mathrm{PbPb}$ are obtained from NLO pp calculations at $\sqrt{s}=5.5 \mathrm{TeV}$ made in the colour evaporation model (CEM) [170], using MRST PDF modified with the EKS98 prescription for nuclear shadowing [171], with renormalisation and factorisation scales $\mu_{R}=\mu_{F}=m_{Q}$, and scaled by $A^{2}(A=208$ for $\mathrm{Pb})$. The resulting (impact-parameter averaged) inclusive quarkonia production cross sections are: $B_{\mu \mu} \sigma_{Q \bar{Q}}=49000,900,300,80,45 \mu \mathrm{b}$ for $J / \psi, \psi^{\prime}, \Upsilon, \Upsilon^{\prime}$, and $\Upsilon^{\prime \prime}$, respectively. The NLO 
double-differential $d^{2} \sigma / d p_{\mathrm{T}} d \varphi$ distributions of $J / \psi$ and $\Upsilon$ are also used for the other states within each quarkonium family.

The two main sources of background in the dimuon invariant mass spectrum are:

1. Uncorrelated decays of charged pions and kaons, which represent about $90 \%$ of the produced charged particles. This source was simulated using input pion and kaon $d^{2} N / d p_{\mathrm{T}} d \eta$ distributions from HIJING, absolutely normalised to give $d N_{c h} /\left.d \eta\right|_{\eta=0}=2500$ (low) and 5000 (high) multiplicities in central PbPb. Both cases are conservative ("pessimistic") estimates, since extrapolations from RHIC data indicate that $d N_{c h} /\left.d \eta\right|_{\eta=0} \approx 2000$ at the LHC.

2. The other source of background muons are open heavy flavour ( $D, B$ mesons) decaying a few $\mathrm{mm}$ away from the interaction vertex. The probability to produce at least one muon at the end of the decay chain of charm (bottom) quarks is $\sim 18 \%$ (38\%) according to PYTHIA 6.025. The double differential $\left(p_{\mathrm{T}}, \eta\right)$ cross-sections are obtained from pp NLO calculations (with CTEQ5M1 PDF, and $\mu_{R}=\mu_{R}=m_{Q}$ ), which give $\sigma_{c \bar{c}, b \bar{b}}=7.5,0.2$ $\mathrm{mb}$ [170], scaled by the nuclear overlap function, $\left\langle T_{P b P b}(b=0 \mathrm{fm})\right\rangle=30.4 \mathrm{mb}^{-1}$, to obtain the expected yields in central $\mathrm{PbPb}$ collisions.

A fast $\mathrm{MC}$ simulation equivalent to $5 \cdot 10^{7} \mathrm{PbPb}$ events has been carried out superimposing the decay dimuon from the five quarkonium resonances on top of the background from the combinatorial decays of $\pi, K$ and open heavy flavour. Each muon track (with a given momentum, pseudorapidity, charge and origin) is weighted by a factor that takes into account the corresponding detector acceptance, as well as trigger and reconstruction efficiency for the two event multiplicities considered (see next section).

\subsubsection{Reconstruction and analysis}

6.1.2.1. Dimuon trigger and acceptance. The response of the CMS detector to muons (as well as long-lived punch through pions and kaons reaching the muon chambers) is parameterised by 2 -dimensional $p, \eta$ acceptance and trigger tables. The particles are fully tracked in CMS using GEANT4 from the vertex to the chambers. Each track is accepted or rejected according to the Level-1,2 heavy-ion dimuon trigger criteria [7] and the corresponding efficiencies, $\varepsilon_{\text {trig }}^{L V 1}(p, \eta)$ and $\varepsilon_{\text {trig }}^{L V L}(p, \eta)$, are computed. Trigger efficiencies are of the order of $\sim 90 \%$ for those $\mu$ reaching the muon chambers. The $J / \psi$ and $\Upsilon$ acceptances are shown as a function of $p_{\mathrm{T}}$ in Fig. 6.1, for two $\eta$ ranges: full detector and central barrel. Because of its relatively low mass, low energy $J / \psi$ 's $\left(p_{\mathrm{T}} \lesssim 4 \mathrm{GeV} / \mathrm{c}\right)$ cannot be detected since their decay muons don't have enough energy to traverse the calorimeters and they are absorbed due to ionisation losses before reaching the muon chambers. For larger $p_{\mathrm{T}}$ values the $J / \psi$ acceptance increases and flattens out at $\sim 15 \%$ for $p_{\mathrm{T}} 12 \mathrm{GeV} / \mathrm{c}$. The $\Upsilon$ acceptance starts at $\sim 40 \%$ at $p_{\mathrm{T}}=0 \mathrm{GeV} / \mathrm{c}$ and remains constant at $15 \%$ (full CMS) or $5 \%$ (barrel) for $p_{\mathrm{T}}>4 \mathrm{GeV} / \mathrm{c}$. The $p_{\mathrm{T}}$-integrated acceptance is about $1 . \%$ for the $J / \psi$ and $21 \%$ for the $\Upsilon$ as obtained from our input theoretical distribution.

6.1.2.2. Dimuon reconstruction efficiency, purity and mass resolution. The dimuon reconstruction algorithm used in the heavy-ion analysis is a version of the regional track finder based on the muons seeded by the muon stations and on the knowledge of the primary vertex, as described in $[172,173]$. It is adapted to deal with the high hit occupancy of the silicon tracker in $\mathrm{PbPb}$ collisions. It uses the muon tracks found in the innermost muon stations to identify hits in the outer CMS tracker layer that can form the starting points (seeds) for the matching muon candidate tracks. The propagation in the tracker is performed from the outer 

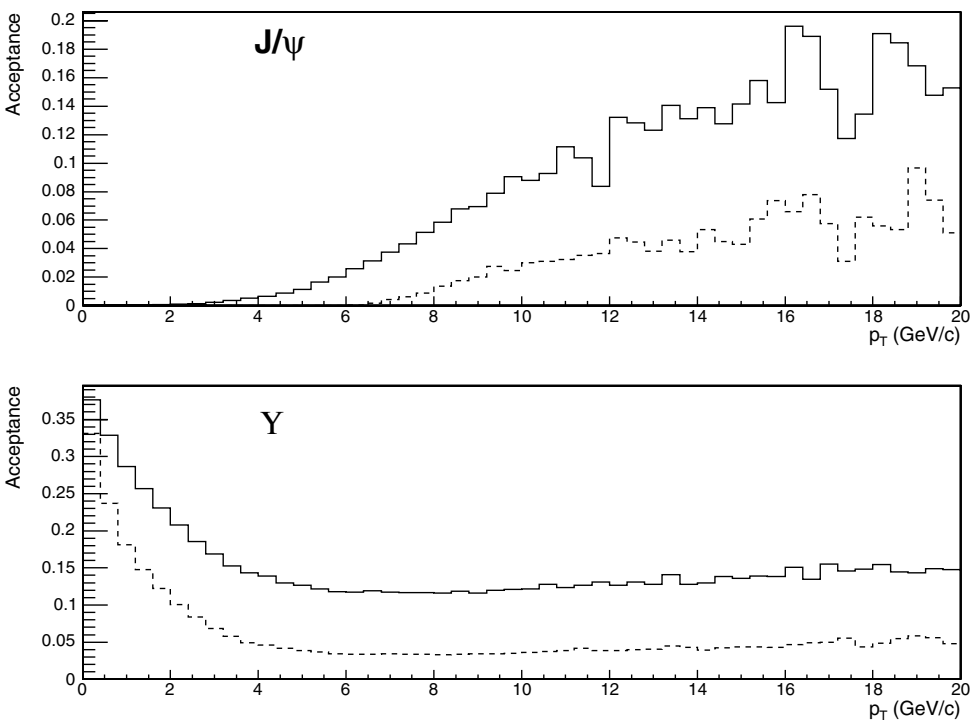

Figure 6.1. $J / \psi$ (top) and $\Upsilon$ (bottom) acceptances as a function of $p_{\mathrm{T}}$, in the full detector (barrel and endcap, $|\eta|<2.4$, full line) and in the barrel alone $(|\eta|<0.8$, dashed line).
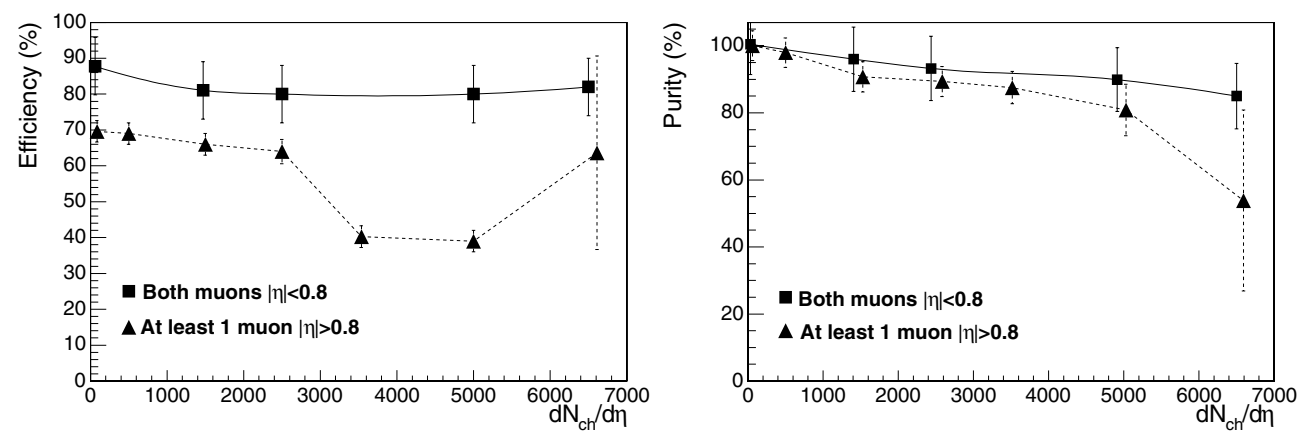

Figure 6.2. $\Upsilon$ reconstruction efficiency (left) and purity (right) as a function of the $\mathrm{PbPb}$ charged particle rapidity density, $d N_{c h} /\left.d \eta\right|_{\eta=0}$.

layer towards the primary vertex, using two-dimensional parametrisation in the transverse and longitudinal planes. The final fit of trajectories is performed with a Kalman-fitter. The efficiency of a given muon pair is: $\varepsilon_{\text {pair }}(p, \eta)=\varepsilon_{\text {track }_{1}} \times \varepsilon_{\text {track }_{2}} \times \varepsilon_{\text {vertex }}$. The dependence of the $\Upsilon$ reconstruction efficiency on the event multiplicity was obtained from a full GEANT simulation using $\Upsilon$ signal dimuon embedded in HIJING PbPb events. Figure 6.2 shows the $\Upsilon$ efficiency and purity (where purity is defined as the ratio of true $\Upsilon$ reconstructed over all $\Upsilon$ reconstructed) as a function of charged-particle multiplicity. In the central barrel, the dimuon reconstruction efficiency is above $\sim 80 \%$ for all multiplicities, whereas the purity decreases slightly with $d N_{c h} / d \eta$ but stays also above $80 \%$ even at multiplicities as high as $d N_{c h} /\left.d \eta\right|_{\eta=0}=6500$. If (at least) one of the muons is detected in the endcaps, the efficiency and purity drop due to stronger reconstruction cuts. Nonetheless, for the maximum $d N_{c h} /\left.d \eta\right|_{\eta=0} \approx 2500$ multiplicities expected in central PbPb at LHC, the efficiency (purity) remains above $65 \%(90 \%)$ even including the endcaps. 

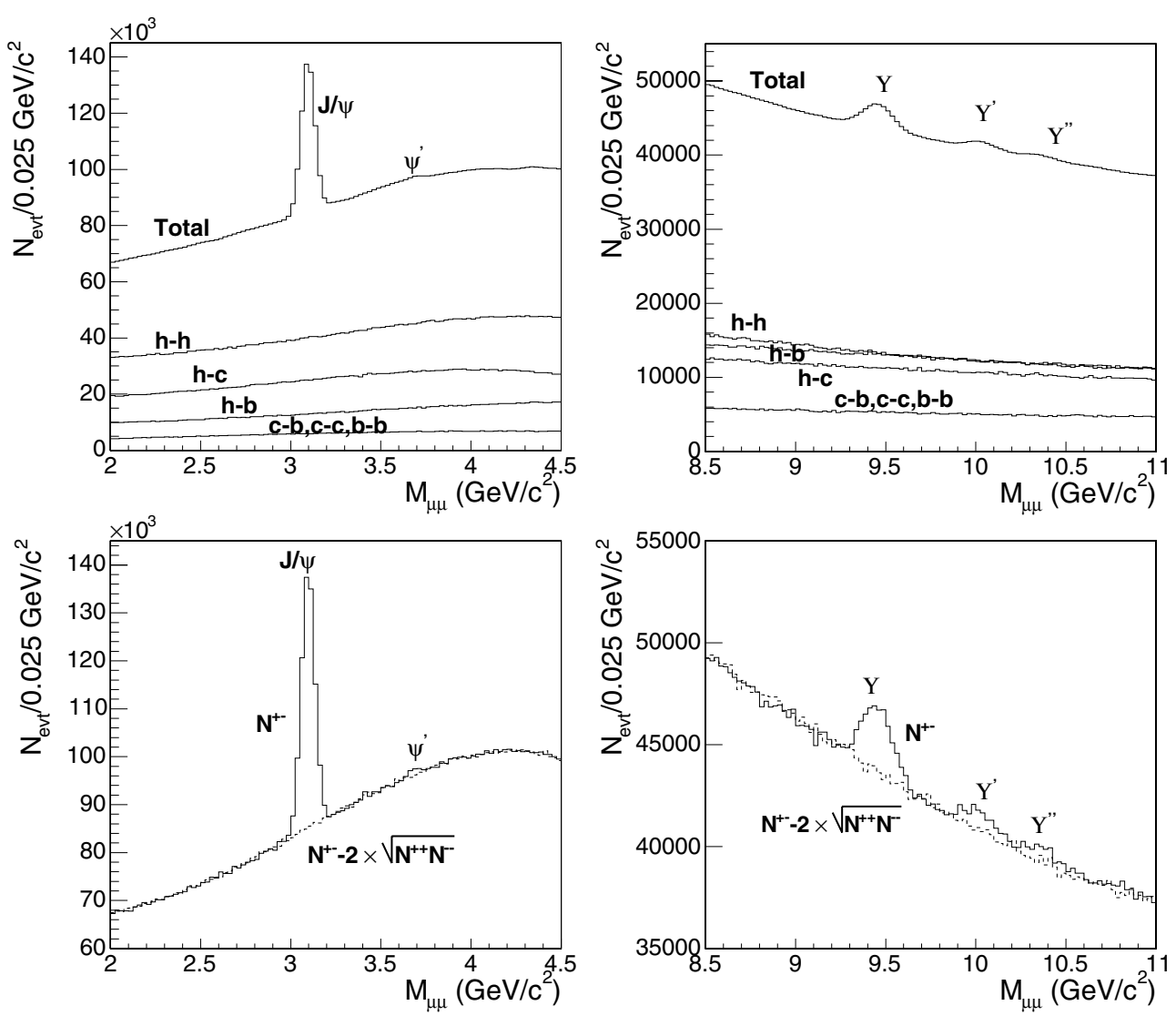

Figure 6.3. Dimuon mass distributions measured within $|\eta|<2.4$ for $\mathrm{PbPb}$ events with $d N_{c h} /\left.d \eta\right|_{\eta=0}=5000$ in the $J / \psi$ (left) and $\Upsilon$ (right) mass regions. The main contributions of the background are shown in the top panels $(h, c, b$ stand for $\pi+K$, charm, bottom decay muons resp.), while the bottom panels also show the like-sign pairs (combinatorial background).

If we only consider muon pairs in the central barrel, $|\eta|<0.8$, the dimuon mass resolution is $\sim 54 \mathrm{MeV} / \mathrm{c}^{2}$ at the $\Upsilon$ mass, as obtained from a Gaussian fit of the reconstructed $\mu \mu m_{\text {inv }}$ distribution (using a detailed MC simulation but without background). In the full pseudorapidity range, the dimuon mass resolution amounts to $\sim 1 \%: 35 \mathrm{MeV} / \mathrm{c}^{2}$ at the $J / \psi$ mass, and $86 \mathrm{MeV} / \mathrm{c}^{2}$ at the $\Upsilon$ mass. These dimuon mass resolutions (the best among the LHC experiments) allow for a clean separation of the different quarkonia states. These values are used to smear the dimuon mass distribution in the fast MC studies.

\subsubsection{Results}

About $5 \times 10^{7} \mathrm{PbPb}$ collisions were simulated. Muons passing the acceptance tables are combined to form pairs and each pair is weighted according to the trigger and reconstruction efficiencies (dependent on the momentum, $\eta$, purity and event multiplicity). Their invariant mass is calculated and smeared as described in the previous section. The obtained dimuon mass distributions are then scaled to $0.5 \mathrm{nb}^{-1}$, corresponding to the $\mathrm{PbPb}$ luminosity integrated in one month with average luminosity $L=0.4 \cdot 10^{27} \mathrm{~cm}^{-2} \mathrm{~s}^{-1}$ and $50 \%$ machine operation efficiency. Figure 6.3 shows the resulting opposite-sign mass distributions, for 

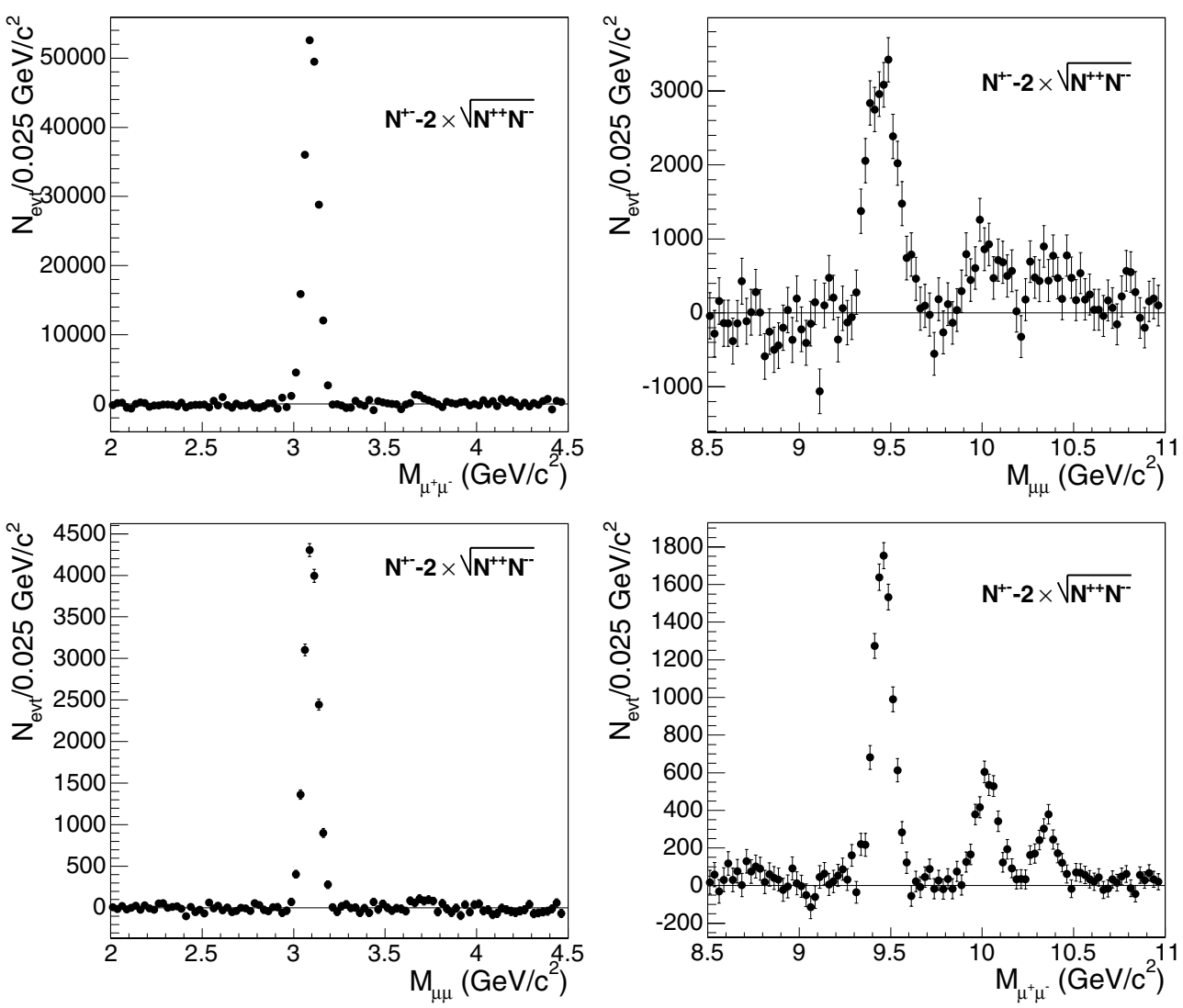

Figure 6.4. Signal dimuon mass distributions after background subtraction in the $J / \psi$ (left) and $\Upsilon$ (right) mass regions expected after one month of $\mathrm{PbPb}$ running. Top panels for $d N_{c h} /\left.d \eta\right|_{\eta=0}=$ 5000 and $|\eta|<2.4$ ("worst" case conditions); bottom panels for $d N_{c h} /\left.d \eta\right|_{\eta=0}=2500$ and $|\eta|<$ 0.8 ("best" measurement conditions).

the high multiplicity case, $d N_{c h} /\left.d \eta\right|_{\eta=0}=5000$ and full acceptance $(\eta<2.4)$. The different quarkonia resonances appear on top of a continuum due to several combinatorial background sources, the main ones being identified in the upper plots ( $h, c$ and $b$ stand for $\pi+K$, charm and bottom decay muons, respectively). Since the CMS trigger and acceptance conditions treat opposite-sign and like-sign muon pairs in the same way, the uncorrelated background can be subtracted using the like-sign pairs: $N^{\mathrm{Sig}}=N^{+-}-2 \sqrt{N^{++} N^{--}}$, shown also in the bottom panels of Fig. 6.3.

Figure 6.4 shows the signal dimuon mass distributions, after background subtraction, for two different scenarios: $d N_{c h} /\left.d \eta\right|_{\eta=0}=5000,|\eta|<2.4$ ("worst" case conditions); and $d N_{c h} /\left.d \eta\right|_{\eta=0}=2500,|\eta|<0.8$ ("best" case). Except for the $\psi^{\prime}$, all quarkonia states are clearly visible. The corresponding signal-to-background ratios and yields (counted within $1 \sigma$ of the resonance peaks) are collected in the Table 6.1 for one month of $\mathrm{PbPb}$ running.

\subsubsection{Conclusions}

With its very broad muon acceptance and precise tracking, CMS will provide significant contributions to heavy ion physics at the LHC. Studies of quarkonium production in $\mathrm{PbPb}$ 
Table 6.1. Signal-to-background ratios and expected quarkonia yields in one month of $\mathrm{PbPb}$ running ( $0.5 \mathrm{nb}^{-1}$ integrated luminosity) for two multiplicity scenarios and two $\eta$ windows.

\begin{tabular}{lllllll}
\hline$d N_{c h} /\left.d \eta\right|_{\eta=0}, \Delta \eta$ & $\mathrm{S} / \mathrm{B}$ & $\mathrm{N}(J / \psi)$ & $\mathrm{S} / \mathrm{B}$ & $\mathrm{N}(\Upsilon)$ & $\mathrm{N}\left(\Upsilon^{\prime}\right)$ & $\mathrm{N}\left(\Upsilon^{\prime \prime}\right)$ \\
\hline $2500,|\eta|<2.4$ & 1.2 & 180000 & 0.12 & 25000 & 7300 & 4400 \\
$2500,|\eta|<0.8$ & 4.5 & 11600 & 0.97 & 6400 & & \\
$5000,|\eta|<2.4$ & 0.6 & 140000 & 0.07 & $20 / 000$ & 5900 & 3500 \\
$5000,|\eta|<0.8$ & 2.75 & 12600 & 0.52 & 6000 & & \\
\hline
\end{tabular}

collisions at $\sqrt{s_{N N}}=5.5 \mathrm{TeV}$, will provide crucial information on the thermodynamical state of QCD medium formed in these collisions, through the expected step-wise "melting" pattern of the different $Q \bar{Q}$ states due to colour screening. These results will also be sensitive to modifications of the low- $x$ nuclear parton distribution functions, as expected in case of gluon saturation.

CMS can reconstruct the charmonium and bottomonium resonances, via their dimuon decay channel, with high efficiencies $(\sim 80 \%)$, good purity $(\sim 90 \%)$ and a very good dimuon mass resolution $\left(54 \mathrm{MeV} / \mathrm{c}^{2}\right.$ at the $\Upsilon$ mass), when both muons are detected in the central barrel $(|\eta|<0.8)$, even in the case of exceptionally high multiplicities $\left(d N_{c h} /\left.d \eta\right|_{\eta=0} \approx 5000\right)$. When considering the full pseudorapidity region $(|\eta|<2.4)$, the mass resolution becomes $\sim 86 \mathrm{MeV} / \mathrm{c}^{2}$ at the $\Upsilon$, and $35 \mathrm{MeV} / \mathrm{c}^{2}$ at the $J / \psi$, with $\sim 50 \%$ dimuon reconstruction efficiencies. The $\Upsilon$ states can be measured all the way down to $p_{\mathrm{T}}=0 \mathrm{GeV} / \mathrm{c}$ with acceptances as large as $40 \%$, while the lower rest mass of the $J / \psi$ state and the large amount of material in the calorimeters absorbs "low" energy decay muons and prevents from measuring $J / \psi$ 's below $p_{\mathrm{T}} \approx 4 \mathrm{GeV} / \mathrm{c}$. At high $p_{\mathrm{T}}$ (above $\sim 12 \mathrm{GeV} / \mathrm{c}$ for the $J / \psi$ and $\sim 4 \mathrm{GeV} / \mathrm{c}$ for the $\Upsilon$ ) the dimuon acceptance flattens out at $15 \%$.

The large aperture of the muon detectors and the precise tracking result in a very good separation between the $Q \bar{Q}$ states in the dimuon mass distributions, and in relatively high statistics and good signal to background ratios $(S / B \approx 1(5), S / B \approx 0.1(1)$ for $J / \psi$ and $\Upsilon$ resp. in the full (central) rapidity range). After one month of $\mathrm{PbPb}$ running $\left(0.5 \mathrm{nb}^{-1}\right)$ we should collect $\sim 180000 \mathrm{~J} / \psi$ and $\sim 25000 \Upsilon$ dimuon, enough to compare central and peripheral $\mathrm{PbPb}$ collisions, and to carry out some differential studies $\left(d N / d y, d N / d p_{\mathrm{T}}\right)$ which will surely contribute significantly to clarify the physics mechanisms behind the production (and "destruction") of quarkonia states in nucleus-nucleus collisions at the LHC. 


\section{Part II. CMS Physics Reach}

\section{Chapter 7. Physics of Strong Interactions}

\subsection{QCD and jet physics}

\subsubsection{Introduction}

With the start-up of LHC, a new domain of energy will be explored and an extrapolation of our current knowledge in the form of the Standard Model may not be sufficient to describe the new measurements. Even in a first data-taking phase with a rather low luminosity, studies of jet physics in the framework of quantum chromodynamics (QCD) will allow to check our current theory against the new data.

Figure 7.1 presents the decomposition of the total jet cross section into the partonic processes for $p \bar{p}$ collisions at the Tevatron and $p p$ collisions at the LHC in dependence of the scaling variable $x_{\mathrm{T}}=2 p_{\mathrm{T}} / \sqrt{s}$, and illustrates the differences in cross section contributions of the PDFs compared to measurements possible today. In Fig. 7.2 the expected statistical uncertainties on differential cross sections for all rapidities are presented for a pilot run with $0.1 \mathrm{fb}^{-1}$ and for a first physics run with $10 \mathrm{fb}^{-1}$. Trigger pre-scales are taken into account. The figure demonstrates that already in the pilot run high statistics will be available up to $1.5 \mathrm{TeV}$ of transverse jet energy.

On the one hand, the measured data have to be corrected for detector effects using fully simulated events. Also, an energy calibration has to be performed on the reconstructed jets which ideally is extracted from data as well, but can also be done employing Monte-Carlo methods. On the other hand, for the theory predictions, which are most precise with respect to the hard parton-parton scattering amplitudes, effects of soft physics modelled in the form of parton showers and hadronisation models with subsequent decays have to be taken into account. Once this is done, parameters of the current theory can be cross-checked or improved in precision by comparing the measured hadronic final state with the corrected theoretical predictions.

\subsubsection{Jet algorithms}

In order to re-establish a link between the observed particles that appear as collimated streams of hadrons in the detector and the hard process, algorithms are defined to group particles that are supposed to come from the same hard parton into jets. The required ingredients of such a jet algorithm are a distance measure to define the separation between objects, a procedure how to decide when objects are to be combined and a recombination scheme explaining how to combine objects. In addition, it has to be specified how the list of input objects has been determined.

Two principal types of algorithms are in common use: Cone type algorithms [174] that traditionally have been employed in hadron-hadron collisions where objects are clustered together that are close in angle around a high-energetic seed, and clustering algorithms where iteratively objects are combined that have the smallest distance of all pairwise combinations possible. The latter have predominantly been used in $e^{+} e^{-}$and $e^{ \pm} p$ collisions, first in the form of the Jade algorithm [175, 176] and nowadays as $k_{\mathrm{T}}$ algorithm [177].

Both algorithms applied in this study use an angular distance measure based on the azimuthal angle $\Phi$ and, instead of the pseudo-rapidity $\eta$, the true rapidity $y=0.5$ $\ln \left(\left(E+p_{z}\right) /\left(E-p_{z}\right)\right)$ which has become an established standard in recent publications 

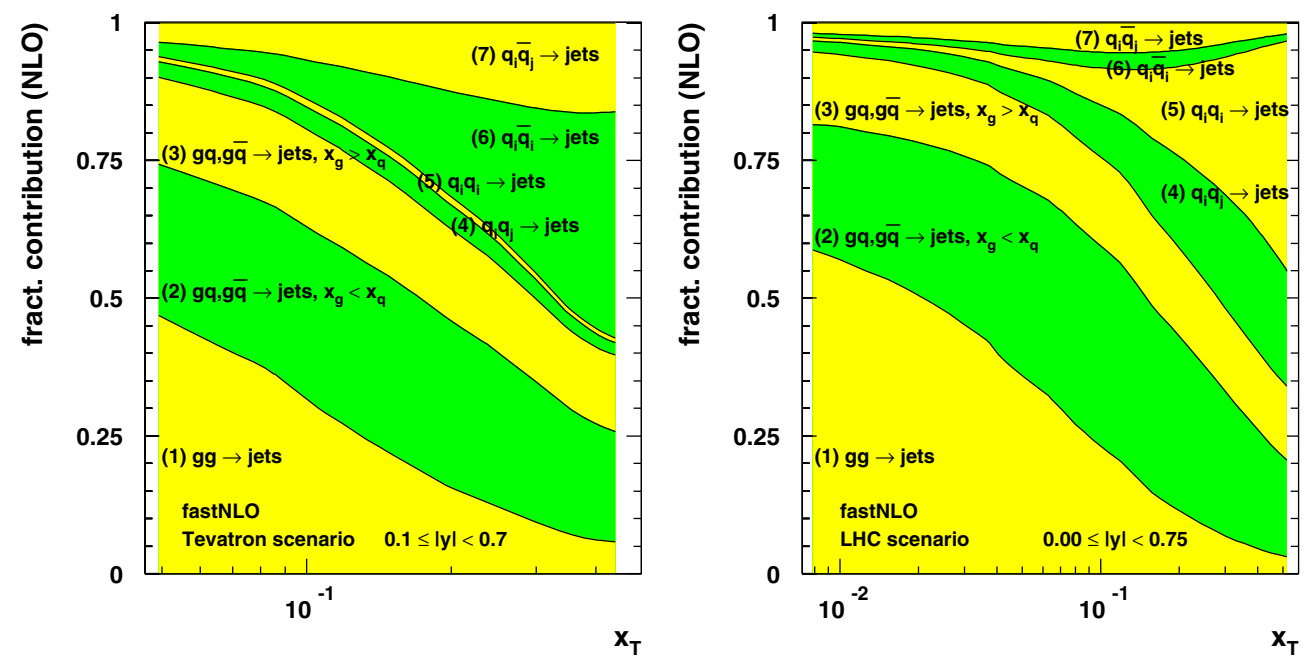

Figure 7.1. Decomposition of the total jet cross section into the partonic processes for $p \bar{p}$ collisions at the Tevatron (left) and $p p$ collisions at the LHC (right). The fractional contributions are shown versus the scaling variable $x_{\mathrm{T}}=2 p_{\mathrm{T}} / \sqrt{s}$
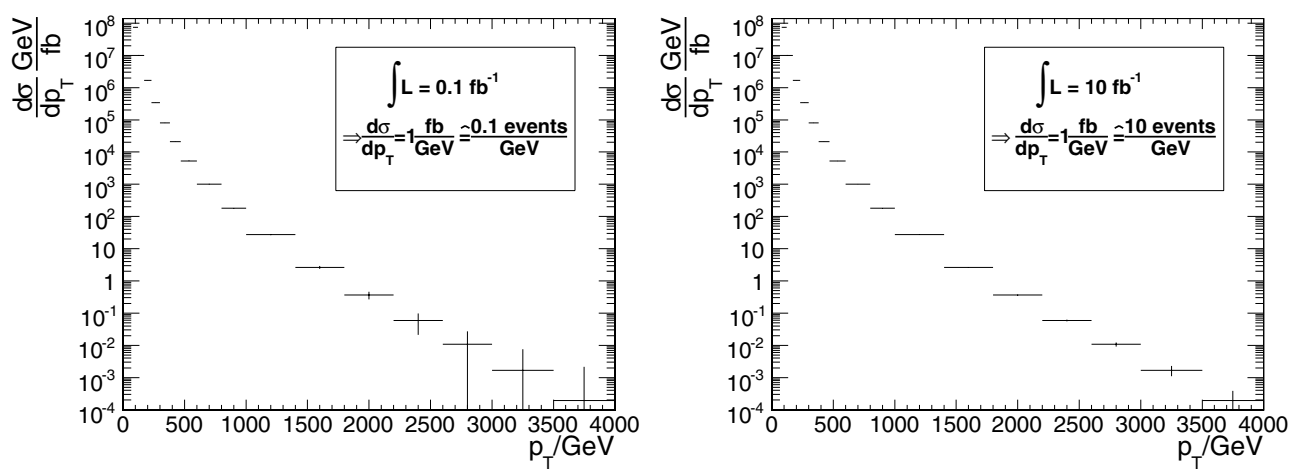

Figure 7.2. Expected statistical uncertainties on differential cross sections for all rapidities; left: for a pilot run with $0.1 \mathrm{fb}^{-1}$, and right: for a physics run with $10 \mathrm{fb}^{-1}$. The central cross section values are taken from a leading-order calculation in dependence of the transverse momenta of the hard interaction.

$[178,179]$. The distance between two objects $i$ and $j$ hence reads

$$
\Delta R_{i j}=\sqrt{\left(\Delta_{i j} \Phi\right)^{2}+\left(\Delta_{i j} y\right)^{2}} .
$$

In addition, the most frequently used recombination scheme, the E scheme, implying a simple four-momentum addition, is employed in both cases.

Two types of jet algorithms are used here. The main results have been achieved with the $k_{\mathrm{T}}$ algorithm defined below, some cross checks have been performed with the midpoint 
cone algorithm:

1. Iterative clustering-type: Inclusive $k_{\mathrm{T}}$ algorithm [180] with

- Distances are evaluated according to the $\Delta R$ scheme, i.e. $d_{i j}=\min \left(p_{\mathrm{T}, i}^{2}, p_{\mathrm{T}, j}^{2}\right) \frac{\Delta R_{i j}^{2}}{D^{2}}$ with $R_{i j}$ as in Eq. 7.1

- Jet resolution parameter $D=1.0$

2. Cone-type: Midpoint cone algorithm [181, 182] with:

- Cone radius $R=0.7$, all objects within a cone have to fulfill $R_{i c} \leqslant R$ with $c$ labelling the four-vector of the current cone.

- Overlap threshold $f_{\text {merge }}=0.50$, i.e. overlapping cone jets are merged when they share more than $50 \%$ of the energy in the less energetic cone

- Search-cone radius fraction $f_{\text {search }}=0.5$, i.e. the first step to find the stable cones (before any splitting/merging is done) is performed with a smaller radius of $f_{\text {search }}^{* R}$

Concerning the $k_{\mathrm{T}}$ algorithm, a jet resolution parameter of $D=1.0$ is, from a theoretical point of view, best comparable to a cone algorithm with $R=0.7$. In order to reduce the sensitivity to the underlying event, it is advantageous to reduce the jet resolution parameter $D$ or the cone radius $R$, respectively.

Note that primarily due to the limited choice of available jet energy calibrations the definition of the midpoint algorithm above has been selected. It does not exactly correspond to the definition given in [181] but to a modified one [182] that is in use by the CDF collaboration [178]. There have been indications that this algorithm leads to an infrared sensitive behaviour [183], so it is recommended to use the original definition of the midpoint algorithm without extra search cone radius.

\subsubsection{Trigger scheme, event selection and phase space}

The level one (L1) and the high level triggers (HLT) required for this analysis are the singlejet triggers which are described in more detail in Section E.4.3.2. QCD jet production has, by several orders of magnitude, the largest cross section, but in contrast to most other analyses QCD jet events are the signal here. Therefore, the sole other selection requirement for this study demands all jets to have a transverse momentum larger than $50 \mathrm{GeV}$. The available phase space is then subdivided into 17 ranges in transverse momentum $p_{\mathrm{T}}$ and five ranges in rapidity $y$, where the focus is mostly on the central region up to 2.5 in rapidity.

\subsubsection{Input data}

The analysed events were generated with PYTHIA [184] and subsequently subjected to the full GEANT-4 based CMS detector simulation and reconstruction programs. Following the analysis setup presented in the Introduction 7.1.1, four classes of input objects to the jet algorithms have been considered: The initial partons of the hard interaction, partons after parton shower (partonic final state, PFS), all stable particles of the hadronic final state (HFS) other than muons or neutrinos and calorimeter towers. The calorimeter towers fulfilling the requirements $E>0.8 \mathrm{GeV}$ and $E_{\mathrm{T}}>0.5 \mathrm{GeV}$ were subjected to the same jet algorithms as the generator particles. If necessary, a matching of generator and calorimeter jets was performed by looking for the pairs closest to each other in distance $d=\sqrt{(\Delta \Phi)^{2}+(\Delta \eta)^{2}}$. 


\subsubsection{Jet energy calibration}

The jet energy calibration has been performed with a MC calibration method implying calibration factors that are applied on a jet by jet basis to the calorimeter jets depending on pseudo-rapidity $\eta$ and transverse momentum $p_{\mathrm{T}}$. The alternative data based technique of gamma-jet calibration, where jet transverse energies are measured against recoiling high energetic photons could not yet be employed for this study.

\subsection{6. $N$ LO calculation}

In order to compare to theoretical predictions of perturbative $\mathrm{QCD}$, calculations of at least next-to-leading order (NLO) precision are required. Here, the program CLOSET++ [185] is employed for the NLO calculation. However, since precise computations in NLO are very time consuming, a more efficient set-up in the form of the fastNLO project [186] is used which allows the fast rederivation of the considered cross section for arbitrary input PDFs and $\alpha_{S}$ values. This is done by separating the PDF dependency from the hard matrix element calculation by interpolating the PDFs between fixed support points in fractional proton momentum $x$ so that the PDF dependency can be evaluated a posteriori from one complete calculation.

Note that neither PYTHIA nor CLOSET++ contain electroweak corrections which may change high $p_{\mathrm{T}}$ cross sections from $1 \mathrm{TeV}$ onwards by up to $30 \%$ [187]. Insofar this study is consistent, but before comparing to real data this has to be taken into account.

\subsubsection{Experimental and theoretical uncertainties}

From the experience at the Tevatron [178, 188, 189], it is known that the jet energy scale with an uncertainty of $3 \%$ represents by far the dominant source of uncertainty for high $p_{\mathrm{T}}$ jet cross sections. Similarly, PDF uncertainties lead to the dominant uncertainty of the jet cross sections from the theoretical side.

According to CMS studies the jet energy scale in this analysis has been varied by $\pm 3 \%$ in order to estimate the impact on the cross section determination. Figure 7.3 presents on the left hand side the corresponding relative experimental uncertainty on the jet cross section for three regions in rapidity. Starting at about $15 \%$ at low $p_{\mathrm{T}}$ it rises up to about $50 \%$ at high $p_{\mathrm{T}}$ for central rapidity. In the two non-central rapidity regions the uncertainties are of comparable size below about $1 \mathrm{TeV}$ of transverse momentum, but get considerably larger for higher $p_{\mathrm{T}}$. In general, a similar behaviour as expected from Tevatron results is observed.

By evaluating the cross section calculation for the error set of the CTEQ6M [12] PDFs the ensuing theoretical uncertainty as shown in fig. 7.3 on the right hand side could be derived. It is of the same order of magnitude as the energy scale uncertainty and rises from about $5 \%$ for low transverse momenta with a minimum of $3 \%$ at $\approx 200 \mathrm{GeV}$ up to $+65 \%$ and $-30 \%$ at the highest transverse momenta for central rapidity.

\subsubsection{Summary and outlook}

The dominant experimental and theoretical uncertainties on the differential inclusive cross sections of jets with high transverse momentum ranging from $80 \mathrm{GeV}$ up to $4000 \mathrm{GeV}$ have been investigated. A variation of $\pm 3 \%$ in the jet energy scale results in an uncertainty of the derived jet cross sections of $15 \%$ at low transverse momenta, increasing up to about $50 \%$ at the highest $p_{\mathrm{T}}$ for central rapidity. The theoretical uncertainty due to the parton density functions of the proton has been found to be of the same order of magnitude and rises from 

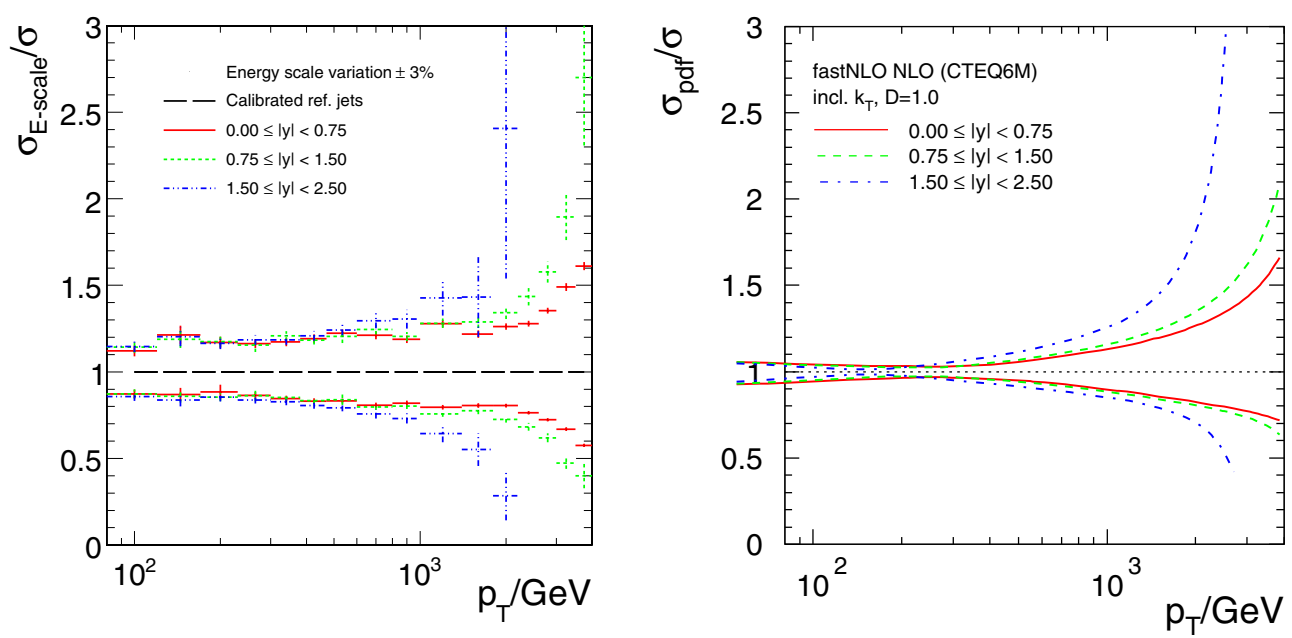

Figure 7.3. Relative systematic uncertainties of the jet cross sections for the $k_{\mathrm{T}}$ algorithm versus $p_{\mathrm{T}}$ due to a change in energy scale of $\pm 3 \%$ for three bins in rapidity (left). The error bars represent the statistical uncertainty. On the right hand side, the relative uncertainties due to an evaluation of the error sets of the CTEQ6M [12] PDFs are shown for the same regions in rapidity.

about $5 \%$ for low transverse momenta with a minimum of $3 \%$ at $\approx 200 \mathrm{GeV}$ up to $+65 \%$ and $-30 \%$ at the highest transverse momenta. For higher rapidities both uncertainties are considerably larger. The results shown have been derived with the $k_{\mathrm{T}}$ jet algorithm, similar values were obtained with the midpoint cone algorithm.

For transverse momenta below about $500 \mathrm{GeV}$ further sources of uncertainties may give significant contributions to the total uncertainty, e.g. corrections due to pile-up, the underlying event and multiple interactions or hadronisation. Theoretical contributions due to scale variations are of the order of 5\% (10\% for transverse momenta larger than $3 \mathrm{GeV})$ for rapidities y below 1.5. Above a rapidity of 1.5 they might be larger especially at the edge of the phase space. In addition, contributions due to $\alpha_{S}$ and electroweak corrections have to be included before comparing to real data.

In the future, it will be possible to run simultaneous fits of $\alpha_{S}$ and the parton density functions, especially the gluon density at high $x$, to the data. To be less sensitive to the jet energy scale other jet related quantities, e.g. jet rates, will be considered. By including other processes into the fit procedure, like $\mathrm{W} / \mathrm{Z}$ production as a luminosity measure or DrellYan reactions to fix the low $x$ gluon density, powerful combined PDF fits to the data of one experiment will become possible.

\subsection{Underlying event studies}

\subsubsection{Definition of the physics process and status of the art}

The "Underlying Event" (UE) in a hard scattering process is everything accompanying an event but the hard scattering component of the collision. A CDF analysis $[190,191]$ showed that the density of particles in the UE of jet events is about a factor of two larger than the density of particles in a typical Minimum Bias (MB) collision. At the LHC the difference might be even larger. 
Hard scattering collider events have a distinct topology and one can use the topological structure of the collision to define regions of the $\eta-\phi$ space that are sensitive to the UE components of the interaction. By comparing different processes such as high transverse momentum jets, "back-to-back" dijet production, or Drell-Yan, one can partially isolate the various components contributing to the UE.

Multiple parton interaction (MPI) models [192], extending the QCD perturbative picture to the soft regime, turn out to be particularly adequate to describe the physics of the UE. In the framework of these models one can regard the observed differences between the UE in a hard scattering process and a MB collision as the effect of the increased probability of partonic interactions for small impact parameter hadron-hadron collisions: one hard scattering implies a small impact parameter collision which makes it more likely that an additional parton-parton interaction will occur. Also, a hard scattering promotes initial and final state gluon radiation which inevitably contributes to the UE.

Examples of MPI models are implemented in the general purpose simulation programs PYTHIA [69], JIMMY [193], and SHERPA [194]. Other successful descriptions of UE and MB at hadron colliders are achieved by alternative approaches like PHOJET [195], which rely on both perturbative QCD and the Dual Parton Models (DPM). The purely phenomenological description available in HERWIG [196] provides a very useful reference of a model not implementing multiple interactions.

The QCD models considered in this study are different settings, called tunes, of relevant parameters in HERWIG and PYTHIA 6.2. One of the PYTHIA tunes is the ATLAS tune [197] and the other (PY Tunes DW) is a tune by R. Field which is similar to PYтHIA Tune A [198]. All these tunes use the CTEQ5L parton distribution functions. Details of the settings are given in reference [199].

Both Tune A and Tune DW fit the CDF Run 1 and Run 2 UE data [190, 191]. Tune DW also fits the CDF Run $1 Z$-boson transverse momentum distribution [200]. Both Tune A and Tune DW use the same multiple parton interaction energy dependence parameter PARP $(90)$ $=0.25$, while the ATLAS tune uses the default value of 0.16 .

The analyses summarised in this section are described in detail in reference [199].

\subsubsection{Underlying event observables discussed for charged jet events}

Charged jets are constructed from the charged particles using a simple clustering algorithm and then the direction of the leading charged particle jet is used to isolate regions of $\eta-\phi$ space that are sensitive to the UE. As illustrated in Fig. 7.4, the direction of the leading charged particle jet, chgjet1, is used to define correlations in the azimuthal angle, $\Delta \phi$. The angle $\Delta \phi=\phi-\phi_{\text {chgjetl }}$ is the relative azimuthal angle between a charged particle and the direction of chgjet1. The "transverse" region is almost perpendicular to the plane of the hard 2-to-2 scattering and is therefore very sensitive to the UE. We restrict ourselves to charged particles in the central region $|\eta|<1$ and consider two $p_{\mathrm{T}}$ thresholds, the nominal CMS cut $p_{\mathrm{T}}>0.9 \mathrm{GeV} / \mathrm{c}$ and a lower threshold with $p_{\mathrm{T}}>0.5 \mathrm{GeV} / \mathrm{c}$.

Figure 7.5 shows the QCD Monte Carlo models predictions for the average density of charged particles, $d N_{\text {chg }} / d \eta d \phi$, and the average charged $P T_{\text {sum }}$ density, $d P T_{\text {sum }} / d \eta d \phi$, respectively, in the "transverse" region for $|\eta|<1$ with $p_{\mathrm{T}}>0.5 \mathrm{GeV} / \mathrm{c}$ and $p_{\mathrm{T}}>0.9 \mathrm{GeV} / \mathrm{c}$ versus the transverse momentum of the leading charged particle jet. The charged particle density is constructed by dividing the average number of charged particles per event by the area in $\eta-\phi$ space (in this case $4 \pi / 3$ ). The charged $P T_{\text {sum }}$ density is the average scalar $p_{\mathrm{T}}$ sum of charged particles per event divided by the area in $\eta-\phi$ space. 

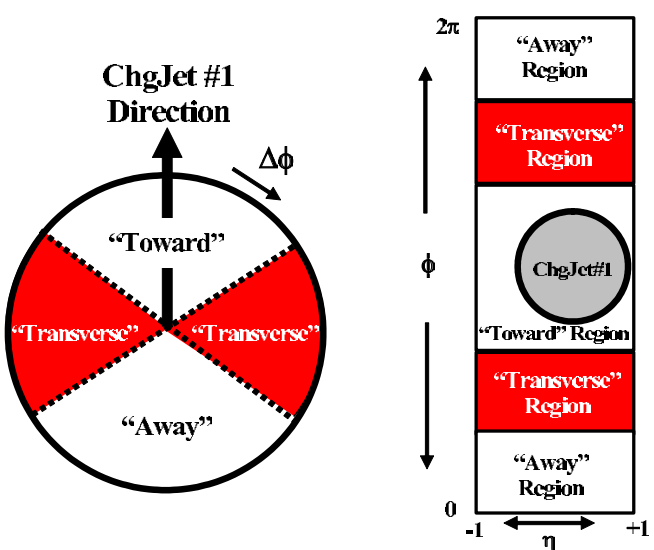

Figure 7.4. Illustration of correlations in azimuthal angle $\phi$ relative to the direction of the leading charged particle jet $(R=0.7)$ in the event, chgjet 1 . The angle $\Delta \phi=\phi-\phi_{\text {chgjet } 1}$ is the relative azimuthal angle between charged particles and the direction of chgjet1. The "transverse" region is defined by $60^{\circ}<|\Delta \phi|<120^{\circ}$ and $|\eta|<1$. We examine charged particles in the range $|\eta|<1$ with $p_{\mathrm{T}}>0.5 \mathrm{GeV} / \mathrm{c}$ or $p_{\mathrm{T}}>0.9 \mathrm{GeV} / \mathrm{c}$.
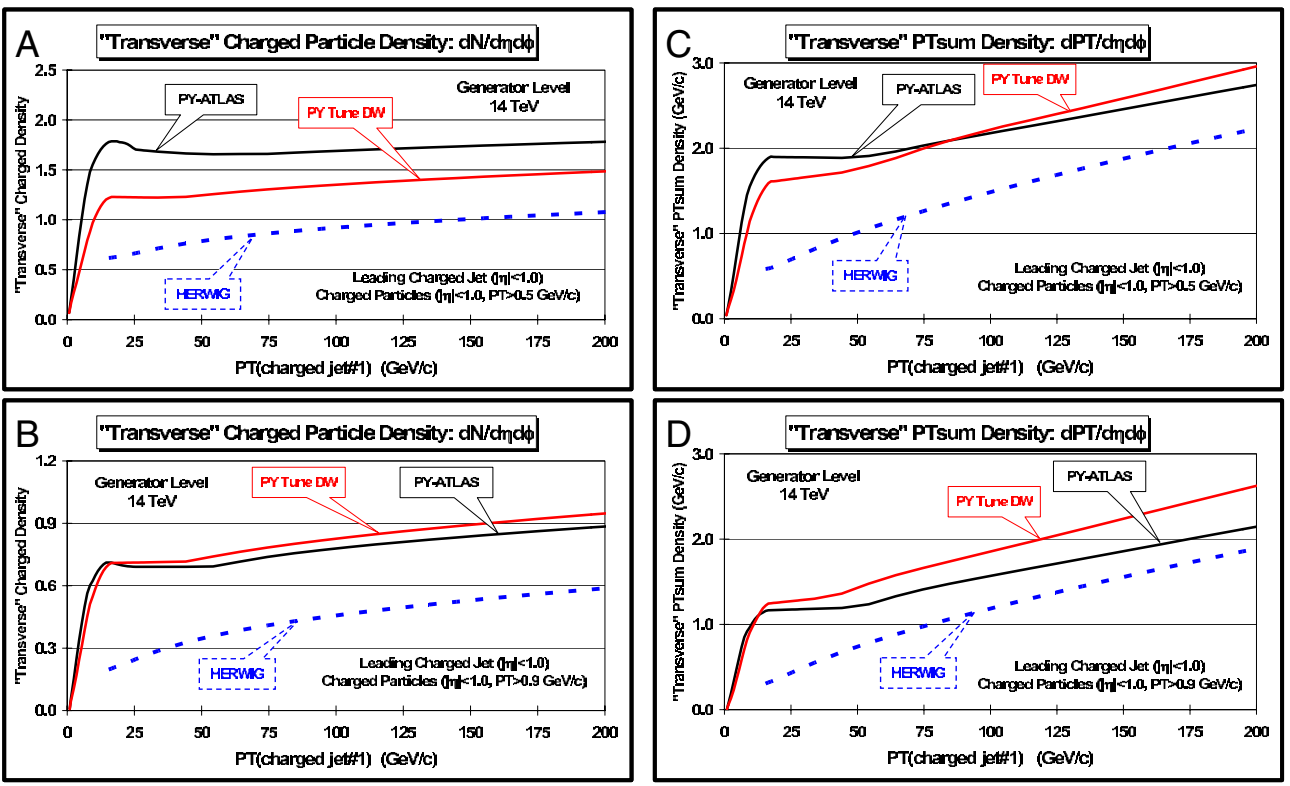

Figure 7.5. QCD Monte Carlo models predictions for charged particle jet production at $14 \mathrm{TeV}$. Left: Average density of charged particles, $d N_{c h g} / d \eta d \phi$, with $|\eta|<1$ in the "transverse" region versus the transverse momentum of the leading charged particle jet for $p_{\mathrm{T}}>0.5 \mathrm{GeV} / \mathrm{c}(A)$ and $p_{\mathrm{T}}>0.9 \mathrm{GeV} / \mathrm{c}(B)$. Right: Average charged $P T_{\text {sum }}$ density, $d P T_{\text {sum }} / d \eta d \phi$, with $|\eta|<1$ in the "transverse" region versus the transverse momentum of the leading charged particle jet for $p_{\mathrm{T}}>0.5 \mathrm{GeV} / \mathrm{c}(C)$ and $p_{\mathrm{T}}>0.9 \mathrm{GeV} / \mathrm{c}(D)$. The QCD models are HERWIG and two PYTHIA6.2 tunes described in the text. 
Due to the multiple parton interactions the PYTHIA tunes rise rapidly and then reach an approximately flat "plateau" region. At very high $P_{\mathrm{T}}$ (chgjet1) they begin to rise again due to initial and final state radiation which increases as the $Q^{2}$ scale of the hard scattering increases. HERWIG has considerably fewer particles in the "transverse" region and predicts a steady rise resulting from initial and final state radiation. The ATLAS tune predicts a larger charged particle density than PYTHIA Tune DW for $p_{\mathrm{T}}>0.5 \mathrm{GeV} / \mathrm{c}$. However, the ATLAS tune and Tune DW have similar charged particle densities for $p_{\mathrm{T}}>0.9 \mathrm{GeV} / \mathrm{c}$. This is because the ATLAS tune has a "softer" charged particle $p_{\mathrm{T}}$ distribution than Tune DW.

\subsubsection{Feasibility studies}

Here we concentrate on the UE measurement that will be performed in nominal CMS conditions at low luminosity [199]. All the studies presented in this section have been obtained applying the GEANT-4 based simulation and reconstruction chain of the CMS experiment.

Events corresponding to Drell-Yan dimuon pairs and leading QCD processes with superimposed low luminosity pile-up have been generated with PYTHIA 6.2 in different $\hat{p}_{\mathrm{T}}$ regions. The relevant PYTHIA 6.2 parameters adopted by CMS in simulation production are documented in [201]. The triggers used to collect Jet and Drell-Yan samples are described in reference [76].

Charged track reconstruction uses the Combinatorial Track Finder [202]. The default algorithm allows to reconstruct tracks with $p_{\mathrm{T}}$ above $0.9 \mathrm{GeV} / \mathrm{c}$. However, the same algorithm can be used in special conditions (with reduced thresholds for the seeds) achieving reasonable performances down to $0.5 \mathrm{GeV} / \mathrm{c}$ [199]. For $\eta \mid<1$, a reconstruction efficiency better than $90 \%$ and a fake rate below $1 \%$ are quoted for charged tracks with $p_{\mathrm{T}}$ above $0.7 \mathrm{GeV} / \mathrm{c}$.

7.2.3.1. The underlying event as observed in charged jet events. The track-based measurement for the scale of the leading interaction allows to keep an acceptable resolution for jet energies below $20 \mathrm{GeV}$, where the calorimetric measurement is dominated by large systematic uncertainties.

In principle MB could be studied from any data selection, getting rid of the leading $p p$ interaction and performing the reconstruction of all the primary vertices from all the other piled-up $p p$ interactions. However, this methodology turns out to be challenging as the resolution on the position of the $p p$ vertices degrades when lowering the total $p_{\mathrm{T}}$ of the associated charged tracks. In this study an MB trigger is defined requiring at least a calorimetric jet of $p_{\mathrm{T}}>20 \mathrm{GeV} / \mathrm{c}$. In order to combine the measurements performed at different leading charged jet scales, on top of the MB trigger, two additional triggers based on the $p_{\mathrm{T}}$ of the leading high level trigger jet are adopted: $p_{\mathrm{T}}>60 \mathrm{GeV} / \mathrm{c}$ and $p_{\mathrm{T}}>120 \mathrm{GeV} / \mathrm{c}$, which will be referred to as JET60 and JET120. Jets are reconstructed with an iterative cone algorithm of radius 0.5 in the pseudorapidity-azimuth space.

Tracks arising from the piled-up interactions are suppressed requiring the extrapolated coordinate along the beam axis to be inside $1 \mathrm{~mm}$ with respect to the primary vertex associated to the leading charged jet. The selection of the $p p$ interaction with the highest $p_{\mathrm{T}}$ charged jet tends to create a small bias on the MB sample, reducing the statistics available at very low $P_{\mathrm{T}}$ (chgjet1).

The definition of the main UE observables have been introduced in Section 7.2.2. The density of charged particles, $d N_{c h g} / d \eta d \phi$, and the charged $P T_{\text {sum }}$ density, $d P T_{\text {sum }} / d \eta d \phi$, with $p_{\mathrm{T}}>0.9 \mathrm{GeV} / \mathrm{c}$ and $|\eta|<1$ in the "transverse" region are reported in Fig. 7.6. Bins of $2 \mathrm{GeV} / \mathrm{c}$ are used up to $P_{\mathrm{T}}$ (chgjet 1$)=20 \mathrm{GeV} / \mathrm{c}$ and bins of $10 \mathrm{GeV} / \mathrm{c}$ above. 

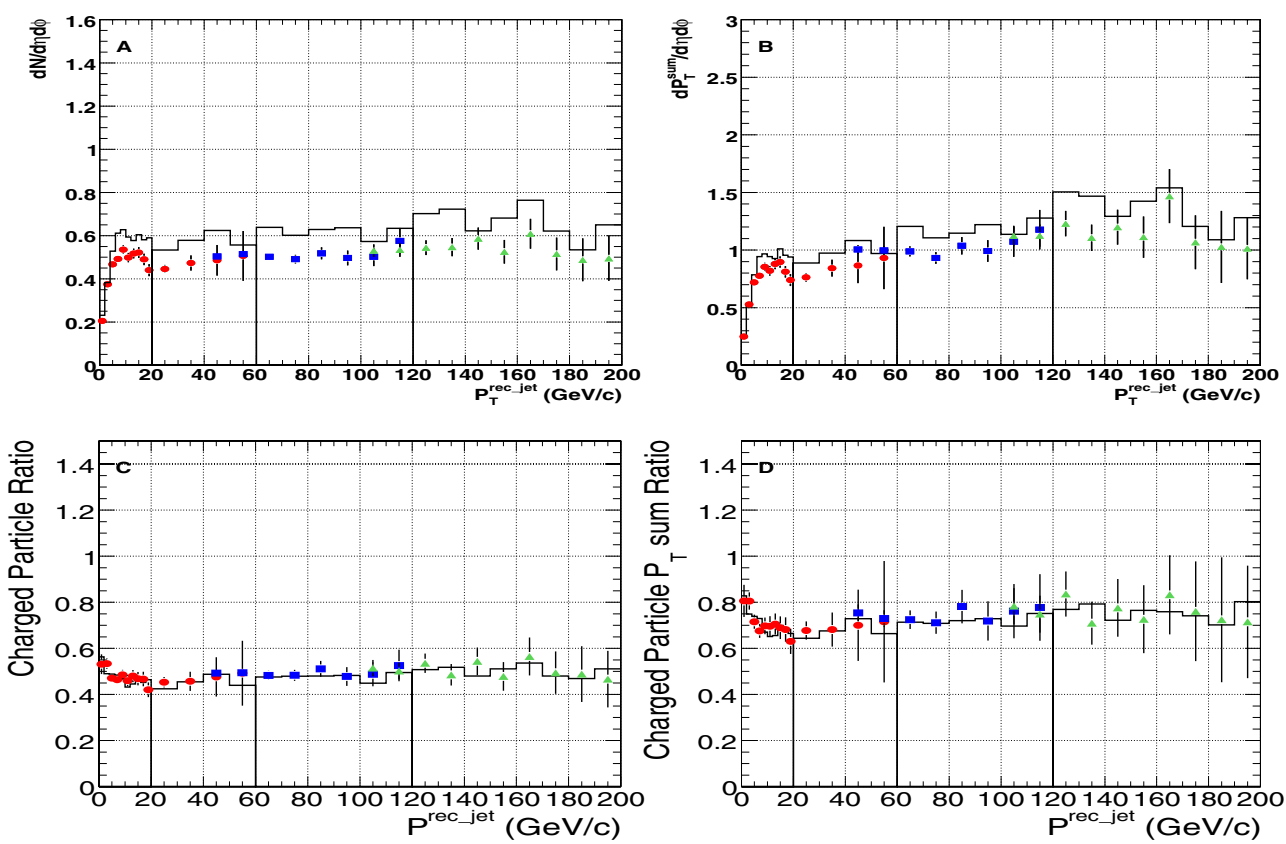

Figure 7.6. Charged jet production at $14 \mathrm{TeV}$. Charged tracks with $|\eta|<1$ in the "transverse" region. Density of charged particles, $d N_{c h g} / d \eta d \phi(A)$ and $P T_{\text {sum }}$ density, $d P T_{\text {sum }} / d \eta d \phi(B)$, with $p_{\mathrm{T}}>0.9 \mathrm{GeV} / \mathrm{c}$ versus the transverse momentum of the leading charged particle jet. Ratio between density of charged particles with $p_{\mathrm{T}}>0.9 \mathrm{GeV} / \mathrm{c}$ and $p_{\mathrm{T}}>0.5 \mathrm{GeV} / \mathrm{c}(C)$ and ratio between $P T_{\text {sum }}$ density with $p_{\mathrm{T}}>0.9 \mathrm{GeV} / \mathrm{c}$ and $p_{\mathrm{T}}>0.5 \mathrm{GeV} / \mathrm{c}(D)$ versus the transverse momentum of the leading charged particle jet. Data from different triggers are superimposed: $($ circles) $=$ MinimumBias; $($ squares $)=$ JET60; $($ triangles) $=$ JET120. The lines show the generator level distributions; the points with error bars correspond to the raw (uncorrected) reconstruction level distributions.

The shapes of uncorrected reconstruction level distributions basically agree with the corresponding generator level ones. The difference in absolute scale (about $-20 \%$ for both $d N_{\text {chg }} / d \eta d \phi$ and $d P T_{\text {sum }} / d \eta d \phi$ ) turns out to be compatible with charged track inefficiencies and fake rates. Further details on these systematic effects, including the calibration and resolution of the leading charged jet have been studied in [199].

Figure 7.6 shows also the ratio between the observables for $p_{\mathrm{T}}>0.9 \mathrm{GeV} / \mathrm{c}$ and $p_{\mathrm{T}}>$ $0.5 \mathrm{GeV} / \mathrm{c}$ in the "transverse" region. These ratios, which are sensitive to the differences between the models and/or to the choice of the tuning for a given model, are also nicely free from the systematic effects enumerated above, and basically do not need to be corrected when comparing to the corresponding generator level observables.

7.2.3.2. The underlying event as observed in Drell-Yan muon-pair production. Drell-Yan muon pair production provides an excellent way to study the UE. Here one studies the outgoing charged particles (excluding the $\mu^{+} \mu^{-}$pair) as a function of the muon-pair invariant mass. After removing the muon-pair everything else is the UE. As for the charged jet production, we restrict ourselves to charged particles in the central region $|\eta|<1$ and consider the two $p_{\mathrm{T}}$ thresholds $p_{\mathrm{T}}>0.5 \mathrm{GeV} / \mathrm{c}$ and $p_{\mathrm{T}}>0.9 \mathrm{GeV} / \mathrm{c}$. 

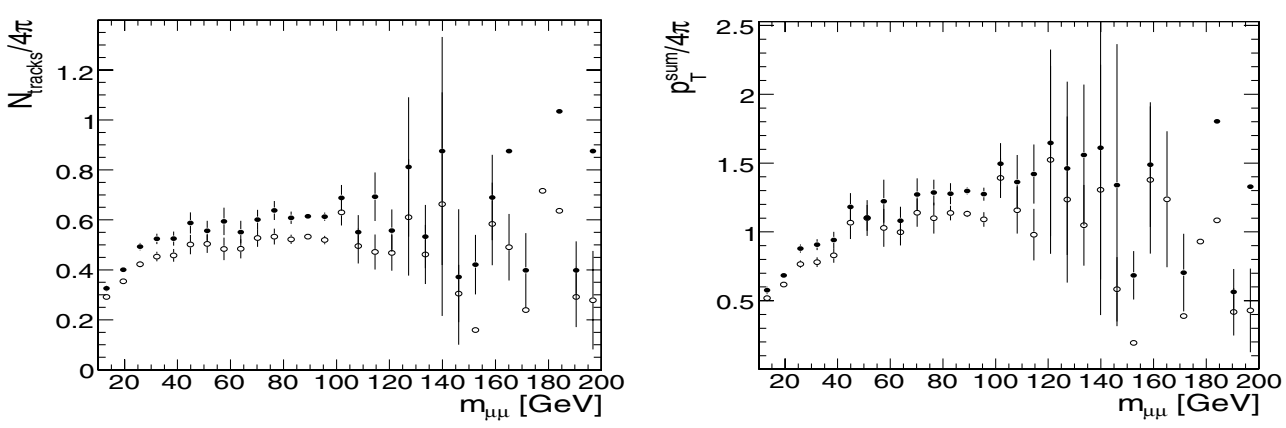

Figure 7.7. Muon-pair production at $14 \mathrm{TeV}$ with two isolated muons. Density of charged particles, $d N_{\text {chg }} / d \eta d \phi($ left $), \quad P T_{\text {sum }}$ density, $d P T_{\text {sum }} / d \eta d \phi($ right $)$, with $p_{\mathrm{T}}>$ $0.9 \mathrm{GeV} / \mathrm{c}$ and $|\eta|<1$ versus the muon-pair invariant mass. (fullcircles) correspond to the generator level distributions; (empty circles) correspond to the raw (uncorrected) reconstruction level distributions.

Single muon and muon-pair CMS triggers ensure very high efficiencies for the studied process. The relative mass shift and the corresponding resolution of the reconstructed muonpair are studied in detail in Ref. [199]. Tracks arising from the piled-up interactions are suppressed requiring the extrapolated coordinate along the beam axis to be inside $1 \mathrm{~mm}$ with respect to the primary vertex associated to the leading muons.

In our study, we require "isolated muons", not to have charged tracks with $p_{\mathrm{T}}>$ $0.9 \mathrm{GeV} / \mathrm{c}$ in a cone of radius $\mathrm{R}=\sqrt{(\Delta \phi)^{2}+(\Delta \eta)^{2}}=0.3$ in the azimuth-pseudorapidity space centred along the direction of the muon. Selecting isolated muons turns out to be essential to reduce the QCD background to negligible levels for $p_{\mathrm{T}}>15 \mathrm{GeV} / \mathrm{c}$, while keeping an efficiency of $76.9 \%$ for Drell-Yan muon-pairs in the same $p_{\mathrm{T}}$ region.

The charge particle density, $d N_{c h g} / d \eta d \phi$, and the charged $P T_{\text {sum }}$ density, $d P T_{\text {sum }} / d \eta d \phi$ with $p_{\mathrm{T}}>0.9 \mathrm{GeV} / \mathrm{c}$ and $|\eta|<1$ in muon-pair production with isolated muons versus the muon-pair invariant mass are shown in Fig. 7.7. Correlations between isolation and UE activity have been studied in Refs. [64, 199].

\subsubsection{Conclusions}

Predictions on the amount of activity in UE at the LHC based on extrapolations from the lower energy data differ greatly. In this study we have demonstrated the feasibility of reference UE measurements at CMS under nominal conditions, assessing our capability to distinguish between the predictions of different models. The UE is studied by examining charged particles in the "transverse" region in charged particle jet production and in the central region of Drell-Yan muon-pair production (after removing the muon-pair).

\subsection{Physics of b-quarks and hadrons}

\subsubsection{Inclusive b-quark production}

7.3.1.1. Introduction. At the LHC new opportunities to improve our understanding of the physics of $b$ quarks will become available because of the high statistics data samples and the high centre-of-mass energy. A study [203] has been performed to investigate methods in CMS of identifying $b$ jets ( $b$ "tagging") in an inclusive sample of events containing jets and at 
least one muon. Here we present the capability to measure the inclusive $b$ quark production cross section as a function of the $B$-hadron transverse momentum and pseudorapidity. An important result of our study is an estimate for the $B$-hadron $p_{\mathrm{T}}$ range reachable at LHC.

Inclusive $b$-quark production has been studied at other proton and electron colliders. The observed shapes of distributions and correlations are reasonably well explained by perturbative QCD. However, the observed cross-sections at the Tevatron (Run I) are larger than QCD predictions [204-211] which is confirmed by Run II data. Similar effects are observed in $\gamma$ p collisions at HERA [212-218] and in $\gamma \gamma$ interactions at LEP [219, 220].

The agreement between experiment and theory has improved due to more precise parton density functions and proper estimates of fragmentation effects [221-226]. But the agreement is not complete and the improvement of the phenomenological description is required using also experimental input.

7.3.1.2. Analysis. This study of the CMS capability to measure the inclusive $b$ production is based on full detector simulation. The generated events are passed through the GEANT4 simulation of CMS. Pile-up corresponding to low-luminosity LHC running conditions $\left(\mathcal{L}=2 \times 10^{33} \mathrm{~cm}^{-2} \mathrm{~s}^{-1}\right)$ is also generated.

7.3.1.2.1 Event selection. About 4 million signal and background events were processed, mainly with high transverse momentum of the partons $\left(p_{\mathrm{T}}>50 \mathrm{GeV} / \mathrm{c}\right)$. Samples of QCD jets were used. Jets in those samples cover the full geometrical acceptance in pseudorapidity of the tracking detector, $|\eta|<2.4$. The measurement of the differential cross sections is studied for $B$-hadrons of $p_{\mathrm{T}}>50 \mathrm{GeV} / \mathrm{c}$ and within the fiducial volume of $|\eta|<2.4$. First, the events are required to pass the Level-1 (L1) trigger selection for the single muon trigger stream which accepts events with muons having $p_{\mathrm{T}}>14 \mathrm{GeV} / \mathrm{c}$. The most energetic $B$-hadron inside the phase space defined above is selected. The trigger efficiency is flat as a function of the $B$-hadron pseudorapidity within the Level-1 trigger acceptance of $|\eta|<2$.1. It increases with transverse momentum of the $B$-particle. The average Level-1 trigger efficiency corresponds to the expected value of the branching fractions for the semi-leptonic $b$ quark and $c$ quark decays, about 19\% [54]. At Level-1, the single muon trigger is used. At the High Level Trigger (HLT) we require the "muon $+b$-jet" trigger, fired by non-isolated muons with $p_{\mathrm{T}}>19 \mathrm{GeV} / \mathrm{c}$ and by jets with $E_{\mathrm{T}}>50 \mathrm{GeV} / \mathrm{c},|\eta|<2.4$ and compatible with $b$ tagging.

The event selection requires a $b$-tagged jet in the fiducial volume to be present in the event. $B$ tagging is based on inclusive secondary vertex reconstruction in jets [157]. The tagging algorithms combine several topological and kinematic secondary vertex related variables into a single tagging variable to discriminate between jets originating from $b$ quarks and those from light quarks and gluons.

To measure differential cross sections for inclusive $B$-particle production as a function of its transverse momentum $p_{\mathrm{T}}$ and pseudorapidity $\eta, d \sigma / d p_{\mathrm{T}}$ and $d \sigma / d|\eta|$, we select as the reconstructed $B$-particle candidate the most energetic $b$ tagged jet. Good correspondence between the generated $B$-particle and the reconstructed $b$-tagged jet is observed. The corresponding $p_{\mathrm{T}}$ and pseudorapidity relative resolutions are shown in Fig. 7.8 for $B$-particles with $p_{\mathrm{T}}>170 \mathrm{GeV} / \mathrm{c}$. The resolutions are $13 \%$ and $6 \%$ for $p_{\mathrm{T}}$ and pseudorapidity, respectively.

The efficiency of the $b$ tagging by secondary vertices in jets is shown in Fig. 7.9 as function of the $B$-particle transverse momentum and pseudorapidity. The $b$ tagging efficiency is defined with respect to events passing the Level-1 trigger and with a single muon of $p_{\mathrm{T}}>19 \mathrm{GeV} / \mathrm{c}$ selected. The efficiency decreases with increasing transverse momentum, while being rather flat as function of pseudorapidity. The slow degradation for larger 

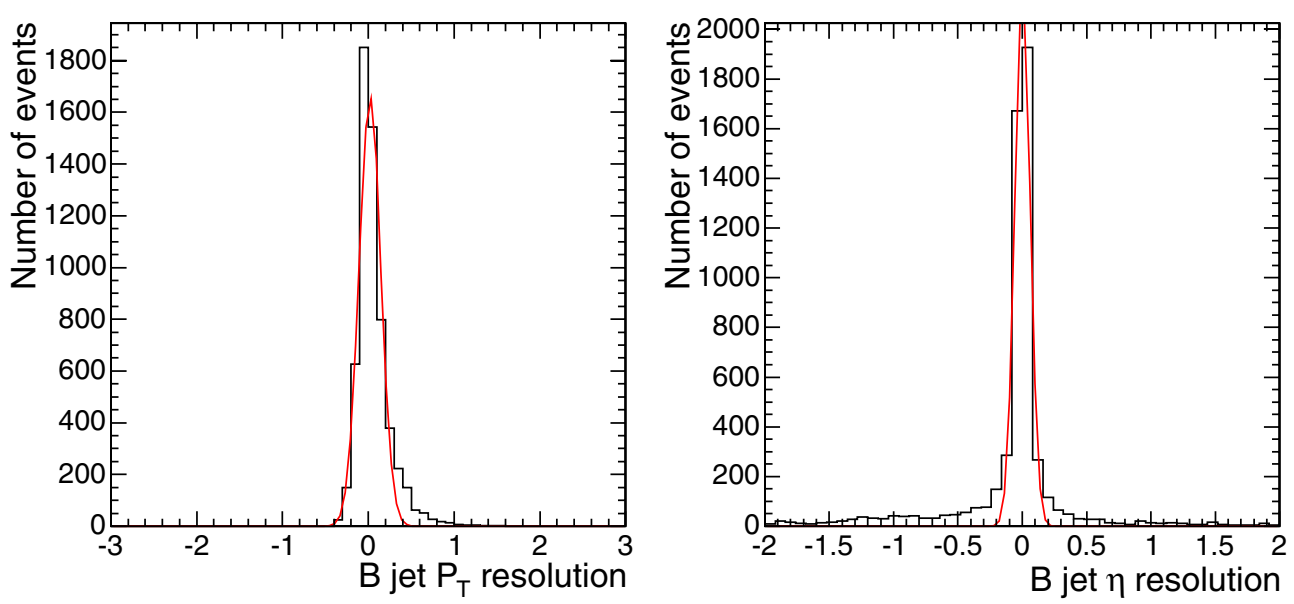

Figure 7.8. Relative resolution, (Reconstructed - True)/True, for $p_{\mathrm{T}}$ and pseudorapidity of $b$ tagged jets in CMS.
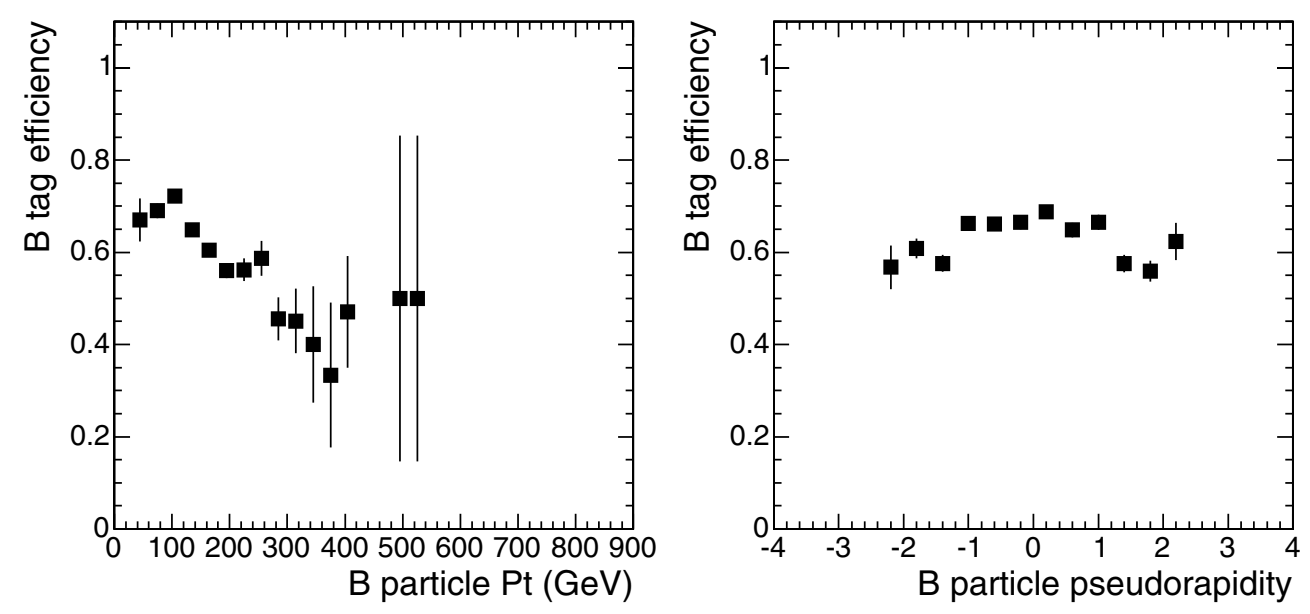

Figure 7.9. The $b$ tagging efficiency versus $p_{\mathrm{T}}$ and pseudorapidity of the generated $B$-particle.

transverse momenta is caused by the worsening of the tracking resolution with increasing $p_{\mathrm{T}}$, an increased track multiplicity from fragmentation and more difficult pattern recognition in dense jets. The average $b$ tagging efficiency is $65 \%$ in the barrel region, while the efficiency is about $10 \%$ less for the endcap region. The muon plus $b$-jet cross-channel trigger has a $4.3 \mathrm{~Hz}$ rate for the signal and a $6.1 \mathrm{~Hz}$ total event rate [203]. This trigger rate corresponds to a low-luminosity LHC run at $\mathcal{L}=2 \times 10^{33} \mathrm{~cm}^{-2} \mathrm{~s}^{-1}$.

To measure the cross section one needs to know the number of selected events, the integrated luminosity, the event sample purity (signal fraction) and the signal efficiency. The signal fraction can be determined from the simulated prediction of the background contribution to the selected event sample. In order to rely less on the absolute prediction for the background one can extract the signal fraction using the prediction of the signal and background shapes for some sensitive variables. A fit to the data distribution using the 


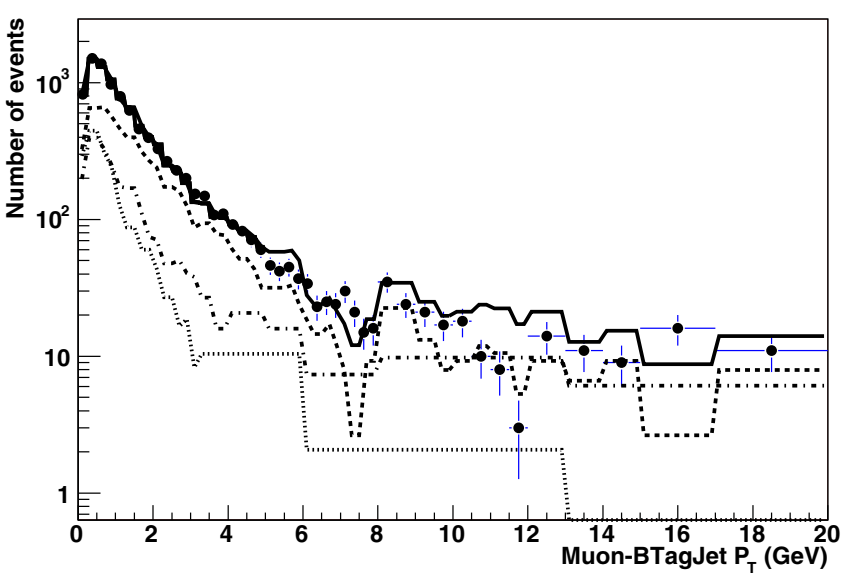

Figure 7.10. Fit of the muon $p_{\mathrm{T}}$ spectrum with respect to the closest $b$ tagged jet. The sample of generated QCD events with " $p_{\mathrm{T}}$-hat" parameter in the range $230<\hat{p_{\mathrm{T}}}<300 \mathrm{GeV} / \mathrm{c}$ is tested. The contributions of tagged muons from $b$ events (dashed curve), $c$ events (dot-dashed curve) and light quark events (dotted curve) as defined by the fit are shown. The solid curve is the sum of the three contributions.

simulated shapes for the signal and background is performed. To do so we apply a lepton tag by selecting inclusive muons.

7.3.1.2.2 Muon tag. Muons are reconstructed in the muon chambers, matched to the inner tracker information and refitted using both subdetectors information. This provides the most precise muon track measurement. Each reconstructed muon is associated to the most energetic $b$ tagged jet. The muon must be closer to this $b$ tagged jet than to any other jet in the event. Otherwise the event is discarded.

In most cases the tagged muon is inside the $b$ jet. The average efficiency of associating the muon with the $b$ tagged jet is $75 \%$.

7.3.1.2.3 Results. We calculate the transverse momentum of the muon with respect to the b-jet axis which effectively discriminates between $b$ events and background. The slopes of the $p_{\mathrm{T}}$ spectra are very different and this is exploited in the fit of the selected events to determine the fractions of the muon sources in the sample.

Figure 7.10 shows an example of the fit of the distribution of the muon $p_{\mathrm{T}}$ with respect to the closest jet, using the expected shapes for the muons from $b$ events, charm events and light quark events. The normalisation of the three contributions are free parameters in the fit. The events in this plot are from a sample of QCD events generated with the PYTHIA " $p_{\mathrm{T}^{-}}$" parameter in the range $230<\hat{p_{\mathrm{T}}}<300 \mathrm{GeV} / \mathrm{c}$. In the fit, the shapes of the distributions were fixed using an independent QCD sample generated with $170<\hat{p_{\mathrm{T}}}<230 \mathrm{GeV} / \mathrm{c}$. The fit results as well as the Monte Carlo input are quoted in Table 7.1. The event fractions are well reproduced within statistical errors. In the actual experiment the shapes will be verified using data at different selection stages. Also the background shape will be derived from the data itself by applying an anti-tag selection (b-suppressed event sample).

In Table 7.2 the $b$ purity, $c \bar{c}$ and light quark event fractions for the different QCD samples are shown. The $b$ purity decreases from about $70 \%$ down to $55 \%$ from low $p_{\mathrm{T}}$ events to the high transverse momentum events. The expected number of $b \bar{b}$ events after event selection is quoted for $10 \mathrm{fb}^{-1}$ of integrated luminosity. For the phase space of $p_{\mathrm{T}}>50 \mathrm{GeV} / \mathrm{c}$ and $|\eta|<2.4$ the event selection will allow for a $b$ event statistics of about 16 million events. We 
Table 7.1. Results of the fit to the distribution of the transverse momentum of the muon with respect to the nearest $b$ tagged jet. The number of beauty, charm and light quark events in the Monte Carlo input are compared to the fit result.

\begin{tabular}{lll}
\hline & $\begin{array}{l}\text { MC input, } \\
230<\hat{p_{\mathrm{T}}}<300 \mathrm{GeV} / \mathrm{c}\end{array}$ & Fit result \\
\hline$N_{\mathrm{b} \overline{\mathrm{b}}}$ & 5250 & $5222 \pm 501$ \\
$N_{\mathrm{c} \overline{\mathrm{c}}}$ & 2388 & $2050 \pm 728$ \\
$N_{\mathrm{uds}}$ & 1740 & $1778 \pm 341$ \\
\hline
\end{tabular}

Table 7.2. $B$ purity and expected number of events after final event selection. The expected number of $b \bar{b}$ events is quoted for $10 \mathrm{fb}^{-1}$ of integrated luminosity.

\begin{tabular}{lcllcl}
\hline$\hat{p}_{\mathrm{T}} \mathrm{GeVc}$ & $\mathrm{N}_{\text {generated }}^{\mathrm{QCD}}$ & $b \bar{b}$ purity, $\%$ & $c \bar{c}$ fraction, $\%$ & $u d s$ fraction, $\%$ & $\mathrm{~N}_{\text {expected }}^{\mathrm{b} \bar{b}}$ \\
\hline $50-80$ & 198993 & 66 & 32 & 2 & $1.4 \mathrm{M}$ \\
$80-120$ & 294986 & 66 & 32 & 2 & $6.1 \mathrm{M}$ \\
$120-170$ & 291982 & 72 & 26 & 2 & $5.1 \mathrm{M}$ \\
$170-230$ & 355978 & 71 & 26 & 3 & $2.4 \mathrm{M}$ \\
$230-300$ & 389978 & 73 & 24 & 3 & $0.9 \mathrm{M}$ \\
$300-380$ & 283983 & 70 & 25 & 5 & $0.3 \mathrm{M}$ \\
$380-470$ & 191989 & 68 & 27 & 5 & $88 \mathrm{k}$ \\
$470-600$ & 190987 & 64 & 29 & 7 & $34 \mathrm{k}$ \\
$600-800$ & 94996 & 60 & 31 & 9 & $10 \mathrm{k}$ \\
$800-1000$ & 89999 & 60 & 30 & 10 & $2.0 \mathrm{k}$ \\
$1000-1400$ & 89998 & 55 & 31 & 14 & $0.5 \mathrm{k}$ \\
\hline
\end{tabular}

conclude that for $B$-hadrons a $p_{\mathrm{T}}$ range up to $1.5 \mathrm{TeV} / \mathrm{c}$ will be accessible with the CMS detector at the LHC.

The background contribution from $t \bar{t}$ events has been estimated from a sample of one million simulated events including all decay modes. The total number of $t \bar{t}$ events passing the selection amounts to 104 thousand events for $10 \mathrm{fb}^{-1}$ of integrated luminosity, corresponding on average to a less than $1 \%$ background contribution. The $t \bar{t}$ background becomes more pronounced for the high $p_{\mathrm{T}}$ part of the inclusive $B$ spectrum. In the region $p_{\mathrm{T}}>500 \mathrm{GeV} / \mathrm{c}$ it amounts to $2.4 \%$.

The total event selection efficiency is about $5 \%$. By correcting for the semi-leptonic branching ratio of $b$ quarks and $c$ quarks it amounts to about $25 \%$ on average. It turns out that the total efficiency is almost independent of transverse momentum and angle of the $B$-particle. Therefore the measurement of the differential cross section is less affected by systematic uncertainties due to bin-by-bin efficiency corrections.

7.3.1.2.4 Systematics Uncertainties. Several potential sources for systematic uncertainties are considered and their impact on the observed cross section is detailed in Table 7.3. The largest uncertainty arises from the $3 \%$ error on the jet energy scale (see Appendix B) which leads to a cross section error of $12 \%$ at $E_{\mathrm{T}}>50 \mathrm{GeV} / \mathrm{c}$. Other important uncertainties arise from the event-selection procedure and the Monte Carlo modelling of the detector response, including the lepton identification and the detector resolution on the energy and angular variables which identify the fiducial volume. The effect of these systematic uncertainties is estimated by varying the corresponding cuts and repeating the fits for the newly selected event samples. It results in an uncertainty of $6 \%$. The expected $b$-tag systematics for $10 \mathrm{fb}^{-1}$ integrated luminosity is 5\% [7]. The luminosity uncertainty is also 5\% [7]. 
Table 7.3. Sources of systematic uncertainty in $\%$ on the inclusive $b$ production cross section measurement. The total systematic uncertainty is calculated by adding all contributions in quadrature.

\begin{tabular}{ll}
\hline Source & uncertainty, $\%$ \\
\hline jet energy scale & 12 \\
event selection & 6 \\
B tagging & 5 \\
luminosity & 5 \\
trigger & 3 \\
muon Br & 2.6 \\
misalignment & 2 \\
muon efficiency & 1 \\
$t \bar{t}$ background & 0.7 \\
fragmentation & 9 \\
total & 18 \\
\hline
\end{tabular}

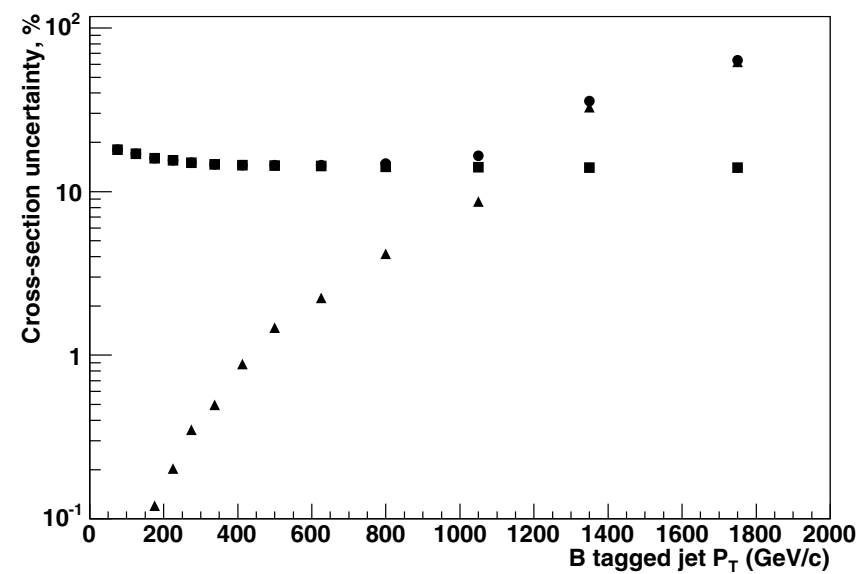

Figure 7.11. The statistical uncertainty for the cross section measurement (triangles), systematic (squares) uncertainty and total (dots) uncertainty as function of the $b$ tagged jet transverse momentum with respect to the beam line. Total uncertainty comprises the statistical and systematic uncertainties added in quadrature.

The trigger efficiency will be determined from the data themselves. We estimate its uncertainty from Monte Carlo studies to be 3.0\%. The experimental uncertainties on the semi-leptonic branching ratio of $b$ quarks [54] is also propagated to the measurement. The impact of the detector misalignment on the CMS $b$ tagging performance has been investigated in [157]. The effect has been found to be small (2\%). The muon detection efficiency can be determined with better than $1 \%$ precision [7]. The $t \bar{t}$ background subtraction uncertainty is conservatively taken as absolute value of the expected $t \bar{t}$ contribution to the considered phase space.

A large contribution is expected from the fragmentation modelling. We estimate the magnitude of the effect from the $\mathrm{D} \varnothing \mathrm{b}$-jet production measurement at Tevatron [211]. This uncertainty propagates to the cross section as a $9 \%$ effect independent of jet $E_{\mathrm{T}}$.

The estimated statistical, systematic and total uncertainty as function of the $b$ tagged jet transverse momentum with respect to the beam line is shown in Fig. 7.11. 
7.3.1.3. Conclusion. The event selection for inclusive $b$ production measurement at CMS will allow to study $b$ production mechanisms on an event sample of 16 million $b$ events for $10 \mathrm{fb}^{-1}$ of integrated luminosity. The $b$ purity of the selected events varies as function of the transverse momentum in a range from $70 \%$ to $55 \%$. Our estimate shows that with the CMS detector we can reach $1.5 \mathrm{TeV} / \mathrm{c}$ as the highest measured transverse momentum of $B$ hadrons.

\subsubsection{Study of $B_{c}$ hadrons}

7.3.2.1. Introduction. The $B_{\mathrm{c}}$ meson is the ground state of the $\bar{b} c$ system, which is doubly heavy flavoured. This unique character provides a window for studying heavy-quark dynamics that is very different from the one of quarkonium. The experimental study of $B_{\mathrm{c}}$ will help us to understand heavy quark dynamics and to test the spin symmetry derived in nonrelativistic quantum chromodynamics (NRQCD) [227-236]. $B_{\mathrm{c}}$ mesons have been observed at the Fermilab Tevatron collider by the CDF collaboration through the decay channel $B_{\mathrm{c}} \rightarrow J / \psi \ell \nu$ [237]. The mass and lifetime are measured to be [238] $\mathrm{M}\left(B_{\mathrm{c}}\right)=6.40 \pm$ 0.39 (stat) $\pm 0.13($ sys $) \mathrm{GeV} / \mathrm{c}^{2}$ and $\tau\left(B_{\mathrm{c}}\right)=0.46_{-0.16}^{+0.18} \pm 0.03($ sys $) \mathrm{ps}$, in agreement with the non-relativistic potential model [239-241] and other approaches [242-244].

Because of the higher colliding energy, the production cross section at the LHC is about a factor of 16 [231] larger than at the Tevatron. As also the LHC luminosity will be higher, CMS has the potential to collect much more $B_{\mathrm{c}}$ mesons than the Tevatron experiments do. We propose to study the $B_{\mathrm{c}}$ meson through $B_{\mathrm{c}} \rightarrow J / \psi \pi, J / \psi \rightarrow \mu^{+} \mu^{-}$. The goal is to measure the mass and lifetime, and to compare the results with theoretical predictions which do have large uncertainties at the moment. More details on the analysis can be found in reference [245].

7.3.2.2. Monte Carlo data samples. A large amount of Monte Carlo data were produced to study the feasibility for CMS to measure the $B_{\mathrm{c}}$ mass and lifetime with the first $\mathrm{fb}^{-1}$. There are two dedicated $B_{\mathrm{c}}$ generators, one is called BICEPS, developed at ITP, Beijing, by Chang et al. [231, 236], and the other is developed at IHEP, Protvino, by Berezhnoy et al. $[239,240]$. Both packages are based on perturbative QCD, and have been integrated into the SIMUB package [130]. PYTHIA [246] can also generate $B_{\mathrm{c}}$ events, but it takes much more CPU time than the dedicated ones. For comparison, the $p_{\mathrm{T}}$ distribution of $B_{\mathrm{c}}$ mesons, generated by PYTHIA, BICEPS and the Protvino package (named Gouz in the plot), are shown in Fig. 7.12. One can see that the Protvino package produces higher $p_{\mathrm{T}}$, while PYTHIA agrees with BICEPS. In order to save CPU time, BICEPS is used to generate $B_{\mathrm{c}}$ events. During generation, only events were retained which contain within $|\eta|<2$ a $B_{\mathrm{c}}$ with $p_{\mathrm{T}}>10 \mathrm{GeV} / \mathrm{c}$, together with a muon of $p_{\mathrm{T}}>4 \mathrm{GeV} / \mathrm{c}$ within $|\eta|<2.2$. After the kinematic cuts, the cross section multiplied by the branching ratio is $1.78 \mathrm{pb}$. $52,000 B_{\mathrm{c}}$ events were produced, corresponding to $29.2 \mathrm{fb}^{-1}$ of integrated luminosity.

Important background sources are $J / \psi$ mesons from decays of other $B$ hadrons and prompt $J / \psi$ mesons. Because of their large cross sections also QCD jets, in particular $b \bar{b} \rightarrow \mu^{+} \mu^{-} X, c \bar{c} \rightarrow \mu^{+} \mu^{-} X$, as well as $W+$ jets and $Z+$ jets have to be considered.

$B$ hadrons that decay into $J / \psi$ were generated with PYTHIA 6.228 with kinematic cuts similar to $B_{\mathrm{c}}$ production, and prompt $J / \psi$ events were generated by PYтHIA 6.324 , where the colour-octet contribution is included.

The full CMS detector simulation and reconstruction was applied to the generated samples. The fast simulation package FAMOs was also used to produce the $B_{\mathrm{c}}$ events, B hadrons, prompt $J / \psi$ and $c \bar{c} \rightarrow \mu^{+} \mu^{-} X$ (Table 7.4). 


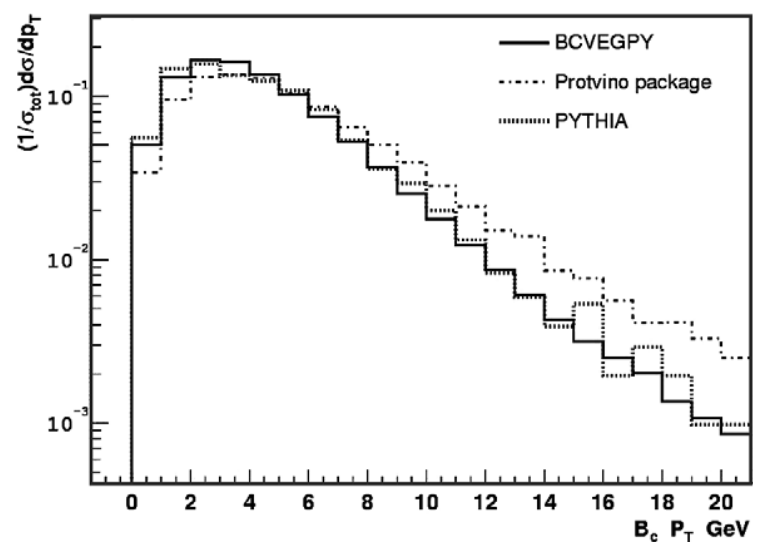

Figure 7.12. Comparison of $p_{\mathrm{T}}$ distributions of $B_{\mathrm{c}}$ mesons for the generator BICEPS, Gouz and PYTHIA.

Table 7.4. The cross section multiplied by the branching ratio after kinematic cuts and the number of events produced for $B$ hadrons and prompt $J / \psi$ and $c \bar{c} \rightarrow \mu^{+} \mu^{-} X$.

\begin{tabular}{lll}
\hline channel & $\sigma \cdot \mathrm{Br} .(\mathrm{pb})$ & $\mathrm{N}$ events \\
\hline$B^{0}$ & 70.3 & 740,000 \\
$B^{+}$ & 70.7 & 740,000 \\
$B_{S}$ & 14.8 & 190,000 \\
$\Lambda_{b}$ & 19.4 & 200,000 \\
prompt $J / \psi$ & 240.3 & 500,000 \\
$c \bar{c} \rightarrow \mu^{+} \mu^{-} \mathrm{X}$ & 1690 & 210,000 \\
\hline
\end{tabular}

Samples corresponding to $10 \mathrm{fb}^{-1}$ of $B$ hadrons, $2 \mathrm{fb}^{-1}$ of prompt $J / \psi$ and $0.12 \mathrm{fb}^{-1}$ of $c \bar{c} \rightarrow \mu^{+} \mu^{-} \mathrm{X}$ events were produced for the analysis. Additional background samples of about 950,000 QCD, 880,000 W+ jets, 710,000 Z+ jets and 100,000 $b \bar{b} \rightarrow \mu^{+} \mu^{-} X$ events were used.

7.3.2.3. Selection. Signal events should have a $b$-jet, a $c$-jet and a $B_{\mathrm{c}}$ meson which decays into a $J / \psi$ and a pion, with the subsequent $J / \psi \rightarrow \mu^{+} \mu^{-}$decay. The selection starts from 2 muon tracks. The $p_{\mathrm{T}}$ of both muons should be larger than $4 \mathrm{GeV} / \mathrm{c}$ and the absolute value of $\eta$ less than 2.2. The two muons should have different charge and share the same vertex. To form a $J / \psi$ candidate the invariant mass of the muons should be in a window between 3.0 and $3.2 \mathrm{GeV} / \mathrm{c}^{2}$. An additional track must be found at the same vertex of the $J / \psi$ which is inconsistent with a muon or an electron. The $p_{\mathrm{T}}$ of it should be larger than $2 \mathrm{GeV} / \mathrm{c}$ and the absolute value of $\eta$ less than 2.4.

The decay length $L_{x y}$, the proper decay length $L_{x y}^{P D L}$ and the error of the decay length $\sigma_{x y}$ are calculated from the $J / \psi$ vertex and the primary vertex in the $x y$-plane. The resolution of the proper decay length is $25 \mu \mathrm{m}$. It is found that the resolution is almost independent of the proper decay length. In order to suppress the prompt backgrounds, the second vertex has to be displaced from the primary one. We require $L_{x y} / \sigma_{x y}>2.5$ and $L_{x y}^{P D L}>60 \mu \mathrm{m}$. In addition, the condition $\cos \theta_{s p}>0.8$ is applied where $\theta_{s p}$ is the opening angle between the second vertex (pointing from the primary vertex) and the reconstructed $B_{\mathrm{c}}$ momentum. Finally, the reconstructed $B_{\mathrm{c}}$ candidate must be in a mass window between 6.25 and $6.55 \mathrm{GeV} / \mathrm{c}^{2}$. 
Table 7.5. Estimated number of signal and background events for $1 \mathrm{fb}^{-1}$.

\begin{tabular}{lllllllll}
\hline$B_{\mathrm{c}}$ & $B^{+}$ & $B_{s}$ & $B_{0}$ & $\operatorname{prompt} J / \psi$ & $\Lambda_{b}$ & $c \bar{c}$ & $b \bar{b}$ & QCD \\
\hline $120 \pm 11$ & $0.7 \pm 0.2$ & 0.1 & $0.9 \pm 0.3$ & 0.1 & 0.1 & 0.01 & 0.01 & $0.7 \pm 0.1$ \\
\hline
\end{tabular}

The number of $B_{\mathrm{c}}$ and background events for $1 \mathrm{fb}^{-1}$ after the selection are listed in Table 7.5. The total number of background events was estimated to be $2.6 \pm 0.4$, mainly from $B$ hadron decays into $J / \psi$. So far tagging of the $b$ jet is not used in the analysis.

Because of the high cross section, the number of produced QCD Monte Carlo events is not sufficient to directly determine the QCD background which is therefore estimated in three steps [245]. At first the efficiency to select two muons is obtained directly from the QCD sample, then the efficiency to reconstruct two muons into a $J / \psi$ candidate is calculated from the $c \bar{c} \rightarrow \mu^{+} \mu^{-} X$ sample, and finally the efficiency for the $J / \psi$ candidate to fake a $B_{\mathrm{c}}$ meson is obtained from the prompt $J / \psi$ sample. The probability of a QCD event to pass the selection cuts is then approximated as the product of the above three efficiencies. In this way, the total number of QCD background for $1 \mathrm{fb}^{-1}$ is estimated to be 0.7 events.

This study which is aimed at the first $\mathrm{fb}^{-1}$ collected with the CMS detector assumes that in this initial phase the dimuon trigger threshold can be set at values such that the applied cut of $p_{\mathrm{T}}>4 \mathrm{GeV} / \mathrm{c}$ on both muons does not introduce a significant inefficiency at trigger level. In case the available trigger bandwidth will prohibit this, more sophisticated High Level Trigger algorithms like a $J / \psi$ mass window could be invoked to restore the trigger efficiency. A detailed study is underway.

7.3.2.4. Mass and lifetime fitting. A kinematic fit was applied to the selected events imposing a $J / \psi$ mass constraint and forcing the two muon tracks as well as the pion track to share the same vertex. After the kinematic fit the invariant mass of the $J / \psi$ - pion system is shown in Fig. 7.13. A Gaussian fit provides a mean value of $6406 \mathrm{MeV} / \mathrm{c}^{2}$, close to the input of 6400 $\mathrm{MeV} / \mathrm{c}^{2}$, and a mass resolution of $22 \mathrm{MeV} / \mathrm{c}^{2}$. The number of signal events in the plot for $1 \mathrm{fb}^{-1}$ is 120 . Backgrounds from $B$ hadrons and prompt $J / \psi$ are included in the plot, while other backgrounds are neglected here.

A binned likelihood fit was done on the proper decay length distribution of the selected $B_{\mathrm{c}}$ events with the likelihood defined as $L=\prod P\left(n_{i}, \mu_{i}\right) . P\left(n_{i}, \mu_{i}\right)$ denotes the Poisson distribution with $n_{i}$ events observed and $\mu_{i}$ events predicted in the $i$-th bin:

$$
\mu=N \cdot \epsilon(x) \cdot \exp (-x / c \tau) \otimes G(x, \sigma)
$$

Here $x$ represents the proper decay length, $N$ and $c \tau$ are the parameters to be fitted and $G(x, \sigma)$ is a Gaussian smearing function with $\sigma$ fixed to $25 \mu \mathrm{m}$ which is the resolution of the proper decay length. The efficiency $\varepsilon(x)$ is obtained from the large $B_{\mathrm{c}}$ sample.

The result of the fit is $c \tau=148.8 \pm 13.1 \mu \mathrm{m}$ which is consistent with the used input value of $150 \mu \mathrm{m}$. The distribution of the proper decay length together with the fit result is shown in Fig. 7.13.

7.3.2.5. Systematic uncertainty. The influence of imperfect detector alignment which is of particular importance at the beginning of the CMS experiment on the track and vertex reconstruction has been studied in $[99,140]$. It will affect the study of $B_{\mathrm{c}}$ in three ways: the momentum scale of muons and pions, the mass resolution and finally the vertex precision. Taking the scale uncertainty to be $\Delta\left(1 / p_{\mathrm{T}}\right)=0.0005 / \mathrm{GeV} / \mathrm{c}$, the resulting uncertainties on the $B_{\mathrm{c}}$ mass is $11 \mathrm{MeV} / \mathrm{c}^{2}$ and $0.2 \mu \mathrm{m}$ on $c \tau$. 

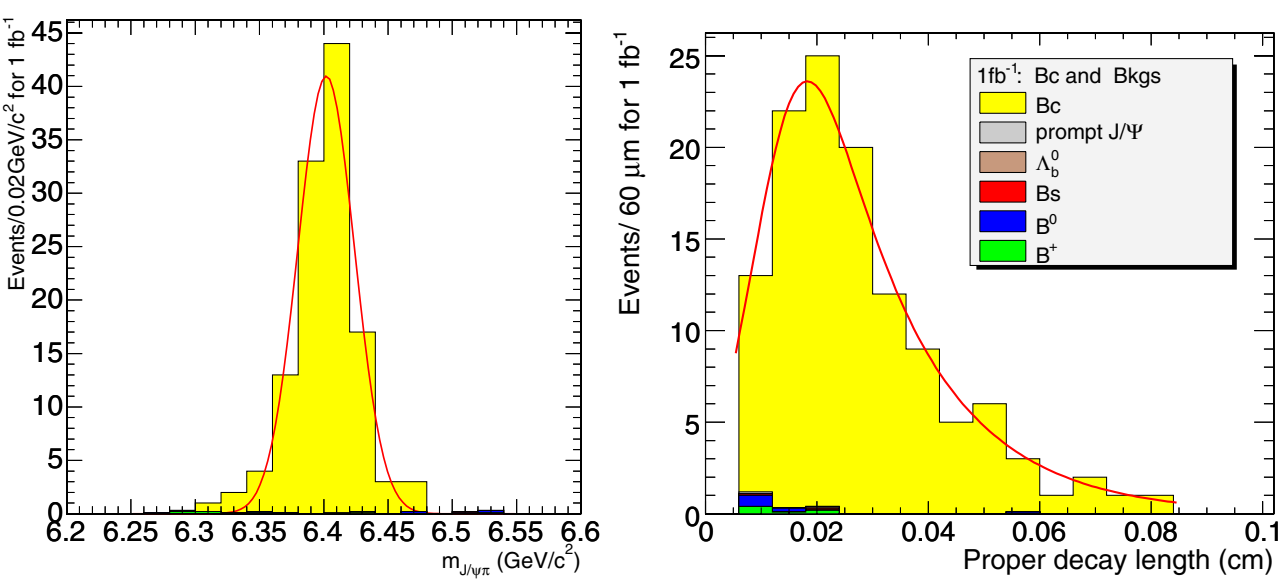

Figure 7.13. Left: The invariant mass of the $J / \psi$ and pion candidate for the selected $B_{\mathrm{c}}$. Right: The $B_{\mathrm{c}}$ proper decay length distribution. Both plots correspond to $1 \mathrm{fb}^{-1}$.

The effect of the muon momentum resolution was estimated following [99] and muon $p_{\mathrm{T}}$-values of 10,100 and $1000 \mathrm{GeV} / \mathrm{c}$ were studied for different $\eta$. The $\Delta p_{\mathrm{T}}$ to be smeared for a muon track from $B_{\mathrm{c}}$ was extrapolated from its $p_{\mathrm{T}}$ and $\eta$ according to [99]. The resulting $B_{\mathrm{c}}$ mass uncertainty is $10 \mathrm{MeV} / \mathrm{c}^{2}$, and $0.8 \mu \mathrm{m}$ on $c \tau$. The error from the vertex uncertainty was determined according to 140 causing an uncertainty on $c \tau$ of $2.4 \mu \mathrm{m}$.

The uncertainty on the efficiency as function of the proper decay length origins from the limited Monte Carlo statistics. By subtracting $\sqrt{N}$ events from the sample ( $N=3600$ events), new efficiencies were calculated and the fit was repeated. The observed difference of $0.1 \mu \mathrm{m}$ on $c \tau$ is taken as systematic uncertainty.

The theoretical uncertainty was estimated from Fig. 7.12 which shows the $p_{\mathrm{T}}$ distributions from different generator packages. The $B_{\mathrm{c}}$ events, generated by BICEPS, were reweighted to agree with the Gouz distribution and the analysis was repeated. The difference on $c \tau$ was found to be $1.5 \mu \mathrm{m}$ which is taken as the error from this source.

To check the sensitivity on the cuts, the muon and pion $p_{\mathrm{T}}$ cuts were changed by one standard deviation of their resolution, about $1.5 \%$ depending on $\eta$. Other cuts like on $\cos \theta_{s p}$ and on the proper decay length were changed by $10 \%$. The resulting mass uncertainty is $0.1 \mathrm{MeV} / \mathrm{c}^{2}$ and $0.2 \mu \mathrm{m}$ on $c \tau$.

In total the systematic uncertainties on the mass and on $c \tau$ are estimated to be $14.9 \mathrm{MeV} / \mathrm{c}^{2}$ and $3.0 \mu \mathrm{m}$, respectively.

7.3.2.6. Conclusion. With the first $\mathrm{fb}^{-1}$ of data CMS is expected to measure the $B_{\mathrm{c}}$ mass with an uncertainty of 22.0 (stat.) \pm 14.9 (syst.) $\mathrm{MeV} / \mathrm{c}^{2}$ and $c \tau$ with 13.1 (stat.) \pm 3.0 (syst.) $\mu \mathrm{m}$, corresponding to a lifetime uncertainty of 0.044 ( fit $) \pm 0.010$ (syst.)ps. About $120 B_{c}^{+} \rightarrow J / \psi \pi^{+}$, with $J / \psi \rightarrow \mu^{+} \mu^{-}$, events would be observed. At the moment, the theoretical calculation is at the leading order without the colour-octet contribution. Therefore, the uncertainties on the total cross section and the $p_{\mathrm{T}}$ distribution are large. In the real data analysis, $J / \psi+$ one track with $J / \psi \rightarrow \mu^{+} \mu^{-}$will be selected as a control sample, $B^{+} \rightarrow J / \psi K^{+}$will be used to estimate the efficiency, and the side band of the $J / \psi$ peak will be used to estimate the background to $B_{\mathrm{c}}$. 


\subsection{Diffraction and forward physics}

\subsubsection{Introduction}

This section outlines the diffractive and forward physics that CMS can do - together with the TOTEM experiment. The CMS and TOTEM detectors involved are presented in Chapter 7 of Volume 1 of the CMS Physics TDR [7].

The combined phase space coverage of the two experiments makes it possible to study many physics subjects in diffractive interactions - from QCD and the investigation of the low- $x$ structure of the proton to the production of SM and MSSM Higgs bosons. Diffractive events are characterised by the fact that the incoming proton(s) emerge from the interaction intact, or excited into a low mass state, with only a small energy loss. Diffractive processes with proton energy losses up to a few per cent are dominated by the exchange of an object with vacuum quantum numbers, the so called Pomeron, now understood in terms of partons from the proton. For larger energy losses, mesonic exchanges - Reggeons and pions become important. The topology of diffractive events is characterised by a gap in the rapidity distribution of final-state hadrons due to the lack of colour of the exchanged object.

Events with a fast proton in the final state can also originate from the exchange of a photon. In particular, forward tagging one leading proton allows the selection of photonproton events with known photon energy; likewise, tagging two leading protons gives access to photon-photon interactions of well known centre-of-mass energy.

Triggering of diffractive/forward events is discussed in [247] and in Appendix E.3. More details on the work presented here can be found in [248].

\subsubsection{The interest of diffractive interactions}

The study of hard diffraction has been pioneered by the UA8 experiment at CERN [249]. There have been major advances in this field recently, largely driven by the study of diffraction at HERA and the Tevatron. The essential results are discussed in [250] and can be summarised as follows:

- Many aspects of hard diffractive processes are well understood in QCD: the presence of a hard scale allows the use of perturbative techniques and thus to formulate the dynamics in terms of quarks and gluons.

- A key to this success are factorisation theorems in electron-proton scattering, which render part of the dynamics accessible to calculation in perturbation theory. The remaining nonperturbative quantities are the so-called diffractive parton distribution functions (dPDFs) and generalised (or "skewed") parton distributions (GPDs). They can be extracted from measurements and contain specific information about small- $x$ partons in the proton that can only be obtained in diffractive processes.

Diffractive parton densities are determined from inclusive diffractive processes and can be interpreted as conditional probabilities to find a parton in the proton when the final state of the process contains a fast proton of given four-momentum. Generalised parton distributions can be accessed in exclusive diffractive processes; they quantify correlations between parton momenta in the proton. Their $t$-dependence is sensitive to the distribution of partons in the transverse plane.

- To describe hard diffractive hadron-hadron collisions is more challenging since factorisation is broken by rescattering between spectator partons. These soft re-interactions can produce additional final-state particles which fill the would-be rapidity gap. When such additional particles are produced, a very fast proton can no longer appear in the final state because of 
energy conservation. The effect is often quantified in terms of the so called "gap survival probability". These rescattering effects are of interest in their own right because of their intimate relation with multiple scattering effects, which at LHC energies are expected to be crucial for understanding the structure of events in hard collisions.

The dynamics of rescattering and multi-gap events is still not completely understood. The available data can be described in terms of an effective, non-linear Pomeron trajectory [251]; its variation with energy would be a consequence of multi-Pomeron exchange effects [252]. Other models, also testable at the LHC have been proposed (see e.g. [253] and references therein). These topics can be pursued in more detail with the CMS-TOTEM data at the LHC.

- A fascinating link has emerged between diffraction and the physics of heavy-ion collisions through the concept of saturation, which offers a new window on QCD dynamics in the regime of high parton densities.

- Perhaps unexpectedly, the production of a SM or MSSM Higgs boson in diffractive $p p$ collisions is drawing more and more attention as a clean channel to study the properties of a light Higgs boson or even to discover it. The central exclusive reaction, $p p \rightarrow p H p$, appears particularly promising.

\subsubsection{A survey of the accessible diffractive/forward processes}

The accessible physics is a function of the integrated luminosity. We assume standard LHC optics with $\beta^{*}=0.5 \mathrm{~m}$ unless stated otherwise. We recall that, in this case, the TOTEM Roman Pots (RP) at $220 \mathrm{~m}$ from the CMS interaction point have coverage for $0.02<\xi<0.2$, where $\xi$ is the proton fractional momentum loss. Near-beam detectors at $420 \mathrm{~m}$ from the interaction point, currently also being considered [254], would cover $0.002<\xi<0.02$.

Low-luminosity $\left(\sim 10^{28}-10^{30} \mathrm{~cm}^{-2} \mathrm{~s}^{-1}\right)$ studies could profit from running with $\beta^{*}>0.5 \mathrm{~m}$, where the $\xi$ coverage of the $220 \mathrm{~m}$ RPs would be wider and the $t$ resolution would improve because of the lower transverse momentum spread of the beam.

7.4.3.1. Inclusive single diffraction and double Pomeron exchange at low luminosity. At modest instantaneous luminosities, up to $10^{32} \mathrm{~cm}^{-2} \mathrm{~s}^{-1}$, inclusive single diffractive (SD) events, $p p \rightarrow p X$, as well as inclusive double-Pomeron exchange (DPE) events, $p p \rightarrow p X p$, can be studied by requiring the presence of one or two rapidity gaps in the event. In the $\xi$ range given above, the scattered proton can be detected and the kinematics of the events fully measured.

The inclusive SD and DPE cross sections, as well as their $M_{X}$ dependence, even in the absence of a hard scale, are important quantities to measure at the LHC. Here $M_{X}$ indicates the mass of the system $X$. These cross sections amount to approximately $15 \%$ and $1 \%$ of the total proton-proton cross section, respectively; their energy dependence is a fundamental parameter of (non-perturbative) QCD. In addition, since diffractive events constitute a major fraction of the pile-up events, their measurement is mandatory to be able to properly simulate and understand high-luminosity data, where, at instantaneous luminosities of $10^{34} \mathrm{~cm}^{-2} \mathrm{~s}^{-1}$, approximately 35 pile-up events are superimposed, on average, to any event.

7.4.3.2. SD and DPE production of dijets, vector bosons and heavy quarks. The study of SD and DPE events in which the diffractively excited state includes high- $E_{\mathrm{T}}$ jets, heavy quarks or vector bosons opens up the possibility of accessing dPDFs and GPDs. The comparison of the DPE and SD rates for these processes may also give information on the hard diffractive 
factorisation breaking at LHC (see Section 7.4.2). A few examples of these processes are given here.

Production of dijets. The measurement of the reaction $p p \rightarrow p X j j$ ( $j$ indicates a jet) has been used for the first time by $\mathrm{CDF}$ to measure the diffractive structure function in antiprotonproton collisions [255]. A similar measurement is possible at LHC with wider kinematic coverage (CDF: $\xi>0.035)$ and larger minimum jet $E_{\mathrm{T}}$. For $E_{\mathrm{T}}>45 \mathrm{GeV}$, of the order of $10^{8}$ events per $\mathrm{fb}^{-1}$ can be expected.

Production of heavy quarks. Inclusive DPE production of $t \bar{t}$ pairs has been studied in the case in which the final state contains one muon and four jets (i.e. with one top quark decaying to $b$ plus lepton and neutrino, and the other to three jets). The analysis required the detection of both final-state protons. The expected number of events is of order $1-100$ for $10 \mathrm{fb}^{-1}$, depending on the theoretical model assumed.

SD and DPE production of $B$-mesons has also been looked at, with $B \rightarrow J / \psi X$ and $J / \psi \rightarrow \mu^{+} \mu^{-}$. Here the number of expected events is much larger, of the order of a few events per $10 \mathrm{fb}^{-1}$ in the DPE case and thousands in the SD case.

Inclusive DPE production of $\mathbf{W}$ bosons. Inclusive DPE production of $W$ bosons, $p p \rightarrow$ $p X W p$, is also sensitive to the dPDFs of the proton and is a relatively abundant process that can be studied at instantaneous luminosities where pile-up is small. In these conditions, the requirement that two final state protons be measured in the $220 \mathrm{~m}$ RPs suppresses both the QCD background and the inclusive $W$ production. Several thousand events with $W \rightarrow e v$ or $W \rightarrow \mu \nu$ are expected, after cuts, for an integrated luminosity of $1 \mathrm{fb}^{-1}$. This process, in conjunction with SD production of $W$ bosons, can be used to study hard diffractive factorisation breaking using the LHC data alone, as mentioned above.

7.4.3.3. SM and MSSM central exclusive Higgs production. As the delivered luminosity reaches tens of $\mathrm{fb}^{-1}$, the central exclusive production process (DPE) becomes a tool to search for new physics, delivering signal to background ratios of order 0.1-1 for Standard Model (SM) Higgs production [256] and more than an order of magnitude larger for certain supersymmetric (MSSM) scenarios.

By central exclusive, we refer to the process $p p \rightarrow p \phi p$, where there are large rapidity gaps between the outgoing protons and the decay products of $\phi$. There are three primary reasons why this process is attractive. Firstly, if the outgoing protons remain intact and scatter through small angles, then, under some general assumptions, the central system $\phi$ is produced in the $J_{Z}=0, \mathrm{C}$ and $\mathrm{P}$ even state. Secondly, the mass of the central system can be determined very accurately from a measurement of the transverse and longitudinal momentum components of the outgoing protons alone. This means an accurate determination of the mass irrespective of the decay mode of the centrally produced particle. Thirdly, the process delivers excellent signal to background ratios, due to the combination of the $J_{Z}=0$ selection rules, the mass resolution, and the simplicity of the event in the central detectors. An additional attractive property of central exclusive production is its sensitivity to $\mathrm{CP}$ violating effects in the couplings of the object $\phi$ to gluons.

The left panel of Fig. 7.14 shows the cross section times the branching ratio for central exclusive production of a Standard Model Higgs, with $H \rightarrow b \bar{b}$ and $H \rightarrow W W$, as a function of the Higgs mass for different theoretical approaches. The $b \bar{b}$ mode is particularly interesting for masses close to the current exclusion limit. The right panel of Fig. 7.14 shows the acceptance assuming various combinations of RPs at $220 \mathrm{~m}$ and near-beam detectors at $420 \mathrm{~m}$. Both protons can be detected in the $220 \mathrm{~m}$ stations only for Higgs masses larger than $280 \mathrm{GeV} / \mathrm{c}^{2}$; this reflects the $\xi$ range for which the $220 \mathrm{~m}$ RPs have acceptance, 

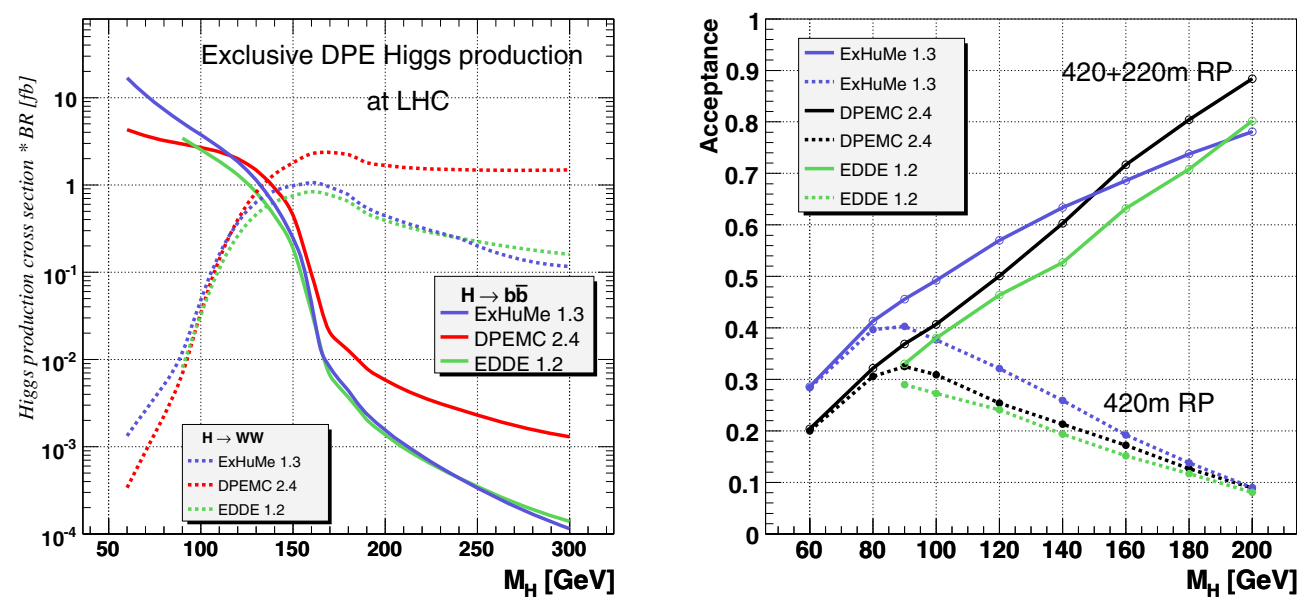

Figure 7.14. Left: The cross section for the exclusive production of the Higgs boson as a function of the Higgs boson mass for $H \rightarrow b \bar{b}$ and $H \rightarrow W W$. The different curves were obtained with the generators Exhume1.3 [259], DPEMC2.4 [260] and EDDE1.2 [261]. Right: Acceptance for the $420 \mathrm{~m}$ detectors alone and for the combination of the $220 \mathrm{~m}$ and $420 \mathrm{~m}$ detectors as a function of the Higgs boson mass.

$0.02<\xi<0.2$ (the mass of the centrally produced Higgs is related to the $\xi$ via $M_{H}^{2}=\xi_{1} \xi_{2} s$, with $\xi_{1}, \xi_{2}$ the fractional momentum losses of the two protons). However, asymmetric events with one proton at low $\xi$ and another at large $\xi$ can be detected by the combination of the $220 \mathrm{~m}$ and $420 \mathrm{~m}$ detectors $(0.002<\xi<0.02)$.

Central exclusive production is generally an attractive way of searching for any new particles that couple strongly to glue. An example studied in [257] is the scenario in which the gluino is the lightest supersymmetric particle. In such models, there should exist a spectrum of gluino-gluino bound states which can be produced in the central exclusive channel. Likewise, central exclusive production of radions, the fields introduced in the Randall-Sundrum model of five-dimensional quantum gravity, has been studied [258].

$\boldsymbol{H} \rightarrow \boldsymbol{b} \overline{\boldsymbol{b}}$. The analysis is based on the requirement of two back-to-back central $b$-tagged jets in addition to the detection of both final-state protons yielding a mass of the central system consistent with that calculated from the protons alone. The event yield is very low, about $2-4$ events per $30 \mathrm{fb}^{-1}$ after all cuts, depending on the model. The non-resonant continuum $b$-jet background is largely suppressed by the $J_{Z}=0$ rule. The residual background, mostly due to dijet production ( $g g \rightarrow$ dijets) and diffractive $g g \rightarrow b \bar{b}$ production, is a function of the mass resolution, which is about $1.6 \%$ for the ' $420+420$ ' combination and $5.6 \%$ for the ' $220+420$ ' combination (for $M_{H}=120 \mathrm{GeV} / \mathrm{c}^{2}$ ). The number of expected background events is of order 10 for $30 \mathrm{fb}^{-1}$.

$\boldsymbol{H} \rightarrow \boldsymbol{W W}$. In this case, the suppression of the background does not rely primarily on the mass resolution of the RPs. There are three main categories of $W W$ events. Events in which at least one of the $W$ bosons decays to an electron or a muon are the simplest, and pass the Level-1 trigger thanks to the high- $p_{\mathrm{T}}$ final-state lepton. This holds also if one of the $W$ bosons decays into a tau, which subsequently decays leptonically. The four-jet mode occurs approximately half of the time; here, however, the RP information is necessary already at Level-1. The expected event yields range between 1 and 7 events for $30 \mathrm{fb}^{-1}$, depending on the mass. Irreducible backgrounds are small and controllable. 
MSSM Higgs. Double proton tagging is especially beneficial in the MSSM case. The $b$-jet channel is very important in the 'intense coupling regime' of $\operatorname{MSSM}\left(M_{h} \approx M_{A} \approx M_{H} \approx\right.$ $100 \mathrm{GeV} / \mathrm{c}^{2}$ ) [262]: couplings of the Higgs to $g g, W W^{*}, Z Z^{*}$ are strongly suppressed, making the discovery challenging by conventional means. Rates for central exclusive production of the two scalar $\left(0^{+}\right)$MSSM Higgs bosons $(h, H)$ are more than a factor 10 larger than for the SM Higgs. The enhancement for $H \rightarrow b \bar{b}$ is by orders of magnitude in the $M_{h}$-max scenario for $M_{H} \approx 180-250 \mathrm{GeV} / \mathrm{c}^{2}$; likewise for $h \rightarrow b \bar{b}$ and $h \rightarrow \tau \tau$ for $M_{h} \approx 90-130 \mathrm{GeV} / \mathrm{c}^{2}$ [263]. In the small $\alpha_{\text {eff }}$ scenario, $h \rightarrow b \bar{b}$ and $h \rightarrow \tau \tau$ can be heavily suppressed for large $\tan \beta$ and for $M_{h} \approx 120 \mathrm{GeV} / \mathrm{c}^{2}$ [263], whereas $h \rightarrow W W$ may be enhanced by up to a factor 4 compared to the SM predictions. Also, the pseudo-scalar $\left(0^{-}\right)$ Higgs boson (A) is practically not produced in the central exclusive channel, yielding a clean separation of the scalar and pseudo-scalar Higgs bosons, impossible in conventional channels. The good missing mass resolution allows to resolve $h, H$ and, if enough statistics is available, measure their widths. This makes central exclusive production a possible discovery channel. Central exclusive production is also interesting in the ' 3 -way mixing' scenario of CP-violating MSSM [264]: here the 3 neutral Higgs bosons are nearly degenerate, mix strongly and have masses close to $120 \mathrm{GeV} / \mathrm{c}^{2}$.

Central exclusive production, with its good mass resolution via the scattered protons, may allow disentangling the Higgs bosons by studying the production lineshape. Explicit $\mathrm{CP}$-violation in the Higgs sector causes an asymmetry in the azimuthal distributions of tagged protons (via the interference of P-even and P-odd amplitudes) - a measurement unique at the LHC $[262,265]$.

7.4.3.4. High-energy photon interactions. A significant fraction of events at the LHC involves photon interactions at energies above the electroweak scale [266]. The protons radiating the photon often survive the collision intact and are scattered at angles comparable to the beam angular divergence. Detection of such events at the LHC will open up a new field of high-energy photon physics, which is briefly outlined below. By requiring the detection of one or two forward protons like in diffractive interactions, photon-photon and photon-proton interactions can be selected. The photon fluxes, and the effective luminosities of photon-photon and photon-proton collisions are well known [267, 268]. The average proton energy loss is larger and the proton scattering angle smaller in photon exchanges than for the diffractive case. This can be used to establish relative contributions of these two processes.

Two-photon exclusive production of $\mathbf{W}$ and $\mathbf{Z}$ boson pairs. The cross section for the production of $W$ pairs via photon-photon interactions, $p p \rightarrow p p W W$, is slightly above $100 \mathrm{fb}$; in almost half of these events both forward protons are produced within the acceptance of the TOTEM RPs. About 100 events per $10 \mathrm{fb}^{-1}$ with leptonic $W$ decays can be detected in CMS. This allows a precise study of the gauge couplings, in particular of the $\gamma \gamma W W$ coupling. The expected sensitivity to anomalous quartic gauge couplings (QGCs) will surpass the LEP and Tevatron limits by orders of magnitude. A deviation from the Standard Model predictions would also allow a clean detection of anomalous $W W$ production as predicted e.g. by A. White's theory of the supercritical Pomeron [269]. Two-photon production of $Z$ pairs, $p p \rightarrow p p Z Z$, is not allowed at the SM tree level, but yields similar sensitivities to the anomalous QGCs in this channel.

Two-photon exclusive production of pairs of SUSY particles. The cross sections for production of pairs of charginos, sleptons and charged Higgs bosons via photon-photon fusion at the LHC decrease rapidly with the masses of these particles [269]. This limits the 
scope of SUSY searches to particle masses below $150-200 \mathrm{GeV} / \mathrm{c}^{2}$. However, the very clean environment of this reaction makes it attractive compared to other production mechanisms; the final state typically consists of two opposite-sign leptons and of missing $p_{\mathrm{T}}$. The main background is due to the exclusive production of $W$ pairs discussed above.

Two-photon production of doubly charged Higgs bosons (appearing in GUTs) is strongly enhanced, and leads to exclusive final states with two pairs of same-sign leptons.

Two-photon lepton pair production. Exclusive production of lepton pairs - a purely QED process at low $|t|$ - may serve for calibration of the $p p$ luminosity; it may also be used for calibration of the momentum measurement of the scattered proton. Thousands of exclusive muon pairs are expected to be reconstructed in CMS for an integrated luminosity of $1 \mathrm{fb}^{-1}$. The striking signature of extremely small muon acoplanarity angles of less than about $10 \mathrm{mrad}$ may be exploited already at the trigger level.

Single $\mathbf{W}$ and single top photoproduction. The cross section for single $W$ photoproduction, $p p \rightarrow p W j X$, reaches almost $100 \mathrm{pb}$. This process can be therefore studied already at low luminosity. It also provides a means to study rescattering effects [268]. At higher luminosities, studies of high mass $W j$ states will be possible; for $W j$ invariant masses above $1 \mathrm{TeV}$, tens of events are expected to be detected in CMS (and tagged by TOTEM) per $10 \mathrm{fb}^{-1}$. This will allow to search for, as an example, an anomalous triple gauge coupling $\gamma W W$. This process is the main background in the search for anomalous photoproduction of single top.

Associated WH and top pair photoproduction. The associated photoproduction of a SM Higgs boson and a $W$ boson has a cross section of about $20 \mathrm{fb}$ for Higgs mass below $180 \mathrm{GeV} / \mathrm{c}^{2}$. About $50 \%$ of the forward protons are tagged by TOTEM, and events with leptonic $W$ decay can be triggered efficiently in CMS. The cross section for photoproduction of top pairs is slightly above $1 \mathrm{pb}$. Top pair production is the main background for $W H$ production, and in the photoproduction case the signal-to-background ratio for photoproduction of $W H$ pairs is superior to the one in inclusive production.

7.4.3.5. Drell-Yan. The study of forward production of low mass Drell-Yan lepton pairs at the LHC provides a unique opportunity to directly access low- $x$ partons in the proton. In this process, the lepton pair originates from the annihilation of a quark-anti-quark pair whose fractional momenta, $x_{1}$ and $x_{2}$, are related to the dilepton mass, $M$, and rapidity, $y$, through

$$
M^{2}=s x_{1} x_{2} ; \quad x_{1,2}=\frac{M}{\sqrt{s}} \exp ^{ \pm y},
$$

with $\sqrt{s}=14 \mathrm{TeV}$, the centre-of-mass energy of the colliding protons. In order to access low $x$, a large imbalance in fractional momenta is required, boosting the lepton pair to large rapidities.

The CASTOR calorimeter will cover the pseudorapidity range $5.3<\eta<6.6$, corresponding to Bjorken- $x$ values down to $10^{-7}$. With CASTOR alone, it may be possible to obtain a crude estimate of the dilepton mass. With the additional information provided by the $\mathrm{T} 2$ tracker, one can enhance the signal to background ratio by requiring tracks in association to the electromagnetic energy deposits. As T2 will measure both the azimuthal and polar angles of the tracks, a much more accurate measurement of the opening angle (and therefore of the dilepton mass) and a two-dimensional study in $M^{2}$ and $x$ will become possible. 
7.4.3.6. Validation of cosmic-ray generators. The correct simulation of the interaction of primary cosmic rays in the $\mathrm{PeV}$ energy range with the atmosphere is a key tool in the study of cosmic rays. Unfortunately, the available generators differ significantly in their predictions for the energy flow, multiplicity, hadronic energy fraction etc., in particular at high rapidities. These models can be tested at the LHC: a $100 \mathrm{PeV}$ fixed-target collision in air corresponds to the centre-of-mass energy of a $p p$ collision at the LHC. Several generators were used to simulate inelastic and diffractive collisions at CMS: QGSJET [271], SIBYLL [272], DPMJET [273], NEXus [271]. There are significant differences in the predictions, notably in the region covered by CASTOR, T1 and T2. A measurement of these features with CASTOR, $\mathrm{T} 1$ and $\mathrm{T} 2$ may thus be used to validate/tune these generators.

\subsection{Physics with heavy ions}

\subsubsection{High-density QCD: heavy-ion physics}

Quantum Chromodynamics (QCD) is the only existing quantum field theory within the Standard Model, whose collective behaviour, phase diagram and phase transitions, are accessible to study in the laboratory. High-energy nucleus-nucleus collisions offer the only experimental means known so far to concentrate a significant amount of energy $(\mathcal{O}(10 \mathrm{TeV})$ at the LHC) in a "large" volume $\left(\mathcal{O}\left(100 \mathrm{fm}^{3}\right)\right.$ at thermalisation times of $\left.\tau_{0} \approx 1 \mathrm{fm} / c\right)$, allowing the study the many-body dynamics of strongly interacting matter. The programme of highenergy heavy-ion physics addresses several key open questions of the strong interaction:

- Deconfinement and chiral symmetry restoration. Lattice QCD calculations predict a new form of matter at energy densities above $\varepsilon \approx 1 \mathrm{GeV} / \mathrm{fm}^{3}$ consisting of an extended volume of deconfined and bare-mass quarks and gluons: the Quark Gluon Plasma (QGP) [274]. The scrutiny of this new state of matter (equation-of-state, order of the phase transition, ... ) promises to shed light on fundamental questions such as the nature of confinement, the mechanism of mass generation (chiral symmetry breaking, structure of the QCD vacuum) and hadronisation, that still evade a thorough theoretical description due to their highly non-perturbative nature.

- Non-linear parton evolution at small-x. At high energies, hadrons consist of a very dense system of gluons with small (Bjorken) parton fractional momenta $x=p_{\text {parton }} / p_{\text {hadron }}$. At low- $x$, the probability to emit an extra gluon is large $\sim \alpha_{S} \ln (1 / x)$ and non-linear gluon-gluon fusion processes start to dominate the parton evolution in the hadronic wave functions. Whereas at values of $x \gtrsim 10^{-3}$, the parton evolution with $Q^{2}($ or $\ln (1 / x))$ is described by the usual DGLAP (or BFKL) equations, at lower values of $x$ and around $Q_{s}^{2} \sim 3 G e V^{2} / c^{2}$, such a saturated configuration is theoretically described in terms of the "Colour Glass Condensate" (CGC) picture [275]. Since the nonlinear growth of the gluon density depends on the transverse size of the system, the effects of gluon saturation are expected to set in earlier (at higher $x$ ) for heavy nuclei than for free nucleons.

In addition, the study of heavy-ion collisions has interesting connections to other research areas such as:

- Early Universe cosmology. The quark-hadron phase transition took place some $10 \mu$ s after the Big-Bang and was the most important event taking place in the Universe between the electro-weak (or SUSY) transition $\left(\tau \sim 10^{-10} \mathrm{~s}\right.$ ) and Big Bang nucleosynthesis (BBN, at $\tau \sim 200 \mathrm{~s}$ ). Depending on the order of the QCD phase transition, several cosmological implications such as the formation of strangelets and cold dark-matter (WIMP) clumps or baryon fluctuations leading to inhomogeneous nucleosynthesis, have been postulated [276]. 
- High-energy cosmic-ray physics. The energy and mass of cosmic particles with energies above $10^{14} \mathrm{eV}$ can only be measured via the ground-based detection of "extended air showers" (EAS) generated in upper-atmosphere interactions of cosmic rays (protons and ions up to $\mathrm{Fe}$ ) with air (N,O nuclei). The interpretation of the EAS (and the related astro-particle phenomena) relies heavily on the accurate modelling of hadronic multiparticle production in proton-nucleus $(\mathrm{p}+\mathrm{N}, \mathrm{p}+\mathrm{O})$ and nucleus-nucleus $(\mathrm{He}+\mathrm{N}, \mathrm{N}+\mathrm{N}, \mathrm{Fe}+\mathrm{N})$ collisions in the $\mathrm{TeV}$ range. Direct measurements at $\mathrm{LHC}$ are needed in order to calibrate and tune the EAS models and correctly extrapolate their predictions to the highest cosmicray energies measured $\left(\sim 10^{20} \mathrm{eV}\right)$.

- Gauge/String duality. Theoretical calculations based on the AdS/CFT correspondence permit to obtain results in strongly coupled $\left(g^{2} N_{c} \gg 1\right)$ gauge theories (QCD-like: SUSY $\mathcal{N}=4$ Yang-Mills) in terms of a dual gravity theory. Recent applications of this formalism have allowed, for the first time, to compute finite temperature QCD transport coefficients (such as the ratio of the QGP viscosity over entropy density, $\eta / s$ ) experimentally accessible, from black hole thermodynamics calculations [277].

\subsubsection{Hard probes of QCD matter at LHC}

Nucleus-nucleus collisions at the LHC offer a unique opportunity for studying strongly interacting matter at values of energy and particle densities never reached before. The factor of 30 increase in energy between RHIC and the LHC $\left(\sqrt{s}_{N N}=5.5 \mathrm{TeV}\right.$ for $\left.\mathrm{PbPb}\right)$ leads to copious production of hard QCD probes: high- $p_{\mathrm{T}}$ hadrons, jets, quarkonia, direct photons, etc., arising from parton-parton scatterings with large squared momentum transfer, $Q^{2}$. Such perturbative processes take place at time scales $\tau \approx 1 / p_{\mathrm{T}} \lesssim 0.1 \mathrm{fm} / c$, and involve primary partons with fractional momenta of order $x \sim 10^{-3}\left(10^{-5}\right)$ at central (forward) rapidities. The produced hard probes are, thus, sensitive to initial-state modifications of the low- $x$ parton distribution functions, as well as to final-state effects while propagating through the bulk matter formed in the collision.

The contribution of CMS to the heavy-ion physics programme at LHC is extremely competent based on a number of unique experimental capabilities including:

(i) Very large acceptance at midrapidity $(|\eta|<2.5$, full $\phi)$ for layered detection of charged hadrons (with the best momentum resolution for charged tracks at LHC) and neutral hadrons as well as muons, electrons, and photons over a wide range of $p_{\mathrm{T}}$.

(ii) The best mass resolution of any LHC detector for quarkonia $(J / \psi, \Upsilon)$ measurements leading to clean separation of the various states, improved signal over background, and large reconstructed yields.

(iii) Complete electromagnetic and hadronic calorimetry since day-1 for full jet triggering and reconstruction over $|\eta|<3$ and $\Delta \phi=2 \pi$ with a large statistical significance for single jet and jet $+X$ channels $(X=$ jet, $\gamma, Z)$, and for full $\mathrm{b}$ - and c- jet identification, allowing detailed studies of "jet quenching" phenomena.

(iv) Unparalleled forward physics (low- $x$ QCD) capabilities thanks to the forward hadronic calorimeter HF $(3<|\eta|<5)$, CASTOR-TOTEM $(5.5<|\eta|<6.6)$, and Zero-DegreeCalorimeter $(|\eta|>8.1$ for neutrals) detector systems.

(v) A DAQ system capable of delivering almost every PbPb event to the High Level Trigger allowing maximum flexibility to select rare probes at the highest multiplicities expected at the LHC.

Among the various perturbative probes accessible to measurement, we focus on this report on the quarkonia detection via the $\mu^{+} \mu^{-}$decay channel. Other experimental 
capabilities, in the hard (notably jet reconstruction in the heavy-ion environment), soft (hadron multiplicities, elliptic flow ...), and low- $x$ (e.g. quarkonia photoproduction in electromagnetic $\mathrm{PbPb}$ interactions) sectors will be discussed in detail in CMS Physics TDR addendum for Heavy Ions.”

\subsubsection{Gluon saturation and QGP colour screening via Quarkonia}

The production of heavy-quarks at LHC proceeds mainly via gluon-gluon fusion processes and, as such, is sensitive to nuclear modifications of the gluon density at low- $x$. At $\sqrt{s}_{N N}=$ $5.5 \mathrm{TeV}$, the average fraction of the nucleon momentum carried by the interacting parton producing a $J / \psi$ at mid (forward) rapidity is $\langle x\rangle \approx 3 \cdot 10^{-3}\left(10^{-5}\right)$. Such a kinematical domain is well in the regime where gluon saturation effects and departures from linear $Q^{2}$ (DGLAP) and $\ln (1 / x)$ (BFKL) evolutions should be observable. In addition, the final-state formation of $Q \bar{Q}$ bound states is expected to be suppressed in a deconfined medium due to colour screening of the heavy-quark potential. Recent finite-temperature lattice QCD calculations exhibit a substantial reduction of the heavy-quark internal energy $U_{Q \bar{Q}}$, with increasing temperature. The ground-state charmonium state $(J / \psi)$ has been found to dissolve slightly below $2 \cdot T_{\text {crit }} \approx 330 \mathrm{MeV}$, whereas much higher dissociation temperatures, $T_{\text {diss }} \approx 4 \cdot T_{\text {crit }}$ reachable at LHC, are needed to dissociate the $\Upsilon$. Although $J / \psi$ suppression has been indeed observed in central A+A collisions both at CERN-SPS and RHIC energies, competing mechanisms to colour deconfinement (hadronic co-movers interactions and charm quark recombination) have been proposed to explain the observed cross-sections. At variance with charmonia states, the study of the much heavier bottomonia spectroscopy accessible at LHC is free from the distorting hadronic and coalescence contributions, and is directly sensitive to the temperature conditions of the produced partonic medium.

CMS has focused on the quarkonia detection through their decays to muon pairs. The good muon momentum resolution translates in an $\Upsilon$ mass resolution of $\sigma=54 \mathrm{MeV} / \mathrm{c}^{2}$ (in the central barrel region $|\eta|<0.8$ ), the best of all the LHC detectors. This good resolution provides a clean separation between the members of the $\Upsilon$ family with a consequent improvement in the signal to background ratio, even in head-on $\mathrm{PbPb}$ collisions with particle multiplicities as large as $N_{c h} /\left.d \eta\right|_{\eta=0}=5000$. The expected signal/background ratios are $S / B \approx 1(5), S / B \approx 0.1(1)$ for $J / \psi$ and $\Upsilon$ respectively in the full $(|\eta|<0.8)$ rapidity range. In the absence of initial- or final-state medium effects, production cross sections of $B_{\mu \mu} \sigma=50 \mathrm{mb}$ and $300 \mu \mathrm{b}$ respectively will be measured in minimum bias $\mathrm{PbPb}$ collisions. The expected reconstructed yields for both charmonium and bottomonium resonances after background subtraction, in one-month data taking (with $50 \%$ overall efficiency) and nominal $\mathrm{PbPb}$ luminosity $\left(0.5 \mathrm{nb}^{-1}\right)$, are $\mathcal{O}\left(1.5 \cdot 10^{5}\right), \mathcal{O}\left(2 \cdot 10^{4}\right)$ respectively. These statistics will allow detailed quantitative studies of quarkonia production as a function of $p_{\mathrm{T}}$, rapidity and/or centrality. Any departure from the expected "vacuum" cross-sections will provide valuable information on the initial-state modifications of the nuclear parton (especially, gluon) distribution functions, as well as on the thermodynamical state of the produced medium from the predicted "melting" pattern of different quarkonia states due to colour screening. 


\section{Chapter 8. Physics of Top Quarks}

\subsection{Selection of $\bar{t} \bar{t}$ events and measurement of the cross sections}

\subsubsection{Introduction}

The goal of top physics at the LHC is to characterise the properties of this heaviest fermion of the Standard Model by measuring observables in its production and decay exploiting all possible decay channels. Important examples are the production cross section and the mass and spin properties of the top quark.

Most of the top quarks at the LHC will be produced as $t \bar{t}$ pairs. The $t \bar{t}$ production cross section is estimated to be $830 \mathrm{pb}$ [278] at NLO and the dominant production mechanisms are gluon-gluon fusion $(\approx 90 \%)$ and quark-anti-quark annihilation $(\approx 10 \%)$. Within the Standard Model the top quark decays almost exclusively to a $W$ boson and a $b$ quark. The decays of the $t \bar{t}$ system are then classified according to the decays of the $W^{+} W^{-}$system as dileptonic, semileptonic or fully hadronic. The $W$ can decay into leptons, $e^{-} \bar{v}_{e}, \mu^{-} \bar{v}_{\mu}, \tau^{-} \bar{v}_{\tau}$, or into quarks, $u \bar{d}^{\prime}, c \bar{s}^{\prime}$, where the charge conjugate is implicit. Neglecting QCD corrections, branching fractions of $9 / 81(11.1 \%)$ for the dileptonic, 36/81 (44.4\%) for the semi-leptonic and 36/81 $(44.4 \%)$ for the fully hadronic decay channel are obtained.

For our studies we use PYTHIA for the simulation of signal and background events. As it includes spin correlation in $t \bar{t}$ production also samples generated with TopREX are used for signal events.

\subsubsection{Dileptonic channel}

8.1.2.1. Event selection for $1 \mathrm{fb}^{-1}$. The very clean signature of this channel combined with a high signal-to-background ratio makes it possible to select $t \bar{t}$-events with simple kinematic cuts. The selection is therefore suitable for the expected early performance of the CMS detector and will allow to establish the signal as well as to measure the top mass at an early stage of the experiment.

For an integrated luminosity of $1 \mathrm{fb}^{-1}$ about 54000 signal events are expected according to the leading-order estimate of PYTHIA. The main backgrounds with a final state mimicking the signal are $Z, W W, W Z$ and $Z Z$ production accompanied by jets. Furthermore, events from semi-leptonic and fully-hadronic top-quark pair production with misidentified leptons and leptons from b-quark jets eventually constitute the dominating background. Here, dilepton events with $\mathrm{W}$ bosons decaying into $\tau$-leptons are considered signal events if the $\tau$ lepton decays leptonically. Details of the analysis can be found in Reference [279].

Events are required to pass the Level-1 and High Level Trigger, in particular the single and dilepton subtriggers. In addition to trigger criteria, events must contain at least two jets and two oppositely charged leptons. Electrons are identified using an electron likelihood method combining various electromagnetic shower variables and track-to-supercluster-matching criteria. After this pre-selection about 15000 signal events are left in a $1 \mathrm{fb}^{-1}$ data set with a signal over background ratio of $S / B=1 / 10$. The most important background at this stage consists of $Z+$ jets production with an accepted cross section of about $120 \mathrm{pb}$ and a similar final state.

Isolation criteria reduce the contribution from misidentified leptons and leptons from b-jets. For a lepton candidate no other track or calorimeter hits amounting to $10 \%$ or more of the lepton $p_{\mathrm{T}}$ are allowed in a cone of $\Delta R<0.2$. Two charged leptons are then chosen with a discriminant based on the likelihood ratio in case of an electron, the energy deposited in a cone of $\Delta R=0.2$ around the lepton axis and the $p_{\mathrm{T}}$ of the lepton. 

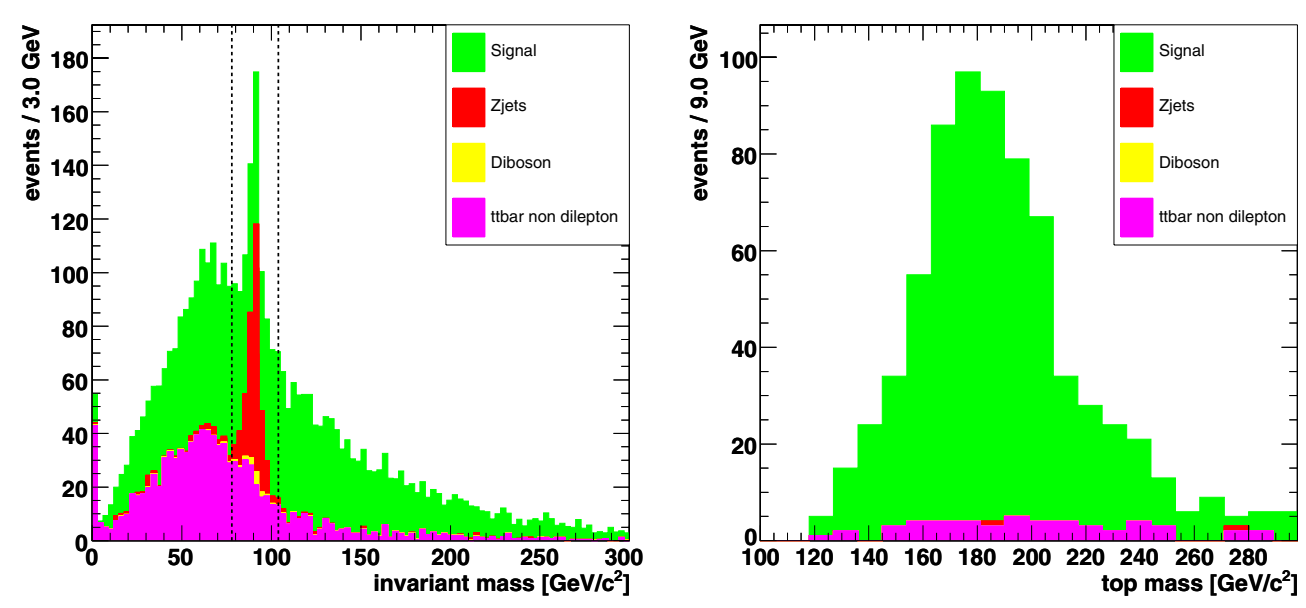

Figure 8.1. Left: Invariant mass of the two lepton candidates indicating the cut window to remove $Z+$ jets events. Right: Most likely top mass after selection for $1 \mathrm{fb}^{-1}$.

Both $b$-jets are selected with a discriminator based on the jet $p_{\mathrm{T}}$, the invariant mass of tracks inside the jet and the output of the combined b-tagging algorithm [157]. Using this scheme the correct jets and leptons of the signal are selected for more than $90 \%$ of the events, if they could be reconstructed. It has been shown in reference [157] that, during the first data taking phases of the LHC, the degradation in b-tagging performance is still acceptable. This implies that the b-tagging results presented here remain essentially correct.

Figure 8.1 shows the invariant mass of the two lepton candidates. The $Z$ mass peak of the invariant mass distribution of two same type leptons is used to remove the contamination due to $Z+$ jets events. As a further improvement a cut on the $b$-tag discriminator is applied to the two selected jets.

The non-dilepton $t \bar{t}$ events usually contain more jets with a $p_{\mathrm{T}}$ greater than $30 \mathrm{GeV} / \mathrm{c}$ but do not contain two high $p_{\mathrm{T}}$ leptons. The second lepton candidate is considerably softer than the corresponding lepton from the signal decay channel. So a cut on the lower transverse momentum lepton is imposed with $p_{\mathrm{T}}>20 \mathrm{GeV} / \mathrm{c}$. The two neutrinos in the decay of the $W$ bosons lead to significant missing transverse energy $E_{\mathrm{T}}^{\text {miss }}$ whereas the decay of $Z$ bosons into electrons or muons does not generate $E_{\mathrm{T}}^{\text {miss }}$. The cut $E_{\mathrm{T}}^{\text {miss }}>40 \mathrm{GeV}$ further improves the signal to background ratio. At this stage about 1800 signal events are left with a signal over background ratio of $S / B=7.3 / 1$.

The kinematics of the $t \bar{t}$ dilepton events yield an underconstrained equation system due to the two undetected neutrinos in the final state. However if, all other kinematic quantities have been measured it is possible to make a fit imposing $m_{W}$ and assuming a top mass parameter in the range of 100 to $300 \mathrm{GeV} / \mathrm{c}^{2}$. A weight can then be assigned to the different solutions obtained [279]. Figure 8.1 shows the distribution of the most likely top mass for signal and background events in the range $100 \mathrm{GeV} / \mathrm{c}^{2}<m_{t}<300 \mathrm{GeV} / \mathrm{c}^{2}$.

The event topology of most of the background events passing the previous cuts does not satisfy the dilepton kinematical constraints. Therefore considering only candidates which give a mass estimate in the range of 100 to $300 \mathrm{GeV} / \mathrm{c}^{2}$ further reduces the background and raises the signal over background ratio to about $S: B=12: 1$. The remaining background essentially contains only non-dilepton $t \bar{t}$ events. In a dataset equivalent to $1 \mathrm{fb}^{-1}, 657$ signal events are selected with an overall efficiency of $1.2 \%$. 
We conclude that a measurement of the $t \bar{t}$ cross section and the top mass (see Section 8.2.1) in the dileptonic channel will be possible already with a modest amount of luminosity [279].

8.1.2.2. Event selection for higher luminosities. The trigger is based on the presence of one muon or electron which covers with high efficiency all the possible final states in this channel. The selection of events in this channel then requires after trigger selection the presence of just two oppositely charged leptons with $E_{\mathrm{T}}>20 \mathrm{GeV}$ within pseudorapidity ranges of \pm 2.4 and \pm 2.5 for muons and electrons respectively. Details are available in [279].

The reconstruction efficiency is good for both for muons and electrons. More than $97 \%$ of the generated muons are correctly reconstructed in the considered range, as well as $90 \%$ of the electrons, with $p_{\mathrm{T}}$ above $20 \mathrm{GeV} / \mathrm{c}$ [279]. An electron is considered isolated if the total uncorrected $E_{\mathrm{T}}$ of the jets within a cone $\Delta R \leqslant 0.3$, minus the lepton $E_{\mathrm{T}}$, is less than $30 \%$ of the lepton $E_{\mathrm{T}}$. In a similar way a muon is considered isolated, if the sum of the $p_{\mathrm{T}}$ of all the tracks present in a cone of $\Delta R \leqslant 0.3$ minus $p_{\mathrm{T}}$ of the muon is less than $2 \mathrm{GeV} / \mathrm{c}$. Candidate events must have $E_{\mathrm{T}}^{\text {miss }}>40 \mathrm{GeV}$. The analysis requires at least two jets with uncorrected $E_{\mathrm{T}}>20 \mathrm{GeV}$ detected within $|\eta|<2.5$, where a jet is defined as a fixed-cone cluster with a cone size of $R=0.5$. Jets produced by electrons are discarded before applying the previous selection by removing those which have an electromagnetic supercluster within $\Delta R=0.2$ with a ratio between the electromagnetic energy of that supercluster and the uncorrected jet energy above 0.75 .

$b$-tagging techniques based on the explicit reconstruction of a secondary vertex in a jet [157] are used to further suppress backgrounds in which no jets from b-quarks are present. The dominant backgrounds to dilepton $t \bar{t}$ events are those which have real leptons, real $E_{\mathrm{T}}^{\text {miss }}$ and jets originating from initial or final state radiation, arising mainly from dibosons ( $W W$, $W Z$, and $Z Z)+$ jets production, and also from top quark decays, either from the semi-leptonic channel or from tau decays producing leptons. This kind of backgrounds are expected to be determined using MC simulation. Instrumental backgrounds, are characterised in general by their large cross sections but not having real $E_{\mathrm{T}}^{\text {miss }}$, among them are: $Z+$ jets, DrellYan $\left(Z / \gamma^{\star} \rightarrow \ell^{+} \ell^{-}\right)$production, "fake" leptons in $W \rightarrow \ell \nu+$ jet events where a jet is falsely reconstructed as a lepton candidate. In principle it is harder to estimate their contribution to the final sample using MC simulation.

After this selection an efficiency close to $5 \%$ is obtained, with a very high rejection of all the backgrounds considered at the level of $10^{-3}: 1$ or better, as shown in Table 8.1. A $S / B$ value of 5.5 is obtained, the main background being the one arising from the dilepton channel itself in which at least one of the $W$ decays into $\tau \nu_{\tau}$ and with a subsequent leptonic tau decay.

Different sources of systematic uncertainties have been identified that affect event selection and background determination and thus the cross section measurement. Detailed studies [279] of these sources have been done based mainly on the results of the studies performed in [7] and [201]. Among the most important experimental sources are uncertainties on the jet energy scale and the b-tag efficiency. The impact of theoretical and phenomenological uncertainties such as those on hadron fragmentation and PDF have been studied using samples generated with different PYTHIA parameters and simulated and reconstructed with the CMS fast simulation and reconstruction program. The uncertainty in the cross section coming from the luminosity estimation was taken as $3 \%$ as expected for $10 \mathrm{fb}^{-1}$ integrated luminosity. As the non- $t \bar{t}$ background is small it does not contribute significantly to the uncertainty. The results are summarised in Table 8.2 and lead to an estimated total error on the $t \bar{t}$ cross section measured in the dileptonic channel using electrons and muons of $\Delta \sigma_{t \bar{t}} / \sigma_{t \bar{t}}=11 \%$ (syst) $\pm 0.9 \%$ (stat) $\pm 3 \%$ (luminosity). 
Table 8.1. Cumulative effect of the different selection criteria applied to the simulated $t \bar{t}$ dilepton sample (electrons and muons) and simulated backgrounds. The column denoted as $\tau$ corresponds to $t \bar{t}$ dilepton sample in which at least one $W$ decays into a $\tau$ lepton. The numbers correspond to LO accepted cross sections in $\mathrm{pb}$.

\begin{tabular}{lcclllll}
\hline & Signal & $\tau$ & $W W$ & $W Z$ & $Z Z$ & $Z+$ jets & other $t \bar{t}$ \\
\hline Before selection & 24.3 & 30.4 & 7.74 & 0.89 & 0.11 & 3912 & 438 \\
Level-1 + HLT & 19.4 & 15.1 & 4.4 & 0.37 & 0.07 & 657 & 92 \\
2 jets $E_{\mathrm{T}}>20 \mathrm{GeV}$ & 11.5 & 9.8 & 0.6 & 0.012 & 0.006 & 23.9 & 73.1 \\
$E_{\mathrm{T}}^{\text {miss }}>40 \mathrm{GeV}$ & 9.6 & 8.1 & 0.5 & 0.01 & 0.003 & 5.8 & 53.6 \\
Two opp. charged leptons & 3.2 & 0.42 & 0.04 & 0.001 & 0.001 & 1.17 & 0.12 \\
b-tag of two highest $E_{\mathrm{T}}$ jets & 1.12 & 0.15 & 0.002 & $\sim 10^{-4}$ & $\sim 10^{-5}$ & $<0.01$ & 0.05 \\
\hline
\end{tabular}

Table 8.2. Uncertainties in the $t \bar{t}$ dilepton cross section determination for $10 \mathrm{fb}^{-1}$.

\begin{tabular}{lc}
\hline Effect & $\Delta \sigma_{t \bar{t} \text { dil } e / \mu} / \sigma_{t \bar{t} \text { dil } e / \mu}$ \\
\hline Jet Energy Scale & $3.6 \%$ \\
b-tag efficiency & $3.8 \%$ \\
Lepton reconstruction & $1.6 \%$ \\
$E_{\mathrm{T}}^{\text {miss }}$ & $1.1 \%$ \\
ISR and FSR & $2.5 \%$ \\
Pile-Up & $3.6 \%$ \\
Underlying Event & $4.1 \%$ \\
Heavy quark fragmentation & $5.1 \%$ \\
PDF uncertainties & $5.2 \%$ \\
Statistical uncertainty & $0.9 \%$ \\
Integrated luminosity & $3 \%$ \\
\hline
\end{tabular}

8.1.2.3. Top decays to tau leptons. In this section studies performed to select events with $\tau$ leptons in the final state are presented. We consider here dileptonic $t \bar{t}$ decays with one tau lepton decaying into hadrons in the final state $t \bar{t} \rightarrow b \bar{b} \tau v_{\tau} \ell v_{\ell},(\ell=\mathrm{e}, \mu)$. The measurement of the ratio $B R(t \bar{t} \rightarrow \ell \tau+X) / B R(t \bar{t} \rightarrow \ell \ell+X)$ will allow to set new limits on the presence of non-standard physics in top decays. Furthermore, this channel is a source of background for Supersymmetry and Higgs searches, as well as for the other dileptonic top channels.

Tau candidates are selected and identified following the method of the MSSM Higgs and HLT analyses [280], adapting the different selection criteria to the momentum range in which tau candidates are expected to be produced in top decays [279]. The hadronic tau identification efficiency obtained in the dilepton samples is about $30 \%$ using this method as can be seen in Fig. 8.2.

Event selection proceeds in a similar way as in Section 8.1.2.2 but only one isolated lepton (electron or muon) is allowed. One isolated tau candidate separated from the isolated lepton has to be present, and the isolated lepton and the tau candidate must have opposite charges. The effect of these selections are described in detail for the $t \bar{t}$ sample in Table 8.3. $b$-tag for the two accompanying jets is also required. An efficiency close to $2 \%$ is obtained, with a very high rejection of all the backgrounds considered. A $S / B$ value close to 1 is obtained, the main background being the one arising from the $t \bar{t}$ semi-leptonic channel. The majority of the systematic uncertainties are described in Section 8.1.2.2. There is another systematic uncertainty intrinsic to this analysis due to the $\tau$ reconstruction and identification. Based on preliminary studies, we assigned a $12 \%$ uncertainty to the $\tau$ reconstruction and identification. Statistical uncertainty in the cross section determination is about $1.3 \%$ for an 

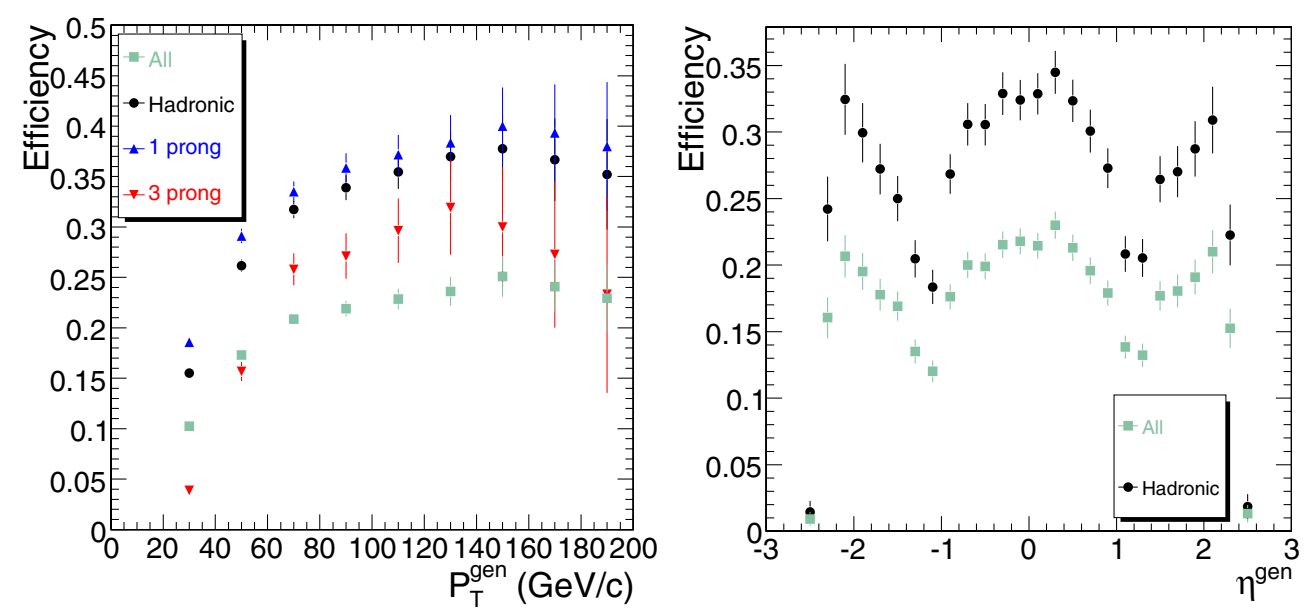

Figure 8.2. Reconstruction efficiency of tau candidates as a function of $p_{\mathrm{T}}$ and $\eta$. Errors are statistical only.

Table 8.3. Cumulative effect of the different selection criteria applied to the simulated $t \bar{t}$ sample. Numbers correspond to LO accepted cross sections.

\begin{tabular}{lcccc}
\hline Cut & \multicolumn{4}{c}{ Efficiency times cross sections (pb) } \\
\cline { 2 - 5 } & $t \bar{t}$ (signal) & $t \bar{t}$ (other dilepton) & $t \bar{t}$ (semi-leptonic) & $t \bar{t}$ (hadronic) \\
\hline Before selection & 15.62 & 38.94 & 218.88 & 218.88 \\
Trigger & 8.61 & 25.40 & 85.90 & 2.08 \\
2 jets & 6.97 & 18.90 & 80.08 & 2.04 \\
$\geqslant 1$ Iso lepton & 4.27 & 13.11 & 34.93 & 0.11 \\
$E_{\mathrm{T}}^{\text {miss }} \geqslant 40 \mathrm{GeV}$ & 3.58 & 10.89 & 26.41 & 0.05 \\
1 lepton & 3.48 & 6.73 & 25.24 & 0.04 \\
$\tau$ cand. with opp. Q & 0.75 & 0.20 & 0.75 & 0.001 \\
$b$-tagging & 0.29 & 0.07 & 0.30 & 0.0005 \\
\hline
\end{tabular}

integrated luminosity of $10 \mathrm{fb}^{-1}$. Then the relative uncertainty in the estimation of the cross

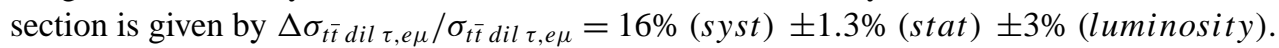

\subsubsection{Semi-leptonic channel}

The semi-leptonic $t \bar{t}$ decay has a final state topology of four hadronic jets of which two originate from a b-quark, an isolated lepton and missing transverse momentum. In this section, we consider the measurement of the cross section of the semi-leptonic $t \bar{t}$ production where the lepton is a muon [281].

Both the Level-1 and the High-Level Trigger selection criteria are applied on the simulated events, resulting in the efficiencies shown in Table 8.4. The single-muon trigger stream was used. The jets are reconstructed from the combined electromagnetic and hadronic calorimeter energy deposits and clustered with the Iterative Cone algorithm using an opening angle of $\Delta R=0.5$. A transverse energy threshold of $0.5 \mathrm{GeV}$ is applied on the input objects 
Table 8.4. Overview of the selection criteria applied. The expected $S / B$ values take into account the respective Leading-Order cross-sections of the processes.

\begin{tabular}{lcccccc}
\hline & Semi-lept. & Other & & & & \\
& $t \bar{t}$ & $t \bar{t}$ & $\mathrm{~W}+4 \mathrm{j}$ & $\mathrm{Wbb}+2 \mathrm{j}$ & $\mathrm{Wbb}+3 \mathrm{j}$ & $\mathrm{S} / \mathrm{B}$ \\
\hline Before selection & $365 \mathrm{k}$ & $1962 \mathrm{k}$ & $82.5 \mathrm{k}$ & $109.5 \mathrm{k}$ & $22.5 \mathrm{k}$ & 5.9 \\
L1 + HLTTrigger & $62.2 \%$ & $5.30 \%$ & $24.1 \%$ & $8.35 \%$ & $8.29 \%$ & 7.8 \\
Four jets $E_{\mathrm{T}}>30 \mathrm{GeV}$ & $25.4 \%$ & $1.01 \%$ & $4.1 \%$ & $1.48 \%$ & $3.37 \%$ & 9.9 \\
$p_{\mathrm{T}}^{\text {lepton }}>20 \mathrm{GeV} / \mathrm{c}$ & $24.8 \%$ & $0.97 \%$ & $3.9 \%$ & $1.41 \%$ & $3.14 \%$ & 10.3 \\
b-tag criteria & $6.5 \%$ & $0.24 \%$ & $0.064 \%$ & $0.52 \%$ & $0.79 \%$ & 25.4 \\
Kinematic fit & $6.3 \%$ & $0.23 \%$ & $0.059 \%$ & $0.48 \%$ & $0.72 \%$ & 26.7 \\
Selected cross section $(\mathrm{pb})$ & 5.21 & 1.10 & 0.10 & 0.08 & 0.05 & 26.7 \\
Scaled $\mathcal{L}=1 \mathrm{fb}^{-1}$ & 5211 & 1084 & 104 & 82 & 50 & 26.7 \\
\hline
\end{tabular}

before clustering. Optimisation of the parameter settings of the clustering algorithms are considered in [282]. Only the jets in the vicinity of the primary vertex are considered in the analyses, rejecting in general those jets with a small transverse momentum. The energy scale of the reconstructed jets is calibrated using the methods described in [283]. Among the list of muon candidates identified flavour, the muon originating directly from the $W$ boson decay is selected following the procedure described in [284]. The transverse momentum components of the unobserved neutrino are estimated via the missing transverse momentum which balances the vectorial sum of the energy deposits in the calorimeter above the transverse energy threshold mentioned.

The event selection consists of a series of sequential cuts on kinematic or topological variables. The event is required to have at least four jets after applying the primary vertex constraint with a calibrated transverse energy, $E_{\mathrm{T}}$, exceeding $30 \mathrm{GeV}$ and within a pseudorapidity in the range of the tracker, $|\eta|<2.4$. If more than four jets match this criterion, the four leading jets are selected as those with the highest $E_{\mathrm{T}}$. Of these four jets, two have to be $b$-tagged according to the method applying a combined $b$-tag variable described in [281, $285,286]$. The selected lepton is required to be within the tracker acceptance and to have a transverse momentum larger than $20 \mathrm{GeV} / \mathrm{c}$.

After classifying two of the four reconstructed jets as b-quark and the other two as light quark jets, only two jet combinations remain to reconstruct the hadronically-decaying top. A kinematic fit [167] was applied on the reconstructed event for both jet combinations forcing the reconstructed $W$ boson mass to its precisely known value. Before applying the kinematic fit the energy scale of the light quark jets is corrected for an overall bias in the reconstructed $W$ boson mass. Following the method described in [287] after the event selection mentioned above, an inclusive jet energy scale correction of $-9.7 \%$ was obtained and applied to light quark jet candidates. The event is finally selected if the fit converged for at least one of the combinations.

The selection efficiency for the signal events is estimated to be $6.28 \pm 0.04 \%$. The fraction of $t \bar{t}$ signal events in the selected sample of inclusive $t \bar{t}$ decays is estimated to be $82.8 \pm 0.2 \%$. The signal-to-background ratio after the event selection is 26.7 , where all $t \bar{t}$ decay channels are considered as signal. Hence the systematic effect of the background contribution is minor. It is shown in [281] that after the event selection topological observables will not help much in differentiating between signal and background. The cross section is therefore estimated from counting events. The statistical uncertainty on the estimated cross section is $1.2 \%, 0.6 \%$ and $0.4 \%$ for integrated luminosities of $1 \mathrm{fb}^{-1}, 5 \mathrm{fb}^{-1}$ and $10 \mathrm{fb}^{-1}$, respectively. 
Table 8.5. Overview of the systematic uncertainties on the cross section.

\begin{tabular}{lccc}
\hline & \multicolumn{3}{c}{$\Delta \hat{\sigma}_{t \bar{t}(\mu)} / \hat{\sigma}_{t \bar{t}(\mu)}$} \\
\cline { 2 - 4 } & $1 \mathrm{fb}^{-1}$ & $5 \mathrm{fb}^{-1}$ & $10 \mathrm{fb}^{-1}$ \\
\hline Simulation samples $\left(\varepsilon_{\text {sim }}\right)$ & & $0.6 \%$ & \\
Simulation samples $\left(F_{\text {sim }}\right)$ & & $0.2 \%$ & \\
Pile-Up (30\% On-Off) & $3.2 \%$ & \\
Underlying Event & $0.8 \%$ & \\
Jet Energy Scale (light quarks) $(2 \%)$ & $1.6 \%$ & \\
Jet Energy Scale (heavy quarks) $(2 \%)$ & & $1.6 \%$ & \\
Radiation $\left(\Lambda_{Q C D}, Q_{0}^{2}\right)$ & & $2.6 \%$ & \\
Fragmentation (Lund b, $\left.\sigma_{q}\right)$ & & $1.0 \%$ & \\
b-tagging $(5 \%)$ & & $7.0 \%$ & \\
Parton Density Functions & $3.4 \%$ & \\
Background level & & $0.9 \%$ & \\
Integrated luminosity & $5 \%$ & \\
Statistical Uncertainty & & $5.4 \%$ \\
Total Systematic Uncertainty & $10 \%$ & $0.6 \%$ & $9.7 \%$ \\
Total Uncertainty & $1.2 \%$ & $10.5 \%$ & \\
\hline
\end{tabular}

Systematic effects are introduced only on the signal events, changing the efficiency of the event selection. Similar effects on the background samples should be a second order effect on the inferred cross section. For the theoretical or phenomenological uncertainties the prescription of [201] was used as described in [281]. The list of systematic uncertainties is shown in Table 8.5. The dominant systematic effects are b-tagging, and in the early stage the uncertainty on the integrated luminosity. For an extended discussion on the studied systematic effects we refer to [281]. As a consequence of the kinematic fit, the uncertainty on both the light- and heavy-quark jet energy scale results in a limited systematic uncertainty, of about $1.6 \%$.

The total relative systematic uncertainty on the cross section is $10.5 \%$ which can be compared to a relative statistical uncertainty of $0.6 \%$ at $5 \mathrm{fb}^{-1}$. The total uncertainty of $10.5 \%$ scales with the integrated luminosity as shown in Fig. 8.3. In this plot it is assumed that the uncertainty on the determination of the integrated luminosity scale as the inverse square root of the integrated luminosity. At an integrated luminosity of about $5 \mathrm{fb}^{-1}$ the total uncertainty is dominated by the uncertainty on the $b$-tagging performance. For the uncertainty on the $b$-tagging efficiency a conservative $5 \%$ is taken according to [286] although the Tevatron experience shows that a value of $2 \%$ can be reached [288, 289].

\subsubsection{Fully hadronic channel}

The fully hadronic final state, characterised by a six-jets topology $t \bar{t} \rightarrow W W b \bar{b} \rightarrow q q q q b \bar{b}$, has the largest branching fraction $(46 \%)$, and kinematics that can be fully reconstructed. However, this channel is affected by a large background from QCD multi-jet production, which makes the isolation of the signal rather challenging, and internal jet-parton permutation uncertainties. Improvements in the signal-to-background ratio are possible by requiring the presence of $b$-quark jets and by selecting central and very high-energy kinematic configurations which are expected for jets arising from the decay of a massive object like the top quark. A specific multi-jet trigger which uses $b$-tagging information has been devised for this analysis and an optimised selection has been applied. The analysis is described in detail in [279]. 


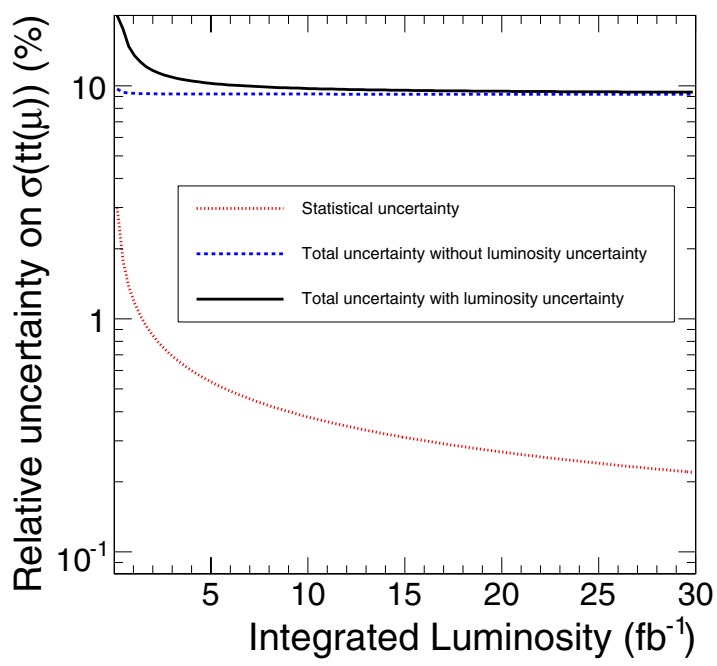

Figure 8.3. Statistical and total uncertainty on the inferred cross section of the process $p p \rightarrow$ $t \bar{t} \rightarrow b q \bar{q} b \mu v_{\mu}$ as a function of the integrated luminosity.

The signal sample consists of 500000 inclusive $t \bar{t}$ events, from which a sub-sample of 230000 fully hadronic $t \bar{t}$ events is extracted. The background consists of 1.5 million multijet events $(\mathrm{QCD})$ generated with $50<\hat{p_{\mathrm{T}}}<470 \mathrm{GeV} / \mathrm{c}$, where the $\hat{p_{\mathrm{T}}}$ symbol indicates the transverse momentum of the most energetic parton of the hard scattering before the final-state radiation processes.

8.1.4.1. Trigger pre-selection and event selection. The trigger pre-selection uses the inclusive jet trigger envisaged in [76] and a special inclusive $b$-jet trigger [290]. The inclusive $b$-jet trigger combines in the first stage the $b$-tagging requirement with an inclusive jet trigger which applies tuned $E_{\mathrm{T}}$ thresholds of $350 \mathrm{GeV}$ for single jets, $150 \mathrm{GeV}$ for 3 -jet and $55 \mathrm{GeV}$ for 4 -jet topologies; then a $b$-tagging based on pixel and regional track and vertex reconstruction is performed on the two most energetic jets. The trigger requires either multiple jets in the event or a $b$-tagged jet among the two highest- $E_{\mathrm{T}}$ jets. After the trigger pre-selection the QCD rate is reduced to $23 \mathrm{~Hz}$, the signal efficiency is $16.8 \%$ and the signal to background ratio, $S / B$, amounts to $1 / 300$.

The selection is designed to optimise the statistical significance $S / \sqrt{S+B}$ for an integrated luminosity of $\mathcal{L}=1 \mathrm{fb}^{-1}$. The first step of the selection requires a topology of $6 \leqslant N_{\text {jet }} \leqslant 8$. For a jet to be counted, the jet pseudorapidity must satisfy $|\eta|<2.4$ and its transverse energy must be greater than $30 \mathrm{GeV}$. Event shape variables, potentially able to separate the signal from the background are then taken into account. The useful ones are centrality, aplanarity and non-leading jet total transverse energy obtained removing the two most energetic jets $\left(\sum_{3} E_{\mathrm{T}}\right)$ of which distributions are shown in Fig. 8.4. After the selection $b$-tagging is applied to the surviving samples of $t \bar{t}$ fully hadronic and QCD events. Selection criteria of at least one $b$-jet and two $b$-jets are considered.

Table 8.6 summarises the selection applied in cascade. The signal-to-background ratio amounts to $1 / 17$ and $1 / 9$ for the 1 and $2 b$-tag samples,respectively, and resulting in signal efficiencies of $3.8 \%$ and $2.7 \%$.

The signal efficiency relative to the total inclusive $t \bar{t}$ sample, to be used in the calculation of the total $t \bar{t}$ production cross section, becomes $2.3 \%$ (1.6\%), respectively for the 1 (2) b-tag requirement. The estimated statistical uncertainty on the cross section is reported in Table 8.7. 

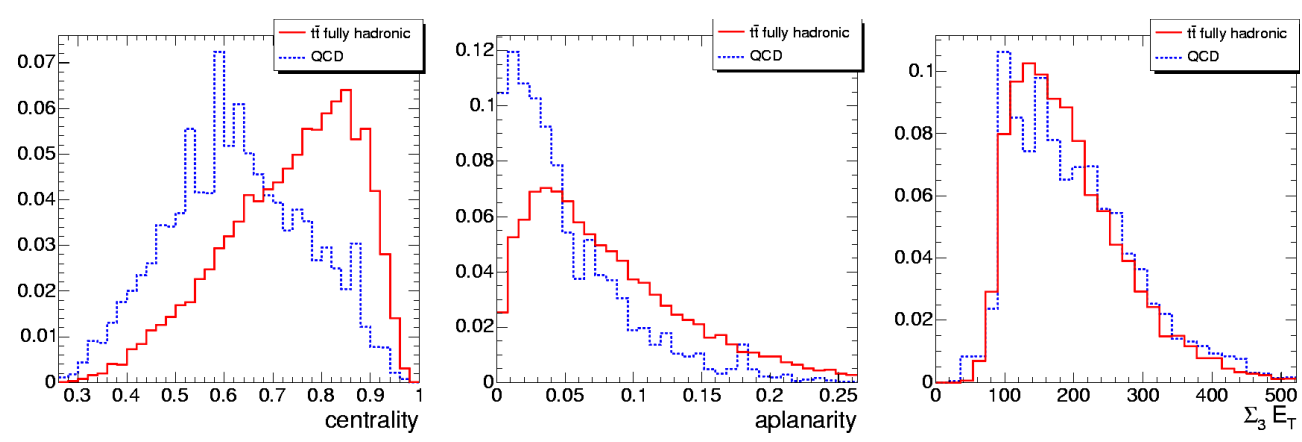

Figure 8.4. Distributions of centrality, aplanarity and $\sum_{3} E_{\mathrm{T}}$ for $t \bar{t}$ and QCD events (normalised to the same area).

Table 8.6. $t \bar{t}$ fully hadronic and QCD effective cross sections, signal-to-background ratio, statistical significance for $1 \mathrm{fb}^{-1}$ and signal efficiency at each step of the selection.

\begin{tabular}{llccccc}
\hline Selection & Requirement & $\sigma \varepsilon[\mathrm{pb}]$ & $\sigma \varepsilon_{\mathrm{QCD}}[\mathrm{pb}]$ & \multicolumn{2}{c}{$S / B$} & \multicolumn{2}{c}{$S / \sqrt{S+B} \varepsilon(\%)$} \\
\hline Before Selection (PYTHIA LO) & & 225 & $25 \mathrm{M}$ & $1 / 10^{5}$ & 0.04 & 100 \\
Trigger & HLT multi-jet $+b$-jet & 38 & 11600 & $1 / 300$ & 11.1 & 16.8 \\
Event & $6 \leqslant N_{\text {jet }} \leqslant 8$ & 35 & 7900 & $1 / 225$ & 12.4 & 15.5 \\
& $E_{\mathrm{T}} \geqslant 30 \mathrm{GeV}$ & 15 & 930 & $1 / 60$ & 15.4 & 6.6 \\
& centrality $\geqslant 0.68$ & 9.9 & 324 & $1 / 33$ & 17.1 & 4.4 \\
& aplanarity $\geqslant 0.024$ & 9.0 & 251 & $1 / 28$ & 17.7 & 4.0 \\
& $\sum_{3} E_{\mathrm{T}} \geqslant 148 \mathrm{GeV}$ & 9.0 & 229 & $1 / 25$ & 18.4 & 4.0 \\
b-tagging & 1 b-tag & 8.6 & 148 & $1 / 17$ & 21.7 & 3.8 \\
& 2 b-tag & 6.0 & 54 & $1 / 9$ & 24.1 & 2.7 \\
\hline
\end{tabular}

Table 8.7. Number of $t \bar{t}$ and QCD events, $t \bar{t}$ efficiency, absolute and relative statistical uncertainties expected on the cross section measurement for an integrated luminosity of $1 \mathrm{fb}^{-1}$.

\begin{tabular}{lrrrcc}
\hline Requirement & \multicolumn{5}{c}{$\mathcal{L}=1 \mathrm{fb}^{-1}$} \\
\cline { 2 - 6 } & $t \bar{t}$ events & QCD events & $\varepsilon(\%)$ & $(\Delta \sigma)_{\text {stat }}[\mathrm{pb}]$ & $(\Delta \sigma / \sigma)_{\text {stat }}(\%)$ \\
\hline 1 b-tag & 11500 & 148000 & 2.3 & 17 & 3.5 \\
2 b-tag & 8000 & 54000 & 1.6 & 15 & 3.0 \\
\hline
\end{tabular}

Sources of systematic uncertainty are studied as described in detail in [201] and [7]. From the experience of CDF and DØ experiments at Tevatron [291], one of the dominating systematic uncertainties arises from jet energy scale. The systematic uncertainty related with the trigger selection is calculated considering contributions from b-tagging and jet energy scale. Table 8.8 summarises the contributions to the total uncertainty on the cross section, which combined lead to a relative uncertainty of $\Delta \sigma / \sigma=3 \%($ stat $)+20 \%($ syst $)+$ $5 \%$ (luminosity).

8.1.4.2. Event selection based on neural net. A more refined selection is based on a neural net exploiting the same variables considered so far. Such approach is attempted in order to investigate the possibility of improving the $S / B$ ratio and/or the efficiency. The previous 
Table 8.8. Contributions to the systematic uncertainty on the $t \bar{t}$ cross section measurement in the fully hadronic channel (cut based approach).

\begin{tabular}{lc}
\hline & $\Delta \sigma / \sigma(\%)$ \\
\hline HLT & 5.9 \\
Pile Up & 10.0 \\
Underlying Event & 4.1 \\
Fragmentation & 1.9 \\
PDF & 4.2 \\
IS/FS Radiation & 7.9 \\
Jet Energy Scale & 11.2 \\
b-tagging & 2.0 \\
Background & 5.0 \\
Integrated Luminosity & 5.0 \\
\hline
\end{tabular}
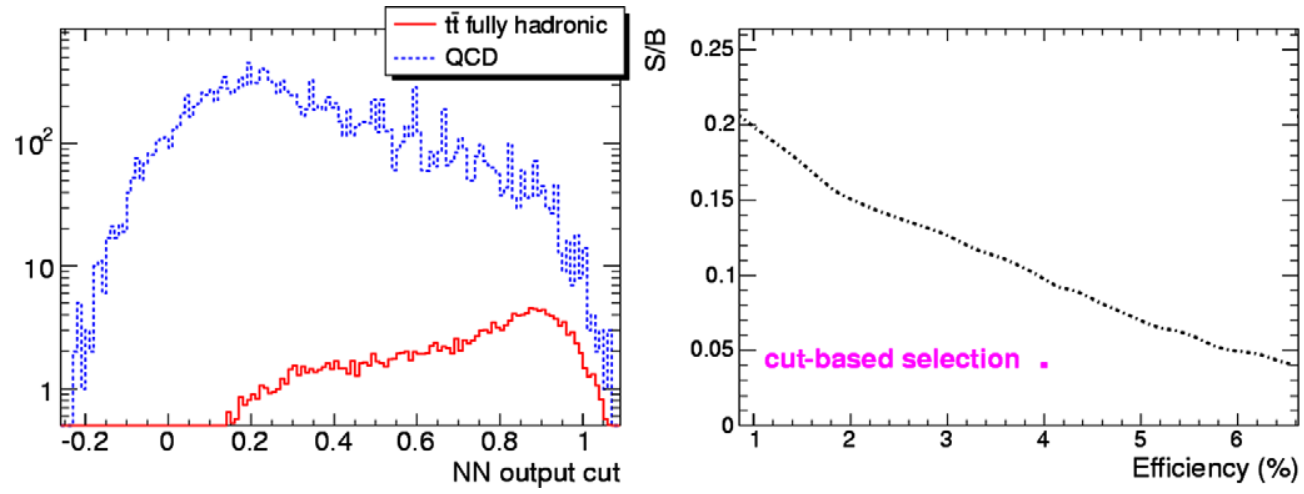

Figure 8.5. Left: distribution of the neural net output for $t \bar{t}$ and QCD. Right: signal-to-background ratio as function of the signal efficiency. For comparison the result of the cut-based selection is also shown.

selection, called "cut-based", could represent a more conservative approach for the first LHC analyses.

The most effective neural network configuration studied is applied to the $t \bar{t}$ and QCD events satisfying the topology request of $6 \leqslant N_{\text {jet }} \leqslant 8$ (jet pseudorapidity $|\eta|<2.4$ ) after a cut on jet transverse energy of $E_{\mathrm{T}}>25 \mathrm{GeV}$ and consists of 6 input nodes: $E_{\mathrm{T}}$ of the first and sixth jet with the jets ordered in increasing $E_{\mathrm{T}}$, centrality, aplanarity, $\sum_{3} E_{\mathrm{T}}$ and sphericity. The performance of the neural net is shown in Fig. 8.5 which compares the output distributions for signal and QCD background. The $S / B$ ratio as a function of the $t \bar{t}$ efficiency is also shown. With respect to the cut-based selection, the request for a neural net output $\geqslant 0.77$ improves the $S / B$ ratio from $1 / 25$ to $1 / 10$ with same efficiency of about $4 \%$.

As done after the cut-based selection, a $b$-tagging is applied to the surviving samples of $t \bar{t}$ fully hadronic and QCD events, and selection criteria of at least one $b$-jet and two $b$-jets are considered. Improved signal-to-background ratio, amounting to $1 / 7(1 / 3)$ respectively for 1 (2) b-tag samples, can be achieved using the neural net keeping the same signal efficiencies of 3.8\% (2.7\%). This means an estimated relative statistical uncertainty on the cross section of $2.3 \%(2.0 \%)$, with the same expected number of $t \bar{t}$ events for an integrated luminosity of $\mathcal{L}=1 \mathrm{fb}^{-1}$. 


\subsection{Measurement of the top quark mass}

\subsubsection{Dileptonic events}

The dilepton channel benefits of a clean signature and a large signal-to-background ratio even though the presence of two neutrinos prevents a direct reconstruction of the top-quark mass. However, the event kinematic retains a large sensitivity to the top mass which can be exploited in various ways. The method presented here is discussed in more detail in [279].

The six unmeasured kinematic quantities corresponding to the momentum components of the two neutrinos are reduced by assuming momentum balance in the transverse plane, by imposing the $m_{W}$ constraint and by requiring both top-quark masses to be equal. The event kinematics can then be written as a fourth order polynomial with the top mass as a parameter. For each candidate event we step through top mass values in the range $100 \mathrm{GeV} / \mathrm{c}^{2} \leqslant m_{t} \leqslant$ $300 \mathrm{GeV} / \mathrm{c}^{2}$ in $1 \mathrm{GeV} / \mathrm{c}^{2}$ steps and weight the kinematic solutions, including their fourfold ambiguity, with the Standard Model expectations of the neutrino momentum spectrum. For each event the most likely solution, i.e. the solution with the highest weight, is retained. The mass distribution of these most likely solutions is shown in Fig. 8.1 for $1 \mathrm{fb}^{-1}$. The figure shows a clear mass peak at the expected value for the fully-simulated and reconstructed events. A Gaussian fit to the signal in a range corresponding to $40 \%$ of the maximum yields $m_{t}=$ $178.5 \pm 1.5 \mathrm{GeV} / \mathrm{c}^{2}$ for an input top mass of $175 \mathrm{GeV} / \mathrm{c}^{2}$, where the uncertainty is statistical. With $10 \mathrm{fb}^{-1}$ the statistical uncertainty will be reduced to $0.5 \mathrm{GeV} / \mathrm{c}^{2}$. The background is small and essentially flat and does not affect the mass determination significantly.

The main systematic effects are due to the assumptions used to reduce the complexity of the kinematic equation system and to detector effects. The dominating systematic effect in the first category is the uncertainty on the initial and final-state radiation which changes the amount of transverse momentum of the $t \bar{t}$-system and the kinematic constraints. This results in an uncertainty on the top mass of $\Delta m_{t}=0.3 \mathrm{GeV} / \mathrm{c}^{2}$ [201]. The zero width approximation for both the $W$ bosons and the top quarks in the equation system gives rise to another shift of about $0.1 \mathrm{GeV} / \mathrm{c}^{2}$.

The expected uncertainty on the jet energy scale for the early data amounts to $15 \%$, independent of the jet $p_{\mathrm{T}}$, which corresponds to an uncertainty of $\Delta m_{t}=4.2 \mathrm{GeV} / \mathrm{c}^{2}$ for the first $1 \mathrm{fb}^{-1}$ of integrated luminosity. This uncertainty is reduced to $2.9 \mathrm{GeV} / \mathrm{c}^{2}$ with an improved calibration in $1-10 \mathrm{fb}^{-1}$ based on photons and jets, especially jets from $W$-boson decays in semi-leptonic and fully-hadronic $t \bar{t}$ events. Further improvement in the knowledge of the jet energy scale after $10 \mathrm{fb}^{-1}$ are expected to reduce this uncertainty to about $1 \mathrm{GeV} / \mathrm{c}^{2}$.

In conclusion, the kinematic reconstruction of the dilepton channel will allow an early measurement of the top-quark mass. Assuming that the goal for a precise jet energy scale determination for $b$-quarks can be achieved the expected precision on the top mass in this channel with $10 \mathrm{fb}^{-1}$ is $\Delta m_{t}=0.5 \mathrm{GeV} / \mathrm{c}^{2}$ (stat) $\pm 1.1 \mathrm{GeV} / \mathrm{c}^{2}$ (sys).

\subsubsection{Semi-leptonic events}

The semi-leptonic $t \bar{t}$ decay is traditionally called the golden channel for measuring the topquark mass. A measurement based on advanced analysis tools is described in detail in [292]. The event reconstruction and initial event selection follows the one of Section 8.1.3. For the event to be selected, exactly two out of the four leading jets are $b$-tagged and the other two need to be anti- $b$-tagged. The four leading jets should not overlap in order to reduce ambiguities in the jet energy scale calibration procedure. The efficiency of each sequential cut is shown in Table 8.9. 
Table 8.9. Overview of the selection criteria applied after the lepton cut $p_{\mathrm{T}}^{\text {lepton }}>20 \mathrm{GeV} / \mathrm{c}$ in Table 8.4.

\begin{tabular}{lllllll}
\hline & Signal & Other $t \bar{t}$ & $\mathrm{~W}+4 \mathrm{j}$ & $\mathrm{Wbb}+2 \mathrm{j}$ & $\mathrm{Wbb}+3 \mathrm{j}$ & $S / B$ \\
\hline Before selection & $365 \mathrm{k}$ & $1962 \mathrm{k}$ & $82.5 \mathrm{k}$ & $109.5 \mathrm{k}$ & $22.5 \mathrm{k}$ & 0.032 \\
b-tag criteria & $5.5 \%$ & $0.21 \%$ & $0.052 \%$ & $0.47 \%$ & $0.70 \%$ & 3.73 \\
No jet overlap & $3.0 \%$ & $0.11 \%$ & $0.027 \%$ & $0.25 \%$ & $0.44 \%$ & 3.87 \\
$P_{\chi^{2}}$-cut 20\% & $1.4 \%$ & $0.039 \%$ & 0.0097 & 0.061 & 0.07 & 5.3 \\
$P_{\text {sign }}$-cut $80 \%$ & $1.2 \%$ & $0.025 \%$ & 0.0085 & 0.052 & 0.05 & 6.8 \\
$P_{\text {comb }}$-cut 50\% & $0.7 \%$ & $0.013 \%$ & 0.0036 & 0.013 & 0. & 8.2 \\
Scaled $\mathcal{L}=1 \mathrm{fb}^{-1}$ & 588 & 64 & 6 & 2 & 0 & 8.2 \\
\hline
\end{tabular}

The amount of events produced via a different $t \bar{t}$ decay channel in the selected event sample is reduced by a likelihood-ratio method combining three kinematic observables resulting in a variable $L_{\text {sign }}$ which is transformed into a probability $P_{\text {sign }}$ for the selected event to be a semi-leptonic muon $t \bar{t}$ event. An extra sequential cut is applied by requiring this probability $P_{\text {sign }}$ to exceed $80 \%$.

Among the four reconstructed jets, three have to be chosen to form the hadronic decaying top quark. The efficiency and purity of this selection was significantly enhanced by applying a second likelihood ratio method combining the information from several sensitive variables. The jet combination with the largest $L_{\text {comb }}$ value is taken as the best pairing. The $L_{c o m b}$ value is transformed into a probability $P_{\text {comb }}$ for the chosen combination to be the correct one. The event probability $P_{\text {comb }}$ is used in the event selection where events are selected if their value for $P_{\text {comb }}$ exceeds $60 \%$, increasing the purity of the selected jet pairings to $81.6 \%$ in the mass window of $25 \mathrm{GeV} / \mathrm{c}^{2}$ around the expected $m_{t}$ of about $175 \mathrm{GeV} / \mathrm{c}^{2}$.

For each jet combination a kinematic fit was applied as described which imposes the Wboson mass for the hadronically-decaying $\mathrm{W}$ boson in the event [167]. Only jet combinations are considered with a probability of the kinematic fit calculated from its $\chi^{2} / n d f$ exceeding $20 \%$. For some events none of the jet combinations fulfill this criterium, therefore reducing the total event selection efficiency. The fraction of fully hadronic $t \bar{t}$ events selected is negligible (less than 0.05 events expected at $1 \mathrm{fb}^{-1}$ ). From this we conclude that the also influence of QCD produced jet events is minor.

When estimating $m_{t}$ from the selected event sample by a simple Gaussian fit in a range of $20 \mathrm{GeV} / \mathrm{c}^{2}$ in both directions around the modal bin, a value of $176.5 \pm 0.65 \mathrm{GeV} / \mathrm{c}^{2}$ is obtained before applying the kinematic fit and $172.2 \pm 0.48 \mathrm{GeV} / \mathrm{c}^{2}$ after applying the kinematic fit, for an input value of $175 \mathrm{GeV} / \mathrm{c}^{2}$. The errors reflect the statistical precision of the available Monte Carlo signal sample. The top quark mass after the kinematic fit is shown in Fig. 8.6.

Rather than developing $m_{t}$ estimators on samples of events, an event-by-event likelihood approach is used to estimate $m_{t}$ from the fitted kinematics of the three jets of the hadronically decaying top quark. The uncertainty on $m_{t}$ for each event is determined from the covariance matrices of the kinematic fit. This uncertainty can either be assumed Gaussian or the full $m_{t}$ range can be explicitly scanned with the kinematic fit.

To obtain information about the true value of $M_{t}$ we convolute the reconstructed resolution function or ideogram with the theoretical expected probability density function $P\left(m_{t} \mid M_{t}\right)$ in the reconstruction space

$$
\mathcal{L}_{i}\left(M_{t}\right)=\int P\left(\left\{\bar{p}_{j}\right\} \mid m_{t}\right) \cdot P\left(m_{t} \mid M_{t}\right) d m_{t}
$$



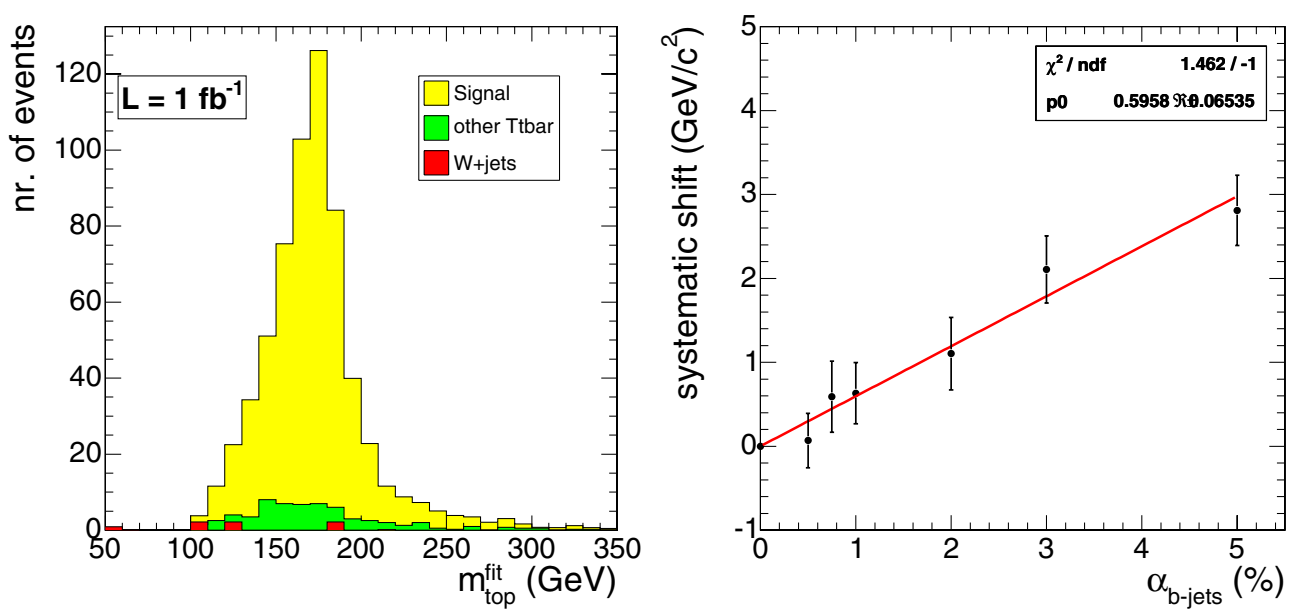

Figure 8.6. Left: Distribution of the mass of the hadronic decaying top quark for the selected events after applying the kinematic fit. Right: Estimated shift in $M_{t}^{\text {FullIdeo }}$ versus a relative shift $\alpha$ applied on the inclusive heavy quark jet energy scale.

where one integrates over the kinematic relevant range of $m_{t}$ to obtain a likelihood function $\mathcal{L}_{i}\left(M_{t}\right)$ for each event $i$. Several contributions are added in the expected density $P\left(m_{t} \mid M_{t}\right)$ : a Breit-Wigner shape for the correct jet combinations $S\left(m_{t} \mid M_{t}\right)$, a parameterised combinatorial background contribution $B_{\text {comb }}\left(m_{t}\right)$ and a parameterised background contribution $B_{\text {proc }}\left(m_{t}\right)$. This results in a function

$$
\begin{aligned}
P\left(m_{t} \mid M_{t}\right)= & P_{\text {sign }} \cdot\left[P_{\text {comb }} \cdot S\left(m_{t} \mid M_{t}\right)\right. \\
& \left.+\left(1-P_{\text {comb }}\right) \cdot B_{\text {comb }}\left(m_{t}\right)\right]+\left(1-P_{\text {sign }}\right) \cdot B_{\text {back }}\left(m_{t}\right)
\end{aligned}
$$

where each contribution is weighted according to the probabilities extracted from the observed event. After combining the likelihoods $\mathcal{L}_{i}\left(M_{t}\right)$ from all selected events, a maximum likelihood method is applied to obtain the best value for the estimator $\hat{M}_{t}$.

The linearity of the estimators have been checked and the slopes are found to be compatible with unity. The width of the pull distribution of the top quark mass estimators $\hat{M}_{t}$ are found to be 0.82 for $\hat{M}_{t}^{\text {fit }}$ (simple fit on reconstructed mass spectrum), 1.04 for $\hat{M}_{t}^{\text {ParIdeo }}$ (convolution with the parameterised ideogram) and 1.02 for $\hat{M}_{t}^{\text {FullIdeo (convolution }}$ with the full scanned ideogram). The resulting top quark mass for the estimator $\hat{M}_{t}^{f i t}$ applied on the simulated events samples with a generated top quark mass of $175 \mathrm{GeV} / \mathrm{c}^{2}$ is $174.16 \pm 0.59 \mathrm{GeV} / \mathrm{c}^{2}$, hence reflecting a bias of $-0.84 \mathrm{GeV} / \mathrm{c}^{2}$. For the convolution method this is $170.65 \pm 0.54 \mathrm{GeV} / \mathrm{c}^{2}$ and $172.42 \pm 0.31 \mathrm{GeV} / \mathrm{c}^{2}$ for respectively the $\hat{M}_{t}^{\text {ParIdeo }}$ and

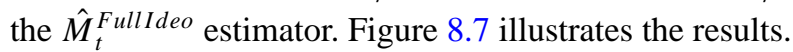

Several systematic effects introduce an uncertainty on the top quark mass estimator. They originate from our understanding of the detector performance, the robustness of the reconstructed objects, for example jets, and the general description of the proton collisions in the simulation. A full description can be found in [292]. The estimation of the systematic uncertainties follows that of the cross section measurement in Section 8.1.3. We conservatively conclude that a total precision on the top quark mass of $1.9 \mathrm{GeV} / \mathrm{c}^{2}$ can be reached with $10 \mathrm{fb}^{-1}$ of data. The uncertainty is dominated by systematic effects like pile-up collisions and the knowledge of the jet energy scale of $b$-quark jets (see Fig. 8.6).

After achieving a better understanding of the accelerator settings and the detector performance, however, the total uncertainty will decrease. Our understanding of the 

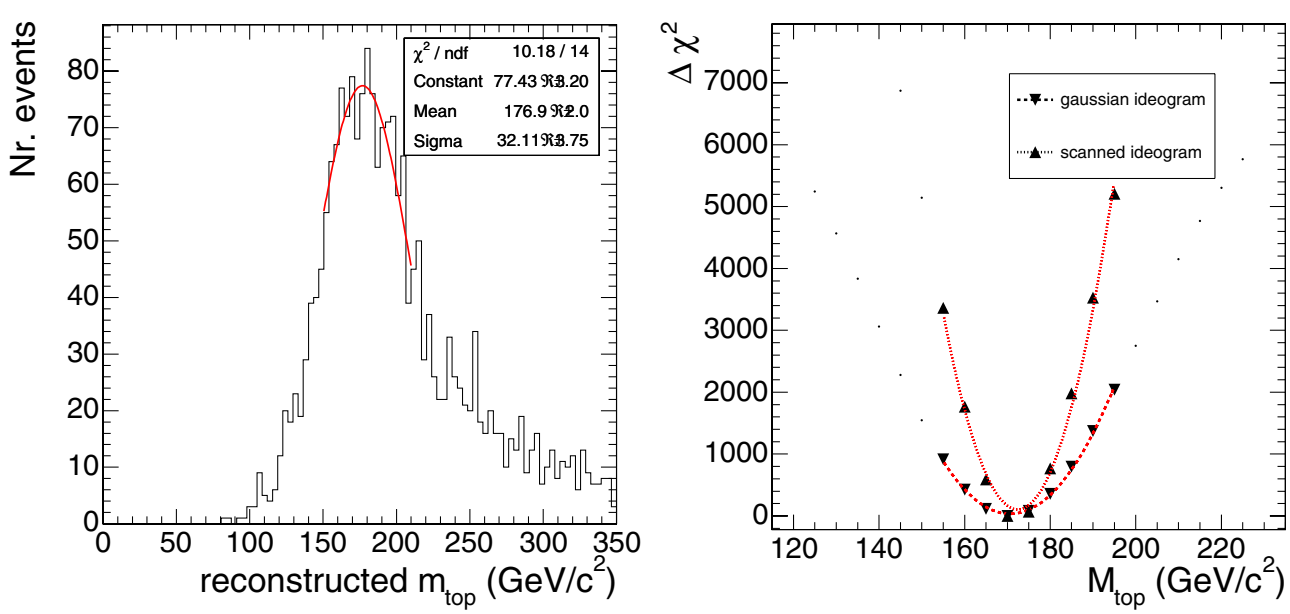

Figure 8.7. Distribution of the mass of the hadronic decaying top quark before the kinematic fit used for the $\hat{M}_{t}^{f i t}$ estimator (left) and the combined $\Delta \chi^{2}\left(M_{t}\right)$ function over all events for both

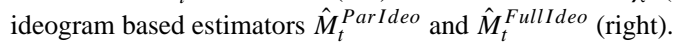

underlying event model will improve in the future significantly when new tuning data become available. The magnitude of pile-up collisions could be monitored to the level of $10 \%$. To take into account the overlap between the pile-up and the jet energy scale uncertainty, the systematic shift due to a $10 \%$ variation in the pile-up collisions is divided by two. The uncertainty on the energy scale of b-quark jets can be extrapolated to about $1.5 \%$ after a better understanding of the detector performance and with the application of advanced tools like energy flow algorithms or selecting jets only in well understood regions in the detector. The measurement of the b-tag efficiency [286] is dominated by systematic uncertainties of radiation effects. The experience at the Tevatron collider [288, 289] illustrates that an uncertainty of $2 \%$ could be reached.

Table 8.10 summarises and combines the extrapolated systematic uncertainties on each of the top quark mass estimators. The uncertainty on the inferred top quark mass of about $1.2 \mathrm{GeV} / \mathrm{c}^{2}$ is dominated by the uncertainty on the energy scale of the $b$-quark jets. This relative uncertainty is taken to be $1.5 \%$ which defines a goal for the performance of jet calibration methods.

\subsubsection{Fully hadronic events}

The selection described in Section 8.1.4.1, including the demand for the two $b$-tags, forms the basis for a selection of fully hadronic $t \bar{t}$ events suitable for a kinematic top-mass reconstruction. An additional cut on the two leading jets, $100 \mathrm{GeV} / \mathrm{c}<p_{\mathrm{T}}<300 \mathrm{GeV} / \mathrm{c}$, is effective against background from mis-reconstructed events and combinatorial background.

The six partons in $p p \rightarrow t \bar{t} \rightarrow b W^{+} \bar{b} W^{-} \rightarrow b q_{1} \bar{q}_{1}^{\prime} \bar{b} q_{2} \bar{q}_{2}^{\prime}$ are matched to six reconstructed jets by picking the matching which minimises the sum of the angular separation between reconstructed jet and matched parton. Only jets satisfying our initial jet-definition, $p_{\mathrm{T}}>$ $30 \mathrm{GeV} / \mathrm{c}$ and $|\eta|<2.4$, as employed in the selection, are taken into account in the matching process. Based on the amount of the angular separation three disjunctive classes of signal events are defined: good (36\%), half-good (45\%) and bad jet-parton-matching (19\%). The first class being the events where all six partons are matched well by jets, the second class where only the three partons from one top are matched well by jets. The reason for the mismatch 
Table 8.10. Overview of all uncertainty components on the top quark mass estimators, extrapolated to a better understanding of both the proton collisions at the LHC and the detector performance.

\begin{tabular}{lccc}
\hline & \multicolumn{3}{c}{ Standard Selection } \\
\cline { 2 - 4 } & $\begin{array}{c}\text { Gaussian Fit } \\
\Delta m_{t}\end{array}$ & Gaussian Ideogram & Full Scan Ideogram \\
& $\left(\mathrm{GeV} / \mathrm{c}^{2}\right)$ & $\Delta m_{t}$ & $\Delta m_{t}$ \\
& 0.32 & $\left(\mathrm{GeV} / \mathrm{c}^{2}\right)$ & $\left(\mathrm{GeV} / \mathrm{c}^{2}\right)$ \\
\hline Pile-Up (5\%) & 0.50 & 0.23 & 0.21 \\
Underlying Event & 2.90 & 0.35 & 0.25 \\
Jet Energy Scale $(1.5 \%)$ & 0.80 & 1.05 & 0.96 \\
Radiation $\left(\lambda Q C D, Q_{0}^{2}\right)$ & 0.40 & 0.27 & 0.22 \\
Fragmentation $\left(\right.$ Lund $\left.\mathrm{b}, \sigma_{q}\right)$ & 0.80 & 0.40 & 0.30 \\
b-tagging $(2 \%)$ & 0.30 & 0.20 & 0.18 \\
Background & 0.12 & 0.25 & 0.25 \\
Parton Density Functions & 3.21 & 0.10 & 0.08 \\
Total Systematical uncertainty & 0.32 & 1.27 & 1.13 \\
Statistical Uncertainty $\left(10 \mathrm{fb}^{-1}\right)$ & 3.23 & 0.36 & 0.21 \\
Total Uncertainty & 1.32 & 1.15 \\
\hline
\end{tabular}

Table 8.11. Distribution of the different signal event classes after jet-pairing and top-choice in the $t \bar{t}$ fully hadronic channel. The label column indicates whether the class is considered signal- or background-like.

\begin{tabular}{lllllr}
\hline reconstruction & pairing & {$[\mathrm{pb}]$} & top-choice & {$[\mathrm{pb}]$} & label \\
\hline good & correct & $0.62(35 \%)$ & always correct & $0.62(35 \%)$ & sig. \\
& wrong & $0.26(14 \%)$ & always wrong & $0.26(14 \%)$ & bkg. \\
half-good & correct & $0.46(25 \%)$ & correct & $0.33(18 \%)$ & sig. \\
& & & wrong & $0.13(7 \%)$ & bkg. \\
& wrong & $0.26(15 \%)$ & always wrong & $0.26(15 \%)$ & bkg. \\
bad & always wrong & $0.20(11 \%)$ & always correct & $0.20(11 \%)$ & bkg. \\
\hline
\end{tabular}

can be traced to parton-level properties, like high $|\eta|$ and low $p_{\mathrm{T}}$, described in more detail in [279].

In order to perform the correct jet pairing, a likelihood variable is constructed from the following event observables: (a) average of the two $W$-boson masses, (b) difference of the two $W$-boson masses, (c) sum of the inter-jet angles of the $W$-boson candidates $\angle\left(q_{1} \bar{q}_{1}^{\prime}\right)+\angle\left(q_{2} \bar{q}_{2}^{\prime}\right)$, (d) difference of the two top-quark masses, (e) sum of the inter-jet angles of the top quark candidates $\angle\left(b q_{1}\right)+\angle\left(b \bar{q}_{1}^{\prime}\right)+\angle\left(q_{1} \bar{q}_{1}^{\prime}\right)+\angle\left(\bar{b} q_{2}\right)+\angle\left(\bar{b} \bar{q}_{2}^{\prime}\right)+\angle\left(q_{2} \bar{q}_{2}^{\prime}\right)$, (f) angle between the direction of the two top-quark candidates. Their distributions are shown in [279]. Taking for each event the pairing with the highest likelihood value yields pairing efficiencies of $71 \%$ for the good and $64 \%$ for the half-good jet-parton-matching.

Only one top per event is chosen for the kinematic mass determination, the choice is once again based on a likelihood variable constructed from the following event observables: (a) $p_{\mathrm{T}}$ of the softest of the three jets of each top-quark candidate (b) mass of the $W$ boson as reconstructed in top decay (c) sum of the inter-jet angles of jets from top decay, $\angle\left(b_{i} q_{i}\right)+\angle\left(b_{i} \bar{q}_{i}^{\prime}\right)+\angle\left(q_{i} \bar{q}_{i}^{\prime}\right)$. Taking the top with the larger likelihood value yields a $72 \%$ efficiency, far greater than the $50 \%$ efficiency of a random choice.

The differentiation of the selected signal events into the now six classes is summarised in Table 8.11, where the six classes are being mapped onto two labels, indicating whether the events are considered signal- or background-like. 


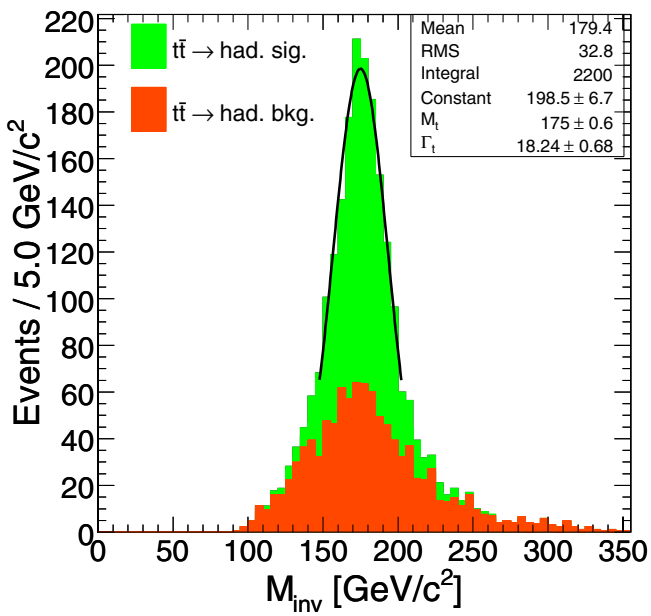

Figure 8.8. Invariant mass distribution of the reconstructed and rescaled, chosen top for both signal classes with a Gaussian fit to the peak.

Table 8.12. Summary of the systematics for the top-mass determination with fully hadronic events.

\begin{tabular}{lc}
\hline & $\Delta m_{t}\left(\mathrm{GeV} / \mathrm{c}^{2}\right)$ \\
\hline Pile Up & 0.4 \\
Underlying Event & 0.6 \\
PDF & 1.4 \\
IS/FS Radiation & 2.3 \\
Fragmentation & 0.9 \\
Jet Energy Scale & 2.3 \\
b-Tagging & 0.3 \\
Background & 2.0 \\
\hline
\end{tabular}

With all the pieces in place a kinematic reconstruction of the top quarks is straightforward and the resulting invariant mass distribution of the chosen top, with the paired non- $b$-jets rescaled such that they yield the $W$-mass, is shown in Figure 8.8.

As expected the signal-like events form a narrow peak, while the wrongly-reconstructed events have a far broader shape. Fitting a Gaussian to the peak of the invariant mass distributions with a fit range corresponding to 0.4 of the peak maximum, as shown in Fig. 8.8 serves as a simple mass estimator. The extracted top-mass is $m_{t}=175.0 \pm 0.6$ (stat.) \pm 4.2 (syst.) $\mathrm{GeV} / \mathrm{c}^{2}$ for an input top-mass of $175 \mathrm{GeV} / \mathrm{c}^{2}$ and an integrated luminosity of $\mathcal{L}=1 \mathrm{fb}^{-1}$.

Already with this amount of data the statistical error becomes negligible compared to the systematic uncertainties which are summarised in Table 8.12. One of the big systematic uncertainties is the QCD background. The $S / B$ in the displayed mass window of Fig. 8.8 is about $2 / 3$, although not shown since the currently available number of simulated events does not allow a determination of the QCD background shape and of the uncertainty it introduces into the top-mass determination. Experience from CDF at the Tevatron [293, 294] indicates that this uncertainty can be understood at the $\sim 2 \mathrm{GeV} / \mathrm{c}^{2}$ level, when using data for background estimation. 


\subsubsection{Top quark mass from $J / \psi$ final states}

8.2.4.1. Introduction. At the LHC the measurement of the top quark mass via direct reconstruction will soon be limited by systematic errors. It is expected that the most severe systematic contributions will be linked to the modelling of the hadronic environment and the knowledge of the jet energies. It would be particularly desirable, therefore, to consider methods for the extraction of $m_{t}$ from the data which could reduce the contribution from these uncertainties considerably. An alternative method, which is making use of exclusive $b$ decays in semi-leptonic top-pair events with the presence of a $J / \psi$ decaying into an electron or muon pair was proposed in [295, 296].

The top quark mass is determined by its correlation with the invariant mass of the reconstructed $J / \psi$ and the lepton from the $\mathrm{W}$ decay coming from the same top decay, $\mathrm{m}_{\mathrm{J} / \psi l}$. The correlation is present because the reconstruction of the $J / \psi$ gives an accurate measurement of the $b$ quark flight direction and its momentum thanks to the relatively high mass of the meson. Moreover, this measure is expected to have an excellent resolution because of the very clean experimental reconstruction of the lepton three-vectors. Details on the analysis presented here can be found in [297].

8.2.4.2. Event generation and selection. Signal events are generated using the TopREX generator [44] and consist of $t \bar{t}$ events where the presence of at least one $J / \psi$ in the final state from the hadronisation of $b$-quarks is required. No distinction is made about the origin of the $J / \psi$; therefore the same samples also contains combinatorial background where the $J / \psi$ is coming from a $b$ quark produced together with a $W$ boson decaying hadronically. Five samples corresponding to five different top masses are generated with a statistics of $200 \mathrm{~K}$ events each. The event hadronisation and the description of the underlying event and the minimum bias is realised with PYTHIA 6.227 [24].

All the signal samples are passed through full detector simulation (ORCA) [10] with a simulation of the minimum bias corresponding to high luminosity data taking. Indeed, the statistics is expected to be so low that the use of high luminosity data must be considered. The same signal samples, and several millions more for studies on systematics, are passed through the fast simulation of the detector (FAMOS) [11]. The shape of the variables used in the selections are fully compatible in both scenarios.

The studied physics backgrounds are generated with the ALPGEN [161] generator and include $W+$ jets, $Z b \bar{b}+$ jets, $W b \bar{b}+$ jets. In these cases the samples are not biased by requiring an explicit $J / \psi$ in the final state, therefore the separation from the signal is studied on the basis of cuts not involving the search for a $J / \psi$ and the contribution of the resulting background is then rescaled taking into account the proper branching fractions. The selection, in terms of signal efficiency, is also cross-checked against $t \bar{t}+$ jets signal generated with ALPGEN, and is found to be consistent.

The main difficulty of the analysis comes from the extremely low branching ratio for a $t \bar{t}$ event to give a final state with a leptonic $J / \psi$. This can be written as:

$$
\begin{aligned}
& B R(t \bar{t}\rightarrow(W b)(W b) \rightarrow(X b)(\ell \nu J / \psi X))=2 \cdot B R(W \rightarrow \ell v) \\
& \cdot B R\left(b(\rightarrow X) \rightarrow B^{ \pm, 0}, B_{s}, B_{\text {baryon }} \rightarrow J / \psi X\right) \cdot B R(J / \psi \rightarrow \ell \ell)
\end{aligned}
$$

where charge conjugation is implicit, $\ell$ indicates either an electron or a muon, and having assumed a $B R(t \rightarrow W b)$ of 1. Replacing the branching ratios with up-to-date numbers [54] one gets for the global branching ratio the value $5.5 \cdot 10^{-4}$ that, in terms of event yield and assuming a cross section for $p p \rightarrow t \bar{t}$ of $830 \mathrm{pb}$, makes approximately 4500 events per $10 \mathrm{fb}^{-1}$. This number does not include neither the trigger and selection efficiency, nor the efficiency for the correct pairing of the $J / \psi$ to the correct lepton from the $W$ decay. 
Table 8.13. Selection performance on signal and expected backgrounds. The first column indicates the channel and its final state, the second the predicted cross section, where the branching ratio for producing at least a $J / \psi$ into leptons from either a $b$ jet or a light jet is accounted for, the third the trigger efficiency, the fourth the selection efficiency, the fifth the expected number of events in $10 \mathrm{fb}^{-1}$, the sixth the classification of the contribution as signal (S), physics background (B) or combinatorial background (C).

\begin{tabular}{|c|c|c|c|c|c|}
\hline Channel & $\mathrm{BR} . \sigma(\mathrm{fb})$ & $\varepsilon_{\text {trig }}(\%)$ & $\varepsilon_{\text {sel }}(\%)$ & Events in $10 \mathrm{fb}^{-1}$ & Class \\
\hline$t \bar{t} \rightarrow(b \rightarrow J / \psi) \ell v-b \ell v$ & 107 & 93.9 & $15.7 \pm 0.4$ & 158 & $\mathrm{~S}+\mathrm{C}$ \\
\hline$t \bar{t} \rightarrow(b \rightarrow J / \psi) \ell v-b \tau \nu$ & 53 & 61.1 & $11.0 \pm 0.8$ & 36 & $\mathrm{~S}$ \\
\hline$t \bar{t} \rightarrow(b \rightarrow J / \psi) \ell v-b q q$ & 320 & 55.3 & $10.9 \pm 0.3$ & 193 & $\mathrm{~S}$ \\
\hline$t \bar{t} \rightarrow(b \rightarrow J / \psi) \tau \nu-b \ell v$ & 53 & 61.1 & $10.6 \pm 0.8$ & 34 & $\mathrm{C}$ \\
\hline$t \bar{t} \rightarrow(b \rightarrow J / \psi) \tau \nu-b \tau \nu$ & 27 & 14.2 & $2.8 \pm 1.2$ & 1 & B \\
\hline$t \bar{t} \rightarrow(b \rightarrow J / \psi) \tau v-b q q$ & 160 & 7.9 & $1.5 \pm 0.5$ & 2 & B \\
\hline$t \bar{t} \rightarrow(b \rightarrow J / \psi) q q-b \ell v$ & 320 & 55.3 & $10.7 \pm 0.3$ & 190 & $\mathrm{C}$ \\
\hline$t \bar{t} \rightarrow(b \rightarrow J / \psi) q q-b \tau \nu$ & 160 & 7.9 & $1.5 \pm 0.5$ & 2 & B \\
\hline$t \bar{t} \rightarrow(b \rightarrow J / \psi) q q-b q q$ & 959 & 0.1 & $0.2 \pm 0.5$ & 0 & $\mathrm{~B}$ \\
\hline$W+N$ jets, $N>1 \rightarrow J / \psi X$ & 394 & 55.3 & $2.1 \pm 0.1$ & 43 & B \\
\hline$W b \bar{b}+$ jets $\rightarrow J / \psi X$ & 196 & 55.3 & $1.6 \pm 0.1$ & 16 & $\mathrm{~B}$ \\
\hline$Z b \bar{b}+$ jets $\rightarrow J / \psi X$ & 23 & 93.9 & $9.4 \pm 0.1$ & 20 & B \\
\hline$b \bar{b} \rightarrow J / \psi X$ & $1.3 \cdot 10^{9}$ & $<2 \cdot 10^{-8}$ & $<1$ & $<2.6$ & B \\
\hline
\end{tabular}

Events are triggered using the inclusive lepton trigger with thresholds described in [76]. The efficiency for triggering signal events is reported in Table 8.13 and is included in all numbers presented here. In events passing the trigger thresholds a $J / \psi$ is searched for by looking for same-flavour, opposite-sign leptons with invariant mass in the range $[2.8,3.2] \mathrm{GeV} / \mathrm{c}^{2}$ and forming an angle greater than 2 and lower than 35 degrees. No isolation requirements must be imposed on these leptons. The efficiency for reconstructing a $J / \psi$ at this stage is $(0.386 \pm 0.007)$ and $(0.114 \pm 0.004)$ for the muon and electron channels, respectively. It is limited by the low momenta of the leptons and because they are produced inside a jet, making the reconstruction more difficult, particularly for electrons.

If a $J / \psi$ is found in an event, the isolated lepton with the highest $p_{\mathrm{T}}$ and higher than $20 \mathrm{GeV} / \mathrm{c}$ is considered as the lepton candidate from the $W$ decay. The isolation discriminant is defined as the sum of the energies in the electromagnetic and hadronic calorimeters in a cone of opening angle $\Delta R=0.3$ around the lepton candidate. The selection requires that the isolation energy is less than $15 \mathrm{GeV}$ for electrons and less than $20 \mathrm{GeV}$ for muons.

We define as background all contributions from processes not resulting in the decay chain $t \rightarrow W b \rightarrow \ell v J / \psi X$. We call physics background the contribution from processes other than $t \bar{t}$ (semi)leptonic and as combinatorial background the irreducible part of $t \bar{t}$ (semi)leptonic where the $J / \psi$ is wrongly associated to the lepton not coming from the $W$ in the same top decay. Any physics background needs to mimic a final state with the presence of a $J / \psi$ and an isolated and energetic lepton. The obvious candidates are bosons in association with jets. It is important to distinguish between $b$ jets and light jets, which produce $J / \psi$ at very different rates, suppressing the contribution of processes with light jets very much. To remove these contributions the total scalar sum of the transverse jet momenta is required to be greater than $100 \mathrm{GeV} / \mathrm{c}$. This cut is not applied if two isolated leptons are found, in order to preserve dileptonic $t \bar{t}$ events. If the flavour of the two leptons is the same, an explicit cut to remove the presence of leptonic $Z$ is made, vetoing events where the invariant mass of the two leptons is between 85 and $97 \mathrm{GeV} / \mathrm{c}^{2}$. To further reduce soft background the cut on the transverse momentum of the isolated lepton is brought to $40 \mathrm{GeV} / \mathrm{c}$, making the analysis less sensitive 

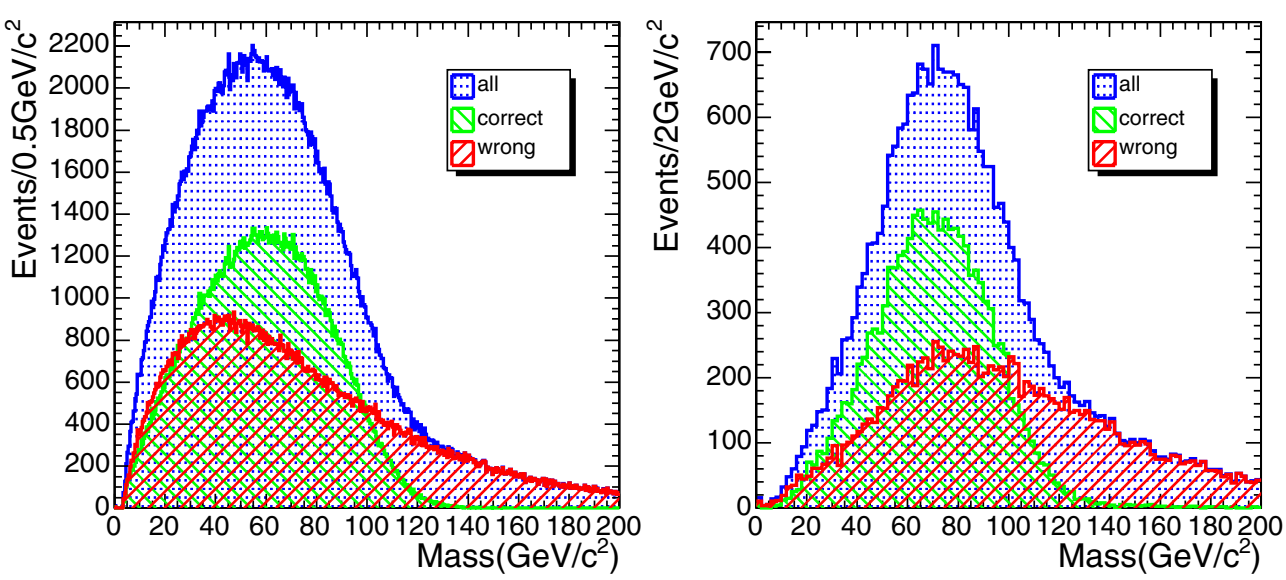

Figure 8.9. Three-lepton mass distribution for $m_{t}=175 \mathrm{GeV} / \mathrm{c}^{2}$ at generator level (left) and after detector simulation and reconstruction (right). In the pictures the components coming from correct and wrong lepton pairing - from both combinatorial and physics backgrounds - are shown.

also to systematic effects involving soft QCD. Table 8.13 presents, in terms of predicted cross sections, efficiencies and events yields per $10 \mathrm{fb}^{-1}$, the performance of the analysis.

8.2.4.3. Reconstruction of $\mathrm{m}_{\mathrm{J} / \psi \ell}$ and statistical performance. In order to estimate the correct invariant mass $J / \psi$-lepton it would be necessary to efficiently discriminate between right pairings, where both particles are coming from the decay of the same top, and from wrong pairings where, in $t \bar{t}$ events, they come from the two different top decays. In the present analysis, in order to increase the available statistics, we propose not to attempt any separation of the combinatorial but, instead, to use the full distribution containing both signal and background.

Figure 8.9 shows the three-lepton invariant mass in $t \bar{t}$ events at generator level without selection and at full reconstruction after the selection described in the previous section. The distribution of the components of signal and background from $t \bar{t}$ are shown, where the Monte Carlo truth is used to judge when the correct pairing is made. No equivalent distribution can be done for non- $t \bar{t}$ backgrounds since no $J / \psi$ is present in those samples. To take this into account the pure background shape is scaled up according to the extra contribution of non $t \bar{t}$ background (Table 8.13), in the hypothesis that the shape of the two samples are the same. Uncertainty in the background description will then be translated into a systematic contribution on the measurement.

The observable most sensitive to the top mass is the position of the maximum of the three-lepton mass distribution. It is determined via a fit of the full shape with a polynomial function of fourth degree. The range chosen for the fit is centred around the maximum and goes from 20 to $120 \mathrm{GeV} / \mathrm{c}^{2}$. The error on the maximum of the fitted polynomial is determined by propagating the errors on the fitted coefficients and taking into account their correlation. As a cross check, an alternative way of fitting the signal with a Gaussian was tried. In this case the background is first subtracted on a bin-by-bin basis making use of an average background distribution determined by using all the simulated samples. The results obtained are comparable.

The fitted maxima are expected to be correlated to the input value of the top mass. This correlation is proven and fitted by a line (Fig. 8.10). The two results at fast and full simulation are in impressive agreement. The correlation curves can be used to estimate the expected 

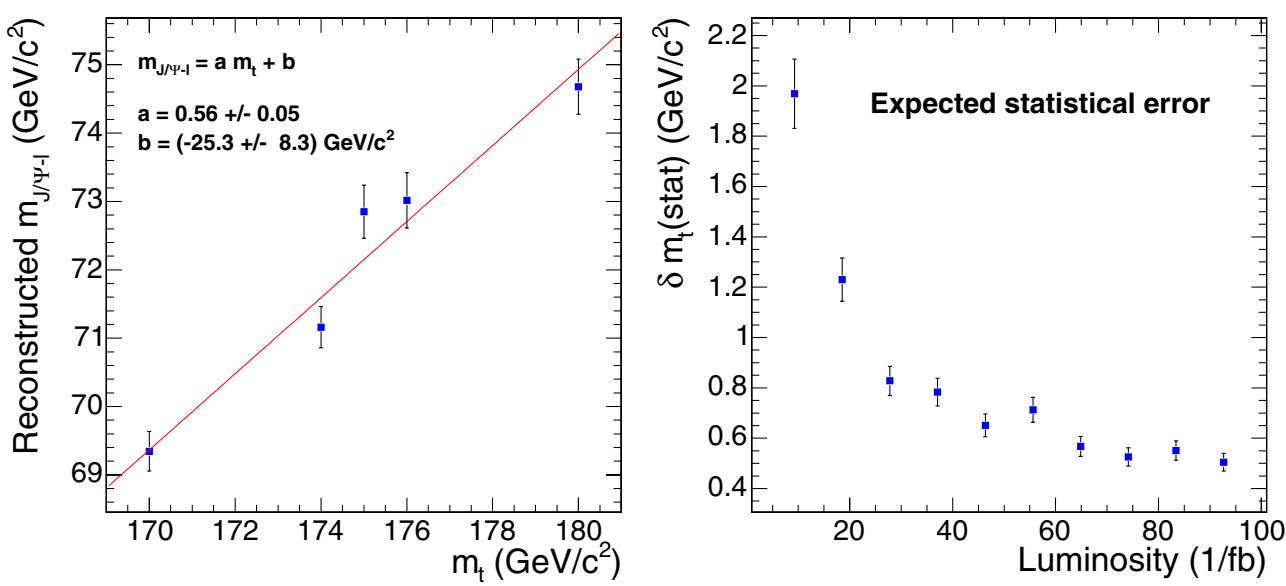

Figure 8.10. Left: correlation between the reconstructed three-lepton invariant mass and the input top mass at full simulation. Right: expected statistical error on the top quark mass as a function of the integrated luminosity.

statistical error on the top mass as a function of the available amount of data. This is done by using the number of events expected according to Table 8.13, and the result is presented in Fig. 8.10. From the figure it can be concluded that the measurement of the top quark mass with this analysis can become, on the statistical footing, competitive already with other analyses' total error after the first years of data taking. Moreover the measurement is expected to be dominated by systematic errors in the long range, as explained in the next section.

8.2.4.4. Systematic errors. The sources of systematic errors can be divided into two main categories: theoretical and experimental. The former include the description of the hard process and the modelling of radiation, fragmentation and the underlying event in the simulation, whereas the latter includes all experimental sources coming from an imperfect detector description. The sources analysed in what follows are considered as uncorrelated and the corresponding resulting errors on the top mass are summed in quadrature to form the total systematic error. To evaluate the effect of various sources the guidelines described in [201] and in Appendix B are followed.

With the exception of the PDF description, for each of the other sources of theoretical uncertainty and for each change in the simulation parameters an independent signal generation with TOPREX and PYTHIA has been performed, with statistics of a few $100 \mathrm{~K}$ events each, and fast simulated. The variations on the resulting top masses are considered as systematics: when the mass difference with respect to the reference sample is smaller than the associated statistical error, this is conservatively quoted as the systematic error.

For all the experimental sources, smearings and shifts on the observed objects (leptons and jets) are applied after reconstruction and before selection in a consistent way. The observed difference on the top mass is taken as an estimation of the associated systematic uncertainty.

Table 8.14 presents the systematic breakdown on the top mass. The systematics error is dominated by theoretical sources, which are the ones affected by the larger statistical uncertainties, quoted here as systematics.

Putting together the systematic and the statistical error one can conclude that, with maybe exception for the first year of data taking, this measurement will be dominated by systematics, 
Table 8.14. Systematic error breakdown. For each source either the maximum variation from a reference sample or the resulting statistical error on the difference is quoted as a systematic error.

\begin{tabular}{lc}
\hline Source & $\delta m_{t}\left(\mathrm{GeV} / \mathrm{c}^{2}\right)$ \\
\hline$\Lambda_{Q C D}$ & 0.31 \\
$\mathrm{Q}^{2}$ & 0.56 \\
Scale definition & 0.71 \\
b-quark fragmentation & 0.51 \\
Light jet fragmentation & 0.46 \\
Minimum bias/Underlying event & 0.64 \\
Proton PDF & 0.28 \\
Total theoretical & 1.37 \\
Electron E scale & 0.21 \\
Muon p scale & 0.38 \\
Electron E resolution & 0.19 \\
Muon p resolution & 0.12 \\
Jet E scale & 0.05 \\
Jet E resolution & 0.05 \\
Background knowledge & 0.21 \\
Total experimental & 0.54 \\
Total systematic & 1.47 \\
\hline
\end{tabular}

in turn dominated by our poor understanding of the theoretical sources. A total error on the top mass below $2 \mathrm{GeV} / \mathrm{c}^{2}$ can be in reach from the first $20 \mathrm{fb}^{-1}$ already. The present result suggests an uncertainty of $1.5 \mathrm{GeV} / \mathrm{c}^{2}$ with full statistics, but this number is fully dominated by the theory systematics. A precision much better than this is not out of reach since, by the time this measurement will be made, the analysis will be hopefully repeated at (N)NLO and our understanding of the dominating systematics, for instance the minimum bias and the underlying event, will be drastically improved. More dedicated reconstruction techniques and more sophisticated analyses will considerably improve the statistical treatment of the information.

This analysis reduces to a minimum those systematics which are expected to dominate in more traditional estimations of the top mass, especially the ones from direct reconstruction, like the jet energy scale and the knowledge of the b-tagging.

\subsubsection{Summary of top mass determinations}

Measuring the mass of the top quark in different channels allows for a combination of the individual results [298]. As the statistical component in the total uncertainty on $m_{t}$ in each channel is negligible, the correlation between the systematic uncertainties must be determined. The dominant uncertainty arises from the knowledge of the energy scale of $b$-quark jets, a component which is assumed to be fully correlated between decay channels. This uncertainty can however be subdivided in several components: detector understanding, clustering algorithms, related to the modelling of $b$ - and light-quark fragmentation and decay and, finally, the statistical precision of the data-based estimates of the $b$-jet energy scale differentiated versus the pseudo-rapidity and the transverse momentum of the observed jet.

The measurement from the $J / \psi$ final states is however limited by other, mainly theoretical, sources of systematic uncertainties. Therefore a reduction of the uncertainty on $m_{t}$ is expected when combining the direct measurements with the measurement from the $J / \psi$ final states. The knowledge of the top quark mass can be improved by developing alternative 
methods which do not rely on the $b$-jet energy scale $[299,300]$. Accounting for these future improvements an uncertainty of $1 \mathrm{GeV} / \mathrm{c}^{2}$ on the top quark mass is feasible. The combination can be performed by applying techniques described in [301,302].

\subsection{Spin correlation in top-quark pair production}

\subsubsection{Introduction}

Because of its large width of $1.4 \mathrm{GeV} / \mathrm{c}^{2}$ the top quark decays before either hadronisation, governed by the scale $\Lambda_{Q C D}$, or depolarisation, governed by the scale $\Lambda_{Q C D}^{2} / m_{t}$, can take place. This unique feature is used to investigate the spin of the top quark; such investigation is not possible in the case of light quarks, where the spin information is diluted by hadronisation. Moreover, the top quark spin-flip time is much larger than its lifetime and the probability of a spin flip due to emission of one or several gluons via chromomagnetic dipole-transition is very small.

The angular distribution of a daughter particle in top quark decays can be written as [303-305]

$$
\frac{1}{\Gamma} \frac{d \Gamma}{d \cos \theta_{i}}=\frac{1}{2}\left(1+\kappa_{i} \cos \theta_{i}\right)
$$

where the decay angle $\theta_{i}$ is defined as the angle between the direction of motion of the daughter particle $i$ and the chosen spin quantisation axis. As gluon fusion is the dominant production mechanism at the LHC there is no well defined spin axis in the initial state. This leads to a choice of the helicity basis along the top quark momenta in the partonic centreof-mass frame. The spin-analyser quality $\kappa$ of the top quark daughter particle is defined as the degree to which the daughter particle is correlated with the top-quark spin. The analysis presented here is based on the semi-leptonic $t \bar{t}$ decay channel with electrons or muons, which is considered to be the signal. Alternatively, the dileptonic $t \bar{t}$ decay channel can also be considered. The $\kappa$ values for the daughter particles used in this analysis [306], lepton, $b$ quark and the lower energy quark from $W$ decay, are $1,-0.41$ and 0.51 , respectively.

The spin correlation in the semi-leptonic $t \bar{t}$ decay channel can be measured in terms of a double differential lepton and quark angular distribution, which, neglecting higher order QCD corrections, is given by

$$
\frac{1}{N} \frac{d^{2} N}{d \cos \theta_{l} d \cos \theta_{q}}=\frac{1}{4}\left(1-\mathcal{A}_{l} \kappa_{q} \cos \theta_{l} \cos \theta_{q}\right) .
$$

Here, using the helicity basis the lepton and quark angles $\theta_{l}$ and $\theta_{q}$ are obtained by measuring the angle between the decay particle momentum in its parent top quark rest frame and the parent top quark momentum in the $t \bar{t}$ quark pair rest frame. The correlation coefficient

$$
\mathcal{A}=\frac{N_{\|}-N_{X}}{N_{\|}+N_{X}}=\frac{N\left(t_{L} \bar{t}_{L}+t_{R} \bar{t}_{R}\right)-N\left(t_{L} \bar{t}_{R}+t_{R} \bar{t}_{L}\right)}{N\left(t_{L} \bar{t}_{L}+t_{R} \bar{t}_{R}\right)+N\left(t_{L} \bar{t}_{R}+t_{R} \bar{t}_{L}\right)},
$$

where $N_{\|}$and $N_{X}$ give the number of events with parallel and anti-parallel top quark spins, respectively. Two angle combinations are considered: $\theta_{l}$ versus $\theta_{b}$ and $\theta_{l}$ versus $\theta_{q(\text { lower energy) }}$; in the following description these two combinations are denoted as $b-t l-t$ and $q-t l-t$.

\subsubsection{Simulation of $t \bar{t}$ with spin correlation}

A $t \bar{t}$ sample of $3.1 \cdot 10^{6}$ events containing $9.1 \cdot 10^{5}$ semi-leptonic signal events was generated with PYTHIA [24] and reconstructed using ORCA. As PYTHIA does not include spin correlations 

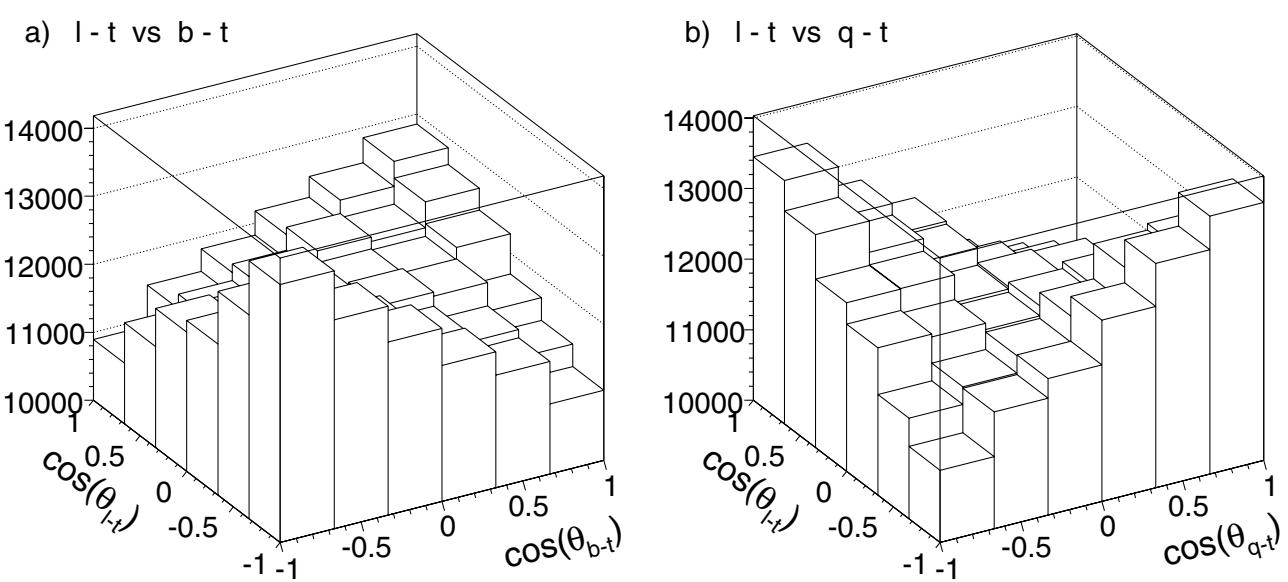

Figure 8.11. Double differential angular distributions obtained from the "analysis" sample, see text.

the events are weighted according to Equation (8.5) with $\mathcal{A}=0.32$ [44] and appropriate values of $\kappa$. Then, this data sample is subdivided into two sub-samples: one is regarded as the "reference" sub-sample (1.61M events), used for determination of the selection efficiency and backgrounds. The other is regarded as the "analysis" sub-sample (1.50M events), used for the measurement of $\mathcal{A}$. This sample provides $436 \mathrm{~K}$ signal events. The double differential angular distributions obtained from the "analysis" sample are presented in Figure 8.11.

The distributions in Figure 8.11 are fitted according to Equation (8.5). The results are $\mathcal{A}_{b-t l-t}=0.321 \pm 0.011$ (stat.) and $\mathcal{A}_{q-t l-t}=0.319 \pm 0.009$ (stat.) which are statistically compatible with the input value of $\mathcal{A}=0.32$.

\subsubsection{Online and offline event selection}

The Level 1 and High Level Triggers select events with a single isolated electron or muon; the trigger efficiency is $55 \%$.

The following requirements are applied in the offline selection: missing transverse energy $E_{\mathrm{T}}^{\text {miss }}>20 \mathrm{GeV}$; at least one isolated lepton with $|\eta|<2.5$, electron with $p_{\mathrm{T}}>27 \mathrm{GeV} / \mathrm{c}$ or muon with $p_{\mathrm{T}}>20 \mathrm{GeV} / \mathrm{c}$; at least four jets with $p_{\mathrm{T}}>30 \mathrm{GeV} / \mathrm{c}$ and $|\eta|<2.5$. Jets are reconstructed with a cone algorithm with $\Delta R=0.5$. At least two jets must be $b$-jets where the tagging efficiency is $66 \%$ for $b$ quarks in $t \bar{t}$ events. This selection results in an overall efficiency of $12 \%$.

The reconstruction of two top quarks includes the following requirements: Two jets that are not $b$-tagged and have an invariant mass in the range $50-135 \mathrm{GeV} / \mathrm{c}^{2}$, consistent with the $W$ mass, are found. A $b$-tag jet which combined with the above reconstructed $W$ gives an invariant mass in the range $130-250 \mathrm{GeV} / \mathrm{c}^{2}$, consistent with the $t$ mass. In addition to the top quark reconstructed above, another top quark is required based on the other $b$-tag jet plus lepton and missing energy combination. The neutrino components are determined by fitting the missing energy components, constrained with $W$ and $t$ quark masses. The azimuthal angle between the two top quarks is required to be greater than 2 rad. This selection results in an overall efficiency of $5 \%$ (Table 8.15 ).

A measure of the selection quality can be obtained by comparing the generated and reconstructed momentum directions expressed in terms of the cosine of the angles defined above. Figure 8.12 presents the differences between the generated and reconstructed cosines 
Table 8.15. The physics processes considered for signal and background. The number of selected events for the non- $t \bar{t}$ processes are scaled to the same $t \bar{t}$ sample luminosity.

\begin{tabular}{lcccc}
\hline Process & Simulated events & $\sigma(\mathrm{pb})$ & Efficiency & Selected events \\
\hline$t \bar{t}$ (signal) & $436 \mathrm{~K}$ & 246 & $5.0 \cdot 10^{-2}$ & 21589 \\
$t \bar{t}$ (background) & $1.07 \mathrm{M}$ & 584 & $4.0 \cdot 10^{-3}$ & 4236 \\
$W W+$ jets & $310 \mathrm{~K}$ & 188 & $4.5 \cdot 10^{-5}$ & 15 \\
$W+$ jets $\left(\hat{p}_{\mathrm{T}}=20-400 \mathrm{GeV} / \mathrm{c}\right)$ & $2.06 \mathrm{M}$ & $43 \mathrm{~K}$ & $3.4 \cdot 10^{-6}$ & 260 \\
$W b t$ semi-leptonic decay & $328 \mathrm{~K}$ & 63.1 & $1.3 \cdot 10^{-3}$ & 144 \\
\hline
\end{tabular}
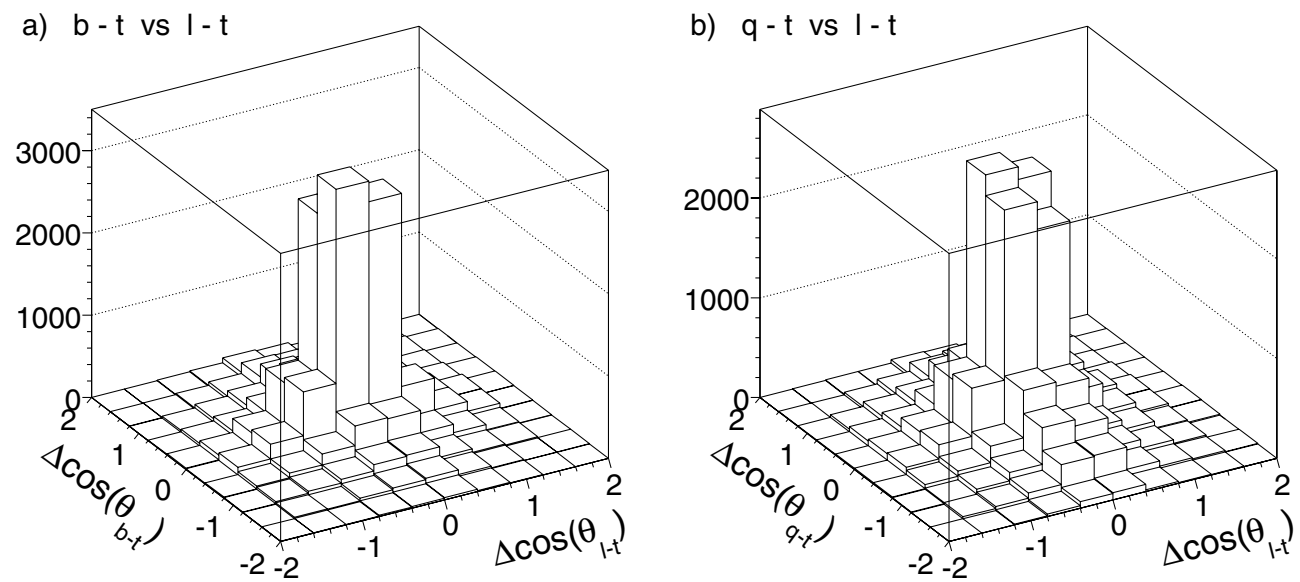

Figure 8.12. Selection quality: Difference between the generated and reconstructed cosine of the analysis angles in the $b-l l-t$ and $q-l l-t$ systems.

of the $b-l l-t$ and $q-l l-t$ systems. Quantifying this selection quality $Q$ as the ratio of the number of events in the four central bins to all bins, one obtains: $Q_{b-t l-t}=52 \%$ and $Q_{q-t l-t}=45 \%$.

The signal-to-background ratio is 4.5 . The main background, detailed in Table 8.15 , is $t \bar{t}$ production with decays different from those treated as the signal. It amounts to $88 \%$ of the total background and is used to model the shape of the total background.

\subsubsection{Estimation of correlation coefficient}

In order to correct for the selection efficiency, an efficiency $(6 \times 6)$ matrix is determined by taking the ratio of the reconstructed double differential angular distribution to the generated one, using the "reference" sample. The final double differential angular distribution is obtained by subtracting, bin-by-bin, the background obtained from the "reference" sample from the total sample of signal plus background obtained from the "analysis" sample. The resulting distributions are corrected for the selection efficiency, Figure 8.13, and fitted using Equation (8.5).

The correlation coefficients obtained from the fit are:

$$
\begin{aligned}
& \mathcal{A}_{b-t l-t}=0.375 \pm 0.100 \text { (stat), } \\
& \mathcal{A}_{q-t l-t}=0.346 \pm 0.079 \text { (stat) }
\end{aligned}
$$

These results agree, within statistical uncertainties, with those obtained from the generated events of Figure 8.11. 

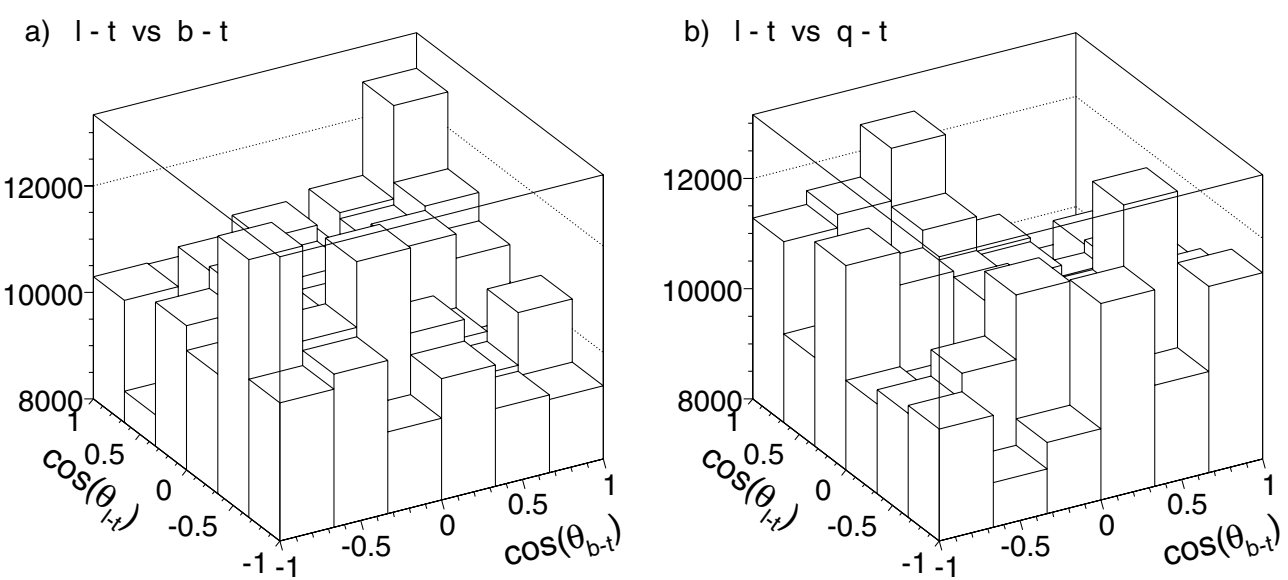

Figure 8.13. Background-subtracted and efficiency-corrected double-differential distribution of the cosine of the analysis angles in the $b-l l-t$ and $q-l l-t$ systems.

The following sources of systematic uncertainties have been evaluated. The choice of the Parton Distribution Function in modelling $t \bar{t}$ production affects the number of $t \bar{t}$ events produced via gluon fusion and that via quark-anti-quark annihilation. The relative variation in $\mathcal{A}$, determined using TopReX with different PDFs (CTEQ6M, MRST2003), is found to be $4 \%$.

The mass of the top quark affects the result of the kinematic fit and the selection. The nominal $m_{t}=175 \mathrm{GeV} / \mathrm{c}^{2}$ is varied by $\pm 5 \mathrm{GeV} / \mathrm{c}^{2}$ [54] using TopReX. The variation in $\mathcal{A}$ is found to be negligible.

The uncertainty on the $t \bar{t}$ cross section affects the shape of the final angular distribution after background subtraction; varying $\sigma(t \bar{t})$ by $10 \%$ results in $1 \%$ relative variation in correlation coefficients.

The uncertainty due to $b$-tagging efficiency is evaluated by varying the $b$-identification discriminant cut. The corresponding relative variation in $\mathcal{A}_{b-t l-t}$ is $-20 \%$, and in $\mathcal{A}_{q-t l-t}$ it is $+6.5 \% /-8.3 \%$.

The jet energy scale uncertainty is evaluated by varying the jet $P_{\mathrm{T}}$. The relative variations in $\mathcal{A}_{b-t l-t}$ and $\mathcal{A}_{q-t l-t}$ are found to be $+7.7 \% /-14 \%$.

Uncertainties in the initial and final state radiation, quark fragmentation, underlying event and pile up rate could result in an underestimation of the number of non- $t \bar{t}$ jets (not originating from top decays). This possible underestimation of jet multiplicity is estimated to be $8 \%$. To estimate the corresponding uncertainty in $\mathcal{A}, 10 \%$ additional jets per event are generated while processing the data sample. These jets are simulated randomly according to the $\eta$ and $p_{\mathrm{T}}$ distributions of non- $t \bar{t}$ jets, obtained from the $t \bar{t}$ Monte Carlo. The relative variations in $\mathcal{A}_{b-t l-t}$ and $\mathcal{A}_{q-t l-t}$ are found to be $-6.3 \%$ and $-5.3 \%$, respectively.

Summing up the systematic uncertainties and using the statistical uncertainties estimated for $10 \mathrm{fb}^{-1}$ of integrated luminosity, the results are:

$$
\begin{aligned}
& \mathcal{A}_{b-t l-t}=0.375 \pm 0.027 \text { (stat.) }{ }_{-0.096}^{+0.055} \text { (syst.), } \\
& \mathcal{A}_{q-t l-t}=0.346 \pm 0.021 \text { (stat.) }{ }_{-0.055}^{+0.026} \text { (syst.). }
\end{aligned}
$$

In summary, the correlation coefficient of top quark spins in $t \bar{t}$ production is measured with a total relative uncertainty (dominated by systematic uncertainties) of $27 \%$ for $\mathcal{A}_{b-t l-t}$ and of $17 \%$ for $\mathcal{A}_{q-t l-t}$. 

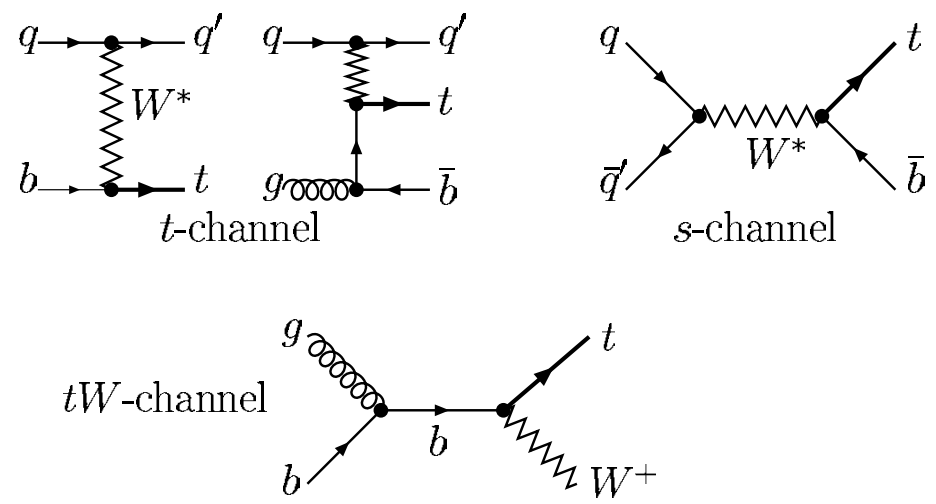

Figure 8.14. Feynman diagrams for the three channels of single top production.

\subsection{Single top quark production}

\subsubsection{Introduction}

The single top production cross section at the LHC is known at NLO level for the tree production mechanisms (see Fig. 8.14, which are classified by the virtuality of the W-boson involved as: $t$-channel $\left(q_{W}^{2}<0\right), s$-channel $\left(q_{W}^{2}>0\right)$, and associated $t W$ production $\left(q_{W}^{2}=M_{W}^{2}\right)$ [307-309]. In all cases, the most dangerous background comes from $t \bar{t}$ process. Other dangerous backgrounds are multi-jet QCD and $\mathrm{W}+$ jets events, but such background is reduced substantially by considering only leptonic decays of the $W^{ \pm}$-bosons from top-quark decays.

All results presented in this Section were done for $10 / \mathrm{fb}^{-1}$ of integrated luminosity.

8.4.1.1. Details on the signal and background simulation. Two generators, SingleTop [310] (based on the CompHEP package [43]) and TopREX [44] were used to generate events for all three single-top production processes. The background processes, namely, $W b \bar{b}$, $W b \bar{b}+j$, and $W+2 j$ were generated with CompHEP, TopReX, MadGrapH [81], and ALPGEN [161] programs as indicated in the Table 8.16. The hard process events containing all needed information were passed to PYTHIA 6.227 [24] for showering, hadronisation and decays of unstable particles. The $t \bar{t}$ and $W+$ jets background events were generated with the same PYTHIA version. All simulations were done with $M_{t}=175 \mathrm{GeV} / \mathrm{c}^{2}$ and $M_{b}=$ $4.7-4.8 \mathrm{GeV} / \mathrm{c}^{2}$, proper considerations of the spin correlations, and the finite $W$-boson and $t$-quark widths. The list of the signal and background process cross sections as well as generators used are given in the Table 8.16. Both the full simulation chain (OSCAR [18] and ORCA [10]) and a fast simulation (FAMOs [11]) were used.

8.4.1.2. Reconstruction algorithms and triggers. Muons are reconstructed by using the standard algorithm combining tracker and muon chamber information as described in [311]; tracker and calorimeter isolation cuts are applied as described in [312]. The electrons are reconstructed by the standard algorithm combining tracker and ECAL information, see [313]. The jets are reconstructed by the Iterative Cone algorithm with the cone size of 0.5 , see [314]; for the calibration both the Monte Carlo (in the t-channel analysis) and the $\gamma+$ jets (in the $t W$ and $s$-channel) methods are used, see [315]. For b-tagging a probability algorithm based on the impact parameter of the tracks is used, as described in [316]. 
Table 8.16. Cross section values (including branching ratio and kinematic cuts) and generators for the signal and background processes (here $\ell=e, \mu, \tau)$. Different generator-level cuts are applied.

\begin{tabular}{llllll}
\hline Process & $\sigma \times \mathrm{BR}, \mathrm{pb}$ & generator & Process & $\sigma \times \mathrm{BR}, \mathrm{pb}$ & generator \\
\hline$t$-ch. $(W \rightarrow \mu \nu)$ & $18(\mathrm{NLO})$ & SINGLETOP & $W b \bar{b}(W \rightarrow \ell \nu)$ & $100(\mathrm{LO})$ & TOPREX \\
$t$-ch. $(W \rightarrow \ell v)$ & $81.7(\mathrm{NLO})$ & TOPREX & $W b \bar{b}+$ jets $(W \rightarrow \mu)$ & $32.4(\mathrm{LO})$ & MAdGRAPH \\
$s$-ch. $(W \rightarrow \ell \nu)$ & $3.3(\mathrm{NLO})$ & TOPREX & $W+2 j(W \rightarrow \mu \nu)$ & $987(\mathrm{LO})$ & COMPHEP \\
$t W(2 W \rightarrow \ell v)$ & $6.7(\mathrm{NLO})$ & TOPREX & $W+2 j(W \rightarrow \ell \nu)$ & $2500(\mathrm{LO})$ & ALPGEN \\
$t W(1 W \rightarrow \ell v)$ & $33.3(\mathrm{NLO})$ & TopReX & $Z / \gamma^{*}\left(\rightarrow \mu^{+} \mu^{-}\right) b \bar{b}$ & $116(\mathrm{LO})$ & COMPHEP \\
$t \bar{t}$ (inclusive) & $833(\mathrm{NLO})$ & PYTHIA & & & \\
\hline
\end{tabular}

The transverse missing energy is reconstructed as follows:

$$
E_{\mathrm{T}}^{\mathrm{miss}}=-\left(\sum \vec{P}_{\mathrm{T}}^{\mu}+\sum \vec{E}_{\mathrm{T}}^{\text {tower }}+\sum\left(\vec{E}_{\mathrm{T}, \mathrm{jet}}^{\mathrm{calib}}\right)-\sum\left(\vec{E}_{\mathrm{T}, \mathrm{jet}}^{\mathrm{raw}}\right)\right)
$$

where $E_{\mathrm{T}}^{\text {tower }}$ is the sum of transverse energy of towers, $E_{\mathrm{T}, \text { jet }}^{\text {calib }}\left(E_{\mathrm{T}, \text { jet }}^{\text {raw }}\right)$ is the transverse energy of calibrated (uncalibrated) jets. For the final states with one isolated lepton the neutrino $\left(E_{\mathrm{T}}^{\mathrm{miss}}\right)$ longitudinal component, $P_{z, v}$, is extracted from the quadratic equation:

$$
M_{W}^{2}=2\left(E_{\mu} \sqrt{P_{z, v}^{2}+\left(E_{\mathrm{T}}^{\mathrm{miss}}\right)^{2}}-\vec{P}_{\mathrm{T}, \mu} \cdot E_{\mathrm{T}}^{\mathrm{miss}}-P_{z, \mu} P_{z, v}\right)
$$

This equation has two solutions:

$$
\begin{aligned}
& P_{z, \nu}^{(1,2)}=\frac{A P_{z, \mu} \pm \sqrt{\Delta}}{P_{\mathrm{T}, \mu}^{2}}, \text { where } A=\frac{M_{W}^{2}}{2}+\vec{P}_{\mathrm{T}, \mu} \cdot E_{\mathrm{T}}^{\mathrm{miss}}, \\
& \Delta=E_{\mu}^{2}\left(A^{2}-\left(E_{\mathrm{T}}^{\mathrm{miss}}\right)^{2} P_{\mathrm{T}, \mu}^{2}\right)
\end{aligned}
$$

Among the two solutions of Equation (8.8) the minimal value of $\left|P_{z, v}\right|$ is used for $W$-boson momentum reconstruction.

About $30 \%$ of the events have negative $\Delta$ values due to the finite detector resolution and to the presence of extra missing energy. In this case for $t$-channel analysis the parameter $M_{W}$ in Equation (8.9) is increased until $\Delta$ becomes zero. Using this value of $M_{W}, P_{z, v}$ is calculated from Equation (8.9). For the $t W$ and $s$-channels analyses, only the real part of $P_{z, v}$ is used for further analysis.

The transverse mass of the $W$-boson is defined as

$$
M_{\mathrm{T}}^{W}=\sqrt{2\left(P_{\mathrm{T}, \mu} E_{\mathrm{T}}^{\text {miss }}-\vec{P}_{\mathrm{T}, \mu} \cdot \vec{E}_{\mathrm{T}}^{\text {miss }}\right)} .
$$

The sum of the transverse momentum vectors of all reconstructed objects

$$
\vec{\Sigma}_{\mathrm{T}} \equiv \vec{P}_{\mathrm{T}, \ell}+\vec{E}_{\mathrm{T}}^{\mathrm{miss}}+\sum \vec{E}_{\mathrm{T}, j e t}
$$

is found to be very effective for signal/background separation.

The "jet charge" $\left(Q_{j}\right)$ is defined as the sum of the charges of the tracks inside the jet cone, weighted over the projections of the track momenta along the jet axis.

The lepton isolation criterion used is to sum the $p_{\mathrm{T}}$ of all the tracks in a cone of $\Delta R<0.2$ around the lepton track, and to reject the event if this sum is greater than $5 \%$ of the lepton $p_{\mathrm{T}}$.

The present study is based on leptonic decay channels $\left(e v_{e}\right.$ or $\left.\mu v_{\mu}\right)$ of the $W$-boson. The signal is triggered by the trigger on leptons. The HLT $p_{\mathrm{T}}$ thresholds from the CMS DAQTDR [76] are assumed: $19 \mathrm{GeV} / \mathrm{c}(29 \mathrm{GeV} / \mathrm{c})$ for the single muon (electron); with $\left|\eta_{\mu}\right| \leqslant 2.1$ and $\left|\eta_{e}\right| \leqslant 2.4$. 
8.4.1.3. The contribution from multi-jet backgrounds. A special treatment is required for QCD events with jets, due to the huge cross section. The currently available samples have very small statistics and typically no events remain after the application of pre-selection cuts. Therefore, in order to estimate the impact of the QCD-background the cuts are applied separately, assuming they are uncorrelated.

For $t$-channel study these cuts are: (a) one isolated muon $\left(p_{\mathrm{T}}>19 \mathrm{GeV} / \mathrm{c}\right)$; (b) $E_{\mathrm{T}}^{\text {miss }}>$ $40 \mathrm{GeV}$ and only two jets; one $B$-jet and one light forward jet. It was found a satisfactory suppression of the multi-jet events as compared to other background process $\left(N_{\mathrm{QCD}} / N_{\mathrm{bckg}}=\right.$ $6924 /\left(8.9 \times 10^{4}\right)=0.078$ (see [317]) and the QCD-background was not considered in the analysis of the $t$ - and $s$-channel single top production.

More detailed investigation of this problem was done for $t W$-channel [318]. The selection cuts are arranged into cut groups whose efficiencies are estimated with the Monte Carlo samples. The product of efficiencies is an indicator of the total efficiency.

Three cut groups are used in the dileptonic channel: lepton, $E_{\mathrm{T}}^{\mathrm{miss}}$, jet. The same procedure is applied on signal sample to find the ratio of total efficiency to the product of efficiencies. The ratio is used to correct the product of efficiencies found in multi-jet sample and the result is 5.6 events. Four cut groups are used in the semi-leptonic channel: jets, leptons, kinematics and finally signal region and $\mathrm{b}$ tagging. The $\mathrm{b}$ tagging requirement is taken out from jets group to have reasonable statistics for the efficiency measurement. By comparing the product of efficiencies with total efficiency of applying cut groups in series, the cut groups are found to be anti-correlated which would result in an over-estimate of the yield. The result of 508 events is kept to be conservative [318].

8.4.1.4. Systematic uncertainties. The following sources of systematic uncertainty are common for all three channels: (i) the theoretical errors to the total rates of the signal is $\Delta_{\text {th }} \approx 4 \%$, rising to $10 \%$ for $t W$. The uncertainties in the background events are assumed to be: $5 \%$ for $t \bar{t}$ [45], $17 \%$ for $W b \bar{b} j, 7 \%$ for $W+$ jets, $5 \%$ for $W j j$ [319], and $5 \%$ for $W b \bar{b}$. (ii) the jet energy scale (JES) uncertainty: using a calibration method based on $t \bar{t}$ events [320], the JES uncertainty after $10 \mathrm{fb}^{-1}$ integrated luminosity is expected to be $\pm 5 \%( \pm 2.5 \%)$ for jets with $p_{\mathrm{T}} \approx 20 \mathrm{GeV} / \mathrm{c}\left(p_{\mathrm{T}}>50 \mathrm{GeV} / \mathrm{c}\right)$. In the region between 20 and $50 \mathrm{GeV} / \mathrm{c}$ a linear dependence is assumed. (iii) b-tagging identification uncertainty: of $\pm 4 \%$ on the overall selection efficiencies is expected on the $b$-tagging efficiencies [157]. (iv) the luminosity uncertainty, expected to be $5 \%$ [321].

\subsubsection{Selection and cross section: $t$-channel}

The final state in $t$-channel includes one isolated muon, missing energy (neutrino), one or two jets from $b$-quarks $\left(B_{\text {jet }}\right)$, and one "forward" hadronic jet. A specific feature of single top events is production of a light jet in the forward/backward direction (see Figs. 8.15) providing an additional possibility for background suppression. The additional $b$-quark is produced with small transverse momentum, making the reconstruction of the associated low- $p_{\mathrm{T}}$ jet and its $b$-tagging very difficult. Therefore, in $t$-channel analysis [317] it is required to have only two hadronic jets in the final state. In this case, the most important background contribution arises from $t \bar{t}$ production and from $W^{ \pm}$-boson production in association with heavy quarks $(W b \bar{b}+$ jet $)$ or light quark jets $(W+$ jets $)$.

8.4.2.1. Analysis of the fully simulated events. The selection requires the presence of only one isolated muon with $p_{\mathrm{T}}>19 \mathrm{GeV} / \mathrm{c}$ and $\left|\eta_{\mu}\right|<2.1$ (HLT selection). Then, it is required: (i) $E_{\mathrm{T}}^{\mathrm{miss}}>40 \mathrm{GeV}$; and (ii) at least two hadronic uncalibrated jets, with $p_{\mathrm{T}}>20 \mathrm{GeV} / \mathrm{c}$. For 

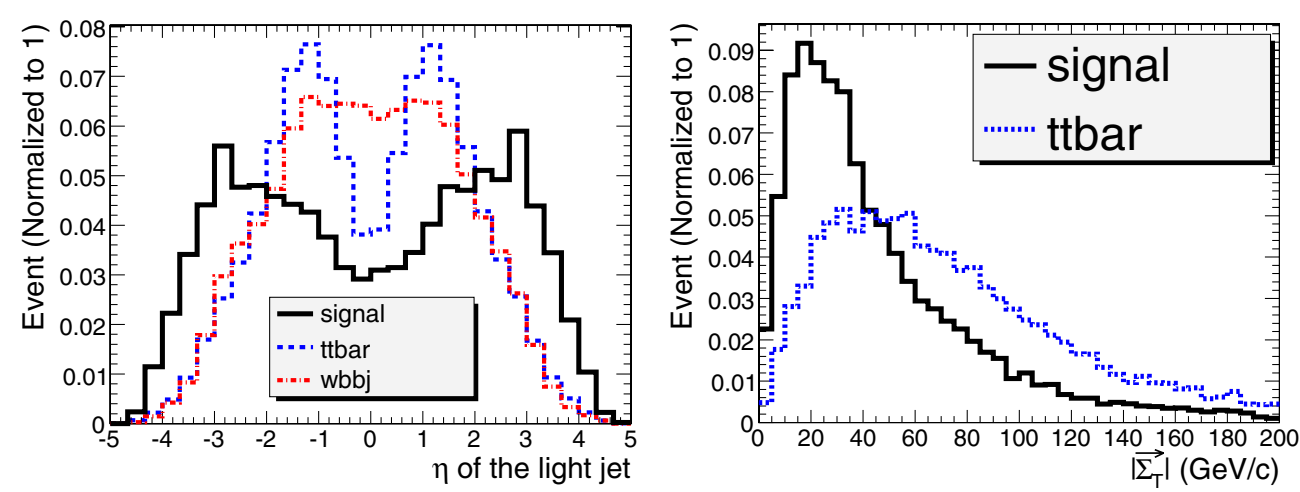

Figure 8.15. The distributions of pseudorapidity $(\eta)$ of the light jet (left), and of $\left|\vec{\Sigma}_{\mathrm{T}}\right|$ (right).

Table 8.17. Number of events ( $t$-channel) and cumulative efficiencies for each cut used in the analysis of $t$-channel single top production. The symbol " $p_{\mathrm{T} B} \times p_{\mathrm{T} j} \times E_{\mathrm{T}}^{\text {miss" }}$ means: $p_{\mathrm{T} B}>$ $35 \mathrm{GeV} / \mathrm{c}, p_{\mathrm{T} j}>40 \mathrm{GeV} / \mathrm{c},\left|\eta_{j}\right|>2.5, E_{\mathrm{T}}^{\mathrm{miss}}>40 \mathrm{GeV}$.

\begin{tabular}{lccccc}
\hline & signal & $t \bar{t}$ & $W b \bar{b} j$ & $W j$ & $W j j$ \\
\hline $\mathrm{N}\left(\right.$ events) at $10 \mathrm{fb}^{-1}$ & $1.8 \times 10^{5}$ & $8.33 \times 10^{6}$ & $3.24 \times 10^{5}$ & $9.7 \times 10^{7}$ & $9.9 \times 10^{5}$ \\
isolated muon & 0.73 & 0.14 & 0.52 & 0.16 & 0.81 \\
$p_{\mathrm{T} B} \times p_{\mathrm{T} j} \times E_{\mathrm{T}}^{\text {miss }}$ & 0.036 & $6.4 \times 10^{-3}$ & $3.4 \times 10^{-3}$ & $9 \times 10^{-6}$ & $3 \times 10^{-3}$ \\
veto on $3^{r d}$ jet & 0.021 & $5.8 \times 10^{-4}$ & $1.6 \times 10^{-3}$ & $4 \times 10^{-6}$ & $1.1 \times 10^{-3}$ \\
$0.0<\Sigma_{\mathrm{T}}<43.5 \mathrm{GeV}$ & 0.018 & $4.1 \times 10^{-4}$ & $1.2 \times 10^{-3}$ & $4 \times 10^{-6}$ & $6.8 \times 10^{-4}$ \\
$50<M_{\mathrm{T}}^{W^{*}}<120$ & 0.015 & $2.2 \times 10^{-4}$ & $9.6 \times 10^{-4}$ & $1 \times 10^{-6}$ & $5.4 \times 10^{-4}$ \\
$110<M_{\text {rec }}(b W)^{*}<210$ & 0.013 & $1.4 \times 10^{-4}$ & $5.8 \times 10^{-4}$ & 0 & $4.1 \times 10^{-4}$ \\
Number of events & 2389 & 1188 & 195 & 0 & 402 \\
\hline
\end{tabular}

further analysis the following additional requirements are: at least one of the selected jets should have the $b$-tag: the second (light) jet should be in the forward region; only two jets (calibrated) with $p_{\mathrm{T}}^{\text {calib }} \geqslant 35 \mathrm{GeV}$ and no other hadronic jets with $p_{\mathrm{T}}^{\text {calib }} \geqslant 35 \mathrm{GeV} / \mathrm{c}$ (jet veto). The GARCON program [63] is used for the final optimisations of the cuts. The signal-overbackground ratio times significance is chosen as an optimisation criterion. Finally, the optimal cut values found are:

- muon: $p_{\mathrm{T}}(\mu)>19.0 \mathrm{GeV} / \mathrm{c}$ and $|\eta(\mu)|<2.1$ and $E_{\mathrm{T}}^{\mathrm{miss}}>40.0 \mathrm{GeV}$;

- b-jet: $p_{\mathrm{T}}>35.0 \mathrm{GeV} / \mathrm{c},|\eta|<2.5$ and Discriminator $>2.4$;

- the light forward: $p_{\mathrm{T}}>40.0 \mathrm{GeV} / \mathrm{c}$ and $|\eta|>2.5$;

- $\left|\vec{\Sigma}_{\mathrm{T}}\right|$ cut window: $(0.0,43.5) \mathrm{GeV} ; 50<M_{\mathrm{T}}^{W}<120 \mathrm{GeV} / \mathrm{c}^{2}$;

- the reconstructed top mass window: $110 \mathrm{GeV} / \mathrm{c}^{2}<M_{\text {rec }}(b W)<210 \mathrm{GeV} / \mathrm{c}^{2}$.

The efficiencies of these cuts and the resulting number of events are given in the Table 8.17. The resulting signal-to-background ratio and the significance are: $N_{S} / N_{B}=1.34$ and $S_{\text {stat }}=N_{S} / \sqrt{N_{S}+N_{B}}=37.0$. The final distribution of the reconstructed top mass is shown in Figure 8.16. The cuts provide a satisfactory background suppression.

The systematic uncertainties (see Section 8.4.1.4) evaluated for $10 \mathrm{fb}^{-1}$ are given in Table 8.18 . In summary, the statistical error is $2.7 \%$, the total systematic error excluding the $5 \%$ luminosity uncertainty is $8 \%$, resulting in a total error of $10 \%$. 

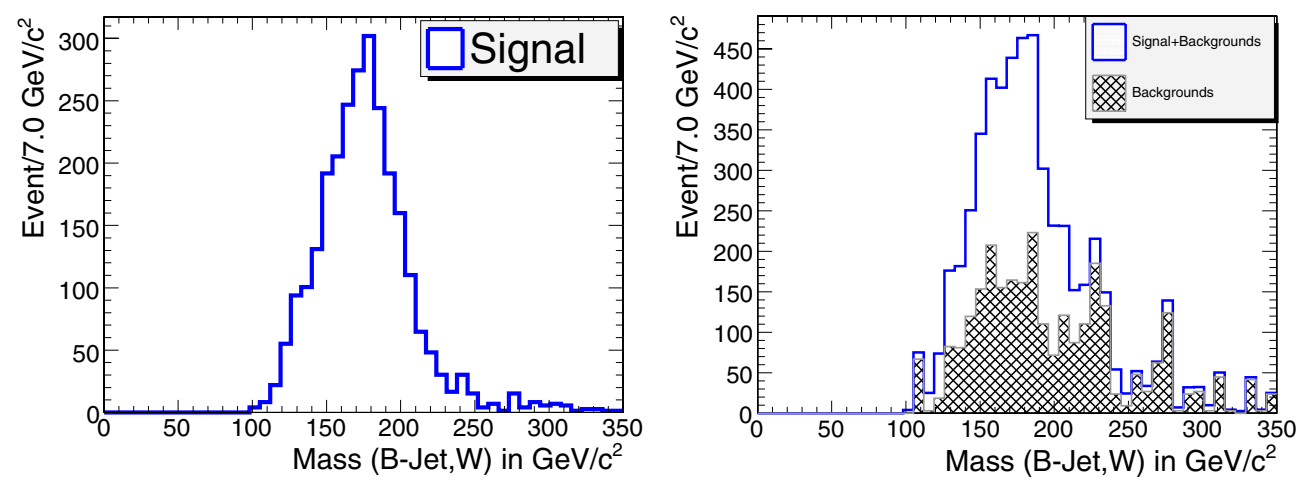

Figure 8.16. The distribution on the reconstructed top mass, for signal only (left) and with background included (right).

Table 8.18. Number of selected events ( $t$-channel) at $10 \mathrm{fb}^{-1}$ with uncertainties due to different sources. $\Delta N_{\text {syst }}$ represents the theoretical, JES and $b$-tagging uncertainties. $\Delta N_{\text {stat }}$ is expected statistical uncertainty.

\begin{tabular}{lrrrcrc}
\hline sample & selected & $\Delta N_{t h}$ & JES & $\Delta N_{\mathrm{b}-\text { tag }}$ & $\Delta N_{\text {syst }}$ & $\Delta N_{\text {stat }}$ \\
\hline$t$-channel & 2389 & 96 & 71 & 96 & 153 & 49 \\
$t \bar{t}$ & 1188 & 59 & 73 & 48 & 105 & 34 \\
$W b \bar{b} j$ & 195 & 33 & 6 & 8 & 35 & 14 \\
$W j j$ & 402 & 20 & 0 & 16 & 26 & 20 \\
\hline
\end{tabular}

\subsubsection{Selection and cross section: $t W$-channel}

The $p p \rightarrow t W$ process contains two $W$-bosons and a $b$-quark in the final state. In this study only leptonic decays of the $W$ 's are considered. The nominal final states are $\ell^{+} \ell^{-} E_{\mathrm{T}}^{\text {miss }} b$ and $\ell^{ \pm} E_{\mathrm{T}}^{\mathrm{miss}} b \mathrm{jj}$ for the dileptonic and semi-leptonic modes, respectively. The dominant background arises from $t \bar{t}$ production. Other backgrounds are $t$ - and $s$-channel single top production, $W b \bar{b}, W+$ jets, $W W+$ jets, and to a lesser extent QCD multi-jet background.

8.4.3.1. Jet quality requirements and extra jet reduction. The most significant difference between $t W$ events and $t \bar{t}$ events is the number of jets in the final state. However, most of the time there are also additional jets due to the underlying event, pile-up or calorimeter noise. These "extra jets" were identified and excluded from the counting by consideration of five jet quality variables (see [318]). It was found that the most discriminating variables are $E_{\mathrm{T}}^{\max }$ (the maximum tower $E_{\mathrm{T}}$ in a cone of 0.5 ) and $N_{\text {track }}$ (the number of associated tracks). A Fisher discriminant [322] $(F)$ is constructed from the jet quality variables to separate real jets from extra jets. Each jet is classified value $F$ into one of three categories: $\operatorname{good}(F<-0.5)$, loose $(|F|<0.5)$ and bad $(F \geqslant 0.5)$ jets. This method yields $84.3 \%$ efficiency on true jets and rejects $86.9 \%$ of extra jets. Only "good" jets and "loose" jets are used in pre-selection and event reconstruction. The jet multiplicity after the extra jet reduction in semi-leptonic channels reveals that the number of good jets peaks at the 2 and 3 jet bins for signal events, and at the 3 and 4 jet bins for $t \bar{t}$ backgrounds.

8.4.3.2. Event selection and reconstruction. The kinematic cuts used for this study are presented in Table 8.19 and Table 8.20. For the semi-leptonic channel, two non- $b$-like jets with $m_{j j}<115 \mathrm{GeV} / \mathrm{c}^{2}$ are used for reconstruction of the $W$-boson (that decays hadronically). In 
Table 8.19. Kinematic cuts used in the dileptonic channel. The final electron and muon should have the opposite charges.

\begin{tabular}{ll}
\hline Leptons & Jets \\
\hline$|\eta(e)|<2.4,|\eta(\mu)|<2.1$ & leading jet: $|\eta|<2.4, p_{\mathrm{T}}>60 \mathrm{GeV} / \mathrm{c}$, disc $>0$ \\
$p_{\mathrm{T}}(e, \mu)>20 \mathrm{GeV} / \mathrm{c}$ & at most one extra jet \\
no other lepton with $p_{\mathrm{T}}>5 \mathrm{GeV} / \mathrm{c}$ & No other jets with $p_{\mathrm{T}}>20 \mathrm{GeV} / \mathrm{c}$ \\
Missing $E_{\mathrm{T}}: E_{\mathrm{T}}^{\text {miss }}>20 \mathrm{GeV}$ & \\
\hline
\end{tabular}

Table 8.20. Kinematic cuts used in the semi-leptonic channel. The presence of a good fourth jet would veto the whole event.

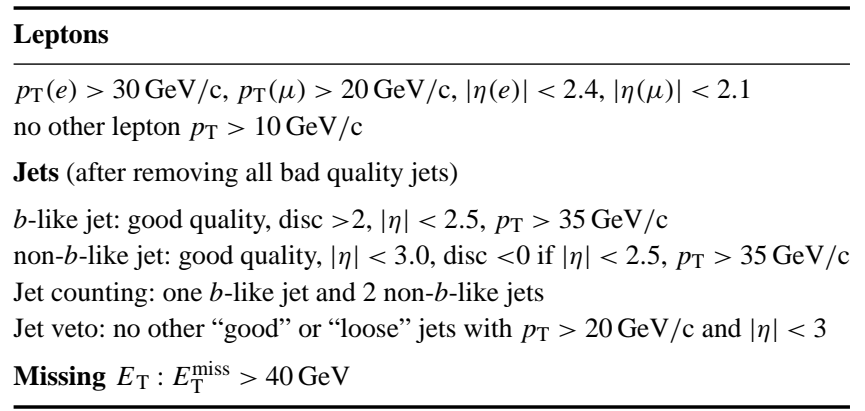

events with a 4th jet that survives jet veto cuts, it is required that the invariant mass of the 4th jet with any of the selected non- $b$-like jets must be outside a window of $M_{W} \pm 20 \mathrm{GeV} / \mathrm{c}^{2}$. For the leptonic decays of the $W$-boson it is required that $M_{\mathrm{T}}^{W}<120 \mathrm{GeV} / \mathrm{c}^{2}$.

To find the correct pairing of $b$-jet and reconstructed $W$-boson (coming from top decay) the following variables were used: the $p_{\mathrm{T}}$ of $(b, W)$ systems; the separation of the $b$-jet with each of the $W$ in $(\eta, \phi)$ space; the "charges" of jets (see Section 8.4.1.2) and $W$ bosons (see Ref. [318] for details). A Fisher discriminant based on these variables is used for discriminating leptonic top events from hadronic top events. A cut of 0.56 is optimal in separating these 2 types of events, and $72 \%$ of the events are correctly paired.

To further enhance the signal to background ratio the following "global" cuts are applied:

- $p_{\mathrm{T}}$ of the reconstructed $t W$ system: $|\vec{\Sigma}(t+W)|<60 \mathrm{GeV} / \mathrm{c}$.

- Scalar sum of transverse energies $H_{\mathrm{T}}: H_{\mathrm{T}}<850 \mathrm{GeV}$.

- Reconstructed top quark mass: $110 \mathrm{GeV} / \mathrm{c}^{2}<m(t)<230 \mathrm{GeV} / \mathrm{c}^{2}$.

- $p_{\mathrm{T}}$ of the reconstructed top quark: $20 \mathrm{GeV} / \mathrm{c}<p_{\mathrm{T}}(t)<200 \mathrm{GeV} / \mathrm{c}$.

8.4.3.3. Efficiencies and expected yields. The efficiencies estimated with Monte Carlo samples are converted to the effective cross sections by multiplying the production cross sections of each process. The effective cross sections, as well as the expected yields with $10 \mathrm{fb}^{-1}$ of data for all signal and background samples, are shown in Table 8.21 and 8.22. The signal to background ratio is found to be 0.37 for dileptonic channel and 0.18 for semi-leptonic channel.

8.4.3.4. The ratio method. The ratio method is developed to reduce systematic uncertainties related to the dominant $t \bar{t}$ background. We define a $t \bar{t}$-rich control region and use ratio of efficiencies to estimate the yield of $t \bar{t}$ in the signal region. The kinematics of $t W$ and $t \bar{t}$ are similar so $t W$ is present in the control region, therefore the ratio of efficiencies for $t W$ is also 
Table 8.21. Summary of cross section times branching ratio times efficiencies at each stage of the analysis for the dileptonic channel. All values are in picobarns The last row is the expected number of events for $10 \mathrm{fb}^{-1}$. Multi-jet background has been estimated separately (see Section 8.4.1.3). When only a limit on the number of events is stated, this is due to MC statistics.

\begin{tabular}{lcccccc}
\hline & $t W$ dil. & $t \bar{t}$ dil. & $t \bar{t}$ oth. & WW dil. & WW oth. & t ch. lept. \\
\hline Production & 6.667 & 92.222 & 737.778 & 11.111 & 88.889 & 81.667 \\
HLT & 4.865 & 74.090 & 346.151 & 7.674 & 27.259 & 41.409 \\
$2 \ell$ & 1.944 & 25.150 & 21.012 & 2.574 & 0.226 & 2.309 \\
Lepton $p_{\mathrm{T}}$ & 0.675 & 7.919 & 0.703 & 0.543 & 0.012 & 0.098 \\
$\leqslant 1$ extra jet & 0.459 & 6.574 & 0.664 & 0.416 & 0.010 & 0.067 \\
Jet $p_{\mathrm{T}}, \eta$ & 0.307 & 5.234 & 0.556 & 0.339 & 0.004 & 0.033 \\
$\geqslant 1 b$-jet & 0.184 & 3.864 & 0.379 & 0.017 & 0.000 & 0.018 \\
$E_{\mathrm{T}}^{\text {miss }}>20$ & 0.170 & 3.640 & 0.349 & 0.017 & 0.000 & 0.016 \\
$\leqslant 2$ jet & 0.150 & 2.734 & 0.221 & 0.015 & 0.000 & 0.012 \\
Final select. & 0.057 & 0.145 & 0.000 & 0.006 & 0.000 & 0.000 \\
Expected events & 567 & 1450 & $\leqslant 55$ & 61 & $\leqslant 10$ & $\leqslant 20$ \\
\hline
\end{tabular}

Table 8.22. Summary of cross section times branching ratio times efficiencies at each stage of the analysis for the semi-leptonic channel. All values are in picobarns. The last row is the expected number of events for $10 \mathrm{fb}^{-1}$.

\begin{tabular}{lccccccccc}
\hline & $\mathrm{tW}$ & $t \bar{t}$ & $\mathrm{tch}$. & $\mathrm{s} \mathrm{ch}$. & $\mathrm{Wbb}$ & $\mathrm{W} 2 \mathrm{j}$ & $\mathrm{W} 3 \mathrm{j}$ & $\mathrm{W} 4 \mathrm{j}$ & Multi-jet \\
\hline Total cross section & 60 & 833 & 245 & 10 & 300 & 7500 & 2166 & 522 & $9.73 \times 10^{9}$ \\
HLT & 18.9 & 263.9 & 39.5 & 1.52 & 34.0 & 1006 & 300 & 73 & $1.86 \times 10^{5}$ \\
Presel. \& isolation & 9.05 & 179.4 & 12.0 & 0.54 & 2.15 & 52 & 35 & 12 & 1325 \\
jet \& lepton p, jet veto & 1.28 & 18.5 & 1.31 & 0.046 & 0.061 & 0.60 & 4.9 & 1.0 & 4.23 \\
$b$-tagging & 0.669 & 6.13 & 0.476 & 0.013 & 0.016 & 0.10 & 0.99 & 0.26 & 0.85 \\
kinematic cuts & 0.223 & 0.999 & 0.047 & 0.002 & 0.003 & 0.017 & 0.101 & 0.008 & 0.105 \\
Signal box cuts & 0.170 & 0.771 & 0.035 & 0.001 & 0.001 & 0.013 & 0.054 & 0.008 & 0.051 \\
Events in 10 fb & 1699 & 7709 & 351 & 14 & 10 & 130 & 539 & 80 & 508 \\
\hline
\end{tabular}

used. The signal and background yield is determined by the following equations:

$$
\begin{aligned}
& S=\frac{R_{t \bar{t}}\left(N_{s}-N_{s}^{o}\right)-\left(N_{c}-N_{c}^{o}\right)}{R_{t \bar{t}}-R_{t W}}, \\
& B=\frac{\left(N_{c}-N_{c}^{o}\right)-R_{t W}\left(N_{s}-N_{s}^{o}\right)}{R_{t \bar{t}}-R_{t W}}+N_{s}^{o} .
\end{aligned}
$$

Here $R_{x}$ is the ratio of efficiencies $R_{x}=\varepsilon_{x}$ (control region) $/ \varepsilon_{x}$ (signal region) for $x=t \bar{t}, t W$; $N_{s}\left(N_{c}\right)$ is total number of events in the signal (control) region; $N_{s}^{o}\left(N_{c}^{o}\right)$ is the estimated number of non-t $t \bar{t}$ background events in the signal (control) region. With $S$ measured with 2 regions and the ratio method, the cross section can be found by $S / \epsilon \mathcal{L}$.

For the ratio method to work it is important to find a control region with similar kinematics except with one more jet. It is expected that systematic uncertainties from PDF, JES and $\mathrm{b}$ tagging cancel to a large extent, while the luminosity uncertainty drops out for the $t \bar{t}$ background. The lepton selection and jet quality requirements in the control region are identical to the signal region. The differences are outlined below.

Dileptonic. A second jet is required with $p_{\mathrm{T}}=20-80 \mathrm{GeV} / \mathrm{c},|\eta|<2.4$ and $b$-tagged (disc $>0$ ). No other jets with $p_{\mathrm{T}}>20 \mathrm{GeV} / \mathrm{c}$ are allowed. The background region is found to be filled by $97.9 \%$ dileptonic $t \bar{t}, 0.4 \%$ other $t \bar{t}$ decays, $1.6 \%$ dileptonic $t W$, and $0.1 \%$ for leptonic t channel single top while $\mathrm{WW}+$ jets yield is negligible. 
Table 8.23. Summary of uncertainties of cross section measurement

\begin{tabular}{llll}
\hline Source & Uncertainty & $\boldsymbol{\Delta} \boldsymbol{\sigma} / \boldsymbol{\sigma}$ (dilept.) & $\boldsymbol{\Delta} \boldsymbol{\sigma} / \boldsymbol{\sigma}$ (semi-lept.) \\
\hline Statistical uncertainty & - & $8.8 \%$ & $7.5 \%$ \\
Integrated luminosity & $5 \%$ & $5.4 \%$ & $7.8 \%$ \\
$t \bar{t}$ cross-section & $9 \%$ & negligible & negligible \\
$t$-channel cross-section & $5 \%$ & negligible & $0.8 \%$ \\
W+jets cross-section & $10 \%$ & not applicable & $3.1 \%$ \\
WW+jets cross-section & $10 \%$ & $1 \%$ & not applicable \\
Jet energy scale & $5 \%-2.5 \%$ & $19.7 \%$ & $9.4 \%$ \\
b tagging efficiency & $4 \%-5 \%$ & $8.7 \%$ & $3.6 \%$ \\
PDF & $1 \sigma$ & $+4 \% /-6.0 \%$ & $1.6 \%$ \\
Pileup & $30 \%$ & $6.1 \%$ & $10.3 \%$ \\
MC statistics & - & $9.9 \%$ & $15.2 \%$ \\
Total uncertainty & & $\pm 23.9 \%$ (syst.) & $\pm 16.8 \%$ (syst.) \\
& & $\pm 9.9 \%$ (MC) & $\pm 15.2 \%$ (MC) \\
\hline
\end{tabular}

Semi-leptonic. It requires 2 jets with $p_{\mathrm{T}}>30,2$ more jets with $p_{\mathrm{T}}>20$, and no bad jets with $p_{\mathrm{T}}>20$. It is required that one of the 2 high- $p_{\mathrm{T}}$ jets is b-tagged (disc $>2$ ), and that both low $-p_{\mathrm{T}}$ jets be not tagged (disc $\left.<0\right)$. The $b-W$ pairing is done in the same way, with a $72 \%$ correct pairing. It is found that the $t \bar{t}$ purity in the control region is $93.9 \%$. The non- $t \bar{t}$ events are mainly composed of $\mathrm{W}+$ jets $(2.8 \%), t W(2.0 \%)$ and $t$-channel single top $(1.2 \%)$. The ratio of efficiencies are found to be $R_{t W}=0.319$ and $R_{t \bar{t}}=3.31$.

\subsubsection{Systematic uncertainties.}

- Theoretical uncertainties. The $t \bar{t}$ cross section does not show up in the ratio method. The effect is $0.8 \%$ for $t$-channel single top and $3.1 \%$ for $W+$ jets. It is found to be negligible for other background.

- Pileup amount. A difference of $30 \%$ between normal pileup and no pileup is used as an estimate of the systematic uncertainty, as was done in [201] for the dileptonic $t \bar{t}$ studies.

Dileptonic mode. The analysis is found to be rather sensitive to the pileup, as the relative shift of the "measured" cross section is $+20.4 \%$ for no pileup, and $-16.2 \%$ for double pileup, while is the difference between the check sample and the reference sample $4.6 \%$ (which has purely statistical origin). The value of $6.1 \%$ is used as the systematic uncertainty.

Semi-leptonic mode. The extracted cross section varies by $+35 \%$ for no pileup and $-63 \%$ for double pile-up so a systematic uncertainty of $10.3 \%$ is obtained. The results for both channels are shown in Table 8.23.

The results from the ratio method were used in the significance calculation. In addition, the uncertainty on the background expectation, evaluated for dileptonic $\left(\Delta_{B} / B= \pm 9.6 \%\right)$ and semi-leptonic $\left(\Delta_{B} / B=+3.6 \% /-4.4 \%\right)$, was taken into account. The resulting significance is 4.2 for the dileptonic channel and 5.1 for the semi-leptonic channel. Combining the two channels gives a total significance of 6.4.

\subsubsection{Selection and cross section: s-channel}

The present analysis of the $s$-channel single top production is based on leptonic channels, i.e. the top is identified and reconstructed by its semi-leptonic decays into $\ell v b$ final states, with $\ell=e, \mu$. For this study, a fast simulation of the CMS detector with FAMOs was used, see $[317,318]$ for details. 
Table 8.24. Efficiencies of the pre-selection cuts, with respect to the initial number of events For all process (except of $t \bar{t})$ the final $W$ decays into charged lepton $(\ell=e, \mu, \tau)$ and neutrino. "HLT" includes the $1 \mu, 1 e$ and $e \times j$ triggers. $N_{e v}$ is the number of events surviving these cuts (the uncertainties are only those due to the limited Monte Carlo statistics).

\begin{tabular}{lccccc}
\hline Cut & $s$-ch. & $t$-ch. & $t \bar{t}$ & $W b \bar{b}$ & $W t(1 W \rightarrow l v)$ \\
\hline "HLT" & $37.5 \pm 0.2 \%$ & $42.5 \pm 0.1 \%$ & $30.1 \pm 0.1 \%$ & $29.4 \pm 0.1 \%$ & $46.5 \pm 0.1 \%$ \\
Isolation & $33.7 \pm 0.2 \%$ & $39.0 \pm 0.1 \%$ & $21.7 \pm 0.1 \%$ & $28.2 \pm 0.1 \%$ & $42.3 \pm 0.1 \%$ \\
$E_{\mathrm{T}}^{\text {miss }}$ cut & $27.3 \pm 0.2 \%$ & $31.9 \pm 0.1 \%$ & $17.4 \pm 0.1 \%$ & $22.6 \pm 0.1 \%$ & $34.4 \pm 0.1 \%$ \\
$M_{\mathrm{T}}^{W}$ cut & $23.2 \pm 0.2 \%$ & $26.3 \pm 0.1 \%$ & $13.6 \pm 0.1 \%$ & $18.4 \pm 0.1 \%$ & $29.2 \pm 0.1 \%$ \\
$N_{j} \geqslant 2 j$ & $11.9 \pm 0.1 \%$ & $11.5 \pm 0.1 \%$ & $11.9 \pm 0.1 \%$ & $0.88 \pm 0.03 \%$ & $18.5 \pm 0.1 \%$ \\
$N_{j}=2 j$ & $8.9 \pm 0.1 \%$ & $8.2 \pm 0.1 \%$ & $1.84 \pm 0.04 \%$ & $0.76 \pm 0.03 \%$ & $7.09 \pm 0.05 \%$ \\
$b$-tag & $3.07 \pm 0.07 \%$ & $0.72 \pm 0.02 \%$ & $0.28 \pm 0.02 \%$ & $0.14 \pm 0.01 \%$ & $0.34 \pm 0.01 \%$ \\
$N_{\mathrm{ev}}$ & $1010 \pm 10$ & $5880 \pm 70$ & $23300 \pm 200$ & $1400 \pm 35$ & $1150 \pm 40$ \\
\hline
\end{tabular}

The signal events are triggered by the single lepton triggers. Since this production mode suffers from low statistics, one could envisage the introduction of a combined trigger $e \times j e t$, with threshold $19 \mathrm{GeV} / \mathrm{c}$ for the electron (in order to make the electronic sample more coherent with the muonic sample) and $45 \mathrm{GeV} / \mathrm{c}$ for the jet. This value has been chosen to be the same as the threshold for the $\tau$-jet in the already existing $e \times \tau-j e t$ trigger.

\subsubsection{Pre-selection. The pre-selection criteria are as follows:}

- The event has to fire at least one of the previously described triggers (including the proposed $e \times j)$.

- The event must contain one isolated lepton $\left(\mu\right.$ or $e$ ) with $p_{\mathrm{T}} \geqslant 19 \mathrm{GeV} / \mathrm{c}$ and $|\eta| \leqslant 2.1$ $(\leqslant 2.4)$ for muons (electrons) and no other lepton above $10 \mathrm{GeV} / \mathrm{c}$.

- Exactly two uncalibrated jets must have $p_{\mathrm{T}} \geqslant 30 \mathrm{GeV} / \mathrm{c}$ and $|\eta| \leqslant 2.5$ and no other jet has to be present with $p_{\mathrm{T}} \geqslant 20 \mathrm{GeV} / \mathrm{c}$.

- Both jets should have a positive b-tagging discriminator value.

- The event should have $E_{\mathrm{T}}^{\mathrm{miss}}>30 \mathrm{GeV}$.

- The transverse mass of the $W$-boson $M_{\mathrm{T}}^{W}$ should be less than $100 \mathrm{GeV} / \mathrm{c}^{2}$.

Details on the effect of the pre-selection cuts are given in Table 8.24. Note, that as in Section 8.4.2, the multi-jet QCD contribution is neglected.

8.4.4.2. Genetic algorithm analysis. The following observables have been chosen in order to further discriminate between signal and background after pre-selection: (i) the jet $b$-tagging discriminants; (ii) the calibrated jet transverse momenta; (iii) the mass of the reconstructed top; (iv) $|\Sigma(t, \bar{b})|$; (v) the scalar sum of the transverse momenta of all the reconstructed objects. The reconstructed top quark is formed by the reconstructed $W$ and one of the two $b$-jets, chosen according to the value of the "jet charge" $\left(Q_{j}\right.$, see Section 8.4.1.2). Since in top decays the $W$ and the original $b$ quark have opposite sign of the charge, the jet with $Q_{j}$ "most opposite" to the $W$ is used for top reconstruction, leading to a probability of $67 \%$ to identify the correct pairing.

The cuts on these variables are optimised by means of the GARCON program [63]. The surviving events after these cuts are shown in cascade in Table 8.25. With this selection, after an integrated luminosity of $10 \mathrm{fb}^{-1}$ one gets: $N_{S} / N_{B} \approx 0.13$. 
Table 8.25. Final cuts and their efficiencies, with respect to the preselected samples, for the signal and the main backgrounds. For $s$ - and $t$-channel and $W b \bar{b}$ samples the final $W$-boson decays into lepton $(e, \mu, \tau)$ and neutrino. $t \bar{t}$ samples includes all $W$-boson decay modes.

\begin{tabular}{lllll}
\hline Cut & $s$-channel & $t$-channel & $t \bar{t}$ & $W b \bar{b}$ \\
\hline $\mathrm{b}-\operatorname{tag}\left(j_{1}\right)>0.4, \mathrm{~b}-\operatorname{tag}\left(j_{2}\right)>0.1$ & $85 \%$ & $75 \%$ & $78 \%$ & $85 \%$ \\
$p_{\mathrm{T}}\left(j_{1}\right)>50 \mathrm{GeV} / \mathrm{c}, p_{\mathrm{T}}\left(j_{2}\right)>50 \mathrm{GeV} / \mathrm{c}$ & $68 \%$ & $53 \%$ & $70 \%$ & $37 \%$ \\
$120<M(l v b)<220 \mathrm{GeV} / \mathrm{c}^{2}$ & $52 \%$ & $34 \%$ & $46 \%$ & $26 \%$ \\
$25<p_{\mathrm{T}}(l v b)<160 \mathrm{GeV} / \mathrm{c}$ & $48 \%$ & $32 \%$ & $43 \%$ & $26 \%$ \\
$\Sigma_{\mathrm{T}}<20 \mathrm{GeV} / \mathrm{c}$ & $35 \%$ & $15 \%$ & $10.6 \%$ & $12.5 \%$ \\
$H_{\mathrm{T}}<340 \mathrm{GeV} / \mathrm{c}$ & $27 \%$ & $10.7 \%$ & $5.4 \%$ & $11.1 \%$ \\
number of surviving events & $273 \pm 4$ & $630 \pm 14$ & $1260 \pm 60$ & $155 \pm 12$ \\
\hline
\end{tabular}

Table 8.26. Number of selected events after $10 \mathrm{fb}^{-1}$ and systematic uncertainties.

\begin{tabular}{lccccccc}
\hline sample & selected & $\Delta \sigma$ & JES & b-tag & $M_{\text {top }}$ & PDF & ISR/FSR \\
\hline$S: s$-channel & 273 & - & \pm 3 & \pm 11 & \pm 1.5 & \pm 2 & \pm 1.5 \\
$B: t$-channel & 630 & \pm 25 & \pm 8 & \pm 25 & - & - & - \\
$B: t \bar{t}$ & 1260 & \pm 63 & \pm 75 & \pm 50 & - & - & - \\
$B: W b \bar{b}$ & 155 & \pm 8 & \pm 7 & \pm 6 & - & - & - \\
\hline
\end{tabular}

8.4.4.3. Systematic uncertainties. In addition to systematics described in Section 8.4.1.4 the following sources of systematic uncertainty are considered:

- Top mass. The variation of $m_{t}$ within $\pm 2 \mathrm{GeV} / \mathrm{c}^{2}$ around top mass $m_{t}=175 \mathrm{GeV} / \mathrm{c}^{2}$ leads to the relative systematic error on the selection efficiency $\sigma_{\text {syst }}^{m_{\mathrm{t}}}=0.5 \%$ for the $s$-channel single top.

- Parton Distribution Functions. To extract the dependence on the PDF uncertainty, two different PDF sets were used: CTEQ61 and CTEQ6M [12]. The result is $\sigma_{\text {syst }}^{\mathrm{PDF}}=0.7 \%$.

- Initial/Final State Radiation Modelling. The model parameters were varied in the ranges $\Lambda_{\mathrm{QCD}}=0.25 \pm 0.1 \mathrm{GeV}$ and $Q_{\max }^{2}$ from 0.25 to $4 \hat{s}$ (see [201]). The extreme values of the efficiencies are taken as systematic error: $\sigma_{\text {syst }}^{\text {rad }}=0.5 \%$.

8.4.4.4. Background normalisation. The $t \bar{t}$ events in Table 8.26 are, in $41 \%$ of the cases, $t \bar{t} \rightarrow l^{+} v b l^{-} \bar{v} \bar{b}$ events with a lepton missed, and in the remain cases $t \bar{t} \rightarrow l^{+} v b q \bar{q}^{\prime} \bar{b}$ events with two jets missed ( $t \bar{t} \rightarrow q \bar{q}^{\prime} b q \bar{q}^{\prime} \bar{b}$ events give a negligible contribution). These two categories of events are very differently affected by the Jet Energy Scale variation. In general, any variation going in the direction of more jets gives a better rejection of the $t \bar{t} \rightarrow l^{+} v b q \bar{q}^{\prime} \bar{b}$ component with respect to the signal, while the $t \bar{t} \rightarrow l^{+} \nu b l^{-} \bar{v} \bar{b}$ events, having two quarks, are affected almost in the same way as the signal.

- $t \bar{t} \rightarrow \ell^{ \pm}+X$ enriched control sample. In this case the difference with respect to Section 8.4.4.1 is the request of three jets instead of two and only the muon channel is used. The selection efficiency for $t \bar{t} \rightarrow \ell^{ \pm}$events is found to be $1.08 \%$. The ratio $R_{c 1}$ between the efficiencies in the main sample and in this control sample is $R_{c 1}=$ 0.0149 , whose variations under JES and b-tagging efficiency systematic shifts are $\Delta R_{c 1}=$ \pm 0.0015 (JES) \pm 0.0003 (b-tag).

- $t \bar{t} \rightarrow \ell^{+\ell^{-}}+X$ enriched control sample. This sample is obtained by the same selection as in Section 8.4.4.1, but two leptons with different flavours with the opposite sign are required. The selection efficiency for $t \bar{t} \rightarrow 2 l$ events is found to be $0.822 \%$. The ratio $R_{c 2}$ between the efficiencies in the main sample and in this control sample is $R_{c 2}=0.0681$, whose 
variations under JES and b-tagging efficiency systematic shifts are $\Delta R_{c 2}= \pm 0.0010$ (JES) \pm 0.0004 (b-tag).

8.4.4.5. Results. The number of the selected signal $\left(N_{S}\right)$ and background $\left(N_{B}\right)$ events and their estimated uncertainties are listed in Table 8.26. The cross section is extracted as

$$
\sigma=\frac{N_{t o t}-b^{0}-R_{c 1}\left(N_{c 1}-b_{c 1}^{0}\right)-R_{c 2}\left(N_{c 2}-b_{c 2}^{0}\right)}{\epsilon L},
$$

where $b^{0}$ is the sum of the non-top backgrounds in the main sample, $N_{c 1}$ and $N_{c 2}$ are the total events selected in the two control regions, and $b_{c 1}^{0}$ and $b_{c 2}^{0}$ are their contamination by non-top backgrounds, single top and other $t \bar{t}$ decays. The statistical error is evaluated to be $18 \%$. The total systematic uncertainty is $31 \%$, where the largest contribution arises from the effect of the JES uncertainty, on the $t \bar{t}$ single lepton background. The use of "Energy Flow" techniques, including the charged tracks information, is expected to significantly reduce this uncertainty. The total error, including also the $5 \%$ luminosity uncertainty, is $36 \%$.

\subsubsection{Conclusion}

Selection strategies have been proposed for all the three single top production modes, and their effectiveness is shown, taking into account the expected statistics after $10 \mathrm{fb}^{-1}$. All analyses will be systematics dominated. For the $s$-channel and $t W$-associated cases, control samples have been proposed in order to constrain the dominant $t \bar{t}$ background.

The resulting signal-to-background ratio and the significance for the $t$-channel are: $N_{S} / N_{B}=1.34$ and $S_{s t a t}=N_{S} / \sqrt{N_{S}+N_{B}}=37.0$, with a statistical error of $2.7 \%$, and a systematic error excluding the $5 \%$ luminosity uncertainty of $8 \%$, resulting in a total error of $10 \%$. For $t W$-channel we expect to reach the significance of 4.2 (5.1) for the dilepton (semileptonic) channel, increasing to 6.4 after combining the two channels. The total uncertainty is $\pm 23.9 \%$ (syst.) $\pm 9.9 \%$ (MC) for dilepton and $\pm 16.8 \%$ (syst.) $\pm 15.2 \%$ (MC) for semi-leptonic channels. The total systematic uncertainty for the $s$-channel is $31 \%$. The total error, including also the $5 \%$ luminosity uncertainty, is $36 \%$.

\subsection{Search for flavour changing neutral currents in top decays}

\subsubsection{Introduction}

The study of Flavour Changing Neutral Current (FCNC) interactions plays an important role in testing the Standard Model (SM) and probing new physics beyond it. The top quark is regarded to be more sensitive to new physics than other fermions, due to its mass close to the electroweak scale. Owing to the GIM mechanism of the SM, top quark FCNC interactions are absent at tree level and extremely small at loop level.

In recent years a lot of work has been done to explore the top quark FCNC couplings. On the theoretical side, various FCNC top quark decays and top-charm associated production at high energy colliders were extensively studied in the SM [323, 324], the Minimal Supersymmetric Standard Model (MSSM) [325-328] and other new physics models [329-333]. In models beyond the SM the top quark FCNC branching fractions may be significantly enhanced. Thus searching for top quark FCNC is a potentially powerful probe of new physics. The CDF and D $\emptyset$ collaborations have reported interesting bounds on the FCNC top quark decays [334-336]. The SM expectations for such top quark FCNC processes are far below the detectable level but the MSSM can enhance them by several orders of magnitude to make them potentially accessible at future collider experiments [337-339]. The theoretical branching ratios and the experimental limits are summarised in Table 8.27. Details of this analysis can be found in [340]. 
Table 8.27. Theoretical branching ratios of FCNC top quark decays in various models and experimental limits.

\begin{tabular}{lccccc}
\hline Decay & SM & two-Higgs & SUSY with $R$ & Exotic Quarks & Exper. Limits (95\% CL) \\
\hline$t \rightarrow g q$ & $5 \times 10^{-11}$ & $\sim 10^{-5}$ & $\sim 10^{-3}$ & $\sim 5 \times 10^{-4}$ & $<0.29$ (CDF+TH) \\
$t \rightarrow \gamma q$ & $5 \times 10^{-13}$ & $\sim 10^{-7}$ & $\sim 10^{-5}$ & $\sim 10^{-5}$ & $<0.0059$ (HERA) \\
$t \rightarrow Z q$ & $\sim 10^{-13}$ & $\sim 10^{-6}$ & $\sim 10^{-4}$ & $\sim 10^{-2}$ & $<0.14$ (LEP-2) \\
\hline
\end{tabular}

\subsubsection{Signal and background generation}

Both the $t \rightarrow \gamma q$ and the $t \rightarrow Z^{0} q$ decay channels are investigated. The channel $t \rightarrow g q$ is not studied because of its very high background. The $t \bar{t}$ signal is generated with TopReX [44], while PYTHIA [184] is used for modelling of quark and gluon hadronisation. The $t \bar{t}$ pair is generated through gluon-gluon and quark-anti-quark annihilation, with subsequent SM decay for one top $(t \rightarrow W b)$ and FCNC decay of the other. Only leptonic decay channels of $Z$ and $W$ bosons are studied, where the lepton could be either $e$ or $\mu$. Hadronic Z/W decays as well as decays to tau leptons are not considered because of the large QCD background. On generator level both top quarks are produced on-shell, with a mass of $m_{t}=175 \mathrm{GeV} / \mathrm{c}^{2}$, including the effects of spin-state correlations on final decay products $\left(\gamma q, Z^{0} q, W b\right)$. Both ISR and FSR are simulated with CTEQ5L PDFs. The generated events are passed through the full detector simulation and digitisation, taking into account low luminosity pile-up.

Several SM processes contributing as background are studied: $t \bar{t}$ production, single top quark production (t-channel), $Z W+$ jets, $W W+$ jets, $Z Z+$ jets, $W+$ jets, $Z+$ jets, $Z b \bar{b}$ and QCD multi-jet production.

\subsubsection{Selection strategies}

The $t \rightarrow \gamma q$ channel is well identified by a high-energy isolated photon accompanying the FCNC top decay. One b-tagged jet and a light jet are also used to distinguish from the standard $t \bar{t}$ decays. For the FCNC $t \rightarrow \gamma q$ channel our main selection cuts are: (a) single electron or single muon' trigger criteria at Level-1 and HLT levels; (b) one isolated $e^{ \pm}$(with $p_{\mathrm{T}}>$ $30 \mathrm{GeV} / \mathrm{c}$ ) or $\mu^{ \pm}$(with $p_{\mathrm{T}}>20 \mathrm{GeV} / \mathrm{c}$ ), and missing transverse energy $E_{\mathrm{T}}^{\text {miss }}>25 \mathrm{GeV}$, forming a transverse invariant mass $M_{\mathrm{T}}(b W)<120 \mathrm{GeV} / \mathrm{c}^{2}$; (c) only one jet compatible with b-jet with $p_{\mathrm{T}}>40 \mathrm{GeV} / \mathrm{c}$, that in combination with the $W$ candidate gives an invariant mass in the range between $110 \mathrm{GeV} / \mathrm{c}^{2}$ and $220 \mathrm{GeV} / \mathrm{c}^{2}$; (d) one single isolated photon with $p_{\mathrm{T}}>50 \mathrm{GeV} / \mathrm{c}$; (e) one light-jet (not compatible with b-jet) with $p_{\mathrm{T}}>50 \mathrm{GeV} / \mathrm{c}$; (f) an invariant mass obtained from the combination of the photon and the light jet that lies in the range between $150 \mathrm{GeV} / \mathrm{c}^{2}$ and $200 \mathrm{GeV} / \mathrm{c}^{2} ;(\mathrm{g})$ the transverse momentum of the photon + light-jet system recoiling against the transverse momentum of the SM-decaying top quark satisfying $\cos \phi(t \bar{t})<-0.95$.

The total efficiency for the signal is $\varepsilon=0.021 \pm 0.002$. Only the SM backgrounds $t \bar{t}$ and EW single top ( $t$-channel) contribute to the accepted background, with $54 \pm 7$ background events accepted for a luminosity of $10 \mathrm{fb}^{-1}$. The uncertainties are statistical only.

Adopting a factorisation method, QCD background is proven to be not dangerous for the analysis: A set of independent cuts (hard jets, isolated hard lepton, isolated hard photon, b-tagging) is applied to both QCD and $t \bar{t}$ background and the efficiencies for single cuts are assumed to factorise. The b-tagging efficiency and the mistagging are $30 \%$ and $0.5 \%$. The number of surviving QCD events for this pre-selection is found to be 42 for a luminosity of $10 \mathrm{fb}^{-1}$, and the efficiency on the $t \bar{t}$ sample amounts to $2.5 \%$. Assuming that after these 

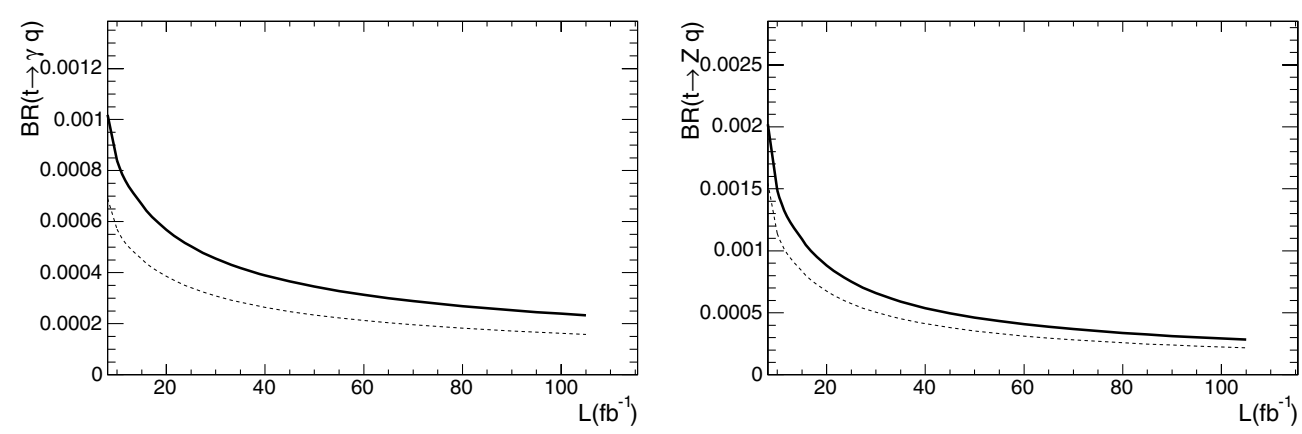

Figure 8.17. Branching Ratios of a FCNC signal detectable at the 5 sigma level as a function of the integrated luminosity, for the $q \gamma$ (left) and $q Z$ (right) channels, shown with (solid line) and without (dashed line) systematic uncertainties.

cuts the further efficiency for the QCD backgrounds and $t \bar{t}$ is the same, leads to expect $\simeq 1$ background events.

For the FCNC $t \rightarrow Z^{0} q$ channel our main selection cuts are: (a) 'double electron or double muon' trigger criteria at Level-1 and HLT levels; (b) two isolated $e^{ \pm}$(each with $p_{\mathrm{T}}>$ $20 \mathrm{GeV} / \mathrm{c}$ ) or $\mu^{ \pm}$(each with $p_{\mathrm{T}}>10 \mathrm{GeV} / \mathrm{c}$ ), having an invariant mass $\pm 10 \mathrm{GeV} / \mathrm{c}^{2}$ around the nominal $Z^{0}$ mass; (c) third lepton ( $e$ with $p_{\mathrm{T}}>20 \mathrm{GeV} / \mathrm{c}$ or $\mu$ with $p_{\mathrm{T}}>15 \mathrm{GeV} / \mathrm{c}$ ), which, in combination with the missing transverse energy $\left(E_{\mathrm{T}}^{\text {miss }}>20 \mathrm{GeV}\right)$ have a transverse mass less than $120 \mathrm{GeV} / \mathrm{c}^{2}$; (d) only one jet compatible with $b$ jet with $p_{\mathrm{T}}>40 \mathrm{GeV} / \mathrm{c}$; (e) invariant mass of candidate $W$ and $b$ jet in the range [110-220] $\mathrm{GeV} / \mathrm{c}^{2}$; (f) one lightjet (not compatible with $b$ jet) with $p_{\mathrm{T}}>30 \mathrm{GeV} / \mathrm{c}(\mathrm{g})$ an invariant mass obtained from the combination of the $\mathrm{Z}$ and the light jet that lies in the range between $110 \mathrm{GeV} / \mathrm{c}^{2}$ and $220 \mathrm{GeV} / \mathrm{c}^{2}$; (h) the transverse momentum of the $\mathrm{Z}+$ light-jet system recoiling against the transverse momentum of the SM-decaying top quark satisfying $\cos \phi(t \bar{t})<0$.

The total efficiency for the signal is $\varepsilon=0.041 \pm 0.002$. A total of $1 \pm 1$ background events are accepted for a luminosity of $10 \mathrm{fb}^{-1}$. The SM background $t \bar{t} \rightarrow(\nu l b)(\nu l b)$ is the only background that gives a significant contribution. The uncertainties are statistical only.

\subsubsection{Sensitivity estimation}

For the FCNC sensitivity estimation, it is assumed that new physics is observed when the signal significance is 5 at least. When dealing with a small number of background $(B)$ events with respect to signal ones $(S)$, an appropriate definition of significance is [49]:

$$
S_{12}=2(\sqrt{B+S}-\sqrt{B})
$$

$S_{12}$ defines the probability (in number of sigmas) that a background with expected value $B$ fluctuates above observed number of events $S+B$ with Poisson statistics. The number of signal events for the $t \rightarrow Z q$ and $t \rightarrow \gamma q$ channel can be expressed as:

$$
\begin{aligned}
& S(t \rightarrow Z q)=2 \times B R(t \rightarrow Z q) \times B r(W \rightarrow l \nu) \times B r(Z \rightarrow l l) \times \sigma(t \bar{t}) \times L \times \epsilon(t \rightarrow Z q) \\
& S(t \rightarrow \gamma q)=2 \times B R(t \rightarrow \gamma q) \times B r(W \rightarrow l v) \times \sigma(t \bar{t}) \times L \times \epsilon(t \rightarrow \gamma q)
\end{aligned}
$$

where $\quad L=10 \mathrm{fb}^{-1}, \quad \sigma(t \bar{t})=833 \mathrm{pb}, \quad B R(W \rightarrow l \nu)=0.2136, \quad B R(Z \rightarrow l l)=0.0673$ $(l=e, \mu), \varepsilon$ selection efficiency for the signal. From these formulae, the FCNC branching ratios $B R(t \rightarrow Z q)$ and $B R(t \rightarrow \gamma q)$ can be calculated for a given significance level $S_{12}$. Without the inclusion of systematic uncertainties, the sensitivity for a significance level 
Table 8.28. Effects of systematic uncertainties on the five-sigma observable FCNC branching ratios induced by different sources of systematic uncertainty. The last row indicates the smallest five-sigma observable FCNC branching ratios for $10 \mathrm{fb}^{-1}$ of integrated luminosity including all sources of systematic uncertainty.

\begin{tabular}{lll}
\hline & $t \rightarrow Z q\left(\times 10^{-4}\right)$ & $t \rightarrow \gamma q\left(\times 10^{-4}\right)$ \\
\hline$B R($ stat $)$ & 11.4 & 5.7 \\
jet energy scale & +0.4 & +0.6 \\
b jet mistagging & +0.2 & +1.8 \\
light jet antitagging & +0.5 & +0.9 \\
lepton energy scale & +2.4 & +0.5 \\
$\sigma(t \bar{t})$ & +0.1 & +0.5 \\
MC statistics in B & +2.4 & +1.3 \\
MC statistics in S & +0.7 & +0.5 \\
Luminosity & +0.1 & +0.5 \\
$B R$ (total) & 14.9 & 8.4 \\
\hline
\end{tabular}

of $S_{12}=5$ is $B R(t \rightarrow Z q)=11.4 \times 10^{-4}$ and $B R(t \rightarrow \gamma q)=5.7 \times 10^{-4}$, also shown in Figure 8.17.

The sources of systematic uncertainty are divided into two groups: those related to detector effects and those related to theoretical issues. For both kind of sources, the impact on the selection efficiency and the surviving number of background events is evaluated. Experimental effects considered here include: (a) the lepton energy scale uncertainty, accounted for with relative increase/decrease of the reconstructed photon and electron fourmomenta by \pm 0.005 ; (b) the jet energy scale uncertainty, expected to lie in the range from $\pm 5 \%$ at $p_{\mathrm{T}}=20 \mathrm{GeV} / \mathrm{c}$ to $\pm 2.5 \%$ at $p_{\mathrm{T}}>50 \mathrm{GeV} / \mathrm{c}$, and totally correlated to missing energy uncertainty (assumed to be $\pm 5 \%$, [320]); (c) b-tagging uncertainty (4\% after $10 \mathrm{fb}^{-1}$ integrated luminosity [285]), that is studied by assuming a non-b-tagged jet is actually a b-tagged jet $4 \%$ of the time; (d) uncertainty in anti-tagging b-jet instead of non- $b$ ones (4\% after $10 \mathrm{fb}^{-1}$ integrated luminosity), simulated by assuming a b-tagged jet is a non-b-tagged jet with the same probability.

The impact of the single sources of systematic uncertainty is detailed in Table 8.28. Experimental sources of systematic uncertainties, such as the control of the lepton energy scale and of the b-tagging procedure are expected to be the most significant. The statistical uncertainty on the prediction of the background level of this analysis has a large contribution to the global systematic uncertainty. Refined techniques for the background estimation will reduce this uncertainty once data will be available.

Including all systematic uncertainties, the smallest detectable FCNC branching ratios, for a five-sigma sensitivity and $10 \mathrm{fb}^{-1}$ of luminosity, are $B R(t \rightarrow Z q)=14.9 \times 10^{-4}$ and $B R(t \rightarrow \gamma q)=8.4 \times 10^{-4}$. Under the assumption that the selection efficiency is unaffected by moderate instantaneous luminosity increases (i.e., pile-up), the decrease in the upper limit on the branching fraction with increasing luminosity can be evaluated in a straightforward way. Figure 8.17 shows the branching ratio for both channels as a function of the integrated luminosity. An improvement in the branching ratio limits by a factor of 2 is expected for a luminosity increase by a factor of 5 . 


\section{Chapter 9. Electroweak Physics}

\subsection{Production of $W$ and $Z$ bosons}

\subsubsection{Introduction}

The reactions $p p \rightarrow W+X$ and $p p \rightarrow Z+X$ with subsequent leptonic decays of the massive electroweak vector bosons, $W \rightarrow \ell v$ and $Z \rightarrow \ell^{+} \ell^{-}$, have a large cross section and are theoretically well understood. Cross sections above $10 \mathrm{nb}(1 \mathrm{nb})$ are expected at the LHC for the $W \rightarrow \ell v\left(Z \rightarrow \ell^{+} \ell^{-}\right)$channel in the fiducial region of the CMS detector. Hence these reactions are useful for many purposes, including a precise luminosity monitor, a highstatistics detector calibration tool and to demonstrate the performance of the CMS experiment. These reactions will be among the first to be measured at the LHC.

Here we discuss prospects for precise measurements of the reactions $p p \rightarrow Z+X$ and $p p \rightarrow W+X$ at the LHC using the decays of the gauge bosons into electrons and muons. Studies have been performed based on Monte Carlo samples generated with PYTHIA including realistic detector simulation and addressing the most relevant systematic effects. The potentially most dangerous background in these analyses consists of QCD events with leptons from hadron decays or tracks misidentified as leptons. However, these lepton candidates are associated to jets and can be largely suppressed using isolation algorithms.

Robust criteria are developed which allow for a low-background event selection which is rather insensitive to detector inhomogeneities. This robust selection is considered as especially useful for the CMS startup phase. The results show that a determination of the $W$ and $Z$ rates with an experimental precision on the percent level is feasible already in the early phase of the experiment.

\subsection{2. $W / Z$ into electrons}

The process $\mathrm{pp} \rightarrow \mathrm{ZX}$ and $\mathrm{pp} \rightarrow \mathrm{WX}$ with subsequent decay of $Z$ and $W$ into electrons is studied using the full CMS detector simulation and analysis scheme. The aim is to define some baseline selection which is suppressing background to a very small level and detector inhomogeneities can be controlled. This selection can thus be considered as especially useful for the CMS startup phase. Details can be found in [341].

Electron (positron) candidates are selected with the following criteria [313]:

- The minimal $E_{\mathrm{T}}$ of the electromagnetic cluster has to be larger than $20 \mathrm{GeV}$ with $\left|\eta_{\text {cluster }}\right|<$ 1.4 for barrel electron candidates and $1.6<\left|\eta_{\text {cluster }}\right|<2.4$ for endcap electron candidates.

- The cluster should be consistent with the shower shape expected for electromagnetic showers. The spread of the electromagnetic shower along the $\eta$ direction is rather insensitive to bremsstrahlung, thus allowing a good separation of signal and background shower shapes. Therefore it is required that the spread of the electromagnetic shower in $\eta$ with respect to $\eta$ of the supercluster, $\sigma_{\eta \eta}$, is smaller than 0.01 .

- The energy deposit in the associated hadron calorimeter cluster should be very small. For this selection the ratio $E_{\mathrm{Had}} / E_{\mathrm{EM}}$ has to be smaller than 0.05 .

- In order to be identified as an electron, a reconstructed track has to be matched with the cluster such that $\Delta R<0.15$ (where $\Delta R=\sqrt{\Delta \phi^{2}+\Delta \eta^{2}}$ ). Furthermore, it is required that the ratio of the cluster energy and the track momentum, $E / P$, is larger than 0.9 and that $|1 / E-1 / P|<0.02$.

- Finally, it is required that the electron candidate is isolated. The transverse momentum sum of all other tracks found within a cone radius $\Delta R$ of 0.35 divided by the electron candidate 

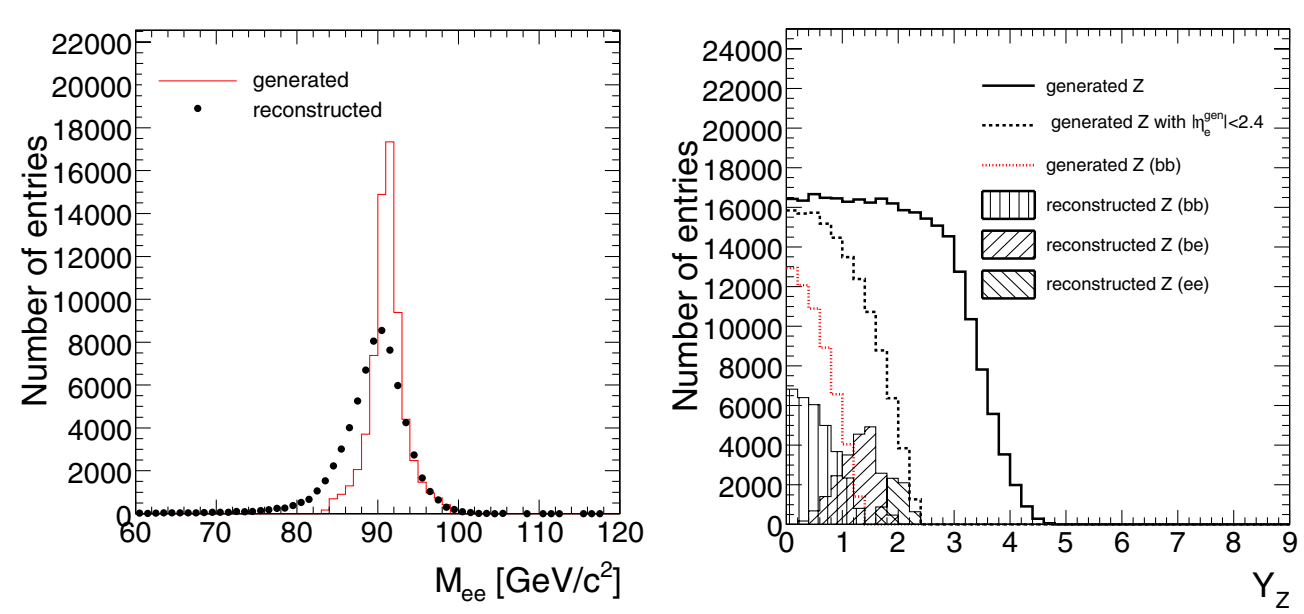

Figure 9.1. Left: Reconstructed and generated $Z$ mass distribution with all cuts. Right: Generated rapidity distribution for all $Z$ candidates and for those where both electrons were generated within the geometrical acceptance of the electromagnetic calorimeter. For comparison, the rapidity distribution of the finally accepted $Z$ events is already shown here.

transverse supercluster energy has to be smaller than 0.2. Only tracks with a transverse momentum above $1.5 \mathrm{GeV} / \mathrm{c}$ and with at least four hits in the central tracker which are close to the interaction vertex are considered.

9.1.2.1. $p p \rightarrow Z \rightarrow$ ee $X$ Selection. We analyse events where one $\mathrm{e}^{+} \mathrm{e}^{-}$pair consistent with the $Z$ mass is found (if more than two electrons pass the selection criteria, only those two with the highest transverse momenta are considered). The generated and reconstructed mass distribution are shown in Figure 9.1 (left). For now, the "electron" clusters are not corrected for bremsstrahlung within the tracker and the reconstructed $Z$ peak is found to be about $1 \mathrm{GeV}$ lower than the generated one.

Using this selection, the rapidity distribution of the accepted $Z$ events is shown in Fig. 9.1 (right). In addition, the rapidity distribution of the potentially accepted $Z$ bosons, separated for the three cases where both decay electrons are within the acceptance of the barrel calorimeter (BB) $\left|\eta_{\mathrm{BB}}\right|<1.4$, both within the endcaps (EE) $1.6<\left|\eta_{\mathrm{EE}}\right|<2.4$ or one within the barrel and the other one in the endcaps (EB) are also shown. In the case that both generated electrons are in the barrel, a $Z$ detection efficiency of about $60 \%$ is reached.

Here the electron efficiency is defined by the ratio of reconstructed electrons from accepted $Z$ events to the number of electrons from generated $Z$ events, where the generated electrons fulfilled the condition $\left|\eta_{\text {gen }}^{\mathrm{e}}\right|<1.4$. Fig. 9.2 (left) shows the efficiency distribution for all supermodules folded such that the local $\phi$ angle for all odd supermodules goes from 0-20 degrees and for all even supermodules from 20-40 degrees.

The efficiency drop of about $10 \%$ between the supermodules is clearly visible with the available sample of $Z$ events corresponding to roughly $0.2 \mathrm{fb}^{-1}$. Similar inefficiencies were found in the $\eta$ direction at supermodule boundaries. From the analysis of the reconstruction efficiency as function of the phi angle, we get an efficiency of $27.1 \% \pm 0.4 \%$ (if the inter-supermodule regions are excluded) while the average over the whole phi range is $26.5 \pm 0.4 \%$.

The average $Z$ efficiency, when both electrons are generated and reconstructed in the barrel calorimeter, is found to be $57.3 \pm 0.2 \%$ (where the uncertainties are from the finite number of Monte Carlo events). Half the efficiency loss is caused by the 

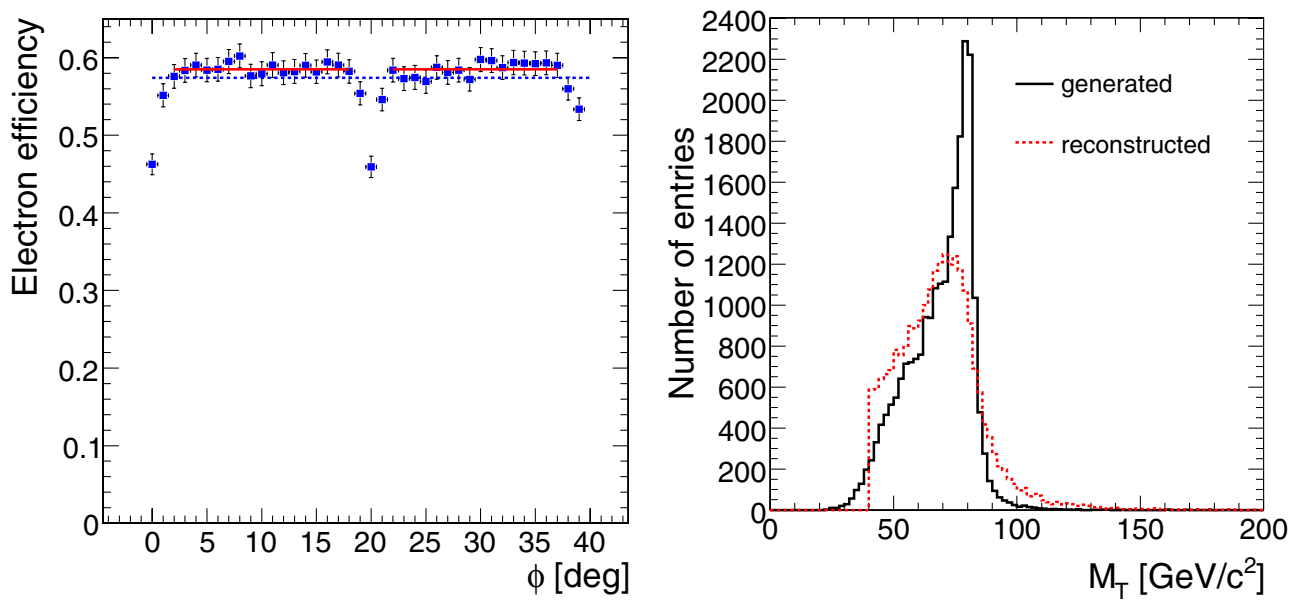

Figure 9.2. Left: The electron reconstruction efficiency in $\mathrm{Z} \rightarrow \mathrm{e}^{+} \mathrm{e}^{-}$events as a function of $\phi$, all even and odd numbered supermodules are folded such that the odd (even) numbered supermodules always cover local $\phi$ angles from 0 to 20 degrees and from 20 to 40 degrees respectively. The dotted line corresponds to the average efficiency $57.3 \pm 0.2 \%$ over the whole $\phi$ range and the solid lines correspond to the average efficiency $58.4 \pm 0.2 \%$ with the gap regions excluded. Right: Generated (solid line) and reconstructed (dashed line) transverse $W$ mass. The $W$ transverse mass is reconstructed from the electron four-momentum and the missing transverse energy. In this plot, only events with no reconstructed jet above $20 \mathrm{GeV}$ transverse energy are included.

shower-shape requirement, and another quarter by the energy-momentum matching requirement. If events, where at least one electron is reconstructed within the gaps, are removed, the average efficiency is found to be $58.4 \pm 0.2 \%$. Assuming that the produced electrons must be homogeneous in $\phi$ and that the effects from geometrical gaps can be monitored with some reasonable statistics, it should be straightforward to correct for the detector gaps. Already with the available statistics used for this study, the corrections for the efficiency loss in the gaps can certainly be determined with a relative accuracy smaller than about $25 \%$. This number is estimated from comparing the minimal efficiency in the gap and the efficiency in the non-gap regions.

We conclude that already with a few 100000 reconstructed $Z$ events, collected at the early stage of the experiment, an efficiency determination with a systematic accuracy of better than 1-2\% should be possible. Obviously, with the much larger statistics of a few million $Z$ events, these uncertainties can be further reduced. Once data from the CMS detector becomes available, these cuts can be applied on one electron and varied on the other electron to compare the selection efficiency in data and Monte Carlo simulation. This can be used to further improve the detector simulation and to better access systematic uncertainties.

9.1.2.2. $p p \rightarrow W \rightarrow e v X$ Selection. In order to pass the $\mathrm{W} \rightarrow \mathrm{ev}$ selection, events must have exactly one electron candidate in the barrel fulfilling the requirements described above, and missing transverse energy associated with the neutrino: a cut on the transverse mass of the e $v$ system is applied. The transverse mass $m_{\mathrm{T}}$ is defined as follows:

$$
m_{\mathrm{T}}=\sqrt{2 p_{\mathrm{T}}^{(e)} p_{\mathrm{T}}^{(\nu)}(1-\cos \Delta \phi)}
$$

where $p_{\mathrm{T}}^{(\mathrm{e}, v)}$ is the (reconstructed) transverse momentum of the electron and the neutrino respectively and $\Delta \phi$ is the azimuthal angle between the electron and the neutrino. 
The missing transverse energy can be determined in several ways, for example:

1. From the vector sum of all clusters in the calorimeter.

2. From the vector sum of hard objects only.

In the electromagnetic calorimeter, the electron transverse energy can be measured accurately. However, the reconstructed transverse missing energy shows a significant bias.

Suspecting that low energy objects (randomly distributed across the detector) are responsible for this bias, we follow the second approach: We select reconstructed jets with a transverse energy above $20 \mathrm{GeV}$ and absolute pseudorapidity less than 2.4 and reconstruct the missing transverse energy only from these jets and the electron. Here we use uncalibrated jets, i.e. whenever we refer to the jet energy we mean raw jet energy.

To study this possibility in more detail, we split our sample into events without jets (as defined in the previous paragraph) and events with one or more jets. Note that in the case of zero accepted jets, only the electron is used to calculate the neutrino transverse energy which is then very close to the electron transverse energy (pointing into opposite directions in $\phi$ ). The transverse mass is equal to twice the electron transverse energy in this case.

No systematic bias is found with this method and the mean value is close to zero. We thus use this method to reconstruct the neutrino transverse energy. The reconstructed $W$ transverse mass is shown in Fig. 9.2 (right). For the purpose of this analysis and the counting of resonant $W$ events, we require the transverse mass to lie in the interval 60 to $100 \mathrm{GeV} / \mathrm{c}^{2}$.

We consider two sources of systematic uncertainties here: The uncertainty due to inhomogeneities in the detector geometry and the uncertainty related to the jet veto. We expect that the uncertainty from the reconstruction efficiency as function of the electron azimuthal angle for the efficiency correction will be similar as for the $Z$ selection.

To address the effect of the scale uncertainty of the absolute calibration on the jet definition, we investigated the changes in the selection efficiency when moving the threshold transverse energy for the jet definition. It follows that for a cut on the transverse jet energy at $20 \mathrm{GeV}$, the efficiency slope is roughly $0.1 \%$ (absolute) per $\mathrm{GeV}$, corresponding to a relative uncertainty of about $0.25 \%$ per $\mathrm{GeV}$.

Assuming a jet energy scale uncertainty of $15 \%$ at the LHC startup we obtain an efficiency uncertainty of $0.75 \%$ relative. For $5 \%$ uncertainty in the jet energy scale expected after the final detector calibration), this value reduces to $0.25 \%$.

The efficiency change due to the jet veto can also be estimated directly from $\mathrm{Z} \rightarrow \mathrm{e}^{+} \mathrm{e}^{-}$ events (applying a jet veto to these events). In the future, this can be done directly from the data recorded with the CMS detector. Thus with the expected large data samples of $\mathrm{Z} \rightarrow \mathrm{e}^{+} \mathrm{e}^{-}$, remaining differences between data and Monte Carlo can be studied and corrected with very small uncertainties.

\subsubsection{W/Z into muons}

Simple sets of cuts can be used in CMS to select large statistics samples of $Z \rightarrow \mu \mu$ and $W \rightarrow \mu \nu$ events with high purity. They are described in detail in Ref. [342] and summarised here.

The $Z \rightarrow \mu \mu$ selection criteria have been chosen to minimise uncertainties from the muon chamber response and from the matching between the inner tracker and the muon spectrometer. The basic idea is to accept events in which one of the muons is reconstructed as an isolated track in the central tracker detector, even if no associated track in the muon spectrometer is present. This results in a more uniform efficiency as a function of the pseudorapidity, as observed in Fig. 9.3 (left). From the kinematics point of view only muons with 

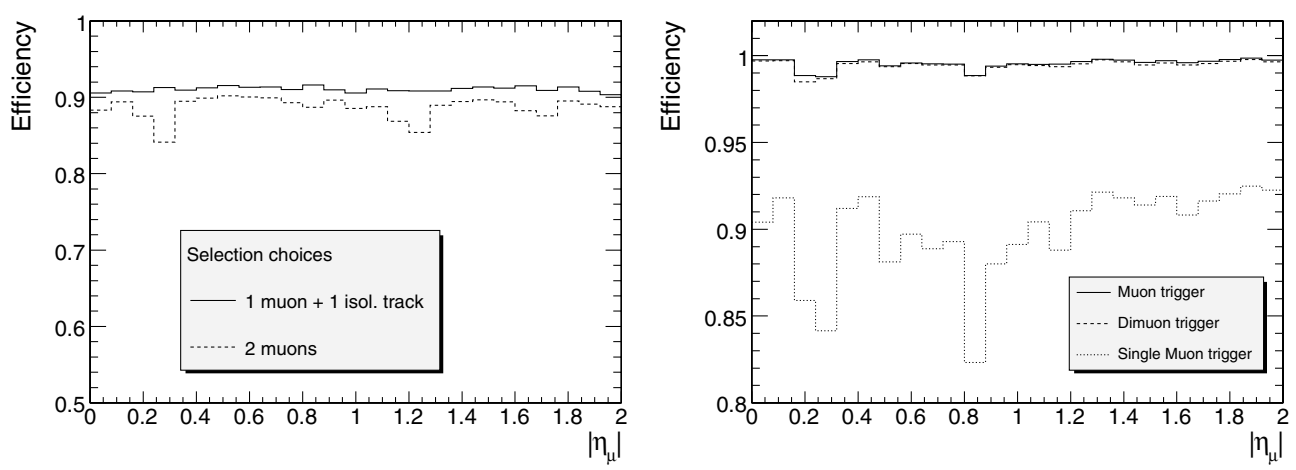

Figure 9.3. Left: Muon efficiency as a function of pseudo-rapidity in the selected $Z \rightarrow \mu \mu$ sample. Two cases are considered: a selection using only muons reconstructed in the muon chambers (dashed histogram) and the selection described in the text (solid histogram), which also accepts isolated tracks in the inner tracker. For this test, no HLT trigger criteria have been applied. Right: HLT efficiency on the selected $Z \rightarrow \mu \mu$ sample as a function of the pseudorapidity of one of the muons. All but the HLT trigger criteria have been applied. The regions at $|\eta| \approx 0.25$ and $|\eta| \approx 0.8$, with a slightly lower trigger efficiency, are visible. The fraction of events triggered by dimuon and single-muon triggers are also shown.

$p_{\mathrm{T}}>20 \mathrm{GeV}$ and pseudorapidity $\left|\eta_{\mu}\right|<2.0$ are considered in the present analysis. A dimuon mass window of $\pm 3 \Gamma_{Z}=7.5 \mathrm{GeV}$ around the reconstructed $\mathrm{Z}$ mass is used. Figure 9.3 (right) shows the efficiency of the HLT criteria on the selected sample as a function of the muon pseudo-rapidity. One can clearly observe two regions with smaller efficiency, around $|\eta| \approx 0.25$ and $|\eta| \approx 0.8$, where transitions between two muon wheels take place. The efficiency is dominated by the dimuon component, which represents a unique tool to study the performance of the single-muon subtrigger, which is of relevance for other selections, like $W \rightarrow \mu \nu$.

Even if the rate of $W \rightarrow \mu \nu$ events is expected to be larger than the $Z \rightarrow \mu \mu$ rate by an order of magnitude, the experimental context is more demanding due to a lower trigger efficiency, only moderate transverse missing energy in the event, the absence of a precise mass constraint and a full dependence on tracker and muon spectrometer behaviours. This will lead to larger experimental uncertainties, which can be studied with the $Z \rightarrow \mu \mu$ data samples. The selection of $W \rightarrow \mu \nu$ events uses the same $\eta$ cut but a higher $p_{\mathrm{T}}$ threshold, $25 \mathrm{GeV}$, due to the higher threshold for the single-muon trigger. Figure 9.4 shows the transverse invariant mass distribution of the muon- $E_{\mathrm{T}}^{\text {miss }}$ system in $W \rightarrow \mu \nu$ events, compared to QCD expectations.

Systematic uncertainties in the determination of $Z \rightarrow \mu \mu$ and $W \rightarrow \mu \nu$ acceptances are summarised in Tables 9.1 and 9.2. The various sources of uncertainties are discussed in detail in Ref. [342]. Most of them are evaluated for a CMS detector calibrated with $1 \mathrm{fb}^{-1}$. The experimental components are well under control in the case of the $Z \rightarrow \mu \mu$ selection, with the limited knowledge on the track efficiency as the dominant source. In the $W \rightarrow \mu \nu$ case, many of them contribute at a similar level, with $E_{\mathrm{T}}^{\text {miss }}$ providing the largest uncertainty. Concerning theoretical sources, the boson $p_{\mathrm{T}}$ uncertainties are the dominant contribution. They are estimated from a comparison between LO and NLO CMS simulations using MC@NLO as event generator [343], as shown in Fig. 9.5.

The results of the study can be summarised in terms of cross section measurement accuracies, for $1 \mathrm{fb}^{-1}$ of integrated luminosity, as follows: $\Delta \sigma / \sigma(p p \rightarrow Z+X \rightarrow$ $\mu \mu+X)=0.13$ (stat) \pm 2.3 (syst.) \pm 10 (lumi)\% and $\Delta \sigma / \sigma(p p \rightarrow W+X \rightarrow \mu \nu+X)=$ 0.04 (stat.) \pm 3.3 (syst.) \pm 10 (lumi)\%, where luminosity represents the dominant uncertainty which will eventually decrease to $5 \%$ with more integrated luminosity. 


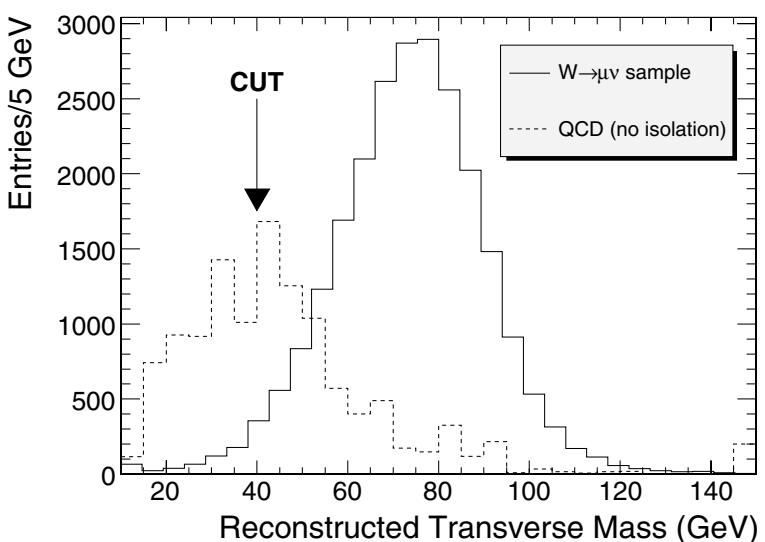

Figure 9.4. Transverse invariant mass reconstructed in $W \rightarrow \mu v$ events. In order to observe the shape of the QCD background with more statistics, the HLT muon isolation criteria have not been applied to obtain the plot. The position of the lower cut $\left(M_{\mu \mu}>40 \mathrm{GeV} / \mathrm{c}^{2}\right)$ is indicated with an arrow.

Table 9.1. Relative systematic uncertainties on the acceptance for the $Z \rightarrow \mu \mu$ sample.

\begin{tabular}{ll}
\hline Source & Uncertainty $(\%)$ \\
\hline Tracker efficiency & 1 \\
Magnetic field knowledge & 0.03 \\
Tracker alignment & 0.14 \\
Trigger efficiency & 0.2 \\
Jet energy scale uncertainties & 0.35 \\
Pile-up effects & 0.30 \\
Underlying event & 0.21 \\
Total exp. & 1.1 \\
PDF choice (CTEQ61 sets) & 0.7 \\
ISR treatment & 0.18 \\
$p_{\mathrm{T}}$ effects (LO to NLO) & 1.83 \\
Total PDF/ISR/NLO & 2.0 \\
Total & 2.3 \\
\hline
\end{tabular}

QCD backgrounds seem to be under control, even if final checks with data will be necessary to determine the level of background with more precision.

Therefore, rates within the fiducial volume of the detector can be determined with high accuracy, even for the first stages of the LHC $(\approx 2.3 \%$ for $Z \rightarrow \mu \mu$ and $\approx 3.3 \%$ for $W \rightarrow \mu \nu)$. These uncertainties will be significantly reduced with the use of the next generation of NLO Monte Carlos and final detector calibrations, and allow these reactions to be used to determine the luminosity.

\subsubsection{Parton distribution functions and parton luminosities}

The production of inclusive $W$ and $Z$ events is theoretically well understood and the couplings to quarks and leptons have been measured with accuracies of $1 \%$ or better. Thus, it follows from the previous sections that a precise counting of $W \rightarrow e v, \mu \nu$ and $Z \rightarrow e e, \mu \mu$ events is 
Table 9.2. Relative systematic uncertainties on the acceptance for the $W \rightarrow \mu v$ sample.

\begin{tabular}{ll}
\hline Source & Uncertainty (\%) \\
\hline Tracker efficiency & 0.5 \\
Muon efficiency & 1 \\
Magnetic field knowledge & 0.05 \\
Tracker alignment & 0.84 \\
Trigger efficiency & 1.0 \\
Transverse missing energy & 1.33 \\
Pile-up effects & 0.32 \\
Underlying event & 0.24 \\
Total exp. & 2.2 \\
PDF choice (CTEQ61 sets) & 0.9 \\
ISR treatment & 0.24 \\
$p_{\mathrm{T}}$ effects (LO to NLO) & 2.29 \\
Total PDF/ISR/NLO & 2.5 \\
Total & 3.3 \\
\hline
\end{tabular}
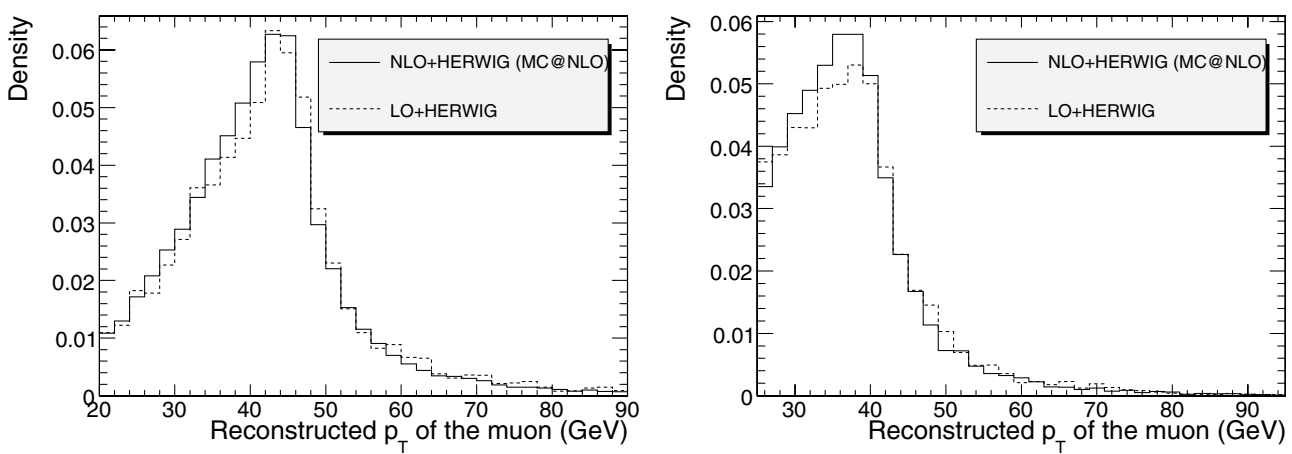

Figure 9.5. Left: Comparison between LO and NLO predictions for the muon $p_{\mathrm{T}}$ distribution in $Z \rightarrow \mu \mu$ selected events. Both histograms have been normalised to the total number of events generated in the fiducial volume: $\left|\eta_{\mu}\right|<2.5, p_{\mathrm{T}_{\mu}}^{\max }>20 \mathrm{GeV} / \mathrm{c}, p_{\mathrm{T} \mu}^{\min }>10 \mathrm{GeV} / \mathrm{c}$ and $M_{Z}-6 \Gamma_{Z}<M_{\mu \mu}<M_{Z}+6 \Gamma_{Z}$ Right: Comparison between LO and NLO predictions for the muon $p_{\mathrm{T}}$ distribution in $W \rightarrow \mu \nu$ selected events. Both histograms have been normalised to the total number of events generated in the fiducial volume: $\left|\eta_{\mu}\right|<2.5$.

equivalent to a precise measurement of the quantity

$$
\int_{q, \bar{q} \text { partons }} \mathrm{d} x_{1} \mathrm{~d} x_{2} \sigma_{q \bar{q} \rightarrow W, Z} \times L_{p p} \times P D F\left(x_{1}, x_{2}, Q^{2}\right),
$$

where $L_{p p}$ is the LHC integrated luminosity, $\sigma_{q \bar{q} \rightarrow W, Z}$ is the cross section for inclusive $W$ or $Z$ production at the partonic level and $P D F\left(x_{1}, x_{2}, Q^{2}\right)$ denotes the probability to produce quarks and anti-quarks with proton fractions $x_{1}$ and $x_{2}$ at a scale $Q^{2}$. The prospect studies of Ref. [342], summarised in Table 9.3, show that uncertainties on the parton distribution functions (PDF) have a relatively small influence on the experimental acceptance for the rates, but a large effect on the global rate expectations.

We conclude from Table 9.3 that a comparison between theory and experiment with a 6-7\% accuracy is possible. This comparison provides a measurement of the integrated luminosity $L_{p p}$ with a similar level of precision. The small theoretical uncertainties on the experimentally measured rate (from the acceptance uncertainty) allow precise measurements of cross section ratios, such as $\sigma(p p \rightarrow Z Z+X) / \sigma(p p \rightarrow Z+X)$, in which 
Table 9.3. Estimated uncertainties in the rate and in the acceptance for the $p p \rightarrow Z+X \rightarrow \mu \mu+X$ and $p p \rightarrow W+X \rightarrow \mu v+X$ processes. The global rate is referred to the fiducial volumes used in Ref. [342], which include a pseudorapidity cut of $\left|\eta_{\mu}\right|<2.5$.

\begin{tabular}{lll}
\hline & $Z \rightarrow \mu \mu$ & $W \rightarrow \mu \nu$ \\
\hline Global rate uncertainty $(\%)$ & +5.8 & +5.6 \\
Acceptance uncertainty $(\%)$ & +0.4 & -7.4 \\
& -0.7 & +0.6 \\
\hline
\end{tabular}

PDF and luminosity uncertainties cancel. Current studies within theoretical and experimental communities [344] aim to a further reduction of uncertainties associated to PDFs. Finally, PDF validity tests and further reductions in the acceptance uncertainty (below the percent level) will require dedicated studies of the lepton rapidity distributions observed in data, like those suggested in Ref. [345].

\subsection{Muon pairs from the Drell-Yan process}

\subsubsection{Introduction}

In the Standard Model, the production of lepton pairs in hadron-hadron collisions, the Drell-Yan (DY) process [346], is described by $s$-channel exchange of photons or $Z$ bosons. The parton cross section in the lepton-pair centre-of-mass system has the form:

$$
\frac{\mathrm{d} \sigma}{\mathrm{d} \Omega}=\frac{\alpha^{2}}{4 s}\left[A_{0}\left(1+\cos ^{2} \theta\right)+A_{1} \cos \theta\right]
$$

where $\sigma=\frac{4 \pi \alpha^{2}}{3 s} A_{0}$ and $A_{\mathrm{FB}}=\frac{3}{8} \frac{A_{1}}{A_{0}}$ are the total cross section and the forward-backward asymmetry, and $\theta$ is angle of lepton in the dilepton rest frame with respect to the quark direction. The terms $A_{0}$ and $A_{1}$ are fully determined by the electroweak couplings of the initial- and final-state fermions. At the $Z$ peak the $Z$ exchange is dominating and the interference term is vanishing. At higher energies both photon and $Z$ exchange contribute and the large value of the forward-backward asymmetry is due to the interference between the neutral currents. Fermion-pair production above the $Z$ pole is a rich search field for new phenomena at present and future high energy colliders. The differential cross section is sensitive to manifestation of new physics from a multi-TeV scale by adding new amplitudes or through their interference with the neutral currents of the SM. At hadron colliders the parton cross sections are folded with the parton density functions (PDF): $p p \rightarrow l_{1} l_{2}$

$$
\frac{\mathrm{d}^{2} \sigma}{\mathrm{d} M_{l l} \mathrm{~d} y}\left[p p \rightarrow l_{1} l_{2}+X\right] \approx \sum_{i j}\left(f_{i / p}\left(x_{1}\right) f_{j / p}\left(x_{2}\right)+(i \leftrightarrow j)\right) \hat{\sigma}
$$

where $\hat{\sigma}$ is the cross section for the partonic subprocess $i j \rightarrow l_{1} l_{2}, M_{l l}=\sqrt{\tau s}=\sqrt{\hat{s}}$ the mass of the lepton-pair system, $y$ the rapidity of the lepton pair, $x_{1}=\sqrt{\tau} e^{y}$ and $x_{2}=\sqrt{\tau} e^{-y}$ the parton momentum fractions, and $f_{i / p(\bar{p})}\left(x_{i}\right)$ the probability to find a parton $i$ with momentum fraction $x_{i}$ in the proton.

The total cross section and the forward-backward asymmetry are function of observables which are well measured experimentally for final states containing $e^{+} e^{-}$or $\mu^{+} \mu^{-}$: the invariant mass and the rapidity of the final-state lepton pair. This allows to reconstruct the centre-of-mass energy of the initial partons, even if their flavours are unknown. For a $\left(x_{1} \geqslant x_{2}\right)$ pair of partons we have 4 combinations of up- or down-type quarks initiating the interaction: $u \bar{u}, \bar{u} u, d \bar{d}, \bar{d} d$. In $p p$ collisions the anti-quarks come always from the sea and the quarks can 
Table 9.4. $x_{1}$ and $x_{2}$ for different masses and rapidities.

\begin{tabular}{|c|c|c|c|c|c|c|c|c|}
\hline$y$ & 0 & 2 & 4 & 0 & 2 & 4 & 0 & 2 \\
\hline & \multicolumn{3}{|c|}{$\mathrm{M}=91.2 \mathrm{GeV} / \mathrm{c}^{2}$} & \multicolumn{3}{|c|}{$\mathrm{M}=200 \mathrm{GeV} / \mathrm{c}^{2}$} & \multicolumn{2}{|c|}{$\mathrm{M}=1000 \mathrm{GeV} / \mathrm{c}^{2}$} \\
\hline ( & & 0.0481 & & 0.01 & 0.1056 & & 0.0714 & 0.5278 \\
\hline$x_{2}$ & 0.0065 & 0.0009 & 0.0001 & 0.0143 & 0.0019 & 0.0003 & 0.0714 & 0.0097 \\
\hline
\end{tabular}

have valence or sea origin. The $x$-range probed depends on the mass and rapidity of the lepton pair as shown in Table 9.4.

The results presented here extend the studies for the LHC SM workshop (see [158] and references therein), using more data and the CMS full detector simulation and reconstruction. More details can be found in [347].

\subsubsection{Cross section measurements}

Simulation of Drell-Yan events in proton-proton collisions at $14 \mathrm{TeV}$ centre-of-mass energy is performed with PYTHIA 6.217 using the CTEQ5L parton distribution functions. The possible contributions from higher-order terms in the dimuon production cross section are taken into account by using a $K$ factor of 1.3 as calculated with the program PHOZPRMs [348]. Eleven samples of 10000 events each with different cut-off values on the dimuon invariant mass are generated: $M_{i n v} \geqslant 0.2,0.5,1,1.5,2,2.5,3,3.5,4,4.5,5 \mathrm{TeV} / \mathrm{c}^{2}$. Only events with at least two muons in the pseudorapidity range $|\eta| \leqslant 2.5$, with transverse momentum $p_{\mathrm{T}} \geqslant 7 \mathrm{GeV} / \mathrm{c}$ are preselected. No cuts on isolation of muons are made at the pre-selection stage. The total efficiency for dimuon pre-selection, $\varepsilon$, is about $87 \%$ for a mass of $1 \mathrm{TeV} / \mathrm{c}^{2}$ and $96 \%$ for a mass of $5 \mathrm{TeV} / \mathrm{c}^{2}$. To simulate the detector geometry, materials and particle propagation inside the detector, the GEANT 4-based simulation of the CMS detector is used.

The trigger simulation is based on the on-line reconstruction algorithms. Events are selected by the single- and double-muon triggers. This means that at least one muon candidate is within pseudorapidity region $|\eta| \leqslant 2.1$. The total efficiency of triggering including reconstruction and trigger selection efficiency is $98 \%$ at $1 \mathrm{TeV}$. There is significant decrease in trigger efficiency after applying calorimeter isolation cuts (down by 15\%). The tracker isolation practically does not affect the trigger efficiency. Thus the additional cuts on calorimeter and tracker isolation of muon tracks are not applied in this analysis.

The off-line muon reconstruction algorithm is applied only to events which have passed trigger selection. At the off-line level two muons inside the CMS acceptance $|\eta| \leqslant 2.4$ are required. The overall efficiency of the full reconstruction procedure taking into account trigger and off-line reconstruction inefficiency is between $97 \%$ and $93 \%$ for a mass range of 0.2 to $5 \mathrm{TeV} / \mathrm{c}^{2}$, as shown in Fig. 9.6 (left). In the case of an ideal detector the mass resolution smearing for fully-reconstructed events is between $1.8 \%$ and $6 \%$ for the same mass range, Fig. 9.6 (right). The effect of misalignment on the mass resolution varies from $1.1 \%$ up to $2.3 \%$ (1.3\%) for the First Data (Long Term) scenarios at the $Z$ and from 5\% up to 25\% (6\%) for $3 \mathrm{TeV} / \mathrm{c}^{2}$.

The cross sections of Drell-Yan production for the simulated CMS runs are shown in Table 9.5. The non-reducible backgrounds considered are vector boson pair production $Z Z$, $W Z, W W, t \bar{t}$ production etc. The simulation and pre-selection of background events is done with the same cuts as for the signal above. In the SM the expected leading-order cross section of these events is negligible in comparison with the Drell-Yan one, see Table 9.5. 

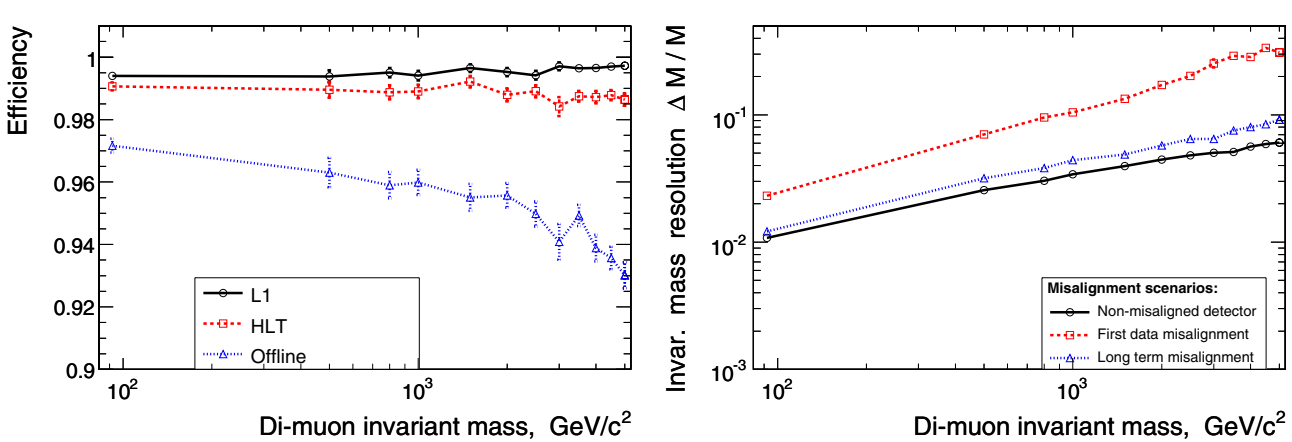

Figure 9.6. Left: dimuon reconstruction efficiency, and right: invariant mass resolution; both as function of the invariant mass cut.

Table 9.5. Leading-order cross sections of Drell-Yan, preselected Drell-Yan, dibosons ( $Z Z, Z W$, $W W$ ) and $t \bar{t}$ events in fb. The CTEQ5L parton distributions are used.

\begin{tabular}{lllllll}
\hline$M_{\mu^{+} \mu^{-}}, \mathrm{TeV} / \mathrm{c}^{2}$ & $\geqslant 1.0$ & $\geqslant 1.5$ & $\geqslant 2.0$ & $\geqslant 2.5$ & $\geqslant 3.0$ & $\geqslant 4.0$ \\
\hline Drell-Yan & 6.61 & 1.04 & $2.39 \cdot 10^{-1}$ & $6.53 \cdot 10^{-2}$ & $1.97 \cdot 10^{-2}$ & $2.09 \cdot 10^{-3}$ \\
Pre-sel. D-Y & 5.77 & $9.53 \cdot 10^{-1}$ & $2.24 \cdot 10^{-1}$ & $6.14 \cdot 10^{-2}$ & $1.87 \cdot 10^{-2}$ & $2.00 \cdot 10^{-3}$ \\
Dibosons & $2.59 \cdot 10^{-4}$ & $1.51 \cdot 10^{-4}$ & $5.6 \cdot 10^{-5}$ & $2.26 \cdot 10^{-5}$ & $9.06 \cdot 10^{-6}$ & $1.66 \cdot 10^{-6}$ \\
$t \bar{t}$ & $2.88 \cdot 10^{-4}$ & $2.58 \cdot 10^{-4}$ & $1.55 \cdot 10^{-4}$ & $7.02 \cdot 10^{-5}$ & $2.93 \cdot 10^{-5}$ & $3.65 \cdot 10^{-6}$
\end{tabular}

Table 9.6. Relative errors of the Drell-Yan muon pairs cross section measurements in the fiducial volume.

\begin{tabular}{llllll}
\hline $\begin{array}{l}M_{\mu^{+} \mu^{-}}, \\
\mathrm{TeV} / \mathrm{c}^{2}\end{array}$ & $\begin{array}{l}\text { Detector } \\
\text { smearing }\end{array}$ & $\begin{array}{l}\text { Statistical } \\
1 \mathrm{fb}^{-1}\end{array}$ & $\begin{array}{l}\text { Statistical } \\
10 \mathrm{fb}^{-1}\end{array}$ & $\begin{array}{l}\text { Statistical } \\
100 \mathrm{fb}^{-1}\end{array}$ & Theor. Syst. \\
\hline$\geqslant 0.2$ & $8 \cdot 10^{-4}$ & 0.025 & 0.008 & 0.0026 & 0.058 \\
$\geqslant 0.5$ & 0.0014 & 0.11 & 0.035 & 0.011 & 0.037 \\
$\geqslant 1.0$ & 0.0049 & 0.37 & 0.11 & 0.037 & 0.063 \\
$\geqslant 2.0$ & 0.017 & & 0.56 & 0.18 & 0.097 \\
$\geqslant 3.0$ & 0.029 & & & 0.64 & 0.134 \\
\hline
\end{tabular}

The $\tau \tau$ background (from $\tau$ decaying to $\mu$ and neutrinos) is $0.8 \%$ at the $\mathrm{Z}$ pole and $0.7 \%$ for masses above $1 \mathrm{TeV} / \mathrm{c}^{2}$. The background from Drell-Yan production of $q \bar{q}$ pairs (mostly semi-leptonic $\mathrm{b}$ or $\mathrm{c}$ decays) is $0.3 \%$ at the $\mathrm{Z}$ pole without applying any isolation cuts and below $0.1 \%$ for masses above $1 \mathrm{TeV} / \mathrm{c}^{2}$. The other background sources are negligible. If the need arises they can be further suppressed by acoplanarity and isolation cuts in the tracker.

The main experimental systematic effects in the cross section measurement arise from the total muon inefficiency and momentum resolution. The latter is very important at high mass as smearing from lower masses from the steeply falling Drell-Yan spectrum can contaminate the high mass measurements, especially if the tails of the momentum resolution are not under control. The main sources of systematic uncertainties on the momentum resolution come from the alignment of the muon chambers and the central tracker, both at start-up and high luminosity.

The statistical errors for 1,10 and $100 \mathrm{fb}^{-1}$ runs, the systematic uncertainty due to smearing in the detector and from theory side are given in Table 9.6. The modification of the measured cross section due to uncertainty of the mass resolution does not exceed $2.9 \%$ which is reached for a mass of $3 \mathrm{TeV} / \mathrm{c}^{2}$, see Table 9.6 . This has been estimated by applying 
an additional smearing to the dimuon mass (see $[99,347])$. The misalignment does not affect the efficiency of dimuon reconstruction for any masses [99]. Taking into account the trigger efficiency changes from $98.5 \%$ to $97 \%$ for masses from 0.2 to $5 \mathrm{TeV} / \mathrm{c}^{2}$, very conservatively we may assign half of this change with mass, i.e., $0.75 \%$, as a systematic uncertainty.

An important ingredient in the cross section measurement is the precise determination of the luminosity. A promising possibility is to go directly to the parton luminosity [345] by using the $W^{ \pm}(Z)$ production of single (pair) leptons. New estimates show that in this way the systematic error on $\sigma_{D Y}^{\text {high } Q^{2}}$ relative to $\sigma_{Z}$ can be reduced to $\approx 5-12 \%$ [349].

On the theory side we consider several sources of systematic uncertainties. Higher order QCD corrections are often taken into account with $K$-factor of 1.3 as calculated with the program PHOZPRMS [348]. It is expected that the total value of additional NNLO contributions does not exceed $8 \%$.

A full-scale analysis of experimental data (comparison data with theory, taking into account acceptance corrections for precise measurement of $\sigma$ and $A_{F B}$ at large centre-ofmass energies $\hat{\mathrm{s}}$ ) requires good knowledge of the different types of genuine electroweak (EW) radiative corrections to the DY process: vertex, propagator, EW boxes. A complete one-loop parton cross section calculation has been included in [158] and confirmed in [350]. The EW corrections change the cross section by 10-20\%. The calculation [105] of the weak radiative corrections to the Drell-Yan processes due to additional heavy bosons contributions shows that these corrections are about $2.9 \%$ to $9.7 \%$ for mass region between $0.2 \mathrm{TeV} / \mathrm{c}^{2}$ and $5 \mathrm{TeV} / \mathrm{c}^{2}$.

The phenomenological origin of PDF gives one additional systematic error. First of all, estimates of cross section obtained by using different sets of structure functions do not give exactly the same values. The results vary within $\pm 7 \%$ for $M_{l l} \geqslant 1 \mathrm{TeV} / \mathrm{c}^{2}$. The internal PDF uncertainties are estimated using the LHAPDF library [95, 351]. The PDF-dependence of the acceptance efficiency is estimated by using the PDF sets CTEQ5L, CTEQ6L and MRST2001E. The changes in the acceptance efficiency are up to $0.5 \%$. The ambiguity in the acceptance efficiency due to internal PDF uncertainties is larger, but less than $1.4 \%$ for any mass region.

The summary of the estimated systematic uncertainties as function of the dilepton mass is given in Fig. 9.7. The CMS experiment has excellent potential to measure the cross section for dimuon pairs up to the highest masses that will be accessible at the LHC, and to test the Standard Model up to very high momentum transfers in a new and unexplored energy range. Current uncertainties from theory are larger than the experimental uncertainties. The statistical errors will dominate for invariant masses larger than $2 \mathrm{TeV} / \mathrm{c}^{2}$ even for $100 \mathrm{fb}^{-1}$.

\subsubsection{Prospects on the measurement of the forward-backward asymmetry}

To measure the forward-backward asymmetry we need the original quark and anti-quark directions of the initiating partons, but these are not known in the case of $p p$ experiments, where the initial state is symmetric. In Ref. $[96,112]$ it is shown that it is possible to approximate the quark direction with the boost direction of the dimuon system with respect to the beam axis. This is due to the fact that the valence quarks have on average larger momentum than the sea anti-quarks, and therefore the dimuon boost direction approximates the quark direction. The most unambiguous tagging occurs for large dimuon rapidity.

The approximation of the original quark direction for $p p$ collisions leads to a flattening out of the original asymmetry $(\approx 0.61$ for Drell-Yan events) by a factor of almost 2 . However, using multi-dimensional fits [111] or reweighting techniques depending on the mistag and acceptance which are under development, we can measure the original asymmetry. 


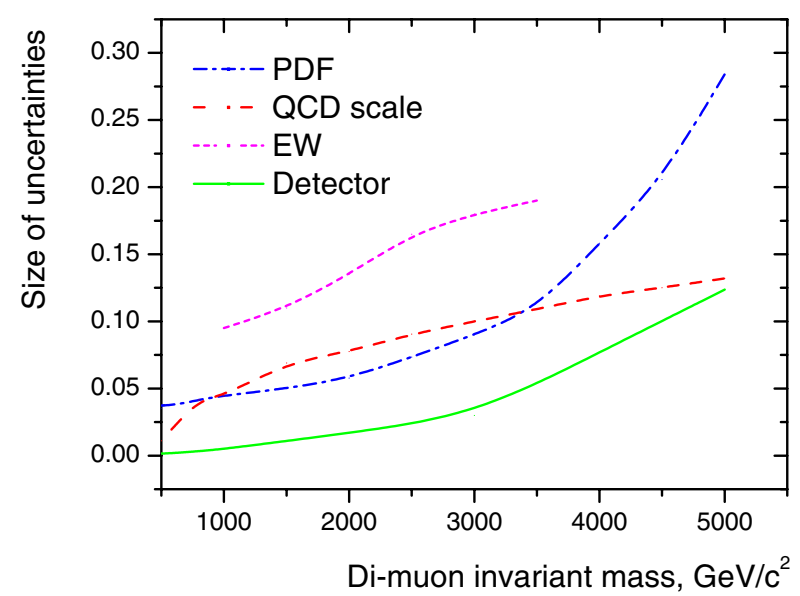

Figure 9.7. Size of the EW corrections and the cross section uncertainties from PDFs, hard process scale and detector understanding as a function of the dimuon invariant mass cut.

The accuracy of asymmetry measurements depends on:

- statistical uncertainty which grows with rising mass cut value, as the number of events for integrated luminosity of e.g. $\int \mathcal{L} \mathrm{d} t=100 \mathrm{fb}^{-1}$ decreases with mass;

- systematic uncertainty from the variation of the mistag probabilities for various PDF sets, typically below $10 \%$.

We expect the systematic uncertainty to dominate the statistical one for integrated luminosity of $\int \mathcal{L} d t=100 \mathrm{fb}^{-1}$ and dimuon masses around $500 \mathrm{GeV} / \mathrm{c}^{2}$, while the statistical one to be more important for dimuon mass cuts above $1000 \mathrm{GeV} / \mathrm{c}^{2}$.

\subsection{Determination of the $W$ mass}

\subsubsection{Introduction}

The precise measurement of the mass of the $W$ boson constitutes an important consistency check of the Standard Model and, together with the top quark mass, is sensitive to supersymmetric corrections. Such a precision measurement of the $W$ mass at the LHC becomes feasible because a huge sample of data available at the LHC will guarantee a nearly negligible statistical uncertainty and a good control of the systematic effects. Extrapolating from traditional approaches based on the reconstruction of the transverse mass $m_{\mathrm{T}}=\sqrt{2 p_{\mathrm{T}}^{l} p_{\mathrm{T}}^{v}\left(1-\cos \left(p_{\mathrm{T}}^{l}, p_{\mathrm{T}}^{v}\right)\right)}$ in leptonic $\mathrm{W}$ decays, the most relevant contributions to the systematic uncertainties come from the lepton energy or momentum scale, the lepton energy or momentum resolution, the modelling of the system recoiling against the $W$ boson, the parton distribution functions, the $W$ intrinsic width, from radiative decays and from backgrounds. To accomplish a competitive measurement of the $W$ boson mass, new strategies must be considered [352]. The most promising one consists in predicting the distribution of experimental observables sensitive to the $W$ mass, such as the transverse momentum of the charged lepton $\left(p_{\mathrm{T}}^{l}\right)$ and the transverse mass of the boson from the corresponding distribution measured in $Z$ boson decays into two charged leptons. The concept of transverse mass measurement can be applied to $Z$ boson events by regarding one of the reconstructed 
leptons as missing energy. The theoretical description of both decays is very similar and the resulting distributions in transverse mass are comparable for a wide range in kinematics.

The advantage of this approach, conceptually discussed in [353], is that most of the experimental and theoretical uncertainties, being common between $W$ and $Z$, cancel in the comparison, leading to a global reduction of the systematic uncertainty. The drawback is a larger statistical uncertainty due to the smaller production rate of $Z$ bosons decaying to charged leptons. Yet a statistical precision of order $10 \mathrm{MeV} / \mathrm{c}^{2}$ and $30 \mathrm{MeV} / \mathrm{c}^{2}$ for an integrated luminosity of $10 \mathrm{fb}^{-1}$ and $1 \mathrm{fb}^{-1}$ respectively is anticipated. In order not to be limited by statistics, the analyses are performed using large data samples produced with the fast simulation of the CMS experiment [11]. Smaller samples of fully simulated events are used for cross checks.

Two different ways to relate $Z$ to $W$ boson events are considered. One is based on the comparison of the same experimental observables in $W$ - and $Z$-events scaled to the boson masses. The sensitivity of this method, which can take advantage of the precision calculation of the theoretical ratio of the $W$ and $Z$ boson differential production cross-sections, is fully addressed in the analysis of transverse energy distribution of the electrons from $W \rightarrow e v$ decays. An alternative approach considered in the analysis of $W \rightarrow \mu v$ events consists of predicting $W$ boson distributions from $Z$-events by means of kinematic transformations of measured $Z$ events, parameterised as a function of the boson masses and widths. This more phenomenological approach is exploited in the analysis of the transverse mass distributions, and relies less on the theoretical prediction of the boson $p_{\mathrm{T}}$.

\subsubsection{Event selections}

In order to obtain a clean signal of $W \rightarrow 1 v$ decays, events that passed the High Level Trigger (HLT) for single leptons are required to satisfy the following selection cuts: one isolated muon with $p_{\mathrm{T}}>25 \mathrm{GeV} / \mathrm{c}$ within the pseudo-rapidity region $|\eta|<2.3$ or one isolated electron with $p_{\mathrm{T}}>25 \mathrm{GeV} / \mathrm{c}$ and within $|\eta|<2.4$; missing transverse energy $E_{\mathrm{T}}^{\text {miss }}>25 \mathrm{GeV}$; no jets in the event with $p_{\mathrm{T} j e t}>30 \mathrm{GeV} / \mathrm{c}$; the transverse momentum of the system recoiling against $W$ has to be lower than $20 \mathrm{GeV} / \mathrm{c}$, measured from the lepton $p_{\mathrm{T}}$ and the missing transverse energy.

The difference in minimum $p_{\mathrm{T}}$ of the charged lepton is determined by the single lepton trigger threshold. The last two selection cuts are intended to select $W$ bosons produced with a small transverse momentum. The selection efficiency is about $15 \%$ for the electron channel and $25 \%$ for the muon channel, with a background at the percent level, dominated by leptonic $Z$ decays with one lepton outside the acceptance, as shown in Fig. 9.8.

$Z$ events used to predict the $W$ distribution are also selected from the sample of events passing the HLT for single leptons. $Z$ candidates contain a pair of identified charged leptons consistent with the $Z$ mass hypothesis [352]. One of the two leptons, randomly chosen, is removed from the event to mimic a $W$ decay. The same selections discussed above are then applied, with the cut values on the lepton quantities (minimum lepton $p_{\mathrm{T}}$ and event missing transverse energy) scaled by the ratio $M_{Z} / M_{W}$. This choice is intended to minimise kinematic and acceptance differences in $Z$ and $W$ events and thus the theoretical uncertainties implied by the above mentioned approaches.

\subsection{3. $W \rightarrow e v$}

The analysis strategy is based on the prediction of the experimental distribution of the electron transverse energy in $W$ events scaled to the boson mass from the corresponding distribution 

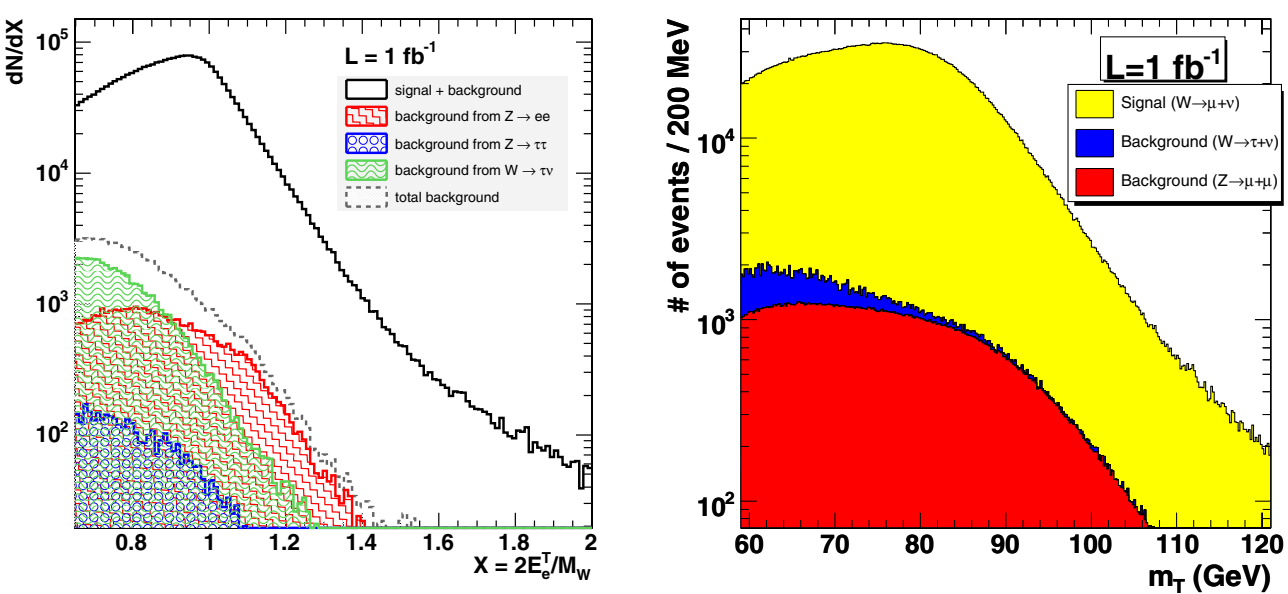

Figure 9.8. W events and main backgrounds for $1 \mathrm{fb}^{-1}$. Left: Electron scaled transverse energy distribution in $W \rightarrow e v$ decays and the backgrounds from $Z \rightarrow e^{+} e^{-}$, from $Z \rightarrow \tau^{+} \tau^{-}$and from $W \rightarrow \tau \nu$ for $1 \mathrm{fb}^{-1}$. Right: Transverse mass distribution in the muon channel with the fractions of $Z^{0} / \gamma^{*} \rightarrow \mu^{+} \mu^{-}$(red/grey) $W \rightarrow \tau \nu$ (blue/dark), and $\mathrm{W} \rightarrow \mu \nu$ (yellow/light) events.

measured for $Z$ bosons decaying into $e^{+} e^{-}$pairs, along with the theoretical ratio between the $W$ and $Z$ cross-sections, calculated at a fixed perturbative order. Ideally, the differential cross section for the $W$ boson can be predicted from the one measured for $Z$ boson by scaling the lepton transverse momenta with the boson masses, $p_{\mathrm{T}}^{\text {lept }, \mathrm{Z}}=M_{Z} / M_{W} p_{\mathrm{T}}^{\text {lept, } \mathrm{W}}$, as:

$$
\left.\frac{\mathrm{d} \sigma^{W}}{\mathrm{~d} p_{\mathrm{T}}^{\text {lept }, \mathrm{W}}}\right|_{\text {pred }}=\left.\frac{M_{Z}}{M_{W}} R(X) \frac{\mathrm{d} \sigma^{Z}}{\mathrm{~d} p_{\mathrm{T}}^{\text {lept }, \mathrm{Z}}}\left(p_{\mathrm{T}}^{\text {lept }, \mathrm{Z}}=\frac{M_{Z}}{M_{W}} p_{\mathrm{T}}^{\text {lept }, \mathrm{W}}\right)\right|_{\text {meas }},
$$

where $R(X)=\frac{\mathrm{d} \sigma^{W}}{\mathrm{~d} X^{W}} / \frac{\mathrm{d} \sigma^{Z}}{\mathrm{~d} X^{Z}}$ is the ratio, deduced from theoretical calculations, between the differential cross sections in terms of the scaled variable $X^{V}=\frac{p_{\mathrm{T}}^{\text {lept }, V}}{M_{V}}$, with $V=\mathrm{W}, \mathrm{Z}$. The parameter $M_{W}$ can be extracted by fitting this prediction to the distribution for $W$ events observed in the experiment. In practice, additional corrections to $R(X)$ are needed to account for the acceptance to $Z$ and $W$ events and for the experimental resolution. This calls for a detailed understanding of the detector response by means of Monte Carlo simulations compared to control samples. Clearly, the definition of $R(X)$ is the most critical aspect and must include both detector effects and theoretical predictions.

The results for $1 \mathrm{fb}^{-1}$ of integrated luminosity using the technique just described are shown in Fig. 9.9. The statistical precision of the method is determined from the resulting $\chi^{2}$ distribution. The evaluation of the systematic uncertainties affecting the measurement of the $W$ mass is performed by determining the distortions implied by the different systematic effects mentioned above. The effects of instrumental origin have been studied by fixing $R(X)$ to the theoretical prediction exactly describing the samples of generated events (i.e. an exact knowledge of the theory is assumed) and by introducing distortions and biases in the detector response. The resulting shift in $M_{W}$ is assumed as the systematic uncertainty associated to the effect. The detector response to electrons, the largest source of systematic uncertainty of instrumental origin with this method, can be determined with the required precision from $Z \rightarrow e e$ events.

The prediction of the lepton transverse spectrum is plagued by large radiative QCD corrections. Yet, in the method adopted, large cancellations occur and $R(X)$ can be reliably 

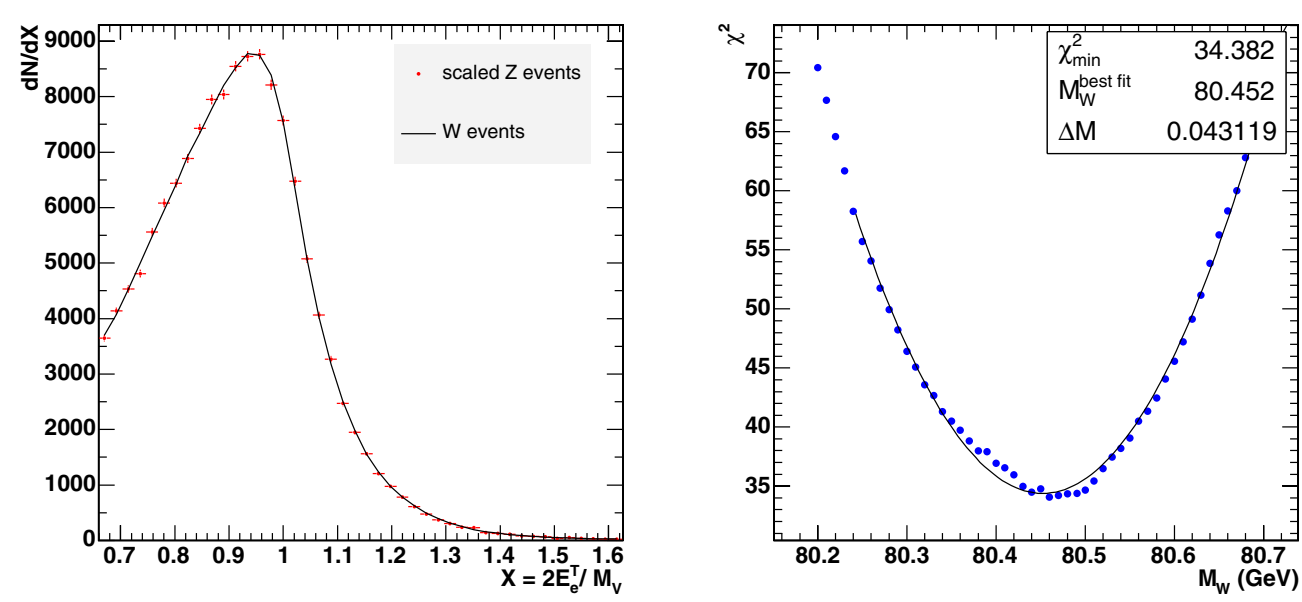

Figure 9.9. Comparison of the scaled electron $E_{\mathrm{T}}$ spectra for $Z$ (dots) and $W$ boson (line) events (left) and $\chi^{2}$ dependence on $M_{W}$ (right) for $1 \mathrm{fb}^{-1}$ of integrated luminosity.

predicted. The uncertainty related to the missing orders in the perturbative expansion can be quantified by the dependence of the available NLO prediction on the choice of the renormalisation and factorisation scales. A conservative figure of $30 \mathrm{MeV} / \mathrm{c}^{2}$ for the mass uncertainty is deduced. This will become the dominant error at $10 \mathrm{fb}^{-1}$. Yet the reduction of this error by extending the calculation one order higher in $\alpha_{S}$ is technically feasible [353].

\subsection{4. $W \rightarrow \mu \nu$}

As a complementary method, the transverse mass distribution of $\mathrm{W}$ events in the muon channel is modelled from $\mathrm{Z} \rightarrow \mu^{+} \mu^{-}$events by a kinematic transformation. In the rest frame of the $Z$ boson, the lepton momenta are scaled such that their invariant mass distribution represents that of the $W$ boson [352]. After removing one randomly chosen muon to mimic a neutrino, the whole system is boosted back into the detector frame, thus obtaining a template for the expected distribution of $W$ events, which depends on the $W$ and $Z$ boson masses and widths as parameters. By iterating the procedure for different $\mathrm{W}$ boson masses, the best agreement with the observed transverse mass distribution in $W$ events is determined using a $\chi^{2}$ criterion. In practice, weighting factors take into account unavoidable differences between the $W$ and $Z$ samples, such as the acceptance for the second lepton, photon radiation, and differences in $\eta$ and $p_{\mathrm{T}}$ of $W$ and $Z$ bosons. Thus perfect agreement of the distributions at the nominal $W$ mass and for the simulated detector is ensured, while systematic effects are studied by introducing distortions of experimental or theoretical origin. The resulting shifts in the extracted $W$ mass are taken as the related systematic uncertainties.

The dominant systematic error arises from scale and resolution uncertainties in the missing energy determined from the calorimeters. These can be controlled by using the $Z$ sample, where the boson $p_{\mathrm{T}}$ can be measured from the two charged leptons, as is shown in Fig. 9.10. The observed differences of $2 \%$ on the scale and $5 \%$ on the resolution are taken as the systematic uncertainties.

\subsubsection{Expected precision and systematic uncertainties}

The expected size of various detector effects for the early detector operation, after the analysis of an initial integrated luminosity of $1 \mathrm{fb}^{-1}$, and for a better detector understanding 

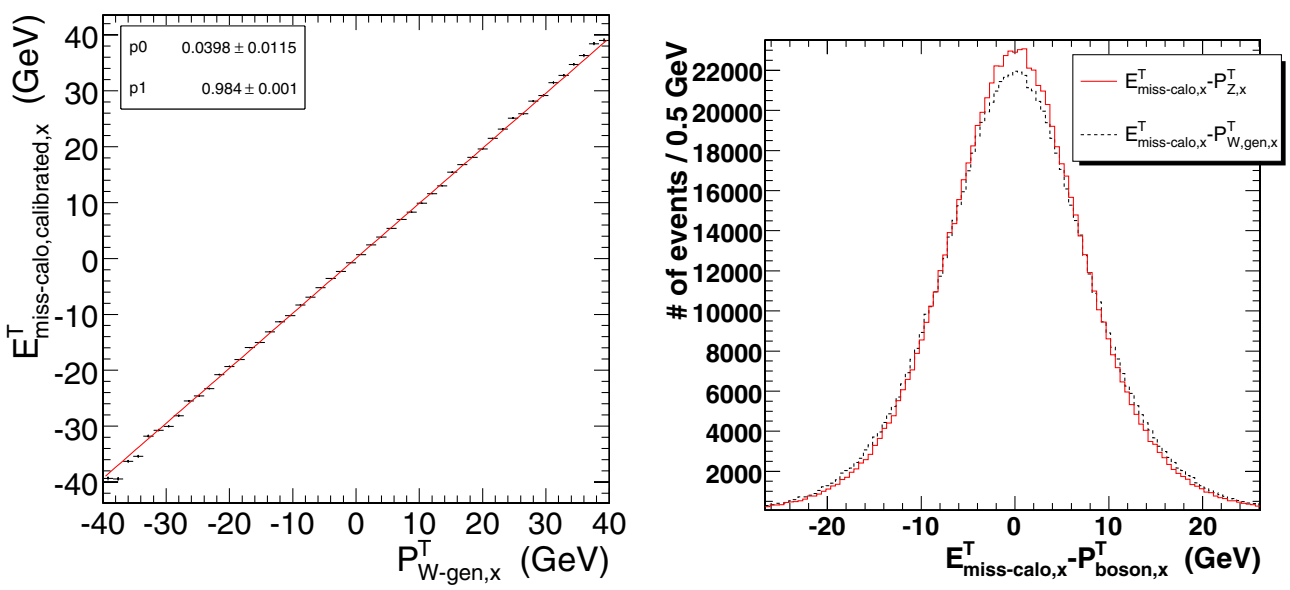

Figure 9.10. Left: $x$-component of the calibrated missing transverse energy in the calorimeters using the reconstructed muon $p_{\mathrm{T}}$ in $\mathrm{Z}$ events, as a function of the transverse $W$ boson momentum at generator level. The slope of a fitted straight line is 0.98. Right: Difference between the reconstructed missing energy in the calorimeters and the measured muon $p_{\mathrm{T}}$ in $Z$ events (red/grey line) or the $W$ boson $p_{\mathrm{T}}$ at generator level (black dashed line). The RMS of the distribution is $8.15 \mathrm{GeV}$ for $\mathrm{Z}$ events and $8.65 \mathrm{GeV}$ for $\mathrm{W}$ events.

expected after employing an integrated luminosity of $10 \mathrm{fb}^{-1}$, is shown in Table 9.7 for the scaled $p_{\mathrm{T}}$-lepton method applied to the electron channel, and for the muon channel using the transformation method.

The measurements of the $W$ mass by means of $W \rightarrow e v$ and $W \rightarrow \mu \nu$ decays are largely independent. Common experimental uncertainties arise from the systematics involving the missing transverse energy in the calorimeters.

Based on the estimated systematic errors, it is clear that the scaled $p^{T}$-lepton method suffers less from experimental systematic errors than the transformation method. If systematic uncertainties arising from the theoretical prediction of the transverse momenta of the $Z$ and $W$ bosons can be brought to a level of $\approx 10 \mathrm{MeV} / \mathrm{c}^{2}$, the scaled $p^{T}$-lepton method is clearly the first choice. Using the scaled $p_{\mathrm{T}}$-lepton method in the muon channel leads to a better statistical precision of $30 \mathrm{MeV} / \mathrm{c}^{2}$ for $1 \mathrm{fb}^{-1}$ due to the higher acceptance for muons compared to electrons. The total instrumental uncertainty of the $p_{\mathrm{T}}$-lepton method applied to the muon channel is estimated from the findings in the electron channel and amounts to about $25 \mathrm{MeV} / \mathrm{c}^{2}$ for the initial measurement with an integrated luminosity of $1 \mathrm{fb}^{-1}$. Uncertainties due to the recoil modelling are fully correlated with the electron channel. The component of the experimental error in common with the electrons amounts to about $20 \mathrm{MeV} / \mathrm{c}^{2}$. Clearly, all theoretical uncertainties are of similar size and also correlated between the electron and muon channels.

The transformation method has the advantage of providing templates for observables in $W$ events from measured observables in $Z$ events. In particular, the measurement of the transverse momentum of $Z$ bosons and the cross checks on the modelling of the missing energy are of vital importance to quantify systematic uncertainties.

The combination of the electron and muon channels brings the statistical uncertainty to a final precision of better than $10 \mathrm{MeV} / \mathrm{c}^{2}$ for an integrated luminosity of $10 \mathrm{fb}^{-1}$, and a systematic uncertainty of instrumental origin below $20 \mathrm{MeV} / \mathrm{c}^{2}$ should be within reach. 
Table 9.7. Expected systematic uncertainties on $M_{\mathrm{W}}$ for the scaled $E^{T}$-lepton method with electrons (upper part) and for the $Z$ transformation method applied to the muon channel (lower part). The first column lists the systematic effect considered, the second and third columns show the assumed detector uncertainty for an initial integrated luminosity of $1 \mathrm{fb}^{-1}$ and the resulting uncertainty on $M_{W}$. The last two columns show the extrapolation to an integrated luminosity of $10 \mathrm{fb}^{-1}$, when the detector understanding is assumed to have significantly improved.

\begin{tabular}{|c|c|c|c|c|}
\hline \multirow[t]{2}{*}{ Source of uncertainty } & uncertainty & $\Delta M_{W}\left[\mathrm{MeV} / \mathrm{c}^{2}\right]$ & uncertainty & $\Delta M_{W}\left[\mathrm{MeV} / \mathrm{c}^{2}\right]$ \\
\hline & \multicolumn{2}{|c|}{ with $1 \mathrm{fb}^{-1}$} & \multicolumn{2}{|c|}{ with $10 \mathrm{fb}^{-1}$} \\
\hline & \multicolumn{4}{|c|}{ scaled lepton- $p_{\mathrm{T}}$ method applied to $\mathrm{W} \rightarrow \mathrm{e} v$} \\
\hline statistics & & 40 & & 15 \\
\hline background & $10 \%$ & 10 & $2 \%$ & 2 \\
\hline electron energy scale & $0.25 \%$ & 10 & $0.05 \%$ & 2 \\
\hline scale linearity & $0.00006 / \mathrm{GeV}$ & 30 & $<0.00002 / \mathrm{GeV}$ & $<10$ \\
\hline energy resolution & $8 \%$ & 5 & $3 \%$ & 2 \\
\hline MET scale & $2 \%$ & 15 & $<1.5 \%$ & $<10$ \\
\hline MET resolution & $5 \%$ & 9 & $<2.5 \%$ & $<5$ \\
\hline recoil system & $2 \%$ & 15 & $<1.5 \%$ & $<10$ \\
\hline total instrumental & & 40 & & $<\mathbf{2 0}$ \\
\hline PDF uncertainties & & 20 & & $<10$ \\
\hline$\Gamma_{W}$ & & 15 & & $<15$ \\
\hline \multirow[t]{2}{*}{$p_{\mathrm{T}}^{\mathrm{W}}$} & & 30 & & 30 (or NNLO) \\
\hline & \multicolumn{4}{|c|}{ transformation method applied to $\mathrm{W} \rightarrow \mu \nu$} \\
\hline statistics & & 40 & & 15 \\
\hline background & $10 \%$ & 4 & $2 \%$ & negligible \\
\hline momentum scale & $0.1 \%$ & 14 & $<0.1 \%$ & $<10$ \\
\hline $1 / p^{T}$ resolution & $10 \%$ & 30 & $<3 \%$ & $<10$ \\
\hline acceptance definition & $\eta$-resol. & 19 & $<\sigma_{\eta}$ & $<10$ \\
\hline calorimeter $E_{\mathrm{T}}^{\text {miss }}$, scale & $2 \%$ & 38 & $\leqslant 1 \%$ & $<20$ \\
\hline calorimeter $E_{\mathrm{T}}^{\text {miss }}$, resolution & $5 \%$ & 30 & $<3 \%$ & $<18$ \\
\hline detector alignment & & 12 & - & negligible \\
\hline total instrumental & & 64 & & $<\mathbf{3 0}$ \\
\hline PDF uncertainties & & $\approx 20$ & & $<10$ \\
\hline$\Gamma_{W}$ & & 10 & & $<10$ \\
\hline
\end{tabular}

\subsection{Multi-boson production}

\subsubsection{Introduction}

The study of multiple gauge-boson production at the $\mathrm{TeV}$ scale constitutes a unique opportunity to test the Standard Model of Electroweak interactions at the highest possible energies. The production of $W^{ \pm} Z^{0}$ and $W^{ \pm} \gamma$ events at the LHC probes the triple gaugeboson couplings and therefore the non-Abelian gauge symmetry of the Standard Model. On the other hand, no neutral gauge-boson couplings exist in the Standard Model, thus anomalies in $Z^{0} Z^{0}$ and $Z^{0} \gamma$ production, hinting at large $s$-channel contributions, could be the first indirect manifestation of New Physics. In the following, the selections of $W^{ \pm} Z^{0}$ and $Z^{0} Z^{0}$ events are described, their signal-over-background ratio discussed and the outlook for an early measurement of multiple gauge-boson production is assessed. Further details are given in Ref. [354].

The multi-lepton final states of multiple gauge-boson production are an important background in the search for New Physics, in particular Supersymmetry. A sound 
understanding of their production process is therefore needed in the first phase of LHC datataking before any discovery can be claimed. In particular, $Z^{0} Z^{0}$ production is an irreducible background to the most-coveted discovery at the LHC: the Standard Model Higgs boson. Its early measurement is therefore important.

The cross sections for multiple gauge-boson production at the LHC are of about $50 \mathrm{pb}$ for the $W^{ \pm} Z^{0}$ channel and $20 \mathrm{pb}$ for the $Z^{0} Z^{0}$ channel [158]. These large cross sections and the clean signature of fully-leptonic final states make $W^{ \pm} Z^{0}$ and $Z^{0} Z^{0}$ production observable in the early LHC data. Final states where the gauge bosons decay into electrons and muons are considered: $e^{ \pm} e^{+} e^{-}, \mu^{ \pm} e^{+} e^{-}, e^{ \pm} \mu^{+} \mu^{-}$and $\mu^{ \pm} \mu^{+} \mu^{-}$for $W^{ \pm} Z^{0}$ production and $e^{+} e^{-} e^{+} e^{-}$for the $Z^{0} Z^{0}$ channel. The competing background processes are the Standard Model production of gauge bosons and top quarks, which also yield leptonic final states.

\subsubsection{Signal definition and modelling}

Both the $W^{ \pm} Z^{0}$ and $Z^{0} Z^{0}$ analyses focus on on-shell gauge bosons. On-shell production of the $W^{ \pm} Z^{0}$ final state proceeds mainly through the $s$-channel, involving a $W W Z$ triple gauge-boson coupling. Additional contributions from the $W^{ \pm} \gamma^{*}$ final state through a $W W \gamma$ coupling are effectively suppressed by constraining the mass of the observed lepton pair to be compatible with a $Z^{0}$ boson. The PYTHIa Monte Carlo generator [24] is used to model $W^{ \pm} Z^{0}$ production and subsequent decay into fully-leptonic final states. Gauge-boson decays into tau leptons are also included. These tau leptons are left free to decay into either leptons or hadrons.

Four-electron final-states can originate from $Z^{0} Z^{0}$ production as well as via either $Z^{0} \gamma^{*}$ or $\gamma^{*} \gamma^{*}$ production. The requirement of on-shell boson is enforced by considering only electron-positron pairs with a mass between 70 and $110 \mathrm{GeV} / \mathrm{c}^{2}$. The PYTHIA Monte Carlo is used to generate events of this process, with the additional requirement that the electrons have a rapidity $|\eta|<2.7$ and a transverse momentum $p_{\mathrm{T}}>5 \mathrm{GeV} / \mathrm{c}$. Of all generated events, $72 \%$ are classified as $Z^{0} Z^{0}$ signal while $26 \%$ are ascribed to the $Z^{0} \gamma^{*}$ process and $2 \%$ to the $\gamma^{*} \gamma^{*}$ process.

Taking into account the branching fraction into leptons, $\mathcal{B}$, and the kinematic requirements, $\varepsilon_{K I N}$, the relevant NLO cross sections using the MCFM [56] Monte Carlo are:

$$
\begin{aligned}
& \sigma_{N L O} \times \mathcal{B} \times \epsilon_{K I N}\left(p p \rightarrow W^{+} Z^{0} \rightarrow \ell^{+} \ell^{+} \ell^{-}\right)=1034 \mathrm{fb} \\
& \sigma_{N L O} \times \mathcal{B} \times \epsilon_{K I N}\left(p p \rightarrow W^{-} Z^{0} \rightarrow \ell^{-} \ell^{+} \ell^{-}\right)=630 \mathrm{fb} \\
& \sigma_{N L O} \times \mathcal{B} \times \epsilon_{K I N}\left(p p \rightarrow Z^{0} Z^{0} \rightarrow e^{+} e^{-} e^{+} e^{-}\right)=18.7 \mathrm{fb}
\end{aligned}
$$

The NLO corrections correspond to $k$-factors of 1.9 and 1.4 for $W^{ \pm} Z^{0}$ and $Z^{0} Z^{0}$ production, respectively. The NNLO box-diagram contribution to $Z^{0} Z^{0}$ production is not taken into account.

Three-lepton final-states from $W^{ \pm} Z^{0}$ and $Z^{0} Z^{0}$ production are collected with high efficiency by the Level-1 and HLT electron and muon triggers. The Level-1 and HLT efficiencies for events retained by the selections discussed below is $100 \%$ [76].

\subsubsection{Background processes}

The background to the selection of $W^{ \pm} Z^{0}$ and $Z^{0} Z^{0}$ events comprises other processes with multiple leptons in the final states, some of which might be due to fake signals. The most copious sources of multiple leptons at the LHC are $t \bar{t}$ and $Z^{0} b \bar{b}$ production. The cross section of these processes is large: $830 \mathrm{pb}$ and $1492 \mathrm{pb}$, respectively, as calculated with MCFM at NLO. 
These processes may have two leptons in the final states from leptonic decays of the $W$ bosons arising from $t \rightarrow W b$ decays or of the $Z^{0}$ boson, respectively. The other leptons can be produced in the direct or cascade decays of the $b$ quarks. The $Z^{0} b \bar{b}$ process is modelled with the CompHEP Monte Carlo generator $[43,355]$ and the $t \bar{t}$ process with the TopReX Monte Carlo program [44]. In addition, the special case in which four electrons are produced in $t \bar{t}$ events is considered in detail and modelled with PYTHIA. Contributions from $W t$ and $Z c \bar{c}$ to the selected samples are negligible.

Events from $Z^{0} Z^{0}$ production also constitute a background to the $W^{ \pm} Z^{0}$ selection. Events from the $Z^{0} \gamma^{*}$ and $\gamma^{*} \gamma^{*}$ processes are a background for both the $W^{ \pm} Z^{0}$ and $Z^{0} Z^{0}$ analyses.

\subsection{4. $W^{ \pm} Z^{0}$ selection}

Events with three charged leptons, either electrons or muons, with $p_{\mathrm{T}}>10 \mathrm{GeV} / \mathrm{c}$ and $|\eta|<2.5$, are considered by the $W^{ \pm} Z^{0}$ selection. All possible $Z^{0}$-boson candidates from same-flavours opposite-charge lepton pairs are formed. Events are retained if the mass of the $Z^{0}$ candidate is within $20 \mathrm{GeV} / \mathrm{c}^{2}$ of the $Z^{0}$-boson mass, $m_{Z}$. These criteria effectively suppress $Z^{0}$ decays into tau leptons. The background from $Z^{0} Z^{0}$ final states is reduced by rejecting events with a second $Z^{0}$ candidate with a mass within $40 \mathrm{GeV} / \mathrm{c}^{2}$ of $m_{Z}$. The remaining lepton is associated to the $W^{ \pm}$-boson decay; its transverse momentum must be larger than $20 \mathrm{GeV}$. This criterion results in lower efficiencies for the $W^{ \pm}$boson decays in tau leptons. The highest- $p_{\mathrm{T}}$ lepton associated to the $Z^{0}$ boson must satisfy $p_{\mathrm{T}}>15 \mathrm{GeV} / \mathrm{c}$. If the event contains more than three leptons, the lepton with highest $p_{\mathrm{T}}$ is chosen as originating from the $W^{ \pm}$. The signal efficiency after these cuts is $9.2 \%$ while the $t \bar{t}, e^{+} e^{-} b \bar{b}$ and $\mu^{+} \mu^{-} b \bar{b}$ efficiencies are $0.7 \%, 0.4 \%$ and $0.6 \%$, respectively.

Leptons from the decay of $b$ quarks in the background processes are produced in a higher-multiplicity environment and isolation criteria suppress the background contamination. Electrons associated to the $\mathrm{W}^{ \pm}$boson must have no other charged track with $p_{\mathrm{T}}>2 \mathrm{GeV} / \mathrm{c}$ within a $\Delta R=0.3$ cone around their direction. All muon candidates must have an energy measured in the calorimeters within a $\Delta R=0.3$ cone around their direction smaller than $5 \mathrm{GeV}$ and the sum of the $p_{\mathrm{T}}$ of tracks within a $\Delta R=0.25$ cone smaller than $2 \mathrm{GeV} / \mathrm{c}$. The significance of the lepton impact parameter in the plane transverse to the beam, $S_{I P}$, discriminates against leptons from heavy-quark decays. This variable is defined as the ratio between the measured impact parameter and its uncertainty and is required to satisfy $S_{I P}<3$. The signal efficiency after these cuts is $7.3 \%$ while the $t \bar{t}, e^{+} e^{-} b \bar{b}$ and $\mu^{+} \mu^{-} b \bar{b}$ efficiencies are $0.07 \%, 0.008 \%$ and $0.03 \%$, respectively.

The $t \bar{t}$ and $Z^{0} b \bar{b}$ final states are associated with one or more hard jets and their contribution is reduced by removing events containing at least a jet with $E_{\mathrm{T}}>25 \mathrm{GeV}$. Only jets outside cones of $\Delta R=0.3$ around the three leptons are considered. The reconstructed mass of the $Z^{0}$ boson is required to be within $10 \mathrm{GeV} / \mathrm{c}^{2}$ of $m_{Z}$, leading to the total efficiencies presented in Table 9.8 .

\subsection{5. $Z^{0} Z^{0}$ selection}

The $Z^{0} Z^{0}$ selection is based on events with four electrons, identified from superclusters in the electromagnetic calorimeter matched with a charged track. The transverse momenta of the electron candidates, ordered from the largest to the smallest, have to be above $30 \mathrm{GeV} / \mathrm{c}$, $20 \mathrm{GeV} / \mathrm{c}, 15 \mathrm{GeV} / \mathrm{c}$ and $10 \mathrm{GeV} / \mathrm{c}$, respectively. This cut suppresses the contribution from the $Z^{0} \gamma^{*}$ and $\gamma^{*} \gamma^{*}$ final states and reduces by $30 \%$ and $60 \%$ the $t \bar{t}$ and $Z^{0} b \bar{b}$ backgrounds, 
Table 9.8. Yield of the $W^{ \pm} Z^{0}$ selection for an integrated luminosity of $1 \mathrm{fb}^{-1}$. Signal efficiencies include gauge-boson decays into tau leptons.

\begin{tabular}{lcccccl}
\hline & $e^{ \pm} e^{+} e^{-}$ & $\mu^{ \pm} e^{+} e^{-}$ & $e^{ \pm} \mu^{+} \mu^{-}$ & $\mu^{ \pm} \mu^{+} \mu^{-}$ & Total & Efficiency \\
\hline$W^{ \pm} Z^{0} \rightarrow \ell^{ \pm} \ell^{+} \ell^{-}$ & 14.8 & 26.9 & 28.1 & 27.0 & 96.8 & $6.1 \%$ \\
$Z^{0} Z^{0}$ & 0.63 & 1.54 & 1.50 & 1.51 & 5.18 & $4.7 \%$ \\
$t \bar{t}$ & 0.93 & 1.55 & - & 0.31 & 2.79 & $0.02 \%$ \\
$\mu^{+} \mu^{-} b \bar{b}$ & - & - & 6.54 & 4.9 & 11.4 & $0.005 \%$ \\
$e^{+} e^{-} b \bar{b}$ & 1.21 & 1.82 & - & - & 3.03 & $0.005 \%$ \\
\hline
\end{tabular}

Table 9.9. Yield of the $Z^{0} Z^{0}$ selection for integrated luminosities of $1 \mathrm{fb}^{-1}$ and $10 \mathrm{fb}^{-1}$. The last row indicates the signal significance, which include systematic effects.

\begin{tabular}{llll}
\hline & Efficiency & $N_{\text {events }} / 1 \mathrm{fb}^{-1}$ & $N_{\text {events }} / 10 \mathrm{fb}^{-1}$ \\
\hline$Z^{0} Z^{0}$ & $38 \%$ & 7.1 & 71.1 \\
$Z^{0} \gamma^{*}$ & $4.5 \%$ & 0.16 & 1.60 \\
$Z^{0} b \bar{b}$ & $0.07 \%$ & 0.08 & 0.84 \\
$t \bar{t}$ & $0.06 \%$ & 0.12 & 1.22 \\
$S_{L}$ & & 4.8 & 13.1 \\
\hline
\end{tabular}

respectively. Leptons from $b$ quarks decays in the $t \bar{t}$ and $Z^{0} b \bar{b}$ background processes are produced in association with hadrons. Their contribution is reduced by requiring the electrons to be isolated: the ratio between the energy deposited in the hadronic and the electromagnetic calorimeters must be below $8 \%$; no more than two other charged track with $p_{\mathrm{T}}>2 \mathrm{GeV} / \mathrm{c}$ must be within a $\Delta R=0.3$ cone around the electron; $\Sigma_{i}\left(p_{\mathrm{T}}^{i}-E_{\mathrm{T}}\right)_{i} / E_{\mathrm{T}}<0.34$, where $E_{\mathrm{T}}$ is the transverse energy of the electron candidate and the sum runs on all tracks with $p_{\mathrm{T}}>2 \mathrm{GeV} / \mathrm{c}$ within a $\Delta R=0.3$ cone around the electron.

Electron-positron pairs are combined to form $Z^{0}$ candidates. Pairs with reconstructed masses between 50 and $120 \mathrm{GeV} / \mathrm{c}^{2}$ are retained. Of the two possible $Z^{0} Z^{0}$ pairings, the one where the $Z^{0}$ candidate masses are closest to $m_{Z}$ is chosen. This pairing is correct for almost all events with two on-shell $Z^{0}$ bosons. For $2.5 \%$ of the events, more than four electrons are present and only the $Z^{0} Z^{0}$ pairing which contains the highest- $p_{\mathrm{T}}$ electron is retained. Table 9.9 presents the signal and background selection efficiencies.

\subsubsection{Systematic uncertainties}

For the first $1 \mathrm{fb}^{-1}$ of integrated luminosity, the total systematic uncertainties on the $W^{ \pm} Z^{0}$ and $Z^{0} Z^{0}$ cross section measurements are $17.4 \%$ and $12.9 \%$, respectively. These figures include a $10 \%$ uncertainties on the determination of the integrated luminosity.

The most important sources of systematic uncertainties are lepton identification and isolation, and background subtraction. A $2 \%$ uncertainty on the efficiency of each lepton propagates to an uncertainty on the cross section between $2.6 \%$ and $7.8 \%$, according to the channel. Background subtraction dominates the $W^{ \pm} Z^{0}$ systematics with an uncertainty of $12 \%$, while it accounts for a $1.3 \%$ uncertainty in the $Z^{0} Z^{0}$ channel. An additional uncertainties of $5 \%$ on the jet energy scale affects the $W^{ \pm} Z^{0}$ channel, while an uncertainty of $1 \%$ on the trigger efficiency affects both channels.

The significance of the observation of the $W^{ \pm} Z^{0}$ and $Z^{0} Z^{0}$ signals in the first $1 \mathrm{fb}^{-1}$ is not sensitive to the luminosity uncertainty. It is affected by all other sources of systematic uncertainty listed above, with a total effect of $14.8 \%$ and $14.2 \%$ on the two channels, 

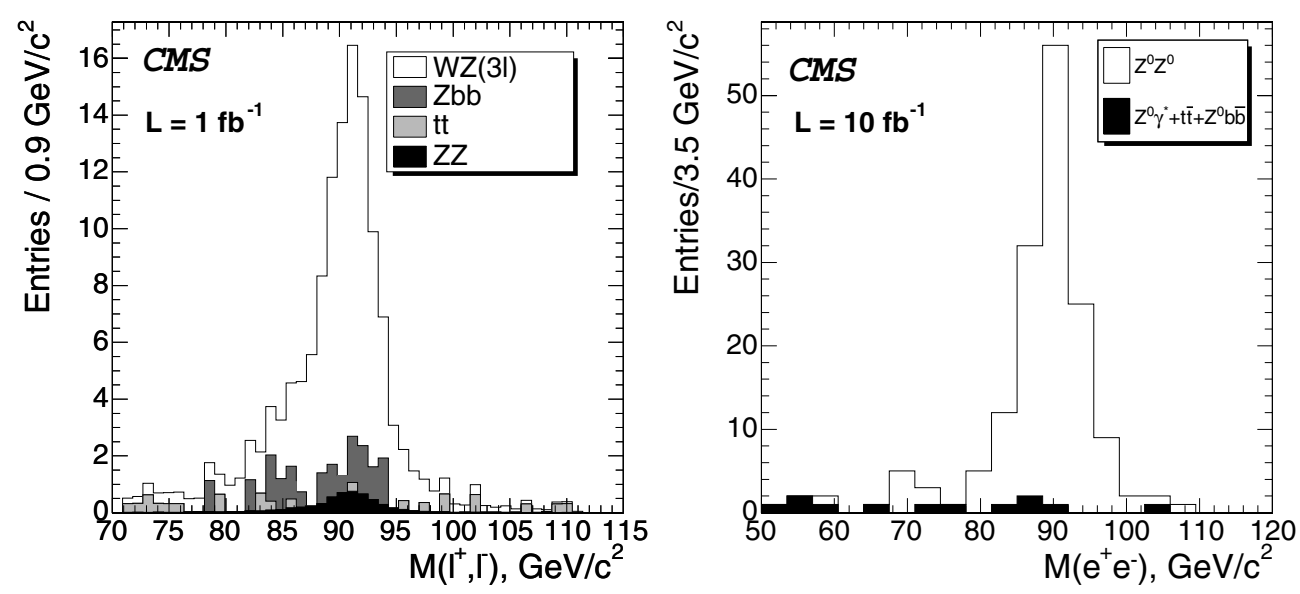

Figure 9.11. Left: Distribution of the mass of the $Z^{0}$ candidates for events retained by the $W^{ \pm} Z^{0}$ selection, for an integrated luminosity of $1 \mathrm{fb}^{-1}$. Right: Distribution of the mass of the $Z^{0}$ candidates, two entries per event, retained by the $Z^{0} Z^{0}$ selection, for an integrated luminosity of $10 \mathrm{fb}^{-1}$.

respectively. These uncertainties include additional PDF and QCD uncertainties in the Monte Carlo modelling, contributing $3.7 \%$ and $6.4 \%$ for the $W^{ \pm} Z^{0}$ and $Z^{0} Z^{0}$ selections, respectively.

\subsubsection{Results}

Figure 9.11 left presents the mass distribution of the $Z^{0}$ candidates in the $W^{ \pm} Z^{0}$ channel for an integrated luminosity of $1 \mathrm{fb}^{-1}$ before the last requirement of a $\pm 10 \mathrm{GeV} / \mathrm{c}^{2}$ window is applied. A large signal-over-background ratio is observed, as shown in Table 9.8.

Figure 9.11 right shows the mass distribution of the $Z^{0}$ candidates, two entries per event, selected by the $Z^{0} Z^{0}$ selection for an integrated luminosity of $10 \mathrm{fb}^{-1}$. Table 9.9 lists the selection yield for $1 \mathrm{fb}^{-1}$ and $10 \mathrm{fb}^{-1}$. The selection results into an almost background-free signal sample, which will constitute a valuable input to assess the background in the search for the Higgs boson.

Both the $W^{ \pm} Z^{0}$ and $Z^{0} Z^{0}$ final states can be selected with high purity. A significance of 12.8 and 4.8 , respectively, is expected in the first $1 \mathrm{fb}^{-1}$ of integrated luminosity, including systematic uncertainties. The $W^{ \pm} Z^{0}$ channel can be observed with a significance of 5 , including systematic effects, in an integrated luminosity of $150 \mathrm{pb}^{-1}$.

This study of multiple gauge-boson production and couplings at the LHC will be extended to include the $W^{ \pm} \gamma$ and $Z^{0} \gamma$ channels, as well as the other flavours of $Z^{0} Z^{0}$ fully-leptonic decays.

In conclusion, the large signal-over-background ratios achieved by the $W^{ \pm} Z^{0}$ and $Z^{0} Z^{0}$ selections suggest that early observation of these channels will take place at the LHC start up. In addition, precise investigations of triple gauge-boson couplings will be possible with the first $10 \mathrm{fb}^{-1}$ of LHC data. 


\section{Chapter 10. Standard Model Higgs Bosons}

\subsection{Introduction}

The Higgs mechanism is a cornerstone of the Standard Model (SM) and its supersymmetric extensions. The introduction of the fundamental Higgs field [356-359 ] renders the standard electroweak theory weakly interacting up to high energy scales without violating the unitarity bounds of scattering amplitudes [360-363]. Due to spontaneous symmetry breaking in the Higgs sector the electroweak gauge bosons $W, Z$ as well as the fermions acquire masses through the interaction with the Higgs fields. Since the gauge symmetry, though hidden, is still preserved, the theory of electroweak interactions is renormalisable [364-368]. In the Standard Model one weak isospin Higgs doublet is introduced and leads to the existence of one elementary Higgs particle after electroweak symmetry breaking. The Higgs couplings to the electroweak gauge bosons and all fermions grow with their masses. The only unknown parameter of the Higgs boson itself is the value of its mass $M_{H}$. Once this is known, all production and decay properties of the SM Higgs boson will be fixed [20, 369, 370 ]. The search for the Higgs boson is a crucial endeavour for establishing the standard formulation of the electroweak theory.

Although the Higgs mass cannot be predicted in the Standard Model, there are several constraints deduced from consistency conditions on the model [371-381 ]. Upper bounds can be derived from the requirement that the Standard Model can be extended up to a scale $\Lambda$, before perturbation theory breaks down and new non-perturbative phenomena dominate the predictions of the theory. If the SM is required to be weakly interacting up to the scale of grand unified theories (GUTs), which is of $\mathcal{O}\left(10^{16} \mathrm{GeV}\right)$, the Higgs mass has to be less than $\sim 190 \mathrm{GeV} / \mathrm{c}^{2}$. For a minimal cut-off $\Lambda \sim 1 \mathrm{TeV} / \mathrm{c}^{2}$ a universal upper bound of $\sim 700 \mathrm{GeV} / \mathrm{c}^{2}$ can be obtained from renormalisation group analyses [371-378] and lattice simulations of the SM Higgs sector [379-381 ]. This issue can be rephrased by stating that the Higgs sector has to be trivial, if the cut-off is extended to arbitrary magnitudes. Triviality means the absence of Higgs self-interactions.

If the top quark mass is large, the Higgs self-coupling can become negative and the Higgs potential deeply negative, thus rendering the SM vacuum unstable. The negative contribution of the top quark, however, can be compensated by a positive contribution due to the Higgs self-interaction, which is proportional to the Higgs mass. For a given top mass $m_{t}=175 \mathrm{GeV} / \mathrm{c}^{2}$ a lower bound of $\sim 60 \mathrm{GeV} / \mathrm{c}^{2}$ can be obtained for the Higgs mass, if the SM remains weakly interacting up to scales $\Lambda \sim 1 \mathrm{TeV} / \mathrm{c}^{2}$. For $\Lambda \sim M_{G U T}$ this lower bound is enhanced to $M_{H} \gtrsim 130 \mathrm{GeV} / \mathrm{c}^{2}$. However, the assumption that the vacuum is metastable, with a lifetime larger than the age of the Universe, decreases these lower bounds significantly for $\Lambda \sim 1 \mathrm{TeV} / \mathrm{c}^{2}$, but only slightly for $\Lambda \sim M_{G U T}[378$ ].

The direct search in the LEP2 experiments via the process $e^{+} e^{-} \rightarrow Z H$ yields a lower bound of $114.4 \mathrm{GeV} / \mathrm{c}^{2}$ on the Higgs mass [62]. After LEP2 the search for the SM Higgs particle is continued at the Tevatron for Higgs masses up to $\sim 130 \mathrm{GeV} / \mathrm{c}^{2}$ [382 ] and the LHC for Higgs masses up to the theoretical upper limit [383, 384].

The Higgs decay modes can be divided into two different mass ranges. For $M_{H} \lesssim$ $135 \mathrm{GeV} / \mathrm{c}^{2}$ the Higgs boson mainly decays into $b \bar{b}$ and $\tau^{+} \tau^{-}$pairs with branching ratios of about $85 \%$ and $8 \%$ respectively (see Fig. 10.1, right plot). The decay modes into $c \bar{c}$ and gluon pairs, with the latter mediated by top and bottom quark loops, accumulate a branching ratio of up to about $10 \%$, but do not play a relevant role at the LHC. The QCD corrections to the Higgs decays into quarks are known up to three-loop order [385-391] and the electroweak corrections up to NLO [392-395]. The latter are also valid for leptonic decay modes. One of 

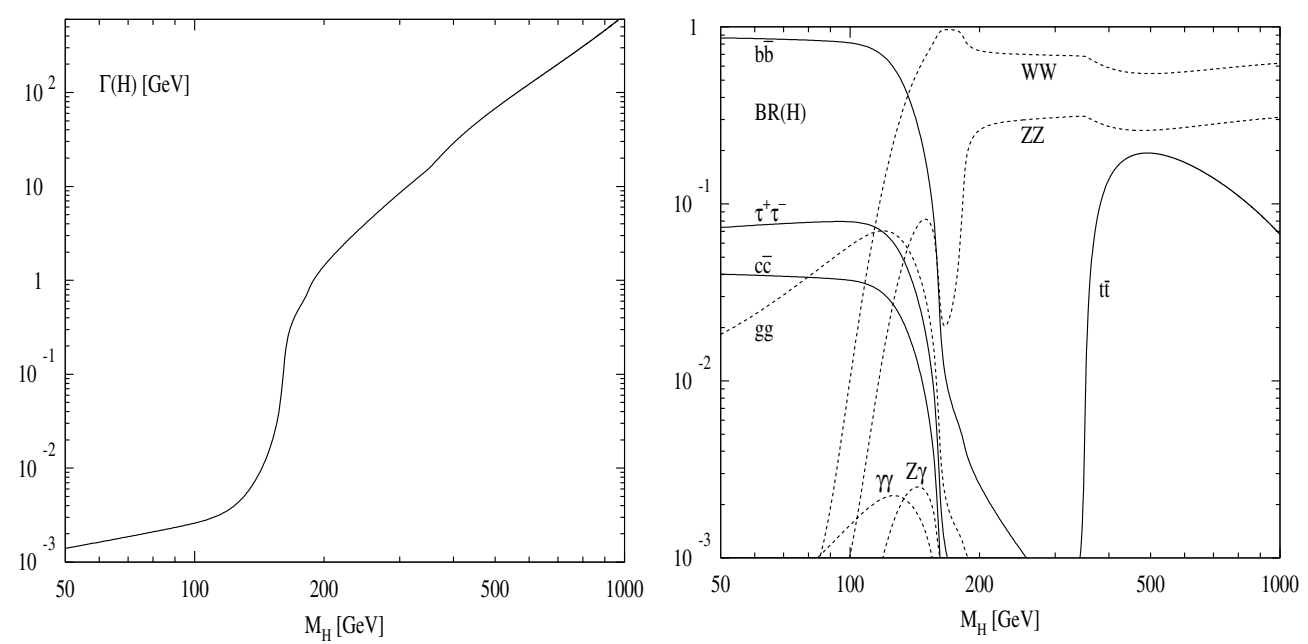

Figure 10.1. Left plot: total decay width (in $\mathrm{GeV} / \mathrm{c}^{2}$ ) of the SM Higgs boson as a function of its mass. Right plot: Branching ratios of the dominant decay modes of the SM Higgs particle. All relevant higher-order corrections are taken into account.

the most important Higgs decays in this mass range at the LHC is the decay into photon pairs, which is mediated by $W$, top and bottom quark loops. It reaches a branching fraction of up to $2 \times 10^{-3}$. The NLO QCD [396-402] and electroweak [403-405] corrections are known. They are small in the Higgs mass range relevant for the LHC.

For Higgs masses above $135 \mathrm{GeV} / \mathrm{c}^{2}$ the main decay modes are those into $W W$ and $\mathrm{ZZ}$ pairs, where one of the vector bosons is off-shell below the corresponding kinematical threshold. These decay modes dominate over the decay into $t \bar{t}$ pairs, the branching ratio of which does not exceed $\sim 20 \%$ as can be inferred from Fig. 10.1 (right plot). The electroweak corrections to the $W W, Z Z$ decays are of moderate size [392, 393, 406, 407]. The total decay width of the Higgs boson, shown in Fig. 10.1 (left plot), does not exceed about $1 \mathrm{GeV} / \mathrm{c}^{2}$ below the $W W$ threshold. For very large Higgs masses the total decay width grows up to the order of the Higgs mass itself so that the interpretation of the Higgs boson as a resonance becomes questionable. This Higgs mass range coincides with the upper bound of the Higgs mass from triviality. [408]

The dominant Higgs production mechanism at the LHC will be the gluon-fusion process

$$
p p \rightarrow g g \rightarrow H
$$

which provides the largest production cross section for the whole Higgs mass range of interest. This process is mediated by top and bottom quark loops (Fig. 10.2a). Due to the large size of the top Yukawa couplings and the gluon densities gluon fusion comprises the dominant Higgs boson production mechanism for the whole Higgs mass range.

The QCD corrections to the top and bottom quark loops have been known a long time including the full Higgs and quark mass dependences [409-411]. They increase the total cross section by $50-100 \%$. The limit of very heavy top quarks provides an approximation within $\sim 10 \%$ for all Higgs masses [20,369,370,409-412]. In this limit the NLO QCD corrections have been calculated before [409-411,413-416] and recently the NNLO QCD corrections [417-420] with the latter increasing the total cross section further by $\sim 20 \%$. A full massive NNLO calculation is not available, so that the NNLO results can only be trusted 


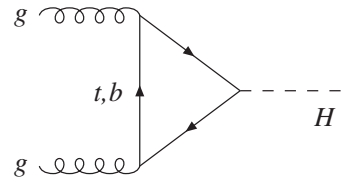

(a)

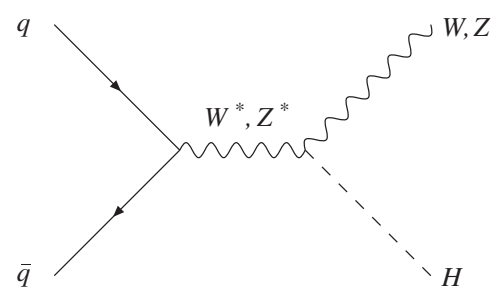

(c)

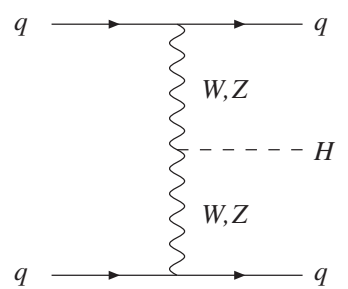

(b)

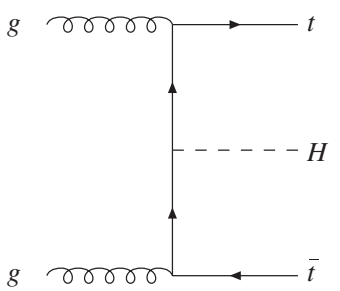

(d)

Figure 10.2. Typical diagrams for all relevant Higgs boson production mechanisms at leading order: (a) gluon fusion, (b) vector boson fusion, (c) Higgs-strahlung, (d) Higgs bremsstrahlung off top quarks.

for small and intermediate Higgs masses. The approximate NNLO results have been improved by a soft-gluon resummation at the next-to-next-to-leading log (NNLL) level, which yields another increase of the total cross section by 10\% [421]. Electroweak corrections have been computed, too, and turn out to be small [403,422-425]. The theoretical uncertainties of the total cross section can be estimated as $\sim 20 \%$ at NNLO due to the residual scale dependence, the uncertainties of the parton densities and due to neglected quark mass effects.

At LO the Higgs boson does not acquire any transverse momentum in the gluon fusion process, so that Higgs bosons with non-vanishing transverse momentum can only be produced in the gluon fusion process, if an additional gluon is radiated. This contribution is part of the real NLO corrections to the total gluon fusion cross section. The LO $p_{\mathrm{T}}$ distribution of the Higgs boson is known including the full quark mass dependence $[426,427]$. The NLO corrections, however, are only known in the heavy quark limit, so that they can only be trusted for small and moderate Higgs masses and $p_{\mathrm{T}}$ [428-443]. In this limit a NLL soft gluon resummation has been performed [433-443], which has recently been extended to the NNLL level [444-448] thus yielding a reliable description of the small $p_{\mathrm{T}}$ range. It should be noted that these results are only reliable, if the top quark loops provide the dominant contribution and $p_{\mathrm{T}}$ is not too large. In the regions where the NLO and resummed results are valid the theoretical uncertainties have been reduced to $\mathcal{O}(20 \%)$.

For large Higgs masses the $W$ and $Z$ boson-fusion processes [449-451] (see Fig. 10.2b)

$$
p p \rightarrow q q \rightarrow q q+W W / Z Z \rightarrow q q H
$$

become competitive. These processes are relevant in the intermediate Higgs mass range, too, since the additional forward jets offer the opportunity to reduce the background processes significantly. Since at NLO there is no colour exchange between the two quark lines, the NLO QCD corrections can be derived from the NLO corrections to deep inelastic leptonnucleon scattering. They turn out to be $\mathcal{O}(10 \%)$ for the total cross section $[20,369,370,452]$. 


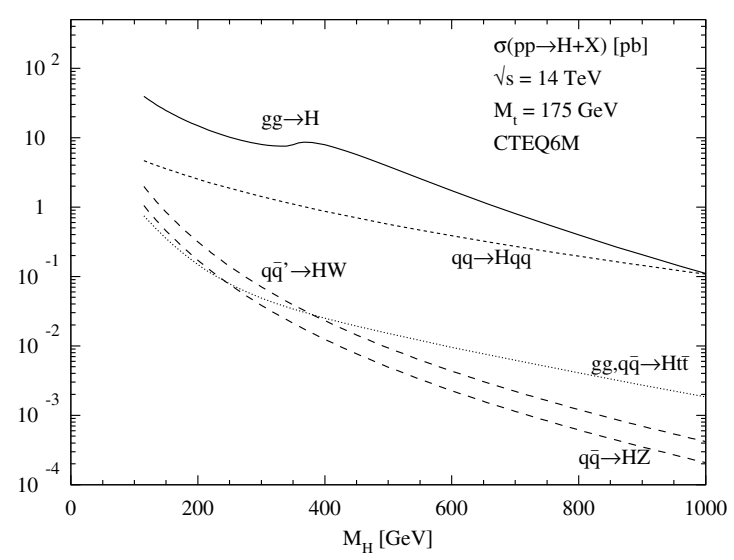

Figure 10.3. Higgs production cross sections at the LHC for the various production mechanisms as a function of the Higgs mass. The full QCD-corrected results for the gluon fusion $g g \rightarrow H$, vector-boson fusion $q q \rightarrow V V q q \rightarrow H q q$, vector-boson bremsstrahlung $q \bar{q} \rightarrow V^{*} \rightarrow H V$ and associated production $g g, q \bar{q} \rightarrow H t \bar{t}$ are shown.

Quite recently the NLO corrections to the differential cross sections have been computed, too, resulting in modifications of the relevant distributions by up to $\sim 30 \%$ [453]. The residual uncertainties are of $\mathcal{O}(5 \%)$.

In the intermediate mass range $M_{H} \lesssim 2 M_{Z}$ Higgs-strahlung off $W, Z$ gauge bosons [454, 455] (see Fig. 10.2c)

$$
p p \rightarrow q \bar{q} \rightarrow Z^{*} / W^{*} \rightarrow H+Z / W
$$

provides alternative signatures for the Higgs boson search. Since only the initial state quarks are strongly interacting at LO, the NLO QCD corrections can be inferred from the Drell-Yan process. They increase the total cross section by $\mathcal{O}(30 \%)[20,369,370,456]$. Recently this calculation has been extended up to NNLO [457]. The NNLO corrections are small. Moreover, the full electroweak corrections have been obtained in Ref. [458] resulting in a decrease of the total cross sections by $5-10 \%$. The total theoretical uncertainty is of $\mathcal{O}(5 \%)$.

Higgs radiation off top quarks (see Fig. 10.2d)

$$
p p \rightarrow q \bar{q} / g g \rightarrow H t \bar{t}
$$

plays a significant role for smaller Higgs masses below $\sim 150 \mathrm{GeV} / \mathrm{c}^{2}$. The $\mathrm{LO}$ cross section has been computed a long time ago [459-463]. During the last years the full NLO QCD corrections have been calculated resulting in a moderate increase of the total cross section by $\sim 20 \%$ at the LHC $[162,464,465]$. These results confirm former estimates based on an effective Higgs approximation [466]. The effects on the relevant parts of final state particle distribution shapes are of moderate size, i.e. $\mathcal{O}(10 \%)$, too, so that former experimental analyses are not expected to alter much due to these results. All SM Higgs production cross sections including NLO QCD corrections are shown in Fig. 10.3.

In the following Standard Model Higgs boson analyses the NLO cross sections and branching ratios for the Higgs boson calculated with the programs HDECAY [41], HIGLU [40], $\mathrm{Vv} 2 \mathrm{H}, \mathrm{v} 2 \mathrm{HV}$ and HQQ [20] are used, as well as the NLO cross sections for the background processes, when available. 


\subsection{Higgs boson channels}

\subsubsection{Inclusive Higgs boson production with $\mathrm{H} \rightarrow \mathrm{ZZ}^{(*)} \rightarrow \mathrm{e}+\mathrm{e}^{-} \mu^{+} \mu^{-}$}

10.2.1.1. Introduction. The $\mathrm{H} \rightarrow \mathrm{ZZ}^{(*)} \rightarrow 4 \ell$ channel has a very clean signature with relatively small backgrounds and is therefore an important discovery channel for the Higgs boson for a large range of masses. This channel is also important for the measurement of the mass and width of the Higgs boson.

A detailed description of the analysis can be found in [467].

10.2.1.2. Event generation. All Monte Carlo event samples used in the analysis were generated using the PYTHIA [69] event generator, except for the $Z \mathrm{~b} \bar{b}\left(e^{+} e^{-} b \bar{b}\right.$ and $\left.\mu^{+} \mu^{-} b \bar{b}\right)$ background samples which were generated with COMPHEP [355].

Higgs-boson production was simulated through leading order gluon-gluon scattering and vector-boson fusion. Monte Carlo samples were produced for 18 values of the Higgs boson mass $m_{H}$ ranging from $115 \mathrm{GeV} / \mathrm{c}^{2}$ to $200 \mathrm{GeV} / \mathrm{c}^{2}$ in $10 \mathrm{GeV} / \mathrm{c}^{2}$ steps, and from $200 \mathrm{GeV} / \mathrm{c}^{2}$ to $600 \mathrm{GeV} / \mathrm{c}^{2}$ in $50 \mathrm{GeV} / \mathrm{c}^{2}$ steps.

Three background processes which yield the same signature of two electrons and two muons in the final state, with significant cross-section times branching ratio, are considered:

1. $\mathrm{q} \overline{\mathrm{q}} / \mathrm{gg} \rightarrow t \bar{t} \rightarrow \mathrm{W}^{+} \mathrm{W}^{-} \mathrm{b} \overline{\mathrm{b}} \rightarrow \mathrm{e}^{+} \mathrm{e}^{-} \mu^{+} \mu^{-}$.

2. $\mathrm{q} \overline{\mathrm{q}} / \mathrm{gg} \rightarrow \mathrm{Zb} \overline{\mathrm{b}} \rightarrow \mathrm{e}^{+} \mathrm{e}^{-} \mu^{+} \mu^{-}$.

3. $\mathrm{q} \overline{\mathrm{q}} \rightarrow \mathrm{ZZ}^{\star} / \gamma^{\star} \rightarrow \mathrm{e}^{+} \mathrm{e}^{-} \mu^{+} \mu^{-}$.

For the $t \bar{t}$ and $\mathrm{Zb} \overline{\mathrm{b}}$ backgrounds, no restrictions are applied on $\mathrm{b}$ decays prior to the preselection. Only events with $\left|\eta_{b}\right|<2.5$ were generated for the $\mathrm{Zb} \overline{\mathrm{b}}$ background. For the $\mathrm{Zb} \overline{\mathrm{b}}$ and $\mathrm{ZZ}^{\star} / \gamma^{\star}$ backgrounds, $m_{\gamma^{\star}}$ is required to be greater $5 \mathrm{GeV} / \mathrm{c}^{2}$.

For the $\mathrm{ZZ}^{\star} / \gamma^{\star}$ background, only the $t$-channel production through $\mathrm{q} \overline{\mathrm{q}}$ fusion is simulated. In order to account for contributions from all NLO diagrams and from the NNLO gluon fusion $\left(\mathrm{gg} \rightarrow \mathrm{ZZ} Z^{\star} / \gamma^{\star}\right)$, all events are re-weighted at analysis level with an $m_{4 \ell}$ dependent K-factor, calculated [51][468] using MCFM.

The potential background contribution from $\mathrm{Zc} \overline{\mathrm{c}} \rightarrow \mathrm{e}^{+} \mathrm{e}^{-} \mu^{+} \mu^{-}$was also investigated using fully simulated events and was shown to be negligible.

For all Monte Carlo samples, a pre-selection is applied at generator level with the following requirements:

1. Final state contains $\mathrm{e}^{+} \mathrm{e}^{-} \mu^{+} \mu^{-}$.

2. $p_{\mathrm{T}}(\mathrm{e})>5 \mathrm{GeV} / \mathrm{c}$ and $|\eta(\mathrm{e})|<2.5$ for both electrons.

3. $p_{\mathrm{T}}(\mu)>3 \mathrm{GeV} / \mathrm{c}$ and $|\eta(\mu)|<2.4$ for both muons.

The cross-section times branching ratio and the cross-section times branching ratio times pre-selection efficiency, are shown for the signal as a function of $m_{H}$ in Fig. 10.4. The NLO cross-section and the cross-section times branching ratio times pre-selection efficiency are shown for each background process in Table 10.1 .

10.2.1.3. Online selection. Events selected by the dimuon or the dielectron triggers are considered. This choice follows from the presence of an on-shell Z-boson in most events. The additional use of single-electron and single-muon triggers does not increase the significance of the results.

The efficiencies of the Level-1 and High Level Triggers are shown for the signal as a function of $m_{H}$ in Fig. 10.5. The corresponding trigger efficiencies for background processes are shown in Table 10.2. 


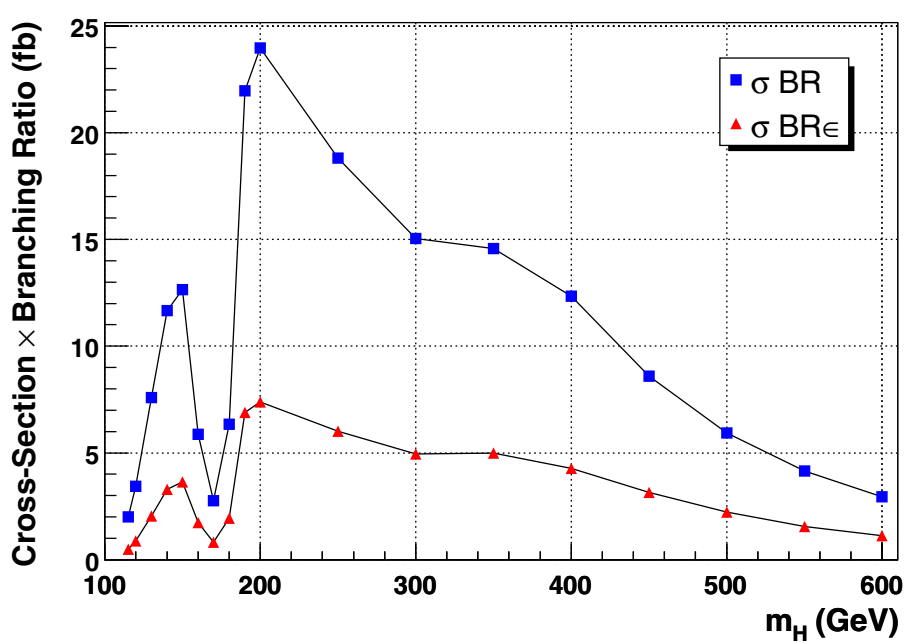

Figure 10.4. Cross-section times branching ratio, and cross-section times branching ratio times pre-selection efficiency for $\mathrm{H} \rightarrow \mathrm{ZZ}^{(\star)} \rightarrow 2 \mathrm{e} 2 \mu$.

Table 10.1. NLO cross-section and the cross-section times branching ratio times pre-selection efficiency for the three background process.

\begin{tabular}{lcc}
\hline Process & $\sigma_{\mathrm{NLO}}(\mathrm{pb})$ & $\sigma_{\mathrm{NLO}} \times \mathrm{BR} \times \varepsilon(\mathrm{fb})$ \\
\hline$t \bar{t} \rightarrow \mathrm{W}^{+} \mathrm{W}^{-} \mathrm{b} \overline{\mathrm{b}} \rightarrow \mathrm{e}^{+} \mathrm{e}^{-} \mu^{+} \mu^{-}$ & 840 & 744 \\
$\mathrm{e}^{+} \mathrm{e}^{-} \mathrm{b} \overline{\mathrm{b}} \rightarrow \mathrm{e}^{+} \mathrm{e}^{-} \mu^{+} \mu^{-}$ & 276 & 262 \\
$\mu^{+} \mu^{-} \mathrm{b} \overline{\mathrm{b}} \rightarrow \mathrm{e}^{+} \mathrm{e}^{-} \mu^{+} \mu^{-}$ & 279 & 128 \\
$\mathrm{ZZ}^{\star} \gamma^{\star} \rightarrow \mathrm{e}^{+} \mathrm{e}^{-} \mu^{+} \mu^{-}$ & 28.9 & 37.0 \\
\hline
\end{tabular}
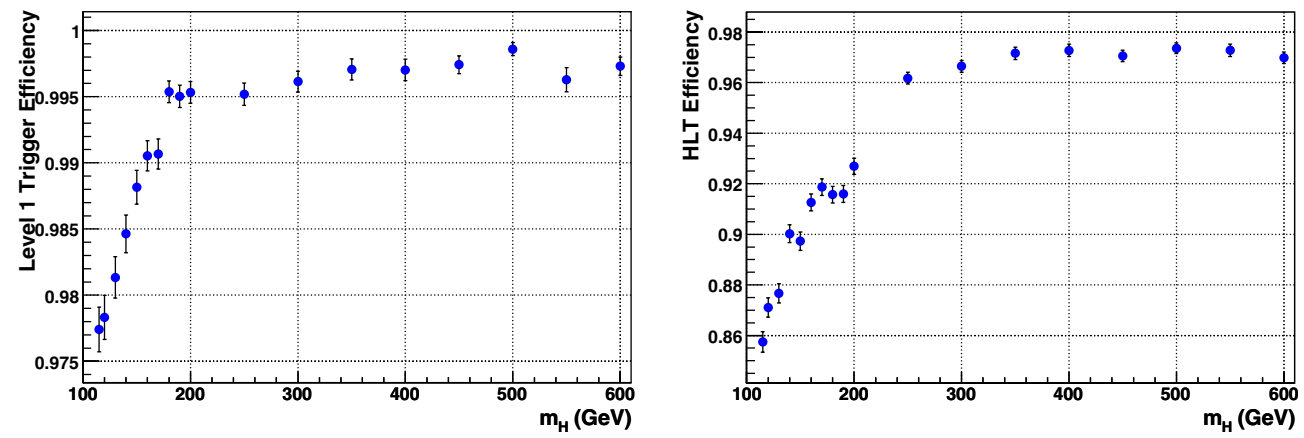

Figure 10.5. Efficiency of the Level-1 and High Level Triggers for the Higgs signal. Monte Carlo Statistical uncertainties are shown.

Table 10.2. Efficiency of the Level-1 and High Level Triggers for each of the three background processes. Monte Carlo Statistical uncertainties are shown.

\begin{tabular}{lccc}
\hline & $t \bar{t}$ & $\mathrm{Zbb}$ & $\mathrm{ZZ} / \gamma^{*}$ \\
\hline Level-1 Trigger efficiency (\%) & $95.1 \pm 0.1$ & $92.3 \pm 0.1$ & $97.9 \pm 0.2$ \\
HLT efficiency (\%) & $39.9 \pm 0.1$ & $65.8 \pm 0.1$ & $89.6 \pm 0.4$ \\
\hline
\end{tabular}


10.2.1.4. Offline event selection. Offline reconstruction of electrons and muons is performed using standard algorithms. It is required that four leptons of type $\mathrm{e}^{+} \mathrm{e}^{-} \mu^{+} \mu^{-}$are reconstructed.

The two largest backgrounds after the HLT, $t \bar{t}$ and $\mathrm{Zb} \bar{b}$, are reducible, since unlike the Higgs signal, two of the leptons will be associated with $b$-jets and will therefore be displaced relative to the primary vertex and will not be isolated. These two considerations can be used to powerfully cut against these processes, whereas the $\mathrm{ZZ}^{\star} / \gamma^{\star}$ background is irreducible by such means. Kinematic cuts are then applied, which further reduce all three backgrounds.

Vertex and Impact Parameter. Three criteria are applied:

1. The transverse distance of the $\mu^{+} \mu^{-}$vertex from the beam line is required to be less than $0.011 \mathrm{~cm}$.

2. The three-dimensional distance between the $\mu^{+} \mu^{-}$vertex and the $\mathrm{e}^{+} \mathrm{e}^{-}$vertex is required to be less than $0.06 \mathrm{~cm}$.

3. The transverse impact parameter significance of all leptons required to be less than 7 .

For events passing this selection, the primary vertex is reconstructed by performing a fit to the tracks of the four reconstructed leptons. The lepton tracks are then refitted using the reconstructed vertex position as an additional point, in order to obtain a more accurate measurement of the momentum at the primary vertex.

Isolation. A cut is applied on the sum of the $p_{\mathrm{T}}$ of reconstructed tracks with $p_{\mathrm{T}}>0.9 \mathrm{GeV} / \mathrm{c}$ and at least five hits, which satisfy the following conditions:

1. The track lies within the region defined by the sum of cones of size $\Delta \mathrm{R}=0.25$ around each of the four leptons and lies outside veto cones of size $\Delta R=0.015$ around each lepton.

2. The track is consistent with originating from the reconstructed primary vertex to within $|\Delta z|<0.2 \mathrm{~cm}$, where $\Delta z$ is the difference between the $z$ position of the point of closest approach of the track to the reconstructed vertex, and the $z$ position of the reconstructed vertex.

Kinematic Cuts. The following kinematic cuts are applied:

1. Lower thresholds on the transverse momenta of each of the four reconstructed leptons.

2. Upper and lower thresholds on the invariant masses of the reconstructed $\mathrm{e}^{+} \mathrm{e}^{-}$and $\mu^{+} \mu^{-}$ pairs.

3. Upper and lower thresholds on the invariant mass of the four reconstructed leptons.

These kinematic thresholds, together with the threshold on $\Sigma p_{\mathrm{T}}$ for tracker isolation are optimised simultaneously using MINUIT, such that the log-likelihood ratio:

$$
S_{L}=\sqrt{2 \ln Q}, \quad \text { where } \quad Q=\left(1+\frac{N_{S}}{N_{B}}\right)^{N_{S}+N_{B}} e^{-N_{S}}
$$

is maximised. The optimisation is performed separately for each Higgs mass.

10.2.1.5. Results. Tables 10.3 and 10.4 show the production cross-section, cross-section times branching ratio, cross-section times branching ratio times pre-selection efficiency and the cross-section times branching ratio times efficiency after each stage of the online and offline event selection, for Higgs masses of $140 \mathrm{GeV} / \mathrm{c}^{2}$ and $200 \mathrm{GeV} / \mathrm{c}^{2}$, respectively. Values are shown for signal and for each of the three background processes. For all values of $m_{H}$, the background after all selections is strongly dominated by ZZ* $/ \gamma^{*}$. For low $m_{H} t \bar{t}$ and $\mathrm{Zb} \bar{b}$ each 
Table 10.3. Production cross-section (NLO), cross-section times branching ratio, cross-section times branching ratio times pre-selection efficiency and cross-section times branching ratio times efficiency after each stage of the online and offline event selection, for $m_{H}=140 \mathrm{GeV} / \mathrm{c}^{2}$, for signal and backgrounds. All values in fb, except for expected number of events. Uncertainties are statistical only.

\begin{tabular}{lllcl}
\hline & Signal & $t \bar{t}$ & $\mathrm{Zb} \overline{\mathrm{b}}$ & $\mathrm{ZZ} / \gamma^{*}$ \\
\hline Production cross-section (NLO) & $33.6 \times 10^{3}$ & $840 \times 10^{3}$ & $555 \times 10^{3}$ & $28.9 \times 10^{3}$ \\
$\sigma \times \mathrm{BR}(4$ lepton final state) & 11.6 & - & & 367.5 \\
Pre-selection: $\sigma \times \mathrm{BR} \times \varepsilon$ & $3.29 \pm 0.04$ & $743 \pm 2$ & $390 \pm 1$ & $37.0 \pm 0.4$ \\
Level-1 trigger & $3.24 \pm 0.04$ & $707 \pm 2$ & $360 \pm 1$ & $36.3 \pm 0.4$ \\
High Level trigger & $2.91 \pm 0.03$ & $282 \pm 1$ & $237 \pm 1$ & $32.5 \pm 0.4$ \\
$\mathrm{e}^{+} \mathrm{e}^{-} \mu^{+} \mu^{-}$reconstructed & $2.23 \pm 0.03$ & $130 \pm 1$ & $141 \pm 1$ & $24.1 \pm 0.3$ \\
Vertex and impact parameter cuts & $2.01 \pm 0.03$ & $18.9 \pm 0.3$ & $18.4 \pm 0.2$ & $21.5 \pm 0.3$ \\
Isolation cuts & $1.83 \pm 0.03$ & $1.34 \pm 0.07$ & $5.8 \pm 0.1$ & $20.0 \pm 0.3$ \\
Lepton $p_{\mathrm{T}}$ cuts & $1.61 \pm 0.03$ & $0.40 \pm 0.04$ & $0.56 \pm 0.03$ & $17.6 \pm 0.3$ \\
Z mass window cuts & $1.35 \pm 0.02$ & $0.20 \pm 0.03$ & $0.23 \pm 0.02$ & $13.8 \pm 0.3$ \\
Higgs mass window cuts & $1.17 \pm 0.02$ & $0.02 \pm 0.01$ & $0.025 \pm 0.007$ & $0.15 \pm 0.03$ \\
Expected events for $\int \mathcal{L}=10 \mathrm{fb}^{-1}$ & $11.7 \pm 0.2$ & $0.2 \pm 0.1$ & $0.25 \pm 0.07$ & $1.5 \pm 0.3$ \\
\hline
\end{tabular}

Table 10.4. Production cross-section (NLO), cross-section times branching ratio, cross-section times branching ratio times pre-selection efficiency and cross-section times branching ratio times efficiency after each stage of the online and offline event selection, for $m_{H}=200 \mathrm{GeV} / \mathrm{c}^{2}$, for signal and backgrounds. All values in fb, except for expected number of events. Uncertainties are statistical only.

\begin{tabular}{llccc}
\hline & Signal & $t \bar{t}$ & $\mathrm{Zb} \overline{\mathrm{b}}$ & $\mathrm{ZZ}^{*} / \gamma^{*}$ \\
\hline Production cross-section (NLO) & $17.9 \times 10^{3}$ & $840 \times 10^{3}$ & $555 \times 10^{3}$ & $28.9 \times 10^{3}$ \\
$\sigma \times \mathrm{BR}(4$ lepton final state) & 23.8 & - & - & 367.5 \\
Pre-selection: $\sigma \times \mathrm{BR} \times \varepsilon$ & $7.39 \pm 0.09$ & $743 \pm 2$ & $390 \pm 1$ & $37.0 \pm 0.4$ \\
Level-1 trigger & $7.36 \pm 0.09$ & $707 \pm 2$ & $360 \pm 1$ & $36.3 \pm 0.4$ \\
High Level trigger & $6.82 \pm 0.08$ & $282 \pm 1$ & $237 \pm 1$ & $32.5 \pm 0.4$ \\
$\mathrm{e}^{+} \mathrm{e}^{-} \mu^{+} \mu^{-}$reconstructed & $5.51 \pm 0.07$ & $130 \pm 1$ & $141 \pm 1$ & $24.1 \pm 0.3$ \\
Vertex and impact parameter cuts & $5.03 \pm 0.07$ & $18.9 \pm 0.3$ & $18.4 \pm 0.2$ & $21.5 \pm 0.3$ \\
Isolation cuts & $4.92 \pm 0.07$ & $5.1 \pm 0.1$ & $12.3 \pm 0.2$ & $21.3 \pm 0.3$ \\
Lepton $p_{\mathrm{T}}$ cuts & $4.78 \pm 0.07$ & $1.93 \pm 0.09$ & $1.78 \pm 0.06$ & $18.7 \pm 0.3$ \\
Z mass window cuts & $4.45 \pm 0.07$ & $0.15 \pm 0.03$ & $0.12 \pm 0.02$ & $14.4 \pm 0.3$ \\
Higgs mass window cuts & $3.64 \pm 0.06$ & $0.006 \pm 0.005$ & $0.006 \pm 0.003$ & $1.61 \pm 0.09$ \\
Expected events for $\int \mathcal{L}=10 \mathrm{fb}^{-1}$ & $36.4 \pm 0.6$ & $0.06 \pm 0.05$ & $0.06 \pm 0.03$ & $16.1 \pm 0.9$ \\
\hline
\end{tabular}

contribute around $10-15 \%$ to the total residual background, whereas for $m_{H}>200 \mathrm{GeV} / \mathrm{c}^{2}$, $\mathrm{ZZ}^{*} / \gamma^{*}$ constitutes more than $99 \%$.

Figure 10.6 shows the invariant mass of the four reconstructed leptons before and after the application of the offline selection, for signal events for $m_{H}=140 \mathrm{GeV} / \mathrm{c}^{2}$ (left) and $m_{H}=$ $200 \mathrm{GeV} / \mathrm{c}^{2}$ (right), and for the three background processes.

Figure 10.7 shows the final cross-section times branching ratio times efficiency for selected events, for signal and background, as a function of the Higgs boson mass. The number of expected events passing all selections for $10 \mathrm{fb}^{-1}$ of integrated luminosity is shown in Table 10.5 for several values of the Higgs boson mass.

Significance. Figure 10.8 shows the $S_{c P}$ significance after all selection cuts for integrated luminosities of $10 \mathrm{fb}^{-1}$ and $30 \mathrm{fb}^{-1}$, with and without the systematic uncertainty on the background estimation taken into account. The background systematic uncertainty will be 

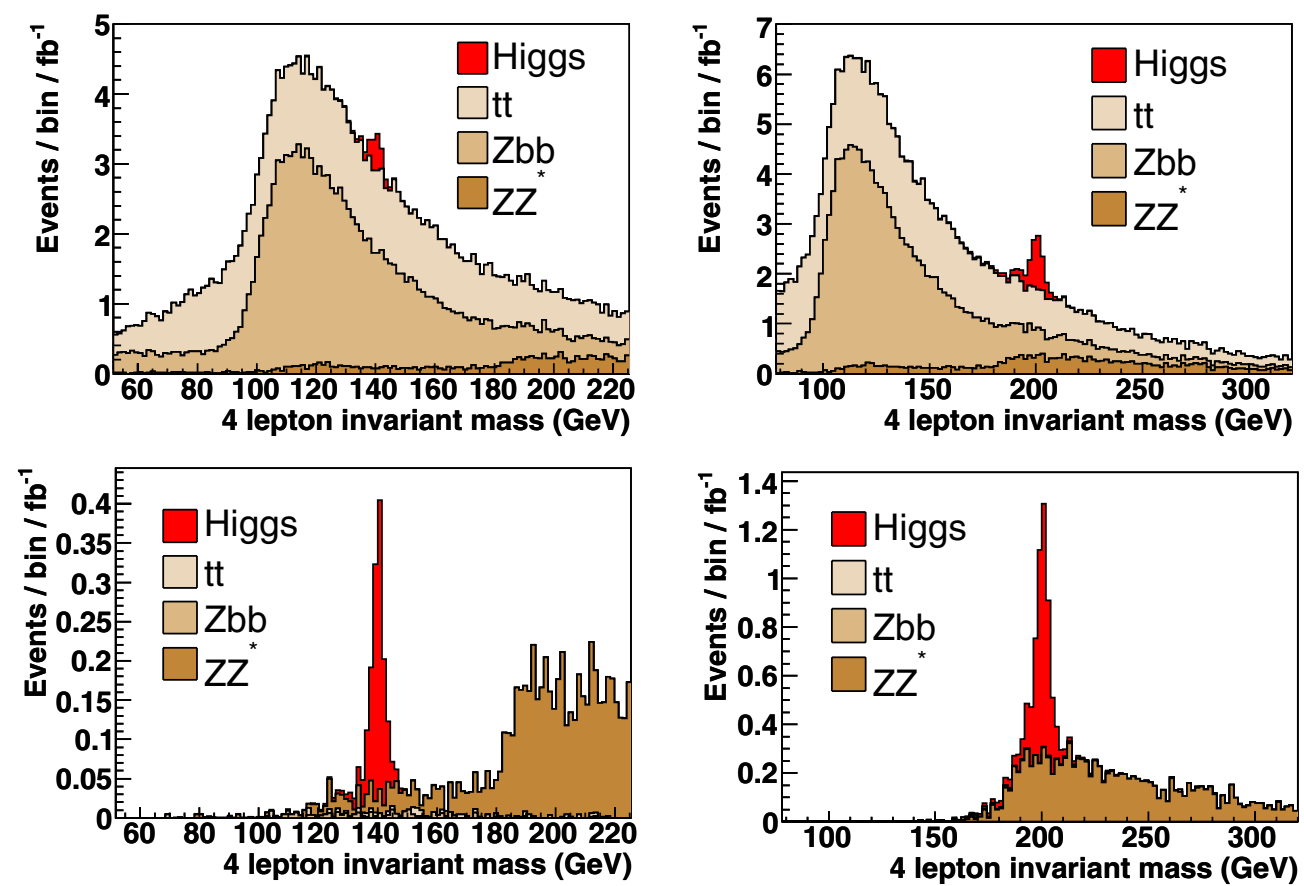

Figure 10.6. Invariant mass of the four reconstructed leptons before (top) and after (bottom) the application of the offline selection, for signal events for $m_{H}=140 \mathrm{GeV} / \mathrm{c}^{2}$ (left) and $m_{H}=$ $200 \mathrm{GeV} / \mathrm{c}^{2}$ (right), and for the three background processes.

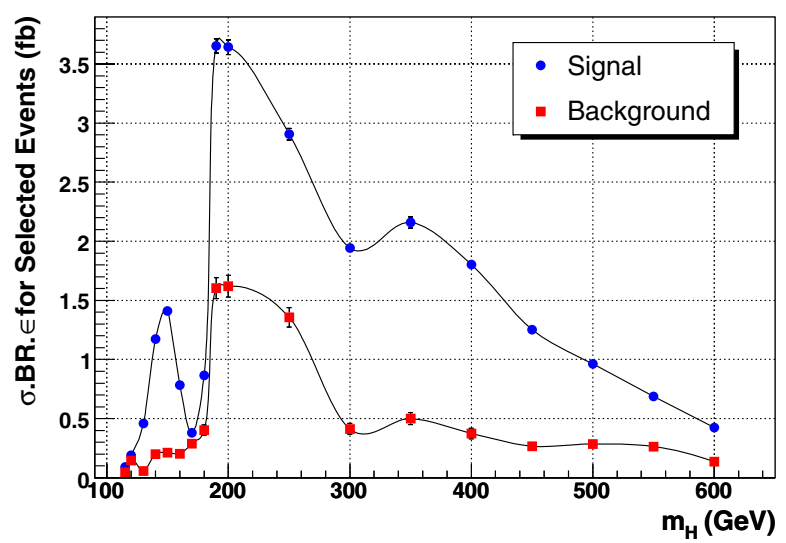

Figure 10.7. Cross-section times branching ratio times efficiency after all selections.

Table 10.5. Expected number of events from signal and background processes after all selections for an integrated luminosity of $10 \mathrm{fb}^{-1}$.

\begin{tabular}{lrrrrrrrrrrrr}
\hline$m_{H}\left(\mathrm{GeV} / \mathrm{c}^{2}\right)$ & 120 & 130 & 140 & 150 & 160 & 170 & 180 & 200 & 250 & 300 & 400 & 500 \\
\hline $\mathrm{N}$ signal for $10 \mathrm{fb}^{-1}$ & 1.9 & 4.6 & 11.7 & 14.1 & 7.8 & 3.8 & 8.7 & 36.4 & 29.1 & 19.4 & 18.0 & 9.6 \\
$\mathrm{~N}$ back for $10 \mathrm{fb}^{-1}$ & 1.5 & 0.6 & 2.0 & 2.1 & 2.0 & 2.9 & 4.0 & 16.2 & 13.6 & 4.1 & 3.7 & 2.6 \\
\hline
\end{tabular}




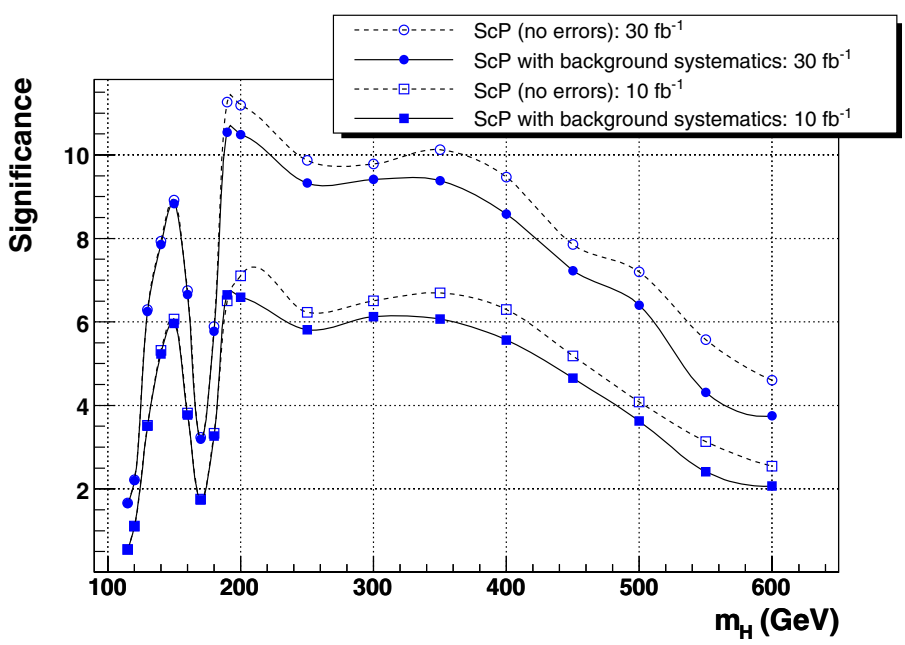

Figure 10.8. The $S_{C P}$ significance after all selection cuts for integrated luminosities of $10 \mathrm{fb}^{-1}$ and $30 \mathrm{fb}^{-1}$, with and without the systematic uncertainty on the background estimation taken into account.

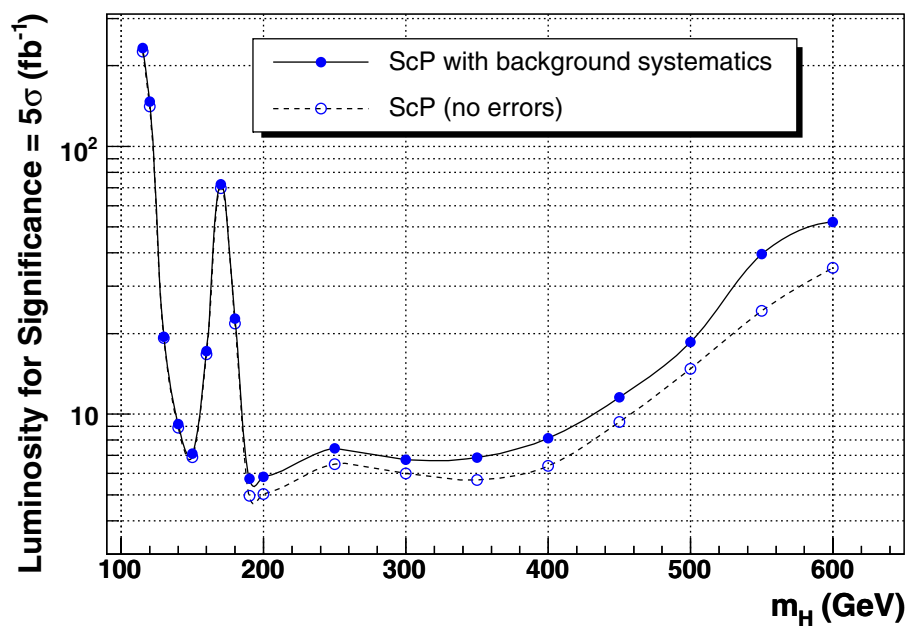

Figure 10.9. Integrated luminosity required to obtain a significance of $5 \sigma$ using the $\mathrm{H} \rightarrow \mathrm{ZZ}(*)$ $\rightarrow 2 \mathrm{e} 2 \mu$ channel, with and without the systematic uncertainty on the background estimation taken into account.

discussed in Section 10.2.1.6. Figure 10.9 shows the integrated luminosity required to obtain a significance of $5 \sigma$ using the $\mathrm{H} \rightarrow \mathrm{ZZ}^{(*)} \rightarrow 2 \mathrm{e} 2 \mu$ channel alone, with and without the background systematic uncertainty. It can be seen that a significance of $5 \sigma$ can be achieved with less than $30 \mathrm{fb}^{-1}$ of integrated luminosity for a Higgs boson with mass in the range $130 \leqslant m_{H} \leqslant 500 \mathrm{GeV} / \mathrm{c}^{2}$, excluding a gap of about $15 \mathrm{GeV} / \mathrm{c}^{2}$ close to $m_{H} / 170 \mathrm{GeV} / \mathrm{c}^{2}$ for which close to $100 \mathrm{fb}^{-1}$ is required. If the Higgs boson mass lies in the range $190 \leqslant m_{H} \leqslant$ $400 \mathrm{GeV} / \mathrm{c}^{2}, 5 \sigma$ significance can be attained with less than $8 \mathrm{fb}^{-1}$ of integrated luminosity. 

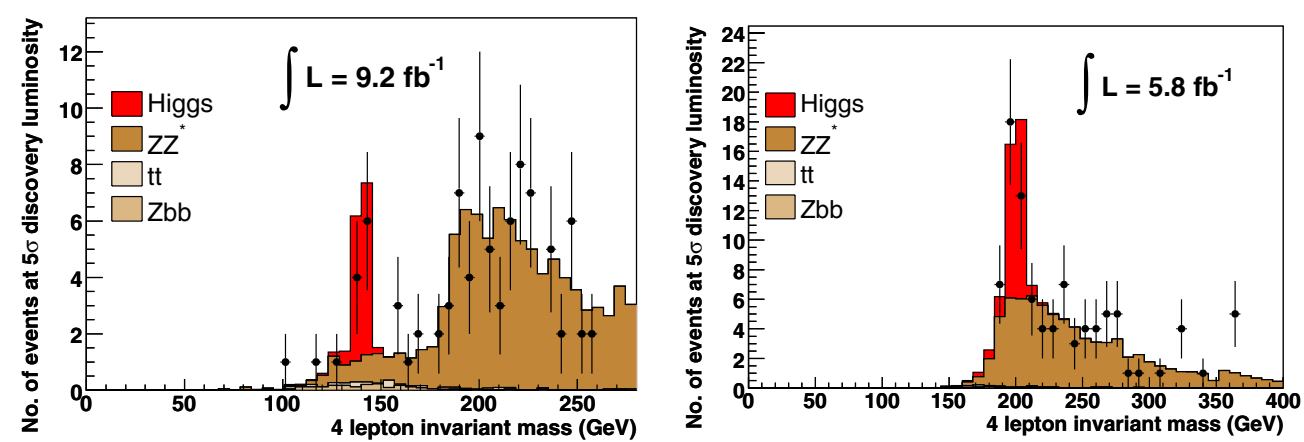

Figure 10.10. Number of expected events for signal and background for an integrated luminosity corresponding to a discovery significance of $5 \sigma$, for Higgs boson masses of 140 and $200 \mathrm{GeV} / \mathrm{c}^{2}$. The results of a simulated experiment are also shown to illustrate the statistical power of the analysis and the determination of the background normalisation from data.

10.2.1.6. Evaluation of background from data. The background normalisation can be estimated from data by using the sidebands in the reconstructed four-lepton invariant mass distribution. Figure 10.10 shows the number of expected events from the signal and background Monte Carlo simulations for an integrated luminosity corresponding to a discovery significance of $5 \sigma$, for Higgs boson masses of 140 and $200 \mathrm{GeV} / \mathrm{c}^{2}: 9.2$ and $5.8 \mathrm{fb}^{-1}$, respectively. Figure 10.10 also shows the results of a simulated experiments with these luminosities.

The number of background events measured from the data within the signal region, $N_{\text {Data }}^{I N}$, is calculated as:

$$
N_{\text {Data }}^{I N}=\alpha_{M C} N_{\text {Data }}^{\text {OUT }}, \quad \text { where } \quad \alpha_{M C}=\frac{N_{M C}^{I N}}{N_{M C}^{O U T}} .
$$

$N_{\text {Data }}^{\text {OUT }}$ is the number observed events lying outside the signal region and $\alpha_{M C}$ is the ratio of the number of background events inside the signal region $\left(N_{M C}^{I N}\right)$ to outside the signal region $\left(N_{M C}^{O U T}\right)$, as determined from the background Monte Carlos.

The uncertainty on the number of background events in the signal region measured using this method is given by:

$$
\Delta B=\Delta B_{\text {Stat }} \oplus \Delta B_{\text {Theory }}, \quad \text { where } \quad \Delta B_{\text {Stat }}=\alpha \sqrt{N_{\text {Data }}^{\text {OUT }}} .
$$

$\Delta B_{\text {Stat }}$ provides the dominant contribution to the uncertainty. Taking $N_{\text {Data }}^{\text {OUT }}$ as the expected number of events outside the signal region for an integrated luminosity corresponding to $5 \sigma$ significance, the value $\Delta B_{\text {Stat }}$ varies between $2 \%$ and $13 \%$ for $m_{H}<200 \mathrm{GeV} / \mathrm{c}^{2}$ and increases to around $30 \%$ for high $m_{H}$ where the statistics in the sidebands are low.

$\Delta B_{\text {Theory }}$ is the theoretical uncertainty on the shape of the $m_{4 \ell}$ distribution for the $\mathrm{ZZ}^{*} / \gamma^{*}$ background. The value is taken from [51], which takes into account PDF and QCD scale uncertainties in the $\mathrm{ZZ}^{*} / \gamma^{*}$ production cross-section at NLO, and varies between 0.5 and $4.5 \%$ for the range Higgs boson masses considered.

10.2.1.7. Measurement of the properties of the Higgs boson. The $\mathrm{H} \rightarrow \mathrm{ZZ}^{(*)} \rightarrow 4 \ell$ channel can be used to evaluate the mass, width and production cross-section of the Higgs boson.

Mass Measurement. The statistical uncertainty on the Higgs boson mass measurement is given by $\Delta$ stat $=\sigma_{\text {Gauss }} / \sqrt{N_{S}}$, where $\sigma_{\text {Gauss }}$ is the measured Gaussian width of the four 


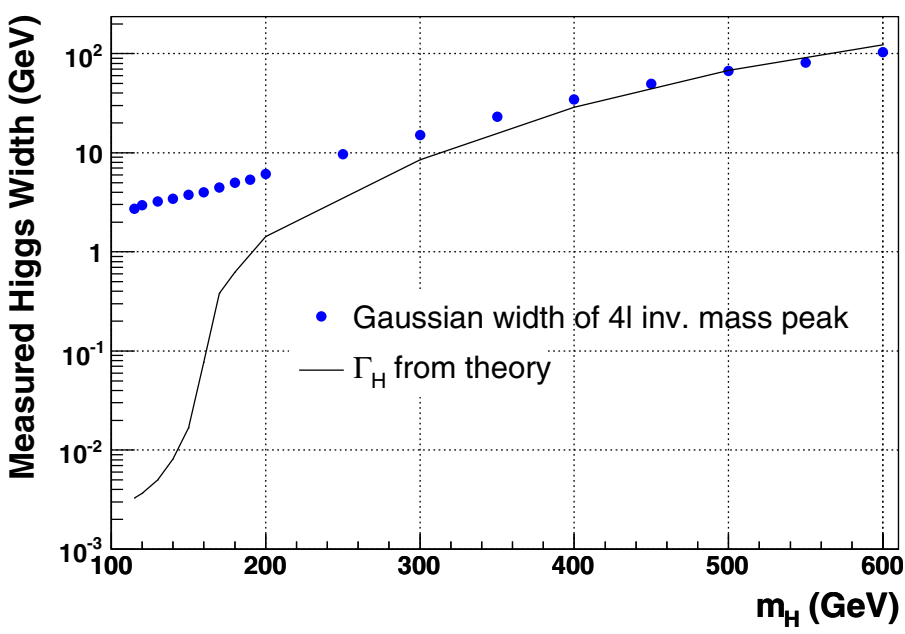

Figure 10.11. Measured width of the Higgs boson mass peak, obtained from a Gaussian fit to the peak, as a function of the true Higgs mass. The true width from theory is also shown.

Table 10.6. Statistical uncertainty on the measurement of the mass, width and production cross-section of the Higgs boson.

\begin{tabular}{llllllllll}
\hline$m_{H}\left(\mathrm{GeV} / \mathrm{c}^{2}\right)$ & 115 & 120 & 130 & 140 & 150 & 160 & 170 & 180 & 190 \\
\hline$\Delta_{\text {Stat }}\left(m_{H}\right)(\%)$ & 0.722 & 0.512 & 0.335 & 0.206 & 0.193 & 0.256 & 0.388 & 0.27 & 0.134 \\
$\Delta_{\text {Stat }}\left(\Gamma_{H}\right)(\%)$ & - & - & - & - & - & - & - & 54.8 & 17.6 \\
$\Delta_{\text {Stat }}\left(\sigma_{H}\right)(\%)$ & 75 & 55.6 & 28.6 & 18.2 & 16.5 & 23.1 & 39.2 & 23.7 & 11.5 \\
$m_{H}\left(\mathrm{GeV} / \mathrm{c}^{2}\right)$ & 200 & 250 & 300 & 350 & 400 & 450 & 500 & 550 & 600 \\
$\Delta_{\text {Stat }}\left(m_{H}\right)(\%)$ & 0.145 & 0.207 & 0.328 & 0.408 & 0.588 & 0.896 & 1.25 & 1.62 & 2.43 \\
$\Delta_{\text {Stat }}\left(\Gamma_{H}\right)(\%)$ & 14.4 & 7.38 & 8.2 & 5.43 & 5.8 & 5.91 & 6.52 & 6.61 & 8.36 \\
$\Delta_{\text {Stat }}\left(\sigma_{H}\right)(\%)$ & 11.5 & 13 & 14.4 & 13.8 & 14.9 & 18 & 21.2 & 25.9 & 32.3 \\
\hline
\end{tabular}

lepton invariant mass peak from the signal Monte Carlo and $N_{S}$ is the expected number of signal events passing all selections. The value, as a fraction of the true mass, is shown in Table 10.6, for an integrated luminosity and $30 \mathrm{fb}^{-1}$, as a function of $m_{H}$.

Width Measurement. Figure 10.11 shows the measured width of the Higgs boson mass peak, obtained from the Gaussian fit, as a function of $m_{H}$. The true width from theory $\Gamma_{H}$ is also shown. The measured width is a convolution of the natural width and the experimental resolution. It can be seen that for $m_{H}$ less than around $200 \mathrm{GeV} / \mathrm{c}^{2}$, the measured width is completely dominated by the experimental resolution. The statistical uncertainty on the width measurement is given by $\Delta s t a t=\sigma_{\text {Gauss }} / \sqrt{2 N_{S}}$, where $\sigma_{\text {Gauss }}$ is the measured Gaussian width of the peak and $N_{S}$ is the expected number of signal events passing all selections. The value, as a fraction of the true width, is shown in Table 10.6, for an integrated luminosity and $30 \mathrm{fb}^{-1}$, as a function of $m_{H}$. The direct measurement the Higgs boson width is possible with $\Delta$ stat $<30 \%$ for $m_{H} \geqslant 200 \mathrm{GeV} / \mathrm{c}^{2}$.

Production Cross-Section Measurement. The Higgs boson production cross-section can be determined from the number of observed events $N_{o b s}$ after all selections, given the efficiency $\varepsilon$ of the event selection and the integrated luminosity $\mathcal{L}$ :

$$
\sigma=\frac{N_{o b s}}{\mathcal{L} \epsilon} .
$$


The total uncertainty on the cross-section measurement is given by:

$$
\Delta \sigma^{2}=\Delta s t a t^{2}+\Delta s y s t^{2}+\Delta \mathcal{L}^{2}+\Delta B^{2}
$$

where $\Delta$ stat, $\Delta$ syst, $\Delta \mathcal{L}$ and $\Delta \mathrm{B}$ are the statistical uncertainty, the systematic uncertainty from the event selection, the uncertainty on the luminosity measurement and the background systematic uncertainty, respectively.

The statistical uncertainty $\Delta$ stat is shown in Table 10.6 for an integrated luminosity $30 \mathrm{fb}^{-1}$, as a function of $m_{H}$.

The total systematic uncertainty arising from the offline reconstruction and event selection can be summarised as:

$$
\Delta s y s t^{2}=2 \Delta \epsilon_{\mathrm{e}}^{2}+2 \Delta \epsilon_{\mu}^{2}+\Delta \epsilon_{i s o}^{2}
$$

where $\Delta \varepsilon_{\mathrm{e}}$ is the uncertainty in the reconstruction efficiency for electrons, estimated to be around $1 \%$ per electron [469], $\Delta \varepsilon_{\mu}$ is the uncertainty in the muon reconstruction efficiency, which has been shown to be measurable to be better than $1 \%$ per muon [51], and $\Delta \varepsilon_{\text {iso }}$ is the uncertainty in the efficiency of the isolation cut, estimated in the $\mathrm{H} \rightarrow \mathrm{ZZ}^{(*)} \rightarrow 4 \mu$ analysis [51] to be around $2 \%$ per event. This gives a total uncertainly $\Delta s y s t=3 \%$.

The uncertainty on the measurement of the LHC luminosity $\Delta \mathcal{L}$ is expected to be around $3 \%$ at the $30 \mathrm{fb}^{-1}$. The background uncertainty $\Delta \mathrm{B}$ is discussed in Section 10.2.1.6.

\subsubsection{Inclusive Higgs boson production with $\mathrm{H} \rightarrow \mathrm{WW}^{*} \rightarrow 2 \ell 2 v$}

The Higgs $\mathrm{H} \rightarrow \mathrm{WW}^{(*)} \rightarrow 2 \ell 2 v$ decay into two Ws and subsequently into two leptons $(\mathrm{H} \rightarrow \mathrm{WW} \rightarrow \ell \nu \ell \nu)$ is the discovery channel for Higgs boson masses between $2 \mathrm{~m}_{\mathrm{W}}$ and $2 \mathrm{~m}_{\mathrm{Z}}$ [470]. In this mass range, the Higgs to WW branching ratio is close to one, leading to large number of events. The signature of this decay is characterised by two leptons and missing energy. However, since no narrow mass peak can be reconstructed, good understanding of the background together with a high signal to background ratio is needed. The most important backgrounds, which give similar signature as the signal (i.e. two leptons and missing energy), are the continuum WW production and the $\bar{t} \bar{t}$ production. To reduce these backgrounds, one has to require a small opening angle between the leptons in the transverse plane and apply a jet veto.

A detailed description of the analysis can be found in [471].

10.2.2.1. Signal and background generation. The signal samples were generated using PYTHIA. The two major Higgs production modes for the mass range studied, gluon and vector boson fusion were generated. The $\mathrm{p}_{\mathrm{t}}(H)$ spectrum predicted by PYTHIA was reweighted to the MC@NLO prediction, defining $\mathrm{p}_{\mathrm{t}}$ dependent k-factors, as proposed in [472].

For the backgrounds, continuum vector boson production (WW, ZZ, WZ) was generated using PYTHIA. The $\mathrm{p}_{\mathrm{t}}(\mathrm{WW})$ spectrum was reweighted using the same technique than for the signal. A NLO cross section of respectively $16 \mathrm{pb}, 50 \mathrm{pb}$ and $114 \mathrm{pb}$ was taken for ZZ, WZ and WW. WW production via gluon box diagram, ggWW, was generated using a parton Monte Carlo provided by N. Kauer and linked to PYTHIA for the parton shower [70]. Top production $(\bar{t} \bar{t}$ and $\mathrm{tWb}$ ) was generated using TopREX. NLO cross sections of respectively $840 \mathrm{pb}$ and $33.4 \mathrm{pb}$ were used for $\mathrm{t} \overline{\mathrm{t}}$ and $\mathrm{tWb}$ [473].

10.2.2.2. Signal reconstruction. The signal signature is characterised by two leptons in the final state with opposite charge, missing energy and no jet. The leptons, either electrons or muons, are required to have $\mathrm{p}_{\mathrm{t}}>20 \mathrm{GeV} / \mathrm{c}$ and $|\eta|<2$. 
Muons candidates are asked to be isolated: The energy left in the calorimeters around the muon candidate within a $\Delta \mathrm{R}=0.3$ cone must be smaller than $5 \mathrm{GeV}$ and the sum of the $\mathrm{p}_{\mathrm{t}}$ of the tracks within a $\Delta \mathrm{R}=0.25$ cone around the muon candidate must be smaller than $2 \mathrm{GeV}$.

Electrons candidates are reconstructed combining tracks and ECAL clusters. They must fulfill in addition the following identification requirements:

- The electron must deposit small energy in the HCAL: $\mathrm{E}_{\text {hcal }} / E_{\text {ecal }}<0.05$ ?

- The electron track and cluster must be precisely matched:

in direction: $\left|\eta_{\text {track }}-\eta_{\mathrm{SC} \text { corr }}\right|<0.005$ and $\left|\phi_{\text {track prop }}-\phi_{\mathrm{SC}}\right|<0.02^{43}$ in magnitude: $\mathrm{E} / \mathrm{p}>0.8$ and $|1 / \mathrm{E}-1 / \mathrm{p}|<0.02$

The electron candidate must be also isolated by requiring, $\sum_{\text {tracks }} \mathrm{p}_{\mathrm{t}}($ track $) / \mathrm{E}_{\mathrm{t}}(\mathrm{SC})<$ 0.05 , where the sum runs on all the tracks (excluding electron) which have:

- $\Delta \mathrm{R}_{\mathrm{SC}-\text { track }}<0.2$ (at vertex);

- $\mathrm{p}_{\mathrm{t}}^{\text {track }}>0.9 \mathrm{GeV} / \mathrm{c}$;

- $\left|\mathrm{z}_{\text {track }}-\mathrm{z}_{\text {electron }}\right|<0.2 \mathrm{~cm}$.

Finally a cut on the impact parameter significance in the transverse plane is applied in order to reduce the b $\bar{b}$ background. Each lepton is required to have $\sigma_{\mathrm{IP}}<3$ where $\sigma_{\mathrm{IP}}$ is the impact parameter significance. The two leptons are also required to come from the same vertex by asking $\left|z_{\text {lep } 1}-z_{\text {lep } 2}\right|<0.2 \mathrm{~cm}$.

With this lepton selection, the contribution of reducible backgrounds like $\mathrm{W}+$ jet where one jet is misidentified as a lepton or b $b \bar{b}$ is expected to be less than $5 \mathrm{fb}$ after all cuts applied.

Missing energy is reconstructed by summing the raw energy of all ECAL and HCAL towers, and correcting for muons. Since a jet veto is applied in the signal selection, further correction on the missing energy did not bring a significant improvement.

Jets are reconstructed using a Cone algorithm of size $\Delta R=0.5$ and requiring its component calorimeter towers to have $\mathrm{E}_{\mathrm{T}}^{\text {tow }}>0.5 \mathrm{GeV}$ and $\mathrm{E}^{\text {tow }}>0.8 \mathrm{GeV}$. Since jets are reconstructed to be vetoed, no energy calibration was applied. For the events studied, $\mathrm{E}_{\mathrm{T}}(\mathrm{jet}) \approx(1.5-2) \cdot E_{\mathrm{T}}($ raw $)$. To veto electrons and Bremsstrahlung photons, the jets are also required to be away from the leptons $\left(\Delta \mathrm{R}_{\text {jet-lepton }}>0.5\right)$.

For jets with a raw energy between 15 and $20 \mathrm{GeV}$ an additional cut on their track content was applied in order to reduce the contamination from fake jets coming from the underlying event. For this, the so-called alpha parameter is defined, as the ratio of the sum of $\mathrm{p}_{\mathrm{t}}$ of tracks from the signal vertex inside the jet over the transverse jet energy in the calorimeter. For a perfect detector, the alpha parameter of a jet would be around 0.66, as in mean two third of a jet are charged particles. This ratio is smeared and reduced by the detector energy resolution and not $100 \%$ efficiency of the charged particle reconstruction in the tracker. In a fake jet, the sum of $\mathrm{p}_{\mathrm{t}}$ of tracks from the signal vertex inside the fake jet is small, leading to an alpha parameter around zero.

Alpha is determined using only tracks that are 'inside' the jet, i.e. with $\Delta \mathrm{R}_{\text {track-jet }}<0.5$ and coming from the event vertex ${ }^{44}$, fulfilling $\left|z_{\text {trk }}-z_{\mathrm{vtx}}\right|<0.4 \mathrm{~cm}$. Finally, these tracks should have more than 5 hits and $p_{t}>2 \mathrm{GeV} / \mathrm{c}$. Alpha is then defined as alpha $=\frac{\sum \mathrm{p}_{\mathrm{t}}(\text { tracks })}{\mathrm{E}_{\mathrm{T}} \text { (jet) }}$. If its raw energy lies between 15 and $20 \mathrm{GeV}$ a jet is then required to have $\alpha>0.2$ to be kept.

\footnotetext{
${ }^{43}$ Where $\phi_{\text {track prop }}$ is the track angle propagated in the magnetic field up to the ECAL cluster position.

44 The event vertex is defined as the mean $\mathrm{z}$ position of the two leptons.
} 


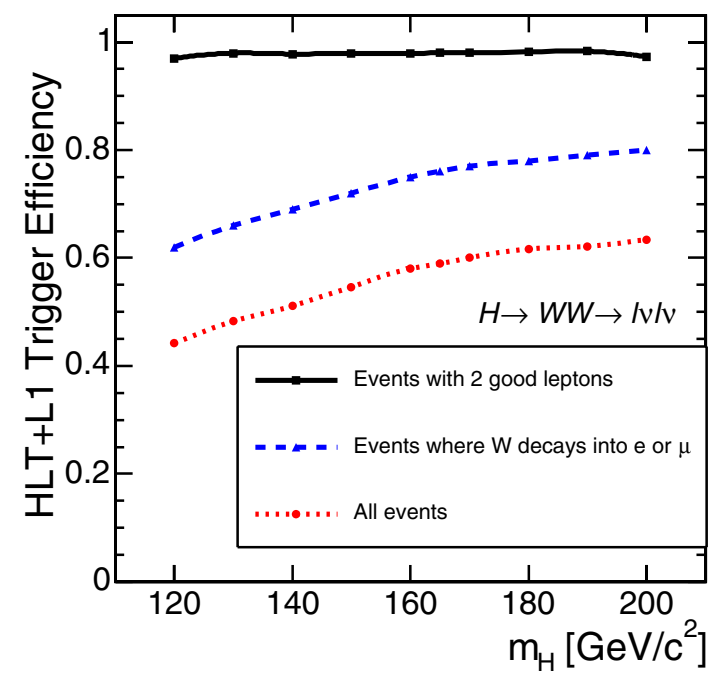

Figure 10.12. Trigger efficiencies (L1+HLT) as a function of the Higgs mass on all events (dotted line), on events where the $\mathrm{W}$ decays in electrons and muons (dashed line) and on events with exactly two leptons passing the lepton selection cuts (solid line).

10.2.2.3. Event selection and results. Events are first required to pass globally the Level-1 trigger and at least one of the following HLT triggers: single electron, double electron, single muon or double muon trigger.

Figure 10.12 shows the Level-1 trigger efficiency (blue dashed curve) and the combined L1+HLT trigger efficiencies (red dotted curve) as a function of the Higgs mass. To estimate the numbers of 'useful events' rejected by the trigger it is interesting to look at the trigger efficiency on events having exactly two leptons which fulfill the lepton selection cuts defined before. This is shown by the solid black curve on Fig. 10.12. In this case, the trigger efficiency is higher than $95 \%$ on the full mass range and is around $100 \%$ for $\mu \mu$ final state, whereas for ee final state it is around $96 \%$.

Then each event has to contain exactly two opposite charge leptons with $\mathrm{p}_{\mathrm{t}}>20 \mathrm{GeV} / \mathrm{c}$ and $|\eta|<2$ passing the cuts described before. The following kinematic selections were applied:

- $\mathrm{E}_{\mathrm{t}}^{\mathrm{miss}}>50 \mathrm{GeV}$

- $\phi_{\ell \ell}<45^{\circ}$ (angle between the leptons in the transverse plane)

- $12 \mathrm{GeV} / \mathrm{c}^{2}<\mathrm{m}_{\ell \ell}<40 \mathrm{GeV} / \mathrm{c}^{2}$ (the invariant mass of the two leptons)

- no jet with $\mathrm{E}_{\mathrm{t}}^{\mathrm{raw}}>15 \mathrm{GeV}$ and $|\eta|<2.5$

- $30 \mathrm{GeV} / \mathrm{c}<\mathrm{p}_{\mathrm{t}}^{\ell \max }<55 \mathrm{GeV} / \mathrm{c}$ (lepton with the maximal $\mathrm{p}_{\mathrm{t}}$ )

- $\mathrm{p}_{\mathrm{t}}^{\ell \min }>25 \mathrm{GeV} / \mathrm{c}$ (lepton with the minimal $\mathrm{p}_{\mathrm{t}}$ ).

These cuts were optimised for a Higgs mass of $165 \mathrm{GeV} / \mathrm{c}^{2}$. The expected number of events for the signal for three different Higgs masses and the different backgrounds in $\mathrm{fb}$ are given in Table 10.7. The first column shows the signal times branching ratio for the different processes, the second one shows the number of events passing the trigger requirement, the third one the number of events with two opposite charge leptons passing the lepton selection cuts and the last one the number of events after all selection cuts are applied. Figure 10.13, left shows the $\phi_{\ell \ell}$ distribution for the signal plotted on the top of the sum of all background when all selection cuts are applied except the one on $\phi_{\ell \ell}$. 
Table 10.7. The expected number of events for the signal for three different Higgs masses and the different backgrounds given in fb. The first column shows the number of expected events after HLT requirement, the second one after having found two opposite charge leptons and the last one the number of events after all selection cuts are applied.

\begin{tabular}{lclll}
\hline Reaction $\mathrm{pp} \rightarrow X$ & $\sigma_{\mathrm{NLO}} \times \mathrm{BR}$ & $\mathrm{L} 1+\mathrm{HLT}$ & 2 leptons & All cuts \\
\hline$\ell=\mathrm{e}, \mu, \tau$ & $\mathrm{pb}$ & \multicolumn{3}{c}{ Expected event rate in fb } \\
$\mathrm{H} \rightarrow \mathrm{WW} \rightarrow \ell \ell, \mathrm{m}_{\mathrm{H}}=160 \mathrm{GeV} / \mathrm{c}^{2}$ & 2.34 & $1353(58 \%)$ & $359(27 \%)$ & $42(12 \%)$ \\
$\mathrm{H} \rightarrow \mathrm{WW} \rightarrow \ell \ell, \mathrm{m}_{\mathrm{H}}=165 \mathrm{GeV} / \mathrm{c}^{2}$ & 2.36 & $1390(59 \%)$ & $393(28 \%)$ & $46(12 \%)$ \\
$\mathrm{H} \rightarrow \mathrm{WW} \rightarrow \ell \ell, \mathrm{m}_{\mathrm{H}}=170 \mathrm{GeV} / \mathrm{c}^{2}$ & 2.26 & $1350(60 \%)$ & $376(28 \%)$ & $33(8.8 \%)$ \\
$\mathrm{qq} \rightarrow \mathrm{WW} \rightarrow \ell \ell$ & 11.7 & $6040(52 \%)$ & $1400(23 \%)$ & $12(0.9 \%)$ \\
$\mathrm{gg} \rightarrow \mathrm{WW} \rightarrow \ell \ell$ & 0.48 & $286(60 \%)$ & $73(26 \%)$ & $3.7(5.1 \%)$ \\
$\mathrm{tt} \rightarrow \mathrm{WWbb} \rightarrow \ell \ell$ & 86.2 & $57400(67 \%)$ & $15700(27 \%)$ & $9.8(0.06 \%)$ \\
$\mathrm{tWb} \rightarrow \mathrm{WWb}(\mathrm{b}) \rightarrow \ell \ell$ & 3.4 & $2320(68 \%)$ & $676(29 \%)$ & $1.4(0.2 \%)$ \\
$\mathrm{ZW} \rightarrow \ell \ell \ell$ & 1.6 & $1062(66 \%)$ & $247(23 \%)$ & $0.50(0.2 \%)$ \\
$\mathrm{ZZ} \rightarrow \ell \ell, v v$ & 1.5 & $485(32 \%)$ & $163(34 \%)$ & $0.35(0.2 \%)$ \\
$\mathrm{Sum}$ backgrounds & 105 & $67600(64 \%)$ & $18300(27 \%)$ & $28(0.2 \%)$ \\
\hline
\end{tabular}

10.2.2.4. Background normalisation and systematics. The following procedure for background normalisation is proposed.

- Top background normalisation. Two procedures are proposed. A first possibility is to define a sample with the same lepton and missing energy cuts as for the signal selection but requiring two b-tagged jets with $\mathrm{E}_{\mathrm{t}}>20 \mathrm{GeV}$. A second possibility is to apply the same kinematic cuts on the leptons and require two additional jets with respectively $\mathrm{E}_{\mathrm{T}}^{\text {raw }}>50 \mathrm{GeV}$ and $\mathrm{E}_{\mathrm{T}}^{\mathrm{raw}}>30 \mathrm{GeV}$. In this case, only e $\mu$ final states are considered in order to avoid a contamination from Drell-Yan. Both methods are expected to give an error of about $16 \%$ on $\overline{\mathrm{tt}}$ estimate for a luminosity of $5 \mathrm{fb}^{-1}$.

- WW background normalisation. A normalisation region can be defined for WW by keeping the same cuts than the signal but requiring $\phi_{\ell \ell}<140$ and $\mathrm{m}_{\ell \ell}>60 \mathrm{GeV} / \mathrm{c}^{2}$. Moreover only opposite flavour leptons are considered in order to reduce the Drell-Yan and WZ contribution. A systematic error of about $17 \%$ is expected with a luminosity of $5 \mathrm{fb}^{-1}$, dominated by statistical uncertainty. Figure 10.13 right shows the $\phi_{\ell \ell}$ distribution for the different process in this normalisation region.

- WZ background normalisation. WZ can be normalised by keeping the same signal cut and requiring an additional lepton in the final state. The cuts on $\phi_{\ell \ell}$ and $\mathrm{m}_{\ell \ell}$ are removed. An accuracy of about $20 \%$ is expected on this background with $5 \mathrm{fb}^{-1}$.

- $g g W W$ and $t W b$ normalisation. The contribution of these backgrounds will be estimated using Monte Carlo prediction, since they represent only a small fraction of signal events. The error on $\mathrm{ggWW}$ is about $30 \%$ whereas the one on $\mathrm{tWb}$ is about $22 \%$, both largely dominated by theoretical errors.

Taking into account the sum of the different backgrounds, an overall error of $13 \%$ is found on the total background. These results are calculated for a luminosity of $5 \mathrm{fb}^{-1}$. For luminosities of 1,2 and $10 \mathrm{fb}^{-1}$, the total systematic errors scale to $19 \%, 16 \%$ and $11 \%$ respectively. Table 10.8 show the signal to background ratio for the different Higgs masses together with the luminosity needed for a $5 \sigma$ discovery, with and without the inclusion of background uncertainties. For Higgs masses of $120-140 \mathrm{GeV} / \mathrm{c}^{2}$ and $190-200 \mathrm{GeV} / \mathrm{c}^{2}$, the background errors are too high to get a significant signal.

Figure 10.14 shows the signal to background ratio (left) and the luminosity needed for a $5 \sigma$ discovery (right) as a function of the Higgs mass. A signal of more than $5 \sigma$ significance 

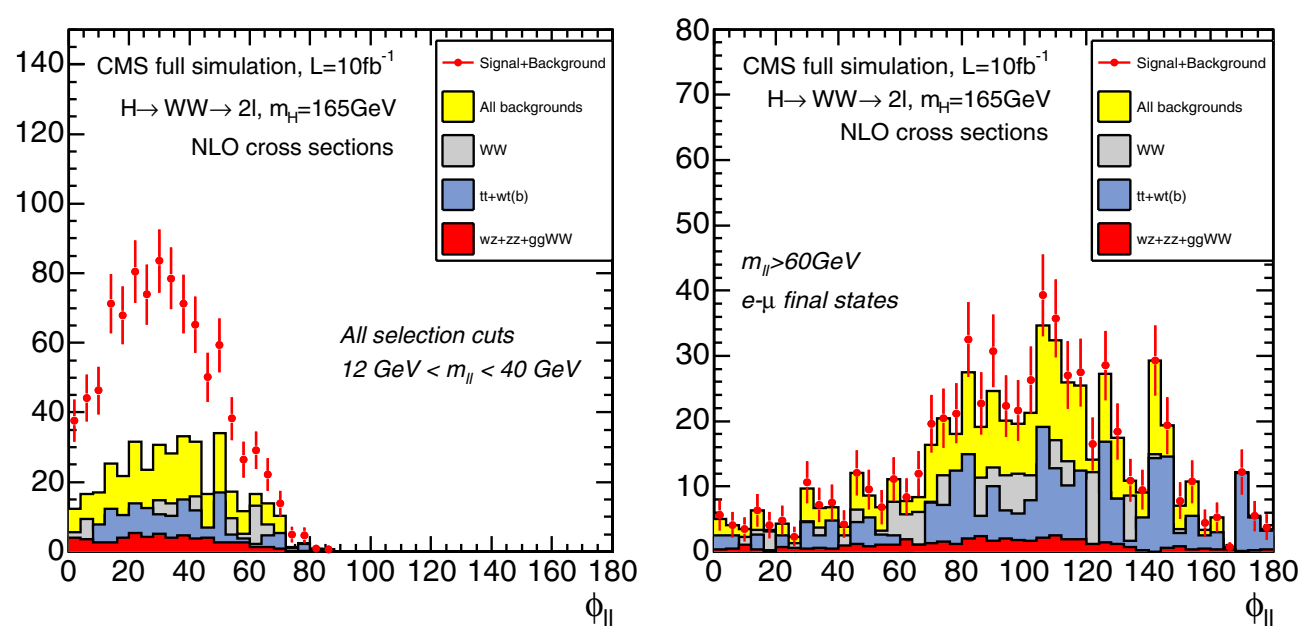

Figure 10.13. The angle between the leptons in the transverse plane for the signal and the different background and a luminosity of $10 \mathrm{fb}^{-1}$, (Left) For the signal cuts taking out the one on $\varphi_{\ell \ell}$. (Right) For the WW background normalisation region where all signal cuts are applied except the one on the lepton invariant mass, which was set to $\mathrm{m}_{\ell \ell}>60 \mathrm{GeV} / \mathrm{c}^{2}$ and only electron-muon final states are kept.

Table 10.8. The signal to background ratio for the different Higgs masses together with the luminosity needed for a $5 \sigma$ discovery, with and without the inclusion of background uncertainties. Also the statistical errors due to the restricted Monte Carlo statistics are taken into account.

\begin{tabular}{|c|c|c|c|c|c|}
\hline \multirow[t]{2}{*}{$\mathrm{m}_{\mathrm{H}}[\mathrm{GeV}]$} & \multirow[t]{2}{*}{$\mathrm{S} / \mathrm{B}$} & \multicolumn{2}{|c|}{ Significance for $5 \mathrm{fb}^{-1}$} & \multirow{2}{*}{$\begin{array}{c}\mathcal{L}_{\text {disc }}[\mathrm{fb}] \\
\text { no bkg syst }\end{array}$} & \multirow{2}{*}{$\begin{array}{c}\mathcal{L}_{\text {disc }}[\mathrm{fb}] \\
\text { with bkg sys }\end{array}$} \\
\hline & & no bkg syst & with bkg syst & & \\
\hline 150 & 0.61 & 6.6 & 4.0 & 3.0 & 8.2 \\
\hline 160 & 1.51 & 14 & 7.7 & 0.58 & 1.1 \\
\hline 165 & 1.66 & 15 & 8.3 & 0.50 & 0.90 \\
\hline 170 & 1.19 & 11 & 6.3 & 0.88 & 1.7 \\
\hline 180 & 0.65 & 6.7 & 3.7 & 2.7 & 7.3 \\
\hline
\end{tabular}

could be already observed with a luminosity of $7 \mathrm{fb}^{-1}$ for a Higgs mass between 150 and $180 \mathrm{GeV} / \mathrm{c}^{2}$. For a Higgs mass of $165 \mathrm{GeV} / \mathrm{c}^{2}$ the luminosity needed for a $5 \sigma$ discovery is expected to be less than $1 \mathrm{fb}^{-1}$.

10.2.2.5. Selection optimisation for $\mathrm{M}_{\mathrm{H}}$ in the $130-150 \mathrm{GeV} / \mathrm{c}^{2}$ mass range with $e^{+} e^{-} v v$ final state. A dedicated optimisation for the $e^{+} e^{-} v v$ final state in the mass range of $130 \leqslant$ $\mathrm{M}_{\mathrm{H}} \leqslant 150 \mathrm{GeV} / \mathrm{c}^{2}$ has been performed [474]. The largest significance is searched assuming a known $\mathrm{M}_{\mathrm{H}}$. The latest developments in detailed electron reconstruction are used and allow a good rejection of the $\mathrm{W}+$ jets background which is characterised by the misidentification of a jet as an electron. New kinematical variables have been designed to reduce the $W+$ jets background as well as the contribution from Drell-Yan events with recoiling jets (Z+jets). For instance, in the signal, the two electrons tend to be close to each other, and the dielectron system is essentially emitted in the central region. On the contrary, in the $\mathrm{Z}+$ jets background, the dielectron pair is emitted uniformly in $\eta$, and the electrons candidates in the $\mathrm{W}+$ jets backgrounds are well separated. Other selection criteria relying on the absence of a true source of missing transverse energy in the $\mathrm{Z}+$ jets events have been introduced: in the events where 

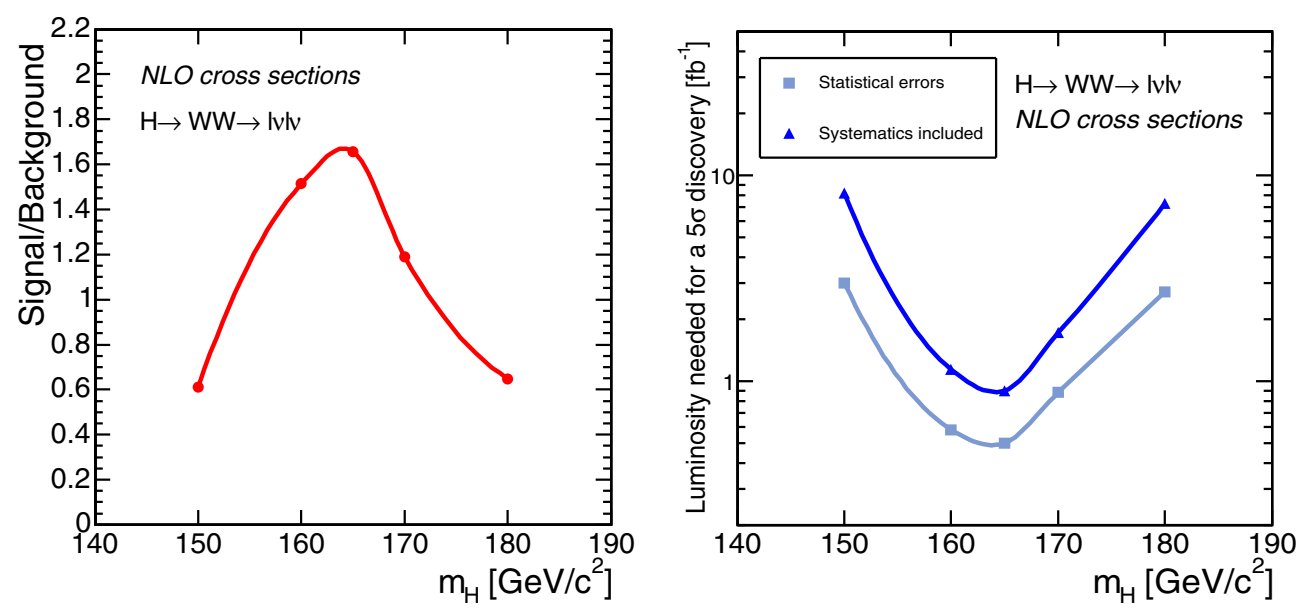

Figure 10.14. Signal to background ratio for a luminosity of $5 \mathrm{fb}^{-1}$ (left) and the luminosity needed for a $5 \sigma$ discovery (right) as a function of different Higgs masses for the $\mathrm{H} \rightarrow \mathrm{WW}$ channel.
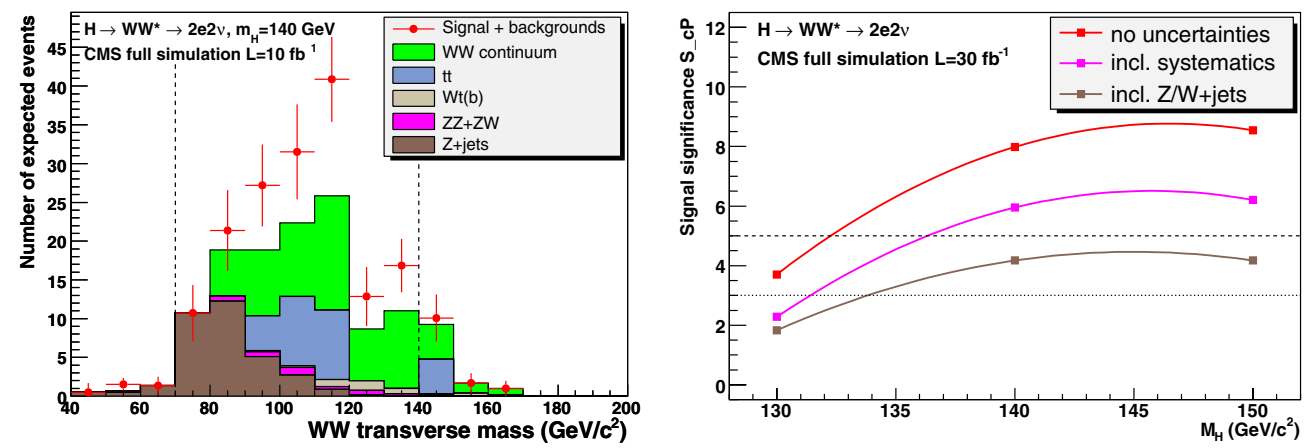

Figure 10.15. Left: the reconstructed $W W$ transverse mass for the $140 \mathrm{GeV}$ Higgs signal selection with $10 \mathrm{fb}^{-1}$. The dashed lines show the window of events entering in the signal significance calculation. Right: the signal significance as function of the Standard Model Higgs mass for an integrated luminosity of $30 \mathrm{fb}^{-1}$.

the missing transverse energy is mis-measured, it is usually in the same direction as the leading jet. Similarly, the imbalance of the missing energy and the dilepton system in the transverse plane is exploited.

Both $\mathrm{W}+$ jets and $\mathrm{Z}+\mathrm{jets}$ backgrounds are thus explicitly reduced to a manageable level. Fig. 10.15 (left) shows the reconstructed WW transverse mass for the $140 \mathrm{GeV}$ Higgs signal selection with $10 \mathrm{fb}^{-1}$. Figure 10.15 (right) shows the signal significance as function of the Standard Model Higgs mass for the integrated luminosity of $30 \mathrm{fb}^{-1}$ with and without systematics taken into account. A $3 \sigma$ observation is possible for Higgs masses from $135 \mathrm{GeV}$. A $5 \sigma$ discovery is reached with $60 \mathrm{fb}^{-1}$.

\subsubsection{The vector boson fusion production with $\mathrm{H} \rightarrow \tau \tau \rightarrow \ell+\tau$ jet $+E_{\mathrm{T}}^{\text {miss }}$}

In the early parton level simulation studies [475, 476] and fast detector simulation studies of ATLAS and CMS [477] it was shown that the Higgs boson production in the vector boson 
fusion $\mathrm{qq} \rightarrow \mathrm{qqH}$ (qqH or $\mathrm{VBF}$ ) and decay into $\tau$ lepton pair could be the discovery channel with $\sim 30 \mathrm{fb}^{-1}$. The cross section measurement of $\mathrm{qqH}, \mathrm{H} \rightarrow \tau \tau, \mathrm{WW}, \gamma \gamma$ channels will significantly extend the possibility of the Higgs boson coupling measurement $[478,479]$ and provide the possibility of the indirect measurement of the light Higgs boson width [478]. In the MSSM the $\mathrm{qqH}(\mathrm{h}), \mathrm{H}(\mathrm{h}) \rightarrow \tau \tau$ channel could be discovered in the largest region of the $\mathrm{M}_{\mathrm{A}}-\tan \beta$ parameter plane $[475,480]$. The forward jet tagging and the central jet veto are the key selections of the VBF Higgs boson channels. The study of the observability of the VBF Higgs boson production and $\mathrm{H} \rightarrow \tau \tau \rightarrow \ell+$ jet decay with the full detector simulation is presented in the following. A detailed description of the analysis can be found in [481].

10.2.3.1. Signal and background generation and pre-selections. The signal events were generated using PYTHIA for four different values of the Higgs boson mass: 115, 125, 135 and $145 \mathrm{GeV} / \mathrm{c}^{2}$. The Higgs boson was forced to decay to two $\tau$ leptons with one $\tau$ decaying to leptons and the other $\tau$ to hadrons. The TAUOLA package was used to simulate the $\tau$ polarisation.

For background events, following processes are considered:

$Q C D 2 \tau+2 / 3 j$

The QCD production of $2 \tau+2$ jet and +3 jet events with the invariant mass of two $\tau$ leptons, $\mathrm{M}_{\tau \tau}>70 \mathrm{GeV} / \mathrm{c}^{2}$, was generated using ALPGEN with CTEQ5L PDF. Given the limit of the detector acceptance and requirements in the course of the event reconstruction, all jets were required to satisfy $\mathrm{p}_{\mathrm{Tj}}>20 \mathrm{GeV},\left|\eta_{\mathrm{j}}\right|<5.0$ and $\left|\Delta \mathrm{R}_{\mathrm{jj}}\right|>0.5$. Further pre-selections were applied on the two highest $\mathrm{p}_{\mathrm{T}}$ jets $(\mathrm{j} 1$ and $\mathrm{j} 2)$ reflecting the offline VBF selection cuts: $\left|\Delta \eta_{\mathrm{j} 1 \mathrm{j} 2}\right|>4.0, \mathrm{M}_{\mathrm{j} 1 \mathrm{j} 2}>600 \mathrm{GeV} / \mathrm{c}^{2}$. Then the events $2 \tau+2 \mathrm{j}$ and $2 \tau+3 \mathrm{j}$ were added together with the MLM prescription in PYTHIA to avoid double counting of the jets. The TAUOLA package was used in PYTHIA to force one $\tau$ lepton to decay leptonically and the other hadronically.

Electro Weak (EW) production of $2 \tau+2 j$

The EW production of two $\tau$ 's with $\mathrm{M}_{\tau \tau}>70 \mathrm{GeV} / \mathrm{c}^{2}$ and two jets in the final state was generated using MADGRAPH with CTEQ5L PDF. Soft pre-selections were applied during generation with MADGRAPH on the kinematics of the jets: $\mathrm{p}_{\mathrm{Tj}}>20 \mathrm{GeV} / \mathrm{c}$ and $\mathrm{M}_{\mathrm{jj}}>$ $500 \mathrm{GeV} / \mathrm{c}^{2}$. Further pre-selection cuts were applied on jets and $\tau$ 's given the limit of the detector acceptance and requirements of the event reconstruction: $\left|\eta_{\mathrm{j}}\right|<5.2,\left|\Delta \mathrm{R}_{\mathrm{jj}}\right|>0.5$, $\left|\Delta R_{\tau \tau}\right|>0.4$. The showering and hadronisation of the MADGRAPH parton level events were carried out using PYTHIA where all decay modes of the $\tau$ lepton were open.

$W+j e t s$

The $\mathrm{W}+3 \mathrm{j}$ and $\mathrm{W}+4 \mathrm{j}$ events with $\mathrm{W} \rightarrow \mu \nu$ decays were generated using ALPGEN with CTEQ5M PDF. In addition to the kinematical cuts on jets used for the QCD Z + jets production described above, further pre-selections were made based on the lepton properties with $\left|\eta_{\ell}\right|<3$ and $\mathrm{p}_{\mathrm{T} \ell}>10 \mathrm{GeV} / \mathrm{c}$. The MLM prescription was applied in PYTHIA.

$t \bar{t} \rightarrow W b W b$

The $\overline{\mathrm{t}}$ background was generated using PyTHIA, TOPREX, ALPGEN, CompHEP and MAdGraph. All leptonic W decays were included and no kinematical pre-selection was applied.

10.2.3.2. Event reconstruction and selection. Events are triggered at Level 1 by the single isolated e, single $\mu$ and combined e- $\tau$ triggers. At the High Level the following triggers are used: the single isolated e, single $\mu$, combined e- $\tau$ and combined $\mu-\tau$ triggers. 
In the off-line analysis the electron and muon candidates were selected and for the electron candidates three additional requirements are applied: $\mathrm{E} / \mathrm{p}>0.9$, tracker isolation, $\left(\sum_{0.01<\Delta R<0.2}^{t r k} \mathrm{p}\right) / \mathrm{E}<0.05$, and $\mathrm{E}_{\mathrm{T}}$ of the hottest HCAL tower, $\mathrm{E}_{\mathrm{T}}^{\mathrm{Htow}}<2 \mathrm{GeV}$. The highest $\mathrm{p}_{\mathrm{T}}$ off-line lepton candidate with $\mathrm{p}_{\mathrm{T}}>15 \mathrm{GeV} / \mathrm{c}$ is then selected. The lepton track is used to identify tracks originating from the signal vertex. The tracks are used for the electron isolation, $\tau$ tagging and in central jet veto. A track is associated to the signal vertex if its $\mathrm{z}$ impact parameter lies within $|\Delta \mathrm{z}|<0.2 \mathrm{~cm}$ from that of the lepton track.

The $\tau$-jet identification is seeded from the L1/HLT $\tau$ candidates. A jet is formed around each candidate which does not coincide with the identified electron, and the jet is passed through a series of $\tau$-tagging criteria. The $\tau$ tagging used in HLT (Ref. [76]) has been adapted to offline use with parameters $R_{m}=0.1, R_{s}=0.07, R_{i}=0.45, p_{T}^{\text {ltr }}=6 \mathrm{GeV} / \mathrm{c}$ and $\mathrm{p}_{\mathrm{T}}^{\mathrm{i}}=1 \mathrm{GeV} / \mathrm{c}$. The charge of the $\tau$-jet is required to be opposite of the lepton charge, and $\mathrm{E}_{\mathrm{T}}^{\mathrm{Htow}}>2 \mathrm{GeV}$ is required if the jet coincides with any of the electron candidates. A further cut is applied on the transverse energy of the $\tau$-jet, $\mathrm{E}_{\mathrm{T}}>30 \mathrm{GeV}$.

The jets from the VBF process are identified as the two highest $\mathrm{E}_{\mathrm{T}}$ calorimeter jets with $\mathrm{E}_{\mathrm{Tj}}>40 \mathrm{GeV}$, excluding the electron and the $\tau$-jet. The jets are required to satisfy: $\left|\eta_{\mathrm{j}}\right|<4.5$, $\eta_{\mathrm{j} 1} \times \eta_{\mathrm{j} 2}<0, \Delta \eta_{\mathrm{j} 1 \mathrm{j} 2}>4.5, \Delta_{\phi \mathrm{j} 1 \mathrm{j} 2}<2.2$, and the invariant mass, $\mathrm{M}_{\mathrm{j} 1 \mathrm{j} 2}>1 \mathrm{TeV}$. The jets after these selections will be referred to as tagging jets.

A cut is applied on the transverse mass of the lepton- $\mathrm{E}_{\mathrm{T}}^{\text {miss }}$ system, $\mathrm{M}_{\mathrm{T}}\left(\mathrm{lep}, \mathrm{E}_{\mathrm{T}}^{\mathrm{miss}}\right)<$ $40 \mathrm{GeV}$, in order to reject backgrounds with $\mathrm{W} \rightarrow \ell v$ decays.

The central jet veto was applied. An event is vetoed if there is an additional jet (j3) with $\mathrm{E}_{\mathrm{T} j 3}^{\mathrm{raw}}>10 \mathrm{GeV}$ in the rapidity gap between the two tagging jets, satisfying the following:

- $\left(\eta_{\min }+0.5\right)<\eta_{\mathrm{j} 3}<\left(\eta_{\max }-0.5\right)$

where $\eta_{\min }$ and $\eta_{\max }$ correspond to the tagging jets which has smaller and larger value of $\eta$ respectively.

- $\alpha_{\mathrm{j} 3}=\sum \mathrm{p}_{\mathrm{Ttrk}} / \mathrm{E}_{\mathrm{Tj} 3}^{\mathrm{raw}}>0.1$

where $\mathrm{p}_{\mathrm{T} \text { trk }}$ is the $\mathrm{p}_{\mathrm{T}}$ of the track originating from the signal vertex, which lie within the 0.5 cone around the jet axis, and $\mathrm{E}_{\mathrm{Tj} 3}^{\mathrm{raw}}$ is the raw $\mathrm{E}_{\mathrm{T}}$ of the jet measured in the calorimeter.

$\alpha_{\mathrm{j} 3}$ is defined for each additional jet, and the one which satisfies the first criteria and has the highest $\alpha_{\mathrm{j} 3}$ is considered for the veto.

The invariant mass of the two reconstructed $\tau$ 's is calculated as described in the MSSM $\mathrm{H}(\mathrm{A}) \rightarrow \tau \tau$ analysis (Section 5.2) using the collinear approximation of the visible part of $\tau$ 's and neutrinos. The $\mathrm{E}_{\mathrm{T}}^{\mathrm{miss}}$ is reconstructed by summing the $\mathrm{E}_{\mathrm{T}}$ of the calorimeter towers and the muon candidates, and applying the jet energy corrections (Type $1 \mathrm{E}_{\mathrm{T}}^{\mathrm{miss}}$ ). The events were accepted if $\mathrm{E}_{v 1, v 2}>0$.

10.2.3.3. Expected number of events. The efficiency of each reconstruction and selection step and the cumulative cross section expected at the LHC are given in Table 10.9. The total selection efficiencies are, $0.32 \%, 0.34 \%, 0.42 \%, 0.39 \%$, for the signal events with the Higgs boson masses, $\mathrm{M}_{\mathrm{H}}=115,125,135$ and $145 \mathrm{GeV} / \mathrm{c}^{2}$ respectively.

For the $\mathrm{W}+3 / 4 \mathrm{j}$ background, the efficiencies of some selection cuts have been obtained from factorisation of cuts. The trigger and the lepton identification are carried out as other samples, and the remaining steps are carried out in two uncorrelated parallel streams - A: $\mathrm{VBF}$ and $\mathrm{M}_{\mathrm{T}}\left(\right.$ lep, $\mathrm{E}_{\mathrm{T}}^{\mathrm{miss}}$ ) cuts, $\mathrm{B}$ : central jet veto, $\tau$ tagging and mass calculation - after preselections of forward jets and $\tau$-jet candidates.

10.2.3.4. Reconstructed mass and fit. The distribution of the invariant mass of two reconstructed $\tau$ 's for different samples is shown in Fig. 10.16, where the signal sample with 
Table 10.9. Cumulative cross sections in fb after successive selection cuts. The efficiency (\%) of each cut is listed inside the brackets. The entry, "valid mass", corresponds to the fraction remained after the calculation of the di $\tau$ mass when some events are lost due to the negative reconstructed neutrino energies. For the $\mathrm{W}+3 / 4 \mathrm{j}$ samples, efficiencies are obtained from factorisation of cuts and the $\tau$-jet ID efficiency includes the $\mathrm{p}_{\mathrm{T}}$ cut, and the number of events at $30 \mathrm{fb}^{-1}$ (indicated by*) is calculated for all leptonic decay modes of $\mathrm{W}$.

\begin{tabular}{|c|c|c|c|c|c|}
\hline \multirow[b]{3}{*}{ Selection } & \multicolumn{5}{|c|}{ cross section, $\sigma[\mathrm{fb}]$ ( $\%$ from previous cut) } \\
\hline & \multirow{2}{*}{$\begin{array}{l}\text { signal } \\
\mathrm{M}_{\mathrm{H}}=135\end{array}$} & \multicolumn{4}{|c|}{ background } \\
\hline & & $\mathrm{EW} 2 \tau+2 \mathrm{j}$ & $\mathrm{QCD} \tau \tau+2 / 3 \mathrm{j}$ & $\mathrm{W}+3 / 4 \mathrm{j}$ & $\mathrm{t} \overline{\mathrm{t}} \rightarrow \mathrm{WbWb}$ \\
\hline Starting $\sigma$ & 82.38 & 299. & 1615. & $14.45 \times 10^{3}$ & $86 \times 10^{3}$ \\
\hline Level-1 & $46.50(56.5)$ & $179.8(60.1)$ & $543.8(33.7)$ & 9186. (63.6) & $71.39 \times 10^{3}(83.0)$ \\
\hline L1+HLT & $24.60(52.9)$ & $58.81(32.7)$ & $201.3(37.0)$ & 6610. (71.9) & $55.42 \times 10^{3}(77.6)$ \\
\hline lepton ID & $23.34(94.9)$ & $50.67(86.2)$ & $187.4(93.1)$ & 6549. (99.1) & $54.08 \times 10^{3}(97.6)$ \\
\hline lepton $\mathrm{p}_{\mathrm{T}}$ & $23.16(99.3)$ & $49.13(97.0)$ & $185.6(99.0)$ & 6543. (99.9) & $53.54 \times 10^{3}(99.0)$ \\
\hline$\tau$-jet ID & $8.276(35.7)$ & $10.49(21.3)$ & $39.64(21.4)$ & $(0.21)$ & $5.056 \times 10^{3}(9.4)$ \\
\hline$\tau$-jet $\mathrm{p}_{\mathrm{T}}$ & $6.422(77.6)$ & $7.360(70.2)$ & $24.25(61.2)$ & - & $3.215 \times 10^{3}(63.6)$ \\
\hline Valid mass & $4.461(69.5)$ & $4.232(57.5)$ & $14.49(59.8)$ & (17.4) & $848.6(26.4)$ \\
\hline VBF cuts & $0.545(12.2)$ & $0.391(9.2)$ & $1.666(11.5)$ & (11.0) & $2.738(0.3)$ \\
\hline $\mathrm{M}_{\mathrm{T}}\left(\mathrm{lep}, \mathrm{E}_{\mathrm{T}}^{\text {miss }}\right)$ & $0.423(77.6)$ & $0.322(82.4)$ & $1.382(83.0)$ & $(30.5)$ & $0.942(34.4)$ \\
\hline Central Jet Veto & $0.344(81.3)$ & $0.230(71.4)$ & $0.555(39.7)$ & $(28.9)$ & $0.224(23.8)$ \\
\hline $\mathrm{N}$ events at $30 \mathrm{fb}^{-1}$ & 10.3 & 6.9 & 16.6 & $1.5^{*}$ & 6.7 \\
\hline
\end{tabular}

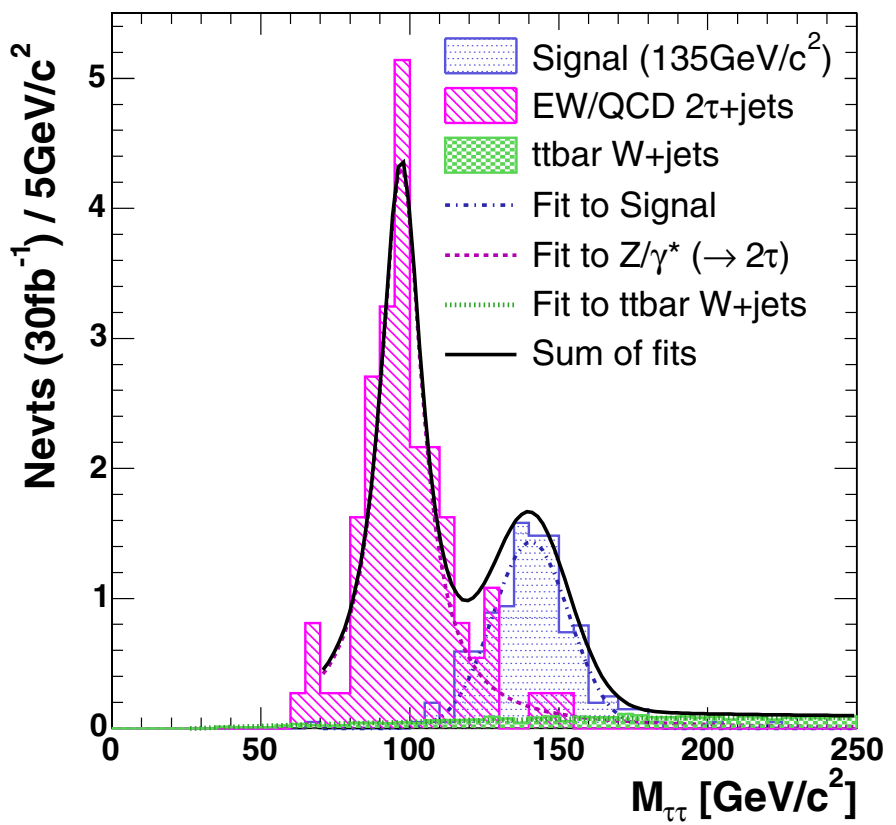

Figure 10.16. The invariant mass of two reconstructed $\tau$ 's. The number of entries in each histogram is normalised to the expected number of events at an integrated luminosity of $30 \mathrm{fb}^{-1}$.

the Higgs boson mass, $\mathrm{M}_{\mathrm{H}}=135 \mathrm{GeV} / \mathrm{c}^{2}$ is used. A Gaussian function is used to fit the signal distribution, a Breit-Wigner function for the $2 \tau+$ jets background from EW and QCD processes, and a second order polynomial for the reducible background from $\mathrm{W}+\mathrm{jets}$ and $\overline{\mathrm{t}}$ events. The Higgs boson mass resolution is $9.1 \%$. 
Table 10.10. The production cross section and significance of the expected number of signal events within the optimum mass window for each of the four different simulated masses of the Higgs boson.

\begin{tabular}{lllll}
\hline $\mathrm{M}_{\mathrm{H}}[\mathrm{GeV}]$ & 115 & 125 & 135 & 145 \\
\hline Production $\sigma[\mathrm{fb}]$ & $4.65 \times 10^{3}$ & $4.30 \times 10^{3}$ & $3.98 \times 10^{3}$ & $3.70 \times 10^{3}$ \\
$\sigma \times \mathrm{BR}(\mathrm{H} \rightarrow \tau \tau \rightarrow l j)[\mathrm{fb}]$ & 157.3 & 112.9 & 82.38 & 45.37 \\
$\mathrm{~N}_{\mathrm{S}}$ at $30 \mathrm{fb}^{-1}$ & 10.5 & 7.8 & 7.9 & 3.6 \\
$\mathrm{~N}_{\mathrm{B}}$ at $30 \mathrm{fb}^{-1}$ & 3.7 & 2.2 & 1.8 & 1.4 \\
Significance at $30 \mathrm{fb}^{-1}\left(\sigma_{\mathrm{B}}=7.8 \%\right)$ & 3.97 & 3.67 & 3.94 & 2.18 \\
Significance at $60 \mathrm{fb}^{-1}\left(\sigma_{\mathrm{B}}=5.9 \%\right)$ & 5.67 & 5.26 & 5.64 & 3.19 \\
\hline
\end{tabular}

10.2.3.5. Signal significance. The significance is calculated using a window with a fixed width of $40 \mathrm{GeV} / \mathrm{c}^{2}$, which slides in $5 \mathrm{GeV} / \mathrm{c}^{2}$ steps. An optimum window position which maximises the significance is chosen for each of the four different masses of Higgs boson. The numbers of signal and background events within the window, $\mathrm{N}_{\mathrm{S}}$ and $\mathrm{N}_{\mathrm{B}}$, are estimated from the fits to individual samples. The method $S_{c P}$ (Ref. [79]) is used for calculating the significance, including the systematic uncertainty of $7.8 \%$ for $30 \mathrm{fb}^{-1}$ and $5.9 \%$ for $60 \mathrm{fb}^{-1}$. The results are summarised in Table 10.10.

It is envisaged that the shapes of the two background distributions will be extracted experimentally from the LHC data in a region unaffected by the signal contribution, using some relaxation of selection cuts. Since the number of background events in the signal region will be estimated using real data, the fitting procedure is the only contribution to the uncertainty in the significance estimate. The fit uncertainty has been evaluated by performing MC trials, randomly generating a mass distribution from the original fit functions and re-fitting the distribution at each trial. With the data, the Higgs boson mass will be estimated by repeating the fitting procedure for different mass hypotheses and finding the value where the $\chi^{2}$ of the fit is minimised.

\subsubsection{Searching for standard model Higgs via vector boson fusion in $\mathrm{H} \rightarrow \mathrm{W}^{+} \mathrm{W}^{-} \rightarrow \ell^{ \pm} v j j$ with $\mathrm{m}_{\mathrm{H}}$ from 120 to $250 \mathrm{GeV} / \mathrm{c}^{2}$}

The signal topology of Higgs boson with $\mathrm{H} \rightarrow \mathrm{W}^{+} \mathrm{W}^{-} \rightarrow \ell \nu j j$ via vector boson fusion has been shown as a good potential discovery channel for the medium-high mass range $\left(\mathrm{m}_{\mathrm{H}}>300 \mathrm{GeV} / \mathrm{c}^{2}\right)$. The final state is characterised as two forward jets, two central jets from $\mathrm{W}$ hadronic decay, and one high $\mathrm{p}_{\mathrm{T}}$ lepton and missing transverse energy $\left(\mathrm{E}_{\mathrm{T}}^{\mathrm{miss}}\right)$ from the $\mathrm{W}$ leptonic decay. Extending the use of this channel to the low mass range $\left(\mathrm{m}_{\mathrm{H}}<\right.$ $300 \mathrm{GeV} / \mathrm{c}^{2}$ ) makes valuable physics analysis possible and is complementary to the Higgs boson search using $\mathrm{H} \rightarrow \mathrm{W}^{+} \mathrm{W}^{-} \rightarrow \ell \nu \ell \nu$, especially for $160<\mathrm{m}_{\mathrm{H}}<180 \mathrm{GeV} / \mathrm{c}^{2}$, where $\mathrm{H} \rightarrow \mathrm{ZZ}^{*}$ branching ratio is highly suppressed due to the opening of $\mathrm{H} \rightarrow \mathrm{W}^{+} \mathrm{W}^{-}$decay with two on-shell $\mathrm{W}$ bosons.

The result of this section shows that in the Higgs boson mass range between 140 and $200 \mathrm{GeV} / \mathrm{c}^{2}$, a significance of $\sim 5 \sigma$ can be achieved with integrated luminosity of $30 \mathrm{fb}^{-1}$. Major backgrounds include $\overline{\mathrm{t}}+$ jets, $\mathrm{W}+\mathrm{t} \overline{\mathrm{b}}(\overline{\mathrm{t}} \mathrm{b}), \mathrm{W}+$ jets, $\mathrm{Z}+$ jets, $\mathrm{WW} / \mathrm{WZ} / \mathrm{ZZ}+$ jets, and QCD events. For WW + jets, the QCD and Electroweak (EW) processes are generated separately. A detailed description of the analysis can be found in [482].

10.2.4.1. Event selection strategy. Major difficulties concerning the low mass Higgs analysis using $\ell \nu \mathrm{jj}$ final state include: many background processes of very large cross section have one 


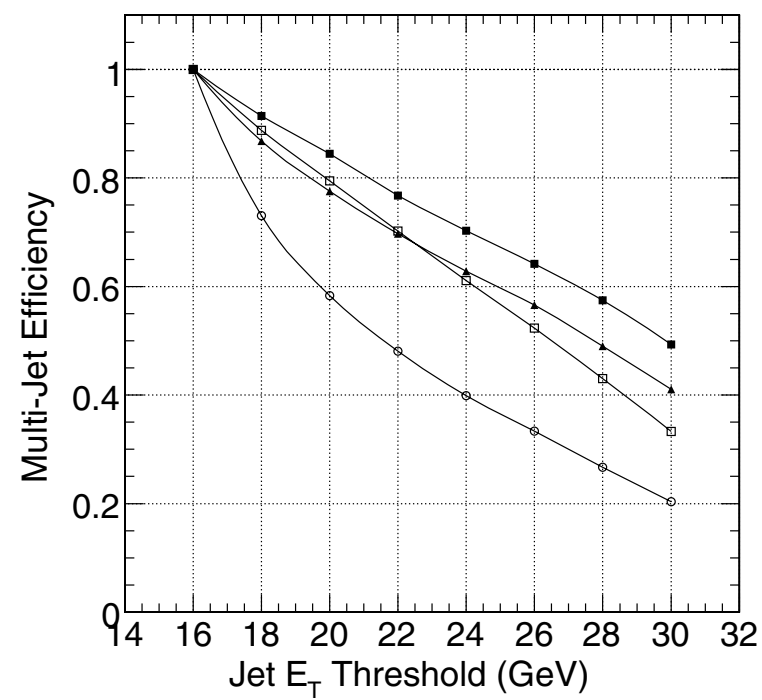

Figure 10.17. Multiple jet selection efficiency (requiring at least 4 jets in an event) as a function of jet $\mathrm{E}_{\mathrm{T}}$ threshold. The efficiency is normalised to the rate with jet $\mathrm{E}_{\mathrm{T}}$ threshold of $16 \mathrm{GeV}$ for each sample. The physics channels include: $\bar{t}+$ jets (solid square), $\mathrm{W}+3$ jets (open circle), $\mathrm{W}+4 \mathrm{jets}$ (solid triangle), and VBF Higgs with $\mathrm{m}_{\mathrm{H}}=170 \mathrm{GeV} / \mathrm{c}^{2}$ (open square).

lepton and multiple jets in the final states; simulating the requisite huge number of background events is both a computing and analysis challenge; hard selection cuts and heavy exploitation of physics signal characteristics are necessary to suppress backgrounds and enhance the statistical significance of the signal, which can lead to large systematic uncertainties; the relatively low Higgs boson mass domain limits the application of high jet $\mathrm{E}_{\mathrm{T}}$ thresholds that would normally be used to suppress backgrounds, in contrast to the situation at high mass; low $\mathrm{E}_{\mathrm{T}}^{\mathrm{miss}}$ and low $\mathrm{E}_{\mathrm{T}}$ jets affect the resolution of Higgs mass. To meet these challenges, a robust reconstruction and selection strategy is developed.

Low $\mathrm{p}_{\mathrm{T}}$ objects are ignored (e.g. leptons with $\mathrm{p}_{\mathrm{T}}<10 \mathrm{GeV} / \mathrm{c}$ and jets with $\mathrm{E}_{\mathrm{T}}<25 \mathrm{GeV}$ ). The jet $\mathrm{E}_{\mathrm{T}}$ threshold is chosen around $25 \mathrm{GeV}$ where there is a stable signal to background ratio $(\mathrm{S} / \mathrm{B})$, so that the systematic uncertainty of jet energy scale is minimised (Fig. 10.17). Due to a number of soft jets in the central detector region, the hadronic $\mathrm{W}$ reconstruction looks for a dijet mass with the smallest deviation from the true $\mathrm{W}$ boson mass. The extra jet veto after forward jet tagging and hadronic $\mathrm{W}$ reconstruction is applied. Two schemes are studied: full extra jet veto $\left(\mathrm{N}_{\text {extra }}<1\right)$ and loose extra jet veto $\left(\mathrm{N}_{\text {extra }}<2\right)$. The full extra jet veto is very powerful in reducing the $\mathrm{t} \overline{\mathrm{t}}+$ jets and $\mathrm{W}+$ jets background.

The selection chain is divided into two major steps: basic selection (Table 10.11) and optimised selection. This strategy helps optimise the selection cuts and factorise the selection efficiency to evaluate the systematic uncertainty and QCD background efficiency.

The optimised selection for $\mathrm{m}_{\mathrm{H}} \geqslant 160 \mathrm{GeV} / \mathrm{c}^{2}\left(\mathrm{~m}_{\mathrm{H}}<160 \mathrm{GeV} / \mathrm{c}^{2}\right)$ includes 3 steps:

- $\mathrm{E}_{\mathrm{T}}^{\mathrm{FH}}>45(40) \mathrm{GeV}, \mathrm{E}_{\mathrm{T}}^{\mathrm{FL}}>35(30) \mathrm{GeV}, \Delta \eta>4.2$, and $\mathrm{m}_{\mathrm{jj}}>1000 \mathrm{GeV} / \mathrm{c}^{2} . \mathrm{E}_{\mathrm{T}}^{\mathrm{FH}}\left(\mathrm{E}_{\mathrm{T}}^{\mathrm{FL}}\right)$ is the high (low) jet $\mathrm{E}_{\mathrm{T}}$ threshold for forward jets.

- $\mathrm{E}_{\mathrm{T}}^{\mathrm{CH}}>30 \mathrm{GeV}, \mathrm{E}_{\mathrm{T}}^{\mathrm{CL}}>25 \mathrm{GeV}, \Delta \mathrm{m}_{\mathrm{W}}<20 \mathrm{GeV} / \mathrm{c}^{2}\left(30<\mathrm{m}_{\mathrm{W}}<90 \mathrm{GeV} / \mathrm{c}^{2}\right)$, and $\mathrm{N}_{\text {extra }}<1$. $\mathrm{E}_{\mathrm{T}}^{\mathrm{CH}}\left(\mathrm{E}_{\mathrm{T}}^{\mathrm{CL}}\right)$ is the high (low) jet $\mathrm{E}_{\mathrm{T}}$ threshold for central jets that are used for hadronic-W reconstruction. 
Table 10.11. Summary of basic event selection cuts.

\begin{tabular}{ll}
\hline Selection & Configuration \\
\hline Lepton selection & calorimeter-based e $/ \mu$ isolation \\
& $30<\mathrm{p}_{\mathrm{T}}<120 \mathrm{GeV} / \mathrm{c}$ \\
& $\Delta \mathrm{R}_{\ell, \mathrm{j}}>0.5$ \\
& $\mathrm{~N}_{\mathrm{jet}} \geqslant 4$ jets with $\mathrm{E}_{\mathrm{T}}>25 \mathrm{GeV}$ \\
& $\mathrm{E}_{\mathrm{T}}^{\text {miss }}>30 \mathrm{GeV}$ \\
& $\mathrm{E}_{\mathrm{T}}>30 \mathrm{GeV}$ \\
& $\eta_{1} \cdot \eta_{2}<0$ \\
Forward jet tagging & $\eta_{1}-\eta_{2} \mid>3.8$ \\
& $\mathrm{~m}_{\mathrm{jj}}>800 \mathrm{GeV} / \mathrm{c}^{2}$ \\
& $\Delta \mathrm{m}_{\mathrm{W}}<25 \mathrm{GeV} / \mathrm{c}^{2}\left(\mathrm{~m}_{\mathrm{H}} \geqslant 160 \mathrm{GeV} / \mathrm{c}^{2}\right)$ \\
& $30<\mathrm{m}_{\mathrm{W}}<90 \mathrm{GeV} / \mathrm{c}^{2}\left(\mathrm{~m}_{\mathrm{H}}<160 \mathrm{GeV} / \mathrm{c}^{2}\right)$ \\
Hadronic-W & select dijet with the least $\Delta \mathrm{m}_{\mathrm{W}}$ \\
& using lepton and $\mathrm{E}_{\mathrm{T}}^{\text {miss }}$ \\
Leptonic-W & select Leptonic-W candidates of \\
& smaller $\Delta \mathrm{R}(\mathrm{Leptonic}-\mathrm{W}, \mathrm{Hadronic}-\mathrm{W})$ \\
\hline
\end{tabular}

Table 10.12. Cross section (fb) of the signal and background in optimised selection with $\mathrm{m}_{\mathrm{H}} \geqslant$ $160 \mathrm{GeV} / \mathrm{c}^{2}$ for full extra jet veto.

\begin{tabular}{lllll}
\hline Channels & Basic Selection & Step 1 & Step 2 & Step 3 \\
\hline VBF Higgs $\left(\mathrm{m}_{\mathrm{H}}=160\right)$ & 16.15 & 9.531 & 4.580 & 2.989 \\
VBF Higgs $\left(\mathrm{m}_{\mathrm{H}}=170\right)$ & 15.99 & 9.814 & 4.828 & 3.006 \\
VBF Higgs $\left(\mathrm{m}_{\mathrm{H}}=180\right)$ & 16.28 & 9.916 & 4.711 & 2.738 \\
VBF Higgs $\left(\mathrm{m}_{\mathrm{H}}=190\right)$ & 14.16 & 9.363 & 4.294 & 2.340 \\
VBF Higgs $\left(\mathrm{m}_{\mathrm{H}}=200\right)$ & 13.78 & 8.626 & 4.341 & 1.983 \\
VBF Higgs $\left(\mathrm{m}_{\mathrm{H}}=210\right)$ & 13.43 & 8.211 & 4.080 & 1.571 \\
VBF Higgs $\left(\mathrm{m}_{\mathrm{H}}=220\right)$ & 13.35 & 8.227 & 4.128 & 1.259 \\
VBF Higgs $\left(\mathrm{m}_{\mathrm{H}}=250\right)$ & 10.71 & 6.900 & 3.426 & 0.810 \\
$\mathrm{tt}+$ jets & 1494.2 & 626.5 & 16.751 & 1.232 \\
$\mathrm{WW}+$ jets $(\mathrm{QCD})$ & 9.27 & 1.265 & 0.422 & $<0.008$ \\
$\mathrm{WW}+$ jets $(\mathrm{EW})$ & 7.88 & 9.683 & 4.454 & $<0.0277$ \\
$\mathrm{ZZ}+$ jets & 1.00 & 0.269 & 0.0245 & $<0.001$ \\
$\mathrm{ZW}+$ jets & 7.23 & 2.335 & 0.223 & $<0.001$ \\
$\mathrm{~W}+\mathrm{tb}(\overline{\mathrm{tb}})$ & 92.8 & 35.21 & 4.427 & $<0.05787$ \\
$\mathrm{~W}+4 \mathrm{j}(\mathrm{W} \rightarrow \mathrm{e} / \mu / \tau+v)$ & 1110.8 & 583.0 & 72.066 & 0.323 \\
$\mathrm{Z}+4 \mathrm{j}(\mathrm{Z} \rightarrow \mathrm{ee} / \mu \mu)$ & 82.3 & 3.713 & 0.141 & 0.0104 \\
$\mathrm{Z}+3 \mathrm{j}(\mathrm{Z} \rightarrow \mathrm{ee} / \mu \mu)$ & 72.4 & 2.313 & 0.233 & $<0.0067$ \\
$\mathrm{Sum}$ of Background & 3579.7 & 1492.5 & 167.38 & 1.565 \\
\hline
\end{tabular}

- $\mathrm{E}_{\mathrm{T}}^{\text {miss }}(\mathrm{qqWW})<40 \mathrm{GeV}, \Delta \mathrm{R}$ (lepton,Hadronic-W) $<2.0$, and $\Delta \mathrm{R}$ (Leptonic-W, Hadronic$\mathrm{W})<1.0 . \mathrm{E}_{\mathrm{T}}^{\text {miss }}(\mathrm{qqWW})$ is the $\mathrm{E}_{\mathrm{T}}^{\text {miss }}$ of $\mathrm{qqWW}$ system that includes reconstructed Higgs boson and two forward jets.

The efficiency of basic selection and three steps of optimised selection is summarised in Table 10.12 and 10.13 for $\mathrm{m}_{\mathrm{H}} \geqslant 160 \mathrm{GeV} / \mathrm{c}^{2}$ and $\mathrm{m}_{\mathrm{H}}<160 \mathrm{GeV} / \mathrm{c}^{2}$ respectively. Loose extra jet veto with tightening cuts: $\mathrm{m}_{\mathrm{jj}}>1200 \mathrm{GeV} / \mathrm{c}^{2}$ and $\Delta \mathrm{R}$ (lepton,Hadronic-W) $<1.6$, gives a conservative result.

The reconstructed Higgs boson mass distributions for signal plus background and background are shown in Fig. 10.18 for $\mathrm{M}_{\mathrm{H}}=160 \mathrm{GeV} / \mathrm{c}^{2}$ (left) and $\mathrm{M}_{\mathrm{H}}=170 \mathrm{GeV} / \mathrm{c}^{2}$ (right) for $60 \mathrm{fb}^{-1}$. 
Table 10.13. Cross section ( $\mathrm{fb}$ ) of signal and background in optimised selection with $\mathrm{m}_{\mathrm{H}}<160 \mathrm{GeV} / \mathrm{c}^{2}$ for full extra jet veto.

\begin{tabular}{lllll}
\hline Channels & Basic Selection & Step 1 & Step 2 & Step 3 \\
\hline VBF Higgs $\left(\mathrm{m}_{\mathrm{H}}=120\right)$ & 1.28 & 0.951 & 0.363 & 0.231 \\
VBF Higgs $\left(\mathrm{m}_{\mathrm{H}}=130\right)$ & 4.03 & 3.004 & 1.125 & 0.664 \\
VBF Higgs $\left(\mathrm{m}_{\mathrm{H}}=140\right)$ & 7.12 & 5.520 & 2.369 & 1.656 \\
VBF Higgs $\left(\mathrm{m}_{\mathrm{H}}=150\right)$ & 11.01 & 8.345 & 3.505 & 2.317 \\
$\mathrm{t}+$ jets & 1483.0 & 859.5 & 20.94 & 0.493 \\
$\mathrm{WW}+$ jets $(\mathrm{QCD})$ & 9.70 & 4.215 & 0.422 & $<0.004$ \\
$\mathrm{WW}+$ jets $(\mathrm{EW})$ & 7.94 & 11.21 & 5.395 & $<0.0277$ \\
$\mathrm{ZZ}+$ jets & 0.96 & 0.465 & 0.0979 & $<0.001$ \\
$\mathrm{ZW}+$ jets & 7.45 & 3.781 & 0.334 & $<0.01$ \\
$\mathrm{~W}+\mathrm{tb}(\overline{\mathrm{tb}})$ & 101.5 & 54.37 & 6.799 & $<0.0289$ \\
$\mathrm{~W}+4 \mathrm{j}(\mathrm{W} \rightarrow \mathrm{e} / \mu / \tau+v)$ & 1110.7 & 778.5 & 118.9 & 0.667 \\
$\mathrm{Z}+4 \mathrm{j}(\mathrm{Z} \rightarrow \mathrm{ee} / \mu \mu)$ & 81.3 & 4.700 & 0.152 & 0.00522 \\
$\mathrm{Z}+3 \mathrm{j}(\mathrm{Z} \rightarrow \mathrm{ee} / \mu \mu)$ & 70.0 & 3.160 & 0.353 & $<0.01333$ \\
Sum of Background & 3630.6 & 2066.5 & 267.2 & 1.164 \\
\hline
\end{tabular}
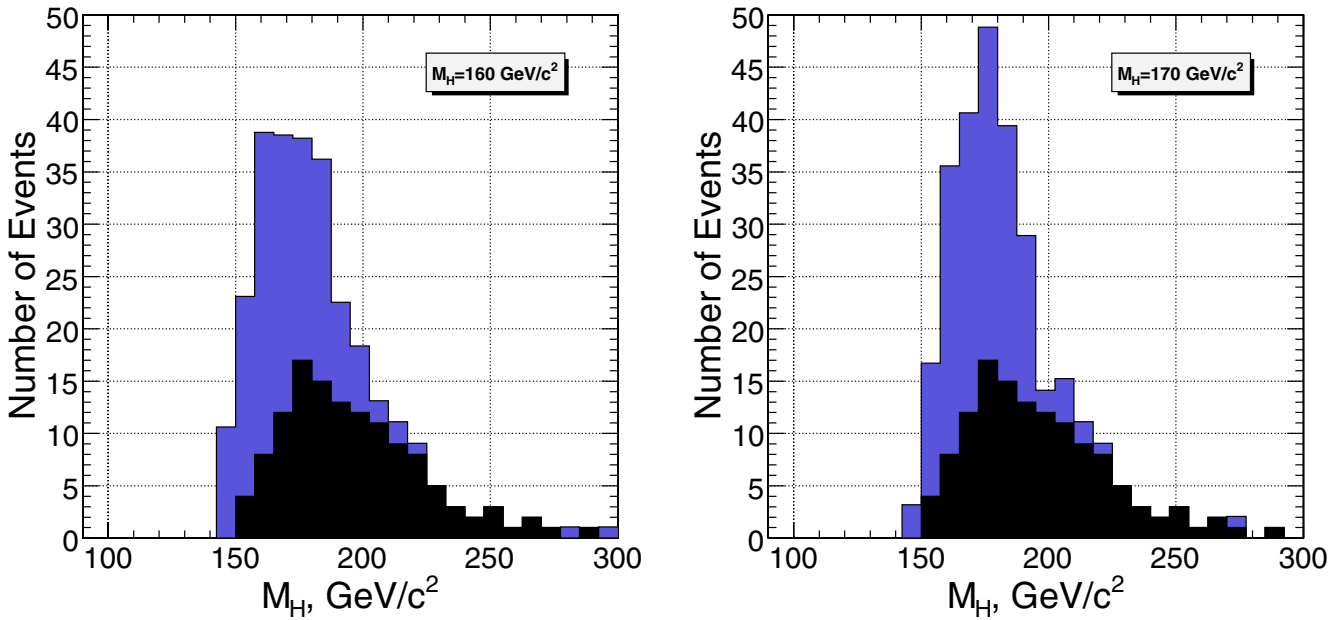

Figure 10.18. The Higgs boson mass reconstruction of signal plus background (blue/grey) and background (black) for $\mathrm{M}_{\mathrm{H}}=160 \mathrm{GeV} / \mathrm{c}^{2}$ (left) and $\mathrm{M}_{\mathrm{H}}=170 \mathrm{GeV} / \mathrm{c}^{2}$ (right).

The overall QCD multi-jet contamination is estimated with the factorisation of the selections as 2-5 events for an upper limit with $60 \mathrm{fb}^{-1}$, which causes possible 2-4\% increase of background, which has almost no change in the significance.

10.2.4.2. Detector systematic uncertainties and control. Several calorimeter level systematic uncertainties have significant impact on this channel including: jet energy scale and resolution, $\mathrm{E}_{\mathrm{T}}^{\mathrm{miss}}$ scale and resolution, and calorimeter-based lepton isolation cut. Their impacts on the rate of signal (S), background (B) and S/B are summarised in Table. 10.14. The total detector level systematic uncertainty is about $16 \%$ in the absolute rate of background in the final result. 
Table 10.14. Systematic Uncertainties due to Jet and $\mathrm{E}_{\mathrm{T}}^{\mathrm{miss}}$

\begin{tabular}{lccc}
\hline Source & $\mathrm{S}$ & $\mathrm{B}$ & $\mathrm{S} / \mathrm{B}$ \\
\hline Jet energy scale & $10.6 \%$ & $14.5 \%$ & $5.2 \%$ \\
Jet energy resolution & $0.1 \%$ & $2.0 \%$ & $2.0 \%$ \\
$\mathrm{E}_{\mathrm{T}}^{\text {miss }}$ & $2.5 \%$ & $1.2 \%$ & $1.7 \%$ \\
Lepton isolation & $1.4 \%$ & $1.3 \%$ & $0.5 \%$ \\
\hline
\end{tabular}

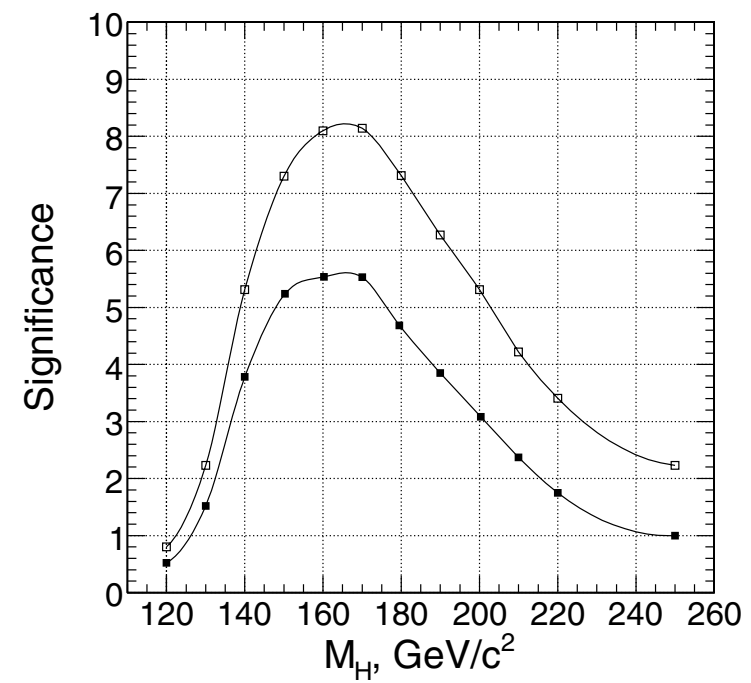

Figure 10.19. The signal significance for $30 \mathrm{fb}^{-1}$. The high (low) curves correspond to full (loose) extra jet veto.

The data driven technique is able to significantly reduce the detector level systematic uncertainties. For example, the largest uncertainty comes from the selection efficiency with respect to lowest jet $\mathrm{E}_{\mathrm{T}}$ threshold. The event rate of the background near this threshold can be measured from data and used to tune the MC prediction, which leaves much less uncertainty due to the systematic bias of jet energy scale. Ignoring the uncertainty in the rate for from lowest jet $\mathrm{E}_{\mathrm{T}}$ threshold, the uncertainty of jet energy scale only causes about $5.5 \%$ error in the rest of the selection chain which immediately reduces the total detector level systematic uncertainty down to $10 \%$ level.

10.2.4.3. Discovery potential. The signal significance for $30 \mathrm{fb}^{-1}$ after optimised selection cuts is shown in Fig. 10.19 for the Higgs boson masses between 120 and $250 \mathrm{GeV} / \mathrm{c}^{2}$. The background systematic uncertainty of $16 \%$ as discussed in the previous section is included.

\subsubsection{Vector boson fusion production with $\mathrm{H} \rightarrow \gamma \gamma$}

A detailed description of the analysis can be found in [483].

10.2.5.1. Signal and background generation and simulation. The Higgs boson production from the vector boson fusion $\mathrm{qq} \rightarrow \mathrm{qqH}$ and $\mathrm{H} \rightarrow \gamma \gamma$ decay was generated by PYTHIA for the Higgs boson masses, $\mathrm{M}_{\mathrm{H}}=115,120,130,140$ and $150 \mathrm{GeV} / \mathrm{c}^{2}$. 
Table 10.15. Cross sections of different types of background.

\begin{tabular}{ll}
\hline Background process & Cross section $(\mathrm{pb})$ \\
\hline QCD hadronic jets & $2.8^{*} 10^{7}$ \\
Gluon fusion & 83 \\
Drell-Yan & $4.1 \times 10^{3}$ \\
$\gamma \gamma+2$ jets, QCD & 47.24 \\
$\gamma \gamma+2$ jets, EW & 0.33 \\
$\gamma+3$ jets, QCD & 5970 \\
$\gamma+3$ jets, EW & 5.15 \\
\hline
\end{tabular}

The backgrounds considered are:

- QCD multi-jet production, where an electromagnetic energy deposit results from the decay of neutral hadrons (especially isolated $\pi^{0} \mathrm{~s}$ ) in a jet. It was generated by PYTHIA with $\hat{\mathrm{p}_{\mathrm{T}}}>50 \mathrm{GeV} / \mathrm{c}$.

- Drell-Yan $e^{-} e^{+}$production (generated with PYTHIA) which could mimic photons when correspondent electron tracks will not be assigned to the clusters in the ECAL during the reconstruction.

- Diphoton production from the gluon fusion (box diagram) when two additional jets from the initial state radiation are presented in the event. It was generated by PYTHIA with $\hat{\mathrm{p}_{\mathrm{T}}}>20 \mathrm{GeV} / \mathrm{c}$.

- QCD and Electro Weak (EW) pp $\rightarrow \gamma \gamma+2$ jets process generated with ComPHEP.

- QCD and EW pp $\rightarrow \gamma+3$ jets generated with CoMPHEP.

Table 10.15 shows the cross sections of different types of backgrounds.

Generator level pre-selections for QCD multi-jet background. Selection based on the generated particles was devised, aimed at selecting events which could produce in the detector two electromagnetic showers consistent with isolated photons. In order to apply cuts on the invariant mass of the two candidates an attempt to estimate lower and upper limits to the energy of the candidates that will be reconstructed after the simulation was done.

The idea of this pre-selection, is to pick up events that will give rise to energy depositions in ECAL large enough and isolated enough to be important for this analysis. Pre-selection algorithm is getting all particles which might deposit electromagnetic energy in ECAL, and looking around each particle in a narrow cone, to find another, may be less energetic particles which will make deposits in ECAL as well, and will potentially be reconstructed as one cluster. In addition to that, a very loose tracker isolation was applied: three charged particles were required in a cone $\Delta R=0.2$ around the "cluster candidate", described above, per one "cluster candidate", and no more than 6 per two first most energetic candidates.

After that some other cuts were applied for the "cluster candidates" as well, $p_{\mathrm{T}}>$ $37.5 \mathrm{GeV} / \mathrm{c}$ for most energetic one and $p_{\mathrm{T}}>22.5 \mathrm{GeV} / \mathrm{c}$ for the second most energetic one. The invariant mass of the first most energetic and second most energetic "cluster candidates" should be more than $90 \mathrm{GeV} / \mathrm{c}^{2}$ for the purpose of this analysis.

Generator level pre-selections for $\gamma+3$ jets and $\gamma \gamma+2$ jets backgrounds. At COMPHEP partonic level event generation the following cuts were applied:

- $p_{\mathrm{T}}^{\gamma}>20 \mathrm{GeV} / \mathrm{c}$

- $p_{\mathrm{T}}^{j}>20 \mathrm{GeV} / \mathrm{c}$

- $\Delta R_{i j}>0.4$

- at least one pair of jets must exist with the jets in the opposite hemispheres with the rapidity gap greater than 3.5 . 
Table 10.16. Number of generated and simulated events for different types of background.

\begin{tabular}{lcccc}
\hline $\begin{array}{l}\text { Background } \\
\text { process }\end{array}$ & $\begin{array}{c}\text { Number of } \\
\text { generated events }\end{array}$ & $\begin{array}{c}\text { Rejection with } \\
\text { pre-selections }\end{array}$ & $\begin{array}{c}\text { Number of } \\
\text { simulated events }\end{array}$ & $\begin{array}{c}\mathrm{L}_{\text {intg }} \\
\left(\mathrm{fb}^{-1}\right)\end{array}$ \\
\hline QCD multi-jets & $31.2 \times 10^{9}$ & 6048 & $4.5 \mathrm{M}$ & $\sim 1$ \\
Gluon fusion $^{2}$ & $2.25 \times 10^{6}$ & 2 & $1 \mathrm{M}$ & $\sim 52$ \\
Drell-Yan e $^{+} \mathrm{e}^{-}$ & $1.0 \times 10^{6}$ & 1 & $1 \mathrm{M}$ & 0.25 \\
$\gamma \gamma+2$ jets, QCD & $0.5 \times 10^{6}$ & 2.56 & $200 \mathrm{k}$ & 6 \\
$\gamma \gamma+2$ jets, EW & $41 \times 10^{3}$ & 1 & $41 \mathrm{k}$ & 120 \\
$\gamma+3$ jets, QCD & $0.3 \times 10^{6}$ & 7.8 & $40 \mathrm{k}$ & 0.05 \\
\hline
\end{tabular}

The CTEQ5L PDF set was used; the factorisation and renormalisation scales were set to $50 \mathrm{GeV}$. Hadronisation was done by PYTHIA and the same pre-selections were applied as it was described above for QCD multi-jet background. Rejection factors of PYTHIA pre-selections are 2.5 for $\gamma \gamma+2$ jets dataset and 7.8 for $\gamma+3$ jets dataset.

The signal and background events passed the full detector simulation and digitisation with pile-up for luminosity $2 \times 10^{33} \mathrm{~cm}^{-2} \mathrm{~s}^{-1}$. The numbers of generated and fully simulated events are shown in Table 10.16 for different types of background. In the last column the corresponding equivalent integrated luminosity is shown.

10.2.5.2. Event reconstruction and selection. The events were triggered by the doubleisolated electron trigger at Level 1 and HLT.

Photons are reconstructed with the hybrid algorithm in the ECAL barrel and with the island algorithm in the ECAL endcap. Both photon candidates had to match Level 1 trigger photon candidates, such that, the distance $\mathrm{R}\left(\mathrm{R}=\sqrt{\delta \eta^{2}+\delta \phi^{2}}\right)$ between the photon candidate and trigger object be less than 0.5 . The transverse energies of the two photon candidates were required to be greater than $40 \mathrm{GeV}$ and $25 \mathrm{GeV}$ respectively. The fiducial volume in rapidity was restricted to $|\eta|<1.4442$ in the barrel and $1.566<|\eta|<2.5$ in the endcap for both photon candidates.

Three different algorithms were studied for the Higgs boson vertex reconstruction:

- $\mathbf{P}_{\mathrm{T}}$ balance. The $\mathrm{P}_{\mathrm{T}}$ balance for charged particle tracks along the reconstructed Higgs boson direction is defined as $\mathrm{P}_{\mathrm{T}}^{B}=-\Sigma \mathrm{P}_{\mathrm{Ti}} \cos \theta_{i}$, where $\theta_{i}$ is the angle between the Higgs boson and track $i$ direction in the transverse plane

- Maximal $\mathbf{P}_{\mathrm{T}}$. The primary vertex is selected as the vertex with the track of highest $\mathrm{P}_{\mathrm{T}}$

- Number of charged particle tracks above $\mathrm{P}_{\mathrm{T}}$ cutoff in pixel vertex. The primary vertex is selected as the vertex with a largest number of tracks.

To compare different vertex reconstruction algorithms, the number of events reconstructed in a $5 \mathrm{GeV} / \mathrm{c}^{2}$ mass window are determined. The $\mathrm{P}_{\mathrm{T}}$ balance and Maximal $\mathrm{P}_{\mathrm{T}}$ algorithms give exactly the same number of events, while track counting algorithm gives a few percent less efficiency. The Higgs boson efficiency in $5 \mathrm{GeV} / \mathrm{c}^{2}$ mass window is improved by $15 \%$.

The photon candidates were required to be isolated in the tracker and calorimeter. The tracker isolation criteria are based on the number of charged particle tracks with $\mathrm{p}_{\mathrm{T}}$ greater than a $\mathrm{p}_{\mathrm{T}}$ threshold, $\mathrm{p}_{\mathrm{T}}^{\text {thresh }}$, calculated in a cone $\mathrm{R}\left(\mathrm{R}=\sqrt{\delta \eta^{2}+\delta \phi^{2}}\right)$ around the photon 
candidate. The algorithm contains three parameters:

- The size of the cone $\mathrm{R}$ around the photon candidate, wherein the number of charged tracks is counted.

- The $\mathrm{p}_{\mathrm{T}}$ threshold, $\mathrm{p}_{\mathrm{T}}^{\text {thresh }}$. Only charged particle tracks with $\mathrm{p}_{\mathrm{T}}$ greater than $\mathrm{p}_{\mathrm{T}}^{\text {thresh }}$ are considered in isolation calculations.

- The 'number of tracks' threshold $\mathrm{N}^{\text {thresh }}$. If the number of charged particle tracks in cone $\mathrm{R}$ with $\mathrm{p}_{\mathrm{T}}$ greater than the chosen $\mathrm{p}_{\mathrm{T}}^{\text {thresh }}$ is greater than $\mathrm{N}^{\text {thresh }}$, then the photon candidate is considered non-isolated, otherwise isolated.

The jet rejection factor is very sensitive to the 'number of tracks' threshold, $\mathrm{N}^{\text {thresh }}$. By increasing $\mathrm{N}^{\text {thresh }}$ from 0 to 1 , the Higgs boson signal efficiency is improved by $6-10 \%$, but the jet rejection factor drops by a factor of $\sim 2$. Therefore, the parameter $\mathrm{N}^{\text {thresh }}$ was fixed to zero. The cone size $\mathrm{R}=0.30$ and $\mathrm{p}_{\mathrm{T}}^{\text {thresh }}=1.5 \mathrm{GeV} / \mathrm{c}$ were used in this study.

The isolation of the photon candidates in the electromagnetic calorimeter is also required. The isolation criteria is based on the sum of transverse energies deposited in basic clusters in some cone $\mathrm{R}\left(\mathrm{R}=\sqrt{\delta \eta^{2}+\delta \phi^{2}}\right)$ around the photon candidate. The basic clusters that belong to the photon candidate's supercluster are not counted as part of the sum. The algorithm contains four parameters:

- The size of the cone $\mathrm{R}$ around the photon candidate wherein the transverse energies deposited in the basic clusters are summed.

- The transverse energy sum threshold $\mathrm{E}_{\mathrm{T}}^{\text {thresh }}$. If the sum of transverse energies is below this threshold, the photon candidate is considered isolated, otherwise non-isolated.

- The ratio, $r$, of the transverse energy sum in all surrounded basic clusters to the transverse energy of the most energetic super cluster.

- The ratio $(\mathrm{H} / \mathrm{E})$ of the energy deposited in the HCAL behind the super-cluster to the energy of the super-cluster.

There is no strong dependence of the jet rejection factor on the cone size $\mathrm{R}$, though slightly better rejection factors are empirically obtained for a cone size $R=0.30-0.35$. The cone size $R=0.30$ is used in this study. The transverse energy sum thresholds, $E_{T}^{\text {thresh }}$, were chosen to be $1.2 \mathrm{GeV}$ in the barrel and $1.6 \mathrm{GeV}$ in the endcap. Finally, the photon candidate must pass the cuts: $r<0.01$ and $\mathrm{H} / \mathrm{E}<0.1$.

Jet tagging was done based on the jets reconstructed with the iterative cone algorithm using cone size 0.7. The two highest $\mathrm{E}_{\mathrm{T}}$ jets were chosen and initial selection cuts were applied:

- $\mathrm{E}_{\mathrm{T}}^{\mathrm{jet}}>20 \mathrm{GeV},\left|\eta_{j e t}\right| \leqslant 4.5, \Delta R_{\gamma j e t} \geqslant 0.5$

- $\Delta \eta_{\text {jets }}=\left|\eta_{\text {jet } 1}-\eta_{\text {jet } 2}\right| \geqslant 4.0, \eta_{\text {jet } 1} \times \eta_{\text {jet } 2}<0$

Two additional cuts were applied to the already selected two forward jets in order to reduce the background even more than it was done with forward jet tagging procedure:

- $\mathrm{E}_{\mathrm{T}}^{\text {jet1 }}>50 \mathrm{GeV}$, where $\mathrm{E}_{\mathrm{T}}^{\mathrm{jet} 1}$ is the transverse momentum of the first most energetic forward jet, selected by forward jet tagging procedure, described above.

- $\mathrm{E}_{\mathrm{T}}^{\mathrm{jet} 2}>35 \mathrm{GeV}$, where $p_{t}^{\text {jet } 2}$ is the transverse momentum of the second most energetic forward jet, selected by forward jet tagging procedure, described above.

- $\mathrm{m}_{\mathrm{j} 1 \mathrm{j} 2}>500 \mathrm{GeV} / \mathrm{c}^{2}$, where $\mathrm{m}_{\mathrm{j} 1 \mathrm{j} 2}$ is the invariant mass of the two most energetic forward jets, selected by forward jet tagging procedure, described above.

- Two photons must in the $\eta$ region between the two forward jets: $\min \left(\eta_{\text {jet1 }}, \eta_{\text {jet2 }}\right)+0.7<$ $\eta_{\gamma 1,2}<\max \left(\eta_{\text {jet } 1}, \eta_{\text {jet } 2}\right)-0.7$. 
Table 10.17. The number of signal and background events and signal significance after all selections within the $5 \mathrm{GeV} / \mathrm{c}^{2}$ mass window around the considered Higgs boson masses for $60 \mathrm{fb}^{-1}$. The $\Delta \mathrm{N}_{\mathrm{b}}$ is the background uncertainty estimated from the side bands.

\begin{tabular}{llllll}
\hline & $\begin{array}{l}m_{H}=115 \\
\mathrm{GeV} / \mathrm{c}^{2}\end{array}$ & $\begin{array}{l}m_{H}=120 \\
\mathrm{GeV} / \mathrm{c}^{2}\end{array}$ & $\begin{array}{l}m_{H}=130 \\
\mathrm{GeV} / \mathrm{c}^{2}\end{array}$ & $\begin{array}{l}m_{H}=140 \\
\mathrm{GeV} / \mathrm{c}^{2}\end{array}$ & $\begin{array}{l}m_{H}=150 \\
\mathrm{GeV} / \mathrm{c}^{2}\end{array}$ \\
\hline$N_{s}$ & 20.2 & 21.1 & 19.1 & 15.7 & 11.2 \\
$\gamma+3$ jets (QCD) & 2.7 & 4.7 & 3.5 & 2.0 & 5.8 \\
$\gamma+3$ jets (EW) & 2.5 & 2.5 & 2.5 & 2.5 & 2.5 \\
$\gamma \gamma+2$ jets (QCD) & 11.2 & 13.2 & 9.85 & 8.9 & 4.6 \\
$\gamma \gamma+2$ jets (EW) & 10 & 7.0 & 7.0 & 11.0 & 2.0 \\
Drell-Yan & 0 & 0 & 0 & 0 & 0 \\
$\mathrm{~N}_{\mathrm{b}}$ & 26.0 & 26.2 & 21.4 & 28.2 & 14.9 \\
$\Delta \mathrm{N}_{\mathrm{b}}$ & 2.8 & 3.2 & 2.4 & 3.0 & 1.8 \\
$S$ & 3.07 & 3.15 & 3.21 & 2.32 & 2.30 \\
\hline
\end{tabular}

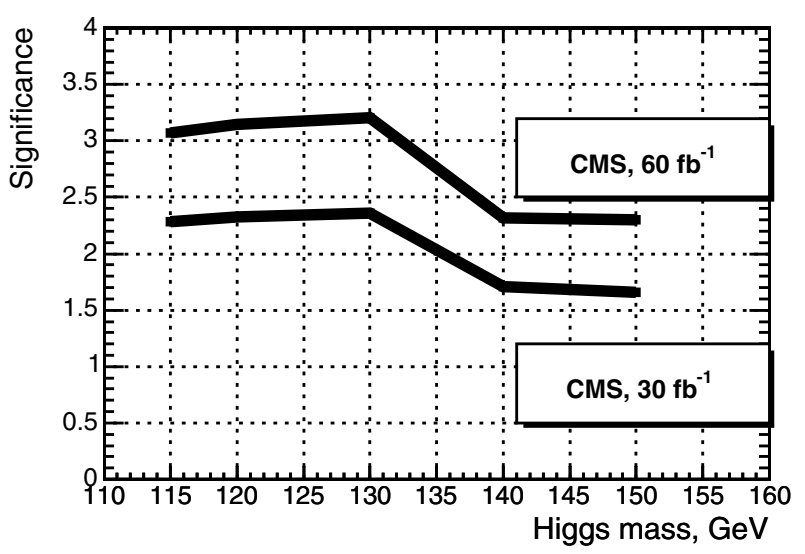

Figure 10.20. Signal significance for 30 and $60 \mathrm{fb}^{-1}$.

10.2.5.3. Results. After all selections the contribution of the QCD multi-jet events and diphoton events from gluon fusion was found to be negligible. Due to the lack of Monte Carlo statistics only upper limits were estimated conservatively for the contribution from QCD and EW $\gamma+3$ jets backgrounds. Table 10.17 shows the number of signal and background events after all selections within $5 \mathrm{GeV} / \mathrm{c}^{2}$ mass window around the considered Higgs boson masses for $60 \mathrm{fb}^{-1}$. The $\Delta \mathrm{N}_{\mathrm{b}}$ shown in the Table is the background uncertainty estimated from the side bands around the Higgs boson mass peak.

The signal significance with the background uncertainty taken into account is shown in Fig. 10.20 for 30 and $60 \mathrm{fb}^{-1}$.

\subsubsection{Associated $\mathrm{WH}$ production with $\mathrm{H} \rightarrow \mathrm{WW}^{(*)} \rightarrow 2 \ell 2 v$}

The cross-section for this process exhibits a maximum near the Higgs boson mass of $160-180 \mathrm{GeV} / \mathrm{c}^{2}$ due to the combined behaviour of the production cross-section and the Higgs boson branching ratio. The intermediate mass region between $120 \mathrm{GeV} / \mathrm{c}^{2}$ and $190 \mathrm{GeV} / \mathrm{c}^{2}$, 
Table 10.18. Background processes considered into the present analysis. The cross-section includes the decay of $\mathrm{W}$ and $\mathrm{Z}$ bosons into leptons. The generator and the number of events processed are also shown together with the corresponding weight for a luminosity of $1 \mathrm{fb}^{-1}$.

\begin{tabular}{lllll}
\hline Background & Cross-section & Generator & MC statistic & weight $\left(1 \mathrm{fb}^{-1}\right)$ \\
\hline $\mathrm{WWW}\left(31^{ \pm}\right)$ & $4.95 \mathrm{fb}$ & COMPHEP & 10000 & $5.19 \times 10^{-4}$ \\
$\mathrm{WZ}\left(3 l^{ \pm}\right)$ & $1.71 \mathrm{pb}$ & PYTHIA & 50000 & $3.46 \times 10^{-2}$ \\
$\mathrm{ZZ}\left(4 l^{ \pm}\right)$ & $0.17 \mathrm{pb}$ & PYTHIA & 50000 & $3.67 \times 10^{-3}$ \\
$\mathrm{t} \mathrm{t}\left(l^{+} l^{-} \mathrm{b} \overline{\mathrm{b}}\right)$ & $90.9 \mathrm{pb}$ & TOPREX & 100000 & 0.93 \\
$\mathrm{Wt}\left(1^{+} 1^{-} \mathrm{b}\right)$ & $5.25 \mathrm{pb}$ & TOPREX & 50000 & 0.11 \\
\hline
\end{tabular}

where the cross-section exceeds $300 \mathrm{fb}$ was investigated using the events containing three leptons, electrons and muons (including leptonic tau decays), in the final state.

A detailed description of the analysis can be found in [484].

10.2.6.1. Signal and background generation. The Higgs boson with masses of 115, 125, 130, 140, 150, 160, 170, 180 and $190 \mathrm{GeV} / \mathrm{c}^{2}$ has been considered. Events were generated with PYTHIA for each of the nine Higgs boson masses, without any kinematical cut. W bosons are forced to decay leptonically $(e, \mu, \tau)$.

All Standard-Model processes likely to produce three leptons must be considered as background for this analysis. This includes events where three leptons are actually produced in the hard process but also events with a fake or missed lepton. One particular case is the production of leptons in the semi-leptonic decay of a B meson. In the present analysis, we considered the production of WWW, WZ, ZZ, $\mathrm{t}$, and Wt. Most of the processes are simulated with PYTHIA, except for WWW, which is generated with COMPHEP, and Wt generated with TOPREX. In all cases, PYTHIA is used for the hadronisation step. Table 10.18 shows the crosssection, the generator used and the number of events produced.

10.2.6.2. Selection streams at Level-1 and HLT. The global (cumulative) trigger efficiency after Level-1 and HLT is found to reach $72 \%$ for a $140 \mathrm{GeV} / \mathrm{c}^{2} \mathrm{Higgs}$ boson using the full trigger table. Main contributions come from single and double leptonic (e and $\mu$ ) triggers $(65 \%)$. There is a small contribution from the missing transverse energy trigger $\left(E_{\mathrm{T}}^{\mathrm{miss}}\right)$ and from combined ( $e$ and $\tau$ ) and ( $\mu$ and $\tau$ ) triggers, further reduced by the event selection, which favours multi-leptonic patterns. For this analysis, events are selected by the triggers known to have the highest impact on the total efficiency: single- and double-electron and muon triggers. Figure 10.21(a) shows the efficiency for each (exclusive) trigger pattern, given the above choice of interesting bits.

Details about the efficiency for each type of event (defined from the number of muons, electrons and taus in the event) are given in Fig. 10.21(b). Events containing one or more muons are more easily retained (efficiency reaches $85 \%$ for events with three muons) while tau events are only marginally selected (efficiency: 12\%). Efficiency rises slightly with the Higgs boson mass, from $58 \%$ at $115 \mathrm{GeV} / \mathrm{c}^{2}$ to $74 \%$ at $190 \mathrm{GeV} / \mathrm{c}^{2}$.

Table 10.19 shows the trigger efficiency for each source of background. Efficiency of the single- and double-electron and muon triggers, varies from $64 \%$ to $73 \%$, which is the same magnitude as the trigger efficiency for signal events. It is $15 \%$ (for $t \bar{t}$ ) to $5 \%$ (for $\mathrm{ZZ}$ ) less efficient than the inclusive High-Level trigger.

10.2.6.3. Off-line selection. Electrons and muons are reconstructed using default offline reconstruction algorithms. For electrons, additional quality cuts are applied: the energy 


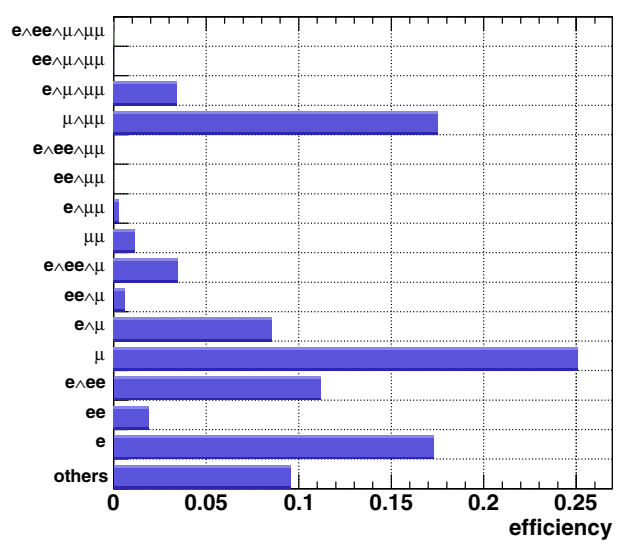

(a)

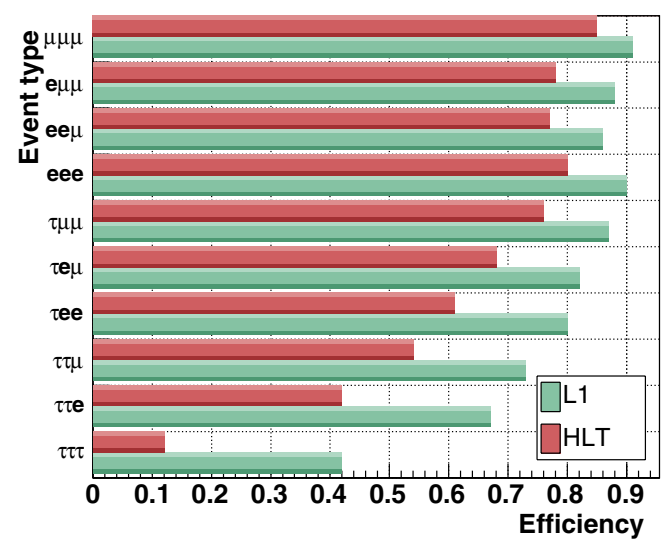

(b)

Figure 10.21. (a) Trigger efficiency by trigger pattern, for the signal. Efficiency is calculated as $N_{x} / N_{H L T}$, where $x$ is one of the $2^{4}$ exclusive trigger classes. "Others" stands for unconsidered trigger patterns; (b) Trigger efficiency for each class of Monte-Carlo events. Results are given after Level-1 and after HLT. Efficiency is computed as the ratio between the number of triggered events and the total number of generated events.

Table 10.19. Trigger efficiency for each source of background. Efficiency at HLT with the restricted trigger set $(e, e e, \mu, \mu \mu)$ used in the present analysis is also shown.

\begin{tabular}{lllc}
\hline Background & Level-1 efficiency & HLT efficiency & $e, e e, \mu, \mu \mu$ HLT efficiency \\
\hline $\mathrm{WWW}\left(31^{ \pm}\right)$ & 0.87 & 0.79 & 0.73 \\
$\mathrm{Wt}\left(1^{+} 1^{-} \mathrm{b}\right)$ & 0.88 & 0.78 & 0.67 \\
$\mathrm{WZ}\left(3 l^{ \pm}\right)$ & 0.8 & 0.72 & 0.65 \\
$\mathrm{ZZ}\left(4 l^{ \pm}\right)$ & 0.78 & 0.69 & 0.64 \\
$\mathrm{t}\left(l^{+} l^{-} \mathrm{b} \overline{\mathrm{b}}\right)$ & 0.91 & 0.79 & 0.65 \\
\hline
\end{tabular}

measured by ECAL and the momentum obtained by the tracker must agree within $50 \%$, and the ratio of energy measured by HCAL and ECAL must be lower than 0.15. Only leptons with $\mathrm{p}_{\mathrm{T}}^{\ell}>14 \mathrm{GeV} / \mathrm{c}$ are retained. A first set of selection criteria is applied to select signallike topologies, requiring three and only three leptons, for a total charge of either +1 or -1 . A cut on the distance in the $\mathrm{z}$ direction between the points of closest approach of lepton tracks to the beam is applied to ensure that all of the three leptons are coming from the same interaction. The two closest (in the $\eta-\phi$ plane) opposite-sign leptons are then assigned to the Higgs boson decay. The angle between leptons attributed to the Higgs boson can be used to distinguish signal and background. The acollinearity between two leptons is defined as the angle between the two leptons, in the space, and their acoplanarity is defined as the same angle projected onto the transverse plane. Both the acollinearity $\left(\theta_{\text {aco }}<1.75 \mathrm{rad}\right)$ and the acoplanarity $\left(0.1 \mathrm{rad}<\phi_{\text {aco }}<0.75 \mathrm{rad}\right)$ between the leptons are used, as they provide complementary information. Leptons required to be isolated in the tracker, i.e. the angle between the lepton's track and the closest track with $\mathrm{p}_{\mathrm{T}}$ above $3 \mathrm{GeV} / \mathrm{c}$ must be more than 0.2 .

A jet veto is applied rejecting events with a jet, reconstructed with the iterative cone algorithm (using cone size of 0.7 ) with raw $\mathrm{E}_{\mathrm{T}}$ above $25 \mathrm{GeV}$ in the central region, $|\eta|<2.1$. An additional $\mathrm{B}$ veto is applied, imposing that no single B-jet is reconstructed by the default combined B-tag algorithm. This removes low-energy $\mathrm{b}$ jets passing the jet veto. 
Table 10.20. Summary of the optimised selection cuts. The cross-section for the signal and backgrounds, for each step in the selection, is given in fb. An upper limit for the $W t$ and $t \bar{t}$ crosssections is given when no simulated event remains.

\begin{tabular}{|c|c|c|c|c|c|c|c|}
\hline \multicolumn{2}{|c|}{ Cut } & \multirow{2}{*}{$\begin{array}{l}\text { Signal (fb) } \\
140 \mathrm{GeV} / \mathrm{c}^{2}\end{array}$} & \multicolumn{5}{|c|}{ Background (fb) } \\
\hline Id. & Type & & $t \bar{t}$ & Wt & ZW & $\mathrm{ZZ}$ & WWW \\
\hline 0 & Level-1 and HLT & 12.24 & 72067 & 4115.8 & 1238.4 & 118.438 & 3.91 \\
\hline 1 & $N_{\text {lept }}=3, \Sigma \mathrm{Q}_{\ell}= \pm 1$ & 3.81 & 16432.7 & 680.0 & 339.4 & 34.65 & 1.05 \\
\hline 2 & Lepton cuts & 2.67 & 5629.1 & 245.3 & 245.9 & 23.53 & 0.70 \\
\hline 3 & Angular cuts & 0.87 & 400.6 & 15.0 & 18.3 & 2.29 & 0.11 \\
\hline 4 & $\mathrm{~B}$ veto & 0.43 & 3.85 & 0.42 & 9.77 & 1.19 & 0.06 \\
\hline 5 & Jet veto & 0.27 & $<1.93$ & 0.31 & 7.26 & 0.58 & 0.04 \\
\hline 6 & $\mathrm{Z}$ veto & 0.21 & $<1.93$ & 0.21 & 0.40 & 0.08 & 0.03 \\
\hline $7-9$ & Topological & 0.13 & $<1.93$ & $<0.11$ & 0.06 & 0.01 & 0.02 \\
\hline
\end{tabular}

A cut on the invariant mass of any pair of leptons compatible with the $\mathrm{Z}$ hypothesis (via charge flavour and invariant mass constraints, $\left.M_{Z} \notin[65] \mathrm{GeV} / \mathrm{c}^{2}\right)$ is used to reject $\mathrm{ZZ}$ and WZ events. Finally, kinematical cuts are used: $\not_{\mathrm{T}}>50 \mathrm{GeV} / \mathrm{c}, M_{\mathrm{T}}\left(W_{3}\right)>40 \mathrm{GeV} / \mathrm{c}^{2}$ and $\sum \vec{p}_{\mathrm{T}}^{\ell}>40 \mathrm{GeV} / \mathrm{c}$, where $\sum \vec{p}_{\mathrm{T}}^{\ell}$ is the transverse momentum of the vector sum of momenta of all three leptons, and $M_{\mathrm{T}}\left(W_{3}\right)$ is the reconstructed transverse mass of the associated W boson:

$$
M_{\mathrm{T}}\left(W_{3}\right)=\sqrt{2 * p_{\mathrm{T}}^{l_{3}} p_{\mathrm{T}}\left(1-\cos \Delta \phi_{l_{3} p_{\mathrm{T}}}\right)}
$$

with $p_{\mathrm{T}}^{l_{3}}$ being the transverse momentum of the lepton not associated to the Higgs boson, $\not p_{\mathrm{T}}$ the missing transverse momentum, and $\Delta \phi_{l_{3} p_{\mathrm{T}}}$ the polar angle between the lepton and the missing transverse momentum. Optimised cuts are summarised in Table 10.20.

The Higgs boson transverse mass is computed from the two chosen leptons and from the missing transverse momentum:

$$
M_{\mathrm{T}}(H)=\sqrt{M_{\mathrm{T}}^{l l^{2}}+2 E_{\mathrm{T}}^{l l} p_{\mathrm{T}}-2 P_{\mathrm{T}}^{l l} \not_{\mathrm{T}} \cos \Delta \phi_{l l p_{\mathrm{T}}}},
$$

Figure 10.22 shows the distribution of $\mathrm{M}_{\mathrm{T}}(\mathrm{H})$ for the signal, on top of remaining background, after all cuts for a Higgs boson mass of $140 \mathrm{GeV} / \mathrm{c}^{2}$ and an integrated luminosity of $100 \mathrm{fb}^{-1}$. The cumulated efficiency (including trigger and event selection) depends on the Higgs boson mass hypothesis. Starting at $0.5 \%$ for a mass hypothesis of $115 \mathrm{GeV} / \mathrm{c}^{2}$, the efficiency rises to a maximum at the "WW resonance" (1.3\%). Beyond the WW production threshold, efficiency drops since $\mathrm{W}$ bosons start to be boosted in the Higgs boson frame, which influences the angular distribution of leptons. Efficiency in that region could certainly be improved by optimising the analysis for a Higgs boson mass of $190 \mathrm{GeV} / \mathrm{c}^{2}$.

10.2.6.4. Systematic uncertainties. Systematic sources considered in this study are related to the normalisation of backgrounds, to the reconstruction, the event selection, the luminosity and the structure functions of protons.

Background will be normalised to signal-free regions of the phase-space. By looking at the acoplanarity distribution when the angular cuts are not applied, data can be fitted to a sum of signal and background shapes. For that purpose, the signal is described by a sigmoid distribution, while the background remains constant. The Monte Carlo distribution for signal and background are first fitted independently, and the shapes obtained that way are used to fit data from pseudo-experiments (Figure 10.23). The uncertainty on the background normalisation is then related to the uncertainty on the background level in that fit. The 


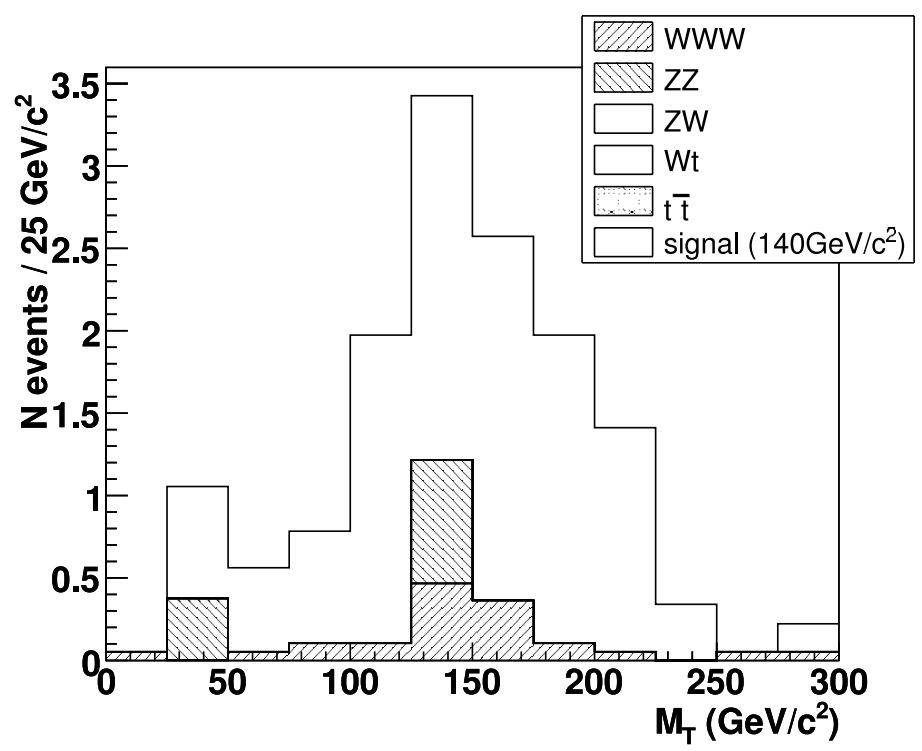

Figure 10.22. Reconstructed transverse mass from Equation (10.4) for a $140 \mathrm{GeV} / \mathrm{c}^{2}$ Higgs boson and an integrated luminosity of $100 \mathrm{fb}^{-1}$.

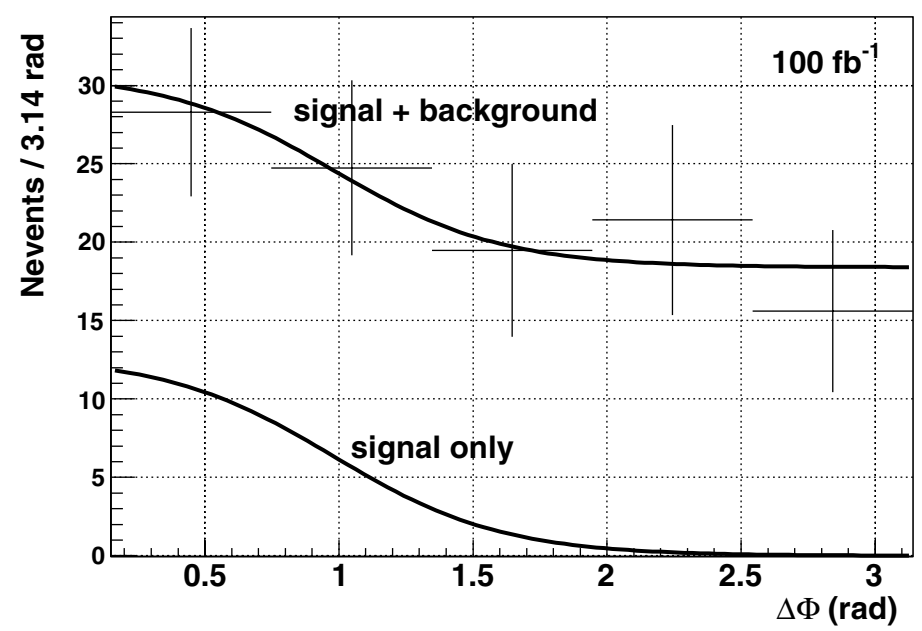

Figure 10.23. Distribution of the acoplanarity for pseudo-experiments, fitted by a signal+ background shape, as described in the text.

uncertainty on the background level is found to be $15 \%$ for an integrated luminosity of $100 \mathrm{fb}^{-1}$, and rises up to $20 \%$ for $30 \mathrm{fb}^{-1}$. That value will be used in the following.

Reconstruction and selection uncertainties arise from the jet veto, the $b$ veto and lepton reconstruction. Experience from Tevatron tells that a typical $2 \%$ uncertainty on lepton reconstruction efficiency has to be considered, while 5\% uncertainty comes from lepton isolation [485] Since three leptons are present in our analysis, a 12\% uncertainty from lepton reconstruction and selection has been taken. The additional uncertainties from the jet veto and the $b$ veto will be assumed to be $5 \%$ each. 
(a)

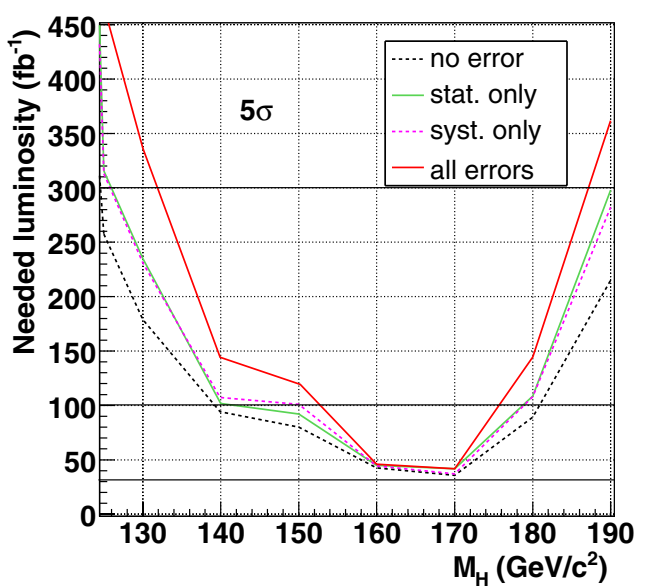

(b)

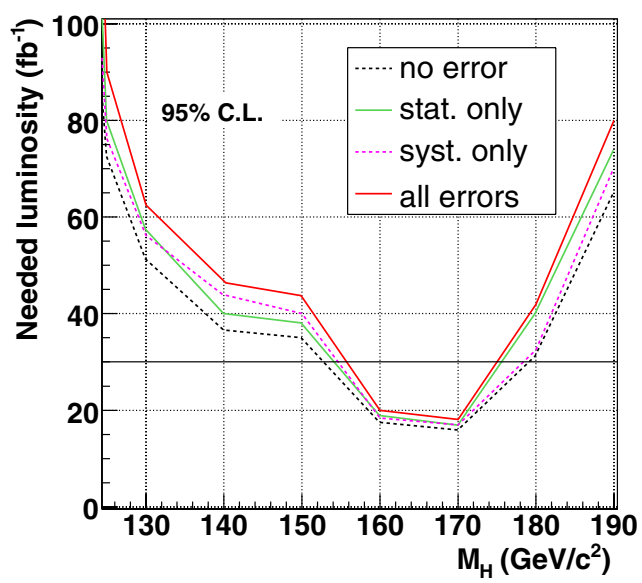

Figure 10.24. (a) Luminosity needed to obtain a $5 \sigma$ significance using the likelihood-ratio method, with systematics only, Monte-Carlo statistical uncertainties only, or with both effects considered; (b) luminosity needed to exclude a Higgs boson at 95\%C.L. if no excess is observed, using the same method.

To take into account other uncertainties related to the event selection, cuts are varied within the resolution of the associated quantity. The signal efficiency and background rejection are found to be stable with respect to such variations. A conservative value of $3 \%$ for the associated uncertainty is considered in the following.

The last uncertainty considered comes from the product of the luminosity and the proton structure functions, known as the parton luminosity. Considering these two quantities separately, a 5\% uncertainty on the luminosity is assumed, while the uncertainty from the proton parton distribution function (PDF) is taken to be 4\% [486]. This latter uncertainty is reduced for the process considered, for which the mid-x region (where uncertainties are small) dominates.

The additional source of systematic uncertainties arising from the limited Monte Carlo statistics is also considered in the following result. With the likelihood ratio method used in the analysis, this is done bin per bin in the distributions of signal and background, so that a single value cannot be quoted. For the time being, this has a large impact on the results, but this effect will easily be reduced in the future, as more events become available.

10.2.6.5. Signal significance. In order to integrate the effect of systematic uncertainties and to exploit the discriminative power from the transverse mass distribution, the likelihood-ratio method $\left(S_{C L}\right)$ is used. Figure 10.24(a) shows the luminosity needed to obtain a $5 \sigma$ significance using this method, with systematics only, with Monte Carlo statistical uncertainties, or with both effects considered. Figure 10.24(b) shows the luminosity needed to exclude a Higgs boson at 95\%C.L. if no excess is observed, using the same method. Less than $50 \mathrm{fb}^{-1}$ are required in most of the mass range, while only $20 \mathrm{fb}^{-1}$ are needed at $170 \mathrm{GeV} / \mathrm{c}^{2}$.

One important motivation for studying this channel is also that it is one of the only allowed signatures for a fermiophobic Higgs boson model. If the Higgs boson does not couple to fermions, the usual gluon-fusion diagrams are indeed forbidden, as well as $b \bar{b}$ decays. A fermiophobic Higgs boson will present a large cross-section at low mass, as the branching ratio does not drop down as in the Standard Model. Figure. 10.25(a) shows the luminosity 
(a)

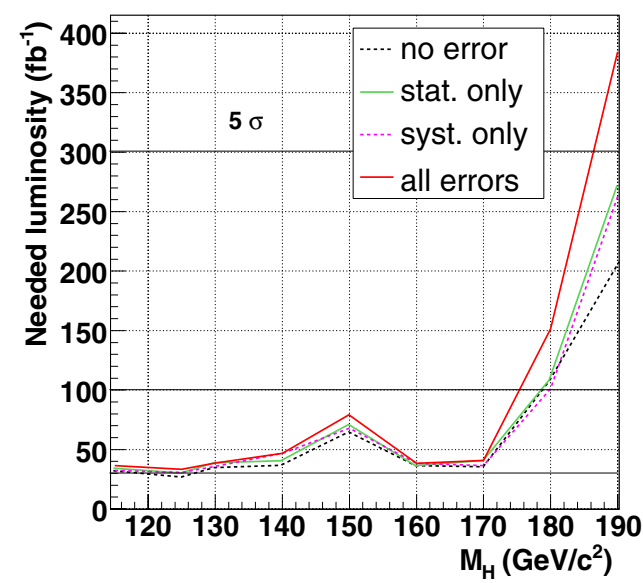

(b)

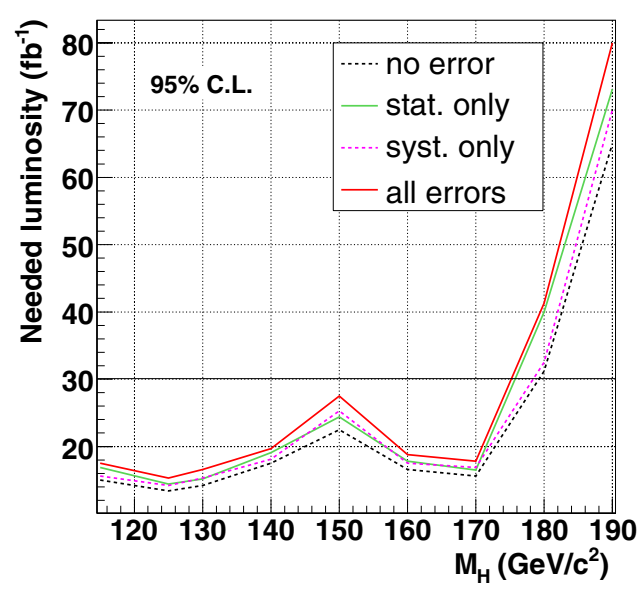

Figure 10.25. Results obtained using the benchmark fermiophobic model; (a) Luminosity needed to obtain a $5 \sigma$ significance using the likelihood-ratio method, with systematics only, Monte-Carlo statistical uncertainties only, or with both effects considered; (b) luminosity needed to exclude a Higgs boson at 95\%C.L. if no excess is observed, using the same method.

needed to obtain a $5 \sigma$ significance for a fermiophobic Higgs boson. Compared to Fig. 10.24, the needed luminosity is found to be similar in the most favourable mass region for the Standard Model (around $170 \mathrm{GeV} / \mathrm{c}^{2}$ ) and above, but far better results are obtained in the low mass region. After $100 \mathrm{fb}^{-1}$, all masses between the LEP limit and $175 \mathrm{GeV} / \mathrm{c}^{2}$ will be covered by this analysis alone. Figure 10.25 (b) shows the luminosity needed to exclude a fermiophobic Higgs boson at 95\%C.L. if no excess is observed. In the absence of signal, less than $30 \mathrm{fb}^{-1}$ are required to reject any fermiophobic Higgs boson up to $175 \mathrm{GeV} / \mathrm{c}^{2}$.

\subsubsection{Associated $\overline{\mathrm{tt}} \mathrm{H}$ production with $\mathrm{H} \rightarrow \gamma \gamma$}

10.2.7.1. Introduction. A Higgs boson produced in association with a $\bar{t} \bar{t}$ pair, with an $\mathrm{H} \rightarrow \gamma \gamma$ decay would share a fully reconstructible mass peak with the inclusive $\mathrm{H} \rightarrow \gamma \gamma$ signature. But like the $\mathrm{WH}$ and $\mathrm{ZH}$ channels [487], the signature could contain an isolated high-transverse- momentum charged lepton which can be used both to discriminate against QCD background and reconstruct the primary vertex; the associated production channels could hence be less dependent on photon energy resolution. In particular, the presence of two top quarks would tend to produce high-multiplicity events, which could offer additional discriminating power against light jet QCD background. In the case of the two-Higgsdoublet MSSM, the gluon fusion Higgs boson production channel could in fact be subject to suppression with respect to the associated production channels in the case of top-stop degeneracy ("maximal mixing") [488]. Prior generator-level studies for the detection of the SM [489] and MSSM [490] Higgs bosons in CMS via this channel have indicated a signalto-background ratio of approximately 1. A full simulation study in the ATLAS Physics Technical Design Report [491] has predicted a signal significance of $S / \sqrt{B}=4.3-2.8$ for $\mathrm{m}_{H}=100-140 \mathrm{GeV} / \mathrm{c}^{2}$ with a signal efficiency of $\sim 30 \%$. A more recent, related ATLAS study involving a 2-photon signature accompanied by missing energy [492] has indicated, for $100 \mathrm{fb}^{-1}$, a signal-to-background ratio of $\sim 2$ for $\mathrm{m}_{H}=120 \mathrm{GeV} / \mathrm{c}^{2}$. 
Table 10.21. Estimated number of signal events for $\overline{\mathrm{t}} \mathrm{H}, \mathrm{H} \rightarrow \gamma \gamma$, assuming NLO production cross sections [162], Higgs boson branching ratios to two photons [21], and one electron or muon from top decay (including from tau lepton decays).

\begin{tabular}{lll}
\hline Higgs Boson Mass $\left(\mathrm{GeV} / \mathrm{c}^{2}\right)$ & After $30 \mathrm{fb}^{-1}$ & After $100 \mathrm{fb}^{-1}$ \\
\hline 115 & 20.80 & 69.33 \\
120 & 19.61 & 65.36 \\
130 & 15.96 & 53.20 \\
140 & 11.20 & 37.33 \\
\hline
\end{tabular}

10.2.7.2. Signal production cross-sections, event rates and event generation. Production cross-sections for $\overline{\mathrm{t}} \mathrm{t}$ have been calculated at next-to-leading order [162, 464, 465]. Taking the branching ratio for $\mathrm{H} \rightarrow \gamma \gamma$ from HDECAY [21] and assuming in addition that the decay of exactly one of the top quarks yields a lepton (electron or muon) from $\mathrm{W}^{ \pm} \rightarrow 1+v_{l}$ (including the possibility of tau lepton decays to muons or electrons), we estimate for several Higgs boson masses the number of signal events for 30 and $100 \mathrm{fb}^{-1}$ (Table 10.21).

Signal events were generated with both the MADGRAPH [81,493,494] and ALPGEN [161, 495, 496] LO exact matrix element generators, for each of the Higgs boson masses shown in Table 10.21. Events from both generators were found to yield comparable LO crosssection and kinematical distributions. The LO cross-sections were also found to agree with those from the program HQQ [20] at the percent level. The samples analysed were those generated with ALPGEN. For the current study all signal events have been generated such that exactly one of the two $\mathrm{W}$ bosons from the two top quarks decays leptonically.

10.2.7.3. Background processes considered and event generation. Standard Model processes resulting in both irreducible and reducible backgrounds have been identified. A background is called irreducible if it is capable of giving rise to the same signature on the particle level as that searched for in a signal event, that is to say, a lepton and two photons $(1 \gamma \gamma)$. Special care has been taken to properly treat the irreducible $\overline{\mathrm{t}} \gamma \gamma$ background. Feynman diagrams of three possible types of $\mathrm{t} \overline{\mathrm{t}} \gamma \gamma$ processes considered are shown in Fig. 10.26. In the first case, called "Type 1", both photons are radiated from either outgoing top quarks or incoming partons. In the third case, called "Type 3", both are radiated from top quark decay products. The second case, "Type 2" combines one photon radiated according to "Type 1" with the second radiated according to "Type 3". (A fourth process arises from both photons being radiated from different decay products of the same top quark; for the relevant event selection (see pertinent section below) with $m_{\gamma \gamma}>70 \mathrm{GeV} / \mathrm{c}^{2}$ we have verified that this contribution is completely negligible). The Types 2 and 3 processes, as well as the process $\mathrm{W} \gamma \gamma+4$ jets, previously unavailable in any matrix element generator, have been specifically added to ALPGEN for this and future studies. Where applicable in the ALPGEN samples, top quarks and $\mathrm{W}$ bosons are decayed within ALPGEN which assures preservation of spin correlation information which could impact kinematical distributions.

Table 10.22 lists the considered irreducible background processes, the generators used to either generate or cross-check event samples, the LO cross-section with statistical errors, the number of events expected for $30(100) \mathrm{fb}^{-1}$ of integrated luminosity, the number of events generated, simulated, reconstructed and analysed as well as the equivalent integrated luminosity, which ranges from 400 to over $6000 \mathrm{fb}^{-1}$. The cross-sections reflect pre-selection criteria imposed at generator-level which are described in the next section. In the processes involving real top quarks as well as in the $\mathrm{W} \gamma \gamma+4 \mathrm{j}$ process, one top quark/the $\mathrm{W}$ boson was forced to decay leptonically, and the stated cross-section therefore implicitly includes 


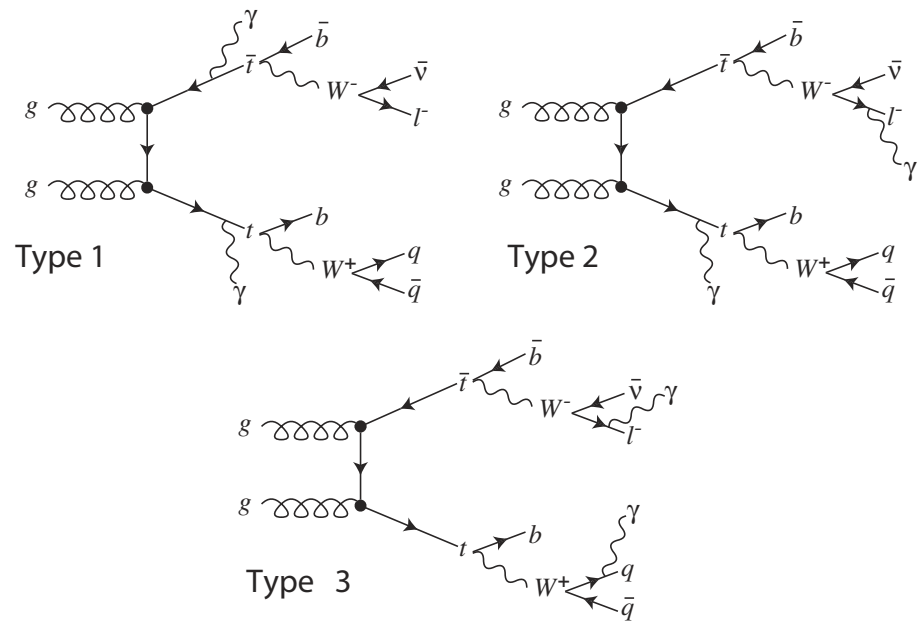

Figure 10.26. A sub-sample of the relative Feynman graphs illustrating the three types of $t \bar{t} \gamma \gamma$ processes.

Table 10.22. Cross-sections at leading order (statistical errors in parentheses), number of events generated, simulated and reconstructed, and equivalent integrated luminosity for the irreducible backgrounds considered.

\begin{tabular}{lllllllll}
\hline Process & $\begin{array}{l}\sigma \times \mathrm{BR}[\mathrm{fb}] \\
(1 \mathrm{~W} \rightarrow l \nu)\end{array}$ & $\mathrm{Ngen}$ & $\mathrm{N} 30 \mathrm{fb}^{-1}$ & $\mathrm{~N} 100 \mathrm{fb}^{-1}$ & Generator & $\begin{array}{l}\text { N simul./ } \\
\text { reconstr. }\end{array}$ & $\begin{array}{l}\text { N Anal. } \\
\text { Lumi. [fb }{ }^{-1} \text { ] }\end{array}$ \\
\hline $\mathrm{tt} \gamma \gamma 1$ & $1.6(\leqslant 1 / \mathrm{mil})$ & 52202 & 48 & 160 & AL,MG & 10000 & 4695 & 6250 \\
$\mathrm{tt} \gamma \gamma 2$ & $6.1(\leqslant 1 \%)$ & 6238 & 183 & 610 & $\mathrm{AL}$ & 6000 & 5109 & 1000 \\
$\mathrm{tt} \gamma \gamma 3$ & $4.9(\leqslant 1 \%)$ & 2967 & 147 & 490 & $\mathrm{AL}$ & 2500 & 2250 & 510 \\
$\mathrm{~W} \gamma \gamma 4 \mathrm{j}$ & $11.5(1.2 \%)$ & 4587 & 345 & 1150 & $\mathrm{AL}$ & 4500 & 3957 & 400 \\
\hline
\end{tabular}

the relevant branching ratio. The effect of the inclusion of background Types 2 and 3 is to augment the total initial contribution (before selection) from $\mathrm{t} \overline{\mathrm{t}} \gamma \gamma$ by approximately one order of magnitude.

A background is called reducible if at least one element of the final-state signature is mistakenly identified due to incomplete detector coverage or other instrumental effects. This could arise if one or more electrons or jets are misidentified as photons, or a jet as an electron or a muon. It has been heretofore possible to evaluate only the irreducible backgrounds discussed above with acceptable statistics, so only these will be presented here. Low-statistics tests on most of the reducible background processes have been performed, and strong requirements have been implemented in the following selection in order to veto them.

10.2.7.4. Event simulation and reconstruction. All generated signal and background events were fragmented and hadronised with PYTHIA [69, 246] version 6.227, using the CTEQ5L [12] PDFs. They were then simulated, digitised and reconstructed using the standard CMS tools. All samples were digitised with high-luminosity $\left(10^{34} \mathrm{~cm}^{-2} \mathrm{~s}^{-1}\right)$ pile-up.

10.2.7.5. Description of generator-level pre-selections. No generator-level pre-selections were made on signal events. For the irreducible background events, the following 
pre-selection was made:

- $\mathrm{m}_{\gamma \gamma} \geqslant 80 \mathrm{GeV} / \mathrm{c}^{2}$ for all four processes;

- $\mathrm{p}_{\mathrm{T} \gamma} \geqslant 20 \mathrm{GeV} / \mathrm{c},\left|\eta_{\gamma}\right| \leqslant 2.5$ (MADGRAPH) or $\mathrm{p}_{\mathrm{T} \gamma} \geqslant 15 \mathrm{GeV} / \mathrm{c},\left|\eta_{\gamma}\right| \leqslant 2.7$ (ALPGEN) for all four processes;

- $\mathrm{p}_{\mathrm{T} l} \geqslant 15 \mathrm{GeV} / \mathrm{c}$ for all processes except $\mathrm{tt} \gamma \gamma 1$;

- $\mathrm{p}_{\mathrm{T} j} \geqslant 15 \mathrm{GeV} / \mathrm{c},\left|\eta_{j, l}\right| \leqslant 2.7, \Delta \mathrm{R}(\mathrm{l}, \mathrm{j}$ or $\mathrm{j}, \mathrm{j}$ or $\gamma, \mathrm{j}$ or $\gamma, \gamma) \geqslant 0.3$ for the process $\mathrm{W} \gamma \gamma 4 \mathrm{j}$, where ' $\mathrm{j}$ ' refers to one of the four additional light quark jets;

where $\mathrm{p}_{\mathrm{T}}$ refers to the transverse momentum of the particle, $\eta$ its pseudorapidity and $\Delta R=\sqrt{\left(\Delta \eta^{2}+\Delta \phi^{2}\right)}$ where $\phi$ is the azimuthal angle.

The intersection (most restrictive set) of the above generator-level criteria except that pertaining to the additional light quark jets was then imposed on all signal and background event samples at the particle level.

10.2.7.6. Event selections. The events are selected by the single and diphoton triggers at Level-1 and High Level Triggers (HLT) configured for high luminosity $\left(10^{34} \mathrm{~cm}^{-2} \mathrm{~s}^{-1}\right)$.

A prior study of this channel at particle-level [497] found that reliance on $\mathrm{p}_{\mathrm{T}}$ alone to identify the two Higgs boson photon candidates results in considerable sidebands (at approximately the $10 \%$ level) in the two-photon invariant mass distribution in signal events. It is the choice of the second (lower in $\mathrm{p}_{\mathrm{T}}$ ) photon which is overwhelmingly contaminated by these combinatorial photons, which originate approximately $80 \%$ from $\pi^{0} \mathrm{~s}, 10 \%$ from $\eta \mathrm{s}$, a few percent from $\omega \mathrm{s}$, and the remainder from other particles. Fully $80 \%$ of these fake Higgs photon 'mother' particles appear to come from parton showers whose origin is one of the two final-state top quarks, and as such are peculiar to the $\bar{t} \mathrm{H}$ process. The other $20 \%$ come from showering from the initial-state partons and hence are common to all the associated production channels. For reconstructed signal events, the misidentification percentage grows to $\sim 30 \%$ (see the pertinent curve in Fig. 10.28(left)).

To improve the Higgs photon selection procedure, we have evaluated the performance of the photon isolation variables investigated and used by the $\mathrm{H} \rightarrow \gamma \gamma$ inclusive analysis [7]. We obtain the best results by considering linear combinations of the variables 'ECALIso' (the sum of $\mathrm{E}_{\mathrm{T}}$ of ECAL basic clusters within a cone after removing the $\mathrm{E}_{\mathrm{T}}$ of those basic clusters constructed with the Island algorithm included in the supercluster matched $(\Delta R<0.2)$ with the offline photon itself) and 'HCALIso' (the sum of $\mathrm{E}_{\mathrm{T}}$ of HCAL calorimeter towers within a cone centred on the photon candidate), as illustrated in Fig. 10.27(right).

For this study, the two highest- $\mathrm{p}_{\mathrm{T}}$ Offline Photons satisfying the following requirement on the isolation energy Iso $=$ HCALIso $+\left(2 .{ }^{*}\right.$ ECALIso $)$ were retained as Higgs photon candidates:

- For photons in the barrel: Iso $<25 \mathrm{GeV}$,

- For photons in the endcap: Iso $<22 \mathrm{GeV}$,

with $\Delta R<0.25$ for ECALIso and $\Delta R<0.3$ for HCALIso (see comparison of performance with different isolation cone radii in Fig. 10.27 (left). These values yield approximately $95 \%$ efficiency for true Higgs photons ${ }^{45}$ and less than $40 \%$ for combinatorial photons. This strategy successfully restores approximately one-half of the true Higgs photon pairs previously lost to misidentification when selection based on only photon $\mathrm{p}_{\mathrm{T}}$ is used, as is demonstrated by Fig. 10.28.

45 "True Higgs photons" are considered to be those Offline Photons lying within a cone of radius $\Delta R<0.1$ of one of the two particle-level photons coming from the Higgs boson decay. 

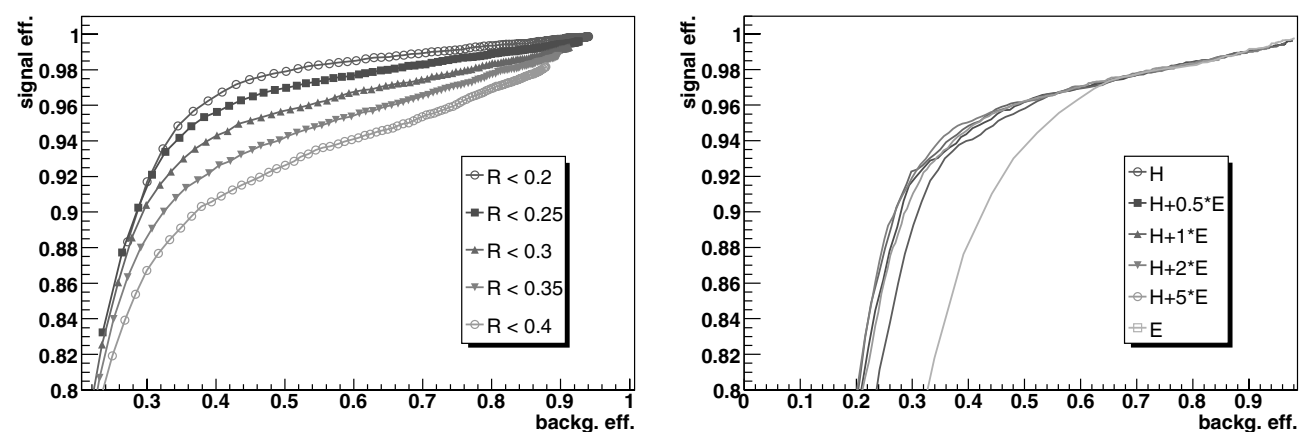

Figure 10.27. Left: Efficiency for true Higgs photons vs. that for combinatorial photons in the ECAL barrel for the ECAL isolation variable, for different isolation cone sizes. Right: Efficiency for true Higgs photons vs. that for combinatorial photons in the ECAL endcap, for several linear combinations of isolation variables.
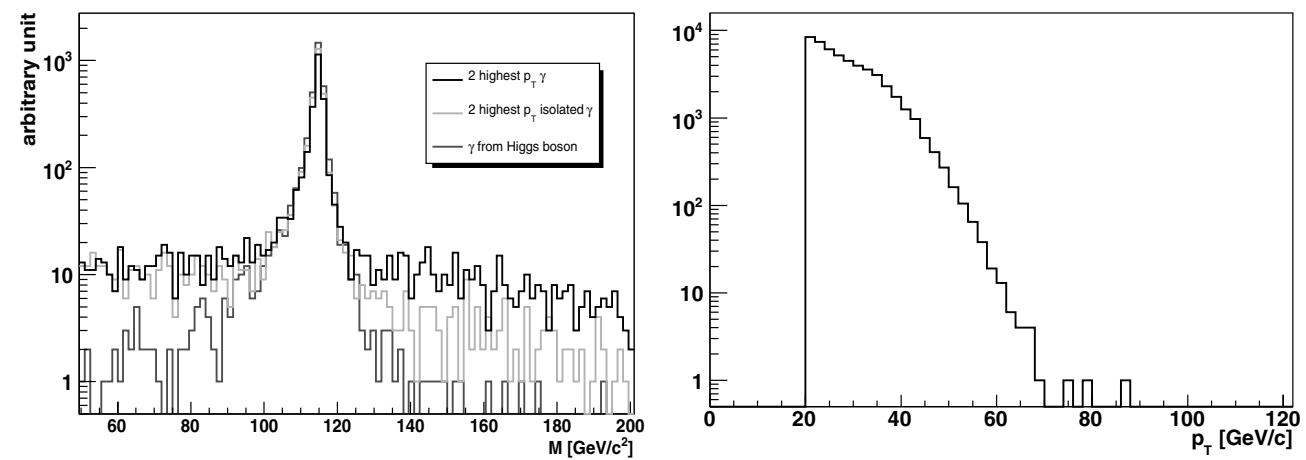

Figure 10.28. Left: Invariant mass of the two Higgs photon candidates selected according to $\mathrm{p}_{\mathrm{T}}$ alone (dark grey curve), $\mathrm{p}_{\mathrm{T}}$ and isolation (light grey curve), and where both candidates are geometrically matched to particle-level Higgs photons (medium grey curve). Right: Distribution of the $p_{\mathrm{T}}$ of jets from pile-up events. Jets not matched to generated particle jets from the signal are considered to be pile-up jets.

A similar technique is employed for the selection of candidate leptons from top quark decays (via a $\mathrm{W}$ boson). We obtain the best performance in selecting 'true' $\mathrm{W}$ leptons ${ }^{46}$ with the previously-defined ECALIso variable for Offline Electrons and with transverse momentum of tracks in a cone of radius $\Delta R<0.25$ ('IsoByTkPt025') for GlobalMuons. We retain as the $\mathrm{W}$-decay (top) lepton candidate the highest- $\mathrm{E}_{\mathrm{T}}$ OfflineElectron or highest- $\mathrm{p}_{\mathrm{T}}$ GlobalMuon satisfying the following requirement:

- For electrons, ECALIso $<5 \mathrm{GeV}$,

- For muons, IsoByTkPt02 <9 GeV.

These values yield $\sim 92 \%$ efficiency for 'true' W leptons and approximately $35 \%$ for combinatorial leptons. In the selection criteria involving photons described below, as well as those involving leptons described thereafter, the pertinent distributions are constructed using

46 As for Higgs photons, considered to be those OfflineElectrons or GlobalMuons lying within a cone of radius $\Delta R<0.1$ of a particle-level electron or muon from a W boson which itself is a decay product of one of the final-state top quarks. 
the Higgs photon and $\mathrm{W}$ lepton candidates selected according to the procedure combining $\mathrm{p}_{\mathrm{T}}$ and isolation described above.

After selection of the two Higgs photon and one W lepton candidates, the remainder of the selection aims for a signal efficiency of between 85 and $95 \%$ per selection criterion. Five variables involving the Higgs photon candidates have demonstrated effective performance: the $\mathrm{p}_{\mathrm{T}}$ of each of the two OfflinePhoton candidates $\left(\mathrm{p}_{\mathrm{T} \gamma 1}, \mathrm{p}_{\mathrm{T} \gamma 2}\right)$, the sum of their $\mathrm{p}_{\mathrm{T}}\left(\mathrm{p}_{\mathrm{T} \gamma 1}+p_{\mathrm{T} \gamma 2}\right)$, the angular distance between them $\left(\Delta R_{\gamma_{1}, \gamma_{2}}\right)$, and $\cos \theta^{*}$, where $\tan \theta^{*}=\frac{\left|\vec{p}_{i}\right| \sin \theta_{i}}{\gamma\left(\left|\vec{p}_{i}\right| \cos \theta_{i}-\beta E_{i}\right)}$, and $\vec{p}_{i}$ and $\theta_{i}$ refer respectively to the momentum of and the 3 -space angle between either of the two Higgs photon candidate directions and the direction of their joint 4-vector, in the laboratory frame (the scalar nature of the Higgs boson should assure a flat distribution of this variable for signal events, and one peaked in the forward and backward directions for background events). We have established the following eventwise selection involving the two Higgs photon candidates:

- $\mathrm{p}_{\mathrm{T} \gamma 1, \gamma 2} \geqslant 50,18 \mathrm{GeV} / \mathrm{c}$

- $\mathrm{p}_{\mathrm{T} \gamma 1}+p_{\mathrm{T} \gamma 2} \geqslant 85 \mathrm{GeV} / \mathrm{c}$

- $\Delta R_{\gamma_{1}, \gamma_{2}} \leqslant 3.2$

- $\cos \theta^{*} \leqslant 0.85$.

Three variables involving the $\mathrm{W}$ lepton candidates have demonstrated effective performance: the $\mathrm{E}_{\mathrm{T}}$ (OfflineElectron) or $\mathrm{p}_{\mathrm{T}}$ (OfflineMuon) of the candidate, and the angular distances between the candidate and each of the two Higgs photon candidates $\left(\Delta R_{\gamma_{1}, \text { lepton }}, \Delta R_{\gamma_{2}, \text { lepton }}\right.$ ). We have established the following eventwise selection involving the $\mathrm{W}$ lepton candidate:

- $\mathrm{p}_{\text {Tlepton }} \geqslant 15 \mathrm{GeV} / \mathrm{c}$

- $\Delta R_{\gamma_{1}, \text { lepton }}, \Delta R_{\gamma_{2}, \text { lepton }} \geqslant 0.3,1.0$.

In order to remove part of the irreducible backgrounds studied here and also eventually to remove backgrounds from QCD processes, we take advantage of the high jet multiplicity of our signal events as well as the presence of two real top quarks yielding b-quark jets as decay products. Jets including those possibly corresponding to b-quarks are constructed with the iterative cone algorithm [7] with a cone radius of $\Delta R=0.5$. A discriminant (BtagDisc) is then calculated for each candidate b-quark jet with the Combined BTag [7] b-quark-tagging algorithm. We require the presence of a minimum number of jets having a value of $p_{\mathrm{T}}$ greater than $60 \mathrm{GeV} / \mathrm{c}$, which permits the removal of jets from pile-up from consideration (we consider a reconstructed jet to be from pile-up if it is not geometrically matched with a particle-level jet, which has been constructed using the same algorithm and parameters as the reconstruction-level jets). Figure 10.28 (right) shows the $p_{\mathrm{T}}$ distribution of the jets thus attributed to pile-up in a signal sample with $\mathrm{m}_{H}=115 \mathrm{GeV} / \mathrm{c}^{2}$. We require $\geqslant 4$ jets with $p_{\mathrm{T}}>60 \mathrm{GeV}$.

To specifically target the $\mathrm{W}+2 \gamma+$ jets background (and eventually other non-b-quark reducible backgrounds), we make limited use of tagging of b-quark jets. We require that at least one candidate jet having $p_{\mathrm{T}}>60 \mathrm{GeV}$ have BtagDisc $>0.8$.

10.2.7.7. Performance of off-line selection. Tables 10.23 and 10.24 show the progression of the signal $\left(\mathrm{m}_{H}=115 \mathrm{GeV} / \mathrm{c}^{2}\right)$ and background samples through the selection. Prior to checking for the Level-1 and HLT decision, we apply the pre-selection at particle-level described in Section 10.2.7.5 to all signal and background samples. The number of surviving events is expressed as an effective cross-section in $\mathrm{fb}$. The final results are also expressed as numbers of surviving signal and total background events with statistical errors, for both 30 and $100 \mathrm{fb}^{-1}$. 
Table 10.23. Progression of the signal $\left(\mathrm{m}_{H}=115 \mathrm{GeV} / \mathrm{c}^{2}\right)$ and background samples through the trigger portion of the selection, expressed as an effective cross-section in fb. Efficiencies with respect to the previous sequential requirement are expressed as percentages.

\begin{tabular}{llllll}
\hline Requirement & $\mathrm{M}_{\mathrm{H}}=115 \mathrm{GeV} / \mathrm{c}^{2}$ & $\mathrm{tt} \gamma \gamma 1$ & $\mathrm{tt} \gamma \gamma 2$ & $\mathrm{t} \gamma \gamma 3$ & $\mathrm{~W} 2 \gamma 4 \mathrm{j}$ \\
\hline Before selection & $0.693(100.0)$ & $1.59(100.0)$ & $6.12(100.0)$ & $4.95(100.0)$ & $11.4(100.0)$ \\
Pre-selection & $0.533(76.8)$ & $1.4(87.9)$ & $5.05(82.5)$ & $3.94(79.6)$ & $11.3(98.9)$ \\
L1 + HLTAccept & $0.517(97.0)$ & $1.34(95.4)$ & $4.71(93.4)$ & $3.36(85.7)$ & $10.5(93.0)$ \\
HLT $\gamma \gamma, \gamma$ accept & $0.508(98.3)$ & $1.30(96.9)$ & $4.57(96.9)$ & $3.25(96.6)$ & $10.0(96.0)$ \\
\hline
\end{tabular}

Table 10.24. Progression of the signal $\left(\mathrm{m}_{H}=115 \mathrm{GeV} / \mathrm{c}^{2}\right)$ and background samples through the offline portion of the selection, expressed as an effective cross-section in fb. Efficiencies with respect to the previous sequential requirement are expressed as percentages.

\begin{tabular}{llllllr}
\hline Requirement & Criterion & $\mathrm{M}_{\mathrm{H}}=115 \mathrm{GeV} / \mathrm{c}^{2}$ & $\mathrm{tt} \gamma \gamma 1$ & $\mathrm{tt} \gamma \gamma 2$ & $\mathrm{tt} \gamma \gamma 3$ & $\mathrm{~W} 2 \gamma 4 \mathrm{j}$ \\
\hline Number of $\gamma$ 's & $\geqslant 2$ & $0.506(100.0)$ & $1.29(100.0)$ & $4.56(100.0)$ & $3.24(100.0)$ & $10.0(100.0)$ \\
Number isolated $\gamma$ 's & $\geqslant 2$ & $0.482(95.2)$ & $1.22(94.0)$ & $3.96(86.8)$ & $2.53(78.2)$ & $9.58(95.7)$ \\
$\mathrm{pT} \gamma_{1}(\mathrm{GeV} / \mathrm{c})$ & $>50$ & $0.432(90.0)$ & $1.04(85.3)$ & $3.14(79.4)$ & $1.48(58.5)$ & $7.90(82.5)$ \\
$\mathrm{pT} \gamma_{2}(\mathrm{GeV} / \mathrm{c})$ & $>18$ & $0.386(89.2)$ & $0.88(84.7)$ & $2.25(71.6)$ & $1.03(69.7)$ & $6.72(85.0)$ \\
$\mathrm{pT} \gamma_{1}+\mathrm{pT} \gamma_{2}(\mathrm{GeV} / \mathrm{c})$ & $>85$ & $0.379(98.2)$ & $0.847(96.3)$ & $2.17(96.5)$ & $0.926(89.8)$ & $6.40(95.3)$ \\
$\Delta R\left(\gamma_{1} \gamma_{2}\right)(\mathrm{GeV} / \mathrm{c})$ & $<3.2$ & $0.364(96.4)$ & $0.738(87.2)$ & $1.86(85.9)$ & $0.719(77.7)$ & $5.30(82.8)$ \\
$\cos \theta^{*}$ & $<0.85$ & $0.332(91.4)$ & $0.589(79.8)$ & $1.48(79.5)$ & $0.583(81.0)$ & $4.36(82.3)$ \\
$\mathrm{pT}$ & $>15$ isolated $(\mathrm{GeV})$ & $0.238(72.2)$ & $0.443(75.2)$ & $0.984(66.4)$ & $0.387(66.4)$ & $3.15(72.3)$ \\
$\Delta R\left(\gamma_{1} l\right)$ & $>0.3$ & $0.236(99.0)$ & $0.441(99.5)$ & $0.925(94.0)$ & $0.321(83.0)$ & $3.14(99.6)$ \\
$\Delta R\left(\gamma_{2} l\right)$ & $>1.0$ & $0.208(87.4)$ & $0.389(88.2)$ & $0.607(65.7)$ & $0.163(50.7)$ & $2.34(74.6)$ \\
$\mathrm{N}_{\mathrm{jets}} p_{\mathrm{T}}>60 \mathrm{GeV}$ & $\geqslant 4$ & $0.179(86.2)$ & $0.338(87.0)$ & $0.455(74.9)$ & $0.110(67.6)$ & $1.79(76.6)$ \\
$\mathrm{Btag}$ Disc for $\geqslant 1 \mathrm{jet}$ & $>0.8$ & $0.110(61.6)$ & $0.217(64.0)$ & $0.276(60.7)$ & $0.051(46.0)$ & $0.294(16.4)$ \\
$\mathrm{M}_{H}+/-1.5 \mathrm{GeV} / \mathrm{c}^{2}$ & & $0.074(67.1)$ & $0.005(2.51)$ & $0.011(3.86)$ & $<0.002(3.92)$ & $<0.003(1.02)$ \\
$\mathrm{N}_{\text {evts }}$ at $30 \mathrm{fb}^{-1}$ & & $2.22+/-0.10$ & & & $0.483+/-0.158$ & \\
$\mathrm{~N}_{\text {evts }}$ at $100 \mathrm{fb}^{-1}$ & & $7.42+/-0.334$ & & & $1.61+/-0.53$ & \\
\hline
\end{tabular}

10.2.7.8. Uncertainties, systematic errors, and strategy for background measurement from data. To estimate the systematic error on the surviving signal cross-section, the following global source of error is applied directly to the estimated number of signal events:

- Luminosity $<3 \%$.

The error due to the inclusion/non-inclusion of initial and final-state photon radiation at the fragmentation/hadronisation level as well as that due to the matrix element generator used (ALPGEN or MADGRAPH) was found to be insignificant.

We have also considered the following sources of uncertainty relevant to the detector:

- Electron/Photon/Muon identification: $1 \%$ per identified object.

- Efficiency to tag jets containing b quarks: $5 \%$ per identified b-quark jet.

- Uncertainty on the jet energy scale: $3 \%$.

Only the effect of the uncertainty on the jet energy scale is evaluated by propagation through the selection, and yields a net uncertainty of $+1.6 /-3.9 \%$ for a Higgs boson mass of $115 \mathrm{GeV} / \mathrm{c}^{2}$. All the above contributions are summed in quadrature and a systematic error of $+6.3 /-7.2 \%$ is obtained for the number of signal events for a Higgs boson mass of $115 \mathrm{GeV} / \mathrm{c}^{2}$. The uncertainty on the number of surviving background events, calculated below and amounting to an average of $\pm 15 \%$, is finally added to the above quadratic sum yielding an error of $+16.3 /-16.6 \%$ on the number of events in a peak containing both signal and background events, corresponding to the case of a signal cross-section measurement. 

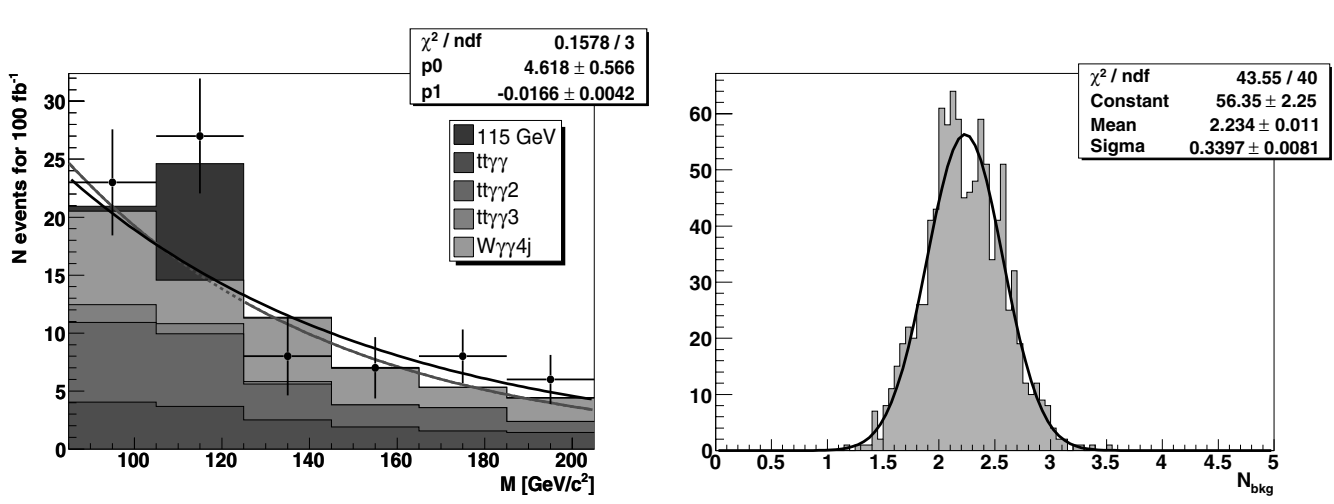

Figure 10.29. Left: Background estimation from the fit of the sidebands: Example of a gedanken experiment giving a possible set of real data points consistent with the Poisson distribution of the simulated number of surviving background events. The fit through these points is superimposed on the original fit. Right: Distribution of the estimated number of background events from the fit of the gedanken experiments.

Table 10.25. The signal selection efficiency, the total number of surviving signal and background events at $100 \mathrm{fb}^{-1}$ with statistical errors (from the number of generated events), the number of background events estimated from the fit with the fit error, and the signal significance calculated using the $\mathrm{ScP}$ estimator without and with the background uncertainty evaluated from the fit.

\begin{tabular}{lllll}
\hline Higgs Boson Mass (GeV) & 115 & 120 & 130 & 140 \\
\hline Sig. Selection Eff. (\%) & 10.7 & 11.2 & 11.3 & 11.3 \\
Number Signal & $7.42 \pm 0.33$ & $7.33 \pm 0.33$ & $5.96 \pm 0.27$ & $4.21 \pm 0.19$ \\
Total Number Bcgkd & $1.61 \pm 0.53$ & $2.79 \pm 0.62$ & $1.98 \pm 0.66$ & $1.10 \pm 0.51$ \\
Total Number Bcgkd from fit w. syst. & $2.23 \pm 0.34$ & $1.94 \pm 0.32$ & $1.60 \pm 0.22$ & $1.39 \pm 0.22$ \\
Signal Significance (ScP) & 3.541 & 3.662 & 3.257 & 2.510 \\
Signal Significance (ScP) w. syst. & 3.414 & 3.523 & 3.184 & 2.453 \\
\hline
\end{tabular}

The background spectrum can be obtained from the sidebands surrounding the positions of the putative Higgs boson masses and fit to a decreasing exponential function (shown by the grey curve in Fig. 10.29 (left)). The bin width has been chosen to be large enough $\left(20 \mathrm{GeV} / \mathrm{c}^{2}\right)$ to have a sufficient number of events for an integrated luminosity of $100 \mathrm{fb}^{-1}$ (The bin centred around the generated Higgs boson mass is not used for the fitting procedure). The number of background events and its error are estimated by fitting test distributions obtained with the average of the bin contents according to a Poisson distribution (gedanken experiments corresponding to possible future real data sets). One such fit is shown in the black curve in Fig. 10.29 (left). The mean and width of the gaussian fit of the distribution thus obtained (Fig. 10.29 (right)) yield respectively estimates of the number of background events and its systematic error, which are used to compute the signal significance.

10.2.7.9. Results. Table 10.25 shows, for each of the four Standard Model Higgs boson mass values considered, the signal selection efficiency, the total number of surviving signal and background events at $100 \mathrm{fb}^{-1}$ with statistical errors (from the number of generated events), the number of background events estimated from the fit with the fit error, and the signal significance calculated using the ScP estimator [498] with and without the background uncertainty evaluated from the fit. 

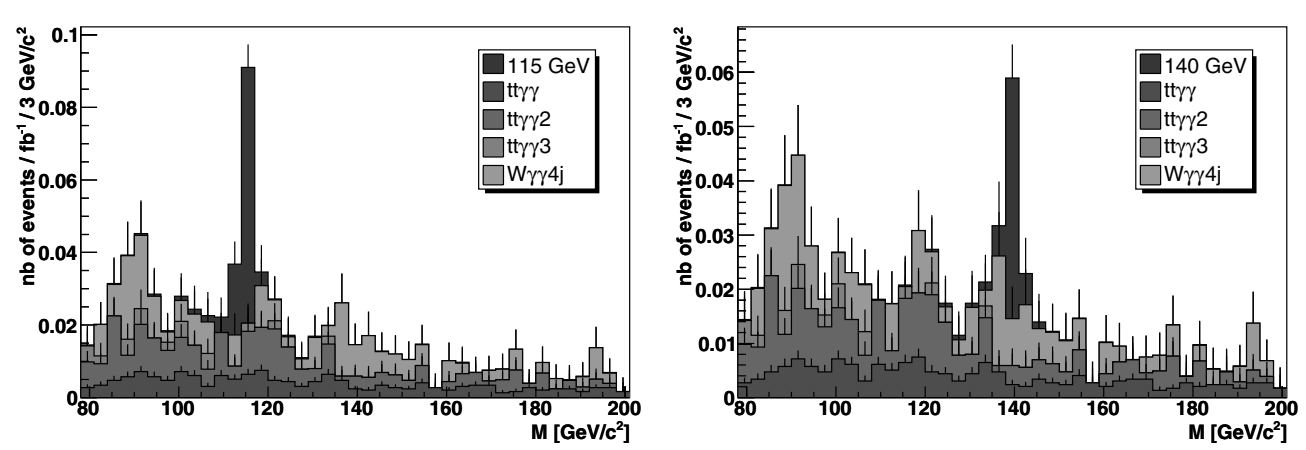

Figure 10.30. The diphoton mass after all selections for signal of $\mathrm{m}_{H}=115 \mathrm{GeV} / \mathrm{c}^{2}$ (left) and $140 \mathrm{GeV} / \mathrm{c}^{2}$ (right) added to the surviving backgrounds.

Figure 10.30 shows the diphoton mass distribution of the signal added to the background after all selections for Higgs boson masses $115 \mathrm{GeV} / \mathrm{c}^{2}$ (left) and $140 \mathrm{GeV} / \mathrm{c}^{2}$ (right).

10.2.7.10. Conclusion. A full-reconstruction-level sequential study has been performed for the channel $\overline{\mathrm{t}} \mathrm{H}$, with $\mathrm{H} \rightarrow \gamma \gamma$, taking into account irreducible backgrounds not previously studied. The ratio of signal to background events is approximately 4:1 representing a factor of 2 improvement over prior CMS and ATLAS studies. Signal observability in excess of $3 \sigma$ is indicated for masses up to $130 \mathrm{GeV} / \mathrm{c}^{2}$ with full simulation and reconstruction and with estimated systematic errors taken into account for $100 \mathrm{fb}^{-1}$ of integrated luminosity.

\subsubsection{Associated WH, ZH production with $\mathrm{H} \rightarrow \gamma \gamma$}

Compared to the gluon-gluon fusion channel $\mathrm{gg} \rightarrow \mathrm{H} \rightarrow \gamma \gamma$, the associated production channels WH/ZH [499,500] suffer from a much lower production cross section. Several advantages, however, make these channels attractive when the decay of the gauge boson results in a charged lepton: requiring an additional relatively high transverse-momentum lepton greatly reduces the significant QCD background in the $\gamma \gamma$ topology and improves the primary vertex reconstruction [501]. In the context of supersymmetric models, maximal mixing in the stop sector could result in a strong suppression of the $\mathrm{gg} \rightarrow \mathrm{h}$ signal, which the associated production channels would not be subject to [ 502]. The searched-for final state is therefore comprised of 2 isolated photons and at least one isolated electron or muon. Prior generator-level or fast simulation studies $[489,490,503,504]$ conclude to the possibility of a discovery at the LHC in this channel.

A detailed description of the analysis can be found in [505].

10.2.8.1. Event generation and reconstruction. All the process considered in this study have been simulated at the leading order. Signal events were generated by the matrix element generator COMPHEP [43] for Higgs boson masses ranging from 90 to $150 \mathrm{GeV} / \mathrm{c}^{2}$, in steps of $5 \mathrm{GeV} / \mathrm{c}^{2}$. Total cross-sections have been rescaled accordingly to the NLO calculation [20]. $\mathrm{K}$-factors from 1.15 to 1.16 are obtained on the whole mass range. Branching ratios for $\mathrm{H} \rightarrow$ $\gamma \gamma$ were taken from HDECAY program [21]. The irreducible backgrounds from the processes $\mathrm{W} \gamma \gamma$ et $\mathrm{Z} \gamma \gamma$ were also generated with CompHEP, with the same $\mathrm{K}$-factors applied as those pertinent to the signal. Fragmentation and hadronisation was performed by PYTHIA [246]. 
The reducible background processes $\gamma \gamma, \gamma$-jet, $\mathrm{W} \gamma, \mathrm{b} \overline{\mathrm{b}}$, and $\mathrm{t} \overline{\mathrm{t}}$, retained due to their capacity to mimic the $l \gamma \gamma$ signal, have been generated with PYTHIA and leading order crosssections were considered, except for the $\mathrm{t} \overline{\mathrm{t}}$ production where a NLO cross section of $840 \mathrm{pb}$ is used [278].

To ensure an efficient generation and preserve sufficient statistics of the most signal like events, a pre-selection is applied at generator level. Three electromagnetic candidates or two electromagnetic candidates and one muon candidate with $E_{\mathrm{T}}>20 \mathrm{GeV}$ and $|\eta|<2.7$ are required. An electromagnetic candidate is obtained by clustering electrons and photons in a $\Delta \eta=0.09, \Delta \phi=0.09$ window. Muon candidates are either $\mu, \tau, \pi$, or K particles.

The generated events were passed through the GEANT3 simulation of CMS [25]. The events were then digitised and reconstructed with the standard CMS software [506] with the addition of pile-up event corresponding to the high luminosity phase $\left(\mathcal{L}=10^{34} \mathrm{~cm}^{-2} \mathrm{~s}^{-1}\right)$.

10.2.8.2. Trigger selection. Events are required to pass the global Level 1 trigger [506] and the double photon High Level Trigger (HLT) [76] configured for the high luminosity phase. The trigger efficiencies for the preselected signal events are higher than $95 \%$ on the whole Higgs boson mass range $\left(90-150 \mathrm{GeV} / \mathrm{c}^{2}\right)$ as shown in Table 10.26 and 10.27.

10.2.8.3. Offline event selection. To suppress the reducible backgrounds, four discriminant combined variables are first constructed using a likelihood ratio method to estimate the isolation of the photons, the quality of the lepton reconstruction, the isolation of the lepton and the QCD/multi-jets nature of the event. The reference histograms for the four likelihoods are all produced on independent simulated event samples after a very loose pre-selection requiring two offline photons and one electron or muon reconstructed by the standard algorithms. Photon candidates with a matching seed in the pixel detector are rejected. The two photons with the highest transverse energy are assigned to the Higgs boson decay. Several isolation variables [507] were tested in the likelihood and the best performance is obtained with the sum of the transverse energy of the basic clusters within a cone $\Delta R<0.3$ around the photon, excluding the basic clusters belonging to the photon supercluster and the sum of the transverse energy of the HCAL towers within a cone $\Delta R<0.3$ around the photon.

Then the offline lepton with the highest $E_{\mathrm{T}}$ is selected. The reconstruction quality of the electron is carefully checked. The four variables yielding the most significant discriminating power are the ratio $E / p$ between the electron energy as measured in the calorimeter and its momentum measured by the tracker, the hadronic energy fraction $E_{\text {had }} / E$, the distance $\Delta \eta$ between the track and the associated supercluster and the ratio $R_{9}$ between the sum of the energies of 9 crystals $(3 \times 3$ matrix centred on the maximum-energy crystal) and the energy of the corresponding supercluster. In the case of muons the purity obtained by the standard CMS reconstruction algorithms has already proven sufficient; therefore, no additional criteria are applied.

For the lepton isolation, similar calorimeter variables as for photons are used. In addition, the number of pixel lines within a cone $\Delta R<0.3$ around the lepton improves the discriminative power of the likelihood.

Finally a global discriminant variable against multi-jet background is constructed. The rejection of $\pi^{0}$ faking signal photons, effective against QCD backgrounds, has been accomplished by a neural network procedure exploiting the information on the lateral profile of the electromagnetic shower. Variables involving the multiplicity of reconstructed objects in the electromagnetic calorimeter improve the discriminating power.

The results of the selection applied on the four combined variables are presented in Tables 10.26 and 10.27. The multi-jet backgrounds are entirely suppressed. To obtain a more 
Table 10.26. Expected rates (in fb) after each stage of the event selection for signals $\left(m_{\mathrm{H}}=\right.$ $120 \mathrm{GeV} / \mathrm{c}^{2}$ ) and irreducible backgrounds. Errors are statistical only.

\begin{tabular}{lllll}
\hline & $\mathrm{WH}$ & $\mathrm{ZH}$ & $\mathrm{W} \gamma \gamma$ & $\mathrm{Z} \gamma \gamma$ \\
\hline$\sigma . \mathrm{BR}$ & 0.810 & 0.137 & - & - \\
Pre-selection: $\sigma . \mathrm{BR} . \varepsilon$ & 0.460 & 0.0440 & 13.58 & 18.92 \\
Double photons HLT & $0.439 \pm 0.005$ & $0.0423 \pm 0.0004$ & $8.80 \pm 0.04$ & $12.13 \pm 0.07$ \\
2 isolated photons & $0.387 \pm 0.005$ & $0.0370 \pm 0.0004$ & $7.14 \pm 0.04$ & $6.51 \pm 0.04$ \\
1 good quality lepton & $0.331 \pm 0.004$ & $0.0350 \pm 0.0003$ & $5.56 \pm 0.04$ & $4.58 \pm 0.03$ \\
Lepton isolation & $0.299 \pm 0.004$ & $0.0318 \pm 0.0003$ & $4.83 \pm 0.04$ & $4.11 \pm 0.03$ \\
QCD rejection & $0.281 \pm 0.004$ & $0.0273 \pm 0.0003$ & $4.50 \pm 0.04$ & $3.53 \pm 0.03$ \\
$80<m_{\gamma \gamma}<160$ & $0.271 \pm 0.004$ & $0.0259 \pm 0.0003$ & $2.04 \pm 0.02$ & $1.42 \pm 0.02$ \\
\hline
\end{tabular}

Table 10.27. Expected rates (in $\mathrm{fb}$ ) after each stage of the event selection for reducible backgrounds. Contributions of the different $\mathrm{p}_{\mathrm{T}}$ bins are summed. Errors are statistical only.

\begin{tabular}{lccccc}
\hline & $\gamma \gamma$ & $\mathrm{W} \gamma$ & $\mathrm{b} \overline{\mathrm{b}}$ & $\mathrm{t} \overline{\mathrm{t}}$ & $\gamma$-jet (jet) \\
\hline$\sigma . \mathrm{BR}$ & $1.1 \times 10^{5}$ & $5.79 \times 10^{3}$ & $1.78 \times 10^{9}$ & $86.2 \times 10^{3}$ & $1.21 \times 10^{8}$ \\
Pre-selection: $\sigma . \mathrm{BR} . \varepsilon$ & 270.1 & 26.5 & $2.96 \times 10^{5}$ & $6.00 \times 10^{3}$ & $7.16 \times 10^{4}$ \\
Double photons HLT & $197.7 \pm 1.0$ & $16.8 \pm 0.1$ & $77120 \pm 764$ & $1948 \pm 17$ & $35045 \pm 256$ \\
2 isolated photons & $161.6 \pm 0.8$ & $9.97 \pm 0.07$ & $682 \pm 72$ & $31.2 \pm 2.2$ & $7235 \pm 115$ \\
1 good quality lepton & $27.3 \pm 0.3$ & $7.98 \pm 0.07$ & $311 \pm 49$ & $23.5 \pm 1.9$ & $2552 \pm 68$ \\
Lepton isolation & $9.8 \pm 0.2$ & $6.59 \pm 0.06$ & $(0.87)$ & $14.2 \pm 1.5$ & $209 \pm 20$ \\
QCD rejection & $7.6 \pm 0.2$ & $5.74 \pm 0.06$ & $(0.003)$ & $(0.35)$ & $(6.6)$ \\
$80<m_{\gamma \gamma}<160$ & $3.2 \pm 0.1$ & $2.40 \pm 0.04$ & $(0.001)$ & $(0.26)$ & $(3.7)$ \\
\hline
\end{tabular}

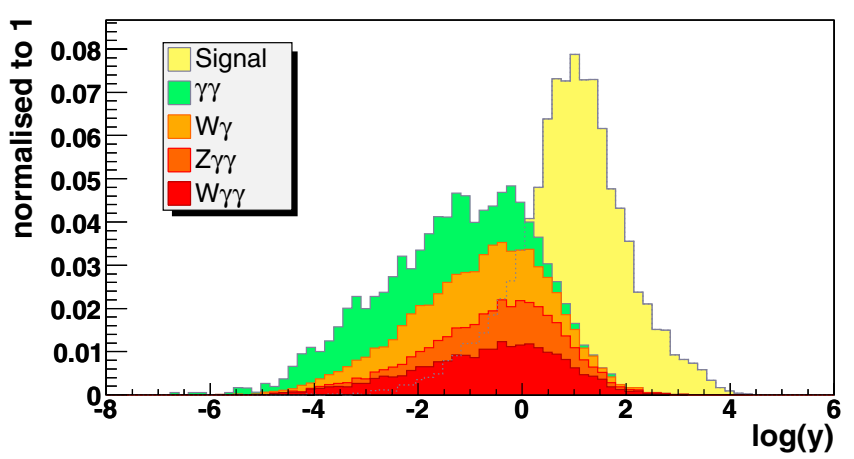

Figure 10.31. Distribution of the final combined variable for the signal $\left(m_{\mathrm{H}}=120 \mathrm{GeV} / \mathrm{c}^{2}\right)$ and for the background. The optimal working point is obtained with a cut $\log (y)>0.35$.

precise estimation of these backgrounds, the cut factorisation method has been applied and the result is given between parentheses in Table 10.27. After rejecting events outside the $80-160 \mathrm{GeV} / \mathrm{c}^{2}$ diphoton mass window, the expected rate of events is $0.297 \pm 0.003 \mathrm{fb}$ for signal and $13.1 \pm 2.6 \mathrm{fb}$ for background. Some simple kinematical variables are then used to form a final likelihood. The best discrimination was obtained with the transverse energy of the photons and of the lepton, the $\Delta R$ distances between lepton and each photon, the missing transverse energy, and the $\Delta \Phi$ angle between the directions of the missing transverse energy and of the highest $E_{\mathrm{T}}$ photon. The distribution of the resulting combined variable $y$ is shown in Fig. 10.31 for a Higgs boson mass of $120 \mathrm{GeV} / \mathrm{c}^{2}$. 

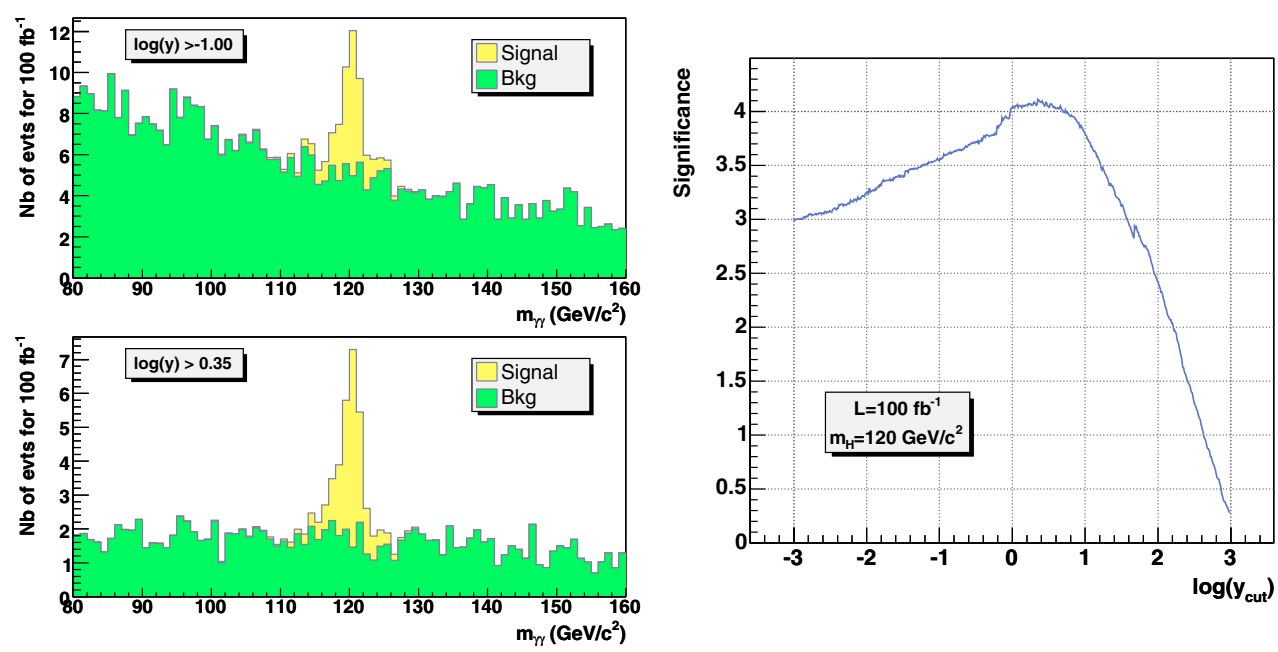

Figure 10.32. Left: Reconstructed $\gamma \gamma$ mass for different selection values on the final combined variable $y$ for an integrated luminosity of $100 \mathrm{fb}^{-1}$. Right: Statistical significance as a function of the cut on the combined variable $\log (y)$, for $m_{\mathrm{H}}=120 \mathrm{GeV} / \mathrm{c}^{2}$ and an integrated luminosity of $100 \mathrm{fb}^{-1}$. The highest significance is obtained with a cut $\log (y)>0.35$.

Table 10.28. Optimal working points for different Higgs boson mass hypotheses. The significance and the expected number of signal and background events are given for an integrated luminosity of $100 \mathrm{fb}^{-1}$.

\begin{tabular}{llllllllllll}
\hline $\begin{array}{l}m_{\mathrm{H}} \\
\left(\mathrm{GeV} / \mathrm{c}^{2}\right)\end{array}$ & $\begin{array}{l}\text { working point } \\
\log (y)>\end{array}$ & significance & $\mathrm{WH}$ & $\mathrm{ZH}$ & $\mathrm{W} \gamma \gamma$ & $\mathrm{Z} \gamma \gamma$ & $\mathrm{W} \gamma$ & $\gamma \gamma$ & $\gamma$-jet & $\mathrm{t} \overline{\mathrm{t}}$ & $\mathrm{b} \overline{\mathrm{b}}$ \\
\hline 115 & 0.41 & $4.30 \sigma$ & 22.1 & 1.8 & 49.3 & 30.9 & 33.0 & 10.2 & 1.7 & 0.16 & $10 \times 10^{-5}$ \\
120 & 0.35 & $4.09 \sigma$ & 20.7 & 1.6 & 51.2 & 36.2 & 34.5 & 12.4 & 1.9 & 0.15 & $10 \times 10^{-5}$ \\
130 & 0.68 & $3.64 \sigma$ & 14.6 & 1.3 & 30.7 & 16.9 & 18.7 & 6.0 & 1.4 & 0.10 & $4 \times 10^{-5}$ \\
140 & 0.99 & $3.35 \sigma$ & 11.4 & 1.0 & 18.9 & 10.3 & 10.6 & 3.7 & 1.0 & 0.04 & $1 \times 10^{-5}$ \\
150 & 0.83 & $2.87 \sigma$ & 10.4 & 0.9 & 20.2 & 11.7 & 12.3 & 5.4 & 1.1 & 0.03 & $3 \times 10^{-5}$ \\
\hline
\end{tabular}

10.2.8.4. Statistical method and optimisation. The statistical methods developed by the LEP Higgs working group [508,509] are used in this analysis to optimise the selection criteria and determine the statistical significance of the final results. To form the test-statistic, the three obvious variables to be used are the counting rates, the $\gamma \gamma$ invariant mass and the kinematic likelihood variable $y$. The limited statistics of the MC events prohibit however the use of a two-dimensional method for the determination of the Higgs boson discovery potential. So, only the counting rates and shape of the reconstructed $\gamma \gamma$ mass distribution will be used along with a cut on the combined likelihood variable $y$. The optimal working point is the $y$ cut value which maximises the discovery potential as shown in Fig. 10.32.

The list of the optimal working points obtained for the different Higgs boson mass hypotheses is given in Table 10.28. The significance and the expected number of signal and background events are given for a luminosity of $100 \mathrm{fb}^{-1}$. For the $\gamma$-jet, $\mathrm{t} \overline{\mathrm{t}}$ and $\mathrm{b} \overline{\mathrm{b}}$ backgrounds, the rates are estimated by the method of cut factorisation.

10.2.8.5. Use of real data in sidebands: systematic uncertainties. The signal is characterised by a strongly peaked diphoton mass and the $m_{\gamma \gamma}$ distribution of the background is quite 


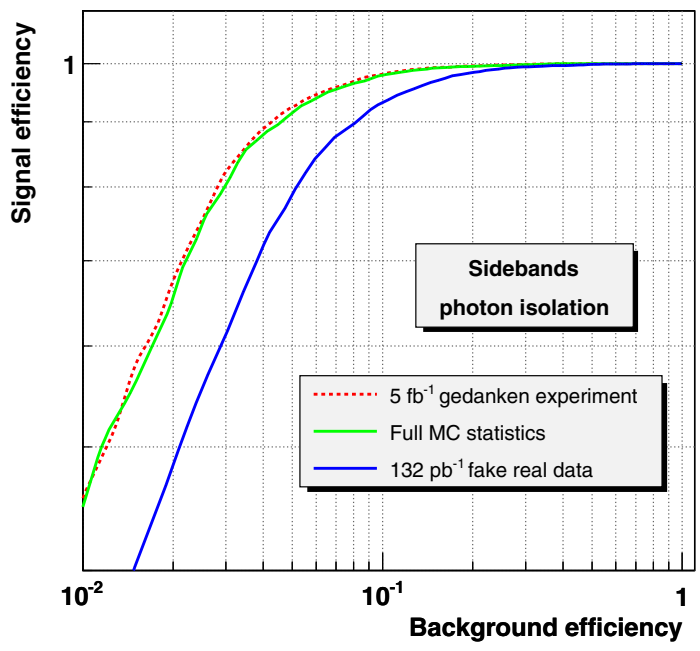

Figure 10.33. Comparison of the performance obtained when optimising the photon isolation likelihood with a sample of $132 \mathrm{fb}^{-1}$ of "fake real data" taken in the $20<m_{\gamma \gamma}<80 \mathrm{GeV} / \mathrm{c}^{2}$ sideband (dash-dotted line) with the performance obtained by the standard analysis using the full MC statistics (solid line). To increase the available statistics in the sideband, gedanken experiments were generated for an equivalent luminosity of $5 \mathrm{fb}^{-1}$. The results of the optimisation on these sideband events is represented by the dotted line.

smooth at the considered working points. Therefore, when real data will be available, the data taken in $m_{\gamma \gamma}$ sidebands will be used to optimise the likelihood analysis and to estimate the background.

Likelihood optimisation with sideband events. No kinematic observables were used to construct the four primary likelihoods aimed at rejecting multi-jet events to avoid correlations with the diphoton mass. If the shapes of the distributions of the variables used in the likelihoods are sufficiently similar for different diphoton mass regions, then data taken outside the signal region can be used to optimise the likelihoods. To test the feasibility of the method, a sample of "fake real data" (the number of MC events for each background is equal to the expected number of events for a given luminosity) taken in the $20<m_{\gamma \gamma}<80 \mathrm{GeV} / \mathrm{c}^{2}$ sideband is used to produce the reference S/B histograms of the likelihoods. The equivalent luminosity of the sample is limited to $132 \mathrm{pb}^{-1}$ by the available statistics and is composed of $4682 \mathrm{~b} \overline{\mathrm{b}}, 465 \gamma$-jet, $222 \mathrm{t} \overline{\mathrm{t}}, 2 \gamma \gamma, 1 \mathrm{~W} \gamma$ and $1 \mathrm{Z} \gamma \gamma$ events. The performance obtained with the likelihood on the events in the $80-160 \mathrm{GeV} / \mathrm{c}^{2}$ band is compared to the results obtained by the standard analysis optimised with the full MC statistics available. For the four global discriminant variables, up to $20 \%$ loss of efficiency is observed for the same rejection power. The degradation of the performance is mainly due to the insufficient statistics of $\gamma$-jet and $t \bar{t}$ events in $132 \mathrm{pb}^{-1}$ of data. To increase the size of the "fake data sample", gedanken experiments were generated using the fitted shapes of the variables used in the likelihoods (correlations between the variables are neglected). The results are presented in Fig. 10.33 for the photon isolation likelihood. An integrated luminosity of $5 \mathrm{fb}^{-1}$ will be sufficient to optimise the four primary likelihoods with the real data taken in the $m_{\gamma \gamma}$ sideband and to reproduce the results obtained when using the full MC statistics.

Background measurement from data. The $m_{\gamma \gamma}$ distribution of the background is smooth enough to be easily fit in the sideband to estimate the background in the signal region. 

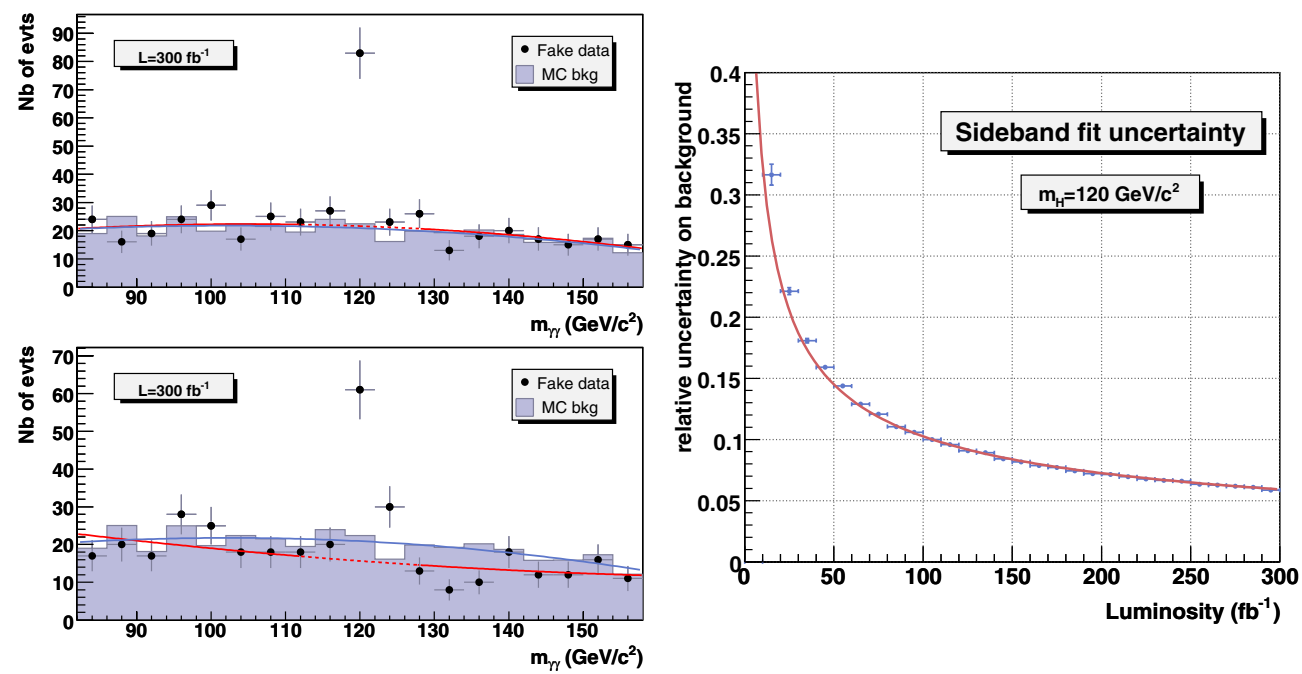

Figure 10.34. Left: Background measurement in the signal region with a fit on the $m_{\gamma \gamma}$ sideband. The fit of the full MC statistics is represented by the solid light gray line. The fit of the fake data (dark grey) is performed on the sidebands (i.e. after the exclusion of the signal window represented by the dotted line). Two gedanken experiments are represented for an integrated luminosity of $300 \mathrm{fb}^{-1}$ and a $120 \mathrm{GeV} / \mathrm{c}^{2}$ Higgs boson mass hypothesis. Right: Relative uncertainty on the background estimation by the sideband fit method as a function of the integrated luminosity with LHC running at high luminosity for a Higgs boson mass of $120 \mathrm{GeV} / \mathrm{c}^{2}$.

To optimise the method (size and position of the window, bin width, choice of the fit function) and to estimate the uncertainty on background, 10000 signal + background pseudoexperiments were generated for each Higgs boson mass and luminosity hypothesis, as illustrated in Fig. 10.34. For a luminosity of $100 \mathrm{fb}^{-1}$ and a Higgs boson mass of $120 \mathrm{GeV} / \mathrm{c}^{2}$, the background is measured with a precision of $11 \%$, and with a precision of $6.6 \%$ for $300 \mathrm{fb}^{-1}$.

Systematic uncertainties for signal and cross-section measurement. The theoretical crosssection error due to the scale variation are estimated to $\pm 3 \%$ for $\mathrm{WH}$ and $\mathrm{ZH}$ production for all considered Higgs boson masses [20]. The uncertainty on the parton density function of the CTEQ collaboration [12] is almost constant for the associated production $\mathrm{q} \overline{\mathrm{q}} \rightarrow \mathrm{VH}$ at the LHC and of the order of $4 \%$ over a Higgs boson mass range between 100 and $200 \mathrm{GeV} / \mathrm{c}^{2}$ [510]. The error on the measured luminosity is expected to be $3 \%$ for luminosity above $30 \mathrm{fb}^{-1}$. The error on the lepton or photon reconstruction and identification has been estimated to $1 \%$ for each photon and lepton. An error of $5 \%$ on the missing transverse energy, see Appendix B, propagated in the final likelihood gives a $-1.08 \%+0.49 \%$ variation of the final signal rate for $m_{\mathrm{H}}=120 \mathrm{GeV} / \mathrm{c}^{2}$. The quadratic sum of all these errors gives a $6 \%$ total error on the expected signal rate.

In the case of a Higgs boson discovery, this channel will be used to measure the crosssection times the branching ratio:

$$
\sigma_{s} \times B R=\frac{N_{s}}{\epsilon_{\text {sel }} \mathcal{L}}=\frac{N-N_{b}^{f i t}}{\epsilon_{\text {sel }} \mathcal{L}}
$$

where $N_{S}$ is the number of signal events given by the difference between the total number $N$ of observed events and the number $N_{b}^{f i t}$ of background events measured by the sideband fit. 

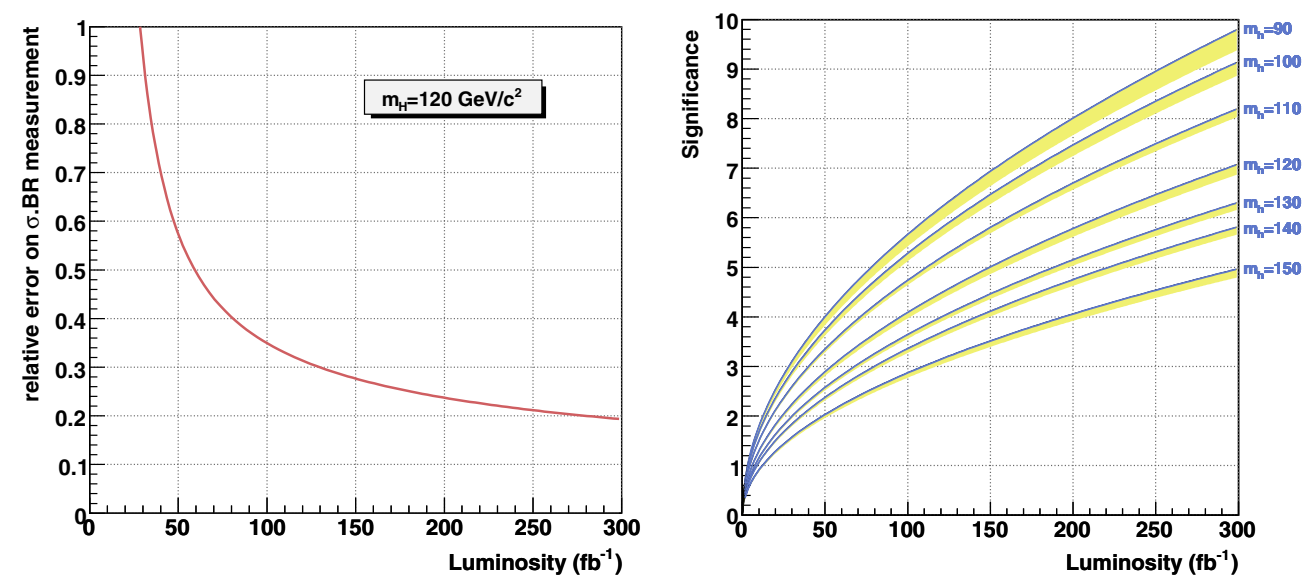

Figure 10.35. Left: Precision on the measurement of the product of cross-section and branching ratio as a function of the integrated luminosity with LHC running at high luminosity for a $120 \mathrm{GeV} / \mathrm{c}^{2}$ Higgs boson. Right: Statistical significance for different Higgs boson mass hypotheses as a function of the integrated luminosity with LHC running at high luminosity. The $1 \sigma$ systematic uncertainty is represented by the grey (yellow online) band.
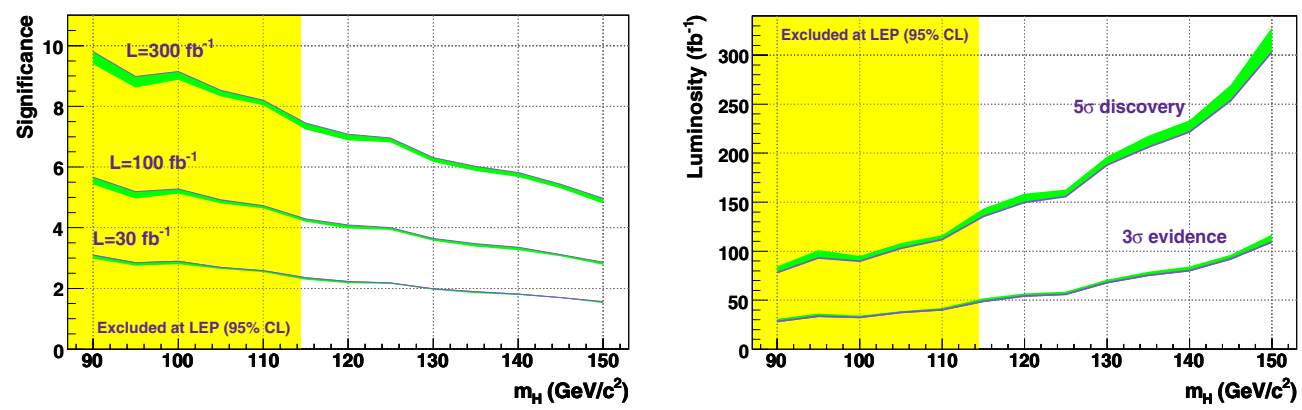

Figure 10.36. Statistical significance (left) and luminosity needed for a $5 \sigma$ or $3 \sigma$ observation (right) as a function of $m_{\mathrm{H}}$. The $1 \sigma$ systematic uncertainty is represented by the grey (yellow online) bands.

The total uncertainty on the measure is given by:

$$
\left(\frac{\Delta\left(\sigma_{s} \times B R\right)}{\sigma_{s} \times B R}\right)^{2}=\left(\frac{\Delta N}{N-N_{b}^{f i t}}\right)^{2}+\left(\frac{\Delta N_{b}^{f i t}}{N-N_{b}^{f i t}}\right)^{2}+\left(\frac{\Delta \mathcal{L}}{\mathcal{L}}\right)^{2}+\left(\frac{\Delta \epsilon_{s e l}}{\epsilon_{\text {sel }}}\right)^{2}
$$

The expected precision on the $\sigma \times B R$ measurement is represented as a function of the integrated luminosity in Fig. 10.35 . For a $120 \mathrm{GeV} / \mathrm{c}^{2}$ Higgs boson, the product of the crosssection and branching ratio will be measured with a precision of $35 \%$ after one year of LHC running at high luminosity, and with a precision of $19 \%$ after three years of high luminosity running.

10.2.8.6. Results for the Standard Model Higgs boson. The statistical significance is represented as a function of the luminosity in Fig. 10.35 for different $m_{\mathrm{H}}$ hypothesis. The statistical significance and the luminosity needed for a $5 \sigma$ or $3 \sigma$ observation are represented as a function of $m_{\mathrm{H}}$ in Fig. 10.36. One year of high luminosity running allows the observation 


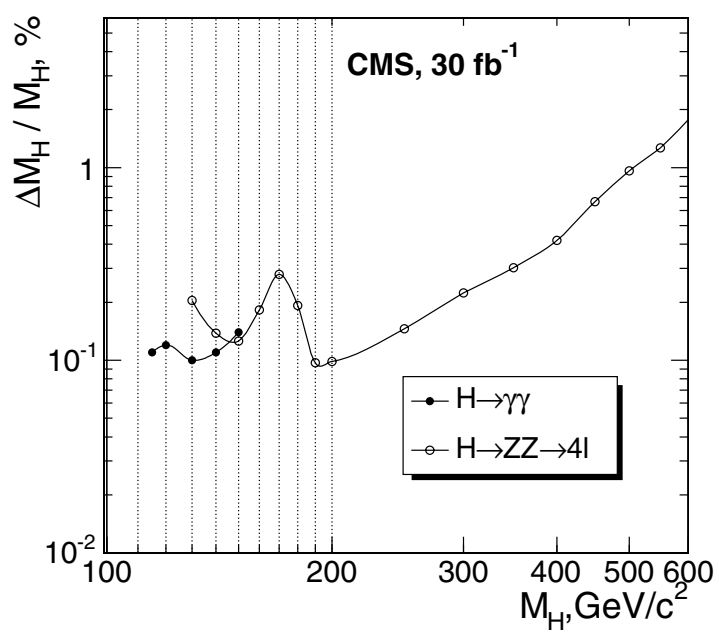

Figure 10.37. The statistical precision of the Higgs boson mass measurement for the $30 \mathrm{fb}^{-1}$ using inclusive Higgs boson production $\mathrm{pp} \rightarrow \mathrm{H}+\mathrm{X}$ and the $\mathrm{H} \rightarrow \gamma \gamma$ and $\mathrm{H} \rightarrow \mathrm{ZZ} \rightarrow 4 \ell$ decay modes.

at $3 \sigma$ of the SM Higgs boson up to $m_{\mathrm{H}}=150 \mathrm{GeV} / \mathrm{c}^{2}$, and three years of running at high luminosity are required to reach a $5 \sigma$ discovery.

\subsection{Discovery reach}

\subsubsection{Accuracy of the Higgs boson mass measurement}

Figure 10.37 shows the statistical precision of the Higgs boson mass measurement for the $30 \mathrm{fb}^{-1}$ using inclusive Higgs boson production $\mathrm{pp} \rightarrow \mathrm{H}+\mathrm{X}$ and the $\mathrm{H} \rightarrow \gamma \gamma$ and $\mathrm{H} \rightarrow$ $\mathrm{ZZ} \rightarrow 4 \ell$ decay modes.

\subsubsection{Discovery reach for the Standard Model Higgs boson}

This section summarises the discovery reach for the Standard Model Higgs boson. The NLO cross sections and branching ratios for the Higgs boson calculated with the programs HDECAY [41], HIGLU [40], VV2H, V2HV and HQQ [20] are used, as well as the NLO cross sections for the background processes, when available.

Figure 10.38 shows the integrated luminosity needed for the $5 \sigma$ discovery of the inclusive Higgs boson production $\mathrm{pp} \rightarrow \mathrm{H}+\mathrm{X}$ with the Higgs boson decay modes $\mathrm{H} \rightarrow \gamma \gamma, \mathrm{H} \rightarrow$ $\mathrm{ZZ} \rightarrow 4 \ell$, and $\mathrm{H} \rightarrow \mathrm{WW} \rightarrow 2 \ell 2 \nu$.

Figure 10.39 shows the signal significance as a function of the Higgs boson mass for $30 \mathrm{fb}^{-1}$ of the integrated luminosity for the different Higgs boson production and decay channels.

\subsubsection{Study of CP properties of the Higgs boson using angle correlation in the} $\Phi \rightarrow Z Z \rightarrow \mathrm{e}^{+} \mathrm{e}^{-} \mu^{+} \mu^{-}$process

The most general $\Phi V V$ coupling $\left(V=W^{ \pm}, Z^{0}\right)$ for spin-0 Higgs boson $\Phi$ ( $\Phi$ means the Higgs particle with unspecified $C P$-parity, while $H(h)$ and $A$ mean the scalar and 


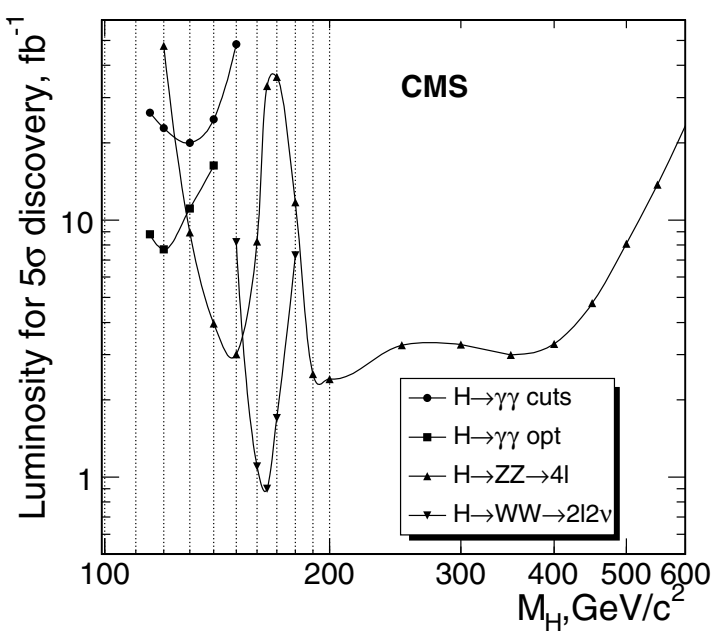

Figure 10.38. The integrated luminosity needed for the $5 \sigma$ discovery of the inclusive Higgs boson production $\mathrm{pp} \rightarrow \mathrm{H}+\mathrm{X}$ with the Higgs boson decay modes $\mathrm{H} \rightarrow \gamma \gamma, \mathrm{H} \rightarrow \mathrm{ZZ} \rightarrow 4 \ell$, and $\mathrm{H} \rightarrow \mathrm{WW} \rightarrow 2 \ell 2 v$

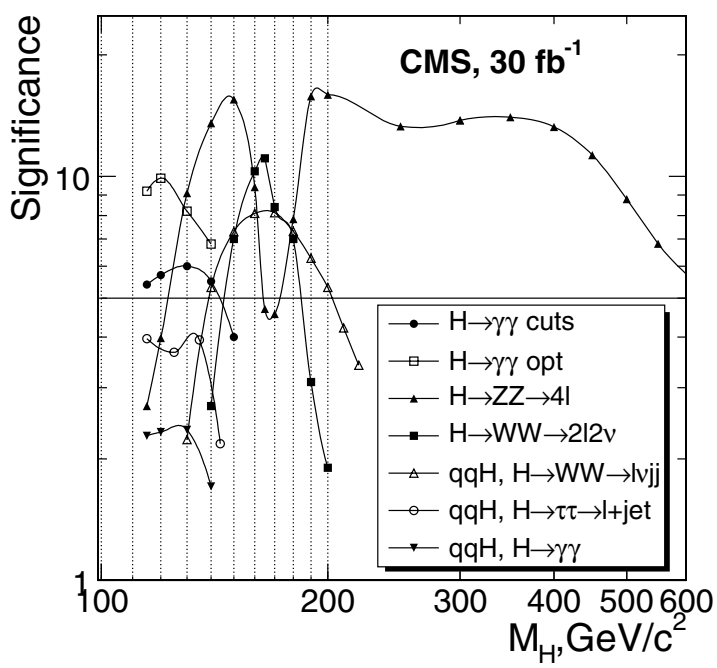

Figure 10.39. The signal significance as a function of the Higgs boson mass for $30 \mathrm{fb}^{-1}$ of the integrated luminosity for the different Higgs boson production and decay channels.

pseudoscalar Higgs particles, respectively) looks as follows [511-514 ]:

$$
\mathcal{C}_{\Phi V V}^{J=0}=\kappa \cdot g^{\mu \nu}+\frac{\zeta}{m_{V}^{2}} \cdot p^{\mu} p^{\nu}+\frac{\eta}{m_{V}^{2}} \cdot \epsilon^{\mu \nu \rho \sigma} k_{1 \rho} k_{2 \sigma},
$$

where $k_{1}, k_{2}$ are four-momenta of vector bosons $V$ and $p \equiv k_{1}+k_{2}$ is four-momentum of the Higgs boson. In the present analysis a simplified version of above $\Phi V V$ coupling (Eq. 10.5) is studied with a Standard-Model-like scalar and a pseudoscalar contributions (i.e. $\kappa, \eta \neq 0$ and $\zeta=0$ ). To study deviations from the Standard Model $\Phi Z Z$ coupling we take $\kappa=1^{47}$.

${ }^{47}$ The $\Phi V V$ coupling with $\kappa=1$ and arbitrary $\eta$ is implemented in the PYTHI a generator. 


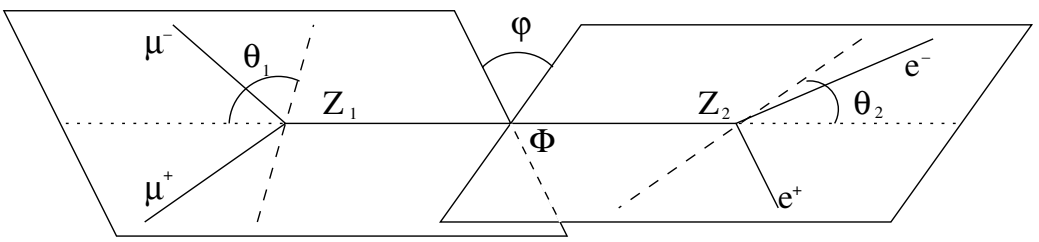

Figure 10.40. Definitions of the angles in the $\Phi \rightarrow \mathrm{ZZ} \rightarrow \mathrm{e}^{+} \mathrm{e}^{-} \mu^{+} \mu^{-}$process.

The decay width for the $\Phi \rightarrow Z Z \rightarrow\left(\ell_{1} \bar{\ell}_{1}\right)\left(\ell_{2} \bar{\ell}_{2}\right)$ process consists now of three terms: a scalar one (denoted by $H$ ), a pseudoscalar one $\sim \eta^{2}$ (denoted by $A$ ) and the interference term violating $\mathrm{CP} \sim \eta($ denoted by $I)$ :

$$
d \Gamma(\eta) \sim H+\eta I+\eta^{2} A .
$$

This way the Standard Model scalar $(\eta=0)$ and the pseudoscalar (in the limit $|\eta| \rightarrow \infty$ ) contributions could be recovered. It is convenient to introduce a new parameter $\xi$, defined by $\tan \xi \equiv \eta$, which is finite and has values between $-\pi / 2$ and $\pi / 2$. Expressions for $H, A$ and $I$ can be found in article [512 ].

In study of the CP-parity of the Higgs boson two angular distributions were used. The first one is a distribution of the angle $\varphi$ (called plane or azimuthal angle) between the planes of two decaying $Z s$ in the Higgs boson rest frame. The negatively charged leptons were used to fix plane orientations. The second one is a distribution of the polar angle $\theta$, in the $Z$ rest frame, between momentum of negatively charged lepton and the direction of motion of $Z$ boson in the Higgs boson rest frame (Figure 10.40).

The analysis was performed for scalar, pseudoscalar and CP-violating Higgs boson states, the latter for $\tan \xi= \pm 0.1, \pm 0.4, \pm 1$ and \pm 4 .

A detailed description of the analysis can be found in [515].

10.3.3.1. Generation and event selections. The production and decay of the scalar, pseudoscalar and CP-violating Higgs boson states were generated using PYTHIA [69] for three masses of the Higgs boson, $\mathrm{M}_{\Phi}=200,300$ and $400 \mathrm{GeV} / \mathrm{c}^{2}$. Backgrounds and event selections are the same as in the analysis of the Standard Model Higgs boson $\mathrm{H} \rightarrow \mathrm{ZZ} \rightarrow$ $\mathrm{e}^{+} \mathrm{e}^{-} \mu^{+} \mu^{-}$described in Section 10.2.1. The reconstructed angular distributions after all selections for the signal with mass $\mathrm{M}_{\Phi}=300 \mathrm{GeV} / \mathrm{c}^{2}$ for various values of the parameter $\xi$, and for the background are shown in Fig. 10.41 at $60 \mathrm{fb}^{-1}$. The Standard-Model signal crosssection and branching ratio were used for the signal normalisation in Fig. 10.41.

10.3.3.2. Determination of the parameter $\xi$. The parameter $\xi$ was determined by maximisation of the likelihood function $\mathcal{L}(\xi, R)$, which was constructed from angular distributions and invariant mass distribution of four leptons, for the signal and the background. The function depends on two parameters: $\xi$ describing CP property of the signal, and $R$ describing fraction of the signal in the data sample. The function has the following form:

$$
\mathcal{L}(\xi, R) \equiv 2 \sum_{x_{i} \in \text { data }} \log \mathcal{Q}\left(\xi, R ; x_{i}\right),
$$

where $\mathcal{Q}\left(\xi, R ; x_{i}\right) \equiv R \cdot \mathcal{P} \mathcal{D} \mathcal{F}_{S}\left(\xi ; x_{i}\right)+(1-R) \cdot \mathcal{P} \mathcal{D} \mathcal{F}_{B}\left(x_{i}\right)$. 

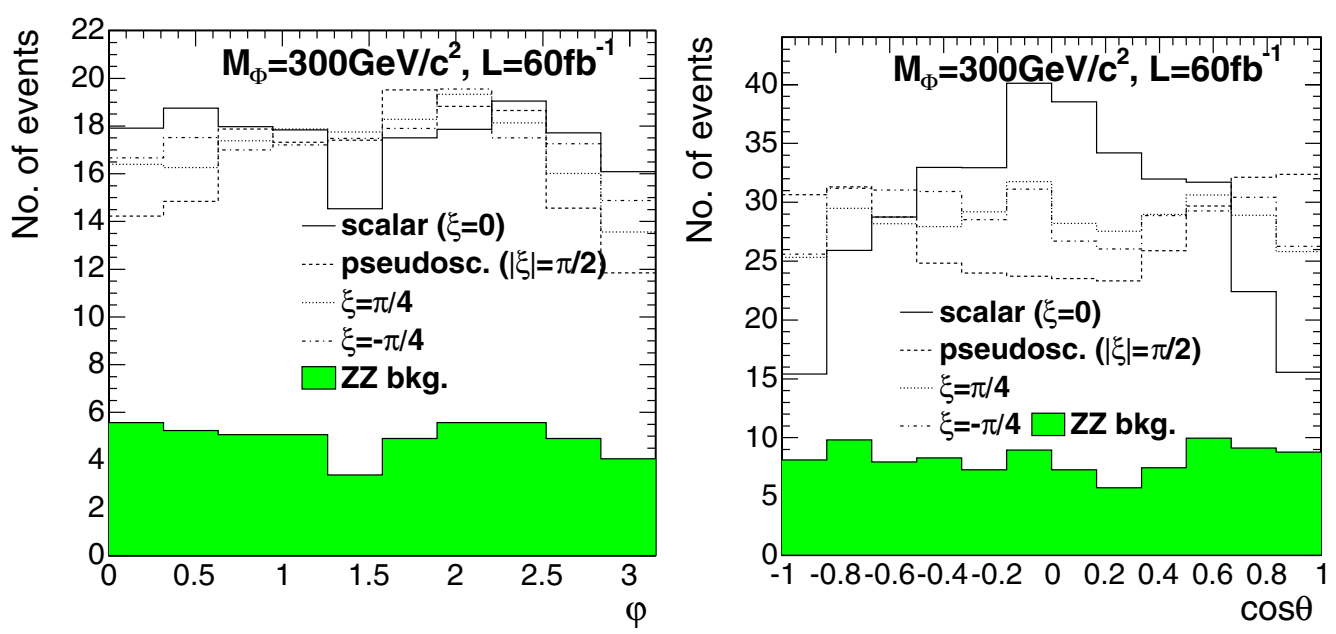

Figure 10.41. The $\varphi$-distributions (left) and the $\theta$-distributions (right) for various values of the parameter $\xi$ after final selections at $60 \mathrm{fb}^{-1}$. Empty histograms - the signal for $\mathrm{M}_{\Phi}=300 \mathrm{GeV} / \mathrm{c}^{2}$ and $\xi=0$ (scalar), $\xi=-\pi / 4, \xi=+\pi / 4$ and $|\xi|=\pi / 2$ (pseudoscalar). The filled histogram the ZZ background. The Standard-Model signal cross-section and branching ratio were used for the signal normalisation.

$\mathcal{P D} \mathcal{F}_{B}\left(x_{i}\right)$ and $\mathcal{P D} \mathcal{F}_{S}\left(\xi ; x_{i}\right)$ are probability density functions for background and signal respectively; $\left\{x_{i}\right\}$ are values of the measured quantities (angles and invariant mass) in the event $i . \mathcal{P} \mathcal{D} \mathcal{F}_{\mathrm{S}}$ are products of probability densities $\mathcal{P}^{M}, \mathcal{P}^{\varphi}, \mathcal{P}^{\cos \theta_{1,2}}$ of four leptons invariant mass and angles $\varphi$ and $\cos \theta_{1,2}: \mathcal{P} \mathcal{D} \mathcal{F} \equiv \mathcal{P}^{M} \cdot \mathcal{P}^{\varphi} \cdot \mathcal{P}^{\cos \theta_{1}} \cdot \mathcal{P}^{\cos \theta_{2}}$. The $\mathcal{P}^{M}, \mathcal{P}^{\varphi}, \mathcal{P}^{\cos \theta_{1,2}}$ are obtained by the Monte Carlo technique, using normalised histograms of given quantities after the final selection.

A part of the function $\mathcal{Q}$ which describes angular distributions of signal depends on the parameter $\xi$, namely from Eq. (10.6) we obtain:

$$
\begin{aligned}
& \mathcal{P}(\xi) \equiv \mathcal{P}_{S}^{\varphi}(\xi) \cdot \mathcal{P}_{S}^{\cos \theta_{1}}(\xi) \cdot \mathcal{P}_{S}^{\cos \theta_{2}}(\xi) \equiv \\
& \left(\mathcal{H}+\tan \xi \cdot \mathcal{I}+\tan ^{2} \xi \cdot a^{2} \mathcal{A}\right) /\left(1+a^{2} \tan ^{2} \xi\right),
\end{aligned}
$$

where $\mathcal{H} \equiv \mathcal{P}_{H}^{\varphi} \cdot \mathcal{P}_{H}^{\cos \theta_{1}} \cdot \mathcal{P}_{H}^{\cos \theta_{2}}$ and $\mathcal{A} \equiv \mathcal{P}_{A}^{\varphi} \cdot \mathcal{P}_{A}^{\cos \theta_{1}} \cdot \mathcal{P}_{A}^{\cos \theta_{2}}$ are probability densities obtained by the Monte Carlo technique for the scalar $(\mathrm{H})$ and the pseudoscalar (A) Higgs boson, respectively. The parameter $a^{2}$ is a (mass dependent) relative strength of the pseudoscalar and scalar couplings. For example $a^{2}=0.51,1.65,1.79$ for $\mathrm{M}_{\Phi}=200,300,400 \mathrm{GeV} / \mathrm{c}^{2}$, respectively. The $\mathcal{I}$ is a normalised product of angular distributions for the CP-violating term. The $\mathcal{I}$ is not always positive, and its integral is equal to zero, so it is not possible to simulate it separately. The $\mathcal{I}$ contribution can be obtained indirectly from the combined probability density for the signal with non-zero value of the parameter $\xi$. For example by introducing $\mathcal{P}_{+} \equiv \mathcal{P}(\pi / 4)=\left(\mathcal{H}+\mathcal{I}+a^{2} \mathcal{A}\right) /\left(1+a^{2}\right)$ and $\mathcal{P}_{-} \equiv \mathcal{P}(-\pi / 4)=\left(\mathcal{H}-\mathcal{I}+a^{2} \mathcal{A}\right) /\left(1+a^{2}\right)$ we have $\mathcal{I}=\left(1+a^{2}\right) / 2 \cdot\left(\mathcal{P}_{+}-\mathcal{P}_{-}\right)$. Finally we obtain:

$$
\mathcal{P}(\xi) \equiv\left[\mathcal{H}+\tan \xi \cdot \frac{1+a^{2}}{2} \cdot\left(\mathcal{P}_{+}-\mathcal{P}_{-}\right)+\tan ^{2} \xi \cdot a^{2} \mathcal{A}\right] /\left(1+a^{2} \tan ^{2} \xi\right)
$$



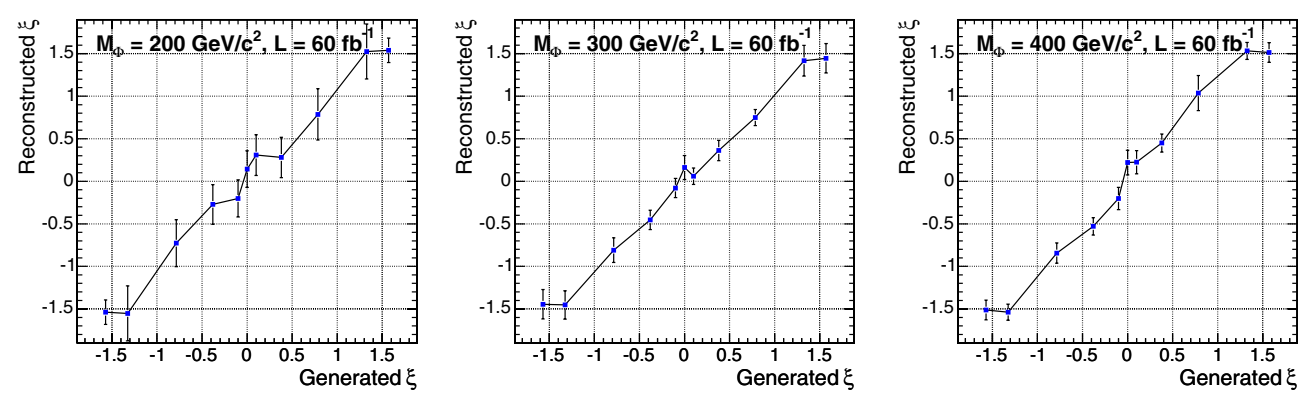

Figure 10.42. Reconstructed value of the parameter $\xi$ as function of the generated value of the parameter $\xi$ for $\mathcal{L}=60 \mathrm{fb}^{-1}$ and Higgs boson masses $\mathrm{M}_{\Phi}=200,300,400 \mathrm{GeV} / \mathrm{c}^{2}$. Uncertainties correspond to one standard deviation. The Standard-Model signal cross-section and branching ratio were used.
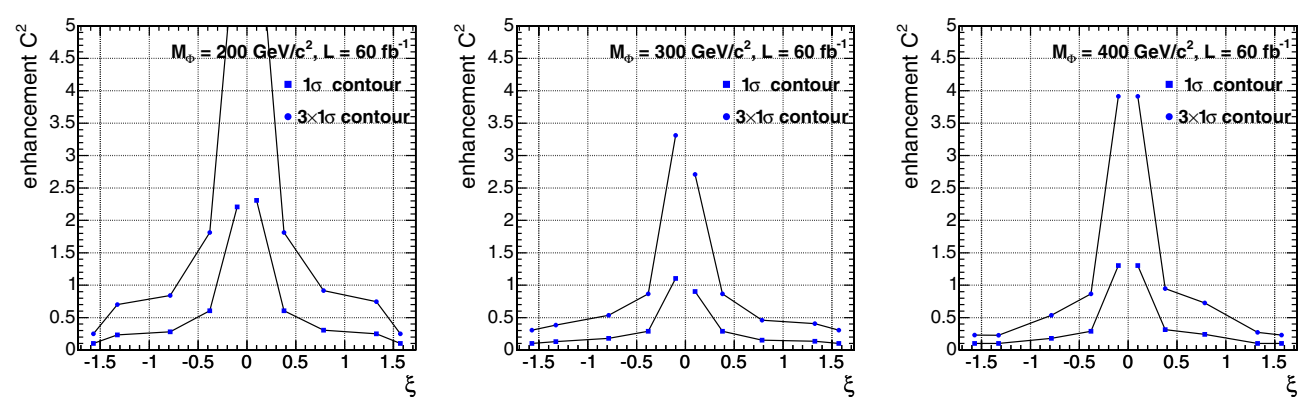

Figure 10.43. The minimal value of the factor $C^{2}$ needed to exclude the Standard Model, scalar Higgs boson at $\mathrm{N} \sigma$ level $(\mathrm{N}=1,3)$ as a function of the parameter $\xi$ for the Higgs boson masses $\mathrm{M}_{\Phi}=200,300$ and $400 \mathrm{GeV} / \mathrm{c}^{2}$ (from left to right) at $60 \mathrm{fb}^{-1}$.

10.3.3.3. Results. After selection all background contributions, but $\mathrm{ZZ} \rightarrow \mathrm{e}^{+} \mathrm{e}^{-} \mu^{+} \mu^{-}$, are negligible, therefore only these events were used to construct probability density function for the background. Signal probability density functions were constructed using samples of scalar Higgs boson $(\mathrm{H})$, pseudoscalar $(\mathrm{A})$ and $\mathcal{P}_{+}, \mathcal{P}_{-}$samples $(\xi= \pm \pi / 4)$.

For each value of the parameter $\xi$ and for each Higgs-boson mass we made 200 pseudo-experiments for the integrated luminosity $\mathcal{L}=60 \mathrm{fb}^{-1}$. For each pseudo-experiment we randomly selected events from the signal and background samples to form a test sample. The number of selected events was given by a Poisson probability distribution with mean defined by the process cross-section, selection efficiency and the examined luminosity. Then we performed a maximisation of the likelihood function $\mathcal{L}(\xi, R)$ for the test sample to obtain a value of the parameter $\xi$. The generated and reconstructed values of the parameter $\xi$ with its uncertainty, obtained for three masses of the Higgs boson are shown in Fig. 10.42. The Standard-Model signal cross-section and branching ratio were used to normalise signal for each value of the parameter $\xi$.

An influence of enhancement (or suppression) factor $C^{2}$ of the Higgs boson production cross section times branching ratio, in respect to the Standard Model

$$
C^{2}=(\sigma \times B r) /\left(\sigma_{S M} \times B r_{S M}\right)
$$

on the accuracy of the $\xi$ measurement and thus, on possibility to exclude the Standard Model, scalar Higgs boson was studied. It was found that the precision of $\xi$ measurement 
is approximately proportional to $1 / C$ (i.e. it depends on square-root of number of events, as one can expect):

$$
\Delta \xi\left(\xi, C^{2}\right) \equiv \frac{\Delta \xi_{S M}(\xi)}{\sqrt{C^{2}}} .
$$

A value of $\Delta \xi_{\mathrm{SM}}(\xi)$ corresponds to the precision of the parameter $\xi$ measurement assuming the Standard Model Higgs boson production cross section times branching ratio. It is shown as the error bars in Fig. 10.42. Figure 10.43 shows the minimal value of the factor $C^{2}$ needed to exclude the SM Higgs boson at $\mathrm{N} \sigma$ level $(\mathrm{N}=1,3)$, where $\mathrm{N}=\xi / \Delta \xi$, as a function of the parameter $\xi$. 


\section{Chapter 11. MSSM Higgs Bosons}

\subsection{Introduction}

Supersymmetric extensions of the SM [516-520] are strongly motivated by the idea of providing a solution of the hierarchy problem in the Higgs sector. They allow for a light Higgs particle in the context of GUTs [521], in contrast with the SM, where the extrapolation requires an unsatisfactory fine-tuning of the SM parameters. Supersymmetry is a symmetry between fermionic and bosonic degrees of freedom and thus the most general symmetry of the $S$-matrix. The minimal supersymmetric extension of the SM (MSSM) yields a prediction of the Weinberg angle in agreement with present experimental measurements if embedded in a SUSY-GUT [522,523]. Moreover, it does not exhibit any quadratic divergences, in contrast with the SM Higgs sector. Owing to the large top quark mass SUSY-GUTs develop electroweak symmetry breaking at the electroweak scale dynamically [524-527]. The lightest supersymmetric particle offers a proper candidate for the Cold Dark Matter content of the universe, if $R$-parity is conserved. Finally, local supersymmetry enforces gravitational interactions.

In the MSSM two isospin Higgs doublets have to be introduced in order to preserve supersymmetry [525, 528,529]. After the electroweak symmetry-breaking mechanism, three of the eight degrees of freedom are absorbed by the $Z$ and $W$ gauge bosons, leading to the existence of five elementary Higgs particles. These consist of two CP-even neutral (scalar) particles $h, H$, one CP-odd neutral (pseudoscalar) particle $A$, and two charged particles $H^{ \pm}$. In order to describe the MSSM Higgs sector one has to introduce four masses $M_{h}, M_{H}, M_{A}$ and $M_{H^{ \pm}}$and two additional parameters, which define the properties of the scalar particles and their interactions with gauge bosons and fermions: the mixing angle $\beta$, related to the ratio of the two vacuum expectation values, $\tan \beta=v_{2} / v_{1}$, and the mixing angle $\alpha$ in the neutral $\mathrm{CP}$-even sector. Due to supersymmetry there are several relations among these parameters, and only two of them are independent at leading order. In the absence of CP-violation they are usually chosen as $M_{A}$ and $\tan \beta$. The other Higgs-boson masses and mixing angles are calculable in terms of the other MSSM parameters. Measuring the masses and angles will constitute an important consistency check of the MSSM.

At tree-level the following mass hierarchies hold: $M_{h}<M_{Z}, M_{A}<M_{H}$ and $M_{W}<$ $M_{H^{ \pm}}$. The tree-level bound on $M_{h}$ receives large corrections from SUSY-breaking effects in the Yukawa sector of the theory. The leading one-loop correction is proportional to $m_{t}^{4}$. The leading logarithmic one-loop term (for vanishing mixing between the scalar top quarks) reads [530-536]

$$
\Delta M_{h}^{2}=\frac{3 G_{\mu} m_{t}^{4}}{\sqrt{2} \pi^{2} \sin ^{2} \beta} \ln \left(\frac{m_{\tilde{t}_{1}} m_{\tilde{t}_{2}}}{m_{t}^{2}}\right),
$$

where $G_{\mu}$ is the Fermi constant, and $m_{\tilde{t}_{1,2}}$ are the two stop masses. Corrections of this kind have drastic effects on the predicted value of $M_{h}$ and many other observables in the MSSM Higgs sector. The higher-order contributions can shift $M_{h}$ by 50-100\% [143, 144, 537-548]. The corrections to the MSSM Higgs boson sector have been evaluated in several approaches. The status of the available calculations can be summarised as follows. For the one-loop part, the complete result within the MSSM is known [530-532, 536, 549-552]. The by far dominant one-loop contribution is the $\mathcal{O}\left(\alpha_{t}\right)$ term due to top and stop loops $\left(\alpha_{t} \equiv h_{t}^{2} /(4 \pi), h_{t}\right.$ being the top-quark Yukawa coupling). Concerning the two-loop effects, their computation is quite advanced and has now reached a stage such that all the presumably dominant contributions are known [143, 538-543, 545-548, 553-563]. They include (evaluated for vanishing external momenta) the strong corrections, $\mathcal{O}\left(\alpha_{t} \alpha_{s}\right)$, and Yukawa corrections, $\mathcal{O}\left(\alpha_{t}^{2}\right)$, to the dominant 


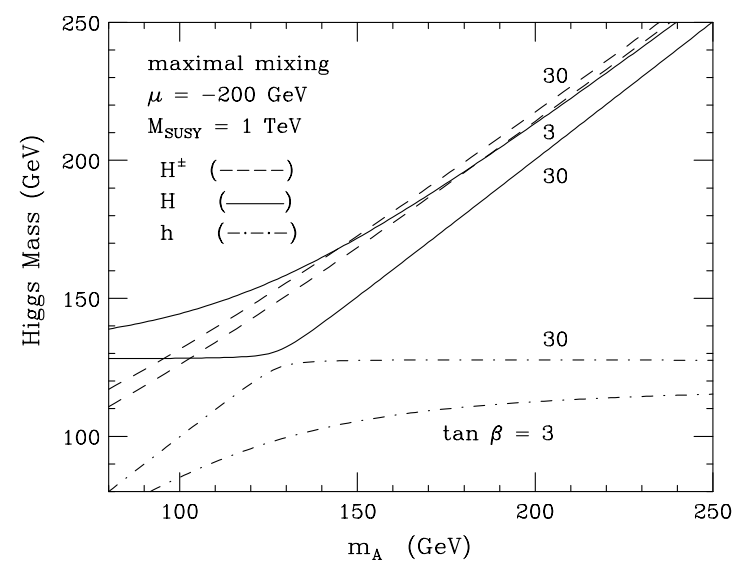

Figure 11.1. The CP-even and charged MSSM Higgs boson masses as a function of $M_{A}$ for $\tan \beta=3$ and 30, including radiative corrections [565].

one-loop $\mathcal{O}\left(\alpha_{t}\right)$ term, as well as the strong corrections to the bottom/sbottom one-loop $\mathcal{O}\left(\alpha_{b}\right)$ term $\left(\alpha_{b} \equiv h_{b}^{2} /(4 \pi)\right)$, i.e. the $\mathcal{O}\left(\alpha_{b} \alpha_{s}\right)$ contribution. The latter can be relevant for large values of $\tan \beta$. For the (s)bottom corrections the all-order resummation of the $\tan \beta$-enhanced terms, $\mathcal{O}\left(\alpha_{b}\left(\alpha_{s} \tan \beta\right)^{n}\right)$, has also been computed. Finally, the $\mathcal{O}\left(\alpha_{t} \alpha_{b}\right)$ and $\mathcal{O}\left(\alpha_{b}^{2}\right)$ corrections have been obtained. The higher-order corrections shift the upper bound of $M_{h}$ to $M_{h} \lesssim 135 \mathrm{GeV}$ $[143,144]$. The remaining theoretical uncertainty on $M_{h}$ has been estimated to be below $\sim 3 \mathrm{GeV}[144,564]$. Besides the masses of the Higgs bosons, also their couplings are affected by large higher-order corrections (see below).

An important feature of the MSSM Higgs sector is that for large pseudoscalar masses $M_{A}$ the light scalar Higgs mass reaches its upper bound and becomes SM-like. Moreover, for large values of $\tan \beta$ the down(up)-type Yukawa couplings are strongly enhanced (suppressed) apart from the region, where the light (heavy) scalar is at its upper (lower) mass bound. The radiatively corrected Higgs masses are depicted in Fig. 11.1.

The LEP experiments have searched for the MSSM Higgs bosons via the Higgs-strahlung process $e^{+} e^{-} \rightarrow Z+h / H$ and the associated production $e^{+} e^{-} \rightarrow A+h / H$ for the neutral Higgs particles and $e^{+} e^{-} \rightarrow H^{+} H^{-}$for the charged Higgs bosons. Neutral Higgs masses $M_{A} \lesssim 91.9 \mathrm{GeV} / \mathrm{c}^{2}$ and $M_{h / H} \lesssim 91 \mathrm{GeV} / \mathrm{c}^{2}$ are excluded [566] as well as charged Higgs masses $M_{H^{ \pm}} \lesssim 78.6 \mathrm{GeV} / \mathrm{c}^{2}[567]$.

The lightest Higgs boson $h$ will mainly decay into $b \bar{b}$ and $\tau^{+} \tau^{-}$pairs, since its mass is below $\sim 135 \mathrm{GeV} / \mathrm{c}^{2}$, see Fig. 11.2a. Close to its upper bound in mass all decay modes as for the SM Higgs boson open up rapidly. For large values of $\tan \beta$ the heavy scalar and pseudoscalar Higgs particles $H, A$ will decay predominantly into $b \bar{b}, \tau^{+} \tau^{-}$pairs, too, due to the enhanced Yukawa couplings for down-type fermions. The branching ratios for the decays into $b \bar{b}$ and $\tau^{+} \tau^{-}$are about $90 \%$ and $10 \%$ respectively. Other heavy scalar Higgs decay modes as $H \rightarrow t \bar{t}, W^{+} W^{-}, Z Z, h h, A A$ develop sizeable branching ratios only for small values of $\tan \beta$ (see Fig. 11.2b) and analogously the pseudoscalar Higgs decays $A \rightarrow t \bar{t}, g g, Z h$ (see Fig. 11.2c). The charged Higgs bosons decay mainly into $\tau \nu_{\tau}$ pairs for $M_{H^{ \pm}} \lesssim 180 \mathrm{GeV} / \mathrm{c}^{2}$ and into $t b$ final states above (see Fig. 11.2d). All other decay modes do not acquire branching ratios larger than a few per cent. The (SUSY-)QCD [385-391, 549, 562, 568] and (SUSY-)electroweak corrections [392-395, 568,569] to the fermionic decay modes are sizeable. In addition to the usual large QCD corrections, significant corrections arise from 

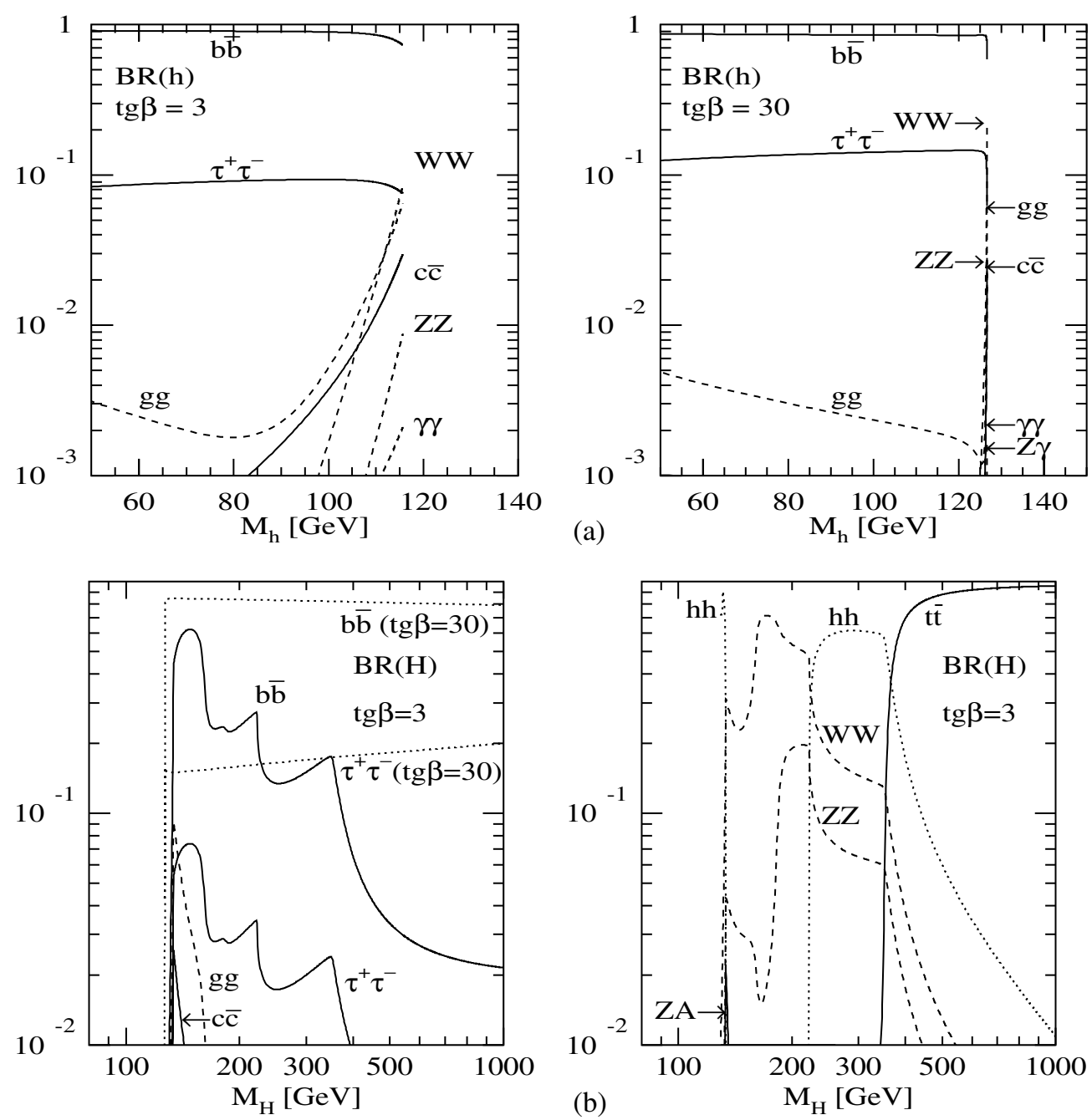

Figure 11.2. Branching ratios of the MSSM Higgs bosons $h, H, A, H^{ \pm}$for non-SUSY decay modes as a function of the masses for two values of $\tan \beta=3,30$ and maximal mixing. The common squark mass has been chosen as $M_{S}=1 \mathrm{TeV} / \mathrm{c}^{2}$. The other SUSY-parameters have been chosen as $M_{2}=m_{\tilde{g}}=\mu=1 \mathrm{TeV} / \mathrm{c}^{2}$ and $A_{t, b}=2783(2483) \mathrm{TeV} / \mathrm{c}^{2}$ for $\tan \beta=3(30)$. (Continued on next page.)

virtual sbottom/stop and gluino/gaugino exchange contributions in the $h, H, A \rightarrow b \bar{b}$ and $H^{ \pm} \rightarrow t b$ decay modes $[549,562,568,569]$. The dominant part of the latter corrections can be absorbed in improved bottom Yukawa couplings. In this way these contributions can also be resummed up to all orders thus yielding reliable perturbative results $[560,563]$. The rare photonic decay modes $h, H, A \rightarrow \gamma \gamma$ are mediated by $W, t, b$ loops as in the SM Higgs case and additional contributions from charged Higgs bosons, charginos and sfermions, if these virtual particles are light enough $[20,369,370]$. The QCD corrections to these decay modes can reach a few per cent in the relevant mass regions [396-402]. If decays into supersymmetric particles, i.e. gauginos and sfermions, are possible, they acquire significant branching ratios and can even be the dominant decay modes [20,369, 370, 570, 571]. In contrast to the SM the 

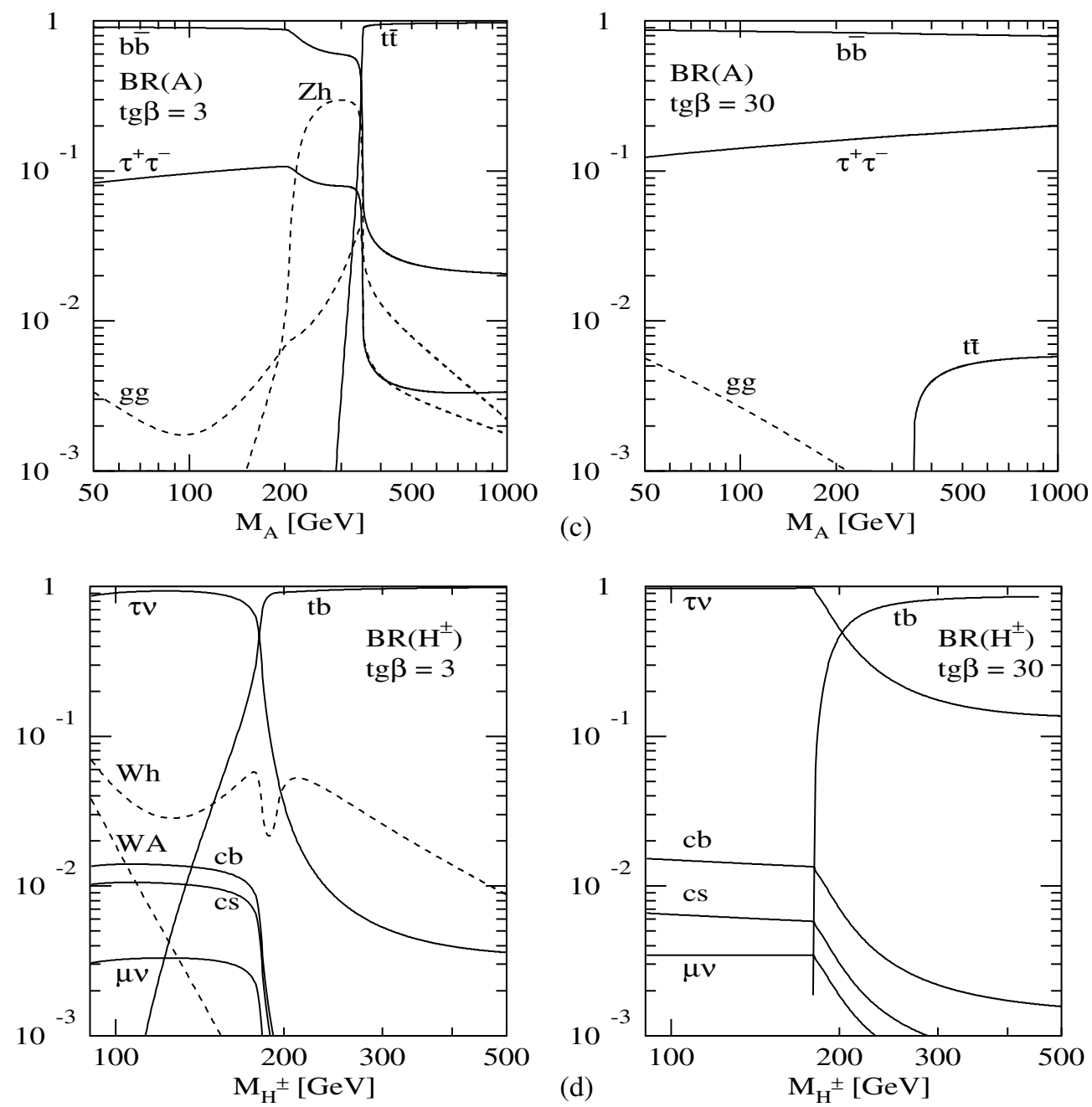

Figure 11.2. Continued.

total widths of the MSSM Higgs bosons do not exceed several tens of GeV, so that the MSSM Higgs particles appear as narrow resonances.

The dominant neutral MSSM Higgs production mechanisms for small and moderate values of $\tan \beta$ are the gluon fusion processes

$$
g g \rightarrow h, H, A
$$

which are mediated by top and bottom loops as in the SM case, but in addition by stop and sbottom loops for the scalar Higgs bosons $h, H$, if the squark masses are below about $400 \mathrm{GeV} / \mathrm{c}^{2}$ [572]. The NLO QCD corrections to the quark loops are known in the heavy quark limit as well as including the full quark mass dependence [409-411,413-416]. They increase the cross sections by up about $100 \%$ for smaller $\tan \beta$ and up to about $40 \%$ for very large $\tan \beta$, where the bottom loop contributions become dominant due to the strongly enhanced bottom Yukawa couplings. The limit of heavy quarks is only applicable for $\tan \beta \lesssim 5$ within about $20-25 \%$, if full mass dependence of the LO terms is taken into 
account $[20,369,370,412]$. Thus the available NNLO QCD corrections in the heavy quark limit [417-420] can only be used for small and moderate $\tan \beta$, while for large $\tan \beta$ one has to rely on the fully massive NLO results [409-411]. The QCD corrections to the squark loops are only known in the heavy squark limit [572] and the full SUSY-QCD corrections in the limit of heavy squarks and gluinos [573-576]. The pure QCD corrections are of about the same size as those to the quark loops thus rendering the total $K$ factor of similar size as for the quark loops alone with a maximal deviation of about 10\% [572]. The pure SUSY-QCD corrections are small [573-576]. The NNLL resummation of the SM Higgs cross section [421] can also be applied to the scalar MSSM Higgs cross sections in the regions, where the heavy quark and squark limits are valid. The same is also true for the NLO QCD corrections to the $p_{\mathrm{T}}$ distributions [428-432] and the NNLL resummation of soft gluon effects [433-443], i.e. for small values of $\tan \beta, M_{H}$ and $p_{\mathrm{T}}$ only. However, for large values of $\tan \beta$ the $p_{\mathrm{T}}$ distributions are only known at LO, since the bottom loops are dominant and the heavy top limit is not valid. An important consequence is that the $p_{\mathrm{T}}$ distributions of the neutral Higgs bosons are softer than for small values of $\tan \beta$ [577].

The vector-boson fusion processes $[449,451]$

$$
p p \rightarrow q q \rightarrow q q+W W / Z Z \rightarrow q q+h / H
$$

play an important role for the light scalar Higgs boson $h$ close to its upper mass bound, where it becomes SM-like, and for the heavy scalar Higgs particle $H$ at its lower mass bound. In the other regions the cross sections are suppressed by the additional SUSY-factors of the Higgs couplings. The NLO QCD corrections to the total cross section and the distributions can be taken from the SM Higgs case and are of the same size [452, 453]. The SUSY-QCD corrections mediated by virtual gluino and squark exchange at the vertices turned out to be small [578].

Higgs-strahlung off $W, Z$ gauge bosons $[454,455]$

$$
p p \rightarrow q \bar{q} \rightarrow Z^{*} / W^{*} \rightarrow H+Z / W
$$

does not play a major role for the neutral MSSM Higgs bosons at the LHC. The NLO [456] and NNLO [457] QCD corrections are the same as in the SM case, and the SUSY-QCD corrections are small [578]. The SUSY-electroweak corrections are unknown.

Higgs radiation off top quarks [459-463]

$$
p p \rightarrow q \bar{q} / g g \rightarrow h / H / A+t \bar{t}
$$

plays a significant role at the LHC for the light scalar Higgs particle only. The NLO QCD corrections are the same as for the SM Higgs boson with modified top and bottom Yukawa couplings and are thus of moderate size [162,464,465]. The SUSY-QCD corrections have been computed recently for the light scalar case [579]. They are of moderate size.

For large values of $\tan \beta$ Higgs radiation off bottom quarks [459-463]

$$
p p \rightarrow q \bar{q} / g g \rightarrow h / H / A+b \bar{b}
$$

constitutes the dominant Higgs production process. The NLO QCD corrections can be taken from the analogous calculation involving top quarks. However, they turn out to be very large [580,581]. The main reason is that the integration over the transverse momenta of the final state bottom quarks generates large logarithmic contributions. The resummation of the latter requires the introduction of bottom quark densities in the proton, since the large logarithms are related to the DGLAP-evolution of these densities. Their DGLAP-evolution 


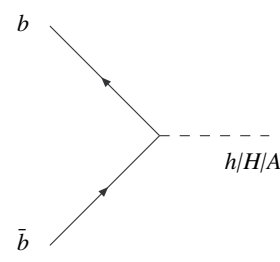

(a)

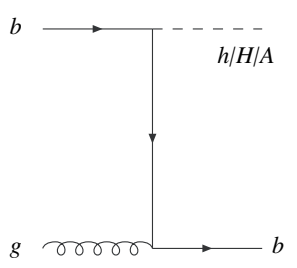

(b)

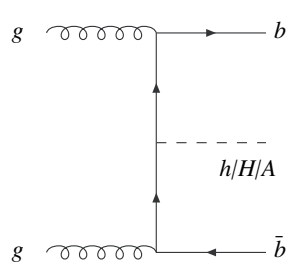

(c)

Figure 11.3. Typical diagrams for all Higgs boson production mechanisms related to Higgs radiation off bottom quarks at leading order: (a) $b \bar{b} \rightarrow h / H / A$, (b) $g b \rightarrow b+h / H / A$, (c) $g g \rightarrow$ $b \bar{b}+h / H / A$

resums them. This leads to an approximate approach starting from the process [582] (see Fig. 11.3a)

$$
p p \rightarrow b \bar{b} \rightarrow h / H / A
$$

at LO, where the transverse momenta of the incoming bottom quarks, their masses and their off-shellness are neglected. The NLO [583,584] and NNLO [585] QCD corrections to this bottom-initiated process are known and of moderate size, if the running bottom Yukawa coupling at the scale of the Higgs mass is introduced. At NNLO the full process $g g \rightarrow h / H / A+b \bar{b}$ (see Fig. 11.3c) contributes for the first time. At this order a proper matching to the fully massive result for this process can be performed $[586,587]$ so that the final expression provides an improved result, which takes into account the resummation of the large logarithms and mass effects. The fully exclusive $g g \rightarrow h / H / A+b \bar{b}$ process, calculated with four active parton flavours in a fixed flavour number scheme (FFNS), and this improved resummed result, calculated with 5 active parton flavours in the variable flavour number scheme (VFNS), will converge against the same value at higher perturbative orders. The best agreement between the NLO FFNS and NNLO VFNS is achieved, if the factorisation scale of the bottom quark densities is chosen as about a quarter of the Higgs mass [588, 589]. If only one of the final state bottom jets accompanying the Higgs particle is tagged, the LO bottom-initiated process is $g b \rightarrow b+h / H / A$ (see Fig. 11.3b), the NLO QCD corrections of which have been calculated $[589,590]$. They turn out to reach $\mathcal{O}(40-50 \%)$. The situation concerning the comparison with the FFNS at NLO is analogous to the total cross section. Agreement within the respective theoretical uncertainties is found for a factorisation scale of about a quarter of the Higgs mass [588]. If both bottom jets accompanying the Higgs boson in the final state are tagged, one has to rely on the fully exclusive calculation for $g g \rightarrow b \bar{b}+h / H / A$.

All neutral MSSM Higgs production cross sections including the NLO QCD corrections are shown in Fig. 11.4.

The dominant charged Higgs production process is the associated production with heavy quarks [591-593] (see Fig. 11.5a)

$$
p p \rightarrow q \bar{q}, g g \rightarrow H^{-}+t \bar{b} \quad \text { and c.c. }
$$

The NLO QCD and SUSY-QCD corrections have very recently been computed [594]. They are of significant size due to the large logarithms arising from the transverse-momentum integration of the bottom quark in the final state and the large SUSY-QCD corrections to the bottom Yukawa coupling. The large logarithms can be resummed by the introduction of bottom quark densities in the proton in complete analogy to the neutral Higgs case. In this 

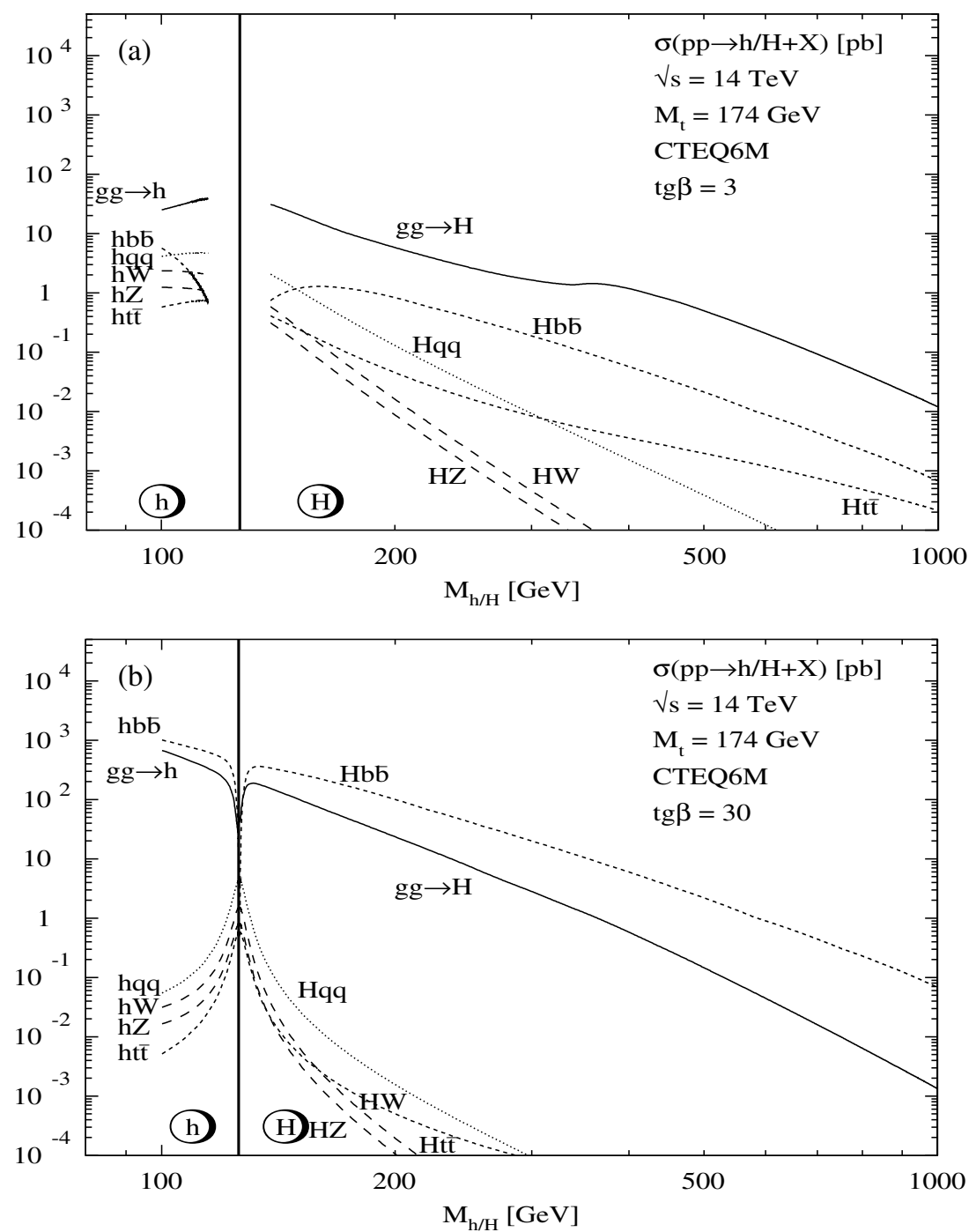

Figure 11.4. Neutral MSSM Higgs production cross sections at the LHC for gluon fusion $g g \rightarrow$ $\Phi$, vector-boson fusion $q q \rightarrow q q V V \rightarrow q q h / q q H$, Higgs-strahlung $q \bar{q} \rightarrow V^{*} \rightarrow h V / H V$ and the associated production $g g, q \bar{q} \rightarrow b \bar{b} \Phi / t \bar{t} \Phi$, including all known QCD corrections. (a) $h, H$ production for $\tan \beta=3$, (b) $h, H$ production for $\tan \beta=30$, (c) $A$ production for $\tan \beta=3$, (d) $A$ production for $\tan \beta=30$. The same parameters as in Fig. 11.2 have been adopted. (Continued on next page.)

approach the LO process is $g b \rightarrow H^{-} t$ and the charge conjugate. The NLO SUSY-QCD corrections have been derived in [595-598] and found to be of significant size. This process, however, relies on the same approximations as all bottom-initiated processes. A quantitative comparison of the processes $g b \rightarrow H^{-} t$ and $g g \rightarrow H^{-}+t \bar{b}$ at NLO is missing so far.

The second important charged Higgs production process is charged Higgs pair production in a Drell-Yan type process (see Fig. 11.5b)

$$
p p \rightarrow q \bar{q} \rightarrow H^{+} H^{-}
$$



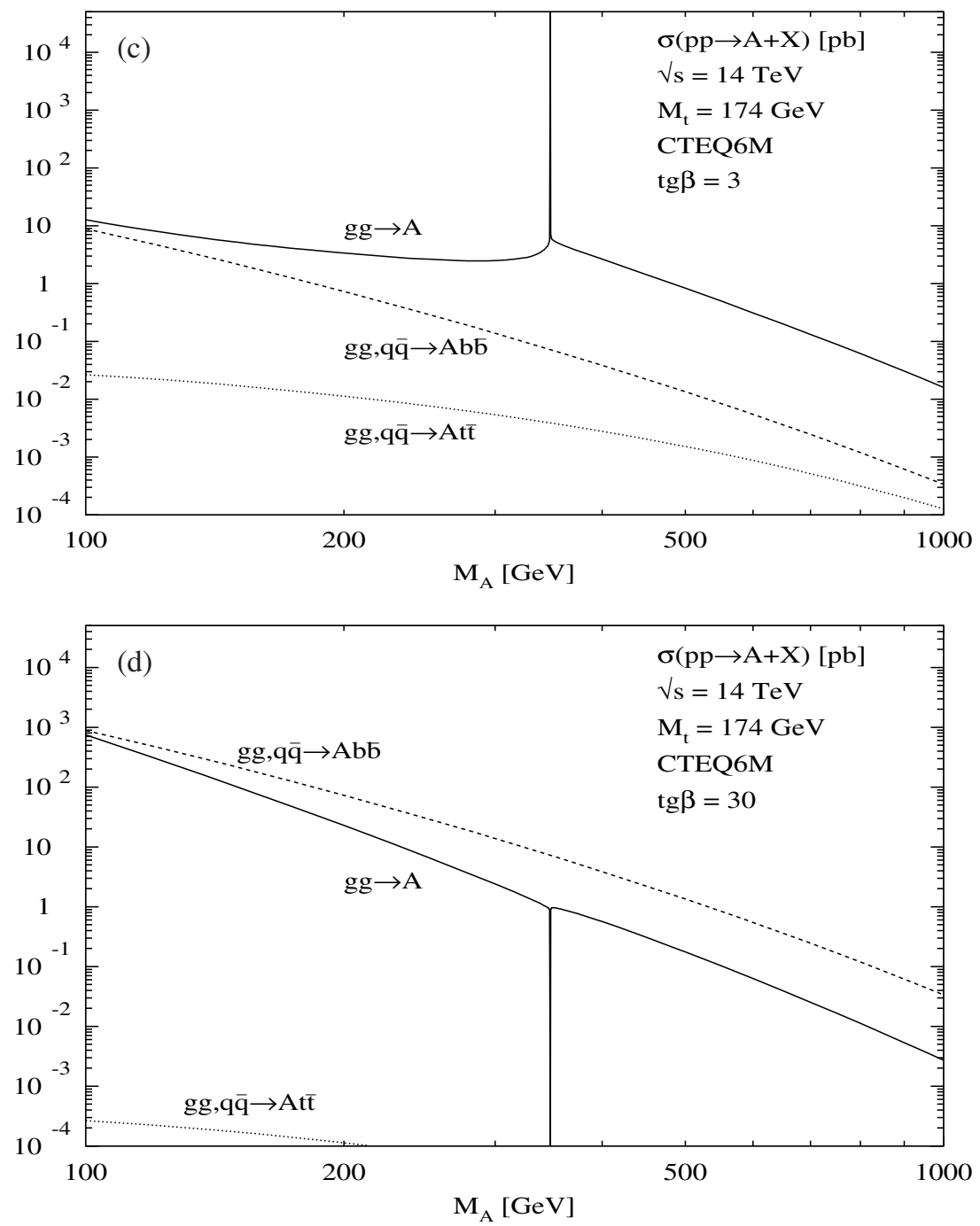

Figure 11.4. Continued.

which is mediated by $s$-channel photon and Z-boson exchange. The NLO QCD corrections can be taken from the Drell-Yan process and are of moderate size as in the case of the neutral Higgs-strahlung process discussed before. The genuine SUSY-QCD corrections, mediated by virtual gluino and squark exchange in the initial state, are small [578].

Charged Higgs pairs can also be produced from $g g$ initial states by the loop-mediated process [599-603] (see Fig. 11.5c)

$$
p p \rightarrow g g \rightarrow H^{+} H^{-}
$$

where the dominant contributions emerge from top and bottom quark loops as well as stop and sbottom loops, if the squark masses are light enough. The NLO corrections to this process are unknown. This cross section is of similar size as the bottom-initiated process [603] (see Fig. 11.5e)

$$
p p \rightarrow b \bar{b} \rightarrow H^{+} H^{-}
$$




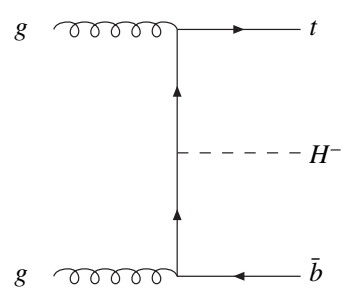

(a)

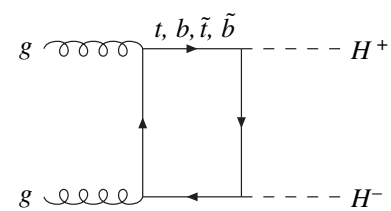

(c)

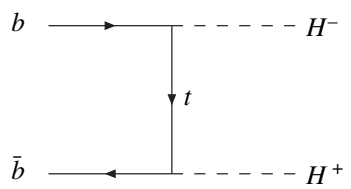

(e)

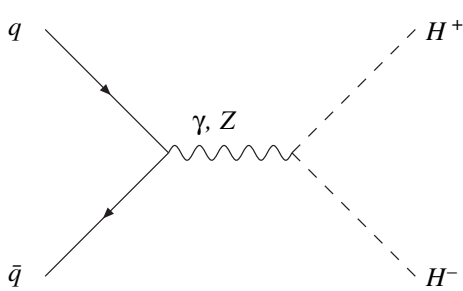

$(b)$

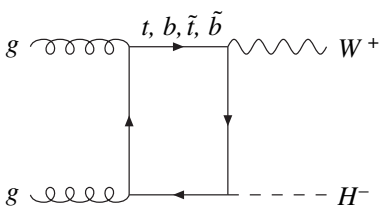

(d)

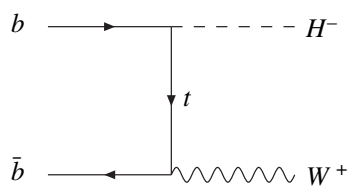

(f)

Figure 11.5. Typical diagrams for charged Higgs boson production mechanisms at leading order: (a) $g g \rightarrow H^{-} t \bar{b}$, (b) $q \bar{q} \rightarrow H^{+} H^{-}$, (c) $g g \rightarrow H^{+} H^{-}$, (d) $g g \rightarrow W^{+} H^{-}$, (e) $b \bar{b} \rightarrow H^{+} H^{-}$, (f) $b \bar{b} \rightarrow W^{+} H^{-}$.

which relies on the approximations required by the introduction of the bottom densities as discussed before and is known at NLO [604]. The SUSY-QCD corrections are of significant size. The pure QCD corrections and the genuine SUSY-QCD corrections can be of opposite sign.

Finally, charged Higgs bosons can be produced in association with a $W$ boson [605-607] (see Fig. 11.5d)

$$
p p \rightarrow g g \rightarrow H^{+} W^{-} \quad \text { and c.c. }
$$

which is generated by top-bottom quark loops and stop-sbottom loops, if the squark masses are small enough. This process is known at LO only. The same final state also arises from the process $[605,606,608]$ (see Fig. 11.5f)

$$
p p \rightarrow b \bar{b} \rightarrow H^{+} W^{-} \quad \text { and c.c. }
$$

which is based on the approximations of the VFNS. The QCD corrections have been calculated and turn out to be of moderate size $[609,610]$.

\subsection{Higgs boson channels}

\subsubsection{Associated $b \bar{b} H$ production with $H \rightarrow \tau \tau \rightarrow e^{ \pm} \mu^{\mp}+E_{\mathrm{T}}^{\text {miss }}$}

Compared to the hadronic and semi-leptonic final states described in Section 5.2, the fully leptonic final states are suppressed by relatively small branching ratio $\operatorname{BR}(\tau \rightarrow \mu \nu \nu) \sim 0.174$ and $\operatorname{BR}(\tau \rightarrow e \nu \nu) \sim 0.178$, but the signal is clean and easy to trigger. 
The signal consists of events in which the Higgs boson decays into two tau leptons which in turn decay leptonically. Two possibilities exist, either to select any-two-lepton final states, which have larger signal rate, or electron + muon final states for which the background is easier to suppress. Here the electron + muon final state is chosen.

The main backgrounds for $\mathrm{H} / A \rightarrow \tau \tau$ with $\mathrm{e} \mu$ final state are the Drell-Yan $\tau \tau$ production, the $\mathrm{t} \overline{\mathrm{t}}$ and the $\mathrm{Wt}$ production where the $\mathrm{W}$ boson coming from top quark decay decays leptonically, the $\tau \tau \bar{b} \bar{b}$ production, and the $b \bar{b}$ background with $\mathrm{b}$ quarks decaying semileptonically. Other backgrounds are pairs of vector bosons WW or WZ decaying into leptonic final states, but their contribution is small. The $\tau \tau$ c $\bar{c}$ background is also found negligible. The most biggest background arises from those $\bar{t} \bar{t}$ and Drell-Yan events which involve genuine $\tau$ 's and $b$ jets and produce events very similar to the signal. No SUSY particle background is assumed.

A detailed description of the analysis can be found in [611].

11.2.1.1. Event generation. The Higgs boson signal is generated with PYTHIA [246]. The signal cross sections and branching ratios are calculated with FeYNHiggs [142]. TAUOLA package [155] is used for leptonic $\tau$ decays in the signal events.

The Drell-Yan $\tau \tau$ production, $\mathrm{b} \overline{\mathrm{b}}$, WW, WZ and ZZ backgrounds are generated with PYTHIA. The Drell-Yan $\tau \tau$ next-to-leading order cross section of $1891 \mathrm{pb}$ calculated with the program MCFM [56] for $\mathrm{M}_{\tau \tau}>80 \mathrm{GeV} / \mathrm{c}^{2}$ is used. The $\tau \tau \mathrm{b} \overline{\mathrm{b}}$ background is generated with COMPHEP [43] with no $\mathrm{p}_{\mathrm{T}}$ and $\eta$ cuts applied on $\mathrm{b}$ quarks and the leading order cross section calculated with COMPHEP are used. The $\mathrm{Z} / \gamma^{*}$ generation is split into two bins of generated $\tau \tau$ mass $\mathrm{m}_{\tau \tau}: 80-100 \mathrm{GeV} / \mathrm{c}^{2}$ and $>100 \mathrm{GeV} / \mathrm{c}^{2}$, and the $\tau \tau \mathrm{b} \bar{b}$ is generated in the $\tau \tau$ mass bins of $60-100 \mathrm{GeV} / \mathrm{c}^{2}$ and $>100 \mathrm{GeV} / \mathrm{c}^{2}$.

The $\bar{t} \bar{t}$ background is generated with TopReX [44] and PYTHIA and the single top (Wt) events are generated with TopReX. A cross section of 840 and $60 \mathrm{pb}$ is used for $\overline{\mathrm{t}}$ and $\mathrm{Wt}$ events, respectively.

11.2.1.2. Level-1 and HLT selections. The events are triggered with the single and the double electron and muon triggers. The $p_{\mathrm{T}}$ threshold for single muons is $19 \mathrm{GeV} / \mathrm{c}$, for single electrons $26 \mathrm{GeV} / \mathrm{c}$, for double muons $7 \mathrm{GeV} / \mathrm{c}$ and for double electrons $14.5 \mathrm{GeV} / \mathrm{c}$. The Level 1 trigger efficiency for the signal of $\mathrm{M}_{\mathrm{A}}=200 \mathrm{GeV} / \mathrm{c}^{2}$ is 0.96 , and the overall trigger efficiency including the HLT is 0.82 . The corresponding trigger efficiencies for the Drell-Yan $\tau \tau$, the $\tau \tau \mathrm{b} \bar{b}$, the $t \bar{t}$ and the Wt backgrounds are $0.18,0.29,0.68$ and 0.68 , respectively.

In the future also a combined e+mu trigger with symmetric thresholds of $10 \mathrm{GeV} / \mathrm{c}$ for the electron and muon will be included. No large gain is expected since events passing e+mu trigger are most probably already triggered by the single muon trigger.

11.2.1.3. Offline selections. The basic event selection is a requirement of two isolated leptons (one e and one $\mu$ ) with $\mathrm{p}_{\mathrm{T}}>20 \mathrm{GeV} / \mathrm{c}$ in the central detector acceptance region $|\eta|<2.5$ coming from a reconstructed primary vertex (PV). The electron candidates are required to pass electron identification cuts described in [156]. The efficiency for the electron identification is about $90 \%$ for electrons passing the trigger. The leptons are defined isolated when there are no other tracks from the primary vertex with $\mathrm{p}_{\mathrm{T}}>1 \mathrm{GeV} / \mathrm{c}$ within a cone $\Delta R=\sqrt{\Delta \varphi^{2}+\Delta \eta^{2}} \leqslant 0.4$ around the lepton. The $\mathrm{p}_{\mathrm{T}}$ cut and the isolation reduce efficiently the backgrounds with soft leptons $(\mathrm{b} \overline{\mathrm{b}}, \mathrm{c} \overline{\mathrm{c}}, .$.$) .$

The $b$ jets associated with the Higgs boson provide a powerful tool to separate the $b \bar{b} \mathrm{H} / \mathrm{A}$ events from the Drell-Yan background. The Drell-Yan background in which $Z / \gamma^{*}$ decay into a tau pair has a large cross section compared to the Higgs production. However, these 
events are mostly produced with no associated jets, and if they have associated jets they are mostly light quark and gluon jets. Therefore the Drell-Yan background can be suppressed by requiring a reconstructed jets present in the event, and even further by requiring that the associated jets are identified as $b$ jets. The $b$ jets associated with the Higgs bosons are generally very soft, which makes their tagging a challenging task. For low jet $\mathrm{E}_{\mathrm{T}}$ values the track multiplicity and momenta tend to be low, and many jets do not have enough significant tracks to be identified as $\mathrm{a} b$ jet. As a consequence the $\mathrm{b}$ tagging efficiency is not very high. The $b$ tagging efficiency of $43 \%$ per jet for the signal events with $2 \%$ of the mistagging rate is found.

The $t \bar{t}$ background cannot be suppressed with $\mathrm{b}$ tagging due the presence of two energetic genuine $b$ jets in the event. In fact, the jet reconstruction and the b-tagging efficiencies are higher for $\mathrm{b}$ jets in $\mathrm{t} \overline{\mathrm{t}}$ events than for those associated with the signal. This can be exploited using a central jet veto: if more than one jet is found, the event is rejected. The threshold of $20 \mathrm{GeV}$ is set on the calibrated $\mathrm{E}_{\mathrm{T}}$ for the jets within the tracker acceptance region, $|\eta|<2.5$. A suppression factor of 8 is obtained against the $\bar{t} \bar{t}$ background with an efficiency of $60 \%$ for the signal.

A missing energy measurement is needed for estimating the fraction of the energy carried away by neutrinos. This information is used in the Higgs boson mass reconstruction. The amount of missing transverse energy is small and close to the detector resolution.

The $\tau$ 's from the Higgs boson with $\mathrm{M}_{\mathrm{A}}=200 \mathrm{GeV} / \mathrm{c}^{2}$ travel on average about $5 \mathrm{~mm}$ before they decay. Therefore the leptons coming from $\tau$ decays are displaced relative to the primary vertex [612]. The track impact parameter measurements in the transverse plane for the two leptons are combined quadratically into one variable $\sigma_{i p}=\sigma_{i p}\left(\tau_{1}\right) \oplus \sigma_{i p}\left(\tau_{2}\right)$, where $\sigma_{i p}\left(\tau_{1}, \tau_{2}\right)$ are significances of the lepton impact parameters. The leptons in $\bar{t} \bar{t}$ background come mostly from $\mathrm{W}$ decays. The $\mathrm{tt}_{\mathrm{t}}$ events with two intermediate $\tau$ 's cannot be suppressed by using impact parameter.

The neutrinos-charged lepton collinear approximation method for the mass reconstruction in $\mathrm{H} / A \rightarrow \tau \tau$ is described in section 5.2.5. The mass reconstruction is possible when the two leptons are not in a back-to-back configuration. The back-to-back events are removed with a cut on the angle between the two leptons in the transverse plane $\Delta \varphi(e, \mu)<175^{\circ}$. Uncertainties of the missing transverse energy measurement can lead to negative neutrino energies. For the signal $\sim 40 \%$ of events are lost when the positive neutrino energies are required. This requirement, however, yields a further suppression of the $\bar{t}$ and $\mathrm{Wt}$ backgrounds, since for these backgrounds the neutrinos are generally not emitted along the lepton directions. The efficiencies of $E_{v 1, v 2}>0$ cut for these backgrounds are about $17 \%$ and $15 \%$, respectively. The reconstructed $\tau \tau$ mass with $30 \mathrm{fb}^{-1}$ after all selections, but the mass window, is shown in Fig. 11.6. In the figure the signal of $\mathrm{M}_{\mathrm{A}}=140, \tan \beta=20$ and $200 \mathrm{GeV} / \mathrm{c}^{2}, \tan \beta=25$ in the $\mathrm{m}_{\mathrm{h}}^{\max }$ scenario and the backgrounds are presented.

11.2.1.4. Expected number of events. Table 11.1 shows the cross section times branching ratio for the backgrounds for each step of the selections. The signal cross sections for $\mathrm{M}_{\mathrm{A}}=140,200$ and $250 \mathrm{GeV} / \mathrm{c}^{2}$ and $\tan \beta=20$ in the $\mathrm{m}_{\mathrm{h}}^{\max }$ scenario are shown in Table 11.2. The expected number of events with $30 \mathrm{fb}^{-1}$ after all cuts, but mass window, is also shown in Tables 11.1 and 11.2. The expected number of events after all cuts including the mass window is shown for the signal and the total background in Table 11.3.

11.2.1.5. Systematic uncertainties and the discovery reach. The uncertainty of the event selection efficiency is related to the uncertainty of the lepton identification efficiency, the jet 

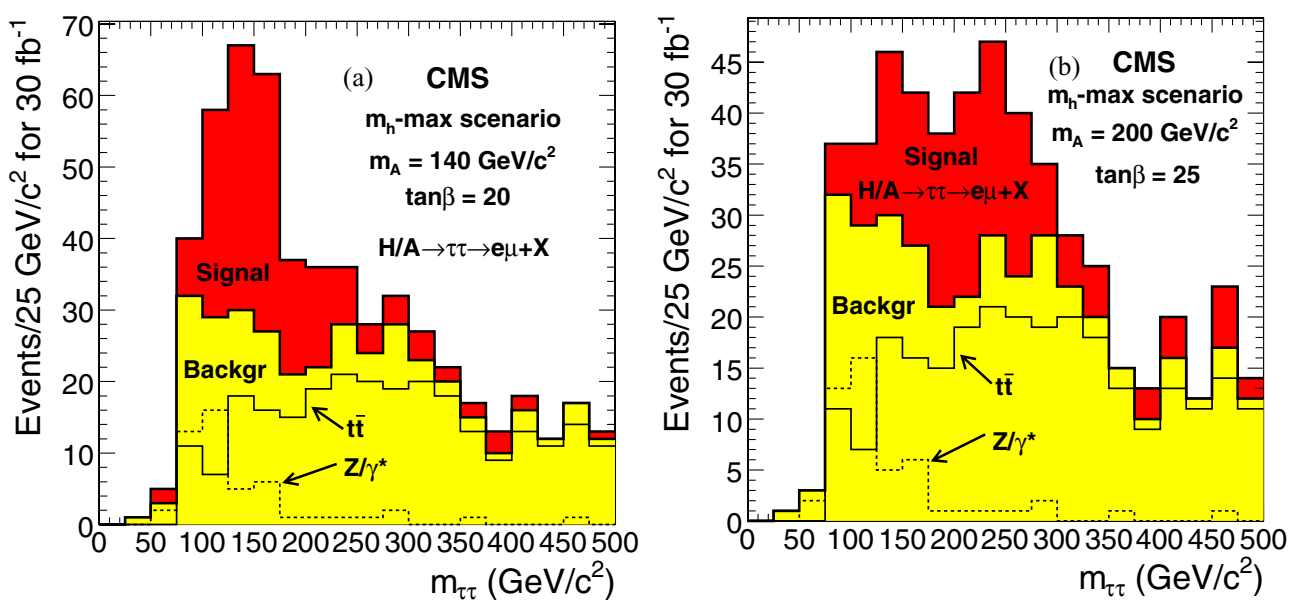

Figure 11.6. The $\tau \tau$ reconstructed mass with $30 \mathrm{fb}^{-1}$ after all selections, but the mass window. The signal in the $\mathrm{m}_{\mathrm{h}}^{\max }$ scenario and the backgrounds are shown for (a) $\mathrm{M}_{\mathrm{A}}=140 \mathrm{GeV} / \mathrm{c}^{2}$, $\tan \beta=20$ and (b) $\mathrm{M}_{\mathrm{A}}=200 \mathrm{GeV} / \mathrm{c}^{2}$ and $\tan \beta=25$.

Table 11.1. The background cross section times branching ratio (in pb) for each step of the selections. The expected number of events at $30 \mathrm{fb}^{-1}$ is also shown.

\begin{tabular}{lllllll}
\hline & $\mathrm{Z}, \gamma^{*}$ & $\mathrm{bbZ}, \gamma^{*}$ & $\mathrm{tt}$ & $\mathrm{tW}$ & $\mathrm{bb}$ & $\mathrm{VV}$ \\
\hline$\sigma \times B R$ & 233.1 & 3.422 & 86.2 & 6.16 & 36170 & 7.88 \\
Level 1 & 83.9 & 1.85 & 72.2 & 5.37 & 811 & 5.16 \\
$\mathrm{HLT}$ & 42.6 & 0.981 & 53.7 & 4.17 & 78.0 & 4.10 \\
reconstructed PV & 40.8 & 0.952 & 53.3 & 4.11 & 78.1 & 3.92 \\
isol e $+\mu, \mathrm{p}_{\mathrm{T}}$ cut & 1.10 & 0.0270 & 5.65 & 0.452 & 0.0378 & 0.288 \\
$\mathrm{Q}_{\mathrm{e}}+\mathrm{Q}_{\mu}=0$ & 1.09 & 0.0268 & 5.62 & 0.451 & 0.0374 & 0.248 \\
$\sigma_{\text {ip }}(e) \oplus \sigma_{\text {ip }}(\mu)$ & 0.296 & 0.00745 & 0.791 & 0.0550 & 0.0254 & 0.0255 \\
$\mathrm{~N}$ jets $>0$ & 0.0127 & 0.00527 & 0.778 & 0.0509 & 0.00654 & 0.0115 \\
$\mathrm{~b}$ tagging & 0.00457 & 0.00289 & 0.608 & 0.0341 & 0.00312 & 0.000547 \\
jet veto & 0.00344 & 0.00124 & 0.0745 & 0.0166 & 0.000179 & 0.000265 \\
$\Delta \varphi(e, \mu)$ & 0.00295 & 0.00116 & 0.0696 & 0.0159 & 0.000142 & 0.000259 \\
$\mathrm{E}_{\nu 1, \nu 2}>0$ & 0.00124 & 0.000486 & 0.0119 & 0.00246 & 0.0000661 & 0.0000546 \\
$\mathrm{~N}_{\mathrm{ev}}$ at $30 \mathrm{fb}^{-1}$ & 37.1 & 14.6 & 355.8 & 73.7 & 2.0 & 1.6 \\
\hline
\end{tabular}

energy and the missing energy scale and the b tagging efficiency. The jet energy and the missing energy scale uncertainty gives the uncertainty of $7.3 \%$ on the $\bar{t} \bar{t}$ background, which is the dominant background. The uncertainty of the lepton identification efficiency of $2 \%$ is used for both electrons and muons. The uncertainty of the b tagging efficiency, $5 \%$, can be estimated from $\bar{t} \bar{t}$ events as in Ref. [83]. The 5\% uncertainty of the mistagging efficiency is assumed [613]. The 5.8\% uncertainty of the theoretical prediction of the $\bar{t} \bar{c}$ cross section is taken. The total systematic uncertainty including the luminosity uncertainty $3 \%$ yields a $12 \%$ uncertainty for the total background.

The signal significance $S$ with $30 \mathrm{fb}^{-1}$ for the signal of $M_{A}=140,200$ and $250 \mathrm{GeV} / \mathrm{c}^{2}$ and $\tan \beta=20$ in the $\mathrm{m}_{\mathrm{h}}^{\max }$ scenario is shown in Table 11.2 without and with the background systematic uncertainty taken into account. Figure 11.7 shows the discovery reach in the $\mathrm{M}_{\mathrm{A}}-\tan (\beta)$ plane in the $\mathrm{m}_{\mathrm{h}}^{\max }$ scenario with $30 \mathrm{fb}^{-1}$. The lower (upper) curve corresponds to the case when the background systematic uncertainty is not taken (taken) into account. 
Table 11.2. The signal cross section times branching ratio (in pb) for $\mathrm{M}_{\mathrm{A}}=140,200$ and $250 \mathrm{GeV} / \mathrm{c}^{2}$ and $\tan \beta=20$ in the $\mathrm{m}_{\mathrm{h}}^{\max }$ scenario for each step of the selections. The expected number of events at $30 \mathrm{fb}^{-1}$ is also shown.

\begin{tabular}{llll}
\hline $\mathrm{m}_{\mathrm{A}}$ & 140 & 200 & 250 \\
\hline$\sigma \times B R(\mathrm{pb})$ & 3.468 & 1.123 & 0.493 \\
$\mathrm{~L} 1$ & 3.238 & 1.079 & 0.479 \\
$\mathrm{HLT}$ & 2.585 & 0.923 & 0.419 \\
reconstructed PV & 2.434 & 0.866 & 0.395 \\
isol $\mathrm{e}^{+} \mu, \mathrm{p}_{\mathrm{T}}$ cut & 0.258 & 0.116 & 0.0613 \\
$\mathrm{Q}_{\mathrm{e}}+\mathrm{Q}_{\mu}=0$ & 0.256 & 0.116 & 0.0612 \\
$\sigma_{\text {ip }}(e) \oplus \sigma_{\mathrm{ip}}(\mu)$ & 0.0859 & 0.044 & 0.0260 \\
$\mathrm{~N}$ jets $>0$ & 0.0375 & 0.0216 & 0.0130 \\
$\mathrm{~b}$ tagging & 0.0177 & 0.0104 & 0.00649 \\
jet veto & 0.0115 & 0.00619 & 0.00390 \\
$\Delta \varphi(e, \mu)$ & 0.0106 & 0.00554 & 0.00351 \\
$\mathrm{E}_{\nu 1, \nu 2}>0$ & 0.00601 & 0.00340 & 0.00222 \\
$\mathrm{~N}_{\mathrm{ev}}$ at $30 \mathrm{fb}^{-1}$ & 180 & 102 & 67 \\
\hline
\end{tabular}

Table 11.3. The expected number of the signal plus background and the background events in a given mass windows for $30 \mathrm{fb}^{-1}$ and the signal significance $S$ without and with the background systematic uncertainty taken into account.

\begin{tabular}{llllll}
\hline & $\Delta \mathrm{m}_{\tau \tau}$ & $\mathrm{N}_{\mathrm{S}}+\mathrm{N}_{\mathrm{B}}$ & $\mathrm{N}_{\mathrm{B}}$ & $\mathrm{S}_{\text {no syst. }}$ & $\mathrm{S}_{\text {syst. }}$ \\
\hline $\mathrm{m}_{\mathrm{A}}=140 \mathrm{GeV} / \mathrm{c}^{2}, \tan \beta=20$ & $100-200 \mathrm{GeV} / \mathrm{c}^{2}$ & 225 & 107 & 9.9 & 7.3 \\
$\mathrm{~m}_{\mathrm{A}}=200 \mathrm{GeV} / \mathrm{c}^{2}, \tan \beta=20$ & $140-250 \mathrm{GeV} / \mathrm{c}^{2}$ & 163 & 109 & 4.8 & 3.1 \\
$\mathrm{~m}_{\mathrm{A}}=250 \mathrm{GeV} / \mathrm{c}^{2}, \tan \beta=20$ & $160-380 \mathrm{GeV} / \mathrm{c}^{2}$ & 244 & 204 & 2.7 & 1.4 \\
\hline
\end{tabular}

\subsubsection{Associated $b \bar{b} H$ production with $H \rightarrow \mu^{+} \mu^{-}$}

The Higgs boson production in association with b quarks, $\mathrm{pp} \rightarrow \mathrm{b} \overline{\mathrm{b}} \phi(\phi=\mathrm{h}, \mathrm{H}, \mathrm{A})$ followed by the $\phi \rightarrow \mu \mu$ decay can provide the best measurement for the mass and width of the heavy MSSM Higgs bosons $\mathrm{H}$ and $\mathrm{A}$. At high $\tan \beta$ the natural width, sensitive to the $\tan \beta$ value, is comparable or dominates the dimuon mass experimental resolution, thus the measured width can be used to constrain the $\tan \beta$.

This analysis uses the dimuon trigger (Level-1 and HLT) stream. Despite of the small $\phi \rightarrow \mu \mu$ branching ratio $\left(\simeq 10^{-4}\right)$ the precise measurement of the dimuon mass in off-line provides an excellent possibility to suppress the t⿱t background. The associated Higgs boson production with $\mathrm{b}$ quarks is exploited to suppress the huge Drell-Yan $\mu \mu$ background using the $\mathrm{b}$ tagging. Irreducible background from $\mu \mu \mathrm{b} \overline{\mathrm{b}}$ process was also considered and found to be small.

The analysis was performed in the $\mathrm{m}_{\mathrm{h}}^{\max }$ scenario for three regions of $\mathrm{M}_{\mathrm{A}}$ :

- the so-called decoupling regime, $\mathrm{M}_{\mathrm{A}} \gg \mathrm{M}_{\mathrm{h}}$, where $\mathrm{M}_{\mathrm{A}} \sim \mathrm{M}_{\mathrm{H}}$. The Higgs bosons $\mathrm{A}$ and $\mathrm{H}$ with $\mathrm{M}_{\mathrm{A}(\mathrm{H})} \geqslant 150 \mathrm{GeV} / \mathrm{c}^{2}$ and $\tan \beta \geqslant 15$ were generated.

- the "intensive-coupling regime" $\mathrm{M}_{\mathrm{A}} \sim \mathrm{M}_{\mathrm{h}}$ defined in [614,615], where the three neutral Higgs bosons have comparable masses, $\mathrm{M}_{\mathrm{A}} \simeq \mathrm{M}_{\mathrm{H}} \simeq \mathrm{M}_{\mathrm{h}}$ The $\mathrm{h}, \mathrm{A}$ and $\mathrm{H}$ bosons were generated for three mass points of $\mathrm{M}_{\mathrm{A}}=125,130$ and $135 \mathrm{GeV} / \mathrm{c}^{2}$ at $\tan \beta=30$.

- the low $\mathrm{M}_{\mathrm{A}}$ regime, $\mathrm{M}_{\mathrm{A}}<\mathrm{M}_{\mathrm{h}}$, where $\mathrm{M}_{\mathrm{A}} \sim \mathrm{M}_{\mathrm{h}}$. The Higgs bosons $\mathrm{h}$ and $\mathrm{A}$ were generated at $\mathrm{M}_{\mathrm{A}}=100 \mathrm{GeV} / \mathrm{c}^{2}$ and $\tan \beta \geqslant 20$ points. 


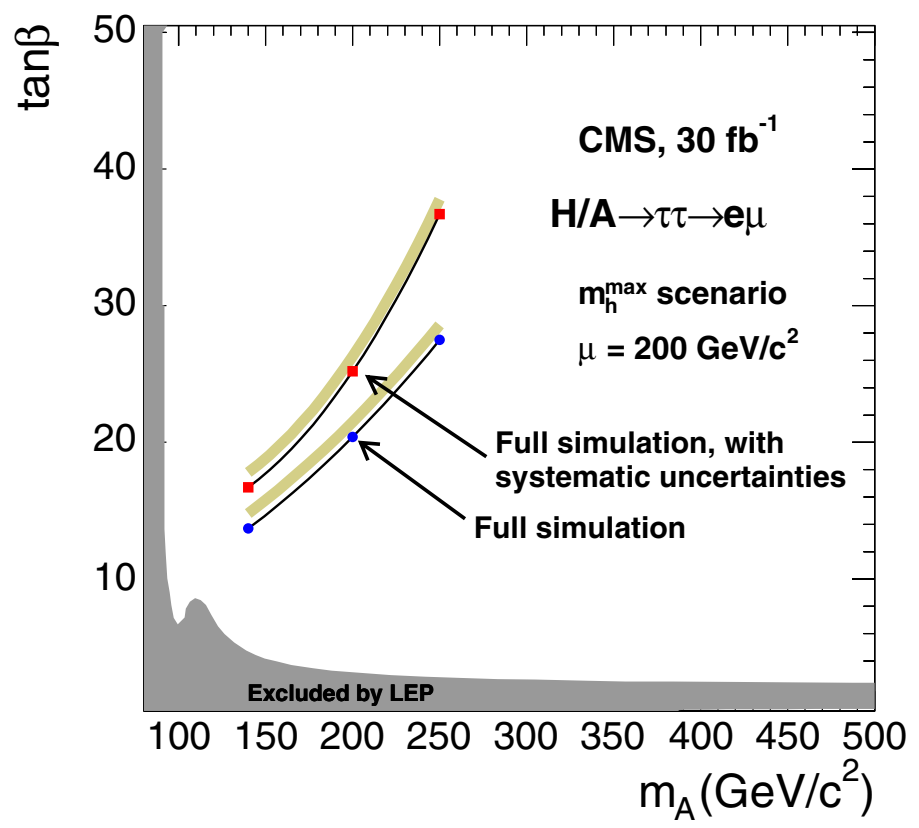

Figure 11.7. The discovery region for $\mathrm{gg} \rightarrow \mathrm{b} \overline{\mathrm{b}} \mathrm{H} / \mathrm{A}, \mathrm{H} / A \rightarrow \tau \tau \rightarrow e \mu+\mathrm{X}$ channel in $\mathrm{M}_{\mathrm{A}}$-tan $\beta$ in the $\mathrm{m}_{\mathrm{h}}^{\max }$ scenario with $30 \mathrm{fb}^{-1}$.

11.2.2.1. Event generation. The Higgs boson production $\mathrm{pp} \rightarrow \mathrm{b} \overline{\mathrm{b}} \phi$ and decay was generated with PYтHIA for the decoupling and low $\mathrm{M}_{\mathrm{A}}$ regimes. For the "intensivecoupling regime" events were generated by COMPHEP as described in [615]. The Higgs boson production cross section and branching ratio were evaluated using FeynHiggs 2.3.2 [142-144]. The mass relations between $\mathrm{A}, \mathrm{H}$ and $\mathrm{h}$ bosons and widths were obtained with HDECAY [41] for the "intensive-coupling regime".

The Drell-Yan and $\bar{t} \bar{t}$ backgrounds were generated with PYTHIA. The Drell-Yan events with $\mathrm{b}$ quarks in the final state were excluded to avoid double counting with $\mu \mu \mathrm{b} \overline{\mathrm{b}}$ background generated with COMPHEP.

\subsubsection{Offline selection.}

Muon identification. The signal is characterised by two well reconstructed, isolated muons. Therefore the event is accepted if there are at least two muons, with opposite charge, both satisfying the following conditions:

- muon transverse momentum $p_{\mathrm{T}}>20 \mathrm{GeV} / \mathrm{c}$;

- a cone of $\Delta R=\sqrt{\Delta \eta^{2}+\Delta \phi^{2}}=0.35$ is defined around the reconstructed muon track. Then the variable $\mathrm{E}_{\text {iso }}$ is evaluated as the sum of the energies measured by all the detectors (tracker, ECAL, HCAL) inside this cone with muon momentum excluded. The muon is defined isolated if $\mathrm{E}_{\text {iso }}<10 \mathrm{GeV}$.

Rejection of $\mathbf{t} \overline{\mathbf{t}}$ background. The rejection of $\overline{\mathrm{t}} \mathrm{t}$ events is based on two selection cuts and exploits the presence of the neutrino in the top decay chain and of two well reconstructed energetic jets. 
The event is accepted if the following conditions are satisfied:

- the missing transverse energy is less than $40 \mathrm{GeV}$;

- the jets, reconstructed with the Iterative Cone Algorithm [314], must have transverse energy less than $45 \mathrm{GeV}$ and $|\eta|<5.0$.

B tagging. The presence of $\mathrm{b}$ jets in the Higgs boson production is exploited to suppress Drell-Yan $\mu \mu$ background, which otherwise be dominant, especially for dimuon invariant masses below $200 \mathrm{GeV} / \mathrm{c}^{2}$.

The $\mathrm{b}$ quarks in signal events are mainly produced in the forward region, with lower $p_{\mathrm{T}}$ with respect to the $\mathrm{b}$ quarks coming from $\mathrm{t} \overline{\mathrm{t}}$ background.

Two different strategies, based on two distinct cuts, have been developed for the $b$ tagging:

1. The event must contain at least one jet tagged as $b$ jet with the Combined B-Tagging algorithm [616]. This algorithm has been designed to tag mainly central $b$ jets of high transverse energy, thus it is not optimised for the $b$ jets of the signal. In the following this cut will be refereed to as hard b-tag.

2. The tracks in the event are classified as good tracks if they satisfy:

- at least 6 hits in the tracker of which at least two belonging to the pixel detectors;

- transverse momentum $p_{\mathrm{T}}>2.4 \mathrm{GeV} / \mathrm{c}$;

- pseudorapidity $|\eta|<2.4$;

- transverse impact parameter IP $<0.5 \mathrm{~cm}$;

- track fit quality $\chi^{2} / n d f<5$.

The event must contain at least two good tracks with transverse impact parameter (IP) in the range $0.01<I P<0.1 \mathrm{~cm}$ (only one track if $0.02<I P<0.075 \mathrm{~cm}$ ).

The first strategy consists on applying selection 1) only. The second strategy is the logical OR between selection 1) and 2) (this strategy will be refereed to as soft b-tag).

Results have been calculated for both selections and the one with the best signal significance has been considered.

11.2.2.3. Fitting procedure. Figure 11.8 shows the distribution of reconstructed dimuon invariant mass after all selections for the backgrounds and, as an example, for the signal of $\mathrm{M}_{\mathrm{A}}=150 \mathrm{GeV} / \mathrm{c}^{2}$ and $\tan \beta=40$. The plot has been obtained assuming an integrated luminosity of $30 \mathrm{fb}^{-1}$ and the hard b-tag. The signal is visible as a peak over a background that exponentially decreases with increasing $M_{\mu \mu}$.

The background is estimated by fitting the dimuon mass distribution in the off-peak regions, where the signal is not present. To identify this region, the TSpectrum class in root is used: this class allows to find a signal peak over a background distribution.

The function used in this analysis to parameterise the background has three free parameters:

$f_{B}\left(M_{\mu \mu} ; P_{0}, P_{1}, P_{2}\right)=P_{0} \times \frac{\Gamma_{Z}}{2 \pi\left(\left(M_{\mu \mu}-M_{Z}\right)^{2}+\left(\frac{\Gamma_{Z}}{2}\right)^{2}\right)}+P_{1}+P_{2} \times M_{\mu \mu}$.

After the background parametrisation function is determined by fitting the background in the off-peak region, a binned likelihood fit method, with three free parameters, is applied over the whole $\mathrm{M}_{\mu \mu}$ range using the function:

$f_{\text {tot }}\left(M_{\mu \mu} ; M_{A}, \sigma_{\mu \mu}, \Gamma_{A}, N_{S}\right)=\left(N_{T O T}-N_{S}\right) \times p d f_{B}\left(M_{\mu \mu}\right)+N_{S} \times V\left(M_{\mu \mu} ; M_{A}, \sigma_{\mu \mu}, \Gamma_{A}\right)$ 


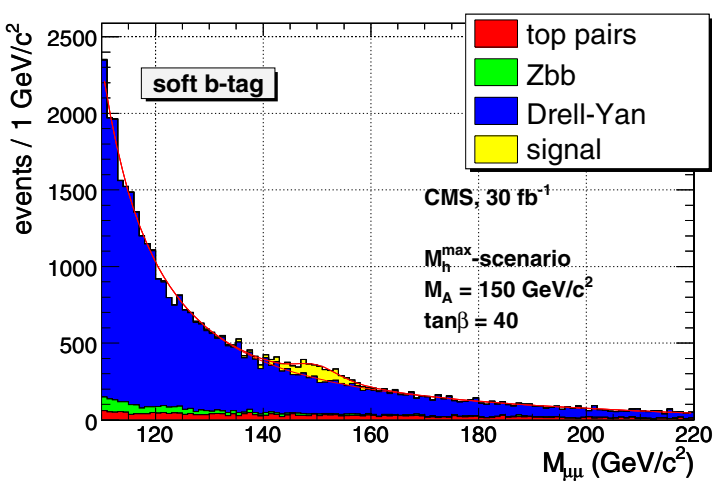

Figure 11.8. Fitting procedure applied to the dimuon reconstruction mass for the main background and for the signal sample with $M_{A}=150 \mathrm{GeV} / \mathrm{c}^{2}$ and $\tan \beta=40$.

Table 11.4. Effect of the selection cuts on the background and signal cross section (all values in pb). Efficiency w.r.t. previous cut in \% is shown in brackets. The no cut value for the top pair background refers to the inclusive $t \bar{t}$ production.

\begin{tabular}{lllll}
\hline & top pairs & $\begin{array}{l}\text { Drell-Yan } \\
M_{\mu \mu}>115 \mathrm{GeV} / \mathrm{c}^{2}\end{array}$ & $\begin{array}{l}\text { Zbb } \\
M_{\mu \mu}>100 \mathrm{GeV} / \mathrm{c}^{2}\end{array}$ & $\begin{array}{l}\text { signal } \\
\mathrm{M}_{\mathrm{A}}=130, \tan \beta=30\end{array}$ \\
\hline No cuts & 840 & 27.8 & 1.05 & 0.309 \\
pre-selection cut & $20.9(2.5)$ & $13.0(46.8)$ & $0.778(74.1)$ & $0.245(79.2)$ \\
Level-1 & $19.8(94.7)$ & $11.9(91.3)$ & $0.720(92.5)$ & $0.226(92.2)$ \\
HLT & $17.1(86.1)$ & $11.8(99.3)$ & $0.712(98.9)$ & $0.223(98.7)$ \\
Muon Id & $5.23(30.7)$ & $10.4(87.9)$ & $0.569(79.9)$ & $0.183(81.8)$ \\
Missing Et & $1.20(23)$ & $9.51(91.7)$ & $0.503(88.4)$ & $0.163(89.2)$ \\
Jet Veto & $0.317(26.4)$ & $8.37(88.1)$ & $0.418(83.1)$ & $0.138(84.5)$ \\
Soft b-tag & $0.238(75.2)$ & $0.916(10.9)$ & $0.146(35.0)$ & $0.0424(30.9)$ \\
$\mathrm{N}_{\mathrm{ev}}$ at 30 fb & 7140 & 27480 & 4380 & 1272 \\
$\mathrm{Hard} \mathrm{b}$-tag $^{-1}$ & $0.173(54.7)$ & $0.0697(0.83)$ & $0.0616(14.7)$ & $0.0154(11.2)$ \\
$\mathrm{N}_{\mathrm{ev}}$ at 30 fb & 5190 & 2091 & 1848 & 462 \\
\hline
\end{tabular}

where $p d f_{B}\left(M_{\mu \mu}\right)$ is the probability distribution function for the background with fixed parameters, and the second is the Voigt function, i.e. the convolution function between Gaussian and Breit-Wigner functions. The three free parameters are the number of signal events $\left(N_{S}\right)$, the MSSM Higgs boson mass $\left(M_{A}\right)$ and width $\left(\Gamma_{A}\right)$. The quantity $\sigma_{\mu \mu}$ is the CMS resolution for $M_{\mu \mu}$ and it's value is found from the fit of the $\mathrm{Z}$ peak in the Drell-Yan distribution.

To estimate the significance for the potential discovery of the Higgs boson, the likelihood fit is performed in the signal + background hypothesis $\left(L_{S+B}\right)$ and in the background hypothesis $\left(L_{B}\right)$. The significance is defined [102] as:

$$
S_{L}=\sqrt{2\left(\ln L_{S+B}-\ln L_{B}\right)}
$$

11.2.2.4. Results. Table 11.4 summarises the selection cut efficiency for background and signal. The first set of cuts, down to the Jet Veto cut, is always applied. After that two different b-tags are considered. 
Table 11.5. Significance for the decoupling regimes.

\begin{tabular}{llll}
\hline Luminosity $\left(\mathrm{fb}^{-1}\right)$ & $\tan \beta=30$ & $\tan \beta=40$ & $\tan \beta=50$ \\
\hline \multicolumn{4}{l}{$M_{A}=150 \mathrm{GeV} / \mathrm{c}^{2}-$ soft b-tag } \\
10 & - & 6.5 & 7.9 \\
20 & 7.2 & 10.3 & 12.1 \\
30 & 9.7 & 13.0 & 15.4 \\
& $M_{A}=150 \mathrm{GeV} / \mathrm{c}^{2}-$ hard b-tag \\
10 & 3.8 & 5.7 & 6.7 \\
20 & 6.2 & 7.3 & 9.8 \\
30 & 8.8 & 9.8 & 13.1 \\
& & $M_{A}=200 \mathrm{GeV} / \mathrm{c}^{2}-$ soft b-tag & \\
20 & - & 3.1 & 5.2 \\
30 & - & 4.7 & 5.7 \\
\hline
\end{tabular}

Table 11.6. Significance for the intensive coupling regime as a function of the integrated luminosity, for different $M_{A}$ values.

\begin{tabular}{llll}
\hline Luminosity $\left(\mathrm{fb}^{-1}\right)$ & $M_{A}=125 \mathrm{GeV} / \mathrm{c}^{2}$ & $M_{A}=130 \mathrm{GeV} / \mathrm{c}^{2}$ & $M_{A}=135 \mathrm{GeV} / \mathrm{c}^{2}$ \\
\hline 20 & 7.1 & 5.4 & 5.1 \\
30 & 9.8 & 7.6 & 7.1 \\
\hline
\end{tabular}

The systematic effects may be introduced by the experimental technique to fit the background. To estimate such effects, the fitting procedure has been repeated fixing one of the parameters to the measured value increased by its error.

Decoupling regime. Table 11.5 shows the significance as a function of $\tan \beta$, for an Higgs mass of 150 and $200 \mathrm{GeV} / \mathrm{c}^{2}$. In general, where the fitting procedure works properly, the significance is greater then five. Best results are obtained for low values of $M_{A}$ (as the cross section increases with decreasing Higgs mass) and for high values of $\tan \beta$ (the cross section is proportional to $\tan ^{2} \beta$ ).

Low $M_{A}$ regime. In the low $M_{A}$ regime the background is large due to the presence of the $Z^{0}$ peak, thus the signal peak is hidden for the integrated luminosity considered in this study. Better results could be obtained in the LHC high luminosity phase.

Intensive coupling regime. The intensive coupling regime is interesting because all the three neutral Higgs bosons contribute to the signal peak of dimuon mass. Each Higgs boson has rather small intrinsic width (less then $3 \mathrm{GeV} / \mathrm{c}^{2}$ for $\tan \beta=30$ ) which is smaller then the mass difference. However, once the mass resolution is taken into account, it becomes impossible to separate the three peaks.

The significance, on the other hand, is quite good despite the vicinity of the $Z^{0}$ peak, because the signal cross section is large, thus the discovery can be already done with an integrated luminosity of $20 \mathrm{fb}^{-1}$. Table 11.6 summarises the significance obtained for the three signal samples as a function of the integrated luminosity.

Figure 11.9 shows the discovery contour plot in the plane $\left(M_{A}, \tan \beta\right)$ obtained with this analysis. The signal significance inside the grey area is $>5$ with an integrated luminosity of $30 \mathrm{fb}^{-1}$. The structure of the contour plot near the minimum is due to the features of the signal in the intense coupling regime. The dashed line refers to the analysis without systematic uncertainties. It must be pointed out that the contour of the grey area does not correspond to a significance equal to 5 for $M_{A}<180 \mathrm{GeV} / \mathrm{c}^{2}$. The contour for $M_{A}<180 \mathrm{GeV} / \mathrm{c}^{2}$ is actually 


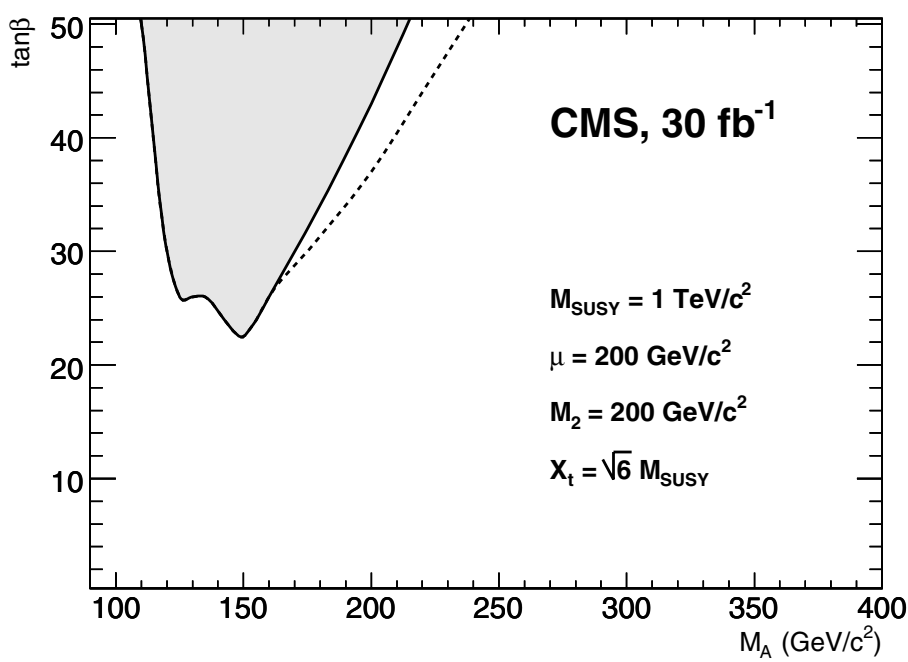

Figure 11.9. Discovery contour plot for the MSSM neutral Higgs in dimuon analysis. The signal significance inside the grey area is $>5$ with an integrated luminosity of $30 \mathrm{fb}^{-1}$.

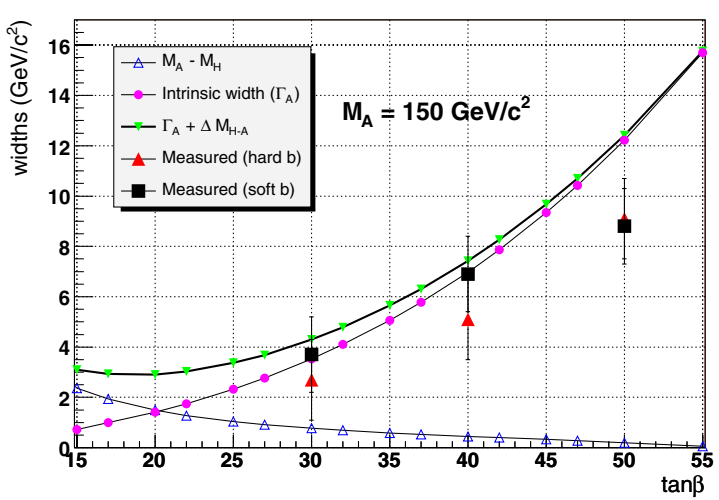

Figure 11.10. The comparison between the expected Higgs boson width and the measured one as a function of $\tan \beta$ for $M_{A}=150 \mathrm{GeV} / \mathrm{c}^{2}$.

determined by the possibility to perform a successful fit to the data, due to the low statistics and the contour plot corresponds to a significance which is actually slightly larger than 5 . Only for $M_{A}>180 \mathrm{GeV} / \mathrm{c}^{2}$ the contour corresponds to the signal significance equal to 5 . This explains why the effect of the inclusion of the systematic uncertainty is visible only in this mass range. For $M_{A}<180 \mathrm{GeV} / \mathrm{c}^{2}$, the fit fails even if systematic uncertainties are not included in the analysis, and the contour plot does not change.

11.2.2.5. $\tan \beta$ measurement. The peculiar feature of the dimuon channel at high $\tan \beta$ is the possibility of the direct measurement of the Higgs boson width, $\Gamma_{\mathrm{H} / \mathrm{A}}$, which is sensitive to $\tan \beta$ value. Therefore, it is possible to constrain $\tan \beta$ using the measured width. Figures 11.10 compares the intrinsic Higgs boson width (shown as solid circles) with the measured one (solid triangles and solid squares) for $M_{A}=150 \mathrm{GeV} / \mathrm{c}^{2}$. Fitting the mass distribution with a Voigt function, the contribution to the Higgs peak from the muon 


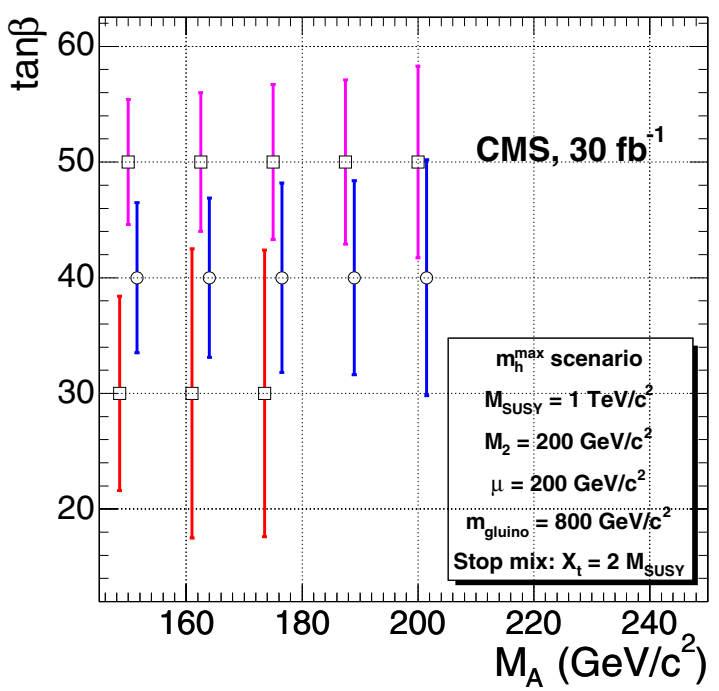

Figure 11.11. Uncertainty on the $\tan \beta$ measurement obtained from the Higgs boson width measurement with an integrated luminosity of $30 \mathrm{fb}^{-1}$.

invariant mass resolution is subtracted. However, another effect must be taken in account: the degeneracy of the two neutral Higgs bosons, A and $\mathrm{H}$, is not perfect. The value of $\mathrm{M}_{\mathrm{A}}-\mathrm{M}_{\mathrm{H}}$ is plotted as a function of $\tan \beta$ (open triangles). The effect is particularly evident for $\mathrm{M}_{\mathrm{A}}=150 \mathrm{GeV} / \mathrm{c}^{2}$ and for low $\tan \beta$, where the mass difference is greater then the intrinsic width. Thus the measured effective width is not the intrinsic one, but it is the sum of the intrinsic width and of Higgs mass difference (inverted triangles): $\Gamma_{A}+\left(M_{H}-M_{A}\right)$.

Figure 11.11 shows the uncertainty on the $\tan \beta$ measurement that can be obtained if the MSSM relation between the Higgs boson width and $\tan \beta$ is exploited in the $\mathrm{m}_{\mathrm{h}}^{\mathrm{max}}$ scenario. A theoretical uncertainty of $15 \%$ [560] is included. The $\tan \beta$ can be further constrained using the cross section measurement and exploiting the $\tan \beta$ dependance, $\sigma \times \mathrm{Br} \sim \tan ^{2} \beta_{\text {eff }}$.

\subsubsection{Associated $b \bar{b} H$ production with $H \rightarrow b \bar{b}$}

At high $\tan \beta$ the associated $\mathrm{b} \overline{\mathrm{b}} \mathrm{H} / \mathrm{A}$ production followed by the $\mathrm{H} / \mathrm{A} \rightarrow \mathrm{b} \overline{\mathrm{b}}$ decay has the biggest cross section. Nevertheless, the challenge of observing this channel is driven by the huge QCD multi-jet background expected for the final signature of two soft b-jets from associated Higgs boson production plus two hard b jets from the Higgs boson decay.

In this analysis [617] a study of the observability of this channel is performed using the fast simulation framework of CMS, FAMOS [11]. Signal is also studied with the full GEANT4 [9] CMS detector simulation [8] which allows to validate the fast simulation samples.

This channel can be considered as a cross-check for the discovery once it is known which Higgs boson mass (observed for instance in $b \bar{b} H / A \rightarrow b \bar{b} \tau^{+} \tau^{-}$channel) must be looked at. In combination with the $\tau \tau$ mode it can be used to evaluate the ratio of $A(H) b \bar{b}$ and $A(H) \tau \tau$ Yukawa couplings.

11.2.3.1. Event generation. Signal events $b \bar{b} H, H \rightarrow b \bar{b}$ were produced using PYTHIA for 4 values of $\mathrm{M}_{\mathrm{A}}: 200,500,600$ and $800 \mathrm{GeV} / \mathrm{c}^{2}$. The signal cross sections and branching ratios were calculated with FeynHiggs 2.3.2 [142-144] in the $\mathrm{m}_{\mathrm{h}}^{\max }$ scenario. The $\tan \beta$ value chosen 
Table 11.7. Off-line selection cuts on $\mathrm{E}_{\mathrm{T}}$ of the jets (in $\mathrm{GeV}$ ) for different Higgs boson mass values considered.

\begin{tabular}{|c|c|c|c|c|}
\hline $\mathrm{M}_{\mathrm{A}}$ & 200 & 500 & 600 & 800 \\
\hline $\mathrm{E}_{\mathrm{T}}^{j 1}$ & 90 & 200 & 220 & 260 \\
\hline $\mathrm{E}_{\mathrm{T}}^{j 2}$ & 80 & 180 & 200 & 240 \\
\hline $\mathrm{E}_{\mathrm{T}}^{j 4}$ & \multicolumn{4}{|c|}{30} \\
\hline
\end{tabular}

for generation was 50. In the considered $\mathrm{M}_{\mathrm{A}}$-tan $\beta$ region, $\mathrm{A}$ and $\mathrm{H}$ Higgs bosons have almost the same mass and can not be distinguished.

Among the Standard Model processes, backgrounds for this channel come mainly from QCD multi-jet production which includes events with four real $b$ jets. Background has been generated with PYTHIA QCD dijet production processes where additional jets are produced from gluon splitting and from the initial and the final state radiation in PYTHIA.

The generation of backgrounds has been weighted in order to get a similar statistics in the whole relevant $\hat{\mathrm{p}_{\mathrm{T}}}$ range. Production was split in $\hat{\mathrm{p}_{\mathrm{T}}}$ bins of $50 \mathrm{GeV} / \mathrm{c}$ from 50 to $1000 \mathrm{GeV} / \mathrm{c}$.

11.2.3.2. Event pre-selection. About 800 million Monte-Carlo events were generated and passed to a pre-selection, requiring a final state containing at least three heavy (b or c) quarks and four jets reconstructed with PYCELL PYTHIA jet finder in the $|\eta|<4.5$ region, using cone size of 0.5. The thresholds $\mathrm{E}_{\mathrm{T}_{2}}>50 \mathrm{GeV} / \mathrm{c}$ and $\mathrm{E}_{\mathrm{T}_{4}}>10 \mathrm{GeV} / \mathrm{c}$ were applied on the second and fourth highest $\mathrm{E}_{\mathrm{T}}$ jet respectively. The $\mathrm{QQ}+\mathrm{jj}$ background (with $\mathrm{Q}=\mathrm{b}, \mathrm{c}$ and $\mathrm{j}=$ light quark or gluon) was estimated to be less than 10\% of the total QCD multi-jet background after final selection cuts. After pre-selection, around 30 million events were passed to the detector simulation.

11.2.3.3. Online selection. This channel is triggered at Level 1 by the standard single and multi-jet triggers. At High Level, the inclusive single b-jet trigger [618] stream has been used. The implementation of the High Level double b-jet trigger and relaxing the jet energy thresholds could improve the observability of the signal, especially for low mass Higgs boson $\left(\sim 200 \mathrm{GeV} / \mathrm{c}^{2}\right)$.

11.2.3.4. Off-line selection. Analysis has been performed with fast simulated signal and background samples where pile-up was not included, once it was checked with full simulation on signal events that its effect was not significant after requiring jets with reconstructed $\mathrm{E}_{\mathrm{T}}>30 \mathrm{GeV}$.

The jets are reconstructed with the iterative cone algorithm [314] using cone size of 0.5. The calorimeter towers with the energy thresholds tuned to minimise the fake jet rate were used as an input for the jet finder. The jet energy corrections were applied using Monte Carlo calibration [619].

The event was required to have at least four jets with the transverse energy of 1st, 2nd and 4th jet greater than thresholds depending upon the $\mathrm{M}_{\mathrm{A}}$ point considered, according to Table 11.7. The cut on the 4th jet $\mathrm{E}_{\mathrm{T}}$ is motivated by reliability of the analysis simulation without pile-up.

Subsequently, the jets were required to be in the range of the tracker acceptance, $|\eta|<2.4$. Combined $b$ tagging as described in [616] has been used. At least three b-tagged jets (with discriminant variable $>2$ ), among the 4 highest $\mathrm{E}_{\mathrm{T}}$ jets, are requested in the analysis; two of them must be the two highest $\mathrm{E}_{\mathrm{T}}$ jets. It would also have been possible to be less restrictive 
Table 11.8. Signal selection cumulative efficiencies for $\mathrm{M}_{\mathrm{A}}=600 \mathrm{GeV} / \mathrm{c}^{2}, \tan \beta=50$ and background cumulative efficiencies. The signal to background ratio, $\mathrm{S} / \mathrm{B}$, is also shown.

\begin{tabular}{llll}
\hline Selection & Signal efficiency & Background efficiency & S/B (full mass range) \\
\hline None & 1 & 1 & $1.85 \times 10^{-7}$ \\
Pre-selection & $5.14 \mathrm{E}-01$ & $5.94 \mathrm{E}-03$ & $1.60 \times 10^{-5}$ \\
At least 4 jets & $5.01 \mathrm{E}-01$ & $5.85 \mathrm{E}-03$ & $1.58 \times 10^{-5}$ \\
$\mathrm{E}_{\mathrm{T}}^{j 1}$ & $3.10 \mathrm{E}-01$ & $1.57 \mathrm{E}-04$ & $3.66 \times 10^{-4}$ \\
$\mathrm{E}_{\mathrm{T}}^{j 2}$ & $1.86 \mathrm{E}-01$ & $4.76 \mathrm{E}-05$ & $7.21 \times 10^{-4}$ \\
$\mathrm{E}_{\mathrm{T}}^{j 4}$ & $1.02 \mathrm{E}-01$ & $3.24 \mathrm{E}-05$ & $5.82 \times 10^{-4}$ \\
Jets in $|\eta| \leqslant 2.4$ & $8.25 \mathrm{E}-02$ & $2.26 \mathrm{E}-05$ & $6.73 \times 10^{-4}$ \\
b tagging of 1 jet & $3.61 \mathrm{E}-02$ & $2.44 \mathrm{E}-06$ & $2.73 \times 10^{-3}$ \\
b tagging of 2 jets & $1.69 \mathrm{E}-02$ & $2.81 \mathrm{E}-07$ & $1.11 \times 10^{-2}$ \\
b tagging of 3 jets & $8.57 \mathrm{E}-03$ & $5.62 \mathrm{E}-08$ & $2.82 \times 10^{-2}$ \\
centrality $>0.7$ & $7.05 \mathrm{E}-03$ & $3.69 \mathrm{E}-08$ & $3.52 \times 10^{-2}$ \\
\hline
\end{tabular}

and accept events where only three of the four jets are in the tracker acceptance, with the other outside the tracker acceptance, but this option is not considered in this analysis.

Finally, the centrality variable, defined as

$$
\mathrm{C}=\frac{\sum \mathrm{E}_{\mathrm{T}}}{\sqrt{\left(\sum \mathrm{E}\right)^{2}+\left(\sum \mathrm{E}_{\mathrm{z}}\right)^{2}}}
$$

using the four highest $\mathrm{E}_{\mathrm{T}}$ jets in the event, is used to discriminate between signal and background, given its independence from the signal mass. The analysis uses the discrimination power of this variable to reject background events with $\mathrm{C}$ lower than 0.7 .

Table 11.8 summarises the selection cut efficiencies for background and signal. The signal to background ratio, $\mathrm{S} / \mathrm{B}$, is also shown. The event samples used to calculate numbers given in this table are statistically independent from the ones used to optimise the cuts.

11.2.3.5. Signal significance. The criterion for the presence of signal is based on the distribution of the reconstructed Higgs boson mass, considering as mass estimator the invariant mass distribution of the two leading $\mathrm{E}_{\mathrm{T}}$ jets. The signal significance, $S / \sqrt{(B)}$ is calculated in the mass window which maximises this ratio. Figure 11.12 shows the reconstructed Higgs boson mass distribution for signal and background after all selections as expected for $60 \mathrm{fb}^{-1}$.

The signal significances in the optimised mass window after all the cuts applied excluding and including the HLT in the analysis chain, can be found in Table 11.9. The HLT decreases the significance up to a factor 10 for low masses $\left(\mathrm{M}_{\mathrm{A}}=200 \mathrm{GeV} / \mathrm{c}^{2}\right)$. For higher masses, this factor is reduced to less than 2 .

11.2.3.6. Background uncertainty and discovery reach in the $M_{A}-\tan \beta$ plane. Given the low S/B ratio and the similarities of the signal and background distributions, a careful evaluation of the background has to be performed. The best source of background events will come from real data samples, when available, as it is being done at the Tevatron experiments [620]. The QCD multi-jet background will be determined from data by normalising distributions outside of the signal region, once the mass of the Higgs is known from other channels for example. Data will be also used to extract the background shape with possibly the help of Monte Carlo.

Figure 11.13 shows the effect of the background uncertainty on the discovery reach (with two sigma signal significance) in the $\mathrm{M}_{\mathrm{A}}$-tan $\beta$ plane. Different curves correspond to the 


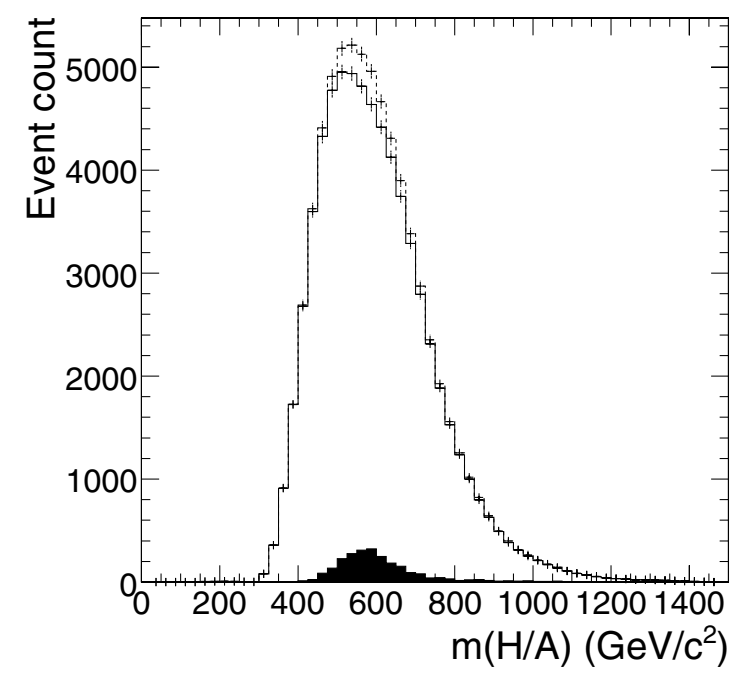

Figure 11.12. The Higgs boson mass distributions after all selections for the signal of $\mathrm{M}_{\mathrm{A}}=$ $600 \mathrm{GeV} / \mathrm{c}^{2}, \tan \beta=50$ in the $\mathrm{m}_{\mathrm{h}}^{\max }$ scenario (black in foreground), background (solid line) and signal plus background (dashed line) for $60 \mathrm{fb}^{-1}$.

Table 11.9. Signal significance $S / \sqrt{B}$ in optimised mass window after all selections with and without HLT filtering included. The last line shows the low limit of $\tan \beta$ where the $5 \sigma$ discovery is possible with $60 \mathrm{fb}^{-1}$ in the absence of systematics.

\begin{tabular}{lcccc}
\hline $\mathrm{M}_{\mathrm{A}}$ & 200 & 500 & 600 & 800 \\
\hline No HLT & 30.9 & 10.4 & 7.7 & 2.3 \\
With HLT & 2.9 & 6.4 & 5.6 & 3.4 \\
$\tan \beta$ where significance is 5 & 71 & 44 & 47 & 62 \\
\hline
\end{tabular}

different assumptions on the background uncertainty, from zero uncertainty to $2 \%$. The signal significance is defined as $s=\frac{S}{\sqrt{B+(\varepsilon B)^{2}}}$, where $\mathrm{S}$ is the number of signal events in the mass window, $\mathrm{B}$ is the number of background events in the same window and $\varepsilon$ is the relative background uncertainty.

The discovery potential of this channel is limited by the low signal-to-background ratio and the similarity of the signal and background distribution shapes. So far, it is not known how well the background can be measured at LHC, thus it is difficult to make predictions about the possibility to observe the MSSM Higgs bosons in the four-b final state.

11.2.4. Charged Higgs boson of $M_{H}<m_{t}$ in $t \bar{t} \rightarrow H^{ \pm} W^{\mp} b \bar{b}$ production with $H^{ \pm} \rightarrow \tau^{ \pm} \nu, \tau \rightarrow v+$ hadrons and $W^{\mp} \rightarrow \ell^{\mp} v$

A detailed description of the analysis can be found in [621].

11.2.4.1. Event generation and cross sections of signal and background events. The charged Higgs boson in the MSSM can be produced in top quark decays, $\mathrm{t} \rightarrow \mathrm{H}^{+} \mathrm{b}$, if $\mathrm{m}_{\mathrm{H}^{ \pm}}<\mathrm{m}_{\mathrm{t}}-\mathrm{m}_{\mathrm{b}}$. The branching ratio of top decay to charged Higgs boson depends on both $\mathrm{m}_{\mathrm{H}^{ \pm}}$and $\tan \beta$ as shown in Fig. 11.14a. The corresponding top decay to $\mathrm{W}^{ \pm} \mathrm{b}$ decreases with increasing $\tan \beta$ so as to keep the sum of branching ratios almost at unity. While the top decay to $\mathrm{H}^{ \pm}$or $\mathrm{W}^{ \pm}$ 


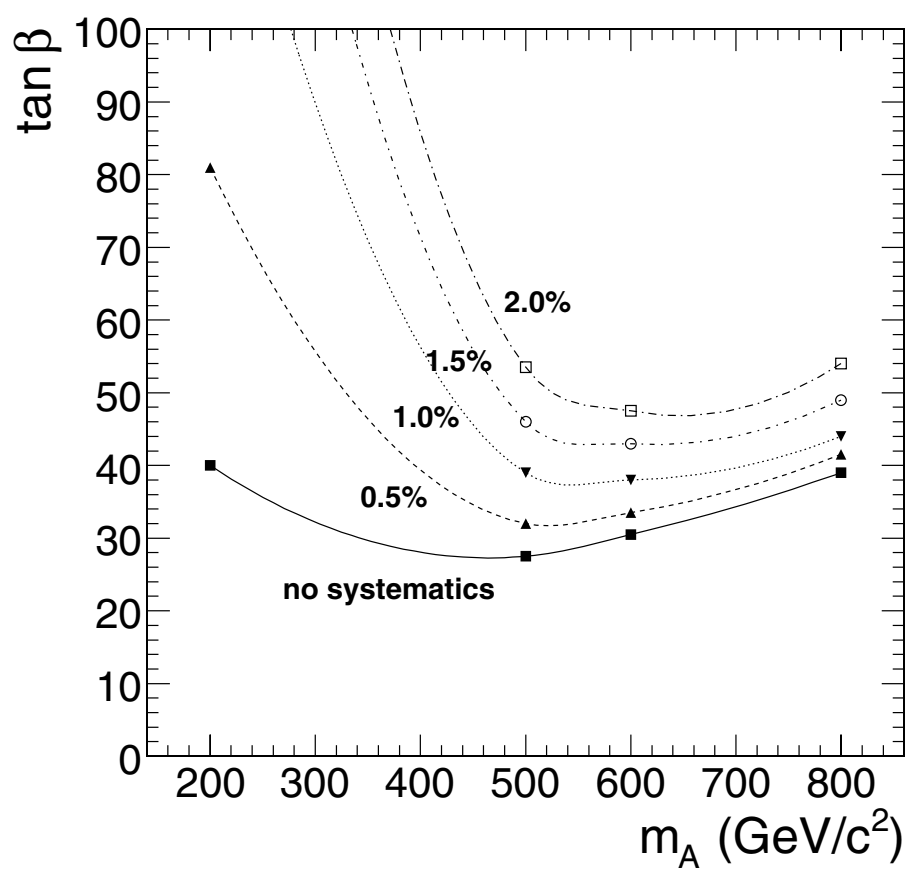

Figure 11.13. Two-sigma significance contours with different assumptions on the background uncertainty at $60 \mathrm{fb}^{-1}$ in the $\mathrm{m}_{\mathrm{h}}^{\max }$ scenario.
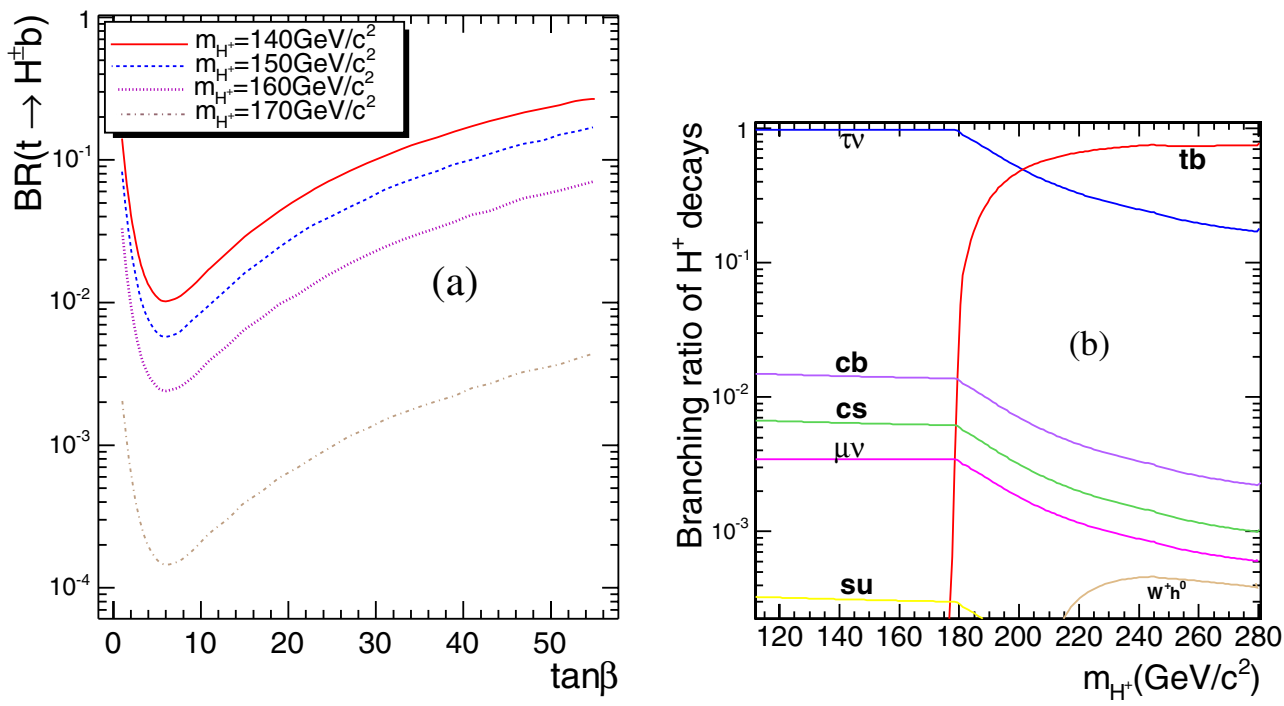

Figure 11.14. (a) Branching ratio of top decay to $\mathrm{H}^{ \pm}$vs $\tan \beta$, and (b) branching ratios for charged Higgs boson decaying to different final states for $\tan \beta=20$.

depends on $\tan \beta$, the light charged Higgs boson decay to $\tau \nu$ is almost independent of $\tan \beta$ (for $\tan \beta>10$ ) and is $\sim 98 \%$ for all $\tan \beta>10$ and $\mathrm{m}_{\mathrm{H}^{ \pm}}<\mathrm{m}_{\mathrm{t}}$ as shown in Fig. 11.14b.

There are two different final states for $\overline{\mathrm{t}} \rightarrow H^{ \pm} W^{\mp} \mathrm{b} \overline{\mathrm{b}}$ events depending on $\mathrm{W}^{ \pm}$decay to leptons or jets. In this analysis the leptonic decay of $\mathrm{W}^{ \pm}$boson is chosen and signal 
Table 11.10. Cross section times branching ratio of $\overline{\mathrm{t}} \rightarrow \mathrm{H}^{ \pm} \mathrm{W}^{\mp} \mathrm{b} \overline{\mathrm{b}} \rightarrow \tau \nu_{\tau} \ell \nu_{\ell} \mathrm{b} \overline{\mathrm{b}}, \tau \rightarrow$ hadrons for $\tan \beta=20$.

\begin{tabular}{lllll}
\hline $\mathrm{m}_{\mathrm{H}^{ \pm}}\left(\mathrm{GeV} / \mathrm{c}^{2}\right)$ & 140 & 150 & 160 & 170 \\
Cross section $[\mathrm{pb}]$ & 10.70 & 5.06 & 1.83 & 0.16 \\
\hline
\end{tabular}

Table 11.11. Cross section times branching ratio of signal events for $m_{H^{ \pm}} \simeq m_{t}$ according to NLO calculations in [597] for $\tan \beta=20$.

\begin{tabular}{lll}
\hline Channel & $\mathrm{gb} \rightarrow \mathrm{tH}^{ \pm} \rightarrow \ell v_{\ell} \mathrm{b} \tau v_{\tau}$ & $\mathrm{gg} \rightarrow \mathrm{tbH}^{ \pm} \rightarrow \ell v_{\ell} \mathrm{bb} \tau \nu_{\tau}$ \\
& $(\tau \rightarrow$ hadrons $)$ & $(\tau \rightarrow$ hadrons $)$ \\
& $\mathrm{m}_{\mathrm{H}^{ \pm}}=170 \mathrm{GeV} / \mathrm{c}^{2}$ & $\mathrm{~m}_{\mathrm{H}^{ \pm}}=170 \mathrm{GeV} / \mathrm{c}^{2}$ \\
Cross section $[\mathrm{pb}]$ & 0.14 & 0.30 \\
\hline
\end{tabular}

Table 11.12. Cross section times branching ratio of background events.

\begin{tabular}{lllll}
\hline & $\overline{\mathrm{t}} \rightarrow W^{+} W^{-} \mathrm{b} \overline{\mathrm{b}}$ & $\overline{\mathrm{t}} \rightarrow W^{+} W^{-} \mathrm{b} \overline{\mathrm{b}}$ & & \\
Channel & $\rightarrow \ell v_{\ell} \tau v_{\tau} \mathrm{b} \overline{\mathrm{b}}$ & $\rightarrow \ell v_{\ell} \ell^{\prime} v_{\ell^{\prime}} \mathrm{b} \overline{\mathrm{b}}$ & $\overline{\mathrm{t}} \rightarrow W^{+} W^{-} \mathrm{b} \overline{\mathrm{b}}$ & $\mathrm{W}^{ \pm}+3$ jets \\
& $(\tau \rightarrow$ hadrons $)$ & $\ell, \ell^{\prime}=\mathrm{e}$ or $\mu$ & $\rightarrow \ell v_{\ell} j j \mathrm{~b} \overline{\mathrm{b}}$ & $\mathrm{W}^{ \pm} \rightarrow \mathrm{e}$ or $\mu$ \\
Cross section $[\mathrm{pb}]$ & 25.8 & 39.7 & 245.6 & 840 \\
\hline
\end{tabular}

events are triggered by the single lepton trigger (e or $\mu$ ). The $\tau$ lepton is forced to decay to hadrons. Table 11.10 shows the cross section times branching ratio of $\bar{t} \overline{\mathrm{t}} \rightarrow \mathrm{H}^{ \pm} \mathrm{W}^{\mp} \mathrm{b} \overline{\mathrm{b}}$ events for $\tan \beta=20$. In this analysis for $\mathrm{m}_{H^{ \pm}}=170 \mathrm{GeV} / \mathrm{c}^{2}$ both $\mathrm{t} \overline{\mathrm{t}}+\mathrm{gb}$ and $\mathrm{gg} \rightarrow \mathrm{tb} \mathrm{H}^{ \pm}$production processes were used for comparison. The NLO cross section times branching ratio of signal events with $m_{H^{ \pm}} \simeq m_{t}$ is listed in Table 11.11.

The background channels consist of $\bar{t}$ events with at least a single lepton (e or $\mu$ ) and $\tau$-jets or jets which could fake $\tau$-jets, $\mathrm{W}^{ \pm}+3$ jet events and also single top (Wt) events which have a small contribution. The cross section of main background channels are shown in Table 11.12.

The $\bar{t}, \mathrm{gb} \rightarrow \mathrm{tH}^{ \pm}$and $\mathrm{gg} \rightarrow \mathrm{t} \bar{b} \mathrm{H}^{ \pm}$processes were generated by PYTHIA. The $\mathrm{Wt}$ background was generated with TOPREX and the $\mathrm{W}+3 \mathrm{j}$ background was generated by MADGRAPH. The production cross sections for the background processes were normalised to the NLO cross sections (except $\mathrm{W}+3$ jet).

11.2.4.2. Online event selection and offline reconstruction. Events are triggered by the single lepton triggers (e or $\mu$ ) at Level 1 and HLT.

In the offline $\geqslant 3$ jets are required to suppress $\mathrm{W}^{ \pm}+$njets background with $\mathrm{n}<3$. The jet reconstruction is performed using the iterative cone algorithm and the jet energy corrections, evaluated from $\gamma+$ jet calibration, were applied. A jet is accepted if it has calibrated $\mathrm{E}_{\mathrm{T}}>$ $40 \mathrm{GeV}$. Only one b-tagged jet is required in this analysis.

Since events are triggered by lepton from $\mathrm{W} \rightarrow \ell \nu$ decay, $\tau$ jets are identified with an offline $\tau$-tagging algorithm which uses Level $1 \tau$ objects as seeds for $\tau$-jet reconstruction. The first, highest $\mathrm{E}_{\mathrm{T}}$, jet satisfying the conditions of $\mathrm{E}_{\mathrm{T}}>20 \mathrm{GeV}$ and hottest HCAL tower $\mathrm{E}_{\mathrm{T}}>2 \mathrm{GeV}$ is used as a $\tau$ candidate. A matching cone with $\mathrm{R}_{\mathrm{m}}=0.1$, an isolation cone with $\mathrm{R}_{\mathrm{i}}=0.4$ and a signal cone with $\mathrm{R}_{\mathrm{S}}=0.07$ are defined for checking isolation requirements in the tracker. The ECAL isolation requirement is defined as

$\mathrm{P}_{\text {isol. }}=\sum_{\text {crystals, } \Delta \mathrm{R}_{\text {crystal }, \tau-\text { jet }}<0.4} \mathrm{E}_{\mathrm{T}_{\text {crystal }}}-\sum_{\text {crystals, }, \Delta \mathrm{R}_{\text {crystal }, \tau-\text { jet }}<0.13} \mathrm{E}_{\mathrm{T}_{\text {crystal }}}<5.6 \mathrm{GeV}$. 
Table 11.13. List of selection cuts and their efficiencies for signal events with $\mathrm{m}_{\mathrm{H}^{ \pm}}<170 \mathrm{GeV} / \mathrm{c}^{2}$ for $\tan \beta=20$. Numbers in each row show the remaining cross section after applying the corresponding cut. Numbers in parentheses are relative efficiencies in percent.

\begin{tabular}{|c|c|c|c|}
\hline & $\begin{array}{l}\overline{\mathrm{t}} \rightarrow \mathrm{H}^{ \pm} \mathrm{W}^{\mp} \mathrm{b} \overline{\mathrm{b}} \\
\rightarrow \ell \nu_{\ell} \tau \nu_{\tau} \mathrm{b} \overline{\mathrm{b}} \\
\mathrm{m}_{\mathrm{H}^{ \pm}}=140 \mathrm{GeV} / \mathrm{c}^{2}\end{array}$ & $\begin{array}{l}\overline{\mathrm{t}} \overline{\mathrm{t}} \rightarrow \mathrm{H}^{ \pm} \mathrm{W}^{\mp} \mathrm{b} \overline{\mathrm{b}} \\
\rightarrow \ell v_{\ell} \tau v_{\tau} \mathrm{b} \overline{\mathrm{b}} \\
\mathrm{m}_{\mathrm{H}^{ \pm}}=150 \mathrm{GeV} / \mathrm{c}^{2}\end{array}$ & $\begin{array}{l}\overline{\mathrm{t}} \rightarrow \mathrm{H}^{ \pm} \mathrm{W}^{\mp} \mathrm{b} \overline{\mathrm{b}} \\
\rightarrow \ell v_{\ell} \tau v_{\tau} \mathrm{b} \overline{\mathrm{b}} \\
\mathrm{m}_{\mathrm{H}^{ \pm}}=160 \mathrm{GeV} / \mathrm{c}^{2}\end{array}$ \\
\hline$\sigma \times \mathrm{BR}[\mathrm{fb}]$ & $10.7 \times 10^{3}$ & 5060 & 1830 \\
\hline L1 + HLT & $5170.5(48.3)$ & $2456.3(48.5)$ & $888.9(48.6)$ \\
\hline$\geqslant 3$ jets & $1889.7(36.5)$ & $795.0(32.4)$ & 264.3(29.7) \\
\hline$\geqslant 1 \mathrm{~b}$ jet & $1103.5(58.4)$ & $427.4(53.8)$ & $131.4(49.7)$ \\
\hline$<2$ b jets & $883.0(80.0)$ & $358.7(83.9)$ & 119.2(90.7) \\
\hline L1 $\tau$ exists & $878.4(99.5)$ & $357.4(99.6)$ & $119.0(99.8)$ \\
\hline$\tau$-jet reconstruction & $875.0(99.6)$ & $356.5(99.7)$ & $118.8(99.8)$ \\
\hline Hottest HCAL tower & $778.0(88.9)$ & $316.1(88.6)$ & $105.9(89.1)$ \\
\hline \multicolumn{4}{|l|}{$\mathrm{E}_{\mathrm{T}}>2 . \mathrm{GeV}$} \\
\hline Tracker isolation & $378.2(48.6)$ & $163.5(51.7)$ & $52.7(49.8)$ \\
\hline Ecal isolation & $292.9(77.4)$ & $134.2(82.1)$ & $43.1(81.8)$ \\
\hline$\tau \mathrm{E}_{\mathrm{T}}>40 \mathrm{GeV}$ & $244.3(83.4)$ & $113.0(84.2)$ & $36.5(84.7)$ \\
\hline $\mathrm{p}_{\text {leading track }} / \mathrm{E}_{\tau-\text { jet }}>0.8$ & $102.3(41.9)$ & $50.7(44.8)$ & $16.8(45.9)$ \\
\hline $\mathrm{Q}(\ell)+\mathrm{Q}(\tau)=0$ & $88.0(86.0)$ & $42.4(83.6)$ & $14.6(87.0)$ \\
\hline $\mathrm{E}_{\mathrm{T}}^{\mathrm{miss}}>70 \mathrm{GeV}$ & $51.0(58.0)$ & $25.4(59.9)$ & $9.2(63.3)$ \\
\hline $\begin{array}{l}\text { Expected Number of } \\
\text { events after } 10 \mathrm{fb}^{-1}\end{array}$ & 510 & 254 & 92 \\
\hline
\end{tabular}

When the tracker and ECAL isolation cuts are applied, the $\tau$-jet $\mathrm{E}_{\mathrm{T}}$ is required to be more than $40 \mathrm{GeV}$ and the leading track of $\tau$ jet is required to carry at least $80 \%$ of the visible $\tau$-lepton energy; finally the charges of the $\tau$ lepton and the lepton in the event should satisfy the requirement $\mathrm{Q}(\ell)+\mathrm{Q}(\tau)=0$.

The missing $\mathrm{E}_{\mathrm{T}}$ is reconstructed with the energy corrections applied to jets (Type 1 $\left.\mathrm{E}_{\mathrm{T}}^{\text {miss }}[147,148]\right)$ and a cut on the reconstructed missing $\mathrm{E}_{\mathrm{T}}\left(\mathrm{E}_{\mathrm{T}}^{\text {miss }}>70 \mathrm{GeV}\right)$ is applied as a rejection tool against background events, especially $\mathrm{W}^{ \pm}+3$ jets.

11.2.4.3. Selection efficiencies and expected number of events. Tables $11.13,11.14,11.15$ show the selection cuts and their efficiencies for signal and background samples. Other background events such as Wbb, Zbb with $\mathrm{W} \rightarrow \ell v(\ell=\mathrm{e}, \mu)$ and $\mathrm{Z} \rightarrow \mathrm{ee}$, or $\tau \tau$ turned out to be negligible. Single top background contribution is also small but was considered in the analysis for signal significance calculations.

11.2.4.4. Systematic uncertainties. The systematic uncertainties in the signal significance calculation include the experimental selection uncertainty of the background events and the theoretical cross section calculation uncertainty of the $t \mathrm{t}$ and single top background. The $t \bar{t}$ background uncertainty is taken into account as in Eq. 11.7:

$\Delta_{\text {sys. }}^{\mathrm{tf}}=\Delta_{\text {lepton reconstruction }} \oplus \Delta_{\geqslant 3 \text { jet selection }} \oplus \Delta_{1 \text { b-jet tagging }} \oplus \Delta_{1 \tau \text { tagging }} \oplus \Delta_{\text {lumi. }} \oplus \Delta_{\text {theo. }}^{\mathrm{tt}}$.

The $\mathrm{W}^{ \pm}+3$ jets background is assumed to be measured from the real data. The uncertainty of the measurement is estimated by propagating the contribution of events counted in the background area to the signal area and cancelling the common selection cuts 
Table 11.14. List of selection cuts and their efficiencies for signal events with $\mathrm{m}_{\mathrm{H}^{ \pm}}=170 \mathrm{GeV} / \mathrm{c}^{2}$ for $\tan \beta=20$. Numbers in each row show the remaining cross section after applying the corresponding cut. Numbers in parentheses are relative efficiencies in percent.

\begin{tabular}{|c|c|c|c|}
\hline & $\begin{array}{l}\mathrm{t} \overline{\mathrm{t}} \rightarrow \mathrm{H}^{ \pm} \mathrm{W}^{\mp} \mathrm{b} \overline{\mathrm{b}} \\
\rightarrow \ell \nu_{\ell} \tau \nu_{\tau} \mathrm{b} \overline{\mathrm{b}} \\
\mathrm{m}_{\mathrm{H}^{ \pm}}=170 \mathrm{GeV} / \mathrm{c}^{2}\end{array}$ & $\begin{array}{l}\mathrm{gb} \rightarrow \mathrm{tH}^{ \pm} \\
\rightarrow \ell v_{\ell} \tau v_{\tau} \mathrm{b} \\
\mathrm{m}_{\mathrm{H}^{ \pm}}=170 \mathrm{GeV} / \mathrm{c}^{2}\end{array}$ & $\begin{array}{l}\mathrm{gg} \rightarrow \mathrm{tb} \mathrm{H}^{ \pm} \\
\rightarrow \ell \nu_{\ell} \tau \nu_{\tau} \mathrm{bb} \\
\mathrm{m}_{\mathrm{H}^{ \pm}}=170 \mathrm{GeV} / \mathrm{c}^{2}\end{array}$ \\
\hline$\sigma \times \mathrm{BR}[\mathrm{fb}]$ & 157 & 140 & 297 \\
\hline $\mathrm{L} 1+\mathrm{HLT}$ & $78.0(49.7)$ & $70.5(50.4)$ & $145.4(48.9)$ \\
\hline$\geqslant 3$ jets & $23.2(29.7)$ & $21.7(30.7)$ & $55.3(38.0)$ \\
\hline$\geqslant 1$ bjet & $11.5(49.4)$ & $11.7(54.1)$ & $31.9(57.7)$ \\
\hline$<2$ b jets & 10.9(94.8) & $10.0(85.5)$ & $25.8(80.9)$ \\
\hline L1 $\tau$ exists & $10.8(99.8)$ & $10.0(99.6)$ & $25.7(99.4)$ \\
\hline$\tau$-jet reconstruction & $10.8(99.9)$ & $10.0(99.9)$ & 25.5(99.1) \\
\hline $\begin{array}{l}\text { Hottest HCAL tower } \\
\mathrm{E}_{\mathrm{T}}>2 . \mathrm{GeV}\end{array}$ & $9.6(88.4)$ & $8.9(88.8)$ & $22.6(88.9)$ \\
\hline Tracker isolation & $4.9(51.3)$ & $5.1(57.2)$ & $11.4(50.5)$ \\
\hline Ecal isolation & $4.2(84.9)$ & $4.3(84.5)$ & $9.6(84.4)$ \\
\hline$\tau \mathrm{E}_{\mathrm{T}}>40 . \mathrm{GeV}$ & $3.8(90.9)$ & $3.9(90.6)$ & $8.6(89.2)$ \\
\hline $\mathrm{p}_{\text {leading track }} / \mathrm{E}_{\tau-\text { jet }}>0.8$ & $1.6(41.7)$ & $1.8(45.9)$ & $3.4(39.6)$ \\
\hline $\mathrm{Q}(\ell)+\mathrm{Q}(\tau)=0$ & $1.3(84.4)$ & $1.6(87.2)$ & $2.8(82.6)$ \\
\hline $\mathrm{E}_{\mathrm{T}}^{\mathrm{miss}}>70 \mathrm{GeV}$ & $0.8(61.7)$ & $1.0(65.2)$ & $1.6(55.3)$ \\
\hline $\begin{array}{l}\text { Expected Number of events } \\
\text { after } 10 \mathrm{fb}^{-1}\end{array}$ & 8 & 10 & 16 \\
\hline
\end{tabular}

Table 11.15. List of selection cuts and their efficiencies for background events. Numbers in each row show the remaining cross section after applying the corresponding cut. Numbers in parentheses are relative efficiencies in percent.

\begin{tabular}{|c|c|c|c|c|}
\hline & $\begin{array}{l}\mathrm{t} \overline{\mathrm{t}} \rightarrow \mathrm{W}^{+} \mathrm{W}^{-} \mathrm{b} \overline{\mathrm{b}} \\
\rightarrow \ell v_{\ell} \tau v_{\tau} \mathrm{b} \overline{\mathrm{b}}\end{array}$ & $\begin{array}{l}\mathrm{t} \overline{\mathrm{t}} \rightarrow \mathrm{W}^{+} \mathrm{W}^{-} \mathrm{b} \overline{\mathrm{b}} \\
\rightarrow \ell v_{\ell} \ell^{\prime} \nu_{\ell^{\prime}} \mathrm{b} \overline{\mathrm{b}}\end{array}$ & $\begin{array}{l}\mathrm{t} \overline{\mathrm{t}} \rightarrow \mathrm{W}^{+} \mathrm{W}^{-} \mathrm{b} \overline{\mathrm{b}} \\
\rightarrow \ell v_{\ell} j j \mathrm{~b} \overline{\mathrm{b}}\end{array}$ & $\begin{array}{l}\mathrm{W}^{ \pm}+3 \text { jets } \\
\mathrm{W}^{ \pm} \rightarrow \ell v_{\ell}\end{array}$ \\
\hline$\sigma \times \mathrm{BR}[\mathrm{fb}]$ & $25.8 \times 10^{3}$ & $39.8 \times 10^{3}$ & $245.6 \times 10^{3}$ & $840 . \times 10^{3}$ \\
\hline L1 + HLT & $12101.2(46.9)$ & 28429.1(71.4) & $99506.6(40.5)$ & $287280(34.2)$ \\
\hline$\geqslant 3$ jets & $5105.2(42.2)$ & $11306.6(39.8)$ & 66038.6(66.4) & $114050(39.7)$ \\
\hline$\geqslant 1 \mathrm{~b}$ jet & $3428.3(67.1)$ & $7622.0(67.4)$ & $43433.0(65.8)$ & $24292.7(21.3)$ \\
\hline$<2$ b jets & $2325.7(67.8)$ & $5262.7(69.0)$ & $29003.4(66.8)$ & $21207.5(87.3)$ \\
\hline L1 $\tau$ exists & 2310.7(99.3) & $5233.7(99.4)$ & $28698.8(98.9)$ & 20613.7(97.2) \\
\hline$\tau$-jet reconstruction & 2303.6(99.7) & $5224.4(99.8)$ & $28465.0(99.2)$ & 19438.7(94.3) \\
\hline $\begin{array}{l}\text { Hottest HCAL tower } \\
\mathrm{E}_{\mathrm{T}}>2 . \mathrm{GeV}\end{array}$ & 2034.1(88.3) & $3850.6(73.7)$ & 26635.1(93.6) & $17125.5(88.1)$ \\
\hline Tracker isolation & 798.7(39.3) & $1120.6(29.1)$ & $6653.3(25.0)$ & $5411.7(31.6)$ \\
\hline Ecal isolation & $545.6(68.3)$ & $519.5(46.3)$ & $2952.8(44.4)$ & $2554.3(47.2)$ \\
\hline$\tau \mathrm{E}_{\mathrm{T}}>40 . \mathrm{GeV}$ & $405.8(74.4)$ & $341.8(65.8)$ & $1946.8(65.9)$ & $1312.9(51.4)$ \\
\hline $\mathrm{p}_{\text {leadingtrack }} / E_{\tau-\text { jet }}>0.8$ & $123.5(30.4)$ & $131.9(38.6)$ & $377.9(19.4)$ & $224.5(17.1)$ \\
\hline $\mathrm{Q}(\ell)+\mathrm{Q}(\tau)=0$ & $95.7(77.5)$ & $56.7(43.0)$ & $78.8(20.9)$ & $27.1(12.1)$ \\
\hline $\mathrm{E}_{\mathrm{T}}^{\mathrm{miss}}>70 \mathrm{GeV}$ & $51.6(53.9)$ & $29.3(51.8)$ & $36.6(46.4)$ & $10.7(39.3)$ \\
\hline $\begin{array}{l}\text { Expected Number of } \\
\text { events after } 10 \mathrm{fb}^{-1}\end{array}$ & 516 & 293 & 366 & 107 \\
\hline
\end{tabular}

uncertainties. Eq. 11.8 describes how systematic uncertainties are taken into account in $\mathrm{W}+3$ jets cross section measurement.

$\Delta_{\text {sys. }}^{\mathrm{W}^{ \pm}+3 \text { jets }}=\Delta_{\text {stat. }} \oplus \frac{\Delta \mathrm{N}_{\mathrm{B}}^{\mathrm{t} \overline{\mathrm{t}}}}{\mathrm{N}_{\mathrm{B}}^{\mathrm{W}^{ \pm}+3 \text { jets }}} \oplus \Delta_{3 \text { non-b-jet }} \oplus \Delta_{\text {b-jet mistagging }} \oplus \Delta_{\tau \text { mistagging }}$. 
Table 11.16. The values of different selection uncertainties for $t \bar{t}$ and $\mathrm{W}^{ \pm}+3$ jets background events at $30 \mathrm{fb}^{-1}$

\begin{tabular}{ll}
\hline Scale uncertainty of $t \bar{t}$ cross section & $5 \%$ \\
PDF uncertainty of $t \bar{t}$ cross section & $2.5 \%$ \\
b tagging & $5 \%$ \\
$\tau$ tagging & $4 \%$ \\
Lepton identification & $2 \%$ \\
Jet energy scale & $3 \%$ \\
Mistagging a non-b jet as a b jet & $5 \%$ \\
Mistagging a jet as a $\tau$ jet & $2 \%$ \\
Non-b-jet identification (anti-b-tagging) & $5 \%$ \\
Luminosity uncertainty & $5 \%$ \\
\hline
\end{tabular}
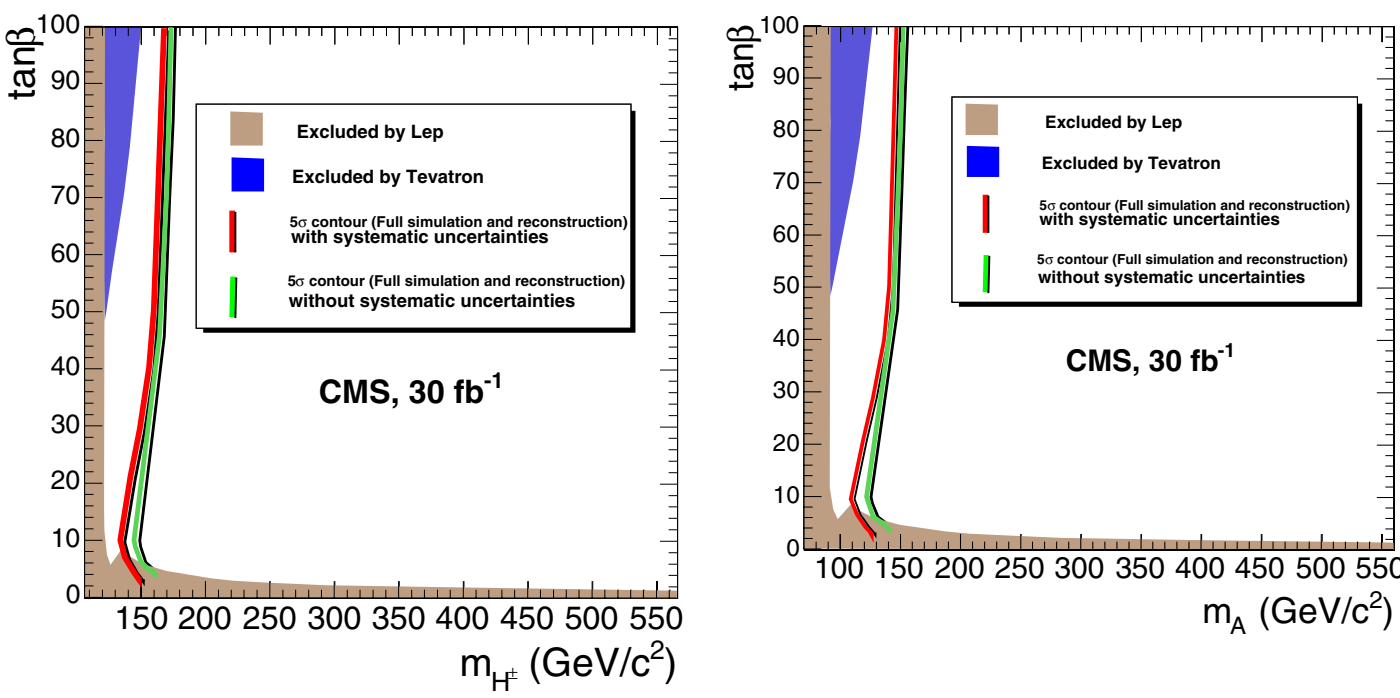

Figure 11.15. The $5 \sigma$ contour in the $\left(\mathrm{M}_{\mathrm{H}^{+}}, \tan \beta\right)$ plane for light charged Higgs boson discovery at $30 \mathrm{fb}^{-1}$ including the effect of systematic uncertainties.

Figure 11.16. The $5 \sigma$ contour in the $\left(\mathrm{M}_{\mathrm{A}}, \tan \beta\right)$ plane for light charged Higgs boson discovery at $30 \mathrm{fb}^{-1}$ including the effect of systematic uncertainties.

Table 11.16 lists different sources of systematic uncertainties and their used values corresponding to $30 \mathrm{fb}^{-1}$ in this analysis.

11.2.4.5. Discovery reach in the $M_{A\left(H^{ \pm}\right)}-\tan \beta$ plane. Figures 11.15 and 11.16 show the $5 \sigma$ discovery region in the $\left(\mathrm{M}_{\mathrm{H}^{+}}, \tan \beta\right)$ and $\left(\mathrm{M}_{\mathrm{A}}, \tan \beta\right)$ planes including the systematic uncertainties. It should be noted that this analysis is systematics dominated and there could be alternative approaches where the systematic uncertainties cancel down to a reasonable level.

11.2.5. Charged Higgs boson of $M_{H}>m_{t}$ in $g g \rightarrow t b H^{ \pm}$production with $H^{ \pm} \rightarrow \tau^{ \pm} v, \tau \rightarrow$ hadrons $v$ and $W^{\mp} \rightarrow j j$

The $\mathrm{H}^{ \pm} \rightarrow \tau^{ \pm} v_{\tau}$ decay mode with fully hadronic final state of the charged Higgs boson in the associated production with a top quark has been shown to lead to a clean and almost background-free signature at large $\tan \beta$ in several particle level [622] and fast 
simulation [383, 384, 623, 624] studies. The advantages of this decay mode in association with top quark are the large missing transverse energy from $\mathrm{H}^{ \pm}$, the possibility to disentangle the hadronic $\tau$ decay from the hadronic jets, the possibility to reconstruct the top mass to suppress the multi-jet backgrounds, and, in particular, $\tau$ helicity correlations favouring the $\mathrm{H}^{ \pm} \rightarrow \tau^{ \pm} \nu_{\tau}$ decay over the $\mathrm{W}^{ \pm} \rightarrow \tau^{ \pm} \nu_{\tau}$ decay (from the $\mathrm{tt}$ background). The main backgrounds are due to genuine $\tau$ 's in multi-jet events from $\mathrm{tt}_{\mathrm{t}}$ with $\mathrm{t}_{1} \rightarrow \mathrm{b} \tau \nu_{\tau}, \mathrm{t}_{2} \rightarrow$ bqq, Wt with $\mathrm{W}_{1} \rightarrow \tau \nu_{\tau}$, $\mathrm{W}_{2} \rightarrow \mathrm{qq}^{\prime}$ and $\mathrm{W}+3$ jets with $\mathrm{W} \rightarrow \tau \nu_{\tau}$. The hadronic QCD multi-jet events can lead to a background through fake $\tau$ 's and the uncertainty of $\mathrm{E}_{\mathrm{T}}^{\mathrm{miss}}$ measurement.

A detailed description of the analysis can be found in [625].

11.2.5.1. Helicity correlations. The polarisation states for the $\tau^{+}$from $\mathrm{H}^{+} \rightarrow \tau^{+} \nu_{\tau}$ and from $\mathrm{W}^{+} \rightarrow \tau^{+} v_{\tau}$ are opposite due to the spin-parity properties of the decaying particle. The angular distribution of a pion from the $\tau^{ \pm} \rightarrow \pi^{ \pm} v$ decay in the CM frame has the form $\left(1+\mathrm{P}_{\tau} \cos \theta\right)$, which leads to more energetic pions in the laboratory frame for the signal $\left(\mathrm{P}_{\tau}=1\right)$ than for the background $\left(\mathrm{P}_{\tau}=-1\right)[622,626]$. The $\tau^{ \pm} \rightarrow \pi^{ \pm} \nu_{\tau}$ decay channel presents $12.5 \%$ of the hadronic decay modes. Similarly, the signal pions are more energetic in the $\tau$ decays to vector mesons and subsequent decays to one charged pion in the longitudinal polarisation states of the vector meson, $\tau^{ \pm} \rightarrow \rho_{\mathrm{L}}^{ \pm} \nu_{\tau} \rightarrow \pi^{ \pm} \pi^{\circ} \nu_{\tau}(26 \%)$ and $\tau^{ \pm} \rightarrow \mathrm{a}_{1 \mathrm{~L}}^{ \pm} \nu_{\tau} \rightarrow \pi^{ \pm} \pi^{\circ} \pi^{\circ} \nu_{\tau}(7.5 \%)$. For the transverse polarisation states of the vector meson the situation is opposite with more energetic pions from the background. The small contributions from $\mathrm{K}^{*}$ and $\mathrm{K}$ in the $\tau$ decays lead to similar effects. The helicity correlations can be expressed as a function of the $\tau$-jet momentum fraction carried by the charged pion $\mathrm{R}_{\tau}=\mathrm{p}_{\pi} / \mathrm{p}_{\tau \text { jet }}$. As is shown in Refs. [622, 626] the $\tau^{ \pm} \rightarrow \pi^{ \pm} \nu_{\tau}$ decay leads to a $\delta$-function at $\mathrm{R}_{\tau}=1$, the $\rho_{\mathrm{L}}^{ \pm} \nu_{\tau} \rightarrow \pi^{ \pm} \pi^{\circ} \nu_{\tau}$ has contributions at $\mathrm{R}_{\tau} \sim 1$ and $\mathrm{R}_{\tau} \sim 0, \rho_{\mathrm{T}}^{ \pm} \nu_{\tau} \rightarrow \pi^{ \pm} \pi^{\circ} \nu_{\tau}$ and $\mathrm{a}_{1 \mathrm{~T}}^{ \pm} \nu_{\tau} \rightarrow \pi^{ \pm} \pi^{\circ} \pi^{\circ} \nu_{\tau}$ have largest contributions around $\mathrm{R}_{\tau} \sim 0.5$ while $\mathrm{a}_{1 \mathrm{~L}}^{ \pm} \nu_{\tau} \rightarrow \pi^{ \pm} \pi^{\circ} \pi^{\circ} \nu_{\tau}$ peaks at $\mathrm{R}_{\tau} \sim 0$.

11.2.5.2. Event generation and simulation. The $\mathrm{gb} \rightarrow \mathrm{tH}^{ \pm}$and $\mathrm{gg} \rightarrow \mathrm{tbH}^{ \pm}$processes contribute to the production of a heavy single charged Higgs boson in association with top quark. In the $\mathrm{gb} \rightarrow \mathrm{tH}^{ \pm}$process the $\mathrm{b}$ quark is considered as a massless parton of the incoming proton. Logarithmic factors of the form $\log \left(\mathrm{p}_{\mathrm{T}}^{\mathrm{b}} / \mathrm{m}_{\mathrm{b}}\right)$, due to the collinear $\mathrm{b}$ quarks, can be resumed to give a well defined cross section. The $\mathrm{gg} \rightarrow \mathrm{tbH}^{ \pm}$process, where the bottom quarks from the incoming gluons are considered massive, is of the order $\alpha_{\mathrm{s}}^{2}$ and is part of the next-to-leading order (LNO) corrections to the leading order (LO) process $\mathrm{gb} \rightarrow \mathrm{tH}^{ \pm}$. These processes lead to somewhat different dynamics of the final state objects, visible in particular as a more energetic associated b quark in the $\mathrm{gg} \rightarrow \mathrm{tbH}^{ \pm}$process [627]. Near the top threshold, $\mathrm{m}_{\mathrm{H}^{ \pm}} \sim \mathrm{m}_{\mathrm{t}}$, only the exclusive process $\mathrm{gg} \rightarrow \mathrm{tbH}^{ \pm}$can lead to a correct event description. As the correct description of merging these two processes is not possible in the full simulation, signal events were generated with the $\mathrm{gg} \rightarrow \mathrm{tbH}^{ \pm}$process over the full mass range with PYTHIA [69]. The cross sections were normalised to the NLO results of Refs. [597, 628]. The mass of the charged Higgs boson and the $\mathrm{H}^{ \pm} \rightarrow \tau \nu_{\tau}$ branching fraction were calculated with FeynHiggs2.3.2 [142-144] in the $\mathrm{m}_{\mathrm{h}}^{\max }$ scenario. The $\bar{t} \overline{\mathrm{t}}$ background was generated with PYTHIA, the Wt background with ToPREX [44], the W+3jet background with MADGRAPH [81] and the QCD multi-jet background with PYTHIA. The production cross sections for the background processes were normalised to the NLO cross sections (except $\mathrm{W}+3 \mathrm{jet}$ ). Pre-selections at the particle level, requiring at least one jet with $\mathrm{E}_{\mathrm{T}}>80 \mathrm{GeV}$, reconstructed with the PYTHIA PYCELL routine with a cone size of 0.5 , and containing at least one charged hadron with $\mathrm{p}_{\mathrm{T}}>60 \mathrm{GeV} / \mathrm{c}$, were applied to the $\mathrm{t} \overline{\mathrm{t}}$ and $\mathrm{Wt}$ backgrounds. The $\tau$ decays were performed with TAUOLA [155] for the signal and backgrounds. The $\tau$ from 
Table 11.17. Cross section times branching fraction for $\mathrm{gg} \rightarrow \mathrm{tbH}^{ \pm}, \mathrm{H}^{ \pm} \rightarrow \tau^{ \pm} \nu, \tau \rightarrow$ hadrons + $\nu$, efficiency for the selection cuts and final number of events for $\mathrm{m}_{\mathrm{T}}\left(\tau\right.$ jet, $\left.\mathrm{E}_{\mathrm{T}}^{\mathrm{miss}}\right)>100 \mathrm{GeV} / \mathrm{c}^{2}$ and for $\Delta \phi\left(\tau\right.$ jet, $\left.\mathrm{E}_{\mathrm{T}}^{\mathrm{miss}}\right)>60^{\circ}$ with an integrated luminosity of $30 \mathrm{fb}^{-1}$ for the signal events with $\mathrm{m}_{\mathrm{H}^{ \pm}}=170,180,200$ and $400 \mathrm{GeV} / \mathrm{c}^{2}$ and $\tan \beta=30$.

\begin{tabular}{|c|c|c|c|c|}
\hline $\begin{array}{l}\mathrm{m}_{\mathrm{H}^{ \pm}}\left(\mathrm{GeV} / \mathrm{c}^{2}\right) \\
\sigma(\mathrm{NLO}) \times \mathrm{BR}(\mathrm{fb})\end{array}$ & $\begin{array}{l}171.6 \\
1359\end{array}$ & $\begin{array}{l}180.4 \\
1238\end{array}$ & $\begin{array}{l}201.0 \\
776\end{array}$ & $\begin{array}{l}400.4 \\
38\end{array}$ \\
\hline Level-1 trigger & $729.9(53.7 \%)$ & $688.1(55.6 \%)$ & $451.3(58.2 \%)$ & $28.5(75.6 \%)$ \\
\hline HLT trigger & $121.0(16.6 \%)$ & $128.6(18.7 \%)$ & $95.9(21.2 \%)$ & $12.1(42.4 \%)$ \\
\hline Primary vertex & $119.9(99.1 \%)$ & $127.5(99.2 \%)$ & $95.1(99.2 \%)$ & $12.0(99.2 \%)$ \\
\hline Isolated lepton veto & $94.4(78.8 \%)$ & $104.2(81.7 \%)$ & $78.2(82.2 \%)$ & $10.1(85.0 \%)$ \\
\hline $\mathrm{E}_{\mathrm{T}}^{\mathrm{miss}}>100 \mathrm{GeV}$ & $66.7(70.6 \%)$ & $70.0(67.2 \%)$ & $53.3(68.2 \%)$ & $8.2(80.7 \%)$ \\
\hline $\mathrm{E}_{\mathrm{T}}^{\tau \mathrm{jet}}>100 \mathrm{GeV}$ & $33.7(50.5 \%)$ & $36.7(52.4 \%)$ & $27.8(52.1 \%)$ & $6.7(81.8 \%)$ \\
\hline $\mathrm{R}_{\tau}>0.8$ & $11.2(33.4 \%)$ & $11.6(31.5 \%)$ & $9.5(34.2 \%)$ & $2.3(34.2 \%)$ \\
\hline 1 or 3 signal tracks & $10.7(95.3 \%)$ & $11.2(97.1 \%)$ & $9.1(95.9 \%)$ & $2.2(97.0 \%)$ \\
\hline Tracker isolation & $10.0(93.2 \%)$ & $10.5(94.0 \%)$ & $8.6(94.9 \%)$ & $2.1(93.7 \%)$ \\
\hline ECAL isolation & $9.4(94.4 \%)$ & $10.0(95.0 \%)$ & $8.3(95.7 \%)$ & $2.0(95.8 \%)$ \\
\hline $\mathrm{E}_{\mathrm{T}}^{\max (\mathrm{HCAL} \text { cell })}>2 \mathrm{GeV}$ & $9.1(96.5 \%)$ & $9.4(93.3 \%)$ & $7.9(95.5 \%)$ & $2.0(98.7 \%)$ \\
\hline $\mathrm{IP}_{\mathrm{T}}^{\text {leading track }}<0.3 \mathrm{~mm}$ & $9.0(97.8 \%)$ & $9.2(98.2 \%)$ & $7.8(99.0 \%)$ & $2.0(99.3 \%)$ \\
\hline $\mathrm{N}_{\text {hits }}^{\text {leading track }} \geqslant 10$ & $8.6(95.9 \%)$ & $8.4(96.5 \%)$ & $7.4(94.6 \%)$ & $2.0(96.5 \%)$ \\
\hline$\geqslant 3$ jets, $\mathrm{E}_{\mathrm{T}}>20 \mathrm{GeV}$ & $6.4(74.4 \%)$ & $7.2(80.9 \%)$ & $5.7(77.4 \%)$ & $1.4(71.9 \%)$ \\
\hline $140<\mathrm{m}_{\mathrm{top}}<210 \mathrm{GeV} / \mathrm{c}^{2}$ & $4.6(72.6 \%)$ & $4.8(67.2 \%)$ & $3.6(63.7 \%)$ & $0.93(66.6 \%)$ \\
\hline $\mathrm{b}$ discriminator $>1.5$ & $2.0(43.7 \%)$ & $2.0(39.9 \%)$ & $1.6(42.7 \%)$ & $0.37(40.3 \%)$ \\
\hline $\mathrm{E}_{\mathrm{T}}^{\mathrm{bjet}}>30 \mathrm{GeV}$ & $1.9(93.2 \%)$ & $1.8(95.2 \%)$ & $1.4(91.6 \%)$ & $0.33(88.2 \%)$ \\
\hline Jet veto, $\mathrm{E}_{\mathrm{T}}^{\mathrm{jet}}>25 \mathrm{GeV}$ & $0.65(35.2 \%)$ & $0.63(34.6 \%)$ & $0.52(36.4 \%)$ & $0.14(40.9 \%)$ \\
\hline $\mathrm{E}_{\mathrm{T}}^{\mathrm{Higgs}}>50 \mathrm{GeV}$ & $0.61(91.9 \%)$ & $0.63(100 \%)$ & $0.52(100 \%)$ & $0.13(95.1 \%)$ \\
\hline $\mathrm{m}_{\mathrm{T}}>100 \mathrm{GeV} / \mathrm{c}^{2}$ & $0.47(77.3 \%)$ & $0.49(78.4 \%)$ & $0.39(74.9 \%)$ & $0.12(94.8 \%)$ \\
\hline $\mathrm{N}^{\mathrm{ev}}, \mathrm{m}_{\mathrm{T}}>100 \mathrm{GeV} / \mathrm{c}^{2}$ & $14.1 \pm 3.4$ & $14.7 \pm 3.2$ & $11.7 \pm 2.3$ & $3.6 \pm 0.5$ \\
\hline$\Delta \phi\left(\tau, \mathrm{E}_{\mathrm{T}}^{\mathrm{miss}}\right)>60^{0}$ & $0.20(31.9 \%)$ & $0.18(28.5 \%)$ & $0.28(53.9 \%)$ & $0.12(93.1 \%)$ \\
\hline $\mathrm{N}^{\mathrm{ev}}, \Delta \phi\left(\tau, \mathrm{E}_{\mathrm{T}}^{\mathrm{miss}}\right)>60^{0}$ & $6.0 \pm 2.2$ & $5.4 \pm 2.0(28.5 \%)$ & $8.3 \pm 2.0$ & $3.6 \pm 0.5$ \\
\hline
\end{tabular}

$\mathrm{H}^{ \pm}$was forced to decay to hadrons in the signal samples while all $\tau$ decays were generated for the backgrounds.

The analysis was based on event samples from full detector simulation and digitisation at low luminosity $2 \times 10^{33} \mathrm{~cm}^{-2} \mathrm{~s}^{-1}$.

11.2.5.3. Event selection. Due to an energetic $\tau$ jet from $\mathrm{H}^{ \pm}$the $\mathrm{gg} \rightarrow \mathrm{tbH}^{ \pm}, \mathrm{H}^{ \pm} \rightarrow \tau^{ \pm} v$ $\left(\tau \rightarrow\right.$ hadrons $\nu, \mathrm{W}^{\mp} \rightarrow \mathrm{jj}$ ) events can be most efficiently triggered at the Level-1 with a single $\tau$-jet trigger [76, 280]. At the HLT, a combined $\mathrm{E}_{\mathrm{T}}^{\mathrm{miss}}-\tau$ trigger was used. For this trigger the $\tau$-jet identification was performed in the full tracker (Tracker Tau trigger) [146]. Efficiencies of the Level 1 and HLT triggers are shown in Tables 11.17 and 11.18 for the signal and backgrounds, respectively. Purity of the $\tau$ trigger for the signal events is higher than $80 \%$.

In the off-line reconstruction the transverse mass from the $\tau$ jet and missing transverse energy requires a fully hadronic event, where $\mathrm{E}_{\mathrm{T}}^{\mathrm{miss}}$ originates mainly from the $\mathrm{H}^{ \pm}$. Other sources of $\mathrm{E}_{\mathrm{T}}^{\mathrm{miss}}$ in the signal events are the leptonic $\mathrm{W}$ decays and the semi-leptonic $\mathrm{b}$ quark decays. The events with leptonic $\mathrm{W}$ decays can be removed with a veto on isolated leptons. The reconstructed electrons and muons were first required to be isolated in the tracker demanding that no track with $\mathrm{p}_{\mathrm{T}}>1 \mathrm{GeV} / \mathrm{c}$ was found in a cone of $\Delta \mathrm{R}=0.4$ around the lepton direction. The fraction of events containing at least one muon candidate with $\mathrm{p}_{\mathrm{T}}>15 \mathrm{GeV} / \mathrm{c}$ is $24.1 \%$. An isolated muon is found in $8.9 \%$ of the signal events. 
Table 11.18. Cross section times branching fraction, efficiency for the selection cuts and final number of events for $\mathrm{m}_{\mathrm{T}}\left(\tau\right.$ jet, $\left.\mathrm{E}_{\mathrm{T}}^{\mathrm{miss}}\right)>100 \mathrm{GeV} / \mathrm{c}^{2}$ and for $\Delta \phi\left(\tau\right.$ jet, $\left.\mathrm{E}_{\mathrm{T}}^{\text {miss }}\right)>60^{\circ}$ with an integrated luminosity of $30 \mathrm{fb}^{-1}$ for the $\mathrm{t} \overline{\mathrm{t}}, \mathrm{Wt}, \mathrm{W}^{ \pm}+3$ jets and QCD multi-jet backgrounds background.

\begin{tabular}{|c|c|c|c|}
\hline & $\mathrm{t} \overline{\mathrm{t}}$ & Wt & $\mathrm{W}^{ \pm}+3$ jets \\
\hline$\sigma(\mathrm{NLO}) \times \mathrm{BR}(\mathrm{fb})$ & 123820 & 9140 & $4.19 \times 10^{5}$ \\
\hline Pre-selection & $6440(5.2 \%)$ & $237.6(2.6 \%)$ & \\
\hline Level-1 trigger & $4730(73.4 \%)$ & $185.6(78.1 \%)$ & $1.25 \times 10^{5}(29.8 \%)$ \\
\hline HLT trigger & $320(6.9 \%)$ & $20.5(11.1 \%)$ & $4.19 \times 10^{3}(3.4 \%)$ \\
\hline Primary vertex & $319(99.8 \%)$ & $20.4(99.7 \%)$ & $4190(100 \%)$ \\
\hline Isolated lepton veto & $314(89.4 \%)$ & $18.4(89.9 \%)$ & $3456(82.5 \%)$ \\
\hline $\mathrm{E}_{\mathrm{T}}^{\mathrm{miss}}>100 \mathrm{GeV}$ & $267.4(85.1 \%)$ & $15.9(86.6 \%)$ & $2674(77.1 \%)$ \\
\hline $\mathrm{E}_{\mathrm{T}}^{\tau \text { jet }}>100 \mathrm{GeV}$ & $167.4(62.6 \%)$ & $10.7(67.2 \%)$ & $1280(69.2 \%)$ \\
\hline $\mathrm{R}_{\tau}>0.8$ & $35.5(21.2 \%)$ & $2.53(23.7 \%)$ & $175.4(13.7 \%)$ \\
\hline 1 or 3 signal tracks & $31.2(88.0 \%)$ & $2.37(93.7 \%)$ & $149.3(85.1 \%)$ \\
\hline Tracker isolation & $27.8(89.1 \%)$ & $2.18(91.9 \%)$ & $132.9(89.2 \%)$ \\
\hline ECAL isolation & $26.1(93.7 \%)$ & $2.07(94.9 \%)$ & $125.1(94.1 \%)$ \\
\hline $\mathrm{E}_{\mathrm{T}}^{\max (\mathrm{HCAL} \text { cell })}>2 \mathrm{GeV}$ & $24.1(92.4 \%)$ & $1.95(94.2 \%)$ & $105.1(84.0 \%)$ \\
\hline $\mathrm{IP}_{\mathrm{T}}^{\text {leading track }}<0.3$ & $21.4(88.8 \%)$ & $1.92(98.3 \%)$ & $88.4(84.1 \%)$ \\
\hline $\mathrm{N}_{\text {hits }}^{\text {leading track }} \geqslant 10$ & $19.9(92.9 \%)$ & $1.81(94.4 \%)$ & $84.6(95.7 \%)$ \\
\hline$\geqslant 3$ jets, $\mathrm{E}_{\mathrm{T}}>20 \mathrm{GeV}$ & $17.3(87.0 \%)$ & $1.04(57.6 \%)$ & $67.5(79.8 \%)$ \\
\hline $140<\mathrm{m}_{\mathrm{top}}<210 \mathrm{GeV} / \mathrm{c}^{2}$ & $12.2(70.4 \%)$ & $0.71(67.7 \%)$ & $26.6(39.4 \%)$ \\
\hline $\mathrm{b}$ discriminator $>1.5$ & $5.81(47.7 \%)$ & $0.34(48.1 \%)$ & $1.09(4.1 \%)$ \\
\hline $\mathrm{E}_{\mathrm{T}}^{\mathrm{bjet}}>30 \mathrm{GeV}$ & $5.27(90.6 \%)$ & $0.30(89.2 \%)$ & $0.82(75.1 \%)$ \\
\hline Jet veto, $\mathrm{E}_{\mathrm{T}}^{\mathrm{jet}}>25 \mathrm{GeV}$ & $1.48(28.1 \%)$ & $0.24(78.0 \%)$ & $0.14(17.2 \%)$ \\
\hline $\mathrm{E}_{\mathrm{T}}^{\mathrm{Higgs}}>50 \mathrm{GeV}$ & $1.44(97.1 \%)$ & $0.23(98.6 \%)$ & $0.14(98.3 \%)$ \\
\hline $\mathrm{m}_{\mathrm{T}}\left(\tau\right.$ jet, $\left.\mathrm{E}_{\mathrm{T}}^{\mathrm{miss}}\right)>100 \mathrm{GeV} / \mathrm{c}^{2}$ & $0.03(2.0 \%)$ & $0.003(1.3 \%)$ & $0.02(10.3 \%)$ \\
\hline Events for $\mathrm{m}_{\mathrm{T}}>100 \mathrm{GeV} / \mathrm{c}^{2}$ & $0.86 \pm 0.33$ & $0.09 \pm 0.04$ & $0.60 \pm 0.60$ \\
\hline$\Delta \phi\left(\tau\right.$ jet, $\left.\mathrm{E}_{\mathrm{T}}^{\mathrm{miss}}\right)>60^{\circ}$ & $0.01(1.0 \%)$ & $9.2 \times 10^{-4}(0.4 \%)$ & $0.013(6.7 \%)$ \\
\hline Events for $\Delta \phi\left(\tau\right.$ jet, $\left.\mathrm{E}_{\mathrm{T}}^{\mathrm{miss}}\right)>60^{\circ}$ & $0.30 \pm 0.25$ & $0.03 \pm 0.02$ & $0.39 \pm 0.39$ \\
\hline
\end{tabular}

About $84 \%$ of these muons were found to originate from $\mathrm{W} \rightarrow \mu v_{\mu}$. The fraction of events containing at least one electron candidate with $\mathrm{p}_{\mathrm{T}}>15 \mathrm{GeV} / \mathrm{c}$ is $72.4 \%$ and an isolated electron candidate $41.7 \%$. The final electron identification was done following the methods described in Ref. [156]. The fraction of events removed with a veto on the identified electrons is $7.9 \%$, from which $93.3 \%$ are due to genuine electrons from $\mathrm{W} \rightarrow \mathrm{ev}$.

The missing transverse energy $\left(\mathrm{E}_{\mathrm{T}}^{\text {miss }}\right)$ was reconstructed from the full calorimeter response summing the calorimeter towers and applying the jet energy corrections (Type 1 $\left.\mathrm{E}_{\mathrm{T}}^{\text {miss }}[147,148]\right)$. The hadronic jets with $\mathrm{E}_{\mathrm{T}}^{\text {raw }}>20 \mathrm{GeV}$ were calibrated using the corrections from $\gamma+$ jet calibration. The $\tau$ jet was reconstructed in the calorimeter around the Level- $1 \tau$-jet direction in a cone of 0.4 applying energy corrections evaluated for one- and three-prong $\tau$ decays. The offline $\mathrm{E}_{\mathrm{T}}$ cut on the $\tau$ jet was taken to be $\mathrm{E}_{\mathrm{T}}^{\tau \text { jet }}>100 \mathrm{GeV}$, close to the Level1 threshold of $93 \mathrm{GeV}$. The tracks were reconstructed inside the jet reconstruction cone. The leading track was searched for in a cone of $\mathrm{R}_{\mathrm{m}}=0.1$ around the $\tau$-jet direction. For an efficient isolation against the hadronic jets a small signal cone of $R_{S}=0.04$ was selected. The isolation cone size was taken to be the same as in the HLT Tau trigger, $R_{i}=0.4$. The $\tau$-jet isolation in the electromagnetic calorimeter was also applied as described in [280]. The fraction of signal events with $\mathrm{m}_{\mathrm{H}^{ \pm}}=200 \mathrm{GeV} / \mathrm{c}^{2}$, where the one-prong (three-prong) $\tau$ decays lead to one (three) reconstructed track(s) with $\mathrm{p}_{\mathrm{T}}>1 \mathrm{GeV} / \mathrm{c}$ in the signal cone, was found to be in 
92.3\% (64\%). Accidental track reconstruction problems, like shared hits, can lead to fake large- $\mathrm{p}_{\mathrm{T}}$ tracks in the hadronic jets $[7,280]$. These fake leading tracks are possible in the hadronic multi-jet events but can appear also in the $t \bar{t}$, Wt and $\mathrm{W}+3$ jet backgrounds if the $\mathrm{E}_{\mathrm{T}}$ of the $\tau$ jet is below the trigger threshold and the event is triggered with a $\tau$-like hadronic jet. The fake tracks can be suppressed with an upper bound in the transverse impact parameter of the leading track $\left(\mathrm{IP}_{\mathrm{T}}^{\text {leading track }}<0.3 \mathrm{~mm}\right)$ and requiring at least 10 hits in the full tracker. The fraction of the $\tau \rightarrow$ ev $\nu$ events passing the full $\tau$ selection was found to be $3 \%$ for the $t \bar{t}$ background. This contamination can be efficiently suppressed requiring that the most energetic HCAL tower inside the $\tau$-jet candidate $\left(\mathrm{E}_{\mathrm{T}}^{\max (\mathrm{HCAL} \text { cell })}\right)$ has the transverse energy greater than $2 \mathrm{GeV}$ [280].

The $\tau$ helicity correlations are best exploited requiring the leading track to carry at least $80 \%$ of the $\tau$ jet energy. The efficiencies for the $t \bar{t}$ and Wt events, shown in Tables 11.17 and 11.18, are affected by the pre-selection cuts and do not show the expected background suppression for $\mathrm{R}_{\tau}>0.8$. This cut suppresses the three-prong $\tau$ decays leaving $3.1 \%$ as the fraction of three-prong $\tau$ decays for the signal events with $m_{\mathrm{H}^{ \pm}}=200 \mathrm{GeV} / \mathrm{c}^{2}$ after all selection cuts.

Due to a limited MC statistics, the trigger simulation was not used in the estimation of the QCD multi-jet background. Events with at least one jet with $\mathrm{E}_{\mathrm{T}}>100 \mathrm{GeV}$, containing a track with $\mathrm{p}_{\mathrm{T}}>80 \mathrm{GeV} / \mathrm{c}$, were used for further analysis. Efficiency for this selection was found to be $5.55 \times 10^{-3}$ for the QCD multi-jet events generated within the $\hat{\mathrm{p}}_{\mathrm{T}}$ interval of $170<\hat{\mathrm{p}}_{\mathrm{T}}<380 \mathrm{GeV} / \mathrm{c}$. The $\tau$ selection cuts, except the $\mathrm{E}_{\mathrm{T}}^{\mathrm{jet}}$ threshold, are not correlated with the $\mathrm{E}_{\mathrm{T}}^{\mathrm{miss}}$ cut. Therefore the selection was factorised to $\mathrm{E}_{\mathrm{T}}^{\mathrm{miss}}$ and $\tau$ selections. The efficiency of the $\tau$-selection cuts on the pre-selected events was found to be $1.65 \%$. Combined with the pre-selection, the full $\tau$-selection efficiency for the hadronic multi-jet events in the $\hat{\mathrm{p}}_{\mathrm{T}}$ interval considered was found to be $9.2 \times 10^{-5}$.

The $\mathrm{gg} \rightarrow \mathrm{tbH}^{ \pm}$events contain two $\mathrm{b}$ jets, one from the decay of the top quark and one associated $b$ jet from the production process. The associated $b$ quark is preferentially emitted in the forward directions and is distributed at smaller $\mathrm{p}_{\mathrm{T}}$ values than the $\mathrm{b}$ quark from top decay. In about $20 \%$ of the signal events, however, this b quark is more energetic than the $\mathrm{b}$ quark from the top decay thus contaminating the spectrum of the identified $\mathrm{b}$ jet for the top reconstruction. The event reconstruction was performed for events where at least three hadronic jets with $\mathrm{E}_{\mathrm{T}}^{\mathrm{jet}}>20 \mathrm{GeV}$ were found. A probabilistic secondary vertex algorithm with a discriminator cut was used for $b$ tagging [157]. The fraction of events where the best $\mathrm{b}$-tagged jet is the $\mathrm{b}$ jet from $\mathrm{t} \rightarrow \mathrm{bW}$ was found to be $61 \%$. The corresponding fractions for the associated $\mathrm{b}$ jets and the quark jets from $\mathrm{W} \rightarrow \mathrm{qq}$ decay were found to be $\sim 26 \%$ and $\sim 8 \%$, respectively.

The top-quark mass was reconstructed minimising the $\chi^{2}$ distribution made from the reconstructed and nominal top and $\mathrm{W}$ masses, $\chi^{2}=\left(\left(\mathrm{m}_{\mathrm{jj}}-\mathrm{m}_{\mathrm{W}}\right) / \sigma_{\mathrm{W}}\right)^{2}+\left(\left(\mathrm{m}_{\mathrm{jjj}}-\mathrm{m}_{\mathrm{top}}\right) / \sigma_{\mathrm{top}}\right)^{2}$, where $m_{\mathrm{jj}}$ and $\mathrm{m}_{\mathrm{jjj}}$ are the invariant masses of all two- and three-jet combinations in the event and $\sigma_{\mathrm{W}}$ and $\sigma_{\text {top }}$ are the gaussian widths of the reconstructed true $\mathrm{W}$ and top mass distributions. The jet assigned to the top but not to the $\mathrm{W}$ presents the $\mathrm{b}$ jet from top. For a better reconstruction efficiency, in the presence of a significant contamination from the associated $b$ quark, any of the three jets assigned to the top were tagged requiring the value of the discriminator greater than 1.5 and $\mathrm{E}_{\mathrm{T}}>30 \mathrm{GeV}$. A mass resolution of $\sim 11 \%$ and a mean reconstructed mass of $\sim 176 \mathrm{GeV} / \mathrm{c}^{2}$ were obtained, with a fraction of about $40 \%$ of correct jet assignments. For a further suppression of the $\mathrm{t} \overline{\mathrm{t}}$ background, the ordinal jets after top reconstruction were searched for within $|\eta|<2.5$ and a jet veto was applied. The $\mathrm{E}_{\mathrm{T}}$ threshold for the jet veto was set to $25 \mathrm{GeV}$. The efficiency of this method has decreased compared to 


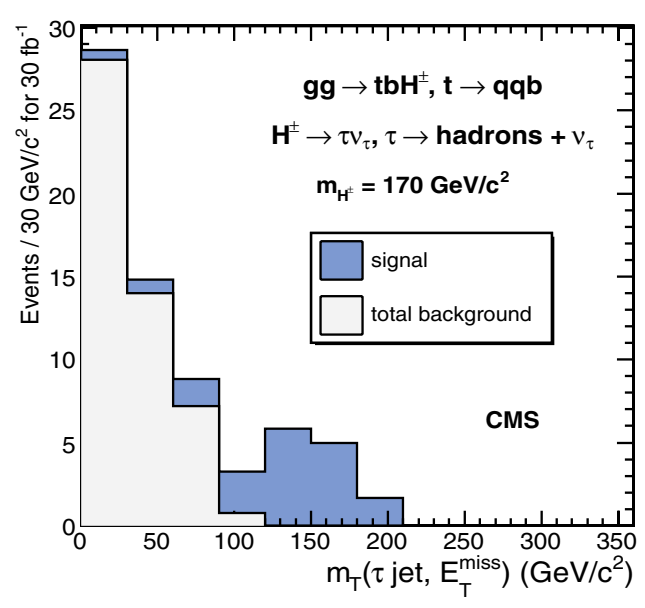

Figure 11.17. Transverse mass reconstructed from the $\tau$ jet and missing transverse energy for the $\mathrm{gg} \rightarrow \mathrm{tbH}^{ \pm}$, $\mathrm{t} \rightarrow \mathrm{bW}, \mathrm{W}^{\mp} \rightarrow \mathrm{jj}$ signal (dark histogram) with $\mathrm{m}_{\mathrm{H}^{ \pm}}=$ $170 \mathrm{GeV} / \mathrm{c}^{2}, \tan \beta=30$ and for the total background (light histogram) for an integrated luminosity of $30 \mathrm{fb}^{-1}$.

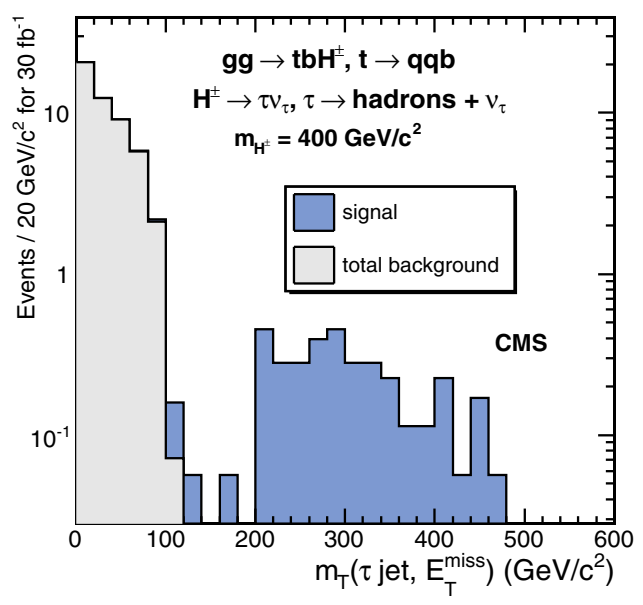

Figure 11.18. Transverse mass reconstructed from the $\tau$ jet and missing transverse energy for the $\mathrm{gg} \rightarrow \mathrm{tbH}^{ \pm}$, $\mathrm{t} \rightarrow \mathrm{bW}, \mathrm{W}^{\mp} \rightarrow \mathrm{jj}$ signal (dark histogram) with $\mathrm{m}_{\mathrm{H}^{ \pm}}=$ $400 \mathrm{GeV} / \mathrm{c}^{2}, \tan \beta=30$ and for the total background (light histogram) for an integrated luminosity of $30 \mathrm{fb}^{-1}$.

the fast simulation results [623] mainly due to more energetic associated $\mathrm{b}$ jets in $\mathrm{gg} \rightarrow \mathrm{btH}^{ \pm}$ with respect to the $\mathrm{gb} \rightarrow \mathrm{tH}^{ \pm}$events.

For the $\mathrm{t} \overline{\mathrm{t}}, \mathrm{Wt}$ and $\mathrm{W}+3$ jet backgrounds the configuration with large $\mathrm{E}_{\mathrm{T}}^{\text {miss }}$ and large $\mathrm{E}_{\mathrm{T}}^{\tau \text { jet }}$ can be reached only for strongly boosted $\mathrm{W}$. Therefore to suppress the background from events triggered with a fake $\tau$ from a hadronic jet recoiling against the genuine $\tau$ jet, a lower bound $\left(\mathrm{E}_{\mathrm{T}}^{\mathrm{H}}>50 \mathrm{GeV}\right)$ was set on the Higgs boson $\mathrm{p}_{\mathrm{T}}$ reconstructed from the $\tau$ jet and the missing transverse energy.

The large $\mathrm{E}_{\mathrm{T}}$ thresholds lead to an almost two-body (Jacobian peak) situation between the $\tau$ jet and missing transverse energy. Therefore an upper edge can be expected in the transverse mass $\mathrm{m}_{\mathrm{T}}=\sqrt{2 \times \mathrm{E}_{\mathrm{T}}^{\tau \text { jet }} \times \mathrm{E}_{\mathrm{T}}^{\text {miss }} \times\left(1-\Delta \phi\left(\tau \text { jet, } \mathrm{E}_{\mathrm{T}}^{\mathrm{miss}}\right)\right)}$ at $\mathrm{m}_{\mathrm{H}^{ \pm}}$for the signal and at $\mathrm{m}_{\mathrm{W}}$ for the $\mathrm{t} \overline{\mathrm{t}}$, Wt and $\mathrm{W}+3$ jet backgrounds. The boost required for the $\mathrm{t} \overline{\mathrm{t}}, \mathrm{Wt}$ and $\mathrm{W}+3$ jet backgrounds to pass the $\mathrm{E}_{\mathrm{T}}$ thresholds, leads to small opening angles $\Delta \phi\left(\tau\right.$ jet, $\left.\mathrm{E}_{\mathrm{T}}^{\mathrm{miss}}\right)$ in the transverse plane. Requiring $\Delta \phi>60^{\circ}$ removes most of the remaining background for $\mathrm{m}_{\mathrm{T}}<100 \mathrm{GeV} / \mathrm{c}^{2}$. The $\mathrm{m}_{\mathrm{T}}$ distributions for the signal and total background are shown in Figs. 11.17 and 11.18 for $\mathrm{m}_{\mathrm{H}^{ \pm}}=170$ and $400 \mathrm{GeV} / \mathrm{c}^{2}$ and $\tan \beta=30$, without a cut on $\Delta \phi\left(\tau\right.$ jet, $\left.\mathrm{E}_{\mathrm{T}}^{\mathrm{miss}}\right)$.

Tables 11.17 and 11.18 show the cross sections and efficiency for the selection cuts for the signal events with $\mathrm{m}_{\mathrm{H}^{ \pm}}=170,180,200$ and $400 \mathrm{GeV} / \mathrm{c}^{2}$ and $\tan \beta=30$. The trigger efficiency and the efficiency of the primary vertex reconstruction are also shown. Table 11.18 shows the same for the $\bar{t}$, Wt and $\mathrm{W}+3$ jet backgrounds. For the QCD multi-jet background the number of events where at least three jets are found after the $\mathrm{E}_{\mathrm{T}}^{\mathrm{miss}}$ and $\tau$ selections was estimated without the $\tau$ selection cuts. At this level of selection the QCD multi-jet events can be assumed to be similar to the $\mathrm{W}+3 \mathrm{jet}$ events at the same selection level. Therefore the efficiency of the remaining selection cuts was taken from the $\mathrm{W}+3$ jet events yielding an estimate of $0.1 \pm 0.1$ events for $\mathrm{m}_{\mathrm{T}}\left(\tau\right.$ jet, $\left.\mathrm{E}_{\mathrm{T}}^{\mathrm{miss}}\right)>100 \mathrm{GeV} / \mathrm{c}^{2}$.

11.2.5.4. Systematic uncertainties on background determination. The background in the signal region $\mathrm{m}_{\mathrm{T}}\left(\tau\right.$ jet, $\left.\mathrm{E}_{\mathrm{T}}^{\mathrm{miss}}\right)>100 \mathrm{GeV} / \mathrm{c}^{2}$ may arise from two main sources, the tail due to measurement uncertainties in the backgrounds with $\mathrm{W} \rightarrow \tau v$ decays, and the possibility of 
Table 11.19. Value of $\tan \beta$, cross section times branching fraction for $g g \rightarrow \mathrm{tbH}^{ \pm}, \mathrm{H}^{ \pm} \rightarrow \tau^{ \pm} \nu$, $\tau \rightarrow$ hadrons $+v$, number of selected signal events and the statistical significance (S) for the total background of $1.7 \pm 1.0$ events with $\left(\mathrm{S}_{\text {syst. }}\right)$ and without $\left(\mathrm{S}_{\text {no syst. }}\right)$ background uncertainty, for the signal with $\mathrm{m}_{\mathrm{H}^{ \pm}}=170$ to $600 \mathrm{GeV} / \mathrm{c}^{2}\left(\mathrm{~m}_{A}=150\right.$ to $\left.600 \mathrm{GeV} / \mathrm{c}^{2}\right)$ and for an integrated luminosity of $30 \mathrm{fb}^{-1}$

\begin{tabular}{lllllll}
\hline $\mathrm{m}_{\mathrm{H}^{ \pm}}\left(\mathrm{GeV} / \mathrm{c}^{2}\right)$ & 171.6 & 180.4 & 201.0 & 300.9 & 400.7 & 600.8 \\
$\tan \beta$ & 30 & 30 & 30 & 30 & 50 & 50 \\
$\sigma(\mathrm{NLO}) \times \mathrm{BR}(\mathrm{fb})$ & 1359.2 & 1237.6 & 775.5 & 118.3 & 104.9 & 15.7 \\
Events for $30 \mathrm{fb}^{-1}$ & $14.1 \pm 1.6$ & $14.7 \pm 3.2$ & $11.7 \pm 2.3$ & $8.3 \pm 1.2$ & $10.0 \pm 1.4$ & $2.0 \pm 0.2$ \\
$\mathrm{~S}_{\text {no syst. }}$ & 6.4 & 6.6 & 5.5 & 4.2 & 4.9 & 1.2 \\
$\mathrm{~S}_{\text {syst. }}$ & 5.0 & 5.2 & 4.3 & 3.3 & 3.8 & 1.0 \\
\hline
\end{tabular}

fake $\tau$ jets, mainly in the QCD multi-jet events. The level of the backgrounds with $\mathrm{W} \rightarrow \tau \nu$ decays can be measured from data exploiting the precise muon momentum measurement in the $\mathrm{W}+3$ jets, $\mathrm{W} \rightarrow \mu \nu$ events, selecting events in the tail of the transverse mass distribution. The probability of a hadronic jet faking the $\tau$ jet can be measured exploiting the $\gamma+$ jet events, as proposed in Ref. [280]. For this work a Monte-Carlo method was chosen assuming that the probability of the background events to migrate to the signal area depends mainly on the precision of the jet energy and $\mathrm{E}_{\mathrm{T}}^{\mathrm{miss}}$ measurements. The systematic uncertainty due to the energy scale was estimated varying the jet energy and the $\mathrm{E}_{\mathrm{T}}^{\mathrm{miss}}$ values with the expected energy scale uncertainties yielding the average values of $3 \%$ and $2 \%$ for the uncertainties on the efficiency of the $\mathrm{E}_{\mathrm{T}}^{\mathrm{miss}}$ cut and the efficiency of the selection of three hadronic jets for top reconstruction, respectively. The uncertainty of the $\tau$ identification has been estimated to be $8 \%$ for the $\mathrm{E}_{\mathrm{T}}$ interval of $\tau$ jets from $\mathrm{Z} \rightarrow \tau \tau$ decays [149]. For the b-tagging uncertainty a conservative estimate of $5 \%$ was taken. The theoretical uncertainty on the $\mathrm{t} \overline{\mathrm{t}}$ cross section due to a variation of the scale and PDF has been estimated to be 5.6\% [159]. These values yield $11 \%$ for the total systematic uncertainty for the $t \bar{t}$ background. For the $\mathrm{W}+3$ jet and QCD multi-jet backgrounds the uncertainties due to present MC statistics strongly dominate the measurement uncertainties and therefore the MC statistical uncertainties were used. The total number of background events in the signal region $\mathrm{m}_{\mathrm{T}}\left(\tau\right.$ jet, $\left.\mathrm{E}_{\mathrm{T}}^{\mathrm{miss}}\right)>100 \mathrm{GeV}$, is $1.7 \pm 1.0$ events, including the systematic and MC uncertainties.

11.2.5.5. Discovery potential. Table 11.19 shows the number of signal events for $\mathrm{m}_{\mathrm{H}^{ \pm}}=170$ to $300 \mathrm{GeV} / \mathrm{c}^{2}$ with $\tan \beta=30$ and for $\mathrm{m}_{\mathrm{H}^{ \pm}}=400$ to $600 \mathrm{GeV} / \mathrm{c}^{2}$ with $\tan \beta=50$ and the signal significance $(\mathrm{S})$ calculated according to Poisson statistics $[498]$ with $\left(\mathrm{S}_{\text {syst }}\right)$ and without $\left(\mathrm{S}_{\text {no syst. }}\right)$ background uncertainty for the total background of $1.7 \pm 1.0$ events. The cut in the transverse mass $\mathrm{m}_{\mathrm{T}}\left(\tau\right.$ jet, $\left.\mathrm{E}_{\mathrm{T}}^{\mathrm{miss}}\right)>100 \mathrm{GeV} / \mathrm{c}^{2}$ is used to select the signal area. The results are shown for an integrated luminosity of $30 \mathrm{fb}^{-1}$. For the $\mathrm{t} \overline{\mathrm{t}}$ background the estimated systematic uncertainty of $11 \%$ is included. Figure 11.19 shows the $5 \sigma$-discovery region in the $\mathrm{m}_{\mathrm{A}}-\tan \beta$ plane in the maximal mixing scenario with $\mu=200 \mathrm{GeV} / \mathrm{c}^{2}$ with and without systematic uncertainties at $30 \mathrm{fb}^{-1}$.

\subsubsection{Charged Higgs boson of $\mathrm{M}_{\mathrm{H}}>m_{\mathrm{t}}$ in $\mathrm{gg} \rightarrow \mathrm{tbH}^{ \pm}$production with $\mathrm{H}^{ \pm} \rightarrow \mathrm{tb}$}

The branching fractions for the decay channels of the charged Higgs boson depend strongly on its mass (see Fig. 11.2). For masses above $m_{\mathrm{t}}+m_{\mathrm{b}}$, the channel $\mathrm{H}^{ \pm} \rightarrow$ tb opens up. Two production channels and corresponding final states were considered in the search for charged Higgs bosons in the $\mathrm{H}^{ \pm} \rightarrow$ tb decay channel [629]:

$$
\begin{aligned}
& \mathrm{gb} \rightarrow \mathrm{tH}^{ \pm} \rightarrow \mathrm{ttb} \rightarrow \mathrm{W}^{+} \mathrm{W}^{-} \mathrm{bbb} \rightarrow \mathrm{qq}^{\prime} \mu v_{\mu} \mathrm{bbb}, \\
& \mathrm{gg} \rightarrow \mathrm{tH}^{ \pm} \mathrm{b} \rightarrow \mathrm{ttbb} \rightarrow \mathrm{W}^{+} \mathrm{W}^{-} \mathrm{bbbb} \rightarrow \mathrm{qq}^{\prime} \mu v_{\mu} \mathrm{bbbb} .
\end{aligned}
$$




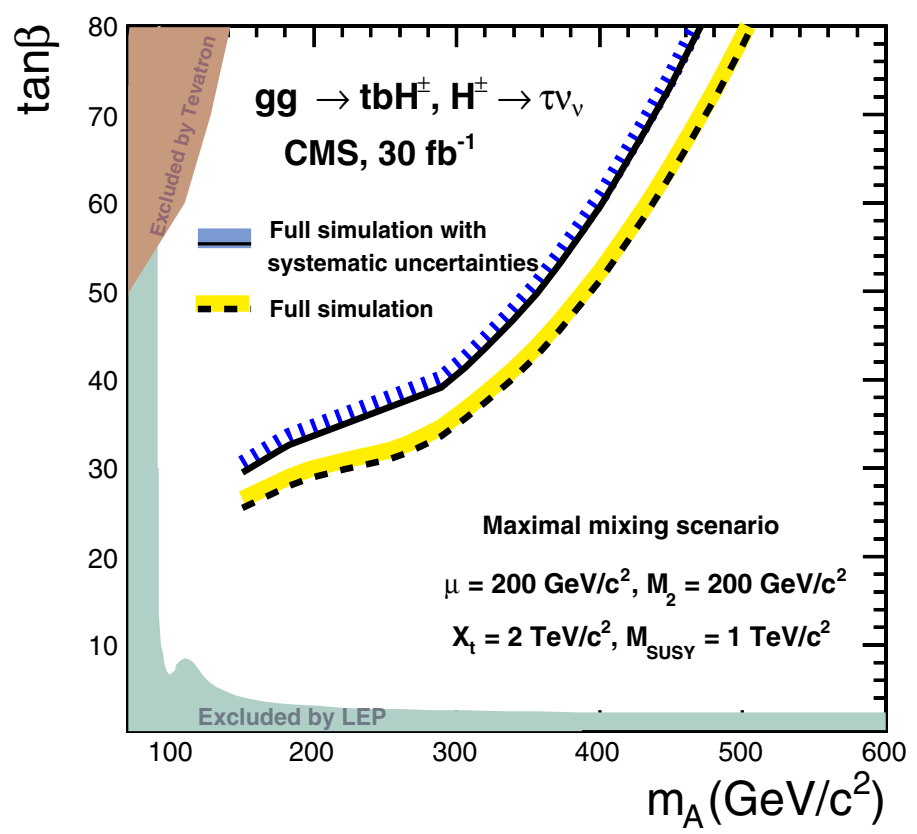

Figure 11.19. The $5 \sigma$-discovery region in the $\mathrm{m}_{\mathrm{A}}$-tan $\beta$ plane for $\mathrm{gg} \rightarrow \mathrm{tbH}^{ \pm}, \mathrm{H}^{ \pm} \rightarrow \tau \nu_{\tau}$ with an integrated luminosity of $30 \mathrm{fb}^{-1}$ in the maximal mixing scenario with $\mu=200 \mathrm{GeV} / \mathrm{c}^{2}$. The discovery regions with and without systematic uncertainties are shown. The regions excluded by the LEP and Tevatron searches are also shown in the figure.

These final states are the most interesting from the experimental point of view because an isolated muon is present to trigger on and the branching fraction into this decay is high $(\sim 30 \%)$.

The inclusive final state (11.9) is studied using triple $b$ tagging within the parameterised simulation framework of CMS [11]. The final state (11.10), where a fourth $b$ jet is resolved in the detector, is studied with full GEANT4 [9] CMS detector simulation [8]. Production of the $\mathrm{H}^{ \pm}$bosons through heavy sparticle cascades is not taken into account. In addition, supersymmetric particles are supposed to be heavy enough, such that supersymmetric decays of the $\mathrm{H}^{ \pm}$can be neglected.

A detailed description of the analysis can be found in [629].

11.2.6.1. Signal and background simulation. Events from the process (11.9) are modelled by considering the initial $\mathrm{b}$ quark as a massless parton from the corresponding parton density in the proton. On the other hand, events from the process (11.10) are described with massive spectator $b$ quarks.

The calculation of the total signal cross section was performed at NLO [628], starting from the process (11.9). When calculating the cross section for both processes (11.9) and (11.10) to all orders, however, one expects to obtain the same result, as they both describe the same physics. Therefore, for both processes, the cross section was rescaled to the NLO result for the $\mathrm{pp} \rightarrow \mathrm{tH}^{ \pm} \mathrm{X}$ channel.

The signal cross section is sensitive to the two parameters $\tan \beta$ and $m_{\mathrm{H}^{ \pm}}$(Fig. 11.20). The cross section is enhanced at small and large values of $\tan \beta$, with a minimum at $\tan \beta=\sqrt{m_{\mathrm{t}} / m_{\mathrm{b}}} \approx 6$. Furthermore, the cross section decreases rapidly with rising $m_{\mathrm{H}^{ \pm}}$. The generation of both processes (11.9) and (11.10) was performed with PYTHIA [69], forcing the 

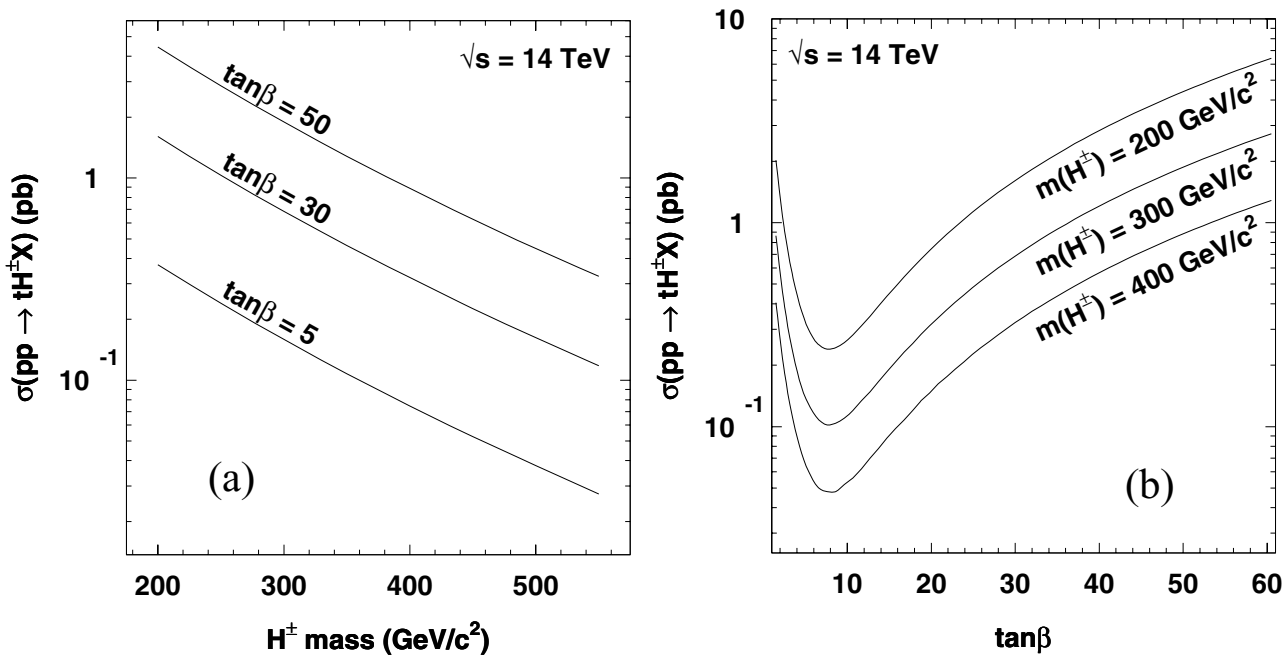

Figure 11.20. NLO cross section for $\mathrm{pp} \rightarrow \mathrm{tH}^{ \pm} \mathrm{X}$ as a function of (a) $m_{\mathrm{H}^{ \pm}}$and (b) $\tan \beta$.

decay $\mathrm{H}^{ \pm} \rightarrow$ tb of the charged Higgs boson. The branching fraction $\mathrm{BR}\left(\mathrm{H}^{ \pm} \rightarrow\right.$ tb) for this decay process was calculated with HDECAY 3.0 [41].

The main background to charged Higgs boson production and decay through $\mathrm{pp} \rightarrow$ $\mathrm{tH}^{ \pm}(\mathrm{b}) \rightarrow \mathrm{ttb}(\mathrm{b})$ is the Standard Model top-quark pair production with additional jets. Other potential multi-jet backgrounds are much smaller and neglected.

In the case of process (11.9), the leading order background comes from SM pp $\rightarrow$ tt'b and $\mathrm{pp} \rightarrow \mathrm{t} \mathrm{t}+$ jet production, where in the latter the extra jet is misidentified as a $b$ jet. The event simulation was performed using the matrix element generator MadGraph/MadEvent [81], interfaced to PYTHIA for parton shower, fragmentation and hadronisation, with a cut $p_{\mathrm{T}}>$ $10 \mathrm{GeV} / \mathrm{c}$ on the transverse momentum and $|\eta|<2.5$ on the pseudorapidity of the extra jet. This resulted in a cross section of $678 \mathrm{pb}$.

The background for process (11.10) consists of the irreducible $\mathrm{pp} \rightarrow \mathrm{t} \mathrm{t} b \bar{b}$ and the reducible $\mathrm{pp} \rightarrow \mathrm{t} \mathrm{tjj}$ process, where in the latter two jets are misidentified as $\mathrm{b}$ jets. Both these backgrounds were simulated using the COMPHEP generator [43]. The generator level cuts $p_{\mathrm{T}}>15 \mathrm{GeV}$ and $|\eta|<3$ were applied on the partons produced in association with the t⿱t pair. A separation cut $\Delta R>0.3$ was also imposed. This resulted in a cross section of $3.285 \mathrm{pb}$ for the $\mathrm{pp} \rightarrow \mathrm{t} \overline{\mathrm{t}} \mathrm{b}$ process and $507.8 \mathrm{pb}$ for $\mathrm{pp} \rightarrow \mathrm{t} \mathrm{tj} j$ production. Care was taken to avoid double counting between the $\mathrm{pp} \rightarrow \mathrm{t} \overline{\mathrm{t}} \mathrm{b} \overline{\mathrm{b}}$ and $\mathrm{pp} \rightarrow \mathrm{t} \mathrm{tjj}$ processes and the cross section for $\mathrm{pp} \rightarrow \mathrm{t} \overline{\mathrm{t} j \mathrm{j}}$ was scaled to the result from a similar ALPGEN generation, where a jet matching technique was applied to more rigourously handle the transition between the hard interaction and the parton shower.

11.2.6.2. Event selection and reconstruction. On the final states (11.9) and (11.10) a basic event selection is applied on the reconstructed objects (Tables 11.20 and 11.21). Events passing the single muon HLT trigger are required to have at least one muon with $p_{\mathrm{T}}>20 \mathrm{GeV}$ and $|\eta|<2.5$, at least respectively five or six calibrated jets with $\mathrm{E}_{\mathrm{T}}>25 \mathrm{GeV}$ and $|\eta|<2.5$ and at least respectively three or four of these jets tagged as $b$ jet with a secondary vertexbased algorithm [157].

In both final states (11.9) and (11.10) the best jet association is selected with a likelihood ratio technique, which combines information from kinematical properties of the extra jets, 
Table 11.20. Event selection yield for $\tan \beta=30$ and an integrated luminosity of $30 \mathrm{fb}^{-1}$.

\begin{tabular}{llllllll}
\hline $30 \mathrm{fb}^{-1}$ & $\mathrm{t} \overline{\mathrm{b}} / \mathrm{t} \overline{\mathrm{tj}}$ & $\mathrm{gb} \rightarrow \mathrm{tH}^{ \pm}(\tan \beta=30)$ \\
\hline$m_{\mathrm{H}^{ \pm}}\left(\mathrm{GeV} / \mathrm{c}^{2}\right)$ & & 263 & 311 & 359 & 408 & 457 & 506 \\
cross section $\times \mathrm{BR}(\mathrm{pb})$ & 678 & 0.850 & 0.570 & 0.377 & 0.251 & 0.169 & 0.116 \\
\# events before cuts & $20.3 \mathrm{M}$ & 25489 & 17088 & 11319 & 7529 & 5063 & 3472 \\
single muon HLT & $17 \%$ & $16 \%$ & $16 \%$ & $16 \%$ & $16 \%$ & $16 \%$ & $16 \%$ \\
1 muon & $95 \%$ & $95 \%$ & $95 \%$ & $95 \%$ & $96 \%$ & $96 \%$ & $96 \%$ \\
5 jets & $18 \%$ & $35 \%$ & $42 \%$ & $44 \%$ & $46 \%$ & $49 \%$ & $51 \%$ \\
3 b-tagged jets & $6 \%$ & $27 \%$ & $29 \%$ & $30 \%$ & $32 \%$ & $31 \%$ & $29 \%$ \\
\# remaining events & 32880 & 364 & 314 & 230 & 171 & 116 & 80 \\
\hline
\end{tabular}

Table 11.21. Event selection yield for $\tan \beta=30$ and an integrated luminosity of $30 \mathrm{fb}^{-1}$.

\begin{tabular}{lllllllll}
\hline $30 \mathrm{fb}^{-1}$ & $\mathrm{t} \mathrm{tb} \overline{\mathrm{b}}$ & $\mathrm{t} \mathrm{tjj}$ & $\mathrm{gg} \rightarrow \mathrm{tbH}^{ \pm}(\tan \beta=30)$ & & \\
\hline$m_{\mathrm{H}^{ \pm}}\left(\mathrm{GeV} / \mathrm{c}^{2}\right)$ & & & 263 & 311 & 359 & 408 & 457 & 506 \\
cross section $\times \mathrm{BR}(\mathrm{pb})$ & 2.386 & 235.8 & 0.850 & 0.570 & 0.377 & 0.251 & 0.169 & 0.116 \\
\# events before cuts & 71580 & $7.07 \mathrm{M}$ & 25489 & 17088 & 11319 & 7529 & 5063 & 3472 \\
single muon HLT & $19 \%$ & $19 \%$ & $13 \%$ & $13 \%$ & $13 \%$ & $13 \%$ & $13 \%$ & $13 \%$ \\
1 muon & $96 \%$ & $97 \%$ & $96 \%$ & $95 \%$ & $97 \%$ & $97 \%$ & $97 \%$ & $97 \%$ \\
6 jets & $19 \%$ & $23 \%$ & $19 \%$ & $23 \%$ & $25 \%$ & $26 \%$ & $28 \%$ & $31 \%$ \\
4 b-tagged jets & $7 \%$ & $0.55 \%$ & $6 \%$ & $5 \%$ & $7 \%$ & $7 \%$ & $5 \%$ & $6 \%$ \\
\# remaining events & 179 & 1623 & 37 & 24 & 25 & 18 & 9 & 8 \\
\hline
\end{tabular}

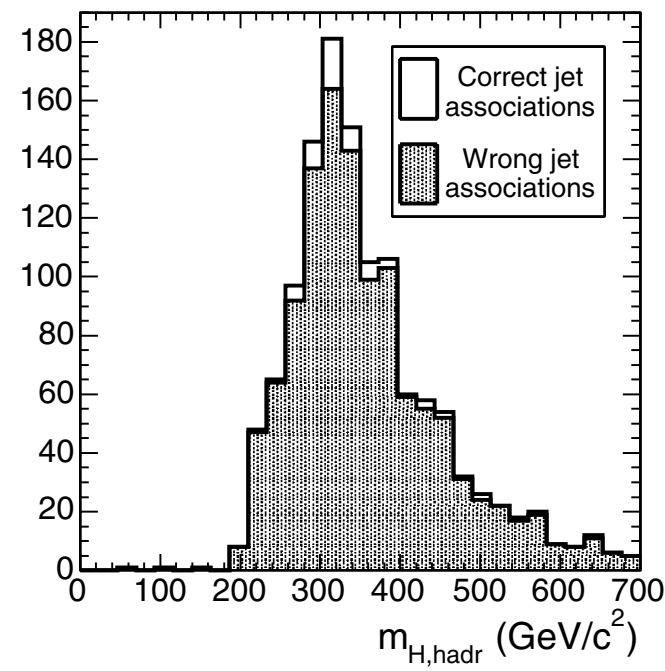

Figure 11.21. Reconstructed $\mathrm{H}^{ \pm}$mass with hadronically decaying top for the chosen jet association $\left(m_{\mathrm{H}^{ \pm}}=311 \mathrm{GeV} / \mathrm{c}^{2}\right)$.

b-tagging of all jets and the result of a kinematic fit on the $\bar{t}$ system, imposing both $\mathrm{W}^{ \pm}$ and $t$ mass constraints. Starting from the chosen jet association the Higgs boson mass was reconstructed. An ambiguity remains, as it is not possible to know which top quark candidate the additional $b$ jet should be combined with. In Fig. 11.21 the reconstructed charged Higgs boson mass with hadronically decaying top is shown for correct and wrong jet pairings in the case of three tagged $\mathrm{b}$ jets and for $m_{\mathrm{H}^{ \pm}}=311 \mathrm{GeV} / c^{2}$. Due to the large combinatorial 


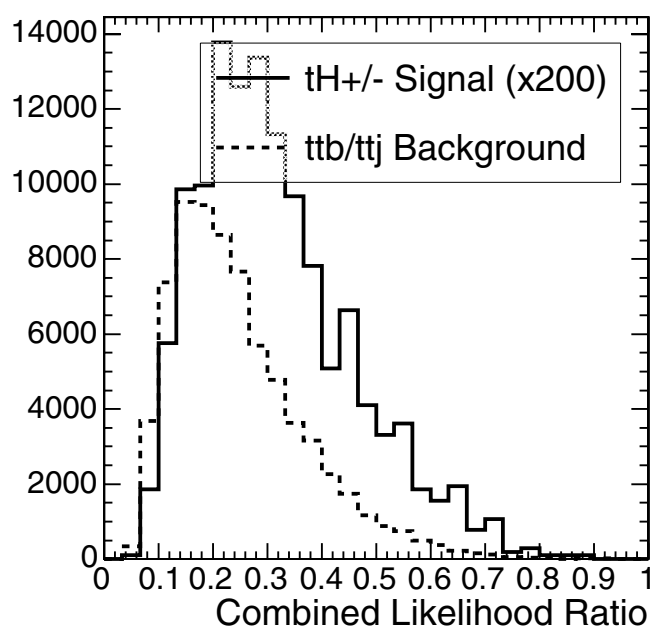

Figure 11.22. Distribution of the discriminator used to distinguish between signal 11.9 and background.

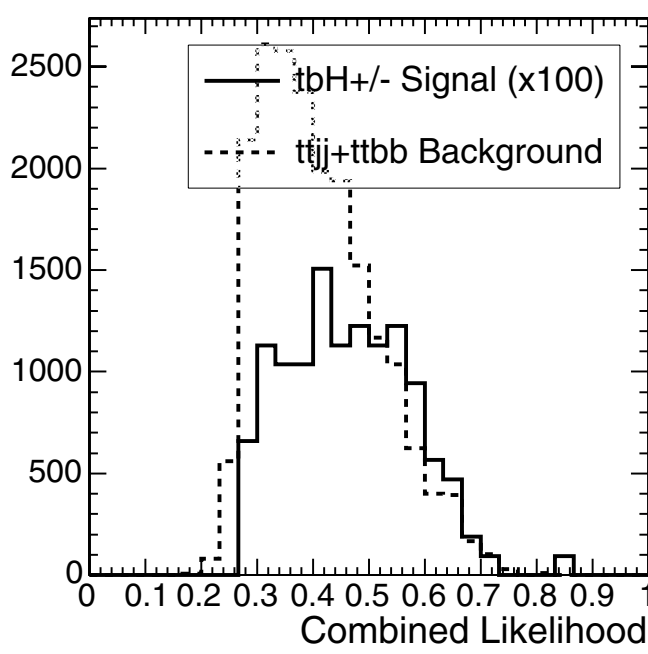

Figure 11.23. Distribution of the discriminator used to distinguish between signal 11.10 and background.

background, the mass information is of limited use for the separation between signal and background, and is therefore not used further on in the analysis.

11.2.6.3. Background suppression. To suppress the large $\bar{t} \bar{t}+$ jets background, observables were identified that have different properties for signal and background events. These observables were combined into an overall discriminator. In the case of process (11.9) the b-tagging information for the extra jet was used, together with the $p_{\mathrm{T}}$ of the softest jet from the $\mathrm{W}^{ \pm}$decay and the ratio of the $E_{\mathrm{T}}$ of the sixth jet and the fifth. For the process (11.10) only the b-tagging information for the two extra jets was used. In Figs. 11.22 and 11.23 the resulting discriminator distributions are shown for the process (11.9) and (11.10) respectively.

11.2.6.4. Discovery reach and systematics. A cut on the discriminating variables of Figs. 11.22 and 11.23 was optimised to obtain the maximal statistical significance for an integrated luminosity of $30 \mathrm{fb}^{-1}$. The signal cross section required for a significance of 5 , corresponding to a discovery, was derived and translated into a minimal value of $\tan \beta$ needed for a discovery for a given value of $m_{\mathrm{A}}$. Performing this analysis and optimisation at different values of $m_{\mathrm{A}}$ a discovery contour was obtained in the MSSM $\left(\tan \beta, m_{\mathrm{A}}\right)$ plane.

The background is large in both final states and therefore the effect of systematic uncertainties on the knowledge of the background is important. A possible way to estimate the background level from data is to require one b-tagged jet less. After such a selection it is possible to calculate the expected number of background events plus its uncertainty, when tagging a third or fourth $b$ jet. Optimistically the uncertainty on the mistag rate can be taken as $5 \%$. Possible large theoretical uncertainties related to this method, like the ratio of events with real extra $b$ jets and events with only light extra jets, should still be accounted for.

Depending on the expected systematic uncertainty on the background level the maximal significance was searched. In Fig. 11.24 the discovery contours are plotted for the final states (11.9) and (11.10) respectively, when supposing perfect knowledge of the background cross section $(\varepsilon=0)$, a $1 \%$ uncertainty $(\varepsilon=0.01)$, and a $3 \%$ uncertainty $(\varepsilon=0.03)$. From 

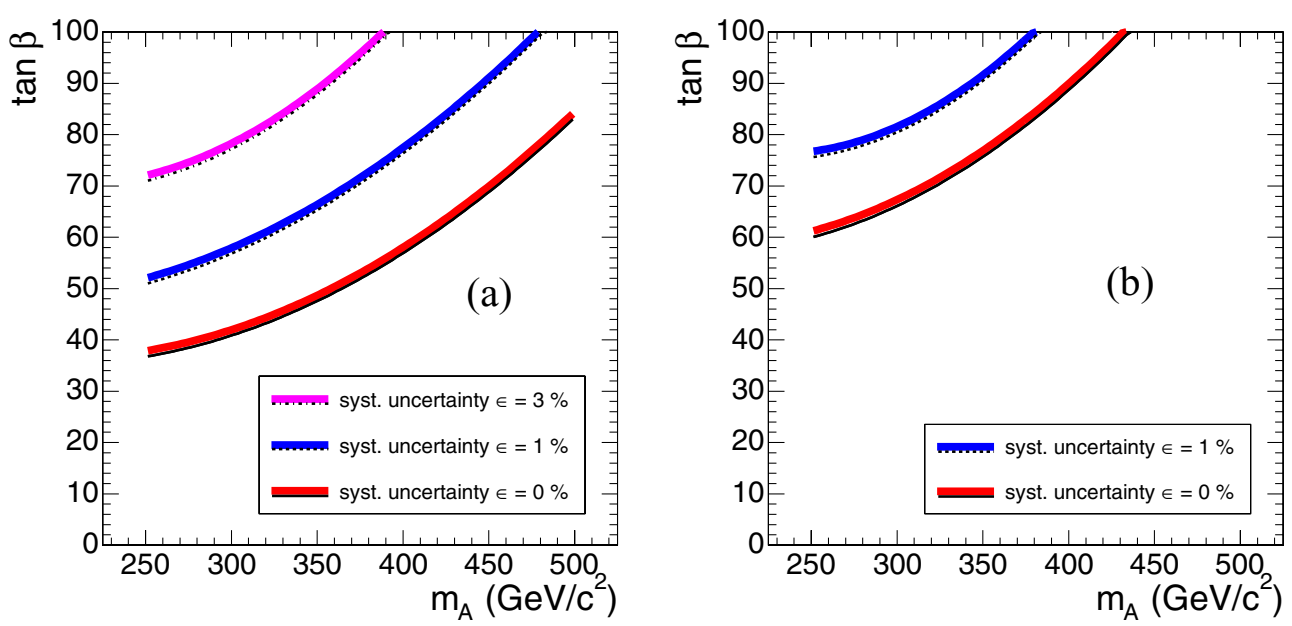

Figure 11.24. Discovery contour for the charged Higgs boson in the $\mathrm{H}^{ \pm} \rightarrow$ tb decay for $30 \mathrm{fb}^{-1}$, (a) applying $3 \mathrm{~b}$ tags, (b) applying $4 \mathrm{~b}$ tags; systematic uncertainties on the background of $\varepsilon=0 \%$, $\varepsilon=1 \%$ and $\varepsilon=3 \%$ are taken into account.

the above estimate of the systematic uncertainty on the number of background events, the conclusion is drawn that, neglecting SUSY cascade decays, no visibility for this channel is obtained in the MSSM parameter space during the low luminosity phase of LHC.

\subsubsection{Search for the $A \rightarrow Z h$ decay with $Z \rightarrow \ell^{+} \ell^{-}, h \rightarrow b \bar{b}$}

The observation of the CP-odd pseudo-scalar Higgs (A) via its decay into a $\mathrm{Z}$ boson and the lighter CP-even scalar Higgs ( $h$ ) followed by $\mathrm{Z} \rightarrow \mathrm{e}^{+} \mathrm{e}^{-}, \mu^{+} \mu^{-}$and $\mathrm{h} \rightarrow \mathrm{b} \overline{\mathrm{b}}$ decays provides an interesting way to detect $\mathrm{A}$ and $\mathrm{h}$ simultaneously. The largest branching ratio of the $\mathrm{A} \rightarrow \mathrm{Zh}$ appears for low $\tan \beta$ and $m_{Z}+m_{h} \leqslant m_{A} \leqslant 2 m_{\text {top }}$ mass region. The main production mechanism for $\mathrm{A}$ at low $\tan \beta$ is via $\mathrm{gg}, \mathrm{qq} \rightarrow \mathrm{A}$.

The decays of the $\mathrm{A}$ into charginos and neutralinos $(\mathrm{A} \rightarrow \chi \chi)$, however, can dominate at certain values of $\mu$ and $\mathrm{M}_{2}$ (Higgs-Higgsino and SU(2) gaugino mass parameters) since the masses of charginos and neutralinos as well as their couplings to the Higgs bosons depend on $\mu$ and $\mathrm{M}_{2}$ (in addition to $\tan \beta$ and $\mathrm{M}_{\mathrm{A}}$ ). Large values of $\mu$ and $\mathrm{M}_{2}$ are more favourable for the observation of the $\mathrm{A} \rightarrow \mathrm{Zh}$ channel.

In Fig. 11.25 the production cross section multiplied by the appropriate branching ratios (including $\mathrm{Z} \rightarrow \mathrm{e}^{+} \mathrm{e}^{-}, \mu^{+} \mu^{-}$and $\mathrm{h} \rightarrow \mathrm{b} \overline{\mathrm{b}}$ decays) is shown as a function of $\mathrm{M}_{\mathrm{A}}$ in the $\mathrm{m}_{\mathrm{h}}^{\max }$ scenario with $\mu=\mathrm{M}_{2}=200 \mathrm{GeV} / \mathrm{c}^{2}$ and $\mu=\mathrm{M}_{2}=600 \mathrm{GeV} / \mathrm{c}^{2}$ for two values of $\tan \beta, 1$ and 5. One can see that the difference in the total cross sections for the two choices of the $\mu$ and $\mathrm{M}_{2}$ parameters can be as large as one order of magnitude. The $\mathrm{A} \rightarrow \mathrm{Zh}$ analysis and the discovery reach presented below was evaluated in the $\mathrm{m}_{\mathrm{h}}^{\max }$ scenario with $\mu=\mathrm{M}_{2}=600 \mathrm{GeV} / \mathrm{c}^{2}$.

11.2.7.1. Event generation, simulation and reconstruction. The Higgs boson production processes, $\mathrm{gg} \rightarrow \mathrm{A}$ and $\mathrm{pp} \rightarrow \mathrm{A} \mathrm{b} \overline{\mathrm{b}}$, were generated using PYTHIA 6.225 [69] for three values of $\mathrm{M}_{\mathrm{A}}\left(250,300,350 \mathrm{GeV} / \mathrm{c}^{2}\right)$ and two values of $\tan \beta(1.0,5.0)$. No pre-selection at generation level was applied. The Standard Model backgrounds considered are: the $\mathrm{Zb} \overline{\mathrm{b}}$ generated with COMPHEP [355] and ZZ, ZW, Z+jets, W+jets and tt generated with PYTHIA 6.215. Events were fully simulated and digitised with pile-up corresponding to a luminosity of $2 \times 10^{33} \mathrm{~cm}^{-2} \mathrm{~s}^{-1}$. 

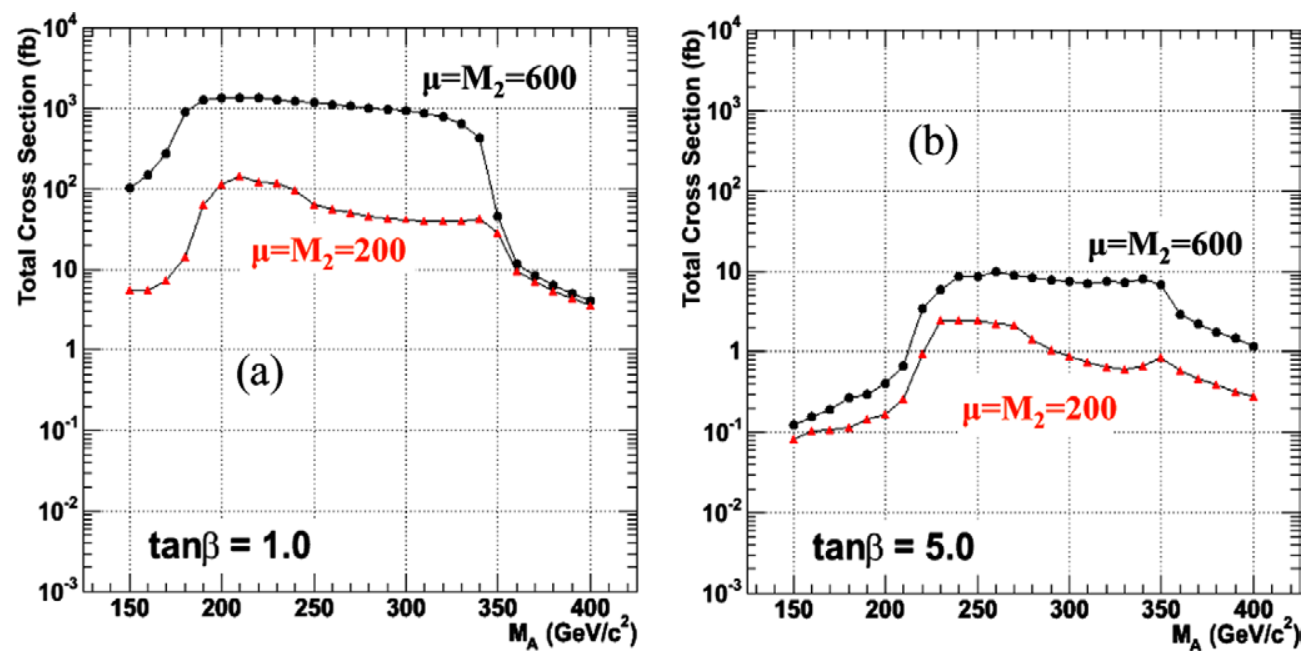

Figure 11.25. The production cross-section multiplied by appropriate branching ratios as a function of $\mathrm{M}_{\mathrm{A}}$ in the $\mathrm{m}_{\mathrm{h}}^{\max }$ scenario with $\mu=\mathrm{M}_{2}=600 \mathrm{GeV} / \mathrm{c}^{2}$ (circles) and $\mu=\mathrm{M}_{2}=$ $200 \mathrm{GeV} / \mathrm{c}^{2}$ (triangles) for (a) $\tan \beta=1$ and (b) $\tan \beta=5$.

Offline reconstruction of electrons, muons, jets and $b$ tagging were performed using standard algorithms.

11.2.7.2. Online selection. The events are required to pass the global Level-1 (L1) and High Level Trigger (HLT) dimuon or dielectron selections since there will always be a real $\mathrm{Z}$ in the event decaying into two high $\mathrm{p}_{\mathrm{T}}$ electrons or muons. The inclusion of the single muon and electron triggers does not improve the discovery reach in the $\mathrm{M}_{\mathrm{A}}-\tan \beta$ plane.

11.2.7.3. Off-line event selection. The baseline selection requires two opposite sign high $\mathrm{p}_{\mathrm{T}}$ isolated leptons (e or $\mu$ ) and two high $\mathrm{E}_{\mathrm{T}}$ tagged b-jets separated from the leptons with $\Delta \mathrm{R}(\ell, \mathrm{j})>0.7$. Muons must have $|\eta|<2.4$ and electrons should be in the ECAL fiducial region $(|\eta|<2.5$ with $1.444<|\eta|<1.566$ region excluded). The event is required to have small missing $\mathrm{E}_{\mathrm{T}}$ and reconstructed invariant mass of the leptons close to the $\mathrm{Z}$ mass in order to reject a significant fraction of the $\mathrm{t} \overline{\mathrm{t}}$ background.

Table 11.22 summarises the basic selection variables and thresholds. The variation of the signal significance with the change of the $\mathrm{p}_{\mathrm{T}}$ thresholds on the electrons, muons and b-jets, and the thresholds on the b-tagging discriminant for the two tagged jets has been checked. No significant variation was found with small changes of the cut values presented in Table 11.22.

11.2.7.4. Results. The selection efficiencies for the signal vary from $5 \%$ to $12 \%$ depending on the $\mathrm{M}_{\mathrm{A}}$ and $\tan \beta$ values as well as the production mechanism. The details can be found in [630]. The next-to-leading order (NLO) background cross sections before and after selections are shown in Table 11.23.

The signal and the background distributions of $\mathrm{M}_{\mathrm{b} \overline{\mathrm{b}}}$ and $\mathrm{M}_{\ell^{+} \ell^{-} \mathrm{b} \overline{\mathrm{b}}}$ after selections are shown in Fig. 11.26 and Fig. 11.27 respectively for $30 \mathrm{fb}^{-1}$ of integrated luminosity.

11.2.7.5. Systematic uncertainties. The method to evaluate the background from the real data measuring the background in the signal free (normalisation) region is proposed. 
Table 11.22. Selection variables and thresholds

\begin{tabular}{ll}
\hline Selection Variable & Threshold \\
\hline most energetic electron/muon $\mathrm{p}_{\mathrm{T}}$ & $>30 \mathrm{GeV} / \mathrm{c}$ \\
second-most energetic electron/muon $\mathrm{p}_{\mathrm{T}}$ & $>15 \mathrm{GeV} / \mathrm{c}$ \\
most energetic b-jet $\mathrm{E}_{\mathrm{T}}$ & $>25 \mathrm{GeV}$ \\
second-most energetic b-jet $\mathrm{E}_{\mathrm{T}}$ & $>20 \mathrm{GeV}$ \\
missing $\mathrm{E}_{\mathrm{T}}$ & $<60 \mathrm{GeV}$ \\
most energetic b-jet discriminator & $>1.5$ \\
second-most energetic b-jet discriminator & $>0.5$ \\
$\mathrm{Z}_{\text {mass cut }}$ & $84 \mathrm{GeV} / \mathrm{c}^{2}<\mathrm{M}_{Z}<96 \mathrm{GeV} / \mathrm{c}^{2}$ \\
$\mathrm{Z}_{\mathrm{p}}$ & $>30.0 \mathrm{GeV} / \mathrm{c}$ \\
\hline
\end{tabular}

Table 11.23. Background cross sections.

\begin{tabular}{lll}
\hline & $\begin{array}{l}\text { NLO cross sections }(\mathrm{fb}) \\
\text { before selection }\end{array}$ & after selection \\
\hline $\mathrm{Zb} \overline{\mathrm{b}}, \mathrm{Z} \rightarrow e e, \mu \mu, \tau \tau$ & 112830 & 415.26 \\
$\mathrm{t} \overline{\mathrm{t}} \mathrm{W} \rightarrow e v, \mu \nu, \tau \nu$ & 88500 & 70.8 \\
$\mathrm{Z}+\mathrm{jets}, \mathrm{Z} \rightarrow e e, \mu \mu, \tau \tau$ & 5300000 & 83.05 \\
$\mathrm{~W}+$ jets, $\mathrm{W} \rightarrow e v, \mu \nu, \tau \nu$ & 47900000 & 0.0 \\
$\mathrm{ZZ}$ (inclusive) & 14985 & 7.34 \\
$\mathrm{ZW}$ (inclusive) & 49422 & 1.98 \\
\hline
\end{tabular}

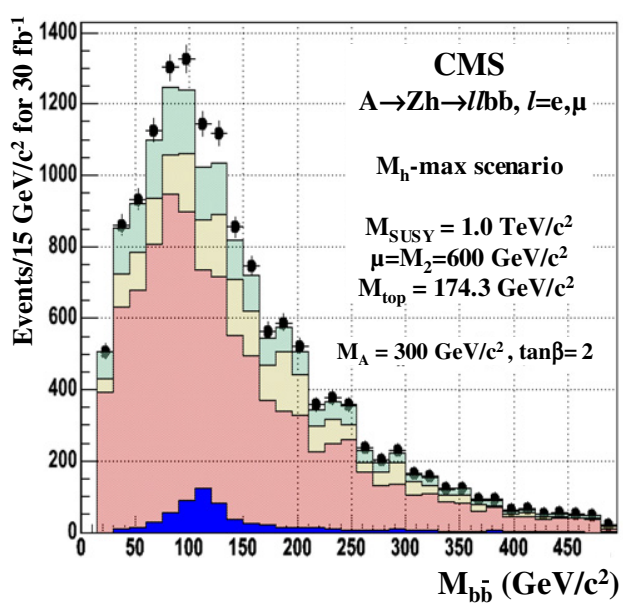

Figure 11.26. Distribution of $\mathrm{M}_{\mathrm{b} \overline{\mathrm{b}}}$ for signal and background after event selection for $30 \mathrm{fb}^{-1}$ of integrated luminosity. Red (dark gray), yellow (light gray) and green (medium gray) distributions represent the $\mathrm{Zb} \bar{b}$, $\bar{t}$ and $\mathrm{Z}+$ jets backgrounds. Blue (black) distribution is the signal $\left(\mathrm{M}_{\mathrm{A}}=300, \tan \beta=2\right)$ and black dots the data (sum of the signal and the background).

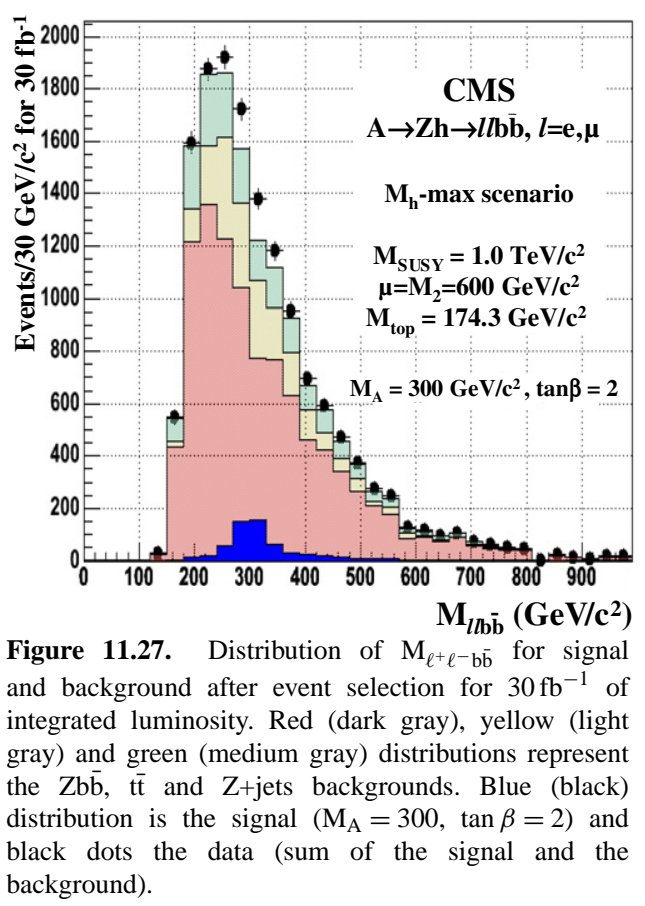



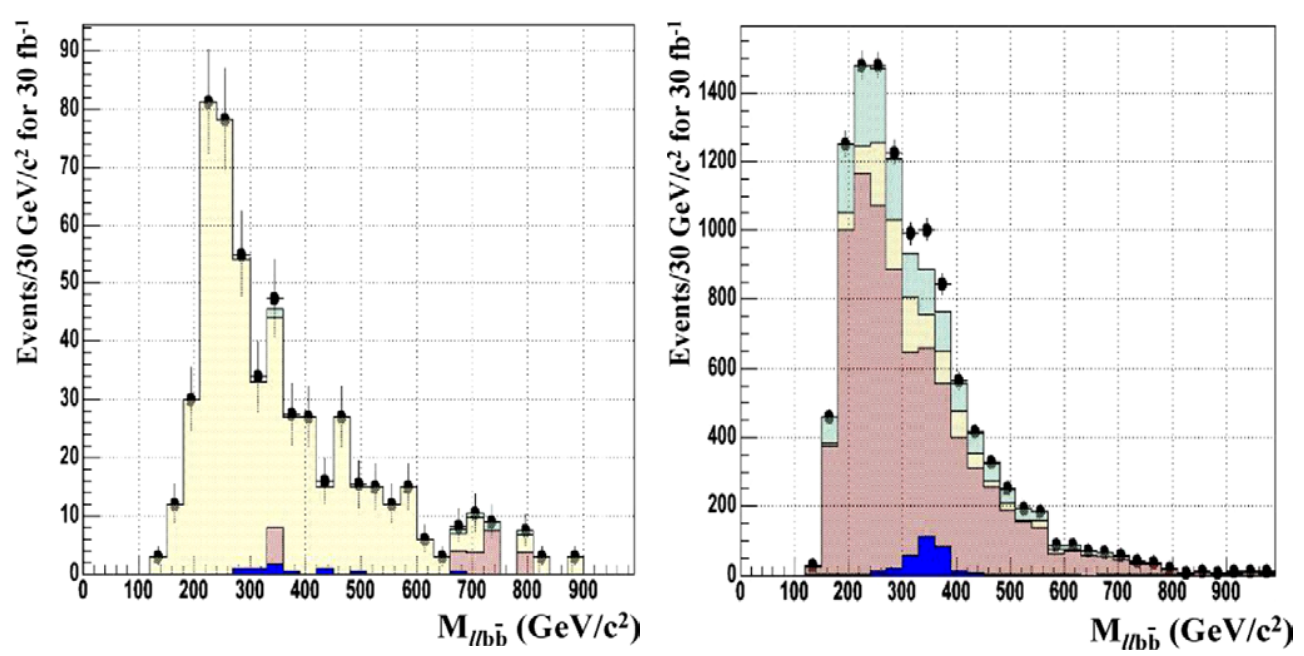

Figure 11.28. Distribution of $\mathrm{M}_{\ell^{+} \ell^{-} \mathrm{b} \overline{\mathrm{b}}}$ in the $\mathrm{t} \overline{\mathrm{t}}$ Figure 11.29. Distribution of $\mathrm{M}_{\ell^{+} \ell^{-} \mathrm{b} \bar{b}}$ used in the $\mathrm{Zb} \overline{\mathrm{b}}$ background normalisation region. Colour code is as in background estimation. Colour code is as in Fig. 11.27. Fig. 11.27.

The background uncertainty then consists of the statistical uncertainty of the background measurement in the normalisation region and the systematic uncertainty of the ratio of the background in the signal and the normalisation region.

The normalisation region for the $\bar{t} \bar{t}$ background is defined by the same selection as for the signal search, except the missing $\mathrm{E}_{\mathrm{T}}$ which is required to be bigger than $120 \mathrm{GeV}$. With such a selection 544 events were found for $30 \mathrm{fb}^{-1}$ with high purity (93.4\%), thus giving the statistical uncertainty of $4.4 \%$. The distribution of $\mathrm{M}_{\ell^{+} \ell^{-} \mathrm{b}}$ in the $\mathrm{t} \overline{\mathrm{t}}$ normalisation region can be seen in Figure 11.28. The contamination comes mainly from $\mathrm{Zb} \bar{b}$ events $(6 \%)$. The $5 \%$ missing $\mathrm{E}_{\mathrm{T}}$ scale uncertainty gives $18.5 \%$ uncertainty on the number of the $\mathrm{t}_{\mathrm{t}}$ events in the signal region. Therefore the overall uncertainty in the estimation of the $\overline{t t}$ background is $19.0 \%$.

For the irreducible $\mathrm{Zb} \overline{\mathrm{b}}$ background a similar idea can be used. In order to suppress the $\overline{\mathrm{t}}$ contribution as much as possible, missing $\mathrm{E}_{\mathrm{T}}<40 \mathrm{GeV}$ was used. Applying a lower cut in the $\mathrm{M}_{\ell^{+} \ell^{-} \mathrm{b}}$ distribution of $500 \mathrm{GeV} / \mathrm{c}^{2}, 920 \mathrm{Zbb}$ events were found with a purity of around $95 \%$ for $30 \mathrm{fb}^{-1}$. Contamination comes mainly from $\mathrm{t} \overline{\mathrm{t}}$ events. The accuracy of measuring the $\mathrm{Zb} \bar{b}$ background is around $3.4 \%$ taking into account only statistics. The distribution of $\mathbf{M}_{\ell^{+} \ell^{-} \mathrm{b} \bar{b}}$ for those events can be seen in Figure 11.29 before the application of the $\mathbf{M}_{\ell^{+} \ell^{-} \mathrm{b} \bar{b}}$ $500 \mathrm{GeV} / \mathrm{c}^{2}$ cut. The uncertainty of $5 \%$ on the missing $\mathrm{E}_{\mathrm{T}}$ scale and the uncertainty of $3 \%$ on the jet energy scale lead to correspondingly $3.6 \%$ and $2.5 \%$ of the uncertainty of the $\mathrm{Zb} \overline{\mathrm{b}}$ background estimate in the signal region. Thus the overall uncertainty in the estimation of the $\mathrm{Zb} \bar{b}$ background is $5.6 \%$.

11.2.7.6. Discovery reach in the $M_{A}-\tan \beta$ plane. Figure 11.30 shows the $5 \sigma$ discovery contours in the $\left(\mathrm{M}_{\mathrm{A}}, \tan \beta\right)$ plane for 30 and $60 \mathrm{fb}^{-1}$ of integrated luminosity in the $\mathrm{m}_{\mathrm{h}}^{\max }$ scenario with $\mu=M_{2}=600 \mathrm{GeV} / \mathrm{c}^{2}$. For the calculation of the signal significance the signal and background events were counted in mass windows of $\pm 1.5 \sigma$ around the reconstructed masses of $\mathrm{M}_{\mathrm{h}}$ and $\mathrm{M}_{\mathrm{A}}$. Since only three different $\mathrm{M}_{\mathrm{A}}$ masses and two $\tan \beta$ values were available, the estimations for the rest of $\mathrm{M}_{\mathrm{A}}, \tan \beta$ parameter space was done using extra/interpolations of the signal efficiencies from the available parameter points. The 


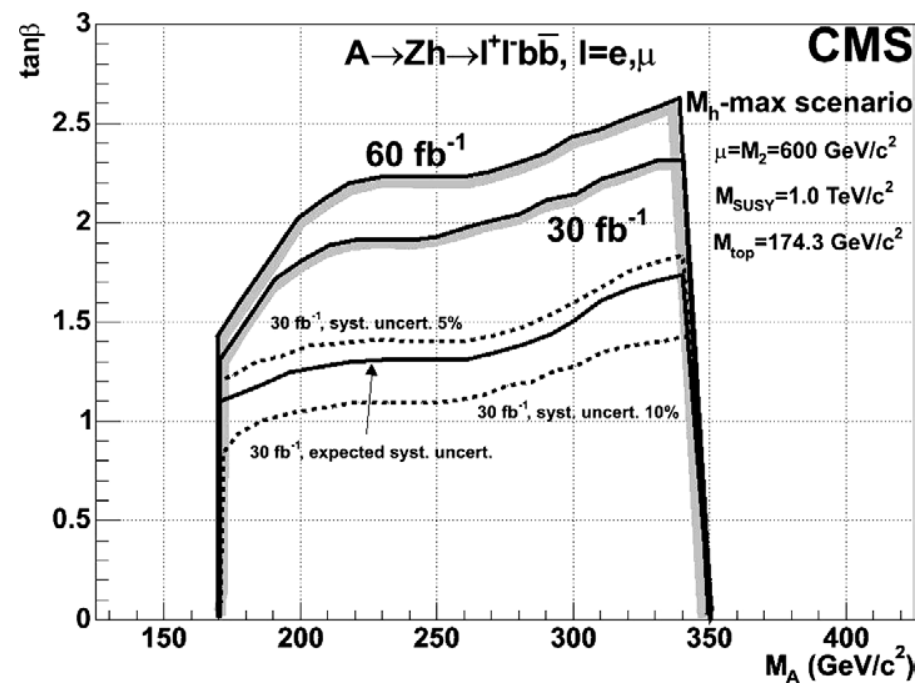

Figure 11.30. The $5 \sigma$ discovery contours for 30 and $60 \mathrm{fb}^{-1}$ integrated luminosity. The effect of underestimation or overestimation of the background systematic uncertainty can be seen in the curve of $30 \mathrm{fb}^{-1}$.

statistical significance for 5, 10\% (dashed lines) as well as the estimated (full line) uncertainty for the background is also shown for $30 \mathrm{fb}^{-1}$ of integrated luminosity.

\subsubsection{Search for $\mathrm{A}^{0} / \mathrm{H}^{0} \rightarrow \chi_{2}^{0} \chi_{2}^{0} \rightarrow 4 \ell+E_{\mathrm{T}}^{\text {miss }}$ channel in $m S U G R A$}

11.2.8.1. Introduction. In some regions of the SUSY parameter space, heavy neutral Higgs bosons can be searched for using their decay modes to supersymmetric particles. This is the case in particular in the difficult low and intermediate $\tan \beta$ region of the parameter space which is not accessible through the $\mathrm{A}^{0} / \mathrm{H}^{0} \rightarrow \tau \tau$ decay channel as the coupling of the Higgs boson to taus is not sufficiently enhanced.

One of the most promising channel is the $\mathrm{A}^{0} / \mathrm{H}^{0}$ decay into a pair of next-to-lightest neutralinos, $\chi_{2}^{0}$, followed by the decay $\chi_{2}^{0} \rightarrow \ell^{+} \ell^{-} \chi_{1}^{0}$ (with $\ell=e, \mu$ ). This process results in a clean four leptons plus missing transverse energy final state:

$$
\mathrm{A}^{0} / \mathrm{H}^{0} \rightarrow \chi_{2}^{0} \chi_{2}^{0} \rightarrow 4 \ell+\mathrm{E}_{\mathrm{T}}^{\mathrm{miss}}
$$

There are two main categories of backgrounds to such process: SUSY and Standard Model backgrounds. In the SUSY category the dominant source of background is the production of leptons from the decays of squarks and gluinos which cascade to charginos and neutralinos. Unlike the neutralinos from the Higgs boson decay, the leptons in this case are produced in association with quarks and gluons. Therefore, the associated large hadronic activity can be used to suppress this type of background. An additional but smaller source of backgrounds come from the direct production of slepton or gaugino pairs via the Drell-Yan processes and the direct production of $\chi_{2}^{0}$ pairs. The rejection of these backgrounds is more difficult, as the hadronic activity in these events is very small. In the Standard Model category, three processes which yield the same signature of 4 leptons in the final state contribute as backgrounds: $Z Z^{*} / \gamma^{*}, Z b \bar{b}$ and $t \bar{t}$. 
Table 11.24. Chosen benchmark points.

\begin{tabular}{lccccc}
\hline Point & $m_{0}\left(\mathrm{GeV} / \mathrm{c}^{2}\right)$ & $m_{1 / 2}\left(\mathrm{GeV} / \mathrm{c}^{2}\right)$ & $A_{0}\left(\mathrm{GeV} / \mathrm{c}^{2}\right)$ & $\tan \beta$ & $\operatorname{sign}(\mu)$ \\
\hline A & 60 & 175 & 0 & 10 & + \\
B & 80 & 200 & 0 & 5 & + \\
C & 50 & 150 & 0 & 5 & + \\
\hline
\end{tabular}

11.2.8.2. Analysis. The study is performed in the minimal Super Gravity constrained version of the MSSM (mSUGRA) [631]. To determine the regions where the signal has a sizeable branching ratio times cross section, a scan of the parameters space $\left(m_{0}, m_{1 / 2}\right)$ for tan $\beta=5,10, \operatorname{sign}(\mu)=+$ and $A_{0}=0$ is performed. Three benchmark points are defined for the evaluation of CMS sensitivity. The corresponding mSUGRA parameters are presented in Table 11.24.

The signal and SUSY background datasets are generated using ISASUGRA and PYTHIA. A pre-selection at generator level is applied, asking for $e^{+} e^{-} \mu^{+} \mu^{-}$final state with $\mathrm{e}(\mu)$ $p_{\mathrm{T}}>7(5) \mathrm{GeV} / \mathrm{c}$ and $|\eta|<2.5$. The fast detector simulation is carried out using FAMOs.

The online selection of the events is a logical or of the dielectron and dimuon triggers. The offline reconstruction of electrons and muons is performed using FAMOS standard algorithms. Events are then analysed as follow:

- $e^{+} e^{-} \mu^{+} \mu^{-}$final state is selected;

- the four leptons are required to be isolated;

- a jet veto is applied, requiring no jets with $\mathrm{E}_{\mathrm{T}}>25 \mathrm{GeV}$ and $|\eta|<5.0$;

- events must have $E_{\mathrm{T}}^{\text {miss }}$ and $p_{\mathrm{T}(\ell \ell \ell \ell)}$ less than $80 \mathrm{GeV} / \mathrm{c}$;

- a $Z$ veto is imposed, i.e. events with a dilepton pair with invariant mass in the range $m_{Z} \pm 10 \mathrm{GeV} / \mathrm{c}^{2}$ are rejected;

- further optimisations are performed by introducing an upper limit to the dilepton invariant masses and by applying a cut on the four lepton invariant mass.

The signal acceptances w.r.t the production cross section times branching ratio are $6.3 \%$, $5.1 \%$ and $2.5 \%$ respectively for point $\mathrm{A}, \mathrm{B}$ and $\mathrm{C}$, whereas the acceptances for SUSY backgrounds are $1.5 \times 10^{-4} \%, 3.6 \times 10^{-4} \%$ and $2.6 \times 10^{-4} \%$ respectively w.r.t. the total the SUSY production cross section.

11.2.8.3. Results. Figure 11.31 shows the invariant mass distribution of the four leptons for the 3 benchmarks points. Results are given for an integrated luminosity of $30 \mathrm{fb}^{-1}$.

Figure 11.32 shows the extrapolated $5 \sigma$-discovery regions in the $\left(m_{0}, m_{1 / 2}\right)$ plane, for an integrated luminosity of $30 \mathrm{fb}^{-1}$. The values of the other mSUGRA parameters are $A_{0}=0$, $\operatorname{sign}(\mu)=+$ and $\tan \beta=5,10$. The complex structure of the high significance region is mainly determined by the effective cross section of $\mathrm{A}^{0} / \mathrm{H}^{0} \rightarrow \chi_{2}^{0} \chi_{2}^{0} \rightarrow 4 \ell+\mathrm{E}_{\mathrm{T}}^{\text {miss }}$. The $\mathrm{A}^{0} / \mathrm{H}^{0}$ could therefore be discovered through their decays to neutralino pairs in the region $150<m_{1 / 2}$ $<250$ and $m_{0}<120$ for $\tan \beta=10$ and in the region $150<m_{1 / 2}<250$ and $30<m_{0}<120$ for $\tan \beta=5$.

\subsection{Discovery reach and measurement of MSSM parameters}

\subsubsection{Benchmark scenarios for MSSM Higgs boson searches}

11.3.1.1. Why benchmarks - which benchmarks? The tree-level values for the CP-even Higgs bosons of the MSSM, $M_{h}$ and $M_{H}$, are determined by $\tan \beta$, the CP-odd Higgs-boson 

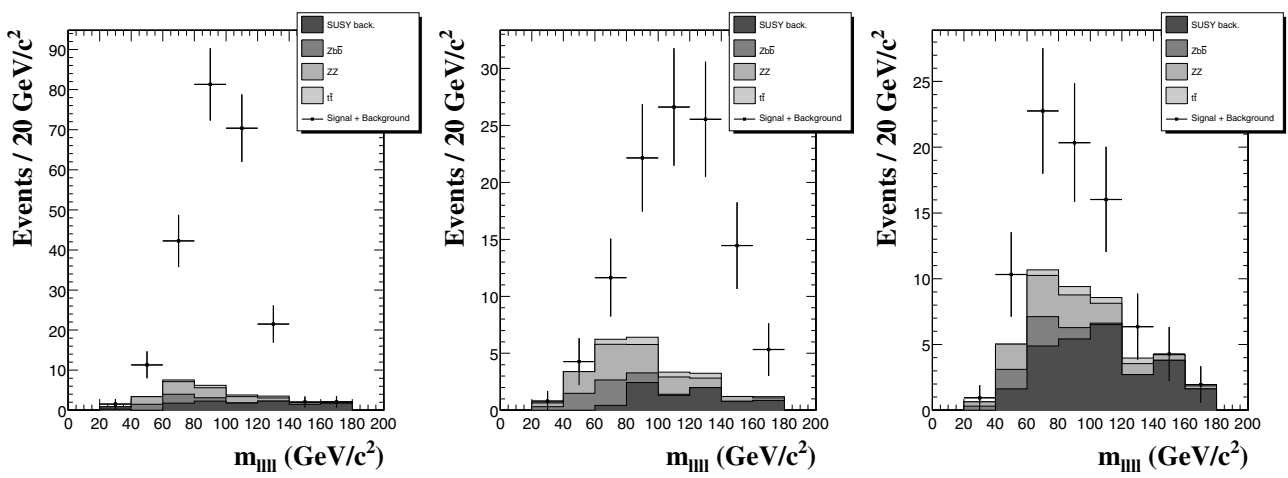

Figure 11.31. Four lepton invariant mass distributions for the 3 benchmark points. Distributions are shown for the signal+backgrounds (points) and for the contribution of each process (histograms).
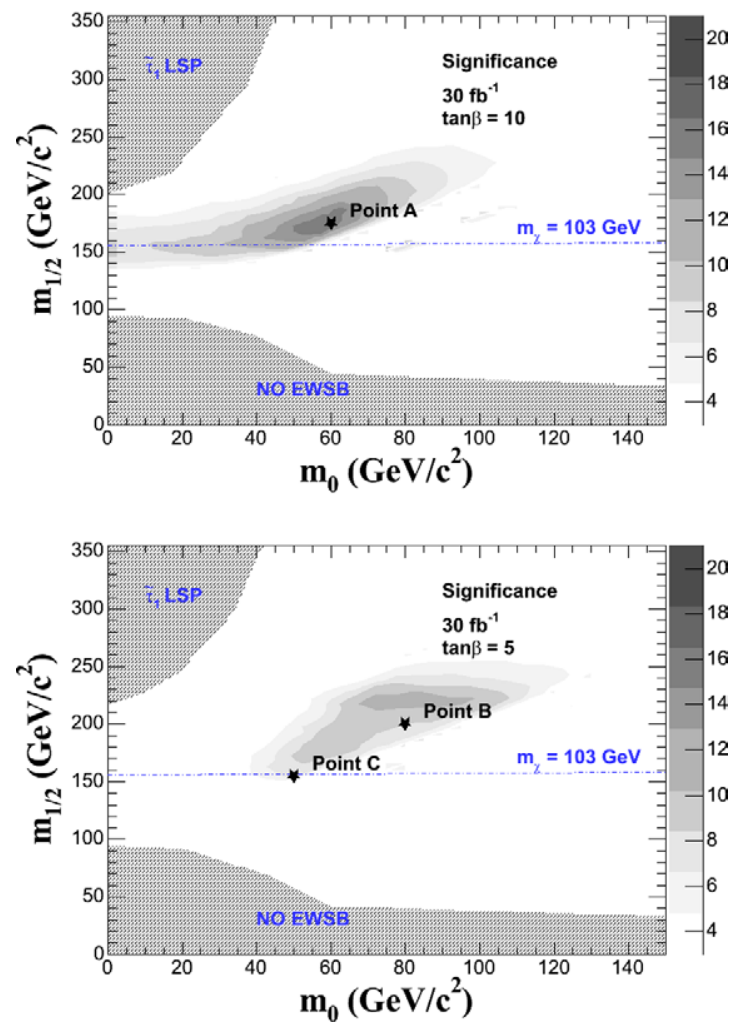

Figure 11.32. For integrated luminosity of $30 \mathrm{fb}^{-1}$ the $5 \sigma$-discovery regions for $\mathrm{A}^{0} / \mathrm{H}^{0} \rightarrow$ $\chi_{2}^{0} \chi_{2}^{0} \rightarrow 4 \ell+\mathrm{E}_{\mathrm{T}}^{\mathrm{miss}}$ channel in the $\left(m_{0}, m_{1 / 2}\right)$ plane for fixed $A_{0}=0, \operatorname{sign}(\mu)=+$ and $\tan \beta=$ 5,10

mass $M_{A}$, and the $Z$ boson mass $M_{Z}$. The mass of the charged Higgs boson, $M_{H^{ \pm}}$, is given in terms of $M_{A}$ and the $W$ boson mass, $M_{W}$. Beyond the tree-level, the main correction to the Higgs boson masses stems from the $t / \tilde{t}$ sector, and for large values of $\tan \beta$ also from the $b / \tilde{b}$ sector, see Section 11.1. Sub-leading corrections come from all other sectors of the MSSM. 
In this way the Higgs sector phenomenology is connected to the full spectrum of the MSSM via radiative corrections.

In the unconstrained version of the MSSM no particular SUSY breaking mechanism is assumed, but rather a parametrisation of all possible soft SUSY breaking terms is used. This leads to more than a hundred parameters (masses, mixing angles, phases) in this model in addition to the ones of the Standard Model. While a detailed scanning over the more-than-hundred-dimensional parameter space of the MSSM is clearly not practicable, even a sampling of three- or four-dimensional parameter space of certain SUSY-breaking models (such as mSUGRA, GMSB or AMSB) is beyond the present capabilities for phenomenological studies, in particular when it comes to simulating experimental signatures within the detectors. For this reason one often resorts to specific benchmark scenarios, i.e. one studies only specific parameter points [632,633] or samples of one- or twodimensional parameter space $[263,634,635]$, which exhibit specific characteristics of the MSSM parameter space. Benchmark scenarios of this kind are often used, for instance, for studying the performance of different experiments at the same collider. Similarly, detailed experimental simulations of MSSM particle production with identical parameters in the framework of different colliders can be very helpful for developing strategies for combining pieces of information obtained at different machines [5].

The question of which parameter choices are useful as benchmark scenarios depends on the purpose of the actual investigation. If one is interested, for instance, in setting exclusion limits on the SUSY parameter space from the non-observation of SUSY signals at the experiments performed up to now, it is useful to use a benchmark scenario which gives rise to "conservative" exclusion bounds. An example of a benchmark scenario of this kind is the $m_{h}^{\max }$-scenario [635] used for the Higgs search at LEP [566]. It gives rise to maximal values of the lightest CP-even Higgs-boson mass (for fixed values of the top-quark mass and the SUSY scale) and thus allows one to set conservative bounds on $\tan \beta$ and $M_{A}$ [544]. Another application of benchmark scenarios is to study "typical" experimental signatures of SUSY models and to investigate the experimental sensitivities and the achievable experimental precisions for these cases. For this purpose it seems reasonable to choose "typical" (a notion which is of course difficult to define) and theoretically well motivated parameters of certain SUSY-breaking scenarios. Examples of this kind are the benchmark scenarios used so far for investigating SUSY searches at the LHC [632, 633] and at the ILC [636]. As a further possible goal of benchmark scenarios, one can choose them so that they account for a wide variety of SUSY phenomenology. For this purpose, it can also be useful to consider "pathological" regions of parameter space or "worst-case" scenarios. Examples for this are the "small $\alpha_{\text {eff }}$ scenario" [635] for the Higgs search at LEP, for which the decay $h \rightarrow b \bar{b}$ or $h \rightarrow \tau^{+} \tau^{-}$can be significantly suppressed.

A related issue concerning the definition of appropriate benchmarks is whether a benchmark scenario chosen for investigating physics at a certain experiment or for testing a certain sector of the theory should be compatible with additional information from other experiments (or concerning other sectors of the theory). This refers in particular to constraints from cosmology (by demanding that SUSY should give rise to an acceptable dark matter density [637-640]) and low-energy measurements such as the rate for $b \rightarrow s \gamma$ [641,642] and the anomalous magnetic moment of the muon, $(g-2)_{\mu}[643,644]$. On the one hand, applying constraints of this kind gives rise to "more realistic" benchmark scenarios. On the other hand, one relies in this way on further assumptions (and has to take account of experimental and theoretical uncertainties related to these additional constraints), and it could eventually turn out that one has inappropriately narrowed down the range of possibilities by applying these constraints. This applies in particular if slight modifications of the model under 
consideration are possible that have a minor impact on collider phenomenology but could significantly alter the bounds from cosmology and low-energy experiments. For instance, the presence of small flavour mixing terms in the SUSY Lagrangian could severely affect the prediction for $\mathrm{BR}(b \rightarrow s \gamma)$, while allowing a small amount of R-parity violation in the model would strongly affect the constraints from dark matter relic abundance while leaving collider phenomenology essentially unchanged. The extent to which additional constraints of this kind should be applied to possible benchmark scenarios is related to the actual purpose of the benchmark scenario. For setting exclusion bounds in a particular sector (e.g. the Higgs sector) it seems preferable to apply constraints from this sector only.

11.3.1.2. The relevant MSSM parameters. Beyond the tree-level, the main correction to the Higgs boson masses and couplings comes from the $t / \tilde{t}$ sector, and for large values of $\tan \beta$ also from the $b / \tilde{b}$ sector. In order to fix our notations, we list the conventions for the inputs from the scalar top and scalar bottom sector of the MSSM: the mass matrices in the basis of the current eigenstates $\tilde{t}_{L}, \tilde{t}_{R}$ and $\tilde{b}_{L}, \tilde{b}_{R}$ are given by

$$
\begin{aligned}
& \mathcal{M}_{\tilde{t}}^{2}=\left(\begin{array}{cc}
M_{\tilde{t}_{L}}^{2}+m_{t}^{2}+\cos 2 \beta\left(\frac{1}{2}-\frac{2}{3} s_{W}^{2}\right) M_{Z}^{2} & m_{t} X_{t} \\
m_{t} X_{t} & M_{\tilde{t}_{R}}^{2}+m_{t}^{2}+\frac{2}{3} \cos 2 \beta s_{W}^{2} M_{Z}^{2}
\end{array}\right), \\
& \mathcal{M}_{\tilde{b}}^{2}=\left(\begin{array}{ll}
M_{\tilde{b}_{L}}^{2}+m_{b}^{2}+\cos 2 \beta\left(-\frac{1}{2}+\frac{1}{3} s_{W}^{2}\right) M_{Z}^{2} & m_{b} X_{b} \\
m_{b} X_{b} & M_{\tilde{b}_{R}}^{2}+m_{b}^{2}-\frac{1}{3} \cos 2 \beta s_{W}^{2} M_{Z}^{2}
\end{array}\right),
\end{aligned}
$$

where

$$
m_{t} X_{t}=m_{t}\left(A_{t}-\mu \cot \beta\right), \quad m_{b} X_{b}=m_{b}\left(A_{b}-\mu \tan \beta\right) .
$$

Here $A_{t}$ denotes the trilinear Higgs-stop coupling, $A_{b}$ denotes the Higgs-sbottom coupling, and $\mu$ is the Higgsino mass parameter.

$\mathrm{SU}(2)$ gauge invariance leads to the relation

$$
M_{\tilde{t}_{L}}=M_{\tilde{b}_{L}} .
$$

For the numerical evaluation, a convenient choice is

$$
M_{\tilde{t}_{L}}=M_{\tilde{b}_{L}}=M_{\tilde{t}_{R}}=M_{\tilde{b}_{R}}=: M_{\mathrm{SUSY}} .
$$

We furthermore use the short-hand notation

$$
M_{S}^{2}:=M_{\mathrm{SUSY}}^{2}+m_{t}^{2} .
$$

Accordingly, the most important parameters for the corrections in the Higgs sector are $m_{t}$, $M_{\text {SUSY }}, X_{t}$ and $X_{b}$ (or equivalently $A_{t}$ and $A_{b}$ ), $\mu$ and $\tan \beta$. The Higgs sector observables furthermore depend on the $\mathrm{SU}(2)$ gaugino mass parameter, $M_{2}$. The other gaugino mass parameter, $M_{1}$, is usually fixed via the GUT relation

$$
M_{1}=\frac{5}{3} \frac{s_{W}^{2}}{c_{W}^{2}} M_{2} .
$$

At the loop level also the gluino mass, $m_{\tilde{g}}$, enters the predictions for the Higgs-boson phenomenology.

It should be noted in this context that the results for Higgs boson sector observables have been obtained in different schemes. Most commonly these are the on-shell (OS) renormalisation scheme (in the Feynman-diagrammatic (FD) approach), and $\overline{\mathrm{MS}}$ scheme (for the renormalisation group (RG) approach) [645]. Owing to the different schemes used in the FD and the RG approach for the renormalisation in the scalar top sector, the parameters 
$X_{t}$ and $M_{\text {SUSY }}$ are also scheme-dependent in the two approaches. This difference between the corresponding parameters has to be taken into account when defining the benchmark scenarios. In a simple approximation the relation between the parameters in the different schemes is at $\mathcal{O}\left(\alpha_{s}\right)$ given by [645]

$$
\begin{aligned}
& M_{S}^{2, \overline{\mathrm{MS}}} \approx M_{S}^{2, \mathrm{OS}}-\frac{8}{3} \frac{\alpha_{s}}{\pi} M_{S}^{2}, \\
& X_{t}^{\overline{\mathrm{MS}}} \approx X_{t}^{\mathrm{OS}}+\frac{\alpha_{s}}{3 \pi} M_{S}\left(8+4 \frac{X_{t}}{M_{S}}-3 \frac{X_{t}}{M_{S}} \log \left(\frac{m_{t}^{2}}{M_{S}^{2}}\right)\right) .
\end{aligned}
$$

At large $\tan \beta$ and large $|\mu|$ the corrections from the $b / \tilde{b}$ sector can become especially important. The leading effects are included in the effective Lagrangian formalism [563]. Numerically this is by far the dominant part of the contributions from the sbottom sector (see also Refs. [547, 548]). The effective Lagrangian is given by

$$
\begin{gathered}
\mathcal{L}=\frac{g}{2 M_{W}} \frac{\bar{m}_{b}}{1+\Delta_{b}}\left[\tan \beta A i \bar{b} \gamma_{5} b+\sqrt{2} V_{t b} \tan \beta H^{+} \bar{t}_{L} b_{R}+\left(\frac{\sin \alpha}{\cos \beta}-\Delta_{b} \frac{\cos \alpha}{\sin \beta}\right) h \bar{b}_{L} b_{R}\right. \\
\left.-\left(\frac{\cos \alpha}{\cos \beta}+\Delta_{b} \frac{\sin \alpha}{\sin \beta}\right) H \bar{b}_{L} b_{R}\right]+ \text { h.c.. }
\end{gathered}
$$

Here $\bar{m}_{b}$ denotes the running bottom quark mass including SM QCD corrections. The pre-factor $1 /\left(1+\Delta_{b}\right)$ in Eq. 11.20 arises from the resummation of the leading corrections to all orders. The function $\Delta_{b}$ consists of two main contributions, an $\mathcal{O}\left(\alpha_{s}\right)$ correction from a sbottom-gluino loop and an $\mathcal{O}\left(\alpha_{t}\right)$ correction from a stop-Higgsino loop. The explicit form of $\Delta_{b}$ in the limit of $M_{S} \gg m_{t}$ and $\tan \beta \gg 1$ reads [563]

$\Delta_{b}=\frac{2 \alpha_{s}}{3 \pi} m_{\tilde{g}} \mu \tan \beta \times I\left(m_{\tilde{b}_{1}}, m_{\tilde{b}_{2}}, m_{\tilde{g}}\right)+\frac{\alpha_{t}}{4 \pi} A_{t} \mu \tan \beta \times I\left(m_{\tilde{t}_{1}}, m_{\tilde{t}_{2}}, \mu\right)$.

The function $I$ is given by

$$
\begin{aligned}
I(a, b, c)= & \frac{1}{\left(a^{2}-b^{2}\right)\left(b^{2}-c^{2}\right)\left(a^{2}-c^{2}\right)}\left(a^{2} b^{2} \log \frac{a^{2}}{b^{2}}+b^{2} c^{2} \log \frac{b^{2}}{c^{2}}+c^{2} a^{2} \log \frac{c^{2}}{a^{2}}\right) \\
& \sim \frac{1}{\max \left(a^{2}, b^{2}, c^{2}\right)} .
\end{aligned}
$$

It becomes obvious that the size and the sign of $\mu$ is especially relevant for this type of corrections.

11.3.1.3. The benchmark scenarios. Since at the tree-level the Higgs sector of the MSSM is governed by two parameters (in addition to $M_{Z}$ and the SM gauge couplings), it seems reasonable to define benchmarks in which all SUSY parameters are fixed and only the two tree-level parameters, $M_{A}$ and $\tan \beta$ are varied. For the search of the heavy MSSM Higgs bosons corrections from the $b / \tilde{b}$ sector can be especially relevant. In this case it is also appropriate to vary $\mu$. We review the definition of the benchmark scenarios as defined in Refs. [263,635]. Another very important parameter is the top-quark mass. For sake of simplicity and to make different analyses readily comparable to each other a fixed value of $m_{t}=175 \mathrm{GeV}$ can be used. Alternatively the current experimental value can be used as input.

The $\boldsymbol{m}_{\boldsymbol{h}}^{\max }$ scenario. This scenario was designed to obtained conservative $\tan \beta$ exclusion bounds [544] at LEP [566]. The parameters are chosen such that the maximum possible 
Higgs-boson mass as a function of $\tan \beta$ is obtained (for fixed $M_{\mathrm{SUSY}}$, and $M_{A}$ set to its maximal value, $\left.M_{A}=1 \mathrm{TeV}\right)$. The parameters are ${ }^{48}$ :

$$
\begin{aligned}
& m_{t}=175 \mathrm{GeV}, \quad M_{\mathrm{SUSY}}=1 \mathrm{TeV}, \quad \mu=200 \mathrm{GeV}, \quad M_{2}=200 \mathrm{GeV}, \\
& X_{t}^{\mathrm{OS}}=2 M_{\mathrm{SUSY}}(\text { FD calculation }), \quad X_{t}^{\overline{\mathrm{MS}}}=\sqrt{6} M_{\mathrm{SUSY}}(\mathrm{RG} \text { calculation }) \\
& A_{b}=A_{t}, \quad m_{\tilde{g}}=0.8 M_{\mathrm{SUSY}} .
\end{aligned}
$$

The no-mixing scenario. This benchmark scenario is the same as the $m_{h}^{\max }$ scenario, but with vanishing mixing in the $\tilde{t}$ sector and with a higher SUSY mass scale to avoid the LEP Higgs bounds $[62,566]$,

$$
\begin{aligned}
& m_{t}=175 \mathrm{GeV}, \quad M_{\mathrm{SUSY}}=2 \mathrm{TeV}, \quad \mu=200 \mathrm{GeV}, \quad M_{2}=200 \mathrm{GeV}, \\
& X_{t}=0 \text { (FD/RG calculation), } \quad A_{b}=A_{t}, \quad m_{\tilde{g}}=0.8 M_{\mathrm{SUSY}} .
\end{aligned}
$$

The gluophobic Higgs scenario. In this scenario the main production cross section for the light Higgs boson at the LHC, $g g \rightarrow h$, is strongly suppressed. This can happen due to a cancellation between the top quark and the stop quark loops in the production vertex (see Ref. [502]). This cancellation is more effective for small $\tilde{t}$ masses and hence for relatively large values of the $\tilde{t}$ mixing parameter, $X_{t}$. The partial width of the most relevant decay mode, $\Gamma(h \rightarrow \gamma \gamma)$, is affected much less, since it is dominated by the $W$ boson loop. The parameters are:

$$
\begin{aligned}
& m_{t}=175 \mathrm{GeV}, \quad M_{\mathrm{SUSY}}=350 \mathrm{GeV}, \quad \mu=300 \mathrm{GeV}, \quad M_{2}=300 \mathrm{GeV}, \\
& X_{t}^{\mathrm{OS}}=-750 \mathrm{GeV} \text { (FD calculation) }, \quad X_{t}^{\overline{\mathrm{MS}}}=-770 \mathrm{GeV} \text { (RG calculation), } \\
& A_{b}=A_{t}, \quad m_{\tilde{g}}=500 \mathrm{GeV} .
\end{aligned}
$$

In the left plot of Fig. 11.33 we show $[\sigma \times \mathrm{BR}]_{\mathrm{MSSM}} /[\sigma \times \mathrm{BR}]_{\mathrm{SM}}$ for the channel $g g \rightarrow h \rightarrow \gamma \gamma$ in the $M_{A}-\tan \beta$-plane. This channel can be strongly suppressed over the whole parameter plane, rendering this detection channel difficult.

The small $\boldsymbol{\alpha}_{\text {eff }}$ scenario. Besides the channel $g g \rightarrow h \rightarrow \gamma \gamma$ at the LHC, other channels for light Higgs searches at the Tevatron and at the LHC rely on the decays $h \rightarrow \bar{b} b$ and $h \rightarrow \tau^{+} \tau^{-}$. If $\alpha_{\text {eff }}$ is small, these two decay channels can be heavily suppressed in the MSSM due to the additional factor $-\sin \alpha_{\text {eff }} / \cos \beta$ compared to the $\mathrm{SM}$ coupling. Such a suppression occurs for large $\tan \beta$ and not too large $M_{A}$ for the following parameters:

$$
\begin{aligned}
& m_{t}=175 \mathrm{GeV}, \quad M_{\mathrm{SUSY}}=800 \mathrm{GeV}, \quad \mu=2.5 M_{\mathrm{SUSY}}, \quad M_{2}=500 \mathrm{GeV}, \\
& X_{t}^{\mathrm{OS}}=-1100 \mathrm{GeV} \text { (FD calculation) }, \quad X_{t}^{\overline{\mathrm{MS}}}=-1200 \mathrm{GeV} \text { (RG calculation), } \\
& A_{b}=A_{t}, \quad m_{\tilde{g}}=500 \mathrm{GeV} .
\end{aligned}
$$

In the right plot of Fig.11.33 we show $[\sigma \times \mathrm{BR}]_{\mathrm{MSSM}} /[\sigma \times \mathrm{BR}]_{\mathrm{SM}}$ for the channel $W W \rightarrow$ $h \rightarrow \tau^{+} \tau^{-}$in the $M_{A}-\tan \beta$-plane. Significant suppression occurs for large $\tan \beta, \tan \beta>20$, and small to moderate $M_{A}, M_{A}<400 \mathrm{GeV}$. Thus, Higgs boson search via the $W W$ fusion channel will be difficult in these parts of the parameter space.

11.3.1.4. Variation of $\mu$. The most sensitive channels for detecting heavy MSSM Higgs bosons at the LHC are the channel $p p \rightarrow H / A+X, H / A \rightarrow \tau^{+} \tau^{-}$(making use of different

\footnotetext{
${ }^{48}$ Better agreement with BR $(b \rightarrow s \gamma)$ constraints is obtained for the other sign of $X_{t}$ (called the "constrained $m_{h}^{\max }$ " scenario). However, this lowers the maximum $M_{h}$ values by $\sim 5 \mathrm{GeV}$.
} 
$\sigma(g g->h) \times B R(h \rightarrow r)$

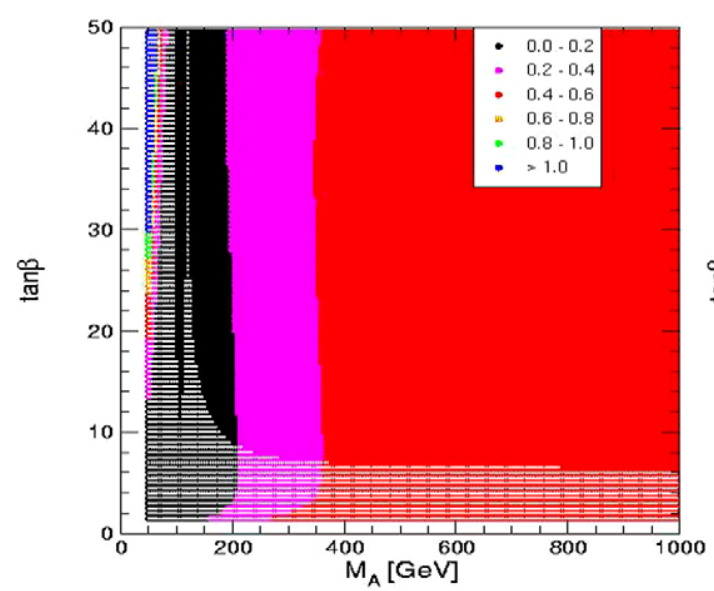

$\sigma(W W h) \times B R(h->\tau \tau)$

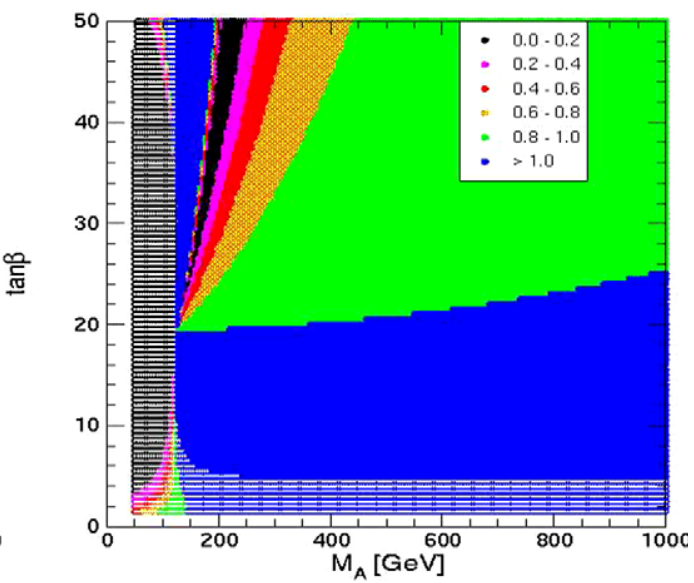

Figure 11.33. $[\sigma \times \mathrm{BR}]_{\mathrm{MSSM}} /[\sigma \times \mathrm{BR}]_{\mathrm{SM}}$ is shown for the channels $g g \rightarrow h \rightarrow \gamma \gamma$ in the gluophobic Higgs scenario (left plot) and $W W \rightarrow h \rightarrow \tau^{+} \tau^{-}$in the small $\alpha_{\text {eff }}$ scenarios (right plot) in the $M_{A}-\tan \beta$-plane. The hatched area is excluded by LEP Higgs searches.

decay modes of the two $\tau$ leptons) and the channel $t H^{ \pm}, H^{ \pm} \rightarrow \tau v_{\tau}$ (for $M_{H^{ \pm}}>m_{t}$ ). These channels show good prospects for $M_{A} \gg M_{Z}$ and large $\tan \beta$.

As discussed above, in this part of the parameter space the corrections from the $b / \tilde{b}$ sector can be very important and thus the size and the sign of $\mu$ can play a dominant role. This lead to the definition of an extension of the $m_{h}^{\max }$ and the no-mixing scenario by the following values of $\mu$ [263]

$$
\mu= \pm 200, \pm 500, \pm 1000 \mathrm{GeV}
$$

allowing both an enhancement and a suppression of the bottom Yukawa coupling and taking into account the limits from direct searches for charginos at LEP. It should be noted that the values $\mu=-500,-1000 \mathrm{GeV}$ can lead to such a large enhancement of the bottom Yukawa coupling that a perturbative treatment is no longer possible in the region of very large values of $\tan \beta$. Some care is therefore necessary to assess up to which values of $\mu$ reliable results can be obtained.

A further variation of the discovery reach is caused by the decays of the heavy Higgs bosons into supersymmetric particles. For a given value of $\mu$, the rates of these decay modes are strongly dependent on the particular values of the weak gaugino mass parameters $M_{2}$ and $M_{1}$. Since the Higgs couplings to neutralinos and charginos depend strongly on the admixture between Higgsino and gaugino states, the rate of these processes is strongly suppressed for large values of $|\mu|>500 \mathrm{GeV}$. In general, the effects of the decays $H / A \rightarrow \tilde{\chi}_{i}^{0} \tilde{\chi}_{j}^{0}, \tilde{\chi}_{k}^{ \pm} \tilde{\chi}_{l}^{\mp}$ only play a role for $M_{A}>|\mu|+M_{1}$. Outside this range the dependence of the rates on $\mu$ is relatively weak.

\subsubsection{Discovery reach in the $\mathrm{M}_{\mathrm{A}}-\tan \beta$ plane}

This section summarises the discovery reach in the $M_{\mathrm{A}}$-tan $\beta$ plane for the charged and the neutral MSSM Higgs bosons in the $\mathrm{m}_{\mathrm{h}}^{\max }$ scenario. The cross sections and branching ratios for the neutral Higgs bosons and the branching ratios for the charged Higgs boson were calculated with FeynHiggs 2.3.2 [142-144]. The next-to-leading order cross section for the 


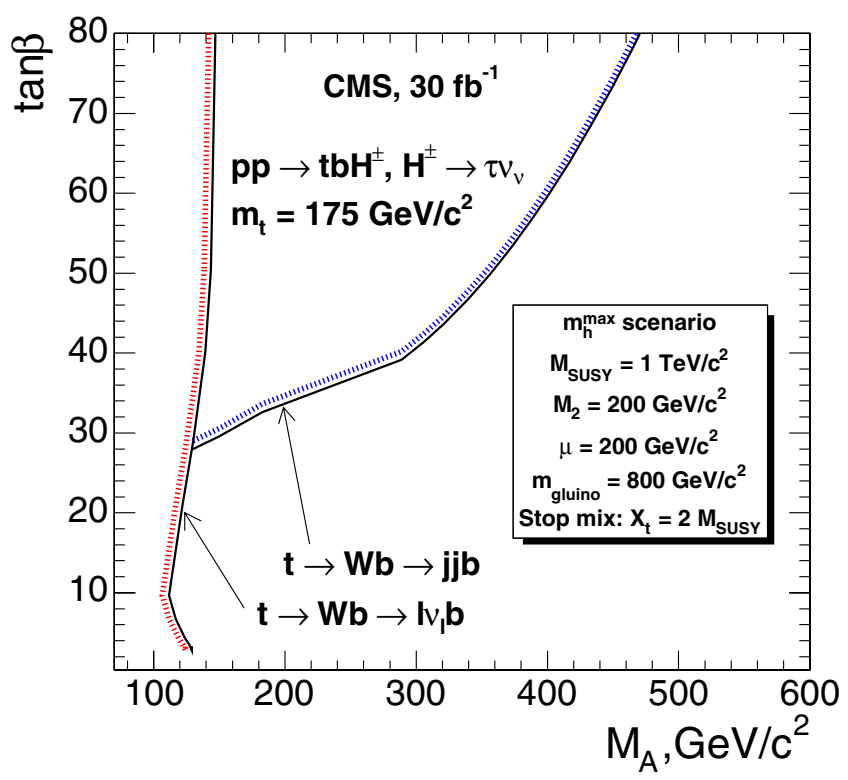

Figure 11.34. The $5 \sigma$ discovery regions for the charged Higgs boson with the $\tau \nu$ decay mode in the $\mathrm{m}_{\mathrm{h}}^{\max }$ scenario.

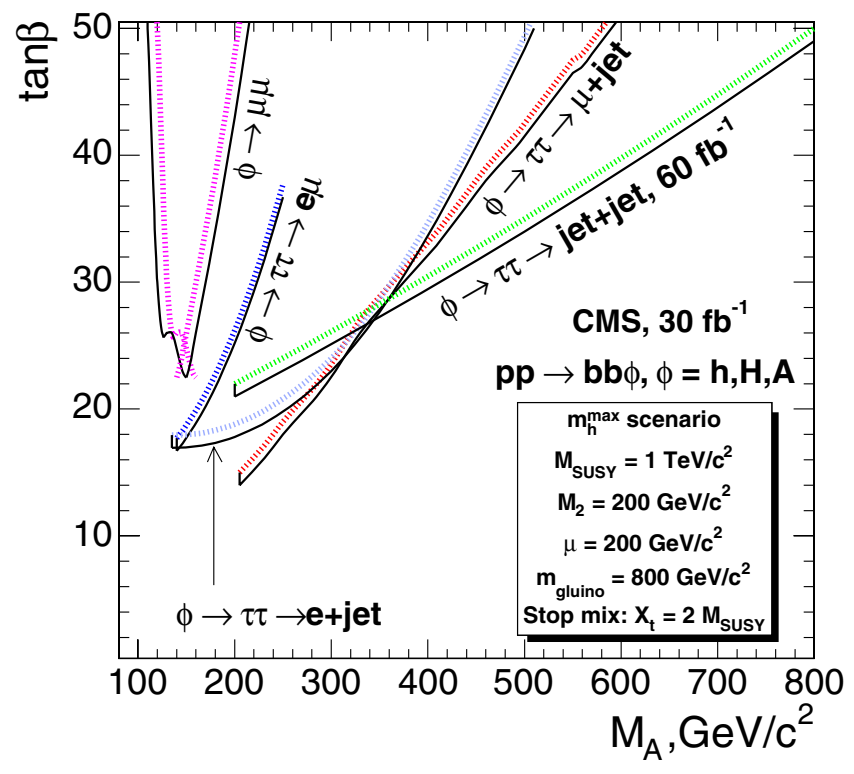

Figure 11.35. The $5 \sigma$ discovery regions for the neutral Higgs bosons $\phi(\phi=\mathrm{h}, \mathrm{H}, \mathrm{A})$ produced in the association with $\mathrm{b}$ quarks $\mathrm{pp} \rightarrow \mathrm{bb} \phi$ with the $\phi \rightarrow \mu \mu$ and $\phi \rightarrow \tau \tau$ decay modes in the $\mathrm{m}_{\mathrm{h}}^{\max }$ scenario.

charged Higgs production was taken from Refs. [628], [597]. The NLO cross sections for the background processes were used, when available.

Figure 11.34 shows the $5 \sigma$ discovery regions for the charged Higgs boson produced in the $\mathrm{pp} \rightarrow \mathrm{tbH}^{ \pm}$process with the $\mathrm{H}^{ \pm} \rightarrow \tau^{ \pm} \nu_{\tau}(\tau \rightarrow$ hadrons) decay mode. Figure 11.35 


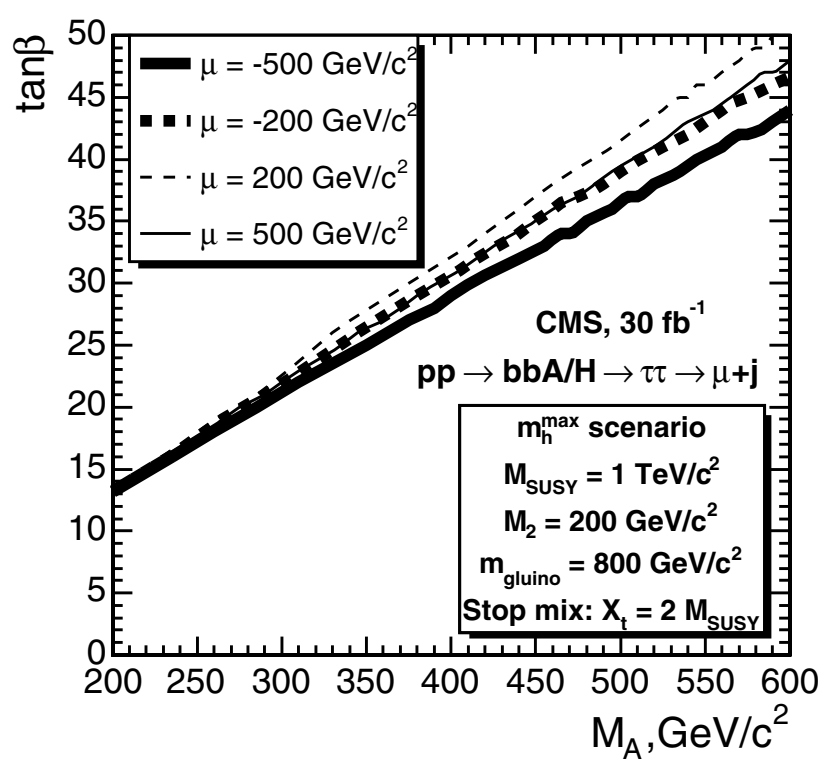

Figure 11.36. Variation of the $5 \sigma$ discovery potential for the neutral Higgs bosons in the $\tau \tau \rightarrow \mu+$ jet decay mode with $\mu$ in the $\mathrm{m}_{\mathrm{h}}^{\max }$ scenario.

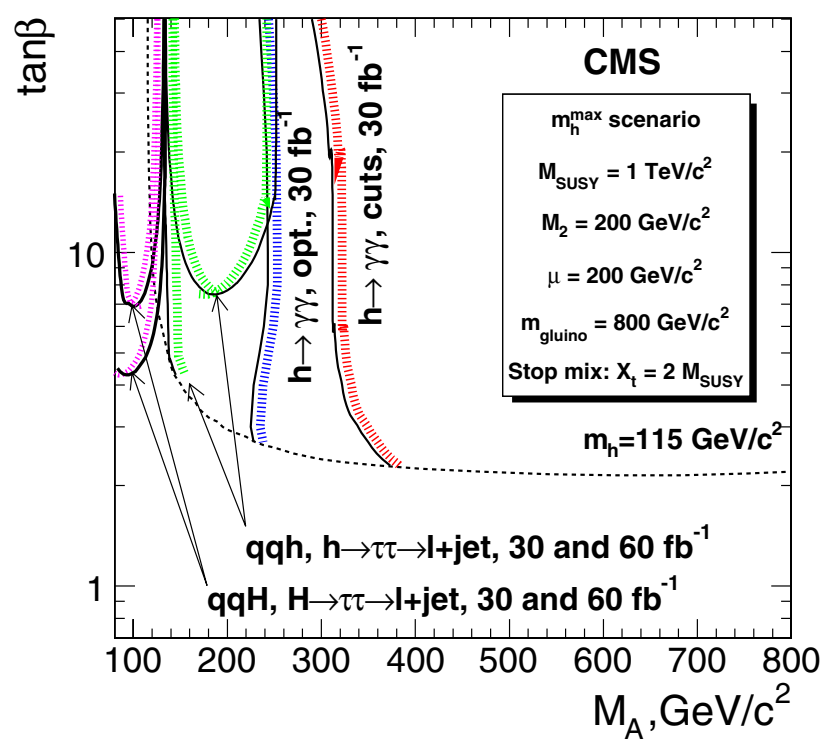

Figure 11.37. The $5 \sigma$ discovery regions for the light, neutral Higgs boson $\mathrm{h}$ from the inclusive $\mathrm{pp} \rightarrow \mathrm{h}+\mathrm{X}$ production with the $\mathrm{h} \rightarrow \gamma \gamma$ decay and for the light and heavy scalar Higgs bosons, $\mathrm{h}$ and $\mathrm{H}$, produced in the vector boson fusion $\mathrm{qq} \rightarrow \mathrm{qqh}(\mathrm{H})$ with the $\mathrm{h}(\mathrm{H}) \rightarrow \tau \tau \rightarrow \ell+$ jet decay in the $\mathrm{m}_{\mathrm{h}}^{\max }$ scenario.

shows the $5 \sigma$ discovery regions for the neutral Higgs boson $\phi(\phi=\mathrm{h}, \mathrm{H}, \mathrm{A})$ produced in the association with $\mathrm{b}$ quarks $\mathrm{pp} \rightarrow \mathrm{b} \overline{\mathrm{b}} \phi$ with the $\phi \rightarrow \mu \mu$ and $\phi \rightarrow \tau \tau$ decay modes. In both figures the discovery reach was evaluated in the $\mathrm{m}_{\mathrm{h}}^{\max }$ scenario with $\mu=200 \mathrm{GeV} / \mathrm{c}^{2}$ (See Section 11.3.1). 
The discovery reach was evaluated also in the extended $\mathrm{m}_{\mathrm{h}}^{\max }$ scenario (see Section 11.3.1.3 and [263]) with the values of $\mu=-200$ and $\pm 500 \mathrm{GeV} / \mathrm{c}^{2}$. The Fig. 11.36 presents the variation of the $5 \sigma$ discovery potential for the neutral Higgs boson produced in the association with $\mathrm{b}$ quarks $\mathrm{pp} \rightarrow \mathrm{b} \overline{\mathrm{b}} \phi$ with the $\phi \rightarrow \tau \tau \rightarrow \mu+\mathrm{jet}$ decay mode. The combination of the effects from supersymmetric radiative corrections and decay modes into supersymmetric particles gives rise to a rather complicated dependence of the discovery contour on $\mu$. This results in a variation of the discovery region, especially for large $M_{A}$ and large $\tan \beta$. For the positive values of $\mu$ the inclusion of the supersymmetric radiative corrections leads to a shift of the discovery region toward higher values of $\tan \beta$.

Figure 11.37 shows the $5 \sigma$ discovery regions for the light, neutral Higgs boson $\mathrm{h}$ from the inclusive $\mathrm{pp} \rightarrow \mathrm{h}+\mathrm{X}$ production with the $\mathrm{h} \rightarrow \gamma \gamma$ decay and for the light and heavy scalar Higgs bosons, $h$ and $\mathrm{H}$, produced in the vector boson fusion $\mathrm{qq} \rightarrow \mathrm{qqh}(\mathrm{H})$ with the $\mathrm{h}(\mathrm{H}) \rightarrow \tau \tau \rightarrow \ell+$ jet decay. 


\section{Chapter 12. Search for Higgs Boson in Non-SUSY Models}

\subsection{Scalar sector of 5D Randall-Sundrum model}

The Randall-Sundrum model (RS) [94, 646] has recently received much attention because it could provide a solution to the hierarchy problem [565], by means of an exponential factor in a five dimensional nonfactorisable metric. In the simplest version the RS model is based on a five dimensional universe with two four-dimensional hypersurfaces (branes), located at the boundary of the fifth coordinate. By placing all the Standard Model fields on the visible brane all the mass terms, which are of the order of the Planck mass, are rescaled by the exponential factor, to a scale of the order of a TeV. The fluctuations in the metric in the fifth dimension are described in terms of a scalar field, the radion, which in general mixes with the Higgs boson. This scalar sector of the RS model is parameterised in terms of a dimensionless Higgs boson radion mixing parameter $\xi$, of the Higgs boson and radion masses $\mathrm{m}_{\mathrm{h}}, \mathrm{m}_{\phi}$ and the vacuum expectation value of the radion field $\Lambda_{\phi}$.

The phenomenology of Higgs boson and radion at LHC has been subject to several studies [647-652] concentrating mainly on Higgs and radion processes. The Higgs boson and radion detection is not guaranteed in all the parameter space region. The presence in the Higgs radion sector of trilinear terms opens the possibility of $\phi \rightarrow \mathrm{hh}$ and $\mathrm{h} \rightarrow \phi \phi$ decays. For example, for $\mathrm{m}_{\mathrm{h}}=120 \mathrm{GeV} / \mathrm{c}^{2}, \Lambda_{\phi}=5 \mathrm{TeV} / \mathrm{c}^{2}$ and $\mathrm{m}_{\phi} \sim 250-350 \mathrm{GeV} / \mathrm{c}^{2}$ the $\mathrm{BR}(\phi \rightarrow \mathrm{hh})$ ranges between 20 and $30 \%$.

The CMS discovery potential is estimated for the decay of the radion in a pair of Higgs bosons, with $\gamma \gamma \mathrm{b} \bar{b}, \tau \tau b \bar{b}$ and $b \bar{b} b \bar{b}$ final states and for an integrated luminosity of $30 \mathrm{fb}^{-1}$. The study has been carried out for the radion mass of $300 \mathrm{GeV} / \mathrm{c}^{2}$ and the Higgs boson mass of $125 \mathrm{GeV} / \mathrm{c}^{2}$. The sensitivity was evaluated in the $\left(\xi, \Lambda_{\phi}\right)$ plane, with systematics uncertainties included.

A detailed description of the analysis can be found in [653]. A brief summary of the analysis and the results is presented below.

\subsubsection{The $\phi \rightarrow$ hh analysis with the $\gamma \gamma b \bar{b}$ and $\tau \tau b \bar{b}$ final states}

Signal events gg $\rightarrow \phi \rightarrow$ hh were generated with PYTHIA. The cross sections and branching ratios were evaluated using rescaled NLO cross sections for the SM Higgs boson and a modified HDECAY program. For the radion and a Higgs boson mass points considered $\left(\mathrm{m}_{\mathrm{h}}=125 \mathrm{GeV} / \mathrm{c}^{2}, \mathrm{~m}_{\phi}=300 \mathrm{GeV} / \mathrm{c}^{2}\right)$ and for $\Lambda_{\phi}=1 \mathrm{TeV} / \mathrm{c}^{2}$ the maximal cross section times branching ratio is $71 \mathrm{fb}$ for $\gamma \gamma \mathrm{b} \bar{b}$ final state. For the $\tau \tau \mathrm{b} \bar{b}$ final state with the topology considered in the analysis, one $\tau$ lepton decaying leptonically and the other $\tau$ lepton decaying hadronically (producing a $\tau$ jet), the maximal cross section times branching ratio is $960 \mathrm{fb}$. This maximal cross section is reached for the radion mixing parameter $\xi=-0.35$.

For the $\gamma \gamma \mathrm{b} \bar{b}$ final state the irreducible backgrounds $\gamma \gamma \mathrm{jj}(\mathrm{j}=\mathrm{u}, \mathrm{d}, \mathrm{s}, \mathrm{g}$ ) (generated with COMPHEP) and the $\gamma \gamma \mathrm{c} \overline{\mathrm{c}}$ and $\gamma \gamma \mathrm{b} \overline{\mathrm{b}}$ (generated with MADGRAPH) were studied. The reducible background from $\gamma+$ three jets and four-jet processes was not evaluated directly, but assumed to be the same as in for the inclusive $h \rightarrow \gamma \gamma$ analysis [19], namely $40 \%$ of the total background after all selection. For the $\tau \tau \mathrm{b} \bar{b}$ final state, the $\bar{t}, \mathrm{Z}+\mathrm{jets}, \mathrm{W}+\mathrm{jets}$ backgrounds

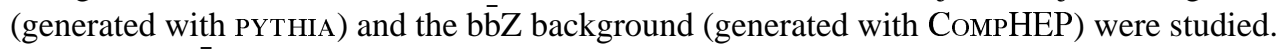

The $\gamma \gamma \mathrm{b} \bar{b}$ events were required to pass the Level-1 and HLT diphoton trigger. In the offline analysis two photon candidates with $\mathrm{E}_{\mathrm{T}}^{\gamma 1, \gamma 2}>40,25 \mathrm{GeV}$ were required to pass tracker cuts and calorimeter isolation cuts. Events with only two calorimeter jets of $\mathrm{E}_{\mathrm{T}}>30 \mathrm{GeV}$ and within $|\eta|<2.4$ were selected. At least one of these jets must be tagged as a b-jet. Finally, the 

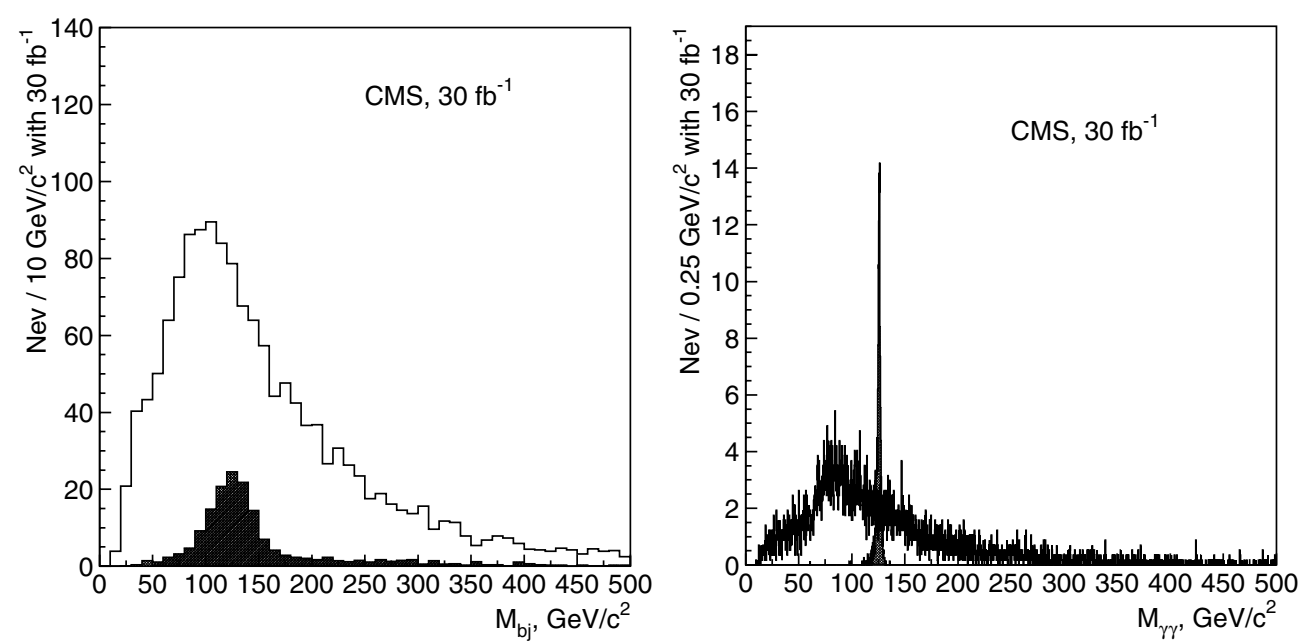

Figure 12.1. The dijet (left plot) and the diphoton (right plot) mass distribution for the background (open histogram) and the signal of $\phi \rightarrow \mathrm{hh} \rightarrow \gamma \gamma \mathrm{b} \bar{b}$ (full black histogram) after all selections except the mass window cuts with $30 \mathrm{fb}^{-1}$. The signal is shown for the maximal cross section times branching ratios point in $\left(\xi-\Lambda_{\phi}\right)$ plane.

diphoton mass, $\mathbf{M}_{\gamma \gamma}$, was required to be in a window of $\pm 2 \mathrm{GeV} / \mathrm{c}^{2}$, the dijet mass, $\mathbf{M}_{\mathrm{j} \mathrm{b}}$, in a window of $\pm 30 \mathrm{GeV} / \mathrm{c}$ and the diphoton-dijet mass, $M_{\gamma \gamma \mathrm{b} \bar{b}}$, in a window $\pm 50 \mathrm{GeV} / \mathrm{c}^{2}$ around the Higgs and Radion mass. Figure 12.1 shows the dijet (left plot) and the diphoton (right plot) mass distribution for the background (open histogram) and the signal of $\phi \rightarrow \mathrm{hh} \rightarrow \gamma \gamma \mathrm{b} \overline{\mathrm{b}}$ (full, black histogram) after all selections except the mass window cuts, and for $30 \mathrm{fb}^{-1}$. The signal is shown for the maximal cross section times branching ratios point in $\left(\xi-\Lambda_{\phi}\right)$ plane. Figure 12.2 (left plot) shows the $\mathbf{M}_{\gamma \gamma \text { bj }}$ distribution for the background (dashed histogram) and for the signal of $\phi \rightarrow \mathrm{hh} \rightarrow \gamma \gamma \mathrm{b} \bar{b}$ plus background (solid histogram) after all selections, and for $30 \mathrm{fb}^{-1}$.

The $\tau \tau \mathrm{b} \bar{b}$ events were selected by the single electron and muon triggers and by the combined e-plus- $\tau$-jet and the $\mu$-plus- $\tau$-jet triggers. In the off-line analysis a lepton and $\tau$-jet identification was performed. The requirements on the jets were similar to the ones used in the $\gamma \gamma \mathrm{b} \bar{b}$ analysis. In addition a cut of the transverse mass of the lepton and missing transverse momentum, $\mathbf{M}_{\mathrm{T}}^{\ell v}<35 \mathrm{GeV} / \mathrm{c}^{2}$ was applied to suppress the $\overline{\mathrm{tt}}$ and $\mathrm{W}+\mathrm{jets}$ backgrounds. The di $\tau$-lepton mass was reconstructed using the missing transverse energy as described in Section 5.2.5. The significance of the discovery was calculated using expected number of the signal and background events after the mass window selections: $100<\mathrm{M}_{\mathrm{bj}}<150 \mathrm{GeV} / \mathrm{c}^{2}$, $100<\mathrm{M}_{\tau \tau}<160 \mathrm{GeV} / \mathrm{c}^{2}$ and $280<\mathrm{M}_{\tau \tau \mathrm{bj}}<330 \mathrm{GeV} / \mathrm{c}^{2}$. Figure 12.2 (right plot) shows the $\mathrm{M}_{\tau \tau \mathrm{bj}}$ distribution for the background (full, grey (yellow) histogram) and for the signal of $\phi \rightarrow \mathrm{hh} \rightarrow \tau \tau \mathrm{b} \overline{\mathrm{b}}$ plus background (points with error bars) after all selections, for $30 \mathrm{fb}^{-1}$. Fitted curves for the background and the signal plus background are superimposed.

The four b-jet final state yields the highest rate for the signal. The maximal cross section times branching ratio at $\Lambda_{\phi}=1 \mathrm{TeV} / \mathrm{c}^{2}$ is $10.3 \mathrm{pb}$, which results in about $3.1 \times 10^{5}$ signal events for $30 \mathrm{fb}^{-1}$. The effective triggering and selection in the off-line analysis of these events is, however a big challenge due to the huge multi-jet background rate. In fact the remaining background is a few orders of magnitude larger than the signal in the relevant mass range. Techniques can be envisaged to normalise the background directly from a signal-free region and predict the number of background events in the signal region. In order to make a $3 \sigma$ 

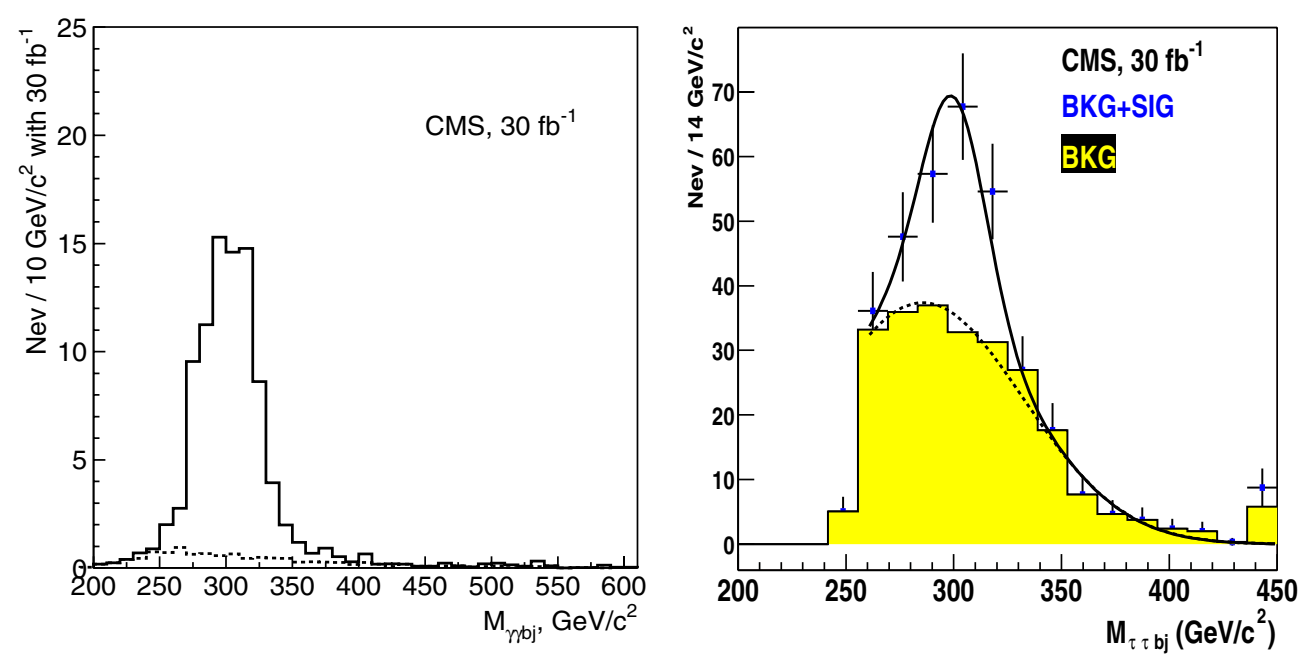

Figure 12.2. Left plot: the $\mathrm{M}_{\gamma \gamma \mathrm{bj}}$ distribution for the background (dashed histogram) and for the signal of $\phi \rightarrow \mathrm{hh} \rightarrow \gamma \gamma \mathrm{b} \overline{\mathrm{b}}$ plus background (solid histogram) after all selections for $30 \mathrm{fb}^{-1}$. Right plot: the $\mathbf{M}_{\tau \tau \text { bj }}$ distribution for the background (full grey (yellow) histogram) and for the signal of $\phi \rightarrow \mathrm{hh} \rightarrow \tau \tau \mathrm{b} \overline{\mathrm{b}}$ plus background (black points with the error bars) after all selections for $30 \mathrm{fb}^{-1}$. The fitted curves for the background and signal plus background are superimposed. On both plots the signal is shown for the maximal cross section times branching ratios point in $\left(\xi-\Lambda_{\phi}\right)$.

discovery, such extrapolation needs to be performed with a precision of about $0.1 \%$, making four b-jet channel essentially hopeless.

The background contribution to the $\gamma \gamma \mathrm{b} \bar{b}$ final state can be determined directly from the $\gamma \gamma$-plus-two-jets data obtained after all selections, except the final mass window cuts on the $\mathbf{M}_{\gamma \gamma}, \mathbf{M}_{\mathrm{j} \overline{\mathrm{b}}}$ and $\mathbf{M}_{\gamma \gamma \mathrm{b} \overline{\mathrm{b}}}$. The signal-to-background ratio is always less than $10 \%$ before the mass cuts are applied. The final cuts on the $\mathbf{M}_{\gamma \gamma}, \mathbf{M}_{\mathrm{j} \overline{\mathrm{b}}}$ and $\mathbf{M}_{\gamma \gamma \mathrm{b} \overline{\mathrm{b}}}$ introduce a systematic uncertainty on the number of the background events expected after these cuts. This uncertainty is determined by the following factors: the energy scale uncertainty for the photons and jets, and the theoretical uncertainty of the shape of the mass distributions due to the scale and PDF uncertainties. Figure 12.3 (left plot) shows the $5 \sigma$ discovery contours for the $\phi \rightarrow \mathrm{hh} \rightarrow \gamma \gamma \mathrm{b} \overline{\mathrm{b}}$ channel for $30 \mathrm{fb}^{-1}$. The solid (dashed) contour shows the discovery region without (with) the effects of the systematic uncertainties.

For the $\tau \tau \mathrm{b} \overline{\mathrm{b}}$ final state the background uncertainty due to the experimental selections was estimated to be between 5\% and 10\% [653]. Figure 12.3 (right plot) shows the $5 \sigma$ discovery contours for the $\phi \rightarrow \mathrm{hh} \rightarrow \tau \tau \mathrm{b} \overline{\mathrm{b}}$ channel for $30 \mathrm{fb}^{-1}$. The two contours corresponds to the variation of the background NLO cross sections due to the scale uncertainty. The 5\% experimental systematics on the background is taken into account.

\subsection{Doubly charged Higgs boson pair production in the Littlest Higgs model}

The main motivation of the Large Hadron Collider (LHC) experiments is to reveal the secrets of electroweak symmetry breaking. If the standard model (SM) Higgs boson will be discovered, the question arises what stabilises its mass against the Planck scale quadratically divergent radiative corrections. The canonical answer to this question is supersymmetry which implies very rich phenomenology of predicted sparticles in the future collider experiments. 

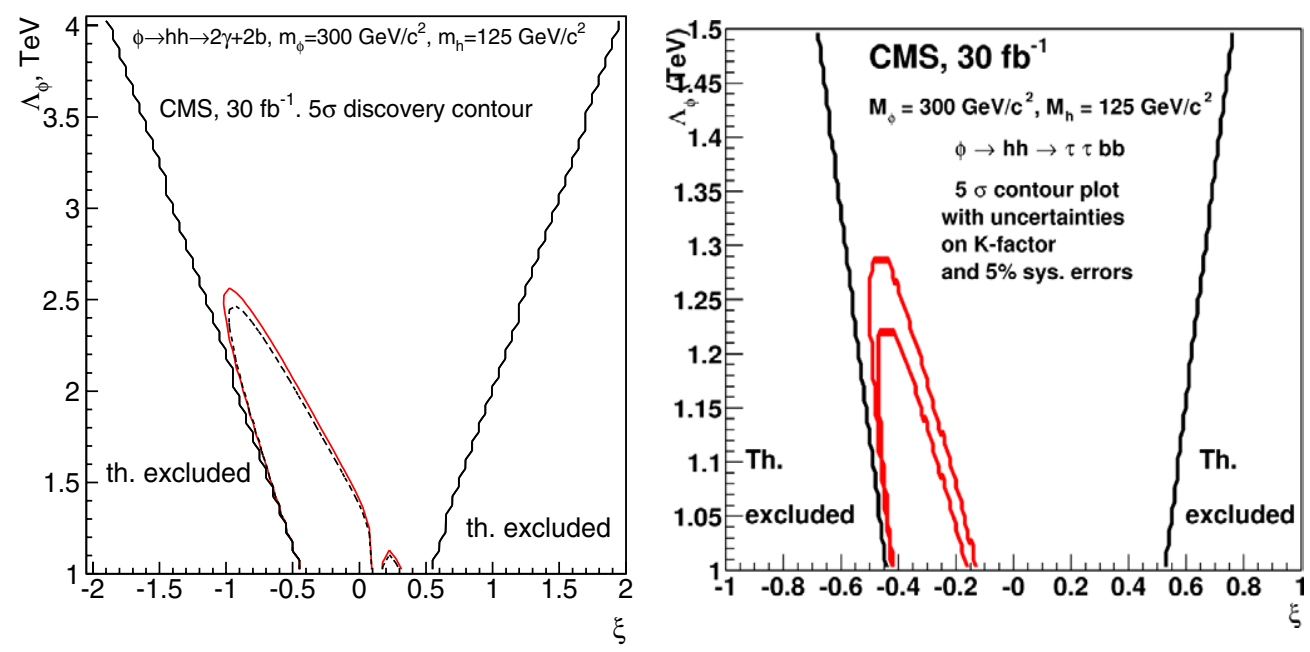

Figure 12.3. Left plot: the $5 \sigma$ discovery contours for the $\phi \rightarrow \mathrm{hh} \rightarrow \gamma \gamma \mathrm{b} \overline{\mathrm{b}}$ channel for $30 \mathrm{fb}^{-1}$. The solid (dashed) contour shows the discovery region without (with) the effects of the systematic uncertainties (find more explanations in the text). Right plot: the $5 \sigma$ discovery contours for the $\phi \rightarrow \mathrm{hh} \rightarrow \tau \tau \mathrm{b} \overline{\mathrm{b}}$ channel for $30 \mathrm{fb}^{-1}$. The two contours corresponds to the variation of the background NLO cross sections due to the scale uncertainty. The 5\% experimental systematics on the background is taken into account (see text).

More recently another possibility of formulating the physics of electroweak symmetry breaking, called the little Higgs, was proposed [654-656]. In those models the SM Higgs boson is a pseudo Goldstone mode of a broken global symmetry and remains light, much lighter than the other new modes of the model which have masses of order the symmetry breaking scale $\mathcal{O}(1) \mathrm{TeV}$. In order to cancel one-loop quadratic divergences to the SM Higgs mass a new set of heavy gauge bosons $W^{\prime}, Z^{\prime}$ with the SM quantum numbers identical to $W Z$, and a vector like heavy quark pair $T, \bar{T}$ with charge $2 / 3$ must be introduced. Notice that those fields are put in by hand in order to construct a model with the required properties. However, the minimal model based on the $S U(5) / S O(5)$ global symmetry, the so-called littlest Higgs model [657], has a firm prediction from the symmetry breaking pattern alone: the existence of another $\mathcal{O}(1) \mathrm{TeV}$ pseudo Goldstone boson $\Delta$ with the $S U(2)_{L} \times U(1)_{Y}$ quantum numbers $\Delta \sim(3,2)$.

Interestingly, the existence of triplet Higgs $\Delta$ might also be required to generate Majorana masses to the left-handed neutrinos [658]. Non-zero neutrino masses and mixing is presently the only experimentally verified signal of new physics beyond the SM. In the triplet neutrino mass mechanism [659] the neutrino mass matrix is generated via

$$
\left(m_{v}\right)_{i j}=\left(Y_{\Delta}\right)_{i j} v_{\Delta},
$$

where $\left(Y_{\Delta}\right)_{i j}$ are the Majorana Yukawa couplings of the triplet to the lepton generations $i, j=e, \mu, \tau$ which are described by the Lagrangian

$$
L=i \bar{\ell}_{L i}^{c} \tau_{2} Y_{\Delta}^{i j}(\tau \cdot \Delta) \ell_{L j}+\text { h.c., }
$$

and $v_{\Delta}$ is the effective vacuum expectation value of the neutral component of the triplet induced via the explicit coupling of $\Delta$ to the SM Higgs doublet $H$ as $\mu \Delta^{0} H^{0} H^{0}$. Here $\mu$ has a dimension of mass. In the concept of seesaw $\mu \sim M_{\Delta}$, and the smallness of neutrino masses is attributed to the very high scale of triplet mass $M_{\Delta}$ via the smallness of $v_{\Delta}=\mu v^{2} / M_{\Delta}^{2}$, where $v=174 \mathrm{GeV}$. 
However, in the littlest Higgs model the triplet mass scale is $\mathcal{O}(1) \mathrm{TeV}$ which alone cannot suppress $v_{\Delta}$. Therefore in this model $\mu \ll M_{\Delta}$, which can be achieved, for example, via shining from extra dimensions as shown in ref. $[660,661]$ or if the triplet is related to the Dark Energy of the Universe [662]. In that case $v_{\Delta} \sim \mathcal{O}(0.1) \mathrm{eV}$ while the Yukawa couplings $Y_{\Delta}$ can be large. For the normally hierarchical light neutrino masses neutrino data implies very small $\Delta$ decay branching fractions to electrons and $B R\left(\Delta^{++} \rightarrow \mu^{+} \mu^{+}\right) \approx B R\left(\Delta^{++} \rightarrow\right.$ $\left.\tau^{+} \tau^{+}\right) \approx B R\left(\Delta^{++} \rightarrow \mu^{+} \tau^{+}\right) \approx 1 / 3$. We remind also that $v_{\Delta}$ contributes to the SM oblique corrections, and the precision data fit $\hat{T}<2 \cdot 10^{-4}$ [663] sets an upper bound $v_{\Delta} \leqslant 1.2 \mathrm{GeV}$ on that parameter.

At LHC $\Delta^{++}$can be produced singly and in pairs. The cross section of the single $\Delta^{++}$ production via the $W W$ fusion process [664] $q q \rightarrow q^{\prime} q^{\prime} \Delta^{++}$scales as $\sim v_{\Delta}^{2}$. In the context of the littlest Higgs model this process, followed by the decays $\Delta^{++} \rightarrow W^{+} W^{+}$, was studied in ref. [91, 665, 666]. The detailed ATLAS simulation of this channel shows [666] that in order to observe $1 \mathrm{TeV} \Delta^{++}$, one must have $v_{\Delta}>29 \mathrm{GeV}$. This is in conflict with the precision physics bound $v_{\Delta} \leqslant 1.2 \mathrm{GeV}$ as well as with the neutrino data. Therefore the $W W$ fusion channel is not experimentally promising for the discovery of very heavy doubly charged Higgs.

On the other hand, the Drell-Yan pair production process [664, 667] $p p \rightarrow \Delta^{++} \Delta^{--}$is not suppressed by any small coupling and its cross section is known up to next to leading order [668] (possible additional contributions from new physics such as $Z^{\prime}$ are strongly suppressed for any practical purposes). Followed by the lepton number violating decays $\Delta^{ \pm \pm} \rightarrow \ell^{ \pm} \ell^{ \pm}$, this process allows to reconstruct $\Delta^{ \pm \pm}$invariant mass from the same charged leptons rendering the SM background to be very small in the signal region. If one also assumes that neutrino masses come from the triplet Higgs interactions, one fixes the $\Delta^{ \pm \pm}$leptonic branching ratios. This allows to test neutrino mass models at LHC.

\subsubsection{Search for the final state with four muons}

12.2.1.1. Introduction. The doubly charged Higgs bosons $\Delta^{ \pm \pm}$pair-produced via the DrellYan process is investigated assuming a branching ratio of $100 \%$ into muons. This provides an almost background free channel.

12.2.1.2. Event generation. The signal events are generated using PYTHIA, with doubly charged Higgs bosons pair-produced through the Drell-Yan process. The Higgs bosons are forced to decay into muons. Datasets are produced for several values of the doubly charged Higgs boson mass, ranging from 100 to $800 \mathrm{GeV} / \mathrm{c}^{2}$.

The leading order (LO) and the next-to-leading order (NLO) cross-sections [668] are shown for the signal as a function of the doubly charged Higgs boson mass in Fig. 12.4.

Important backgrounds for this channel with a four muon final state are:

- $t \bar{t} \rightarrow W^{+} W^{-} b \bar{b} \rightarrow 2 \mu+2 \mu$ (generated with PYTHIA);

- $Z b \bar{b} \rightarrow 2 \mu+2 \mu$ (generated with CoMPHEP);

- $Z Z \rightarrow 2 \mu+2 \mu$ (generated with CoMPHEP);

- $Z Z \rightarrow 2 \tau+2 \mu$ (generated with CoMPHEP).

The $Z Z$ production process includes $\gamma^{*}$. The contribution of background from $b \bar{b}$ production has also been investigated. The $b \bar{b}$ background is the QCD multi-jet background which yields the highest probability to fake events with multiple muons. It has been found that the $b \bar{b}$ background can be neglected after the online selection and a cut which requires four well-reconstructed muons with pseudorapidity $|\eta|<2.1$ and transverse momentum $p_{\mathrm{T}}>$ $8 \mathrm{GeV} / \mathrm{c}$. The $\mathrm{W}$ bosons in the $t \bar{t}$ data sample are forced to decay into electrons, muons and taus. The tau leptons are forced to decay into electrons and muons. The $\mathrm{Z}$ boson in the $Z \bar{b} b$ 


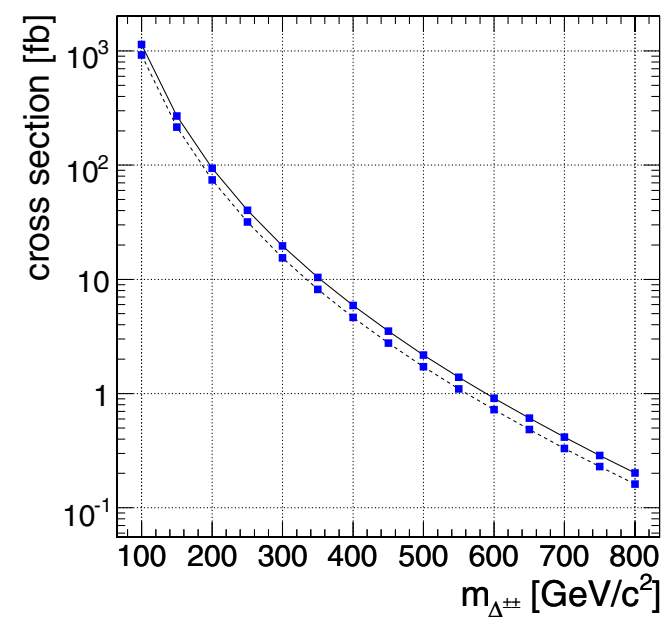

Figure 12.4. The leading order (LO) and the next-to-leading order (NLO) cross-section, for $\mathrm{H}^{++} \mathrm{H}^{--} \rightarrow 4 \mu$.

sample is generated with $m_{Z / \gamma^{*}}>5 \mathrm{GeV} / \mathrm{c}^{2}$ and is forced to decay into muons. The $\mathrm{Z}$ bosons in the $Z Z$ samples are forced to decay into muons and the taus in the $Z Z \rightarrow 2 \tau+2 \mu$ sample decay freely.

On all samples pre-selection cuts are applied at the generation level with the following requirements:

- Final state contains two positive and two negative muons.

- Transverse momentum $p_{\mathrm{T}}(\mu)>3 \mathrm{GeVc}$ and pseudorapidity $|\eta(\mu)|<2.4$ for all muons.

12.2.1.3. Event selection and reconstruction. The events are selected by dimuon trigger at Level 1 and the HLT. The $p_{\mathrm{T}}$ threshold for the dimuon HLT is $7 \mathrm{GeV} / \mathrm{c}$. The Level 1 and HLT efficiency for the signal is $>99 \%$ within uncertainties.

The muons are reconstructed by the Global Muon Reconstructor. At least 4 muons, with a $p_{\mathrm{T}}>8 \mathrm{GeV} / \mathrm{c}$ and $\eta \leqslant 2.1$, are required. The invariant mass of the doubly charged Higgs is reconstructed, by calculating the invariant mass of the two same charge muons with the highest $p_{\mathrm{T}}$, after all cuts.

An event, where two or three muons are generated in one collision, and one or two in another, has also to be considered as background to our four muon signal. To suppress this background a vertex cut has been applied. For each muon in an event the impact point is determined. The impact point is the point of closest approach of the extrapolated muon trajectory to the nominal interaction point. The longitudinal distances $\Delta z_{I P S}$ between the impact point states of all muons in one event are calculated. The biggest calculated $\Delta z_{I P S}$ is required to be smaller than $0.05 \mathrm{~cm}$. This is much smaller than the longitudinal size of the luminous region of the LHC beam of about $5 \mathrm{~cm}$. So this cut rejects events with muons from different collision vertices with a probability of roughly $99 \%$.

12.2.1.4. Results. Table 12.1 and Table 12.2 show the NLO production cross-section without any forced decay, the cross-section times branching ratio times pre-selection efficiency and the cross-section times branching ratio times efficiency after each stage of the online and offline event selection. Table 12.1 shows these values for each of the background samples. 
Table 12.1. The NLO cross sections $\sigma$ for background events with forced decay modes after each stage of the event selection. Errors are statistical only.

\begin{tabular}{lllll}
\hline & $t \bar{t}$ & $Z \bar{b} b$ & $Z Z \rightarrow 4 \mu$ & $Z Z \rightarrow 2 \mu 2 \tau$ \\
\hline Pre-selection [fb] & 232 & 289.8 & 87.4 & 1.63 \\
Level-1 Trigger [fb] & $232 \pm 1$ & $289 \pm 1$ & $87.3 \pm 0.3$ & $1.63 \pm 0.02$ \\
High Level Trigger [fb] & $149 \pm 1$ & $195 \pm 1$ & $69.7 \pm 0.3$ & $1.10 \pm 0.01$ \\
$4 \mu$ reconstructed $\left(p_{\mathrm{T}}>8 \mathrm{GeV} / \mathrm{c},|\eta|<2.1\right)[\mathrm{fb}]$ & $45.1 \pm 0.4$ & $25.1 \pm 0.3$ & $18.5 \pm 0.1$ & $0.25 \pm 0.01$ \\
Impact Point Cut [fb] & $22.8 \pm 0.3$ & $13.1 \pm 0.2$ & $16.9 \pm 0.1$ & $0.22 \pm 0.01$ \\
\hline
\end{tabular}

Table 12.2. Production cross sections (NLO) for signal events with $m_{H^{++}}=300,600,800 \mathrm{GeV} / \mathrm{c}^{2}$ and forced decay into four muons after each stage of the event selection. Errors are statistical only.

\begin{tabular}{llll}
\hline$\Delta^{ \pm \pm}$mass & $300 \mathrm{GeV} / \mathrm{c}^{2}$ & $600 \mathrm{GeV} / \mathrm{c}^{2}$ & $800 \mathrm{GeV} / \mathrm{c}^{2}$ \\
\hline Production cross section(NLO) [fb] & 19.6 & 0.909 & 0.201 \\
Pre-selection [fb] & $17.4 \pm 0.3$ & $0.85 \pm 0.02$ & $0.190 \pm 0.004$ \\
Level-1 Trigger [fb] & $17.3 \pm 0.3$ & $0.85 \pm 0.02$ & $0.190 \pm 0.004$ \\
High Level Trigger [fb] & $17.1 \pm 0.3$ & $0.83 \pm 0.02$ & $0.188 \pm 0.004$ \\
$4 \mu$ reconstructed $\left(p_{\mathrm{T}}>8 \mathrm{GeV} / \mathrm{c},|\eta|<2.1\right)[\mathrm{fd}]$ & $13.0 \pm 0.2$ & $0.70 \pm 0.02$ & $0.158 \pm 0.003$ \\
Impact Point Cut [fd] & $12.5 \pm 0.2$ & $0.67 \pm 0.02$ & $0.153 \pm 0.003$ \\
\hline
\end{tabular}

Table 12.2 show these values for signal samples with doubly charged Higgs masses 300, 600 and $800 \mathrm{GeV} / \mathrm{c}^{2}$.

Figure 12.5 shows the invariant mass spectrum of the reconstructed $\Delta^{ \pm \pm}$before and after the offline cuts, for $m\left(\Delta^{ \pm \pm}\right)=300 \mathrm{GeV} / \mathrm{c}^{2}$ and for $m\left(\Delta^{ \pm \pm}\right)=600 \mathrm{GeV} / \mathrm{c}^{2}$.

12.2.1.5. Statistical interpretation. To interpret the results, the $C L_{s}$ method [508] is applied, which is based on log-likelihood ratios, calculated for all bins of the invariant mass distribution. $C L_{s}$ is defined as ratio of the confidence levels for the signal and background hypotheses $C L_{s}=C L_{s+b} / C L_{b} . C L_{s}$ can be understood as the probability of excluding an existing signal. The $1-C L_{b}$ can be understood as the probability for the background distribution to fake a signal. For high doubly charged Higgs boson masses the amount of simulated background events goes to zero. Nevertheless, zero simulated background events do not necessarily mean zero background events in reality. To estimate the amount of background in this region, empty bins are filled for each background with upper limits to Poisson statistic. Zero background events are compatible with maximal three generated events. Therefore empty bins get filled for each background with three events times the scale factor for an integrated luminosity of $10 \mathrm{fb}^{-1}$. The left plot in Fig. 12.6 shows the $1-C L_{b}$ values for different doubly charged Higgs boson masses. For a doubly charged Higgs Boson mass smaller than $650 \mathrm{GeV} / \mathrm{c}^{2}$ the signal plus background expectation will exceed the background only expectation by more than $5 \sigma$. To claim a discovery, at least three signal events need to be detected. For a mass of $650 \mathrm{GeV} / \mathrm{c}^{2}$ four detectable events remain after all cuts. The right plot in figure 12.6 shows the $C L_{s}$ values for different doubly charged Higgs boson masses. If no signal can be detected for an integrated luminosity of $10 \mathrm{fb}^{-1}$ the existence of a doubly charged Higgs Boson in this decay channel can be excluded with $95 \%$ confidence up to a mass of $760 \mathrm{GeV} / \mathrm{c}^{2}$. The \pm 1 and \pm 2 -sigma bands in figure 12.6 are only for statistical errors.

12.2.1.6. Systematical uncertainties. The uncertainties on the exclusion limit resulting from systematical errors have yet to be studied in detail, once the detector is running. 

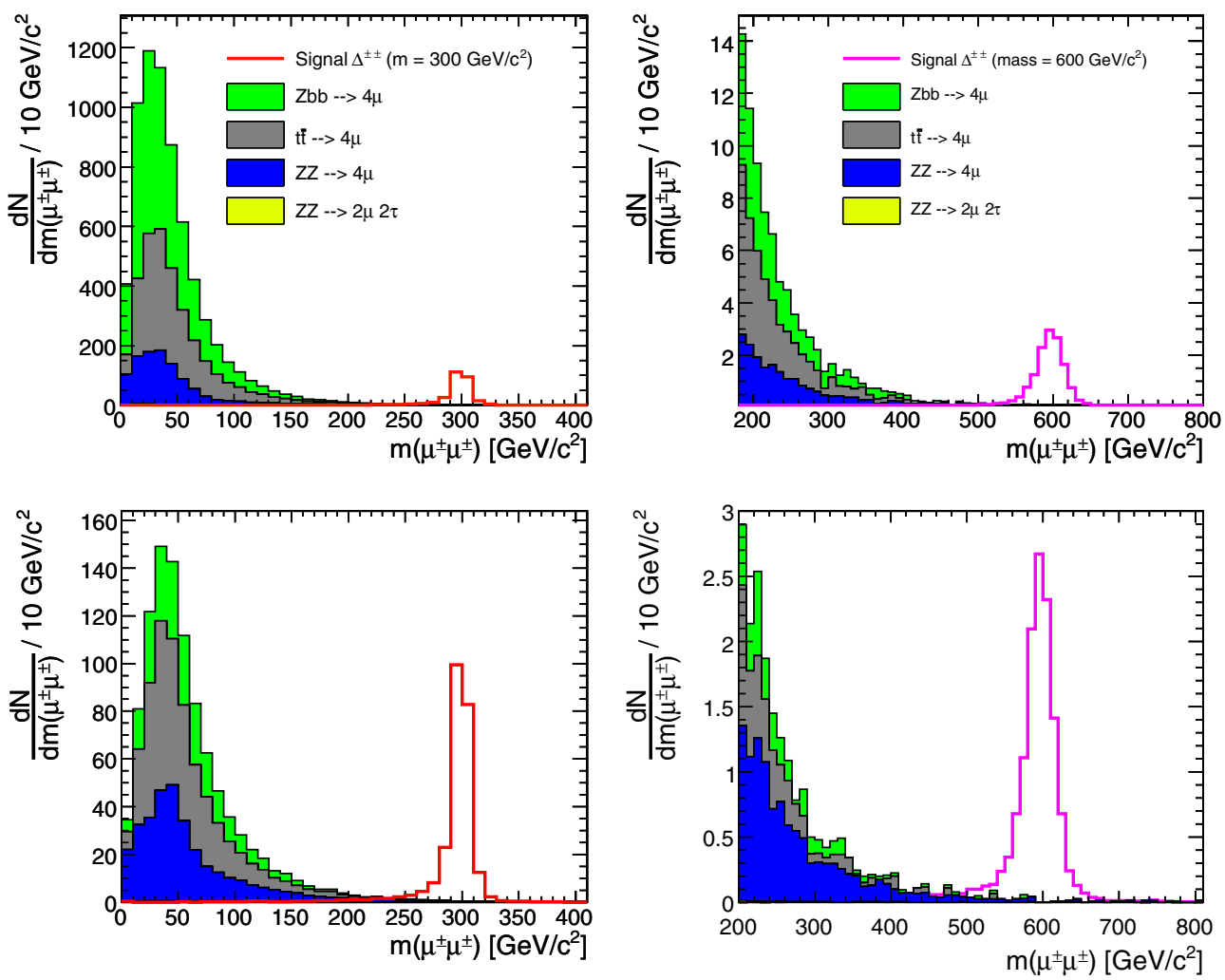

Figure 12.5. The reconstructed $\Delta^{ \pm \pm}$invariant mass after pre-selection and trigger selection (top) and after offline cuts (bottom).
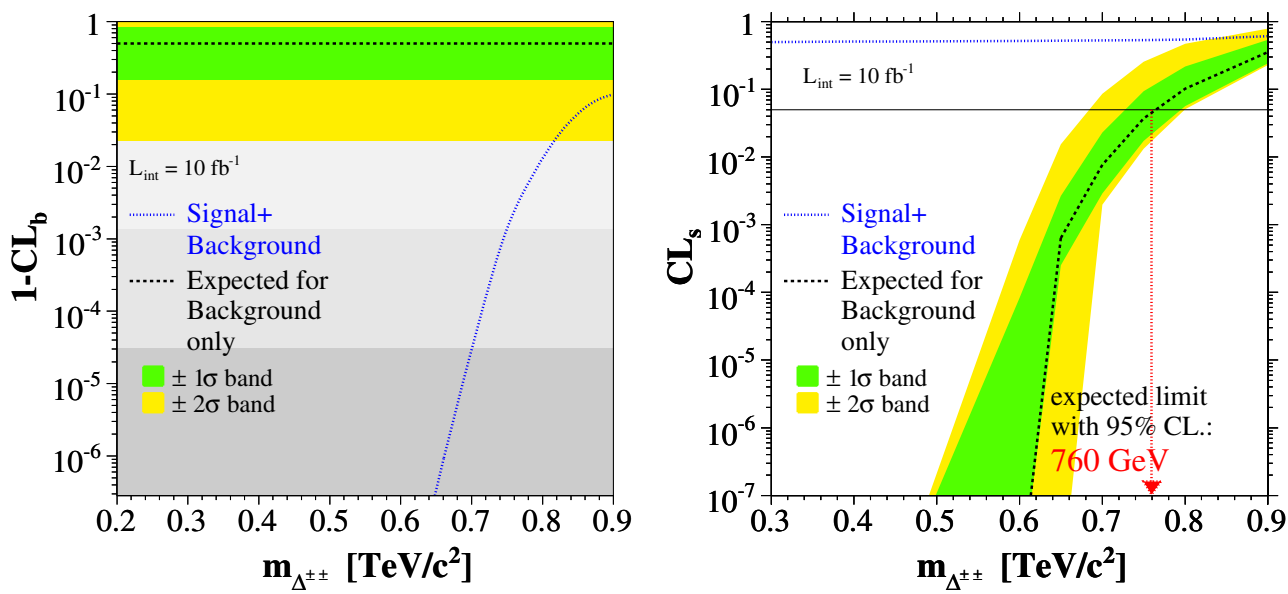

Figure 12.6. $1-C L_{b}$ and $C L_{s}$ as defined in the Log Likelihood Ratio Method after all selection cuts for an integrated luminosity of $10 \mathrm{fb}^{-1}$.

The considered backgrounds are also backgrounds to the Standard Model $H \rightarrow Z Z \rightarrow$ $4 \mu$ process. As this process is one of the benchmark processes of the future CMS detector, this backgrounds are studied in detail. The obtained total uncertainty on the background cross 
Table 12.3. The NLO background processes cross sections used (in fb)

\begin{tabular}{lllll}
\hline background & $t \bar{t} \rightarrow 41$ & $\mathrm{Z} b \bar{b}$ & $\mathrm{ZZ}$ & $t \bar{t} \mathrm{Z}$ \\
Cross section times BR & $88.4 \cdot 10^{3}$ & $52.4 \cdot 10^{3}$ & 229.5 & 650 \\
\hline
\end{tabular}

section is $1 \%$ to $6 \%$. The uncertainty on signal cross section is $10 \%$ to $15 \%$. The uncertainty on the luminosity $\mathcal{L}$ is $\sim 5 \%$ for an integrated luminosity of $10 \mathrm{fb}^{-1}$.

Using a background cross section uncertainty of $6 \%$, a signal cross section uncertainty of $10 \%$ and a luminosity uncertainty of $5 \%$ the approximated uncertainties on the exclusion mass limit and on the discovery mass limit are:

$$
\begin{aligned}
& \text { Exclusion Limit }=\left(760_{-2}^{+0.5}(\mathrm{bkg}) \pm 10(\text { signal }) \pm 4(\text { lumi })\right) \mathrm{GeV} / \mathrm{c}^{2} \\
& \text { Discovery Limit }=\left(650_{-0.3}^{+0.4}(\mathrm{bkg})_{-0.4}^{+3}(\text { signal }) \pm 0.2(\text { lumi })\right) \mathrm{GeV} / \mathrm{c}^{2} .
\end{aligned}
$$

\subsubsection{Search for the final states with $\tau$ leptons}

12.2.2.1. Introduction. In this section, we discuss the doubly charged Higgs boson pairproduction via a Drell-Yan process and investigate decays which involve taus and muons. The branching ratios are assumed to be $1 / 3$ for the following three channels: $\Delta^{ \pm \pm} \rightarrow 2 \mu^{ \pm}, \Delta^{ \pm \pm} \rightarrow$ $\mu^{ \pm} \tau^{ \pm}$and $\Delta^{ \pm \pm} \rightarrow 2 \tau^{ \pm}$. The reasoning comes from recent neutrino mixing measurements. As the neutrino mixing matrix and doubly charged Higgs boson decays are directly related then the appropriate branchings can be determined.

12.2.2.2. Event generation. The doubly charged Higgs boson pair-production via DrellYan process is generated using PYTHIA. Datasets are produced with Higgs boson mass from $200 \mathrm{GeV} / \mathrm{c}^{2}$ to $600 \mathrm{GeV} / \mathrm{c}^{2}$. The taus from Higgs boson decays can decay both leptonically and hadronically while in analysis we only consider hadronic decays.

The backgrounds which were considered for this analysis are as follows:

- $t \bar{t} \rightarrow \mathrm{W}^{+} \mathrm{W} b \bar{b}$ generated by PYTHIA, CompHEP, ALPGEN, TopReX and MAdGRAPH with $\mathrm{W}$ boson decay $\mathrm{W} \rightarrow \ell v(\ell=\mathrm{e}, \mu, \tau)$ forced.

- $t \bar{t} \mathrm{Z} \rightarrow W^{+} W^{-} \mathrm{Z} b \bar{b}$ generated with CompHEP. The $\mathrm{W}$ and $\mathrm{Z}$ bosons are allowed to decay arbitrarily.

- $\mathrm{Zb} \bar{b}$ where the $\mathrm{Z}$ boson decays to muons and $\tau$ leptons, generated with CoMPHEP.

- ZZ generated with PYTHIA, where the Z bosons are forced to decay leptonically $(\mathrm{e}, \mu, \tau)$. The contribution of $\gamma^{*}$ is included with $\mathrm{m}_{\gamma^{*}}>12 \mathrm{GeV} / \mathrm{c}^{2}$.

The next-to-leading order (NLO) cross sections times branching ratios used for the backgrounds can be found in Table 12.3. The Monte Carlo statistics of the generated background exceed $30 \mathrm{fb}^{-1}$ except $\mathrm{Z} b \bar{b}$ background, where it is $8 \mathrm{fb}^{-1}$. Therefore the results will be presented for an integrated luminosity of $10 \mathrm{fb}^{-1}$.

12.2.2.3. Event selection and reconstruction. The events are triggered by the single muon trigger at Level 1 and HLT. After HLT the event is only used if it is possible to reconstruct the event primary vertex. If the primary vertex fails to be reconstructed the event is rejected.

The muons are reconstructed using Global Muon Reconstructor. The $\tau$ leptons are reconstructed using $\tau$-jet candidates and missing transverse energy after selection cuts. The doubly charged Higgs boson invariant mass is reconstructed from the same charge lepton pairs after all selection cuts. 
The selection cuts used on muons are:

- The transverse momentum must be higher than $50 \mathrm{GeV} / \mathrm{c}$. For background events $80 \%$ of muons have $\mathrm{p}_{\mathrm{T}}$ less than $50 \mathrm{GeV} / \mathrm{c}$ while for the signal with Higgs boson mass $200 \mathrm{GeV} / \mathrm{c}^{2}$ it is $27 \%$ and for higher masses it reduces to around $10 \%$.

- The distance to primary vertex in z-direction must not exceed $0.03 \mathrm{~cm}$. It does not cut away any muons from the signal events but limits analysis to leptons coming from the same primary vertex.

The selection cuts used on $\tau$ jets are:

- For $\tau$ jets we consider $\tau$ decays which involve 1 or 3 charged tracks. We use $\tau$-jet candidates which passed the $\tau$-jet filtering algorithms described in [280]. Two isolation criteria are used. Either one or three charged tracks in the signal cone and no charged tracks in the isolation cone or two tracks in signal cone and exactly one charged track in the isolation cone.

- The maximal distance to the primary vertex in the z-direction of any charged track in the $\tau$ jet must not exceed $0.2 \mathrm{~cm}$.

- The transverse energy of the hottest HCAL tower of the $\tau$ jet must be higher than $2 \mathrm{GeV}$. This cut eliminates $86 \%$ of all electrons taken as $\tau$ candidates and only removes $7.5 \%$ of real $\tau$ jets.

- The transverse energy of the $\tau$ jet candidate must exceed $50 \mathrm{GeV}$. It has been chosen to be the same as the cut used on muons.

- No muon track should be in a cone with $\Delta R=0.3$ constructed around the $\tau$-jet candidate. If there is, then the candidate is dropped. This eliminates false $\tau$-jet candidates which are generated when a charged muon track passes the same region as photons or hadrons. With this cut only a few real $\tau$ jets are discarded however most of the false $\tau$ jets coming from this misidentification are rejected.

Missing transverse energy $\left(E_{\mathrm{T}}^{\text {miss }}\right)$ is reconstructed using calorimeter Type $1 E_{\mathrm{T}}^{\text {miss }}\left(E_{\mathrm{T}}^{\text {miss }}\right.$ with the jet energy corrections) and $\mathrm{p}_{\mathrm{T}}$ of muons.

Only events with at least four objects, muons or $\tau$ jets, are accepted. The possible final states are:

- $\Delta^{++} \Delta^{--} \rightarrow 4 \mu$ : this channel is investigated in the previous subsection.

- $\Delta^{++} \Delta^{--} \rightarrow 3 \mu 1 \tau$ : this channel is easily reconstructible as there is only one neutrino and it goes the direction of the $\tau$ jet.

- $\Delta^{++} \Delta^{--} \rightarrow 2 \mu 2 \tau$ : this channel can also be reconstructed using the assumption that the neutrinos go in the same directions as the $\tau$ jets.

- $\Delta^{++} \Delta^{--} \rightarrow 1 \mu 3 \tau$ : this channel can be reconstructed only with very good $E_{\mathrm{T}}^{\text {miss }}$ resolution as it requires an additional assumption that the masses of the two reconstructed Higgs bosons are the same. However the reconstruction is very sensitive to $E_{\mathrm{T}}^{\text {miss }}$ accuracy and often the event has to be dropped due to negative $\tau$-lepton energies.

- $\Delta^{++} \Delta^{--} \rightarrow 4 \tau$ : this channel can not be reconstructed (and triggered by the single muon trigger).

Once the event leptons are reconstructed, some additional selections are performed:

- $\mathrm{Z}$ boson veto: if the odd sign pairing gives an invariant mass of $91 \pm 5 \mathrm{GeV} / \mathrm{c}^{2}$ then these leptons are removed from further use.

- Same charge lepton pairs are reconstructed and only those reconstructed Higgs candidate pairs whose invariant mass difference is within $20 \%$ of each other are considered.

The reconstructed mass of doubly charged Higgs boson is shown on Figure 12.7 for the Higgs boson masses 200 and $500 \mathrm{GeV} / \mathrm{c}^{2}$. 

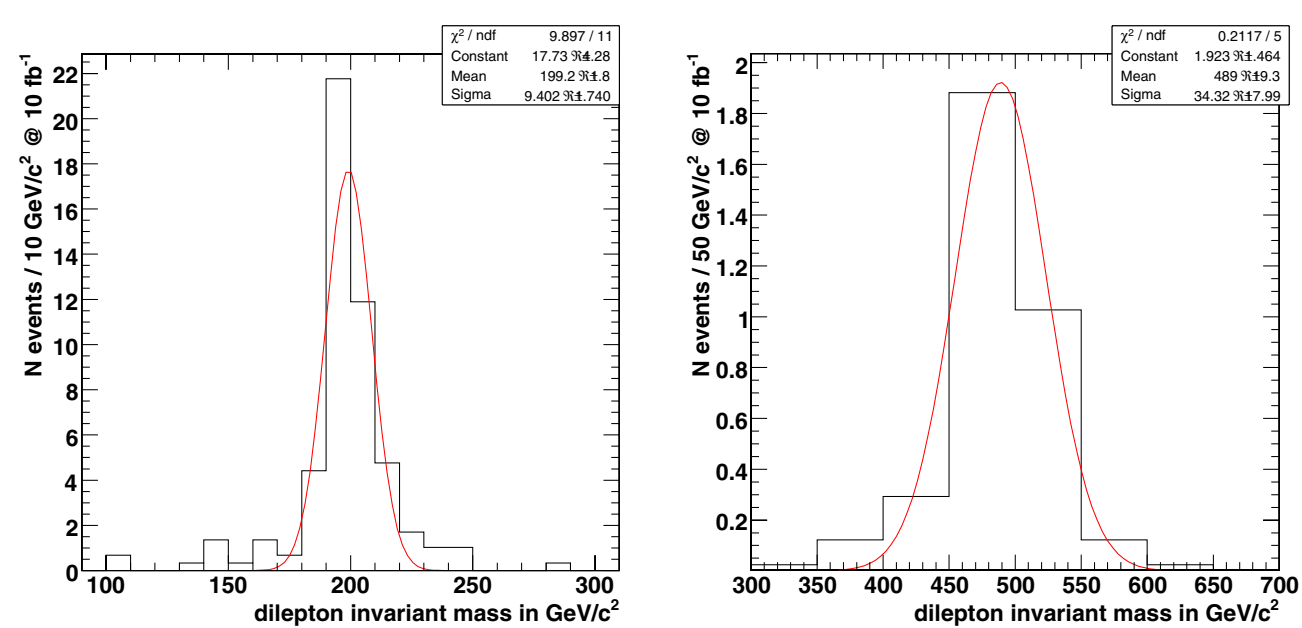

Figure 12.7. The reconstructed invariant mass for $\mathrm{M}\left(\Delta^{ \pm \pm}\right)=200 \mathrm{GeV} / \mathrm{c}^{2}$ and $500 \mathrm{GeV} / \mathrm{c}^{2}$.

Table 12.4. The signal selection efficiencies for different $\Delta^{ \pm \pm}$masses. Total efficiency is the product of the single efficiencies.

\begin{tabular}{lrllll}
\hline$m_{\Delta}^{ \pm \pm}\left(\mathrm{GeV} / \mathrm{c}^{2}\right)$ & \multicolumn{1}{l}{200} & \multicolumn{1}{l}{300} & \multicolumn{1}{l}{400} & \multicolumn{1}{l}{500} & \multicolumn{1}{l}{600} \\
\hline Level 1 and HLT & $83.7 \%$ & $86.0 \%$ & $86.7 \%$ & $85.8 \%$ & $88.3 \%$ \\
Primary vertex & $96.9 \%$ & $98.5 \%$ & $97.0 \%$ & $97.5 \%$ & $98.0 \%$ \\
4 leptons in final state & $10.1 \%$ & $17.2 \%$ & $23.6 \%$ & $24.7 \%$ & $26.7 \%$ \\
two pairs and at least one $\tau$ & $44.9 \%$ & $46.1 \%$ & $41.7 \%$ & $53.2 \%$ & $52.9 \%$ \\
Mass difference & $62.5 \%$ & $77.2 \%$ & $80.4 \%$ & $74.3 \%$ & $63.6 \%$ \\
Total signal efficiency & $2.3 \%$ & $5.1 \%$ & $6.6 \%$ & $8.1 \%$ & $7.7 \%$ \\
\hline
\end{tabular}

12.2.2.4. Selection efficiencies. The upper limit of the signal selection efficiency is given by the fraction of events with $3 \mu 1 \tau, 2 \mu 2 \tau, 1 \mu 3 \tau$ ( $\tau \rightarrow$ hadrons) topology relative to all possible final states with muons and $\tau$ leptons from decays of two Higgs bosons. Assuming the above mentioned branching ratios the upper limit is $\simeq 35 \%$. The fraction of every selected topology is given below:

- $\Delta^{++} \Delta^{--} \rightarrow 3 \mu 1 \tau=2 / 9$ events $\times 0.65=14.4 \%$

- $\Delta^{++} \Delta^{--} \rightarrow 2 \mu 2 \tau=3 / 9$ events $\times 0.65^{2}=14.1 \%$

- $\Delta^{++} \Delta^{--} \rightarrow 1 \mu 3 \tau=2 / 9$ events $\times 0.65^{3}=6.1 \%$.

where 0.65 is the branching ratio of $\tau \rightarrow$ hadrons decays. Table 12.4 summarises the efficiencies of each selection (relative to the previous one) for the signal of different $\Delta^{ \pm \pm}$ masses. The lepton selection efficiency and purity is shown in Table 12.5. Background efficiencies are shown in Table 12.6.

12.2.2.5. Systematic errors. At the integrated luminosity of $10 \mathrm{fb}^{-1}$ the cuts implemented above result in an almost background free signal. For datasets with Monte Carlo statistics above $30 \mathrm{fb}^{-1}$ giving zero Monte Carlo events after all selections $\left(t \bar{t}, Z Z^{*}\right)$ we assume the background to be zero. For $t \bar{t} Z$ background where is one Monte Carlo event passing all cuts, which corresponds to 0.05 expected events when scaled with cross section and luminosity. 
Table 12.5. Single muon and $\tau$ selection efficiencies and purity.

\begin{tabular}{lrrrrr}
\hline$m_{\Delta}^{ \pm \pm}\left(\mathrm{GeV} / \mathrm{c}^{2}\right)$ & \multicolumn{1}{c}{200} & \multicolumn{1}{c}{300} & \multicolumn{1}{c}{400} & \multicolumn{1}{c}{500} & \multicolumn{1}{c}{600} \\
\hline Single $\mu$ selection efficiency & $70.7 \%$ & $82.0 \%$ & $86.1 \%$ & $87.2 \%$ & $89.2 \%$ \\
1 - purity of accepted muons: & $0.1 \%$ & $0.4 \%$ & $0.8 \%$ & $0.7 \%$ & $1.0 \%$ \\
Single $\tau$ selection efficiency & $36.6 \%$ & $42.3 \%$ & $50.6 \%$ & $53.3 \%$ & $53.3 \%$ \\
1 - purity of accepted $\tau$ jets: & $2.2 \%$ & $2.2 \%$ & $4.2 \%$ & $3.6 \%$ & $3.2 \%$ \\
\hline
\end{tabular}

Table 12.6. Selection efficiencies for background. Total efficiency is the product of the single efficiencies.

\begin{tabular}{lllll}
\hline Process & $t \bar{t}$ & $t \bar{t} \mathrm{Z}$ & $\mathrm{ZZ}$ & $\mathrm{Zbb}$ \\
\hline Level 1 and HLT trigger & $40.7 \%$ & $20.3 \%$ & $40.0 \%$ & $42.1 \%$ \\
Primary vertex & $99.3 \%$ & $99.8 \%$ & $96.7 \%$ & $98.2 \%$ \\
4 leptons in final state & $0.0015 \%$ & $0.04 \%$ & $3.0 \%$ & $0.0005 \%$ \\
two pairs and at least one $\tau$ & - & $0.1 \%$ & - & - \\
Mass difference & - & $100 \%$ & - & - \\
Total signal efficiency & - & $0.0008 \%$ & - & - \\
\hline
\end{tabular}

For $\mathrm{Z} b \bar{b}$ background where the Monte Carlo statistics corresponds to $8 \mathrm{fb}^{-1}$ no events passed all cuts. The analysis was repeated with $p_{\mathrm{T}}$ cut on muon $(\tau$ jet) of $40 \mathrm{GeV} / \mathrm{c}, 30 \mathrm{GeV} / \mathrm{c}$ and $20 \mathrm{GeV} / \mathrm{c}$, again with no events passing the cuts, which confirms the assumption that leptons coming from $\mathrm{Z} b \bar{b}$ are too soft to produce a background. Considering the smallness of all backgrounds we assume no background at $10 \mathrm{fb}^{-1}$ for the following analysis.

The systematic uncertainties used for the signal are the following:

- muon misidentification $(\Delta \mu): 1 \%$ per muon;

- muon isolation $\left(\Delta \mu_{i s o l}\right): 2 \%$ per event;

- $\tau$ jets identification $(\Delta \tau): 9 \%$ per $\tau$ jet;

- luminosity $(\Delta \mathcal{L}): 5 \%$;

- PDF and scale $(\Delta \sigma) 10 \%$ (theoretical uncertainty, it is not used for the signal cross section measurement with no background).

As the events are a mixture of different decay modes the total selection efficiency uncertainty $\left(\Delta \varepsilon_{S}\right)$ is calculated per decay channel and then added together with the corresponding weights:

$$
\begin{aligned}
& \Delta 3 \mu 1 \tau=\sqrt{3 \Delta \mu^{2}+\Delta \tau^{2}}=8.2 \%, \\
& \Delta 2 \mu 2 \tau=\sqrt{2 \Delta \mu^{2}+2 \Delta \tau^{2}}=11.4 \%, \\
& \Delta 1 \mu 3 \tau=\sqrt{\Delta \mu^{2}+3 \Delta \tau^{2}}=13.9 \%,
\end{aligned}
$$

giving

$$
\Delta \varepsilon_{S}=\frac{144 \Delta 3 \mu 1 \tau+141 \Delta 2 \mu 2 \tau+61 \Delta 1 \mu 3 \tau}{346}=10.5 \%
$$

The total systematic error for cross section measurement is then

$$
\frac{\Delta \sigma}{\sigma}=\sqrt{\Delta \mu_{i s o l}^{2}+\Delta \mathcal{L}^{2}+\Delta \varepsilon_{S}^{2}}=13 \% .
$$


Table12.7. Expected number of events, NLO cross section with expected statistical and systematic uncertainty of the cross section measurement at $10 \mathrm{fb}^{-1}$, and integrated luminosity needed for exclusion at $95 \% \mathrm{CL}$.

\begin{tabular}{lcccc}
\hline$m_{\Delta}^{ \pm \pm}(\mathrm{GeV})$ & 200 & 300 & 400 & 500 \\
\hline $\mathrm{N}_{\mathrm{ev}}$ expected at $10 \mathrm{fb}^{-1}$ & 26 & 10 & 4 & 2 \\
$\sigma_{\mathrm{NLO}} \pm$ stat \pm syst $(\mathrm{fb})$ & $93.9_{-17.5}^{+19.3} \pm 12.2$ & $19.6_{-5.6}^{+6.6} \pm 2.5$ & $5.9_{-2.5}^{+3.4} \pm 0.8$ & $2.2_{-1.3}^{+1.9} \pm 0.3$ \\
Luminosity for & 1.3 & 3.0 & 7.7 & 16.8 \\
$95 \%$ CL exclusion, $\mathrm{fb}^{-1}$ & & & & \\
\hline
\end{tabular}

The statistical errors were evaluated constructing the shortest Bayesian confidence interval for the confidence level of $67 \%$ [669].

12.2.2.6. Results. The expected number of events at $10 \mathrm{fb}^{-1}$ and the NLO cross section with expected statistical and systematic uncertainty of the cross section measurement are given in Table 12.7. Table 12.7 shows also the integrated luminosity needed for exclusion at 95\% CL. 


\section{Chapter 13. Supersymmetry}

\subsection{Introduction}

This chapter presents the results of analyses by which evidence for supersymmetry could be obtained in CMS during the "low luminosity" period of the LHC. After a brief reminder of the main phenomenological features of SUSY in Section 13.2, Section 13.3 is devoted to the outline of the scope of present searches. The emphasis was not on a complete study of a specific point in the parameter space, but rather on covering all relevant signatures by which SUSY might be discovered. For this purpose, a set of test points have been defined, for which a full simulation of the CMS detector was performed, to serve as basis for the analyses. An algorithm allowing the separation of the sparticle decay chains, used in several analyses, is presented in Section 13.4. Sections 13.5 to 13.12 summarise the searches for SUSY and the reach as a function of luminosity, demonstrating that low mass supersymmetry can be discovered at the LHC with fairly low integrated luminosity for all these signatures in inclusive searches and show the projected reach at the end of the low luminosity run. They are followed by some exclusive studies, mass reconstruction in ditau final states (Section 13.13), tri-lepton final states from direct chargino/neutralino production (Section 13.14) and slepton pair production (Section 13.15). A possible violation of lepton number in $\tilde{\chi}_{2}^{0}$ decay is studied in Section 13.16. Section 13.18 contains some considerations on the robustness of the considered signatures in scenarios beyond mSUGRA, like for non-universal Higgs masses, and shows that the same signatures would still allow the discovery of supersymmetry. The chapter ends with our conclusion on the CMS reach.

\subsection{Summary of supersymmetry}

\subsubsection{The MSSM}

The Minimal Supersymmetry Model (MSSM) contains the minimal extension of the Standard Model (SM) particle content. Its gauge sector is fully determined by Supersymmetry. But the unknown mechanism for breaking Supersymmetry introduces a large number of free parameters [670] and makes this general model intractable. Therefore, several more constrained models have appeared in the literature. Below, we will focus on a version derived from Supergravity with minimal superpotential and Kähler potential, called mSUGRA, which guarantees universality of gaugino and scalar masses and of trilinear couplings at a high scale. Other SUSY breaking models, like Gauge Mediated Supersymmetry Breaking (GMSB) or Anomaly Mediated Supersymmetry Breaking (AMSB) have not been included here. R-parity breaking in SUSY is also not considered.

An earlier summary of the potentialities of the CMS experiment at LHC for the discovery of Supersymmetry has been published in 1998 [671]. The potential of the ATLAS experiment for the discovery of supersymmetry was analysed in [491].

\subsection{2. mSUGRA parameters and spectrum}

The mSUGRA model of supersymmetry is determined by 5 free parameters defined at the Grand Unification (GUT) scale. If it is assumed that the spontaneous gauge symmetry breaking is induced by radiative corrections, the absolute value of $\mu$ is determined from the $Z^{0}$ mass. The free parameters are then:

$$
m_{0}, m_{1 / 2}, A_{0}, \tan \beta, \operatorname{sign}(\mu) .
$$


They are run down to the electroweak scale by Renormalisation Group Equations (RGE) from which the sparticle spectrum, decay branching ratios and production cross sections can be derived.

The gaugino mass parameters $M_{a}$ at the electroweak scale are approximately:

$$
\begin{aligned}
& M_{3} \equiv M_{\tilde{g}} \simeq 2.7 m_{1 / 2} \\
& M_{2}\left(M_{Z}\right) \simeq 0.8 m_{1 / 2} \\
& M_{1}\left(M_{Z}\right) \simeq 0.4 m_{1 / 2}
\end{aligned}
$$

The parameter $M_{3}$ determines the gluino mass (after QCD corrections). The masses of neutralinos $\tilde{\chi}_{i}^{0}(i=1-4)$ and charginos $\tilde{\chi}_{i}^{ \pm}(i=1,2)$ are obtained after diagonalising their mass matrices which are a function of $M_{1}, M_{2}$ and $\mu$. In the mSUGRA framework, the lightest chargino and the two lightest neutralinos are dominantly gaugino-like with masses close to $M_{1}$ and $M_{2}$.

The sfermions of the first two generations have masses given approximately by:

$$
\begin{aligned}
& m_{\tilde{u}_{L}}^{2} \simeq m_{0}^{2}+5.0 m_{1 / 2}^{2}+0.35 \cos 2 \beta M_{Z}^{2} \\
& m_{\tilde{d}_{L}}^{2} \simeq m_{0}^{2}+5.0 m_{1 / 2}^{2}-0.42 \cos 2 \beta M_{Z}^{2} \\
& m_{\tilde{u}_{R}}^{2} \simeq m_{0}^{2}+4.5 m_{1 / 2}^{2}+0.15 \cos 2 \beta M_{Z}^{2} \\
& m_{\tilde{d}_{R}}^{2} \simeq m_{0}^{2}+4.4 m_{1 / 2}^{2}-0.07 \cos 2 \beta M_{Z}^{2} \\
& m_{\tilde{e}_{L}}^{2} \simeq m_{0}^{2}+0.49 m_{1 / 2}^{2}-0.27 \cos 2 \beta M_{Z}^{2} \\
& m_{\tilde{v}_{v}}^{2} \simeq m_{0}^{2}+0.49 m_{1 / 2}^{2}+0.50 \cos 2 \beta M_{Z}^{2} \\
& m_{\tilde{e}_{R}}^{2} \simeq m_{0}^{2}+0.15 m_{1 / 2}^{2}-0.23 \cos 2 \beta M_{Z}^{2}
\end{aligned}
$$

By comparing with the gluino mass, these relations show that the latter cannot be much larger than the squark mass:

$$
M_{\tilde{g}} \lesssim 1.2 m_{\tilde{q}}
$$

This relation (obtained for $m_{0}=0$ ) is not restricted to the mSUGRA case, as it depends primarily on the $\alpha_{S}$ contributions to the running down of the mass parameters from the GUT scale.

The masses of the third family scalars are more complicated as the contributions from Yukawa couplings can no longer be neglected and non-negligible off-diagonal elements between left and right states appear (they are proportional to the fermion masses).

\subsection{Scope of present searches}

\subsubsection{Sparticle production and cascade decays}

If we assume that Supersymmetry is discovered at the LHC, most likely from fully inclusive studies based on large missing energy and jets, it will be very important to investigate all the typical SUSY signatures to help pin down the underlying model.

If the squarks and/or gluinos are kinematically accessible at the LHC, they are expected to have large production rates. The cross sections for the production of a squark (excluding stop) or a gluino at the LHC are displayed in Fig. 13.1. The nearly diagonal lines delimit three regions:

- Region 1: in this region, the gluinos are heavier than any of the squarks. The decay chains of the produced sparticles are expected to be

$$
\tilde{g} \rightarrow \tilde{q} \bar{q}, \tilde{q} \rightarrow q \chi .
$$




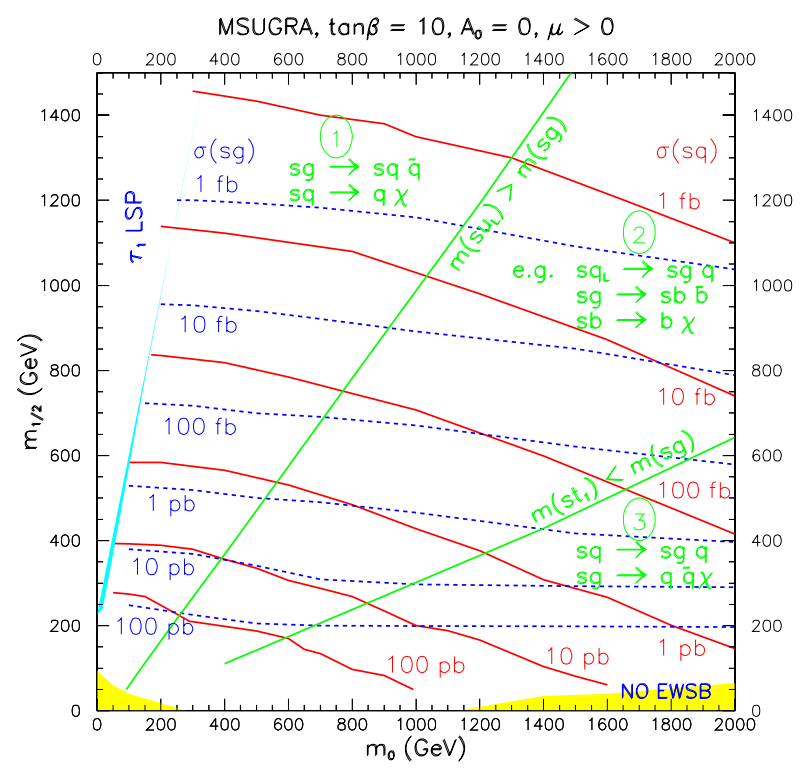

Figure 13.1. Regions of the $m_{0}$ versus $m_{1 / 2}$ plane showing the production cross-sections and with main squark and gluino decays.

- Region 2: in this region some squarks are heavier, other are lighter than the gluino. Hence, rather complicated decay chains are possible, for instance

$$
\tilde{q}_{L} \rightarrow \tilde{g} q, \tilde{g} \rightarrow \tilde{b} \bar{b}, \tilde{b} \rightarrow b \chi
$$

as the $\tilde{q}_{L}$ of the first two generations are expected to be among the heaviest squarks and the $\tilde{b}_{1}$ (and $\tilde{t}_{1}$ ) among the lightest.

- Region 3: in this region, the gluinos are lighter than any of the squarks. A typical decay chain is then

$$
\tilde{q} \rightarrow \tilde{g} q, \tilde{g} \rightarrow q \bar{q} \chi
$$

where the gluino gives rise to a three-body decay mediated by a virtual squark.

They will cascade down to the LSP, here assumed to be stable. In mSUGRA, the lightest two neutralinos are $\tilde{\chi}_{1}^{0}$, which is dominantly bino-like, and $\tilde{\chi}_{2}^{0}$, which is dominantly winolike. The $\tilde{q}_{R}$ then decays almost exclusively directly into $q \tilde{\chi}_{1}^{0}$. But the $\tilde{q}_{L}$ have usually a nonnegligible branching ratio to decay via the $\tilde{\chi}_{2}^{0}$ or $\tilde{\chi}_{1}^{ \pm}$. The decay of the $\tilde{\chi}_{2}^{0}$ will then provide an excellent signature for the events which can be observed in inclusive searches.

The main decay modes of the $\tilde{\chi}_{2}^{0}$, and hence the signatures, are

$$
\begin{aligned}
& \tilde{\chi}_{2}^{0} \rightarrow \tilde{l} l, \\
& \tilde{\chi}_{2}^{0} \rightarrow \tilde{v} v, \\
& \tilde{\chi}_{2}^{0} \rightarrow h^{0} \tilde{\chi}_{1}^{0}, \\
& \tilde{\chi}_{2}^{0} \rightarrow Z^{0} \tilde{\chi}_{1}^{0}, \\
& \tilde{\chi}_{2}^{0} \rightarrow l^{+} l^{-} \tilde{\chi}_{1}^{0}
\end{aligned}
$$

where the last decay is mediated by the exchange of an off-shell $Z^{0}$ or $\tilde{l}$. The first decay corresponds to a gauge interaction coupling a Wino to a slepton-lepton pair and dominates 

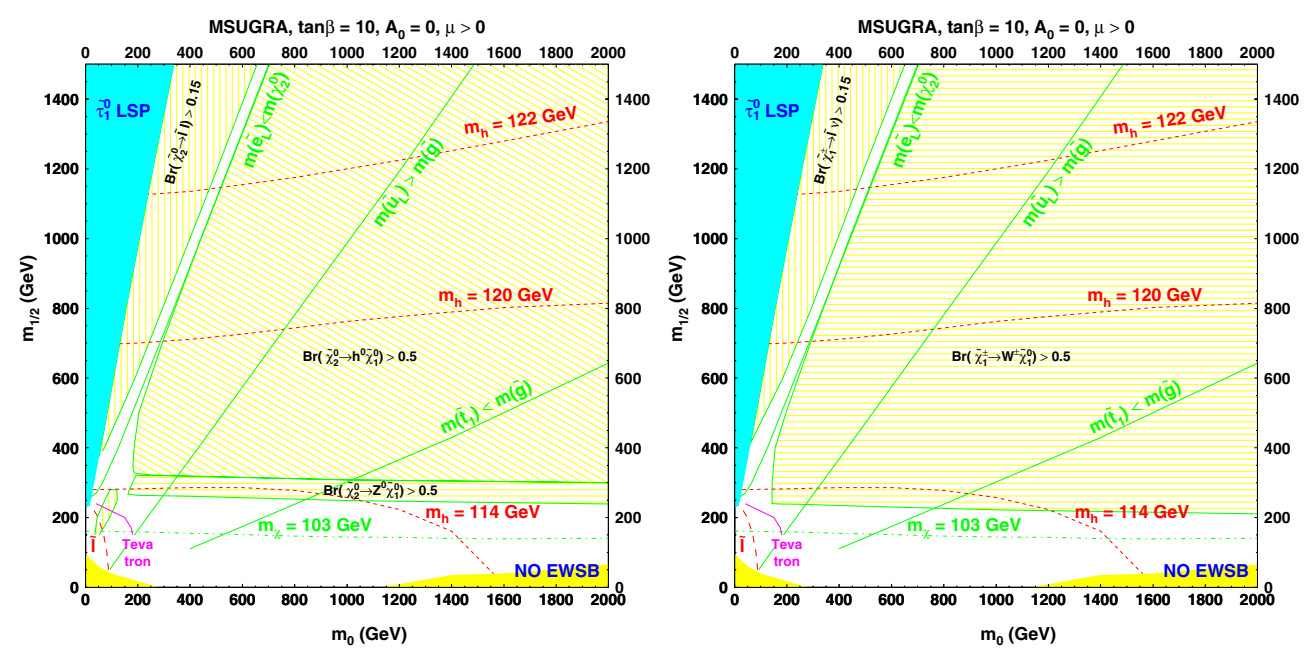

Figure 13.2. Regions of the $m_{0}$ versus $m_{1 / 2}$ plane with main $\chi_{2}^{0}$ decays (left) and main decays of $\tilde{\chi}_{1}^{ \pm}$(right).

if it is kinematically allowed. When this decay is kinematically forbidden and $m_{1 / 2}$ is large enough, so that $m\left(\tilde{\chi}_{2}^{0}\right)-m\left(\tilde{\chi}_{1}^{0}\right)>m\left(h^{0}\right)$, the next preferred decay is to $h^{0}$. This corresponds to a gaugino-Higgsino transition and thus requires a non-zero Higgsino component in at least one of the two neutralinos. If also this decay is kinematically forbidden and the neutralino mass difference is sufficient, the $\tilde{\chi}_{2}^{0}$ decays to a $Z^{0}$ which is suppressed compared to the $h^{0}$ decay because it couples to the Higgsino component of both neutralinos. When also this decay is kinematically forbidden, direct three-body decays take place. The corresponding regions in the $m_{0}$ versus $m_{1 / 2}$ plane are illustrated for a mSUGRA case in Fig. 13.2 (left). The exact boundaries of the areas depend on the assumptions (mSUGRA) and on the value of $\tan \beta$ and the parameter $A$, but their existence is rather generic. It should be emphasised that the existence of these decay modes is a direct consequence of the gauge structure of the theory and is therefore independent of the model details. Their relative importance at a given SUSY point is, however, model dependent.

In addition to the decays via a $\tilde{\chi}_{2}^{0}$, a large fraction of squark decays will proceed via a $\tilde{\chi}_{1}^{ \pm}$ decay, which may lead to

$$
\begin{aligned}
& \tilde{\chi}_{1}^{ \pm} \rightarrow \tilde{l} v, \\
& \tilde{\chi}_{1}^{ \pm} \rightarrow \tilde{v} l, \\
& \tilde{\chi}_{1}^{ \pm} \rightarrow W^{ \pm} \tilde{\chi}_{1}^{0}, \\
& \tilde{\chi}_{1}^{ \pm} \rightarrow H^{ \pm} \tilde{\chi}_{1}^{0}, \\
& \tilde{\chi}_{1}^{ \pm} \rightarrow l^{ \pm} v \tilde{\chi}_{1}^{0},
\end{aligned}
$$

where the last decay is mediated by the exchange of an off-shell $W, \tilde{v}$ or $\tilde{l}$. The localisation of the chargino decay modes in the $\left(m_{0}, m_{1 / 2}\right)$ plane is illustrated for a mSUGRA case in Fig. 13.2 (right).

Further constraints beyond the mSUGRA ones can be imposed, for example the compatibility with the measured relic density. These limit very severely the available parameter space. However, the lack of knowledge of the SUSY breaking mechanism 
Table 13.1. mSUGRA parameter values for the test points. Masses are given in units of $\mathrm{GeV} / \mathrm{c}^{2}$.

\begin{tabular}{lrrccc}
\hline Point & $m_{0}$ & $m_{1 / 2}$ & $\tan \beta$ & $\operatorname{sgn}(\mu)$ & $A_{0}$ \\
\hline LM1 & 60 & 250 & 10 & + & 0 \\
LM2 & 185 & 350 & 35 & + & 0 \\
LM3 & 330 & 240 & 20 & + & 0 \\
LM4 & 210 & 285 & 10 & + & 0 \\
LM5 & 230 & 360 & 10 & + & 0 \\
LM6 & 85 & 400 & 10 & + & 0 \\
LM7 & 3000 & 230 & 10 & + & 0 \\
LM8 & 500 & 300 & 10 & + & -300 \\
LM9 & 1450 & 175 & 50 & + & 0 \\
LM10 & 3000 & 500 & 10 & + & 0 \\
HM1 & 180 & 850 & 10 & + & 0 \\
HM2 & 350 & 800 & 35 & + & 0 \\
HM3 & 700 & 800 & 10 & + & 0 \\
HM4 & 1350 & 600 & 10 & + & 0 \\
\hline
\end{tabular}

encourages the future experiments to prepare themselves to cope with the broadest possible spectrum of situations. Rather than restricting oneself to a very constrained model, it will be important to understand how to detect departures from the SM in a large variety of topologies and to investigate how to reconstruct the sparticle masses and other SUSY parameters. Of course, there is more information available in the events than just the end points, e.g. momentum asymmetries of the decay leptons, branching ratios and total cross section measurements. This additional information have so far not been used to a large extent.

\subsubsection{Test points for $m S U G R A$}

To cover the significantly different experimental signatures, a set of mSUGRA test points have been defined and will be used in the subsequent analyses. First, low mass (LM1 to LM9) test points were chosen to evaluate the sensitivity to SUSY signals in the early period of the LHC but above the Tevatron reach. Then, some high mass test points (HM1 to HM4) near the ultimate reach of the LHC were included.

Their parameters are defined in Table 13.1 and their position in the $\left(m_{0}, m_{1 / 2}\right)$ plane is shown in Fig. 13.3. Points LM1, LM2 and LM6 are compatible with WMAP Cold Dark Matter limits in a strict mSUGRA scenario. The other points are not, but can be made compatible with CDM if universality of the Higgs mass parameters is abandoned (NUHM). Quoted branching ratios are from ISASUGRA7.69 [672] (lepton is $e$ or $\mu$ ). The post-WMAP benchmark points are found in [633], the NUHM points in [673] and the CMS DAQ TDR points in [76].

\section{- Point LM1:}

* Same as post-WMAP benchmark point $\mathrm{B}^{\prime}$ and near DAQ TDR point 4.

$* m(\tilde{g}) \geqslant m(\tilde{q})$, hence $\tilde{g} \rightarrow \tilde{q} q$ is dominant.

$* B\left(\tilde{\chi}_{2}^{0} \rightarrow \tilde{l}_{R} l\right)=11.2 \%, B\left(\tilde{\chi}_{2}^{0} \rightarrow \tilde{\tau}_{1} \tau\right)=46 \%, B\left(\tilde{\chi}_{1}^{ \pm} \rightarrow \tilde{v}_{l} l\right)=36 \%$.

\section{- Point LM2:}

* Almost identical to post-WMAP benchmark point I'.

$* m(\tilde{g}) \geqslant m(\tilde{q})$, hence $\tilde{g} \rightarrow \tilde{q} q$ is dominant $\left(\tilde{b}_{1} b\right.$ is $\left.25 \%\right)$.

$* B\left(\tilde{\chi}_{2}^{0} \rightarrow \tilde{\tau}_{1} \tau\right)=96 \% B\left(\tilde{\chi}_{1}^{ \pm} \rightarrow \tilde{\tau} \nu\right)=95 \%$. 


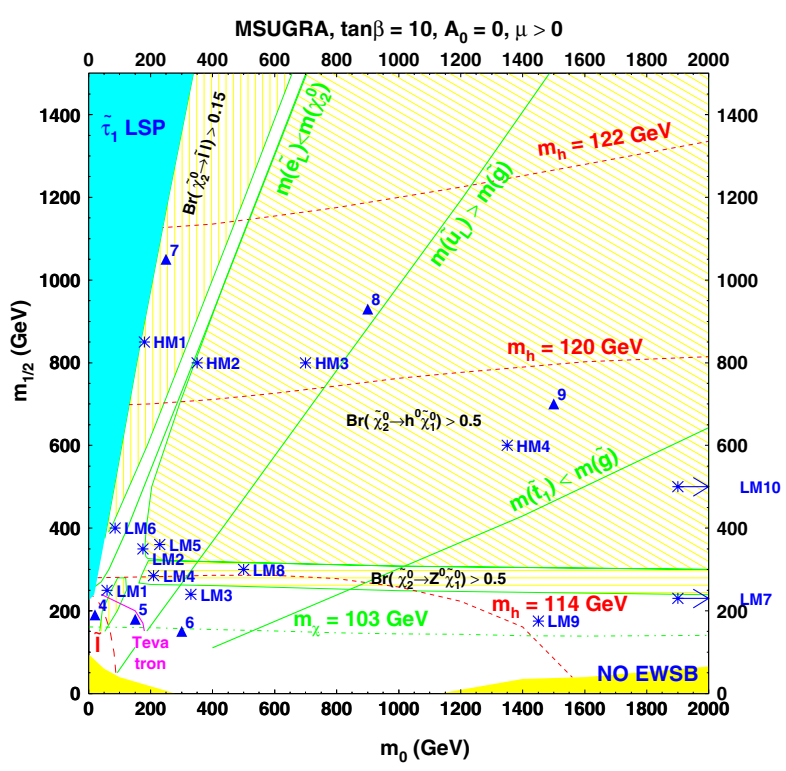

Figure 13.3. Position of the test points in the $m_{0}$ versus $m_{1 / 2}$ plane. The lines in this plane correspond to the assumptions that $\tan \beta=10, A_{0}=0$ and $\mu>0$. The shaded regions are excluded because either the $\tilde{\tau}_{1}$ would be the LSP or because there is not radiative electroweak symmetry breaking. The regions excluded by the LEP limit on the $h^{0}$ or the $\tilde{\chi}_{1}^{ \pm}$masses are delineated by dashed lines. The test CMS points are indicated by stars (LM7 and LM10 are outside the boundaries) and the points used in the CMS DAQ TDR by triangles. Also shown are the regions of interest for the decay of the $\tilde{\chi}_{2}^{0}$.

\section{- Point LM3:}

* Same as NUHM point $\gamma$ and near DAQ TDR point 6.

$* m(\tilde{g})<m(\tilde{q})$, hence $\tilde{g} \rightarrow \tilde{q} q$ is forbidden except $B\left(\tilde{g} \rightarrow \tilde{b}_{1,2} b\right)=85 \%$

$* B\left(\tilde{\chi}_{2}^{0} \rightarrow l l \tilde{\chi}_{1}^{0}\right)=3.3 \%, B\left(\tilde{\chi}_{2}^{0} \rightarrow \tau \tau \tilde{\chi}_{1}^{0}\right)=2.2 \%, B\left(\tilde{\chi}_{1}^{ \pm} \rightarrow W^{ \pm} \tilde{\chi}_{1}^{0}\right)=100 \%$

\section{- Point LM4:}

* Near NUHM point $\alpha$ in the on-shell $Z^{0}$ decay region

$* m(\tilde{g}) \geqslant m(\tilde{q})$, hence $\tilde{g} \rightarrow \tilde{q} q$ is dominant with $\tilde{g} \rightarrow \tilde{b}_{1} b=24 \%$

$* B\left(\tilde{\chi}_{2}^{0} \rightarrow Z^{0} \tilde{\chi}_{1}^{0}\right)=97 \%, B\left(\tilde{\chi}_{1}^{ \pm} \rightarrow W^{ \pm} \tilde{\chi}_{1}^{0}\right)=100 \%$

\section{- Point LM5:}

* In the $h^{0}$ decay region, same as NUHM point $\beta$.

$* m(\tilde{g}) \geqslant m(\tilde{q})$, hence $\tilde{g} \rightarrow \tilde{q} q$ is dominant with $B\left(\tilde{g} \rightarrow \tilde{b}_{1} b\right)=19.7 \%$ and $B\left(\tilde{g} \rightarrow \tilde{t}_{1} t\right)=23.4 \%$

* $B\left(\tilde{\chi}_{2}^{0} \rightarrow h^{0} \tilde{\chi}_{1}^{0}\right)=85 \%, B\left(\tilde{\chi}_{2}^{0} \rightarrow Z^{0} \tilde{\chi}_{1}^{0}\right)=11.5 \%, B\left(\tilde{\chi}_{1}^{ \pm} \rightarrow W^{ \pm} \tilde{\chi}_{1}^{0}\right)=97 \%$

\section{- Point LM6:}

* Same as post-WMAP benchmark point $\mathrm{C}^{\prime}$.

* $m(\tilde{g}) \geqslant m(\tilde{q})$, hence $\tilde{g} \rightarrow \tilde{q} q$ is dominant

* $B\left(\tilde{\chi}_{2}^{0} \rightarrow \tilde{l}_{L} l\right)=10.8 \%, B\left(\tilde{\chi}_{2}^{0} \rightarrow \tilde{l}_{R} l\right)=1.9 \%, B\left(\tilde{\chi}_{2}^{0} \rightarrow \tilde{\tau}_{1} \tau\right)=14 \%$, $B\left(\tilde{\chi}_{1}^{ \pm} \rightarrow \tilde{v}_{l} l\right)=44 \%$

\section{- Point LM7:}

* Very heavy squarks, outside reach, but light gluino.

* $m(\tilde{g})=678 \mathrm{GeV} / \mathrm{c}^{2}$, hence $\tilde{g} \rightarrow 3$-body is dominant 
* $B\left(\tilde{\chi}_{2}^{0} \rightarrow l l \tilde{\chi}_{1}^{0}\right)=10 \%, B\left(\tilde{\chi}_{1}^{ \pm} \rightarrow v l \tilde{\chi}_{1}^{0}\right)=33 \%$

* EW chargino-neutralino production cross-section is about $73 \%$ of total.

\section{- Point LM8:}

* Gluino lighter than squarks, except $\tilde{b}_{1}$ and $\tilde{t}_{1}$

* $m(\tilde{g})=745 \mathrm{GeV} / \mathrm{c}^{2}, M\left(\tilde{t}_{1}\right)=548 \mathrm{GeV} / \mathrm{c}^{2}, \tilde{g} \rightarrow \tilde{t}_{1} t$ is dominant

* $B\left(\tilde{g} \rightarrow \tilde{t}_{1} t\right)=81 \%, B\left(\tilde{g} \rightarrow \tilde{b}_{1} b\right)=14 \%, B\left(\tilde{q}_{L} \rightarrow q \tilde{\chi}_{2}^{0}\right)=26-27 \%$,

$* B\left(\tilde{\chi}_{2}^{0} \rightarrow Z^{0} \tilde{\chi}_{1}^{0}\right)=100 \%, B\left(\tilde{\chi}_{1}^{ \pm} \rightarrow W^{ \pm} \tilde{\chi}_{1}^{0}\right)=100 \%$

\section{- Point LM9:}

* Heavy squarks, light gluino. Consistent with EGRET data on diffuse gamma ray spectrum, WMAP results on CDM and mSUGRA [674]. Similar to LM7.

* $m(\tilde{g})=507 \mathrm{GeV} / \mathrm{c}^{2}$, hence $\tilde{g} \rightarrow 3$-body is dominant

$* B\left(\tilde{\chi}_{2}^{0} \rightarrow l l \tilde{\chi}_{1}^{0}\right)=6.5 \%, B\left(\tilde{\chi}_{1}^{ \pm} \rightarrow v l \tilde{\chi}_{1}^{0}\right)=22 \%$

\section{- Point LM10:}

* Similar to LM7, but heavier gauginos.

* Very heavy squarks, outside reach, but light gluino.

$* m(\tilde{g})=1295 \mathrm{GeV} / \mathrm{c}^{2}$, hence $\tilde{g} \rightarrow 3$-body is dominant

$* B\left(\tilde{g} \rightarrow t \bar{t} \tilde{\chi}_{4}^{0}\right)=11 \%, B\left(\tilde{g} \rightarrow t b \tilde{\chi}_{2}^{ \pm}\right)=27 \%$

\section{- Point HM1:}

* $m(\tilde{g}) \geqslant m(\tilde{q})$, hence $\tilde{g} \rightarrow \tilde{q} q$ is dominant

$* B\left(\tilde{g} \rightarrow \tilde{t}_{1} t\right)=25 \%, B\left(\tilde{q}_{L} \rightarrow q \tilde{\chi}_{2}^{0}\right)=32 \%$, but $B\left(\tilde{t}_{1} \rightarrow t \tilde{\chi}_{2}^{0}\right)=6 \%, B\left(\tilde{t}_{1} \rightarrow t \tilde{\chi}_{3}^{0}\right)=18 \%, B\left(\tilde{t}_{1} \rightarrow t \tilde{\chi}_{4}^{0}\right)=9 \%$,

$* B\left(\tilde{\chi}_{2}^{0} \rightarrow \tilde{l}_{L} l\right)=27 \%, B\left(\tilde{\chi}_{2}^{0} \rightarrow \tilde{\tau}_{1} \tau\right)=14 \%, B\left(\tilde{\chi}_{1}^{ \pm} \rightarrow \tilde{v}_{l} l\right)=37 \%$

\section{- Point HM2:}

* $m(\tilde{g}) \geqslant m(\tilde{q})$, hence $\tilde{g} \rightarrow \tilde{q} q$ is dominant

* $B\left(\tilde{g} \rightarrow \tilde{t}_{1} t\right)=25 \%, B\left(\tilde{q}_{L} \rightarrow q \tilde{\chi}_{2}^{0}\right)=32 \%$, but $B\left(\tilde{t}_{1} \rightarrow t \tilde{\chi}_{2}^{0}\right)=6 \%, B\left(\tilde{t}_{1} \rightarrow t \tilde{\chi}_{3}^{0}\right)=20 \%, B\left(\tilde{t}_{1} \rightarrow t \tilde{\chi}_{4}^{0}\right)=9 \%$,

* $B\left(\tilde{\chi}_{2}^{0} \rightarrow \tilde{\tau}_{1} \tau\right)=78 \%, B\left(\tilde{\chi}_{1}^{ \pm} \rightarrow \tilde{v} \tau+\tilde{\tau}_{1} v\right)=13+76 \%$

\section{- Point HM3:}

* $m(\tilde{g}) \geqslant m(\tilde{q})$, hence $\tilde{g} \rightarrow \tilde{q} q$ is dominant

* $B\left(\tilde{g} \rightarrow \tilde{t}_{1} t\right)=52 \%, B\left(\tilde{q}_{L} \rightarrow q \tilde{\chi}_{2}^{0}\right)=32 \%$, but $B\left(\tilde{t}_{1} \rightarrow t \tilde{\chi}_{2}^{0}\right)=5 \%, B\left(\tilde{t}_{1} \rightarrow t \tilde{\chi}_{3}^{0}\right)=20 \%, B\left(\tilde{t}_{1} \rightarrow t \tilde{\chi}_{4}^{0}\right)=11 \%$,

$* B\left(\tilde{\chi}_{2}^{0} \rightarrow h^{0} \tilde{\chi}_{1}^{0}\right)=94 \%, B\left(\tilde{\chi}_{1}^{ \pm} \rightarrow W^{ \pm} \tilde{\chi}_{1}^{0}\right)=100 \%$

\section{- Point HM4:}

$* m(\tilde{g})<m(\tilde{q})$, hence $\tilde{q} \rightarrow \tilde{g} q$ is important

$* B\left(\tilde{q}_{L} \rightarrow \tilde{g} q\right)=43 \%, B\left(\tilde{q}_{R} \rightarrow \tilde{g} q\right)=77-93 \%, B\left(\tilde{g} \rightarrow \tilde{t}_{1} t\right)=82 \%$,

$* B\left(\tilde{t}_{1} \rightarrow t \tilde{\chi}_{2}^{0}\right)=3 \%, B\left(\tilde{t}_{1} \rightarrow t \tilde{\chi}_{3}^{0}\right)=22 \%, B\left(\tilde{t}_{1} \rightarrow t \tilde{\chi}_{4}^{0}\right)=16 \%$,

* $B\left(\tilde{\chi}_{2}^{0} \rightarrow h^{0} \tilde{\chi}_{1}^{0}\right)=94 \%, B\left(\tilde{\chi}_{4}^{0} \rightarrow h^{0} \tilde{\chi}_{2}^{0}\right)=30 \%, B\left(\tilde{\chi}_{1}^{ \pm} \rightarrow W^{ \pm} \tilde{\chi}_{1}^{0}\right)=100 \%$

The cross sections for the test points are given at NLO and LO from PROSPINO1 in Table 13.2. 
Table 13.2. Cross sections for the test points in pb at NLO (LO) from PROSPINO1

\begin{tabular}{|c|c|c|c|c|c|c|c|}
\hline Point & $M(\tilde{q})$ & $M(\tilde{g})$ & $\tilde{g} \tilde{g}$ & $\tilde{g} \tilde{q}$ & $\tilde{q} \overline{\tilde{q}}$ & $\tilde{q} \tilde{q}$ & Total \\
\hline LM1 & 558.61 & 611.32 & $\begin{array}{l}10.55 \\
(6.489)\end{array}$ & $\begin{array}{l}28.56 \\
(24.18)\end{array}$ & $\begin{array}{l}8.851 \\
(6.369)\end{array}$ & $\begin{array}{l}6.901 \\
(6.238)\end{array}$ & $\begin{array}{l}54.86 \\
(43.28)\end{array}$ \\
\hline LM2 & 778.86 & 833.87 & $\begin{array}{l}1.443 \\
(0.829)\end{array}$ & $\begin{array}{l}4.950 \\
(3.980)\end{array}$ & $\begin{array}{l}1.405 \\
(1.013)\end{array}$ & $\begin{array}{l}1.608 \\
(1.447)\end{array}$ & $\begin{array}{l}9.41 \\
(7.27)\end{array}$ \\
\hline LM3 & 625.65 & 602.15 & $\begin{array}{l}12.12 \\
(7.098)\end{array}$ & $\begin{array}{l}23.99 \\
(19.42)\end{array}$ & $\begin{array}{l}4.811 \\
(3.583)\end{array}$ & $\begin{array}{l}4.554 \\
(4.098)\end{array}$ & $\begin{array}{l}45.47 \\
(34.20)\end{array}$ \\
\hline LM4 & 660.54 & 695.05 & $\begin{array}{l}4.756 \\
(2.839)\end{array}$ & $\begin{array}{l}13.26 \\
(10.91)\end{array}$ & $\begin{array}{l}3.631 \\
(2.598)\end{array}$ & $\begin{array}{l}3.459 \\
(3.082)\end{array}$ & $\begin{array}{l}25.11 \\
(19.43)\end{array}$ \\
\hline LM5 & 809.66 & 858.37 & $\begin{array}{l}1.185 \\
(0.675)\end{array}$ & $\begin{array}{l}4.089 \\
(3.264)\end{array}$ & $\begin{array}{l}1.123 \\
(0.809)\end{array}$ & $\begin{array}{l}1.352 \\
(1.213)\end{array}$ & $\begin{array}{l}7.75 \\
(5.96)\end{array}$ \\
\hline LM6 & 859.93 & 939.79 & $\begin{array}{l}0.629 \\
(0.352)\end{array}$ & $\begin{array}{l}2.560 \\
(2.031)\end{array}$ & $\begin{array}{l}0.768 \\
(0.559)\end{array}$ & $\begin{array}{l}0.986 \\
(0.896)\end{array}$ & $\begin{array}{l}4.94 \\
(3.84)\end{array}$ \\
\hline LM7 & 3004.3 & 677.65 & $\begin{array}{l}6.749 \\
(3.796)\end{array}$ & $\begin{array}{l}0.042 \\
(0.028)\end{array}$ & $\begin{array}{l}0.000 \\
(0.000)\end{array}$ & $\begin{array}{l}0.000 \\
(0.000)\end{array}$ & $\begin{array}{l}6.79 \\
(3.82)\end{array}$ \\
\hline LM8 & 820.46 & 745.14 & $\begin{array}{l}3.241 \\
(1.780)\end{array}$ & $\begin{array}{l}6.530 \\
(5.021)\end{array}$ & $\begin{array}{l}1.030 \\
(0.778)\end{array}$ & $\begin{array}{l}1.385 \\
(1.230)\end{array}$ & $\begin{array}{l}12.19 \\
(8.81)\end{array}$ \\
\hline LM9 & 1480.6 & 506.92 & $\begin{array}{l}36.97 \\
(21.44)\end{array}$ & $\begin{array}{l}2.729 \\
(1.762)\end{array}$ & $\begin{array}{l}0.018 \\
(0.015)\end{array}$ & $\begin{array}{l}0.074 \\
(0.063)\end{array}$ & $\begin{array}{l}39.79 \\
(23.28)\end{array}$ \\
\hline LM10 & 3132.8 & 1294.8 & $\begin{array}{l}0.071 \\
(0.037)\end{array}$ & $\begin{array}{l}0.005 \\
(0.004)\end{array}$ & $\begin{array}{l}0.000 \\
(0.000)\end{array}$ & $\begin{array}{l}0.000 \\
(0.000)\end{array}$ & $\begin{array}{l}0.076 \\
(0.041)\end{array}$ \\
\hline HM1 & 1721.4 & 1885.9 & $\begin{array}{l}0.002 \\
(0.001)\end{array}$ & $\begin{array}{l}0.018 \\
(0.016)\end{array}$ & $\begin{array}{l}0.005 \\
(0.005)\end{array}$ & $\begin{array}{l}0.020 \\
(0.021)\end{array}$ & $\begin{array}{l}0.045 \\
(0.043)\end{array}$ \\
\hline HM2 & 1655.8 & 1785.4 & $\begin{array}{l}0.003 \\
(0.002)\end{array}$ & $\begin{array}{l}0.027 \\
(0.024)\end{array}$ & $\begin{array}{l}0.008 \\
(0.007)\end{array}$ & $\begin{array}{l}0.027 \\
(0.028)\end{array}$ & $\begin{array}{l}0.065 \\
(0.061)\end{array}$ \\
\hline HM3 & 1762.1 & 1804.4 & $\begin{array}{l}0.003 \\
(0.002)\end{array}$ & $\begin{array}{l}0.021 \\
(0.018)\end{array}$ & $\begin{array}{l}0.005 \\
(0.004)\end{array}$ & $\begin{array}{l}0.018 \\
(0.019)\end{array}$ & $\begin{array}{l}0.047 \\
(0.043)\end{array}$ \\
\hline HM4 & 1815.8 & 1433.9 & $\begin{array}{l}0.026 \\
(0.014)\end{array}$ & $\begin{array}{l}0.056 \\
(0.043)\end{array}$ & $\begin{array}{l}0.003 \\
(0.003)\end{array}$ & $\begin{array}{l}0.017 \\
(0.017)\end{array}$ & $\begin{array}{l}0.102 \\
(0.077)\end{array}$ \\
\hline
\end{tabular}

\subsection{Hemisphere algorithm for separation of decay chains}

\subsubsection{Basic idea and goal}

In the MSSM, the primary SUSY particles are heavy and tend to be produced with a large $Q^{2}$, whereas the transverse momentum of their decay products with respect to their initial direction is limited by the magnitude of their mass. Moreover, ignoring $R_{p}$ violation, they are produced in pairs. It may, therefore, be possible to separate the two decay chains by reconstructing the two production directions (in 3D) and collecting the jets and leptons in two clusters according to their "closeness" to these axes. This procedure is inspired by the reconstruction of the thrust or sphericity axis in $e^{+} e^{-}$collisions, except that in hadron collisions two separate axes need to be introduced per event, as the laboratory frame does not coincide with the parton centre of mass frame. Moreover, the back-to-back orientation of the sparticles in the transverse plane cannot be used, as the invisible LSP disturbs significantly the direction of the observable particles.

In hadron colliders like the LHC, the large multiplicity of jets and leptons often lead to a large combinatorial background when trying to reconstruct peaks or to determine end points in effective mass distributions (to reconstruct sparticle masses). Provided the hemisphere algorithm has a large probability to assign correctly the jets to their parents, a reduction of a factor 2 to 4 can be expected in the combinatorial background. 
The proposed algorithm consists of a recursive method going through the following steps:

- Starting off by computing two initial axes (called "seeds" below).

- Associating the objects (jets and leptons) to one of these axes according to a certain criterion (hemisphere association method).

- Recalculating the axes as the sum of the momenta of all the connected objects. In order to converge to a stable solution, the axes are only updated after a full iteration is performed.

- Iterating the association until no objects switch from one group to the other.

\subsubsection{Seeding methods}

Two seeding methods have been tested:

(1) The first axis is chosen as the direction of the highest momentum object and the second axis as the direction of the object with the largest $p \cdot \Delta R$ with respect to the first axis, where $\Delta R$ is defined as

$$
\Delta R=\sqrt{\Delta \phi^{2}+\Delta \eta^{2}} .
$$

(2) The axes are chosen as the directions of the pair of objects which have the largest invariant mass.

\subsubsection{Association methods}

Three association methods are available. An object is assigned to a given axis $\vec{A}$ when:

(1) The scalar product $\vec{p} \cdot \vec{A}$ is maximum, which amounts to choosing the smallest angle

(2) The hemisphere squared masses are minimum, i.e. object $k$ is associated to the hemisphere with mass $m_{i}$ rather than $m_{j}$ if $m_{i k}^{2}+m_{j}^{2} \leqslant m_{i}^{2}+m_{j k}^{2}$. This is equivalent to the requirement

$$
\left(E_{i}-p_{i} \cos \theta_{i k}\right) \leqslant\left(E_{j}-p_{j} \cos \theta_{j k}\right)
$$

(3) The Lund distance measure is minimum, i.e.

$$
\left(E_{i}-p_{i} \cos \theta_{i k}\right) \frac{E_{i}}{\left(E_{i}+E_{k}\right)^{2}} \leqslant\left(E_{j}-p_{j} \cos \theta_{j k}\right) \frac{E_{j}}{\left(E_{j}+E_{k}\right)^{2}} .
$$

In order to converge to a stable solution, the axes are only updated after a full iteration is performed.

\subsubsection{Results}

The performance of the hemisphere assignment was tested on events with production of squarks and/or gluinos. Jets were reconstructed using the Iterative Cone method with $\Delta R=$ 0.5 and calibrated with the "GammaJet" procedure. They were selected when $E_{\mathrm{T}}>30 \mathrm{GeV}$ and $|\eta|<3$.0. The momentum vectors used were from the Monte carlo parton level objects which matched with the jets and/or leptons. Some of the CMS test points were used, namely LM1 (dilepton final states via $\tilde{l}_{R}$ ), LM5 (with decay of $\tilde{\chi}_{2}^{0}$ to $h \rightarrow b \bar{b}$ ) and LM9 (with dileptons from 3-body decays).

The efficiencies quoted below are the ratio between the correctly assigned MC objects and their total number. The correct hemisphere was chosen as the one for which 
Table 13.3. Efficiencies for test point LM1.

\begin{tabular}{lccccc}
\hline Type of jet & all jets & quark jets & gluon jets & q from $\tilde{q}$ & q from $\tilde{g}$ \\
\hline Seed 1, Assoc 1 & $79 \%$ & $80 \%$ & $74 \%$ & $85 \%$ & $69 \%$ \\
Seed 1, Assoc 2 & $80 \%$ & $80 \%$ & $77 \%$ & $85 \%$ & $72 \%$ \\
Seed 2, Assoc 2 & $81 \%$ & $81 \%$ & $78 \%$ & $85 \%$ & $72 \%$ \\
Seed 2, Assoc 3 & $81 \%$ & $81 \%$ & $79 \%$ & $86 \%$ & $73 \%$ \\
\hline
\end{tabular}

Table 13.4. Efficiencies for test points LM1, LM5 and LM9, using the methods Seed 2 and hemisphere association 3 .

\begin{tabular}{lccccc}
\hline Point & all jets & quark jets & gluon jets & q from $\tilde{q}$ & q from $\tilde{g}$ \\
\hline LM1 & $81 \%$ & $81 \%$ & $79 \%$ & $86 \%$ & $73 \%$ \\
LM5 & $77 \%$ & $77 \%$ & $74 \%$ & $87 \%$ & $70 \%$ \\
LM9 & $74 \%$ & $75 \%$ & $69 \%$ & - & $76 \%$ \\
\hline
\end{tabular}

the axis matched most closely the original squark or gluino, after subtracting from it the unobserved $\tilde{\chi}_{1}^{0}$.

The efficiencies of various types of jets for the different algorithms at the test point LM1 are summarised in Table 13.3.

It is seen that all the algorithms behave nearly in the same way, with the combination (seed 1, hemisphere association 1) being slightly worse and (seed 2, hemisphere association 3) slightly better.

The efficiencies obtained for the different test points are listed in Table 13.4 for the different types of jets by using the (seed 2, hemisphere association 3) method. Note that at point LM9 the $\tilde{g}$ undergoes a direct 3-body decay, the $\tilde{q}$ being heavier than the $\tilde{g}$.

From these tests it can be concluded that quark jets from $\tilde{q}$ have a rather high efficiency, $\geqslant 85 \%$, to be correctly assigned to a hemisphere, whereas the quark jets from a $\tilde{g}$ reach only $\gtrsim 70 \%$. This reflects the fact that the latter jets are much softer, on average, than the jets from the $\tilde{q}$ decay.

The same procedure was also applied to leptons ( $e$ or $\mu)$. However, due to their small mass, the leptons barely "feel" the boost and are sent in any direction. The results were only slightly better than the expectation from random association. Some improvement could be obtained, e.g. for $\tilde{\chi}_{2}^{0} \rightarrow e^{+} e^{-} \tilde{\chi}_{1}^{0}$, by treating the lepton pair as a single (massive) object. But this introduces some model dependence.

The power of the hemisphere separation can be further illustrated by the search for Higgs at point LM5. The reconstructed jets selected as above are identified as b-jets by a combined b-tagging method (see Vol. 1, Section 12.2.2) when the discriminant variable is $>1.5$. The invariant mass of all combinations of two b-jets is displayed in Fig. 13.4 (left). The peak from $h^{0} \rightarrow b \bar{b}$ is visible above a large combinatorial SUSY background, mostly due to the production of $\tilde{b} \tilde{b}$ and $\tilde{t} \tilde{t}$ (directly or from cascade decays). After applying the hemisphere separation method, the $2 \mathrm{~b}$ invariant mass combinations are separated into the cases where both b-jets are in the same hemisphere (centre), with a clearly visible Higgs peak, and in opposite hemispheres (right), where almost no sign of Higgs remains. Note that these plots were obtained without selection cuts. This method has been used for the Higgs search in Section 13.10 and in other searches. 

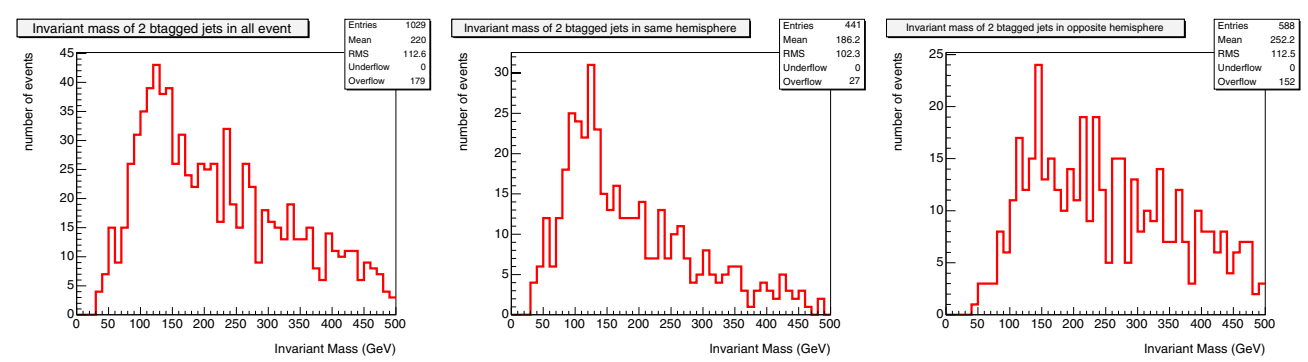

Figure 13.4. $b \bar{b}$ invariant mass distributions in $h^{0}$ production with mass $m_{h}=116 \mathrm{GeV}$ for (left) all combinations, (centre) combinations in the same hemisphere, (right) combinations in opposite hemispheres.

\subsection{Inclusive analysis with missing transverse energy and jets}

The missing transverse energy plus multi-jets final state has been a canonical signature for SUSY searches. This study is a search for the production and decay of gluinos and scalar quarks in $\geqslant 3$-jet events with large missing transverse energy. The large missing energy originates from the two LSPs in the final states of the squark and gluino decays. The three or more hadronic jets result from the hadronic decays of the squarks and/or gluinos. The full analysis is presented in section 4.2. The analysis uses the LM1 test-point at which squark and gluino production has a LO cross section of $49 \mathrm{pb}$. The major Standard Model background components include production of $Z+$ jets with the $Z$ decaying invisibly, $W+$ jets, top-antitop pairs, dibosons, single top and QCD jets. The trigger path used is the missing energy plus jets both at Level-1 and at HLT.

\subsubsection{Analysis path and results}

Events that are accepted after clean-up pre-selection requirements, proceed through the analysis path if they have missing transverse energy $E_{\mathrm{T}}^{\text {miss }}>200 \mathrm{GeV}$ and at least three jets with $E_{\mathrm{T}} \geqslant 30 \mathrm{GeV}$ within $|\eta|<3$. In addition the leading jet is required to be within the central tracker fiducial volume i.e. $|\eta|<1$.7. These requirements directly define the searched for signal signature. The rest of the analysis path is designed based on elimination of the major classes of backgrounds: the QCD production, top-anti-top pairs and the $W / Z$-QCD associated production. In Table 13.5 the path is shown with a remark indicating the reason and aim of each selection step.

A detailed explanation of the analysis path requirements and variables used is given in section 4.2. The global signal efficiency for the analysis is $13 \%$ while the signal to background ratio is $\sim 26$. The results are shown in Table 13.6 for $1 \mathrm{fb}^{-1}$.

In summary the major background components and their uncertainties are as follows:

- $t \bar{t}$ uncertainties: $7 \% E_{\mathrm{T}}^{\text {miss }}$ shape, $22 \% \mathrm{JES}, 13 \%$ statistical;

- $Z \longrightarrow v \bar{v}+$ jets, $W / Z+$ jets: $5 \%$ Luminosity (direct candle normalisation to the data ( $c f$. section 4.2);

- QCD: $E_{\mathrm{T}}^{\mathrm{miss}} 7 \%$ shape, $22 \% \mathrm{JES}, 10 \%$ statistical.

The number of backgrounds events per background component and their uncertainties are tabulated in Table 13.7. Based on the Standard Model background estimates and their uncertainties, a $5 \sigma$ observation of low mass SUSY at LM1 (gluino mass $600 \mathrm{GeV} / \mathrm{c}^{2}$ ) is achievable with $\sim 6 \mathrm{pb}^{-1}$ in events with large missing energy plus multi-jets, using a 
Table 13.5. The $E_{\mathrm{T}}^{\text {miss }}+$ multi-jet SUSY search analysis path.

\begin{tabular}{ll}
\hline Requirement & Remark \\
\hline Level 1 & Level-1 trigger efficiency parametrisation \\
$\mathrm{HLT}, E_{\mathrm{T}}^{\text {miss }}>200 \mathrm{GeV}$ & trigger/signal signature \\
primary vertex $\geqslant 1$ & primary cleanup \\
$F_{\text {em }} \geqslant 0.175, F_{c h} \geqslant 0.1$ & primary cleanup \\
$N_{j} \geqslant 3,\left|\eta_{d}^{1 j}\right|<1.7$ & signal signature \\
$\delta \phi_{\text {min }}\left(E_{\mathrm{T}}^{\mathrm{miss}}-j e t\right) \geqslant 0.3 \mathrm{rad}$, & \\
$R 1, R 2>0.5 \mathrm{rad}$, & \\
$\delta \phi\left(E_{\mathrm{T}}^{\mathrm{miss}}-j(2)\right)>20^{\circ}$ & $\mathrm{QCD}$ rejection \\
$I s o^{\text {lead trk }}=0$ & $\mathrm{ILV}(\mathrm{I}) W / Z / t \bar{t}$ rejection \\
$f_{\text {em }(j(1))}, f_{\text {em }(j(2))}<0.9$ & $\mathrm{ILV}(\mathrm{II}), W / Z / t \bar{t}$ rejection \\
$E_{\mathrm{T}, j(1)}>180 \mathrm{GeV}, E_{\mathrm{T}, j(2)}>110 \mathrm{GeV}$ & signal/background optimisation \\
$H_{\mathrm{T}} \equiv E_{\mathrm{T}(2)}+E_{\mathrm{T}(3)}+E_{\mathrm{T}(4)}+E_{\mathrm{T}}^{\text {miss }}>500 \mathrm{GeV}$ & signal/background optimisation \\
$\mathrm{SUSY} \mathrm{LM} 1$ signal efficiency $13 \%$ & \\
\hline
\end{tabular}

Table 13.6. Selected SUSY and Standard Model background events for $1 \mathrm{fb}^{-1}$.

\begin{tabular}{llllll}
\hline Signal & $t \bar{t}$ & single $t$ & $Z(\rightarrow v \bar{v})+$ jets & $(W / Z, W W / Z Z / Z W)+$ jets & QCD \\
\hline 6319 & 53.9 & 2.6 & 48 & 33 & 107 \\
\hline
\end{tabular}

Table 13.7. Standard Model background components and uncertainties for $1 \mathrm{fb}^{-1}$.

\begin{tabular}{llll}
\hline$t \bar{t}$, single top & $Z(\rightarrow v \bar{v})+$ jets & $(W / Z, W W / Z Z / Z W)+$ jets & QCD \\
\hline $56 \pm 11$ (sys) \pm 7.5 (stat) & $48 \pm 3.5$ (all) & $33 \pm 2.5$ (all) & $107 \pm 25($ sys $) \pm 10($ stat $)$ \\
\hline
\end{tabular}

significance computed with ScPf, defined in Appendix A.1. After $\sim 1.5 \mathrm{fb}^{-1}$ the $W / Z+$ jets backgrounds, including the invisible decays of the $Z$ boson which constitutes a large irreducible background component, can be reliably normalised using the $Z \rightarrow \mu \mu$ and $Z \rightarrow$ $e e+$ multi-jet data candle. The comparison of the signal, total background estimated and its components for the $M_{\text {eff }} \equiv E_{\mathrm{T}(1)}+E_{\mathrm{T}(2)}+E_{\mathrm{T}(3)}+E_{\mathrm{T}(4)}+E_{\mathrm{T}}^{\mathrm{miss}}$ can be found in section 4.2.

To perform the $5 \sigma$ reach scan (Fig. 13.5) in the mSUGRA parameter space, the HM1 test point is used as optimisation reference and the $E_{\mathrm{T}}^{\mathrm{miss}}$ and $H_{\mathrm{T}}$ requirements are raised to $600 \mathrm{GeV}$ and $1500 \mathrm{GeV}$ correspondingly. The analysis efficiency for HM1 is $\sim 12 \%$ while the total Standard Model background for $1 \mathrm{fb}^{-1}$ is 4.36 events with a total uncertainty of $7 \%$. The background composition is $67 \% \mathrm{Z}$ invisible decays, $19 \%$ QCD jets and $14 \% \mathrm{~W} / Z+$ jets.

\subsection{Inclusive muons with jets and missing transverse energy}

We study the production and decay of new particles in mSUGRA via inclusive final states including muons, high $p_{\mathrm{T}}$ jets, and large missing transverse energy. Requiring at least one muon provides a relatively clean experimental signature (complementing searches involving only inclusive jets and missing energy), however requires a well-understood trigger shortly after the LHC start-up. In this work [675], the fully simulated and reconstructed LM1 mSUGRA point is taken as the benchmark for selection optimisation and study of systematic effects. Even though the study was performed within the context of mSUGRA, this method is not specific to the mSUGRA framework and should apply equally well in other contexts. 


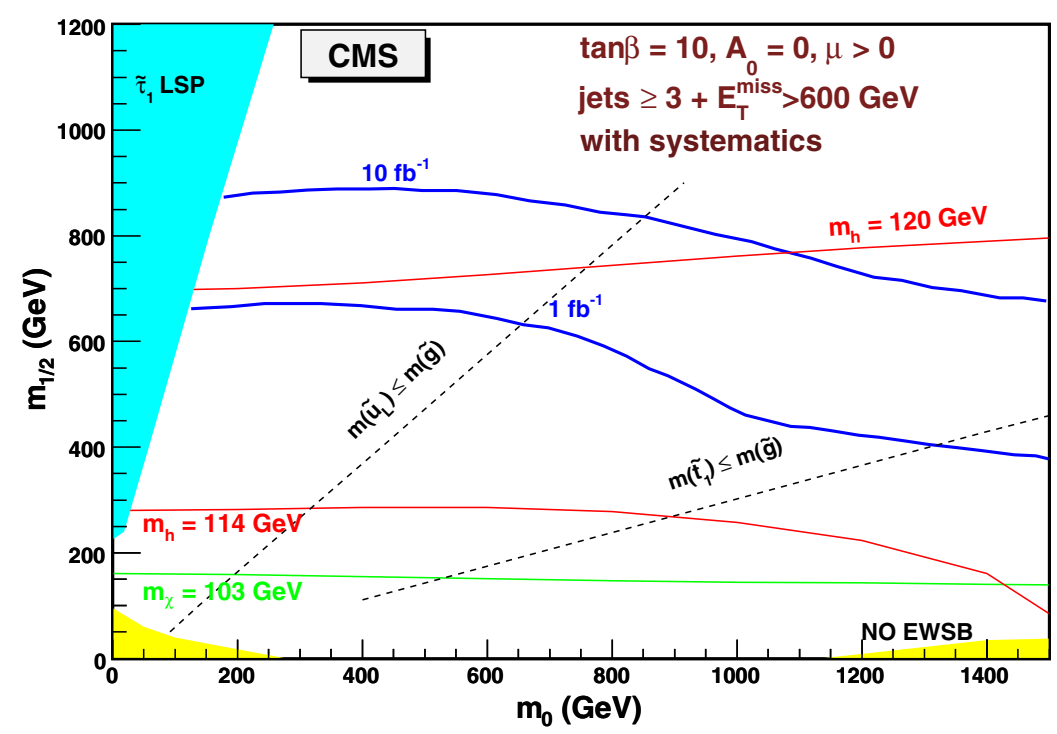

Figure 13.5. $5 \sigma$ reach for 1 and $10 \mathrm{fb}^{-1}$ using multi-jets and missing transverse energy final state.

The strategy employed in this analysis is to optimise a set of selection cuts based on an objective function which provides a reasonable estimate of the significance to exclude the Standard Model null-hypothesis while explicitly including systematic uncertainties (thus avoiding regions of phase space which are prone to systematics). This work uses a Genetic Algorithm (GARCON [63]) for the optimisation of cuts.

\subsubsection{Signal selection and backgrounds considered}

Because this work is an inclusive study of mSUGRA signatures involving at least one muon accompanied by multiple jets and large $E_{\mathrm{T}}^{\mathrm{miss}}$, several Standard Model processes contribute as sources of background and must be taken into account. Accordingly, the main backgrounds studied in this analysis correspond to QCD dijet $(2.8$ million events with $0<$ $\hat{p}_{\mathrm{T}}<4 \mathrm{TeV} / \mathrm{c}$ ), top $(t \bar{t})$ production (3.3 million events), electroweak single-boson production (4.4 million events with $0<\hat{p}_{\mathrm{T}}<4.4 \mathrm{TeV} / \mathrm{c}$ ) and electroweak dibosons production (1.2 million events). All backgrounds used in this work are fully simulated and reconstructed. This work uses only leading order cross-sections, consistently for both signal and all backgrounds. Considering NLO $k$-factors for the signal and background processes do not change the final results significantly.

The CMS trigger system is described in [76], and the current working trigger menu is described in Appendix E. This work uses an event sample which is triggered by either of two HLT triggers: the inclusive isolated single-muon trigger or the isolated dimuon trigger.

The following quality criteria are applied to muons and jets. The leading muon is required to have a transverse momentum above $p_{\mathrm{T}}=30 \mathrm{GeV} / \mathrm{c}$ which ensures that the muon candidate is reconstructed with good efficiency, well above the trigger thresholds. Further, the leading muon is required to be isolated with less than $10 \mathrm{GeV}$ of calorimeter energy within a cone of radius $R=0.3$, reducing the effects due to fake muons, whilst preserving reasonable efficiency for signal acceptance. Finally, the three leading jets must each have an $E_{\mathrm{T}}$ of at least $50 \mathrm{GeV}$ which guarantees that jets are reconstructed with good efficiency. 
Table 13.8. Total number of selected events (for $10 \mathrm{fb}^{-1}$ ) and significance ("Signif.") with systematic uncertainties (but excluding uncertainties due to finite Monte Carlo simulation statistics and higher order QCD effects). "SM" represents the total of all Standard Model backgrounds considered.

\begin{tabular}{lcccccccc}
\hline Sample(s) & Events & Signif. & Sample & Events & Signif. & Sample & Events & Signif. \\
\hline SM & 2.54 & - & LM4 & 246 & 29.2 & LM6 & 277 & 31.6 \\
LM1 & 311 & 34.0 & LM5 & 165 & 22.9 & HM1 & 13 & 5.0 \\
\hline
\end{tabular}

The genetic algorithm GARCON [63] used for the optimisation of cuts results in: $\quad E_{\mathrm{T}}^{\mathrm{miss}}>130 \mathrm{GeV}, \quad E_{\mathrm{T}}^{\mathrm{j} 1}>440 \mathrm{GeV}, \quad E_{\mathrm{T}}^{\mathrm{j} 2}>440 \mathrm{GeV}, \quad\left|\eta^{\mathrm{j} 1}\right|<1.9, \quad\left|\eta^{\mathrm{j} 2}\right|<1.5, \quad\left|\eta^{\mathrm{j} 3}\right|<$ $3, \cos [\Delta \phi(\mathrm{j} 1, \mathrm{j} 2)]<0.2,-0.95<\cos \left[\Delta \phi\left(E_{\mathrm{T}}^{\text {miss }}, \mathrm{j} 1\right)\right]<0.3, \cos \left[\Delta \phi\left(E_{\mathrm{T}}^{\text {miss }}, \mathrm{j} 2\right)\right]<0.85$. Assuming $10 \mathrm{fb}^{-1}$ of collected data, this set of cuts would expect to select a total of 2.54 background events from the Standard Model and 311 signal events from the mSUGRA LM1 benchmark signal point.

\subsubsection{Results for $10 \mathrm{fb}^{-1}$ using full detector simulation and reconstruction}

After all selection cuts have been applied, several effects contribute as systematic uncertainties, including: jet energy scale (10\%), jet energy resolution (5\%), luminosity measurement (5\%), and full GEANT simulation versus fast simulation differences $(5 \%)$, used to determine the analysis reach in mSUGRA parameters in Section 13.6.3). Since this analysis is performed consistently at leading order, the inclusion of higher order effects involving ISR/FSR is not taken into account. A generator-level comparison of the parton shower method for inclusive $t \bar{t}$ used by PYTHIA [69] with the matrix element calculation for $t \bar{t}+1$ jet from CoMPHEP [355] suggests $\mathrm{a} \approx 10 \%$ enhancement in the acceptance of $t \bar{t}+1$ jet events (generated via the matrix element method) compared with inclusive $t \bar{t}$. When combined with other expected effects - such as underlying event (5\%), pile-up (5\%), and parton distribution functions $(5 \%)$ - a total theoretical systematic uncertainty of $\sim 13 \%$ is estimated. The dominant uncertainty $(32 \%)$ arises from an inability to precisely predict the number of background events, due to finite Monte Carlo simulation statistics. We note that by the time $10 \mathrm{fb}^{-1}$ of data is collected, many of the contributing background processes will be measured from real data, thereby reducing this uncertainty. If one includes the uncertainty due to finite Monte Carlo simulation statistics, the total systematic uncertainty for this work is 37\%. Neglecting Monte Carlo simulation statistics, as well as higher order QCD effects, the total systematic uncertainty for this work is $19 \%$.

Table 13.8 shows the main results of this study. For the fully simulated low mass mSUGRA point LM1, and assuming $10 \mathrm{fb}^{-1}$ of data, this work selects an expected 311 signal events (with an efficiency of $0.074 \%$ ) compared with 2.54 expected background events, comprised of $t \bar{t}$ ( 0.73 events), $W+$ jets ( 1.56 events), and $Z+$ jets $(0.24$ events). The separation of signal from background for the different low mass mSUGRA points range in values from 23 to 34 in significance, including systematic uncertainties (but excluding uncertainties related to the limited number of simulated events). Such large values of significance merely indicate that the low mass mSUGRA region will either have been discovered or excluded, long before $10 \mathrm{fb}^{-1}$ of data is collected. We note that shortly after the LHC start-up, the systematic understanding of the CMS detector is expected to be quite different than what is presented in this work, which assumes $\mathcal{L}=10 \mathrm{fb}^{-1}$. Nevertheless, if one assumes a similar systematic understanding and extrapolates the results of this work to early running, the expected 


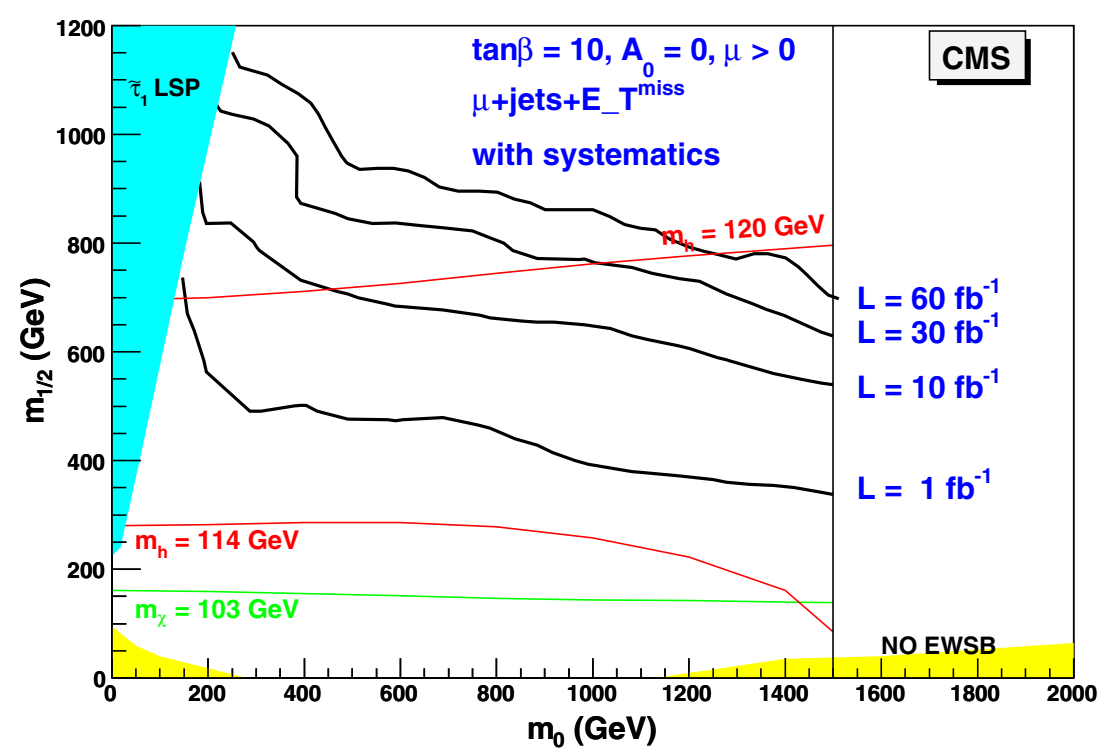

Figure 13.6. CMS discovery reach contours in the $m_{0}-m_{1 / 2}$ plane using inclusive muons with jets and missing energy for $10 \mathrm{fb}^{-1}$ (lower contour), $30 \mathrm{fb}^{-1}$ (middle contour), and $60 \mathrm{fb}^{-1}$ (upper contour) including systematics.

luminosity required to discover the LM1 mSUGRA study point would be $\mathrm{O}(0.1) \mathrm{fb}^{-1}$. Hence, low mass SUSY is a prime candidate for possible discovery during the very early running of the LHC.

\subsubsection{CMS Reach using inclusive muons with jets and missing energy}

Since CMS will have either discovered or excluded the lower mass region well in advance of the time required to collect $10 \mathrm{fb}^{-1}$ of data, the selection cuts for $30 \mathrm{fb}^{-1}$ and $60 \mathrm{fb}^{-1}$ are re-optimised using GARCON to select the HM1 mSUGRA point: $E_{\mathrm{T}}^{\text {miss }}>$ $210 \mathrm{GeV}, E_{\mathrm{T}}^{\mathrm{j} 1}>730 \mathrm{GeV}, E_{\mathrm{T}}^{\mathrm{j} 2}>730 \mathrm{GeV}, \cos [\Delta \phi(\mathrm{j} 1, \mathrm{j} 2)]<0.95, \cos \left[\Delta \phi\left(\mathrm{E}_{\mathrm{T}}^{\mathrm{miss}}, \mathrm{j} 1\right)\right]<$ $-0.2, \cos \left[\Delta \phi\left(E_{\mathrm{T}}^{\text {miss }}, \mathrm{j} 2\right)\right]<0.95$. To estimate the reach for $30 \mathrm{fb}^{-1}$ and $60 \mathrm{fb}^{-1}$, this same cut-set is applied in both cases and results in an estimated Standard Model background yield of $N_{\mathrm{B}}=0.25$ for $30 \mathrm{fb}^{-1}$, and $N_{\mathrm{B}}=0.49$ for $60 \mathrm{fb}^{-1}$. In both cases the uncertainty on the background levels is $\approx 71 \%$, primarily due to a limited number of simulated events; if one neglects that uncertainty, the systematic uncertainty is $\approx 19 \%$.

Fast simulation and reconstruction was also performed in order to scan the plane of universal scalar $\left(m_{0}\right)$ and gaugino $\left(m_{1 / 2}\right)$ masses for fixed mSUGRA parameters: $\tan \beta=10$, $\mu>0$ and $A_{0}=0$. Points were generated on a coarse grid with $\Delta m_{0}=100 \mathrm{GeV} / \mathrm{c}^{2}$ and $\Delta m_{1 / 2}=100 \mathrm{GeV} / \mathrm{c}^{2}$, starting from the point $m_{0}=100 \mathrm{GeV}, m_{1 / 2}=100 \mathrm{GeV}$. Figure 13.6 shows the discovery reach of this analysis (contours correspond to a significance value of 5 ), plotted in the mSUGRA $m_{0}-m_{1 / 2}$ plane. Assuming $10 \mathrm{fb}^{-1}$ of data, CMS can observe SUSY mass scales of over $\approx 1.5 \mathrm{TeV} / \mathrm{c}^{2}$; assuming $30 \mathrm{fb}^{-1}$ of integrated luminosity, several of the high mass CMS SUSY benchmark points become interesting for possible discovery; and, assuming $60 \mathrm{fb}^{-1}$ of integrated luminosity, CMS is able to reach in this channel SUSY mass scales of up to $\approx 2 \mathrm{TeV} / \mathrm{c}^{2}$. 


\subsection{Inclusive analyses with same sign dimuons}

The topology of two same sign isolated muons, high $p_{\mathrm{T}}$ jets, and large missing transverse energy is interesting as it allows for an efficient suppression of the Standard Model backgrounds, and at the same time allows much of the mSUGRA signal to be retained. Likesign leptons can result from several signal processes because the gluino, being a Majorana particle, has equal probability of yielding either a positively or a negatively charged lepton in its decay chain. Squark production is another important source of like-sign dileptons, since the squark charge tends to be determined by the valence quarks in the proton-proton collision. The same-sign muon topology provides a clean experimental signature and has the extra advantage of an anticipated efficient and well-understood dimuon trigger soon after LHC start-up. Even though this study [676] is performed within the context of mSUGRA, this method is not specific to the mSUGRA framework.

The genetic algorithm GARCON [63] is used to determine the optimal set of cuts for each mSUGRA benchmark point. An interval for each physics cut-parameter is then defined corresponding to its minimal cut value and the maximum cut value, determined over all different optimal mSUGRA benchmark point cut-sets. The interval for each cut-parameter is then coarsely binned and the significance systematically calculated for each possible cut combination within this reduced sub-space.

\subsubsection{Signal selection and backgrounds}

Because this work is an inclusive study of mSUGRA signatures involving at least two like-sign muons accompanied by multiple jets and large missing transverse energy, several Standard Model processes contribute as sources of background and must be taken into account. Accordingly, the main backgrounds studied in this analysis correspond to QCD dijet (2.8 million fully simulated events with $\left.0<\hat{p}_{\mathrm{T}}<4 \mathrm{TeV} / \mathrm{c}\right)$, top $(t \bar{t})$ production (3.3 million fully simulated events), electro-weak single boson production (4.4 million fully simulated events with $0<\hat{p}_{\mathrm{T}}<4.4 \mathrm{TeV} / \mathrm{c}$ ) and electro-weak dibosons production (1.2 million fully simulated events). This work uses only leading order cross-sections, consistently for both signal and all backgrounds.

The dimuon HLT trigger (98\% efficient) is required for this analysis. The following selection criteria are applied to muons and jets. The two leading muons are required to be of the same sign and to each have a transverse momentum above $10 \mathrm{GeV} / \mathrm{c}$, ensuring that the muon candidate is reconstructed with good efficiency, above the symmetric thresholds of $7 \mathrm{GeV} / \mathrm{c}$ in the dimuon trigger. Also this analysis requires at least three jets in the event, all of which are required to have $E_{\mathrm{T}}>50 \mathrm{GeV}$.

In order to select the particular SUSY diagrams responsible for prompt same-sign dimuons, we apply the following criteria. Each reconstructed muon is required to be separated by at least $\Delta R \geqslant 0.01$ from the other muons. The muon track fit is required to have $\chi_{\mu}^{2} \leqslant 3$ and the number of hits associated with the muon must be at least 13. Each muon is required to be isolated, both with respect to the tracker and calorimeter. A combined isolation parameter is used to account for correlations between the tracker (IsoByTk) and calorimeter (IsoByCalo) isolation variables, Iso $=\mathrm{IsoByTk}_{\mathrm{s}}+0.75 \times \mathrm{IsoByCalo}_{\mathrm{s}}$, with $\mathrm{Iso}_{\mu 1} \leqslant 10 \mathrm{GeV}$, Iso $\mathrm{Is}_{2} \leqslant$ $6 \mathrm{GeV}$.

In addition to a priori requiring three jets in the event, the cut-set maximising the significance (with GARCON) to discover the lowest significant fully simulated mSUGRA test point is then chosen as the final optimal cut-set: $E_{\mathrm{T}}^{\mathrm{j} 1}>175 \mathrm{GeV}, E_{\mathrm{T}}^{\mathrm{j} 2}>130 \mathrm{GeV}, E_{\mathrm{T}}^{\mathrm{j} 3}>$ $55 \mathrm{GeV}, E_{\mathrm{T}}^{\mathrm{miss}}>200 \mathrm{GeV}$. 
Table 13.9. Total number of selected events (for $\mathcal{L}=10 \mathrm{fb}^{-1}$ ) and significance ("Signif.") with systematic uncertainties. "SM" represents the total of all Standard Model backgrounds considered.

\begin{tabular}{lcccccccc}
\hline Sample(s) & Events & Signif. & Sample & Events & Signif. & Sample & Events & Signif. \\
\hline SM & 1.5 & - & LM5 & 61 & 14.0 & LM10 & 4 & 2.2 \\
LM1 & 341 & $>37.0$ & LM6 & 140 & 22.3 & HM1 & 4 & 2.2 \\
LM2 & 94 & 17.6 & LM7 & 82 & 16.3 & HM2 & 2 & 1.1 \\
LM4 & 90 & 17.2 & LM8 & 294 & 35.9 & & & \\
\hline
\end{tabular}

\subsubsection{Results for full detector simulated mSUGRA samples}

After all selection cuts have been applied the main systematic uncertainty is due to the absolute jet energy scale, which is estimated to be $15 \%$ after $10 \mathrm{fb}^{-1}$. In addition, jet energy resolution (10\%), muon identification efficiency and fake rate (negligible), luminosity (5\%), theory (10\%; cross sections, showering, ISR/FSR, etc.) and full simulation versus fast simulation (5\%, used to determine the analysis reach in mSUGRA parameters in Section 13.7.3) have been evaluated. Since this analysis is performed consistently at leading order, the inclusion of higher order effects involving ISR/FSR is not taken into account. A generator-level comparison of the parton shower method for inclusive $t \bar{t}$ used by PYTHIA [69] with the matrix element calculation for $t \bar{t}+1$ jet from CoMPHEP [355] suggests a $\approx 10 \%$ enhancement in the acceptance of $t \bar{t}+1$ jet events (generated via the matrix element method) compared with inclusive $t \bar{t}$. The total systematic uncertainty on the number of background events is $24 \%$.

Table 13.9 shows the main results of this study. For the fully simulated low mass mSUGRA point LM1, assuming $10 \mathrm{fb}^{-1}$ of data, this work selects an expected 341 signal events (with an efficiency of $0.081 \%$ ) compared with 1.5 expected background events (comprised of $t \bar{t}$ ). For other fully simulated low mass mSUGRA points (excluding LM10) and an integrated luminosity $10 \mathrm{fb}^{-1}$ of data, the selection cuts (collectively optimised over all benchmark points) achieve a separation of signal from background with a statistical significance of between $16 \sigma$ and greater than $37 \sigma$, including systematic uncertainties. Such a large significance merely indicates that the low mass mSUGRA region will either have been discovered or excluded, long before $10 \mathrm{fb}^{-1}$ of data is collected. Hence, low mass SUSY is a prime candidate for possible discovery during the very early running of the LHC. The discovery of high mass SUSY, represented by the fully simulated HM1 and HM2 points, is more difficult and requires more than $10 \mathrm{fb}^{-1}$ of data.

\subsubsection{CMS inclusive reach}

Fast simulation and reconstruction was also performed in order to scan the plane of universal scalar $\left(m_{0}\right)$ and gaugino $\left(m_{1 / 2}\right)$ masses for fixed mSUGRA parameters: $\tan \beta=10, \mu>0$ and $A_{0}=0$. Points were generated on a coarse grid with $\Delta m_{0}=100 \mathrm{GeV} / \mathrm{c}^{2}$ and $\Delta m_{1 / 2}=$ $100 \mathrm{GeV} / \mathrm{c}^{2}$, starting from the point $m_{0}=100 \mathrm{GeV} / \mathrm{c}^{2}, m_{1 / 2}=100 \mathrm{GeV} / \mathrm{c}^{2}$.

The $5 \sigma$ reach of this analysis, including systematic uncertainties, for different integrated luminosities and assuming no re-optimisation of the selection cuts is shown on Fig. 13.7. By the time CMS collects integrated luminosity $30 \mathrm{fb}^{-1}$, the high mass point HM1 becomes interesting for possible discovery. For comparison, $\mathcal{L}=1 \mathrm{fb}^{-1}$ and $\mathcal{L}=100 \mathrm{fb}^{-1}$ are also shown in the figure. Clearly, the systematics for $\mathcal{L}=1 \mathrm{fb}^{-1}$ will be higher than that assumed in this work, nevertheless these results strongly suggest (provided systematics can be brought 


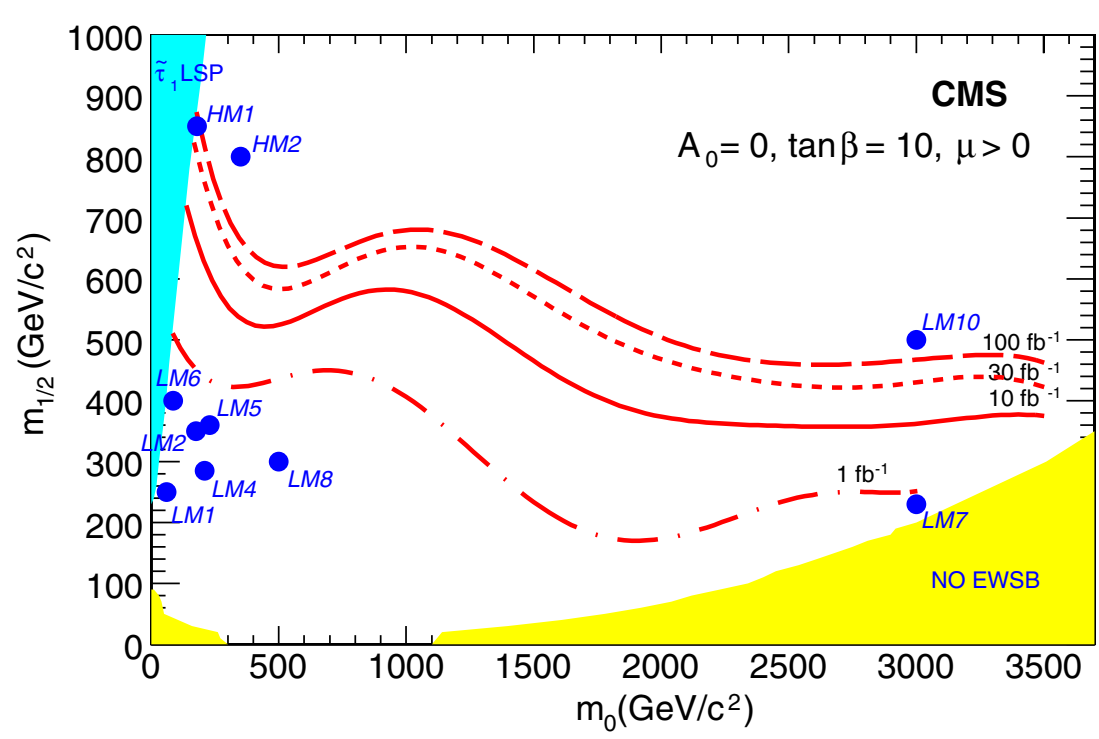

Figure 13.7. CMS reach contours (systematic uncertainties included) in the $\left(m_{0}, m_{1 / 2}\right)$ plane for SUSY processes involving two prompt same-sign muons for $\mathcal{L}=1 \mathrm{fb}^{-1}$ (dot-dashed line), $\mathcal{L}=10 \mathrm{fb}^{-1}$ (solid line), $\mathcal{L}=30 \mathrm{fb}^{-1}$ (short dashed line) $\mathcal{L}=100 \mathrm{fb}^{-1}$ (dashed line). The other mSUGRA parameters are fixed to $\tan \beta=10, \mu>0$ and $A_{0}=0$. Points corresponding to the full detector simulation and reconstruction are also shown (solid circles).

under control) that most of the low mass mSUGRA points are well within reach of CMS during the early running of the LHC.

\subsection{Inclusive analyses with opposite sign dileptons}

Final states with opposite sign dileptons, originating from the decay $\tilde{\chi}_{2}^{0} \rightarrow \tilde{l}_{R} l \rightarrow l^{+} l^{-} \tilde{\chi}_{1}^{0}$ in the cascade decays of squarks and gluinos provide a clean signature of SUSY with isolated leptons, high $p_{\mathrm{T}}$ jets and missing transverse energy [677]. In addition, the dilepton invariant mass distribution for this decay is expected to have a triangular shape with a sharp upper edge, which renders this signature striking and useful for further characterisation of SUSY.

\subsubsection{Signal selection and backgrounds}

The analysis is performed at the LM1 mSUGRA test-point using GEANT-based detailed simulation of the CMS detector [8] and reconstruction [10]. The fast CMS simulation and reconstruction [11] is used to evaluate the discovery reach in the mSUGRA parameter space.

Signal events were generated by ISAJET 7.69 interfaced to PYTHIA 6.225 at the test point LM1, where the NLO cross section at NLO is about $52 \mathrm{pb}$, dominated by the production of $\tilde{q} \tilde{g}$, $\tilde{g} \tilde{g}$ and $\tilde{q} \overline{\tilde{q}}$. The gluino is the heaviest particle and decays to $\tilde{q} q$. While right squarks decay almost directly to the LSP, due to the bino-like nature of the $\tilde{\chi}_{1}^{0}$ at Point LM1, left-handed squarks decay to $\tilde{\chi}_{2}^{0}$ with a branching ratio $\sim 30 \%$.

The SM backgrounds studied consist of $t \bar{t}, W+$ jets, $Z+$ jets, $W W+$ jets, $Z Z+$ jets, $Z b b$ (with leptonic decays of the $Z$ boson), Drell-Yan leptonic events and QCD dijet production processes. 
Table 13.10. Cross section at NLO, selection efficiencies and number of events surviving cuts for signal and background processes.

\begin{tabular}{llrll}
\hline Process & $\sigma(\mathrm{pb})$ & Ev. analysed & $\varepsilon$ & $\mathrm{N}_{e v}$ in $1 \mathrm{fb}^{-1}$ \\
\hline SUSY (LM1) & 52 & $478 \mathrm{k}$ & 0.016 & 853 \\
$t \bar{t}$ & 830 & $913 \mathrm{k}$ & $1.9 \cdot 10^{-4}$ & 155 \\
$W W+$ jets & 188 & $197 \mathrm{k}$ & $1.4 \cdot 10^{-4}$ & 26 \\
$Z+$ jets & $5 \cdot 10^{3}$ & $606 \mathrm{k}$ & $4.8 \cdot 10^{-6}$ & 24 \\
$\mathrm{DY} \rightarrow 2 \mu$ & $3.97 \cdot 10^{3}$ & $916 \mathrm{k}$ & $<1.1 \cdot 10^{-6}$ & $<4$ \\
$\mathrm{DY} \rightarrow 2 \tau$ & $3.97 \cdot 10^{3}$ & $514 \mathrm{k}$ & $1.1 \cdot 10^{-6}$ & 4.5 \\
$Z b b \rightarrow l l b b(l=e, \mu, \tau)$ & 57.4 & $621 \mathrm{k}$ & $8.4 \cdot 10^{-5}$ & 4.83 \\
$\mathrm{P}_{h a t}^{T}>60 \mathrm{GeV} / \mathrm{c}$ & & & & \\
$t \bar{t} b \bar{b}$ & 3.3 & $50 \mathrm{k}$ & $9.8 \cdot 10^{-4}$ & 3.2 \\
$Z Z+$ jets & 11 & $37 \mathrm{k}$ & $2.4 \cdot 10^{-4}$ & 2.7 \\
$W+$ jets & $1.5 \cdot 10^{5}$ & $1765 \mathrm{k}$ & $6.7 \cdot 10^{-9}$ & 1 \\
\hline
\end{tabular}

The SUSY final state studied contains at least two high- $p_{\mathrm{T}}$ isolated leptons, at least two high- $p_{\mathrm{T}}$ jets and large missing transverse energy. The event selection path includes the following requirements:

- the Level-1 and HLT path that requires a single isolated lepton (muon or electron);

- at least two same-flavour opposite-sign (SFOS) isolated leptons $(e$ or $\mu)$ with $p_{\mathrm{T}} \geqslant$ $10 \mathrm{GeV} / \mathrm{c}$ and $\Delta R_{l l} \geqslant 0.2$ and 0.15 for $e e$ and $\mu \mu$, respectively where $\Delta R_{l l}$ is the distance of the two leptons in the $\eta-\phi$ space;

- $E_{\mathrm{T}}^{\text {miss }}>200 \mathrm{GeV}$;

- at least two jets with $p_{\mathrm{T}} \geqslant 100$ and $\geqslant 60 \mathrm{GeV} / \mathrm{c}$ within $|\eta|<3$.

The isolation of the leptons is obtained requiring the sum of $p_{\mathrm{T}}$ of the tracks in a cone of $\Delta R=0.25$ around the lepton track to be less than $5 \mathrm{GeV} / \mathrm{c}$. The $E_{\mathrm{T}}^{\text {miss }}$ is computed from the vectorial sum of the jets and leptons.

These selection criteria result in 853 signal events (which correspond to 913 dilepton pairs) for a luminosity of $1 \mathrm{fb}^{-1}$. The Standard Model background consists of $155 t \bar{t}$ events, 26 events from $\mathrm{WW}+$ jets and 24 events from $\mathrm{Z}+$ jets (Table 13.10). All other backgrounds have been found to be negligible and amount in total to at most 20 events.

\subsubsection{Results for point LMI}

The dilepton invariant mass distribution for $1 \mathrm{fb}^{-1}$ is displayed in Fig. 13.8 showing a clear dilepton edge structure.

The presence of two SFOS leptons can also be due to other processes. Two leptons can result from independent leptonic decays, for example from two charginos or two $W$ 's. In that case the final state contains as many SFOS leptons as different-flavour opposite-sign (DFOS) ones and with identical distributions. The background to the SFOS contribution is removed by subtracting the DFOS events, which leads to the dilepton mass distribution of Figure 13.9. The $t \bar{t}$ and $W W+$ jets backgrounds are also strongly reduced by the flavour subtraction. The resulting dilepton invariant mass distribution is fitted using a triangular function smeared (for resolution effects) with a Gaussian to extract the end-point related to the kinematics of the decay $\tilde{\chi}_{2}^{0} \rightarrow \tilde{l}_{R} l \rightarrow l^{+} l^{-} \tilde{\chi}_{1}^{0}$. The value obtained from $1 \mathrm{fb}^{-1}$ of integrated luminosity is:

$$
M_{l l}^{\max }=80.42 \pm 0.48 \mathrm{GeV} / \mathrm{c}^{2}
$$



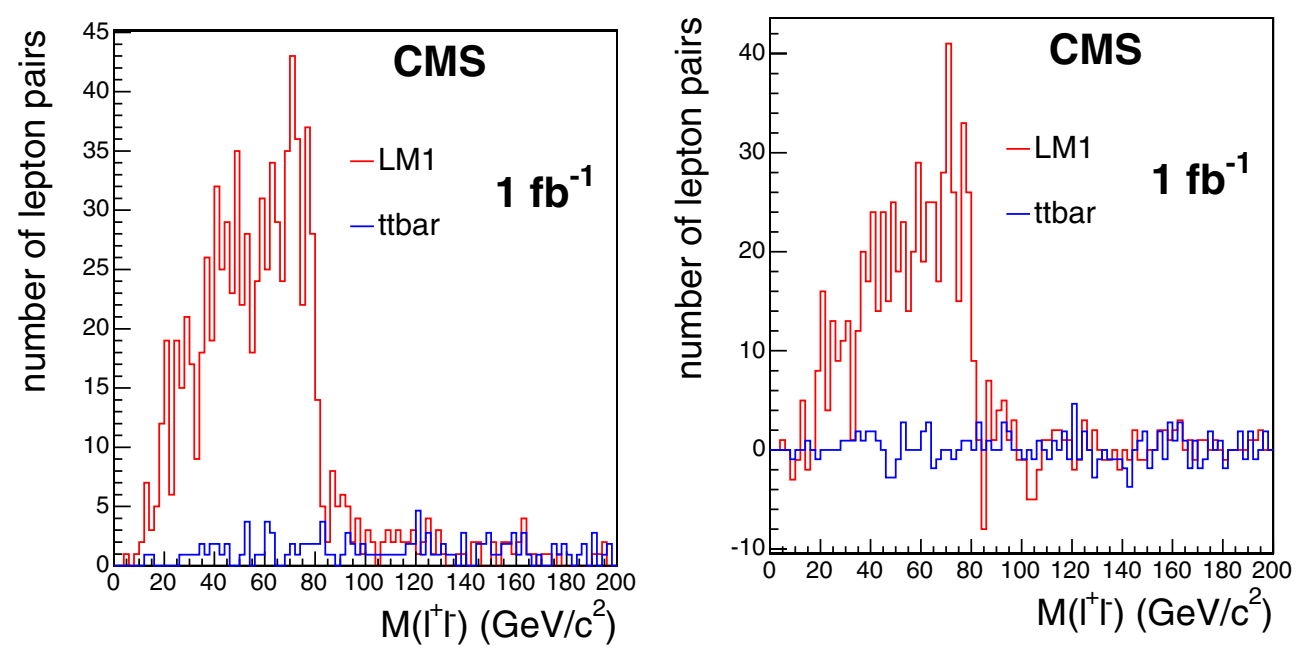

Figure 13.8. Invariant mass distribution of $\mu^{+} \mu^{-}+$ $e^{+} e^{-}$and $\mu^{ \pm} e^{\mp}$ pairs at LM1 for $1 \mathrm{fb}^{-1}$ luminosity. The contribution from the $t \bar{t}$ background is also shown.

Figure 13.9. Invariant mass distribution of $\mu^{+} \mu^{-}+$ $e^{+} e^{-}$and $\mu^{ \pm} e^{\mp}$ pairs at point LM1 for $1 \mathrm{fb}^{-1}$ luminosity after subtracting $e^{+} \mu^{-}$and $\mu^{+} e^{-}$pairs. The contribution from the $t \bar{t}$ background is also shown.

to be compared to the expected value of $81.04 \mathrm{GeV} / \mathrm{c}^{2}$ for the masses $m\left(\tilde{\chi}_{1}^{0}\right)=95, m\left(\tilde{\chi}_{2}^{0}\right)=$ 180 and $m\left(\tilde{l}_{R}\right)=119 \mathrm{GeV} / \mathrm{c}^{2}$. The signal-to-background ratio at point LM1 is 4.1 , the total signal efficiency is $1.6 \%$ and the background composition is $69 \%$ of total ttbar, $11.6 \%$ of total $\mathrm{WW}+$ jets, $10 \% \mathrm{Z}+$ jets, $3 \% \mathrm{DY}, 2 \% \mathrm{Zbb}, 1 \% \mathrm{ttbb}, 1 \% \mathrm{ZZ}+$ jets, fractions the others. The total efficiency for the QCD background is too low to be directly calculated, and is then estimated through a factorisation, considering separately the effects due to the single selection cuts. Although the number of surviving QCD events is expected to be negligible, a residual QCD background is still possible, which will be measured using the real data. A statistical significance of 5 sigma, calculated using $S_{c P}$ defined in Appendix A.1, is achieved with $14 \mathrm{pb}^{-1}$ of integrated luminosity. At this luminosity 12.8 signal events are expected with 3.1 Standard Model background events. Therefore this signature is a strong probe for early discovery of low mass supersymmetry.

Systematic uncertainties have been evaluated under the assumption that control data are used for the Standard Model processes. Hence no uncertainties on the theory cross sections, showering, ISR/FSR, are taken into account. The main systematic uncertainty considered is due to the absolute jet energy scale. $\mathrm{A} \simeq 7 \%$ uncertainty on the jet energy scale for $1 \mathrm{fb}^{-1}$ of data is used while this is expected to be $\simeq 2 \%$ after $10 \mathrm{fb}^{-1}$. After applying the selection cuts this leads to $\mathrm{a} \simeq 20 \%$ systematic uncertainty on the $t \bar{t}$ background and to a $\simeq 8 \%$ systematic uncertainty on the SUSY signal. The electron energy scale uncertainty, expected to be $0.25 \%$, leads to a systematic uncertainty of less than $1 \%$ on the background, and less than $0.1 \%$ on the signal. The total considered systematic uncertainty on the Standard Model background is $20 \%$ at low luminosity, $5 \%$ at high luminosity. The effect on the signal of the Tracker and Muon System misalignment in the first months of LHC run has also been evaluated. The number of selected dimuon (dielectron) pairs is lowered by about $30 \%(10 \%)$ while the total signal selection efficiency is decreased by about $20 \%$. The measurement of the distribution end-point is affected by about $1 \mathrm{GeV} / \mathrm{c}^{2}$. The effect of the electron energy scale uncertainty on the dilepton measurement gives a systematic uncertainty of about $0.15 \mathrm{GeV} / \mathrm{c}^{2}$. 


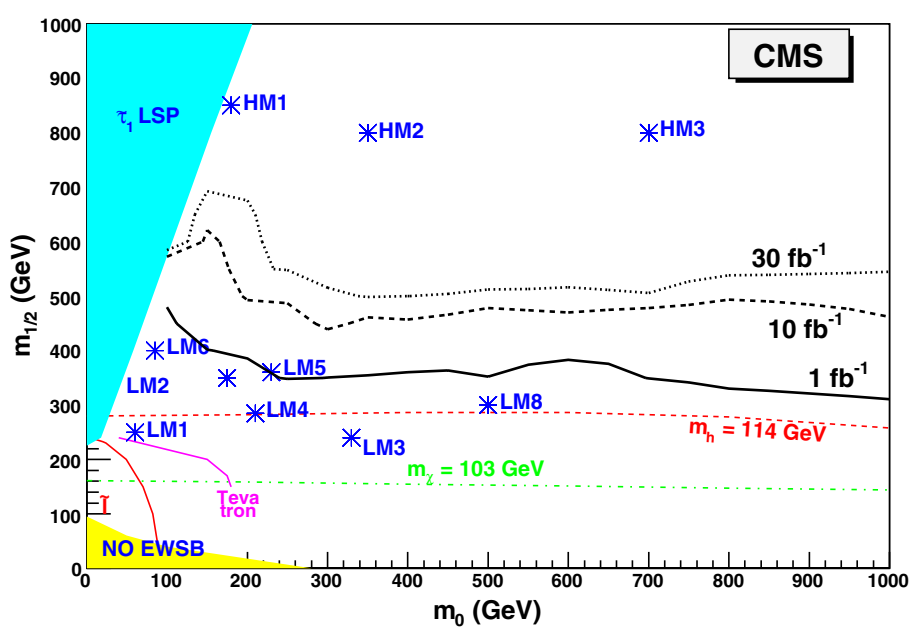

Figure 13.10. $5 \sigma$ discovery reach for the dilepton final state, assuming $\tan \beta=10, A=0, \mu>0$ and $1,10,30 \mathrm{fb}^{-1}$ integrated luminosity (statistical uncertainties only).

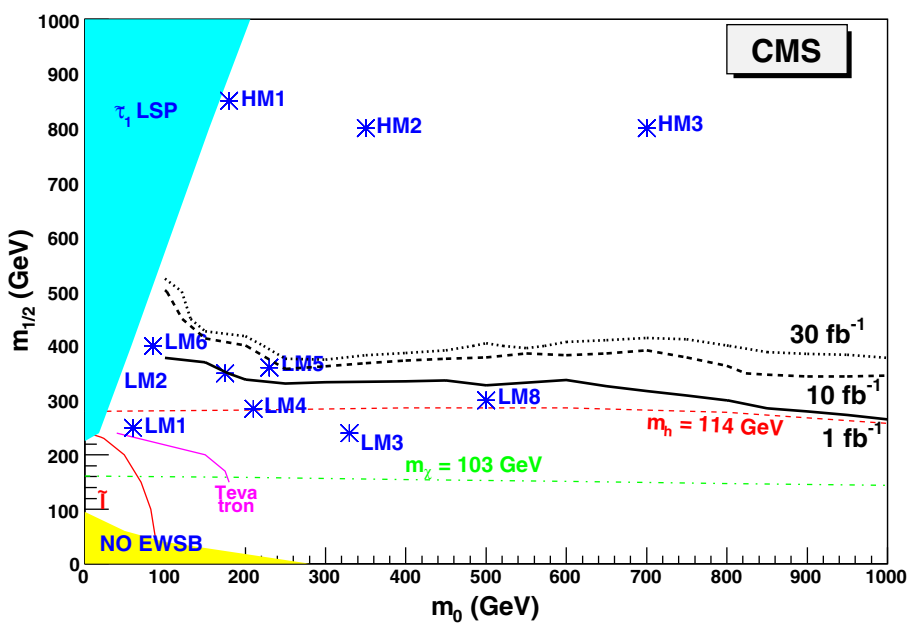

Figure 13.11. $5 \sigma$ discovery reach for $\tan \beta=10$ taking into account background systematic uncertainties.

Taking into account the systematic uncertainties on the Standard Model backgrounds expected after the first $1 \mathrm{fb}^{-1}$ of data, the 5 sigma discovery can be achieved with $17 \mathrm{pb}^{-1}$ of integrated luminosity.

\subsubsection{CMS inclusive reach}

Using the discussed selection path a scan was performed over the mSUGRA parameters in the $\left(m_{0}, m_{1 / 2}\right)$ plane for $\tan \beta=10, A=0, \mu>0$ to determine the $5 \sigma$ discovery reach. The observability of the signal over the Standard Model background uses the dilepton estimates before flavour subtraction. The results of the survey are shown for integrated luminosities of 1,10 and $30 \mathrm{fb}^{-1}$ in Figs. 13.10 and 13.11. It is notable that most of the low mass test-points can be discovered with about $1 \mathrm{fb}^{-1}$. 


\subsection{Inclusive analyses with ditaus}

In this section, $\tilde{\tau}$ production through the $\tilde{\chi}_{2}^{0}$ decays in $\tilde{q}$ or $\tilde{g}$ cascades is investigated. The $\tilde{\tau}$ is produced through $\tilde{\chi}_{2}^{0} \rightarrow \tau^{ \pm} \tilde{\tau}^{\mp}$, which further decays to $\tau \tilde{\chi}_{1}^{0}$ leaving a final state with two taus of opposite sign. The branching fraction of $\tilde{\tau}$ production through $\tilde{\chi}_{2}^{0}$ varying with mSUGRA parameters, the analysis is first carried out at large $\tan \beta$, at the LM2 test point, which parameters are given in Section 13.3.2, where the $\tilde{\chi}_{2}^{0}$ is predicted to decay $95 \%$ of the time into $\tau^{ \pm} \tilde{\tau}^{\mp}$. Results are then generalised to any choice of mSUGRA parameters.

This section studies the opportunity of discovering such a model in the first years of data taking of LHC, with integrated luminosities as low as $0.1 \mathrm{fb}^{-1}$ and up to $10 \mathrm{fb}^{-1}$. The possibility of measuring the SUSY mass spectra associated to this cascade decay (in particular $\tilde{\chi}_{2}^{0}, \tilde{\chi}_{1}^{0}$ and $\tilde{\tau}$ masses) is investigated in Section 13.13.

\subsubsection{Event selection and background studies}

For this analysis, 93.5k events (corresponding to an integrated luminosity of $12.6 \mathrm{fb}^{-1}$ ) were generated at the LM2 test point using ISASUGRA. Those events were further passed through the full simulation of the CMS detector [8] then digitised and reconstructed [10]. The same procedure was applied to the Monte Carlo samples used as SM background in this analysis. However, in some cases, where large statistics were required, the fast simulation program [11] was used. All Monte Carlo samples used in this analysis are produced with leading order Parton Distribution Functions.

Physics processes responsible for $\mathrm{W}$ and $\mathrm{Z}$ production and $t \bar{t}$ which final states may contain several taus and jets are considered as potential background sources. In addition, because of its huge cross section $\left(1.3 \cdot 10^{-4} \mathrm{mb}\right)$ QCD jet production is also considered. The latter can also represent an important source of fake taus as well as fake missing transverse energy $\left(E_{\mathrm{T}}^{\mathrm{miss}}\right)$ due to imprecision in jet energy measurement.

13.9.1.1. Event selection using all reconstructed taus. In this analysis [678], only events passing the JETMET level1 and HLT triggers are accepted. The event selection is then carried out using only the $E_{\mathrm{T}}^{\mathrm{miss}}$, the reconstructed taus and jets. In order to increase the sensitivity of the selection both tau's decaying hadronically and leptonically are considered in this section.

The mSUGRA events are selected with the following requirement:

- $E_{\mathrm{T}}^{\mathrm{miss}}$ larger than $150 \mathrm{GeV}$.

This cut removes a large fraction of Standard Model physics background.

- At least two tau candidates are required.

- At least two jets with $E_{\mathrm{T}}>150 \mathrm{GeV}$.

This requirement is very aggressive on the LM2 events, however it allows to remove most of the Standard Model background.

- $\Delta R$ between any pair of tau's should be smaller than two.

This cut makes use of the fact that in $\tilde{\chi}_{2}^{0}$ decays, taus belonging to a same cascade decay will be produced relatively close to each other while in Standard Model physics processes taus as well as Supersymmetric physics processes such as chargino production (producing one tau in each cascade) tend to be produced in opposite direction. This cut also reduces the amount of wrong pairing.

Both theoretical and experimental systematic uncertainties are considered in this analysis. The theoretical systematic uncertainty is estimated for the signal according to standard CMS guidelines and involves changing the PDF [351] and varying generator parameters governing 
both hard process and fragmentation. Each variation leads to the generation of a new LM2 sample which is then simulated and reconstructed using FaMos and analysed in the same way as the main signal samples. Variations in the number of selected events are then taken as systematic uncertainty. The relative theoretical systematic uncertainty on the signal was found to be $12 \%$. The experimental systematic uncertainties are coming from the Jet energy scale, the $E_{\mathrm{T}}^{\text {miss }}$ and the tau-jet energy scale. These uncertainties are estimated following standard CMS procedure, see appendix B, by varying the jet and tau energies by an amount corresponding to their respective energy scales and redoing the analysis. The uncertainty on $E_{\mathrm{T}}^{\text {miss }}$ is estimated in a similar way by varying the energy of the jets used to estimate $E_{\mathrm{T}}^{\text {miss }}$ within their energy scale. The experimental systematic uncertainty affect the selection of signal events by $11 \%$ for low integrated luminosities (smaller than $1 \mathrm{fb}^{-1}$ ) but for large integrated luminosities the systematic effect is less than $3.2 \%$. The experimental systematic uncertainty on the background is $30 \%$ for integrated luminosities smaller than $1 \mathrm{fb}^{-1}$ and $11 \%$ for larger integrated luminosities.

At $12.67 \mathrm{fb}^{-1}, N_{s}=2735 \pm 273$ (sys) \pm 52 (stat) events from the signal and $N_{b k g}=938 \pm$ 103 (sys) \pm 114 (stat) events from the background survive the selection. 50\% of the remaining background is coming from QCD, $39 \%$ from $t \bar{t}$ and $11 \%$ from $\mathrm{W}+\mathrm{jets}$.

To this selection corresponds a ratio signal over background $S / B=2.9$. The global efficiency of the selection of the signal is around 3\% (of which 88\% are SUSY events with at least two taus), while only $0.001 \%$ of the background remains after selection. Using $S_{c L}$ significance, defined in Appendix A.1, it is possible to estimate that a $5 \sigma$ discovery can be achieved with only $0.07 \mathrm{fb}^{-1}$. Using $S_{c P}$ significance [679], which takes into account systematic uncertainties on the background, a $5 \sigma$ discovery can be expected with a luminosity of $0.125 \mathrm{fb}^{-1}$.

13.9.1.2. Event selection using only reconstructed taus decaying hadronically. If only taus decaying hadronically are used in the selection described in 13.9.1.1, both signal and backgrounds are affected differently.

At $12.67 \mathrm{fb}^{-1}, N_{s}=1447 \pm 144$ (sys) \pm 38 (stat) events from the signal and $N_{b k g}=543 \pm$ 60 (sys) \pm 112 (stat) events from the background survive the selection. $70 \%$ of the remaining background is coming from QCD, $20 \%$ from $t \bar{t}$ and $10 \%$ from $\mathrm{W}+$ jets. To this selection corresponds a ratio signal over background $S / B=2.6$. The global efficiency of the selection of the signal is around $1.5 \%$ (of which $88 \%$ are SUSY events with at least two taus), while only $0.0006 \%$ of the background remains after selection. This time, using $S_{c L}$ a $5 \sigma$ discovery is achieved with only $0.14 \mathrm{fb}^{-1}$. Using $S_{C P}$ significance [679], which takes into account systematic uncertainties on the background, a $5 \sigma$ discovery can be expected with a luminosity of $0.26 \mathrm{fb}^{-1}$.

\subsubsection{Discovery potential of $m S U G R A$ with ditaus final states}

A scan of the mSUGRA $\left(m_{0}, m_{1 / 2}\right)$ parameters plane is performed in order to delimit the mSUGRA parameter region where SUSY could be discovered with this analysis. Because the analysis focuses on ditau final states and since the respective branching ratio to ditaus and to other leptons from SUSY may vary by large amounts in the mSUGRA parameter space, allowing large contamination from leptons into ditaus final states the scan is performed using only hadronic tau decays as described in section 13.9.1.2.

This scan is achieved by generating many mSUGRA samples varying $m_{0}$ and $m_{1 / 2}$ values so that the entire region of the plane $\left(m_{0}, m_{1 / 2}\right)$ below $m_{0}<1500 \mathrm{GeV}$ and $m_{1 / 2}<800 \mathrm{GeV}$ is covered. The samples were generated with ISASUGRA 7.69 then simulated and reconstructed 


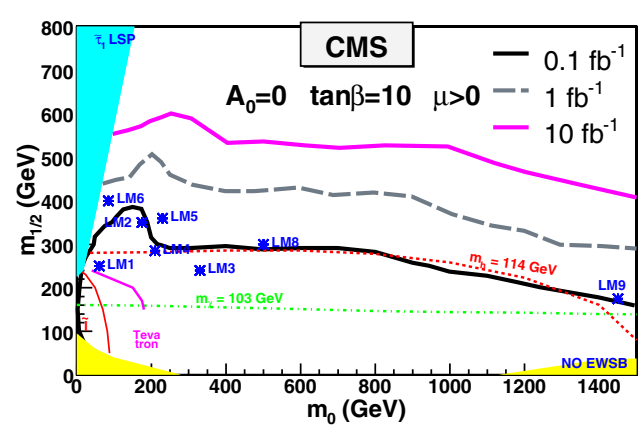

Figure 13.12. Inclusive ditau analysis discovery potential for mSUGRA between 0.1 and $30 \mathrm{fb}^{-1}$ for $\tan \beta=10$ including only statistical uncertainties.

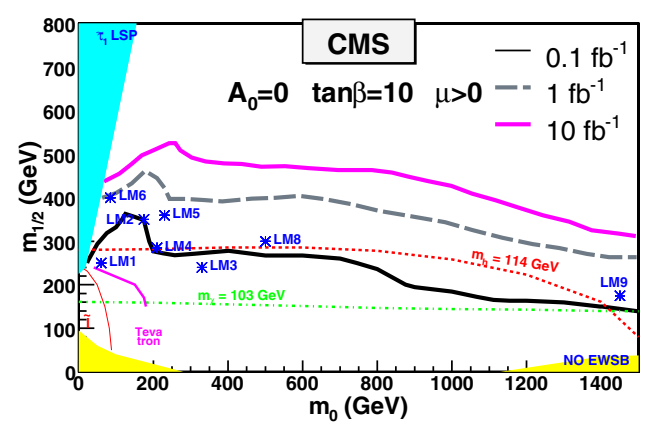

Figure 13.14. Inclusive ditau analysis discovery potential for mSUGRA between 0.1 and $30 \mathrm{fb}^{-1}$ for $\tan \beta=10$ where both statistical and systematic uncertainties are taken into account.

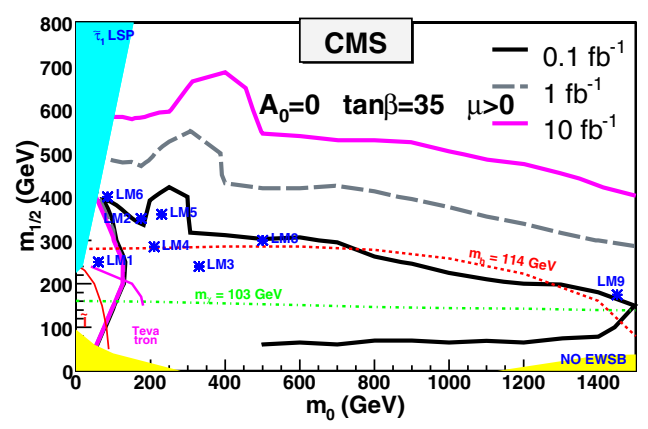

Figure 13.13. Inclusive ditau analysis discovery potential for mSUGRA between 0.1 and $30 \mathrm{fb}^{-1}$ for $\tan \beta=35$ including only statistical uncertainties.

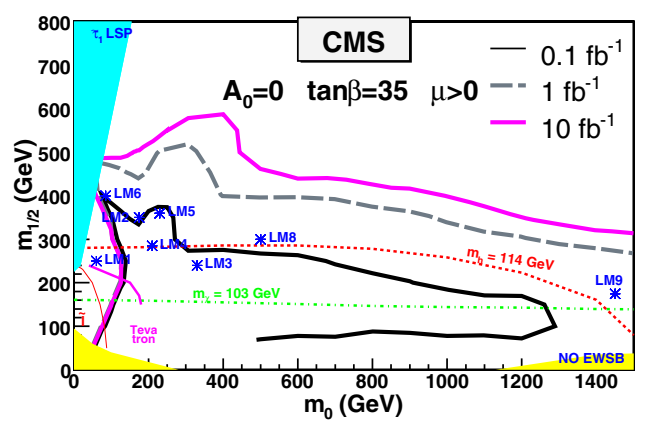

Figure 13.15. Inclusive ditau analysis discovery potential for mSUGRA between 0.1 and $30 \mathrm{fb}^{-1}$ for $\tan \beta=35$ where both statistical and systematic uncertainties are taken into account.

with FAMOS and analysed in the same way as the LM2 sample. The resulting number of events surviving the selection were used to estimate the significance at each point of the mSUGRA parameter plane. Two types of significance are estimated here, $S_{c L}$ which accounts only for statistical uncertainties and $S_{c p}$ which accounts for both statistical and systematics effects on the background. The resulting $5 \sigma$ contours over the mSUGRA $\left(m_{0}, m_{1 / 2}\right)$ parameter plane obtained with $S_{c l}$ for several integrated luminosities between 0.1 and $30 \mathrm{fb}^{-1}$ are shown in Figs. 13.12 and 13.13 for $\tan \beta=10$ and $\tan \beta=35$, respectively. Results obtained with $S_{c p}$ are shown in Figs. 13.14 and 13.15. The region where a $5 \sigma$ discovery is possible is somewhat shrunk, especially for the very early measurement at $0.1 \mathrm{fb}^{-1}$ as a precise knowledge of the jet energy scale and of the measurement of the $E_{\mathrm{T}}^{\mathrm{miss}}$ will still be limited. However, a large region is accessible with larger integrated luminosities.

\subsection{Inclusive analyses with Higgs}

This section describes the potential of the CMS experiment to discover a light supersymmetric Higgs boson $\left(h^{0}\right)$ produced at the end of a cascade of supersymmetric particles starting with the strong production of squarks $(\tilde{q})$ and gluinos $(\tilde{g})$. Because of the cascade production mechanism, the events can be efficiently triggered using inclusive SUSY triggers such as jet $+E_{\mathrm{T}}^{\mathrm{miss}}$, and the dominant $h^{0} \rightarrow b \bar{b}$ decay mode of the Higgs boson can be exploited. 
This analysis focuses on a full CMS detector simulation [8] and event reconstruction [10] at the mSUGRA point LM5, defined in Section 13.3.2. The total SUSY cross section at this parameter point is about $7.75 \mathrm{pb}$ at NLO.

All SUSY channels leading to a light Higgs boson in the final state have been taken into account. The signal events are characterised by at least two b-tagged jets, an important missing transverse energy $\left(E_{\mathrm{T}}^{\text {miss }}\right)$ and multiple hard jets. This signature allows to suppress the majority of the $b \bar{b}$ background due to SM processes (mainly top pair production $t \bar{t}, W^{ \pm}+$jets, $Z^{0}+$ jets).

\subsubsection{Signal selection and backgrounds}

This analysis has been developed based on the CMS reconstruction. The two main algorithms used for the signal reconstruction are the jet reconstruction algorithm (the Iterative cone algorithm with a cone size of 0.5 radians and the GammaJet calibration) and the b-tagging algorithm (Combined b-tagging algorithm, see the PTDR Volume 1, Section 12.2).

A first rejection of the Standard Model backgrounds happens at the online trigger stage. The Level-1 and the High Level Trigger (HLT) efficiencies for the signal and background have been evaluated. The trigger path used for this analysis consists of the Level-1 and HLT Jet $+E_{\mathrm{T}}^{\text {miss }}$ stream. This particular trigger is already an important tool in rejecting Standard Model backgrounds, for example it rejects $96 \%$ of the $t \bar{t}$ background while keeping $79 \%$ of the signal events.

In order to further remove the SM background events and reduce the SUSY background, a number of offline selection cuts are applied: a minimal number of four jets with a transverse energy above $30 \mathrm{GeV}$ is required, of which at least two are b-tagged with high quality (i.e. a b-tag discriminator greater than 1.5).

The mean b-tagging efficiency is found to be $50 \%$ with a mistagging rate of about $1.6 \%$, for $u, d, s$ quarks and gluons, and $12 \%$ for $c$ quarks. The mean $b$ jet energy originating from the Higgs decay is approximately $70 \mathrm{GeV}$, corresponding to a b-tagging efficiency of about $50 \%$ at this energy. This means that approximately $25 \%$ of the signal events will pass the double b-tag criterion.

Other variables have been identified in order to improve the signal over background ratio, in particular for the most problematic $t \bar{t}$ background: the $E_{\mathrm{T}}^{\text {miss }}$, the first, second and third highest jet $P_{t}$. The selection requires a $E_{\mathrm{T}}^{\text {miss }}>200 \mathrm{GeV}$, the highest jet $p_{t}$ in the event $>200 \mathrm{GeV} / \mathrm{c}$, the second highest jet $p_{t}$ in event $>150 \mathrm{GeV} / \mathrm{c}$, the third highest jet $p_{t}$ in event $>50 \mathrm{GeV} / \mathrm{c}$.

Next, in order to select the b-jet pair coming from the Higgs decay, two methods are used. First, the Hemisphere separation technique (see section 13.4) is applied to identify two groups of jets in the detector, each group associated with an initial squark and/or gluino cascade. After that, the b-jet pairing is done only in each of these groups separately, reducing the number of possible combinations by a large factor. In addition, as the Higgs is relatively heavy, its decay products have an important boost leading to a small angle $\Delta R=\sqrt{\Delta \eta^{2}+\Delta \phi^{2}}$ between the two $\mathrm{b}$ jets. Therefore, in case of multiple possible combinations inside one hemisphere, the pair with the smallest $\Delta R$ value within $\Delta R<1.5$ is chosen. This procedure gives an efficiency of around $40 \%$ and strongly suppresses the combinatorial background.

The full selection chain leads to a signal efficiency of about $8 \%$ for all SUSY channels yielding a Higgs. The global rejection factor for $t \bar{t}$ events, including the rejection made by the Jet $+E_{\mathrm{T}}^{\text {miss }}$ trigger, is close to $4.6 \cdot 10^{4}$. No $Z+$ jets, $W+$ jets nor $\mathrm{QCD}$ events from the full simulation samples pass the previously described series of cuts, hence the only remaining background is from $t \bar{t}$. The resulting SUSY signal over SM background ratio is $>70.61 \%$ 


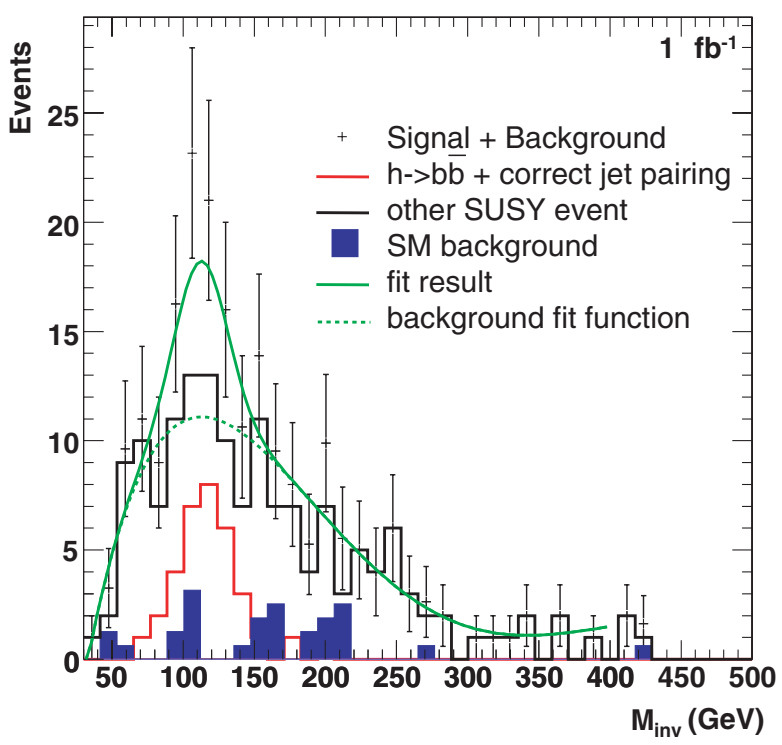

Figure 13.16. Invariant mass distribution of $b \bar{b}$ jets for the search of Higgs final states with $1 \mathrm{fb}^{-1}$.

of the SUSY signal comes from events with a true $h^{0}$, but only part of those have the correct b-jet pairing with both jets from the $h^{0}$.

\subsubsection{Results at LM5 and systematics}

The resulting invariant mass distribution, after the selection cuts described above, is shown in Fig. 13.16. The plot corresponds to the expected statistics equivalent to $1 \mathrm{fb}^{-1}$ of integrated luminosity. A peak around $116 \mathrm{GeV} / \mathrm{c}^{2}$ is visible. The main background is due to the remaining SUSY background events and some $t \bar{t}$ events.

A fit was performed representing the background by a fifth order polynomial and approximating the Higgs signal by a Gaussian. The r.m.s of the Gaussian has been fixed to $18 \mathrm{GeV}$, which is the Higgs mass resolution estimated using the Monte Carlo truth. In real data, this number will be determined from studying b-rich samples such as $t \bar{t}$. The results of the fit for the equivalent of $1 \mathrm{fb}^{-1}$ of data are the following: the Higgs mass is found to be $(112.9 \pm 6.6) \mathrm{GeV} / \mathrm{c}^{2}$ (for a generated mass of $116 \mathrm{GeV} / \mathrm{c}^{2}$ ) and the fraction of signal in the distribution is evaluated to be $0.28 \pm 0.08$. The significance $S_{C L}$, directly extracted from the fraction of signal in the histogram, is found to be 4.5. A significance of 5 should be achieved with approximately $1.5 \mathrm{fb}^{-1}$ luminosity.

For $1 \mathrm{fb}^{-1}$, the jet energy scale and $E_{\mathrm{T}}^{\text {miss }}$ uncertainties have been estimated assuming a linear evolution from $\pm 15 \%$ to $\pm 5 \%$ for low energy jets (below $50 \mathrm{GeV}$ ) and then fixed at $\pm 5 \%$ for higher energy jets. As the $E_{\mathrm{T}}^{\text {miss }}$ is computed from the jets, a correction on the jet energy is automatically propagated to its estimation. The effects are about $15 \%$ on the SUSY event selection and $17 \%$ on the $t \bar{t}$ event rejection respectively. The impact on the Higgs mass measurement have been estimated to be $\pm 7.5 \mathrm{GeV} / \mathrm{c}^{2}$; on the signal fraction, the effect is \pm 0.04 .

Another systematic uncertainty is introduced by the misalignment of the tracker. Both the short and long term misalignment scenarios have been investigated. The short term misalignment corresponds to a displacement of the tracker (strips/pixels) = $(100 \mu \mathrm{m} / 10 \mu \mathrm{m})$, while the long term misalignment takes the following shift of the tracker (strips $/$ pixels $)=(20 \mu \mathrm{m} / 10 \mu \mathrm{m})$ into account. The misalignment of the tracker reduces the 


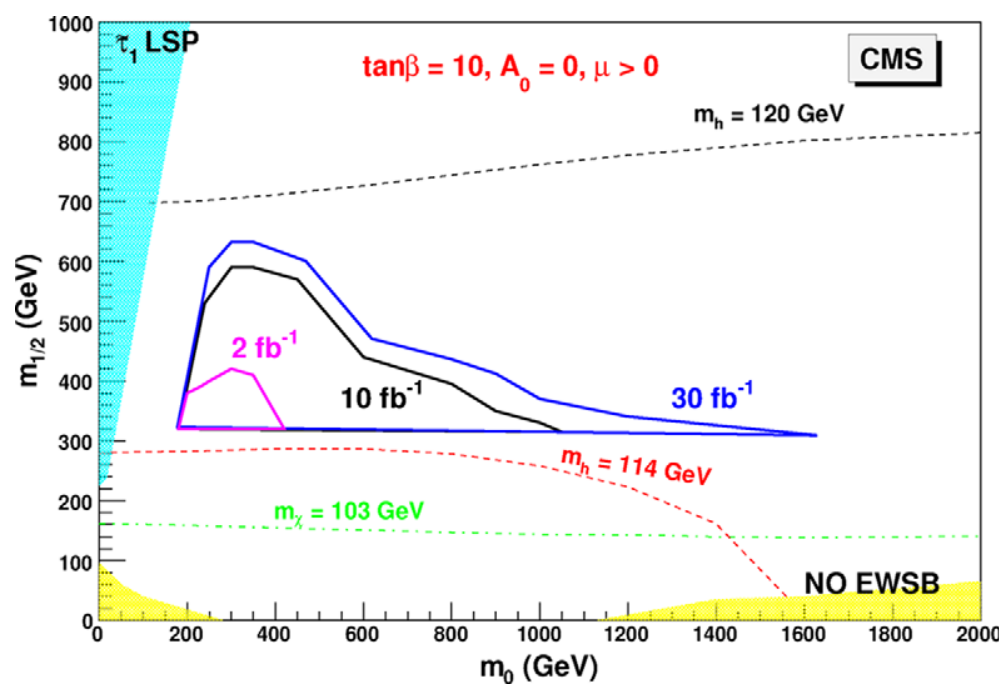

Figure 13.17. Higgs discovery reach in SUSY cascades for 2,10 and $30 \mathrm{fb}^{-1}$.

track reconstruction resolution, which results in a reduced b-tagging efficiency and which in its turn causes a reduced signal event selection efficiency. The long term misalignment scenario results in a drop of the signal selection efficiency of $(\sim 10 \%)$ compared to the case of an aligned detector; for the short term misalignment case, the reduction is $(\sim 17 \%)$. No effect on the position/width of the Higgs mass peak was observed.

Finally, the systematics due to the choice of the background fit function has been estimated to be small (by changing the background function to a third, fourth, sixth or a seventh order polynomial): $\pm 0.3 \mathrm{GeV} / \mathrm{c}^{2}$ on the Higgs mass and \pm 0.01 on the signal fraction.

The final result including all the previously discussed systematics for $1 \mathrm{fb}^{-1}$ of integrated luminosity is then $112.9 \pm 6.6$ (stat) \pm 7.5 (syst) $\mathrm{GeV} / \mathrm{c}^{2}$ for the Higgs mass and $0.28 \pm 0.08$ (stat) \pm 0.04 (syst) for the signal fraction.

\subsubsection{CMS reach for inclusive Higgs production}

After establishing the visibility of the signal for the LM5 point, a scan was performed in the $\left(m_{0}, m_{1 / 2}\right)$ plane in order to determine the region where a $5 \sigma$ discovery could be made with 2,10 and $30 \mathrm{fb}^{-1}$.

First, an effective cross section $(\sigma \times B R(h 0))$ was used (calculated with PROSPINO and ISASUGRA) to obtain an estimate of the reach. Using this first estimate, 40 points were chosen for which the full spectrum was calculated and a fast simulation was performed with FAMOS [11]. The same selection criteria as for LM5 point were applied, and the number of Higgs signal and background events was determined. Given that the background is dominated by SUSY events, the signal and background are similarly affected by the systematic uncertainties and the effect on the significance is small. The same significance definition $\left(S_{C L}\right)$ was used in order to determine the 5-sigma contours. Comparing the ORCA/FAMOS results at LM5, the significances obtained with both programs were found to agree well.

The result of the scan is displayed in the reach plot in Fig. 13.17. Although for $1 \mathrm{fb}^{-1}$ the sensitivity remains below $5 \sigma$, everywhere a sizeable region of the $\left(m_{0}, m_{1 / 2}\right)$ plane, up to $1100(1600) \mathrm{GeV}$ in $m_{0}$ and $600(650) \mathrm{GeV}$ in $m_{1 / 2}$, can be covered with $10(30) \mathrm{fb}^{-1}$. With $2 \mathrm{fb}^{-1}$ of integrated luminosity, a small region of the plane can already be probed. The plot assumes $\tan \beta=10, A_{0}=0$, and a positive sign of $\mu$. 
Table 13.11. Number of events for signal $\left(\tilde{\chi}_{2}^{0} \rightarrow Z^{0}+\tilde{\chi}_{1}^{0}, Z^{0} \rightarrow e^{+} e^{-}, \mu^{+} \mu^{-}\right)$and background before and after selection criteria for $10 \mathrm{fb}^{-1}$. The numbers below $Z j$ specify the range of partonic $p_{\mathrm{T}}$ in $\mathrm{GeV} / \mathrm{c}$.

\begin{tabular}{llllllll}
\hline & $\begin{array}{l}\text { LM4 } \\
\text { with } \tilde{\chi}_{2}^{0}\end{array}$ & $\begin{array}{l}\text { LM4 } \\
\text { no } \tilde{\chi}_{2}^{0}\end{array}$ & ZZj & ZWj & WWj & $t \bar{t}$ & $\begin{array}{l}\mathrm{Zj} \\
85-250\end{array}$ \\
\hline$\sigma \mathrm{NLO}(\mathrm{pb})$ & 0.664 & 17.4 & 15.5 & 51.5 & 270 & 830 & 116.7 \\
$10 \mathrm{fb}^{-1}$ & & & & & & & \\
total events & 6640 & $173.8 \mathrm{~K}$ & $155 \mathrm{~K}$ & $515 \mathrm{~K}$ & $2.7 \mathrm{M}$ & $8.3 \mathrm{M}$ & $1.17 \mathrm{M}$ \\
L1+HLT & 6032 & $81.7 \mathrm{~K}$ & $12.6 \mathrm{~K}$ & $24.4 \mathrm{~K}$ & $174 \mathrm{~K}$ & $973 \mathrm{~K}$ & $462 \mathrm{~K}$ \\
OS leptons & 4489 & 7147 & 9124 & $14.7 \mathrm{~K}$ & $26.3 \mathrm{~K}$ & $268 \mathrm{~K}$ & $331 \mathrm{~K}$ \\
$M_{l l}$ & 3773 & 804 & 6999 & $11.5 \mathrm{~K}$ & 2406 & $23.1 \mathrm{~K}$ & $249 \mathrm{~K}$ \\
$E_{\mathrm{T}}^{\text {miss }}$ & 1420 & 306 & 32 & 24 & 70 & 149 & 44 \\
$\Delta \phi_{l l}$ & 1289 & 264 & 31 & 22 & 47 & 61 & 35 \\
\hline
\end{tabular}

\subsection{Inclusive SUSY search with $Z^{0}$}

\subsubsection{Topology of the signal}

SUSY processes leading to final states with $Z^{0}$ can be detected in CMS using the $Z^{0}$ decays into same flavour opposite sign (SFOS) lepton pairs. The detection of SUSY in the mSUGRA framework through the decay $\tilde{\chi}_{2}^{0} \rightarrow Z^{0}+\tilde{\chi}_{1}^{0}$ is the scope of this study. The mSUGRA testpoint LM4 with the parameters described in Section 13.3 is chosen. The $\tilde{\chi}_{2}^{0}$ is produced mainly through the cascade decays of gluinos $\left(M_{\tilde{g}}=695 \mathrm{GeV}\right)$ and squarks (mainly the $\tilde{b}_{1}$ with $M_{\tilde{b}_{1}}=601 \mathrm{GeV}$ ). The decays of the second neutralino to $Z^{0}$ have a large branching ratio $(\sim 100 \%)$. The signal events are characterised by large missing $E_{\mathrm{T}}$ (due to the undetectable LSP) and the SFOS lepton pair from $Z^{0}$. The analysis details can be found in [680].

The main Standard Model backgrounds originate from the production of one or more $Z^{0}$ bosons in association with jets as well as $t \bar{t}$. In addition SUSY events contain dileptons that do not originate from the above neutralino decay chain and large missing transverse energy. These events are considered as signal for SUSY detection but as background for the $\tilde{\chi}_{2}^{0}$ detection. The following backgrounds were considered in this study: dibosons $(Z Z+j, Z W+j, W W+j)$, inclusive top $(t \bar{t})$ and $Z+$ jets. The signal events were generated interfacing ISAJET 7.69 with PYTHIA. Unless otherwise stated all events are fully simulated and analysed using the CMS full detector simulation [8] and reconstruction [10] packages. The next to leading order (NLO) cross sections of the relevant processes are shown in Table 13.11.

\subsubsection{Event selection}

The following requirements are imposed in order to efficiently select the signal and reject the background events. All criteria were chosen so that the final SUSY search significance estimator $S_{c 1}[102,681]$ for $10 \mathrm{fb}^{-1}$ integrated luminosity is maximised. Very similar requirements maximise also significance estimator $S_{L 2}$ [102] used in the case of $1 \mathrm{fb}^{-1}$ integrated luminosity. The effect of the selection requirements on the signal and on each background sample separately can be seen in Table 13.11 for $10 \mathrm{fb}^{-1}$ integrated luminosity.

- Events are required to pass the HLT dielectron or dimuon triggers.

- An $e^{+} e^{-}$or $\mu^{+} \mu^{-}$pair with lepton $p_{\mathrm{T}}>17 \mathrm{GeV}$ for electrons and $p_{\mathrm{T}}>7 \mathrm{GeV}$ for muons (as per L1 trigger requirements). Each lepton is required to be within $|\eta|<2.4$.

- The SFOS lepton pair invariant mass is required to be consistent with the $Z^{0}$ mass, i.e. $81 \mathrm{GeV}<M_{l l}<96.5 \mathrm{GeV}$. The reconstructed masses for the $e^{+} e^{-}$and the $\mu^{+} \mu^{-}$pairs and 

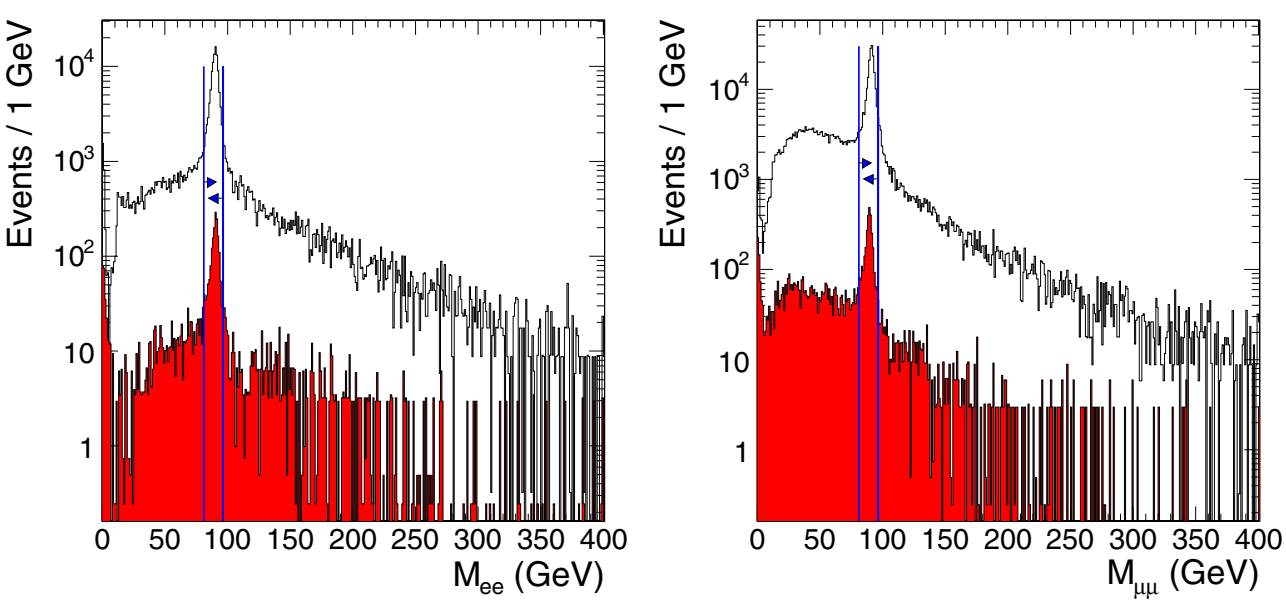

Figure 13.18. Reconstructed masses for (left) $e^{+} e^{-}$and (right) $\mu^{+} \mu^{-}$pairs for the background and for the signal (shaded) events. SUSY events not involving $\tilde{\chi}_{2}^{0}$ are considered signal. The vertical lines denote the imposed mass requirement.
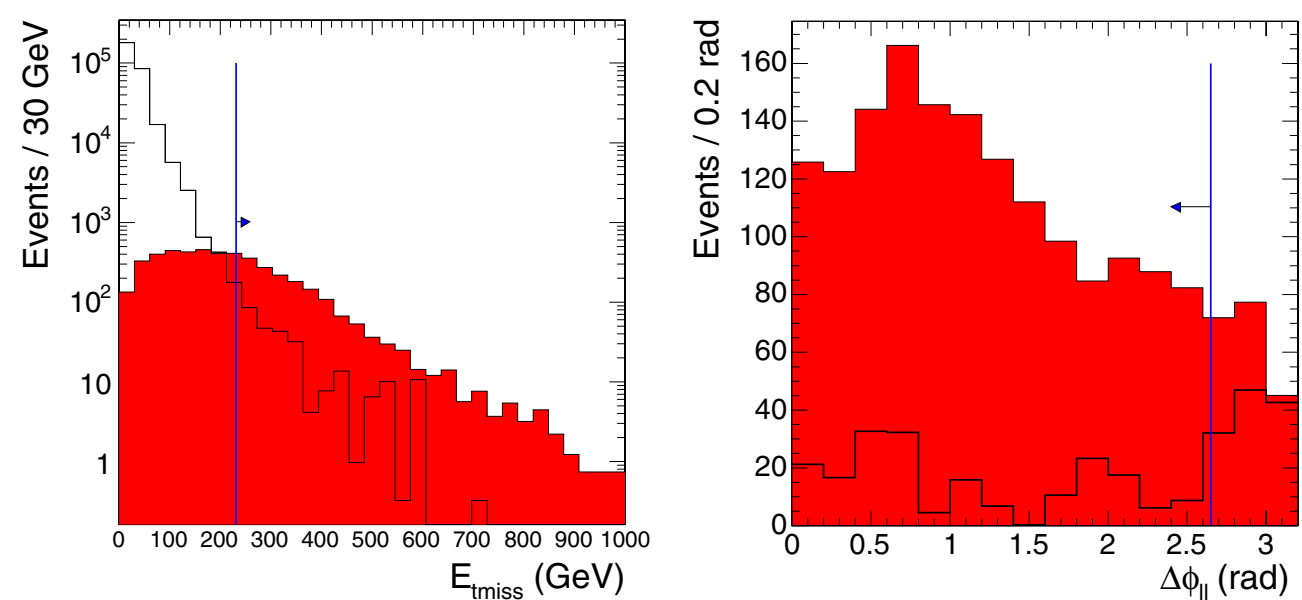

Figure 13.19. $E_{\mathrm{T}}^{\text {miss }}$ (left) and $\Delta \phi$ between the two leptons (right) for background (black line) and signal (shaded) events. SUSY events not involving $\tilde{\chi}_{2}^{0}$ are considered signal. The vertical lines denote the $E_{\mathrm{T}}^{\mathrm{miss}}$ and $\Delta \phi$ requirements.

the mass requirements are shown in Figs. 13.18 (left) and (right) respectively. This cut reduces backgrounds not involving a $Z^{0}(t \bar{t}, \mathrm{WW}+\mathrm{j})$ and the sample of SUSY events not involving $\tilde{\chi}_{2}^{0}$.

- The missing transverse energy $E_{\mathrm{T}}^{\text {miss }}$ is required to be greater $230 \mathrm{GeV}$. This requirement reduces all backgrounds as seen in Fig. 13.19 (left). It allows, however, for enough signal and background events in order to maintain good statistics both for $1 \mathrm{fb}^{-1}$ and for $10 \mathrm{fb}^{-1}$ integrated luminosity.

- The angle $\Delta \phi$ between the two leptons of the lepton pair that reconstructs the mass of $Z^{0}$ is required to be less than $2.65 \mathrm{rad}$. The $\Delta \phi$ distribution is shown in Fig. 13.19 (right) for signal and background. This requirement targets the remainder of the $t \bar{t}$ and the $\mathrm{WW}+\mathrm{j}$ backgrounds that survived the $E_{\mathrm{T}}^{\text {miss }}$ requirement. 

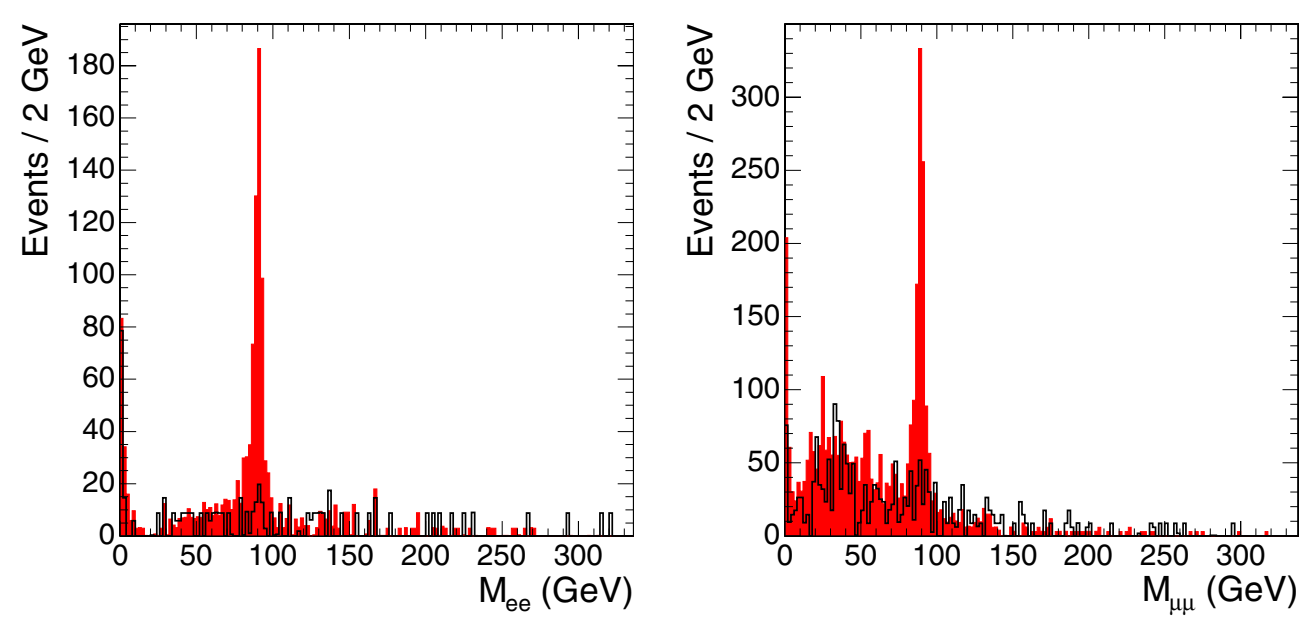

Figure 13.20. Reconstructed masses for (left) $e^{+} e^{-}$and (right) $\mu^{+} \mu^{-}$pairs for the background and for the signal (shaded) events after the cut on $E_{\mathrm{T}}^{\mathrm{miss}}$. SUSY events not involving $\tilde{\chi}_{2}^{0}$ are considered signal.

\subsubsection{Results and systematic uncertainties}

The reconstructed masses for the $e^{+} e^{-}$and the $\mu^{+} \mu^{-}$pairs without the $Z^{0}$ mass cut but after the cut on $E_{\mathrm{T}}^{\text {miss }}$ are shown in Fig. 13.20 (left) and (right) respectively. A clear $Z^{0}$ peak from the signal is observed.

After the application of the above criteria and for $10 \mathrm{fb}^{-1}$ integrated luminosity we have 1553 SUSY events and 196.5 Standard Model background events in the $Z^{0}$ window. This gives a signal over background ratio of 8 and inside the signal events $83 \%$ originate from a $\tilde{\chi}_{2}^{0}$ decay. The total efficiency for $Z^{0}$ events from a $\tilde{\chi}_{2}^{0}$ decay is $19.4 \%$. The background is composed of $31 \% t \bar{t}, 24 \% \mathrm{WW}, 18 \% \mathrm{Zj}, 16 \% \mathrm{ZZ}$ and $11 \% \mathrm{ZW}$.

The significance based on statistical uncertainties only has been evaluated by means of $S_{C L}$, defined in Appendix A.1. A significance of $5 \sigma$ would be reached after $0.06 \mathrm{fb}^{-1}$ if systematic effects were negligible.

When LHC will start running many uncertainties will be controlled from data. In this analysis relevant uncertainties are the lepton $P_{t}$ resolution and the $E_{\mathrm{T}}^{\text {miss }}$ uncertainty. The lepton $P_{t}$ resolution $(\sim 3 \%)$ introduces an uncertainty of $2.7 \%$ in the number of background events. The dominant systematic, however, is the $E_{\mathrm{T}}^{\text {miss }}$ energy scale uncertainty which is estimated to $\sim 5 \%$ and which introduces a $20 \%$ uncertainty in the number of background events, nearly independent of the background channel. The significance was recomputed after including the systematic uncertainties using $S_{c 12 s}$ (see Appendix A.1), which increases the required integrated luminosity for a $5 \sigma$ discovery to $\sim 0.1 \mathrm{fb}^{-1}$.

\subsubsection{CMS reach for inclusive $Z^{0}$ search}

A scan was performed over the mSUGRA $m_{0}, m_{1 / 2}$ parameter space in order to determine the range over which the above analysis can reveal new physics. The test points were taken at high density in the area where the $Z^{0}$ has high production cross section (especially due to the decay $\tilde{\chi}_{2}^{0} \rightarrow Z^{0}+\tilde{\chi}_{1}^{0}$ ). This is an almost horizontal band in the $m_{0}-m_{1 / 2}$ plane between $m_{1 / 2} \sim 240 \mathrm{GeV} / \mathrm{c}^{2}$ and $m_{1 / 2} \sim 340 \mathrm{GeV} / \mathrm{c}^{2}$. Points were also taken at higher and lower $m_{1 / 2}$ values, because there is an excess of lepton pairs created due to SUSY processes. These may have invariant mass close to the $Z^{0}$ mass and pass analysis cuts assisting in the detection 


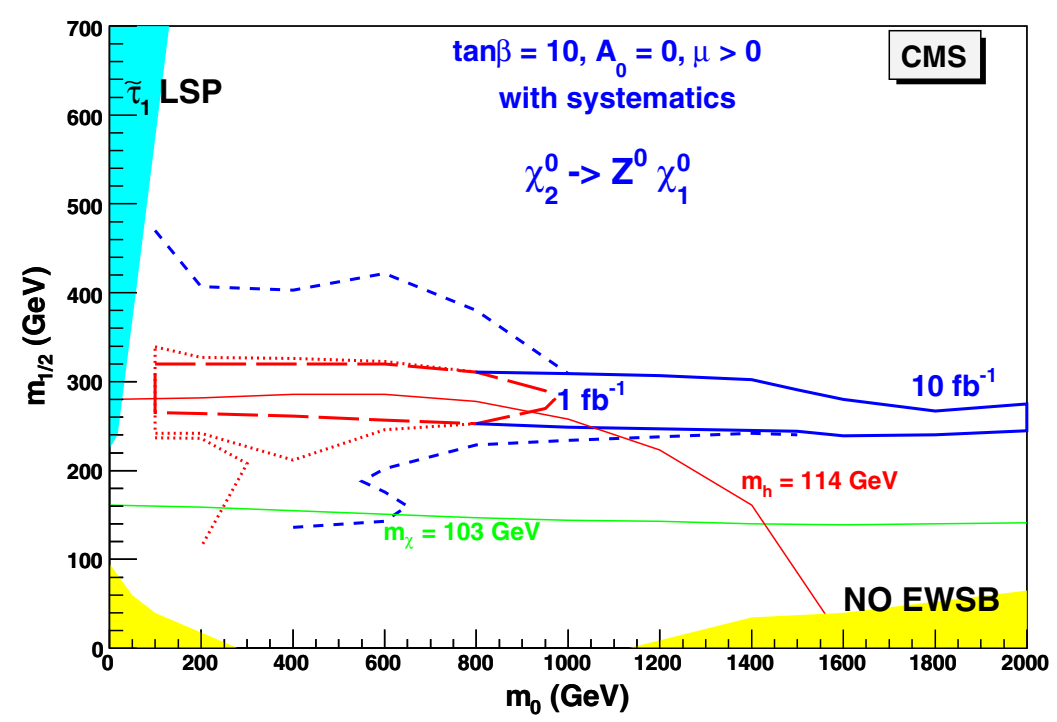

Figure 13.21. The $5 \sigma$ significance contours of final states with $Z^{0}$ for $1 \mathrm{fb}^{-1}$ (dashed line) and $10 \mathrm{fb}^{-1}$ (full line) integrated luminosities, taking into account systematic uncertainties, in the region where the $\tilde{\chi}_{2}^{0} \rightarrow Z^{0} \tilde{\chi}_{1}^{0}$ decay takes place. Also indicated as dotted and short dashed lines are the extensions at higher and lower $m_{1 / 2}$ where the $Z^{0}$ is off-shell.

of SUSY. For each point 2000 events were produced with an OS lepton pair close to the $Z^{0}$ mass. The events were generated interfacing ISAJET 7.69 with PYTHIA 6.227 and they were simulated, reconstructed and analysed using the FAMOS fast simulation package [11]. Systematic uncertainties were taken into account. The $5 \sigma$ significance contour is shown for integrated luminosities of $1 \mathrm{fb}^{-1}$ and $10 \mathrm{fb}^{-1}$ in Fig. 13.21.

\subsection{Inclusive analyses with top}

The supersymmetric partner of the top quark in most of the supersymmetric scenarios is the lightest squark. Finding evidence of its existence can be a clear signature for supersymmetry. In the main part of the allowed $m_{0}-m_{1 / 2}$ plane, the stop can decay to a top plus a neutralino. This neutralino can be either the $\operatorname{LSP}\left(\tilde{\chi}_{1}^{0}\right)$ or a heavier neutralino which decays in turn to a LSP which appears as missing transverse energy $\left(E_{\mathrm{T}}^{\text {miss }}\right)$. Hence in the final state there is at least a top quark plus large $E_{\mathrm{T}}^{\mathrm{miss}}$.

The search for top was tuned on test point LM1, where the stop decays according to

$$
\tilde{t}_{1} \rightarrow t \tilde{\chi}_{2}^{0} \rightarrow t l \tilde{l}_{R} \rightarrow t l l \tilde{\chi}_{1}^{0}
$$

giving rise to a final state which also contains two leptons. Although this analysis consists primarily in a search for an excess of top quarks from any SUSY origin with respect to its SM production, it was also optimised for the selection of events where the top results from the production of $\tilde{t}$.

\subsubsection{Top quark and lepton reconstruction and identification}

Electrons and muons are requested to have $p_{\mathrm{T}} \geqslant 5 \mathrm{GeV} / \mathrm{c}$ and $\eta \leqslant 2.5$.

Electrons are separated from jets by requiring that the ratio of energy deposited in the HCAL to the ECAL $\leqslant 0.1$, the absolute difference in $\eta$ between the electromagnetic cluster 
in the ECAL and the associated track $\Delta \eta \leqslant 0.006$ and the energy weighted spread of the electron shower in $\eta$ be $\sigma_{\eta \eta} \leqslant 0.015$.

Leptons were required to be isolated, namely that the ratio of $p_{\mathrm{T}}$ of the lepton to the $p_{\mathrm{T}}$ sum of other particles inside a cone of size $\Delta R=0.1$ around the lepton track be greater than 2 . Jets were reconstructed from ECAL and HCAL towers using an Iterative cone algorithm with cone size $\Delta R=0.5$ and were selected if their uncalibrated transverse energy $E_{\mathrm{T}} \geqslant 30 \mathrm{GeV}$ in the acceptance of $\eta \leqslant 2.5$. Their energy was calibrated using corrections from photon-jet balancing studies presented in Vol. 1 Section 11.6.3.

In this analysis only hadronic decays of the top quark were considered. A kinematic fit with constraints is utilised to find the best combination of jets to make the top quark. Since the purpose of this analysis is not to measure the top quark mass, its known value was used to constrain the invariant mass of the system of three jets. Among these three jets, one and only one must be tagged as a b-jet and the other two were constrained to be consistent with a hadronically decaying $W$. The fit then consisted in minimising the $\chi^{2}$ as a function of the three jet energies and imposing the top and $W$ mass constraints. The solution was obtained by an iterative method based on Lagrange multipliers. As several combinations may lead to a convergent fit for a given event, only the combination with the best $\chi^{2}$ was kept, with the additional requirement that its $\chi^{2}$ probability was greater than 0.1 .

\subsubsection{Signal selection and backgrounds}

All events were fully simulated [8], digitised with low luminosity pileup and reconstructed [10].

The signal events consisted of an inclusive SUSY sample at the test point LM1 (see Section 13.3.2), where the total cross section at NLO is about $52 \mathrm{pb}$. Top quarks are found in the decay of $\tilde{t}$, but other important sources exist, e.g. $\tilde{b} \rightarrow t \tilde{\chi}_{1}^{ \pm}$. At an integrated luminosity of $1 \mathrm{fb}^{-1}$, the total SUSY production amounts to 52000 events, out of which 8375 contain a top quark.

The main backgrounds, generated with PYTHIA 6.225 [69], consist of $t \bar{t}, W W+$ jets, $W Z+j e t s$ and QCD. In addition, single top generated with TopREX $4.11[44]$ and $W+j e t s$ generated with ALPGEN V2.0 [161] were considered.

The selection of SUSY events containing a top quark was based on the following criteria:

- L1T: every event must pass the first level of the Trigger (L1T) cuts corresponding to "Jet/Met" (a jet with $E_{\mathrm{T}}>88$ and $E_{\mathrm{T}}^{\text {miss }}>46 \mathrm{GeV} / \mathrm{c}$ ).

- HLT: events were required to pass High level Trigger (HLT) cuts (a jet with $E_{\mathrm{T}}>180$ and $E_{\mathrm{T}}^{\text {miss }}>123 \mathrm{GeV}$ ).

- $\geqslant 4$ jets with $E_{\mathrm{T}}^{r a w} \geqslant 30 \mathrm{GeV}$ and $\eta \leqslant 2.5$.

- $\geqslant 1$ b-jet with $E_{\mathrm{T}}^{\text {raw }} \geqslant 30 \mathrm{GeV}$ and $\eta \leqslant 2.5$.

- $E_{\mathrm{T}}^{\text {miss }} \geqslant 150 \mathrm{GeV}$ to suppress $t \bar{t}$ and other SM backgrounds.

- a convergent fit with $P\left(\chi^{2}\right) \geqslant 0.1$.

- $\Delta \Phi$ between the fitted top and $E_{\mathrm{T}}^{\text {miss }} \leqslant 2.6$ rad to suppress semi-leptonic $t \bar{t}$ events.

- $\geqslant 1$ isolated lepton ( $e$ or $\mu$ ) with $p_{\mathrm{T}} \geqslant 5 \mathrm{GeV}$ and $\eta \leqslant 2.5$ to suppress QCD background.

These criteria were simultaneously optimised to reject SM backgrounds and to maximise the ratio of events with a top quark at generator level, called SUSY(with top), to events without top at generator level, called SUSY(no top).

The effect of the cuts is shown in Table 13.12. As a result of the selection, the signal events remaining for a $1 \mathrm{fb}^{-1}$ luminosity consist of 38 events SUSY(with top) and 17 events 
Table 13.12. Effect of different cuts on different samples. In every row, the number of the remaining events after that cut is shown. "No.of.used.events" shows the number of events used in this analysis, "NEve(Nor.xsec) $1 \mathrm{fb}^{-1}$ " is the same number after normalising to the cross section times $1 \mathrm{fb}^{-1}$ and "wT/noT" means $\frac{\text { SUSY (withTop) }}{\text { SUSY(noTop) }}$.

\begin{tabular}{lrrrrrrr}
\hline cut & $\begin{array}{r}\text { SUSY } \\
\text { (withTop) }\end{array}$ & $\begin{array}{r}\text { SUSY } \\
\text { (noTop) }\end{array}$ & ttInc & WW & ZW & Single t & wT/noT \\
\hline x-sec(pb) NLO & 52 & 830 & 269.91 & 51.5 & 250 & - & \\
No.of.used.events & 494261 & 1674500 & 305000 & 70000 & 100000 & - & \\
NEve(Nor.xsec) $1 \mathrm{fb}^{-1}$ & 8375 & 43625 & 830000 & 269910 & 51500 & 250000 & 0.19 \\
L1T (Jet/Met) & 6269 & 33582 & 75806 & 18498 & 598 & 10875 & 0.19 \\
HLT (Jet/Met) & 5070 & 29427 & 14430 & 4733 & 142 & 1750 & 0.17 \\
MET $\geqslant 150 \mathrm{GeV}$ & 4183 & 25677 & 4930 & 2312 & 99 & 653 & 0.16 \\
$n_{b j} \geqslant 1$ & 3457 & 14388 & 3718 & 792 & 32 & 355 & 0.24 \\
$n_{j}^{b}$ or light $\geqslant 4$ & 1789 & 4576 & 769 & 25 & 0 & 33 & 0.39 \\
A convergent Fit & 1335 & 3062 & 557 & 12 & 0 & 28 & 0.44 \\
$\chi^{2}$ probability $>0.1$ & 105 & 69 & 56 & 0 & 0 & 5 & 1.52 \\
$\Delta \phi<2.6$ & 79 & 52 & 12 & 0 & 0 & 5 & 1.51 \\
$n_{l}>0$ & 38 & 17 & 5 & 0 & 0 & 0 & 2.19 \\
\hline
\end{tabular}
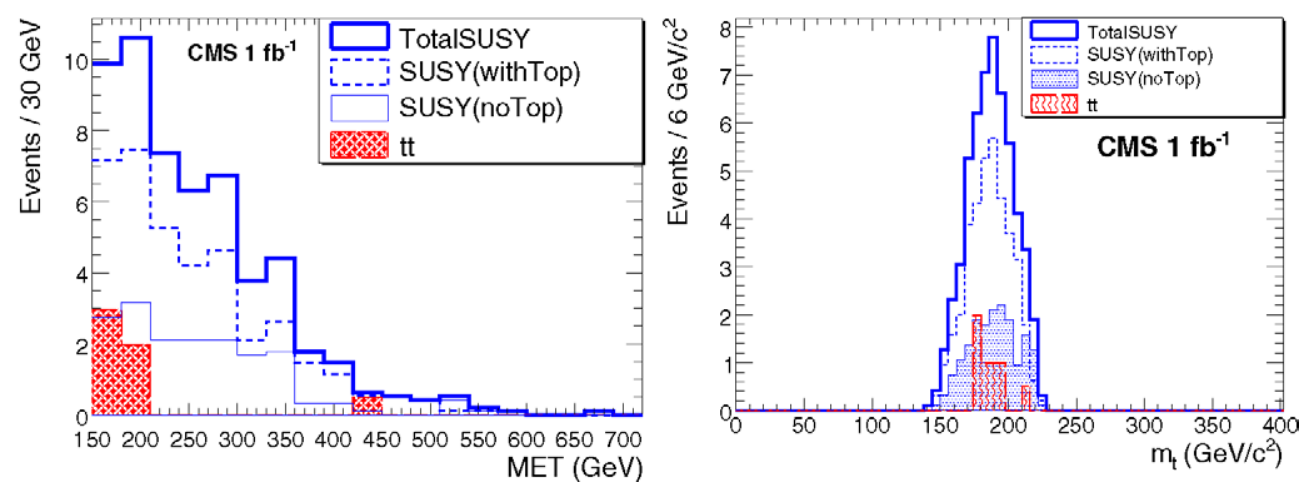

Figure 13.22. (left) Distributions of $E_{\mathrm{T}}^{\text {miss }}$ and (right) fitted top mass after all selection criteria are applied.

SUSY(no top). The remaining backgrounds are 5 events from $t \bar{t}$. The resulting distributions of $E_{\mathrm{T}}^{\mathrm{miss}}$ and of the fitted top mass are displayed in Fig. 13.22.

\subsubsection{Results at point LM1}

The significance of a discovery was computed from statistical uncertainties only using the formula of $S_{c 12}$, defined in Appendix A.1, where the number of signal events, $S$, is the sum of SUSY(with top) and SUSY(no top) and $B$ represents the sum of all SM backgrounds. Using this formula, the integrated luminosity required to make a discovery at point LM1 with a significance of 5 amounts to $\sim 210 \mathrm{pb}^{-1}$.

Many systematic uncertainties (cross section, showering, ISR/FSR, ... ) will be rendered very small by using real data. The main uncertainties remaining will be the absolute jet energy scale (estimated to $5 \%$ for jets and MET in $1 \mathrm{fb}^{-1}$ ), which leads to $5.1 \%$ from jets and $18.3 \%$ from MET in the $t \bar{t}$ sample and the b-tagging efficiency estimated to $8 \%$ for $1 \mathrm{fb}^{-1}$. Adding them in quadrature yields a total systematic uncertainty of $21 \%$, considered common to all backgrounds. It is seen that this remains negligible compared to the statistical uncertainty. 


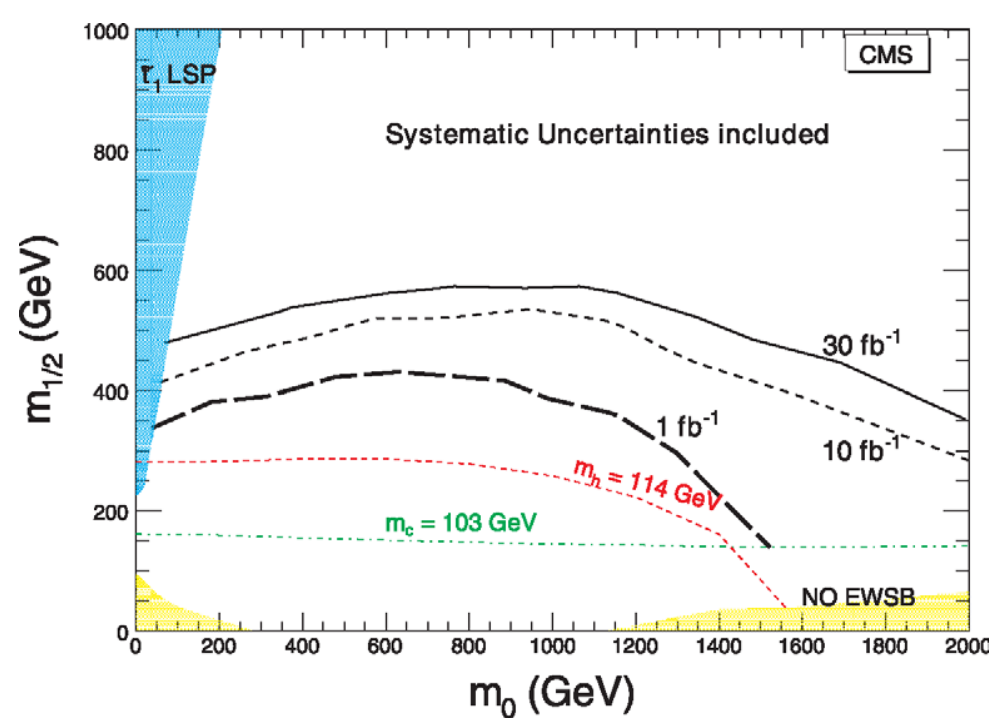

Figure 13.23. The $5 \sigma$ reach in $m_{0}, m_{1 / 2}$ plane with 1,10 and $30 \mathrm{fb}^{-1}$ obtained for final states with a top quark.

\subsubsection{CMS reach for inclusive top search}

The CMS fast simulation, FAMOs, was used to find the reach of CMS in this channel in $m_{0}, m_{1 / 2}$ plane. In total 36 points have been tried. The ntuples were generated by using the CMS-official ISAPYTHIA. The NLO cross sections were derived by PROSPINO [682].

Figure 13.23 shows the $5 \sigma$ reach in $m_{0}, m_{1 / 2}$ plane with 1,10 and $30 \mathrm{fb}^{-1}$.

\subsection{Mass determination in final states with ditaus}

In this section the determination of the sparticle masses using invariant mass distributions in the ditau final state is investigated. The selection of the events is the same as presented in Section 13.9.

\subsubsection{Extraction of mSUGRA mass spectra from the measurement of the end points of} invariant mass distributions

Using the kinematics of the successive two body decays in $\tilde{q} \rightarrow q \tilde{\chi}_{2}^{0} \rightarrow q \tau \tilde{\tau} \rightarrow q \tau \tau \tilde{\chi}_{1}^{0}$, it is possible to express the mass of the sparticles involved in that cascade as a fully resolved system of equations which depends only on the end-point of the invariant mass distributions obtained by combining the leptons and quark-jets observed in the final state.

However, the tau-lepton always decays, producing at least one undetected neutrino. Therefore, instead of observing a triangle-shaped distribution like for the dilepton invariant mass distribution of chapter 13.8, where the end-point coincides with the maximum of the distribution, the absence of the neutrino smears the resulting mass distribution to lower values. Even though the end-point of the distribution remains unchanged, it now lies at the tail of a gaussian-like distribution.

The $\tilde{\chi}_{2}^{0}$ cascade always produces a pair of opposite charge $\tau$ 's, therefore signal samples are obtained by combining opposite charge tau pairs to the two most energetic jets of the event. In $75 \%$ of the cases the quark produced by the decay of the $\tilde{q}$ to $\tilde{\chi}_{2}^{0}$ is among these 


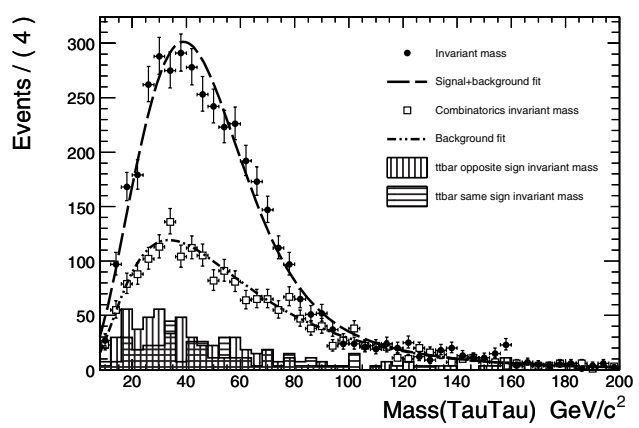

Figure 13.24. Ditau invariant mass distribution.

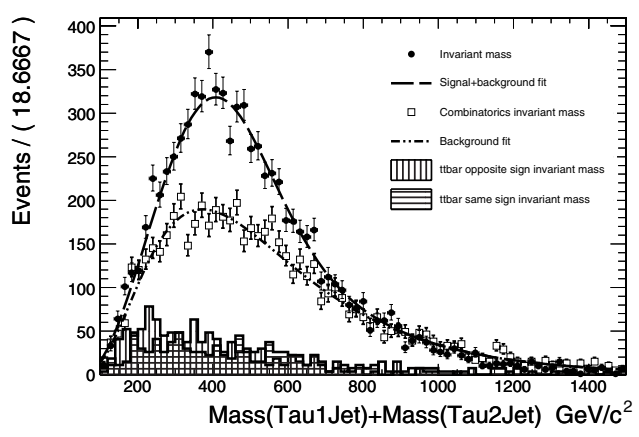

Figure 13.26. $\tau_{1} \mathrm{Jet}+\tau_{2} \mathrm{Jet}$ invariant mass distribution.

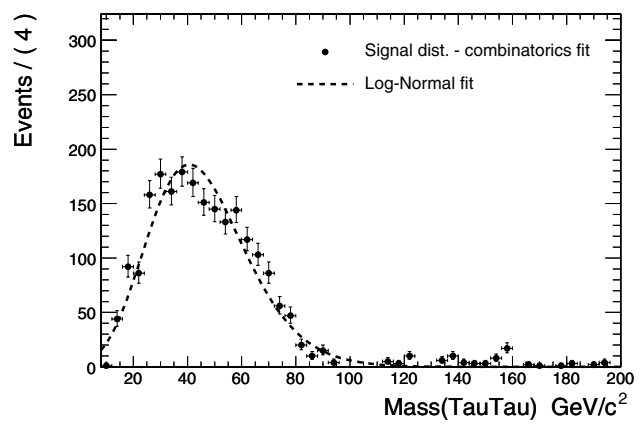

Figure 13.25. Difference between ditau invariant mass distribution and combinatorics fit together with lognormal fit.

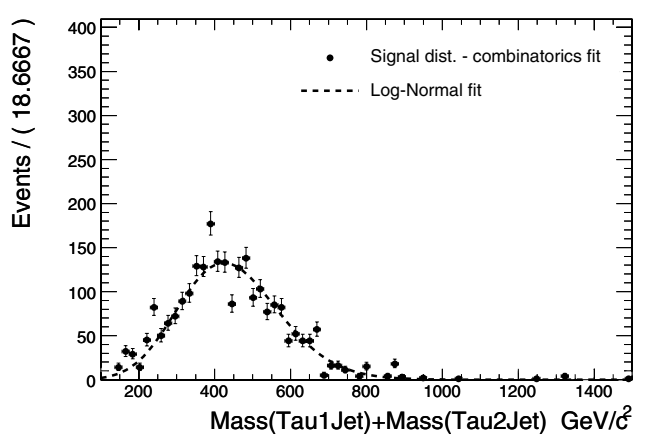

Figure 13.27. Difference between $\tau_{1} \mathrm{Jet}+\tau_{2}$ Jet invariant mass distribution and combinatorics fit together with log-normal fit.

two jets, due to the fact that the $\tilde{q}$ is much heavier than the $\tilde{\chi}_{2}^{0}$. This large number of tau's and jets is responsible for a high combinatorial background. A good description of this combinatorial background, in particular of its tail, is essential for extracting the true endpoints. The combinatorial background in the opposite sign invariant ditau mass is estimated by taking same sign tau pairs. The combinatorial background from the jets is estimated by combining all tau pairs to a jet taken among the 2 most energetic jets of a previous event selected randomly to insure that the jet and tau's are uncorrelated.

Five invariant mass and their associated combinatorial background distributions are then obtained: $M(\tau \tau), M(\tau \tau$ Jet $), M\left(\tau_{1} \mathrm{Jet}\right), M\left(\tau_{2} \mathrm{Jet}\right)$ and $M\left(\tau_{1} \mathrm{Jet}\right)+M\left(\tau_{2} \mathrm{Jet}\right) .\left(\tau_{1}\right.$ is defined as the one which maximises the invariant mass formed by its association with a jet, $M\left(\tau_{1} \mathrm{Jet}\right)>$ $\left.M\left(\tau_{2} J e t\right)\right)$.

The distributions of combinatorial background are first fitted. Then, the resulting fit parameters are used together with a Log-normal distribution, which gives a good description of the tail of the true distributions, to fit the distributions of the signal. Since it is possible to express the log-normal distribution as a function of the end-point, the end-point can be extracted directly from the fit.

The ditau invariant mass and $M\left(\tau_{1} \mathrm{Jet}\right)+M\left(\tau_{2} \mathrm{Jet}\right)$ are fitted first (Figs 13.24-13.27). The three other invariant mass distributions are built using only candidates found to have values for the two previous distributions below the measured end-points. Then, they are fitted using the same procedure. The sparticle masses are evaluated by solving the system of four equations giving the end-points as a function of the sparticle mass [683]. 
Table 13.13. End-point obtained with the lognormal fit together with sparticle masses measured with the end-point technique for LM2 for integrated luminosities around $40 \mathrm{fb}^{-1}$.

\begin{tabular}{lcc}
\hline End-points $(\mathrm{GeV})$ & case $1(\mathrm{GeV})$ & case 2 $(\mathrm{GeV})$ \\
\hline$m\left(\tau_{1} \tau_{2}\right)^{\max }=95 \pm 3$ & $M\left(\tilde{\chi}_{1}^{0}\right)=213 \pm 14$ & $M\left(\tilde{\chi}_{1}^{0}\right)=147 \pm 23$ \\
$m\left(\tau_{1} Q\right)^{\max }=559 \pm 11$ & $M\left(\tilde{\chi}_{2}^{0}\right)=337 \pm 17$ & $M\left(\tilde{\chi}_{2}^{0}\right)=265 \pm 10$ \\
$m\left(\tau_{2} Q\right)^{\max }=298 \pm 7$ & $M(\tilde{\tau})=310 \pm 17$ & $M(\tilde{\tau})=165 \pm 10$ \\
$m\left(\tau_{1} \tau_{2} Q\right)^{\max }=596 \pm 12$ & $M(\tilde{q})=839 \pm 19$ & $M(\tilde{q})=763 \pm 33$ \\
$E_{5}^{\text {meas }}=780 \pm 20$ & $E_{5}^{\text {calc }}=815 \pm 26$ & $E_{5}^{\text {calc }}=765 \pm 30$ \\
\hline
\end{tabular}

Table 13.14. sparticle masses measured with end-point method for LM 2 together with theoretical value.

\begin{tabular}{lll}
\hline & \multicolumn{2}{c}{ LM2 benchmark point } \\
\cline { 2 - 3 } & \multicolumn{1}{c}{ measured } & theory \\
\hline$M\left(\tilde{\chi}_{1}^{0}\right)(\mathrm{GeV})$ & $147 \pm 23($ stat $) \pm 19($ sys $)$ & 138.2 \\
$M\left(\tilde{\chi}_{2}^{0}\right)(\mathrm{GeV})$ & $265 \pm 10($ stat $) \pm 25($ sys $)$ & 265.5 \\
$M(\tilde{\tau})(\mathrm{GeV})$ & $165 \pm 10($ stat $) \pm 20($ sys $)$ & 153.9 \\
$M(\tilde{q})(\mathrm{GeV})$ & $763 \pm 33($ stat $) \pm 58($ sys $)$ & $753-783$ (light $\tilde{q})$ \\
\hline
\end{tabular}

When several solutions are possible for the SUSY mass spectrum (as it is the case here, where two valid solutions exist), the choice is made by comparing the measured $M\left(\tau_{1} \mathrm{Jet}\right)+M\left(\tau_{2} \mathrm{Jet}\right)$ end-point value, $E_{5}$, to the one computed from the sparticle masses found by solving the systems of equations.

The most probable mass hypothesis is then chosen as the one for which $E_{5}$ computed for each mass spectrum is the closest to the measured one. The measured end-point was found to be $780 \pm 20 \mathrm{GeV}$ while the calculations for the mass hierarchy in case 1 and case 2 yield to $815 \pm 26 \mathrm{GeV}$ and $765 \pm 30 \mathrm{GeV}$ respectively (Table 13.14). The second hypothesis, which corresponds to the correct LM2 mass hierarchy, gives a result compatible with the measured end-point value.

Three main systematic uncertainties are considered, the jet energy scale and tau-jet energy scale as well as systematics uncertainties arising from the extraction procedure.

Results obtained are shown in Table 13.14 for $40 \mathrm{fb}^{-1}$, together with LM2 generated sparticle masses. They are found to be in good agreement with the theoretical values. Using a $40 \mathrm{fb}^{-1}$ LM2 sample, it is possible to measure the SUSY mass spectra and in particular $\tilde{\tau}$ mass with a precision of $30 \mathrm{GeV}$.

\subsection{Direct $\chi_{2}^{0} \chi_{1}^{ \pm}$production in tri-leptons}

The exclusive tri-lepton final state appears in $p p \rightarrow \tilde{\chi}_{2}^{0} \tilde{\chi}_{1}^{ \pm}$channel with subsequent three body decays of the second neutralino, $\tilde{\chi}_{2}^{0} \rightarrow \tilde{\chi}_{1}^{0} l l$, and chargino, $\tilde{\chi}_{1}^{ \pm} \rightarrow \tilde{\chi}_{1}^{0} W^{*} \rightarrow \tilde{\chi}_{1}^{0} l v$; or via sleptons in two body decay, $\tilde{\chi}_{2}^{0} \rightarrow l \tilde{l} \rightarrow l \tilde{\chi}_{1}^{0} l$, and $\tilde{\chi}_{1}^{ \pm} \rightarrow l \tilde{v} \rightarrow l \tilde{\chi}_{1}^{0} v, \tilde{\chi}_{1}^{ \pm} \rightarrow v \tilde{l} \rightarrow v \tilde{\chi}_{1}^{0} l$. The final signatures are two Opposite-Sign Same-Flavour (SFOS) leptons $(e, \mu)$ from the neutralino $\tilde{\chi}_{2}^{0}$ decay plus any lepton from the chargino $\tilde{\chi}_{1}^{ \pm}$. Jets are expected to be only due to gluon state radiation or pile up events. In spite of the escaping $\tilde{\chi}_{1}^{0}$, the $E_{\mathrm{T}}^{\text {miss }}$ is relatively small at low $m_{1 / 2}$ and is comparable with the one of SM backgrounds, especially for three body decays at large $m_{0}$. The invariant mass of the SFOS dileptons exhibits a particular shape with a kinematic end point $M_{l l}^{\max }$ that depends upon the event topology, see section 13.3. 


\subsubsection{Datasets}

The tri-lepton cross section $\sigma_{3 l}$ was calculated with ISAJET (7.69) and PYTHIA (6.225 CTEQ5L) at LO, the $\mathrm{K}_{N L O}$ factor calculated with PROSPINO is in the range of 1.30-1.25 (for $m_{\tilde{\chi}_{2}^{0}}=150-300 \mathrm{GeV} / \mathrm{c}^{2}$ ) [684]. The $\sigma_{3 l}$ drops rapidly with the neutralino mass $m_{\tilde{\chi}_{2}^{0}} \sim$ $0.8 m_{1 / 2}, \sigma_{3 l} \sim m_{1 / 2}^{-4}$. This study is restricted to the low $m_{1 / 2}$ region, where $\sigma_{3 l}$ contributes, for instance, $\sim 0.5 \%$ to the total SUSY cross section at $m_{0}>1000 \mathrm{GeV} / \mathrm{c}^{2}$. The three body decays are dominant in this $m_{0}, m_{1 / 2}$ region, except for $m_{0}<150 \mathrm{GeV} / \mathrm{c}^{2}$ and $\tan \beta \leqslant 20$. The kinematic end point in the invariant mass is approximately $M_{l l}^{\max } \sim 0.42 * m_{1 / 2}-18.4$ $\mathrm{GeV} / \mathrm{c}^{2}$ (at $m_{0} \sim 1000 \mathrm{GeV} / \mathrm{c}^{2}$ ), thus moving into the $\mathrm{Z}$-peak region at $m_{1 / 2}>250 \mathrm{GeV} / \mathrm{c}^{2}$ where the SM background is high. Among the CMS benchmark points in this region, LM9 $\left(m_{1 / 2}=175, m_{0}=1450, \tan \beta=50, A_{0}=0\right)$ has the largest cross section, $\sim 3700$ events are produced for $30 \mathrm{fb}^{-1}$, and it was used as a reference.

\subsubsection{Backgrounds and trigger path}

The main background results from the Drell-Yan, $Z+$ jets, $\overline{\mathrm{t}} \rightarrow W b W b, Z W, Z Z, W t+$ jets, $W W+$ jets, $W+$ jets and inclusive SUSY channels. For all backgrounds, except $Z W$ and $Z Z$, some leptons originate from jets, mostly $b \rightarrow l+j$. The background events were produced with PYTHIA (ALPGEN and TOPREX are also used) and their cross section corrected to NLO. The $Z$ and $W$ bosons are forced to decay leptonically to $e, \mu, \tau \rightarrow e, \mu$. The DY and $Z+$ jets cross section is large $\left(\sigma_{D Y, Z j} \sim 10 \mathrm{nb}\right)$ and events were preselected by requiring three leptons with $p_{\mathrm{T}}>5 \mathrm{GeV} / \mathrm{c}$ and $|\eta|<2.4$ at the generator level. The full data samples of $30 \mathrm{fb}^{-1}$ for the LM9 test point and backgrounds are simulated with the CMS fast simulations (FAMOS) validated with smaller statistics samples produced with the full GEANT based simulation (OSCAR, ORCA). Low luminosity pile-up was included.

All events were required to pass Level-1 and HLT triggers. The main trigger paths for LM9 are the dimuons (74\%) and dielectrons (25\%). The trigger efficiency is $86 \%$ at Level-1 and $91 \%$ at HLT for LM9 and is increasing for larger $m_{1 / 2}$ where the leptons become harder. In the off-line selection, at least three isolated leptons in $|\eta|<2.4$ and $P_{\mathrm{T}}^{\mu, e}>10 \mathrm{GeV} / \mathrm{c}$ are required for each event. The leptons are reconstructed using standard reconstruction algorithms. Electrons and muons are required to be isolated, i.e. other tracks may only contribute up to $\sum P_{\mathrm{T}}$ of $1.5 \mathrm{GeV} / \mathrm{c}$ inside a cone of $\Delta R<0.3$. Moreover, for muons the energy deposit in calorimeters should be $E_{\mathrm{T}}<5 \mathrm{GeV}$ in a cone of $\Delta R<0.3$. In addition, electron candidates are required to satisfy quality criteria based on a likelihood function, $>0.65$. The muons and electrons reconstruction efficiencies in ORCA are found to be $78 \%\left(\mathrm{P}_{\mathrm{T}}^{\mu}>5 \mathrm{GeV} / \mathrm{c}\right)$ and $66 \%\left(\mathrm{P}_{\mathrm{T}}^{e}>10 \mathrm{GeV} / \mathrm{c}\right)$ respectively. The jets are reconstructed using an iterative cone algorithm with the seed energies $E_{\mathrm{T}}^{\text {seed }}>0.5 \mathrm{GeV}$ in a cone $\Delta R<0.5$.The $E_{\mathrm{T}}^{\mathrm{miss}}$ was reconstructed from the calorimeter towers. Since the $E_{\mathrm{T}}^{\mathrm{miss}}$ for the signal events is relatively small and its reconstruction at low energy scale is limited by the $\mathrm{E}_{\mathrm{T}}$ resolution, a $E_{\mathrm{T}}^{\text {miss }}$ requirement is not as efficient as in other SUSY channels.

\subsubsection{Analysis path}

The reconstructed events are selected in two steps. First, sequential cuts are applied: 1) No central jets with corrected energy $E_{\mathrm{T}}>30 \mathrm{GeV}$ in $|\eta|<2.4$, 2) Two SFOS isolated leptons $(e, \mu)$ in $|\eta|<2.4$ with $P_{\mathrm{T}}^{\mu}>10 \mathrm{GeV} / \mathrm{c}, P_{\mathrm{T}}^{e}>17 \mathrm{GeV} / \mathrm{c}$ and the dilepton invariant mass below the $\mathrm{Z}$ peak $M_{l l}<75 \mathrm{GeV} / \mathrm{c}^{2}$. 3) The third lepton is with $P_{\mathrm{T}}^{\mu, e}>10 \mathrm{GeV} / \mathrm{c}$ in $|\eta|<2.4$. The evolution of statistics and the efficiencies of the selection cuts are presented in Table 13.15. 
Table 13.15. Evolution of signal and background statistics with the cuts as expected for $30 \mathrm{fb}^{-1}$ The last column gives the results of a neural network selection applied after the sequential cuts.

\begin{tabular}{llllll}
\hline channel & $\begin{array}{l}N_{e v} 30 \mathrm{fb}^{-1} \\
(\sigma \times B R[\mathrm{pb}])\end{array}$ & L1+HLT & No Jets & $\begin{array}{l}2 \text { SFOS+1 } \\
\text { SFOS } M_{l l}<75 \mathrm{GeV} / \mathrm{c}^{2}\end{array}$ & $\begin{array}{l}\mathrm{NN}_{L M 9} \\
\end{array}$ \\
\hline LM1 & $2640(0.088)$ & $1544(58 \%)$ & $864(56 \%)$ & $70(8 \%)$ & $17(24 \%)$ \\
LM7 & $1540(0.051)$ & $1250(82 \%)$ & $738(59 \%)$ & $91(12 \%)$ & $57(62 \%)$ \\
LM9 & $3700(0.125)$ & $2896(78 \%)$ & $1740(60 \%)$ & $239(14 \%)$ & $158(68 \%)$ \\
SUSY & $4 \cdot 10^{5}\left(13.1^{N L O}\right)$ & $2.5 \cdot 10^{5}(63 \%)$ & $1.8 \cdot 10^{4}(7 \%)$ & $34(0.2 \%)$ & $22(65 \%)$ \\
ZW & $5 \cdot 10^{4}\left(1.68^{N L O}\right)$ & $3.6 \cdot 10^{4}(73 \%)$ & $1.9 \cdot 10^{4}(53 \%)$ & $173(1 \%)$ & $44(25 \%)$ \\
ZZ & $4.8 \cdot 10^{3}\left(0.16^{N L O}\right)$ & $3.5 \cdot 10^{3}(73 \%)$ & $1.7 \cdot 10^{3}(48 \%)$ & $38(2.3 \%)$ & $15(39 \%)$ \\
tt & $2.6 \cdot 10^{6}\left(88^{N O}\right)$ & $1.8 \cdot 10^{6}(70 \%)$ & $1.3 \cdot 10^{5}(7 \%)$ & $239(0.2 \%)$ & $89(37 \%)$ \\
Z+jets(31) & $4.6 \cdot 10^{5}\left(15.4^{L O}\right)$ & $3.7 \cdot 10^{5}(80.5 \%)$ & $9.8 \cdot 10^{4}(26.5 \%)$ & $504(0.5 \%)$ & $129(26 \%)$ \\
DY(31) & $4.5 \cdot 10^{5}\left(15.1^{L O}\right)$ & $3.2 \cdot 10^{5}(71 \%)$ & $1.4 \cdot 10^{5}(44 \%)$ & $670(0.5 \%)$ & $131(20 \%)$ \\
Zbb $(31)$ & $8.4 \cdot 10^{4}\left(2.8^{L O}\right)$ & $7.3 \cdot 10^{4}(87 \%)$ & $1.5 \cdot 10^{4}(20 \%)$ & $69(0.6 \%)$ & $18(26 \%)$ \\
Wt+jets & $3 \cdot 10^{5}\left(10^{N L O}\right)$ & $2.1 \cdot 10^{5}(70 \%)$ & $3.9 \cdot 10^{4}(18.5 \%)$ & $52(0.1 \%)$ & $20(38 \%)$ \\
WW+jets & $6 \cdot 10^{5}\left(19.8^{L O}\right)$ & $3.8 \cdot 10^{5}(63 \%)$ & $1.9 \cdot 10^{4}(50 \%)$ & $7(0.04 \%)$ & $2(29 \%)$ \\
Tot. bkg & $\sim 4.9 \cdot 10^{6}$ & & & 1786 & $470(26 \%)$ \\
\hline
\end{tabular}

In a second step the background suppression is improved with a Neural Network (NN). Five networks for DY, $Z+$ jets, $\mathrm{t} \overline{\mathrm{t}}, Z W$ and $Z Z$ backgrounds are trained on the LM9 signal sample using the following variables: $\mathrm{P}_{\mathrm{T}}^{1,2,3}, \sum P_{\mathrm{T}}, \mathrm{M}_{l l}, P_{\mathrm{T}}^{2 l}$ (transverse momentum of two SFOS leptons), $A=\frac{P_{\mathrm{T}}^{1}-P_{\mathrm{T}}^{2}}{P_{\mathrm{T}}^{1}+P_{\mathrm{T}}^{2}}, \Theta_{l l}$ (angle between two SFOS leptons), $\Phi_{l l}$ (angle in transverse plane), $E_{\mathrm{T}}^{\mathrm{miss}}, N_{\text {jets }}$ (number of jets passing the jets veto), $E_{t}^{h j}$ (of the highest $\mathrm{E}_{\mathrm{T}}$ jet), $\eta_{h j}$ (rapidity of the highest jet). The selection cuts on the NN outputs were optimised for the maximum significance at LM9 with the genetic algorithm GARCON [63]. The efficiency of the NN selection is also shown in Table 13.15.

\subsubsection{Results at LM9 and systematics}

After the selection based on cuts the $S_{c p}$ significance calculated for all SFOS pair combination is 6.1 at point LM9 for an integrated luminosity of $30 \mathrm{fb}^{-1}$. The NN improves the $S_{c p}$ for all SFOS combinations to 7.8 .

In addition to the real tri-lepton final state, leptons can be produced in the detector volume from $\pi^{ \pm}, \mathrm{K}^{ \pm}$decays, bremsstrahlung, punch-through or faked by jets. The rate per event of such fake leptons was estimated individually for each background by matching the reconstructed lepton with the generated one and is $\sim 10^{-4}$ for electrons and $\sim 10^{-5}$ for muons. The expected fake leptons substantially increase the background, especially for the preselected channels like DY or $Z+$ jets, by $\sim 221 \pm 48$ events and $\sim 31 \pm 16$ events respectively for the tri-muon final state where the fake rate is smaller. The $S_{c P}$ significance defined in Appendix A.1 including fakes but without other systematic uncertainties for all SFOS combinations and for the tri-muon state at LM9 is 6.5 and 5.1 respectively.

The reconstruction uncertainties related to the jet energy scale $(5 \%)$ and the lepton momentum resolution ( $2 \%$ ) contribute $1 \%$ to the uncertainties on the background. The average theoretical uncertainty from the PDFs, calculated with the LHPDF subsets using the reweighting technique for each background channel, amounts to $1.7 \%$. These uncertainties reduce the significances to 5.8 and 4.8 for the all SFOS pairs and for the tri-muon final state, respectively. However the largest uncertainties are coming from the Monte Carlo statistical 

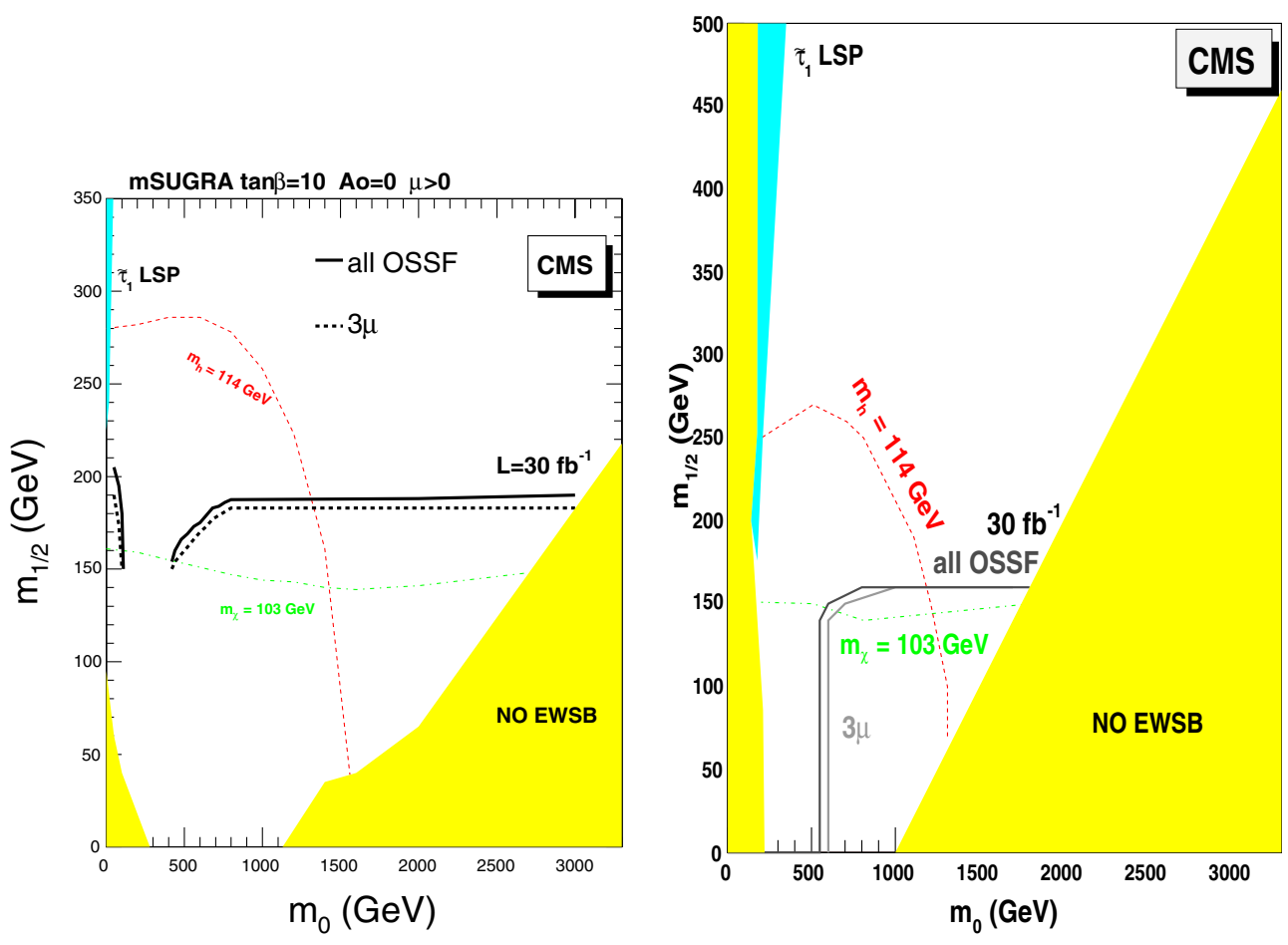

Figure 13.28. Discovery reach of tri-lepton from the $p p \rightarrow \tilde{\chi}_{2}^{0} \tilde{\chi}_{1}^{ \pm}$production at $\mathcal{L}_{\text {int }}=30 \mathrm{fb}^{-1}$ for all SFOS lepton combinations (dashed) and for the tri-muon final state (solid) including systematic uncertainties from reconstruction, for (left) $\tan \beta=10$ and (right) $\tan \beta=50$.

errors in the fake rate estimation which contribute $\sim 7 \%$ to the background uncertainties rendering the signal hardly observable, $S_{c} p \sim 3.3$. These fake rate uncertainties can be reduced with larger simulation samples.

In summary, for the tri-lepton mSUGRA study presented here, the final signal to background ratio is 0.23 , the total signal efficiency is $4.4 \%$ and the background composition is $28 \%$ Drell-Yan, $27 \% Z+$ jets, $19 \% t \bar{t}, 9 \% W Z$, and $17 \% Z Z, W W$, SUSY, $W+$ jets and QCD. The total considered theoretical and reconstruction systematic uncertainties on the Standard Model background is $2.2 \%$. The Monte Carlo statistics systematic errors in the fake rates increases this to $7.5 \%$.

\subsubsection{CMS reach for the tri-lepton final state}

Figure 13.28 shows the $5 \sigma$ discovery reach in $m_{0}$ and $m_{1 / 2}$ plane at $\mathrm{L}_{i n t}=30 \mathrm{fb}^{-1}$ for all SFOS combinations and for the tri-muon final state including the systematic uncertainties due to the reconstruction. The signal can be observed at large $m_{0}>1000 \mathrm{GeV} / \mathrm{c}^{2}$ in a narrow band below $m_{1 / 2}<180 \mathrm{GeV} / \mathrm{c}^{2}$. At low $m_{0}<100 \mathrm{GeV} / \mathrm{c}^{2}$ the two body decays are visible although a better optimisation is possible in this region, see Sections 13.8 and 13.15. The trilepton final state from direct neutralino-chargino production is complementary to the inclusive SFOS dilepton search and provides an additional verification for the leptonic decays of the neutralino at low $m_{1 / 2}$. 


\subsection{Production of $\tilde{l} \tilde{l}$}

The aim of this section is the study of the possibility of detecting sleptons. Note the previous related papers where the sleptons detection was studied at the level of a toy detector [685-689].

\subsubsection{Simulation details}

ISASUSY 7.69 [672] was used for the calculation of coupling constants and cross sections in the leading order approximation for SUSY processes. For the calculation of the next-toleading order corrections to the SUSY cross sections the PROSPINO code [682] was used. Cross sections of the background events were calculated with PYTHIA 6.227 [69] and COMPHEP $4.2 \mathrm{pl}$ [355]. For considered backgrounds the NLO corrections are known and they were used. Official datasets (DST) production was used for the study of CMS test point LM1 and backgrounds ( $\mathrm{t} \bar{t}, \mathrm{ZZ}, \mathrm{WW}, \mathrm{Wt}, \mathrm{Z}$ b $\bar{b}, \mathrm{DY} 2 \mathrm{e}, \mathrm{DY} 2 \tau$ ). For WZ, DY $2 \mu$ and $\mathrm{W}+$ jet backgrounds the events were generated with PYTHIA 6.227. The detector simulation and hits production were made with full CMS simulation [8], digitised and reconstructed [10]. The DY2 $\mu$ and $\mathrm{W}+$ jet backgrounds were simulated with fast simulation [11].

Jets were reconstruction using an iterative cone algorithm with cone size 0.5 and their energy corrected with the GammaJet calibration.

The events are required to pass the Global Level 1 Trigger (L1) and the High Level Trigger (HLT). The events have to pass at least one of the following triggers: single electron, double electron, single muon, double muon.

The CMS fast simulation code was used for the determination of the sleptons discovery plot.

\subsubsection{Sleptons production and decays}

When sleptons are heavy relative to $\tilde{\chi}_{1}^{ \pm}, \tilde{\chi}_{2}^{0}$, they are produced significantly at the LHC through the Drell-Yan mechanism (direct sleptons production), via $q \bar{q}$ annihilation with neutral or charged boson exchange in the s-channel, namely, $p p \rightarrow \tilde{l}_{L} \tilde{l}_{L}, \tilde{l}_{R} \tilde{l}_{R}, \tilde{v} \tilde{v}, \tilde{v} \tilde{l}, \tilde{l}_{L} \tilde{l}_{R}$. The left sleptons decay to charginos and neutralinos via the following (kinematically accessible) decays:

$$
\begin{aligned}
& \tilde{l}_{L}^{ \pm} \rightarrow l^{ \pm}+\tilde{\chi}_{1,2}^{0}, \\
& \tilde{l}_{L}^{ \pm} \rightarrow v_{l}+\tilde{\chi}_{1}^{ \pm}, \\
& \tilde{v} \rightarrow v_{l}+\tilde{\chi}_{1,2}^{0}, \\
& \tilde{v} \rightarrow l^{ \pm}+\tilde{\chi}_{1}^{ \pm} .
\end{aligned}
$$

For right sleptons only decays to neutralino are possible and they decay mainly to LSP:

$$
\tilde{l}_{R}^{ \pm} \rightarrow l^{ \pm}+\tilde{\chi}_{1}^{0} .
$$

If sleptons are light relative to $\tilde{\chi}_{1}^{ \pm}, \tilde{\chi}_{2}^{0}$, they can be abundantly produced, besides the Drell-Yan mechanism, also from chargino and neutralino decays $\tilde{\chi}_{1}^{ \pm}, \tilde{\chi}_{2}^{0}$ (indirect production), equations (13.8), (13.9), (13.13) and (13.14). 


\subsubsection{Signature and backgrounds}

The slepton production and decays described previously lead to the signature with the simplest event topology: two leptons $+E_{\mathrm{T}}^{\mathrm{miss}}+$ jet veto. This signature arises for both direct and indirect slepton pair production. In the case of indirectly produced sleptons not only the event topology with two leptons but with single, three and four leptons is possible. Besides, indirect slepton production from decays of squarks and gluino through charginos, neutralinos can lead to an event topology two leptons $+E_{\mathrm{T}}^{\mathrm{miss}}+(n \geqslant 1)$ jets.

The cut set close to the optimal one is the following:

(a) for leptons:

- $p_{\mathrm{T}}$ - cut on leptons $\left(p_{\mathrm{T}}^{\text {lept }}>20 \mathrm{GeV} / \mathrm{c},|\eta|<2.4\right)$ and lepton isolation within $\Delta R<$ 0.3 cone containing calorimeter cells and tracker;

- effective mass of two opposite-sign and the same-flavour leptons is outside $\left(M_{Z}-\right.$ $\left.15 \mathrm{GeV}, M_{Z}+10 \mathrm{GeV}\right)$ interval;

- $\Phi\left(l^{+} l^{-}\right)<140^{\circ}$ cut on angle between two leptons;

(b) for $E_{\mathrm{T}}^{\text {miss }}$ :

- $E_{\mathrm{T}}^{\mathrm{miss}}>135 \mathrm{GeV}$ cut on missing $E_{\mathrm{T}}$;

- $\Phi\left(E_{\mathrm{T}}^{\mathrm{miss}}, l l\right)>170^{\circ}$ cut on relative azimuthal angle between dilepton and $E_{\mathrm{T}}^{\text {miss }}$;

(c) for jets:

- jet veto cut: $N_{\text {jet }}=0$ for a $E_{\mathrm{T}}^{\text {jet }}>30 \mathrm{GeV}$ (corrected jets) threshold in the pseudorapidity interval $|\eta|<4.5$.

The Standard Model (SM) backgrounds are: $\bar{t}$, WW, WZ, ZZ, Wt, Zb̄̄, DY, W + jet. The main contributions come from WW and $\bar{t} \bar{t}$ backgrounds. There are also internal SUSY backgrounds which arise from $\tilde{q} \tilde{q}, \tilde{g} \tilde{g}$ and $\tilde{q} \tilde{g}$ productions and subsequent cascade decays with jets outside the acceptance or below the threshold. Note that when we are interested in new physics discovery we have to compare the calculated number of SM background events $N_{S M b g}$ with new physics signal events $N_{\text {new physics }}=N_{\text {slept }}+N_{\text {SUSYbg }}$, so SUSY background events increase the discovery potential of new physics.

\subsubsection{Results}

For the point LM1 with the used set of cuts for the integral luminosity $\mathcal{L}=10 \mathrm{fb}^{-1}$ the number of signal events (direct sleptons plus sleptons from chargino/neutralino decays) is $N_{S}=60$, whereas the number of SUSY background events is $N_{S U S Y b g}=4$ and the number of SM background events is $N_{S M b g}=41$. The total signal efficiency is $1.16 \times 10^{-4}$ and the background composition is $1.32 \times 10^{-6}$ of the total ttbar, $1.37 \times 10^{-5}$ of the total WW, $4 \times 10^{-6}$ of the total WZ, $4.4 \times 10^{-5}$ of the total ZZ, $8.1 \times 10^{-6}$ of the total $\mathrm{Wt}, 0$ of the total Zbb, DY, W + jet.

The SUSY background is rather small compared to the signal, so we can assume $N_{S}=N_{\text {direct sleptons }}+N_{\text {chargino/neutralino }}+N_{S U S Y b g}=64$. It corresponds to the significances $S_{c 12}=7.7$ and $S_{c L}=8.3$, defined in Appendix A.1.

Taking into account the systematic uncertainty of $23 \%$ related with in exact knowledge of backgrounds leads to the decrease of significance $S_{c 12}$ from 7.7 to 4.3.

The ratio of the numbers of background events from two different channels $N\left(e^{+} e^{-}+\right.$ $\left.\mu^{+} \mu^{-}\right) / N\left(e^{ \pm} \mu^{\mp}\right)=1.37$ will be used to keep the backgrounds under control.

The CMS discovery plot for two leptons $+E_{\mathrm{T}}^{\mathrm{miss}}+$ jet veto signature is presented in Fig. 13.29. 


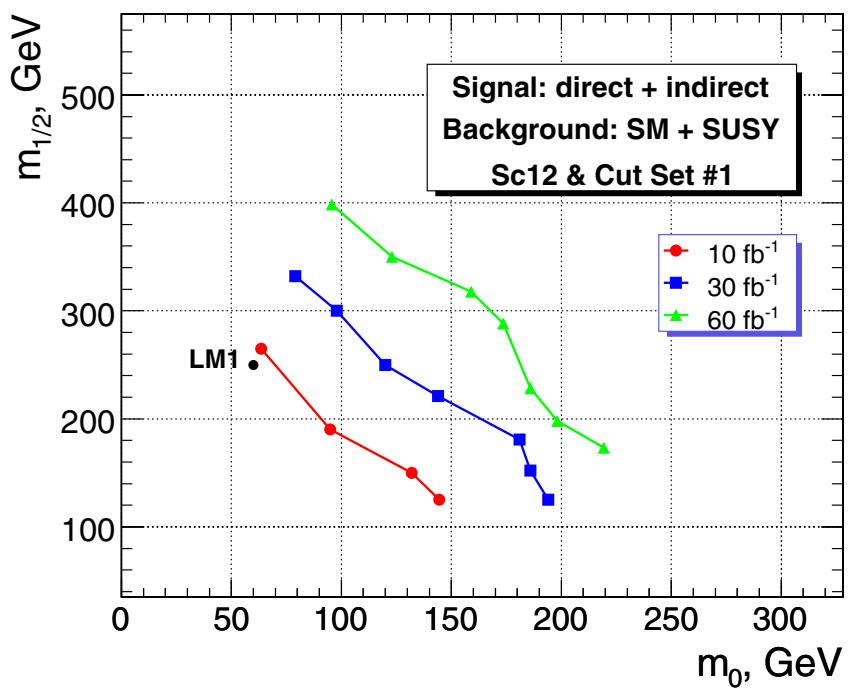

Figure 13.29. Discovery plot $(\tan \beta=10, \operatorname{sign}(\mu)=+, A=0)$ for final states with $l^{+} l^{-}$, missing transverse energy and a jet veto.

\subsection{Lepton flavour violation in neutralino decay}

The aim of this section is the study of the possibility to detect SUSY and Lepton Flavour Violation (LFV) using the $e^{ \pm} \mu^{\mp}+E_{\mathrm{T}}^{\text {miss }}$ signature.

\subsubsection{Signal selection and backgrounds}

The simulation details of this study could be found in the Section 13.15.

The SUSY production $p p \rightarrow \tilde{q} \tilde{q}^{\prime}, \tilde{g} \tilde{g}, \tilde{q} \tilde{g}$ with subsequent decays leads to the event topology $e^{ \pm} \mu^{\mp}+E_{\mathrm{T}}^{\text {miss }}$. In the MSSM with lepton flavour conserving neutralino decays into leptons $\tilde{\chi}_{2,3,4}^{0} \rightarrow l^{+} l^{-} \tilde{\chi}_{1}^{0}$ do not contribute to this signature and contribute only to $l^{+} l^{-}+E_{\mathrm{T}}^{\text {miss }}$ signature (here $l=e$ or $\mu$ ). The main backgrounds which contribute to the $e^{ \pm} \mu^{\mp}$ events are: $\mathrm{tt}, \mathrm{ZZ}, \mathrm{WW}, \mathrm{WZ}, \mathrm{Wt}, \mathrm{Zb} \overline{\mathrm{b}}, \mathrm{DY} 2 \tau, \mathrm{Z}+\mathrm{jet}$. It has been found that $\mathrm{t} \overline{\mathrm{t}}$ background is the biggest one and it gives more than $50 \%$ contribution to the total background.

Our set of cuts is the following:

- $p_{\mathrm{T}}$ - cut on leptons $\left(p_{\mathrm{T}}^{\text {lept }}>20 \mathrm{GeV} / \mathrm{c},|\eta|<2.4\right)$ and lepton isolation within $\Delta R<0.3$ cone.

- $E_{\mathrm{T}}^{\text {miss }}>300 \mathrm{GeV}$ cut on missing $E_{\mathrm{T}}$.

\subsubsection{Results at CMS test points and reach}

For integrated luminosity $\mathcal{L}=10 \mathrm{fb}^{-1}$ the number of background events is $N_{B}=93$. The results for this luminosity are presented in Table 13.16. At point LM1 the signal over background ratio is 3 and the signal efficiency is $6 \times 10^{-4}$. The background composition is $9.5 \times 10^{-6}$ of the total ttbar, $3.4 \times 10^{-6}$ of the total WW, $4 \times 10^{-6}$ of the total WZ, $3.2 \times 10^{-6}$ of the total Wt, $2.2 \times 10^{-6}$ of the total $\mathrm{Z}+$ jet, 0 of the total $\mathrm{ZZ}, \mathrm{Zb} \bar{b}, \mathrm{DY} 2 \tau$.

The CMS discovery plot for the $e^{ \pm} \mu^{\mp}+E_{\mathrm{T}}^{\text {miss }}$ signature is presented in Fig. 13.30.

In the MSSM the off-diagonal components of the slepton mass terms violate lepton flavour conservation. As it was shown in Refs. [690-692] it is possible to look for lepton 
Table 13.16. Number of signal events and significances $S_{c 12}$ [50] and $S_{c L}[100,102]$, defined in Appendix A.1, for $\mathcal{L}=10 \mathrm{fb}^{-1}$

\begin{tabular}{lrrr}
\hline Point & $N$ events & $S_{c 12}$ & $S_{c L}$ \\
\hline LM1 & 329 & 21.8 & 24.9 \\
LM2 & 94 & 8.1 & 8.6 \\
LM3 & 402 & 25.2 & 29.2 \\
LM4 & 301 & 20.4 & 23.1 \\
LM5 & 91 & 7.8 & 8.3 \\
LM6 & 222 & 16.2 & 18.0 \\
LM7 & 14 & 1.4 & 1.4 \\
LM8 & 234 & 16.9 & 18.8 \\
LM9 & 137 & 11.0 & 11.9 \\
\hline
\end{tabular}

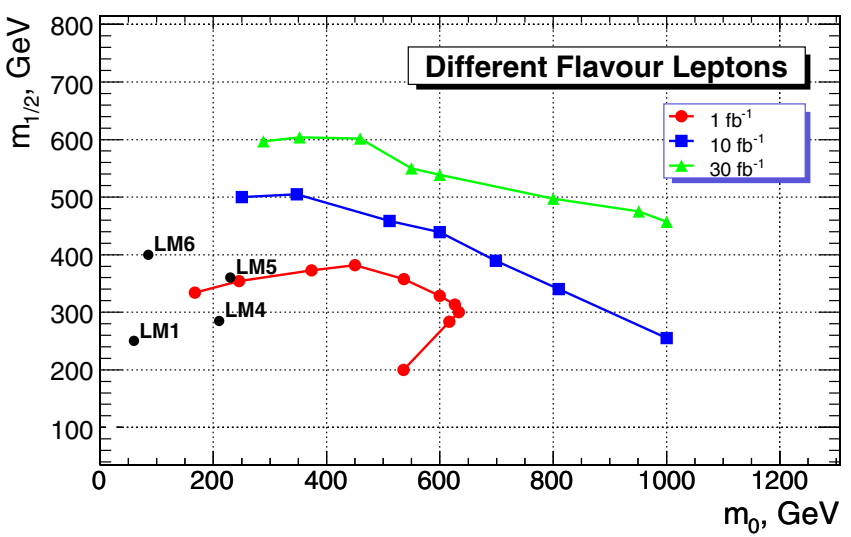

Figure 13.30. Discovery plot $(\tan \beta=10, \operatorname{sign}(\mu)=+, A=0)$ for the luminosities $\mathcal{L}=1$, $10,30 \mathrm{fb}^{-1}$ for the $e^{ \pm} \mu^{\mp}+E_{\mathrm{T}}^{\text {miss }}$ signature.

flavour violation at supercolliders through the production and decays of the sleptons. For the LFV at the LHC one of the most promising processes is the LFV decay of the second neutralino $[693,694] \quad \tilde{\chi}_{2}^{0} \rightarrow \tilde{l} l \rightarrow \tilde{\chi}_{1}^{0} l l^{\prime}$, where the non zero off-diagonal component of the slepton mass matrix leads to the different flavours for the leptons in the final state. By using the above mode, LFV in $\tilde{e}-\tilde{\mu}$ mixing has been investigated in Refs. [693, 694] at a parton model level for a toy detector. In this section we study the perspectives of the LFV detection in CMS on the base of full simulation of both signal and background is studied. To be specific, we study the point LM1. We assume that the LFV is due to nonzero mixing of right-handed smuon and selectron. The signal of the LFV $\tilde{\chi}_{2}^{0}$ decay is two opposite-sign leptons $\left(e^{+} \mu^{-}\right.$or $e^{-} \mu^{+}$) in the final state with the characteristic edge structure. In the limit of lepton flavour conservation, the process $\tilde{\chi}_{2}^{0} \rightarrow \tilde{l} l \rightarrow l l \tilde{\chi}_{1}^{0}$ has the edge structure for the distribution of the lepton-pair invariant mass $m_{l l}$ and the edge mass $m_{l l}^{\max }$ is expressed by the slepton mass $m_{\tilde{l}}$ and the neutralino masses $m_{\tilde{\chi}_{1,2}^{0}}$ as follows:

$$
\left(m_{l l}^{\max }\right)^{2}=m_{\tilde{\chi}_{2}^{0}}^{2}\left(1-\frac{m_{\tilde{l}}^{2}}{m_{\tilde{\chi}_{2}^{0}}^{2}}\right)\left(1-\frac{m_{\tilde{\chi}_{1}^{0}}^{2}}{m_{\tilde{l}}^{2}}\right) .
$$

The SUSY background for the LFV comes from uncorrelated leptons from different squark or gluino decay chains. The SM background comes mainly from

$$
t \bar{t} \rightarrow b W b W \rightarrow b l b l^{\prime} v v^{\prime} .
$$



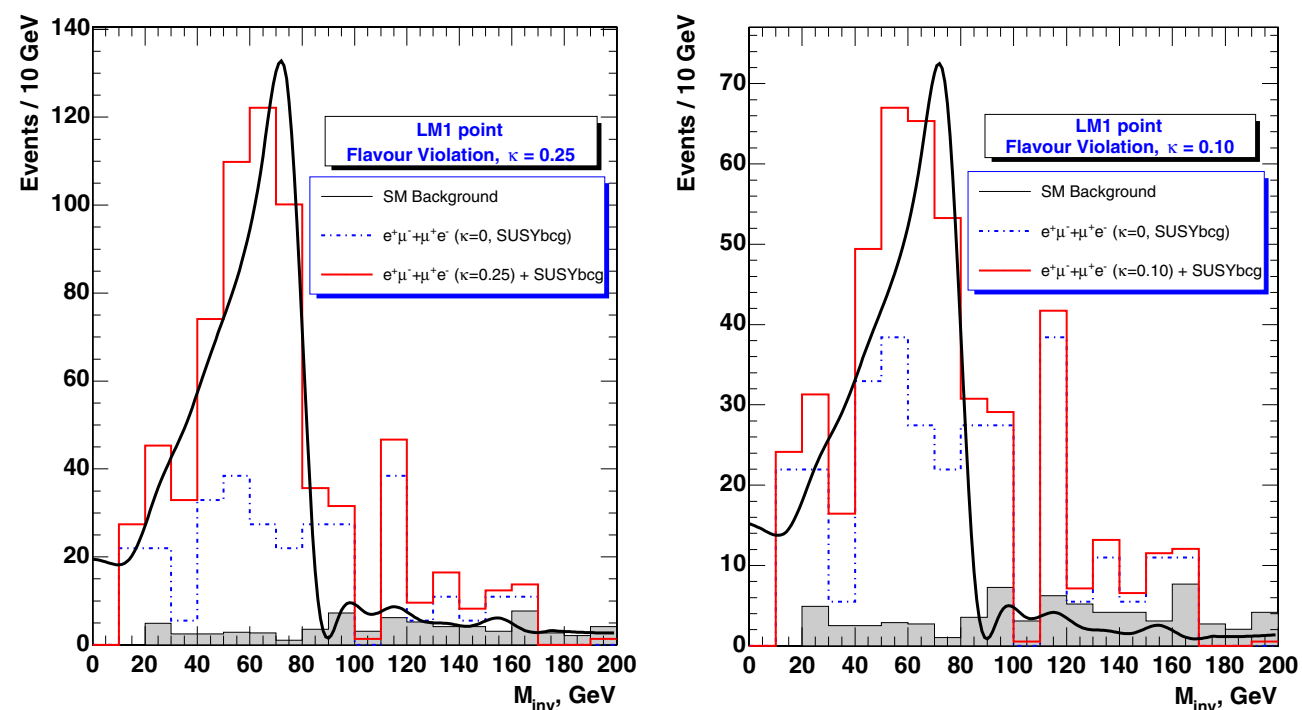

Figure 13.31. The distribution of dilepton invariant mass after selection of two isolated $e^{ \pm} \mu^{\mp}$ leptons with $p_{\mathrm{T}}^{\text {lept }}>20 \mathrm{GeV} / \mathrm{c}$ and $E_{\mathrm{T}}^{\text {miss }}>300 \mathrm{GeV}$ for flavour violation parameter $k=0.25$ (left) and $k=0.1$ (right). The superimposed curves are fits to the invariant mass distribution for the case of $100 \%$ LFV.

The Drell-Yan background from $p p \rightarrow \tau \tau \rightarrow e \mu \ldots$ is negligible. It should be stressed that for the signature with $e^{ \pm} \mu^{\mp}$ in the absence of the LFV we do not have the edge structure for the distribution on the invariant mass $m_{i n v}\left(e^{ \pm} \mu^{\mp}\right)$. As the result of the LFV the edge structure for $e^{ \pm} \mu^{\mp}$ events arises too. Therefore the signature of the LFV is the existence of an edge structure in the $e^{ \pm} \mu^{\mp}$ distribution. The rate for a flavour violating decay is

$$
\operatorname{Br}\left(\tilde{\chi}_{2}^{0} \rightarrow e^{ \pm} \mu^{\mp} \tilde{\chi}_{1}^{0}\right)=\kappa B r\left(\tilde{\chi}_{2}^{0} \rightarrow e^{+} e^{-} \tilde{\chi}_{1}^{0}, \mu^{+} \mu^{-} \tilde{\chi}_{1}^{0}\right),
$$

where

$$
\begin{aligned}
& \operatorname{Br}\left(\tilde{\chi}_{2}^{0} \rightarrow e^{+} e^{-} \tilde{\chi}_{1}^{0}, \mu^{+} \mu^{-} \tilde{\chi}_{1}^{0}\right)=\operatorname{Br}\left(\tilde{\chi}_{2}^{0} \rightarrow e^{+} e^{-} \tilde{\chi}_{1}^{0}\right) \\
& +B r\left(\tilde{\chi}_{2}^{0} \rightarrow \mu^{+} \mu^{-} \tilde{\chi}_{1}^{0}\right) \text {, } \\
& \kappa=2 x \sin ^{2} \theta \cos ^{2} \theta \\
& x=\frac{\Delta m_{\tilde{e} \tilde{\mu}}^{2}}{\Delta m_{\tilde{e} \tilde{\mu}}^{2}+\Gamma^{2}}, \\
& B r\left(\tilde{\chi}_{2}^{0} \rightarrow e^{ \pm} \mu^{\mp}\right)=\operatorname{Br}\left(\tilde{\chi}_{2}^{0} \rightarrow e^{+} \mu^{-}\right)+B r\left(\tilde{\chi}_{2}^{0} \rightarrow e^{-} \mu^{+}\right) .
\end{aligned}
$$

Here $\theta$ is the mixing angle between $\tilde{e}_{R}$ and $\tilde{\mu}_{R}$ and $\Gamma$ is the sleptons decay width. The parameter $x$ is the measure of the quantum interference effect. There are some limits on $\tilde{e}-\tilde{\mu}$ mass splitting from lepton flavour violating processes but they are not very strong.

For $\kappa=0.25, \kappa=0.1$ the distributions of the number of $e^{ \pm} \mu^{\mp}$ events on the invariant mass $m_{i n v}\left(e^{ \pm} \mu^{\mp}\right)$ (see Figure 13.31) clearly demonstrates the existence of the edge structure [695], i.e. the existence of the lepton flavour violation in neutralino decays. It appears that for the point LM1 the use of an additional cut

$$
m_{\text {inv }}\left(e^{ \pm} \mu^{\mp}\right)<85 \mathrm{GeV}
$$



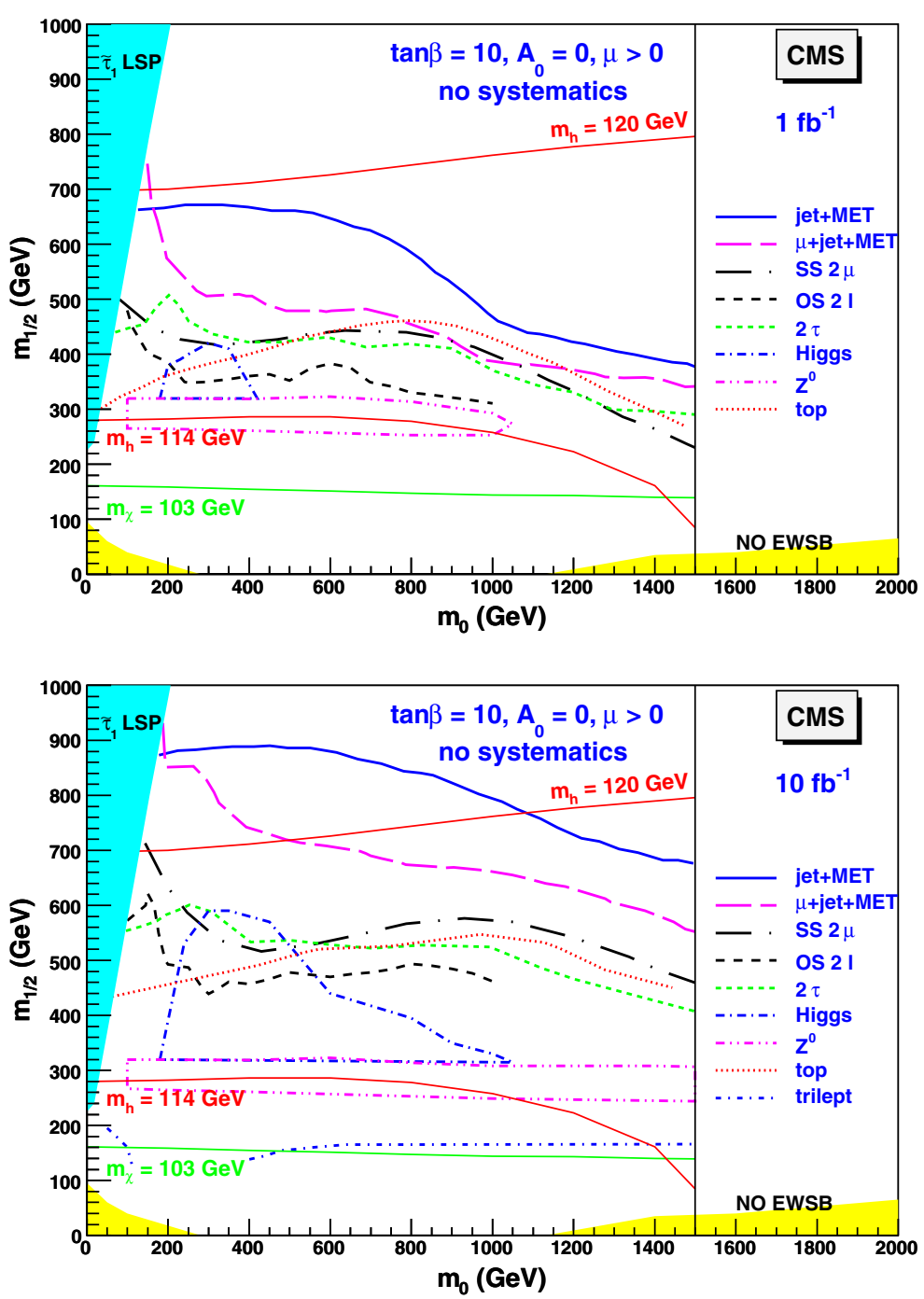

Figure 13.32. Regions of the $m_{0}$ versus $m_{1 / 2}$ plane showing CMS the reach when only statistical uncertainties are taken into account. (Top) for $1 \mathrm{fb}^{-1}$ integrated luminosity, except the Higgs case which assumes $2 \mathrm{fb}^{-1}$. (Bottom) for $10 \mathrm{fb}^{-1}$.

reduces both the SM and SUSY backgrounds and increases the discovery potential in the LFV search. For the point LM1 we found that in the assumption of exact knowledge of the background (both the SM and SUSY backgrounds) for the integrated luminosity $\mathcal{L}=10 \mathrm{fb}^{-1}$ it would be possible to detect LFV at $5 \sigma$ level in $\tilde{\chi}_{2}^{0}$ decays for $\kappa \geqslant 0.04$.

\subsection{Summary of the reach with inclusive analyses}

\subsubsection{Summary of the mSUGRA studies}

In previous sections, several characteristic topologies (or signatures) for MSSM were studied and it was shown that many are already detectable with rather low integrated luminosity 

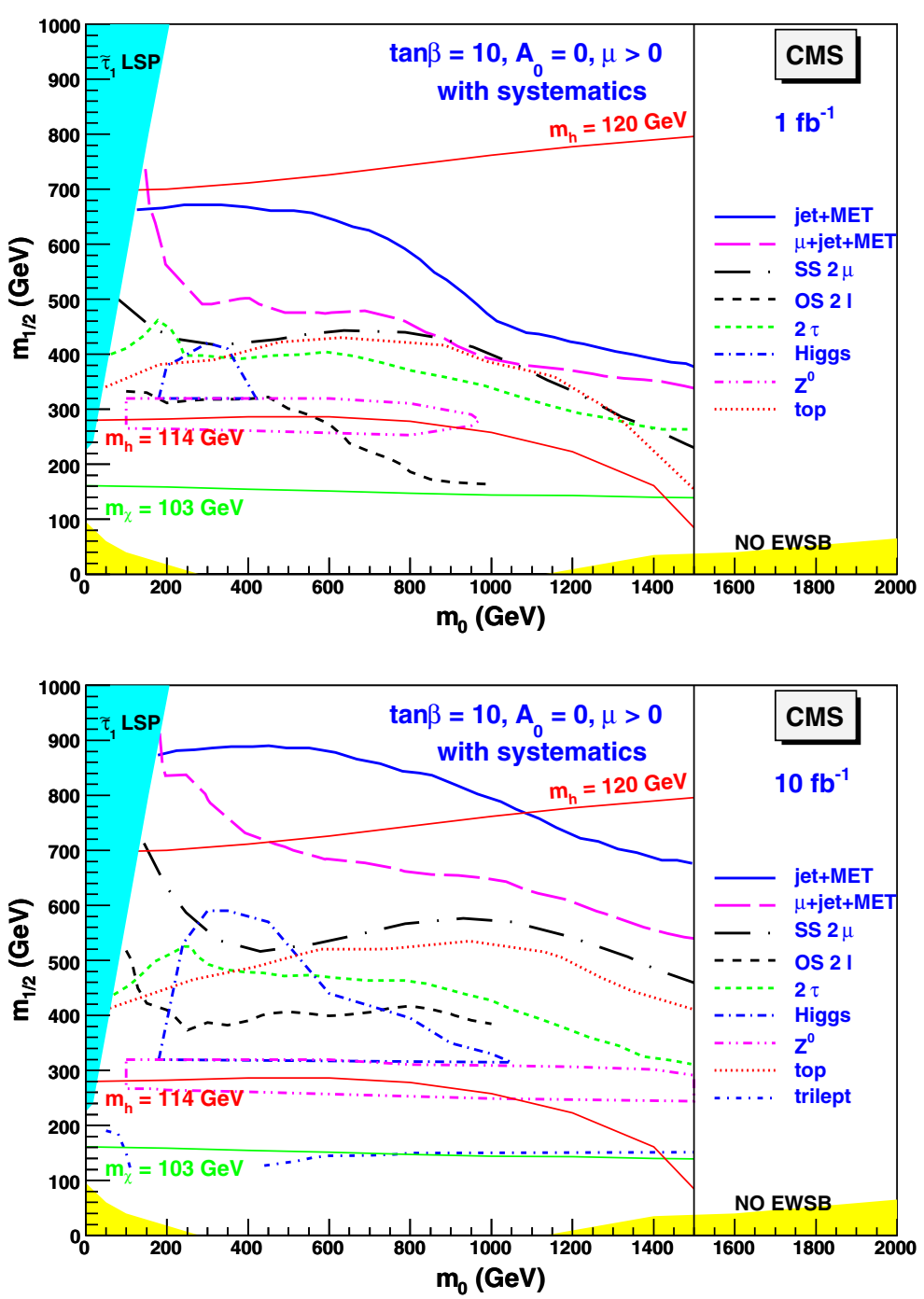

Figure 13.33. Regions of the $m_{0}$ versus $m_{1 / 2}$ plane showing CMS the reach when systematic uncertainties are included. (Top) for $1 \mathrm{fb}^{-1}$ integrated luminosity, except the Higgs case which assumes $2 \mathrm{fb}^{-1}$. (Bottom) for $10 \mathrm{fb}^{-1}$.

(few years of LHC running) over a sizeable part of the parameter space, extending well beyond the Tevatron reach.

The curves in Fig. 13.32 summarise the reach estimated for the various topologies of the preceding sections for integrated luminosities of 1 and $10 \mathrm{fb}^{-1}$ when only statistical uncertainties are taken into account. The same results are shown in Fig. 13.33 when systematic uncertainties are included. It is seen that the systematic uncertainties do not degrade the reach very much for integrated luminosities up to $10 \mathrm{fb}^{-1}$. It should be noted that the analyses have not been reoptimised for the inclusion of systematics nor for higher masses which could be reached with higher luminosity. Moreover, the reach will be further improved by the addition of topologies with electrons, which are presently missing for the muon + jet + MET and same sign dimuon searches. 


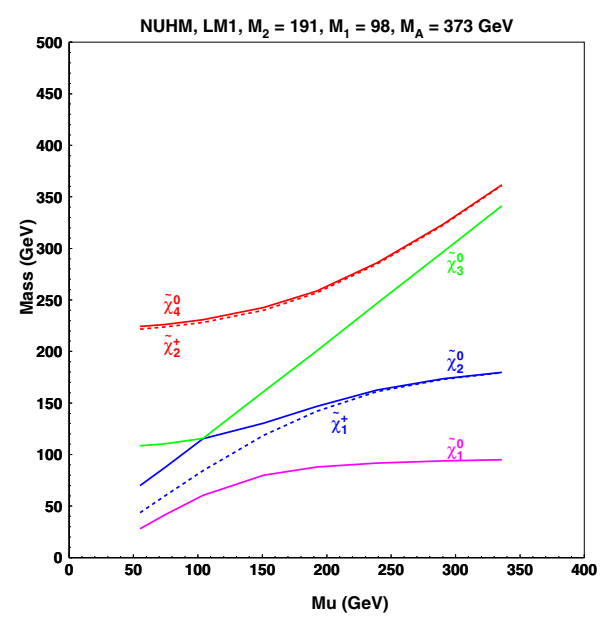

Figure 13.34. Variation of the chargino and neutralino masses as a function of $\mu$ for the CMS test point LM1.

The best reach is obtained with the most inclusive channels, the jets + MET and muons + jet + MET. The range of gluino and squark masses up to about $1.5 \mathrm{TeV}$ can be probed with an integrated luminosity of only $1 \mathrm{fb}^{-1}$ and is extended to about $2 \mathrm{TeV}$ with $10 \mathrm{fb}^{-1}$. Moreover, a large part of the area is covered by several search topologies. The simultaneous observation of a signal in various topologies will help unravel the underlying physics. Examples are the triangular dilepton mass distribution, the observation of the $Z^{0}$ or the $h^{0}$ in less inclusive channels, which provide a hint that their origin may be the decay of a $\tilde{\chi}_{2}^{0}$. If discovered, yet more exclusive analyses should then allow a more quantitative study, e.g. the reconstruction of the sparticle masses and cross section measurements of relevant sub-processes and their ratios.

\subsection{Look beyond mSUGRA}

\subsubsection{Non-universal Higgs masses}

It was emphasised in Section 13.3 that the signatures of SUSY with a stable LSP result from the fundamental Supersymmetry gauge couplings, together with the composition of the lightest charginos and neutralinos. As all previous analyses were based on mSUGRA, it is interesting to verify their robustness when relaxing some of the assumptions which might affect the signal observability. As full generality, including giving up all universality assumptions, would lead to an intractable model, a choice needs to be made. Here, a mild extension is considered whereby the two Higgsino mass parameters at the GUT scale are no longer supposed to be degenerate with the other scalar masses, which is sometimes called the Non Universal Higgs Masses (NUHM [696]) scenario. This scenario is conveniently parameterised in terms of two low scale parameters, the mass of the CP-odd Higgs $\left(m_{A}\right)$ and the parameter $\mu$. More specifically, we will analyse the effect of lowering the value of $\mu$ compared to its mSUGRA value on the observability of the signatures, as this modifies the composition of the charginos and neutralinos as a function of the gaugino and Higgsino fields. For simplicity, $m_{A}$ is kept at a fixed value. As exemplified in Fig. 13.34 for the test point LM1, lowering $\mu$ also lowers the gaugino masses and in particular their splittings, which affect the 

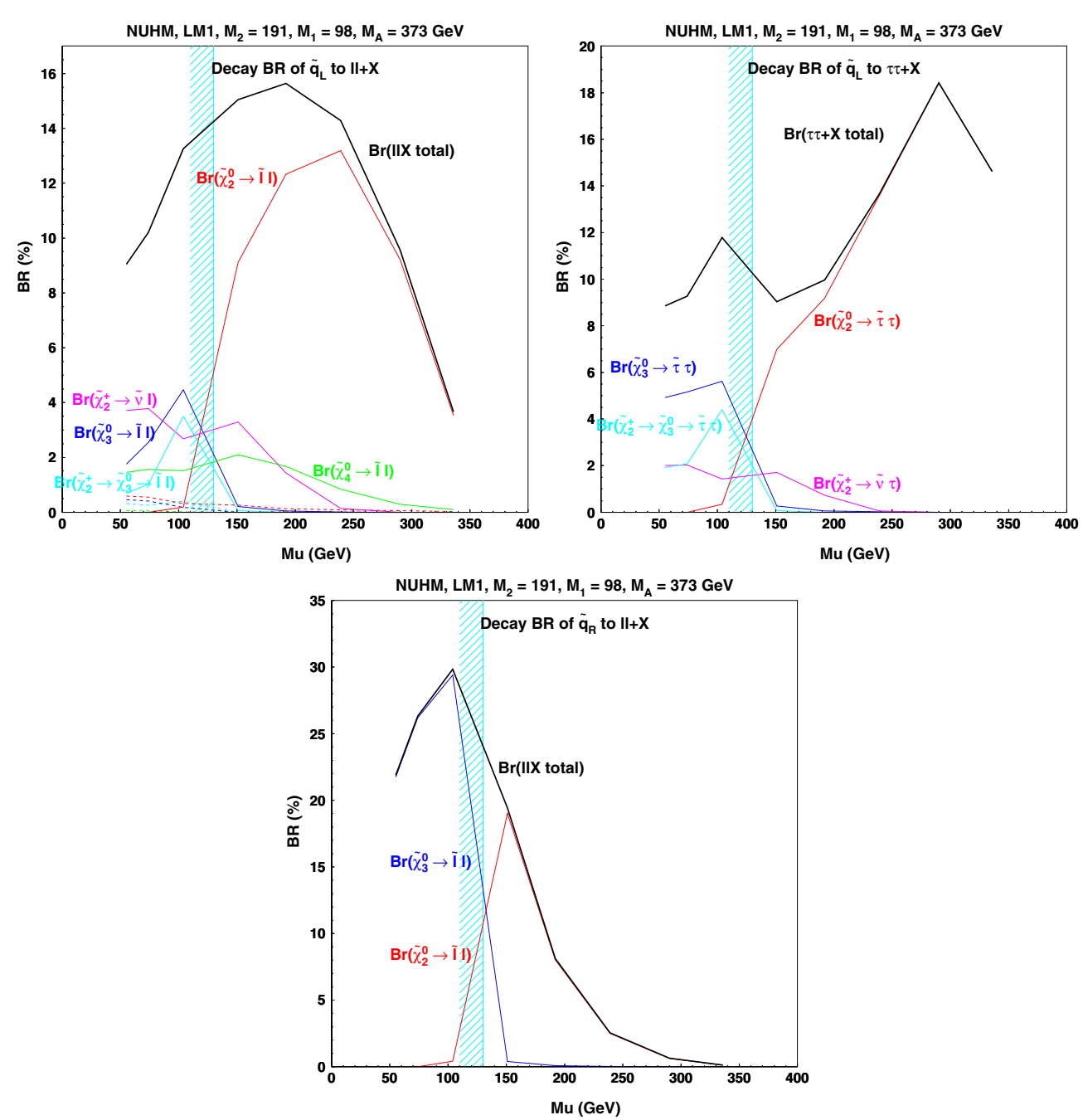

Figure 13.35. Decay branching ratios as a function of $\mu$ for $\tilde{q}_{L}$ into $l l$ and $\tau \tau$ and for $\tilde{q}_{R}$ into $l l$ at the test point LM1.

branching ratios through phase space effects (a similar behaviour is observed for the other test points). The $\tilde{q}$ and $\tilde{l}$ spectra are almost unaffected. As for low values of $\mu$ the lightest chargino becomes lighter than the exclusion from LEP, $\left.m_{(} \tilde{\chi}_{1}^{ \pm}\right) \geqslant 103 \mathrm{GeV}$, this region is excluded and is indicated on Fig. 13.35 by a grey (blue) shaded strip.

13.18.1.1. Signatures at point LM1. The test point LM1 was studied above for its detectability in cascade decays via a $\tilde{\chi}_{2}^{0}$ into $\tilde{l}_{R} l$. Figure 13.35 shows the variation of some branching ratios from the value of $\mu$ near the region where radiative electroweak symmetry breaking is not possible up to its value in mSUGRA.

It is seen that by lowering $\mu, B\left(\tilde{q}_{L} \rightarrow q \tilde{\chi}_{2}^{0} \rightarrow q \tilde{l}_{R} l\right)$ first increases (due to closing the competing decay to $\tilde{v} \nu$ ), then decreases when the $\tilde{\chi}_{2}^{0}$ becomes Higgsino-like, but it remains considerably larger than its mSUGRA value for all values of $\mu$ down to the LEP limit. In 

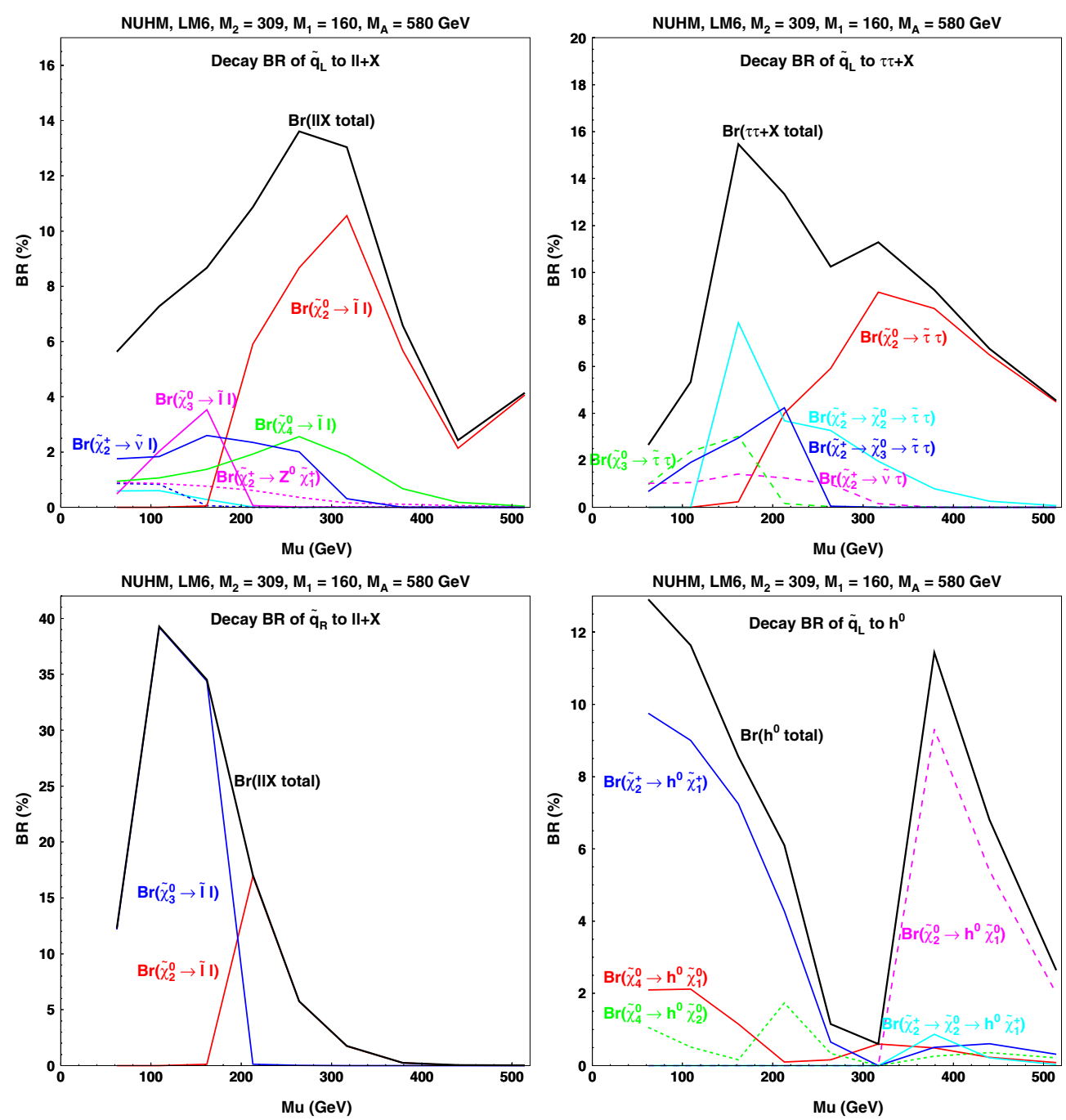

Figure 13.36. Decay branching ratios as a function of $\mu$ for $\tilde{q}_{L}$ into $l l, \tau \tau$ and $h^{0}$ and for $\tilde{q}_{R}$ into $l l$ at the test point LM6.

addition, some new channels open up, like the decay via $\tilde{\chi}_{4}^{0}$ into left and right sleptons and the decay via a $\tilde{\chi}_{2}^{ \pm} \rightarrow \tilde{v}_{l} \bar{l}$ followed by $\tilde{v}_{l} \rightarrow \tilde{\chi}_{1}^{ \pm} l$ (the $\tilde{\chi}_{4}^{0}$ and $\tilde{\chi}_{2}^{ \pm}$become more Wino-like). Other decays via $\tilde{\chi}_{3}^{0}$ might also contribute, but only in the region excluded by LEP.

The branching for the decay to $\tilde{\tau} \tau$ shows qualitatively the same behaviour, but is larger than its mSUGRA value in only a small region of $\mu$. Also here a small contribution from the decay $\tilde{\chi}_{2}^{ \pm} \rightarrow \tilde{v} \tau$ is present at small $\mu$.

It is interesting to note that, although for mSUGRA the $\tilde{q}_{R}$ decays exclusively directly to the LSP, it may have for lower $\mu$ a non negligible branching ratio to $\tilde{\chi}_{2}^{0}$ and also contributes to the dilepton signature.

Finally, there is a non-zero branching ratio for the $\tilde{q}_{L}$ to the light Higgs via the $\tilde{\chi}_{2}^{ \pm}$or $\tilde{\chi}_{4}^{0}$ (not shown), but it remains below $1 \%$ over the whole range of $\mu$ above the LEP limit and will be difficult to detect. 

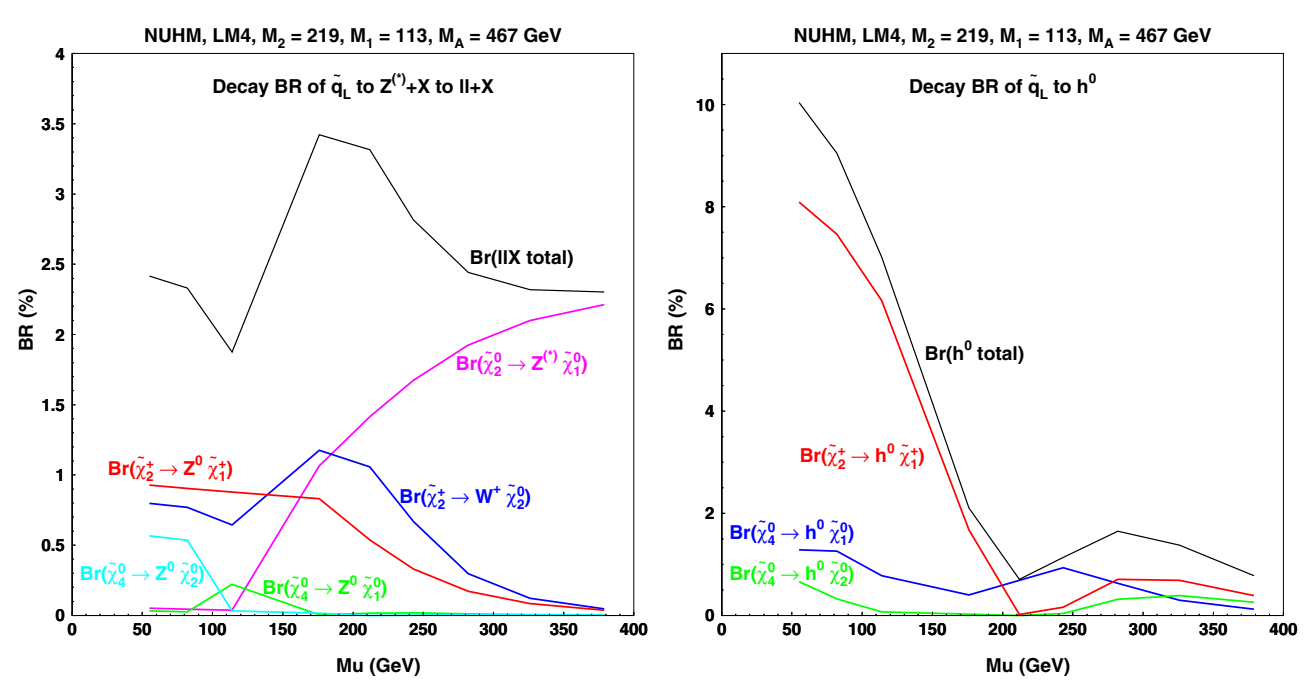

Figure 13.37. Decay branching ratios as a function of $\mu$ for $\tilde{q}_{L}$ into $l l$ and $h^{0}$ at the test point LM4.

13.18.1.2. Signatures at point LM6. The test point LM6 has many features in common with LM1, but the $\tilde{\chi}_{2}^{0}$ decays mainly to $\tilde{l}_{L} l$ with a small admixture of $\tilde{l}_{R} l$. Moreover the decay $\tilde{\chi}_{2}^{0} \rightarrow h^{0} \tilde{\chi}_{1}^{0}$ is kinematically allowed, although suppressed due to the strong gaugino dominance in the $\tilde{\chi}_{1}^{0}$ and $\tilde{\chi}_{2}^{0}$. The variation of the branching ratios as a function of $\mu$ is displayed in Fig. 13.36.

The cascade decays of $\tilde{q}_{L}$ to $\tilde{l} l$ and $\tilde{\tau} \tau$ via $\tilde{\chi}_{2}^{0}$ show grossly the same behaviour as for LM1, with an increase at intermediate values of $\mu$ followed by a decrease at low $\mu$. Again, the contributions from other charginos and neutralinos are non negligible near the LEP exclusion limit. Also $\tilde{q}_{R}$ decays contribute to the dilepton signal via $\tilde{\chi}_{2}^{0}$ and $\tilde{\chi}_{3}^{0}$ intermediate states.

A distinctive feature of LM6 is its production of final states with $h^{0}$. The $\tilde{q}_{L}$ branching ratio via $\tilde{\chi}_{2}^{0} \rightarrow h^{0} \tilde{\chi}_{1}^{0}$, which is only $2 \%$ for mSUGRA increases drastically for lower $\mu$ due to the increased Higgsino components in $\tilde{\chi}_{1}^{0}$ and $\tilde{\chi}_{2}^{0}$, then it drops as the decay becomes kinematically forbidden. After a gap where the branching ratio is below $1 \%$, a strong increase is again visible for lower $\mu$ from the cascade dominated by $\tilde{\chi}_{1}^{ \pm} \rightarrow h_{0} \tilde{\chi}_{1}^{ \pm}$down to the LEP limit. Such an effect is not observed at LM1 due to the smaller spacing of the masses.

13.18.1.3. Signatures at point LM4. Point LM4 was chosen for its characteristic decay of $\tilde{\chi}_{2}^{0}$ into $Z^{0} \tilde{\chi}_{1}^{0}$. Figure 13.37 shows the variation of the branching ratios as a function of $\mu$.

As the decay $\tilde{\chi}_{2}^{0} \rightarrow Z^{0} \tilde{\chi}_{1}^{0}$ requires Higgsino components in both the $\tilde{\chi}_{1}^{0}$ and $\tilde{\chi}_{2}^{0}$, its branching ratio remains above $90 \%$ for all values of $\mu$ allowed by the LEP limit. The branching ratio of the $\tilde{q}_{L}$ into $Z^{(+)}$via a $\tilde{\chi}_{2}^{0}$ decreases mainly due to the decrease of $B\left(\tilde{q}_{L} \rightarrow q \tilde{\chi}_{2}^{0}\right)$ (the $\tilde{\chi}_{2}^{0}$ becomes less gaugino-like). This loss is, however, compensated by the contributions from cascades via $\tilde{\chi}_{2}^{ \pm} \rightarrow W \tilde{\chi}_{2}^{0}$ and $\tilde{\chi}_{2}^{ \pm} \rightarrow Z^{0} \tilde{\chi}_{1}^{ \pm}$and the overall effect is a net increase of the branching ratio of the $\tilde{q}_{L}$ to final states with a $Z^{0}$.

For low values of $\mu$ there is also a contribution to $h^{0}$ final states via the decay $\tilde{\chi}_{2}^{ \pm} \rightarrow$ $h^{0} \tilde{\chi}_{1}^{ \pm}$, but it remains small above the limit imposed by LEP.

13.18.1.4. Signatures at point LM5. At point LM5, the main signature for mSUGRA is provided by the cascade via $\tilde{\chi}_{2}^{0} \rightarrow h^{0} \tilde{\chi}_{1}^{0}$. The variation of the branching ratios with $\mu$ are shown in Fig. 13.38. 

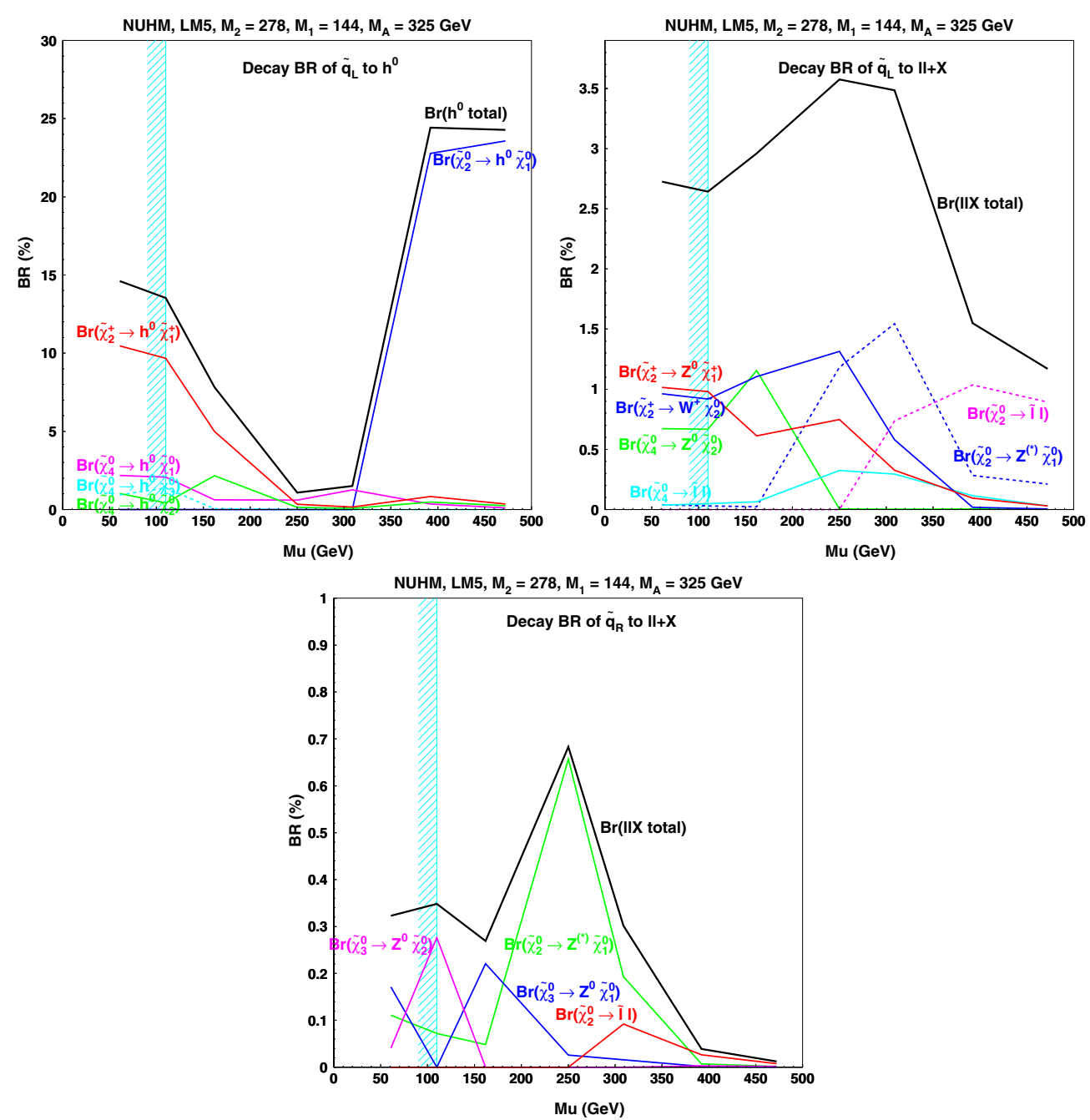

Figure 13.38. Decay branching ratios as a function of $\mu$ for $\tilde{q}_{L}$ into $h^{0}$ and $l l$ and for $\tilde{q}_{R}$ into $l l$ at the test point LM5.

The sharp drop in the branching ratio of $\tilde{\chi}_{2}^{0}$ to $h^{0}$ below the mSUGRA value of $\mu$ results from the decrease in the mass splitting between $\tilde{\chi}_{2}^{0}$ and $\tilde{\chi}_{1}^{0}$ which suppresses the decay to $h^{0}$. For lower values of $\mu$, final states with $h^{0}$ are again produced mainly via the $\tilde{\chi}_{2}^{ \pm} \rightarrow h^{0} \tilde{\chi}_{1}^{ \pm}$. In between these two decay chains, a narrow gap is left where the Higgs branching ratio is less than $2 \%$ and hence very difficult to detect.

It is seen that this loss of sensitivity to Higgs final states is to some extent compensated by an increase of the dilepton final states in the region of the gap. The cascade decays of both $\tilde{q}_{L}$ and $\tilde{q}_{R}$ contribute in this region, the main contributions being through $\tilde{\chi}_{2}^{0} \rightarrow Z^{*} \tilde{\chi}_{1}^{0}$, $\tilde{\chi}_{2}^{ \pm} \rightarrow Z^{0} \tilde{\chi}_{1}^{ \pm}$and $\tilde{\chi}_{2}^{ \pm} \rightarrow W \tilde{\chi}_{2}^{0}$. It gives a branching ratio of up to $3.5 \%$ for the dilepton decay of $\tilde{q}_{L}$ and less than $1 \%$ for $\tilde{q}_{R}$ and hence should be detectable. However, the mixture of intermediate states leading to the dileptons will make the sparticle mass reconstruction very challenging. 
13.18.1.5. Conclusion. It can be concluded that the same flavour dilepton signatures originating from the decay of $\tilde{l} l$ or $Z^{*}$ are quite robust with respect to the chargino and neutralino composition. Lowering $\mu$ with respect to its mSUGRA value, a sizeable increase of the branching ratio is even observed for the test points LM1, LM4 and LM6. The $h^{0}$ signature at point LM5 is less robust and a region with low branching ratio exists at intermediate values of $\mu$. It is compensated by an increase of dilepton final states. It may be noted that the loss of $\tilde{\chi}_{2}^{0}$ decay to $h^{0}$ is due to the reduction of the $\tilde{\chi}_{2}^{0}$ and $\tilde{\chi}_{1}^{0}$ mass splitting. It is therefore a consequence of the low mass spectrum chosen and should disappear at larger values of $m_{1 / 2}$. Another feature of the NUHM scenario is that for small $\mu$ the cascades from $\tilde{q}_{R}$ also contribute to the signatures, unlike the mSUGRA case. Moreover the signatures at low to intermediate $\mu$ tend to be produced by several intermediate neutralino and chargino states. This points to the difficulty of identifying which sparticles are at the origin of the observed end points in the effective mass distributions. 


\section{Chapter 14. Extra Dimensions and New Vector Boson High Mass States}

\subsection{Introduction}

The theoretical and phenomenological landscape of beyond the standard model searches extends to a multitude of exotic tendencies today in collider physics. Most are conceived within one kind or another of extra dimensions and supersymmetric scenarios. The strict or loose dualities between different frameworks for physics "beyond the Standard Model" have a direct experimental consequence: the final states and signatures of the models are very similar. This renders the characterisation of an excess or a deviation a fine and probably long challenge. To mention a couple of examples: the question "is it extra dimensions (e.g. $\mathrm{UED} / \mathrm{TeV}$ ) or is it SUSY?" or "is it a Randall-Sundrum graviton mode or a Z'" is not going to be answered immediately when the excess is observed. The results from all the collider data to date, together with the as yet unobserved Higgs and including the data on the neutrino masses and the composition of the universe, impose a wide program of searches that the LHC experiments are preparing for.

In the present chapter and as well as the "alternatives" chapter that follows, a series of searches is presented with signatures (corresponding to models) as indicated below:

- Dilepton, dijet, diphoton resonances

* using ee, $\mu \mu, \gamma \gamma$, dijets

* searching for $Z^{\prime}$ (leptons, jets), RS Extra Dimensions (leptons, photons, jets), $Z_{K K}$ in $\mathrm{TeV}^{-1}$ (electrons) (can also be interpreted in the context of Little Higgs models)

- Dilepton, dijet continuum modification

* using $\mu \mu$, dijets

* searching for ADD graviton exchange (dimuons), contact interactions (dimuons, dijets)

- Dilepton + dijets

* using ee, $\mu \mu+$ dijets

* searching for heavy neutrino from right-handed $W$ (can also be interpreted in the context of leptoquark searches)

- Single photon + missing $E_{\mathrm{T}}$

$*$ using $\gamma+$ missing $\mathrm{E}_{\mathrm{T}}$

* searching for ADD direct graviton emission (can also be interpreted in the context of GMSB gravitino-type searches)

- Single lepton + missing $E_{\mathrm{T}}$

* using $\mu+$ missing $\mathrm{E}_{\mathrm{T}}$

* searching for $W^{\prime}$ (can also be interpreted in the context of little Higgs or $W_{K K}$ excitation in $\mathrm{TeV}^{-1}$ models)

- Multilepton + multijet

* using top, $W$ and $Z$ reconstruction and constraints

* searching for technicolour, littlest Higgs (can also be interpreted in the context of leptoquark searches)

- Same-sign dileptons

* using $e e, \mu \mu, e \mu$

* searching for same-sign top (can be interpreted in the context of technicolour, charged Higgs or SUSY searches) 
- High multiplicity/sphericity

* searching for microscopic black holes in large extra dimensions scenarios

Although not included here, a number of searches are being developed for signatures that involve heavy highly-ionising charged particles and split-SUSY type R-hadrons as well as low $P_{\mathrm{T}}$ multi-lepton signatures in UED scenarios. Strategies are being developed to extract the Standard Model backgrounds from data and control its systematic uncertainties. Fake rates are being estimated as possible while machine and cosmic ray induced backgrounds are not included although methods to suppress them are being developed.

\subsubsection{Models with heavy vector bosons}

Additional heavy neutral gauge bosons $\left(Z^{\prime}\right)$ are predicted in many superstring-inspired [87, 88] and grand unified theories (GUTs) [89], as well as in dynamical symmetry breaking [90] and "little Higgs" [91] models. There are no reliable theoretical predictions, however, of the $\mathrm{Z}^{\prime}$ mass scale. Current lower limits on the $\mathrm{Z}^{\prime}$ mass are (depending on the model) of the order of $600-900 \mathrm{GeV} / \mathrm{c}^{2}$ [54]. The mass region up to about $1 \mathrm{TeV} / \mathrm{c}^{2}$ is expected to be explored at Run II at the Tevatron [92, 93]. The LHC offers the opportunity to search for $\mathrm{Z}^{\prime}$ bosons in a mass range significantly larger than $1 \mathrm{TeV} / \mathrm{c}^{2}$. In the $\mathrm{Z}^{\prime}$ studies presented here (Sections 14.3 and 14.2) six models which are frequently discussed and whose properties are representative of a broad class of extra gauge bosons are used:

- $\mathrm{Z}_{\mathrm{SSM}}$ within the Sequential Standard Model (SSM), which has the same couplings as the Standard Model $Z^{0}$.

- $\mathrm{Z}_{\psi}, \mathrm{Z} \eta$ and $\mathrm{Z}_{\chi}$, arising in $\mathrm{E}_{6}$ and $\mathrm{SO}(10)$ GUT groups with couplings to quarks and leptons as derived in Refs. [96, 97].

- $\mathrm{Z}_{\mathrm{LRM}}$ and $\mathrm{Z}_{\mathrm{ALRM}}$, arising in the framework of the so-called "left-right" [98] and "alternative left-right" [92, 93] models with couplings as derived in Ref. [92, 93], with the choice of $g_{R}=g_{L}$.

The $W^{\prime}$ search presented in Section 14.4 uses a reference model by Altarelli [697], in which the $W^{\prime}$ is a heavy copy of the $W$, with the very same left-handed fermionic couplings (including CKM matrix elements), while there is no interaction with the Standard Model gauge bosons or with other heavy gauge bosons such as a $Z^{\prime}$.

\subsubsection{Arkani-Hamed-Dimopoulos-Dvali (ADD) models}

ADD refers to the class of models which incorporate the large extra dimensions scenario of Arkani-Hamed, Dvali, and Dimopoulos [698]. These were the first extra dimensions models in which the compactified dimensions can be of macroscopic size, consistent with all current measurements, and they are referred to as "large extra dimensions" models. In the most basic version, $n$ extra spatial dimensions are compactified on a torus with common circumference $R$, and a brane is introduced which extends only in the three infinite spatial directions. Strictly speaking, the brane should have a very small tension (energy per unit volume) in order that it does not significantly warp the extra dimensional space. It is assumed that all standard model fields extend only in the brane. This can be considered as a toy version of what happens in string theory, where chiral gauge theories similar to the standard model are confined to reasonably simple brane configurations in reasonably simple string compactifications [699].

A consequence of these assumptions is that the effective $4 \mathrm{~d}$ Planck scale is related to the underlying fundamental Planck scale of the $4+n$-dimensional theory and to the volume of 
the compactified space. This relation follows from Gauss' law, or by dimensional analysis

$$
M_{\text {Planck }}^{2}=M_{*}^{2+n} R^{n},
$$

where $M_{\text {Planck }}^{2}$ is defined by Newton's constant: $M_{\text {Planck }}=1 / \sqrt{G_{N}}=1.2 \times 10^{19} \mathrm{GeV} / \mathrm{c}^{2}$. $M_{*}^{2+n}$ is defined as the gravitational coupling which appears in the $4+n$-dimensional version of the Einstein-Hilbert action. It is the quantum gravity scale of the higher dimensional theory.

If $M_{\text {Planck }}, M^{*}$ and $1 / R$ are all of the same order, as is usually assumed in string theory, this relation is not very interesting. But it is plausible and experimentally allowed that $M^{*}$ is equal to some completely different scale. Taking $M^{*} \sim 1 \mathrm{TeV} / \mathrm{c}^{2}[700]$ the hierarchy problem of the standard model is translated from an ultraviolet problem to an infrared one. Note that if there is any interface with string theory, ADD-like models must arise from string ground states in which the string scale (and thus the ultraviolet cutoff for gravity) is also in the TeV range. This is difficult to achieve but has been studied in [701].

The ADD scenario renders observations of quantum gravity at the LHC possible. In such models only the graviton, and possibly some non-SM exotics like the right-handed neutrino, probe the full bulk space. There is a Kaluza-Klein (KK) tower of graviton modes, where the massless mode is the standard $4 \mathrm{~d}$ graviton, and the other KK modes are massive spin 2 particles which also couple to SM matter with gravitational strength.

Whereas bremsstrahlung of ordinary gravitons is a completely negligible effect at colliders, the total cross section to produce some massive KK graviton is volume enhanced, and effectively suppressed only by powers of $M^{*}$ and not $M_{\text {Planck. }}$. From Eq. (14.1) it follows:

$$
\sigma \sim \frac{1}{M_{\text {Planck }}^{2}}(E R)^{n} \sim \frac{1}{M_{*}^{2}}\left(E M_{*}\right)^{n},
$$

where $E$ is the characteristic energy of the subprocess.

For graviton phenomenology it is useful to replace the ADD parameter $M^{*}$ by other rescaled parameters. The two most useful choices are taken from the work of Giudice, Rattazzi and Wells (GRZ) [702], and Han, Lykken and Zhang (HLZ) [703]:

$$
\begin{aligned}
& M_{*}^{n+2}=\frac{S_{n-1}}{(2 \pi)^{n}} M_{s}^{n+2}, \\
& M_{*}^{n+2}=\frac{8 \pi}{(2 \pi)^{n}} M_{D}^{n+2},
\end{aligned}
$$

where $M_{s}$ is the HLZ scale, $M_{D}$ is the GRW scale, and $S_{n-1}$ is the surface area of a unit $n$-sphere:

$$
S_{n-1}=\frac{2 \pi^{n / 2}}{\Gamma(n / 2)} .
$$

Both notations are equivalent. To obtain a complete dictionary between ADD, GRZ and HLZ, one also needs to relate the ADD parameter $R$ to those used by the other authors: $R=R_{\mathrm{HLZ}}=2 \pi R_{G R W}$, and take note of the different notations for Newton's constant:

$$
\kappa^{2}=16 \pi G_{N}(\mathrm{HLZ}) ; \quad \overline{\mathrm{M}}_{\mathrm{P}}^{2}=\frac{1}{8 \pi \mathrm{G}_{\mathrm{N}}}(\mathrm{GRW}) .
$$

A Kaluza-Klein graviton mode has a mass specified by an $n$-vector of integers $\vec{k}$ :

$$
m^{2}(\vec{k})=\frac{\vec{k}^{2}}{R_{\mathrm{GRW}}^{2}} .
$$


Let $r=|\vec{k}|$. Then for large $r$ (as is often the relevant case for ADD phenomenology) the number of KK graviton states of a given polarisation with $r \leqslant r_{\max }$ is given by the integral

$$
\begin{aligned}
S_{n-1} \int_{0}^{r_{\max }} d r r^{n-1} & =\frac{1}{n} S_{n-1} r_{\max }^{n} \\
& =\int_{0}^{m_{\max }} \rho(m) d m,
\end{aligned}
$$

where the KK density of states is

$$
\rho(m)=\frac{m^{n-1}}{G_{N} M_{s}^{n+2}} .
$$

$M_{s}$ is the natural scaling parameter for KK graviton production. The density of states formulation can be applied to a much more general class of models than ADD, and can also include graviton wavefunction factors when the extra dimensions are not flat.

Consider an on-shell production of a KK graviton from a $p p$ or collision. To leading order this is a $2 \rightarrow 2$ process with two massless partons in the initial state, plus a massive KK graviton and a massless parton in the final state. Let $p_{1}, p_{2}$ denote the 4-momenta of the initial state partons, $p_{3}$ the 4-momentum of the graviton, and $p_{4}$ the 4-momentum of the outgoing parton. The total cross section for any particular variety of partonic subprocess has the form

$\sigma(1+2 \rightarrow \mathrm{KK}+4)=\int d x_{1} d x_{2} f_{1}\left(x_{1}, \hat{s}\right) f_{2}\left(x_{2}, \hat{s}\right) \int d \hat{t} \int_{0}^{\sqrt{\hat{s}}} d m \rho(m) \frac{d \sigma_{m}}{d \hat{t}}(\hat{s}, \hat{t})$,

where $f_{1}\left(x_{1}, \hat{s}\right), f_{2}\left(x_{2}, \hat{s}\right)$ are the parton distribution functions (PDFs) for the initial state partons, $\hat{s}=x_{1} x_{2} s=\left(p_{1}+p_{2}\right)^{2}$ is the square of the total centre of mass $(\mathrm{cm})$ energy of the subprocess, and $\hat{t}=\left(p_{1}-p_{3}\right)^{2}$ is the usual Mandelstam invariant. The formulae for $d \sigma_{m} / d \hat{t}$, the differential subprocess cross sections for KK gravitons of mass $m$, are given in [702].

14.1.2.1. Graviton production above the cutoff. At the LHC, proton-proton collisions will probe a distribution of partonic subprocess energies $\sqrt{\hat{s}}$. This creates a problem for the consistent analysis of missing energy signatures in the framework of ADD models. These models are simple low energy effective theories which are only valid for $\sqrt{\hat{s}}$ below some cutoff. This cutoff is at most $2 M^{*}$, and could be a factor of a few smaller if the ultraviolet completion of the model is weakly coupled string theory [704]. The same is true for the Lykken-Randall model [705], which is a low energy description of gravity in a single infinite warped extra dimension, valid up to a cutoff $\sim M^{*}$. It is inconsistent to use either type of model to describe LHC collisions with subprocess energies greater than the cutoff.

This problem was first noted by the authors of [702], who suggested replacing the ADD graviton density of states $\rho(m)$ by $\rho(m) \theta\left(\sqrt{\hat{s}}-M_{D}\right)$, where $\theta$ is a step function. This introduces a systematic theory error into the analysis. The size of this error is very sensitive to the values of $M_{D}$ and $n$. For initial LHC data sets, we will be probing the lower range of $M_{D}$ values, beginning at the current $\simeq 1 \mathrm{TeV} / \mathrm{c}^{2}$ bounds from Tevatron and LEP. This increases the theory systematic from the cutoff for any fixed $n$. For fixed $M_{D}$, the theory systematic increases rapidly for increasing $n$. For $n=2$, the theory uncertainty in the total cross section remains below about $20 \%$ even for $M_{D}$ approaching $1 \mathrm{TeV} / \mathrm{c}^{2}{ }^{49}$ For $n=6$ and above, the effect of the cutoff is enormous for modest values of $M_{D}$, because the rapid rise in the graviton density of states is not compensated by the rapid falloff of the pdfs. The theory error for the total cross section in this case can be as large as an order of magnitude.

\footnotetext{
49 To avoid strong astrophysical constraints, $n=2$ ADD models also require an ad hoc infrared cutoff, truncating the massive graviton spectrum for masses below about $20 \mathrm{MeV}$. This has a negligible effect on LHC analysis.
} 
The resolution of this problem depends upon whether or not there is a signal in the missing energy channels (we will not discuss the related problems which arise in channels affected by virtual graviton exchanges). If there is a signal, the optimal procedure is to measure the observables $d^{2} \sigma / d p_{\mathrm{T}} d \eta$ as accurately as possible, perhaps at more than one collider energy as suggested in [706, 707]. No theory systematic should be included in these analyses. Instead, one should use the data to find the best fit form for $\rho(m, \sqrt{\hat{s}})$. Simple trial forms can be obtained, for example, from multiplying the ADD density of states by the form factors obtained in models with strings [704, 708, 709] or branes [710]. For the lower range of $M_{D}$ values, the sensitivity to $n$ suggested in $[706,707]$ will tend to be washed out. This is not a bad outcome, since it is a result of convolving the $n$ dependence with the effects of strings, branes or other new physics. Thus the theory systematic is replaced by likelihood fits to theories of Planck scale physics.

More problematic is the case where there is no graviton signal in a given data set. Since in this case we are trying to set a limit, we need an estimate of the theory systematic. The simplest possibility is to implement the GRW cutoff defined above, and estimate the theory error by varying the cutoff. For ADD with $n \geqslant 6$, one expects to obtain no lower bound at all on $M_{D}$, as noted in [702].

\subsubsection{Virtual graviton exchange}

The second class of collider signals for large extra dimensions is that of virtual graviton exchange[702, 711] in $2 \rightarrow 2$ scattering. This leads to deviations in cross sections and asymmetries in Standard Model processes with difermion final states. It may also give rise to new production processes which are not present at tree-level in the Standard Model, such as $g g \rightarrow \ell^{+} \ell^{-}$. The signature is similar to that expected in composite theories and provides a good experimental tool for searching for large extra dimensions for the case $\sqrt{s}<M_{D}$.

Graviton exchange is governed by the effective Lagrangian

$$
\mathcal{L}=i \frac{4 \lambda}{M_{H}^{4}} T_{\mu \nu} T^{\mu \nu}+\text { h.c. }
$$

The amplitude is proportional to the sum over the propagators for the graviton KK tower which may be converted to an integral over the density of KK states. However, in this case, there is no specific cut-off associated with the process kinematics and the integral is divergent for $n>1$. This introduces a sensitivity to the unknown ultraviolet physics which appears at the fundamental scale. This integral needs to be regulated and several approaches have been proposed: (i) a naive cut-off scheme [702, 711], (ii) brane fluctuations [710], or (iii) the inclusion of full weakly coupled $\mathrm{TeV}$-scale string theory in the scattering process [704, 708]. The most model independent approach which does not make any assumptions as to the nature of the new physics appearing at the fundamental scale is that of the naive cut-off. Here, the cut-off is set to $M_{H} \neq M_{D}$; the exact relationship between $M_{H}$ and $M_{D}$ is not calculable without knowledge of the full theory. The parameter $\lambda= \pm 1$ is also usually incorporated in direct analogy with the standard parametrisation for contact interactions [123] and accounts for uncertainties associated with the ultraviolet physics. The substitution

$$
\mathcal{M} \sim \frac{i^{2} \pi}{M_{\mathrm{Pl}}^{2}} \sum_{\vec{n}=1}^{\infty} \frac{1}{s-m_{\vec{n}}^{2}} \rightarrow \frac{\lambda}{M_{H}^{4}}
$$

is then performed in the matrix element for s-channel KK graviton exchange with corresponding replacements for $\mathrm{t}$ - and $\mathrm{u}$-channel scattering. As above, the Planck scale suppression is removed and superseded by powers of $M_{H} \sim \mathrm{TeV} / \mathrm{c}^{2}$. 
The resulting angular distributions for fermion pair production are quartic in $\cos \theta$ and thus provide a unique signal for spin-2 exchange.

The experimental analyses also make use of the cut-off approach. Using virtual KaluzaKlein graviton exchange in reactions with diphoton, dibosons and dilepton final states, $\left(G_{n} \rightarrow\right.$ $\gamma \gamma, V V, \ell \ell$ ), the LEP and Tevatron experiments exclude exchange scales up to $\sim 1.1 \mathrm{TeV} / \mathrm{c}^{2}$.

In the dimuon studies presented here (14.3.2) with $1 \mathrm{fb}^{-1}$ a 5-sigma effect from the virtual contributions of ADD gravitons to Drell-Yan process is observable for effective fundamental Planck scale of 4.0 TeV and for $n=6$ extra dimensions.

\subsubsection{Inverse TeV sized extra dimensions}

The possibility of $\mathrm{TeV}^{-1}$-sized extra dimensions naturally arises in braneworld theories [700]. By themselves, they do not allow for a reformulation of the hierarchy problem, but they may be incorporated into a larger structure in which this problem is solved. In these scenarios, the Standard Model fields are phenomenologically allowed to propagate in the bulk. This presents a wide variety of choices for model building: (i) all, or only some, of the Standard Model gauge fields exist in the bulk; (ii) the Higgs field may lie on the brane or in the bulk; (iii) the Standard Model fermions may be confined to the brane or to specific locales in the extra dimension. The phenomenological consequences of this scenario strongly depend on the location of the fermion fields. Unless otherwise noted, our discussion assumes that all of the Standard Model gauge fields propagate in the bulk.

The masses of the excitation states in the gauge boson KK towers depend on where the Higgs boson is located. If the Higgs field propagates in the bulk, the zero-mode state of the Higgs KK tower receives a vacuum expectation value (vev) which is responsible for the spontaneous breaking of the electroweak gauge symmetry. In this case, the resulting mass matrix for the states in the gauge boson KK towers is diagonal and the excitation masses are shifted by the mass of the gauge zero-mode, which corresponds to the Standard Model gauge field, giving

$$
m_{\vec{n}}=\left(m_{0}^{2}+\vec{n} \cdot \vec{n} / R_{c}^{2}\right)^{1 / 2}
$$

where $\vec{n}=\left(n_{1}, n_{2}, \ldots\right)$ labels the KK excitation levels. However, if the Higgs is confined to the brane, its vev induces mixing, amongst the gauge KK states of order $\left(m_{0} R_{c}\right)^{2}$. The KK mass matrix must then be diagonalised in order to determine the excitation masses. For the case of 1 extra $\mathrm{TeV}^{-1}$-sized dimension, the coupling strength of the gauge KK states to the Standard Model fermions on the brane is $\sqrt{2} g$, where $g$ is the corresponding Standard Model gauge coupling.

In the case where the Standard Model fermions are rigidly fixed to the brane, they do not feel the effects of the additional dimensions. For models in this class, precision electroweak data place strong constraints on the mass of the first gauge KK excitation. Contributions to electroweak observables arise from the virtual exchange of gauge KK states and a summation over the contributions from the entire KK tower must be performed. For $D>5$, this sum is divergent. In the full higher dimensional theory, some new, as of yet unknown, physics would regularise this sum and render it finite. An example of this is given by the possibility that the brane is flexible or non-rigid, which has the effect of exponentially damping the sum over KK states. Due to our present lack of knowledge of the full underlying theory, the KK sum is usually terminated by an explicit cut-off, which provides a naive estimate of the magnitude of the effects.

Since the $D=5$ theory is finite, it is the scenario that is most often discussed and is sometimes referred to as the 5-dimensional Standard Model (5DSM). In this case, a global 
fit to the precision electroweak data including the contributions from KK gauge interactions yields $m_{1} \sim R_{c}^{-1} \gtrsim 4 \mathrm{TeV} / \mathrm{c}^{2}$. In addition, the $\mathrm{KK}$ contributions to the precision observables allow for the mass of the Higgs boson to be somewhat heavier than the value obtained in the Standard Model global fit. Given the constraint on $R_{c}$ from the precision data set, the gauge KK contributions to the anomalous magnetic moment of the muon are small. The first gauge $\mathrm{KK}$ state can be produced as a resonance at the LHC in the Drell-Yan channel provided $m_{1} \lesssim 6 \mathrm{TeV} / \mathrm{c}^{2}$. In the studies presented here using the $Z_{K K}$ in the dielectron channel a 5-sigma reach for $m_{1} \sim R_{c}^{-1} \sim 4.97 \mathrm{TeV} / \mathrm{c}^{2}$ is obtained with $10 \mathrm{fb}^{-1}$.

In the scenario where the Standard Model fermions are localised at specific points in the extra $\mathrm{TeV}^{-1}$-sized dimensions, the fermions have narrow gaussian-like wave functions in the extra dimensions with width much smaller than $R_{c}^{-1}$. The placement of the different fermions at distinct locations in the additional dimensions, along with the narrowness of their wavefunctions, can then naturally suppress operators mediating dangerous processes such as proton decay. The exchange of gauge KK states in $2 \rightarrow 2$ scattering processes involving initial and final state fermions is sensitive to the placement of the fermions and can be used to perform a cartography of the localised fermions, i.e., measure the wavefunctions and locations of the fermions. At very large energies, it is possible that the cross section for such scattering will tend rapidly to zero since the fermions' wavefunctions will not overlap and hence they may completely miss each other in the extra dimensions.

\subsubsection{Randall-Sundrum (RS) models}

Randall-Sundrum refers to a class of scenarios, also known as warped extra dimensions models, originated by Lisa Randall and Raman Sundrum [94, 646]. In these scenarios there is one extra spatial dimension, and the five-dimensional geometry is "warped" by the presence of one or more branes. The branes extend infinitely in the usual three spatial dimensions, but are sufficiently thin in the warped direction that their profiles are well-approximated by delta functions in the energy regime of interest. If we ignore fluctuations of the branes, we can always choose a "Gaussian Normal" coordinate system, such that the fifth dimension is labelled $y$ and the usual $4 \mathrm{~d}$ spacetime by $x^{\mu}$. The action for such a theory contains, at a minimum, a $5 \mathrm{~d}$ bulk gravity piece and $4 \mathrm{~d}$ brane pieces. The bulk piece has the $5 \mathrm{~d}$ EinsteinHilbert action with gravitational coupling $M^{3}$, and a $5 \mathrm{~d}$ cosmological constant $\Lambda$. The brane pieces are proportional to the brane tensions $V_{i}$, which may be positive or negative. These act as sources for $5 \mathrm{~d}$ gravity, contributing to the $5 \mathrm{~d}$ stress-energy terms proportional to

$$
\sum_{i} V_{i} \delta\left(y-y_{i}\right)
$$

where the $y_{i}$ are the positions of the branes. Combined with a negative $\Lambda$, this results in a curved geometry, with a $5 \mathrm{~d}$ metric of the form:

$$
\begin{aligned}
& g_{\mu \nu}\left(x^{\rho}, y\right)=a^{2}(y) \tilde{g}_{\mu \nu}\left(x^{\rho}\right), \\
& g_{\mu y}=0, \quad g_{y y}=1,
\end{aligned}
$$

where $a(y)$ is called the warp factor, $\tilde{g}$ is a $4 \mathrm{~d}$ metric, and we have made a useful choice of coordinates. Warping refers to the fact that a $4 \mathrm{~d}$ distance $d_{0}$ measured at $y=y_{0}$ is related to an analogous $4 \mathrm{~d}$ distance $d_{1}$ measured at $y=y_{1}$ by $a\left(y_{0}\right) d_{0}=a\left(y_{1}\right) d_{1}$. Thus in RandallSundrum scenarios $4 \mathrm{~d}$ length, time, energy and mass scales vary with $y$.

Most collider physics phenomenology done with warped extra dimensions so far is based upon one very specific model, the original simple scenario called RSI. In this model the extra dimension is compactified to a circle of circumference $2 L$, and then further orbifolded by identifying points related by $y \rightarrow-y$. The fifth dimension then consists of two periodically 
identified mirror copies of a curved $5 \mathrm{~d}$ space extending from $y=0$ to $y=L$. It is assumed that there is a brane at $y=0$, with positive tension $V_{0}$; it is known as the Planck brane strong gravity resides on that brane. There is another brane at $y=L$, with negative tension $V_{L}$, known as the $\mathrm{TeV}$ brane-the entire $4 \mathrm{~d}$ universe is confined to the $\mathrm{TeV}$ brane.

Randall and Sundrum showed that, for a tuned choice of input parameters $V_{0}=-V_{L}=$ $-M^{2} \Lambda$, the $5 \mathrm{~d}$ Einstein equations have a simple warped solution on $0<y<L$ with metric:

$$
\begin{aligned}
& g_{\mu \nu}\left(x^{\rho}, y\right)=\mathrm{e}^{-2 k y} \eta_{\mu \nu}, \\
& g_{\mu y}=0, \quad g_{y y}=1,
\end{aligned}
$$

where $\eta_{\mu \nu}$ is the $4 \mathrm{~d}$ flat Minkowski metric, and $k=\sqrt{-\Lambda}$. Away from the branes, the $5 \mathrm{~d}$ curvature is constant and negative; it is thus equivalent locally to $A d S_{5}$, with the anti-de Sitter radius of curvature given by $1 / k$. At the locations of the branes the curvature is discontinuous, due to the fact that the branes are delta function sources for curvature.

The RSI model is completely described by three parameters: $k, M$, and $L$. Restricting the scenario to a low energy effective description implies considering $k, 1 / L \ll M$. In fact in RSI it is assumed that $k$ is merely parametrically small compared to the $5 \mathrm{~d}$ Planck scale $M$, i.e. $k \sim M / 10$. The effective $4 \mathrm{~d}$ Planck scale, which is the same as the coupling of the graviton zero mode, is given by dimensional truncation:

$$
M_{\text {Planck }}^{2}=\frac{M^{3}}{2 k}\left(1-\mathrm{e}^{-2 k L}\right) .
$$

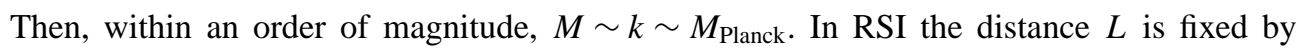
requiring that $a(L) M_{\text {Planck }} \simeq 1 \mathrm{TeV}$, thus $k L \sim 30$. This is not a large extra dimension: its inverse size is comparable to the grand unification scale.

Since the standard model fields live on the $\mathrm{TeV}$ brane as in ADD models, the phenomenology of RSI is concerned with the effects of the massive KK modes of the graviton. These modes as measured on the TeV brane have their mass splittings of the order of a TeV, and have TeV suppressed couplings to the standard model fields. In RSI, the Standard Model is replaced at the TeV scale by a new effective theory in which gravity is still very weak, but there are exotic heavy spin-two particles.

At the LHC the KK gravitons of RSI would be seen as difermion or dibosons resonances, since (unlike the KK gravitons of ADD) the coupling of each $\mathrm{KK}$ mode is only $\mathrm{TeV}$ suppressed [712]. The width of these resonances is controlled by the ratio $c=k / M$; the resonances become more narrow as the coupling parameter $c=k / M$ is reduced, as shown in Fig. 14.1.

The studies presented here focus on dilepton and diphoton final states while results using dijets can be found in Section 14.4.1. Note that due to the spin-2 nature of the graviton its branching ratio to diphotons is roughly twice that of a single dilepton channel.

\subsection{High mass dielectron final states}

This section presents the CMS experiment discovery potential for new heavy resonances, decaying into an electron pair. The $\mathrm{e}^{+} \mathrm{e}^{-}$decay channel provides a clean signature in the CMS detector. The presence of a heavy particle would be detected in CMS by the observation of a resonance peak in the dielectron mass spectrum over the Drell-Yan process $(\mathrm{pp} \rightarrow \gamma / \mathrm{Z} \rightarrow$ $\mathrm{e}^{+} \mathrm{e}^{-}$) which constitutes the main Standard Model background.

Heavy resonances with mass above $1 \mathrm{TeV} / \mathrm{c}^{2}$ are predicted by several models beyond the Standard Model. Three models are considered here: Kaluza-Klein (KK) excitations of a Z boson $\left(\mathrm{TeV}^{-1}\right.$ model, see Section 14.1.4) and KK excitation of a graviton (Randall-Sundrum 


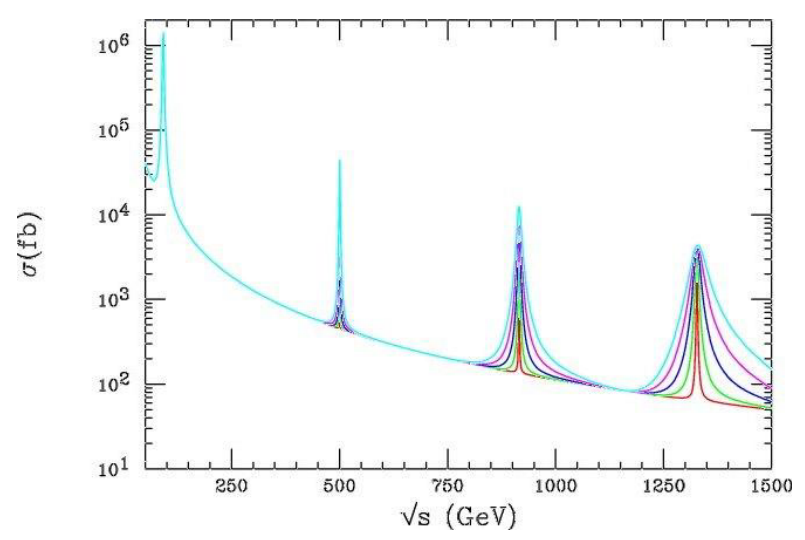

Figure 14.1. The cross section for $e^{+} e^{-} \rightarrow \mu^{+} \mu^{-}$including the exchange of KK gravitons in the RSI model. The narrowest resonances correspond to $k / M=0.05$, the widest to $k / M=0.14$. (Taken from Ref. [713].)

(RS) model, see Section 14.1.5), both predicted in extra dimensions models, and neutral heavy $Z^{\prime}$ boson predicted by Grand Unified Theories (GUT) (see Section 14.1.1). For the $Z^{\prime}$ bosons, 6 models are studied, as for the $Z^{\prime} \rightarrow \mu^{+} \mu^{-}$channel [100] that is discussed in Section 14.3.

Details of the analyses presented in this section can be found in [714] and [715].

\subsubsection{Event selection and correction}

Two electrons are required for this analysis. They are reconstructed as super-clusters (SC) in the ECAL calorimeter in the barrel and the endcap regions [716]. For endcap SC, the energy loss in the preshower detector is taken into account. The two SC with highest energies are selected as the electron candidates.

Reducible backgrounds (like QCD jets and $\gamma$-jets) are suppressed by applying the following requirements:

- The ratio of the HCAL to ECAL energy deposits is required to be $H / E<10 \%$.

- The two SC must be isolated: the total additional transverse energy in a cone of radius $0.1<\Delta R<0.5$ is required to be below $2 \%$ of the SC transverse energy (where $\Delta R=$ $\left.\sqrt{\Delta \eta^{2}+\Delta \phi^{2}}\right)$.

- To identify electrons and reject neutral particles, a track is requested to be associated for each electron candidate. If a track is associated with only one of these SC, the event is however kept if it contains a third SC with $E>300 \mathrm{GeV}$ with an associated track and satisfying the $H / E$ and isolation cuts described above.

The selected events are then corrected for the following effects:

- Saturation correction. For very energetic electrons and photons, saturation occurs in the ECAL electronics because of the limited dynamical range of the Multi-Gain-Pre-Amplifier. The saturation threshold has been established to be at $1.7 \mathrm{TeV}$ in crystals of the barrel and 3.0 TeV in the endcaps. A correction method (for barrel only) has been developed using the energy deposit in crystals surrounding the saturated crystal. The correction allows the energy deposits of clusters suffering from saturation to be estimated with a resolution of about 7\% [717]. 

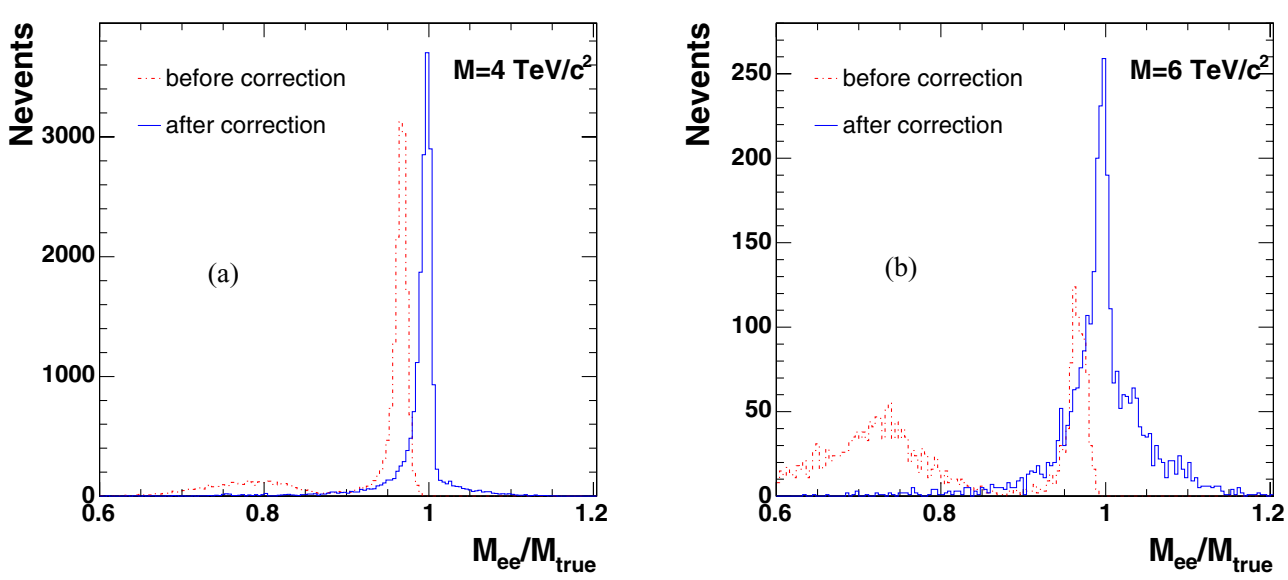

Figure 14.2. Ratio $M_{\mathrm{ee}} / M_{\text {true }}$ before and after corrections for $\mathrm{KK} Z$ boson production, for $M=4 \mathrm{TeV} / \mathrm{c}^{2}$ (a) and $M=6 \mathrm{TeV} / \mathrm{c}^{2}(\mathrm{~b})$.

- Energy correction. The ECAL measured electron energy after preshower, HCAL and saturation corrections, is smaller than the generated energy. Dedicated energy correction factors for very energetic electrons have been determined using calibration files. These factors depend on both energy, $\eta$ and whether saturation occurs or not. The resolution on the corrected SC energy is $0.6 \%$ at $E=1000 \mathrm{GeV}$.

- z-vertex distribution. The measurement in $\eta$ takes into account the knowledge of the $\mathrm{z}$-vertex position.

- FSR recovery. Hard photon emission from Final State Radiation can induce the detection in the event of a third energetic SC If a SC with $E>300 \mathrm{GeV}$ satisfying the $H / E$ and isolation cuts is observed very close to the SC of the electron candidates $(\Delta R<0.1)$, this additional SC is associated to the corresponding electron.

\subsubsection{Mass peak distributions}

The resonance mass is reconstructed from the energies and angles of the 2 electron candidates, after the selection cuts and energy corrections mentioned above. Figures $14.2 \mathrm{a}$ and $14.2 \mathrm{~b}$ show the ratio of the reconstructed and the true masses, $M_{\mathrm{ee}} / M_{\text {true }}$, before and after energy corrections for $\mathrm{KK} \mathrm{Z}$ production with $M=4$ and $6 \mathrm{TeV} / \mathrm{c}^{2}$, respectively. The peaks at low values of $M_{\mathrm{ee}} / M_{\text {true }}$ correspond to events with saturated ECAL electronics. The final resolution on the resonance mass is around $0.6 \%$ for events with no saturation, and $7 \%$ in case of saturation.

Figure 14.3a presents the signal and the Drell-Yan background for KK $Z$ boson production with $M=4 \mathrm{TeV} / \mathrm{c}^{2}$; Fig. $14.3 \mathrm{~b}$ for $Z^{\prime}$ boson production with $M=1.5 \mathrm{TeV} / \mathrm{c}^{2}$; Fig. $14.3 \mathrm{c}$ for graviton production with $M=1.5 \mathrm{TeV} / \mathrm{c}^{2}$ and coupling parameter, defined in Section 14.1.5, $c=0.01$.

\subsubsection{Discovery potential of CMS}

The discovery potential of a new physics resonance is determined using the likelihood estimator $S_{c L}$ (defined in Appendix A.1) based on event counting, suited for small event samples. The discovery limit is defined by $S_{C L}>5$. 

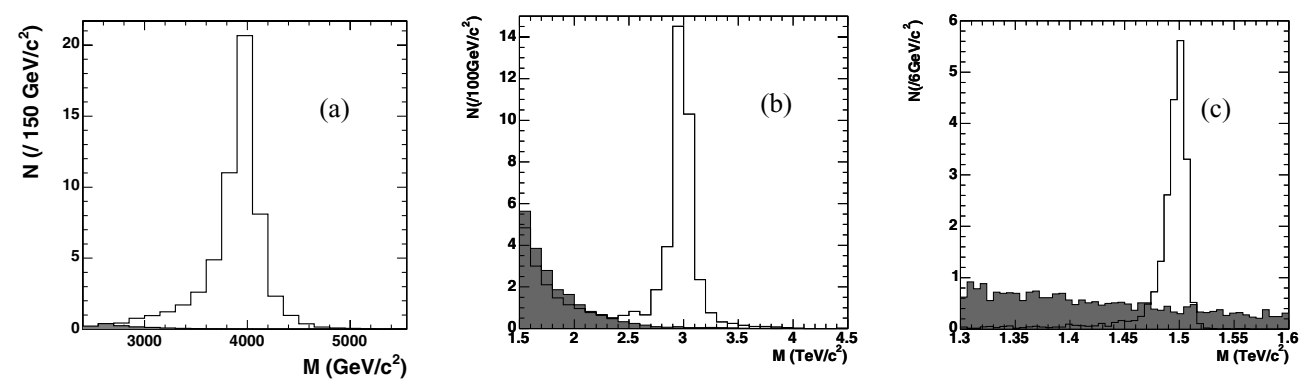

Figure 14.3. Resonance signal (white histograms) and Drell-Yan background (shaded histograms) for KK $Z$ boson production with $M=4.0 \mathrm{TeV} / \mathrm{c}^{2}$ (a), SSM $Z^{\prime}$ boson production with $M=3.0 \mathrm{TeV} / \mathrm{c}^{2}(\mathrm{~b})$, and graviton production with $M=1.5 \mathrm{TeV} / \mathrm{c}^{2}$, coupling parameter $c=0.01$ (c), for an integrated luminosity of $30 \mathrm{fb}^{-1}$.

Table 14.1. Number of events for resonant signal, $N_{\mathrm{s}}$, and for Drell-Yan background, $N_{\mathrm{b}}$, and corresponding significances $S_{c L}$ for an integrated luminosity of $30 \mathrm{fb}^{-1}$. The masses $M$ and the mass windows $M_{\mathrm{w}}$ are in $\mathrm{TeV} / \mathrm{c}^{2}$.

\begin{tabular}{lllllll}
\hline & \multicolumn{2}{c}{ KK $Z$} & $\mathrm{G}, c=0.01$ & $\mathrm{G}, c=0.1$ & \multicolumn{2}{c}{$\mathrm{SSM} Z^{\prime}$} \\
\hline$M$ & 4.0 & 6.0 & 1.5 & 3.5 & 1.0 & 5.0 \\
$M_{\mathrm{w}}$ & $3.5-4.5$ & $5.0-6.7$ & $1.47-1.52$ & $3.30-3.65$ & $0.92-1.07$ & $4.18-5.81$ \\
$N_{\mathrm{s}}$ & 50.6 & 1.05 & 18.8 & 7.30 & 72020 & 0.58 \\
$N_{\mathrm{b}}$ & 0.13 & 0.005 & 4.16 & 0.121 & 85.5 & 0.025 \\
$S$ & 22.5 & 3.0 & 6.39 & 6.83 & 225 & 1.63 \\
\hline
\end{tabular}

The number of signal and background events, $N_{\mathrm{s}}$ and $N_{\mathrm{b}}$, computed for a given mass window around the peak, are presented in Table 14.1 for the three models, together with the corresponding significance, for an integrated luminosity of $30 \mathrm{fb}^{-1}$.

The $5 \sigma$ discovery limits as a function of mass are given in Fig. 14.4a and Fig. 14.4b, for KK $Z$ boson production and $Z^{\prime}$ production (for the 6 considered models), respectively. In the graviton case, the $5 \sigma$ discovery plane as a function of the coupling parameter $c$ and the resonance mass is given in Fig. 14.4c.

For KK $Z$ bosons, a $5 \sigma$ discovery can be achieved for a resonance mass up to $M=$ $4.97 \mathrm{TeV} / \mathrm{c}^{2}$ for an integrated luminosity of $10 \mathrm{fb}^{-1}, M=5.53 \mathrm{TeV} / \mathrm{c}^{2}$ for $30 \mathrm{fb}^{-1}$ and $M=5.88 \mathrm{TeV} / \mathrm{c}^{2}$ for $60 \mathrm{fb}^{-1}$. For gravitons, with an integrated luminosity of $30 \mathrm{fb}^{-1}$, a $5 \sigma$ discovery can be extracted for masses up to $1.64 \mathrm{TeV} / \mathrm{c}^{2}$ for $c=0.01$ and up to $3.81 \mathrm{TeV} / \mathrm{c}^{2}$ for $c=0.1$. For $Z^{\prime}$ boson production, with an integrated luminosity of $30 \mathrm{fb}^{-1}$, a $5 \sigma$ discovery can be extracted for masses up to $3.31 \mathrm{TeV} / \mathrm{c}^{2}$ for model $\psi$ and up to $4.27 \mathrm{TeV} / \mathrm{c}^{2}$ for model ARLM. The $5 \sigma$ discovery limits on the resonance masses for 10,30 and $60 \mathrm{fb}^{-1}$ are summarised in Table 14.2.

For KK $Z$ boson production, the luminosities needed for a five $\sigma$ discovery are 1.5, 4.0, $10.8,29.4$, and $81.4 \mathrm{fb}^{-1}$ for $M=4.0,4.5,5.0,5.5$ and $6.0 \mathrm{TeV} / \mathrm{c}^{2}$, respectively; for SSM $Z^{\prime}$ boson production, they are $0.015,3.0$ and $260 \mathrm{fb}^{-1}$ for $M=1,3$ and $5 \mathrm{TeV} / \mathrm{c}^{2}$; for graviton production, most of the interesting region of the (mass, coupling) plane is already covered with $10 \mathrm{fb}^{-1}$.

For $\mathrm{KK} Z$ and $Z^{\prime}$ production, a $\mathrm{K}$ factor of 1 was conservatively taken for both the signal and the Drell-Yan background, since heavy $Z$ production interferes with $Z / \gamma$ Drell-Yan production. For the graviton analysis, as little interference is present with the Standard Model 

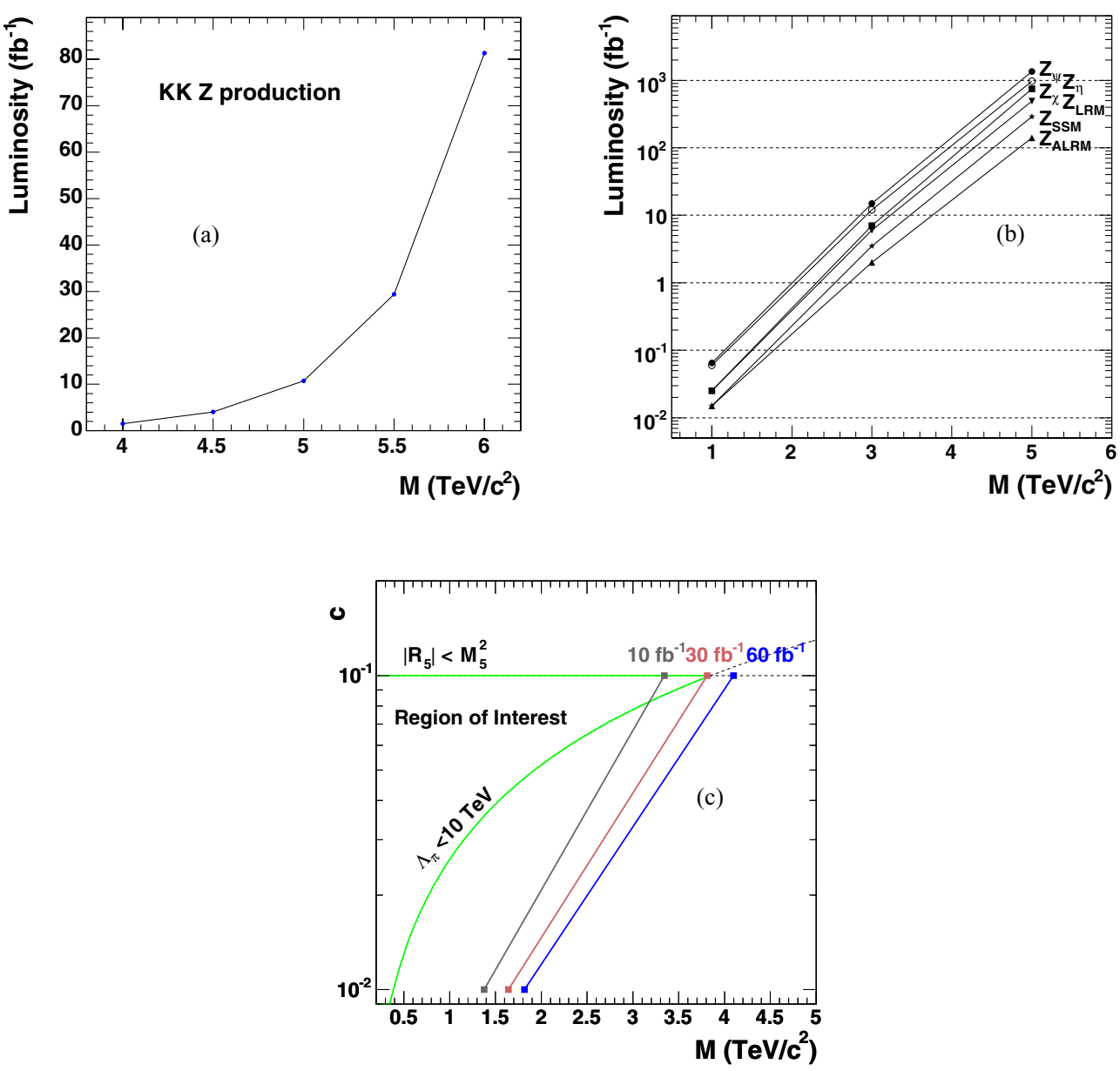

Figure 14.4. Five $\sigma$ discovery limit as a function of the resonance mass for KK $Z$ boson production (a), for the $6 Z^{\prime}$ models (b); five $\sigma$ discovery plane for graviton production as a function of the coupling parameter $c$ and the graviton mass (c).

Table 14.2. The $5 \sigma$ discovery limit on the resonance mass (given in $\mathrm{TeV} / \mathrm{c}^{2}$ ) for the three models, for an integrated luminosity of 10,30 and $60 \mathrm{fb}^{-1}$.

\begin{tabular}{lccc}
\hline Model & \multicolumn{3}{c}{ Luminosity $\left(\mathrm{fb}^{-1}\right)$} \\
\cline { 2 - 4 } & 10 & 30 & 60 \\
\hline KK $Z$ & 4.97 & 5.53 & 5.88 \\
G $(c=0.01)$ & 1.38 & 1.64 & 1.82 \\
G $(c=0.1)$ & 3.34 & 3.81 & 4.10 \\
$Z^{\prime}(\psi)$ & 2.85 & 3.31 & 3.62 \\
$Z^{\prime}($ ALRM $)$ & 3.76 & 4.27 & 4.60 \\
\hline
\end{tabular}

processes, a $\mathrm{K}$ factor of 1.0 is used for the signal and of 1.3 for the Drell-Yan background, in order to take into account the higher order terms in the cross section. The latter number comes from the CDF analysis [718] and is compatible with the $\mathrm{K}$ factor obtained from theoretical computations [348]. 


\subsubsection{Systematic uncertainties}

The uncertainty coming from the choice of the parton distribution function (PDF) was investigated using the set of 20 positive and 20 negative errors, of the CETQ6.1M "best fit" parametrisation [12, 719, 720]. For each event, a weight factor is computed according to the $x_{1}, x_{2}$, and $Q^{2}$ variables, for each of the 40 PDF errors, in the case of graviton production with $M=1.5 \mathrm{TeV} / \mathrm{c}^{2}(c=0.01)$ and $M=3.5 \mathrm{TeV} / \mathrm{c}^{2}(c=0.1)$. The uncertainties on the PDF modify the number of signal events by a factor 1.20 (positive deviations) and 0.86 (negative deviations) for $M=1.5 \mathrm{TeV} / \mathrm{c}^{2}(c=0.01)$. The corresponding numbers for $M=3.5 \mathrm{TeV} / \mathrm{c}^{2}$ $(c=0.1)$ are 1.47 and 0.78 . For the Drell-Yan background, the re-weighting effects on the numbers of events are 1.07 and 0.94 for masses around $1.5 \mathrm{TeV} / \mathrm{c}^{2}$, and 1.19 and 0.88 for masses around $3.5 \mathrm{TeV} / \mathrm{c}^{2}$. For an integrated luminosity of $30 \mathrm{fb}^{-1}$, the significances with the "best fit" and with the positive/negative deviations are equal respectively to 6.40 and $7.25 / 5.78$ for $M=1.5 \mathrm{TeV} / \mathrm{c}^{2}$, and to 6.83 and $8.54 / 5.93$ for $M=3.5 \mathrm{TeV} / \mathrm{c}^{2}$. The main effect of the variation comes from the gluon-fusion contribution to the graviton production cross section. A lower dependence is observed for the $\mathrm{KK} Z$ and $Z^{\prime}$ channels, which are produced by quark-anti-quark annihilation. For KK $Z$ boson production at $M=4 \mathrm{TeV} / \mathrm{c}^{2}$ with an integrated luminosity of $30 \mathrm{fb}^{-1}$, the significances with the "best fit" and with the positive/negative errors are equal respectively to 22.5 and 23.3/21.9.

Changing to 1 the value of the $\mathrm{K}$ factor of the Drell-Yan background for RS graviton production increases the significance from 6.39 to $6.87\left(M=1.5 \mathrm{TeV} / \mathrm{c}^{2}, c=0.01\right)$ and from 6.83 to $7.09\left(M=3.5 \mathrm{TeV} / \mathrm{c}^{2}, c=0.1\right)$. The discovery limits increase respectively from 1.64 to $1.68 \mathrm{TeV} / \mathrm{c}^{2}$ and from 3.81 to $3.84 \mathrm{TeV} / \mathrm{c}^{2}$.

The data themselves will be used to estimate and cross-check the Drell-Yan background at very high energy. For resonance discovery, the number of events in the side-bands of the resonance and their mass dependence will be used to estimate the number of background events under the resonance peak, provided there is enough data in the side-bands. In this approach, the uncertainties on the background cross-sections, the PDF and the luminosity measurement are highly reduced.

\subsubsection{Identification of new particles}

Once a resonance is found, information will be gained on its characterisation from the study of other decay channels, like $\gamma \gamma$ (see Section 14.6), of angular distributions and of asymmetries, in view of the spin determination (see also Section 14.3).

As an example, RS gravitons with spin 2 can be distinguished from the Standard Model background and $Z^{\prime}$ bosons with spin 1 using the distribution of the $\cos \theta^{*}$ variable, computed as the cosine of the polar angle between the electron and the boost direction of the heavy particle in the latter rest frame. In addition to the cuts defined above, the electron and positron candidates are requested to have opposite charges, in order to identify the electron, from which the $\cos \theta^{*}$ variable is computed.

The $\cos \theta^{*}$ distributions for graviton production with $M=1.25 \mathrm{TeV} / \mathrm{c}^{2}, c=0.01$ and $M=2.5 \mathrm{TeV} / \mathrm{c}^{2}, c=0.1$, are presented in Fig. 14.5, for an integrated luminosity of $100 \mathrm{fb}^{-1}$. The error bars represent the corresponding statistical uncertainties, applied to the signal distribution obtained from a large statistics simulation. The spin-2 hypothesis is compared to the spin-1 hypothesis (dashed red curve in the figures), formed by the Drell-Yan production (Figs. 14.5a and 14.5b) or the ALRM $Z^{\prime}$ production (Figs. 14.5c and $14.5 \mathrm{~d})$. For graviton production, the expected background is included in the $\cos \theta^{*}$ distributions. 

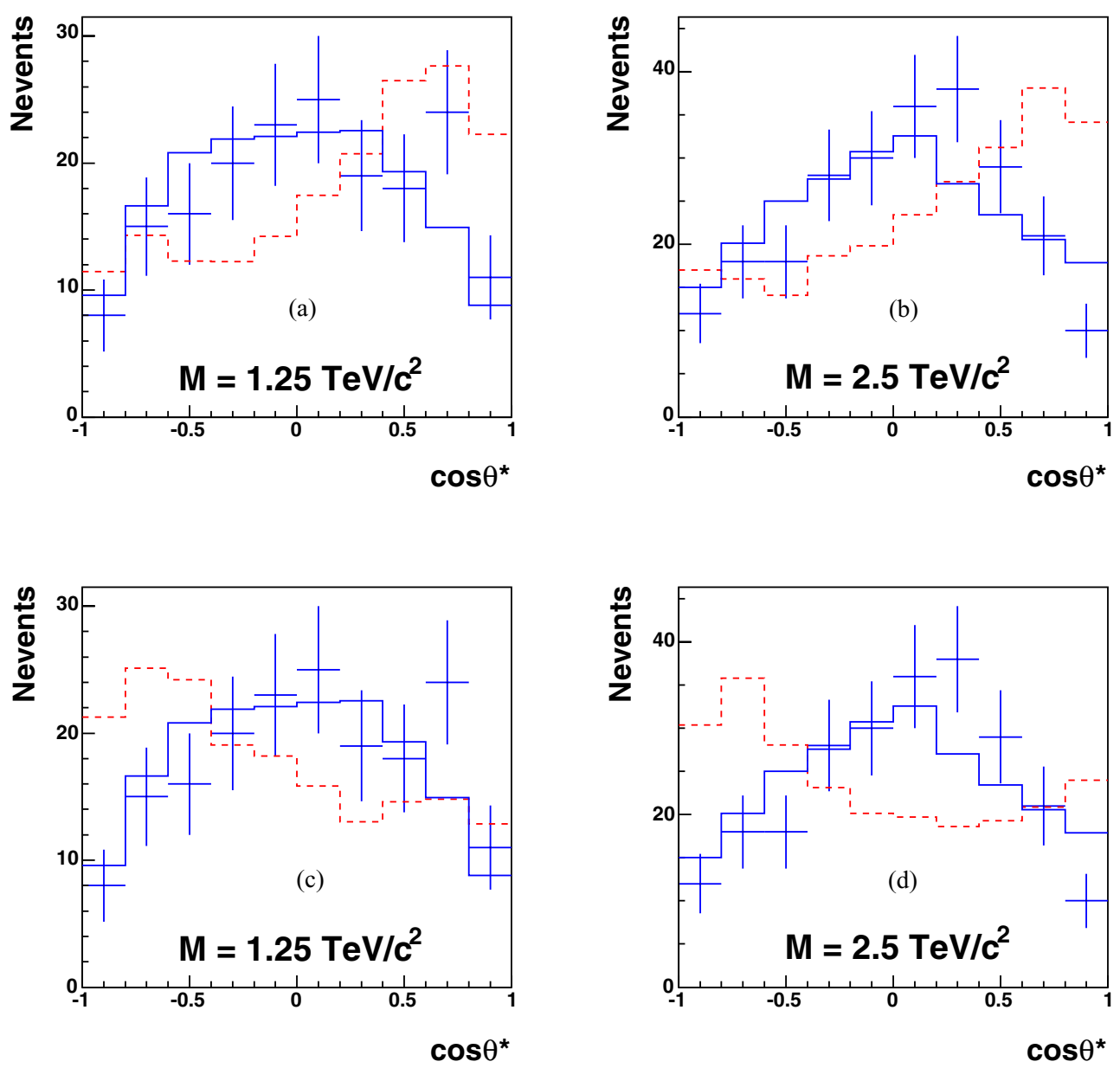

Figure 14.5. Distributions of $\cos \theta^{*}$ for graviton production (full blue curves) and for DrellYan production (dashed red curves) normalised to the signal, for $M=1.25 \mathrm{TeV} / \mathrm{c}^{2}$ (a) and $2.5 \mathrm{TeV} / \mathrm{c}^{2}$ (b), and for $Z^{\prime}$ boson (ALRM model) (dashed red curves), normalised to the signal, for $M=1.25 \mathrm{TeV} / \mathrm{c}^{2}$ (c) and $2.5 \mathrm{TeV} / \mathrm{c}^{2}$ (d), with an integrated luminosity of $100 \mathrm{fb}^{-1}$. The error bars represent the "1-experiment" distribution for the graviton production. The expected background is included in the $\cos \theta^{*}$ distributions.

The spin 2 nature of RS gravitons can be determined in contrast to the Drell-Yan production or the $Z^{\prime}$ boson production for an integrated luminosity of $100 \mathrm{fb}^{-1}$ up to $1.25 \mathrm{TeV} / \mathrm{c}^{2}$ for $c=0.01$ and $2.5 \mathrm{TeV} / \mathrm{c}^{2}$ for $c=0.1$.

\subsection{High mass dimuon final states}

Many scenarios beyond the Standard Model are expected to manifest themselves through modifications in the mass spectrum of high-mass dimuon pairs. The potential of the CMS experiment to discover dimuon decays of a new heavy neutral gauge boson, $Z^{\prime}$, is discussed in Section 3.3.4; the discovery reach for a representative set of $\mathrm{Z}^{\prime}$ models was found to be in the range between 2.9 and $3.8 \mathrm{TeV} / \mathrm{c}^{2}$ for an integrated luminosity of $10 \mathrm{fb}^{-1}$. In this section, we discuss the observability of $\mu^{+} \mu^{-}$final states predicted in two classes of 
large extra dimensions models, RS and ADD. While the RS scenario gives rise to relatively narrow resonances, the ADD model is expected to be observed via non-resonant modifications of the dimuon spectrum; therefore, these two searches require somewhat different experimental approaches. The search for compositeness in the dimuon channel is described in Section 15.2.

Once a new physics is discovered, observables other than dimuon invariant mass can be used to determine the theoretical framework to which it belongs. The measurement of the forward-backward asymmetries of leptonic decay products has long been known as a powerful tool to identify $Z^{\prime}$; some aspects of such a measurement at the LHC are discussed in Section 3.3.5. Spin discrimination of new heavy resonances based on an unbinned likelihood ratio statistic incorporating the angles of the decay products is described in Section 3.3.6.

\subsubsection{The Randall-Sundrum model in the dimuon channel}

We consider the range of RS1 graviton masses in the range $1<m<4 \mathrm{TeV} / \mathrm{c}^{2}$ and the dimensionless coupling constant in the expected theoretical range $0.01 \leqslant c \leqslant 0.1$ [721]. A full simulation with PYTHIA [69] version 6.227 and with the GEANT4-based CMS program [8] and reconstruction with the CMS full-reconstruction package [10], including pile-up of minimum-bias collisions is carried out. We derive both the CMS discovery potential for Randall-Sundrum gravitons and the performance of spin determination in this channel (see details in Ref. [117]). The non-reducible backgrounds are the Drell-Yan process, vector boson pair production $Z Z, W Z, W W, t \bar{t}$ production, etc. In the SM the expected leading-order cross section of the Drell-Yan process dominates the other contributions (see the Section 9.2 for details). The trigger simulation is based on the reconstruction package, using the on-line reconstruction algorithm. We require the single or double muon trigger, no requirement for calorimeter isolation of high- $p_{\mathrm{T}}$ muons is made. The total trigger + reconstruction efficiency varies between $95 \%$ and $90 \%$ for dimuons in the mass range $1<m<4 \mathrm{TeV} / \mathrm{c}^{2}$. Only the events which passed both the Level-1 and HLT cuts are selected. Note that the trigger efficiency is significantly decreased after applying of the calorimeter isolation cuts (down to $15 \%$ ). This drop is caused by electromagnetic showers accompanying high-energy muons. In the following, no cuts on calorimeter isolation of muon tracks are applied at the HLT level.

14.3.1.1. The Randall-Sundrum model discovery potential. The significance estimators used for studying the discovery potential of the RS1 model were $S_{c P}, S_{c L}$ and $S_{L}$, defined in Appendix A.1 (see discussion of $S_{L}$ in Section 3.3.4.1).

Figure 14.6a shows the integrated luminosity required for a $5 \sigma$ discovery as a function of the dimuon mass. The results for different values of integrated luminosity are summarised in Table 14.3 and Fig. 14.6b. The CMS experiment can observe a RS1 graviton with mass up to $2.3 \mathrm{TeV} / \mathrm{c}^{2}$ with an integrated luminosity of $\int L d t=1 \mathrm{fb}^{-1}$ if the coupling $c$ is equal to 0.1 . For $c=0.01$ the mass reach does not exceed $1.9 \mathrm{TeV} / \mathrm{c}^{2}$, even for the asymptotic regime of LHC operation with $\int \mathcal{L} d t=300 \mathrm{fb}^{-1}$. The asymptotic reach limit for $c=0.1$ is 4.5 $\mathrm{TeV} / \mathrm{c}^{2}$.

A combined analysis [721] in the RS1 scenario shows that the value of the coupling constant $c$ is strongly restricted (Fig. 14.6b) due to the theoretical constraints to assure that the model does not introduce a new hierarchy (the scale parameter $\Lambda_{\pi}=M_{P l} e^{k L}<10 \mathrm{TeV} / \mathrm{c}^{2}$ with the symbols defined in Section 14.1.5). The direct comparison of results on a mass reach region for $c$ with the data of the Fig. 14.6 shows that a luminosity of $100 \mathrm{fb}^{-1}$ is needed to test the RS1 model everywhere in $\left(c-M_{\text {grav }}\right)$ space of model parameters. However, these conclusions are not definitive since the initial theoretical constraints are quite arbitrary. 

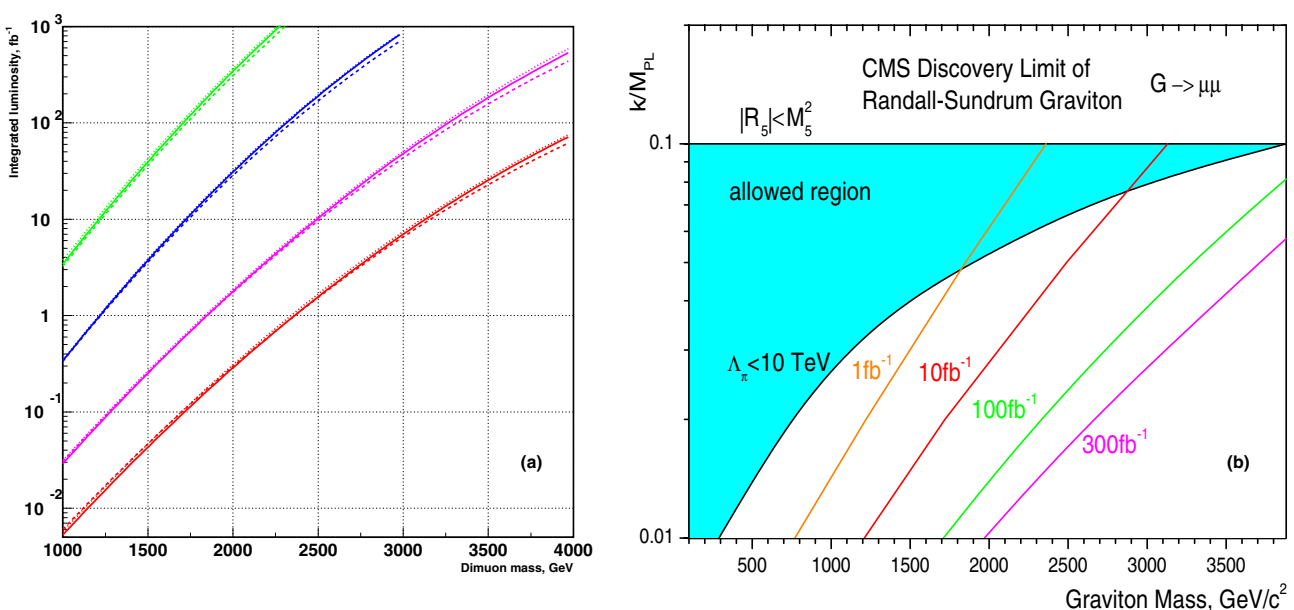

Figure 14.6. (a) Discovery limit for RS1 graviton with $\mu^{+} \mu^{-}$decay mode for different values of RS1 coupling constant $c=0.01,0.02,0.05$ and 0.1 (from top to bottom). Used discovery limit $S>5$ for the $S_{c P}$ estimator (solid lines), $S_{L}$ (dashed lines), $S_{c L}$ (dotted lines). (b) Reach of the CMS experiment as a function of the coupling parameter $c$ and the graviton mass for various values of integrated luminosity. The left part of each curve is the region where significance exceeds $5 \sigma$.

Table 14.3. CMS discovery potential invariant mass reach (in $\mathrm{TeV}$ ) to observe the RS1 graviton in $\mu^{+} \mu^{-}$channel.

\begin{tabular}{llllll}
\hline Coupling constant $c$ & Estimator & $1 \mathrm{fb}^{-1}$ & $10 \mathrm{fb}^{-1}$ & $100 \mathrm{fb}^{-1}$ & $300 \mathrm{fb}^{-1}$ \\
\hline \multirow{3}{*}{0.01} & $S_{c P}$ & 0.75 & 1.20 & 1.69 & 1.95 \\
& $S_{c L}$ & 0.77 & 1.21 & 1.71 & 1.97 \\
0.02 & $S_{L}$ & 0.78 & 1.23 & 1.73 & 1.99 \\
& $S_{c P}$ & 1.21 & 1.72 & 2.30 & 2.63 \\
0.05 & $S_{c L}$ & 1.22 & 1.72 & 2.31 & 2.64 \\
& $S_{L}$ & 1.22 & 1.74 & 2.34 & 2.68 \\
& $S_{c P}$ & 1.83 & 2.48 & 3.24 & 3.67 \\
0.1 & $S_{c L}$ & 1.85 & 2.49 & 3.26 & 3.71 \\
& $S_{L}$ & 1.85 & 2.51 & 3.31 & 3.79 \\
& $S_{c P}$ & 2.34 & 3.11 & 4.12 & 4.52 \\
& $S_{c L}$ & 2.36 & 3.13 & 4.14 & 4.54 \\
& $S_{L}$ & 2.36 & 3.16 & 4.23 & 4.73 \\
\hline
\end{tabular}

14.3.1.2. Systematic uncertainties. The results taking into account the systematic uncertainties are shown in Fig. 14.7. The expected effects of misalignment are considered in two misalignment scenarios: the First Data and the Long Term scenarios [99], which correspond to different stages of the alignment corrections for the positions of the tracker and muon chambers. The current estimate is that the transition to the Long Term scenario can be achieved at an integrated luminosity of about $1 \mathrm{fb}^{-1}$ [86]. In contrast to Fig. 14.6 which assumed a $\mathrm{K}$-factor equal to unity, a K-factor of $K=1.30 \pm 0.05$ is used both for the RS1 signal and Drell-Yan background. Additional variations due to EW corrections, hard-scale and PDF uncertainties have been considered, the details being found in Ref. [117].

14.3.1.3. Spin discrimination in angular analysis. A study of muon angular distributions allows a discrimination between the hypotheses of Graviton (spin-2 particle) and $Z^{\prime}$ (spin-1 particle) - see the discussion and the results in Section 3.3.6. 

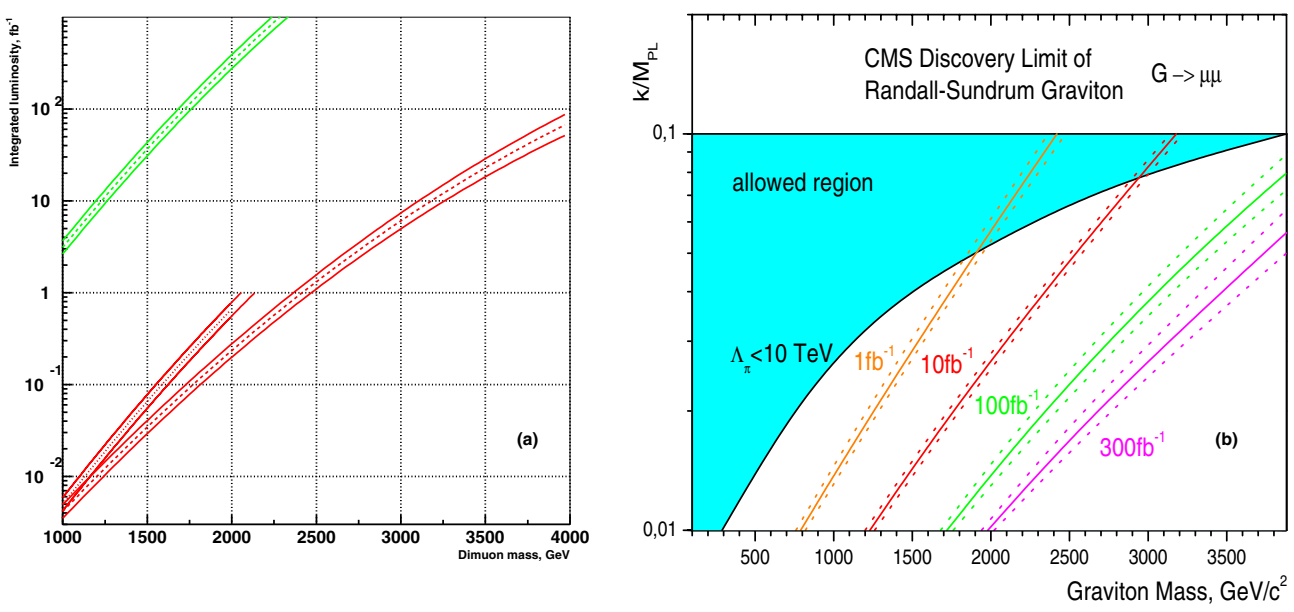

Figure 14.7. (a) Discovery limit for coupling constants $c=0.01,0.1$ (upper and lower curves, respectively) after taking into account the systematic uncertainties including misalignment in two scenarios: the curves ending at integrated luminosity of $1 \mathrm{fb}^{-1}$ correspond the First Data misalignment scenario, the other ones correspond to the Long Term scenario. The ranges show the expected variations due to the systematic uncertainties. (b) The ranges of the expected variations due to the systematic uncertainties for the mass reach of the CMS experiment.

\subsubsection{The ADD model in the dimuon channel}

We consider the fundamental Planck scale of the ADD model in the range of $3.0<$ $M_{S}<10.0 \mathrm{TeV} / \mathrm{c}^{2}$ and numbers of extra dimensions in the range of $3 \leqslant n \leqslant 6$ [698]. The contribution of KK-modes of ADD gravitons to the Drell-Yan processes is computed using the leading-order matrix element [722] which was implemented in STAGEN generator collection as external matrix element in PYTHIA [69] version 6.227. A full simulation [8] of the CMS detector and reconstruction [10], without a pile-up of minimum-bias collision is performed to derive the CMS discovery potential for ADD virtual gravitons (see details in Ref. [723]). The non-reducible backgrounds are the Drell-Yan process, vector boson pair production $Z Z, W Z, W W, t \bar{t}$ production, etc. In the SM the expected leading-order cross section of the Drell-Yan process dominates the other contributions (see Section 9.2 for details). The trigger simulation is realised in the reconstruction package, using the on-line reconstruction algorithm. A single or double muon trigger is required, but no requirement for calorimeter isolation of high- $p_{\mathrm{T}}$ muons is made. The total trigger + reconstruction efficiency varies between $70 \%$ and $90 \%$ for dimuons dependent on the model parameters. Only the events which passed both the Level-1 and HLT cuts are selected.

14.3.2.1. The ADD discovery limit. The CMS discovery potential was estimated using as significance $S_{c P}$ and $S_{c L}$, defined in Appendix A.1. The computed significance values for the ideal detector as a function of a fundamental theory scale, $M_{S}$, are presented in Fig. 41.8 for integrated luminosities of $0.1,1.0,10,100,300,1000 \mathrm{fb}^{-1}$. The main observations are:

- $\int \mathcal{L} \mathrm{d} t=1 \mathrm{fb}^{-1}$, even a low luminosity regime allows us to measure the effect from the virtual contributions of ADD gravitons to Drell-Yan process for an effective fundamental Planck scale up to $4.0 \mathrm{TeV}$ for the most unfavourable case with $n=6$. For a scenario where the number of extra dimensions is $n=3$ the reach limit is extended to $5.8 \mathrm{TeV}$.

- $\int \mathcal{L} \mathrm{d} t=10 \mathrm{fb}^{-1}, M_{S}$ values of 4.8 and $7.2 \mathrm{TeV}$ can be reached for $n=3$ and $n=6$ respectively. 

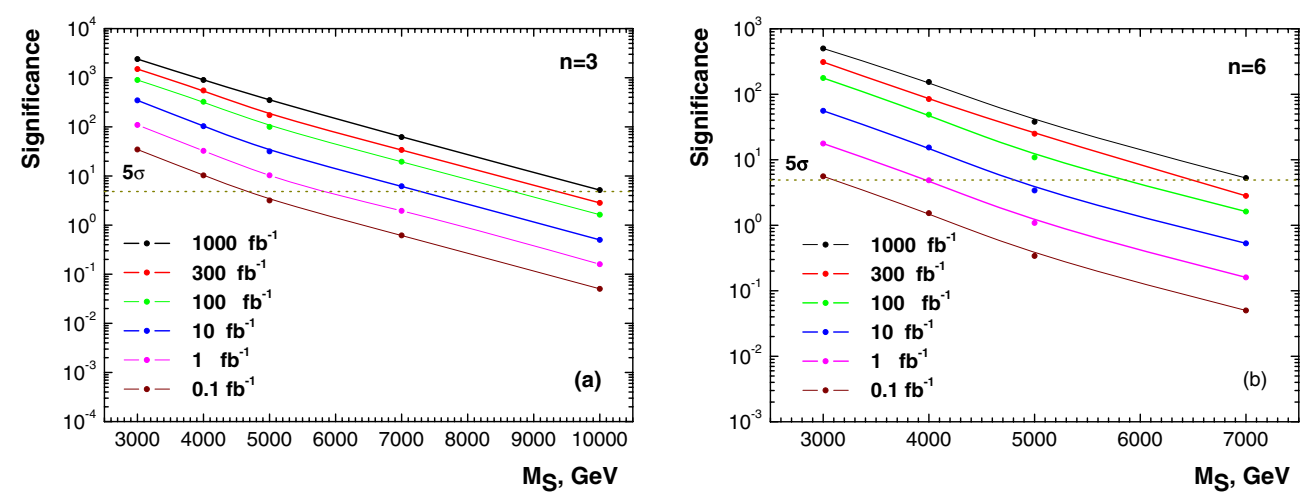

Figure 14.8. Significance as a function of $M_{S}$ for (a) $n=3$ and (b) $n=6$.

- $\int \mathcal{L} \mathrm{d} t=100 \mathrm{fb}^{-1}$, for LHC operation in a high luminosity regime allow the observation of the ADD signal at $5.8 \div 8.7 \mathrm{TeV}$ of model scale dependent on a number of extra dimensions.

- $\int \mathcal{L} \mathrm{d} t=300 \mathrm{fb}^{-1}$, in the asymptotic regime the CMS sensitivity to fundamental Planck scale is increased to values of $6.5 \div 9.3 \mathrm{TeV}$.

14.3.2.2. Systematics. The results taking into account the systematical uncertainties with the $S_{c P}$ estimator are shown in Fig. 14.8. To take into account the misalignment effect two scenario of misalignment were considered during reconstruction procedure: First Data scenario [99] for 0.1 and $1.0 \mathrm{fb}^{-1}$ and Long Term scenario [99] for 10, 100, 300, $1000 \mathrm{fb}^{-1}$. The K-factor of $K=1.30 \pm 0.05$ is used both for ADD signal and Drell-Yan background. Additional variations due to hard-scale and PDF uncertainties as well as trigger and selection uncertainties have been considered, the details being given in Ref. [723].

\subsection{High energy single lepton final states}

\subsubsection{Introduction}

Several theoretical models predict, in addition to the well known electroweak vector bosons $\gamma, W, Z$, further heavy gauge bosons. These additional particles are postulated for example in Left-Right Symmetric Models [724-727], based on the gauge group $\mathrm{SU}(3)_{\mathrm{C}} \times \mathrm{SU}(2)_{\mathrm{L}} \times \mathrm{SU}(2)_{\mathrm{R}} \times \mathrm{U}(1)_{\mathrm{B}-\mathrm{L}}(\mathrm{B}, \mathrm{L}$ : baryon-, lepton-number $)$ in theories predicting a substructure of the known "elementary particles", and in Little Higgs Models [91].

Here we investigate the detection capabilities for a hypothetical heavy partner of the $W$, a charged spin-1 boson $W^{\prime}$. We do not assume one of the specific models mentioned above, but derive the $W^{\prime}$ properties from the Reference Model by Altarelli [697], which has been used in several earlier experiments, so that the resulting limits can be compared easily. In this Reference Model the $W^{\prime}$ is a carbon copy of the $W$, with the very same left-handed fermionic couplings (including CKM matrix elements), while there is no interaction with the Standard Model gauge bosons or with other heavy gauge bosons as a $Z^{\prime}$. Thus the $W^{\prime}$ decay modes and corresponding branching fractions are similar to those for the $W$, with the notable exception of the $\mathrm{t} \overline{\mathrm{b}}$ channel, which opens for $W^{\prime}$ masses beyond $180 \mathrm{GeV}$.

In hadron collisions $W^{\prime}$ bosons can be created through $q \bar{q}$ annihilation, in analogy to $W$ production. Previous searches for the Reference $W^{\prime}$ at LEP and at the Tevatron give rise to lower bounds approaching $1 \mathrm{TeV}$ [728]. 
This analysis is based on the decay $W^{\prime} \rightarrow \mu \nu$, with a branching ratio of roughly $10 \%$. The resulting signature of a high energy muon accompanied by missing energy allows an easy separation of signal and background reactions. More details are found in [729].

\subsubsection{Data samples}

For this study we assume an integrated luminosity of $10 \mathrm{fb}^{-1}$ and an average instantaneous luminosity of $\mathcal{L}=2 \times 10^{33} \mathrm{~cm}^{-2} \mathrm{~s}^{-1}$ corresponding to an average pile-up of $3.5 \mathrm{pp}$-collisions per bunch crossing.

Reference Model $W^{\prime}$ events decaying into muon and neutrino have been generated with PYTHIA v6.227 [69], based on the leading order cross section and the parton density functions CTEQ 5L (leading order) [719]. In total about 300000 events have been produced for $W^{\prime}$ masses between $1 \mathrm{TeV}$ and $8 \mathrm{TeV}$. The product of LO cross section and branching fraction varies between $3.0 \times 10^{3} \mathrm{fb}(1 \mathrm{TeV})$ and $3.3 \times 10^{-4} \mathrm{fb}(8 \mathrm{TeV})$, to be compared with $1.7 \times 10^{7} \mathrm{fb}$ for Standard Model $W$ production and muonic decay. The detector response was simulated with the full CMS simulation [8] and reconstruction [10] software. Both the signal events and the following background samples were analysed: $\mathrm{W} \rightarrow \mu \nu, Z \rightarrow \mu \mu, W W$ inclusive, $Z Z$ inclusive, $Z W$ inclusive, $t \bar{t}$ inclusive. These data sets have been produced in the CMS Data Challenge 2004. On average 3.5 minimum bias reactions have been overlaid to each event.

\subsubsection{Event selection and analysis}

Events have been preselected requiring at least one globally reconstructed muon which pass the trigger criteria.

The final cuts to select $W^{\prime} \rightarrow \mu \nu$ candidate events are:

- muon quality: at least 13 hits along the global track, $\chi^{2} / N_{d o f}<50$ for the fit;

- single muon requirement;

- muon isolation: no additional track $\left(p_{\mathrm{T}}>0.8 \mathrm{GeV}\right)$ within a cone of size $\Delta R=0.17$.

These cuts have been chosen to maximise the signal/background ratio.

For the selected events the transverse mass

$$
M_{\mathrm{T}}=\sqrt{2 p_{\mathrm{T}_{\mu}} E_{\mathrm{T}}^{\text {miss }}\left(1-\cos \Delta \phi_{\mu, E_{\mathrm{T}}^{\text {miss }}}\right)}
$$

is calculated from the muon transverse momentum $p_{\mathrm{T}_{\mu}}$, the missing energy component in the transverse plane $E_{\mathrm{T}}^{\text {miss }}$ and the angular $\Delta \phi_{\mu, E_{\mathrm{T}} \text { miss }}$ between both in this plane. Figure 14.9 shows the resulting distribution for signal $(1$ and $5 \mathrm{TeV})$ and background events. The $W^{\prime}$ boson distributions show a Jacobian peak which is spread out for large $M_{\mathrm{T}}$ due to the detector resolution. It can be seen immediately, that a $1 \mathrm{TeV}$ boson can be discovered or excluded easily, while for higher masses a statistical analysis is needed to quantify the sensitivity.

\subsubsection{Discovery and exclusion potential}

To interpret the results, the $\mathrm{CL}_{\mathrm{s}}$ method [508] is applied, which is based on the likelihood ratios, calculated for all bins of the $M_{\mathrm{T}}$ distribution. $\mathrm{CL}_{\mathrm{s}}$ is defined as ratio of the confidence levels for the signal and background hypotheses, $\mathrm{CL}_{\mathrm{s}}=\mathrm{CL}_{\mathrm{s}+\mathrm{b}} / \mathrm{CL}_{\mathrm{b}}$.

Figure 14.10 shows, that for an integrated luminosity of $10 \mathrm{fb}^{-1}$, a limit of $4.7 \mathrm{TeV}$ at the 95\% CL is reachable, if no signal is present in the CMS data. Both the expected discovery and exclusion limits are displayed in Fig. 14.11 as a function of integrated luminosity and $W^{\prime}$ mass. To investigate the sensitivity to the signal and background cross sections, they have been varied in a wide range; relative changes by factors of 2 and 10, respectively, lead to a lowering of the accessible mass range by about $0.5 \mathrm{TeV}$ in the worst case. 


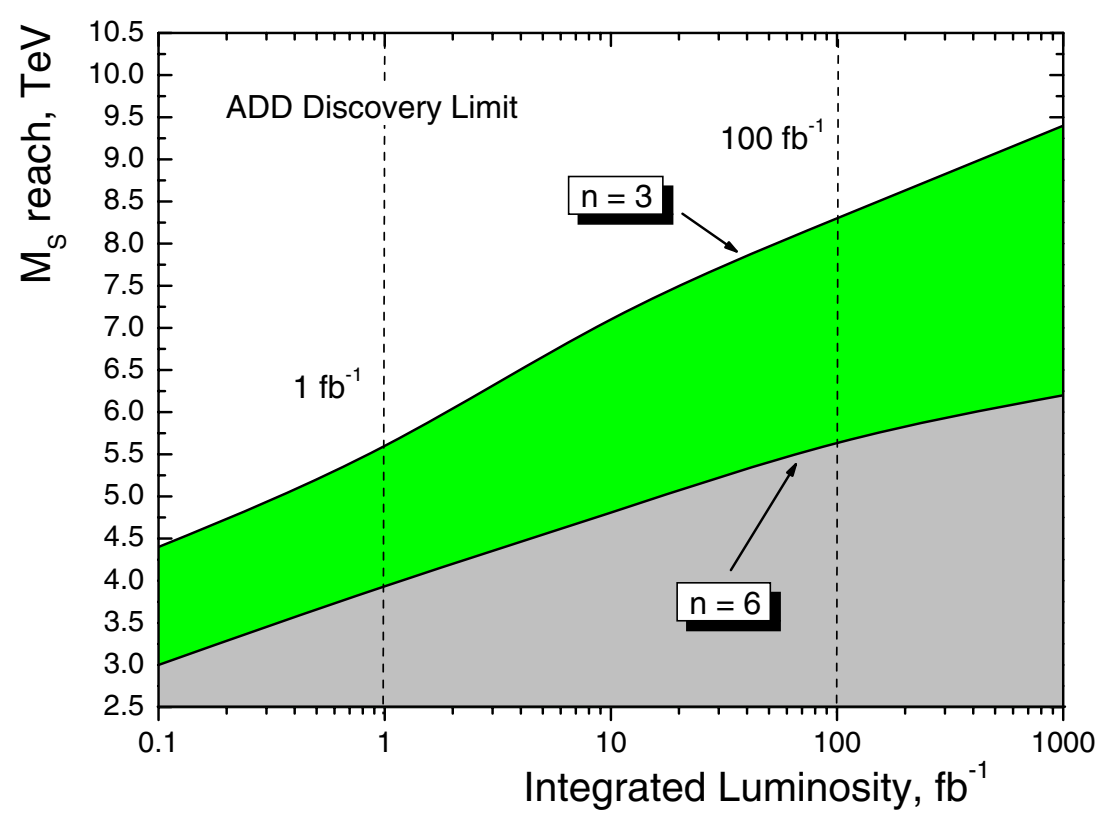

Figure 14.9. $5 \sigma$ limit on $M_{S}$ for the number of extra dimensions $n=3$ and 6 .
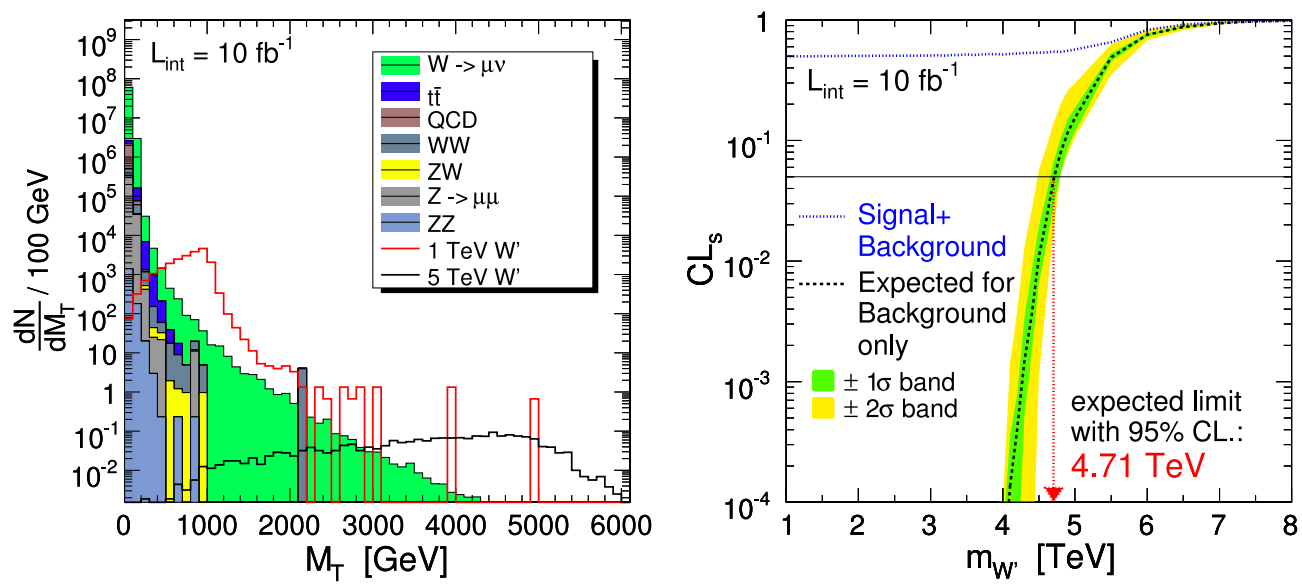

Figure 14.10. (Left) transverse invariant mass spectrum of signal ( 1 and $5 \mathrm{TeV}$, non-stacked) and background (stacked) after applying the selection cuts. (Right) result of the $\mathrm{CL}_{\mathrm{s}}$-method: with an integrated luminosity of $10 \mathrm{fb}^{-1}$. Reference $W^{\prime}$ bosons can be excluded up to a mass of $4.7 \mathrm{TeV}$.

\subsubsection{Systematic uncertainties}

The uncertainties arising from an imperfect knowledge of the PDFs at LHC energies and the error from the hard scale parameters have been investigated by using the Les Houches Accord PDFs [95] and varying the hard scale, respectively. The relative errors on the cross-section of the signal are listed in Table 14.4. The error on the background is comparable to that of the $W^{\prime}$ at the corresponding invariant mass.

The steep falling invariant mass distribution especially of the $W$ background holds a potential danger for the detection of $W^{\prime}$ bosons: if only a small fraction of these events is 

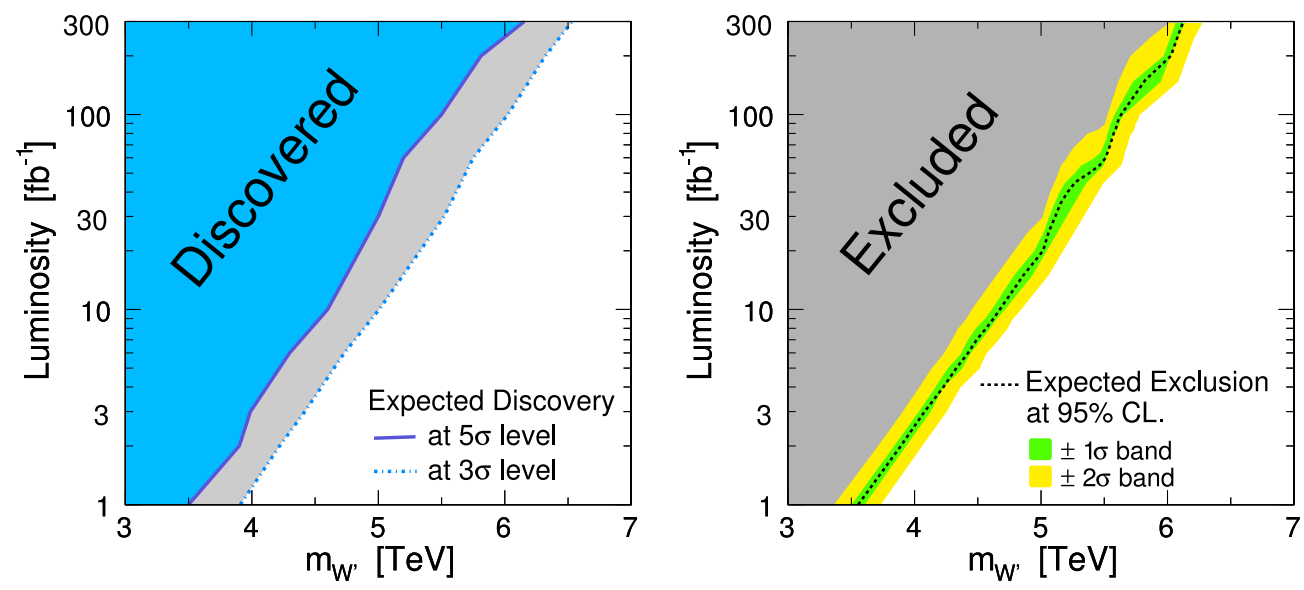

Figure 14.11. The plots show which integrated luminosity is needed to discover (left) or exclude (right) $W^{\prime}$ bosons of a certain mass.

Table 14.4. Relative systematic uncertainties in percent, arising from an imperfect theoretical knowledge (parton density functions, hard scale) and the expected luminosity error for an integrated luminosity of $10 \mathrm{fb}^{-1}$.

\begin{tabular}{lccccc}
\hline \multicolumn{5}{c}{ Systematic Uncertainties } \\
\cline { 2 - 6 } Type & $1 \mathrm{TeV} W^{\prime}$ & $2 \mathrm{TeV} W^{\prime}$ & $3 \mathrm{TeV} W^{\prime}$ & $4 \mathrm{TeV} W^{\prime}$ & $5 \mathrm{TeV} W^{\prime}$ \\
\hline PDF $\Delta \sigma / \sigma$ & +3.6 & +6.8 & +6.2 & +17.1 & +33.7 \\
Hard Scale $\Delta \sigma / \sigma$ & +4.3 & -5.9 & -8.3 & -10.6 & -18.9 \\
Luminosity $\Delta \mathcal{L} / \mathcal{L}$ & -4.1 & -6.5 & +10.4 & +13.1 & +14.8 \\
& $\pm 5 \%$ & $\pm 5 \%$ & $\pm 5 \%$ & $\pm 5 \%$ & $\pm 5 \%$ \\
\hline
\end{tabular}

reconstructed with a by far too large mass, which might result from a mis-measured muon momentum, the detection of a $W^{\prime}$ becomes extremely difficult. Such a behaviour would be visible in non-gaussian tails for example in the $p_{\mathrm{T}}$ resolution distribution. Using a large sample of a $W$ events it could be demonstrated, that the alignment precision expected after an integrated luminosity of $10 \mathrm{fb}^{-1}$ has only a small influence on the non-gaussian tails of the muon $p_{\mathrm{T}}$ resolution distribution.

The luminosity uncertainty at the considered integrated luminosity of $10 \mathrm{fb}^{-1}$ is expected to be $5 \%$, while other experimental errors (neutron background, dead detector components, etc.) are expected to be negligible.

\subsubsection{Summary}

For an integrated luminosity of $10 \mathrm{fb}^{-1}, W^{\prime}$ bosons of the Reference Model can be discovered or excluded up to a mass of $4.5-5 \mathrm{TeV}$, from an analysis of the muonic decay mode.

\subsection{High mass dijet final states}

\subsubsection{Dijet resonances and contact interactions}

Dijet resonances and contact interactions are the two major signals of new physics with dijets. Dijet resonances are direct and compelling observations of a new physical object at a mass $M$, requiring an incoming parton-parton collision energy equal to the mass. Contact interactions 

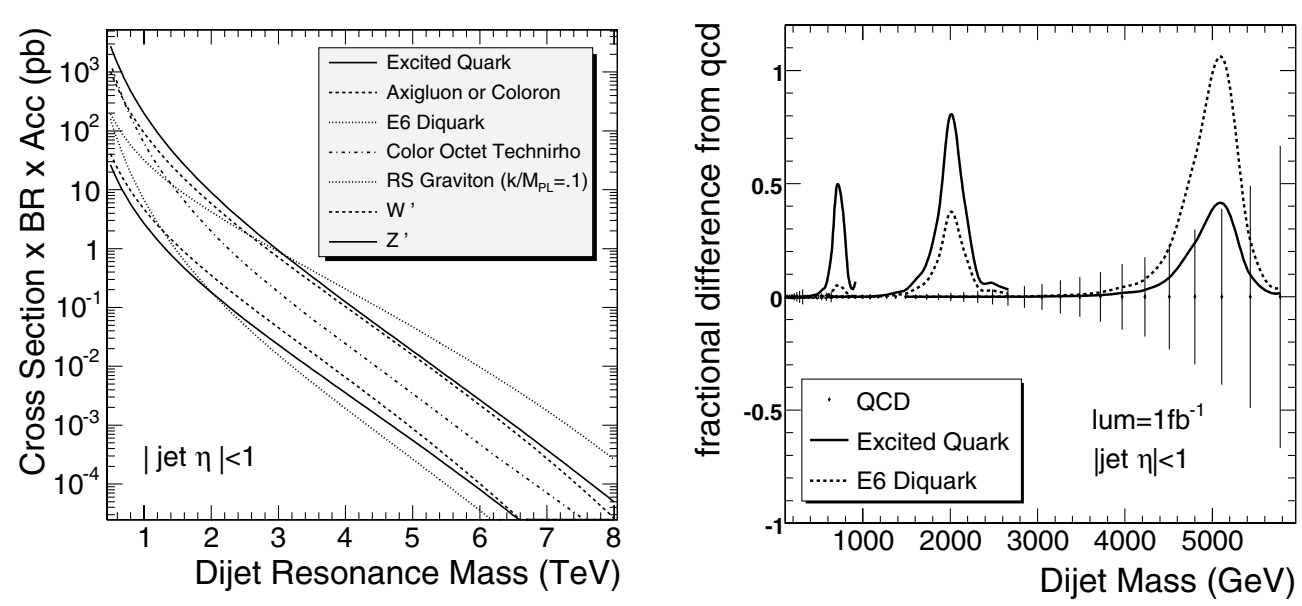

Figure 14.12. (Left) The total cross section times branching ratio times acceptance for dijet resonances from eight different models (see text). (Right) For resonance masses of 0.7, 2.0, and $5.0 \mathrm{TeV} / \mathrm{c}^{2}$, the fractional difference between an excited quark (solid curve) or an E6 diquark (dashed curve) and the QCD dijet background is compared to the QCD statistical errors (vertical lines).

(discussed in Section 15.3) are indirect observations of an energy scale of new physics, $\Lambda$, which can be significantly larger than the available collision energy. Resonances are clear signals but contact interactions are often observed first.

\subsubsection{Dijet resonance search}

We search for processes producing narrow resonances, $X$, decaying to dijets: $p p \rightarrow X \rightarrow$ jet + jet (inclusive) [730]. Our experimental motivation is that LHC is a parton-parton collider, and resonances made from partons must decay to the same partons giving two jets in the final state. The theoretical motivation is broad, since there are many models that predict narrow dijet resonances.

14.5.2.1. Dijet resonance models. In Fig. 14.12 we show the cross section times branching ratio times acceptance calculated to lowest order for eight benchmark models. Here we introduce them in order of descending cross section at low mass. Excited states of composite quarks [731] are strongly produced giving large cross sections $\left(q g \rightarrow q^{*}\right)$. Axigluons $(A)$ [732] or colorons $(C)$ [733] from an additional colour interaction are also strongly produced, but require an anti-quark in the initial state $(q \bar{q} \rightarrow A$ or $C)$ slightly reducing the cross section compared to excited quarks. Diquarks [734] from superstring inspired $E_{6}$ grand unified models are produced with electromagnetic coupling from the valence quarks of the proton $(u d \rightarrow D)$. The cross section for $E_{6}$ diquarks at high mass is the largest of all the models considered, because at high parton momentum the probability of finding a quark in the proton is significantly larger than the probability of finding a gluon or anti-quark. Colour octet technirhos [735] from topcolour-assisted technicolour are produced for either gluons or quarkanti-quark pairs in the initial state through a vector-dominance model of mixing between the gluon and the technirho $\left(q \bar{q}, g g \rightarrow g \rightarrow \rho_{\mathrm{T} 8}\right)$. Randall-Sundrum gravitons [94] from a model of large extra dimensions are produced with a significant cross section at masses below $1 \mathrm{TeV} / \mathrm{c}^{2}$ primarily from gluons in the initial state $(q \bar{q}, g g \rightarrow G)$. Heavy $W$ bosons [736] inspired by left-right symmetric grand unified models have electroweak couplings and 

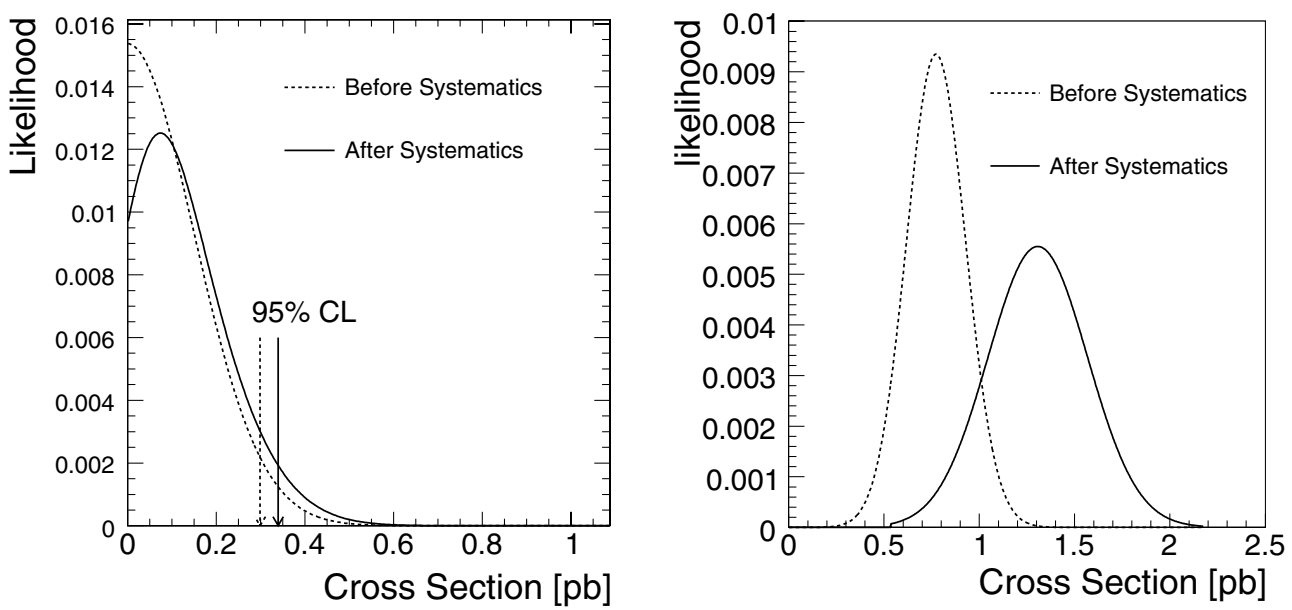

Figure 14.13. Likelihoods for observing a narrow dijet resonance of mass $2 \mathrm{TeV} / \mathrm{c}^{2}$ in a $1 \mathrm{fb}^{-1}$ data sample that contains only QCD background (left) and a data sample that also contains a resonance with a significance of $5 \sigma$ (right) are shown with statistical uncertainties only (dashed) and including systematics (solid).

require anti-quarks for their production $\left(q_{1} \overline{q_{2}} \rightarrow W^{\prime}\right)$ giving small cross sections. Heavy $Z$ bosons [736] inspired by grand-unified models are widely anticipated by theorists, but they are weakly produced, and require an anti-quark in the initial state $\left(q \bar{q} \rightarrow Z^{\prime}\right)$, so their production cross section is around the lowest of the models considered. Lower limits from CDF [120] and D0 [121] on the mass of these models range from 0.4 to $1.0 \mathrm{TeV} / \mathrm{c}^{2}$.

14.5.2.2. Dijet resonance sensitivity estimates. The signal and background dijet mass distributions for narrow resonances were presented in Section 4.1.4. In Fig. 14.12 we demonstrate the size of the signal for excited quarks and $E_{6}$ diquarks compared to the QCD background and it's statistical uncertainty. It is clear that we will be sensitive to such large signals for strongly produced dijet resonances. Here we quantify our sensitivity to any model of narrow dijet resonances. In Fig. 14.13 we show examples of likelihoods for excluding or observing a narrow resonance signal on a QCD background as a function of the signal cross section. In the case where the observed sample is QCD only, the signal likelihood peaks around zero cross section, and the $95 \%$ CL excluded signal cross section is shown. In the case where the observed sample is QCD plus a resonance signal, we have varied the signal size until the Gaussian distributed likelihood is $5 \sigma$ above zero. In Fig. 14.13 we have included estimates of our systematic uncertainties. For a resonance mass of $0.7(5.0) \mathrm{TeV} / \mathrm{c}^{2}$ the systematic uncertainty on the observable signal cross section due to the jet energy uncertainty in the background rate is $15 \%$ (25\%), the uncertainty due to jet resolution in the resonance shape is $10 \%(10 \%)$, the uncertainty due to radiation's affect on the resonance shape is $10 \%(25 \%)$, and the uncertainty due to luminosity is $10 \%(10 \%)$. For resonance masses just above the dijet mass thresholds where the trigger prescale decreases, there is an additional systematic uncertainty from the jet energy uncertainty. Systematic uncertainties have a greater effect on discovery than exclusion, because exclusions occur at a smaller signal cross section and are dominated by statistical uncertainties.

Figure 14.14 demonstrates that the $95 \% \mathrm{CL}$ exclusion and $5 \sigma$ discovery signal cross sections, including statistical uncertainties only, have reasonable values when compared to the size of the QCD statistical errors. Also in Fig. 14.13 we present the resonance cross 

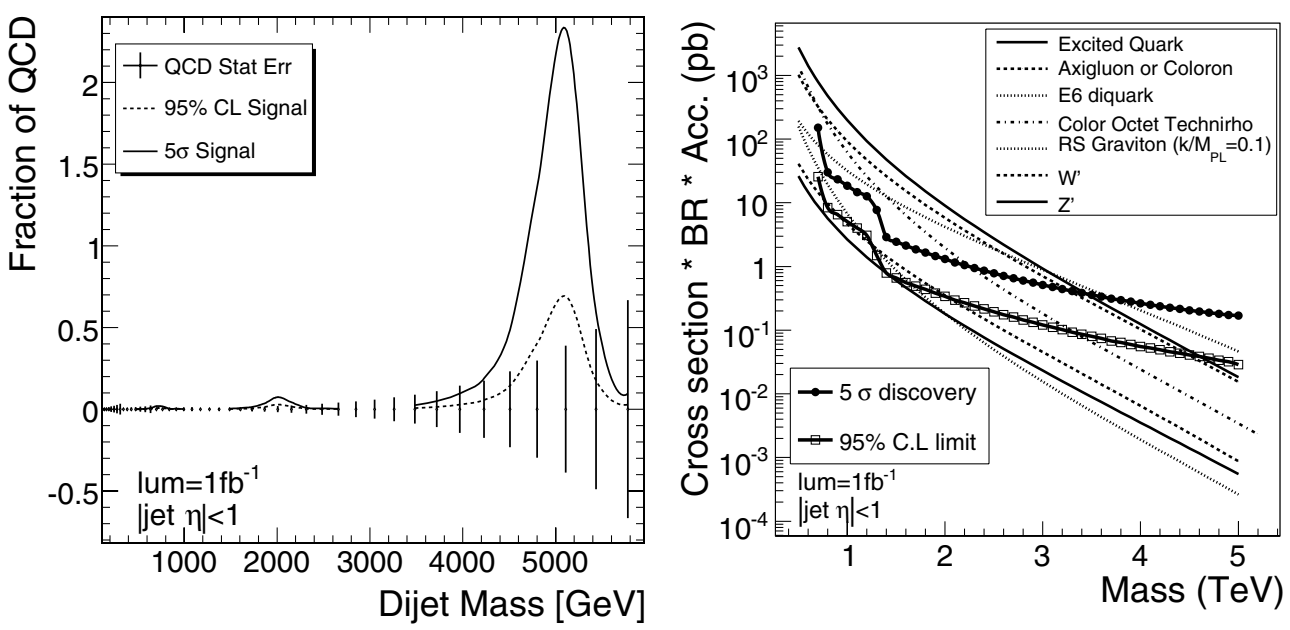

Figure 14.14. (Left) For resonances of mass $0.7,2.0$ and $5.0 \mathrm{TeV} / \mathrm{c}^{2}$, the rate as a fraction of QCD that CMS expects to exclude (dashed) or discover (solid) including statistical uncertainties only. (Right) The resonance cross section that CMS expects to exclude (boxes) or discover (circles), including systematic uncertainties, is compared to the cross section for eight resonance models.

Table 14.5. Sensitivity to dijet resonances with $100 \mathrm{pb}^{-1}, 1 \mathrm{fb}^{-1}$ and $10 \mathrm{fb}^{-1}$. For each resonance model, we show the range of masses we expect to be able to exclude at a confidence level of $95 \%$ or greater, and the range of masses we expect to be able to discover with a significance of $5 \sigma$ or greater. All estimates are with both statistical and systematic uncertainties.

\begin{tabular}{|c|c|c|c|c|c|c|}
\hline \multirow[t]{2}{*}{ Resonance Model } & \multicolumn{3}{|c|}{ 95\% CL Excluded Mass $\left(\mathrm{TeV} / \mathrm{c}^{2}\right)$} & \multicolumn{3}{|c|}{$5 \sigma$ Discovered Mass $\left(\mathrm{TeV} / \mathrm{c}^{2}\right)$} \\
\hline & $100 \mathrm{pb}^{-1}$ & $1 \mathrm{fb}^{-1}$ & $10 \mathrm{fb}^{-1}$ & $100 \mathrm{pb}^{-1}$ & $1 \mathrm{fb}^{-1}$ & $10 \mathrm{fb}^{-1}$ \\
\hline Excited Quark & $0.7-3.6$ & $0.7-4.6$ & $0.7-5.4$ & $0.7-2.5$ & $0.7-3.4$ & $0.7-4.4$ \\
\hline Axigluon or Colouron & $0.7-3.5$ & $0.7-4.5$ & $0.7-5.3$ & $0.7-2.2$ & $0.7-3.3$ & $0.7-4.3$ \\
\hline$E_{6}$ diquarks & $0.7-4.0$ & $0.7-5.4$ & $0.7-6.1$ & $0.8-2.0$ & $0.8-3.7$ & $0.8-5.1$ \\
\hline Colour Octet Technirho & $0.7-2.4$ & $0.7-3.3$ & $0.7-4.3$ & $0.7-1.5$ & $0.7-2.2$ & $0.7-3.1$ \\
\hline Randall-Sundrum & $0.7-1.1$ & $0.7-1.1$ & $0.7-1.1$ & & & \\
\hline Graviton & & $1.3-1.6$ & $\begin{array}{l}1.3-1.6 \\
2.1-2.3\end{array}$ & N/A & N/A & N/A \\
\hline $\mathrm{W}^{\prime}$ & $0.8-0.9$ & $\begin{array}{l}0.8-0.9 \\
1.3-2.0\end{array}$ & $\begin{array}{l}0.8-1.0 \\
1.3-3.2\end{array}$ & N/A & N/A & N/A \\
\hline$Z^{\prime}$ & N/A & N/A & $2.1-2.5$ & N/A & N/A & N/A \\
\hline
\end{tabular}

section values for jet $|\eta|<1$ that CMS can expect to exclude at 95\% CL or discover at $5 \sigma$ significance for an integrated luminosity of $1 \mathrm{fb}^{-1}$. These can be compared with the cross section of any model of narrow dijet resonances, and here we compare with our benchmark models. From Fig. 14.14 we can read off the mass limits or discoveries that are possible with $1 \mathrm{fb}^{-1}$ of data, which are listed in Table 14.5 along with the results of repeating the same analysis for $100 \mathrm{pb}^{-1}$ and $10 \mathrm{fb}^{-1}$. The resonances that are produced via the colour interaction (excited quarks, axigluons, colorons and colour octet technirhos) or from the valence quarks of each proton ( $E_{6}$ diquarks) have large cross sections and can be discovered up to a mass of a few TeV. A single search for resonances in the dijet mass distribution provides CMS with a sensitive test of many different models of the widely anticipated New Physics at the TeV scale. 


\subsection{High mass diphoton final states}

\subsubsection{Introduction}

The study of the Randall-Sundrum (RS) graviton decaying into the two photons is particularly interesting as the detection of such few $\mathrm{TeV} / \mathrm{c}^{2}$ mass resonance in such channel together with its observation in the dilepton channel will sign a RS graviton, distinguishing it from a $\mathrm{Z}^{\prime}$ production. The model is governed by two parameters: the graviton mass $M$ and its coupling to Standard Model particles $c$, the latter being related to the natural width of the resonance.

\subsubsection{Event generation and kinematics pre-selection}

The search for the $\mathrm{G} \rightarrow \gamma \gamma$ signal at LHC is affected by four types of backgrounds:

- The prompt diphoton production from the quark annihilation and gluon fusion diagrams, which provides an intrinsic or 'irreducible' background.

- The $\gamma+$ jets production consisting of two parts: i) prompt photon from hard interaction + the second photon coming from the outgoing quark due to final state radiation and ii) prompt photon from hard interaction + the decay of a neutral hadron (mostly isolated $\pi^{0}$ ) in a jet, which could fake a real photon.

- The background from QCD hadronic jets, where electromagnetic energy deposits result from the decay of neutral hadrons (especially isolated $\pi^{0} \mathrm{~s}$ ) in both jets.

- Drell-Yan process with $e^{+} e^{-}$in a final state which could mimic photons when correspondent electron tracks will not be assigned to the superclusters during the reconstruction.

Generator-level pre-selection and parameters used for QCD and bremsstrahlung backgrounds is described in [737].

\subsubsection{Offline selection and analysis}

The requirements for the analysis were as follows:

1 Two super-clusters (SCs) with $E_{\mathrm{T}}>150 \mathrm{GeV}$ and two HLT trigger bits triggered at the same time: $2 p$ (two photons) and $\mathrm{r} 2 \mathrm{p}$ (two photons relaxed).

2 Calorimeter isolation criteria: for each SC the energy in a cone of $\Delta R=0.5$ (excluding SC itself) should be $<0.02 E_{\mathrm{T}}(S C)$

$3 E(H C A L) / E(E C A L)<0.05$

4 Tracker isolation: the sum of the energy of all tracks in a cone $\Delta R=0.5$ around the SC should be $<0.01 E_{\mathrm{T}}(S C)$

5 Photon energy corrections are done in a simple way so far:

- For E1 energy $<1.7 \mathrm{TeV}$, only a simple energy dependent part of correction is applied (just a shift of the peak).

- For E1 energy > 1.7 TeV, the MGPA saturation correction (1d) was applied (see and [738]).

\subsubsection{K-factors}

To produce the final results and to calculate the expected statistical significance for RS-1 graviton search recently calculated next-to-leading order corrections ( $\mathrm{K}$ factors) to the cross sections of different types of background are used: $\mathrm{K}=1.5$ for quark annihilation [26], $\mathrm{K}=1.2$ for gluon fusion [29], $\mathrm{K}=1$ for the $\gamma+$ hadronic jets [29] and $\mathrm{K}=1$ for QCD jets. For signal, a conservative $\mathrm{K}=1$ value is taken. 
Table 14.6. Number of events passed through the analysis cuts defined above for $M_{G}=$ $1.5 \mathrm{TeV} / \mathrm{c}^{2}, c=0.01$ and $\mathcal{L}=30 \mathrm{fb}^{-1}$. Leading column is non-saturated events, all saturated events, passed through the analysis, were added in brackets, where applied.

\begin{tabular}{lcccccc}
\hline & signal & $\begin{array}{c}\text { Born } \\
(\mathrm{K}=1.5)\end{array}$ & $\begin{array}{c}\text { Box } \\
(\mathrm{K}=1.2)\end{array}$ & $\begin{array}{c}\text { Brem } \\
(\mathrm{K}=1)\end{array}$ & $\begin{array}{c}\text { QCD } \\
(\mathrm{K}=1)\end{array}$ & $\begin{array}{c}\mathrm{DY} \\
(\mathrm{K}=1)\end{array}$ \\
\hline trigger + 2SC & 28.9 & 8.6 & 0.10 & 29.2 & 798.7 & 4.3 \\
+ EM isolation & 24.5 & 5.5 & 0.08 & 20.3 & 361.8 & 3.5 \\
+ HCAL/ECAL & 24.3 & 5.4 & 0.08 & 4.4 & 12.8 & 3.5 \\
+ tracker isolation & 17.6 & $4.2(+0.2)$ & 0.05 & 0.17 & 0.0 & 0.0 \\
\hline
\end{tabular}

Table 14.7. Number of events passed through the analysis cuts defined above for $M_{G}=$ $3.5 \mathrm{TeV} / \mathrm{c}^{2}, c=0.1$ and $\mathcal{L}=30 \mathrm{fb}^{-1}$. Leading column is non-saturated events, all saturated events, passed through the analysis, were added in brackets, where applied.

\begin{tabular}{lcccccc}
\hline & signal & $\begin{array}{c}\text { Born } \\
(\mathrm{K}=1.5)\end{array}$ & $\begin{array}{c}\text { Box } \\
(\mathrm{K}=1.2)\end{array}$ & $\begin{array}{c}\text { Brem } \\
(\mathrm{K}=1)\end{array}$ & $\begin{array}{c}\text { QCD } \\
(\mathrm{K}=1)\end{array}$ & $\begin{array}{c}\mathrm{DY} \\
(\mathrm{K}=1)\end{array}$ \\
\hline trigger + 2SC & 11.6 & 0.20 & $4.4 * 10^{-4}$ & 0.78 & 821.9 & 0.10 \\
+ EM isolation & 10.8 & 0.14 & $3.6 * 10^{-4}$ & 0.32 & 164.4 & 0.095 \\
+ HCAL/ECAL & 10.6 & 0.13 & $3.4 * 10^{-4}$ & 0.016 & 0.0 & 0.095 \\
+ tracker isolation & $8.9(+1.0)$ & $0.10(+0.02)$ & $2.7(+0.24) * 10^{-4}$ & $1.7 * 10^{-3}$ & 0.0 & $7.2 * 10^{-4}$ \\
\hline
\end{tabular}
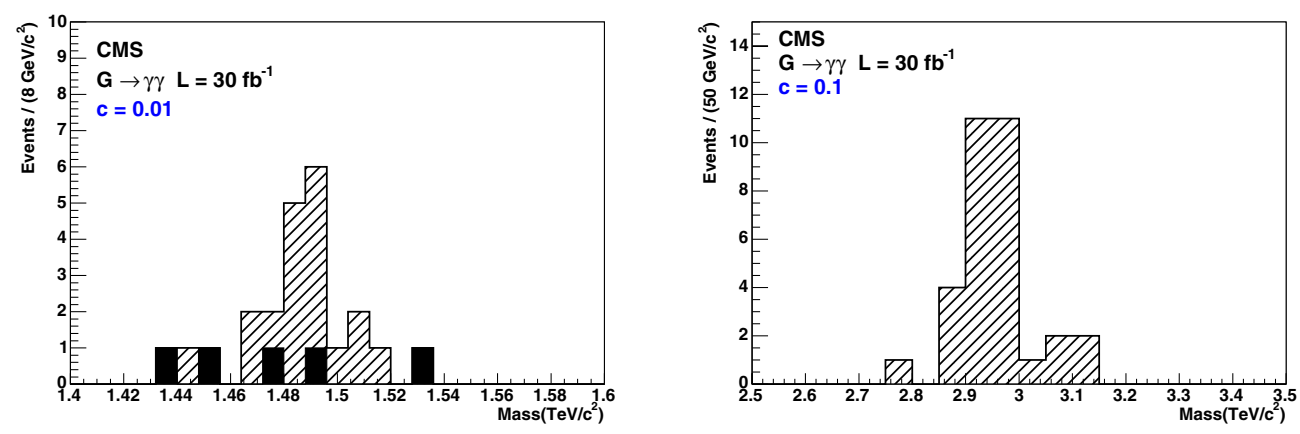

Figure 14.15. Number of events passing all cuts for $\left(1.5 \mathrm{TeV} / \mathrm{c}^{2}, 0.01\right)(\mathrm{left})$ and $\left(3.0 \mathrm{TeV} / \mathrm{c}^{2}, 0.1\right)$ (right) RSI gravitons for $30 \mathrm{fb}^{-1}$ integrated luminosity.

\subsubsection{Results}

The numbers of events passing the analysis cuts described above, for the signal and for the backgrounds, are presented in Table $14.6\left(1.5 \mathrm{TeV} / \mathrm{c}^{2}, 0.01\right)$ and in Table $14.7\left(3.5 \mathrm{TeV} / \mathrm{c}^{2}\right.$, $0.1)$.

Figure 14.15 shows the number of events satisfying all cuts for both signal and backgrounds for the cases $\left(1.5 \mathrm{TeV} / \mathrm{c}^{2}, 0.01\right)$ and $\left(3.0 \mathrm{TeV} / \mathrm{c}^{2}, 0.1\right)$ after $30 \mathrm{fb}^{-1}$ luminosity. The results for one year low luminosity of $10 \mathrm{fb}^{-1}$ are presented in Fig. 14.16.

Taking into account the K-factors described above, the number of events for signal and background and the significance $S_{c L}$ (defined in Appendix A.1) for $c=0.01$ and $c=0.1$ are shown respectively in Tables 14.8 and 14.9 for an integrated luminosity of $30 \mathrm{fb}^{-1}$.

The significance as a function of the graviton mass $\left(M_{G}\right)$ for integrated luminosities of $10 \mathrm{fb}^{-1}, 30 \mathrm{fb}^{-1}$ and $60 \mathrm{fb}^{-1}$ are displayed in Fig. 14.17.

The discovery region in the plane of the coupling parameter $c$ and the graviton mass is shown in Fig. 14.18. 

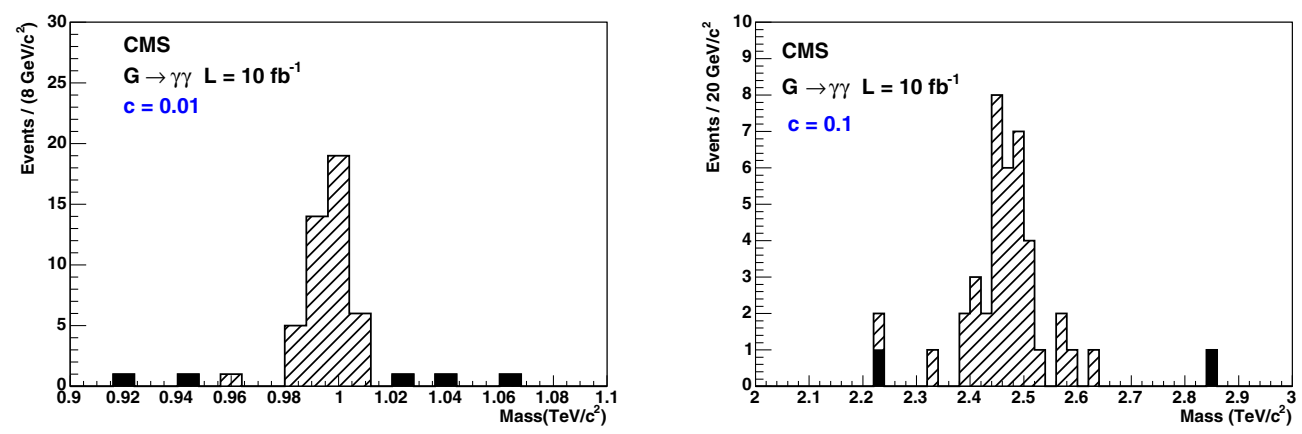

Figure 14.16. Number of events passing all cuts for $\left(1.0 \mathrm{TeV} / \mathrm{c}^{2}, 0.01\right)($ left $)$ and $\left(2.5 \mathrm{TeV} / \mathrm{c}^{2}, 0.1\right)$ (right) RS-1 gravitons for $10 \mathrm{fb}^{-1}$ integrated luminosity.
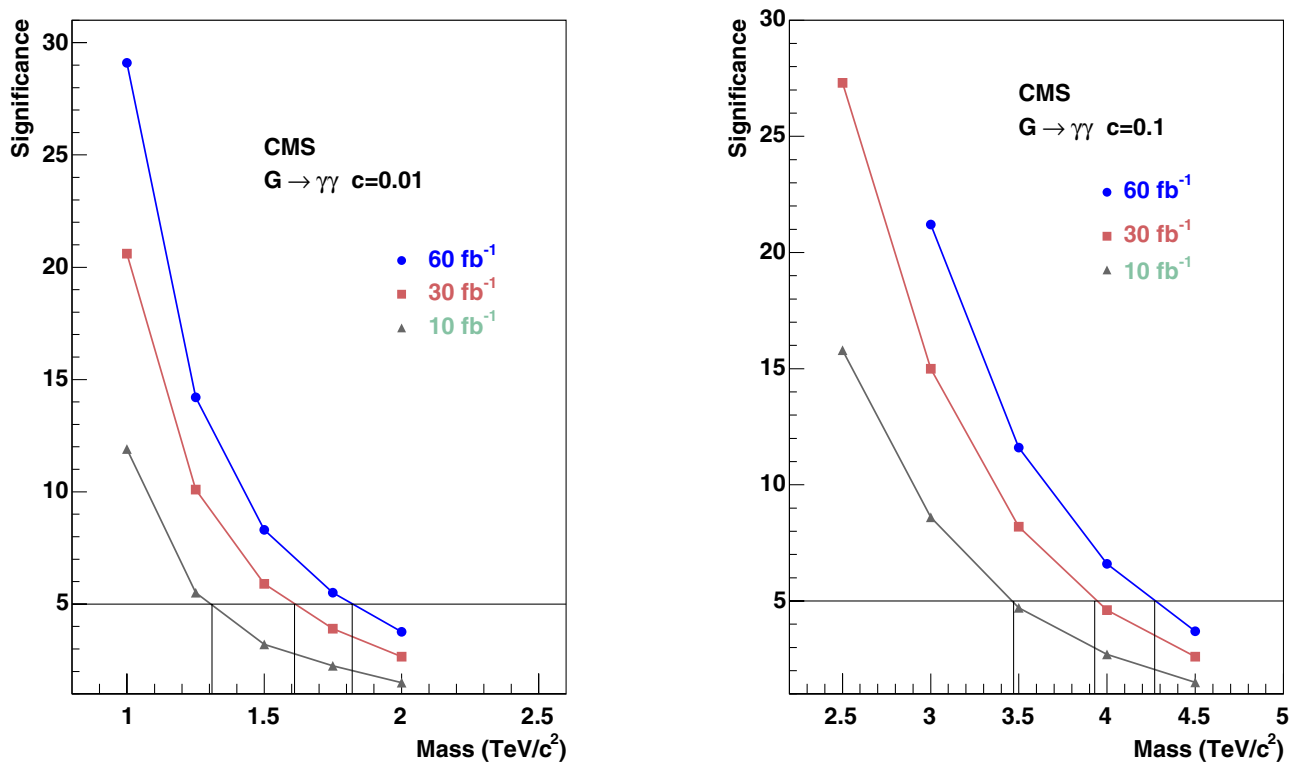

Figure 14.17. Significance as a function of the graviton mass for $10 \mathrm{fb}^{-1}, 30 \mathrm{fb}^{-1}$ and $60 \mathrm{fb}^{-1}$ integrated luminosities, with $\mathrm{c}=0.01$ (left) and $\mathrm{c}=0.1$ (right).

Table 14.8. Significance for $c=0.01$ and $\mathcal{L}=30 \mathrm{fb}^{-1}$.

\begin{tabular}{lccccc}
\hline & $\begin{array}{c}M_{G}=1.0 \\
\mathrm{TeV} / \mathrm{c}^{2}\end{array}$ & $\begin{array}{c}M_{G}=1.25 \\
\mathrm{TeV} / \mathrm{c}^{2}\end{array}$ & $\begin{array}{c}M_{G}=1.5 \\
\mathrm{TeV} / \mathrm{c}^{2}\end{array}$ & $\begin{array}{c}M_{G}=1.75 \\
\mathrm{TeV} / \mathrm{c}^{2}\end{array}$ & $\begin{array}{c}M_{G}=2.0 \\
\mathrm{TeV} / \mathrm{c}^{2}\end{array}$ \\
\hline$N_{s}$ & 135.8 & 44.0 & 17.6 & 7.3 & 3.9 \\
$N_{b k g}$ & 15.0 & 8.8 & 4.6 & 1.8 & 1.2 \\
Significance & 20.6 & 10.1 & 5.9 & 3.9 & 2.6 \\
\hline
\end{tabular}

Table 14.9. Significance for $c=0.1$ and $\mathcal{L}=30 \mathrm{fb}^{-1}$.

\begin{tabular}{lccccc}
\hline & $\begin{array}{c}M_{G}=2.5 \\
\mathrm{TeV} / \mathrm{c}^{2}\end{array}$ & $\begin{array}{c}M_{G}=3.0 \\
\mathrm{TeV} / \mathrm{c}^{2}\end{array}$ & $\begin{array}{c}M_{G}=3.5 \\
\mathrm{TeV} / \mathrm{c}^{2}\end{array}$ & $\begin{array}{c}M_{G}=4.0 \\
\mathrm{TeV} / \mathrm{c}^{2}\end{array}$ & $\begin{array}{c}M_{G}=4.5 \\
\mathrm{TeV} / \mathrm{c}^{2}\end{array}$ \\
\hline$N_{s}$ & 103.8 & 31.6 & 9.9 & 3.44 & 1.11 \\
$N_{b k g}$ & 1.11 & 0.35 & 0.13 & 0.06 & 0.02 \\
Significance & 27.3 & 15.0 & 8.2 & 4.6 & 2.6 \\
\hline
\end{tabular}




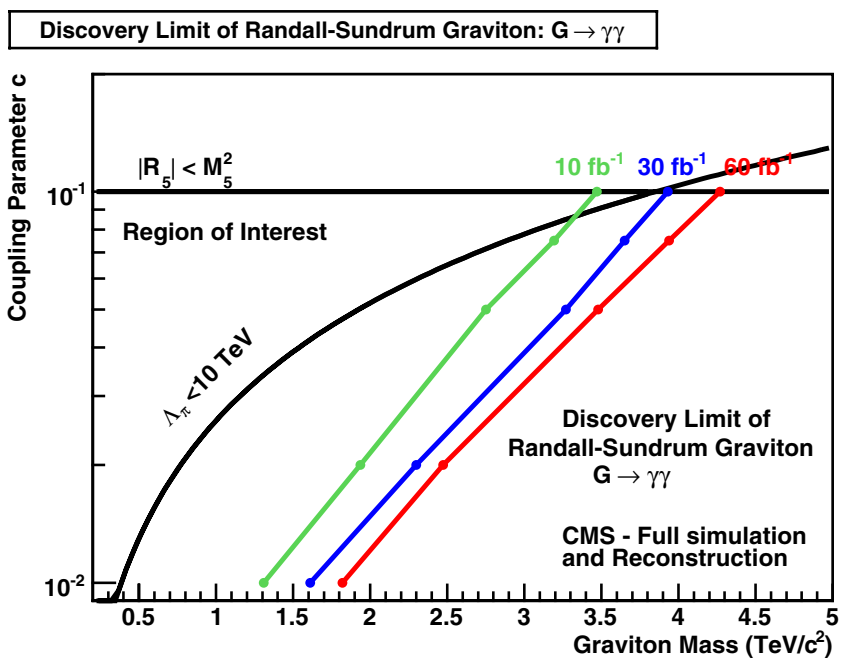

Figure 14.18. Reach of the CMS experiment in the search for the Randall-Sundrum graviton decaying into diphoton channel as a function of the coupling parameter $c$ and the graviton mass for $10 \mathrm{fb}^{-1}, 30 \mathrm{fb}^{-1}$ and $60 \mathrm{fb}^{-1}$. The left part of each curve is the region where the significance exceeds $5 \sigma$.

Table 14.10. Hard scale confidence limits uncertainties for $30 \mathrm{fb}^{-1}$.

\begin{tabular}{lcc}
\hline & $4 \hat{s}$ & $0.25 \hat{s}$ \\
\hline$c=0.01$ & $-62 \mathrm{GeV} / \mathrm{c}^{2}$ & $+56 \mathrm{GeV} / \mathrm{c}^{2}$ \\
$c=0.1$ & $-47 \mathrm{GeV} / \mathrm{c}^{2}$ & $+42 \mathrm{GeV} / \mathrm{c}^{2}$ \\
\hline
\end{tabular}

The discovery region for $60 \mathrm{fb}^{-1}$ extends to $M_{G}=1.82 \mathrm{TeV} / \mathrm{c}^{2}$ if $c=0.01$ and to $M_{G}=4.27 \mathrm{TeV} / \mathrm{c}^{2}$ if $c=0.1$. For $30 \mathrm{fb}^{-1}$ it is $M_{G}=1.61 \mathrm{TeV} / \mathrm{c}^{2}$ if $c=0.01$ and $M_{G}=3.95 \mathrm{TeV} / \mathrm{c}^{2}$ if $c=0.1$. For $10 \mathrm{fb}^{-1}$ it reaches to $M_{G}=1.31 \mathrm{TeV} / \mathrm{c}^{2}$ if $c=0.01$ and $M_{G}=3.47 \mathrm{TeV} / \mathrm{c}^{2}$ if $c=0.1$.

\subsubsection{Systematic uncertainties for $30 \mathrm{fb}^{-1}$}

Several systematic uncertainties and their effect on the mass reach have been evaluated for an integrated luminosity of $30 \mathrm{fb}^{-1}$. The effect of hard scale uncertainties is given in Table 14.10 , computed by multiplying and dividing the scale $\hat{s}$ by a factor 2 . The uncertainties from the pdfs, computed with LHAPDF, amount for $c=0.01$ to $-55 \mathrm{GeV} / \mathrm{c}^{2}$ and for $c=0.1$ to $-152 \mathrm{GeV} / \mathrm{c}^{2}$. There is another source of uncertainties due to the fact, that we have used $\mathrm{K}$-factor $=1.5$ for the Born process, while the most recent measurements at the Tevatron pointed to a $\mathrm{K}$-factor closer to 2 [739]. The effect of such a change on the mass reach is $-50 \mathrm{GeV} / \mathrm{c}^{2}$ for $c=0.01$ and $-30 \mathrm{GeV} / \mathrm{c}^{2}$ for $c=0.1$.

\subsection{Single $\gamma$ final state with $E_{\mathrm{T}}^{\mathrm{miss}}$ from extra dimensions}

\subsubsection{Topology of single-photon final states}

An introduction to the signals involving direct graviton emission in ADD type of extra dimensions frameworks is given is Section 14.3.2. The topology of single photon events can 


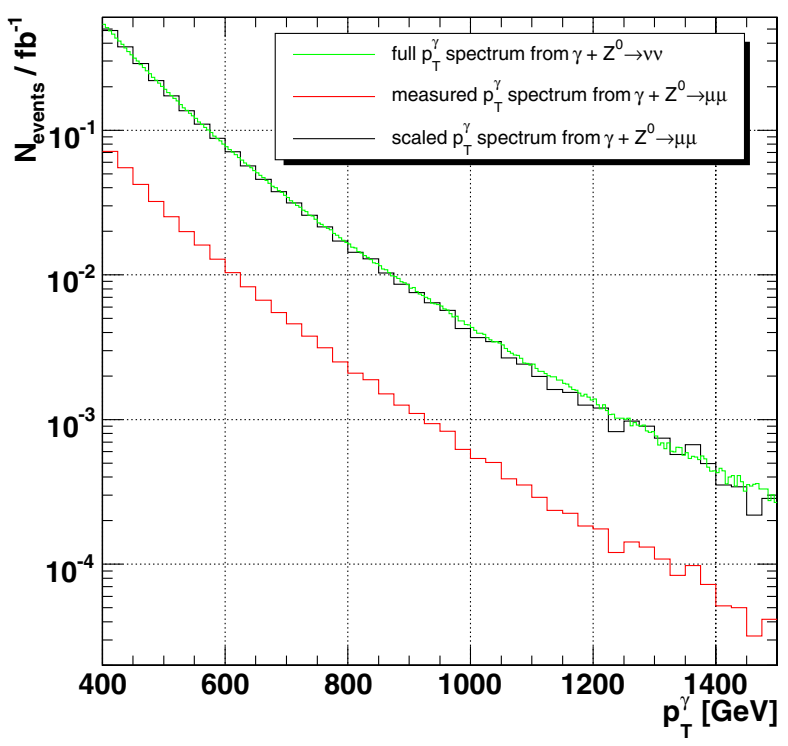

Figure 14.19. Number of expected $p_{\mathrm{T}}^{\gamma}$ events per $25 \mathrm{GeV}$ bin at $1 \mathrm{fb}^{-1}$ from measured $\gamma+Z^{0} \rightarrow \mu^{+} \mu^{-}$events before and after transformation compared with the generator distribution for $\gamma+Z^{0} \rightarrow v_{i} \bar{v}_{i}$ - the transformed muon distribution models the $v_{i} \bar{v}_{i}$ spectrum well.

be identified by:

- a single high $p_{\mathrm{T}}$ photon in the central $\eta$ region;

- high missing $p_{\mathrm{T}}$ back-to-back to the photon in the azimuthal plane with a similar $p_{\mathrm{T}}$ distribution.

These characteristics are not strongly dependent on the ADD model parameters. The details of this analysis can be found in [740].

\subsubsection{Backgrounds from the Standard Model}

All signal and background samples used in the following were simulated using the CMS fast detector simulation [11]. Fully simulated reference samples were generated for the signal and the largest irreducible background, $Z^{0} \gamma \rightarrow \nu \bar{v}+\gamma$. A detailed comparison of the resolution, efficiency and purity of all reconstructed objects used in this analysis to the GEANT-based CMS simulation confirmed that the fast simulation provides a very good approximation of the expected detector response. All samples were consistently generated using a generator level cut in PYTHIA $\hat{p}_{\mathrm{T}}>400 \mathrm{GeV}$. The backgrounds considered in the study are, $Z^{0} \gamma \rightarrow v \bar{v}+\gamma$, $W^{ \pm} \rightarrow \ell v$ where $\ell$ is electron, muon or tau, $W^{ \pm} \gamma \rightarrow e v+\gamma \gamma+\mathrm{Jets}$, QCD, di $\gamma$ and $Z^{0}$ + jets. For the main background, a normalisation method from measured data is developed employing the reconstructed leptonic decays of the $Z^{0}$ into muon and electron pairs.

The detector acceptance for selecting the leptons is parameterised using a twodimensional function $\alpha\left(p_{\mathrm{T}}^{\gamma}, \eta_{\gamma}\right)$. Figure 14.19 shows the measured and the $p_{\mathrm{T}}^{\gamma}$ spectrum from $\gamma+Z^{0} \rightarrow \mu^{+} \mu^{-}$after the (acceptance $\times$efficiency) parameterisation is applied, in comparison with the generator spectrum for $\gamma+Z^{0} \rightarrow v_{i} \bar{\nu}_{i}$ events. For $p_{\mathrm{T}}^{\gamma}>100 \mathrm{GeV} / \mathrm{c}$ there is 1170 $Z^{0} \rightarrow \mu^{+} \mu^{-} / e^{+} e^{-}$events expected after all selection cuts for $30 \mathrm{fb}^{-1}$. These can be used as the candle sample that provides a direct normalisation of the $\gamma+Z^{0} \rightarrow v_{i} \bar{\nu}_{i}$ with a statistical precision of $3 \%$. 


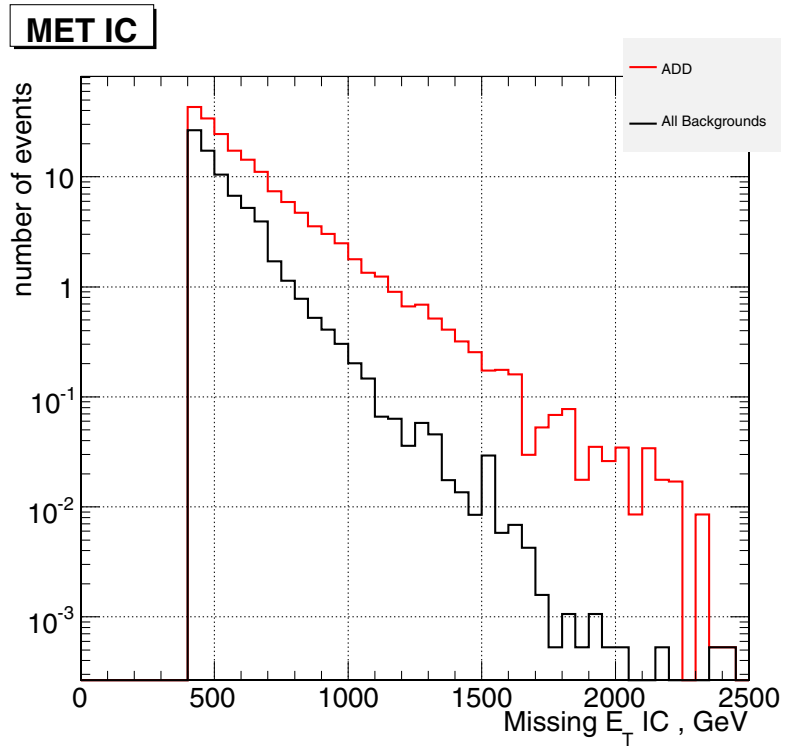

Figure 14.20. Spectrum of the missing $E_{\mathrm{T}}$ for all backgrounds (black histogram) and for an example signal sample $\left(M_{\mathrm{D}}=2.5 \mathrm{TeV}, n=2\right)$. The number of events corresponds to an integrated luminosity of $30 \mathrm{fb}^{-1}$.

\subsubsection{Event selection}

The main trigger path for the selection of signal and background events will be the single photon trigger, both at the Level-1 and the HLT. Presently the single photon trigger has a HLT level threshold of $80 \mathrm{GeV}$, which is far below the selection cut for events with isolated photons above $400 \mathrm{GeV}$ used here. Hence the expected trigger efficiency is close to $100 \%$ and its efficiency can be monitored from data with a $E_{\mathrm{T}}^{\text {miss }}$ trigger which will have a threshold in the range of $200-300 \mathrm{GeV}$, well below the acceptance of the bulk of the signal. Both the topological characteristic and the necessity to reduce the Standard Model background lead to the following selection criteria:

- At least a $E_{\mathrm{T}}^{\text {miss }}>400 \mathrm{GeV}$ is required and the photon $p_{\mathrm{T}}$ has to be above $400 \mathrm{GeV}$.

- $|\eta|$ of the photon $<2.4$.

- $\Delta \phi\left(E_{\mathrm{T}}^{\text {miss }}, \gamma\right)>2.5$.

- A track veto for high $p_{\mathrm{T}}$ tracks $>40 \mathrm{GeV}$ is applied. This is a powerful criterion to reduce all backgrounds containing high-energetic charged particles (such as $e^{ \pm}, \mu^{ \pm}$, jets).

- An Isolated Photon Likelihood criterion is applied to remove residual background from hard photon emission from jets as well as fake photons from jets.

Figure 14.20 shows the missing transverse energy spectra for events surviving the selection path for both the signal and the backgrounds. As expected the $Z^{0} \gamma$ is by far the most dominant component of the background, followed by $W^{ \pm} \gamma$ while the contributions of the other Standard Model backgrounds are small. For all ADD cross section the hard truncation approach is used (see Section 14.1), i.e. events with $M_{G}<M_{D}$ are rejected.

\subsubsection{Systematic uncertainties and discovery potential}

We consider an uncertainty of $2 \%$ for the measurement of the photon $p_{\mathrm{T}}^{\gamma}$ in the electromagnetic calorimeter and an uncertainty of $5 \%$ for the $E_{\mathrm{T}}^{\text {miss }}$ measurement. The 


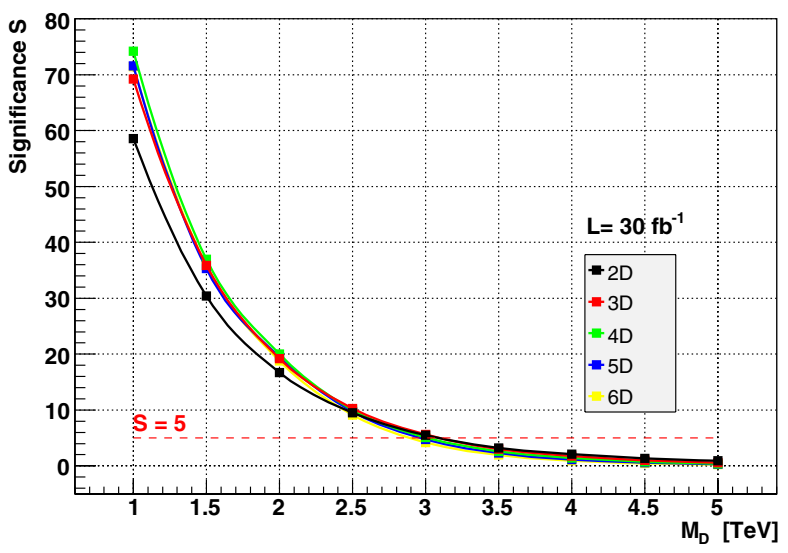

Figure 14.21. Expected significances as function of $M_{\mathrm{D}}$ for different number $n$ of extra dimensions.

resulting decrease of the significance is $1.0 \%$ and $1.6 \%$ respectively. For the main background the systematics can be reduced to the luminosity measurement using the $Z^{0}$ candle calibration method. It can thus be measured with a precision of $3 \%$ after $30 \mathrm{fb}^{-1}$. The $5 \sigma$ discovery reach is achievable for $M_{D}<2.5 \mathrm{TeV} / \mathrm{c}^{2}$ and all values of extra dimensions while for $M_{D}<$ $3 \mathrm{TeV} / \mathrm{c}^{2} 5 \sigma$ reach is achievable for $n$ between 2 and 4 . Figure 14.21 shows the expected significances as function of $M_{\mathrm{D}}$.

\subsection{Black holes}

\subsubsection{Introduction to higher-dimensional black holes}

One of the consequences of large extra dimensions is the possibility to produce microscopic black hole $(\mathrm{BH})$ at LHC energies. Such a BH formed in a $(4+n)$-dimensional space-time has a Schwarzschild radius

$$
r_{s(4+n)}=\frac{1}{\sqrt{\pi} M_{(4+n)}}\left(\frac{M_{B H}}{M_{(4+n)}}\left(\frac{8 \Gamma((n+3) / 2)}{n+2}\right)\right)^{1 /(n+1)}
$$

where $M_{(4+n)}$ is the reduced Planck scale and $n$ is the number of large extra dimensions [741]. A high energy collision of two partons can result in the formation of a $\mathrm{BH}$ when the impact parameter is smaller than $r_{s(4+n)}$. In the semi-classical approach the $\mathrm{BH}$ cross section is given by $\sigma\left(M_{\mathrm{BH}}\right)=\pi r_{s(4+n)}^{2}$ at the parton level. If for low masses $M_{(4+n)}$, i.e. around $2 \mathrm{TeV}$, the $\mathrm{BH}$ production cross sections at the $\mathrm{LHC}$ is in the pb range.

Once produced, these BHs are expected to decay thermally via Hawking radiation [742]. The Hawking temperature for a $\mathrm{BH}$ in $4+n$ dimensions is [743]

$$
T_{(4+n)} \sim M_{(4+n)}\left(M_{(4+n)} / M_{\mathrm{BH}}\right)^{1 /(n+1)} .
$$

These BHs have a very short lifetime typically of $\sim 10^{-27}$ seconds.

$\mathrm{BH}$ events are expected to evaporate democratically by emission of all particle types that exist in nature, independent of their spin, charge, quantum numbers or interaction properties. Therefore they can be a source of new particles. BH physics at the LHC can provide the possibility of probing quantum gravity in the lab. 

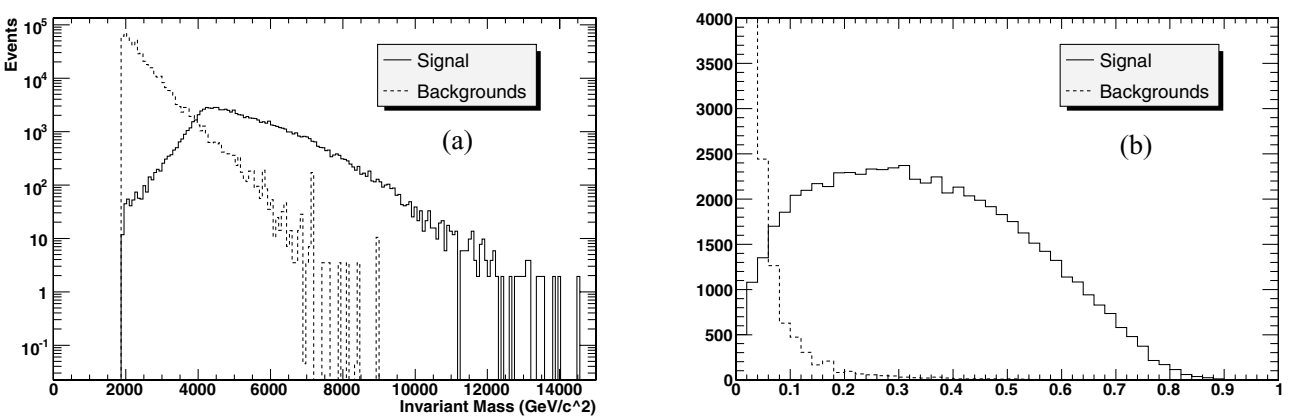

Figure 14.22. (a) Reconstructed invariant mass distribution and (b) event sphericity for black hole and standard model background events.

\subsubsection{Analysis selection path and results}

Black hole event samples were produced using the CHARYBDIs event generator [744]. As a benchmark the case which is analysed has the following parameters: a) $2 \mathrm{TeV} / \mathrm{c}^{2}$ effective Planck scale, b) $4 \mathrm{TeV} / \mathrm{c}^{2}$ minimum and $14 \mathrm{TeV} / \mathrm{c}^{2}$ maximum black hole mass c) 3 extra dimensions. Time evolution during Hawking radiation and gray body effects are included. The detector response was simulated by us using the CMS fast simulation (FAMOs, version 1.4.0) after validation against the detailed CMS GEANT-based simulation. The Standard Model backgrounds taken into account include QCD jets, top production and boson plus jet production. The invariant mass of all final state objects (electrons, photons, jets and muons) in the event is found to be correlated with the input black hole mass. In addition since the black hole formation can only occur if $M_{\mathrm{BH}}>M_{(4+n)}$, the event invariant mass can indicate the effective Planck scale $M_{(4+n)}$. In the benchmark scenario the invariant mass is required to be greater than $2 \mathrm{TeV} / \mathrm{c}^{2}$. BH events are characterised by a high multiplicity of the final state particles, which increase as a function of the BH mass (and decreases as a function of Hawking temperature). In particular the ratio of jets to leptons is found to be 5 to 1 . In this study with a simple jet and lepton multiplicity counting the jet/lepton ratio is formed. The average value of this ratio is found to be 4.5 . The thermal nature of Hawking radiation requires the distribution of $\mathrm{BH}$ remnants to be spherical as shown and a sphericity of 0.28 is required which eliminates drastically the Standard Model backgrounds. The invariant mass distribution and sphericity for the signal and background events is shown in Fig. 14.22.

Events are counted when the total sum of the $P_{\mathrm{T}}$ of all reconstructed objects plus the missing transverse energy is larger than $2500 \mathrm{GeV}$. A study of the Level-1 and HLT trigger path shows that the 4 jet trigger has a $93 \%$ efficiency for the signal events and is used in the analysis.

The event selection criteria applied to the reconstructed events and the efficiencies of the requirements are listed in Table 14.11.

The minimum integrated luminosity needed for $5 \sigma$ significance and for the benchmark point is $\sim 2 \mathrm{pb}^{-1}$. A survey of the parameter space using 25 points shows that for effective Planck scale of 2-3 TeV, minimum black hole mass up to $4 \mathrm{TeV}$ and 2-6 extra dimensions the 5 sigma significance can be obtained with luminosity between fraction of $\mathrm{pb}^{-1}$ and 100 's of $\mathrm{pb}^{-1}$. For effective Planck scale of $4 \mathrm{TeV}$ a few $\mathrm{fb}^{-1}$ is needed for discovery. To account for the systematic uncertainties in the number of signal events, the effect of PDF distribution on cross section is calculated using the CTEQ6 NLO PDF set with the help of LHAPDF interface. PDF uncertainties for the chosen benchmark point is found to be ${ }_{-9.07 \%}^{+24.2 \%}$. Using these uncertainties, the error in significance calculation was computed to be $12 \%$. 
Table 14.11. Event selection and background rejection for signal events and major background processes.

\begin{tabular}{lllllll}
\hline Cut & Signal & $\mathrm{tt}+\mathrm{nJ}$ & $\mathrm{W}+\mathrm{nj}$ & $\mathrm{Z}+\mathrm{nJ}$ & QCD Dijet & WW+nJ \\
\hline Cross Section $(\mathrm{pb})$ & 18.85 & 371 & 896 & 781.84 & 33076.8 & 269.91 \\
Events $\left(10 \mathrm{fb}^{-1}\right)$ & 188500 & $3.71 \times 10^{6}$ & $8.96 \times 10^{6}$ & $7.82 \times 10^{6}$ & $3.31 \times 10^{8}$ & $2.70 \times 10^{6}$ \\
$M$ Inv $>2 \mathrm{TeV} / \mathrm{c}^{2}$ & 18.71 & 13.29 & 6.53 & 3.85 & 2634.94 & 20.53 \\
Tot. Multiplicity $>4$ & 17.72 & 13.25 & 6.43 & 3.84 & 2613.18 & 20.42 \\
Sphericity $>0.28$ & 9.27 & 1.60 & 0.23 & 0.10 & 53.74 & 0.07 \\
Final No. Events $\left(10 \mathrm{fb}^{-1}\right)$ & 92740 & 15990 & 2328 & 982 & 537391 & 740 \\
\hline
\end{tabular}

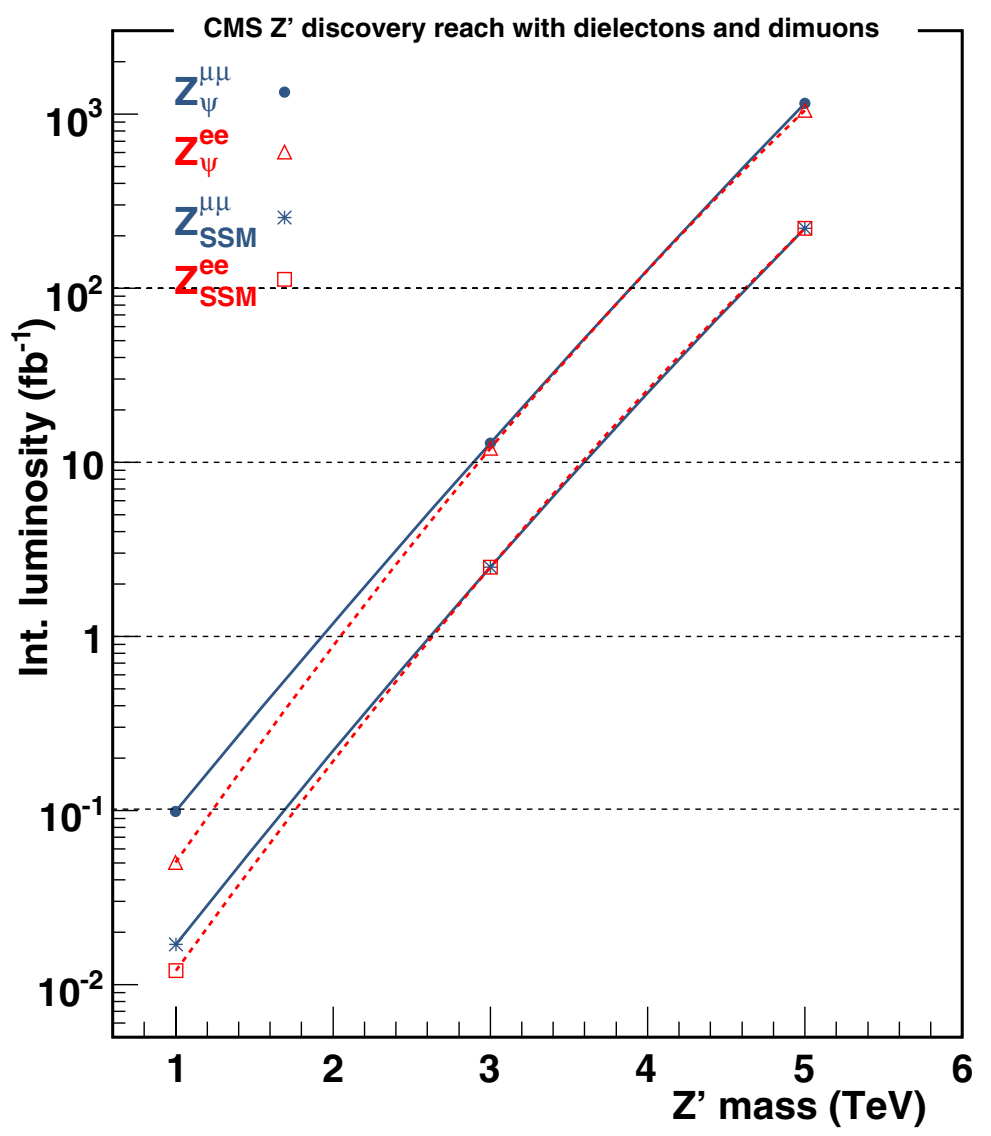

Figure 14.23. $Z^{\prime}$ discovery reach for two of the models studied in the dielectron and dimuon channels. The reach for the rest of the models studied is within the band between the two shown here.

\subsection{Discussion}

The results on Z's and RS gravitons in the channels studied in this chapter are summarised here.

In Fig. 14.23 the summary of the discovery reach in the dielectron and dimuon channels is shown for two representative $Z^{\prime}$ models. The reach for the rest of the models studied lies within the band of the two shown in the figure. The results for the dielectron channel are using 


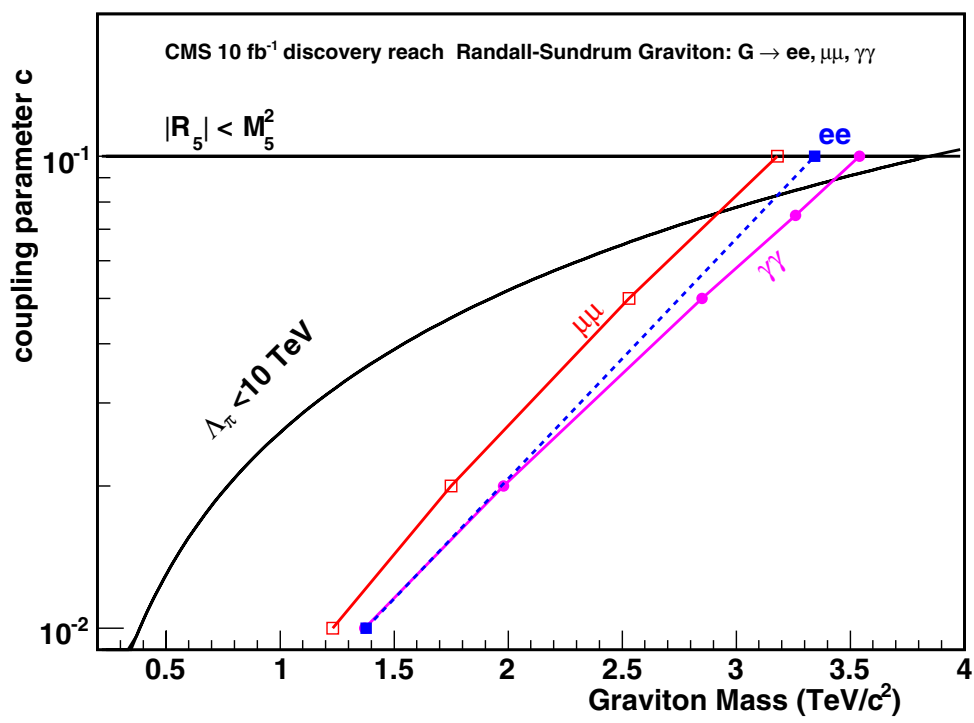

Figure 14.24. RS graviton discovery mass reach as a function of the model coupling parameter in the dielectron, dimuon and diphoton channels for $10 \mathrm{fb}^{-1}$. The dielectron reach is shown as dashed because only the boundary points $(\mathrm{c}=0.01$ and $\mathrm{c}=0.1)$ where studied.

here K-factor of 1.3 for the signal and background in order to be directly compared with the dimuon results ${ }^{50}$. Although the analysis strategies and significance computation is different between the two analyses the results are compatible. For low luminosity and mass reach up to $3 \mathrm{TeV} / \mathrm{c}^{2}$ the muons suffer from misalignment effects which are recovered after $10 \mathrm{fb}^{-1}$. For high mass reach (above $3 \mathrm{TeV} / \mathrm{c}^{2}$ ) the saturation in the ECAL is causing a degradation of the resolution in the dielectron channel. The reach using the dielectron channel is up to $3 \mathrm{TeV}$ better than the dimuons due to less than $1 \%$ resolution. Optimising the analysis in the dielectron channel to extract the background from the data and detailed studies of the saturation is expected to further improve the reach in the dielectron channel for high masses. The combined reach of the two channels requires a detailed analysis and is not presented here. Note that a $1 \mathrm{TeV} / \mathrm{c}^{2} \mathrm{Z}^{\prime}$ is observable with less than $0.1 \mathrm{fb}^{-1}$ for all models and with a single channel while every $\mathrm{TeV} / \mathrm{c}^{2}$ in mass reach corresponds to approximately an order of magnitude increase in integrated luminosity.

In Fig. 14.24 the summary of the RS graviton discovery reach in the dielectron, dimuon and diphoton channels is shown. Here the results for the diphoton channel are using CTEQ6M PDFs to be directly compared with the dielectron and dimuon channels. ${ }^{51}$ Although the branching ratio to photons is roughly twice that of electrons or muons the reach for low coupling and graviton mass is comparable between dielectrons and diphotons due to the QCD and prompt photon backgrounds in the photon channel which are harder to efficiently suppress. For higher masses and coupling the diphoton is leading the reach due to the higher branching ratio. The dimuon channel is trailing the reach compared to the dielectrons merely due to resolution.

\footnotetext{
${ }^{50}$ Recent calculation of K-factors for several of the processes discussed here can be found in reference [745].

${ }^{51}$ In the main analysis the diphoton channel uses CTEQ5L while the dielectron and dimuon analyses use CTEQ6M where the gluon-gluon contribution is enhanced compared to the CTEQ5L; while the Drell-Yan background is largely insensitive to this choice, at low masses the gluon-gluon is the dominant graviton production process while at high masses the $q q$ dominates where CTEQ5L and CTEQ6M are comparable.
} 


\section{Chapter 15. Alternative BSM Signatures}

\subsection{Technicolour}

\subsubsection{The $\rho_{\mathrm{TC}} \rightarrow W+Z$ channel}

Technicolour (TC) provides an alternative to the elementary Higgs mechanism of the Standard Model. It introduces a new strong interaction [746] providing a dynamical nature to Electroweak Symmetry Breaking. Technicolour is a QCD-like force, acting on technifermions at an energy scale $\Lambda_{\mathrm{TC}} \sim v_{\text {weak }}=246 \mathrm{GeV}$. A number $N_{D}$ of technifermion doublet condensates yield the pseudo-Goldstone bosons $\pi_{\mathrm{T} C}$, together with a wide spectroscopy of excited technimesons. The present simulation is performed using the phenomenology of the lowest-lying technihadrons, commonly referenced as the "Technicolour Straw Man" model (TCSM) [735]. The colour-singlet sector includes the spin-zero $\pi_{\mathrm{TC}}$ and the spin-one technimesons $\rho_{\mathrm{TC}}$ and $\omega_{\mathrm{T} C}$. The decay cross-section of the $\rho_{\mathrm{TC}}$ is expressed as an admixture of $\pi_{\mathrm{TC}}$ and the Standard Model $Z$ and $W$ bosons:

$$
\rho_{T C} \rightarrow \cos ^{2} \chi\left\langle\pi_{T C} \pi_{T C}\right\rangle+\cos \chi \sin \chi\left\langle\pi_{T C} V_{L}\right\rangle+\sin ^{2} \chi\left\langle V_{L} V_{L}\right\rangle
$$

where $V_{L}$ is the longitudinal mode of the $V=Z, W$ and $\sin \chi \simeq 1 / \sqrt{N_{D}} \sim 1 / 3$. The branching fraction $\operatorname{BR}\left(\rho_{\mathrm{TC}} \rightarrow W+Z\right)$ is competing with the two first terms in Eq. 15.1, hence changing with $\mathrm{M}\left(\pi_{\mathrm{T} C}\right)$.

The decay channel $\rho_{\mathrm{TC}} \rightarrow W+Z$ is the subject of this analysis [747] as it has the advantage of a very clean final state, namely $3 \ell+v$. The background contributions arise mainly from Standard Model processes involving weak boson production and decays. Other technicolour decay modes that include jets such as $\rho_{\mathrm{TC}} \rightarrow \pi_{\mathrm{TC}}+W$, have higher branching fractions but are much harder to disentangle from the Standard Model background processes.

15.1.1.1. Event selection. All signal and backgrounds samples used in this analysis are generated with PYтнIA 6.2 [24] with the requirement of at least 3 prompt leptons in the CMS fiducial region. The $Z b \bar{b}$ background is generated using CoMPHEP [355] interfaced to PYTHIA. Contributions from processes of type $Z \rightarrow 2 \ell$ plus an additional fake lepton from a jet have been taken into account in the systematic uncertainties, see Sect. 15.1.1.2. A set of 14 different $\rho_{\mathrm{T} C}$ samples are generated within the $\left[\mathrm{M}\left(\rho_{\mathrm{T} C}\right), \mathrm{M}\left(\pi_{\mathrm{T} C}\right)\right]$ phase space.

Nominal CMS Level-1 and High-Level Trigger requirements are applied [76]. The CMS fast simulation [11] is used for detector simulation and event reconstruction. The main reconstructed objects and their efficiencies have been validated against the detailed GEANT-based CMS detector simulation $[8,10]$.

The analysis is designed to reduce the main Standard Model background contributions $W Z, Z Z, Z b \bar{b}$ and $t \bar{t}$, while retaining high signal efficiency. It is summarised as follows:

(i) Lepton selection: 3 high- $p_{\mathrm{T}}$ and isolated electrons or muons.

(ii) Lepton trigger: single- or two-electron or muon mode (Level-1 and HLT).

(iii) $Z$ : same-flavour and opposite-charge $\ell$-pair closest to $\mathrm{M}(Z)$, with $p_{\mathrm{T}}\left(\ell_{1,2}\right)>$ $(30,10) \mathrm{GeV} / \mathrm{c}$.

(iv) $W$ : solution to 3rd lepton with $p_{\mathrm{T}}>10 \mathrm{GeV} / \mathrm{c}+$ Missing $E_{\mathrm{T}}+\mathrm{M}(W)$ constraint.

(v) $\left|\mathrm{M}\left(\ell^{+} \ell^{-}\right)-\mathrm{M}(Z)\right| \leqslant 3 \sigma_{\mathrm{M}_{Z}} \cong 7.8 \mathrm{GeV} / \mathrm{c}^{2}$.

(vi) $p_{\mathrm{T}}(Z)$ and $p_{\mathrm{T}}(W)>30 \mathrm{GeV} / \mathrm{c}$. For benchmark points with $\mathrm{M}\left(\rho_{\mathrm{TC}}\right)=200 \mathrm{GeV} / \mathrm{c}^{2}$, the minimum $p_{\mathrm{T}}(Z)$ and $p_{\mathrm{T}}(W)$ threshold is $10 \mathrm{GeV} / \mathrm{c}$.

(vii) $|\Delta[\eta(Z)-\eta(W)]| \leqslant 1.2$. 

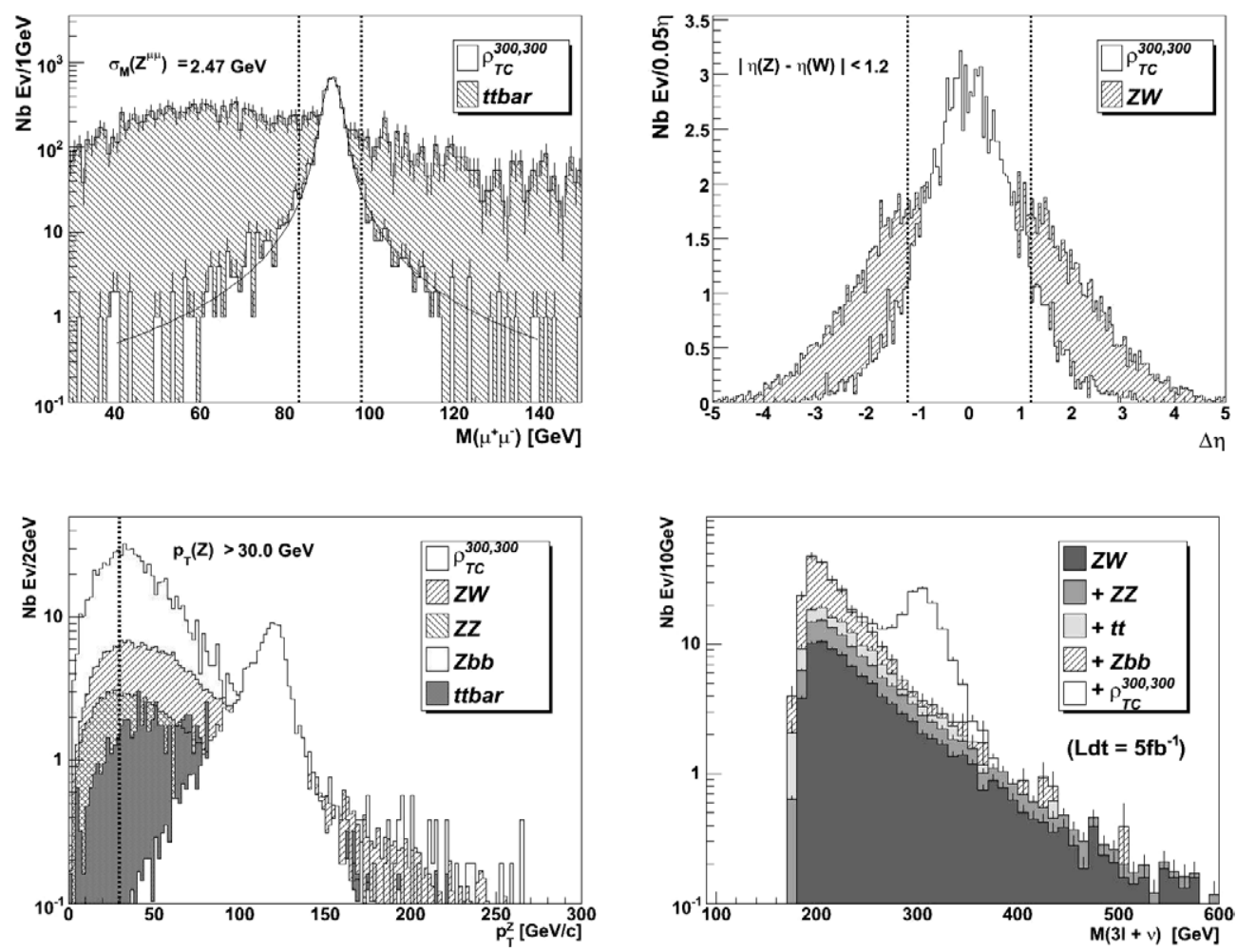

Figure 15.1. (a) $\mathrm{M}\left(\mu^{+} \mu^{-}\right)$for $\rho_{\mathrm{TC}}(300,300)$ and $t \bar{t}$; (b) $\Delta[\eta(\mathrm{Z})-\eta(\mathrm{W})]$ for $\rho_{\mathrm{T} C}(300,300)$ and $W Z$; (c) $p_{\mathrm{T}}(Z)$ for $\rho_{\mathrm{T} C}(300,300)$ and all backgrounds $\left(p_{\mathrm{T}}(W)\right.$ is similar); (d) Reconstructed $\mathrm{M}(3 \ell+v)$ for $\rho_{\mathrm{TC}}(300,300)$ and all backgrounds. The vertical lines indicate the applied requirements.

The $Z$ and $W$ are reconstructed with a purity of $\sim 99 \%$, using the 3 highest- $p_{\mathrm{T}}$ leptons in the event, and the Missing Transverse Energy (MET), obtained as the vector sum of the jets and leptons in the event. The $\mathrm{M}(W)$ constraint yields a 2 fold ambiguity in the $p_{Z}$ component of the reconstructed neutrino: it is found that the most efficient choice for the $\rho_{\mathrm{T} C}$ signal is the minimum $p_{\mathrm{Z}}$ solution. The kinematic cuts are illustrated in Fig. 15.1. The main $t \bar{t}$ reduction is obtained via the $Z$-mass window requirement $(\mathrm{v})$. The irreducible background $W Z \rightarrow 3 \ell+v$ is most efficiently separated from the signal via the $\eta(Z)-\eta(W)$ correlation requirement (vii).

The $p_{\mathrm{T}}$ cut on $Z$ and $W$ further improves the signal to background ratio, however it is kept modest in order to preserve the exponential background hypothesis of the $3 \ell+v$ invariant mass spectrum, used to compute the signal sensitivity. The $\rho_{\mathrm{TC}}(300,300)$ signal and background yields are shown in Fig. 15.1d and the corresponding reconstruction efficiencies are listed in Table 15.1.

15.1.1.2. Signal sensitivity and systematic uncertainties. The sensitivity of each $\rho_{\mathrm{T} C}$ benchmark point is computed by taking into account realistic statistical fluctuations for a given integrated luminosity. The sensitivity estimator is defined as the likelihood-ratio $S_{L}$, defined in Appendix A.1. The signal probability density function (p.d.f.) is assumed Gaussian (dominated by detector resolution) and the background p.d.f. is exponential in all $\rho_{\mathrm{TC}}$ fit regions. The output of the fitting procedure is shown in the contour plot over the 
Table 15.1. $\sigma \times \operatorname{BR}(\ell=e$ or $\mu)$, 3-lepton pre-selection efficiency, total efficiency and final yield within $3 \sigma$ of the signal region $(\mathrm{Nev})$, for $\mathcal{L}=5 \mathrm{fb}^{-1} . \rho_{\mathrm{TC}}(300,300)$ and the main background contributions are shown. The simulation is repeated for all $\rho_{\mathrm{TC}}$ benchmark points.

\begin{tabular}{llccc}
\hline Sample & $\sigma \times \mathrm{BR}(\mathrm{pb})$ & $\varepsilon$ (3-lept) & $\varepsilon(\operatorname{Reco})(\%)$ & $\mathrm{Nev}\left(5 \mathrm{fb}^{-1}\right)$ \\
\hline$\rho_{\mathrm{TC}} \rightarrow W+Z \rightarrow 3 \ell+v$ & 0.13 & 0.635 & $25.88 \pm 0.40$ & 103 \\
$W Z \rightarrow 3 \ell+v$ & 0.39 & 0.471 & $9.91 \pm 0.11$ & 27 \\
$Z Z \rightarrow 4 \ell$ & 0.07 & 0.719 & $15.80 \pm 0.14$ & 10 \\
$Z b \bar{b} \rightarrow 2 \ell+X$ & 332 & 0.046 & $0.23 \pm 0.01$ & 12 \\
$t \bar{t}$ & 489.72 & 0.065 & $0.019 \pm 0.001$ & 8 \\
\hline
\end{tabular}
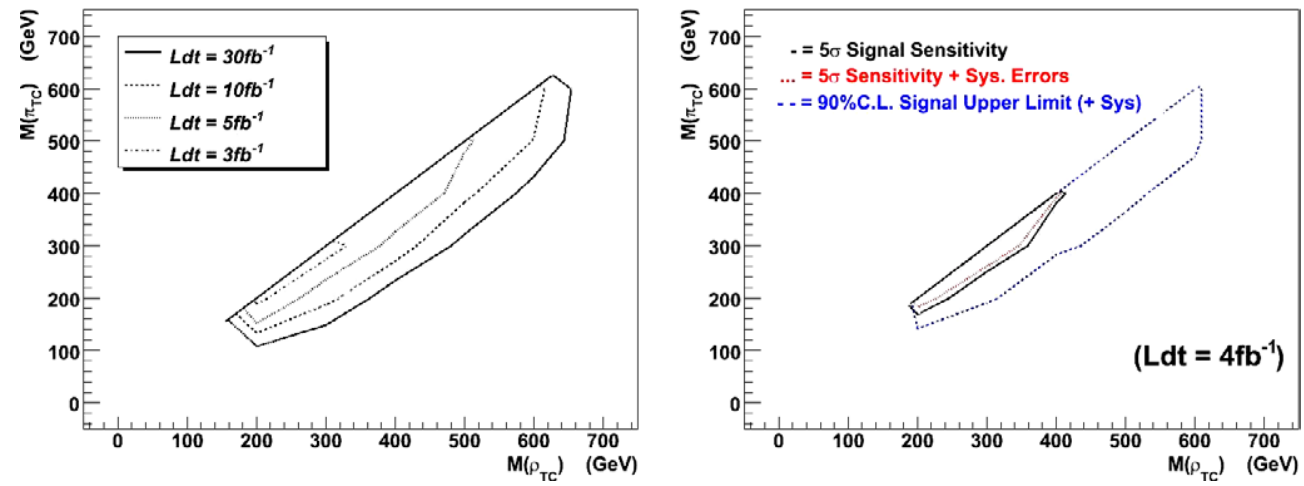

Figure 15.2. Left: Signal $5 \sigma$ Sensitivity curves for various integrated luminosities. Right: sensitivity for $\mathcal{L}=4 \mathrm{fb}^{-1}$ : the dotted (resp. dashed) curve shows the sensitivity (resp. the $90 \%$ C.L. signal upper limit) after including systematic uncertainties.

$\left[\mathrm{M}\left(\rho_{\mathrm{TC}}\right), \mathrm{M}\left(\pi_{\mathrm{T} C}\right)\right]$ phase space in Fig. 15.2 (left), for various integrated luminosities. A signal sensitivity above 5 is expected for $\mathcal{L}=3 \mathrm{fb}^{-1}$ (before including systematic uncertainties).

The $\rho_{\mathrm{TC}}$ sensitivity has been simulated for the early CMS data taking phase. Expected detector related systematic uncertainties for $\mathcal{L}=1 \mathrm{fb}^{-1}$ are taken into account. While no substantial contribution is found from the tracker and muon system misalignment or the calorimeter miscalibration, the accuracy at which the lepton efficiency will be determined from data affects the result: a $2 \%$ uncertainty is considered. Moreover, the lepton fake rate has been simulated on $Z b \bar{b}$ and extrapolated to any $Z+j e t(s)$ type background, in order to take into account additional contaminations from pion/kaon decays or from wrongly identified lepton candidates. A production cross-section of $1047 \mathrm{pb}$ per lepton flavour is assumed for $Z+n$-jets, $n \geqslant 0$. A single lepton fake rate of $\mathrm{O}\left(10^{-3}\right)$ is obtained using the fast simulation [11], affecting the $\rho_{\mathrm{TC}}$ sensitivity as shown below. Finally, a 7.5\% uncertainty on the missing transverse energy measurement is considered. The above uncertainties result in the following relative $\rho_{\mathrm{TC}}$ sensitivity drop:

$\Delta_{\mathrm{SYS}}^{\mathrm{tot}}=\sqrt{\left(\Delta_{\mathrm{SYS}}^{\mathrm{Eff}}\right)^{2}+\left(\Delta_{\mathrm{SYS}}^{\mathrm{Fake}}\right)^{2}+\left(\Delta_{\mathrm{SYS}}^{\mathrm{MET}}\right)^{2}}=\sqrt{(2.7 \%)^{2}+(8.5 \%)^{2}+(6.6 \%)^{2}}=11 \%$.

Introducing K-factors from next-to-leading-order (NLO) expectations for the signal (a K-factor 1.35 is assumed in similarity with the Drell-Yan process) and background leads to a relative signal sensitivity increase of $6 \%$; however the latter estimate has not been included in the final result. 
Table 15.2. Contact interaction models.

\begin{tabular}{|c|c|c|c|c|c|c|c|c|}
\hline \multirow[t]{2}{*}{ Model } & LL & RR & LR & RL & VV & AA & $L L+R R$ & $\mathrm{LR}+\mathrm{RL}$ \\
\hline & \multicolumn{5}{|c|}{ Non-parity conserving } & \multicolumn{3}{|c|}{ Parity conserving } \\
\hline$\eta_{\mathrm{LL}}$ & \pm 1 & 0 & 0 & 0 & \pm 1 & \pm 1 & \pm 1 & 0 \\
\hline$\eta_{\mathrm{RR}}$ & 0 & \pm 1 & 0 & 0 & \pm 1 & \pm 1 & \pm 1 & 0 \\
\hline$\eta_{\mathrm{LR}}$ & 0 & 0 & \pm 1 & 0 & \pm 1 & $\mp 1$ & 0 & \pm 1 \\
\hline$\eta_{\mathrm{RL}}$ & 0 & 0 & 0 & \pm 1 & \pm 1 & $\mp 1$ & 0 & \pm 1 \\
\hline
\end{tabular}

In summary, the technicolour signature $\rho_{\mathrm{T} C} \rightarrow W+Z$ in the context of the Straw Man model is studied. The 5 sigma discovery reach is obtained for an integrated luminosity $\mathcal{L} \simeq 4 \mathrm{fb}^{-1}$.

\subsection{Search for contact interactions with dimuons}

Contact interactions offer a general framework for describing a new interaction with typical energy scale $\Lambda \gg \sqrt{s}$. The presence of operators with canonical dimension $N>4$ in the Lagrangian gives rise to effects $\sim 1 / \Lambda^{N-4}$. Such interactions can occur for instance, if the SM particles are composite, or when new heavy particles are exchanged.

In the following we will consider lepton-pair production. The lowest order flavourdiagonal and helicity-conserving operators have dimension six [123].

The differential cross section takes the form

$$
\frac{d \sigma}{d \Omega}=S M(s, t)+\varepsilon \cdot C_{I n t}(s, t)+\varepsilon^{2} \cdot C_{N e w P h}(s, t)
$$

where the first term is the Standard Model contribution, the second comes from interference between the SM and the contact interaction, and the third is the pure contact interaction effect. The Mandelstam variables are denoted as $s, t$ and $u$.

Usually the coupling is fixed, and the structure of the interaction is parameterised by coefficients for the helicity amplitudes:

$$
\begin{array}{ll}
g & \text { coupling (by convention } \left.g^{2} / 4 \pi=1\right), \\
\left|\eta_{i j}\right| \leqslant 1 & \text { helicity amplitudes }(i, j=\mathrm{L}, \mathrm{R}), \\
\varepsilon & \frac{g^{2}}{4 \pi} \frac{\operatorname{sign}(\eta)}{\Lambda^{2}} \text { for } f \bar{f} .
\end{array}
$$

Some often investigated models are summarised in Table 15.2. The models in the second half of the table are parity conserving, and hence not constrained by the very precise measurements of atomic parity violation at low energies. The results presented in this contribution cover the LL model, which has the highest sensitivity at LHC energies from the models in the first half of the table. More details can be found in [349].

\subsubsection{Analysis}

The topology under study is high-mass muon pairs with opposite sign. More details on the analysis are found in [349]. The Global Muon Reconstructor (GMR, described in PTDR, Volume 1, Section 9.1.2) output is used. The dimuon events are triggered by the single and dimuon triggers. We have processed events, generated to cover the whole region of interest 
up to dimuon masses of $6 \mathrm{TeV} / \mathrm{c}^{2}$, through full simulation with OSCAR and reconstruction with ORCA. The dimuon mass resolution is parameterised in two ways:

- as mass dependent one standard deviation (RMS);

- by fitting the mass resolution with a sum of two Gaussians to account for the long tail of less well reconstructed masses.

The results are remarkably stable as a function of the dimuon mass: the second Gaussian contributes around $14 \%$ and has a standard deviation 3.3 times bigger than the first Gaussian.

Our strategy is to generate events with PYTHIA and apply parametrisations of the dimuon mass efficiency and resolution obtained from full simulation. We have verified our approach by comparing the resulting mass spectra with the ones obtained with OSCAR/ORCA or FAMOS for Drell-Yan and selected contact interactions samples, observing good agreement in all cases.

Two mass regions: $500-1000 \mathrm{GeV}$ and $1000-6000 \mathrm{GeV}$ are considered. The total cross section and the forward-backward asymmetry as function of the dimuon mass are studied. Our analysis shows that the sensitivity to contact interactions comes almost exclusively from the cross section measurements for the LL model.

In order to reduce the systematic uncertainties both on the experimental and theory sides a "double ratio" method is developed. The number of observed events for a given bin in invariant mass is

$$
N_{o b s}=L \cdot \sigma \cdot \varepsilon
$$

where $L$ is the luminosity, $\sigma$ the differential cross section for the given mass bin, and $\varepsilon$ the experimental efficiency. We select a zeroth "normalisation" bin for invariant masses between $250-500 \mathrm{GeV} / \mathrm{c}^{2}$, both well above the $\mathrm{Z}$ pole and in an area well covered by the Tevatron, and define the experimental ratios

$$
R_{i}^{D A T A}=\frac{N_{i}^{D}}{N_{0}^{D}}=\frac{\sigma_{i}^{D} \cdot \varepsilon_{i}^{D}}{\sigma_{0}^{D} \cdot \varepsilon_{0}^{D}} .
$$

Here the cross sections and efficiencies are the ones for the real LHC data. The index $i$ runs for all measured bins with masses above $500 \mathrm{GeV} / \mathrm{c}^{2}$. The luminosity cancels in the ratio. The choice of this mass bin is not random. If we compare the flavour composition of partons initiating the hard interaction (Table 15.3), at the $\mathrm{Z}$ peak 32.1\% are heavier flavours (not $\mathrm{u}$ or $\mathrm{d}$ quarks), with their own parton density functions (PDF) uncertainties. At $250-500 \mathrm{GeV} / \mathrm{c}^{2}$ the $u$ and d quarks are "initiators" already in $85.6 \%$ of the cases, increasing to $96.3 \%$ above $1 \mathrm{TeV} / \mathrm{c}^{2}$, etc. Moreover, at the $\mathrm{Z}$ peak $\mathrm{d}$ quarks are most abundant, while at higher masses u quarks dominate, asymptotically approaching a ratio 4:1. It is clear that our choice of normalisation bin gives flavour composition much closer to the most interesting high mass events, compared to a normalisation using $\mathrm{Z}$ pole events. The PDF uncertainty on cross sections is estimated using LHAPDF [95, 351]. It is interesting to note that this uncertainty reaches a minimum for masses $250-600 \mathrm{GeV} / \mathrm{c}^{2}$, corresponding to medium values of the parton momentum fractions $\mathrm{X}$, reinforcing our choice of normalisation bin.

We define similar ratios for the Monte Carlo (theory) predictions. The absolute values of the cross sections and efficiencies are not important for the ratios, what matters is the shape of these quantities as function of invariant mass. For example, the absolute value of K-factors, a way to compensate for missing higher order $\mathrm{N}(\mathrm{N}) \mathrm{LO}$ terms and enable the comparison of leading order Monte Carlo predictions to data (similarly for the electroweak radiative corrections) disappears from the ratios and only the shape of the K-function as depending on invariant mass remains - a much smaller effect. And part of the uncertainties introduced due to our limited knowledge of PDFs cancels in the ratio, leaving smaller residual uncertainties due to the change of phase space for changing masses. 
Table 15.3. Flavour composition of partons initiating the hard Drell-Yan interaction.The PDF uncertainty on the cross sections (positive and negative asymmetric errors) is estimated using LHAPDF.

\begin{tabular}{lccccccc}
\hline $\begin{array}{l}\text { Mass } \\
{\left[\mathrm{GeV} / \mathrm{c}^{2}\right]}\end{array}$ & $\begin{array}{c}\mathrm{d} \\
{[\%]}\end{array}$ & $\begin{array}{c}\mathrm{u} \\
{[\%]}\end{array}$ & $\begin{array}{c}\mathrm{s} \\
{[\%]}\end{array}$ & $\begin{array}{c}\mathrm{c} \\
{[\%]}\end{array}$ & $\begin{array}{c}\mathrm{b} \\
{[\%]}\end{array}$ & $\begin{array}{c}\text { PDF+ } \\
{[\%]}\end{array}$ & $\begin{array}{c}\text { PDF- } \\
{[\%]}\end{array}$ \\
\hline Z peak & 35.9 & 32.1 & 17.2 & 9.77 & 5.10 & +4.7 & -5.7 \\
$250-500$ & 24.3 & 61.3 & 6.22 & 6.64 & 1.54 & +3.4 & -4.2 \\
$500-600$ & 22.8 & 68.4 & 4.03 & 3.95 & 0.89 & +3.5 & -4.1 \\
$1000+$ & 21.7 & 74.6 & 1.86 & 1.48 & 0.33 & +5.0 & -5.8 \\
$2000+$ & 19.9 & 78.4 & 0.91 & 0.63 & 0.14 & +9.0 & -7.7 \\
\hline
\end{tabular}

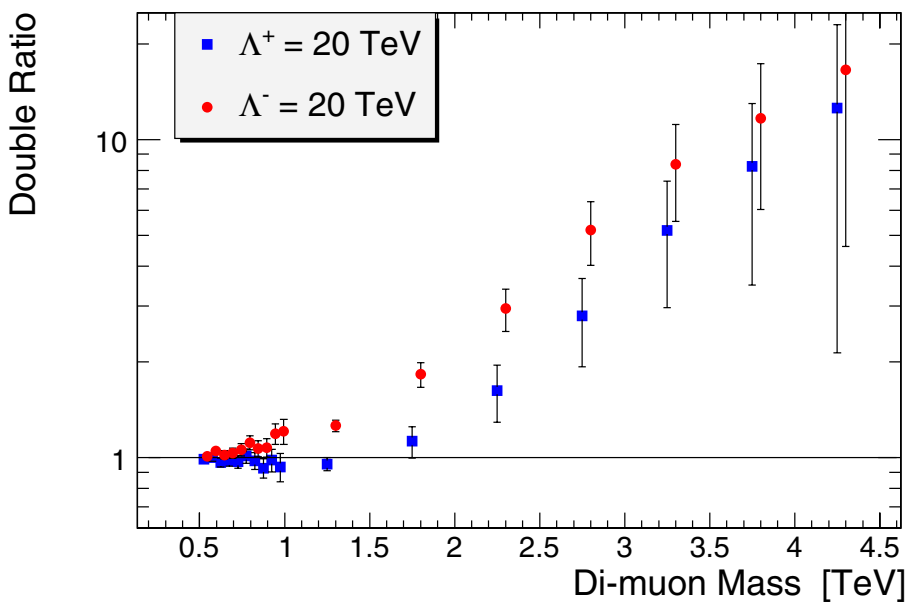

Figure 15.3. Double ratios for contact interactions in the dimuon channel, LL model, scale $\Lambda=20 \mathrm{TeV} / \mathrm{c}^{2}$, positive and negative interference, and luminosity $100 \mathrm{fb}^{-1}$. The errors shown are statistical.

Now let us define the double ratios

$$
D R_{i}=\frac{R_{i}^{D A T A}}{R_{i}^{M C}} .
$$

This method is inspired by a study of Drell-Yan events and extraction of proton and pion PDFs at lower masses [748], as well as by the SuperKamiokande double ratio method for measuring atmospheric neutrino oscillations [749]. If our theory understanding and detector modelling are both perfect, we expect $D R_{i} \equiv 1$. The experimental or Monte Carlo errors introduced in the ratios from the uncertainties in the zeroth bin are negligible, as due to the steeply falling Drell-Yan spectrum this bin has much more data compared to the high mass bins.

An example of double ratios for positive and negative interference is shown in Fig. 15.3. As can be seen, for scale $\Lambda=20 \mathrm{TeV} / \mathrm{c}^{2}$ the expected effects are quite sizable (note the $\log$ scale), with the sensitivity for negative interference starting around dimuon masses of $750 \mathrm{GeV} / \mathrm{c}^{2}$, while for positive interference masses above $2 \mathrm{TeV} / \mathrm{c}^{2}$ are required.

The experimental systematic effects in the cross section measurement are estimated to be $2 \%$ from the total muon efficiency and no more than $1.4 \%$ from momentum resolution. The former can be controlled quite well with the huge sample of $\mathrm{Z}$ events decaying to dimuons, and the effects for $\mathrm{TeV}$ muons are taken into account on top of this. The latter is important at high mass as smearing from lower masses from the steeply falling Drell-Yan spectrum can contaminate the high mass measurements, especially if the tails of the momentum resolution 
Table 15.4. The PDF uncertainty on the cross section ratios (positive and negative asymmetric errors) as estimated using LHAPDF. Clearly normalising to the $250-500 \mathrm{GeV} / \mathrm{c}^{2}$ mass bin is superior compared to a normalisation relative to the $\mathrm{Z}$ peak $\left(70-120 \mathrm{GeV} / \mathrm{c}^{2}\right)$.

\begin{tabular}{lccccc}
\hline & \multicolumn{2}{c}{$\mathrm{R}\left(\frac{M}{250-500}\right)$} & & \multicolumn{2}{c}{$\mathrm{R}\left(\frac{M}{\text { Zpeak }}\right)$} \\
\cline { 2 - 3 } Mass & PDF+ & PDF- & & PDF+ & PDF- \\
{$\left[\mathrm{GeV} / \mathrm{c}^{2}\right]$} & {$[\%]$} & {$[\%]$} & {$[\%]$} & {$[\%]$} \\
\hline $500-600$ & +1.5 & -1.5 & & +4.6 & -4.2 \\
$1000+$ & +5.2 & -4.8 & +7.8 & -7.1 \\
$2000+$ & +10.7 & -7.8 & +12.9 & -9.4 \\
\hline
\end{tabular}

are not under control. It is estimated by varying the two parametrisations of the mass resolution by $\pm 40 \%$, giving consistent results. The main source of systematic uncertainties on the momentum resolution comes from the alignment of the muon chambers and the central tracker, both at start-up and at high luminosity.

The systematic uncertainties from our limited knowledge of PDFs is estimated using the CTEQ6M PDF set from LHAPDF. From Table 15.4 our estimate of the PDF uncertainty on the cross section ratio is ${ }_{-4.8}^{+5.2} \%$ above $1 \mathrm{TeV}$ or ${ }_{-7.8}^{+10.7} \%$ above $2 \mathrm{TeV}$.

The genuine electro-weak radiative corrections change by $\sim 10 \%$ in the relevant mass range $[158,350]$. The $\mathrm{K}$-function changes faster below $250-300 \mathrm{GeV}$. From our normalisation bin to the highest masses first estimates show a change below $8 \%$ on the cross section ${ }^{52}$. Taking conservatively half of these changes with mass as an upper limit on the systematic uncertainty we arrive at $5 \%$ and $4 \%$ respectively.

Combining all effects in quadrature, we arrive conservatively at systematic uncertainties below $2.5 \%$ experimental, $11.5 \%$ from theory, $12 \%$ total at nominal conditions, $15 \%$ shortly after start-up. With the accumulation of data and improved calculations there is hope to improve this number by making progress in our understanding of PDF, electro-weak radiative corrections and K-functions.

The discovery reach for a given model is determined by constructing a negative log-likelihood function combining the deviations between measurements and predictions, including the contact interaction contributions, for all simulated data points. The error on a deviation consists of three parts, which are combined in quadrature: a statistical error, an experimental systematic error and a theoretical uncertainty. The log-likelihood function is integrated in the physically allowed region (all positive $\Lambda$ for positive interference and all negative $\Lambda$ for negative interference) to derive the five standard deviations $\sigma$ discovery reach and one-sided lower limits at $95 \%$ confidence level on the scale.

The discovery reach is summarised in Fig. 15.4. The sensitivity is dominated by the cross section measurement, the contribution of the forward-backward asymmetry is minor. The sensitivity for negative interference is substantially better. Even at the highest luminosities the statistical errors at LHC play a major role, as evident from the comparison of the cases with total systematic uncertainties of 3,15 and 30\%. This is not surprising as the Drell-Yan process is probing directly masses up to $\sim 4-5 \mathrm{TeV} / \mathrm{c}^{2}$, where due to the steeply falling cross sections the statistical errors remain important for all considered luminosities.

\footnotetext{
52 Calculations by M. Schmitt with the program PHOZPRMS [348].
} 
Contact Interactions LL $5 \sigma$ Discovery in CMS at LHC

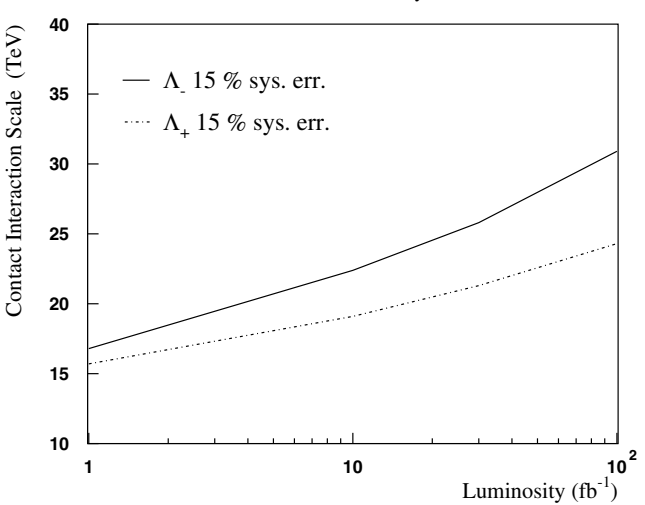

Contact Interactions LL $95 \%$ CL Exclusion in CMS at LHC

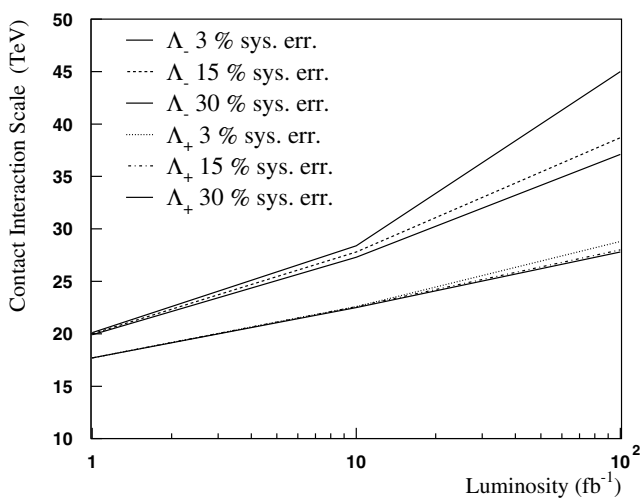

Figure 15.4. Five sigma discovery reach (left) and sensitivity at $95 \%$ CL (right) for contact interactions in the dimuon channel for different luminosities and signs of the interference.

\subsection{Search for contact interactions with dijets}

New physics at a scale $\Lambda$ above the mass of the final state is effectively modelled as a contact interaction. Here the propagator for a particle of mass $M \sim \Lambda$ exchanged between quarks, or exchanged between constituent particles inside two interacting composite quarks, shrinks to a single point and gives a contact interaction. Quark contact interactions, for example those that arise from a left-handed interaction among composite quarks [123, 124], will always produce a rise in rate relative to QCD at high dijet mass or high inclusive jet $E_{\mathrm{T}}$. However, observation in the mass distribution alone requires precise understanding of the QCD rate as a function of dijet mass, which is complicated by the large systematic uncertainties discussed in Section 4.1.6. Angular distributions benefit from much smaller systematic uncertainties. The contact interaction is often more isotropic than the QCD background, since QCD is dominated by t-channel scattering and produces jets predominantly in the forward direction. Our analysis uses the dijet ratio, discussed in section 4.1.5, to measure the angular distribution as a function of dijet mass, and see any contact interactions which affect the dijet angular distribution [750].

15.3.0.1. Contact interaction sensitivity estimates. The QCD background distribution for the dijet ratio was discussed in section 14.5. In Fig. 15.5 we show a smooth dijet ratio for QCD, estimated at 0.6 from the fit to the full simulation. The error bars shown in Fig. 15.5 are the statistical uncertainties expected with $1 \mathrm{fb}^{-1}$ and the jet trigger prescales discussed in section E.4.3.2. The uncertainties are calculated using Poisson statistics at high dijet mass, where few events are expected and Gaussian statistics is less accurate. In Fig. 4.7 we presented a lowest order calculation of both QCD and a contact interaction among left-handed quarks. The signal in Fig. 15.5 is estimated by scaling the lowest order contact interaction calculation of Fig. 4.7 by the ratio of our full simulation prediction for QCD to the lowest order QCD calculation: signal $=$ contact $\times 0.6 / Q C D$. Systematic uncertainties on the dijet ratio are small, as discussed in section 4.1.6 and demonstrated in Fig. 4.8. The calculated chisquared between QCD and the contact interaction signal, including all uncertainties on the dijet ratio, is listed in Table 15.5. In Fig. 15.5 we show the significance in $\sigma$, estimated as $\sqrt{\chi^{2}}$, compared to a smooth fit as a function of $1 / \Lambda^{+}$. The anticipated capability of CMS with $1 \mathrm{fb}^{-1}$ to exclude contact interactions at $95 \% \mathrm{CL}$ or discover them at $5 \sigma$ can be read off Fig. 15.5, and they are listed in Table 15.6. This includes the uncertainty on $\Lambda$ due to the anticipated 5\% uncertainty on the observed jet energy. The same analysis is repeated for $100 \mathrm{pb}^{-1}$ and $10 \mathrm{fb}^{-1}$ and the 

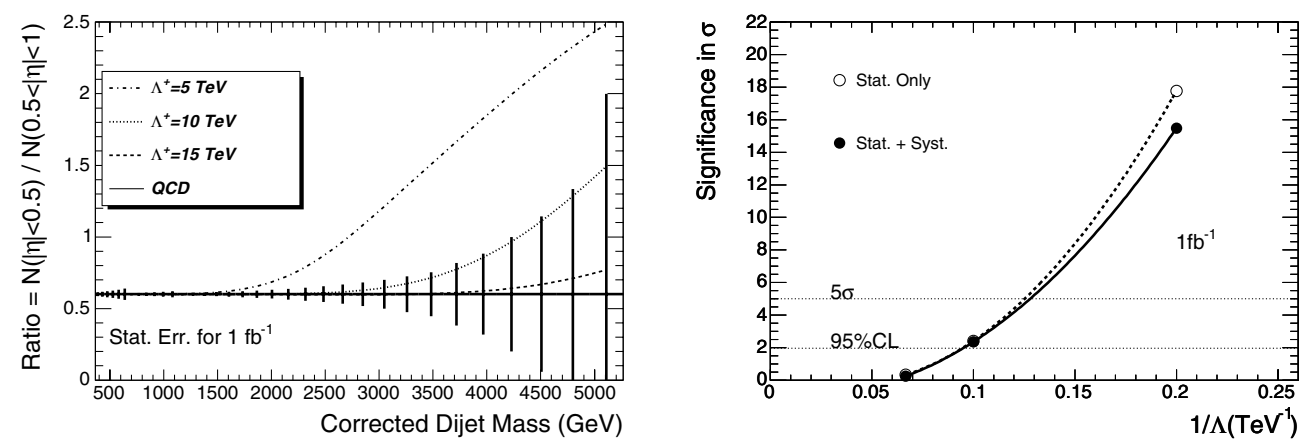

Figure 15.5. Left: The expected value and statistical error of the dijet ratio of QCD in the CMS detector for $1 \mathrm{fb}^{-1}$ (solid) is compared with QCD plus a quark contact interaction at a scale $\Lambda^{+}$of $15 \mathrm{TeV}$ (dashed), $10 \mathrm{TeV}$ (dotted) and $5 \mathrm{TeV}$ (dot-dashed). Right: The significance with statistical uncertainties only (open circles) and with all uncertainties (solid circles) of the difference between QCD alone and QCD plus a quark contact interaction is plotted vs $1 / \Lambda^{+}$and fit with a quadratic function. Horizontal lines show the $5 \sigma$ and $95 \%$ CL levels.

Table 15.5. Chisquared between signal and background. For each luminosity and contact interaction scale considered we list the chisquared between QCD alone and QCD plus a contact interaction, for the case where only statistical uncertainties are included (Stat), and for the case where both statistical and systematic uncertainties are included (All).

\begin{tabular}{llllllllll}
\hline Luminosity & \multicolumn{3}{l}{$100 \mathrm{pb}^{-1}$} & \multicolumn{3}{c}{$1 \mathrm{fb}^{-1}$} & \multicolumn{3}{c}{$10 \mathrm{fb}^{-1}$} \\
\hline$\Lambda^{+}(\mathrm{TeV})$ & 5 & 10 & 15 & 5 & 10 & 15 & 5 & 10 & 15 \\
$\chi^{2}$ (Stat) & 18.3 & 0.090 & 0.0037 & 316 & 5.82 & 0.107 & 3652 & 133 & 4.15 \\
$\chi^{2}$ (All) & 16.7 & 0.082 & 0.0011 & 240 & 5.55 & 0.061 & 1340 & 124 & 3.56 \\
\hline
\end{tabular}

Table 15.6. Sensitivity to contact interactions with $100 \mathrm{pb}^{-1}, 1 \mathrm{fb}^{-1}$, and $10 \mathrm{fb}^{-1}$. We list the largest value of the contact interaction scale we expect to be able to exclude at a confidence level of $95 \%$ or greater, and the largest value we expect to be able to discover with a significance of $5 \sigma$ or greater. Estimates include both statistical and systematic uncertainties.

\begin{tabular}{lllllllll}
\hline & \multicolumn{2}{c}{$95 \%$ CL Excluded Scale } & & \multicolumn{3}{c}{$5 \sigma$ Discovered Scale } \\
\cline { 2 - 4 } \cline { 5 - 6 } Luminosity & $100 \mathrm{pb}^{-1}$ & $1 \mathrm{fb}^{-1}$ & $10 \mathrm{fb}^{-1}$ & & $100 \mathrm{pb}^{-1}$ & $1 \mathrm{fb}^{-1}$ & $10 \mathrm{fb}^{-1}$ \\
\hline$\Lambda^{+}(\mathrm{TeV})$ & $<6.2$ & $<10.4$ & $<14.8$ & $<4.7$ & $<7.8$ & $<12.0$ \\
\hline
\end{tabular}

results are also listed in Table 15.6. The systematic uncertainties on the dijet ratio reduced the CMS sensitivity to a contact interaction between 0.1 and $0.3 \mathrm{TeV} / \mathrm{c}^{2}$ depending on luminosity and level of significance. To see how quickly CMS jet data will extend the search for new physics, we note that with $100 \mathrm{pb}^{-1}$ our anticipated 95\% CL sensitivity, $\Lambda^{+}<6.3 \mathrm{TeV}$, is more than twice the sensitivity of the $\mathrm{D} \varnothing \operatorname{search}\left(\Lambda^{+}<2.7 \mathrm{TeV}\right.$ at $\left.95 \% \mathrm{CL}\right)$ [122]. We note that our contact interaction sensitivity to composite quarks in Table 15.6 is roughly twice our mass resonance sensitivity to excited states of composite quarks in Table 14.5, and is equivalent to observing or excluding a quark radius of order $10^{-18} \mathrm{~cm}$.

\subsection{Heavy Majorana neutrinos and right-handed bosons}

\subsubsection{Introduction}

This study is exploring the left-right (LR) symmetric model $S U_{C}(3) \otimes S U_{L}(2) \otimes S U_{R}(2) \otimes$ $U(1)[724,725,751]$ at LHC. The model embeds the SM at the scale of the order of $1 \mathrm{TeV}$ 
and naturally explains the parity violation in weak interactions as a result of the spontaneously broken parity. It necessarily incorporates three additional gauge bosons $W_{R}$ and $Z^{\prime}$ and the heavy right-handed Majorana neutrino states $N$. The $N$ s can be the partners $\left(N_{l}\right)$ of the light neutrino states $v_{l}(l=e, \mu, \tau)$ and can provide their non-zero masses through the see-saw mechanism [726]. Given the results from the atmospheric, solar and reactor neutrino experiments the LR model is very attractive. In the framework of the LR symmetric model, we have studied the production and the experimental signature of heavy Majorana neutrinos and the associated heavy gauge bosons. The detailed analysis is presented in [752].

Existing experimental data constrain the $Z^{\prime}$ mass to the values $O(1) \mathrm{TeV} / \mathrm{c}^{2}$ [753]. The lower bound on the $W^{\prime}$ mass derived from the $K_{L}-K_{S}$ mass difference is quite stringent, $M_{W^{\prime}} \gtrsim 1.6 \mathrm{TeV}$ [754], however with some uncertainties from the low energy QCD corrections to the kaon system. The direct searches for $W^{\prime}$ at the Tevatron yield bounds $M_{W^{\prime}} \gtrsim 720 \mathrm{GeV} / \mathrm{c}^{2}$ assuming a light (keV-range) $N$, and $M_{W^{\prime}} \gtrsim 650 \mathrm{GeV} / \mathrm{c}^{2}$ assuming $M_{N}<M_{W^{\prime}} / 2$ [755]. These bounds are less stringent in more general LR models.

\subsubsection{Heavy Majorana neutrino production and decay}

The cross sections of $p p \rightarrow W_{R} \rightarrow l+N_{l}+X$ (the process studied here), and $p p \rightarrow Z^{\prime} \rightarrow$ $N_{l}+N_{l}+X$ (where $N_{l} \rightarrow l+j_{1}+j_{2}$ ) depend on the value of the coupling constant $g_{R}$, the parameters of the CKM mixing matrix for the right-handed sector, the $W_{R}-W_{L}$ and $Z^{\prime}-Z$ mixing strengths, and the masses of the partners $N_{l}$ of the light neutrino state. In the study presented here the mixing angles are assumed small, the right-handed CKM matrix is identical to the left-handed one and $g_{R}=g_{L}$. With these assumptions the $Z^{\prime}$ is about 1.7 times heavier than $W_{R}$ and the production cross-section for $p p \rightarrow W_{R} \rightarrow e N_{e}$ is found to be at least one order of magnitude higher than for the $p p \rightarrow Z^{\prime} \rightarrow N_{e} N_{e}$ process. Finally it is assumed that only the lightest $M_{N_{e}}$ is reachable at the LHC. In the case of degenerated masses of $N_{l}$, the channels with $\mu$ 's and $\tau$ 's are open resulting in the increase of the cross section of the process studied here by a factor of $\sim 1.2$. The analysis is performed in the $M_{W_{R}}, M_{N_{e}}$ parameter space. For the benchmark point considered (referred to as (LRRP)) $M_{N_{e}}=500 \mathrm{GeV} / \mathrm{c}^{2}$ and $M_{W_{R}}=2000 \mathrm{GeV} / \mathrm{c}^{2}$.

For the signal event generation and calculation of cross sections, the PYTHIA Monte Carlo program is used that includes the LR symmetric model with the standard assumptions mentioned above and CTEQ5L parton distribution functions. The fraction of $p p \rightarrow W_{R}^{+}$ $\left(p p \rightarrow W_{R}^{-}\right)$reactions as a function of $M_{W_{R}}$ changes from $\simeq 70 \%(\simeq 30 \%)$ at $M_{W_{R}} \simeq$ $1 \mathrm{TeV} / \mathrm{c}^{2}$ to $\simeq 95 \%(\simeq 5 \%)$ at $M_{W_{R}} \simeq 10 \mathrm{TeV} / \mathrm{c}^{2}$. For $W_{R}$ boson masses higher than $M_{W_{R}} \simeq$ $2 \mathrm{TeV} / \mathrm{c}^{2}$ the production of $W_{R}^{+}$boson dominates. The $W_{R}$ mass region above $1 \mathrm{TeV} / \mathrm{c}^{2}$ is studied since smaller masses are excluded by indirect analyses [756].

The signal and background data sample are simulated using the GEANT based CMS full detector simulation [8] and reconstruction package [10].

\subsubsection{Analysis}

The two major backgrounds considered in this study are the $Z+$ jets and $t \bar{t}$ production. In the event selection two isolated electrons and at least two jets are required. The dielectron invariant mass $M_{e e}$ is required to be above $200 \mathrm{GeV} / \mathrm{c}^{2}$ to suppress the $Z+$ jets Standard Model background. The invariant mass of each electron with the two leading jets $M_{e j j}$ ( $M_{N_{e}}^{\text {cand }}$ is formed. The $M_{e e j j}$ ( $W_{R}$ boson candidate) invariant mass is required to be above $1 \mathrm{TeV} / \mathrm{c}^{2}$. After this requirement the Standard Model background is suppressed as shown in Fig. 15.6.

The total $W_{R}$ mass the reconstruction efficiency for $M_{W_{R}}=2 \mathrm{TeV} / \mathrm{c}^{2}$ and for neutrino masses above $500 \mathrm{GeV} / \mathrm{c}^{2}$ is between $20 \%$ and $25 \%$ while for neutrino masses much smaller 

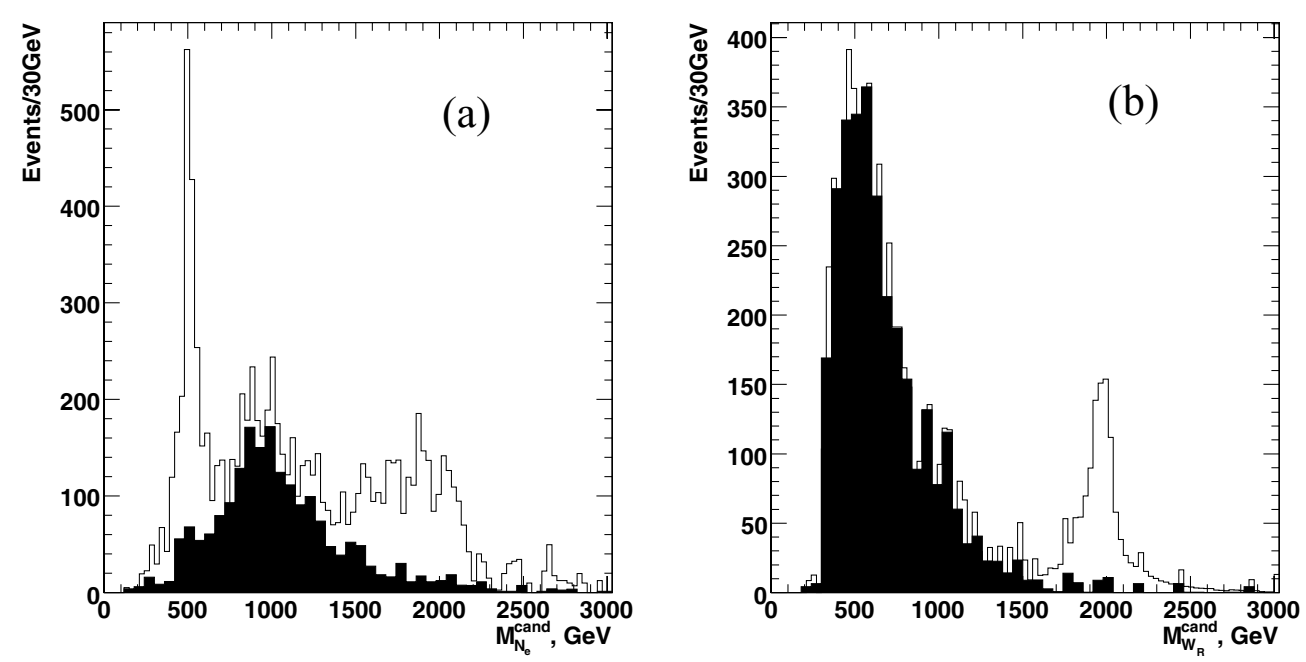

Figure 15.6. $M_{e j j}$ for the signal overlaid with the SM background (shaded histogram) for $30 \mathrm{fb}^{-1}$ : (a) $M_{e e j j}>1 \mathrm{TeV} / \mathrm{c}^{2}$, (b) $M_{e e j j}<1 \mathrm{TeV} / \mathrm{c}^{2}$.

than the $W_{R}$ mass the reconstruction efficiency drops due to the significant overlap of the heavy neutrino decay products in $\eta-\phi$.

\subsubsection{Results}

The 5 sigma discovery contour in the $\left(M_{W_{R}} ; M_{N_{e}}\right)$ plane is shown in Fig. 15.7 for 1 and $30 \mathrm{fb}^{-1}$. With $30 \mathrm{fb}^{-1}$ a 5 sigma observation of $W_{R}$ and $N_{e}$ with masses up to $4 \mathrm{TeV} / \mathrm{c}^{2}$ and $2.4 \mathrm{TeV} / \mathrm{c}^{2}$ respectively can be achieved. The signal at the LRRP test point $\left(W_{R}\right.$ of $2 \mathrm{TeV} / \mathrm{c}^{2}$ and $N_{e} 500 \mathrm{GeV} / \mathrm{c}^{2}$ ) is observable already after one month of running at low luminosity.

\subsection{Little Higgs models}

\subsubsection{Introduction}

The Little Higgs model [656] provides an alternative mechanism of electroweak symmetry breaking keeping a light Higgs boson free from one-loop divergences of SM. It breaks a global symmetry spontaneously and invokes a number of new particles of masses in TeV scale. A heavy singlet quark of charge $2 / 3$, marked as $T$, is the lightest among them and hence we study the viability of its observation with limited integrated luminosity.

The heavy quark $T$ acquires its mass via Yukawa interactions of two gauge groups with couplings $\lambda_{1}$ and $\lambda_{2}$ which are of similar order. $T$ has three dominant decay modes, the corresponding branching ratios following the relation: $B R(T \rightarrow t h)=B R(T \rightarrow t Z)=$ $\frac{1}{2} B R(T \rightarrow b W)$.

\subsubsection{Analysis}

The decay channel $\mathrm{T} \rightarrow \mathrm{tZ}$, with leptonic decays of $\mathrm{Z}$ and $\mathrm{W}$ bosons, provides a clean signature at the LHC environment. This channel has not been previously studied in CMS and the work presented here is a feasibility study. Further details can be found in [757].

The signal samples were generated with PYTHIA 6.227 [24] and the $T$ production was mimicked by activating the fourth quark generation through the $\mathrm{W}-\mathrm{b}$ fusion. The $T$ quark mass 


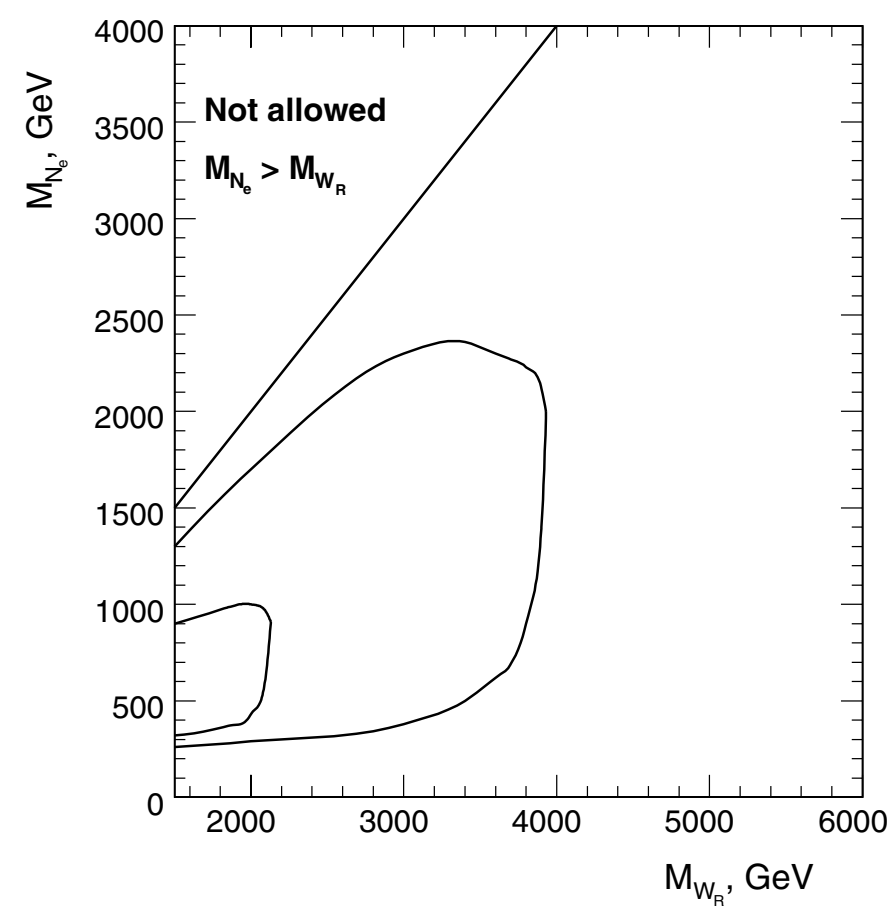

Figure 15.7. CMS discovery potential of the $W_{R}$ boson and right-handed Majorana neutrinos of the Left-Right Symmetric model for the integrated luminosity $L_{t}=30 \mathrm{fb}^{-1}$ (outer contour) and for $L_{t}=1 \mathrm{fb}^{-1}$ (inner contour).

was set to $1 \mathrm{TeV} / \mathrm{c}^{2}$ and was treated as a narrow resonance. The CMS full detector simulation was performed with OSCAR [8] and ORCA [10] while pile-up events corresponding to the low luminosity running period of the LHC were taken into account. The major backgrounds considered in this analysis were: $t \bar{t}, Z W+$ jets, $Z Z+$ jets, $W W+$ jets, $Z \mathrm{~b} \bar{b}$, and $Z+$ jets.

The main selection requirements are summarised below:

- Events are required to pass the "double electron" or "double muon" L1 and HLT trigger criteria.

- Electrons are required to have $p_{\mathrm{T}}>20 \mathrm{GeV} / \mathrm{c}$ and muons $p_{\mathrm{T}}>10 \mathrm{GeV} / \mathrm{c}$.

- The combined transverse momentum of the same flavour opposite sign lepton pair is required to be $p_{\mathrm{T}}^{\ell \ell}>100 \mathrm{GeV} / \mathrm{c}$. The invariant mass of the pair is required to be consistent with the nominal $\mathrm{Z}$ mass within $10 \mathrm{GeV} / \mathrm{c}^{2}$.

- A further third lepton is required in the event $\left(e^{ \pm}\right.$with $p_{\mathrm{T}}>20 \mathrm{GeV} / \mathrm{c}$ or $\mu^{ \pm}$with $\left.p_{\mathrm{T}}>15 \mathrm{GeV} / \mathrm{c}\right)$. The combined transverse momentum of the third lepton with the missing transverse energy is required to be greater than $60 \mathrm{GeV} / \mathrm{c}$. In addition the transverse mass of the third lepton with the missing transverse energy is required to be less than $120 \mathrm{GeV} / \mathrm{c}^{2}$, to be consistent with the $\mathrm{W}$ boson transverse mass.

- Exactly one jet compatible with a $b$-jet and with calibrated transverse momentum more than $30 \mathrm{GeV} / \mathrm{c}$ is required.

- The combined transverse momentum of the $\mathrm{W}$ boson and the $b$-jet should be more than $150 \mathrm{GeV} / \mathrm{c}$, while their invariant mass is required to be in the range (110-220) $\mathrm{GeV} / \mathrm{c}^{2}$.

- The combined $Z W b$ system invariant mass is required to be in the mass range of the search for heavy quark, namely (850-1150) $\mathrm{GeV} / \mathrm{c}^{2}$. 


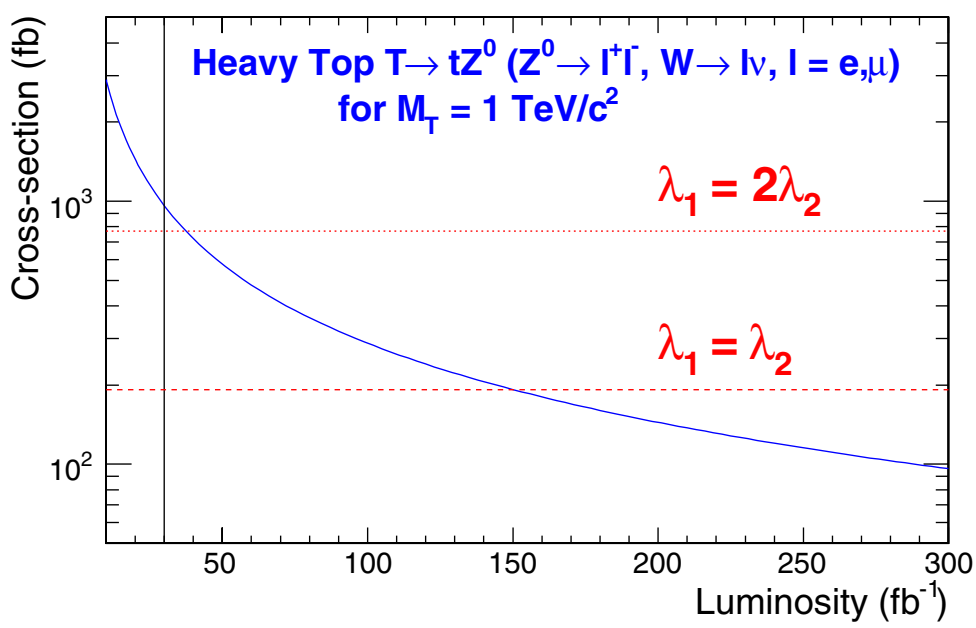

Figure 15.8. Minimum cross section required for a $5 \sigma$ discovery for a heavy quark of mass $M_{\mathrm{T}}=1 \mathrm{TeV} / \mathrm{c}^{2}$ as a function of the luminosity. The horizontal lines correspond to the cross section values for the two cases of $\lambda_{1} / \lambda_{2}$. The vertical line indicates the luminosity of $30 \mathrm{fb}^{-1}$ used for this analysis.

The $\mathrm{SM}$ background $\mathrm{ZZ} \rightarrow$ leptonic, is the only background that gives non-zero contribution (still less than 1 event at luminosity $30 \mathrm{fb}^{-1}$ ). The total efficiency for the signal selection is $(9.7 \pm 0.4) \%$. Assuming the production cross section of $\mathrm{T} \rightarrow \mathrm{tZ}$ to be $192 \mathrm{fb}$ for $M_{\mathrm{T}}=1 \mathrm{TeV} / \mathrm{c}^{2}$ (for the case of $\lambda_{1}=\lambda_{2}$ ) and folding in the branching ratios involved, a total of $N_{S}=2.1 \pm 0.1$ signal events are expected for $30 \mathrm{fb}^{-1}$. This implies that the discovery potential of the channel is rather limited.

The statistical significance of the channel $\left(S_{c 12}\right.$, defined in Appendix A.1) is 2.5 with a signal-to-background ratio of 41 for $30 \mathrm{fb}^{-1}$. Taking into account systematic uncertainties from the electron energy scale, jet and missing energy scale and $b$-tagging efficiency uncertainty, the significance drops down to 2.0. Figure 15.8 shows the signal cross section as a function of the integrated luminosity at the LHC, for establishing at $5 \sigma$ level, single production of a heavy quark of mass $=1 \mathrm{TeV} / \mathrm{c}^{2}$. The luminosity needed for $5 \sigma$ evidence is estimated to be around $150 \mathrm{fb}^{-1}\left(40 \mathrm{fb}^{-1}\right)$ for choices of parameters $\lambda_{1}=\lambda_{2}\left(\lambda_{1}=2 \lambda_{2}\right)$. The vertical line corresponds to the luminosity used for this analysis and demonstrates the inadequacy of statistics for a luminosity of $30 \mathrm{fb}^{-1}$.

\subsection{Same sign top}

At the LHC dileptonic $t \bar{t}(+j e t s)$ events can be selected with a relatively high signal-tonoise ratio and efficiency. Within the clean sample of such events, both leptons (electrons and muons) have an opposite electric charge. In several models beyond the Standard Model however, $t t / \overline{\mathrm{tt}}(+j e t s)$ topologies are predicted where both leptons have an equal electric charge. The signal excess is highly enhanced by the application of a combined likelihood variable described in [284]. The likelihood variable is designed to differentiate the lepton from the $W$ boson decay from leptons arising for example in QCD jets or from fake reconstructions. The signal of new physics can be diluted by the mis-identification of the electric charge of the leptons in Standard Model $t \bar{t}(+j e t s)$ events and the mis-identification of the leptons from the $W$ decay themselves. The observability of an excess of same-sign signals above 
Table 15.7. Overview of the selection criteria applied on the events using simulated events with pile-up collisions included. The expected number of events are rescaled to a dataset of $1 \mathrm{fb}^{-1}$ taking into account the respective Leading-Order cross-sections of the processes.

\begin{tabular}{|c|c|c|c|c|c|c|c|}
\hline & $\mu \mu$ & $\mu e$ and $e e$ & $t \bar{t} \rightarrow \tau+X$ & Other $t \bar{t}$ & $W^{ \pm} W^{\mp}$ & $Z+j e t s$ & $\mathrm{~S} / \mathrm{N}$ \\
\hline Before selection & 6915.0 & 20745.0 & 34606.2 & 485973.2 & 189951.7 & 578033.3 & 0.0078 \\
\hline Trigger & 6114.7 & 16314.8 & 17415.6 & 100137.2 & 41288.4 & 266366.7 & 0.017 \\
\hline Two jets $E_{\mathrm{T}}>25 \mathrm{GeV}$ & 4398.2 & 11982.7 & 13560.9 & 93858.2 & 20593.8 & 66146.7 & 0.032 \\
\hline b-tag criteria & 989.8 & 2485.4 & 2289.6 & 8784.7 & 133.5 & 240.0 & 0.13 \\
\hline Two leptons identified & 888.2 & 30.1 & 375.8 & 801.6 & 1.7 & 73.3 & 1.30 \\
\hline Two leptons selected & 481.5 & 0.07 & 48.4 & 3.01 & 0.4 & 53.3 & 4.7 \\
\hline Efficiency (in \%) & 6.96 & 0.0003 & 0.14 & 0.0006 & 0.00022 & 0.0092 & \\
\hline Opposite-sign & 481.3 & 0 & 48.3 & 2.19 & 0 & 53.3 & \\
\hline \multirow[t]{2}{*}{ Same-sign } & 0.2 & 0.07 & 0.1 & 0.82 & 0.4 & 0 & \\
\hline & $e e$ & $\mu e$ and $\mu \mu$ & $t \bar{t} \rightarrow \tau+X$ & Other $t \bar{t}$ & $W^{ \pm} W^{\mp}$ & $Z+j e t s$ & $\mathrm{~S} / \mathrm{N}$ \\
\hline Before selection & 6915.0 & 20745.0 & 34606.2 & 485973.2 & 189951.7 & 578033.3 & 0.0078 \\
\hline Trigger & 5354.8 & 17074.7 & 17415.6 & 100137.2 & 41288.4 & 266366.7 & 0.015 \\
\hline Two jets $E_{\mathrm{T}}>25 \mathrm{GeV}$ & 3960.9 & 12420.0 & 13560.9 & 93858.2 & 20593.8 & 66146.7 & 0.029 \\
\hline b-tag criteria & 802.7 & 2672.4 & 2289.6 & 8784.7 & 133.5 & 240.0 & 0.11 \\
\hline Two leptons identified & 724.5 & 34.6 & 453.8 & 2283.6 & 73.1 & 126.7 & 0.57 \\
\hline Two leptons selected & 285.0 & 0.3 & 37.5 & 5.2 & 0.8 & 53.3 & 3.1 \\
\hline Efficiency (in \%) & 4.12 & 0.0013 & 0.11 & 0.0011 & 0.00044 & 0.0092 & \\
\hline Opposite-sign & 279.6 & 0.3 & 36.8 & 4.1 & 0.4 & 46.7 & \\
\hline \multirow[t]{2}{*}{ Same-sign } & 5.4 & 0 & 0.7 & 1.1 & 0.4 & 6.7 & \\
\hline & $e \mu$ & $\mu \mu$ and $e e$ & $t \bar{t} \rightarrow \tau+X$ & Other $t \bar{t}$ & $W^{ \pm} W^{\mp}$ & $Z+j e t s$ & $\mathrm{~S} / \mathrm{N}$ \\
\hline Before selection & 13830.0 & 13830.0 & 34606.2 & 485973.2 & 189951.73 & 578033.3 & 0.016 \\
\hline Trigger & 10960.0 & 11469.5 & 17415.6 & 100137.2 & 41288.4 & 266366.7 & 0.030 \\
\hline Two jets $E_{\mathrm{T}}>25 \mathrm{GeV}$ & 8021.8 & 8359.1 & 13560.9 & 93858.2 & 20593.8 & 66146.7 & 0.061 \\
\hline b-tag criteria & 1682.7 & 1792.5 & 2289.6 & 8784.7 & 133.5 & 240.0 & 0.25 \\
\hline Two leptons identified & 1500.6 & 66.4 & 822.1 & 3001.6 & 30.2 & 20.0 & 0.88 \\
\hline Two leptons selected & 722.7 & 0.9 & 85.2 & 6.3 & 0.4 & 0 & 8.3 \\
\hline Efficiency (in \%) & 5.23 & 0.0065 & 0.25 & 0.0013 & 0.00022 & 0 & \\
\hline Opposite-sign & 715.5 & 0.9 & 83.8 & 4.9 & 0 & 0 & \\
\hline Same-sign & 7.2 & 0 & 1.3 & 1.4 & 0.4 & 0 & \\
\hline
\end{tabular}

the mis-reconstruction of the Standard Model background is determined. The details of the analysis are mentioned in [758].

The jets in the final state are reconstructed with an Iterative Cone jet clustering algorithm using a cone size of $\Delta R=0.5$. Input objects for the cones are selected from all calorimeter towers above a pseudo-rapidity dependent energy threshold determined from the average underlying event energy deposits [165]. The energy scale of the reconstructed jets is calibrated with corrections from Monte Carlo studies. The primary vertices in the proton bunch crossing are determined, and the vertex with the highest transverse momentum is taken as the one of the hard scattering. Via a track-based algorithm, jets are rejected if they do not match with this hard primary vertex.

The leptons are reconstructed and identified using the methods described in [284]. A likelihood variable is used to suppress leptons from the heavy flavour quark background exploiting several reconstruction aspects of leptons in the CMS detector. This likelihood is determined for each muon or electron in the final state in order to enhance the purity of choosing the correct lepton from the leptonic $W$ decay. The combined likelihood 


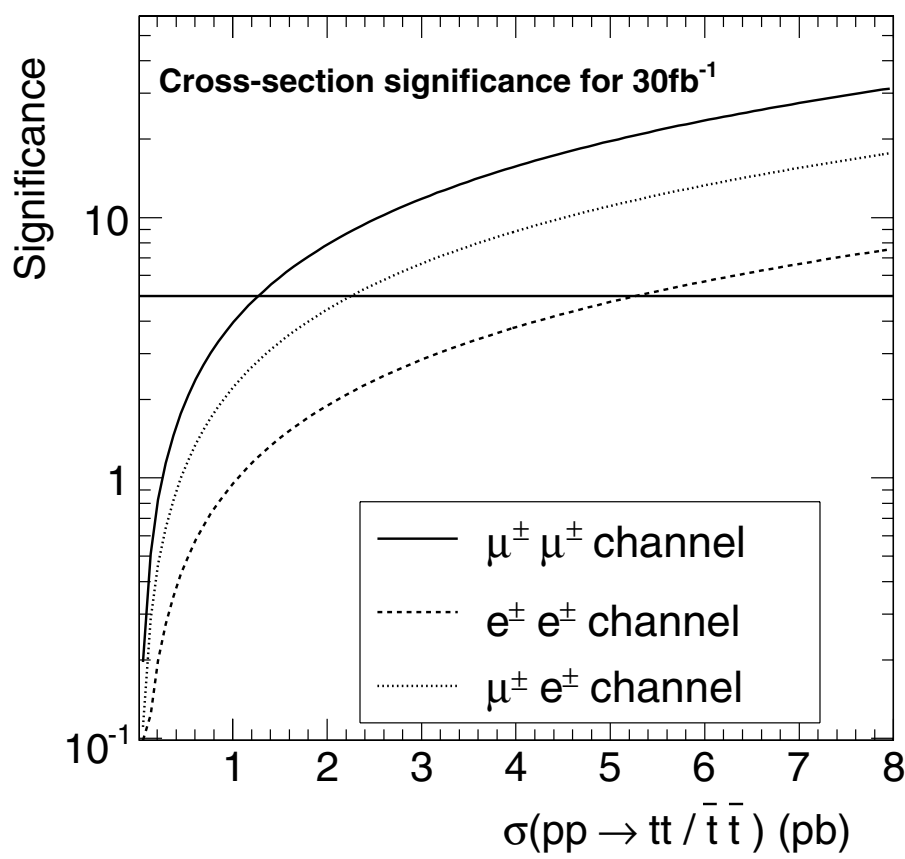

Figure 15.9. For an integrated luminosity of $30 \mathrm{fb}^{-1}$ the significance of the same-sign $t t$ or $\overline{t t}$ excess above the Standard Model events is indicated as a function of the cross-section of the inclusive process $p p \rightarrow t t / \overline{t t}$.

includes observables as tracker isolation, calorimeter isolation, vertex matching significance, transverse momentum of the lepton and angular distance to the closest jet. For the electron likelihood a variable reflecting the reconstruction quality is added. The two muons or electrons having the largest combined likelihood ratio value are taken as the hard leptons of interest.

The inclusive single-muon, single-electron, double muon and double electron triggers are applied as described in [506]. The event should be triggered in at least one of these streams. In total $88.4 \%, 77.4 \%$ and $79.2 \%$ of respectively the $\mu \mu$, the $e e$ and the $\mu e$ signal events remain after applying the trigger criteria. The event is required to have at least 2 jets with a calibrated $E_{\mathrm{T}}$ above $25 \mathrm{GeV}$. These jets need to have a pseudo-rapidity in the range $|\eta|<2.4$ and a b-tag discriminant larger than 0.5 [157]. The reconstructed hard leptons are required to have transverse momentum $p_{\mathrm{T}}$ exceeding $25 \mathrm{GeV} / \mathrm{c}$ in the pseudo-rapidity range of $|\eta|<2.4$ and a combined likelihood variable larger than 0.05 .

In Table 15.7 the efficiencies and signal-to-noise ratios are shown after each selection step. Applying all cuts a signal-to-noise ratio of 4.7, 3.1 and 8.3 is obtained for respectively the $\mu \mu$, the $e e$ and the $e \mu$ final state. Cross-talk between these three considered final states is by construction not possible. As the amount of selected $W W$ and $Z+j e t s$ events in Table 15.7 is small, their contribution is alternatively estimated by multiplying the efficiencies of the event selection without the $b$-tagging and the individual $b$-tagging selection cut efficiency under the assumption that both selection cuts are uncorrelated.

It is illustrated [758] that from the selected topology of dilepton $t \bar{t}$ events, a ratio $R=\frac{N_{++,--}}{N_{+-}}$can be determined which is sensitive to physics beyond the Standard Model. In the ratio the total amount of events with equally charged leptons is divided by the total amount of events with opposite charged leptons. As the efficiency of reconstructing the leptons electric charge is very high, we can neglect the amount of selected $p p \rightarrow t t$ or $p p \rightarrow \overline{t t}$ 
events observed with two opposite-charged leptons. Using the uncertainty on the ratio $R$, the significance of the observation of new physics channels $p p \rightarrow t t$ or $p p \rightarrow \overline{t t}$ is determined as a function of the cross section (see Fig. 15.9). The dimuon channel has a larger sensitivity compared to the decay channels with electrons. This is caused by the electron reconstruction where a large fraction of electron energy clusters are matched with a wrong track resulting in a charge ambiguity.

It is assumed that the new physics processes beyond the Standard Model have a similar kinematic topology compared to the $t \bar{t}$ process, therefore the selection efficiency of the new physics channels is taken equal to that of the Standard Model $t \bar{t}$ process. Several models predict an excess of events with same-sign leptons in this topology, via the process $p p \rightarrow t t / \overline{t t}$ or $p p \rightarrow t t / \overline{t t}+b / c$. These models are motivated by Flavour Changing Neutral Currents (FCNC) [759, 760], topcolour-assisted Technicolour (TC2) [761] or supersymmetry [762]. With a measurement of $R$ these kinematically similar processes $p p \rightarrow t t / \overline{t t}$ can be observed with $30 \mathrm{fb}^{-1}$ of integrated luminosity if they have a cross section above $1 \mathrm{pb}$. Because a ratio of kinematically similar event topologies is measured, most of the experimental and theoretical systematic uncertainties cancel. The uncertainty of the background cross sections on the significances shown in Fig. 15.9 is found to be negligible. A feasibility study is performed to estimate the potential uncertainty on the mis-identification efficiency of the electric charge of electrons and muons from $Z$ boson decays [758]. The effect on the significance of the excess of $t t / \overline{t t}$ events is found to be negligible. 


\section{Appendix A. 95\% CL limits and $5 \sigma$ discoveries}

\section{A.1. Estimators of significance}

Several methods exist to quantify the statistical "significance" of an expected signal at future experiments. Following the conventions in high energy physics, the term significance usually means the "number of standard deviations" an observed signal is above expected background fluctuations. It is understood implicitly that $S$ should follow a Gaussian distribution with a standard deviation of one. In statistics, the determination of the sensitivity is a typical problem of hypothesis testing, aiming at the discrimination between a null-hypothesis $H_{0}$ stating that only background and no signal is present, and a alternative hypothesis $H_{1}$, which states the presence of a signal on top of the background. The "significance level" is the probability to find a value of a suitably constructed test statistic beyond a certain pre-specified critical value, beyond which the validity of $H_{1}$ is assumed. The significance level has to be converted into an equivalent number of Gaussian sigmas to arrive at the common terminology of a high-energy physicist.

Since a signal is usually searched for in many bins of a distribution, and in many channels, a very high value of the significance of a local excess of events must be demanded before an observed "peak" found somewhere in some distribution can be claimed to be an observation of a signal. If the position of the signal peak is not known a-priori and treated as a free parameter in searches for new physics, the probability of background fluctuations is much higher. This is quantified in a case study in Section A. 2 below, and this aspect will need careful consideration in the near future before first data taking at the LHC. The general, somewhat arbitrary convention is that the value of $S$ of a local signal excess should exceed five, meaning that the significance level, or the corresponding one-sided Gaussian probability that a local fluctuation of the background mimics a signal, is $2.9 \times 10^{-7}$.

Here, the recommendations for the procedures to be used for the studies presented in this document are summarised. The aim of many of these studies is the prediction of the average expected sensitivity to the observation of a new signal in a future experiment. The real experiment might be lucky, i.e. observe a higher significance than the average expectation, or a downward fluctuation of the expected signal could lead to a lower observed significance. The proposed methods have been checked in a large number of pseudo-experiments using Monte Carlo simulation in order to investigate whether the probability of a background fluctuation having produced the claimed significance of the discovery is properly described.

Counting methods use the number of signal events, $s$, and the number of background events, $b$, observed in some signal region to define the significance $S$. These event numbers can be turned into a significance, $S_{C P}$, by using either the Poisson distribution for small numbers of events, or, in the high-statistics limit, the Gaussian distribution, leading to

$$
S_{c 1}=\frac{s}{\sqrt{b}}
$$

The significance may also be obtained from the ratio of the likelihoods, $\mathcal{L}_{1}$ and $\mathcal{L}_{0}$, belonging to the hypothesis $H_{0}$ and $H_{1}$,

$$
S_{L}=\sqrt{2 \ln Q}, \text { with } Q=\frac{\mathcal{L}_{0}}{\mathcal{L}_{1}}
$$


This approach is theoretically well founded and is applicable also to the simple approach of the counting method, leading to

$$
S_{c L}=\sqrt{2\left((s+b) \ln \left(1+\frac{s}{b}\right)-s\right)},
$$

which follows directly from the Poisson distribution. In the Gaussian limit of large numbers $s$ and $b, S_{c L}$ becomes equivalent to $S_{c 1}$. The likelihood approach can be extended to include the full shapes of the signal and background distributions for the hypothesis $H_{0}$ and $H_{1}$, and the likelihood may be obtained from binned or unbinned likelihood fits of the backgroundonly and the background-plus-signal hypotheses to the observed distributions of events.

Another estimator,

$$
S_{c 12}=2(\sqrt{s+b}-\sqrt{b}),
$$

has been suggested in the literature [79, 763]. The formula for $S_{c 12}$ is strictly only valid in the Gaussian limit, but tabulated values exist for small statistics.

The presence of systematic errors deserves some special care. Two cases must be separated clearly:

(a) If the background and signal contributions can be determined from the data, e.g. by extrapolating the background level into the signal region from sidebands, systematic errors may be irrelevant, and the systematic errors only influence our ability to predict the average expected sensitivity. In this case, simple propagation of the theoretical errors on $s$ and $b$ applied to the above formulae for the various significances is all that is needed.

(b) If systematic errors on the background will affect the determination of the signal in the real experiment, e.g. because an absolute prediction of the background level or a prediction of the background shape are needed, the theoretical uncertainty must be taken into account when estimating the sensitivity. This can be done by numerical convolution of the Poisson distribution, or the Gaussian distribution in the high-statistics limit, with the probability density function of the theoretical uncertainty. Numerical convolutions of the Poisson distribution with a theoretical error of a Gaussian shape, leading to a variant of $S_{c} P$ including systematic errors, were used for this document [679]. Numerical convolutions of the Poisson distribution with a systematic error of a Gaussian shape, leading to a variant of $S_{c P}$ including systematic errors, were used for this document. The program ScPf [679] computes the significance by Monte Carlo integration with the assumption of an additional Gaussian uncertainty $\Delta b$ on $b$. The significance can be approximated by an extension of $S_{c 12}$ :

$$
S_{c 12 s}=2(\sqrt{s+b}-\sqrt{b}) \frac{b}{b+\Delta b^{2}} .
$$

In the Gaussian limit it leads to

$$
S_{c 1}=s / \sqrt{b+\Delta b^{2}} \text {. }
$$

The most crucial point in this context is a realistic description of the probability density function of the systematic theoretical uncertainty, which can be anything ranging from a flat distribution between $b \pm \Delta b$ to a pathological distribution with a significant non-Gaussian tail, but, in practice, is hardly ever known precisely.

The distribution of a significance estimator $S$ in a series of experiments, its probability density function (p.d.f.), is of prime importance for the calculation of discovery probabilities in the presence of a real signal, or of fake probabilities due to fluctuations of the background. In the large-statistics limit, the likelihood-based significance estimators are expected to follow a $\chi^{2}$-distribution with a number of degrees of freedom given by the difference in the number of free parameters between the alternative hypothesis and the null hypothesis [103]. When 


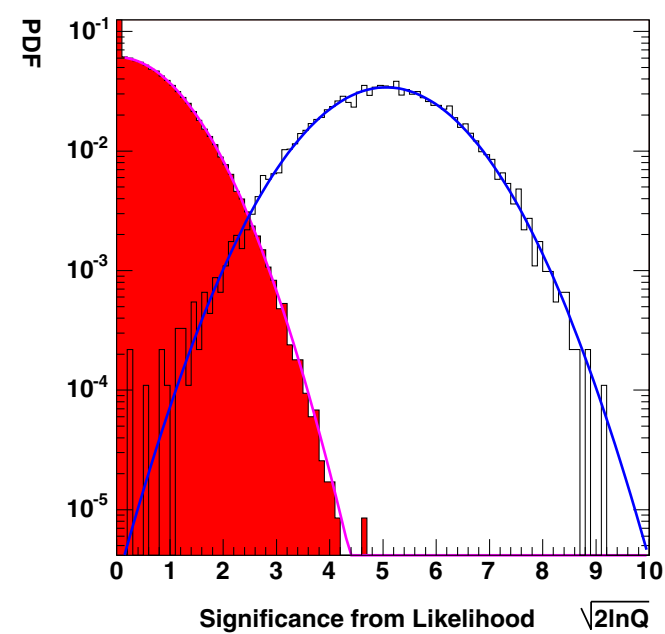

Figure A.1. Probability density functions of the estimator of significance $S_{L}$ for small statistics (11 signal events over a background of 1.5 events). Filled histogram: pure background sample from 200000 toy experiments, open histogram: background plus signal from 10000 toy experiments. Gaussian fits are overlayed; the distribution of $S_{L}$ for the background-only sample has a mean of -0.004 and a width of $\sigma=1.0$, the background-plus-signal sample has a width of 1.1 .

testing for the presence of a signal on top of background at a fixed peak position, $2 \ln Q=S_{L}^{2}$ is expected to follow a $\chi^{2}$ distribution with one degree of freedom, i.e. a standard Gaussian distribution. All of the above estimators have been tested in a large number of toy experiments, see e.g. [60, 100, 102]. In particular the likelihood based estimators were found to be wellbehaved, i.e. the distribution of the values of significance followed the expected behaviour already at moderate statistics, as is shown for one example in Fig. A.1. Good scaling with the square root of the integrated luminosity was also observed in these studies. On the other hand, the estimator $S_{c 1}$ cannot be considered a useful measure of significance at low statistics.

A quantitative comparison as a function of the number of background events for fixed values of $s / \sqrt{b}$ of the various estimators discussed above is shown in Fig. A.2. $S_{c L}$ and $S_{c P}$ are found to agree very well, while $S_{c 12}$ tends to slightly underestimate the significance, a result which was also verified in the above Monte Carlo studies with large samples of toy experiments. While $S_{C L}$ and $S_{C P}$ remain valid independent of the value of $b$, the simpler estimator $S_{c 1}$ can only be used for background levels larger than 50 events.

\section{A.2. On the true significance of a local excess of events}

In searching for new phenomena in a wide range of possible signal hypotheses (e.g. a narrow resonance of unknown mass over a broad range background), a special care must be exercised in evaluating the true significance of observing a local excess of events. In the past, this fact was given substantial scrutiny by statisticians (e.g. [764, 765]) and physicists (e.g., [766-770]) alike. The purpose of this Appendix is to quantify a possible scope of this effect on an example of a search for the Standard Model Higgs boson in the $H \rightarrow Z Z^{(*)} \rightarrow 4 \mu$ decay channel. As the case study, we chose a counting experiment approach widely used in this volume. 


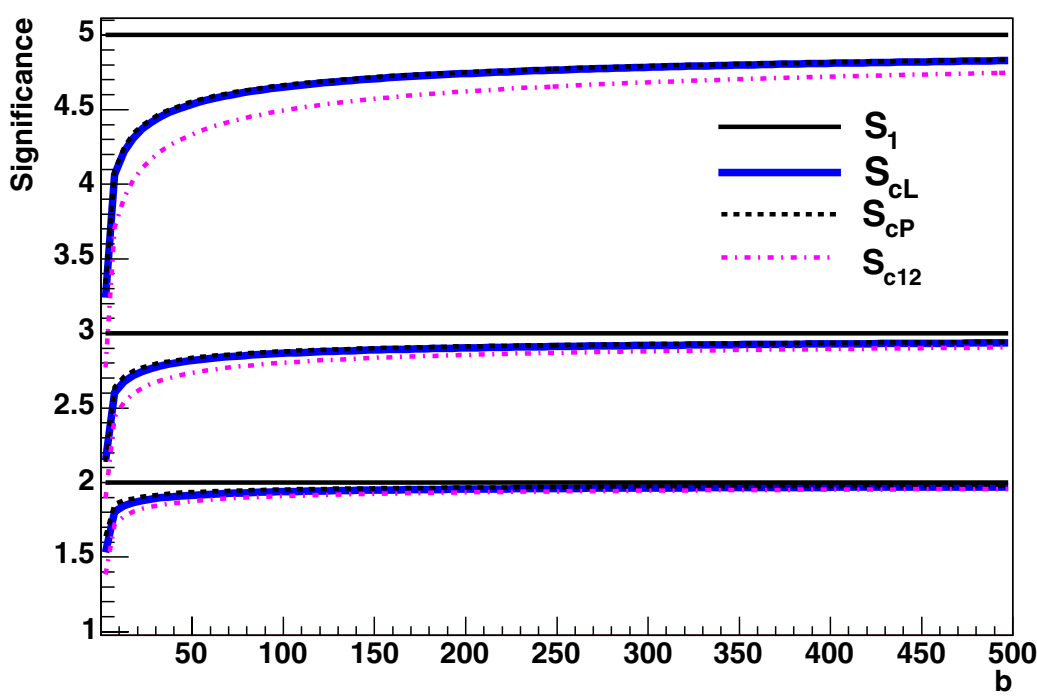

Figure A.2. Comparison of the various significance estimators as a function of the number of background events, $b$. The number of signal events was taken as $s=S_{c 1} \sqrt{b}$, hence the constant black lines represent the value of $S_{c 1}$. As can be seen, $S_{c P}$ and $S_{C L}$ agree perfectly, while $S_{12}$ leads to slightly smaller values of significance. $S_{1}$ significantly overestimates the significance at small event numbers.

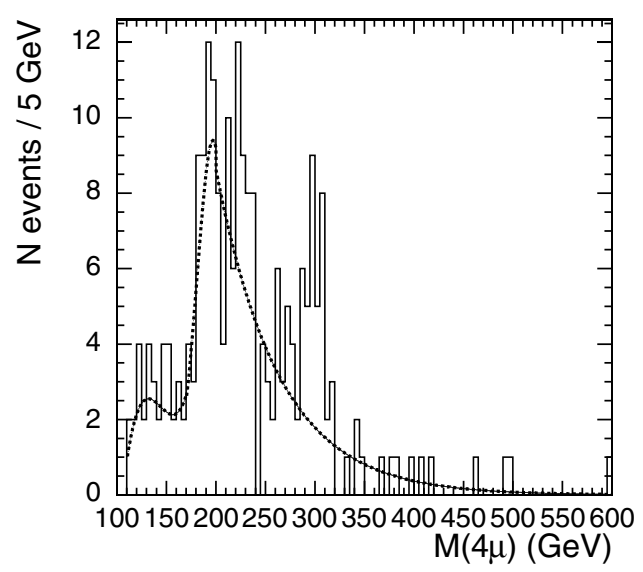

Figure A.3. The background $p d f$ and an example of one pseudo-experiment with a statistical fluctuation appearing just like a signal.

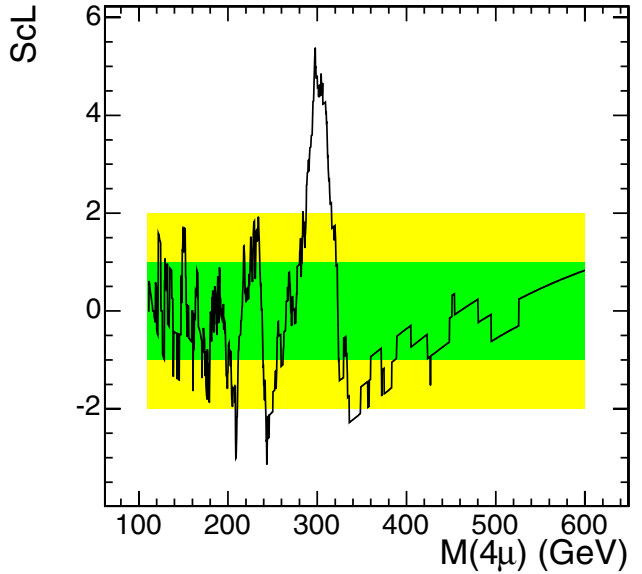

Figure A.4. Profile of the $S_{c L}$ scan corresponding to the pseudo-experiment example shown on the left. Green (inner) and yellow (outer) bands denote $\pm 1 \sigma$ and $\pm 2 \sigma$ intervals. Spikes that can be seen are due to events coming in or dropping off the trial-window, a feature of low-statistics searches.

The dashed line in Fig. A.3 shows the expected $4 \mu$ invariant mass distribution for background at $\mathcal{L}=30 \mathrm{fb}^{-1}$ after applying all the $m_{4 \mu}$-dependent analysis cuts described in Sec. . Using this distribution, we played out $\sim 10^{8}$ pseudo-experiments; an example is shown in Fig. A.3. For each pseudo-experiment, we slid a signal region window across the spectrum looking for a local event excess over the expectation. The size of the window $\Delta m=w\left(m_{4 \mu}\right)$ was optimised and fixed a priori (about $\pm 2 \sigma$ ) to give close to the best significance for a 


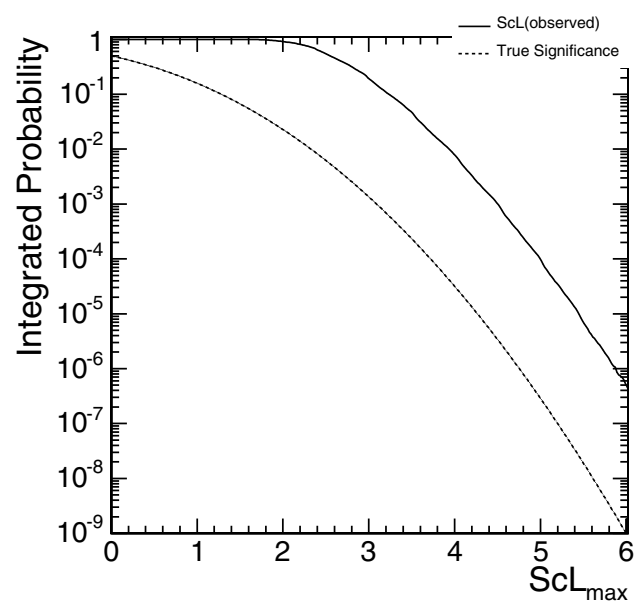

Figure A.5. $S_{c L}$ cumulative probability density function.

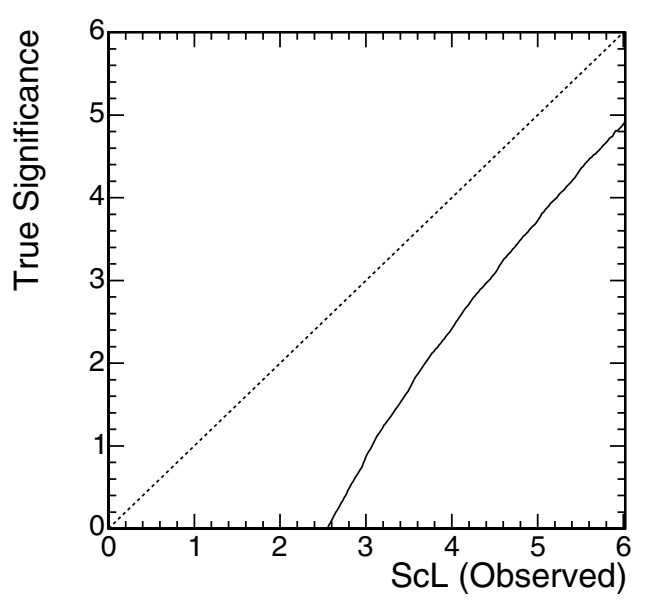

Figure A.6. Local significance "renormalisation" from an observed value to the true significance with a proper probabilistic interpretation.

resonance with a width corresponding to the experimental SM Higgs boson width $\sigma\left(m_{4 \mu}\right)$. The step of probing different values of $m_{4 \mu}$ was "infinitesimally" small $\left(0.05 \mathrm{GeV} / \mathrm{c}^{2}\right)$ in comparison to the Higgs boson width of more than $1 \mathrm{GeV} / \mathrm{c}^{2}$. The scanning was performed in a priori defined range of $115-600 \mathrm{GeV} / \mathrm{c}^{2}$.

We used a significance estimator $S_{c L}=\operatorname{sign}(s) \sqrt{2 n_{o} \ln (1+s / b)-2 s}$, where $b$ is the expected number of background events, $n_{o}$ is the number of observed events, and the signal is defined as $s=n_{o}-b$. This estimator, based on the Log-Likelihood Ratio, is known to follow very closely the true Poisson significance, only slightly over-estimating it in the limit of small statistics [51]. Figure A.4 presents the results of such a scan for the pseudo-experiment shown in Fig. A.3. The maximum value of $S_{c L}, S_{\text {max }}$, and the corresponding mass of a "Higgs boson candidate" obtained in each pseudo-experiment were retained for further statistical studies.

After performing $10^{8}$ pseudo-experiments, the differential probability density function for $S_{\max }$ and its corresponding cumulative probability function $P\left(S_{\max }>S\right)$ (Fig. A.5) were calculated. From Fig. A.5, one can see that the frequency of observing some large values of $S_{c L}$ (solid line) is much higher than its naive interpretation might imply (dashed line). If desired, the actual probability can be converted to the true significance. The result of such "renormalisation" is presented in Fig. A.6. One can clearly see that the required de-rating of significance is not negligible; in fact, it is larger than the effect of including all theoretical and instrumental systematic errors for this channel (see Section 3.1). More details on the various aspects of these studies can be found in [51].

There are ways of reducing the effect. A more detailed analysis of the shape of the $m_{4 \mu}$ distribution will help somewhat. Using the predicted number of signal events $s=s_{\text {theory }}$ in the significance estimator to begin with and, then, for validating the statistical consistency of an excess $n_{o}-b$ with the expectation $s_{\text {theory }}$ will reduce the effect further. One can also use a non-flat prior on the Higgs mass as it comes out from the precision electroweak measurements. Whether one will be able to bring the effect to a negligible level by using all these additional constraints on the signal hypotheses is yet to be seen. The purpose of this Appendix is not to give the final quantitative answer, but rather to assert that these studies must become an integral part of all future search analyses when multiple signal hypotheses are tried. 


\section{Appendix B. Systematic Errors}

\section{B.1. Theoretical uncertainties}

The simulation of events at the LHC is complex and can be conventionally divided into different parts which either involve the description of the interesting physics process or the description of the initial scattering conditions and the physics environment.

The simulation of the hardest part of the physics process is done via matrix element (ME) calculations at a certain order in the coupling constants and continues with the parton showering (PS) of the resulting partons until a cut-off scale, over which the perturbative evolution stops and the fragmentation of the final partons takes on. This cut-off is often referred to as factorisation scale, because it is the scale at which the two processes (showering and fragmentation) are supposed to factorise.

The interesting event is accompanied by the so-called underlying event (UE), term which identifies all the remnant activity from the same proton-proton $(p-p)$ interaction and whose definition often includes ISR as well, and the pile-up, composed by other minimum bias (MB) p-p interactions in the same bunch crossing (up to 25 at high luminosity at the LHC). Moreover, since the initial state is not defined in $\mathrm{p}-\mathrm{p}$ collisions, a proper description of the proton parton density functions (PDFs) should be included in the calculations.

Each of these effects needs to be modelled to the best of our knowledge, and the associated uncertainties need to be determined and propagated to the physics measurements. Moreover, many of the sources are correlated: for instance, fragmentation and showering are obviously dependent on each other, and in turn they assume a certain description of the underlying event. The task of assessing systematics due to theory and modelling can therefore be a difficult one and can sometime contain a certain degree of arbitrariness.

In what follows we propose some guidelines for the estimation of errors coming from the above, trying to divide the systematics sources into wider categories as much uncorrelated as possible: QCD radiation, fragmentation description, PDFs, UE and MB.

In attributing systematic errors we believe that one should use motivated recipes, avoiding unrealistic scenarios which will lead to unnecessarily conservative errors or, much worse, totally arbitrary assumptions.

\section{B.1.1. Hard process description and parametric uncertainties}

The description of the hard process should be done with Monte Carlo tools which are best suited to the specific analysis. For instance, when precise description of hard gluon emission becomes an issue, then next-to-leading order (NLO) generator tools like MC@NLO [771], or higher leading order (LO) $\alpha_{\mathrm{s}}$ generators like COMPHEP [43], MADGRAPH [81], ALPGEN [161], and SHERPA [194] should be considered. This is in general true for both the signal and the background description.

When adopting a ME tool, one should always keep in mind that its output is often (if not always) supposed to be interfaced to PS Monte Carlo such as HERWIG [196], PYTHIA [24] or ISAJET [672], that treat the soft radiation and the subsequent transition of the partons into observable hadrons. One of the most difficult problems is to eliminate double counting where jets can arise from both higher order ME calculations and from hard emission during the shower evolution. Much theoretical progress has been made recently in this field [772-775]. For what concerns the ME/PS matched description of multi-jet final states, a rich spectrum of processes is currently available in ALPGEN. However, adopting general purpose generators like PYTHIA can still be the best option for topologies that are better described in the Leading 
Logarithm Approximation (LLA), for instance in the case of two leading jets and much softer secondary jets. The two different descriptions should be regarded as complementary.

In general, a sensible choice for the selection of the best generation tools can be driven by the HEPCODE data base [776]. However, comparison between different generators is recommended whenever applicable.

Each analysis needs then to make sure that other important effects (e.g. spin correlations in the final state, NLO ME corrections to top decays) are included in the generation mechanism. For example, TopREX [44], as long with some of the Monte Carlo generators already introduced in this section, provides a correct treatment of top quark spin correlations in the final state. Neglecting some of these effects corresponds to introducing an error in the analysis that cannot be considered as coming from a theoretical uncertainty.

For both signal and backgrounds, missing higher orders are a delicate source of uncertainty. Formally, the associated error cannot be evaluated unless the higher order calculation is available. This is often not possible, unless extrapolating by using comparisons with analytical calculations of total or differential cross-sections at the next order, if available. One should keep in mind that simple K-factors are not always enough and that the inclusion of higher orders typically also involves distortions in differential distributions.

Moreover, one should not forget that any Standard Model calculation is performed in certain schemes and that the input parameters are subject to their experimental uncertainties; if the error on most of those and the choice of the renormalisation scheme are expected to give negligible effects in comparison with other uncertainties, this might not be so for the choice of the hard process scale, which we will discuss in the next section, and some of the input parameters.

Among the input parameters, by far the one known with less accuracy will be the top mass. The current uncertainty of about $2 \%$ [777] enters in the LO calculations for processes which involve top or Higgs production. For instance, the total $\bar{t}$ cross-section is known to have a corresponding 10\% uncertainty due to this [45 ]. As far as Higgs production (in association or not with tops) is concerned, gluon-gluon fusion proceeds via a top loop and therefore the total cross-section can have a strong dependence on the top mass when $\mathrm{m}_{\mathrm{H}} \approx 2 \mathrm{~m}_{\mathrm{t}}$. Analyses which include Higgs bosons or top are encouraged to estimate the dependence of the significant observables on the top mass itself. Effects of $m_{t}$ variation on acceptances of these analyses should instead be negligible.

\section{B.1.2. Hard process scale}

The hard process under study drives the definition of the $\mathrm{Q}^{2}$ scale, which directly enters in the parametrisation of PDFs and $\alpha_{\mathrm{s}}$, hence in the expression of the cross sections.

The dependence of the observables on the choice for the $\mathrm{Q}^{2}$ hard process scale is unphysical and should be regarded as one important contribution to the total uncertainty in the theoretical predictions. The sensitivity of the predicted observables to such choice is expected to decrease with the increasing order in which the calculation is performed, and can be tested by changing the hard process scale parameters in the generation (where applicable) using a set of sound values according to the characteristics of the hard process.

A sensible choice for the hard process scale in $2 \rightarrow 1$ processes is often $\hat{s}$, which is the default in general purpose generators like PYTHIA. Alternative choices to quote theoretical uncertainties can be $0.25 \hat{s}$ and $4.0 \hat{s}$. In PYTHIA this can be obtained acting on PARP(34).

For $2 \rightarrow n$ processes, many reasonable alternatives for the $\mathrm{Q}^{2}$ scale definition exist. The PYTHIA default $(\operatorname{MSTP}(32)=8)$, corresponds to the average squared transverse mass of the outgoing objects. It is possible to test the sensitivity on the $\mathrm{Q}^{2}$ scale switching to different options, for example trying $\mathrm{Q}^{2}=\hat{s}(\operatorname{MSTP}(32)=4$ in PYTHIA $)$. 


\section{B.1.3. PDF description}

The parton distribution functions of interacting particles describe the probability density for partons undergoing hard scattering at the hard process scale $Q^{2}$ and taking a certain fraction $x$ of the total particle momentum. Since the $Q^{2}$ evolution can be calculated perturbatively in the framework of QCD, PDFs measurements can be cross checked using heterogeneous DIS, Drell-Yan and jet data, and achieve predictivity for points where no direct measurements are available yet, for example in a large region of the $\left(x, Q^{2}\right)$ space for $\mathrm{p}-\mathrm{p}$ interactions at the LHC energy.

Various approaches are currently available to quote the PDFs of the proton, which propose different solutions for what concerns the functional form, the theoretical scheme, the order of the QCD global analysis (including possible QED corrections), and the samples of data retained in the fits: CTEQ [778], MRST [779], Botje [780], Alekhin [781], etc. The CTEQ and MRST PDFs, including Tevatron jet data in the fits, seem to be well suited for use in Monte Carlo simulations for the LHC.

The best way to evaluate theoretical uncertainties due to a certain proton PDFs is to vary the errors on the parameters of the PDF fit itself. With the Les Houches accord [95] PDF (LHAPDF) errors should be easily propagated via re-weighting to the final observables. However, errors are available only for NLO PDF, whereas in most of the cases only LO tools are available for the process calculation. Correctly performing evaluation of theoretical uncertainties in these cases requires some care. The proposed solution is to adopt CTEQxL (LO) for the reference predictions using CTEQxM (NLO) only to determine the errors.

For analyses which are known to be particularly sensitive to PDFs, like cross-section measurements, it would be also desirable to compare two different sets of PDFs (typically CTEQ vs MRST) taking then the maximum variation as an extra error. This is important since, even considering the error boundaries, different set of PDFs may not overlap in some region of the phase space.

The LHAGLUE interface [95] included from the most recent LHAPDF versions simplifies the use of the Les Houches accord PDF in PYTHIA by the switches MSTP(52) $=2$, $\operatorname{MSTP}(51)=\mathrm{LHAPDF}_{i d}$.

\section{B.1.4. QCD radiation: the parton shower Monte Carlo}

The showering algorithm is basically a numeric Markov-like implementation of the QCD dynamic in the LLA. After the generation of a given configuration at partonic level, the initial state radiation (ISR) and the final state radiation (FSR) are produced following unitary evolutions with probabilities defined by the showering algorithm.

The probability for a parton to radiate, generating a $1 \rightarrow 2$ branching, are given by the Altarelli-Parisi equations [782], however various implementations of the showering algorithm exist in parton shower Monte Carlo, which mostly differ for the definition of the $Q^{2}$ evolution variable (virtuality scale) in the $1 \rightarrow 2$ radiation branching and for the possible prescriptions limiting the phase space accessible to the radiation: PYTHIA, HERWIG, ARIADNE [783], ISAJET etc.

The virtuality scales for both ISR and FSR need to be matched to the hard process scale, the latter setting an upper limit on the former ones; such limit has to be considered in a flexible way, given the level of arbitrariness in the scale definitions. While this matching is somewhat guaranteed if one adopts the same simulation tool for both hard scattering and parton shower, a careful cross check is recommended in all other cases. In general, a critical judgement taking into account the hard process type is needed. Allowing a virtuality scale higher than the hard 
process scale may give rise to double counting. This is the case of $\mathrm{gg} \rightarrow \mathrm{gg}$ processes with additional hard gluons added in the showering. However other processes are safer from this point of view, for instance the case of the $\mathrm{q} \overline{\mathrm{q}} \rightarrow \mathrm{Z}$ process at $\mathrm{LO}$.

Quantum interference effects in hadronic collisions have been observed by CDF [784] and $\mathrm{D} \emptyset[785]$ studying the kinematical correlations between the third jet (regarded as the result of a soft branching in the LLA) and the second one. The implementation of the so called colour coherence in PS Monte Carlo is made in the limit of large number of colours and for soft and collinear emissions, restricting the phase space available to the radiation depending on the developed colour configuration. Different implementations of the colour coherence are available in HERWIG and PYTHIA, while ISAJET doesn't take into account such effects.

The theoretical uncertainty associated to the parton showering descriptions, includes what is normally referred to as ISR or FSR and their interference. In order to achieve practical examples for the recommended parton shower settings, we will consider PYTHIA as the default tool for showering from now on.

Turning OFF ISR and FSR $(\operatorname{MSTP}(61)=0, \operatorname{MSTP}(71)=0$ respectively) or even the interference part $(\operatorname{MSTP}(62)=0, \operatorname{MSTP}(67)=0)$ is certainly a too crude approach and, to a large extent, a totally arbitrary procedure to assess a systematic error. We believe it is much more realistic to vary, according to sound boundaries, the switches regulating the amount and the strength of the radiation of the showering. These can correspond to $\Lambda_{\mathrm{QCD}}$ and the maximum virtuality scales up to which ISR stops and from which FSR starts. It would be important to switch the parameters consistently going from low to high values in both ISR and FSR.

Notice that the radiation parameters were typically fitted at LEP1 together with the fragmentation parameters, benefiting from a much simplified scenario where no ambiguity on the maximum virtuality scale applies, the only relevant energy scale of the problem being $\hat{s}=s$. One has to take into account that while for instance FSR accompanying heavy boson decays at the LHC can be directly related to the LEP experience, FSR in processes like $\mathrm{gg} \rightarrow \mathrm{b} \overline{\mathrm{b}}$ entails additional uncertainties arising from the maximum allowed virtuality scale and ISR/FSR interference. On top of that, additional complications arise from the fact that ISR at hadron machines contributes to the description of the underlying event. Matching two different tunings of the same parameter (in particular PARP(67)) can be very subtle at the LHC.

These are the suggested settings in PYTHIA, which have been cross-checked with the ones adopted by the CDF experiment and also follow the prescription by the main author:

- $\Lambda_{\mathrm{QCD}}$ : PARP(61), PARP(72), PARJ(81) from 0.15 to $0.35 \mathrm{GeV}$ consistently, symmetric with respect to 0.25 . Notice that these settings have been optimised for the CTEQ6L PDFs. In general different ranges apply when changing PDFs. In order to give the user full control on these parameters the option $\operatorname{MSTP}(3)=1$ has to be set, otherwise $\Lambda_{\mathrm{QCD}}$ is assumed to be derived from the PDFs parametrisation.

- $\mathrm{Q}_{\max }^{2}$ : PARP(67) from 0.25 to 4 and $\operatorname{PARP}(71)$ from 1 to 16 going from low to high emission in a correlated way. In doing so one should also make sure that the tuning of the underlying event is not changing at the same time. Possible re-tuning of the underlying event in different radiation scenarios may be needed, in particular for what concerns $\operatorname{PARP}(82)$.

\section{B.1.5. Fragmentation}

Perturbative QCD cannot provide the full description of the transition from primary quarks to observable hadrons, but only the part which involves large momentum transfer. The formation of final hadrons involves a range of interactions which goes above the Fermi scale and where 
the strong coupling constant $\alpha_{\mathrm{s}}$ increases above unity, making it necessary to describe this part in a non-perturbative way, normally referred to as fragmentation or hadronisation.

The non-perturbative description of fragmentation is realised via models, which need to be tuned to experimental data. The data correspond, typically, to event shapes and multiplicities at leptonic machines or to the inclusive jet shapes at hadronic machines. A comprehensive overview of the models can be found in [786].

Fragmentation is said to depend only on the factorisation scale if jet universality is assumed, i.e. assuming that jets fragment in the same way at hadron and lepton machines. Jet universality will be ultimately verified at the LHC; one should clarify whether instrumental effects and the LHC environment will have an impact on the final observables. For instance, the much larger fraction of gluon jets or the different description of the underlying event can change the values of the parameters that regulate the fragmentation. Moreover, for events with high multiplicity of jets it will also be crucial to properly describe fragmentation in conditions where large jet overlapping is to be expected and where inclusive tunings might not be ideal.

The consequence of jet universality is that, once the PS cut-off scale is fixed, the fragmentation description for light quarks should be universal, and the LEP/SLD tunings (or the Tevatron ones) could be used as they are for the LHC.

It is important to underline that the description of the non-perturbative part of the radiation also depends on the way the perturbative one is described. This means that one should not use a tuning of fragmentation done with LO(+LL) tools (typically PYTHIA at LEP) attached to perturbative calculation which are done at higher (or different) order.

B.1.5.1. Light quarks fragmentation. In the absence of LHC data, the best choice is therefore to use a model tuned to the LEP and SLD data [787-789]. It is important to choose the tuning in a consistent way from the same experiment, given that a combined LEP/SLD tuning has never been attempted. As a possibility, suggested by the major success in describing the data and by its extensive use in the experimental collaborations, is the use of PYTHIA, which uses the string (or Lund) fragmentation model [790]. The parameters that we consider more relevant in PYTHIA for the description of fragmentation are the following, where the central value is taken by the fit performed by the OPAL Collaboration, as an example:

$$
\begin{aligned}
& \operatorname{PARJ}(81)=0.250 \\
& \operatorname{PARJ}(82)=1.90 \\
& \operatorname{PARJ}(41)=0.11 \\
& \operatorname{PARJ}(42)=0.52 \\
& \operatorname{PARJ}(21)=0.40
\end{aligned}
$$

where PARJ $(81)\left(\Lambda_{\mathrm{QCD}}\right)$ and $\mathrm{PARJ}(82)\left(\mathrm{Q}_{\mathrm{min}}^{2}\right)$ refer to the radiation part. To properly evaluate a systematic error due to pure fragmentation one should vary only PARJ(42) and PARJ(21) by their respective errors (0.04 and 0.03 for OPAL). The variation should account for the proper parameter correlation if the effect is critical for the analysis. PARJ(41) is totally correlated to $\operatorname{PARJ}(42)$.

Alternatively, or additionally, it would also be important to compare PYTHIA with HERWIG with consistent tunings from LEP [787-789]; in doing so it is important to factorise the UE description (see next section) that can induce important differences in the results.

B.1.5.2. Heavy quarks fragmentation. The description of the heavy quarks fragmentation is important for top physics and for those processes with large b production in the final states. Exclusive channels are particularly influenced by the description of the fragmentation of the b quark. 
The description of the fragmentation of the heavy quarks has been tuned to $\mathrm{Z}$ data at LEP and SLD[778, 791-793] (via a measurement of $x_{B}$ and $x_{D}$ ) and $b \bar{b}$ data at the Tevatron, using different fragmentation functions like Lund, Bowler [794], Peterson [795], Kartvelishvili [796].

In the spirit of fragmentation universality the LEP/SLD tunings can be adopted for the LHC, but with much care. Significant differences among the fitted values in different experiment can point out that the factorisation scale used for the PS is not the same everywhere. One should make sure that the scale used is set consistently with the chosen fragmentation function parameters. This can be done by using the tuning from only one experiment, making sure to also use the main switches of the parton showering, (PARJ $(81)$ and PARJ(82) in PYTHIA).

The fragmentation function that best describes heavy flavour data at LEP is Bowler. With the same OPAL tuning reported above the best fit of the Bowler parameters, $a$ and $b m_{\perp}^{2}$, to data gives:

$$
\begin{aligned}
& b m_{\perp}^{2}=65_{-14}^{+17} \\
& a=15.0 \pm 2.3 .
\end{aligned}
$$

The Bowler model would extend the string model to heavy flavours, describing the corrections in terms of the charm and bottom masses. Unfortunately, no tuning exists in the literature which is capable to describe at the same time light and heavy quark fragmentation, i.e. adopting universal parameters $a=\operatorname{PARJ}(41)$ and $b=\operatorname{PARJ}(42)$ for both light and heavy quarks.

Alternatively, the widely used Peterson function can be used, and its parameters are directly switchable in PYTHIA for just $\mathrm{b}$ and $\mathrm{c}$ fragmentation:

$$
\begin{aligned}
& \operatorname{PARJ}(54)=-0.031 \pm 0.011 \\
& \operatorname{PARJ}(55)=-0.0041 \pm 0.0004
\end{aligned}
$$

where the two parameters correspond, respectively, to $\varepsilon_{\mathrm{c}}$ and $\varepsilon_{\mathrm{b}}$ fitted in the OPAL tuning. The systematic can then be evaluated by varying the errors on the fitted parameters or by comparing with a different fragmentation function like Kartvelishvili, or Lund.

An important feature of the $b$ fragmentation that should be considered by those analyses in the top sector sensitive to the details of the fragmentation, is the way the b fragments in top decays. At the LHC the $b$ from a $t$ is hadronising with a beam remnant, introducing potentially worrying differences with respect to the fragmentation at LEP. The main effects are presented in [797] and are known as cluster collapse, happening when a very low mass strings quarkremnant directly produces hadrons without fragmenting, hence enhancing the original flavour content, and beam drag, which is an angular distortion of hadron distribution toward the end of the string in the remnant. If, under reasonable assumptions on the transverse momentum in top events at the LHC, one can exclude to a large extent the importance of the first effect, beam drag could potentially introduce B meson production asymmetries, even though estimations are keeping the effect at the level of $1 \%$ at the LHC [797].

\section{B.1.6. Minimum bias and underlying event}

Multiple parton interaction models, extending the QCD perturbative picture to the soft regime, turn out to be particularly adequate to describe the physics of minimum bias and underlying event. Examples of these models are implemented in the general purpose simulation programs PYTHIA, HERWIG/JIMMY [193] and SHERPA. Other successful descriptions of underlying event and minimum bias at hadron colliders are achieved by alternative approaches like PHOJET [798], which rely on both perturbative QCD and Double Pomeron Models (DPM). 
Huge progress in the phenomenological study of the underlying event in jet events have been achieved by the CDF experiment at Tevatron [799], using the multiplicity and transverse momentum spectra of charged tracks in different regions in the azimuth-pseudorapidity space defined with respect to the direction of the leading jet. Regions that receive contributions only by the underlying event have been identified. The average charged multiplicity per unit of pseudorapidity in these regions turns out to be significantly higher with respect to the one measured in minimum bias events. This effect, referred to as "pedestal effect", is well reproduced only by varying impact parameters models with correlated parton-parton interactions $(\operatorname{MSTP}(82)>1$ in PYTHIA). Simpler models are definitely ruled out.

The main problem of extrapolating the predictions of the multiple interactions models to the LHC is that some of the parameters are explicitly energy dependent, in particular the colour screening $\mathrm{p}_{\mathrm{T}}$ cut-off (PARP(82) at the tuning energy PARP(89) in PYTHIA). The CDF tuning, often referred to as Tune-A, is not concentrating on this particular aspect. Other works $[197,800]$ have put more emphasis on this issue. However, one of their results is that currently only PYTHIA can be tuned to provide at the same time description of CDF and lower energy minimum bias data from UA5. One of these tunings can be summarised as follows:

- $\operatorname{PARP}(82)=2.9$

- $\operatorname{PARP}(83)=0.5$

- $\operatorname{PAPR}(84)=0.4$

- $\operatorname{PARP}(85)=0.33$

- $\operatorname{PARP}(86)=0.66$

- $\operatorname{PARP}(89)=14000$

- $\operatorname{PARP}(90)=0.16$

- $\operatorname{PARP}(91)=1.0$

- $\operatorname{MSTP}(81)=1$

- $\operatorname{MSTP}(82)=4$.

Sensible estimation of theoretical uncertainties arising from underlying event and minimum bias modelling can be performed assigning $\pm 3 \sigma$ variations to the colour screening $\mathrm{p}_{\mathrm{T}}$ cut-off parameter tuned on minimum bias CDF and UA5 data and extrapolated to the LHC energy [ 800], i.e. varying PARP(82) in the range [2.4-3.4], while keeping the other parameters listed above to their tuned values.

As a new tool for the description of UE and MB we would like to mention PYTHIA 6.3 [801], that allows for new interesting features, including the new $\mathrm{p}_{\mathrm{T}}$-ordered initial- and final-state showers and a new very sophisticated multiple interactions model that achieves description of colliding partons in the proton in terms of correlated multi-parton distribution functions of flavours, colours and longitudinal momenta. However, as stressed by the PYTHIA authors, the new model (PYEVNW) is still not so well explored. Therefore the old model (PYEVNT) is retained as the default choice, with full backward compatibility. Moreover, in the use of PYTHIA 6.3, one should be careful when switching to the new $\mathrm{p}_{\mathrm{T}}$-ordered showers and multiple interaction models, as their parameters are not tuned yet, in particular for what concerns the energy dependence, necessary to get meaningful extrapolations at the LHC energy.

\section{B.1.7. Pile-up and LHC cross sections}

The design parameters of the LHC at both low and high luminosity are such that, on top of possible signal events, additional minimum bias interactions are produced in the same beam crossing, the so-called pile-up effect. 
Pile-up is a purely statistical effect. The number of minimum bias interactions generated in a single beam crossing is a Poissonian distribution that depends on the instantaneous luminosity, which varies of about a factor 2 during a LHC fill. Although luminosity variation is not arising from theoretical uncertainties, it is recommended to cross check the stability of the results against variation of the nominal luminosity.

An issue which can affect the pile-up is the definition of the minimum bias itself. The latter, indeed, may or may not include the diffractive and elastic contributions, with figures for the total cross section which can vary from $100 \mathrm{mb}$ to $50 \mathrm{mb}$ respectively. If the PYTHIA generator is adopted, these two different options correspond to MSEL 2 and MSEL 1, however, in order to get full control on the different contributions to the cross sections, one can use MSEL 2, setting $\operatorname{MSTP}(31)=0$, and providing explicit input through $\operatorname{SIGT}(0,0, \mathrm{~J})$, where the meaning of the index $\mathrm{J}$ is described below:

$$
\begin{array}{ll}
J=0 & \text { Total cross section }(\text { reference value }=101.3 \mathrm{mb}) \\
J=1 & \text { Elastic cross section (reference value }=22.2 \mathrm{mb}) \\
J=2 & \text { Single diffractive cross section XB }(\text { reference value }=7.2 \mathrm{mb}) \\
J=3 & \text { Single diffractive cross section AX }(\text { reference value }=7.2 \mathrm{mb}) \\
J=4 & \text { Double diffractive cross section }(\text { reference value }=9.5 \mathrm{mb}) \\
J=5 & \text { Inelastic, non-diffractive cross section }(\text { reference value }=55.2 \mathrm{mb}) .
\end{array}
$$

Where $\mathrm{J}=0$ has to correspond to the sum of the contributions for $\mathrm{J}=1, \ldots, 5$. With respect to alternative cross section predictions [802], PYTHIA reference values for diffractive cross sections might be slightly shifted on the high side. A possible sound alternative could be to reduce the diffractive cross sections of around 30\%, keeping constant the total cross section.

In order to assess the sensitivity of one analysis to the diffractive variations in the pile-up, at least the two options MSEL 1 and MSEL 2 should be tried. Diffractive contribution will in general result in few additional soft charged particles spiralling in the high magnetic fields of the LHC experiments. This effect is most likely to be relevant in the tracker detectors, where multiple hits in the same layer can be generated by the same track.

\section{B.1.8. Decays}

In contrast to the simple decay models available in the common PS Monte Carlo, alternative hadron decay models exist, for example EVTGEN [803], which have huge collections of exclusive hadron decays up to branching ratios as low as $10^{-4}$.

EVTGEN follows the spin density matrix formalism and has an easily tuneable and upgradeable hadron decay data base which currently constitutes the largest and most refined collection of hadron decay models.

Comparison between the simple default decay models implemented in PS Monte Carlo and those available in EVTGEN should be recommended at least for analyses dealing with $\mathrm{B}$ hadrons or relying on $\mathrm{b}$-tagging. However, since switching to a new hadron decay model could have a deep spin-offs on the exclusive description of the final states (multiplicity of kaons, pions, photons and muons, multiplicity of tracks reconstructed in secondary vertices) it might be worth to study also effects on trigger performances.

The LHC version of EVTGEN was initially provided by the LHCb experiment and is currently maintained by LCG Generator [804]. It comprises an interface to PYTHIA simulation that solves the technical problems of switching between the two different scenarios (i.e. hadron decays performed by PYTHIA, hadron decays performed by EVTGEN). 


\section{B.1.9. LHAPDF and PDF uncertainties}

The detailed investigations of processes at LHC required a well understanding of the systematic theoretical uncertainties [201]. One of the important source of such errors is the parton distribution functions (PDFs).

The Les Houches Accord Parton Density Functions (LHAPDF) package [95] is designed to work with the different PDF sets ${ }^{53}$. In this approach a "fit" to the data is no longer described by a single PDF, but by a PDF set consisting of many individual PDF members. Indeed, PDFs are specified in a parameterised form at a fixed energy scale $Q_{0}$, such as

$$
f\left(x, Q_{0}\right)=a_{0} x^{a_{1}}(1-x)^{a_{2}}\left(1+a_{3} x^{a_{4}} \ldots\right) .
$$

The PDFs at all higher $Q$ are determined by NLO perturbative QCD evolution equations. The total number of PDF parameters $(d)$ could be large (for example, for CTEQ parametrisation one has $d=20$ [12]). Fitting procedure is used for evaluation an effective $\chi^{2}$ function, which can be used to extract the "best fit" (the global minimum of $\chi^{2}$ ) and also to explore the neighbourhood of the global minimum in order to quantify the uncertainties. As a result one has the "best-fit" PDF and $2 d$ subsets of PDF $[12,95]$ :

$$
f_{0}(x, Q), f_{i}^{ \pm}(x, Q)=f\left(x, Q ;\left\{a_{i}^{ \pm}\right\}\right), i=1, \ldots, d .
$$

B.1.9.1. Master equations for calculating uncertainties. Let $X(\{a\})$ be any variable that depends on the PDFs. It can be a physical quantity such as the $W$ production cross section, or a differential distribution.

Let $X_{0}=X\left(\left\{a_{0}\right\}\right)$ be the estimate for $X$ calculated with the best-fit PDF and $X_{i}^{ \pm}$be the observable $X$ calculated with $i$-th subset $f_{i}^{ \pm}(x, Q)$.

Following to CTEQ6 collaboration one can estimate the variation of $X$ by using a master formula [12]:

$$
\Delta X=\sqrt{\sum_{i=1}^{d}\left(X_{i}^{+}-X_{i}^{-}\right)^{2}} .
$$

However, very often many $X_{i}^{+}$and $X_{i}^{-}$have different magnitudes and even signs! This failure of the master formula is a result of the simple observation that the PDF set that minimises the uncertainty in a given observable $X$ is not necessarily the same as the one that minimises the fit to the global data set.

The better estimator for the uncertainty of a generic observable $X$ was proposed in [805]. It is defined as the maximum positive and negative errors on an observable $X$ by

$$
\begin{aligned}
& \Delta X_{+}=\sqrt{\sum_{i=1}^{d}\left(\max \left[\left(X_{i}^{+}-X_{0}\right),\left(X_{i}^{-}-X_{0}\right), 0\right]\right)^{2}}, \\
& \Delta X_{-}=\sqrt{\sum_{i=1}^{d}\left(\max \left[\left(X_{0}-X_{i}^{+}\right),\left(X_{0}-X_{i}^{-}\right), 0\right]\right)^{2}} .
\end{aligned}
$$

In Eqs. (B.4) one sums the maximum deviations on the observable in each of the parameter directions, and hence retain both maximal sensitivity to the parameters that vary most and estimate the range of allowed values of the cross section. Note, that the errors in Table C. 2 were evaluated with this Eq. (B.4).

\footnotetext{
53 Note, at CMS it was recommended to use the CTEQ 5L set for PTDR simulation. Since there is only one CTEQ 5L PDF set (without corresponding subsets), it was recommended to use CTEQ $6 \mathrm{M}$ for evaluation of uncertainties due to PDFs for PTDR estimates and only in a special case can one use another sets (e.g. MRST).
} 


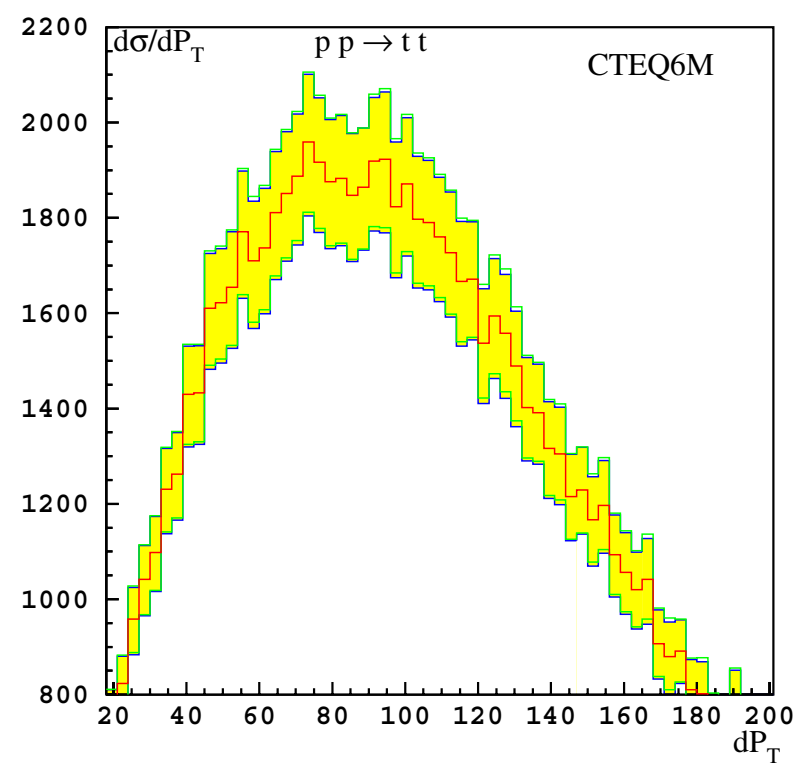

Figure B.1. $d \sigma / d P_{\mathrm{T}}$ distribution for $t \bar{t}$-pair production at LHC. The central histogram corresponds to the 'best-fit' of CTEQ6M PDF, while the shaded area represents the deviation due to PDF uncertainties.

Eq. (B.4 could also be used for calculations of differential distribution. Fig. B.1 presents the differential distribution $d \sigma / d P_{\mathrm{T}}$ for $t \bar{t}$-pair production at LHC.

B.1.9.2. How to calculate $X\left(\left\{a_{i}\right\}\right)$. The most simple and straightforward method is to simulate a sample with the "best-fit" PDFs and then to repeat a such simulation $2 d$ times with different $2 d$ PDF subsets. As a results one gets $(1+2 d)$ samples of unweighted events with different kinematics for each samples. Then use these samples to calculate $(1+2 d)$ values for observable:

$$
\left.X_{0}=\sum_{\text {events }} X_{n}\left(\left\{a_{0}\right\}\right)\right), \quad X_{i}^{ \pm}=\sum_{\text {events }} X_{n}\left(\left\{a_{i}^{ \pm}\right\}\right), \quad i=1, \ldots, d .
$$

In practice, such method requires a large CPU-time and can be recommended only to be used for very few special cases, when a high accuracy is required.

In the second approach ("re-weighting" method) one needs to simulate only one sample with the 'best-fit' PDF. In doing so the additional weights, corresponding to all other PDF subsets are evaluated. This weight is the ratio of the parton luminosity $\left[\operatorname{PDF}\left(\left\{a_{i}\right\}\right)-\right.$ the product of PDFs] evaluated with PDF subset to the parton luminosity, calculated with the 'best-fit' PDF. As a result, for any $n$-event one has $2 d$ additional weights:

$$
\left.w_{(0)}=1 \text { (best fit } \mathrm{PDF}\right), w_{(i)}^{ \pm}=\frac{\operatorname{PDF}\left(\left\{a_{i}^{ \pm}\right\}\right)_{n}}{\operatorname{PDF}\left(\left\{a_{0}\right\}\right)_{n}} ; w_{(i)}^{ \pm}=\mathcal{O}(1) .
$$

The corresponding $(1+2 d)$ values for observable $X$ are evaluated as follows:

$$
\left.X_{0}=\sum_{\text {events }} X_{n}\left(\left\{a_{0}\right\}\right)\right), \quad X_{i}^{ \pm}=\sum_{\text {events }} w_{(i)}^{ \pm} X_{n}\left(\left\{a_{0}\right\}\right)
$$

Contrary to the first method (see (B.5)) these $(1+2 d)$ samples have the events with different weights, but with identical kinematics for each samples. Note, that all additional samples have 
different "total number of events":

$$
N_{0}=\sum_{\text {events }} w_{(0)}(=1), N_{i}^{ \pm}=\sum_{\text {events }} w_{(i)}^{ \pm} \neq N_{0}, \text { and } N_{i}^{ \pm}=\mathcal{O}\left(N_{0}\right)
$$

Starting from CMKIN_6_0_0 version it is possible for each event the evaluation of the additional weights, corresponding to different PDF subsets (i.e. $w_{(i)}^{ \pm}$, see (B.6)). This option is available for CMKIN run with PYTHIA-like generators (PYTHIA, MADGRAPH, COMPHEP, ALPGEN, TopReX, StaGen, etc) and HeRwig. This information is written in /mc_param/ user block after all variables filled by CMKIN and a user (by using of kis_xxx routines).

\section{B.2. Experimental uncertainties}

The systematic uncertainties associated with the detector measurements contributing to an analysis are mostly covered in the corresponding chapters of Volume 1 of this Report [7] and are summarised here.

\section{B.2.1. Luminosity uncertainty}

As discussed in Chapter 8 of [7], the design goal for the precision of the luminosity measurement at CMS is $5 \%$, which is assumed to be achieved after $1 \mathrm{fb}^{-1}$ of data has been collected. For integrated luminosities of less than $1 \mathrm{fb}^{-1}$, it is assumed that the precision is limited to $10 \%$. For studies based on $30 \mathrm{fb}^{-1}$ or more in this Report, it is assumed that further improvement on the uncertainty can be achieved and a $3 \%$ uncertainty is assumed, via e.g. W, $\mathrm{Z}$ based luminosity measurements.

\section{B.2.2. Track and vertex reconstruction uncertainties}

The uncertainty in the silicon track reconstruction efficiency is taken to be $1 \%$ for all tracks. The primary vertex precision along the $z$ coordinate is expected to be about $10 \mu \mathrm{m}$ once $1 \mathrm{fb}^{-1}$ has been collected. The transverse vertex precision is expected to be about $1 \mu \mathrm{m}$.

The effects of uncertainties on the alignment of silicon sensors on track and vertex reconstruction are studied using a dedicated software tool (Section 6.6.4 of [7]) that is able to displace tracker elements according to two scenarios: a "First Data Taking Scenario" with placement uncertainties as expected at LHC start-up from measurements using the laser alignment system for the strip tracker and from in-situ track-based alignment of the pixel detector, and a "Long Term Scenario" appropriate after the first few $\mathrm{fb}^{-1}$ have been collected and a complete track-based alignment has been carried out for all tracker elements.

The effect of the magnetic field uncertainty in the central region of CMS is expected to contribute a momentum scale uncertainty of $0.0003 \mathrm{GeV} / \mathrm{c}$ to $1 / p_{\mathrm{T}}$. When combined with the aggregate effect from alignment uncertainties, the overall momentum scale uncertainty is $0.0005 \mathrm{GeV} / \mathrm{c}$ at start-up.

\section{B.2.3. Muon reconstruction uncertainties}

As with the silicon tracker studies, a dedicated software tool has been developed (Section 3.2.2 of [7]) to study the effects of muon detector placement uncertainties on muon reconstruction. Two scenarios, a "First Data Taking Scenario" with placement uncertainties as expected at LHC start-up and a "Long Term Scenario" appropriate after the first few $\mathrm{fb}^{-1}$, are available and used in analyses sensitive to the alignment precision of the muon detectors. The latter 


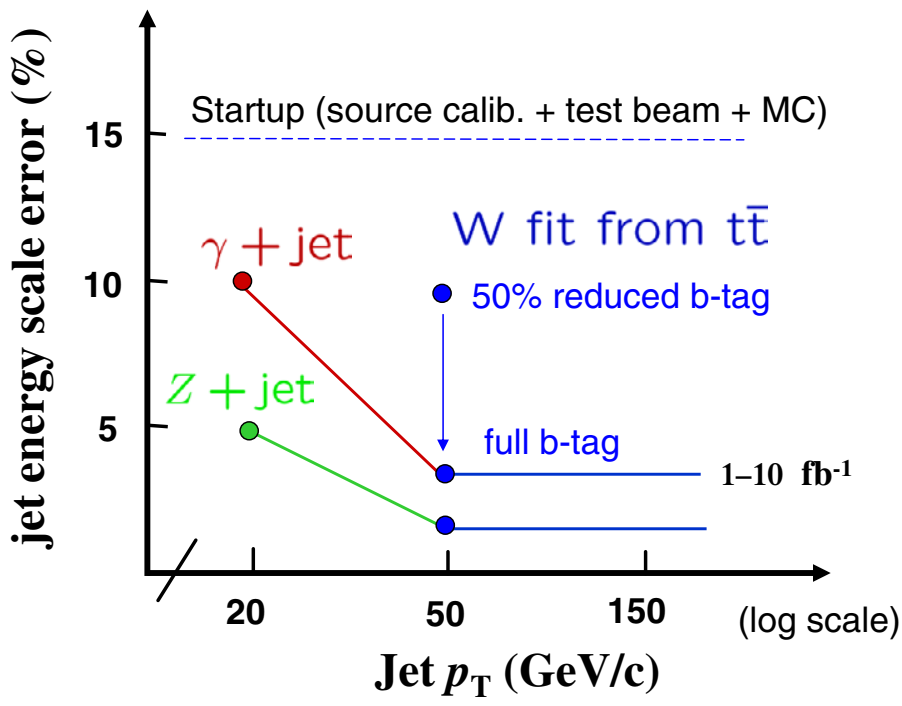

Figure B.2. Jet energy scale uncertainty is applied as a rescaling of the four-momentum of the reconstructed jet $p_{\text {scaled } \pm}^{\mu, \text { jet }}=(1 \pm \alpha) \cdot p_{\text {meas }}^{\mu, j e t}$ where $\alpha$ is the percentage uncertainty plotted above.

scenario describes a detector alignment precision of $200 \mu \mathrm{m}$ in the plane transverse to the beam axis using the laser alignment system and track-based alignment strategies.

The effect of magnetic field uncertainties on the muon momentum will be dominated by the uncertainty in the central region and its impact on the momentum scale determined by fits to the silicon tracker hits for muon momenta well below the $\mathrm{TeV} / \mathrm{c}$ scale.

\section{B.2.4. Electromagnetic calibration and energy scale uncertainties}

The precision to which the ECAL crystals can be intercalibrated from a variety of techniques is discussed in Section 4.4 of [7], and ranges from $0.4-2.0 \%$ using about $5 \mathrm{fb}^{-1}$ of in situ single isolated electron data. A software tool is used to apply calibration constants to the accuracy expected to be obtained with either $1 \mathrm{fb}^{-1}$ or $10 \mathrm{fb}^{-1}$ of integrated luminosity. The absolute energy scale can be determined using the $\mathrm{Z}$ mass constraint in $\mathrm{Z} \rightarrow$ ee decays, and is expected to be measured to a precision of about $0.05 \%$.

\section{B.2.5. Jet and missing transverse energy uncertainties}

The estimated systematic uncertainty on the jet energy scale is shown in Fig. B.2. At startup the accuracy of the jet energy scale relies on the understanding of single-particle test beam calibration and the level of agreement achieved in the data-to-Monte Carlo simulation comparisons of the detector response. The response of an individual tile or crystals is known to limited accuracy from source calibration in the HCAL and test stand measurements for crystals in the ECAL. Hence, given the limitations of the precalibration of the calorimeters, an overall uncertainty of $15 \%$ is expected for the "day-one" absolute energy scale. This applies equally for jet response and the energy scale uncertainty of the missing transverse energy.

In the first $1-10 \mathrm{fb}^{-1}$ of data, the $\gamma+$ jet calibration [283] and the hadronic $\mathrm{W}$ boson mass calibration in top quark pair production events [287] are currently the best estimates for the accuracy on the absolute jet energy scale. The hadronic $\mathrm{W}$ jets in the selected 
sample have a mean $p_{\mathrm{T}}$ that is approximately $50 \mathrm{GeV} / \mathrm{c}$. A lowering of the jet selection threshold increases the effects of the offset correction from pile-up. The systematic on offset corrections and backgrounds puts the absolute jet energy scale at $3 \%$. The jet reconstruction efficiencies are flat above $50 \mathrm{GeV} / \mathrm{c}$, but drop in the low $p_{\mathrm{T}}$ region. The current estimate of the high $p_{\mathrm{T}}$ jet energy scale based on the hadronic $\mathrm{W}$ calibration is $3 \%$. The calorimeter response curves that are required to extrapolate to high $p_{\mathrm{T}}$ are not expected to significantly increase the energy scale uncertainty beyond the $3 \%$ from the $\mathrm{W}$ calibration. In the low $p_{\mathrm{T}}$ region excluded from the hadronic $\mathrm{W}$ analysis, the absolute jet energy scale will be set by the $\gamma+$ jet calibration which will extend down to $20 \mathrm{GeV}$. Below $20 \mathrm{GeV}$, only the singleparticle calibration methods apply and these will have an accuracy of $10 \%$. The recommended treatment for the jet energy systematic in this report is to apply an uncertainty according to this functional form:

$\sigma_{E}^{j e t} / E= \begin{cases}10 \% & p_{\mathrm{T}}<20 \mathrm{GeV} / \mathrm{c} \\ 10 \%-7 \% *\left(p_{\mathrm{T}}-20 \mathrm{GeV} / \mathrm{c}\right) /(30 \mathrm{GeV} / \mathrm{c}) & 20 \mathrm{GeV} / \mathrm{c}<p_{\mathrm{T}}<50 \mathrm{GeV} / \mathrm{c} \\ 3 \% & p_{\mathrm{T}}>50 \mathrm{GeV} / \mathrm{c}\end{cases}$

It is expected that the $\mathrm{Z}+\mathrm{jet}$ sample and further analysis of the hadronic $\mathrm{W}$ systematics will reduce the overall jet energy scale uncertainty, but these analyses remain under active study.

The low $p_{\mathrm{T}}$ region is particularly important for the missing transverse energy (MET) response. As the MET will have significant contributions from low $p_{\mathrm{T}}$ jets and unclustered energy, it is expected that the low $p_{\mathrm{T}}$ component of the MET will not be understood to better than $10 \%$ following the first $1-10 \mathrm{fb}^{-1}$ of data. The recommended treatment of the MET energy scale uncertainty has two approaches (one simple and one more detailed). For a MET which is known to be dominated by low $p_{\mathrm{T}}$ jets and unclustered energy, an uncertainty of $10 \%$ should be applied to the components of the MET uncorrelated to the jet energy scale uncertainty of the jets. This is the simple approach and gives a conservative error on the MET. For events with reconstructed high $p_{\mathrm{T}}$ jets, the contributions to the MET uncertainty are correlated to the jet energy scale uncertainty of the high $p_{\mathrm{T}}$ jets. The recommended treatment of the MET uncertainty is to apply separate uncertainties on the low $p_{\mathrm{T}}$ and high $p_{\mathrm{T}}$ components of the MET. The MET is reconstructed as described in [147] and [148]. This gives a type- 1 correction of the following form:

$$
E_{\mathrm{Tx}(\mathrm{y})}^{\mathrm{miss}}=-\left[E_{\mathrm{Tx}(\mathrm{y})}^{\mathrm{raw}}+\sum_{\text {jets }}\left(p_{\mathrm{Tx}(\mathrm{y})}^{\text {corr. jet }}-p_{\mathrm{Tx}(\mathrm{y})}^{\text {raw jet }}\right)\right]
$$

where $E_{\mathrm{Tx}(\mathrm{y})}^{\mathrm{raw}}$ is the sum over the raw calorimeter tower energies and the jet sum in the equation is over jets with a reconstructed $p_{\mathrm{T}}$ above a given jet $p_{\mathrm{T}}^{\text {cut }}$ selection cut, typically $20-25 \mathrm{GeV} / \mathrm{c}$. The jet $p_{\mathrm{T}}$ is used in these formula to account for the angular separation of the towers included in the jet sum, contributing to the jet mass. Rewriting the above equation in this form

$$
E_{\mathrm{Tx}(\mathrm{y})}^{\mathrm{miss}}=-\left[\left(E_{\mathrm{Tx}(\mathrm{y})}^{\mathrm{raw}}-\sum_{\text {jets }} p_{\mathrm{Tx}(\mathrm{y})}^{\text {raw jet }}\right)_{\text {low } p_{\mathrm{T}}}+\left(\sum_{\text {jet }} p_{\mathrm{Tx}(\mathrm{y})}^{\text {corr. jet }}\right)_{\text {high } p_{\mathrm{T}}}\right]
$$

shows explicitly the low $p_{\mathrm{T}}$ (in the first set of brackets) and the high $p_{\mathrm{T}}$ components (second set of brackets) of the MET. The proposed systematics treatment is to vary the components of the low $p_{\mathrm{T}}$ MET by $10 \%$ scale uncertainty uncorrelated with the high $p_{\mathrm{T}}$ component and to vary the high $p_{\mathrm{T}}$ component according the jet energy scale uncertainty for the measured jets. 
If a subset of the high $p_{\mathrm{T}}$ jets are identified as electromagnetic objects, isolated electrons or photons, then these EM-jets should be given EM-scale energy corrections which are closer to unity than hadronic jet corrections. The energy scale uncertainty on an EM-object will also be much lower than the jet energy scale systematic. Therefore, if the EM-objects are not removed from the jet list, the quoted energy scale uncertainty will be conservative relative to the lower errors associated with separate treatment of identified EM-objects.

In addition to the jet energy scale uncertainty, there are uncertainties on the jet resolution. At startup the jet resolution is estimated to be accurate to $20 \%$ of the quoted resolution based on the test-beam data and simulation studies. The dijet balancing resolution will be determined from data and will further constrain this uncertainty. It is expected that the systematics on the third jet veto and other selection criteria will limit the uncertainty on the jet resolution to $10 \%$ in the $1-10 \mathrm{fb}^{-1}$ dataset. The recommended treatment for this systematic is to add an additional smearing to the jet energy which broadens the overall jet resolution by $10 \%$. This can be done by throwing a Gaussian random number and adding an energy term which is $46 \%$ of the jet resolution. Therefore, the jet-by-jet event-by-event smearing should be done as follows:

$$
E_{\mathrm{T}}^{\text {jet }}=E_{\mathrm{T}}^{\mathrm{jet}}+\operatorname{Gaus}\left[0,0.46 * \sigma\left(E_{\mathrm{T}}, \eta\right)\right]
$$

where $\sigma\left(E_{\mathrm{T}}, \eta\right)$ is the reference jet resolution which for the central barrel is given by (using Monte Carlo simulation derived jet calibrations where $E_{\mathrm{T}}^{\mathrm{MC}}$ is equal to $E_{\mathrm{T}}^{\mathrm{rec}}$ on average)

$$
\sigma\left(E_{\mathrm{T}}^{\mathrm{jet}},|\eta|<1.4\right)=(5.8 \mathrm{GeV}) \oplus\left(1.25 * \sqrt{E_{\mathrm{T}}^{\mathrm{jet}}}\right) \oplus 0.033 * E_{\mathrm{T}}^{\mathrm{jet}}
$$

(terms added in quadrature) and Gaus $\left[0,0.46 * \sigma\left(E_{\mathrm{T}}, \eta\right)\right]$ is a randomly thrown sampling of a normal distribution per jet with a mean of zero and a width of $46 \%$ of the jet resolution and therefore $E_{\mathrm{T}}^{\prime \text { jet }}$ is the smeared jet energy to be used in the estimation of the jet resolution systematic uncertainty of the measurement. The $46 \%$ is chosen so that when added in quadrature to the nominal resolution gives an overall widening of the energy resolution of $10 \%$. The resolutions of the endcap and forward jet regions are found in [165, Table 5]. These are

$$
\begin{aligned}
& \sigma\left(E_{\mathrm{T}}^{\mathrm{jet}}, 1.4<|\eta|<3.0\right)=(4.8 \mathrm{GeV}) \oplus\left(0.89 * \sqrt{E_{\mathrm{T}}^{\mathrm{jet}}}\right) \oplus 0.043 * E_{\mathrm{T}}^{\mathrm{jet}} \\
& \sigma\left(E_{\mathrm{T}}^{\mathrm{jet}}, 3.0<|\eta|<5.0\right)=(3.8 \mathrm{GeV}) \oplus 0.085 * E_{\mathrm{T}}^{\mathrm{jet}}
\end{aligned}
$$

where for these jet resolution fits the stochastic term in the forward region is small compared to the noise and constant terms (hence the missing $\sqrt{E_{\mathrm{T}}^{\text {jet }}}$ term for $3.0<|\eta|<5.0$ ). The shift in the $+10 \%$ direction can be symmetrised to account for the $-10 \%$ shift. Otherwise, the difference between the reconstructed and generated jet energies must be reduced by $10 \%$ in order to estimate the $-10 \%$ uncertainty from the nominal Monte Carlo jet resolution. The jet resolution uncertainty is particularly important when searching for signals that are on a rapidly falling QCD multi-jet $p_{\mathrm{T}}$ spectrum.

\section{B.2.6. Heavy-flavour tagging uncertainties}

A strategy for measuring the b-tag efficiency using an enriched sample of b-jets from tt events, and its estimated precision, is described in Section 12.2.8 of [7]. The relative uncertainty on the b-efficiency measurement is expected to be about 6\% (4\%) in the barrel and $10 \%(5 \%)$ in 
the endcaps for $1 \mathrm{fb}^{-1}\left(10 \mathrm{fb}^{-1}\right)$ of integrated luminosity. These uncertainties correspond to a b-tag working point efficiency of $50 \%$.

The light-quark (and gluon) mis-tag uncertainty is expected to be larger than the $b$ efficiency uncertainty; however, for this Report a global uncertainty of 5\% is assumed for the mis-tag uncertainty. As with the efficiency determination, it is important to identify strategies to measure the mis-tagging probabilities in data as well.

Likewise, a strategy to measure the uncertainty on the efficiency for identifying $\tau$ leptons is described in Section 12.1.4 of [7], and involves comparing the ratio of $Z \rightarrow \tau \tau \rightarrow \mu+$ jet to $\mathrm{Z} \rightarrow \mu \mu$ events. With a $30 \mathrm{fb}^{-1}$ data sample, the relative uncertainty on $\tau$-tagging is estimated to be about $4 \%$. A measurement of the $\tau$ misidentification probability can be determined from a sample of $\gamma+$ jet events, and with a $10 \mathrm{fb}^{-1}$ data sample is expected to have an uncertainty at the level of 4-10\%. 


\section{Appendix C. Monte Carlo Models and Generators}

\section{C.1. Introduction}

This section presents a short description of the basic event generators used in CMS during preparation of the PTDR (see CMS "Generator Tools group" for details). A comprehensive review of the present Monte Carlo models and generators is given elsewhere [806]. Note that only MC generators used in CMS are described here, and a full description of several popular packages (like ISAJET or ACERMC, see [806]) is omitted.

There are several available Monte Carlo event generators for $p p, p A$ and $A A$ collisions, namely HERWIG [196], HIJING [807], ISAJET [672], PYTHIA [69] and SHERPA [808]. Each of these simulates a hadronic final state corresponding to some particular model of the underlying physics. The details of the implementation of the physics are different in each of these generators, however the underlying philosophy of the generators is the same.

The cross section values and the differential distribution for almost all processes are evalueated as follows:

$$
\sigma(p p \rightarrow C X)=\sum_{i j} \int f_{i}^{p}\left(x_{1}, Q^{2}\right) f_{j}^{p}\left(x_{2}, Q^{2}\right) \hat{\sigma}(i j \rightarrow C) \mathrm{d} x_{1} \mathrm{~d} x_{2},
$$

where $f_{i}^{p}\left(x, Q^{2}\right)$ are the Parton Distribution Functions (PDF) of $i$ th parton, that carried a fraction $x$ of the initial proton momentum at a scale $\left(Q^{2}\right) ; \sigma(i j \rightarrow C)$ is the cross section for the hard process (i.e. describing two partons, $i$ and $j$, interaction).

A general scheme of event generation assumes the evaluation of the hard process (the cross section value, the incoming and outgoing particle's momenta and colours), then evolves the event through a parton showering and hadronisation step, and the decay of the unstable particles. The event information (stored in /HEPEVT/ common block [69]) contains the momenta of the final hadrons, leptons and photons and positions of their decay vertexes. Typically such information contains also the characteristics (momenta, colours, KF-codes, mother's and daughter's relations) of all intermediate partons (quarks, gluons, gauge bosons, unstable physical particles, etc) that provide a trace-back the history of particle production inside of an event. By using an acceptance-rejection methods weighted events can be returned.

Parton showering is based on the expansion around the soft and collinear evolution limits and is often ascribed to either the initial or final state. The algorithm used by HERWIG and SHERPA also include some effects due to quantum interference. The events that have more energy in the parton process have more showering, and consequently more jet activity.

The collection of quarks and gluons must then be hadronised into mesons and baryons. This is done differently in each of the event generators, but is described by a set of (fragmentation) parameters that must be adjusted to agree with experimental results. HERWIG looks for colour singlet collections of quarks and gluons with low invariant mass and groups them together; this set then turns into hadrons. PYTHIA splits gluons into quark-anti-quark pairs and turns the resulting set of colour singlet quark-anti-quark pairs into hadrons via a string model. ISAJET simply fragments each quark independently paying no attention to the colour flow.

The dominant cross-section at the LHC consists of events with no hard scattering. There is little detailed theoretical understanding of these minimum-bias events and the event generators must rely on present data. These minimum-bias events are important at LHC, particularly at design luminosity, as they overlap with interesting hard-scattering events. The generators use a different approach in this case. HERWIG uses a parametrisation of data mainly from the CERN $p \bar{p}$ Collider. PYTHIA uses a mini-jet model where the jet cross-section is used at very low 


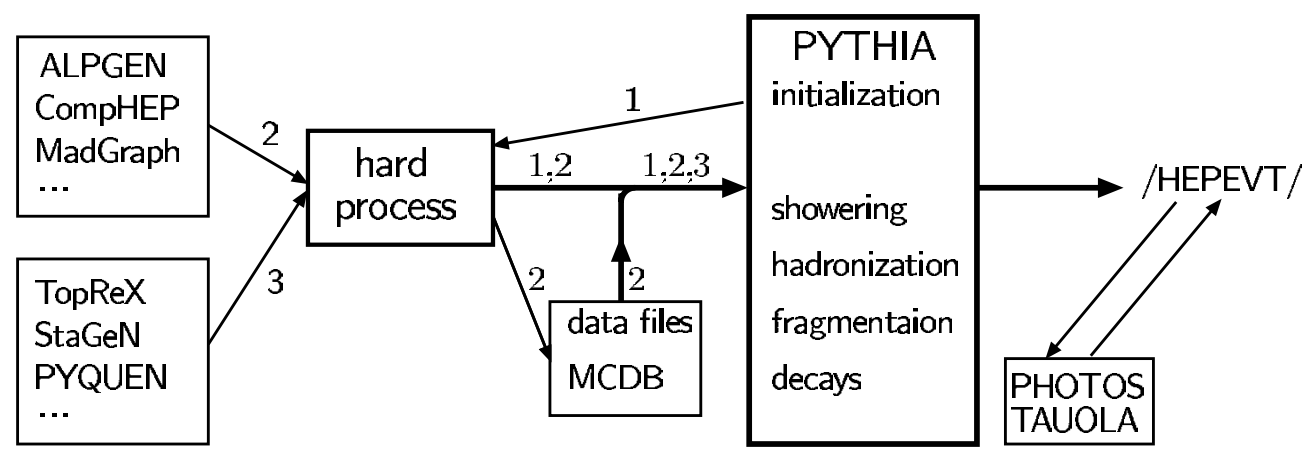

Figure C.1. Purely schematic data flow in PYTHIA and HERWIG.

transverse momenta, i.e the hard scattering process is extrapolated until it saturates the total cross-section. CMS has used the PYTHIA approach with dedicated modifications that agree with present data from Tevatron [69]. The model of the hadronic interactions implemented in the physics generator has a direct impact on physical observables such as jet multiplicity, their average transverse momentum, internal structure of the jets and their heavy flavour content. This led to the choice to use PYTHIA for most processes, allowing for a consistent set of signal and background events to be generated.

Table C. 2 presents the predicted cross-section values for the basic SM processes, as used in the simulations for PTDR. The cross-section values (at leading order) were calculated by using PYTHIA 6.327 with CTEQ5L (default PDF for PTDR) and with CTEQ6M PDFs. $\alpha_{s}$ at 1 st (2nd) order is used with CTEQ5L (CTEQ6M) PDFs. For CTEQ6M the quoted errors are related to the uncertainties due to PDFs (see Subsection B.1.9).

\section{C.2. General scheme of generator usage in CMS}

All event generators, included in CMS simulation software, can be separated into two groups.

The first group (HERWIG, HIJING, ISAJET, PYTHIA) provides the full simulation of events. The basic package explored in CMS is PYTHIA and only few specific processes were simulated with HERWIG or HIJING.

A purely schematic data flow in PYTHIA and HERWIG is presented in Fig. C.1.

After initialisation the package (HERWIG or PYTHIA) calls "hard process" routines (see "1" arrow lines in Fig. C.1). Then information (the momenta of initial and final partons, the colours and $\mathrm{KF}$-codes) is passed to package for parton showering, hadronisation, fragmentation and decays of the unstable particles.

However, all these "full event simulation" generators have very limited number of the hard process matrix elements (typically for $2 \rightarrow 2$ reaction at LO). Therefore, several special generators are used for simulation of many other LO processes. In fact, such packages generate the hard processes kinematic quantities, such as masses and momenta, the spin, the colour connection, and the flavour of initial- and final-state partons. The information is stored in the "Les Houches" format [809] (/HEPEUP/ common block) and is passed to full event simulation package like PYTHIA or HERWIG (see thick "output" line on Fig. C.1).

Three generators, namely ALPGEN [161], COMPHEP [355], and MADGRAPH [81, 493], are widely used for simulation of many processes, especially for the generation of the hard processes with multi-jet final states. For example, ALPGEN allows to generate $Q \bar{Q}$ pair 


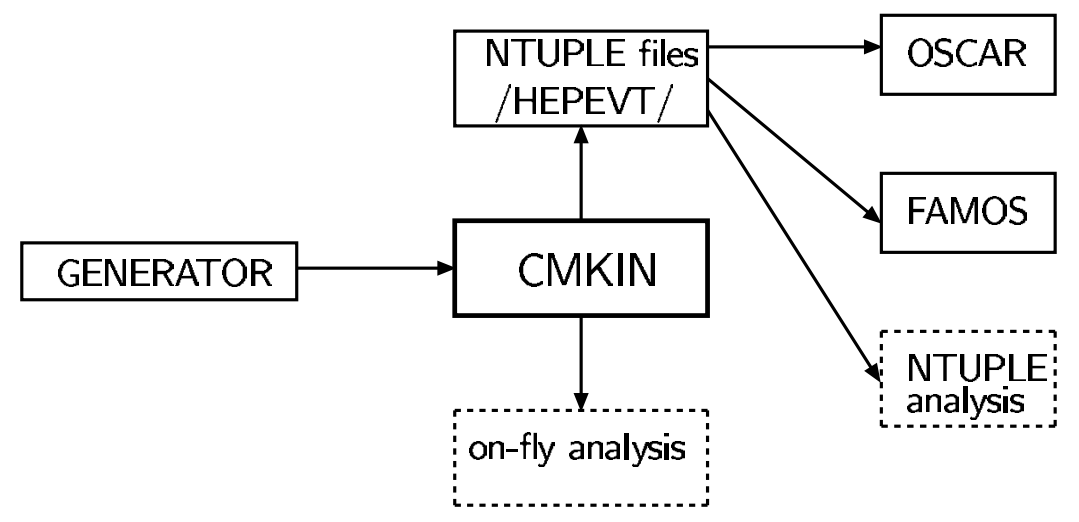

Figure C.2. Illustration of the CMKIN interface.

production with up to 6 jets. Due to the complexity of the matrix elements, describing the multi-jet processes, and a re-weighting procedure the generation of events is very CPU-time consuming. As a result, the information with kinematics is stored in the output files. (see "2" lines on Fig. C.1). Then, like in a generic PYTHIA process, such information is passed to PYTHIA (see thick "output" line on Fig. C.1).

There are several "dedicated generators", TopReX [44], StaGen, SingleTop, cosmic, SIMUb, PHASE, PYQUEN [810, 811], HYDJET [812], EDDE. These generators are used for simulation of several specific process (see below for a short description of these codes). The information with hard processes kinematic quantities is stored in /HEPEUP/ common block [809] and is passed to the "full event simulation" package (see "3" lines on Fig. C.1).

After full simulation of event with PYTHIA or HERWIG the output information is stored in the /HEPEVT/ common block. In addition two special functionality codes provide a better description of photon radiation from a charge final particles (Pнотоs [39]) and $\tau^{ \pm}$-lepton decays (TAUOLA [155]). Typically, these codes read information from /HEPEVT/ common, perform simulation and then add generated information (new particles) into the /HEPEVT/ common block (see Fig. C.1).

\section{C.3. CMKIN}

Almost all generators available in CMS could be used with the CMKIN package. Now the CMKIN is used for OSCAR and FAMOS detector simulation input. This software package provides a common interface between physics event generators and CMS detector simulation (see Fig. C.2). It also provides an environment to make physics plots of generated events. CMKIN provides an interface to a number of physics generators like PYTHIA, ISAJET and HERWIG. It also offers the possibility to use different 'external generators' like aLPGEn [161], CompHEP [355], MadGraph [81, 493] and TopReX [44]. Cosmic muon simulation is available as well. Simple particle generation is also included, i.e. single and double particles as well as simple multi particle events. The interface is based on a common block HEPEVT - a HEP standard to store particle kinematics information for one event [69]. The /HEPEVT/ common block is converted to HBOOK n-tuples. The event output format follows the HEPEVT standard and additional information can be included by the user in the block /MC_PARAM/. 
There is a unified compilation script which is used as follows:

kine_make_ntpl.com <generator> [lhapdf]

where the first parameter can have one of the following values: pythia, herwig, isajet, simple, single, double, simplemulti, cosmic, comphep, alpgen, madgraph, phase, toprex or stagen. The optional second parameter lhapdf is given when the user wants to use LHAPDF library [95].

\section{C.4. Full event simulation generators}

\section{C.4.1. PYTHIA}

The PYTHIA package [69] is a general-purpose generator for hadronic events in $\mathrm{pp}, \mathrm{e}^{+} \mathrm{e}^{-}$ and ep colliders. It contains a subprocess library and generation machinery, initial- and finalstate parton showers, underlying event, hadronisation and decays, and analysis tools. PYTHIA contains around 240 different $2 \rightarrow 2$ (and some $2 \rightarrow 1$ or $2 \rightarrow 3$ ) subprocesses, all at leading order. The subsequent decays of unstable resonances ( $W, Z$, top, Higgs, SUSY, $\ldots$ ) brings up the partonic multiplicity, for many processes with full spin correlations in the decays. The external processes can be evolved through the showering and hadronisation (like internal ones).

The final-state shower is based on forward evolution in terms of a decreasing timelike virtuality $\mathrm{m}^{2}$, with angular ordering imposed by veto. The framework is leading-log, but includes many NLL aspects such as energy-momentum conservation, $\alpha_{s}\left(p_{\perp}^{2}\right)$ and coherence. Further features include gluon polarisation effects and photon emission.

The initial-state shower is based on backward evolution, i.e. starting at the hard scattering and moving backwards in time to the shower initiators, in terms of a decreasing spacelike virtuality $Q^{2}$. Initial and final showers are matched to each other by maximum emission cones.

The composite nature of hadrons (and resolved photons) allows for several partons from each of the incoming hadrons to undergo scatterings. Such multiple parton-parton interactions are instrumental in building up the activity in the underlying event, in everything from charged multiplicity distributions and long-range correlations to minijets and jet pedestals. The interactions are described by perturbation theory, approximated by a set of more or less separate $2 \rightarrow 2$ scatterings; energy conservation and other effects introduce (anti)correlations. The scatterings are colour-connected with each other and with the beam remnants.

The Lund string model, used for hadronisation, is based on a picture with linear confinement, where (anti)quarks or other colour (anti)triplets are located at the ends of the string, and gluons are energy and momentum carrying kinks on the string. The string breaks by the production of new $q \bar{q}$ pairs, and a quark from one break can combine with an anti-quark from an adjacent one to form a colour singlet meson.

Unstable particles are allowed to decay. In cases where better decay models are available elsewhere, e.g. for $\tau^{ \pm}$with spin information or for $B$ hadrons, such decays can be delegated to specialised packages.

At present the parameters from almost all PYTHIA common blocks (see BLOCK DATA PYDATA) could be set via data cards. With the CMKIN these parameters could be set in data card file with the following format (note, that only capital letters should be used):

\begin{tabular}{|c|c|c|}
\hline PYTHIA & CMKIN & COMMENT \\
\hline \multicolumn{3}{|c|}{ parameter } \\
\hline MSEL $=6$ & MSEL6 & $t \bar{t}$ production \\
\hline \multicolumn{3}{|c|}{ one- and two-dimensional arrays } \\
\hline $\operatorname{CKIN}(1)=100$ & CKIN1 = 100 & $\min \cdot \sqrt{\hat{s}}$ \\
\hline i.e. $\operatorname{PMAS}(6,1)=178$ & PMAS6, $1=178$ & top-quark mass \\
\hline
\end{tabular}


- Common cards for CMKIN

Below we present a list of PYTHIA parameters used for full event simulation for PTDR. Some of these parameters correspond to the old multiple interactions scenario, namely Tune A [813].

$\operatorname{MSTP}(2)=1: 1$ (first) $/ 2$ (second) order running $\alpha_{s}$

$\operatorname{MSTP}(33)=0:$ do not include of $K$-factors in hard cross sections

$\operatorname{MSTP}(51)=7:$ PDF set (here is CTEQ5L)

$\operatorname{MSTP}(81)=1:$ multiple parton interactions is switched ON

$\operatorname{MSTP}(82)=4:$ defines the multiple parton interactions model

$\operatorname{PARP}(67)=1:$ amount of initial-state radiation

$\operatorname{PARP}(82)=1.9: P_{\mathrm{T}}$ cut-off for multi-parton interactions

$\operatorname{PARP}(83)=0.5:$ fraction of total hadronic matter in core

$\operatorname{PARP}(84)=0.4:$ radius of core

$\operatorname{PARP}(85)=0.33:$ gluon production mechanism in multiple interactions

$\operatorname{PARP}(86)=0.66:$ gluon prod. mechanism in multiple interactions

$\operatorname{PARP}(88)=0.5$

$\operatorname{PARP}(89)=1000$ : reference energy scale for which $\operatorname{PARP}(82)$ is set

$\operatorname{PARP}(90)=0.160:$ effective $P_{\mathrm{T}}$ cut - off $=[\operatorname{PARP}(82) / \operatorname{PARP}(89)]^{* *} \operatorname{PARP}(90)$

$\operatorname{PARP}(91)=1.0:$ width of Gaussian primordial $k_{\perp}$ distribution inside hadron

$\operatorname{PARJ}(71)=10:$ maximum average $c \tau$ for particles allowed to decay

$\operatorname{MSTJ}(11)=3:$ choice of the fragmentation function

$\operatorname{MSTJ}(22)=2:$ allow to decay those unstable particles

$\operatorname{PMAS}(5,1)=4.8:$ the mass of the $b$-quark

$\operatorname{PMAS}(6,1)=175.0:$ the mass of the $t$-quark

\section{C.4.2. HERWIG}

HERWIG contains a wide range of Standard Model, Higgs and supersymmetric processes [196]. HERWIG uses the parton-shower approach for initial- and final-state QCD radiation, including colour coherence effects and azimuthal correlations both within and between the jets.

In the treatment of supersymmetric processes, HERWIG itself doesn't calculate the SUSY mass spectrum or decay rates, but reads in an input file containing the low-energy parameters (masses, couplings, decays, ....). This file can be written by hand or more conveniently be generated with the ISAWIG program. This program provides an interface to ISAJET (and therefore to all models in ISASUSY and ISASUGRA), to HDECAY (for NLO Higgs decays), and can also add R-parity violating decays.

Colour coherence effects of (initial and final) partons are taken into account in all hard subprocesses, including the production and decay of heavy quarks and supersymmetric particles. HERWIG uses the angular ordered parton shower algorithm which resumes both soft and collinear singularities. HERWIG includes spin correlation effects in the production and decay of top quarks, tau leptons and supersymmetric particles. For the SUSY decays, there is an option for using either the matrix elements (fast) or the full spin correlations. HERWIG uses a cluster hadronisation model based on non-perturbative gluon splitting, and a similar cluster model for soft and underlying hadronic events. This model gives a good agreement with the LEP data on event shapes, but does not fit the identified particle spectrum well.

\section{C.4.3. ISAJET}

ISAJET is a Monte Carlo program which simulates $p p, p \bar{p}, e^{+} e^{-}$interactions at high energies [672]. ISAJET is based on perturbative QCD plus phenomenological models for parton and beam jet fragmentation. At CMS ISAJET is used for calculations of SUSY parameters. 


\section{C.4.4. HIJING}

Hard or semi-hard parton scatterings with transverse momentum of a few $\mathrm{GeV} / \mathrm{c}$ are expected to dominate high energy heavy ion collisions. The HIJING (Heavy Ion Jet INteraction Generator) Monte Carlo model [807] was developed by M Gyulassy and X-N Wang with special emphasis on the role of minijets in $p p, p A$ and $A A$ reactions at collider energies.

Detailed systematic comparison of HIJING results with a very wide range of data demonstrates that a quantitative understanding of the interplay between soft string dynamics and hard QCD interaction has been achieved. In particular, HIJING reproduces many inclusive spectra two particle correlations, and can explain the observed flavour and multiplicity dependence of the average transverse momentum.

\section{C.5. Tree level matrix element generators}

\section{C.5.1. ALPGEN}

ALPGEN is designed for the generation of Standard Model processes in hadronic collisions, with emphasis on final states with large jet multiplicities [161]. It is based on the exact leading order evaluation of partonic matrix elements and $t$ and gauge boson decays with helicity correlations. The code generates events in both a weighted and unweighted mode. Weighted generation allows for high-statistics parton-level studies. Unweighted events can be processed in an independent run through shower evolution and hadronisation programs.

The current available processes are:

- $W / Z / H Q \bar{Q}+N$ jets $(Q=c, b, t)$ with $N \leqslant 4$

- $Q \bar{Q}+N$ jets, with $N \leqslant 6$

- $Q \bar{Q} Q^{\prime} \bar{Q}^{\prime}+N$ jets, with $N \leqslant 4$

- $W+$ charm $+N$ jets, with $N \leqslant 5$

- $N$ jets, $W / Z+N$ jets, with $N \leqslant 6$

- $n W+m Z+l H+N$ jets, with $n+m+l+N \leqslant 8, N \leqslant 3$

- $N \gamma+M$ jets, with $N \geqslant 1, N+M \leqslant 8$ and $M \leqslant 6$

- $H+N$ jets $(N \leqslant 4)$, with the Higgs produced via $g g H$ vertex

- single top production.

\section{C.5.2. COMPHEP}

CoMPHEP [814] is a package for evaluating Feynman diagrams, integrating over multiparticle phase space and generating events with a high level of automation. CoMPHEP includes the Feynman rules for SM and several versions of MSSM (SUGRA, GMSB, MSSM with R-parity violation).

COMPHEP computes squared Feynman diagrams symbolically and then numerically calculates cross sections and distributions. After numerical computation one can generate the unweighted events with implemented colour flow information. The events are in the form of the Les Houches Accord event record [809] to be used in the PYTHIA program for showering and hadronisation.

COMPHEP allows for the computation of scattering processes with up to 6 particles and decay processes with up to 7 particles in the final state. 


\section{C.5.3. MADGRAPH and MADEVENT}

MADEVENT [81] is a multi-purpose, tree-level event generator which is powered by the matrix element generator MADGRAPH [493]. Given a user process, MADGRAPH automatically generates the amplitudes for all the relevant subprocesses and produces the mappings for the integration over the phase space. This process-dependent information is packaged into MADEVENT, and a stand-alone code is produced. It allows the user to calculate cross sections and to obtain unweighted events automatically. Once the events have been generated - event information, (e.g. particle id's, momenta, spin, colour connections) is stored in the "Les Houches" format [809]. Events may be passed directly to a shower Monte Carlo program (interfaces are available for HERWIG and PYTHIA).

The limitation of the code are related to the maximum number of final state QCD particles. Currently, the package is limited to ten thousand diagrams per subprocess. So, for example, $W+5$ jets is close to its practical limit. At present, only the Standard Model Feynman rules are implemented and the user has to provide his/her own rules for beyond Standard Model physics, such as MSSM.

\section{C.5.4. TopReX}

The event generator TopREX [44] provides the simulation of several important processes in $p p$ and $p \bar{p}$ collisions, not implemented in PYTHIA. In the matrix elements used in TopREX the decays of the final $t$-quarks, $W^{ \pm}, Z$ and charged Higgs bosons are also included. The final top quark could decay into SM channel $\left(t \rightarrow q W^{+}, q=d, s, b\right), b$-quark and charged Higgs $\left(t \rightarrow b H^{+}\right)$and the channels with flavour changing neutral current (FCNC): $t \rightarrow u(c) V$, $V=g, \gamma, Z$. The implemented matrix elements take into account spin polarisations of the top quark, that provides a correct description of the differential distributions and correlations of the top quarks decay products.

\section{C.6. Supplementary packages}

\section{C.6.1. РнотоS}

PHoTos is a universal package to simulate QED photon radiative corrections [39]. The precision of the generation may in some cases be limited, in general it is not worse than the complete double bremsstrahlung in LL approximation. The infrared limit of the distributions is also correctly reproduced. The action of the algorithm consists of generating, with internally calculated probability, bremsstrahlung photon(s), which are later added to the /HEPEVT/ record. Kinematic configurations are appropriately modified. Energy-momentum conservation is assured. When using PHOTOS, the QED bremsstrahlung of the principal generator must be switched off. For example in case of PYTHIA one has to use MSTJ 41=1.

\section{C.6.2. TAUOLA}

TAUOLA is a package for simulation of the $\tau^{ \pm}$-lepton decays [155]. It uses the PHOTOs package to simulate radiative corrections in the decay. The TAUOLA interface is made with the PYTHIA generator. This interface evaluates also the position of $\tau$-lepton decay (i.e. the information on the production vertex of the decay products of $\tau$-lepton). 


\section{C.6.3. PYQUEN}

The event generator PYQUEN (PYthia QUENched) [810, 811] provides the simulation of rescattering and energy loss of hard partons in dense QCD-matter (quark-gluon plasma) created in ultrarelativistic heavy ion collisions. The approach relies on an accumulative energy losses, when gluon radiation is associated with each scattering in expanding medium together including the interference effect by the modified radiation spectrum $d E / d \ell$ as a function of decreasing temperature $T$. The model is implemented as fast Monte Carlo tool, to modify standard PYTHIA jet event.

\section{C.6.4. HYDJET}

The event generator HYDJET [812] (HYDrodynamics + JETs) provides the fast simulation of heavy ion events at LHC energy including longitudinal, transverse and elliptic flow effects together with jet production and jet quenching (rescattering and energy loss of hard partons in dense QCD-matter, quark-gluon plasma). The model merges a fast generator of flow effects HYDRO [815] with PYTHIA (for jet production) and PYQUEN [810, 811] (for jet quenching) by simulating full heavy ion event as a superposition of soft, hydro-type state and hard multi-jets.

First of all, HYDJET calculates the number $N^{\text {hard }}$ of hard nucleon-nucleon sub-collisions and number $N^{\text {part }}$ nucleons-participants (at given impact parameter $b$ of $A A$ collision and minimum $P_{\mathrm{T}}$ of hard parton scattering) and generates the initial parton spectra by calling PYTHIA $N^{\text {hard }}$ times (fragmentation off). After each jet parton affected by medium-induced rescattering and energy loss according with PYQUEN model. In the end of each PYTHIA subevent adding new (in-medium emitted) gluons into PYTHIA parton list and rearrangements of partons to update string formation are performed. Then PYQUEN forms final hadrons with PYEXEC subroutine (fragmentation on). Finally, HYDJET calculates the multiplicity of soft, hydro-induced part of the event and add new particles in the end of the event record.

\section{C.7. K-factors for dilepton production}

Some event generators such as PYTHIA do not employ the most advanced matrix-element calculations. They must be reasonably fast since in most applications, many millions of events must be generated. Experimenters apply an ad-hoc correction or "kludge" called the $K$-factor so that the cross-section value used for, say, the production of muon pairs, is correct. This $K$-factor amounts to the ratio of a highly accurate cross-section calculation to a less accurate one, typically a leading-order calculation:

$$
K_{\mathrm{NLO}}=\frac{\sigma_{\mathrm{NLO}}}{\sigma_{\mathrm{LO}}} \quad \text { and } \quad K_{\mathrm{NNLO}}=\frac{\sigma_{\mathrm{NNLO}}}{\sigma_{\mathrm{LO}}} .
$$

Clearly the $K$-factor reflects the accuracy of the better theoretical calculation, and there can be significant differences between $K_{\mathrm{NNLO}}$ and $K_{\mathrm{NLO}}$. The most significant contributions to the $K$-factor come from QCD radiative corrections are expected to be on the order of $10 \%$ or more. Usually one does not include electroweak radiative corrections in the $K$-factor.

We have examined the $K$-factor for the Drell-Yan production of charged lepton pairs, as well as the signal for new $Z^{\prime}$ neutral gauge bosons. The program PHOZPRMs is used to compute mass-dependent cross-sections [348], and a generalised version called WUWD is used to study $Z^{\prime}$ cross-sections [816]. We checked carefully the differential cross-section, $d \sigma / d M$ obtained from PHOZPRMS with the program RESBOS [817, 818] and found very good agreement. We use the MRST parton distribution functions [819] for these calculations. Very similar results are obtained using CTEQ6M [12]. 


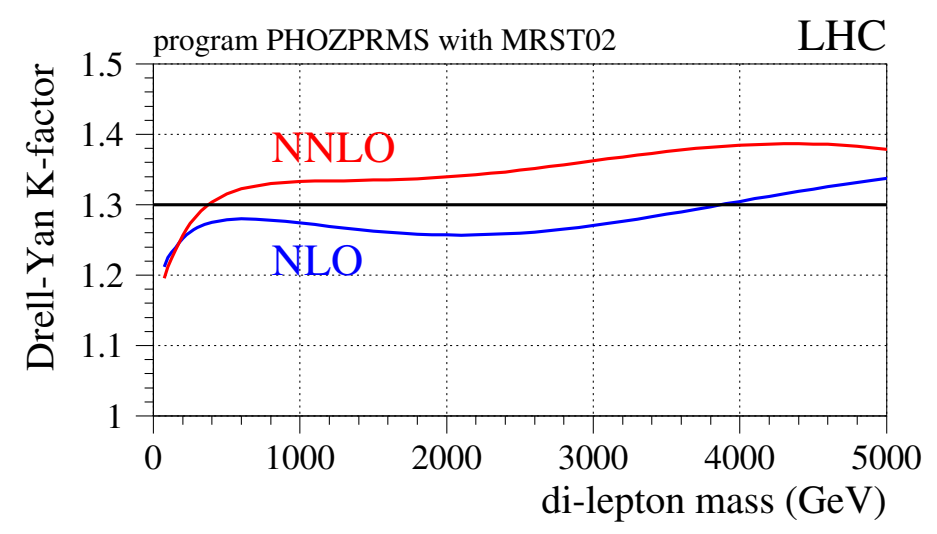

Figure C.3. $K$-factors as a function of mass for the LHC.

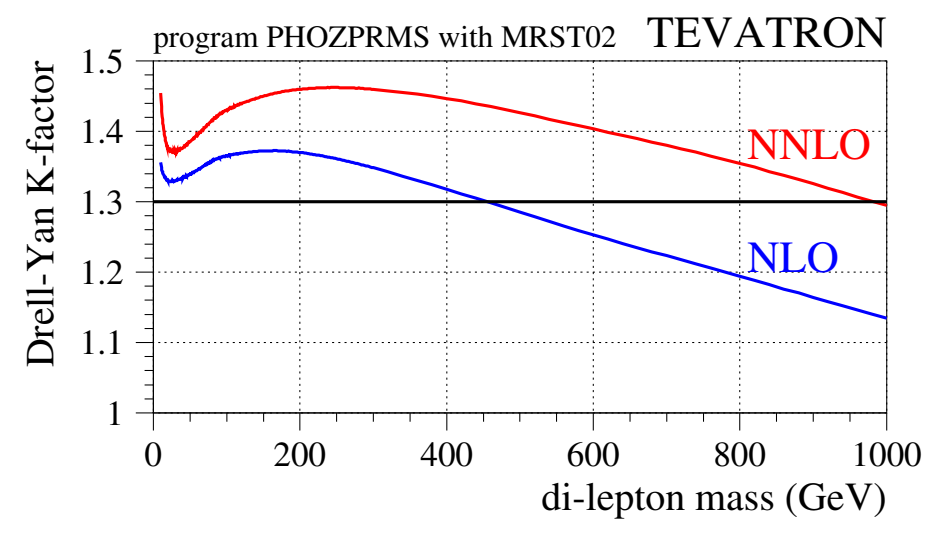

Figure C.4. $K$-factors as a function of mass for the Tevatron.

Usually experimenters use a constant value for the $K$-factor, but in fact this is not accurate. The variation of the $K$-factor with mass is substantial, as shown in Fig. C.3. (There is a similar, though different, variation in the $K$-factor for Drell-Yan production at the Tevatron - see Fig. C.4.) Notice that $K_{\mathrm{NLO}} \neq K_{\mathrm{NNLO}}$, in general, and the difference can be as large as $7 \%$. A number of values for the $K$-factor are listed in Table C.1.

It is customary to take the difference $K_{\mathrm{NNLO}}-K_{\mathrm{NLO}}$ as a measure of the theoretical uncertainty due to missing higher orders. According to the results obtained with PHOzPRMS, this uncertainty is on the order of $5 \%$. It is interesting to compare this to the uncertainty coming from the parton distribution functions (PDFs). We used the CTEQ6M set which contains "error" PDFs with which one can estimate this uncertainty [12]. The relative uncertainty of the Drell-Yan cross-section as a function of mass is shown in Fig. C.5. The positive and negative variations of the cross-section were summed separately. The error bands show the full uncertainty obtained from the twenty error-PDFs - no rescaling was done to take into account the fact that these error-PDF's correspond to $2 \sigma$ variations of the PDF parameters. One sees that the PDF uncertainty varies from about $3 \%$ at low masses to $20 \%$ toward the upper reach of the LHC. Of course, these uncertainties will be reduces as data from HERA, the Tevatron and fixed-target experiments are used to improve the PDFs. 
Table C.1. Values for $K_{\mathrm{NNLO}}, K_{\mathrm{NLO}}$ and $K_{\mathrm{NNLO}} / K_{\mathrm{NLO}}$ as a function of mass.

\begin{tabular}{llll}
\hline mass $\left(\mathrm{GeV} / \mathrm{c}^{2}\right)$ & $K_{\mathrm{NNLO}}$ & $K_{\mathrm{NLO}}$ & $K_{\mathrm{NNLO}} / K_{\mathrm{NLO}}$ \\
\hline 100 & 1.212 & 1.225 & 0.989 \\
200 & 1.256 & 1.252 & 1.003 \\
300 & 1.286 & 1.268 & 1.014 \\
400 & 1.303 & 1.275 & 1.022 \\
600 & 1.323 & 1.280 & 1.033 \\
800 & 1.330 & 1.278 & 1.040 \\
1000 & 1.333 & 1.274 & 1.046 \\
2000 & 1.339 & 1.257 & 1.065 \\
3000 & 1.362 & 1.270 & 1.073 \\
4000 & 1.385 & 1.304 & 1.061 \\
5000 & 1.378 & 1.338 & 1.031 \\
\hline
\end{tabular}

Table C.2. Leading order cross sections for some typical process at the LHC calculated by using PYTHIA 6.327 with CTEQ5L (default PDF for PTDR) and with CTEQ6M PDFs. $P_{0}$ denotes $\hat{p}_{\mathrm{T}}$-min. for the hard process.

\begin{tabular}{|c|c|c|c|}
\hline process & cross section & comment & \\
\hline$\sigma_{\text {tot }}(p p \rightarrow X)$ & $110 \pm 10 \mathrm{mb}$ & different models & \\
\hline$\sigma_{\text {tot }}(p p \rightarrow X)$ & $111.5 \pm 1.2_{-2.1}^{+4.1} \mathrm{mb}$ & COMPETE Coll. & \\
\hline process & CTEQ5L & CTEQ6M & comment \\
\hline$Z$-boson & $48.69 \mathrm{nb}$ & $50.1_{-4.76 \%}^{+4.19 \%} \mathrm{nb}$ & \\
\hline$Z+\operatorname{jet}(g+q)$ & $13.94 \mathrm{nb}$ & $12.73_{-3.94 \%}^{+3.16 \%} \mathrm{nb}$ & $P_{0}=20 \mathrm{GeV}$ \\
\hline$q \bar{q} \rightarrow Z \gamma$ & $44.21 \mathrm{pb}$ & $46.7_{-4.22 \%}^{+3.93 \%} \mathrm{nb}$ & $P_{0}=20 \mathrm{GeV}$ \\
\hline$W^{ \pm}$-boson & $158.5 \mathrm{pb}$ & $161.3_{-4.93 \%}^{+4.32 \%} \mathrm{nb}$ & \\
\hline$W^{ \pm}+\operatorname{jet}(g+q)$ & $41.42 \mathrm{nb}$ & $37.24_{-4.10 \%}^{+3.34 \%} \mathrm{nb}$ & $P_{0}=20 \mathrm{GeV}$ \\
\hline$W^{ \pm} \gamma$ & $56.21 \mathrm{pb}$ & $56.42_{-4.38 \%}^{+4.11 \%} \mathrm{nb}$ & $P_{0}=20 \mathrm{GeV}$ \\
\hline$W^{+} W^{-}$ & $69.69 \mathrm{pb}$ & $75.0_{-4.03 \%}^{+3.87 \%} \mathrm{pb}$ & \\
\hline$W^{ \pm} Z$ & $26.69 \mathrm{pb}$ & $28.76_{-4.03 \%}^{+3.93 \%} \mathrm{pb}$ & \\
\hline$q \bar{q} \rightarrow Z Z$ & $11.10 \mathrm{pb}$ & $10.78_{-4.21 \%}^{+4.02 \%} \mathrm{pb}$ & \\
\hline$W Q \bar{Q}$ & \multicolumn{3}{|c|}{$m_{b}=4.8 \mathrm{GeV}, m_{c}=1.5 \mathrm{GeV}$, TopReX } \\
\hline$W^{ \pm} c \bar{c}$ & $1215 \mathrm{pb}$ & $1086_{-4.53 \%}^{+4.12 \%} \mathrm{pb}$ & $M_{c \bar{c}} \geqslant 3.0 \mathrm{GeV}$ \\
\hline$W^{ \pm} c \bar{c}$ & $33.5 \mathrm{pb}$ & $31.3_{-4.18 \%}^{+4.00 \%} \mathrm{pb}$ & $M_{c \bar{c}} \geqslant 50 \mathrm{GeV}$ \\
\hline$W^{ \pm} b \bar{b}$ & $328 \mathrm{pb}$ & $297_{-4.37 \%}^{+4.04 \%} \mathrm{pb}$ & $M_{b \bar{b}} \geqslant 9.6 \mathrm{GeV}$ \\
\hline$W^{ \pm} b \bar{b}$ & $34.0 \mathrm{pb}$ & $31.3_{-4.18 \%}^{+4.00 \%} \mathrm{pb}$ & $M_{b \bar{b}} \geqslant 50 \mathrm{GeV}$ \\
\hline$Z b \bar{b}, m_{b}=4.62 \mathrm{GeV}$ & $789.6 \pm 3.66 \mathrm{pb}$ & MCFM & $M_{b \bar{b}} \geqslant 9.24 \mathrm{GeV}$ \\
\hline dijet processes & $819 \mu \mathrm{b}$ & $583_{-6.02 \%}^{+4.78 \%} \mu \mathrm{b}$ & $P_{0}=20 \mathrm{GeV}$ \\
\hline$\gamma+$ jet & $182 \mathrm{nb}$ & $135_{-6.14 \%}^{+4.92 \%} \mathrm{nb}$ & $P_{0}=20 \mathrm{GeV}$ \\
\hline$\gamma \gamma$ & $164 \mathrm{pb}$ & $137_{-5.65 \%}^{+4.14 \%} \mathrm{pb}$ & $P_{0}=20 \mathrm{GeV}$ \\
\hline$b \bar{b}, m_{b}=4.8 \mathrm{GeV}$ & $479 \mu \mathrm{b}$ & $187_{-13.2 \%}^{+9.7 \%} \mu \mathrm{b}$ & \\
\hline$t \bar{t}, m_{t}=175 \mathrm{GeV}$ & $488 \mathrm{pb}$ & $493_{-3.31 \%}^{+3.24 \%} \mathrm{pb}$ & \\
\hline$t \bar{t}, m_{t}=175 \mathrm{GeV}$ & $830 \pm 90 \mathrm{pb}$ & NLO+NNLO & \\
\hline$t \bar{t} b \bar{b}$ & $10 \mathrm{pb}$ & & AcerMC 1.2 \\
\hline inclusive Higgs & $m_{H}=150 \mathrm{GeV}$ & $23.8 \mathrm{pb}$ & \\
\hline inclusive Higgs & $m_{H}=500 \mathrm{GeV}$ & $3.8 \mathrm{pb}$ & \\
\hline
\end{tabular}

The variation of the $K$-factors with mass comes in part because of the $Z$-resonance. The size of the $Z$-peak relative to the continuum production of lepton pairs is therefore relevant. This relative size depends on the coupling of the $Z$-boson to the up and down quarks in 


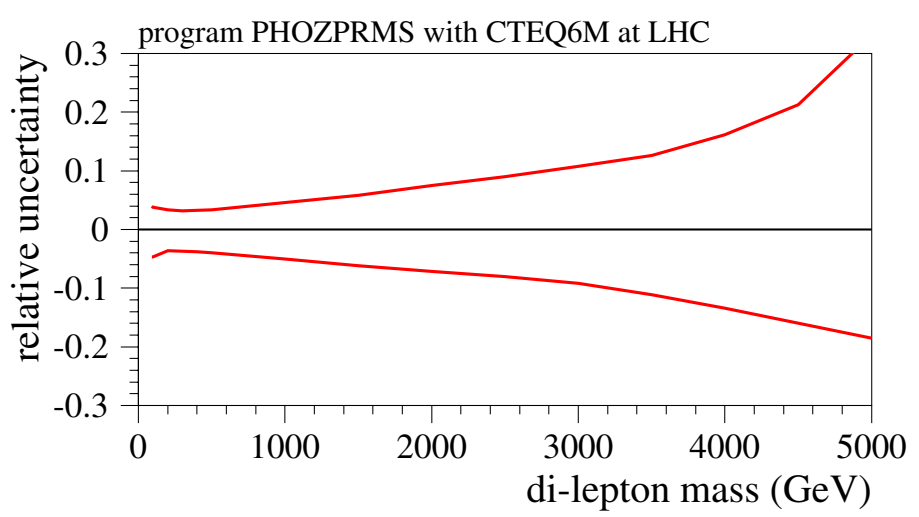

Figure C.5. Uncertainty from the parton distribution functions, evaluated using the CTEQ6M set.

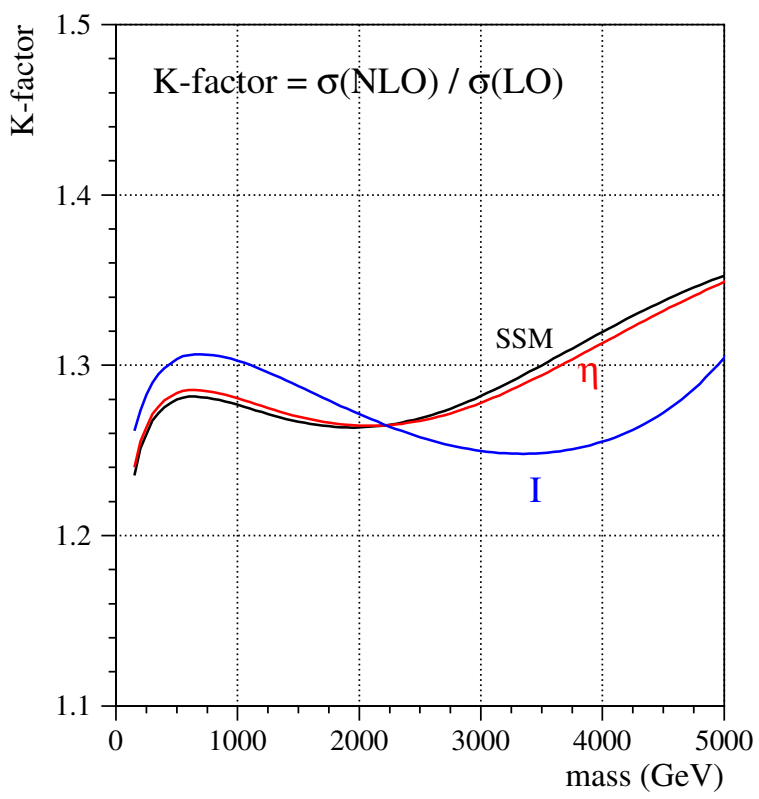

Figure C.6. $K$-factors as a function of mass of a new $Z^{\prime}$ resonance, for two cases: $\eta$ and $I$ (see text). The curve 'SSM' refers to a sequential Standard Model $Z$ '.

the proton. There is practically no uncertainty on those couplings, and they are completely determined in the Standard Model. However, if a new $Z^{\prime}$ resonances is present, its couplings will not be known a priori. Thus it is interesting to consider to what extent the $K$-factor will depend on those couplings.

We have considered two examples of possible $Z^{\prime}$ resonances, and computed $K_{\mathrm{NLO}}$ as a function of the resonance mass, as shown in Fig. C.6. The first model, labelled " $\eta$," illustrates the case of a $Z^{\prime}$ which couples primarily to up-quarks, and the second one, labelled "I," couples mainly to down-quarks [816]. As is clear from the figure, the radiative corrections as a function of mass are quite different in these two extreme cases. Thus, there will be an ambiguity in the cross-section measurement of a new $Z^{\prime}$ resonance at the level of about $5 \%$ until the relative couplings of that $Z^{\prime}$ to up and down quarks can be established. 


\section{Appendix D. GARCON: Genetic Algorithm for Rectangular Cuts OptimizatioN}

Typically HEP analysis has quite a few selection criteria (cuts) to optimise for example a significance of the "signal" over "background" events: transverse energy/momenta cuts, missing transverse energy, angular correlations, isolation and impact parameters, etc. In such cases simple scan over multi-dimensional cuts space (especially when done on top of a scan over theoretical predictions parameters space like for SUSY e.g.) leads to CPU time demand varying from days to many years... One of the alternative methods, which solves the issue is to employ a Genetic Algorithm (GA), see e.g. [820-822].

We wrote a code, GARCON [63], which automatically performs an optimisation and results stability verification effectively trying $\sim 10^{50}$ cut set parameters/values permutations for millions of input events in hours time. Examples of analyses are presented in this Physics TDR; see, for example, Sections 3.1, 8.4.1, 13.6, 13.7, 13.14 and recent papers $[51,317,675,676]$.

The GARCON program among many other features allows user:

- to select an optimisation function among known significance estimators, as well as to define user's own formula, which may be as simple as signal to background ratio, or a complicated one including different systematic uncertainties separately on different signal and background processes, different weights per event and so on;

- to define a precision of the optimisation;

- to restrict the optimisation using different kind of requirements, such us minimum number of signal/background events to survive after final cuts, variables/processes to be used for a particular optimisation run, number of optimisations inside one run to ensure that optimisation converges/finds not just a local maximum(s), but a global one as well (in case of a complicated phase space);

- to automatically verify results stability.

GARCON, like GA-based programs in general, exploits evolution-kind algorithms and uses evolution-like terms:

- Individual is a set of qualities, which are to be optimised in a particular environment or set of requirements. In HEP analysis case Individual is a set of lower and upper rectangular cut values for each of variables under study/optimization.

- Environment or set of requirements of evolutionary process in HEP analysis case is a Quality Function (QF) used for optimisation of individuals. The better QF value the better is an Individual. Quality Function may be as simple as $S / \sqrt{B}$, where $S$ is a number of signal events and $\mathrm{B}$ is a total number of background events after cuts, or almost of any degree of complexity, including systematic uncertainties on different backgrounds, etc.

- A given number of individuals constitute a Community, which is involved in evolution process.

- Each individual involved in the evolution: breeding with possibility of mutation of new individuals, death, etc. The higher is the QF of a particular individual, the more chances this individual has to participate in breeding of new individuals and the longer it lives (participates in more breeding cycles, etc.), thus improving community as a whole.

- Breeding in HEP analysis example is a producing of a new individual with qualities (set of $\mathrm{min} / \mathrm{max}$ cut values) taken in a defined way from two "parent" individuals.

- Death of an individual happens, when it passes over an age limit for it's quality: the bigger it's quality, the more it lives. 
- Cataclysmic Updates may happen in evolution after a long period of stagnation in evolution, at this time the whole community gets renewed and gets another chance to evolve to even better quality level. In HEP analysis case it corresponds to a chance to find another local and ultimately a global maximum in terms of quality function. Obviously, the more complicated phase space of cut variables is used the more chances exist that there are several local maximums in quality function optimisation.

- There are some other algorithms involved into GAs. For example mutation of a new individual. In this case newly "born" individual has not just qualities of its "parents", but also some variations, which in terms of HEP analysis example helps evolution to find a global maximum, with less chances to fall into a local one. There are also random creation mechanisms serving the same purpose.

There is nothing special involved in GARCON input preparation. One would need to prepare a set of arrays for each background and a signal process of cut variable values for optimisation. Similar to what is needed to have to perform a classical eye-balling cut optimisation.

In comparison to other automatised optimisation methods GARCON output is transparent to user: it just says what rectangular cut values are optimal and recommended in an analysis. Interpretation of these cut values is absolutely the same as with eye-balling cuts when one selects a set of rectangular cut values for each variable in a "classical" way by eye.

All-in-all it is a simple yet powerful ready-to-use tool with flexible and transparent optimisation and verification parameters setup. It is publicly available along with a paper on it [63] consisting of an example case study and user's manual. 


\section{Appendix E. Online Selection}

\section{E.1. Introduction}

The CMS trigger menu depends upon the luminosity delivered by the LHC and the available bandwidth between and out of the systems. The LHC luminosity is expected to start at $\mathcal{L}=10^{32} \mathrm{~cm}^{-2} \mathrm{~s}^{-1}$ in 2007 and gradually rise to $\mathcal{L}=10^{34} \mathrm{~cm}^{-2} \mathrm{~s}^{-1}$ by 2010 . The CMS data acquisition can be operated with one to eight slices of Event Filter Farms that execute HighLevel Trigger (HLT) algorithms. It is expected that we start with one slice in 2007, allowing a bandwidth of $12.5 \mathrm{kHz}$ between Level-1 and HLT, and build up to the full eight slices by 2010, when the Level-1 to HLT bandwidth can be raised to $100 \mathrm{kHz}$. It is assumed that the data logging capability after the HLT selection will remain constant at a rate between $100 \mathrm{~Hz}$ to $150 \mathrm{~Hz}^{54}$. The Level-1 and HLT algorithms will be configured to operate with the lowest possible thresholds making the best use of the available bandwidth.

Here we focus solely on trigger studies for $\mathcal{L}=2 \times 10^{33} \mathrm{~cm}^{-2} \mathrm{~s}^{-1}$. The scenario of operation assumes that CMS uses four DAQ slices capable of $50 \mathrm{kHz}$. While the actual choice of trigger thresholds, especially at HLT, depends strongly upon the physics of interest at the time of operation, we propose here an example set of trigger menus within the constraints of the data acquisition system. An effort has been made to optimise the Level-1 and HLT thresholds coherently, taking into account possible bandwidth limitations.

The structure of this note is as follows: first we overview the object-identification algorithms used for these studies. The emphasis is given to the changes that have been introduced since a similar study was performed in the DAQ TDR [76]. We then introduce a series of new trigger paths, aiming at increasing the event yield for various physics analyses. The central idea is to exploit various multi-object (or cross-channel) triggers in an attempt to improve the rejection and, at the same time, lower the kinematic thresholds of the corresponding objects. We finally present the performance of the triggers, and we calculate the overlap among them and the total HLT output rate.

\section{E.2. Description of trigger tools}

\section{E.2.1. Level-1 reconstruction}

There have been no significant changes in the Level-1 algorithms since the DAQ TDR. We have introduced an $H_{\mathrm{T}}$ algorithm which sums the corrected jet $E_{\mathrm{T}}$ of all the jets found above a programmable threshold, within $|\eta|<5$. It does not account for $E_{\mathrm{T}}$ carried by muons and neutrinos.

The Level-1 strategy is the following: We have made an effort to keep the thresholds at the same levels, or even reduce them in order to be able to study cross-channel triggers (typically appearing with lower kinematic cuts). The notable exception is the tau triggers, where an increase in the HCAL noise and the usage of a new pile-up model in the simulation do affect the Level-1 $\tau$ identification tools, and therefore the related trigger rates. We have introduced additional Level-1 conditions for all HLT paths. The determination of thresholds and prescales is a compromise between the desire to distribute reasonably the available L1 bandwidth to the various triggers, and the need to optimise the L1 and HLT thresholds coherently in well-defined trigger paths.

\footnotetext{
54 At the time of the writing of this document, several scenarios for the HLT output rate, the disk requirements for the storage manager and the associated cost are under discussion.
} 


\section{E.2.2. HLT reconstruction}

Well defined Level-1 terms are used in order to obtain triggers whose behaviour and efficiency can be studied with real data. We have replaced some of the Level-1 conditions with respect to the DAQ TDR with new Level-1 terms when this leads to more reasonable trigger paths or triggers that are more stable and carry less of a bias. The optimisation of the thresholds for the various triggers has been a compromise between the physics needs of the CMS experiment and the total HLT rate available. This study serves only as an intermediate step in a long-term trigger study project. Further improvements in the reconstruction tools, better optimisation of the thresholds, implementation of additional triggers and a CMS-wide discussion of the allocation of the HLT bandwidth to the physics groups according to the priorities of the experiment, are foreseen.

A general and detailed description of the HLT system can be found in Ref. [76]. Here we summarise the recent modifications of the HLT tools, and the expected changes in the rates of the various triggers with respect to the earlier studies.

- Muons: The muon algorithm has not changed, with the exception of the drift-tube local reconstruction and segment building. Therefore, no significant changes in the rates of single- and dimuon trigger paths are expected. The option of constructing muon triggers without isolation has been added.

- Electrons-Photons. Here the most important change is that all saturated trigger towers at Level-1 are now considered isolated. This increases both the signal efficiency and the background. At HLT, the photon rate can be reduced by increasing the thresholds or by applying some isolation cuts. For the electrons the options include a matching with pixel lines and tracks, as well as isolation requirements in the hadron calorimeter and the tracker. A study of the algorithm optimisation can be found in Ref. [7]. An improvement of the rejection power of the electron-photon algorithms is achieved with a simultaneous decrease of the HLT thresholds. Similar enhancements are expected for cross-channel triggers where one of the objects under consideration is an electron or a photon.

- Jets and $\boldsymbol{E}_{\mathbf{T}}^{\mathrm{miss}}$. The main jet-finder algorithm (Iterative Cone with $R=0.5$ ) has not been modified. Some optimisations of the tower thresholds have been added, and the jet corrections have been updated ("Scheme C"). Similarly, there are no major algorithm changes for $E_{\mathrm{T}}^{\text {miss }}$, however it has been ensured that all triggers including a $E_{\mathrm{T}}^{\text {miss }}$ object do not have any off-line corrections applied. Another improvement that has been recently introduced is the ability to construct acoplanar triggers by combining two jets, or a jet and a $E_{\mathrm{T}}^{\text {miss }}$ object that do not lie "back-to-back" Details of the physics algorithms can be found in Refs. [165] and [148].

- $\boldsymbol{b}$-jets. The algorithm now uses muon information for fast rejection. Further improvements have been made for faster decisions and for an increased efficiency in fully hadronic final states. The documentation for the $b$-jet HLT algorithm can be found in Ref. [290].

- Taus: The HLT $\tau$ algorithm has not changed. However, the increase in the Level-1 rate does propagate into the HLT. The isolation parameters for the electromagnetic calorimeter and the tracker have been tuned after recent studies performed by the Higgs group, described in Ref. [280]. The overall rate for $\tau$-related triggers is expected to be slightly increased.

A new addition to the HLT reconstruction tools is the $H_{\mathrm{T}}$ algorithm. It sums the corrected jet $E_{\mathrm{T}}$ of all the $E_{\mathrm{T}}>5 \mathrm{GeV}$ jets found within $|\eta|<5$, along with the energy of the $p_{\mathrm{T}}>5 \mathrm{GeV} / \mathrm{c}$ HLT muons found in the event, and the $E_{\mathrm{T}}^{\text {miss }}$ computed using the calorimeter deposits. It is meant to be driven off the corresponding L1 $H_{\mathrm{T}}$ term. 


\section{E.3. Triggering with forward detectors}

\section{E.3.1. Objective}

We discuss ${ }^{55}$ the feasibility of a special forward detectors trigger stream, with target output rate of $\mathcal{O}(1) \mathrm{kHz}$ at $\mathrm{L} 1$ and $\mathcal{O}(1) \mathrm{Hz}$ on the HLT, as well as the potential of the already foreseen CMS L1 trigger streams for retaining events with diffractive processes.

The proposed forward detectors trigger stream combines the information of the central CMS detector with that from detectors further downstream of the CMS IP. The forward detectors considered are the TOTEM T1 and T2 tracker telescopes as well as the TOTEM Roman Pot (RP) detectors up to $220 \mathrm{~m}$ downstream of CMS [823, 824]. Information from TOTEM will be available to the CMS L1 trigger. We also consider detectors at a distance of $420 \mathrm{~m}$, in the cryogenic region of the LHC ring, currently being studied by the FP420 project [254].

Topologically, diffractive events are characterised by a gap in the rapidity distribution of final-state hadrons. In addition, the fractional momentum loss, $\xi$, of diffractively scattered protons peaks at $\xi=0$ ("diffractive peak"). The TOTEM RP detectors will permit to measure protons in the region $0.2>\xi>0.02$. Detectors at a distance of $420 \mathrm{~m}$ from the IP would provide a coverage of $0.02>\xi>0.002$, complementary to that of the TOTEM detectors, but cannot be included in the Level-1 trigger without an increase in the Level-1 latency of $3.2 \mu \mathrm{s}$ (though a special, long latency running mode might be feasible at lower luminosities).

The studies discussed in the following assume that the RP detectors are $100 \%$ efficient in detecting all particles that emerge at a distance of at least $10 \sigma_{\text {beam }}+0.5 \mathrm{~mm}$ from the beam axis $(1.3 \mathrm{~mm}$ at $220 \mathrm{~m}, 4 \mathrm{~mm}$ at $420 \mathrm{~m})$. Their acceptance was calculated for the nominal LHC optics $\left(\beta^{*}=0.55 \mathrm{~m}\right)$, version V6.5 [825, 826], and by way of a simulation program that tracks particles through the accelerator lattice [827]. LHC bunches with $25 \mathrm{~ns}$ spacing were assumed.

The results presented below do not depend on the specific hardware implementation of the TOTEM T1, T2 and RP detectors; they hold for any tracker system with the T1, T2 $\eta$ coverage in conjunction with RPs at $220 \mathrm{~m}$ from the IP.

\section{E.3.2. Level-1 trigger rates for forward detectors trigger stream}

E.3.2.1. 2-Jet conditions. A particularly interesting and challenging diffractive channel is the central exclusive production of a Higgs Boson, $p p \rightarrow p H p$, with Higgs mass close to the current exclusion limit. The dominant decay of a SM Higgs Boson of mass $\sim 120 \mathrm{GeV} / \mathrm{c}^{2}$ is into two $b$-quarks and generates 2 jets with at most $60 \mathrm{GeV} / \mathrm{c}$ transverse momentum each. In order to retain as large a signal fraction as possible, as low an $E_{\mathrm{T}}$ threshold as possible of the Level-1 2-jet trigger is desirable. In practice, the threshold value cannot be chosen much lower than $40 \mathrm{GeV}$ per jet. The Level-1 trigger applies cuts on the calibrated $E_{\mathrm{T}}$ value of the jet. Thus, a threshold of $40 \mathrm{GeV}$ corresponds to $20-25 \mathrm{GeV}$ in reconstructed $E_{\mathrm{T}}$, i.e. to values where noise starts becoming sizable.

For luminosities of $10^{32} \mathrm{~cm}^{-2} \mathrm{~s}^{-1}$ and above, the Level-1 rate from standard QCD processes for events with at least 2 central jets $(|\eta|<2.5)$ with $E_{\mathrm{T}}>40 \mathrm{GeV}$ exceeds by far the target output rate of $\mathcal{O}(1) \mathrm{kHz}$. Thus additional conditions need to be employed to reduce the rate from QCD processes. The efficacy of several conditions was investigated [247, 248, 828-830]. In the following, the corresponding rate reduction factors are always quoted with respect to the rate of QCD events that contain at least 2 central jets with $E_{\mathrm{T}}>40 \mathrm{GeV}$ per jet.

\footnotetext{
55 These studies were carried out in collaboration with TOTEM.
} 
Table E.1. Reduction of the rate from standard QCD processes for events with at least 2 central Level-1 jets with $E_{\mathrm{T}}>40 \mathrm{GeV}$, achievable with requirements on the tracks seen in the RP detectors. Additional rate reductions can be achieved with the $H_{\mathrm{T}}$ condition and with a topological condition. Each of them yields, for all luminosities listed, an additional reduction by about a factor 2 .

\begin{tabular}{|c|c|c|c|c|c|c|c|c|c|}
\hline \multirow{3}{*}{$\begin{array}{l}\text { Luminosity } \\
{\left[\mathrm{cm}^{-2} \mathrm{~s}^{-1}\right]}\end{array}$} & \multirow{3}{*}{$\begin{array}{l}\text { Pile-up } \\
\text { events } \\
\text { per BX }\end{array}$} & \multirow{3}{*}{$\begin{array}{l}\text { Level-1 2-jet } \\
\text { rate }[\mathrm{kHz}] \text { for } \\
E_{\mathrm{T}}>40 \mathrm{GeV}\end{array}$} & \multirow{3}{*}{$\begin{array}{l}\text { Total } \\
\text { reduction } \\
\text { needed }\end{array}$} & \multicolumn{6}{|c|}{ Reduction when requiring track in RPs at } \\
\hline & & & & \multicolumn{2}{|r|}{$220 \mathrm{~m}$} & \multirow[t]{2}{*}{$420 \mathrm{~m}$} & \multicolumn{2}{|c|}{$\begin{array}{l}220 \& 420 \mathrm{~m} \\
\text { (asymmetric) }\end{array}$} & \multirow[t]{2}{*}{$\begin{array}{l}420 \& \\
420 \mathrm{~m}\end{array}$} \\
\hline & & & & \multicolumn{2}{|r|}{$\xi<0.1$} & & \multicolumn{2}{|c|}{$\xi<0.1$} & \\
\hline $1 \times 10^{32}$ & 0 & 2.6 & 2 & 370 & & & & & \\
\hline $1 \times 10^{33}$ & 3.5 & 26 & 20 & 7 & 15 & 27 & 160 & 380 & 500 \\
\hline $2 \times 10^{33}$ & 7 & 52 & 40 & 4 & 10 & 14 & 80 & 190 & 150 \\
\hline $5 \times 10^{33}$ & 17.5 & 130 & 100 & 3 & 5 & 6 & 32 & 75 & 30 \\
\hline $1 \times 10^{34}$ & 35 & 260 & 200 & 2 & 3 & 4 & 17 & 39 & 10 \\
\hline
\end{tabular}

The QCD background events were generated with the Pythia Monte Carlo generator. In order to assess the effect when the signal is overlaid with pile-up, a sample of 500,000 pile-up events was generated with Pythia. This sample includes inelastic as well as elastic and single diffractive events. Pythia underestimates the number of final state protons in this sample. The correction to the Pythia leading proton spectrum described in [831] was used to obtain the results discussed in the following.

Given a Level-1 target rate for events with 2 central Level- 1 jets of $\mathcal{O}(1) \mathrm{kHz}$, a total rate reduction between a factor 20 at $1 \times 10^{33} \mathrm{~cm}^{-2} \mathrm{~s}^{-1}$ and 200 at $1 \times 10^{34} \mathrm{~cm}^{-2} \mathrm{~s}^{-1}$ is necessary. Table E. 1 summarises the situation for luminosities between $10^{32} \mathrm{~cm}^{-2} \mathrm{~s}^{-1}$ and $10^{34} \mathrm{~cm}^{-2} \mathrm{~s}^{-1}$, and for different RP detector conditions: a track at $220 \mathrm{~m}$ on one side of the IP (single-arm $220 \mathrm{~m}$ ), without and with a cut on $\xi$; a track at $420 \mathrm{~m}$ on one side of the IP (single-arm $420 \mathrm{~m}$ ); a track at $220 \mathrm{~m}$ and $420 \mathrm{~m}$ (asymmetric); a track at $420 \mathrm{~m}$ on both sides of the IP (doublearm $420 \mathrm{~m}$ ). Because the detectors at $220 \mathrm{~m}$ and $420 \mathrm{~m}$ have complementary coverage in $\xi$, the asymmetric condition in effect selects events with two tracks of very different $\xi$ value, in which one track is seen at $220 \mathrm{~m}$ on one side of the IP and a second track is seen on the other side at $420 \mathrm{~m}$. If not by the L1 trigger, these asymmetric events can be selected by the HLT and are thus of highest interest. At luminosities where pile-up is present, the rate reduction achievable with the RP detector conditions decreases because of the diffractive component in the pile-up.

A collimator located in front of the LHC magnet Q5, planned to be operative at higher luminosities, will have an effect on the acceptance of the RP detectors resembling that of a $\xi$ cut. This effect has not been taken into account in Table E.1.

Using T1 and T2 as vetoes in events with 2 central Level-1 jets was found to be effective only in the absence of pile-up [832].

In addition to the $E_{\mathrm{T}}$ values of individual Level-1 jets, the CMS Calorimeter Trigger has at its disposal the scalar sum, $H_{\mathrm{T}}$, of the $E_{\mathrm{T}}$ values of all jets. Requiring that essentially all the $E_{\mathrm{T}}$ be concentrated in the two central Level-1 jets with highest $E_{\mathrm{T}}$, i.e. $\left[E_{\mathrm{T}}^{1}+E_{\mathrm{T}}^{2}\right] / H_{\mathrm{T}}>0.9$ ( $H_{\mathrm{T}}$ condition), corresponds to imposing a rapidity gap of at least 2.5 units with respect to the beam direction. This condition reduces the rate of QCD events by approximately a factor 2 , independent of the presence of pile-up and with only a small effect on the signal efficiency.

A further reduction of the QCD rate could be achieved with the help of a topological condition. The 2-jet system has to balance the total momentum component of the two protons along the beam axis. In signal events with asymmetric $\xi$ values, the proton seen on one side 
Table E.2. Estimated threshold values that result in a $\mathrm{L} 1$ output rate of $\sim 1 \mathrm{kHz}$, for various conditions on central CMS detector quantities and on tracks seen in the RP detectors at $220 \mathrm{~m}$ and $420 \mathrm{~m}$.

\begin{tabular}{|c|c|c|c|c|}
\hline \multirow[b]{2}{*}{ L1 condition } & \multicolumn{4}{|c|}{$\begin{array}{l}\mathrm{L} 1 E_{\mathrm{T}} \text { or } p_{\mathrm{T}} \text { threshold }[\mathrm{GeV}] \text { at } \mathcal{O}(1) \mathrm{KHz} \\
\mathrm{L} 1 \text { output rate for luminosity }\left[\mathrm{cm}^{-2} \mathrm{~s}^{-1}\right]\end{array}$} \\
\hline & $1 \times 10^{33}$ & $2 \times 10^{33}$ & $5 \times 10^{33}$ & $1 \times 10^{34}$ \\
\hline $1 \mathrm{Jet}$ & 115 & 135 & 160 & 190 \\
\hline 2 Jet & 90 & 105 & 130 & 150 \\
\hline $1 \mathrm{Jet}+220 \mathrm{~s}$ & 90 & 115 & 155 & 190 \\
\hline $2 \mathrm{Jet}+220 \mathrm{~s}$ & 65 & 90 & 125 & 150 \\
\hline $1 \mathrm{Jet}+220 \mathrm{~d}$ & 55 & 85 & 130 & 175 \\
\hline $2 \mathrm{Jet}+220 \mathrm{~d}$ & 30 & 60 & 100 & 140 \\
\hline $1 \mathrm{Jet}+220 \mathrm{~s}(\mathrm{c})$ & 70 & 90 & 150 & 185 \\
\hline $2 \mathrm{Jet}+220 \mathrm{~s}(\mathrm{c})$ & 60 & 70 & 115 & 145 \\
\hline $1 \mathrm{Jet}+220 \mathrm{~d}(\mathrm{c})$ & 30 & 65 & 110 & 155 \\
\hline $2 \mathrm{Jet}+220 \mathrm{~d}(\mathrm{c})$ & 20 & 45 & 85 & 125 \\
\hline $1 \mathrm{Jet}+420 \mathrm{~s}$ & 65 & 90 & 125 & 165 \\
\hline $2 \mathrm{Jet}+420 \mathrm{~s}$ & 45 & 70 & 100 & 130 \\
\hline $1 \mathrm{Jet}+420 \mathrm{~d}$ & 20 & 40 & 80 & 115 \\
\hline $2 \mathrm{Jet}+420 \mathrm{~d}$ & $<10$ & 30 & 60 & 90 \\
\hline $1 \mu+220 \mathrm{~s}$ & 12 & 16 & 23 & $>100$ \\
\hline $1 \mu+220 \mathrm{~d}$ & 4 & 9 & 17 & 80 \\
\hline $1 \mu+220 \mathrm{~s}(\mathrm{c})$ & - & 11 & 22 & 100 \\
\hline $1 \mu+220 \mathrm{~d}(\mathrm{c})$ & - & 6 & 13 & 30 \\
\hline $1 \mu+420 \mathrm{~s}$ & 7 & 11 & 14 & 37 \\
\hline $1 \mu+420 \mathrm{~d}$ & $<2$ & 4 & 7 & 14 \\
\hline
\end{tabular}

in the RP detectors at $220 \mathrm{~m}$ distance is the one with the larger $\xi$ and thus has lost more of its initial momentum component along the beam axis. Hence the jets tend to be located in the same $\eta$-hemisphere as the RP detectors that detect this proton. A trigger condition requiring that $\left[\eta^{j e t 1}+\eta^{j e t 2}\right] \times \operatorname{sign}\left(\eta^{220 m R P}\right)>0$ reduces the QCD background by a factor 2 , independent of pile-up, and with no loss in signal efficiency.

A reduction of the QCD rate to levels compatible with a Level-1 output target rate of $\mathcal{O}$ (1) $\mathrm{kHz}$ by including RP detectors at a distance of $220 \mathrm{~m}$ from the CMS IP thus appears feasible for luminosities up to $2 \times 10^{33} \mathrm{~cm}^{-2} \mathrm{~s}^{-1}$, as long as a $\xi$ cut can be administered in the L1 trigger.

E.3.2.2. Other conditions. The effect of combining already foreseen Level-1 trigger conditions with conditions on the RP detectors is illustrated in Table E.2 [829]. Single- and double-arm RP detector conditions are indicated with 's' and 'd' endings, respectively. Entries marked with a '(c)' indicate thresholds applicable if a cut on $\xi<0.1$ is implemented for the $\mathrm{RP}$ detectors at $220 \mathrm{~m}$. The jet conditions consider all Level-1 jets with $|\eta|<5$.

A further rate reduction by approximately a factor two can be obtained at luminosities with negligible pile-up by imposing a rough large rapidity gap cut at L1. This was implemented by requiring that there be no forward jets, i.e. jets in the HF, in either hemisphere in the event.

\section{E.3.3. Level-1 signal efficiencies}

Of the Level-1 conditions discussed so far, only those based on the RP detectors have a significant impact on the signal efficiency. Of further interest is the question how many signal events are being retained by the already foreseen trigger streams, notably the muon trigger. 

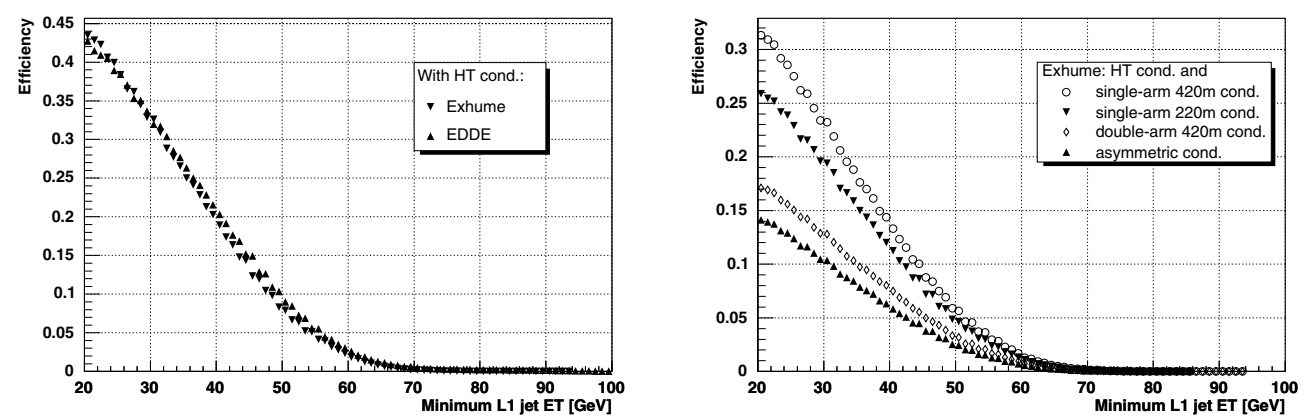

Figure E.1. L1 selection efficiency for $p p \rightarrow p H p$ and $H\left(120, \mathrm{GeV} / \mathrm{c}^{2}\right) \rightarrow b \bar{b}$ as function of the $E_{\mathrm{T}}$ threshold value when at least 2 central Level-1 jets with $E_{\mathrm{T}}$ above threshold are required. All plots are for the non-pile-up case and the $H_{\mathrm{T}}$ condition has been applied. Left: Comparison between the EDDE and Exhume Monte Carlo generators, without applying any additional RP conditions. Right: Comparison of the effect of different RP conditions on the efficiency in the Exhume Monte Carlo sample.

E.3.3.1. Central exclusive Higgs production $\left(H\left(120 \mathrm{GeV} / \mathrm{c}^{2}\right) \rightarrow b \bar{b}\right)$. In order to study the effect of the Level-1 trigger selection on the Higgs signal, signal samples of 100,000 events with central exclusive production of a Higgs Boson were generated with the Monte Carlo programs EDDE [261] (version 1.1) and Exhume [259] (version 1.0).

Figure E.1 shows the Level-1 selection efficiency as a function of the $E_{\mathrm{T}}$ threshold values when at least 2 central Level-1 jets with $E_{\mathrm{T}}$ above threshold are required [829]. For a threshold of $40 \mathrm{GeV}$ per jet, Exhume and EDDE both yield an efficiency of about $20 \%$. The plot on the right-hand side overlays the efficiency curves obtained with Exhume when the 2-jet condition is combines with RP detector conditions. With an $E_{\mathrm{T}}$ threshold of $40 \mathrm{GeV}$ per jet, the single$\operatorname{arm} 220 \mathrm{~m}(420 \mathrm{~m})$ condition results in an efficiency of the order $12 \%(15 \%)$, the double-arm $420 \mathrm{~m}$ condition in one of $8 \%$ and the asymmetric condition in one of $6 \%$. This also means that, even without the possibility of including the RP detectors at $420 \mathrm{~m}$ from the CMS IP in the Level-1 trigger, $6 \%$ of the signal events can be triggered on with the single-arm $220 \mathrm{~m}$ condition, but will have a track also in the $420 \mathrm{~m}$ detectors that can be used in the HLT.

An alternative trigger strategy is to exploit the relatively muon-rich final state from $B$ decays: about $20 \%$ of the events have at least a muon in the final state. Requiring at least one (two) L1 muon(s) with $p_{\mathrm{T}}$ above $14 \mathrm{GeV} / \mathrm{c}(3 \mathrm{GeV} / \mathrm{c})$ yields an efficiency of $6 \%(2 \%)$. Demanding at least 1 muon and 1 jet, the latter with $E_{\mathrm{T}}>40 \mathrm{GeV}$, is a condition not yet foreseen in the CMS trigger tables. For a muon $p_{\mathrm{T}}$ threshold of $3 \mathrm{GeV} / \mathrm{c}$, the rate at a luminosity of $10^{33} \mathrm{~cm}^{-2}$ is slightly less than $3 \mathrm{kHz}$, and about half of the decays with muons in the final state (i.e. $9 \%$ ) are retained [830].

E.3.3.2. Central exclusive Higgs production $\left(H\left(140 \mathrm{GeV} / \mathrm{c}^{2}\right) \rightarrow W W\right)$. For SM Higgs Boson masses above $120 \mathrm{GeV} / \mathrm{c}^{2}$, the $H \rightarrow W W$ branching ratio becomes sizable; in this case the final state contains high- $p_{\mathrm{T}}$ leptons that can be used for triggering. Efficiencies are in general high [830]. About $23 \%$ of the events have at least one muon in the final state. Approximately $70 \%$ of these (i.e. $16 \%$ ) are retained by requiring at least one muon with a $p_{\mathrm{T}}$ threshold of $14 \mathrm{GeV} / \mathrm{c}$. An extra $\approx 10 \%$ (i.e. $2 \%$ ) would be retained by implementing the muon/jet slot discussed above with thresholds of $3 \mathrm{GeV} / \mathrm{c}$ on the muon $p_{\mathrm{T}}$ and $40 \mathrm{GeV}$ on the jet $E_{\mathrm{T}}$. 


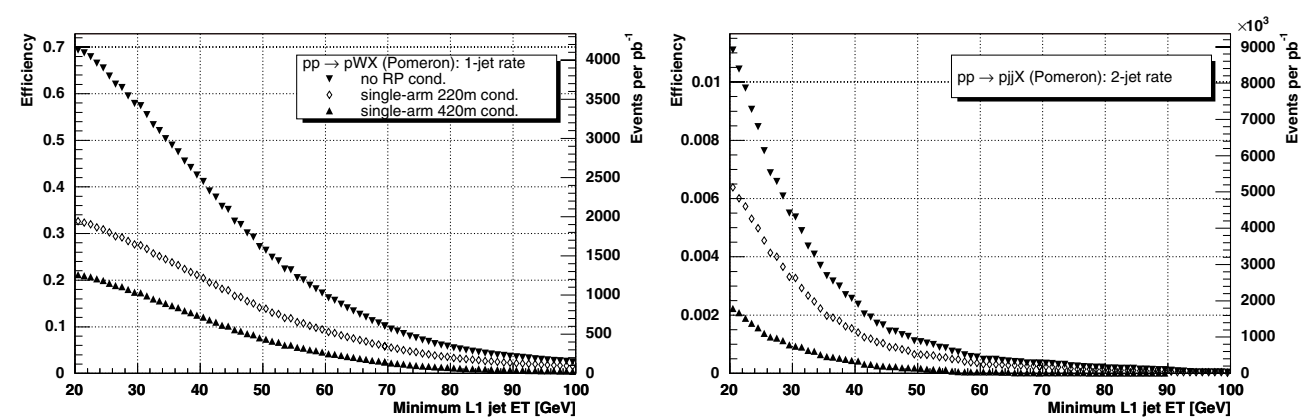

Figure E.2. L1 selection efficiency as function of the $E_{\mathrm{T}}$ threshold value for $p p \rightarrow p W X$ (left) and $p p \rightarrow p j j X$ (right), when at least one (left) or two (right) Level-1 jets $(|\eta|<5$ ) above threshold are required. All plots are for the non-pile-up case.

E.3.3.3. Single diffractive hard processes. Double-Pomeron exchange processes constitute only a small part of the diffractive cross section. Hard single-diffraction, $p p \rightarrow p X$, where only one proton remains intact and the other is diffractively excited, have much higher cross sections than hard double-Pomeron exchange events. Efficiencies have been studied for $p p \rightarrow p X$, with $X$ containing a $W$ or a $Z$ boson that decay to jets and to muons, as well as with $X$ containing a dijet system. Samples of 100,000 signal events each were generated with the POMwig Monte Carlo generator [833] (version 1.3).

For two example processes, Figure E.2 shows the efficiency as a function of the Level1 threshold value, normalised to the number of events where for the diffractively scattered proton $0.001<\xi<0.2$ holds [829]. Three different trigger conditions are considered: trigger on central detector quantities alone (i), trigger on central detector quantities in conjunction (ii) with the single-arm $220 \mathrm{~m}$ condition, and (iii) with the single-arm $420 \mathrm{~m}$ condition. Also shown is the number of events expected to pass the L1 selection per $\mathrm{pb}^{-1}$ of LHC running. A significant part of events is retained when a proton is required in the $220 \mathrm{~m}$ RPs.

\section{E.3.4. Effect of pile-up, beam-halo and beam-gas backgrounds}

Pile-up effects are included in all rate and efficiency studies presented. In the $220 \mathrm{~m}$ stations, 0.055 protons/pile-up event are expected on average, in the $420 \mathrm{~m}$ stations, 0.012 protons/pileup event. At a luminosity of $10^{34} \mathrm{~cm}^{-2} \mathrm{~s}^{-1}$, there are 35 pile-up events on average; this entails, on average, 2 extra tracks in the $220 \mathrm{~m}$ stations and less than one in the $420 \mathrm{~m}$ stations.

The effect from beam-halo and beam-gas events on the Level-1 rate is not yet included in the studies discussed here. Preliminary estimates suggest that they are chiefly a concern for any trigger condition based solely on the forward detectors. For any trigger condition that includes a requirement on central CMS detector quantities the size of their contribution is such that they do not lead to a significant increase of the Level-1 output rate.

\section{E.3.5. HLT strategies}

Jets are reconstructed at the HLT with an iterative cone $(R<0.5)$ algorithm. The Level-1 selection cuts are repeated with HLT quantities. The following conditions are imposed [829]:

(A) The event pass the single-arm $220 \mathrm{~m}$ Level- 1 condition with $\xi<0.1$ cut. As demonstrated in Table E.1, this condition reduces the Level-1 output rate to below $\mathcal{O}(1) \mathrm{kHz}$. Additional 
Table E.3. Results of HLT selection.

\begin{tabular}{llll}
\hline HLT selection condition & $\mathrm{A}+\mathrm{B}+\mathrm{C}$ & $\mathrm{A}+\mathrm{B}+\mathrm{D}$ & $\mathrm{A}+\mathrm{B}+\mathrm{C}+\mathrm{E}$ \\
\hline HLT rate at $1 \times 10^{33} \mathrm{~cm}^{-2} \mathrm{~s}^{-1}$ & $15 \mathrm{~Hz}$ & $20 \mathrm{~Hz}$ & $<1 \mathrm{~Hz}$ \\
line HLT rate at $2 \times 10^{33} \mathrm{~cm}^{-2} \mathrm{~s}^{-1}$ & $60 \mathrm{~Hz}$ & $80 \mathrm{~Hz}$ & $1 \mathrm{~Hz}$ \\
e Signal eff. $H(120) \mathrm{GeV} / \mathrm{c}^{2} \rightarrow b \bar{b}$ & $11 \%$ & $7 \%$ & $6 \%$ \\
\hline
\end{tabular}

rate reduction factors of $\sim 300(\sim 1000)$ at $1(2) \times 10^{33} \mathrm{~cm}^{-2} \mathrm{~s}^{-1}$ are needed to reach the HLT target output rate of $\mathcal{O}(1) \mathrm{Hz}$.

(B) The two jets are back-to-back in the azimuthal angle $\phi(2.8<\Delta \phi<3.48 \mathrm{rad})$, and have $\left(E_{\mathrm{T}}^{1}-E_{\mathrm{T}}^{2}\right) /\left(E_{\mathrm{T}}^{1}+E_{\mathrm{T}}^{2}\right)<0.4$, and $E_{\mathrm{T}}>40 \mathrm{GeV}$ for each jet.

(C) The proton fractional momentum loss $\xi$ is evaluated with the help of calorimeter quantities [834-836]:

$$
\xi_{+-}=(1 / \sqrt{s}) \Sigma_{i} E_{T i} \exp \left(\mp \eta_{i}\right)
$$

where the sum runs over the two jets and the,+- signs denote the two hemispheres. The result is compared with the $\xi$ value measured by the RP detectors. At present, no simulation of the RP reconstruction is available. As estimate of the $\xi$ resolution, $15 \%$ $(10 \%)$ is assumed at $220 \mathrm{~m}(420 \mathrm{~m})$. Events are rejected if the difference between the two values of $\xi$ is larger than $2 \sigma$.

(D) At least one of the two jets is $b$-tagged.

(E) A proton is seen at $420 \mathrm{~m}$.

The case without pile-up presents no difficulty: essentially no QCD background events survive the selection. If conditions $\mathrm{A}+\mathrm{B}+\mathrm{C}$ are applied, the signal efficiency for $p p \rightarrow p H p$ with $H\left(120 \mathrm{GeV} / \mathrm{c}^{2}\right) \rightarrow b \bar{b}$ is at $11 \%$ essentially unchanged with respect to the Level-1 selection, but the HLT output rate exceeds the target output rate, see Table E.3. If $b$-tagging is required but no $\xi$ matching (conditions $\mathrm{A}+\mathrm{B}+\mathrm{D}$ ), the efficiency drops to $7 \%$, without any improvement in the rate reduction. The combination of conditions $\mathrm{A}+\mathrm{B}+\mathrm{C}+\mathrm{E}$ finally leads to the targeted HLT output rate of $\mathcal{O}(1) \mathrm{Hz}$, without any loss in signal efficiency compared to L1.

\section{E.4. High-Level Trigger paths}

We are starting with the DAQ-TDR trigger table as the baseline. This includes single- and double-triggers for the basic objects $(e, \gamma, \mu, \tau)$ along with jets and $b$-jets. Some crosschannel triggers are also present. We are expanding the cross-channel "menu" by introducing additional triggers. We introduce an $H_{\mathrm{T}}$ algorithm, which we combine with other objects. We are also adding a series of central single-jets, non-isolated muons, and a diffractive trigger discussed earlier.

\section{E.4.1. Level-1 conditions}

Table E.4 summarises the Level-1 conditions used to drive all the trigger paths. A pseudo "L1 bit number" has been assigned for easy reference in the following sections.

\section{E.4.2. Evolution of DAQ-TDR triggers}

The trigger paths that have been studied in Ref. [76] have been inherited and constitute the "bulk" of this next iteration of the CMS Trigger Menu for $\mathcal{L}=2 \times 10^{33} \mathrm{~cm}^{-2} \mathrm{~s}^{-1}$. 
Table E.4. Level-1 conditions used in High Level Trigger paths.

\begin{tabular}{|c|c|c|c|}
\hline Level-1 bit \# & Trigger & $(\mathrm{GeV})$ & Prescale \\
\hline 0 & Single $\mu$ & 14 & 1 \\
\hline 1 & Double $\mu$ & 3 & 1 \\
\hline 2 & Single isolated $e \gamma$ & 23 & 1 \\
\hline 3 & Double isolated $e \gamma$ & 11 & 1 \\
\hline 4 & Double $e \gamma$ (isolated/non-isolated) & 19 & 1 \\
\hline 8 & Single central jet & 177 & 1 \\
\hline 9 & Single forward jet & 177 & 1 \\
\hline 10 & Single $\tau$-jet & 100 & 1 \\
\hline 11 & 2 central jets & 130 & 1 \\
\hline 12 & 2 forward jets & 130 & 1 \\
\hline 13 & $2 \tau$-jets & 66 & 1 \\
\hline 14 & 3 central jets & 86 & 1 \\
\hline 15 & 3 forward jets & 86 & 1 \\
\hline 16 & $3 \tau$-jets & 40 & 1 \\
\hline 17 & 4 central jets & 70 & 1 \\
\hline 18 & 4 forward jets & 70 & 1 \\
\hline 19 & $4 \tau$-jets & 30 & 1 \\
\hline 26 & (isolated) $e \gamma+\tau$ & 14,52 & 1 \\
\hline 31 & $H_{\mathrm{T}}$ & 300 & 1 \\
\hline 32 & $E_{\mathrm{T}}^{\mathrm{miss}}$ & 60 & 1 \\
\hline 33 & Single jet (central, forward or $\tau$ ) & 140 & 10 \\
\hline 34 & Single jet (central, forward or $\tau$ ) & 60 & 1000 \\
\hline 35 & Single jet (central, forward or $\tau$ ) & 20 & 100000 \\
\hline 36 & Single jet (central, forward or $\tau$ ) & 150 & 1 \\
\hline 37 & 2 jets (central, forward or $\tau$ ) & 100 & 1 \\
\hline 38 & 3 jets (central, forward or $\tau$ ) & 70 & 1 \\
\hline 39 & 4 jets (central, forward or $\tau$ ) & 50 & 1 \\
\hline
\end{tabular}

Modifications (optimisation of isolation cuts and thresholds) have been made for certain of the triggers, to reflect changes in the physics algorithms, or the improved understanding of the background from Monte Carlo (MC) simulations. The proposed Trigger Tables includes:

- Muons. The standard muon triggers include calorimeter-based isolation at L2, and both calorimeter and tracker isolation at L3. The $p_{\mathrm{T}}$ thresholds remain at $19 \mathrm{GeV} / \mathrm{c}$ for the single-muon and $(7,7) \mathrm{GeV} / \mathrm{c}$ for the dimuon trigger. A second set of relaxed single- and double-muons has been added with $p_{\mathrm{T}}>37 \mathrm{GeV}$ and $p_{\mathrm{T}}>10 \mathrm{GeV}$, respectively. The main motivation here is Drell-Yan studies. In general, physics analyses that do not need a low $p_{\mathrm{T}}$ muon but do suffer from the isolation requirement on the muon. The reduced rejection caused by the removal of the isolation cuts is compensated by the higher- $p_{\mathrm{T}}$ thresholds on the muons, without affecting the event yield for the physics signal. The relaxed triggers have the advantage that the muons here are immune to radiative losses for the higher energy spectrum $\left(p_{\mathrm{T}}>500 \mathrm{GeV} / \mathrm{c}\right)$. Both isolated and relaxed triggers run off the corresponding non-isolated single- and double-muon bits at L1.

- Electrons. The $p_{\mathrm{T}}$ threshold remains at $26 \mathrm{GeV} / \mathrm{c}$ for the single electron trigger and has a new value of $(12,12) \mathrm{GeV} / \mathrm{c}$ for the dielectron trigger. An additional relaxed dielectron trigger appears with $p_{\mathrm{T}}>19 \mathrm{GeV} / \mathrm{c}$. The single-electron and double-electron triggers run off the corresponding Level-1 bits.

- Photons. The new $p_{\mathrm{T}}$ thresholds are $80 \mathrm{GeV} / \mathrm{c}$ for the single-photon trigger and (30, 20) $\mathrm{GeV} / \mathrm{c}$ for the diphoton trigger (both relaxed and non-relaxed flavours). A few prescaled 
single- and double-photon triggers have also been introduced, for the purpose of studying trigger efficiencies. The photon HLT algorithms run off the corresponding Level-1 e $\gamma$ bits (single- and double-triggers).

- Taus. The single- $\tau$ trigger runs off the corresponding Level-1 bit. The double- $\tau$ trigger is driven by the .OR. -ing of the single- and double- $\tau$ trigger bits at L1. There is no explicit kinematic cut on the tau at HLT. There is, however, a match-to-track requirement in addition to the $p_{\mathrm{T}}>100(66) \mathrm{GeV} / \mathrm{c} \mathrm{L} 1$ precondition for the inclusive (double) tau trigger. The single- $\tau$ has also a $E_{\mathrm{T}}^{\text {miss }}>65 \mathrm{GeV}$ requirement at HLT.

- Tau and electron. The Level- 1 condition is the corresponding $\tau+e \gamma$ trigger. The $p_{\mathrm{T}}$ threshold remains at $16 \mathrm{GeV} / \mathrm{c}$ for the electron. There is no explicit $p_{\mathrm{T}}$ cut for the $\tau$ at HLT, but there is the match-to-track requirement for the $\tau$ candidate.

- Jets. The Level-1 conditions for the single-, double-, triple- and quadruple-jet triggers have been simplified considerably. Single jet triggers run off an OR . of a central-, forward- or tau-jet trigger at L1. Double-, triple- and quadruple-jet triggers use an .OR. of the all the Level-1 terms requiring the same number of jets or less. For example, the triple-jet trigger is driven by an OR . of the single-, double- and triple-jet Level-1 bits. In all cases, jets can be found in either the central or the forward region of the detector, and they include the $\tau$ candidates. The additional $p_{\mathrm{T}}$ cuts at HLT are: 400 (single), 350 (double), 195 (triple) and 80 (quadruple) GeV. The new double-jet trigger is expected to have a large overlap with the single-jet trigger path. However, it is useful for testing the additional bias introduced by the requirement for a second jet in the event. A series of prescaled triggers have also been introduced, which are discussed later (Sec. E.4.3.2).

- $\boldsymbol{b}$-jet. This trigger is also based on the logical .OR . of the single-, double-, triple- and quadruple-jet Level-1 terms. At HLT, we have the additional requirement that the event is consistent with $b$-content. The $E_{\mathrm{T}}$ cut for the HLT jets is one of the following: $350 \mathrm{GeV}$ if the event has one jet, $150 \mathrm{GeV}$ if the event has three jets, or $55 \mathrm{GeV}$ if the event has four jets.

- Jet and $\boldsymbol{E}_{\mathrm{T}}^{\text {miss. The }} E_{\mathrm{T}}$ thresholds are 180 and $80 \mathrm{GeV}$, respectively. The Level-1 condition is a single $E_{\mathrm{T}}^{\text {miss }}$ object above $60 \mathrm{GeV}$.

\section{E.4.3. New triggers}

E.4.3.1. Cross-channel triggers. The trigger studies presented in the DAQ TDR [76] have been the most comprehensive CMS effort to date to calculate rates for various trigger paths across many physics channels. For those studies the focus has been the optimisation of the rejection of the individual object-id algorithms (muon, electron, tau, etc.) rather than the combination of them into more powerful trigger tools. However, single (or even double) trigger objects are limited by the rate and, therefore, have their thresholds often higher than desired for many physics analyses. If the signal contains more than one trigger objects, using trigger paths combining different objects may yield a considerable gain by allowing lower trigger thresholds and higher efficiency. Cross-channel triggers can be much more stable and less prone to rate fluctuations from operating conditions. The correlations among trigger objects can help reduce difficult backgrounds and instrumental fakes. The additional advantage is that such cross-channel triggers have noticeably lower rates than the single trigger channels and therefore contribute fairly little to the overall bandwidth.

Some cross-channel triggers have already been considered and their rates estimated [76], such as $\tau+e$ and $\tau+E_{\mathrm{T}}^{\text {miss }}$, motivated by the Higgs searches with hadronic decays of $\tau$ and leptons, and jet $+E_{\mathrm{T}}^{\text {miss }}$, important for searches of super-symmetric particles. The new addition 
to the Trigger Menu, expanding the scope of Higgs searches, is a combined $\tau+\mu$ trigger with $p_{\mathrm{T}}$ thresholds at 40 and $15 \mathrm{GeV} / \mathrm{c}$, respectively. It is driven by the single- $\mu$ Level- 1 bit.

We are presenting here a few additional cross-channel triggers, along with the physics motivation.

- A new category of triggers introduced here is the acoplanar dijet and jet $+E_{\mathrm{T}}^{\mathrm{miss}}$ for SUSY signals. The gain is the lower thresholds that become possible because of the topology constraint. Possible biases should be studied, so these triggers are meant to run in parallel with the standard jet and jet $+E_{\mathrm{T}}^{\text {miss }}$ triggers without the acoplanarity requirements. We introduce a double-jet trigger with $E_{\mathrm{T}}$ thresholds at $(200,200) \mathrm{GeV}$ and $|\Delta \phi|<2.1$, and a new jet $+E_{\mathrm{T}}^{\text {miss }}$ trigger with $E_{\mathrm{T}}$ thresholds at $(100,80) \mathrm{GeV}$ and $|\Delta \phi|<2.1$. The former is driven by an .OR. of the single- and double-jet requirements at Level-1 (bits 36, 37). The latter is driven by a simple $E_{\mathrm{T}}^{\mathrm{miss}}>60 \mathrm{GeV}$ Level- 1 requirement.

- " $E_{\mathrm{T}}^{\text {miss }}+X$ " triggers. A combination of an $E_{\mathrm{T}}^{\text {miss }}$ object with an $H_{\mathrm{T}}$ cut, one (or more) jet or lepton may be the only way to access $E_{\mathrm{T}}^{\text {miss }}$-enhanced triggers if there are problems $(e . g$. instrumental fakes) that prevent CMS from running an inclusive $E_{\mathrm{T}}^{\text {miss }}$ trigger. At this point we have implemented:

* Multi-jets and $E_{\mathrm{T}}^{\text {miss }}$. These will be useful for SUSY studies, just like the series of jet triggers. However, the additional $E_{\mathrm{T}}^{\text {miss }}$ requirement allows us to lower the thresholds on the jets, and therefore increase the sensitivity of the analyses. We introduce here a dijet $+E_{\mathrm{T}}^{\text {miss }}$ trigger with $E_{\mathrm{T}}^{\text {jet }}>155 \mathrm{GeV}, E_{\mathrm{T}}^{\text {miss }}>80 \mathrm{GeV}$, a triple - jet $+E_{\mathrm{T}}^{\text {miss }}$ trigger with $E_{\mathrm{T}}^{\text {jet }}>85 \mathrm{GeV}, E_{\mathrm{T}}^{\text {miss }}>80 \mathrm{GeV}$ and a quadruple - jet $+E_{\mathrm{T}}^{\text {miss }}$ trigger with $E_{\mathrm{T}}^{\mathrm{jet}}>35 \mathrm{GeV}, E_{\mathrm{T}}^{\text {miss }}>80 \mathrm{GeV}$. These all run off the single Level-1 requirement for $E_{\mathrm{T}}^{\text {miss }}>60 \mathrm{GeV}$.

$* H_{\mathrm{T}}+E_{\mathrm{T}}^{\text {miss }}$ and $H_{\mathrm{T}}+e$. It is difficult to contain the rate for an inclusive $H_{\mathrm{T}}$ trigger without any additional cuts. The requirement for a $E_{\mathrm{T}}^{\mathrm{miss}}$ cut or an additional electron in the event allows us to access events with lower $E_{\mathrm{T}}^{\text {miss }}$ or softer electrons. This can give an increased efficiency for $W+$ jets, top physics, SUSY cascades, and other similar physics channels. Here we propose an $H_{\mathrm{T}}+E_{\mathrm{T}}^{\text {miss }}$ trigger with $H_{\mathrm{T}}>350 \mathrm{GeV}$, $E_{\mathrm{T}}^{\text {miss }}>80 \mathrm{GeV}$ and an $H_{\mathrm{T}}+e$ trigger with $H_{\mathrm{T}}>350 \mathrm{GeV}$ and $p_{\mathrm{T}}>20 \mathrm{GeV} / \mathrm{c}$ for the electron. They are both driven by the $E_{\mathrm{T}}^{\text {miss }}>60 \mathrm{GeV}$ condition at L1.

Some additional cross-channel triggers that have not been included in this Trigger Table iteration but should be considered in future trigger studies are:

- An $e+\mu$ trigger is of interest in many studies, for example:

$* q q H, H \rightarrow \tau \tau \rightarrow 2 \ell$, with an expected gain thanks to the lower lepton thresholds compared to the single-electron and single-muon trigger paths,

* many SUSY decays including leptons in the final state,

* top measurements in the double leptonic channel $(t \bar{t} \rightarrow b \bar{b} \ell \nu \ell v)$, gaining sensitivity at the lower $p_{\mathrm{T}}$ spectrum, and

$* B_{s} \rightarrow \ell \ell$, to allow for the lepton-number-violating channel to be studied.

- $E_{\mathrm{T}}^{\text {miss }}+\ell$. The idea here is to exploit the presence of a $W$ boson or a top decay in many channels. This could be used in many SM channels where lowering the lepton threshold extends the range of the measurement. For example:

* top measurement in the double leptonic and semi-leptonic channels,

$*$ single top production, and

* $W$ measurements.

Furthermore, this is a typical signature of an event containing super-symmetric particles. 

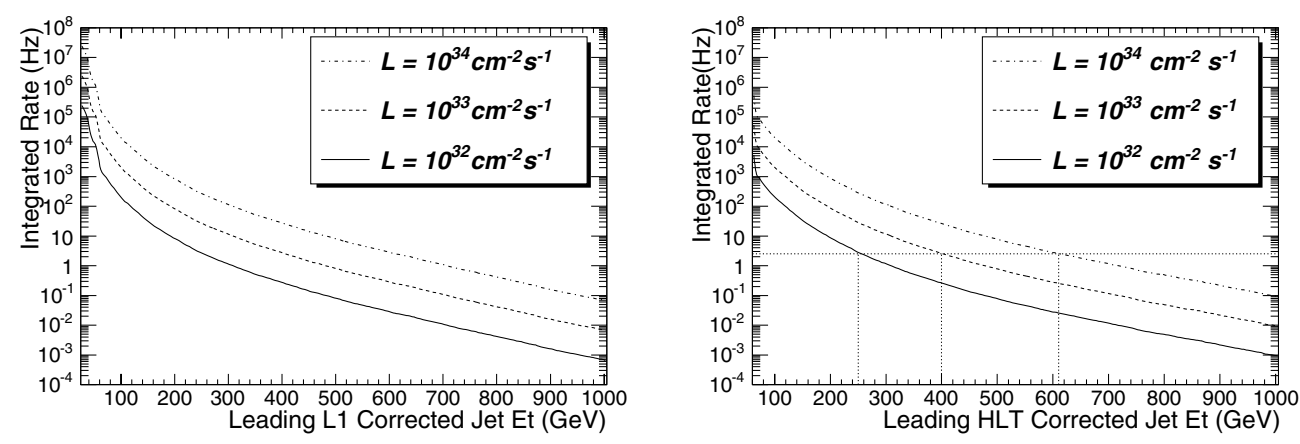

Figure E.3. The integrated trigger rates at Level-1 (left) and HLT (right) above the $E_{\mathrm{T}}$ thresholds for the highest $E_{\mathrm{T}}$ jet is plotted versus the $E_{\mathrm{T}}$ threshold for three luminosity scenarios: $\mathcal{L}=$ $10^{32} \mathrm{~cm}^{-2} \mathrm{~s}^{-1}$ (solid), and $\mathcal{L}=10^{33} \mathrm{~cm}^{-2} \mathrm{~s}^{-1}$ (dashed), and $\mathcal{L}=10^{34} \mathrm{~cm}^{-2} \mathrm{~s}^{-1}$ (dot-dashed). HLT thresholds that give $2.5 \mathrm{~Hz}$ are shown by vertical dotted lines.

- Triggers combining a lepton and a jet, or a lepton and a $b$-jet could be of interest for top measurements. The $\ell+$ jet signature is also very common in super-symmetric events.

- Finally, a combination of a lepton and a photon $(e+\gamma$ and $\mu+\gamma)$ is ideal for Flavour Changing Neutral Current analyses, exploiting the extraordinary capabilities of CMS in detecting photons. These triggers allow to lower the thresholds on the lepton and the photon, increasing the event yield compared to the single- $e, \mu$ or $\gamma$ trigger paths.

E.4.3.2. Single jet triggers. In this section we propose the single jet trigger paths. These have been driven by the needs of the inclusive jet and dijet analysis. The full study can be found in Ref. [118]. Here we summarise conclusions, along with a short description of the strategy for adjusting thresholds and prescales as the luminosity changes. This study looks at the evolution of the single-jet triggers for various luminosities. It serves as an example of how to preserve the long-term continuity of the triggers used for physics analyses. It is, therefore, interesting and instructive beyond the strict scope of the single-jet trigger suite.

To measure jet spectra down to low jet $E_{\mathrm{T}}$ and dijet mass requires multiple triggers, of roughly equal total rate, and with appropriately chosen $E_{\mathrm{T}}$ thresholds and prescales. In Fig. E.3 we show estimates of the Level-1 and HLT single jet trigger rates vs. corrected jet $E_{\mathrm{T}}$. In Table E.5 we show the single jet trigger paths from Level-1 to HLT including thresholds, prescales and estimates of the rates. We find that the maximum allowed HLT rate is the constraining factor for triggering on jets. For luminosity $\mathcal{L}=10^{32} \mathrm{~cm}^{-2} \mathrm{~s}^{-1}$, $\mathcal{L}=10^{33} \mathrm{~cm}^{-2} \mathrm{~s}^{-1}$ and $\mathcal{L}=10^{34} \mathrm{~cm}^{-2} \mathrm{~s}^{-1}$ the highest $E_{\mathrm{T}}$ threshold at HLT was chosen to give a rate of roughly $2.5 \mathrm{~Hz}$, as illustrated in Fig. E.3, so that four triggers would saturate an allowed jet rate of roughly $10 \mathrm{~Hz}$ at HLT.

The highest $E_{\mathrm{T}}$ threshold in each scenario is not prescaled. Lower thresholds are prescaled and are chosen at roughly half the $E_{\mathrm{T}}$ of the next highest threshold. This allows reasonable statistics in the overlap between the two samples, necessary for measuring trigger efficiencies and producing a continuous jet spectrum. Note that the total L1 jet rate required is only around $0.3 \mathrm{KHz}$, a small fraction of the Level- 1 total bandwidth. Since we are limited by HLT, not L1, for each trigger path the Level-1 thresholds are chosen low enough to have a Level-1 trigger efficiency of more than $95 \%$ at the corresponding HLT threshold in the path, as shown in Figure E.4. This strategy utilizes ten times more bandwidth at L1 than at HLT to insure that all of the resulting HLT sample has high enough trigger efficiency to be useful for analysis. 
Table E.5. Single jet trigger table showing path names, trigger thresholds in corrected $E_{\mathrm{T}}$, prescales, and estimated rates at Level-1 and HLT for four different luminosity scenarios.

\begin{tabular}{|c|c|c|c|c|c|c|}
\hline \multirow[b]{2}{*}{ Path } & \multicolumn{4}{|c|}{ L1 } & \multicolumn{2}{|c|}{ HLT } \\
\hline & $\begin{array}{l}E_{\mathrm{T}} \\
\text { Cut } \\
(\mathrm{GeV})\end{array}$ & $\begin{array}{l}\text { Unpres. } \\
\text { Rate } \\
(\mathrm{KHz})\end{array}$ & $\begin{array}{r}\text { Prescale } \\
\text { (N) }\end{array}$ & $\begin{array}{l}\text { Presc. } \\
\text { Rate } \\
(\mathrm{KHz})\end{array}$ & $\begin{array}{l}E_{\mathrm{T}} \\
\text { Cut } \\
\text { GeV) }\end{array}$ & $\begin{array}{l}\text { Rate } \\
(\mathrm{Hz})\end{array}$ \\
\hline \multicolumn{7}{|c|}{ Single Jet Triggers in Scenario 1: $\mathcal{L}=10^{32} \mathrm{~cm}^{-2} \mathrm{~s}^{-1}$} \\
\hline High & 140 & 0.044 & 1 & 0.044 & 250 & 2.8 \\
\hline Med & 60 & 3.9 & 40 & 0.097 & 120 & 2.4 \\
\hline Low & 25 & $2.9 \times 10^{2}$ & 2,000 & 0.146 & 60 & 2.8 \\
\hline \multicolumn{7}{|c|}{ Single Jet Triggers in Scenario 2: $\mathcal{L}=10^{33} \mathrm{~cm}^{-2} \mathrm{~s}^{-1}$} \\
\hline Ultra & 270 & 0.019 & 1 & 0.019 & 400 & 2.6 \\
\hline High & 140 & 0.44 & 10 & 0.044 & 250 & 2.8 \\
\hline Med & 60 & 39 & 400 & 0.097 & 120 & 2.4 \\
\hline Low & 25 & $2.9 \times 10^{3}$ & 20,000 & 0.146 & 60 & 2.8 \\
\hline \multicolumn{7}{|c|}{ Single Jet Triggers in Scenario 3: $\mathcal{L}=2 \times 10^{33} \mathrm{~cm}^{-2} \mathrm{~s}^{-1}$} \\
\hline Ultra & 270 & 0.038 & 1 & 0.038 & 400 & 5.2 \\
\hline High & 140 & 0.88 & 20 & 0.044 & 250 & 2.8 \\
\hline Med & 60 & 78 & 800 & 0.097 & 120 & 2.4 \\
\hline Low & 25 & $5.8 \times 10^{3}$ & 40,000 & 0.146 & 60 & 2.8 \\
\hline \multicolumn{7}{|c|}{ Single Jet Triggers in Scenario 4: $\mathcal{L}=10^{34} \mathrm{~cm}^{-2} \mathrm{~s}^{-1}$} \\
\hline Super & 450 & 0.014 & 1 & 0.014 & 600 & 2.8 \\
\hline Ultra & 270 & 0.19 & 10 & 0.019 & 400 & 2.6 \\
\hline High & 140 & 4.4 & 100 & 0.044 & 250 & 2.8 \\
\hline Med & 60 & $3.9 \times 10^{2}$ & 4,000 & 0.097 & 120 & 2.4 \\
\hline Low & 25 & $2.9 \times 10^{4}$ & 200,000 & 0.146 & 60 & 2.8 \\
\hline
\end{tabular}

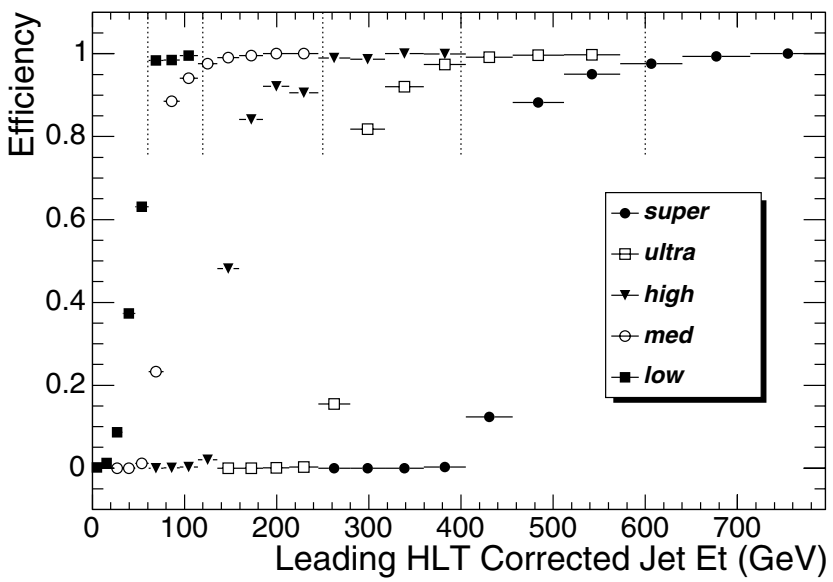

Figure E.4. The efficiency for passing the Level-1 jet trigger is shown as a function of HLT corrected jet $E_{\mathrm{T}}$ for each of the trigger paths shown in table E.5. The Level-1 thresholds were chosen to give an efficiency of greater than $95 \%$ at the corresponding HLT threshold.

Table E.5 illustrates a trigger strategy to maintain the continuity of jet analysis as the luminosity increases over a time span of years. The most important feature is that each luminosity scenario maintains the thresholds introduced in the previous scenario, allowing 
combination of trigger samples over time. For the prescaled thresholds, we may increase the prescales, either in discrete steps or dynamically, to maintain the allowed HLT rate with increasing luminosity. However, to maintain maximum sensitivity to new physics, the highest $E_{\mathrm{T}}$ threshold must never be prescaled. For example, in table E.5 when the luminosity increases by only a factor of 2 from $\mathcal{L}=10^{33} \mathrm{~cm}^{-2} \mathrm{~s}^{-1}$ to $\mathcal{L}=2 \times 10^{33} \mathrm{~cm}^{-2} \mathrm{~s}^{-1}$, we double the prescales on the prescaled triggers but don't change either the threshold or the prescale of the highest $E_{\mathrm{T}}$ trigger labelled Ultra. This allows us to maintain stability of the single jet trigger thresholds, and analyses that depend on them, with only modest increases in the total rate for single jets. When the HLT rate in the unprescaled trigger becomes intolerably high, a higher $E_{\mathrm{T}}$ threshold unprescaled trigger is introduced, and the old unprescaled trigger can then be prescaled as necessary.

For the particular case of single-jet triggers: To commission the calorimeters, or perform a one-time jet study, it may be desirable to have more jets. If we want to write more than roughly $10 \mathrm{~Hz}$ of single jets at HLT, we can still use the same suite of single-jets, but lower the prescales to obtain more jets at low $E_{\mathrm{T}}$. This is preferable to moving the threshold for the unprescaled trigger, or any of the triggers, and ending up with a special trigger that is only applicable for a given running period and difficult to combine with other samples.

For $\mathcal{L}=2 \times 10^{33} \mathrm{~cm}^{-2} \mathrm{~s}^{-1}$, the suggested jet thresholds have been studied again in the scope of the global High-Level trigger analysis (Sec. E.5) and new Level-1 prescales and rates have been determined. For the trigger table proposed in this study, we have chosen four triggers, with $E_{\mathrm{T}}$ thresholds of 400, 250, 120 and $60 \mathrm{GeV}$, amd prescales of 1, 10, 1000 and 100000 , respectively.

E.4.3.3. Other triggers. The remaining triggers that have been introduced since the DAQ TDR are:

- Inclusive $E_{\mathrm{T}}^{\text {miss }}$ trigger. As discussed earlier, this is a difficult trigger that is subject to the good understanding and control of the detector noise. We suggest here a single $E_{\mathrm{T}}^{\text {miss }}$ trigger with $E_{\mathrm{T}}>91 \mathrm{GeV}$, driven by the $E_{\mathrm{T}}^{\text {miss }}>60 \mathrm{GeV} \mathrm{L1}$ condition. This is just an indicative value, rather on the low side, as $E_{\mathrm{T}}^{\mathrm{miss}}$ rates appear lower compared to Ref. [76]. It is foreseen that additional $E_{\mathrm{T}}^{\mathrm{miss}}$ triggers with different thresholds and prescales will be introduced in the future.

- Diffractive trigger. This trigger is different than all others described earlier in that it uses the TOTEM detector [823, 824]. At Level-1 we ask for two central jets with $E_{\mathrm{T}}>40 \mathrm{GeV}$, along with a proton tagged with the $220 \mathrm{~m}$ Roman Pot. At HLT, a similar dijet cut and a "back-to-back" azimuthal condition are applied. We also require that we have a consistent measurement of the proton energy loss $\xi$ in the two hemispheres (within $2 \sigma$, measured at the Roman Pots). A final condition for a tagged proton seen by the $420 \mathrm{~m}$ Roman Pot brings the HLT rate down to $\mathcal{O}(1) \mathrm{Hz}$. This trigger is discussed in detail in Sec. E.3.

\section{E.5. Performance}

The performance of the trigger system is studied by using simulated data that has been digitised with appropriate pileup ${ }^{56}$, taking into account both the inelastic $(55.2 \mathrm{mb})$ and the diffractive $(24.1 \mathrm{mb})$ cross sections. To reduce the amount of simulation time, about 50 million

\footnotetext{
56 We have estimated the average number of in-time interactions per bunch crossing to be 5 for $\mathcal{L}=2 \times$ $10^{33} \mathrm{~cm}^{-2} \mathrm{~s}^{-1}$. Additional, out-of-time interactions have been ignored.
} 
Table E.6. Description and sizes of MC Samples used for the trigger studies. The contribution to the HLT rate does not include pre-scaled triggers.

\begin{tabular}{|c|c|c|c|c|}
\hline Sample description & $\begin{array}{l}\text { Cuts } \\
\text { (Momenta in } \mathrm{GeV} / \mathrm{c} \text { ) }\end{array}$ & $\begin{array}{l}\text { Cross section } \\
(\mathrm{mb})\end{array}$ & \# of events & $\begin{array}{l}\text { HLT rate } \\
(\mathrm{Hz})\end{array}$ \\
\hline $\begin{array}{l}\text { Minimum bias with } \\
\text { in-time pile-up; } \\
\langle \# \text { of interactions }\rangle=5\end{array}$ & - & 79.3 & 50000000 & - \\
\hline QCD & $\hat{p_{\mathrm{T}}} \in[15,20]$ & $1.46 \times 10^{0}$ & 49491 & \\
\hline QCD & $\hat{p_{\mathrm{T}}} \in[20,30]$ & $6.32 \times 10^{-1}$ & 49244 & \\
\hline QCD & $\hat{p_{\mathrm{T}}} \in[30,50]$ & $1.63 \times 10^{-1}$ & 49742 & \\
\hline QCD & $\hat{p_{\mathrm{T}}} \in[50,80]$ & $2.16 \times 10^{-2}$ & 99486 & \\
\hline QCD & $\hat{p_{\mathrm{T}}} \in[80,120]$ & $3.08 \times 10^{-3}$ & 96238 & \\
\hline QCD & $\hat{p_{\mathrm{T}}} \in[120,170]$ & $4.94 \times 10^{-4}$ & 99736 & \\
\hline QCD & $\hat{p_{\mathrm{T}}} \in[170,230]$ & $1.01 \times 10^{-4}$ & 99226 & \\
\hline QCD & $\hat{p_{\mathrm{T}}} \in[230,300]$ & $2.45 \times 10^{-5}$ & 99481 & \\
\hline QCD & $\hat{p_{\mathrm{T}}} \in[300,380]$ & $6.24 \times 10^{-6}$ & 98739 & \\
\hline QCD & $\hat{p_{\mathrm{T}}} \in[380,470]$ & $1.78 \times 10^{-6}$ & 46491 & \\
\hline QCD & $\hat{p_{\mathrm{T}}} \in[470,600]$ & $6.83 \times 10^{-7}$ & 47496 & \\
\hline QCD & $\hat{p_{\mathrm{T}}} \in[600,800]$ & $2.04 \times 10^{-7}$ & 48986 & \\
\hline \multirow[t]{2}{*}{ QCD } & $\hat{p_{\mathrm{T}}} \in[800,1000]$ & $3.51 \times 10^{-8}$ & 45741 & \\
\hline & Partial total & & 930099 & $55.3 \pm 6.9$ \\
\hline$W \rightarrow e v$ & 1 electron with $|\eta|<2.7, p_{\mathrm{T}}>25$ & $7.9 \times 10^{-6}$ & 3944 & $9.7 \pm 0.2$ \\
\hline$Z \rightarrow e e$ & 2 electrons with $|\eta|<2.7, p_{\mathrm{T}}>5$ & $8.2 \times 10^{-7}$ & 4000 & $1.4 \pm 0.0$ \\
\hline $\begin{array}{l}p p \rightarrow \text { jet }(\mathrm{s})+\gamma, \\
\hat{p_{\mathrm{T}}}>30 \mathrm{GeV} / \mathrm{c}\end{array}$ & jet: $p_{\mathrm{T}}>20, \gamma: p_{\mathrm{T}}>30$ & $2.5 \times 10^{-6}$ & 4000 & $1.0 \pm 0.0$ \\
\hline$W \rightarrow \mu \nu$ & 1 muon with $|\eta|<2.5, p_{\mathrm{T}}>14$ & $9.8 \times 10^{-6}$ & 4000 & $14.0 \pm 0.3$ \\
\hline$Z \rightarrow \mu \mu$ & 2 muons with $|\eta|<2.5, p_{\mathrm{T}}>20,10$ & $7.9 \times 10^{-7}$ & 2941 & $1.5 \pm 0.0$ \\
\hline$p p \rightarrow \mu+X$ & 1 muon with $p_{\mathrm{T}}>3$ & $2.4 \times 10^{-2}$ & 839999 & $25.5 \pm 1.2$ \\
\hline
\end{tabular}

minimum bias events were simulated and reused in random combinations. It was ensured that these events do not cause triggers by themselves to avoid over estimating the rates due to this reuse of events.

In the following sections we list trigger rates along with their statistical uncertainties. These take into account the luminosity-dependent weight of the events from the different samples, the corresponding cross sections and the $\hat{p_{\mathrm{T}}}$ of the main interaction and the pile-up contribution. They do not take into account the uncertainties of these individual factors, i.e. no systematic effects are studied here.

The Level-1 calorimeter trigger object rate studies are performed using QCD data that has been generated in several bins of $\hat{p_{\mathrm{T}}}$. A special event-weighting procedure has been applied to properly take into account the cross sections of the sub-samples. The Level- 1 muon and $E_{\mathrm{T}}^{\text {miss }}$ rate studies are performed using a purely minimum bias sample.

The HLT rates are estimated using specially enriched samples. For the triggers invoking muons, electrons and photons we have used a minimum bias sample enriched in muons, as well as $W \rightarrow e / \mu \nu, Z \rightarrow e e / \mu \mu$ and jet(s) $+\gamma \mathrm{MC}$ datasets. For the triggers including jets we have used QCD samples. These samples also contribute to the electron and photon triggers. Events triggered exclusively with muons have been excluded from the QCD samples, to avoid double-counting with the muon-enriched sample. Table E.6 summarises the MC samples used for the trigger studies, and their corresponding contribution to the HLT rate. A more detailed breakdown of the contributions to the electron, photon and muon trigger rates from 
Table E.7. Trigger table showing Level-1 rates for DAQ TDR chosen thresholds for $\mathcal{L}=2 \times 10^{33} \mathrm{~cm}^{-2} \mathrm{~s}^{-1}$. Whenever the "95\% efficiency point" is reported in DAQ TDR, we also give the actual kinematic threshold that has been applied.

\begin{tabular}{|c|c|c|c|c|}
\hline Trigger & $95 \%$ Eff. point & Threshold (GeV) & Rate $(\mathrm{kHz})$ & Cumulative Rate $(\mathrm{kHz})$ \\
\hline Single $e \gamma$ & 29 & 23.4 & $3.38 \pm 0.23$ & $3.4 \pm 0.2$ \\
\hline Double $e \gamma$ & 17 & 11.5 & $0.85 \pm 0.12$ & $4.0 \pm 0.3$ \\
\hline Single $\mu$ & - & 14 & $2.53 \pm 0.20$ & $6.5 \pm 0.3$ \\
\hline Double $\mu$ & - & 3 & $4.05 \pm 0.26$ & $10.3 \pm 0.4$ \\
\hline Single $\tau$ & 86 & 93 & $3.56 \pm 0.24$ & $9.7 \pm 0.4$ \\
\hline Double $\tau$ & 59 & 66 & $1.97 \pm 0.18$ & $10.6 \pm 0.4$ \\
\hline 1-, 3-, 4-jets & $177,86,70$ & $135,58,45$ & $2.43 \pm 0.20$ & $11.9 \pm 0.4$ \\
\hline $\mathrm{Jet}+E_{\mathrm{T}}^{\text {miss }}$ & - & 88,46 & $1.07 \pm 0.13$ & $12.2 \pm 0.4$ \\
\hline$e \gamma+\tau$ & - & 21,45 & $3.64 \pm 0.24$ & $12.9 \pm 0.5$ \\
\hline & \multicolumn{3}{|c|}{ Level-1 Trigger Total } & $12.9 \pm 0.5$ \\
\hline
\end{tabular}

the different samples is discussed later (Sec. E.5.3-rates). For our calculations, we have used the standard HLT physics algorithms (ORCA/_8/_13/_3 [10]) for the implementation of all trigger paths. At the time of this writing, this includes the latest algorithms and jet calibrations. For the global evaluation of the trigger rates we have used the "HLT steering code"

\section{E.5.1. Level-1 rates}

The background at Level-1 is entirely dominated by strong interactions. The muon rates at Level-1 are dominated by low $p_{\mathrm{T}}$ muons which are reconstructed as high $p_{\mathrm{T}}$ muons due to limited resolution at the trigger level. For the electron/photon trigger the rate is dominated by jets that fragment to high $E_{\mathrm{T}} \pi^{0} \mathrm{~s}$. The jet rates are dominated by true jets in the QCD events. The $E_{\mathrm{T}}^{\text {miss }}$ background is due to the limited energy resolution, and pile-up of minimum bias interactions.

We first produce a trigger table with Level-1 rates for DAQ TDR chosen thresholds for comparison. For the calculations we use a sample of 2 million minimum bias crossings with an average of 5 events per crossing, constructed from the minbias events, without reuse of events. The out-of-time pile-up is neglected. Even though there are small differences for the individual triggers, the integral rate is consistent with the rates reported in Ref. [76]. This comparison serves as a cross-check and is a necessary intermediate step before the introduction of new trigger terms. Table E.7 summarises the Level-1 rate calculations for the DAQ TDR triggers with the new MC samples. Besides the "95\% efficiency points" (used throughout the DAQ TDR), the applied L1 thresholds are also given.

For the new trigger table: We select several thresholds for each trigger object type and quote corresponding rates and prescales for $\mathcal{L}=2 \times 10^{33} \mathrm{~cm}^{-2} \mathrm{~s}^{-1}$. For the single objects we have added a series of prescaled triggers to determine the efficiency turn-on. For the multi-object triggers we have picked the lowest common threshold that is allowed for the allocated bandwidth. For the cross-channel triggers we have attempted to keep the lepton thresholds as low as possible, within the allocated bandwidth based on the physics needs of the experiment. The prescales are chosen such that the simulated rate at all times falls below the DAQ bandwidth taking into account a safety factor of 3 . The total Level-1 rate for all triggers (including prescaled ones) is $22.6 \pm 0.3 \mathrm{kHz}$. 
Table E.8. Comparison of HLT bandwidth given to various trigger paths calculated in this study with the DAQ TDR. See text for details on different kinematic cuts and changes in the HLT algorithms.

\begin{tabular}{lcc}
\hline Trigger & DAQ TDR Rate $(\mathrm{Hz})$ & New Rate $(\mathrm{Hz})$ \\
\hline Inclusive $e$ & 33.0 & $23.5 \pm 6.7$ \\
$e-e$ & 1.0 & $1.0 \pm 0.1$ \\
Relaxed $e-e$ & 1.0 & $1.3 \pm 0.1$ \\
Inclusive $\gamma$ & 4.0 & $3.1 \pm 0.2$ \\
$\gamma-\gamma$ & 5.0 & $1.6 \pm 0.7$ \\
Relaxed $\gamma-\gamma$ & 5.0 & $1.2 \pm 0.6$ \\
Inclusive $\mu$ & 25.0 & $25.8 \pm 0.8$ \\
$\mu$ - $\mu$ & 4.0 & $4.8 \pm 0.4$ \\
$\tau+E_{\mathrm{T}}^{\text {miss }}$ & 1.0 & $0.5 \pm 0.1$ \\
$\tau+e$ & 2.0 & $<1.0$ \\
Double Pixel $\tau$ & 1.0 & $4.1 \pm 1.1$ \\
Double Tracker $\tau$ & 1.0 & $6.0 \pm 1.1$ \\
Single jet & 1.0 & $4.8 \pm 0.0$ \\
Triple jet & 1.0 & $1.1 \pm 0.0$ \\
Quadruple jet & 7.0 & $8.9 \pm 0.2$ \\
jet $+E_{\mathrm{T}}^{\text {miss }}$ & 5.0 & $3.2 \pm 0.1$ \\
$b$-jet (leading jet) & 5.0 & $10.3 \pm 0.3$ \\
$b$-jet (2 ${ }^{\text {nd }}$ leading jet) & 5.0 & $8.7 \pm 0.3$ \\
\hline
\end{tabular}

\section{E.5.2. Level-1 trigger object corrections}

The trigger decisions are based on $E_{\mathrm{T}}$ of the objects reconstructed by various algorithms. Unfortunately, the energy deposition in the calorimeter and the size of the trigger towers, are not entirely uniform. We have used fits to the reconstructed-to-generated $E_{\mathrm{T}}$ ratios to correct for non-uniformity of the response for jets and electron/photon candidates found at all levels of trigger [830]. This correction procedure adjusts the mean response to the generated level.

The energy response of the calorimeters and the limited number of bits used in trigger calculations result in a finite resolution for the reconstructed trigger objects. Similarly, misalignments of the tracking systems and the limited number of patterns in the muon trigger look-up-tables also result in a finite resolution. To avoid systematic problems in understanding the trigger efficiency turn-on with the $E_{\mathrm{T}}$ of the trigger objects, it is envisioned that only data where high trigger efficiency is assured is used for analysis.

\section{E.5.3. HLT rates}

A rough comparison of the HLT bandwidth given to various triggers, calculated with the latest algorithms and the ones reported in Ref. [76] is shown in Table E.8. It must be noted that not only thresholds but also other cuts are different in the two trigger studies. Furthermore, additional changes in the HLT algorithms (summarised in Sec. E.2.2) must be taken into account. This comparison serves only as a consistency check. It reaffirms that despite the evolution of the CMS reconstruction algorithms over the years, trigger rates remain under control and that no major bandwidth changes are expected.

Table E. 10 shows in a similar way the contributions to the single and double standard and relaxed muon rates from the various MC samples.

The contributions to the single and double electron and photon trigger rates at HLT from the various MC samples is given at Table E.9-egamma. The main contributions to the single 
Table E.9. Contributions to the HLT rates for the electron and photon triggers from the various MC datasets.

\begin{tabular}{lcrlcc}
\hline Trigger & Threshold $(\mathrm{GeV})$ & \multicolumn{5}{c}{ Rates $(\mathrm{Hz})$} \\
\cline { 3 - 6 } & & QCD & $W \rightarrow e v$ & $Z \rightarrow e e$ & jet(s) $+\gamma$ \\
\hline Inclusive $e$ & 26 & $12.6 \pm 6.7$ & $9.7 \pm 0.2$ & $1.2 \pm 0.0$ & - \\
$e-e$ & 12,12 & $0.1 \pm 0.1$ & - & $1.0 \pm 0.0$ & - \\
Relaxed $e-e$ & 19,19 & $0.3 \pm 0.1$ & - & $1.0 \pm 0.0$ & - \\
Inclusive $\gamma$ & 80 & $1.1 \pm 0.2$ & - & - & $2.0 \pm 0.1$ \\
$\gamma-\gamma$ & 30,20 & $1.3 \pm 0.8$ & - & - & $0.3 \pm 0.0$ \\
Relaxed $\gamma-\gamma$ & 30,20 & $0.9 \pm 0.6$ & - & - & $0.3 \pm 0.0$ \\
\hline
\end{tabular}

Table E.10. Contributions to the HLT rates for the muon triggers from the various MC datasets.

\begin{tabular}{lcccc}
\hline Trigger & \multirow{2}{*}{$\begin{array}{c}\text { Threshold } \\
(\mathrm{GeV})\end{array}$} & Enriched- $\mu$ sample & $W \rightarrow \mu \nu$ & $Z \rightarrow \mu \mu$ \\
\cline { 3 - 5 } & & $10.9 \pm 0.8$ & $13.4 \pm 0.3$ & $1.5 \pm 0.0$ \\
Inclusive $\mu$ & 19 & $5.1 \pm 0.5$ & $5.7 \pm 0.1$ & $1.1 \pm 0.0$ \\
Relaxed $\mu$ & 37 & $3.4 \pm 0.4$ & - & $1.3 \pm 0.0$ \\
$\mu-\mu$ & 7,7 & $7.1 \pm 0.5$ & - & $1.4 \pm 0.0$ \\
Relaxed $\mu-\mu$ & 10,10 & & & \\
\hline
\end{tabular}

Table E.11. The Level-1 Trigger Menu at $\mathcal{L}=2 \times 10^{33} \mathrm{~cm}^{-2} \mathrm{~s}^{-1}$. Individual and cumulative rates are given for the different trigger paths and selected kinematic thresholds.

\begin{tabular}{llll}
\hline Trigger & $\begin{array}{l}\text { Level-1 Threshold } \\
(\mathrm{GeV})\end{array}$ & $\begin{array}{l}\text { Level-1 Rate } \\
(\mathrm{kHz})\end{array}$ & $\begin{array}{l}\text { Cumulative Level-1 Rate } \\
(\mathrm{kHz})\end{array}$ \\
\hline Inclusive $e \gamma$ & 22 & $4.2 \pm 0.1$ & $4.2 \pm 0.1$ \\
Double $e \gamma$ & 11 & $1.1 \pm 0.1$ & $5.1 \pm 0.1$ \\
Inclusive $\mu$ & 14 & $2.7 \pm 0.1$ & $7.8 \pm 0.2$ \\
Double $\mu$ & 3 & $3.8 \pm 0.1$ & $11.4 \pm 0.2$ \\
Inclusive $\tau$ & 100 & $1.9 \pm 0.1$ & $13.0 \pm 0.2$ \\
Double $\tau$ & 66 & $1.8 \pm 0.1$ & $14.1 \pm 0.2$ \\
$1-, 2-, 3-, 4-\mathrm{jets}$ & $150,100,70,50$ & $1.8 \pm 0.1$ & $14.8 \pm 0.3$ \\
$H_{\mathrm{T}}$ & 300 & $1.2 \pm 0.1$ & $15.0 \pm 0.3$ \\
$E_{\mathrm{T}}^{\text {miss }}$ & 60 & $0.3 \pm 0.1$ & $15.1 \pm 0.3$ \\
$H_{\mathrm{T}}+E_{\mathrm{T}}^{\text {miss }}$ & 200,40 & $0.7 \pm 0.1$ & $15.3 \pm 0.3$ \\
jet $+E_{\mathrm{T}}^{\text {miss }}$ & 100,40 & $0.8 \pm 0.1$ & $15.4 \pm 0.3$ \\
$\tau+E_{\mathrm{T}}^{\text {miss }}$ & 60,40 & $2.7 \pm 0.1$ & $17.4 \pm 0.3$ \\
$\mu+E_{\mathrm{T}}^{\text {miss }}$ & 5,30 & $0.3 \pm 0.1$ & $17.6 \pm 0.3$ \\
$e \gamma+E_{\mathrm{T}}^{\text {miss }}$ & 15,30 & $0.7 \pm 0.1$ & $17.7 \pm 0.3$ \\
$\mu+$ jet & 7,100 & $0.1 \pm 0.1$ & $17.8 \pm 0.3$ \\
$e \gamma+$ jet & 15,100 & $0.6 \pm 0.1$ & $17.8 \pm 0.3$ \\
$\mu+\tau$ & 7,40 & $1.2 \pm 0.1$ & $18.4 \pm 0.3$ \\
$e \gamma+\tau$ & 14,52 & $5.4 \pm 0.2$ & $20.7 \pm 0.3$ \\
$e \gamma+\mu$ & 15,7 & $0.2 \pm 0.1$ & $20.7 \pm 0.3$ \\
Prescaled & & & $22.6 \pm 0.3$ \\
& Total Level-1 Rate & & $22.6 \pm 0.3$ \\
\hline & & &
\end{tabular}


Table E.12. The High-Level Trigger Menu at $\mathcal{L}=2 \times 10^{33} \mathrm{~cm}^{-2} \mathrm{~s}^{-1}$ for an output of approximately $120 \mathrm{~Hz}$. The $E_{\mathrm{T}}$ values are the kinematic thresholds for the different trigger paths.

\begin{tabular}{|c|c|c|c|c|}
\hline Trigger & $\begin{array}{l}\text { Level-1 } \\
\text { bits used }\end{array}$ & $\begin{array}{l}\text { Level-1 } \\
\text { Prescale }\end{array}$ & $\begin{array}{l}\text { HLT Threshold } \\
(\mathrm{GeV})\end{array}$ & $\begin{array}{l}\text { HLT Rate } \\
(\mathrm{Hz})\end{array}$ \\
\hline Inclusive $e$ & 2 & 1 & 26 & $23.5 \pm 6.7$ \\
\hline$e-e$ & 3 & 1 & 12,12 & $1.0 \pm 0.1$ \\
\hline Relaxed $e-e$ & 4 & 1 & 19,19 & $1.3 \pm 0.1$ \\
\hline Inclusive $\gamma$ & 2 & 1 & 80 & $3.1 \pm 0.2$ \\
\hline$\gamma-\gamma$ & 3 & 1 & 30,20 & $1.6 \pm 0.7$ \\
\hline Relaxed $\gamma-\gamma$ & 4 & 1 & 30,20 & $1.2 \pm 0.6$ \\
\hline Inclusive $\mu$ & 0 & 1 & 19 & $25.8 \pm 0.8$ \\
\hline Relaxed $\mu$ & 0 & 1 & 37 & $11.9 \pm 0.5$ \\
\hline$\mu-\mu$ & 1 & 1 & 7,7 & $4.8 \pm 0.4$ \\
\hline Relaxed $\mu-\mu$ & 1 & 1 & 10,10 & $8.6 \pm 0.6$ \\
\hline$\tau+E_{\mathrm{T}}^{\mathrm{miss}}$ & 10 & 1 & $65\left(E_{\mathrm{T}}^{\text {miss }}\right)$ & $0.5 \pm 0.1$ \\
\hline Pixel $\tau-\tau$ & 10,13 & 1 & - & $4.1 \pm 1.1$ \\
\hline Tracker $\tau-\tau$ & 10,13 & 1 & - & $6.0 \pm 1.1$ \\
\hline$\tau+e$ & 26 & 1 & 52,16 & $<1.0$ \\
\hline$\tau+\mu$ & 0 & 1 & 40,15 & $<1.0$ \\
\hline$b$-jet (leading jet) & $36,37,38,39$ & 1 & $350,150,55$ (see text) & $10.3 \pm 0.3$ \\
\hline$b$-jet ( $2^{\text {nd }}$ leading jet $)$ & $36,37,38,39$ & 1 & $350,150,55$ (see text) & $8.7 \pm 0.3$ \\
\hline Single-jet & 36 & 1 & 400 & $4.8 \pm 0.0$ \\
\hline Double-jet & 36,37 & 1 & 350 & $3.9 \pm 0.0$ \\
\hline Triple-jet & $36,37,38$ & 1 & 195 & $1.1 \pm 0.0$ \\
\hline Quadruple-jet & $36,37,38,39$ & 1 & 80 & $8.9 \pm 0.2$ \\
\hline$E_{\mathrm{T}}^{\text {miss }}$ & 32 & 1 & 91 & $2.5 \pm 0.2$ \\
\hline jet $+E_{\mathrm{T}}^{\mathrm{miss}}$ & 32 & 1 & 180,80 & $3.2 \pm 0.1$ \\
\hline acoplanar 2 jets & 36,37 & 1 & 200,200 & $0.2 \pm 0.0$ \\
\hline acoplanar jet $+E_{\mathrm{T}}^{\text {miss }}$ & 32 & 1 & 100,80 & $0.1 \pm 0.0$ \\
\hline 2 jets $+E_{\mathrm{T}}^{\text {miss }}$ & 32 & 1 & 155,80 & $1.6 \pm 0.0$ \\
\hline 3 jets $+E_{\mathrm{T}}^{\text {miss }}$ & 32 & 1 & 85,80 & $0.9 \pm 0.1$ \\
\hline 4 jets $+E_{\mathrm{T}}^{\text {miss }}$ & 32 & 1 & 35,80 & $1.7 \pm 0.2$ \\
\hline Diffractive & Sec. E.3 & 1 & 40,40 & $<1.0$ \\
\hline$H_{\mathrm{T}}+E_{\mathrm{T}}^{\text {miss }}$ & 31 & 1 & 350,80 & $5.6 \pm 0.2$ \\
\hline$H_{\mathrm{T}}+e$ & 31 & 1 & 350,20 & $0.4 \pm 0.1$ \\
\hline Inclusive $\gamma$ & 2 & 400 & 23 & $0.3 \pm 0.0$ \\
\hline$\gamma-\gamma$ & 3 & 20 & 12,12 & $2.5 \pm 1.4$ \\
\hline Relaxed $\gamma-\gamma$ & 4 & 20 & 19,19 & $0.1 \pm 0.0$ \\
\hline Single-jet & 33 & 10 & 250 & $5.2 \pm 0.0$ \\
\hline Single-jet & 34 & 1000 & 120 & $1.6 \pm 0.0$ \\
\hline Single-jet & 35 & 100000 & 60 & $0.4 \pm 0.0$ \\
\hline \multicolumn{4}{|c|}{ Total HLT rate } & $119.3 \pm 7.2$ \\
\hline
\end{tabular}

electron trigger come from the QCD and $W \rightarrow e v$ samples, whereas for the single photon trigger the primary source is the jet(s) $+\gamma$ events.

\section{E.5.4. Trigger tables}

Table E.11 summarises the Level-1 triggers used in this study, their kinematic thresholds, the individual and cumulative rates. We have assumed a DAQ capability of $50 \mathrm{kHz}$, taking into account a safety factor of 3 . 


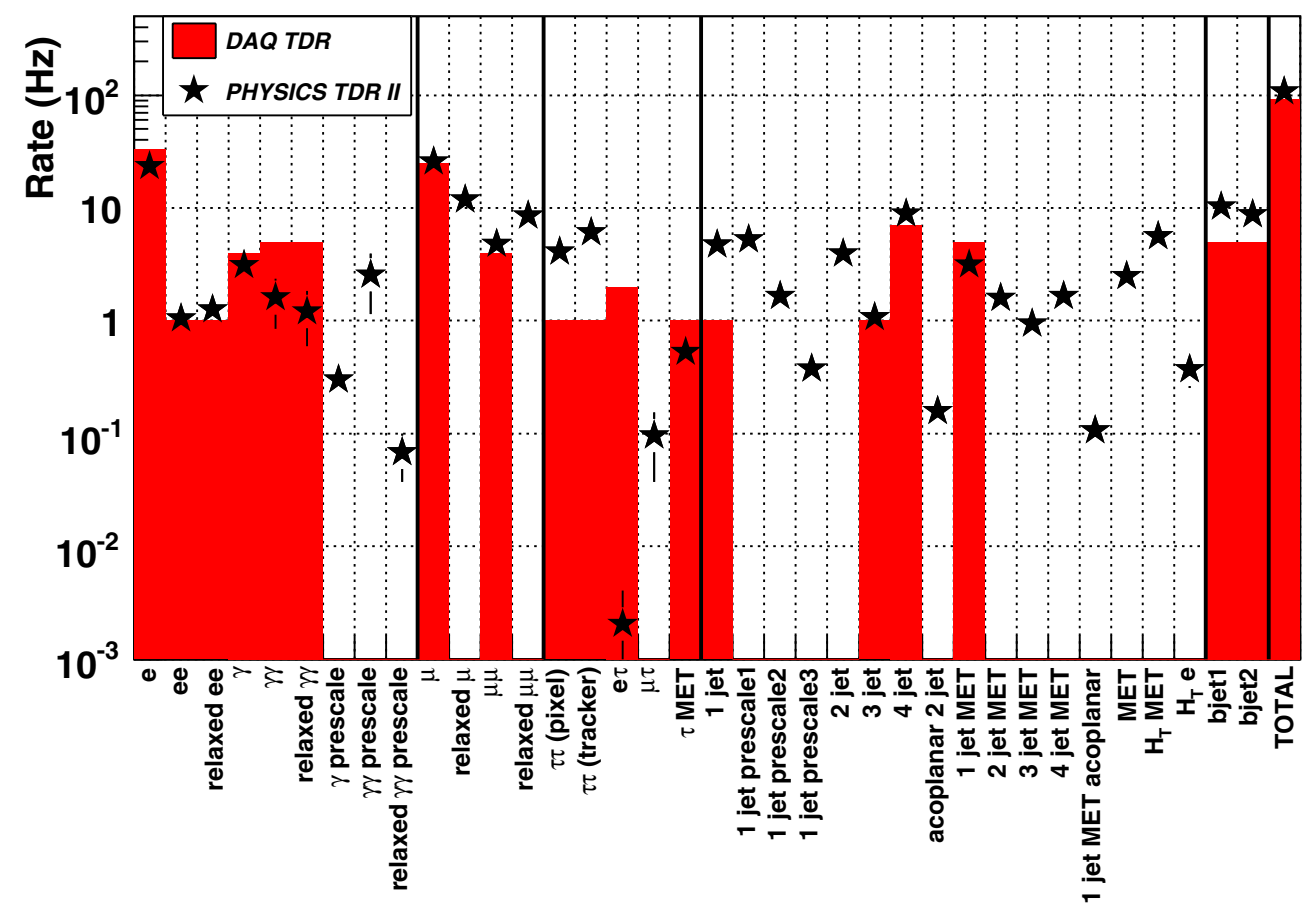

Figure E.5. Heuristic comparison of HLT bandwidth assigned to various trigger paths calculated in this study with the DAQ TDR. For the triggers introduced in this study the DAQ TDR entries appear empty. See text for details on different kinematic cuts and changes in the HLT algorithms.

Table E.12 gives the full list of trigger paths proposed for $\mathcal{L}=2 \times 10^{33} \mathrm{~cm}^{-2} \mathrm{~s}^{-1}$ that have been described earlier for an HLT output rate of approximately $120 \mathrm{~Hz}$.

Fig. E.5 shows a graphic representation of the HLT bandwidth assigned to all trigger paths presented in this study. For the triggers that appeared in the DAQ TDR, the corresponding rates are overlaid, in a heuristic comparison. 


\section{Glossary}

ADC

AdS

ALEPH

ALICE

ALPGEN

ATLAS

BMU

BR

BX

BXN

CASTOR

CDF

CL

CLHEP

CMKIN

CMS

CMSIM

CMSSW

CPT

CPU

CompHEP

CSC

CVS

DØ

DAQ

DELPHI

DESY

DST

DT

DY

EB

ECAL

ED

EE

EM

EMU

ES

EW

FAMOS

FLUKA

FNAL

FSR
Analog to Digital Converter

Anti de Sitter space

An experiment at LEP

A Large Ion Collider Experiment at the LHC

Monte Carlo event generator for multi-parton processes in hadronic collisions

A Toroidal LHC ApparatuS experiment

Barrel Muon system

Branching Ratio

Bunch Crossing

Bunch Crossing Number

Calorimeter in the forward region of CMS

Collider Detector Facility experiment at the FNAL Tevatron

Confidence Level

Class Library for HEP

CMS Kinematics Package (legacy Fortran)

Compact Muon Solenoid experiment

CMS Simulation Package (legacy Fortran)

CMS Software framework

Computing, Physics, TriDAS and software projects of CMS

Central Processing Unit

Monte Carlo event generator for high-energy physics collisions

Cathode Strip Chamber muon system

Concurrent Versions System

Experiment at the FNAL Tevatron

Data Acquisition

An experiment at LEP

Deutsches Elektronen SYnchrotron laboratory, Hamburg

Data Summary Tape - a compact event format

Drift Tube muon system

Drell-Yan

Electromagnetic Calorimeter (Barrel)

Electromagnetic Calorimeter

Extra Dimensions

Electromagnetic Calorimeter (Endcap)

Electromagnetic

Endcap Muon system

Endcap preShower detector

ElectroWeak

CMS Fast Simulation

Computer program for hadron shower calculations

Fermi National Accelerator Laboratory, USA

Final State Radiation 


\begin{tabular}{|l} 
Gb \\
GB \\
GCALOR \\
GEANT \\
GMSB \\
GUT
\end{tabular}

H1

HAD

HCAL

HB

HE

HEP

HEPEVT

HERA

HERWIG

HF

HI

HIJING

HLT

HO

IGUANA

I/O

IP

ISR

JES

Kalman Filter

kb

kB

L1

L3

LCG

LED

LEP

LHC

LHCb

LHCC

LHEP

LL

LO

LOI

LPC

LS

LSP
Gigabit $\left(10^{9}\right.$ bits $)$

Gigabyte $\left(10^{9}\right.$ bytes $)$

Computer program for hadron shower calculations

Detector simulation framework and toolkit

Gauge Mediated Symmetry Breaking

Grand Unified Theory

An experiment at the DESY HERA collider

Hadronic

Hadron Calorimeter

Hadron Calorimeter (Barrel)

Hadron Calorimeter (Endcap)

High Energy Physics

HEP Event (generated event format)

Electron-proton collider at DESY

Hadron Emission Reactions With Interfering Gluons, a Monte

Carlo event generator for high-energy physics collisions

Hadron Calorimeter (Forward)

Heavy Ion(s)

Heavy Ion Jet INteraction Generator, Monte Carlo event generator for heavy-ion collisions

High-Level Trigger

Hadron Calorimeter (Outer Barrel)

Interactive Graphics for User ANAlysis - used for the CMS

Event Display Package

Input/Output

Impact Parameter, also Impact Point or Internet Protocol

Initial State Radiation, also Intersecting Storage Ring collider at CERN

Jet Energy Scale

Computational method for fitting tracks

kilobit $\left(10^{3}\right.$ bits $)$

kilobytes $\left(10^{3}\right.$ bytes $)$

Level-1 hardware-based trigger

An experiment at LEP

LHC Computing Grid (a common computing project)

Large Extra Dimenstions, also Light Emitting Diode

Large Electron Positron collider at CERN

Large Hadron Collider

Large Hadron Collider Beauty experiment

LHC (review) Committee

Physics model of GEANT4

Leading Logarithm, also Log Likelihood

Leading Order calculation

Letter Of Intent

LHC Physics Center, Fermilab

Like-Sign

Lightest Supersymmetric Particle 
Mb

MB

MC

ME

MET

metadata

MIP

MSUGRA

MSSM

MTCC

ndf

NLO

NN

NNLO

NS

OO

OPAL

ORCA

OS

OSCAR

P5

PAW

PB

PC

PD

PDF

PRS

PS

PV

PYTHIA

QCD

QED

QGSP

RecHit

RHIC

RMS

ROOT

RPC
Megabit (10 bits)

Muon system (Barrel), also Mother Board or Megabyte ( $10^{6}$ bytes)

Monte Carlo simulation program/technique, also Mini-Crate of DT system

Muon system (Endcap), also Matrix Element or Monitoring

Element

Missing Transverse Energy

Data describing characteristics of other data

Minimum Ionizing Particle

Minimal SUper GRAvity model of supersymmetry

Minimal SuperSymmetric Model

Magnet Test Cosmic Challenge

number of degrees of freedom

Next-to-Leading Order calculation

Neural Network

Next-to-Next-to-Leading Order calculation

Numbering Scheme

Object Oriented

An experiment at LEP

Object-oriented Reconstruction for CMS Analysis

Opposite-Sign, also Operating System

Object-oriented Simulation for CMS Analysis and

Reconstruction

Point 5 collision area of LHC

Physics Analysis Workstation (legacy interactive analysis

application)

Petabyte $\left(10^{5}\right.$ bytes $)$

Personal Computer

Pixel Detector

Parton Density Function, also Probability Distribution

Function (p.d.f.)

Physics Reconstruction and Selection groups

Proton Synchrotron, also Parton Showers

Primary Vertex

Monte Carlo event generator for high-energy physics collisions

Quantum Chromodynamics

Quantum Electrodynamics

Physics model of GEANT4

Reconstructed hit in a detector element

Relativistic Heavy Ion Collider (at Brookhaven, USA)

Root Mean Square

An object-oriented data analysis framework

Resistive Plate Chamber muon system 
SLT

SM

S/N

SPS

SS

SST

SUSY

SV

T1, T2

TAG

Tb

TB

TDR

TEC

TIB

TID

TOB

TOTEM

TPD

TriDAS

UA1

UA2

UE

UED

VBF

VPT

WWW

ZDC

ZEUS
Soft Lepton Tag

Standard Model, also SuperModule (ECAL) or Storage

Manager (DAQ)

Signal to Noise ratio

Super Proton Synchrotron collider at CERN

Same-Sign

Silicon Strip Tracker

SUperSYmmetry

Secondary Vertex

Tracking telescopes of TOTEM

Event index information such as run/event number, trigger bits, etc.

Terabit $\left(10^{12}\right.$ bits $)$

Terabyte $\left(10^{12}\right.$ bytes)

Technical Design Report

Tracker EndCap

Tracker Inner Barrel

Tracker Inner Disks

Tracker Outer Barrel

Separate experiment at P5 for forward physics

Tracker Pixel Detector

Trigger and Data Acquisition project

An experiment at the CERN SPS collider

An experiment at the CERN SPS collider

Underlying Event

Universal Extra Dimensions

Vector Boson Fusion

Vacuum PhotoTriode

World Wide Web

Zero Degree Calorimeter

An experiment at the DESY HERA collider 


\section{References}

Blue font in the online PDF indicates links to full-text articles.

DOI numbers are given for some published articles, for which the full text may be accessed by prefixing the number with http://dx.doi.org/

CMS Notes are available at http://cms.cern.ch/iCMS/ unless otherwise noted.

[1] TLS Group 1995 The Large Hadron Collider Conceptual Design CERN-AC-95-05 Preprint hep-ph/0601012

[2] CMS Collaboration 1992 The Compact Muon Solenoid Letter of Intent CERN/LHCC 1992-3, LHCC/I 1

[3] CMS Collaboration 1994 The Compact Muon Solenoid Technical Proposal CERN/LHCC 94-38, LHCC/P1

[4] ATLAS and CMS Collaboration, Branson J G et al 2002 High transverse momentum physics at the Large Hadron Collider: The ATLAS and CMS Collaborations Eur. Phys. J. direct C 4 N1 (Preprint hep-ph/0110021)

[5] LHC/LC Study Group Collaboration, Weiglein G et al 2004 Physics interplay of the LHC and the ILC Preprint hep-ph/0410364

[6] Krasnikov N and Matveev V 2004 Search for new physics at LHC Phys. Usp. 47643 (Preprint hep-ph/0309200)

[7] CMS Collaboration 2006 The CMS Physics Technical Design Report, Volume 1 CERN/LHCC 2006-001, CMS TDR 8.1

[8] OSCAR: CMS Simulation Package Home Page http://cmsdoc.cern.ch/oscar

[9] GEANT4 Collaboration, Agostinelli S et al 2003 GEANT4: A simulation toolkit Nucl. Instrum. Methods A 506 250-303

[10] ORCA: CMS reconstruction Package Site located at http://cmsdoc.cern.ch/orca

[11] CMS Collaboration, Acosta D et al 2006 CMS Physics Technical Design Report, Volume 1, Section 2.6: Fast simulation p 55 CERN/LHCC 2006-001

[12] Pumplin J et al 2002 New generation of parton distributions with uncertainties from global QCD analysis J. High. Energy Phys. JHEP07(2002)012 (Preprint hep-ph/0201195)

[13] Martin A D, Roberts R G, Stirling W J and Thorne R S 2002 MRST2001: Partons and alpha(s) from precise deep inelastic scattering and Tevatron jet data Eur. Phys. J. C 2373 (Preprint hep-ph/0110215)

[14] Alekhin S et al 2005 HERA and the LHC - A workshop on the implications of HERA for LHC physics: Proceedings Part A

[15] Seez C et al 1990 Photon decay modes of the intermediate mass Higgs Proceedings of the Large Hadron Collider Workshop (Aachen, 4-9 October, 1990), CERN 90-10 volume II ed G Jarlskog and D Rein

[16] CMS Collaboration 1997 The Electromagnetic Calorimeter Technical Design Report CERN/LHCC, 97-033 CMS TDR 4, Addendum CERN/LHCC 2002-027

[17] Favara A and Pieri M 1997 Confidence Level Estimation and Analysis Optimization Preprint DFF 278/4/1997

[18] L3 Collaboration, Acciarri M et al 1997 Search for the Standard Model Higgs Boson in $\mathrm{e}^{+} \mathrm{e}^{-}$Interactions at $161 \mathrm{GeV}<\sqrt{s}<172 \mathrm{GeV}$ Phys. Lett. B 411373

[19] CMS Collaboration, Pieri M et al 2006 Inclusive search for the Higgs boson in the $H \rightarrow \gamma \gamma$ channel $C M S$ Note AN 2006/112

[20] Spira M 1998 QCD effects in Higgs physics Fortsch. Phys. 46 203-284 (Preprint hep-ph/9705337)

[21] Djouadi A, Kalinowski J and Spira M 1998 HDECAY: a program for Higgs boson decays in the Standard Model and its supersymmetric extension Comput. Phys. Commun. 108 56-74 (doi:10.1016/S0010-4655(97)00123-9)

[22] CMS Collaboration, Dubinin M et al 2006 The vector boson fusion production with $H \rightarrow \gamma \gamma$ CMS Note 2006/097

[23] CMS Collaboration, Lethuillier M 2006 Search for SM Higgs boson with $\mathrm{W} / \mathrm{Z}+\mathrm{H}, \mathrm{H} \rightarrow 2 \gamma$ channel CMS Note $2006 / 110$

[24] Sjostrand T, Lonnblad L and Mrenna S 2001 PYTHIA 6.2: Physics and manual Preprint hep-ph/0108264

[25] CMSIM home page http://cmsdoc.cern.ch/cmsim/cmsim.html

[26] Giele W et al 2002 The QCD/SM working group: Summary report Preprint hep-ph/0204316

[27] Dobbs M et al 2004 The QCD/SM working group: Summary report Preprint hep-ph/0403100

[28] Binoth T 2000 Two photon background for Higgs boson searches at the LHC Preprint hep-ph/0005194

[29] Bern Z, Dixon L J and Schmidt C 2002 Isolating a light Higgs boson from the di-photon background at the LHC Phys. Rev. D 66074018 (Preprint hep-ph/0206194) 
[30] Binoth T, Guillet J P, Pilon E and Werlen M 2002 A next-to-leading order study of photon pion and pion pair hadro-production in the light of the Higgs boson search at the LHC Eur. Phys. J. Direct C 4 (doi:10.1007/s1010502c0007)

[31] CMS Collaboration, Agostino L et al 2006 HLT Selection of Electrons and Photons CMS Note 2006/078

[32] CMS Collaboration, Meschi E et al 2001 Electron Reconstruction in the CMS Electromagnetic Calorimeter CMS Note 2001/034

[33] CMS Collaboration, Marinelli N 2006 Track Finding and Identification of Converted Photons CMS Note 2006/005

[34] CMS Collaboration, Litvin V et al 2002 The Rejection of Background to the HGG Process Using Isolation Criteria Based on Information from the Calorimeter and Tracker CMS Note 2002-030

[35] CMS Collaboration, Pieri M et al 2006 Distinguishing Isolated Photos from Jets CMS Note 2006/007

[36] The LEP Collaborations: ALEPH, DELPHI, L3 and OPAL1998 Lower Bound for the Standard Model Higgs Boson Mass from Combining the Results of the Four LEP Experiments CERN EP 98-046

[37] CMS Collaboration, Baffioni S et al 2006 Discovery potential for the SM Higgs boson in the $\mathrm{H} \rightarrow \mathrm{ZZ}^{(*)} \rightarrow \mathrm{e}^{+} \mathrm{e}^{-} \mathrm{e}^{+} \mathrm{e}^{-}$decay channel CMS Note $2006 / 115$

[38] Barberio E, van Eijk B and Was Z 1991 PHOTOS: A Universal Monte Carlo for QED radiative corrections in decays Comput. Phys. Commun. 66 115-128

[39] Barberio E and Was Z 1994 PHOTOS: A Universal Monte Carlo for QED radiative corrections. Version 2.0 Comput. Phys. Commun. 79 291-308 (doi:10.1016/0010-4655(94)90074-4)

[40] Spira M 1995 HIGLU: A Program for the Calculation of the Total Higgs Production Cross Section at Hadron Colliders via Gluon Fusion including QCD Corrections Preprint hep-ph/9510347

[41] Djouadi A, Kalinowski J and Spira M 1998 HDECAY: A Program for Higgs Boson Decays in the Standard Model and its Supersymmetric Extension Comput. Phys. Commun. 108 56-74 (Preprint hep-ph/9704448)

[42] Zecher C et al 1994 Leptonic Signals from off-shell Z Boson Pairs at Hadron Colliders Preprint hep-ph/9404295

[43] CompHEP Collaboration, Boos E et al 2004 CompHEP 4.4: Automatic computations from Lagrangians to events Nucl. Instrum. Meth. A 534 250-259 (Preprint hep-ph/0403113)

[44] Slabospitsky S R and Sonnenschein L 2002 TopReX generator (version 3.25): Short manual Comput. Phys. Commun. 148 87-102 (doi:10.1016/S0010-4655(02)00471-X)

[45] Beneke M et al 2000 Top quark physics Preprint hep-ph/0003033

[46] CMS Collaboration, Baffioni S, Charlot C, Ferri F, Futyan D, Meridiani P, Puljak I, Rovelli C, Salerno R and Sirois Y 2006 Electron Reconstruction in CMS CMS Note 2006/040

[47] Abdullin S et al 2006 Study of PDF and QCD scale uncertainties in $\mathrm{H} \rightarrow \mathrm{ZZ}^{(*)} \rightarrow 4 \mu$ events at the LHC CMS Note 2006/068 See also hep-ph/0604120, Les Houches Physics at TeV Colliders 2005, Standard Model and Higgs working group: Summary report. April 2006

[48] Meridiani P and Paramatti R 2006 Use of $Z \rightarrow e^{+} e^{-}$events for ECAL calibration CMS Note 2006/039

[49] Bartsch V and Quast G 2006 Expected signal observability at future experiments CMS Note 2005-004

[50] Bityukov S and Krasnikov N 1998 New physics discovery potential in future experiments Mod. Phys. Lett. A $133235-3249$

[51] Abdullin S et al 2006 Search Strategy for the Standard Model Higgs Boson in the $\mathrm{H} \rightarrow \mathrm{ZZ}{ }^{(\star)} \rightarrow 4 \mu$ Decay Channel using $M(4 \mu)$-Dependent Cuts CMS Note 2006/122

[52] Karimaki V et al 2004 CMKIN v3 User's Guide CMS IN 2004-016

[53] Spira M 2005 'SM Higgs boson production cross sections at NLO' and 'SM Higgs boson BRs'Available at http://cmsdoc.cern.ch/ anikiten/cms-higgs/

[54] Particle Data Group, Eidelman S et al 2004 Review of Particle Physics Phys. Lett. B 5921 (doi:10.1016/j.physletb.2004.06.001)

Particle Data Group, Yao W-M et al 2006 Review of Particle Physics J. Phys. G: Nucl. Part. Phys. 33 (doi:10.1088/0954-3899/33/1/001)

[55] Maltoni F 2005 Theoretical Issues and Aims at the Tevatron and LHC Proceedings of the 1st Hadron Collider Physics Symposium (HCP 2005) (Les Diablerets, Switzerland 2005 July) Available at http://hcp-2005.web.cern.ch/HCP-2005

[56] Campbell J M $2001 \mathrm{~W} / \mathrm{Z}+\mathrm{B}$ anti-B/jets at NLO using the Monte Carlo MCFM Talk given at 36th Rencontres de Moriond on QCD and Hadronic Interactions (Les Arcs, France, 17-24 March 2001 ) Preprint hep-ph/0105226

[57] Abdullin S et al 2006 Relative Contributions of t- and s-Channels to the ZZ $\rightarrow 4 \mu$ Process CMS Note 2006/057, See also hep-ph/0604120, Les Houches Physics at TeV Colliders 2005, Standard Model and Higgs working group: Summary report. April 2006 
[58] Bartalini P et al 2006 NLO vs. LO: kinematical differences for signal and background in the $\mathrm{H} \rightarrow \mathrm{ZZ}^{*} \rightarrow 4 \mu$ analysis CMS Note 2006/130

[59] Acosta D et al 2006 Measuring Muon Reconstruction Efficiency from Data CMS Note 2006/060

[60] Aldaya M, Arce P, Caballero J, de la Cruz B, Garcia-Abia P, Hernandez J M, Josa M and Ruiz E 2006 Discovery potential and search strategy for the Standard Model Higgs boson in the $H \rightarrow Z Z^{\star} \rightarrow 4 \mu$ decay channel using a mass-independent analysis CMS Note 2006/106

[61] Read A 2000 Modified Frequentist Analysis of Search Results (The CLs Method) 1st Workshop on Confidence Limits, CERN, Geneva CERN-EP/2000-005

[62] LEP Working Group for Higgs boson searches Collaboration, Barate R et al 2003 Search for the standard model Higgs boson at LEP Phys. Lett. B 565 61-75 (Preprint hep-ex/0306033)

[63] Abdullin S and Drozdetskiy A et al 2006 GARCON: Genetic Algorithm for Rectangular Cuts Optimization. User's manual for version 2.0 Preprint hep-ph/0605143 See also http://drozdets.home.cern.ch/drozdets/home/genetic/

[64] Abdullin S et al 2006 Sensitivity of the Muon Isolation Cut Efficiency to the Underlying Event Uncertainties CMS Note 2006/033, See also hep-ph/0604120, Les Houches Physics at TeV Colliders 2005, Standard Model and Higgs working group: Summary report. April 2006

[65] Aldaya M et al 2006 A method for determining the mass, cross-section and width of the Standard Model Higgs boson using the $\mathrm{H} \rightarrow \mathrm{ZZ}^{*} \rightarrow 4 \mu$ decay channel CMS Note 2006/107

[66] Bartsch V Simulation of Silicon Sensors and Study of the Higgs Decay

[67] Dittmar M and Dreiner H 1997 How to find a Higgs boson with a mass between $155 \mathrm{GeV}$ to to $180 \mathrm{GeV}$ at the CERN LHC Phys. Rev. D 55 167-172 (Preprint hep-ph/9608317) (doi:10.1103/PhysRevD.55.167)

[68] Dittmar M and Dreiner H 1997 LHC Higgs Search with $l^{+} v l^{-} \bar{v}$ final states CMS Note 1997/083

[69] Sjostrand Tet al 2001 High-energy-physics event generation with PYTHIA 6.1 Comput. Phys. Commun. 135 238-259 (doi:10.1016/S0010-4655(00)00236-8)

[70] Binoth T, Ciccolini M, Kauer N and Kramer M 2005 Gluon-induced W W background to Higgs boson searches at the LHC J. High. Energy Phys. JHEP03(2005)065 (Preprint hep-ph/0503094)

[71] Davatz G et al 2004 Effective k-factors for $H \rightarrow W W^{(*)} \rightarrow 2 \mu 2 v$ at the LHC J. High. Energy Phys. JHEP0405(2004)009 (Preprint hep-ph/0402218) (doi:10.1088/1126-6708/2004/05/009)

[72] Frixione S and Webber B R 2002 Matching NLO QCD computations and parton shower simulations J. High. Energy Phys. JHEP0206(2002)029 (Preprint hep-ph/0204244)

[73] Davatz G, Dittmar M and Giolo-Nicollerat A S 2006 Standard Model Higgs Discovery Potential of CMS in the $H \rightarrow W W^{(*)} \rightarrow l v l v$ Channel CMS Note 2006/047

[74] Campbell J M and Ellis R K 1999 An update on vector boson pair production at hadron colliders Phys. Rev. D 60113006 (doi:10.1103/PhysRevD.60.113006)

[75] Campbell J and Tramontano F 2005 Next-to-leading order corrections to Wt production and decay Nucl. Phys. B 726 109-130 (doi:10.1016/j.nuclphysb.2005.08.015)

[76] CMS Collaboration 2002 The TriDAS Project Technical Design Report, Volume 2: Data Acquisition and High-Level Trigger CERN/LHCC 2002-26, CMS TDR 6.2

[77] Drollinger V, Gasparini U, Torassa E and Zanetti M 2006 Physics study of the Higgs decay chanel $H \rightarrow W W^{(*)} \rightarrow 2 \mu 2 v$ CMS Note 2006/055

[78] Chekanov S V 2002 Jet algorithms: a mini review Proceedings of the 14th Topical Conference on Hadron Collider Physics (HCP 2002) September-October (Karlsruhe, Germany)

[79] Bityukov S I and Krasnikov N V 2002 Uncertainties and discovery potential in planned experiments $C M S C R$ $2002 / 05$

[80] Davatz G, Giolo-Nicollerat A and Zanetti M 2006 Systematics uncertainties of the top background in the $H \rightarrow W W$ channel CMS Note 2006/048

[81] Maltoni F and Stelzer T 2003 MadEvent: Automatic event generation with MadGraph J. High. Energy Phys. JHEP02(2003)027 (doi:10.1088/1126-6708/2003/02/027)

[82] Kauer N 2004 Top background extrapolation for $H \rightarrow W W$ searches at the LHC Preprint hep-ph/0404045

[83] Lowette S, D’Hondt J, Heyninck J and Vanlaer P 2006 Offline Calibration of b-Jet Identification Efficiencies CMS Note 2006/013

[84] Drollinger V et al 2005 Modeling the production of W pairs at the LHC CMS Note 2005/024

[85] Anastasiou C et al 2004 High precision QCD at hadron colliders: Electroweak gauge boson rapidity distribution at NNLO Phys. Rev. D 69

[86] Belotelov I et al 2006 Simulation of misalignment scenarios for CMS tracking devices CMS Note 2006-008

[87] Cvetic M and Langacker P 1996 Implications of abelian extended gauge structures from string models Phys. Rev. D 54 3570-3579 (Preprint hep-ph/9511378) 
[88] Cvetic M and Langacker P 1996 New gauge bosons from string models Mod. Phys. Lett. A 11 1247-1262 (Preprint hep-ph/9602424)

[89] Leike A 1999 The phenomenology of extra neutral gauge bosons Phys. Rept. 317 143-250 (Preprint hep-ph/9805494)

[90] Hill C T and Simmons E H 2003 Strong dynamics and electroweak symmetry breaking Phys. Rept. 381 235-402 (Preprint hep-ph/0203079)

[91] Han T, Logan H, McElrath B and Wang L-T 2003 Phenomenology of the little Higgs model Phys. Rev. D 67 095004 (doi:10.1103/PhysRevD.67.095004)

[92] Cvetic M and Godfrey S 1996 Discovery and identification of extra gauge bosons Electroweak symmetry breaking and new physics at the TeV scale ed T L Barlow and S Dawson (World Scientific) 383-415 Preprint hep-ph/9504216

[93] Godfrey S 2001 Update of discovery limits for extra neutral gauge bosons at hadron colliders Proceedings of Snowmass 2001 (Snowmass, Colorado: June-July ) p P344 Preprint hep-ph/0201093

[94] Randall L and Sundrum R 1999 A large mass hierarchy from a small extra dimension Phys. Rev. Lett. 83 3370-3373 (doi:10.1103/PhysRevLett.83.3370)

[95] Whalley M R, Bourilkov D and Group R C 2005 The Les Houches accord PDFs (LHAPDF) and LHAGLUE Preprint hep-ph/0508110

[96] Rosner J L 1987 Off peak lepton asymmetries from new Zs Phys. Rev. D 352244

[97] Rosner J L 1996 Forward-Backward asymmetries in hadronically produced lepton pairs Phys. Rev. D 54 1078-1082 (Preprint hep-ph/9512299)

[98] Mohapatra R N, Goldstein S A and Money D (eds) 2002 Unification and supersymmetry: The Frontiers of quark - lepton physics 3rd ed. (Springer-Verlag)

[99] Belotelov I et al 2006 Influence of misalignment scenarios on muon reconstruction CMS Note 2006-017

[100] Cousins R, Mumford J and Valuev S 2005 Detection of $Z^{\prime}$ gauge bosons in the dimuon decay mode in CMS CMS Note 2005-002

[101] Cousins R, Mumford J and Valuev V 2006 Detection of $Z^{\prime}$ gauge bosons in the dimuon decay mode in CMS CMS CR 2004/050

[102] Bartsch V and Quast G 2005 Expected signal observability at future experiments CMS Note 2005-004

[103] Wilks S S 1938 The large-sample distribution of the likelihood ratio for testing composite hypotheses Annals of Math. Stat. 960

[104] Baur U, Brein O, Hollik W, Schappacher C and Wackeroth D 2002 Electroweak radiative corrections to neutral-current Drell-Yan processes at hadron colliders Phys. Rev. D 65033007 (Preprint hep-ph/0108274)

[105] Zykunov V 2005 Weak radiative corrections to Drell-Yan process for large invariant mass of di-lepton pair Preprint hep-ph/0509315

[106] De Roeck A and Slabospitsky S Evaluation of the PDF Uncertainties (in CMS) available at http://cmsdoc.cern.ch/cms/PRS/gentools/www/pdfuncert/uncert.html

[107] Cerminara G private communication

[108] Langacker P, Robinett R W and Rosner J L 1984 New heavy gauge bosons in $p p$ and $p \bar{p}$ collisions Phys. Rev. D 301470

[109] Rosner J L 1989 Observability of charge asymmetries for lepton pairs produced in present collider experiments Phys. Lett. B 22185

[110] Cheng T P and Li L F 1988 Gauge theory of elementary particle physics ( Oxford University Press)

[111] Cousins R, Mumford J and Valuev V 2005 Measurement of Forward-Backward Asymmetry of Simulated and Reconstructed $\mathrm{Z}^{\prime} \rightarrow \mu^{+} \mu^{-}$Events in CMS CMS Note 2005-022

[112] Dittmar M 1997 Neutral current interference in the TeV region: The experimental sensitivity at the LHC Phys. Rev. D 55 161-166 (Preprint hep-ex/9606002)

[113] Wulz C-E 1996 Z' at LHC Proceedings of the 1996 DPF/DPB Summer Study on New Directions in High-Energy Physics available at http://www.slac.stanford.edu/pubs/snowmass96

[114] Collins J C and Soper D E 1977 Angular distribution of dileptons in high-energy hadron collisions Phys. Rev. D 162219

[115] Allanach B C, Odagiri K, Parker M A and Webber B R 2000 Searching for narrow graviton resonances with the ATLAS detector at the Large Hadron Collider J. High. Energy Phys. JHEP09(2000)019 (Preprint hep-ph/0006114)

[116] Cousins R, Mumford J, Tucker J and Valuev V 2005 Spin discrimination of new heavy resonances at the LHC J. High. Energy Phys. JHEP11(2005)046 (doi:10.1088/1126-6708/2005/11/046)

[117] Belotelov I et al 2006 Search for Randall-Sandrum Graviton Decay into Muon Pairs CMS Note 2006/104

[118] Esen S and Harris R M 2006 Jet Triggers and Dijet Mass CMS Note 2006/069 
[119] CMS Collaboration, Acosta D et al 2006 CMS Physics Technical Design Report, Volume 1, Section 11.3: Monte Carlo Corrections CERN/LHCC, 2006-001 p 409

[120] CDF Collaboration, Abe F et al 1997 Search for new particles decaying to dijets at CDF Phys. Rev. D 55 5263-5268 (doi:10.1103/PhysRevD.55.R5263)

[121] DØ Collaboration, Abazov V M et al 2004 search for new particles in the two-jet decay channel with the DØdetector Phys. Rev. D 69111101 (Preprint hep-ex/0308033)

[122] DØ Collaboration, Abbott B et al 1999 The dijet mass spectrum and a search for quark compositeness in $\bar{p} p$ collisions at $\sqrt{s}=1.8-\mathrm{TeV}$ Phys. Rev. Lett. 82 2457-2462 (doi:10.1103/PhysRevLett.82.2457)

[123] Eichten E, Lane K D and Peskin M E 1983 New tests for quark and lepton substructure Phys. Rev. Lett. 50 811-814 (doi:10.1103/PhysRevLett.50.811)

[124] Lane K D 1996 Electroweak and flavor dynamics at hadron colliders Preprint hep-ph/9605257

[125] CDF Collaboration, Abe F et al 1996 Measurement of dijet angular distributions at CDF Phys. Rev. Lett. 77 5336-5341 (doi:10.1103/PhysRevLett.77.5336)

[126] CMS Collaboration, Acosta D et al 2006 CMS Physics Technical Design Report, Volume 1, Section 11.6.1: Data-driven calibration strategy CERN/LHCC, 2006-001 p 421

[127] CMS Collaboration, Acosta D et al 2006 CMS Physics Technical Design Report, Volume 1, Section 11.6.2: Dijet Balancing CERN/LHCC, 2006-001 p 422

[128] CDF Collaboration, Acosta D et al 2005 Measurement of the lifetime difference between $B_{s}$ mass eigenstates Phys. Rev. Lett. 94101803 (Preprint hep-ex/0412057) (doi:10.1103/PhysRevLett.94.101803)

[129] DØ Collaboration 2006 Measurement of the lifetime difference in the $B_{s}$ system DØ Conference note 5052

[130] Belkov A and Shulga S 2004 Studies of angular correlations in the decays $B_{s}^{0} \rightarrow J / \psi \phi$ by using the SIMUB generator Comput. Phys. Commun. 156 221-240 (Preprint hep-ph/0310096) (doi:10.1016/S0010-4655(03)00465-X)

[131] Dighe A S, Dunietz I and Fleischer R 1999 Extracting CKM phases and $B_{s}-\bar{B}_{s}$ mixing parameters from angular distributions of non-leptonic B decays Eur. Phys. J. C 6 647-662 (doi:10.1007/s100529800954)

[132] Dighe A S, Dunietz I, Lipkin H J and Rosner J L 1996 Angular distributions and lifetime differences in $B_{s} \rightarrow J / \psi \phi$ decays Phys. Lett. B 369 144-150 (doi:10.1016/0370-2693(95)01523-X)

[133] CDF Collaboration, Abe F et al 1997 Production of $J / \psi$ mesons from $\chi_{c}$ meson decays in $p \bar{p}$ collisions at $\sqrt{s}=1.8$ TeV Phys. Rev. Lett. 79 578-583 (doi:10.1103/PhysRevLett.79.578)

[134] Cano-Coloma B and Sanchis-Lozano M A 1997 Charmonia production in hadron colliders and the extraction of colour-octet matrix elements Nucl. Phys. B 508 753-767 (Preprint hep-ph/9706270) (doi:10.1016/S0550-3213(97)00660-3)

[135] Cucciarelli S, Konecki M, Kotlinski D and Todorov T 2006 Track reconstruction, primary vertex finding and seed generation with the Pixel Detector CMS Note 2006/026

[136] Prokofiev K and Speer T 2005 A kinematic fit and a decay chain reconstruction library CERN Yellow Report http://doc.cern.ch/yellowrep/2005/2005-002/p411.pdf, 2005-002 Proc. of the 2004 Conference for Computing in High-Energy and Nuclear Physics (CHEP 04) (Interlaken, Switzerland, 2004) available at http://indico.cern.ch

[137] Dunietz I, Fleischer R and Nierste U 2001 In pursuit of new physics with $B_{s}$ decays Phys. Rev. D 63114015 (doi:10.1103/PhysRevD.63.114015)

[138] BABAR Collaboration, Aubert B et al 2005 Ambiguity-free measurement of $\cos (2 \beta)$ : Time-integrated and time-dependent angular analyses of $B \rightarrow J / \psi K \pi$ Phys. Rev. D 71032005 (doi:10.1103/PhysRevD.71.032005)

[139] Dunietz I, Quinn H, Snyder A, Toki W and Lipkin H J 1991 How to extract CP-violating asymmetries from angular correlations Phys. Rev. D 43 2193-2208 (doi:10.1103/PhysRevD.43.2193)

[140] Vanlaer P et al 2006 Impact of CMS Silicon Tracker Misalignment on Track and Vertex Reconstruction CMS Note 2006/029

[141] Speer T et al 2006 Vertex Fitting in the CMS Tracker CMS Note 2006/032

[142] Heinemeyer S, Hollik W and Weiglein G 2000 FeynHiggs: A program for the calculation of the masses of the neutral CP-even Higgs bosons in the MSSM Comput. Phys. Commun. 124 76-89 (Preprint hep-ph/9812320)

[143] Heinemeyer S, Hollik W and Weiglein G 1999 The masses of the neutral CP-even Higgs bosons in the MSSM: accurate analysis at the two-loop level Eur. Phys. J. C 9 343-366 (Preprint hep-ph/9812472)

[144] Degrassi G, Heinemeyer S, Hollik W, Slavich P and Weiglein G 2003 Towards high-precision predictions for the MSSM Higgs sector Eur. Phys. J. C 28 133-143 (Preprint hep-ph/0212020)

[145] Lehti S 2006 Study of bbZ as a benchmark for MSSM bbH CMS Note 2006/099 
[146] Bagliesi G, Dutta S, Gennai S, Kalinowski A, Konecki M, Kotlinski D, Moortgat F, Nikitenko A, Wendland $\mathrm{L}$ and Wakefield S 2006 Tau jet reconstruction and tagging at high level trigger and off-line CMS Note 2006/028

[147] Kunori S, Kinnunen R and Nikitenko A 2001 Missing transverse energy measurement with jet energy correctioins CMS Note 2001/040

[148] Pi H et al 2006 Measurement of missing transverse energy with the CMS detector at the LHC CMS Note 2006/035

[149] Kalinowski A and Nikitenko A 2006 Measurement of the $\tau$-tagging efficiency using the $Z \rightarrow \tau \tau \rightarrow \mu+$ hadrons + X events CMS Note 2006/074

[150] Gennai S, Nikitenko A and Wendland L 2006 Search for MSSM Heavy Neutral Higgs Boson in $\tau \tau \rightarrow$ two Jet Decay Mode CMS Note 2006/126

[151] Rizzi A, Palla F and Segneri G 2006 Track impact parameter based b-tagging with CMS CMS Note 2006/019

[152] Kalinowski A, Konecki M and Kotlinski D 2006 Search for MSSM Heavy Neutral Higgs Boson in $\tau \tau \rightarrow \mu$ + jet Decay Mode CMS Note 2006/105

[153] Kalinowski A, Konecki M and Kotliński D 2006 Search for MSSM heavy neutral Higgs boson in $\tau+\tau \rightarrow \mu+$ jet decay mode CMS Note 2006/105

[154] Kinnunen R and Lehti S 2006 Search for the Heavy Neutral MSSM Higgs Bosons with the H/A $\rightarrow \tau \tau \rightarrow$ Electron plus Jet Decay Mode CMS Note 2006/075

[155] Jadach S, Was Z, Decker R and Kuhn J H 1993 The tau decay library TAUOLA: Version 2.4 Comput. Phys. Commun. 76 361-380 (doi:10.1016/0010-4655(93)90061-G)

[156] Giolo-Nicollerat A-S 2006 CMS Note

[157] Weiser C 2006 A Combined Secondary Vertex Based B-Tagging Algorithm in CMS CMS Note 2006/014

[158] Haywood S et al 1999 Electroweak physics Preprint hep-ph/0003275 In 'Standard Model Physics (and more) at the LHC', Geneva, 1999

[159] Chakraborty D, Konigsberg J and Rainwater D L 2003 Review of top quark physics Ann. Rev. Nucl. Part. Sci. 53 301-351 (Preprint hep-ph/0303092)

[160] Benedetti D, Cucciarelli S, Hill C, Incandela J, Koay S, Riccardi C, Santocchia A, Schmidt A, Torre P and Weiser C 2006 Search for $H \rightarrow b b$ in association with a $t$ pair at CMS CMS Note 2006/119

[161] Mangano M L, Moretti M, Piccinini F, Pittau R and Polosa A D 2003 ALPGEN, a generator for hard multiparton processes in hadronic collisions J. High. Energy Phys. JHEP07(2003)001 (Preprint hep-ph/0206293) (doi:10.1088/1126-6708/2003/07/001)

[162] Beenakker W, Dittmaier S, Kraemer M, Pluemper B, Spira M and Zerwas P 2003 NLO QCD corrections to $t \bar{t} H$ production in hadron collisions Nucl. Phys. B 653 151-203 (Preprint hep-ph/0211352)

[163] Roberfroid V et al 2006 Validation and status of the HLT Steering Code and default menu CMS IN 2006/001

[164] James E, Maravin Y, Mulders M and Neumeister N 2006 Muon Identification in CMS CMS Note 2006/010

[165] Heister A, Kodolova O, Konoplianikov V, Petrushanko S, Rohlf J, Tully C and Ulyanov A 2006 Measurement of Jets with the CMS Detector at the LHC CMS Note 2006/036

[166] Santocchia A 2006 Optimization of Jet Reconstruction Settings and Parton-Level Correction for the ttH Channel CMS Note 2006/059

[167] D'Hondt J, Lowette S, Buchmuller O, Cucciarelli S, Schilling F P, Spiropulu M, Mehdiabadi S, Benedetti D and Pape L 2006 Fitting of Event Topologies with External Kinematic Constraints in CMS CMS Note 2006/023

[168] Mangano M private communication

[169] Wang X-N and Gyulassy M 1991 HIJING: A Monte Carlo model for multiple jet production in p p, p A and A A collisions Phys. Rev. D 44 3501-3516

[170] Bedjidian M et al 2004 Hard probes in heavy ion collisions at the LHC: heavy flavour physics Preprint hep-ph/0311048

[171] Eskola K J, Kolhinen V J and Salgado C A 1999 The scale dependent nuclear effects in parton distributions for practical applications Eur. Phys. J. C 9 61-68 (Preprint hep-ph/9807297)

[172] Kodolova O, Bedjidian M and Petrouchanko S 1999 Dimuon reconstruction in heavy ion collisions using a detailed description of CMS geometry CMS Note 1999-004

[173] Bedjidian M and Kodolova O 2006 Quarkonia measurements in heavy-ion collisions in CMS CMS Note 2006/089

[174] Sterman G and Weinberg S 1977 Jets from Quantum Chromodynamics Phys. Rev. Lett. 391436

[175] JADE Collaboration, Bartel W et al 1986 Experimental studies on multi-jet production in $\mathrm{e}^{+} \mathrm{e}^{+}$annihilation at petra energies Z. Phys. C 3323

[176] JADE Bethke $\mathrm{S}$ et al 1988 Experimental investigation of the energy dependence of the strong coupling strength Phys. Lett. B 213235 
[177] Catani S, Dokshitzer Y L, Olsson M, Turnock G and Webber B R 1991 New clustering algorithm for multi-jet cross-sections in $\mathrm{e}^{+} \mathrm{e}^{+}$annihilation Phys. Lett. B $269432-438$

[178] CDF Run II Collaboration, Abulencia A et al 2005 Measurement of the inclusive jet cross section in $p \bar{p}$ interactions at $\sqrt{s}=1.96 \mathrm{TeV}$ using a cone-based jet algorithm Preprint hep-ex/0512020

[179] DØ, Collaboration, Strohmer R 2006 Inclusive jet cross-sections and dijet azimuthal decorrelations with DØPoS HEP 2005051 (Preprint hep-ex/0601016)

[180] Butterworth J, Couchman J, Cox B and Waugh B 2003 KtJet: A C ${ }^{++}$implementation of the K(T) clustering algorithm Comput. Phys. Commun. 153 85-96 (Preprint hep-ph/0210022)

[181] ed U Baur, R K Ellis and D Zeppenfeld 2000 QCD and Weak Boson Physics in Run II (Batavia, IL, USA FERMILAB) Prepared for Physics at Run II: QCD and Weak Boson Physics Workshop: Final General Meeting, Batavia, Illinois, 4-6 November, 1999

[182] Ellis S D, Huston J and Tonnesmann M 2001 On building better cone jet algorithms eConf C 010630 P513 (Preprint hep-ph/0111434)

[183] Wobisch M private communication

[184] Sjostrand T 1994 High-energy physics event generation with PYTHIA 5.7 and JETSET 7.4 Comput. Phys. Commun. 82 74-90

[185] Nagy Z 2002 Three-jet cross sections in hadron hadron collisions at next-to-leading order Phys. Rev. Lett. 88 122003 (Preprint hep-ph/0110315)

[186] Kluge T, Rabbertz K and Wobisch M fast NLO—fast pQCD calculations for hadron-induced processes To be published, available at http://hepforge.cedar.ac.uk/fastnlo

[187] Moretti S, Nolten M R and Ross D A 2005 Weak corrections and high E(T) jets at Tevatron Preprint hep-ph/0503152

[188] DØ Collaboration, Abazov V M et al 2002 The inclusive jet cross-section in $\mathrm{p}$ anti-p collisions at $\mathrm{s}^{* *}(1 / 2)=1.8-\mathrm{TeV}$ using the $\mathrm{k}(\mathrm{T})$ algorithm Phys. Lett. B 525 211-218 (Preprint hep-ex/0109041)

[189] DØ Collaboration, Abbott B et al $2001 \mathrm{High}-p_{\mathrm{T}}$ jets in $\bar{p} p$ collisions at $\sqrt{s}=630 \mathrm{GeV}$ and $1800 \mathrm{GeV}$ Phys. Rev. D 64032003 (doi:10.1103/PhysRevD.64.032003)

[190] CDF Collaboration, Affolder A A et al 2002 Charged jet evolution and the underlying event in proton anti-proton collisions at 1.8-TeV Phys. Rev. D 65092002

[191] CDF Collaboration, Acosta D et al 2004 The underlying event in hard interactions at the Tevatron anti- $p p$ collider Phys. Rev. D 70072002 (Preprint hep-ex/0404004)

[192] Sjostrand T and van Zijl M 1987 A multiple interaction model for the event structure in hadron collisions Phys. Rev. D 362019

[193] Butterworth J, Forshaw J R and Seymour M 1996 Multiparton interactions in photoproduction at HERA Z. Phys. C 72 637-646 (Preprint hep-ph/9601371)

[194] Gleisberg T et al 2004 SHERPA 1.alpha, a proof-of-concept version J. High. Energy Phys. JHEP02(2004)056 (Preprint hep-ph/0311263)

[195] Bopp F W, Engel R and Ranft J 1998 Rapidity gaps and the PHOJET Monte Carlo Preprint hep-ph/9803437

[196] Corcella G et al 2001 HERWIG 6: An event generator for hadron emission reactions with interfering gluons (including supersymmetric processes) J. High. Energy Phys. JHEP01(2001)010 (Preprint hep-ph/0011363) (doi:10.1088/1126-6708/2001/01/010)

[197] Buttar C M, Clements D, Dawson I and Moraes A 2004 Simulations of minimum bias events and the underlying event, MC tuning and predictions for the LHC Acta Phys. Polon. B 35 433-441

[198] CDF Collaboration, Field R 2005 Min-bias and the underlying event in Run 2 at CDF Acta Phys. Polon. B 36 167-178

[199] Acosta D et al 2006 The underlying event at the LHC CMS Note 2006/067

[200] CDF Collaboration, Abe F et al 1991 Measurement of the Z-boson $p_{\mathrm{T}}$ distribution in $\bar{p} p$ collisions at $\sqrt{s}=1.8$ TeV Phys. Rev. Lett. 67 2937-2941 (doi:10.1103/PhysRevLett.67.2937)

[201] Bartalini P, Chierici R and De Roeck A 2005 Guidelines for the estimation of theoretical uncertainties at the LHC CMS Note 2005/013

[202] Adam W, Mangano B, Speer T and Todorov T 2006 Track reconstruction in the CMS tracker CMS Note 2006/041

[203] Andreev V P, Cline D.B and Otwinowski S 2006 Inclusive $b$ quark production CMS Note 2006/120

[204] CDF Collaboration, Abe F et al 1993 Measurement of the bottom quark production cross section using semileptonic decay electrons in $p \bar{p}$ collisions at $\sqrt{s}=1.8$ TeV Phys. Rev. Lett. 71500 (doi:10.1103/PhysRevLett.71.500)

[205] CDF Collaboration, Abe F et al 1994 Measurement of the $B$ meson and $b$ quark cross sections at $\sqrt{s}=1.8$ TeV using the exclusive decay $B^{0} \rightarrow J / \psi K^{*}(892)^{0}$ Phys. Rev. D 504252 (doi:10.1103/PhysRevD.50.4252) 
[206] CDF Collaboration, Abe F et al 1995 Measurement of the B Meson Differential Cross Section $d \sigma / d p_{\mathrm{T}}$ in $p \bar{p}$ Collisions at $\sqrt{s}=1.8 \mathrm{TeV}$ Phys. Rev. Lett. 751451 (doi:10.1103/PhysRevLett.75.1451)

[207] CDF Collaboration Abe F et al 2002 Measurement of the $B^{+}$total cross section and $B^{+}$differential cross section $d \sigma / d p_{\mathrm{T}}$ in $p \bar{p}$ collisions at $\sqrt{s}=1.8 \mathrm{TeV}$ Phys. Rev. D 65052005 (doi:10.1103/PhysRevD.65.052005)

[208] CDF Collaboration, Abe $\mathrm{F}$ et al 2002 Measurement of the ratio of $b$ quark production cross sections in $\bar{p} p$ collisions at $\sqrt{s}=630 \mathrm{GeV}$ and $\sqrt{s}=1800 \mathrm{GeV}$ Phys. Rev. D 66032002 (doi:10.1103/PhysRevD.66.032002)

[209] DØ Collaboration, Abachi S et al 1995 Inclusive $\mu$ and $b$-quark production cross-sections in $p \bar{p}$ collisions at $\sqrt{s}=1.8$ TeV Phys. Rev. Lett. 743548 (doi:10.1103/PhysRevLett.74.3548)

[210] DØ Collaboration, Abbott B et al 2000 The $b \bar{b}$ production cross section and angular correlations in $p \bar{p}$ collisions at $\sqrt{s}=1.8 \mathrm{TeV}$ Phys. Lett. B 487264 (doi:10.1016/S0370-2693(00)00844-3)

[211] Dø Collaboration, Abbott B et al 2000 Cross section for $b$-Jet production in $\bar{p} p$ collisions at $\sqrt{s}=1.8 \mathrm{TeV}$ Phys. Rev. Lett. 855068 (doi:10.1103/PhysRevLett.85.5068)

[212] H1 Collaboration, Adloff C et al 1999 Measurement of open beauty production at HERA Phys. Lett. B 467 156 (doi:10.1016/S0370-2693(99)01099-0)

[213] H1 Collaboration, Adloff C et al 2001 Erratum Phys. Lett. B 518331

[214] ZEUS Collaboration, Breitweg J et al 2001 Measurement of open beauty production in photoproduction at HERA Eur. Phys. J. C 18 625-637 (doi:10.1007/s100520100571)

[215] H1 Collaboration, Aktas A et al 2005 Measurement of F2(c anti-c) and F2(b anti-b) at high Q**2 using the H1 vertex detector at HERA Eur. Phys. J. C 40 349-359 (Preprint hep-ex/0411046)

[216] ZEUS Collaboration, Chekanov $S$ et al 2004 Bottom photoproduction measured using decays into muons in dijet events in e p collisions at $\mathrm{s} * *(1 / 2)=318 \mathrm{GeV}$ Phys. Rev. D 70012008

[217] ZEUS Collaboration, Chekanov S et al 2004 Measurement of beauty production in deep inelastic scattering at HERA Phys. Lett. B 599 173-189 (Preprint hep-ex/0405069)

[218] H1 Collaboration, Aktas A et al 2005 Measurement of beauty production at HERA using events with muons and jets Eur. Phys. J. C 41 453-467 (Preprint hep-ex/0502010)

[219] L3 Collaboration, Acciarri $\mathrm{M}$ et al 2001 Measurements of the cross sections for open charm and beauty production in $\gamma \gamma$ collisions at $\sqrt{s}=189 \mathrm{GeV}-202 \mathrm{GeV}$ Phys. Lett. B 50310 (doi:10.1016/S0370-2693(01)00134-4)

[220] L3 Collaboration, Achard P et al 2005 Measurement of the cross section for open-beauty production in photon photon collisions at LEP Phys. Lett. B 61971

[221] Nason P, Dawson S and Ellis R K 1988 The total cross-section for the production of heavy quarks in hadronic collisions Nucl. Phys. B 303607

[222] Nason P, Dawson S and Ellis R K 1989 The one particle inclusive differential cross-section for heavy quark production in hadronic collisions Nucl. Phys. B 327 49-92

[223] Beenakker W, Van Neerven W L, Meng R, Schuler G A and Smith J 1991 QCD corrections to heavy quark production in hadron-hadron collisions Nucl. Phys. B 351 507-560 (doi:10.1016/S0550-3213(05)80032-X)

[224] Cacciari M, Frixione S, Mangano M L, Nason P and Ridolfi G 2004 QCD analysis of first b cross section data at 1.96-TeV J. High. Energy Phys. JHEP07(2004)033 (doi:10.1088/1126-6708/2004/07/033)

[225] Mangano M L 2005 The saga of bottom production in proton antiproton collisions AIP Conf. Proc. 753 247-260 (Preprint hep-ph/0411020)

[226] Frixione S 2004 Bottom production Preprint hep-ph/0408317

[227] Weinberg S 1990 Unitarity vonstraints on CP nonconservation in Higgs exchange Phys. Rev. D 42 860-866

[228] Lavoura L 1993 Maximal CP violation via Higgs boson exchange Int. J. Mod. Phys. A 8 375-390

[229] Jenkins E, Luke M E, Manohar A V and Savage M J 1993 Semileptonic $B_{c}$ decay and heavy quark spin symmetry Nucl. Phys. B 390 463-473 (Preprint hep-ph/9204238)

[230] Kiselev V V, Kovalsky A E and Likhoded A K $2000 B_{c}$ decays and lifetime in QCD sum rules Nucl. Phys. B 585 353-382 (doi:10.1016/S0550-3213(00)00386-2)

[231] Wu X-G, Chang C-H, Chen Y-Q and Fang Z-Y 2003 The meson $B_{c}$ annihilation to leptons and inclusive light hadrons Phys. Rev. D 67094001 (doi:10.1103/PhysRevD.67.094001)

[232] Chang C-H and Chen Y-Q 1992 The Production of $B_{c}$ or $\bar{B}_{c}$ meson associated with two heavy quark jets in $Z^{0}$ boson decay Phys. Rev. D 46 3845-3855 (doi:10.1103/PhysRevD.46.3845)

[233] Chang C-H and Chen Y-Q 1993 Hadronic production of the $B_{c}$ meson at TeV energies Phys. Rev. D 484086 (doi:10.1103/PhysRevD.48.4086)

[234] Chang C-H, Chen Y-Q, Han G-P and Jiang H-T 1995 On hadronic production of the $B_{c}$ meson Phys. Lett. B 36478 (doi:10.1016/0370-2693(95)01235-4) 
[235] Chang C-H, Chen Y-Q and Oakes R J 1996 Comparative study of the hadronic production of $B_{c}$ mesons Phys. Rev. D 544344 (doi:10.1103/PhysRevD.54.4344)

[236] Chang C-H and Wu X-G 2004 Uncertainties in estimating hadronic production of the meson $\mathrm{B} / \mathrm{c}$ and comparisons between TEVATRON and LHC Eur. Phys. J. C 38 267-276 (doi:10.1140/epjc/s2004-02015-0)

[237] CDF Collaboration, Abe F et al 1998 Observation of the $B_{c}$ meson in $p \bar{p}$ collisions at $\sqrt{s}=1.8 \mathrm{TeV}$ Phys. Rev. Lett. 81 2432-2437 (doi:10.1103/PhysRevLett.81.2432)

[238] CDF Collaboration, Acosta D et al 2006 Evidence for the exclusive decay $B_{c}^{ \pm} \rightarrow J / \psi \pi^{ \pm}$and measurement of the mass of the $B_{c}$ meson Phys. Rev. Lett. 96082002 (doi:10.1103/PhysRevLett.96.082002)

[239] Berezhnoi A V, Kiselev V V, Likhoded A K and Onishchenko A I 1997 B ${ }_{c}$ meson at LHC Phys. Atom. Nucl. 60 1729-1740 (Preprint hep-ph/9703341)

[240] Gouz I P, Kiselev V V, Likhoded A K, Romanovsky V I and Yushchenko O P 2004 Prospects for the $B_{c}$ studies at LHCb Phys. Atom. Nucl. 67 1559-1570 (doi:10.1134/1.1788046)

[241] Chang C-H and Chen Y-Q 1994 Decays of the $B_{c}$ meson Phys. Rev. D 49 3399-3411 (doi:10.1103/PhysRevD.49.3399)

[242] Kwong W-K and Rosner J L 1991 Masses of new particles containing b quarks Phys. Rev. D 44 212-219 (doi:10.1103/PhysRevD.44.212)

[243] Eichten E J and Quigg C 1994 Mesons with beauty and charm: Spectroscopy Phys. Rev. D 49 5845-5856 (Preprint hep-ph/9402210)

[244] Chen Y-Q and Kuang Y-P 1992 Improved QCD motivated heavy quark potentials with explicit $\Lambda \overline{M S}$ dependence Phys. Rev. D 46 1165-1171 (doi:10.1103/PhysRevD.46.1165)

[245] Chen G, Meng X and Tao J 2006 Feasibility to study the $B_{c}$ meson at CMS CMS Note 2006/118

[246] Sjostrand T, Lonnblad L, Mrenna S and Skands P 2003 PYTHIA 6.3: Physics and manual Preprint hep-ph/0308153

[247] Grothe M et al 2006 Triggering on Forward Physics CMS Note 2006/054. Also available as TOTEM NOTE 2006-01

[248] CMS/TOTEM Collaboration, CMS/TOTEM document on diffractive and forward physics, in preparation 2006

[249] UA8 Collaboration, Bonino R et al 1988 Evidence for transverse jets in high mass diffraction Phys. Lett. B 211239

[250] Arneodo M and Diehl M 2005 Diffraction for non-believers Preprint hep-ph/0511047

[251] UA8 Collaboration, Brandt A et al 1998 Measurements of single diffraction at $\sqrt{s}=630 \mathrm{GeV}$ : Evidence for a non-linear $\alpha(t)$ of the pomeron Nucl. Phys. B 514 3-44 (doi:10.1016/S0550-3213(97)00813-4)

[252] Erhan S and Schlein P E 2000 Inelastic diffraction data and the Pomeron trajectory Phys. Lett. B 481 177-186 (doi:10.1016/S0370-2693(00)00467-6)

[253] Goulianos K 2005 Twenty years of diffraction at the TevatronPresented at 11th international conference on elastic and diffractive scattering: towards high energy frontiers: the 20th anniversary of the blois workshops Preprint hep-ph/0510035

[254] FP420 Collaboration, Albrow M G et al 2005 FP420: A proposal to investigate the feasibility of installing proton tagging detectors in the $420 \mathrm{~m}$ region of the LHC CERN/LHCC, 2005-025

[255] CDF Collaboration, Affolder A A et al 2000 Diffractive dijets with a leading antiproton in anti-p p collisions at $\sqrt{s}=1800 \mathrm{GeV}$ Phys. Rev. Lett. 84 5043-5048

[256] De Roeck A, Khoze V A, Martin A D, Orava R and Ryskin M G 2002 Ways to detect a light Higgs boson at the LHC Eur. Phys. J. C 25 391-403 (Preprint hep-ph/0207042)

[257] Khoze V, Martin A and Ryskin M 2002 Prospects for new physics observations in diffractive processes at the LHC and Tevatron Eur. Phys. J. C 23 311-327 (Preprint hep-ph/0111078)

[258] Kisselev A, Petrov V and Ryutin R 2005 5-dimensional quantum gravity effects in exclusive double diffractive events Phys. Lett. B 630 100-107 (doi:10.1016/j.physletb.2005.09.059)

[259] Monk J and Pilkington A 2005 ExHuME: a Monte Carlo event generator for exclusive diffraction Preprint hep-ph/0502077

[260] Boonekamp M et al 2005 Monte-Carlo generators for central exclusive diffraction Proceedings of the HERA-LHC Workshop (CERN/DESY, January, 2005) available at http://www.desy.de/ heralhc/proceedings/wg4montecarlo.pdf

[261] Ryutin R 2004 EDDE Monte Carlo event generator Preprint hep-ph/0409180

[262] Kaidalov A, Khoze V A, Martin A D and Ryskin M G 2003 Central exclusive diffractive production as a spin parity analyser: From hadrons to Higgs Eur. Phys. J. C 31 387-396 (Preprint hep-ph/0307064)

[263] Carena M, Heinemeyer S, Wagner C E M and Weiglein G 2006 MSSM Higgs boson searches at the Tevatron and the LHC: Impact of different benchmark scenarios Eur. Phys. J. C 45 797-814 (Preprint hep-ph/0511023) 
[264] Ellis J R, Lee J S and Pilaftsis A 2005 Diffraction as a CP and lineshape analyzer for MSSM Higgs bosons at the LHC Phys. Rev. D 71075007 (Preprint hep-ph/0502251)

[265] Petrov A, Ryutin R, Sobol A and Guillaud J-P 2005 Azimuthal angular distributions in EDDE as spin-parity analyser and glueball filter for LHC J. High. Energy Phys. JHEP06(2005)007 (Preprint hep-ph/0409118)

[266] Piotrzkowski K 2001 Tagging two-photon production at the LHC Phys. Rev. D 63071502 (Preprint hep-ex/0009065)

[267] Budnev V, Ginzburg I, Meledin G and Serbo V 1974 The Two photon particle production mechanism. physical problems. applications. equivalent photon approximation Phys. Rept. 15 181-281 (doi:10.1016/0370-1573(75)90009-5)

[268] Khoze V, Martin A and Ryskin M 2002 Photon-exchange processes at hadron colliders as a probe of the dynamics of diffraction Eur. Phys. J. C 24 459-468 (Preprint hep-ph/0201301) (doi:10.1007/s10052-002-0964-4)

[269] White A R 2005 The physics of a sextet quark sector Phys. Rev. D 72036007 (Preprint hep-ph/0412062)

[270] Piotrzkowski K 2002 High energy two-photon interactions at the LHC Preprint hep-ex/0201027

[271] Kalmykov N N, Ostapchenko S S and Pavlov A I 1997 Quark-gluon string model and EAS simulation problems at ultra-high energies Nucl. Phys. Proc. Suppl. B 52 17-28

[272] Engel R, Gaisser T K, Stanev T and Lipari P 1999 Air shower calculations with the new version of SIBYLL Prepared for 26th International Cosmic Ray Conference (ICRC 99) (Salt Lake City, Utah, 17-25 August 1999)

[273] Roesler S, Engel R and Ranft J The Event generator DPMJET-III at cosmic ray energies Prepared for 27th International Cosmic Ray Conference (ICRC 2001) (Hamburg, Germany, 7-15 Aug 2001)

[274] Karsch Frithjof 2002 Lattice QCD at high temperature and density Lect. Notes Phys. 583 209-249 (Preprint hep-lat/0106019)

[275] Iancu Edmond and Venugopalan Raju 2003 The color glass condensate and high energy scattering in QCD Preprint hep-ph/0303204

[276] Schwarz Dominik J 2003 The first second of the universe Annalen Phys. 12 220-270 (Preprint astro-ph/0303574)

[277] Starinets Andrei O 2005 Transport coefficients of strongly coupled gauge theories: Insights from string theory Preprint nucl-th/0511073

[278] Bonciani Roberto, Catani Stefano, Mangano Michelangelo L and Nason Paolo 1998 NLL resummation of the heavy-quark hadroproduction cross-section Nucl. Phys. B 529 424-450 (Preprint hep-ph/9801375)

[279] Davids M et al 2006 Measurement of top-pair cross section and top-quark mass in the di-lepton and full-hadronic channels with CMS CMS Note http://cms.cern.ch/iCMS/jsp/openfile.jsp?tp=draft\&files=2907_ttbar.pdf2006/077

[280] Gennai S et al 2006 Tau jet reconstruction and tagging at High Level Trigger and off-line CMS Note http://cms.cern.ch/iCMS/jsp/openfile.jsp?type=NOTE\&year=2006\&files=NOTE2006_028.pdf2006/028

[281] D'Hondt J, Heyninck J and Lowette S 2006 Measurement of the cross section of single leptonic $t \bar{t}$ events CMS Note http://cms.cern.ch/iCMS/jsp/openfile.jsp?type=NOTE\&year=2006\&files=NOTE2006_064.pdf2006/064

[282] Buttar C et al 2006 Les Houches Physics at TeV Colliders 2005, Standard Model and Higgs working group: Summary report Preprint hep-ph/0604120

[283] Konoplianikov V, Kodolova O and Ulyanov A 2006 Jet calibration using $\gamma+$ jet events in the CMS Detector CMS Note http://cms.cern.ch/iCMS/jsp/openfile.jsp?type=NOTE\&year=2006\&files=NOTE2006_042.pdf2006/042

[284] D'Hondt J et al 2006 Electron and muon reconstruction in single leptonic $t \bar{t}$ events CMS Note http://cms.cern.ch/iCMS/jsp/openfile.jsp?type=NOTE\&year=2006\&files=NOTE2006_024.pdf2006/024

[285] CMS Collaboration, Acosta D et al 2006 CMS Coll. Physics TDR Vol. I, Section 12.2.8 CERN/LHCC 2006-001

[286] Lowette S, D'Hondt J, Heyninck J and Vanlaer P 2006 Offline calibration of b-jet identification efficiency, CERN-CMS-NOTE -2006-013

[287] D'Hondt J, Lowette S, Heyninck J and Kasselmann S 2006 Light quark jet energy scale calibration using the W mass constraint in single-leptonic $t \bar{t}$ events CERN-CMS-NOTE -2006-025

[288] The CDF Collaboration, Acosta $\mathrm{D}$ et al 2005 Measurement of the $t \bar{t}$ cross section in $p \bar{p}$ collisions at $\sqrt{s}=1.96 \mathrm{TeV}$ using kinematic characteristics of lepton plus jets events Phys. Rev. D 71072005 (doi:10.1103/PhysRevD.71.072005)

[289] DØ Collaboration, Abazov V M et al 2005 Measurement of the $t \bar{t}$ cross section in $p \bar{p}$ collisions at $\sqrt{s}=1.96$ $\mathrm{TeV}$ using kinematic characteristics of lepton plus jets events Phys. Lett. B 62655 (doi:10.1016/j.physletb.2005.08.105) 
[290] Vos M and Palla F 2006 B-tagging in the High Level Trigger CMS Note http://cmsdoc.cern.ch/documents/06/note06_030.pdf2006-030

[291] CDF and DØ, Wicke D 2005 Top pair production cross-section measurement in the all-hadronic channel at CDF and DØCollaboration Int. J. Mod. Phys. A 20 3183-3186 (Preprint hep-ex/0411009) (doi:10.1142/S0217751X05026091)

[292] D’Hondt J, Heyninck J and Lowette S 2006 Top Quark mass measurement in single-leptonic $t \bar{t}$ events CMS Note http://cms.cern.ch/iCMS/jsp/openfile.jsp?type=NOTE\&year=2006\&files=NOTE2006_066.pdf2006/066

[293] CDF Collaboration, Abe F et al 1997 First Observation of the All-Hadronic Decay of $t \bar{t}$ pairs Phys. Rev. Lett. 79 1992-1997 (doi:10.1103/PhysRevLett.79.1992)

[294] Tevatron Electroweak Working Group Collaboration 2006 Combination of CDF and DØresults on the mass of the top quark Preprint hep-ex/0603039

[295] Kharchilava Avto 2000 Top mass determination in leptonic final states with J/psi Phys. Lett. B476 73-78 (Preprint hep-ph/9912320)

[296] Grenier P 2001 ATLAS Physics Note 2001-023

[297] Chierici R and Dierlamm A 2006 Determination of the top mass in exclusive JPsi decays CMS Note http://cmsdoc.cern.ch/documents/06/note06_058.pdf2006-058

[298] Tevatron Electroweak Working Group Collaboration 2006 Combination of CDF and DØ results on the mass of the top quark Preprint hep-ex/0603039

[299] Hill C S, Incandela J R and Lamb J M 2005 A method for measurement of the top quark mass using the mean decay length of $b$ hadrons in $t$ anti- $t$ events Phys. Rev. D 71054029 (Preprint hep-ex/0501043)

[300] Borjanovic I et al 2005 Investigation of top mass measurements with the ATLAS detector at LHC Eur. Phys. J. C39S2 63-90 (Preprint hep-ex/0403021)

[301] Lyons Louis, Gibaut Duncan and Clifford Peter 1988 How to combine correlated estimates of a single physical quantity Nucl. Instrum. Methods A 270110

[302] Valassi A 2003 Combining correlated measurements of several different physical quantities Nucl. Instrum. Methods A 500 391-405

[303] Mahlon G and Parke S 1996 Angular correlation in top quark pair production and decay at hadron collider Phys. Rev. D 53 4886-4896 (Preprint hep-ph/9512264)

[304] Stelzer T and Willenbrock S 1996 Spin correlation in top-quark production at hadron collider Phys. Lett. B 374 169-172 (Preprint hep-ph/9512292)

[305] Brandenburg A 1996 Spin-spin correlations of top quark pairs at Hadron colliders Phys. Lett. B 387 626-632 (Preprint hep-ph/9606379)

[306] Baarmand M, Mermerkaya H and Vodopiyanov I 2006 Measurement of spin correlation in top quark pair production in semi-leptonic final state CMS Note 2006/111

[307] Tait T 2000 The $t W^{-}$mode of single top production Phys. Rev. D 61034001 (Preprint hep-ph/9909352) (doi:10.1103/PhysRevD.61.034001)

[308] Belyaev A and Boos E 2001 Single top quark t W + X production at the LHC: A closer look Phys. Rev. D 63 034012 (Preprint hep-ph/0003260)

[309] Zhu S 2002 Next-to-leading order QCD corrections to b g $\rightarrow \mathrm{t} \mathrm{W}$ - at the CERN Large Hadron Collider Phys. Lett. B 524 283-288

[310] Boos E, Bunichev V, Dudko L, Savrin V and Sherstnev A 2005 A simulation method of the electroweak top quark production events in the NLO approximation: a Monte-Carlo generator 'singletop'abstract available at http://www.npi.msu.su/eng/science.php3?sec=preprint\&ref_pp=1262

[311] CMS Collaboration, Acosta D et al 2006 CMS Physics Technical Design Report, Volume 1, Section 9.1.2: Global muon reconstruction CERN/LHCC, 2006-001 p 333

[312] CMS Collaboration, Acosta D et al 2006 CMS Physics Technical Design Report, Volume 1, Section 9.3: Muon identification CERN/LHCC, 2006-001 p 351

[313] CMS Collaboration, Acosta D et al 2006 CMS Physics Technical Design Report, Volume 1, Section 10.4: Electron reconstruction and selection CERN/LHCC, 2006-001 p 390

[314] CMS Collaboration, Acosta D et al 2006 CMS Physics Technical Design Report, Volume 1, Section 11.2.1: Iterative cone CERN/LHCC, 2006-001 p 408

[315] CMS Collaboration, Acosta D et al 2006 CMS Physics Technical Design Report, Volume 1, Section 11.6.3: $\gamma+$ jet events CERN/LHCC, 2006-001 423

[316] CMS Collaboration, Acosta D et al 2006 CMS Physics Technical Design Report, Volume 1, Section 12.2: b-tagging tools CERN/LHCC, 2006-001 p 461

[317] Abramov V et al 2006 Selection of single top events with the CMS detector at LHC CMS Note 2006/084

[318] Yeh P et al 2006 Search for W-associated Production of Single Top Quarks in CMS CMS Note 2006/086 
[319] Campbell J, Ellis R K and Rainwater D L 2003 Next-to-leading order QCD predictions for W + 2jet and Z + 2jet production at the CERN LHC Phys. Rev. D 68094021 (doi:10.1103/PhysRevD.68.094021)

[320] CMS Collaboration, Acosta D et al 2006 CMS Coll. Physics Technical Design Physics, Volume I, Section 11.6.5 CERN/LHCC, 2006-001

[321] CMS Collaboration, Acosta D et al 2006 CMS Physics Technical Design Physics, Volume I, Section 8.5: Sources of systematic effects CERN/LHCC, 2006-001 p 229

[322] Fisher R A 1936 The use of multiple measurements in taxonomic problems Annuals of Eugenics 7 179-188

[323] Mele B, Petrarca S and Soddu A 1998 A new evaluation of the $t \rightarrow c H$ decay width in the standard model Phys. Lett. B 435 401-406 (Preprint hep-ph/9805498)

[324] Huang C-S, Wu X-H and Zhu S-H 1999 Top-charm associated production at high energy $e^{+} e^{-}$colliders in standard model Phys. Lett. B 452 143-149 (Preprint hep-ph/9901369)

[325] de Divitiis G M, Petronzio R and Silvestrini L 1997 Flavour changing top decays in supersymmetric extensions of the standard model Nucl. Phys. B 504 45-60 (Preprint hep-ph/9704244)

[326] Guasch J and Sola J 1999 FCNC top quark decays: A door to SUSY physics in high luminosity colliders? Nucl. Phys. B 562 3-28 (Preprint hep-ph/9906268)

[327] Eilam G, Gemintern A, Han T, Yang J M and Zhang X 2001 Top quark rare decay $t \rightarrow c h$ in R-parity-violating SUSY Phys. Lett. B 510 227-235 (Preprint hep-ph/0102037) (doi:10.1016/S0370-2693(01)00598-6)

[328] Li C S, Zhang X-M and Zhu S H 1999 SUSY-QCD effect on top charm associated production at linear collider Phys. Rev. D 60077702 (Preprint hep-ph/9904273)

[329] Bejar S, Guasch J and Sola J 2001 FCNC top quark decays beyond the standard model Preprint hep-ph/0101294

[330] Diaz R A, Martinez R and Alexis Rodriguez J 2001 The rare decay $t \rightarrow c \gamma$ in the general 2HDM type III Preprint hep-ph/0103307

[331] Han T and Hewett J L 1999 Top charm associated production in high energy $e^{+} e^{-}$collisions Phys. Rev. D 60 074015 (Preprint hep-ph/9811237)

[332] del Aguila F, Aguilar-Saavedra J A and Miquel R 1999 Constraints on top couplings in models with exotic quarks Phys. Rev. Lett. 82 1628-1631 (Preprint hep-ph/9808400)

[333] Aguilar-Saavedra J A and Nobre B M 2003 Rare top decays $t \rightarrow c \gamma, t \rightarrow c g$ and CKM unitarity Phys. Lett. B 553 251-260 (Preprint hep-ph/0210360)

[334] CDF and DØ Collaboration, Paulini M 1996 Heavy flavor physics from top to bottom Preprint hep-ex/9701019

[335] CDF Collaboration, Abe $\mathrm{F}$ et al 1998 Search for flavor-changing neutral current decays of the top quark in $p \bar{p}$ collisions at $\sqrt{s}=1.8 \mathrm{TeV}$ Phys. Rev. Lett. 80 2525-2530 (doi:10.1103/PhysRevLett.80.2525)

[336] Heinson A 1996 Future top physics at the Tevatron and LHC Preprint hep-ex/9605010

[337] Aguilar-Saavedra J A and Branco G C 2000 Probing top flavour-changing neutral scalar couplings at the CERN LHC Phys. Lett. B 495 347-356 (Preprint hep-ph/0004190)

[338] Aguilar-Saavedra J A 2001 Top flavour-changing neutral coupling signals at a linear collider Phys. Lett. B 502 115-124 (Preprint hep-ph/0012305)

[339] Aguilar-Saavedra J A and Riemann T 2001 Probing top flavor-changing neutral couplings at TESLA Preprint hep-ph/0102197

[340] Karafasoulis K et al 2006 Study of flavour changing neutral currents in top quark decays with the CMS detector CMS Note 2006/093

[341] Dissertori G et al 2006 How accurately can we count the number of $p p \rightarrow Z X$ and $p p \rightarrow W X$ events using decays to electrons CMS Note 2006/124

[342] Alcaraz J 2006 Measurement of $\mathrm{Z} \rightarrow \mu^{+} \mu^{-}$and $\mathrm{W} \rightarrow \mu v$ rates in CMS CMS Note 2006/082

[343] Frixione S and Webber B R 2006 The MC@ NLO 3.2 event generator Preprint hep-ph/0601192

[344] For further details see Proceedings of the 2004/2005 HERA-LHC workshop

[345] Dittmar M, Pauss F and Zurcher D 1997 Towards a precise parton luminosity determination at the CERN LHC Phys. Rev. D 56 7284-7290 (Preprint hep-ex/9705004)

[346] Drell S D and Yan T-M 1970 Massive lepton pair production in hadron-hadron collisions at high-energies Phys. Rev. Lett. 25 316-320 (doi:10.1103/PhysRevLett.25.316)

[347] Belotelov I et al 2006 Study of Drell-Yan di-muon production with the CMS detector CMS Note 2006/123

[348] Hamberg R, van Neerven W L and Matsuura T 1991 A Complete calculation of the order $\alpha_{s}^{2}$ correction to the Drell-Yan $K$-factor Nucl. Phys. B 359 343-405 (doi:10.1016/0550-3213(91)90064-5)

[349] Bourilkov D 2006 Compositeness search with di-muons in CMS CMS Note 2006/085

[350] Baur U and Wackeroth D 2003 Electroweak radiative corrections to weak boson production at hadron colliders Nucl. Phys. Proc. Suppl. 116 159-163 (Preprint hep-ph/0211089) 
[351] Bourilkov D 2003 Study of parton density function uncertainties with LHAPDF and PYTHIA at LHCPrepared for the LHC/LC Study Group Meeting (Geneva, Switzerland, 9 May 2003) Preprint hep-ph/0305126

[352] Buege V et al 2006 Prospects for the precision measurement of the $\mathrm{W}$ mass with the CMS detector CMS Note 2006-061

[353] Giele W T and Keller S 1998 Determination of W boson properties at hadron colliders Phys. Rev. D 57 4433-4440 (Preprint hep-ph/9704419)

[354] Brigljević V et al 2006 Study of di-boson production with the CMS detector at the LHC CMS Note 2006/108

[355] Pukhov A et al 1999 CompHEP: A package for evaluation of Feynman diagrams and integration over multi-particle phase space. User's manual for version 33 Preprint hep-ph/9908288

[356] Higgs P W 1964 Broken symmetries, massless particles and gauge fields Phys. Lett. 12 132-133

[357] Higgs P W 1966 Spontaneous symmetry breakdown without massless bosons Phys. Rev. 145 1156-1163

[358] Englert F and Brout R 1964 Broken symmetry and the mass of gauge vector mesons Phys. Rev. Lett. 13 321-322

[359] Guralnik G S, Hagen C R and Kibble T W B 1964 Global conservation laws and massless particles Phys. Rev. Lett. 13 585-587

[360] Cornwall J M, Levin D N and Tiktopoulos G 1973 Uniqueness of spontaneously broken gauge theories Phys. Rev. Lett. 30 1268-1270 (doi:10.1103/PhysRevLett.30.1268)

[361] Cornwall J M, Levin D N and Tiktopoulos G 1974 Derivation of gauge invariance from high-energy unitarity bounds on the S-Matrix Phys. Rev. D 101145

[362] Llewellyn Smith C H 1973 High-energy behavior and gauge symmetry Phys. Lett. B $46233-236$

[363] Joglekar S D 1974 S-matrix derivation of the Weinberg model Ann. Phys. 83427

[364] Veltman M 1968 Perturbation theory of massive Yang-Mills fields Nucl. Phys. B 7 637-650

[365] 't Hooft G 1971 Renormalization of massless Yang-Mills fields Nucl. Phys. B 33 173-199

[366] 't Hooft G 1971 Renormalizable lagrangians for massive Yang-Mills fields Nucl. Phys. B 35 167-188

[367] 't Hooft G and Veltman M J G 1972 Regularization and renormalization of gauge fields Nucl. Phys. B 44 189-213

[368] 't Hooft G and Veltman M J G 1972 Combinatorics of gauge fields Nucl. Phys. B 50 318-353

[369] Djouadi A 2005 The anatomy of electro-weak symmetry breaking I: The Higgs boson in the standard model Preprint hep-ph/0503172

[370] Djouadi A 2005 The anatomy of electro-weak symmetry breaking II: The Higgs bosons in the minimal supersymmetric model Preprint hep-ph/0503173

[371] Cabibbo N, Maiani L, Parisi G and Petronzio R 1979 Bounds on the fermions and higgs boson masses in grand unified theories Nucl. Phys. B 158295

[372] Chanowitz M S, Furman M A and Hinchliffe I 1978 Weak interactions of ultraheavy fermions Phys. Lett. B 78285

[373] Flores R A and Sher M 1983 Upper limits to fermion masses in the Glashow-Weinberg-Salam model Phys. Rev. D 271679

[374] Lindner M 1986 Implications of triviality for the standard model Zeit. Phys. C 31295

[375] Sher M 1989 Electroweak Higgs potentials and vacuum stability Phys. Rept. 179 273-418

[376] Sher M 1993 Precise vacuum stability bound in the standard model Phys. Lett. B 317 159-163 (Preprint hep-ph/9307342)

[377] Altarelli G and Isidori G 1994 Lower limit on the Higgs mass in the standard model: an update Phys. Lett. B 337 141-144

[378] Espinosa J and Quiros M 1995 Improved metastability bounds on the standard model Higgs mass Phys. Lett. B 353 257-266 (Preprint hep-ph/9504241)

[379] Hasenfratz A, Jansen K, Lang C B, Neuhaus T and Yoneyama H 1987 The triviality bound of the four component phi**4 model Phys. Lett. B 199531

[380] Kuti J, Lin L and Shen Y 1988 Upper bound on the Higgs mass in the standard model Phys. Rev. Lett. 61678

[381] Luscher M and Weisz P 1989 Scaling laws and triviality bounds in the lattice $\varphi^{4}$ theory (III) $n$-component model Nucl. Phys. B 318705 (doi:10.1016/0550-3213(89)90637-8)

[382] Higgs Working Group Collaboration, Carena M et al 2000 Report of the Tevatron Higgs working group Preprint hep-ph/0010338

[383] ATLAS Collaboration, 1994 Technical Design Report CERN/LHCC, 94-14, ATLAS TDR 14

[384] Abdullin S et al 2005 Summary of the CMS potential for the Higgs boson discovery Eur. Phys. J. C 39S2 41-61 (doi:10.1140/epjcd/s2004-02-003-9)

[385] Braaten E and Leveille J P 1980 Higgs boson decay and the running mass Phys. Rev. D 22715 
[386] Sakai N 1980 Perturbative QCD Corrections to the hadronic decay width of the Higgs boson Phys. Rev. D 222220

[387] Inami T and Kubota T 1981 Renormalization group estimate of the Hadronic decay width of the Higgs boson Nucl. Phys. B 179171

[388] Gorishnii S G, Kataev A L and Larin S A 1984 The width of Higgs Boson decay into Hadrons: three loop corrections of strong interactions Sov. J. Nucl. Phys. 40 329-334

[389] Drees M and Hikasa K-i 1990 Heavy quark thresholds in Higgs physics Phys. Rev. D 411547

[390] Drees M and Hikasa K 1990 Note on QCD corrections to Hadronic Higgs decay Phys. Lett. B 240455

[391] Chetyrkin K G 1997 Correlator of the quark scalar currents and Gamma(tot)(H $\rightarrow$ hadrons) at $\mathrm{O}($ alpha(s)**3) in pQCD Phys. Lett. B 390 309-317 (Preprint hep-ph/9608318)

[392] Fleischer J and Jegerlehner F 1981 Radiative Corrections to Higgs Decays in the Extended Weinberg-Salam Model Phys. Rev. D 23 2001-2026

[393] Bardin D Y, Vilensky B M and Khristova P K 1991 Calculation of the Higgs boson decay width into fermion pairs Sov. J. Nucl. Phys. 53 152-158

[394] Dabelstein A and Hollik W 1992 Electroweak corrections to the fermionic decay width of the standard Higgs boson Z. Phys. C 53 507-516

[395] Kniehl B A 1992 Radiative corrections for $H \rightarrow f \bar{f}(\gamma)$ in the standard model Nucl. Phys. B 376 3-28 (doi:10.1016/0550-3213(92)90065)-J

[396] Zheng H-Q and Wu D-D 1990 First order QCD corrections to the decay of the Higgs boson into two photons Phys. Rev. D 42 3760-3763

[397] Djouadi A, Spira M, van der Bij J and Zerwas P 1991 QCD corrections to gamma gamma decays of Higgs particles in the intermediate mass range Phys. Lett. B 257 187-190

[398] Dawson S and Kauffman R P 1993 QCD corrections to H $\rightarrow$ gamma gamma Phys. Rev. D 47 1264-1267

[399] Djouadi A, Spira M and Zerwas P 1993 Two photon decay widths of Higgs particles Phys. Lett. B 311 255-260 (Preprint hep-ph/9305335)

[400] Melnikov K and Yakovlev O I 1993 Higgs $\rightarrow$ two photon decay: QCD radiative correction Phys. Lett. B 312 179-183 (Preprint hep-ph/9302281)

[401] Inoue M, Najima R, Oka T and Saito J 1994 QCD corrections to two photon decay of the Higgs boson and its reverse process Mod. Phys. Lett. A 9 1189-1194

[402] Steinhauser M 1996 Corrections of $\mathrm{O}\left(\mathrm{alpha}(\mathrm{s})^{* * 2}\right.$ ) to the decay of an intermediate-mass Higgs boson into two photons Preprint hep-ph/9612395

[403] Djouadi A, Gambino P and Kniehl B A 1998 Two-loop electroweak heavy-fermion corrections to Higgsboson production and decay Nucl. Phys. B 523 17-39 (Preprint hep-ph/9712330)

[404] Aglietti U, Bonciani R, Degrassi G and Vicini A 2004 Two-loop light fermion contribution to Higgs production and decays Phys. Lett. B 595 432-441 (Preprint hep-ph/0404071)

[405] Degrassi G and Maltoni F 2005 Two-loop electroweak corrections to the Higgs-boson decay $\mathrm{H} \rightarrow$ gamma gamma Preprint hep-ph/0504137

[406] Kniehl B A 1991 Radiative corrections for $H \rightarrow Z Z$ in the standard model Nucl. Phys. B 352 1-26 (doi:10.1016/0550-3213(91)90126-I)

[407] Kniehl B A 1991 Radiative corrections for $H \rightarrow W^{+} W^{-}(\gamma)$ in the standard model Nucl. Phys. B 357 439-466 (doi:10.1016/0550-3213(91)90476-E)

[408] Georgi H, Glashow S, Machacek M and Nanopoulos D 1978 Higgs bosons from two gluon annihilation in proton proton collisions Phys. Rev. Lett. 40692 (doi:10.1103/PhysRevLett.40.692)

[409] Graudenz D, Spira M and Zerwas P M 1993 QCD corrections to Higgs boson production at proton proton colliders Phys. Rev. Lett. 70 1372-1375 (doi:10.1103/PhysRevLett.70.1372)

[410] Spira M, Djouadi A, Graudenz D and Zerwas P 1993 SUSY Higgs production at proton colliders Phys. Lett. B 318 347-353 (doi:10.1016/0370-2693(93)90138-8)

[411] Spira M, Djouadi A, Graudenz D and Zerwas P 1995 Higgs boson production at the LHC Nucl. Phys. B 453 17-82 (Preprint hep-ph/9504378) (doi:10.1016/0550-3213(95)00379-7)

[412] Kramer M, Laenen E and Spira M 1998 Soft gluon radiation in Higgs boson production at the LHC Nucl. Phys. B 511 523-549 (Preprint hep-ph/9611272) (doi:10.1016/S0550-3213(97)00679-2)

[413] Djouadi A, Spira M and Zerwas P 1991 Production of Higgs bosons in proton colliders: QCD corrections Phys. Lett. B 264 440-446

[414] Dawson S 1991 Radiative corrections to Higgs boson production Nucl. Phys. B 359 283-300

[415] Kauffman R P and Schaffer W 1994 QCD corrections to production of Higgs pseudoscalars Phys. Rev. D 49 551-554 (Preprint hep-ph/9305279)

[416] Dawson S and Kauffman R 1994 QCD corrections to Higgs boson production: nonleading terms in the heavy quark limit Phys. Rev. D 49 2298-2309 (Preprint hep-ph/9310281) 
[417] Harlander R V and Kilgore W B 2002 Next-to-next-to-leading order Higgs production at hadron colliders Phys. Rev. Lett. 88201801 (Preprint hep-ph/0201206)

[418] Harlander R V and Kilgore W B 2002 Production of a pseudo-scalar Higgs boson at hadron colliders at next-to-next-to leading order J. High. Energy Phys. JHEP10(2002)017 (Preprint hep-ph/0208096)

[419] Anastasiou C and Melnikov K 2002 Higgs boson production at hadron colliders in NNLO QCD Nucl. Phys. B 646 220-256 (Preprint hep-ph/0207004)

[420] Ravindran V, Smith J and van Neerven W L 2003 NNLO corrections to the total cross section for Higgs boson production in hadron hadron collisions Nucl. Phys. B 665 325-366 (Preprint hep-ph/0302135)

[421] Catani S, de Florian D, Grazzini M and Nason P 2003 Soft-gluon resummation for Higgs boson production at hadron colliders J. High. Energy Phys. JHEP07(2003)028 (Preprint hep-ph/0306211)

[422] Djouadi A and Gambino P 1994 Leading electroweak correction to Higgs boson production at proton colliders Phys. Rev. Lett. 73 2528-2531 (Preprint hep-ph/9406432)

[423] Chetyrkin K G, Kniehl B A and Steinhauser M 1997 Virtual top-quark effects on the H $\rightarrow$ b anti-b decay at next-to-leading order in QCD Phys. Rev. Lett. 78 594-597 (Preprint hep-ph/9610456)

[424] Chetyrkin K G, Kniehl B A and Steinhauser M 1997 Three-loop O(alpha(s)**2 G(F) M(t)**2) corrections to hadronic Higgs decays Nucl. Phys. B 490 19-39 (Preprint hep-ph/9701277)

[425] Ghinculov A and van der Bij J J 1996 The Higgs resonance shape in gluon fusion: Heavy Higgs effects Nucl. Phys. B 482 59-72 (Preprint hep-ph/9511414)

[426] Ellis R K, Hinchliffe I, Soldate M and van der Bij J J 1988 Higgs Decay to tau + tau-: A Possible Signature of Intermediate Mass Higgs Bosons at the SSC Nucl. Phys. B 297221

[427] Baur U and Glover E W N 1990 Higgs Boson Production at Large Transverse Momentum in Hadronic Collisions Nucl. Phys. B 339 38-66

[428] Schmidt C R $1997 H \rightarrow g g g(g q \bar{q})$ at two loops in the large- $M_{t}$ limit Phys. Lett. B 413 391-395 (doi:10.1016/S0370-2693(97)01102-7)

[429] de Florian D, Grazzini M and Kunszt Z 1999 Higgs production with large transverse momentum in hadronic collisions at next-to-leading order Phys. Rev. Lett. 82 5209-5212 (Preprint hep-ph/9902483)

[430] Ravindran V, Smith J and Van Neerven W L 2002 Next-to-leading order QCD corrections to differential distributions of Higgs boson production in hadron hadron collisions Nucl. Phys. B 634 247-290 (Preprint hep-ph/0201114)

[431] Glosser C J and Schmidt C R 2002 Next-to-leading corrections to the Higgs boson transverse momentum spectrum in gluon fusion J. High. Energy Phys. JHEP12(2002)016 (Preprint hep-ph/0209248)

[432] Anastasiou C, Melnikov K and Petriello F 2005 Fully differential Higgs boson production and the di-photon signal through next-to-next-to-leading order Nucl. Phys. B 724 197-246 (Preprint hep-ph/0501130)

[433] Catani S, D’Emilio E and Trentadue L 1988 The Gluon Form-Factor to Higher Orders: Gluon Gluon Annihilation at Small Q-Transverse Phys. Lett. B 211 335-342

[434] Hinchliffe I and Novaes S F 1988 On the Mean Transverse Momentum of Higgs Bosons at the SSC Phys. Rev. D 38 3475-3480

[435] Kauffman R P 1991 Higgs boson p(T) in gluon fusion Phys. Rev. D 44 1415-1425

[436] Kauffman R 1992 Higher order corrections to Higgs boson p(T) Phys. Rev. D $451512-1517$

[437] Balazs C and Yuan C P 2000 Higgs boson production at the LHC with soft gluon effects Phys. Lett. B 478 192-198 (Preprint hep-ph/0001103)

[438] Berger E L and Qiu J-w 2003 Differential cross section for Higgs boson production including all-orders soft gluon resummation Phys. Rev. D 67034026 (Preprint hep-ph/0210135)

[439] Kulesza A and Stirling W J 2003 Non-perturbative effects and the resummed Higgs transverse momentum distribution at the LHC J. High. Energy Phys. JHEP12(2003)056 (Preprint hep-ph/0307208)

[440] Kulesza A, Sterman G and Vogelsang W 2004 Joint resummation for Higgs production Phys. Rev. D 69 014012 (Preprint hep-ph/0309264)

[441] Gawron A and Kwiecinski J 2004 Resummation effects in Higgs boson transverse momentum distribution within the framework of unintegrated parton distributions Phys. Rev. D 70014003 (Preprint hep-ph/0309303)

[442] Watt G, Martin A and Ryskin M 2004 Unintegrated parton distributions and electroweak boson production at hadron colliders Phys. Rev. D 70014012 (Preprint hep-ph/0309096)

[443] Lipatov A and Zotov N 2005 Higgs boson production at hadron colliders in the $\mathrm{k}(\mathrm{T})$-factorization approach Eur. Phys. J. C 44 559-566 (Preprint hep-ph/0501172)

[444] de Florian D and Grazzini M 2000 Next-to-next-to-leading logarithmic corrections at small transverse momentum in hadronic collisions Phys. Rev. Lett. 85 4678-4681 (Preprint hep-ph/0008152) 
[445] Catani S, De Florian D and Grazzini M 2001 Higgs production at hadron colliders in (almost) NNLO QCD Bologna 2001, Deep Inelastic Scattering (Bologna, Italy, April, 2001) pp 518-521 Preprint hep-ph/0106049

[446] Catani S, de Florian D and Grazzini M 2001 Universality of non-leading logarithmic contributions in transverse momentum distributions Nucl. Phys. B 596 299-312 (Preprint hep-ph/0008184)

[447] Bozzi G, Catani S, de Florian D and Grazzini M 2003 The q(T) spectrum of the Higgs boson at the LHC in QCD perturbation theory Phys. Lett. B 564 65-72 (Preprint hep-ph/0302104)

[448] Bozzi G, Catani S, de Florian D and Grazzini M 2006 Transverse-momentum resummation and the spectrum of the Higgs boson at the LHC Nucl. Phys. B 737 73-120 (Preprint hep-ph/0508068)

[449] Cahn R N and Dawson S 1984 Production of Very Massive Higgs Bosons Phys. Lett. B 136196

[450] Hikasa K-i 1985 Heavy Higgs Production in e+ e- and e- e- Collisions Phys. Lett. B 164385

[451] Altarelli G, Mele B and Pitolli F 1987 Heavy Higgs production at future colliders Nucl. Phys. B 287 205-224

[452] Han T, Valencia G and Willenbrock S 1992 Structure function approach to vector boson scattering in $p \mathrm{p}$ collisions Phys. Rev. Lett. $693274-3277$ (Preprint hep-ph/9206246)

[453] Figy T, Oleari C and Zeppenfeld D 2003 Next-to-leading order jet distributions for Higgs boson production via weak-boson fusion Phys. Rev. D 68073005 (Preprint hep-ph/0306109)

[454] Glashow S, Nanopoulos D and Yildiz A 1978 Associated production of Higgs bosons and Z particles Phys. Rev. D 18 1724-1727

[455] Kunszt Z, Trocsanyi Z and Stirling W J 1991 Clear signal of intermediate mass Higgs boson production at LHC and SSC Phys. Lett. B 271 247-255

[456] Han T and Willenbrock S 1991 QCD correction to the $\mathrm{p} \mathrm{p} \rightarrow \mathrm{W} \mathrm{H}$ and Z H total cross- sections Phys. Lett. B $273167-172$

[457] Brein O, Djouadi A and Harlander R 2004 NNLO QCD corrections to the Higgs-strahlung processes at hadron colliders Phys. Lett. B 579 149-156 (Preprint hep-ph/0307206)

[458] Ciccolini M L, Dittmaier S and Kramer M 2003 Electroweak radiative corrections to associated W H and Z H production at hadron colliders Phys. Rev. D 68073003 (Preprint hep-ph/0306234)

[459] Raitio R and Wada W W 1979 Higgs Boson production at large transverse momentum in QCD Phys. Rev. D 19941

[460] Ng J N and Zakarauskas P 1984 A QCD Parton calculation of conjoined production of Higgs Bosons and heavy flavors in p anti-p collision Phys. Rev. D 29876

[461] Kunszt Z 1984 Associated production of heavy Higgs boson with top quarks Nucl. Phys. B 247339

[462] Gunion J F 1991 Associated top anti-top Higgs production as a large source of W H events: Implications for Higgs detection in the lepton neutrino gamma gamma final state Phys. Lett. B $261510-517$

[463] Marciano W J and Paige F E 1991 Associated production of Higgs bosons with t anti-t pairs Phys. Rev. Lett. $662433-2435$

[464] Beenakker W, Dittmaier S, Kraemer M, Pluemper B, Spira M and Zerwas P 2001 Higgs radiation off top quarks at the Tevatron and the LHC Phys. Rev. Lett. 87201805 (Preprint hep-ph/0107081)

[465] Dawson S, Orr L H, Reina L and Wackeroth D 2003 Associated top quark Higgs boson production at the LHC Phys. Rev. D 67071503 (Preprint hep-ph/0211438)

[466] Dawson S and Reina L 1998 QCD corrections to associated Higgs boson production Phys. Rev. D 57 5851-5859 (Preprint hep-ph/9712400)

[467] Futyan D, Fortin D and Giordano D 2006 Search for the Standard Model Higgs Boson in the Two-Electron and Two-Muon Final State with CMS CMS Note 2006/136

[468] Bartalini P et al 2006 NLO vs. LO: kinematical differences for signal and background in the $\mathrm{H} \rightarrow \mathrm{ZZ}^{(*)} \rightarrow 4 \mu$ analysis CMS Note $2006 / 130$

[469] Baffioni S et al 2006 Discovery potential for the SM Higgs boson in the $\mathrm{H} \rightarrow \mathrm{ZZ}^{(*)} \rightarrow \mathrm{e}^{+} \mathrm{e}^{-} \mathrm{e}^{+} \mathrm{e}^{-}$decay channel CMS Note 2006/115

[470] Dittmar M and Dreiner H K 1997 How to find a Higgs boson with a mass between $155-\mathrm{GeV}$ to $180-\mathrm{GeV}$ at the LHC Phys. Rev. D 55 167-172 (Preprint hep-ph/9608317)

[471] Davatz G, Dittmar M and Giolo-Nicollerat A-S 2006 Standard Model Higgs Discovery Potential of CMS in $\mathrm{H} \rightarrow \mathrm{WW} \rightarrow \ell v \ell v$ Channel CMS Note 2006/047

[472] Davatz G, Dissertori G, Dittmar M, Grazzini M and Pauss F 2004 Effective K-factors for $g g \rightarrow H \rightarrow W W \rightarrow \ell \nu \ell v$ at the LHC J. High. Energy Phys. JHEP05(2004)009 (Preprint hep-ph/0402218)

[473] Campbell J and Tramontano F 2005 Next-to-leading order corrections to W t production and decay Nucl. Phys. B 726 109-130 (Preprint hep-ph/0506289)

[474] CMS Collaboration, Beaudette F et al 2006 Search for a Light Standard Model Higgs Boson in the $\mathrm{H} \rightarrow W W^{(*)} \rightarrow e^{+} v e^{-} \bar{v}$ Channel CMS Note 2006/114 
[475] Plehn T, Rainwater D L and Zeppenfeld D 2000 A method for identifying $H \rightarrow \tau \tau \rightarrow e^{ \pm} \mu_{P_{\mathrm{T}}}^{\mp}$ at the CERN LHC Phys. Rev. D 61093005 (doi:10.1103/PhysRevD.61.093005)

[476] Rainwater D L, Zeppenfeld D and Hagiwara K 1999 Searching for $H \rightarrow \tau \tau$ in weak boson fusion at the LHC Phys. Rev. D 59014037 (doi:10.1103/PhysRevD.59.014037)

[477] Cavalli D et al 2002 The Higgs working group: Summary report Preprint hep-ph/0203056

[478] Zeppenfeld D, Kinnunen R, Nikitenko A and Richter-Was E 2000 Measuring Higgs boson couplings at the LHC Phys. Rev. D 62013009 (Preprint hep-ph/0002036)

[479] Duhrssen M et al 2004 Extracting Higgs boson couplings from LHC data Phys. Rev. D 70113009 (Preprint hep-ph/0406323)

[480] Plehn T, Rainwater D L and Zeppenfeld D 1999 Probing the MSSM Higgs sector via weak boson fusion at the LHC Phys. Lett. B 454 297-303 (Preprint hep-ph/9902434)

[481] Foudas C, Nikitenko A and Takahashi M 2006 Observation of the Standard Model Higgs boson via $\mathrm{H} \rightarrow \tau \tau \rightarrow$ lepton+jet Channel CMS Note 2006/088

[482] Pi H, Avery P, Rohlf J, Tully C and Kunori S 2006 Search for Standard Model Higgs Boson via Vector Boson Fusion in the $\mathrm{H} \rightarrow \mathrm{W}^{+} \mathrm{W}^{-}$to $\ell^{ \pm} v \mathrm{jj}$ with $120<\mathrm{m}_{\mathrm{H}}<250 \mathrm{GeV} / c^{2}$ CMS Note 2006/092

[483] Dubinin M, Litvin V, Ma Y, Newman H and Pieri M 2006 Vector Boson Fusion Production with $\mathrm{H} \rightarrow \gamma \gamma$ CMS Note 2006/097

[484] Delaere C 2006 Study of associated WH production with $\mathrm{H} \rightarrow \mathrm{WW}^{*}$ in the 3 leptons final state CMS Note 2006/053

[485] CDF Collaboration, Acosta D et al 2005 Measurement of the ttbar Production Cross Section in ppbar Collisions at sqrt(s) $=1.96 \mathrm{TeV}$ using Lepton + Jets Events with Secondary Vertex b-tagging Phys. Rev. D 71052003

[486] Djouadi A and Ferrag S 2003 PDF Uncertainties In Higgs Production At Hadron Colliders Preprint hep-ph/0310209

[487] Higgs Working Group Collaboration, Assamagan K et al 2004 The Higgs working group: Summary report 2003 Preprint hep-ph/0406152

[488] Belanger G, Boudjema F and Sridhar K 2000 SUSY Higgs at the LHC: Large stop mixing effects and associated production Nucl. Phys. B 568 3-39 (Preprint hep-ph/9904348)

[489] Dubinin M, Ilyin V and Savrin V 1997 Light Higgs Boson Signal at LHC in the reaction pp $\rightarrow \gamma \gamma+$ jet and pp $\rightarrow \gamma \gamma$ +lepton CMS Note 1997/101

[490] Kinnunen R and Denegri D 1997 Expected SM/SUSY Higgs Observability in CMS CMS Note 1997/057

[491] ATLAS Collaboration, 1999 ATLAS Detector and Physics Performance. Technical Design Report. Vol. 2 CERN/LHCC, CERN-LHCC -99-15

[492] Beauchemin P, Azuelos G and Burgess C 2004 Dimensionless coupling of bulk scalars at the LHC J. Phys. G30 N17 (doi:10.1088/0954-3899/30/10/N01)

[493] Stelzer T and Long W F 1994 Automatic generation of tree level helicity amplitudes Comput. Phys. Commun. 81 357-371 (doi:10.1016/0010-4655(94)90084-1)

[494] Murayama H, Watanabe I and Hagiwara K HELAS: HELicity amplitude subroutines for Feynman diagram evaluations KEK-91-11

[495] Mangano M, Moretti M and Pittau R 2002 Multijet matrix elements and shower evolution in hadronic collisions: $W b \bar{b}+n$-jets as a case study Nucl. Phys. B 632 343-362 (Preprint hep-ph/0108069) (doi:10.1016/S0550-3213(02)00249-3)

[496] Caravaglios F, Mangano M, Moretti M and Pittau R 1999 A new approach to multi-jet calculations in hadron collisions Nucl. Phys. B 539 215-232 (Preprint hep-ph/9807570)

[497] Buttar C et al Les Houches physics at TeV colliders 2005, standard model, QCD, EW, and Higgs working group: Summary report 2006 Preprint hep-ph/0604120

[498] Bityukov S, Erofeeva S, Krasnikov N and Nikitenko A 2005 Program for evaluation of significance, confidence intervals and limits by direct calculation of probabilities Proceedings of PhyStat 2005

[499] Lethuillier M et al 2006 Search for a neutral Higgs boson with WH / ZH, $\mathrm{H} \rightarrow \gamma \gamma$ channel CMS Note $2006 / 110$

[500] Ravat O 2004 Etude du Calorimètre électromagnétique de l'expérience CMS et recherche de bosons de Higgs neutres dans le canal de production associée PhD Thesis, IPN, Lyon, 2004. LYCEN-T2004-29

[501] Graham D J 1995 An algorithm using tracks to locate the two photon vertex at high luminosity CMS TN $1995 / 115$

[502] Djouadi A 1998 Squark effects on Higgs boson production and decay at the LHC Phys. Lett. B 435 101-108 (Preprint hep-ph/9806315)

[503] Eynard G 1998 Study of associated Higgs boson production $\mathrm{HW}, \mathrm{Ht} \overline{\mathrm{t}}, \mathrm{HZ} \rightarrow \gamma \gamma+\mathrm{e}^{ \pm} / \mu^{ \pm}+\mathrm{X}$ with the ATLAS detector at LHC. (In French) PhD Thesis, Annecy, CERN-THESIS -2000-036 
[504] Beauchemin P-H and Azuelos G 2004 Search for the Standard Model Higgs Boson in the $\gamma \gamma+E_{\mathrm{T}}^{\text {miss }}$ channel ATL-PHYS-2004-028

[505] Lethuillier M, Agram J-L, Baty C, Gascon-Shotkin S, Perries S and Ravat O 2006 Search for a Neutral Higgs Boson with WH/ZH, $\mathrm{H} \rightarrow \gamma \gamma$ Channel CMS Note 2006/110

[506] CMS Collaboration 2000 The TriDAS Project Technical Design Report, Volume 1: The Trigger Systems CERN/LHCC, 2000-38, CMS TDR 6.1

[507] CMS Collaboration, Acosta D et al 2006 CMS Physics Technical Design Report, Volume 1, Section 10.3.2: Photon isolation CERN/LHCC, 2006-001 p 376

[508] Read A L 2002 Presentation of search results: The CL(s) technique J. Phys. G 28 2693-2704

[509] Junk T 1999 Confidence level computation for combining searches with small statistics Nucl. Instrum. Meth. A 434 435-443 (Preprint hep-ex/9902006)

[510] Djouadi A and Ferrag S 2004 PDF uncertainties in Higgs production at hadron colliders Phys. Lett. B 586 345-352 (Preprint hep-ph/0310209)

[511] Dell'Aquila J R and Nelson C A 1986 Simple tests for $C P$ or $P$ violation by sequential decays: $V_{1} V_{2}$ modes with decays into $\bar{\ell}_{A} \ell_{B}$ and/or $\bar{q}_{A} q_{B}$ Phys. Rev. D 33101 (doi:10.1103/PhysRevD.33.101)

[512] Skjold A and Osland P 1994 Signals of CP violation in Higgs decay Phys. Lett. B 329 305-311 (Preprint hep-ph/9402358)

[513] Choi S Y, Miller D J, Muhlleitner M M and Zerwas P M 2003 Identifying the Higgs spin and parity in decays to Z pairs Phys. Lett. B 553 61-71 (Preprint hep-ph/0210077)

[514] Buszello C P, Fleck I, Marquard P and van der Bij J J 2004 Prospective analysis of spin- and CP-sensitive variables in $\mathrm{H} \rightarrow \mathrm{ZZ} \rightarrow$ 1(1)+ 1(1)- 1(2)+ 1(2)- at the LHC Eur. Phys. J. C 32 209-219 (Preprint hep-ph/0212396)

[515] Bluj M 2006 A Study of Angular Correlations in $\mathrm{H} \rightarrow \mathrm{ZZ} \rightarrow 2 \mathrm{e} 2 \mu$ CMS Note 2006/094

[516] Wess J and Zumino B 1974 Supergauge transformations in four-dimensions Nucl. Phys. B 70 39-50

[517] Fayet P and Ferrara S 1977 Supersymmetry Phys. Rept. 32 249-334

[518] Nilles H P 1984 Supersymmetry, supergravity and particle physics Phys. Rept. 1101

[519] Barbieri R 1988 Looking beyond the standard model: the supersymmetric option Riv. Nuovo Cim. 11N4 1-45

[520] Haber H E and Kane G L 1985 The search for supersymmetry: probing physics beyond the standard model Phys. Rept. 11775

[521] Witten E 1981 Mass hierarchies in supersymmetric theories Phys. Lett. B 105267

[522] Dimopoulos S, Raby S and Wilczek F 1981 Supersymmetry and the scale of unification Phys. Rev. D 24 $1681-1683$

[523] Ibanez L E and Ross G G 1981 Low-energy predictions in supersymmetric grand unified theories Phys. Lett. B 105439

[524] Ibanez L E and Ross G G $1982 S U(2)_{L} \times U(1)$ Symmetry breaking as a radiative effect of supersymmetry breaking in GUTs Phys. Lett. B 110 215-220 (doi:10.1016/0370-2693(82)91239-4)

[525] Inoue K, Kakuto A, Komatsu H and Takeshita S 1982 Aspects of grand unified models with softly broken supersymmetry Prog. Theor. Phys. 68927

[526] Alvarez-Gaume L, Claudson M and Wise M B 1982 Low-energy supersymmetry Nucl. Phys. B 20796

[527] Ellis J R, Nanopoulos D V and Tamvakis K 1983 Grand unification in simple supergravity Phys. Lett. B 121 123

[528] Dimopoulos S and Georgi H 1981 Softly broken supersymmetry and SU(5) Nucl. Phys. B 193150

[529] Sakai N 1981 Naturalness in supersymmetric 'GUTS' Zeit. Phys. C 11153

[530] Okada Y, Yamaguchi M and Yanagida T 1991 Upper bound of the lightest Higgs boson mass in the minimal supersymmetric standard model Prog. Theor. Phys. 85 1-6

[531] Haber H E and Hempfling R 1991 Can the mass of the lightest Higgs boson of the minimal supersymmetric model be larger than $\mathrm{m}(\mathrm{Z})$ ? Phys. Rev. Lett. 66 1815-1818

[532] Ellis J R, Ridolfi G and Zwirner F 1991 Radiative corrections to the masses of supersymmetric Higgs bosons Phys. Lett. B 257 83-91

[533] Barbieri R, Frigeni M and Caravaglios F 1991 The supersymmetric Higgs for heavy superpartners Phys. Lett. B 258 167-170

[534] Yamada A 1991 Radiative corrections to the Higgs masses in the minimal supersymmetric standard model Phys. Lett. B $263233-238$

[535] Brignole A, Ellis J R, Ridolfi G and Zwirner F 1991 The supersymmetric charged Higgs boson mass and LEP phenomenology Phys. Lett. B 271 123-132

[536] Chankowski P H, Pokorski S and Rosiek J 1992 Charged and neutral supersymmetric Higgs boson masses: Complete one loop analysis Phys. Lett. B 274 191-198 
[537] Espinosa J R and Quiros M 1991 Two loop radiative corrections to the mass of the lightest Higgs boson in supersymmetric standard models Phys. Lett. B 266 389-396

[538] Hempfling R and Hoang A H 1994 Two loop radiative corrections to the upper limit of the lightest Higgs boson mass in the minimal supersymmetric model Phys. Lett. B 331 99-106 (Preprint hep-ph/9401219)

[539] Casas J A, Espinosa J R, Quiros M and Riotto A 1995 The lightest Higgs boson mass in the minimal supersymmetric standard model Nucl. Phys. B 436 3-29 (Preprint hep-ph/9407389)

[540] Carena M, Espinosa J R, Quiros M and Wagner C 1995 Analytical expressions for radiatively corrected Higgs masses and couplings in the MSSM Phys. Lett. B 355 209-221 (Preprint hep-ph/9504316)

[541] Carena M, Quiros M and Wagner C E M 1996 Effective potential methods and the Higgs mass spectrum in the MSSM Nucl. Phys. B 461 407-36 (Preprint hep-ph/9508343)

[542] Heinemeyer S, Hollik W and Weiglein G 1998 QCD corrections to the masses of the neutral CP-even Higgs bosons in the MSSM Phys. Rev. D 58091701 (Preprint hep-ph/9803277)

[543] Heinemeyer S, Hollik W and Weiglein G 1998 Precise prediction for the mass of the lightest Higgs boson in the MSSM Phys. Lett. B 440 296-304 (Preprint hep-ph/9807423)

[544] Heinemeyer S, Hollik W and Weiglein G 2000 Constraints on tan(beta) in the MSSM from the upper bound on the mass of the lightest Higgs boson J. High. Energy Phys. JHEP06(2000)009 (Preprint hep-ph/9909540)

[545] Espinosa J R and Zhang R-J 2000 Complete two-loop dominant corrections to the mass of the lightest CP-even Higgs boson in the minimal supersymmetric standard model Nucl. Phys. B 586 3-38 (Preprint hep-ph/0003246)

[546] Brignole A, Degrassi G, Slavich P and Zwirner F 2002 On the $\mathcal{O}\left(\alpha_{t}^{2}\right)$ two-loop corrections to the neutral Higgs boson masses in the MSSM Nucl. Phys. B 631 195-218 (doi:10.1016/S0550-3213(02)00184-0)

[547] Brignole A, Degrassi G, Slavich P and Zwirner F 2002 On the two-loop sbottom corrections to the neutral Higgs boson masses in the MSSM Nucl. Phys. B 643 79-92 (Preprint hep-ph/0206101)

[548] Heinemeyer S, Hollik W, Rzehak H and Weiglein G 2005 High-precision predictions for the MSSM Higgs sector at $\mathcal{O}\left(\alpha_{b} \alpha_{s}\right)$ Eur. Phys. J. C 39 465-481 (doi:10.1140/epjc/s2005-02112-6)

[549] Coarasa J A, Jimenez R A and Sola J 1996 Strong effects on the hadronic widths of the neutral Higgs Bosons in the MSSM Phys. Lett. B 389 312-320 (Preprint hep-ph/9511402)

[550] Chankowski P H, Pokorski S and Rosiek J 1994 Complete on-shell renormalization scheme for the minimal supersymmetric Higgs sector Nucl. Phys. B 423 437-496 (Preprint hep-ph/9303309)

[551] Dabelstein A 1995 The One loop renormalization of the MSSM Higgs sector and its application to the neutral scalar Higgs masses Z. Phys. C 67 495-512 (Preprint hep-ph/9409375)

[552] Brignole A 1992 Radiative corrections to the supersymmetric neutral Higgs boson masses Phys. Lett. B 281 284-294

[553] Haber H E, Hempfling R and Hoang A H 1997 Approximating the radiatively corrected Higgs mass in the minimal supersymmetric model Z. Phys. C 75 539-554 (Preprint hep-ph/9609331)

[554] Zhang R-J 1999 Two-loop effective potential calculation of the lightest CP-even Higgs-boson mass in the MSSM Phys. Lett. B 447 89-97 (Preprint hep-ph/9808299)

[555] Espinosa J R and Zhang R-J 2000 MSSM lightest CP-even Higgs boson mass to O(alpha(s) alpha(t)): The effective potential approach J. High. Energy Phys. JHEP03(2000)026 (Preprint hep-ph/9912236)

[556] Degrassi G, Slavich P and Zwirner F 2001 On the neutral Higgs boson masses in the MSSM for arbitrary stop mixing Nucl. Phys. B 611 403-422 (Preprint hep-ph/0105096)

[557] Hempfling R 1994 Yukawa coupling unification with supersymmetric threshold corrections Phys. Rev. D 49 $6168-6172$

[558] Hall L J, Rattazzi R and Sarid U 1994 The Top quark mass in supersymmetric SO(10) unification Phys. Rev. D 50 7048-7065 (Preprint hep-ph/9306309)

[559] Carena M, Olechowski M, Pokorski S and Wagner C 1994 Electroweak symmetry breaking and bottom-top Yukawa unification Nucl. Phys. B 426 269-300 (Preprint hep-ph/9402253)

[560] Guasch J, Hafliger P and Spira M 2003 MSSM Higgs decays to bottom quark pairs revisited Phys. Rev. D 68 115001 (Preprint hep-ph/0305101)

[561] Dedes A, Degrassi G and Slavich P 2003 On the two-loop Yukawa corrections to the MSSM Higgs boson masses at large tan(beta) Nucl. Phys. B 672 144-162 (Preprint hep-ph/0305127)

[562] Eberl H, Hidaka K, Kraml S, Majerotto W and Yamada Y 2000 Improved SUSY QCD corrections to Higgs boson decays into quarks and squarks Phys. Rev. D 62055006 (Preprint hep-ph/9912463)

[563] Carena M, Garcia D, Nierste U and Wagner C 2000 Effective Lagrangian for the $\bar{t} b H^{+}$interaction in the MSSM and charged Higgs phenomenology Nucl. Phys. B 577 88-120 (doi:0.1016/S0550-3213(00)00146-2) 
[564] Heinemeyer S, Hollik W and Weiglein G 2006 Electroweak precision observables in the minimal supersymmetric standard model Phys. Rept. 425 265-368 (Preprint hep-ph/0412214)

[565] Carena M and Haber H E 2003 Higgs boson theory and phenomenology. ((V)) Prog. Part. Nucl. Phys. 50 63-152 (Preprint hep-ph/0208209)

[566] ALEPH, DELPHI, L3, OPAL Collaboration 2006 LEP Working Group for Higgs Boson Searches, Search for neutral MSSM Higgs bosons at LEP Preprint hep-ex/0602042

[567] LEP Higgs Working Group for Higgs boson searches Collaboration 2001 Search for charged Higgs bosons: Preliminary combined results using LEP data collected at energies up to $209 \mathrm{GeV}$ Preprint hep-ex/0107031

[568] Dabelstein A 1995 Fermionic decays of neutral MSSM Higgs bosons at the one loop level Nucl. Phys. B 456 25-56 (Preprint hep-ph/9503443)

[569] Heinemeyer S, Hollik W and Weiglein G 2000 Decay widths of the neutral CP-even MSSM Higgs bosons in the Feynman-diagrammatic approach Eur. Phys. J. C 16 139-153 (Preprint hep-ph/0003022)

[570] Djouadi A, Janot P, Kalinowski J and Zerwas P M 1996 SUSY Decays of Higgs Particles Phys. Lett. B 376 220-226 (Preprint hep-ph/9603368)

[571] Djouadi A, Kalinowski J, Ohmann P and Zerwas P M 1997 Heavy SUSY Higgs bosons at e+ e- linear colliders Z. Phys. C 74 93-111 (Preprint hep-ph/9605339)

[572] Dawson S, Djouadi A and Spira M 1996 QCD Corrections to SUSY Higgs Production: The Role of Squark Loops Phys. Rev. Lett. 77 16-19 (Preprint hep-ph/9603423)

[573] Harlander R V and Steinhauser M 2003 Hadronic Higgs production and decay in supersymmetry at next-to-leading order Phys. Lett. B 574 258-268 (Preprint hep-ph/0307346)

[574] Harlander R and Steinhauser M 2003 Effects of SUSY-QCD in hadronic Higgs production at nextto-next-to-leading order Phys. Rev. D 68111701 (Preprint hep-ph/0308210)

[575] Harlander R V and Steinhauser M 2004 Supersymmetric Higgs production in gluon fusion at next-to-leading order J. High. Energy Phys. JHEP09(2004)066 (Preprint hep-ph/0409010)

[576] Harlander R V and Hofmann F 2005 Pseudo-scalar Higgs production at next-to-leading order SUSY-QCD Preprint hep-ph/0507041

[577] Langenegger U, Spira M, Starodumov A and Trueb P 2006 SM and MSSM Higgs boson production: spectra at large transverse momentum Preprint hep-ph/0604156

[578] Djouadi A and Spira M 2000 SUSY-QCD corrections to Higgs boson production at hadron colliders Phys. Rev. D 62014004 (Preprint hep-ph/9912476)

[579] Wu P et al 2005 NLO supersymmetric QCD corrections to t anti-t h0 associated production at hadron colliders Preprint hep-ph/0505086

[580] Dittmaier S, Kramer M and Spira M 2004 Higgs radiation off bottom quarks at the Tevatron and the LHC Phys. Rev. D 70074010 (Preprint hep-ph/0309204)

[581] Dawson S, Jackson C B, Reina L and Wackeroth D 2004 Exclusive Higgs boson production with bottom quarks at hadron colliders Phys. Rev. D 69074027 (Preprint hep-ph/0311067)

[582] Dicus D A and Willenbrock S 1989 Higgs boson production from heavy quark fusion Phys. Rev. D 39751

[583] Dicus D, Stelzer T, Sullivan Z and Willenbrock S 1999 Higgs boson production in association with bottom quarks at next-to-leading order Phys. Rev. D 59094016 (Preprint hep-ph/9811492)

[584] Balazs C, He H-J and Yuan C P 1999 QCD corrections to scalar production via heavy quark fusion at hadron colliders Phys. Rev. D 60114001 (Preprint hep-ph/9812263)

[585] Harlander R V and Kilgore W B 2003 Higgs boson production in bottom quark fusion at next-tonext-to-leading order Phys. Rev. D 68013001 (Preprint hep-ph/0304035)

[586] Aivazis M A G, Olness F I and Tung W-K 1994 Leptoproduction of heavy quarks. 1. General formalism and kinematics of charged current and neutral current production processes Phys. Rev. D 50 3085-3101 (Preprint hep-ph/9312318)

[587] Aivazis M A G, Collins J C, Olness F I and Tung W-K 1994 Leptoproduction of heavy quarks. 2. A unified QCD formulation of charged and neutral current processes from fixed target to collider energies Phys. Rev. D 50 3102-3118 (Preprint hep-ph/9312319)

[588] Campbell J et al 2004 Higgs boson production in association with bottom quarks Preprint hep-ph/0405302 Contributed to 3rd Les Houches Workshop: Physics at TeV Colliders, Les Houches, France, 26 May-6 June 2003

[589] Campbell J, Ellis R K, Maltoni F and Willenbrock S 2003 Higgs boson production in association with a single bottom quark Phys. Rev. D 67095002 (Preprint hep-ph/0204093)

[590] Dawson S, Jackson C B, Reina L and Wackeroth D 2005 Higgs boson production with one bottom quark jet at hadron colliders Phys. Rev. Lett. 94031802 (doi:10.1103/PhysRevLett.94.031802)

[591] Bawa A C, Kim C S and Martin A D 1990 Charged Higgs production at hadron colliders Z. Phys. C 47 75-82 
[592] Borzumati F, Kneur J-L and Polonsky N 1999 Higgs-strahlung and R-parity violating slepton-strahlung at hadron colliders Phys. Rev. D 60115011 (Preprint hep-ph/9905443)

[593] Belyaev A, Garcia D, Guasch J and Sola J 2002 Prospects for heavy supersymmetric charged Higgs boson searches at hadron colliders J. High. Energy Phys. JHEP06(2002)059 (Preprint hep-ph/0203031)

[594] Wu P et al 2006 NLO supersymmetric QCD corrections to the t anti-b H-associated production at hadron colliders Phys. Rev. D 73015012 (Preprint hep-ph/0601069) (doi:10.1103/PhysRevD.73.015012)

[595] Zhu S-H 2003 Complete next-to-leading order QCD corrections to charged Higgs boson associated production with top quark at the CERN large hadron collider Phys. Rev. D 67075006 (doi:10.1103/PhysRevD.67.075006)

[596] Plehn T Charged Higgs boson production in bottom-gluon fusion Prepared for 10th International Conference on Supersymmetry and Unification of Fundamental Interactions (SUSY02), Hamburg, Germany, 17-23 June 2002

[597] Berger E L, Han T, Jiang J and Plehn T 2005 Associated production of a top quark and a charged Higgs boson Phys. Rev. D 71115012 (Preprint hep-ph/0312286)

[598] Gao G, Lu G, Xiong Z and Yang J M 2002 Loop effects and non-decoupling property of SUSY QCD in $g b \rightarrow t H^{-}$Phys. Rev. D 66015007 (doi:10.1103/PhysRevD.66.015007)

[599] Willenbrock S S D 1987 Pair production of supersymmetric charged Higgs bosons Phys. Rev. D 35173

[600] Krause A, Plehn T, Spira M and Zerwas P M 1998 Production of charged Higgs boson pairs in gluon-gluon collisions Nucl. Phys. B 519 85-100 (Preprint hep-ph/9707430)

[601] Jiang Y, Han L, Ma W-G, Yu Z-H and Han M 1997 Pair production of charged Higgs bosons in gluon-gluon collisions J. Phys. G23 385-400 (Preprint hep-ph/9703275)

[602] Brein O and Hollik W 2000 Pair production of charged MSSM Higgs bosons by gluon fusion Eur. Phys. J. C 13 175-184 (Preprint hep-ph/9908529)

[603] Barrientos Bendezu A A and Kniehl B A $2000 \mathrm{H}^{+} \mathrm{H}^{-}$pair production at the large hadron collider $\mathrm{Nucl}$. Phys. B 568 305-318 (doi:10.1016/S0550-3213(99)00732-4)

[604] Hou H-S et al 2005 Pair production of charged Higgs bosons from bottom-quark fusion Phys. Rev. D 71 075014 (doi:10.1103/PhysRevD.71.075014)

[605] Barrientos Bendezu A A and Kniehl B A $1999 W^{ \pm} H^{\mp}$ associated production at the large hadron collider Phys. Rev. D 59015009 (Preprint hep-ph/9807480) (doi:10.1103/PhysRevD.59.015009)

[606] Brein O, Hollik W and Kanemura S 2001 The MSSM prediction for $W^{ \pm} H^{\mp}$ production by gluon fusion Phys. Rev. D 63095001 (Preprint hep-ph/0008308)

[607] Barrientos Bendezu A A and Kniehl B A 2001 Squark loop correction to $W^{ \pm} H^{\mp}$ associated hadroproduction Phys. Rev. D 63015009 (Preprint hep-ph/0007336)

[608] Dicus D, Hewett J, Kao C and Rizzo T G $1989 W^{ \pm} H^{\mp}$ production at hadron colliders Phys. Rev. D 40787

[609] Hollik W and Zhu S-h $2002 \mathcal{O}\left(\alpha_{s}\right)$ corrections to $b \bar{b} \rightarrow W^{ \pm} H^{\mp}$ at the CERN large hadron collider Phys. Rev. D 65075015 (Preprint hep-ph/0109103) (doi:10.1103/PhysRevD.65.075015)

[610] Zhao J, Li C S and Li Q 2005 SUSY-QCD corrections to $W^{ \pm} H^{\mp}$ associated production at the CERN large hadron collider Phys. Rev. D 72114008 (Preprint hep-ph/0509369)

[611] Lehti S 2006 Study of $\mathrm{H} / \mathrm{A} \rightarrow \tau \tau \rightarrow \mathrm{e} \mu+\mathrm{X}$ in CMS CMS Note 2006/101

[612] Lehti S 2002 Study of $g g \rightarrow b \bar{b} H_{S U S Y}, H_{S U S Y} \rightarrow \tau \tau \rightarrow \ell \ell+X$ CMS Note 2002/035

[613] Bloch D private communication

[614] Boos E, Djouadi A, Muhlleitner M and Vologdin A 2002 The MSSM Higgs bosons in the intense-coupling regime Phys. Rev. D 66055004 (Preprint hep-ph/0205160)

[615] Boos E, Djouadi A and Nikitenko A 2004 Detection of the neutral MSSM Higgs bosons in the intense-coupling regime at the LHC Phys. Lett. B 578 384-393 (Preprint hep-ph/0307079)

[616] CMS Collaboration, Acosta D et al 2006 CMS Physics Technical Design Report, Volume 1, Section 12.2.3: Combined secondary vertex tag CERN/LHCC, 2006-001 p 466

[617] Fernandez J 2006 Search for MSSM heavy neutral Higgs bosons in the four-b final state CMS Note 2006/080

[618] CMS Collaboration, Acosta D et al 2006 CMS Physics Technical Design Report, Volume 1, Section 12.2.6: HLT b tag CERN/LHCC, 2006-001 p 474

[619] CMS Collaboration, Acosta D et al 2006 CMS Physics Technical Design Report, Volume 1, Section 11.6.4 Parton-level corrections CERN/LHCC, 2006-001 p 428

[620] DØ Collaboration, Abazov V M et al 2005 Search for neutral supersymmetric Higgs bosons in multijet events at $\sqrt{s}=1.96 \mathrm{TeV}$ Phys. Rev. Lett. 95151801 (doi:10.1103/PhysRevLett.95.151801)

[621] Baarmand M, Hashemi M and Nikitenko A 2006 Light Charged Higgs Discovery Potential of CMS in the $\mathrm{H}^{+} \rightarrow \tau v$ with single lepton trigger CMS Note 2006/056

[622] Roy D 1999 The hadronic tau decay signature of a heavy charged Higgs boson at LHC Phys. Lett. B 459 607-614 (Preprint hep-ph/9905542) 
[623] Kinnunen R 2004 Study of $A / H \rightarrow \tau \tau$ and $H^{ \pm} \rightarrow \tau v$ in CMS Czech. J. Phys. 54 A93-A101

[624] Assamagan K A and Coadou Y 2002 The hadronic tau decay of a heavy $H^{ \pm}$in ATLAS Acta Phys. Polon. B 33 707-720

[625] Baarmand M, Hashemi M and Nikitenko A 2006 Search for the heavy charged MSSM Higgs bosons with the $\mathrm{H}^{+} \rightarrow \tau v$ decay mode in fully hadronic final state CMS Note 2006/100

[626] Roy D P 2004 Looking for the charged Higgs boson Mod. Phys. Lett. A 19 1813-1828 (Preprint hep-ph/0406102)

[627] Alwall J and Rathsman J 2004 Improved description of charged Higgs boson production at hadron colliders J. High Energy Phys. JHEP12(2004)050 (Preprint hep-ph/0409094)

[628] Plehn T 2003 Charged Higgs boson production in bottom gluon fusion Phys. Rev. D 67014018 (Preprint hep-ph/0206121)

[629] Lowette S, D’Hondt J and Vanlaer P 2006 Charged MSSM Higgs boson observability in the $H^{ \pm} \rightarrow t b$ Decay CMS Note 2006/109

[630] CMS Collaboration, Anagnostou G and Daskalakis G 2006 Search for the MSSM A $\rightarrow$ Zh decay with $\mathrm{Z} \rightarrow \ell^{+} \ell^{-}, \mathrm{h} \rightarrow$ bb CMS Note 2006/063

[631] CMS Collaboration, Charlot C, Salerno R and Sirois Y 2006 Observability of the heavy neutral SUSY Higgs Bosons decaying into neutralinos CMS Note 2006/125

[632] Allanach B C et al 2002 The snowmass points and slopes: benchmarks for SUSY searches Eur. Phys. J. C 25 113-123 (Preprint hep-ph/0202233)

[633] Battaglia M et al 2004 Updated post-WMAP benchmarks for supersymmetry Eur. Phys. J. C 33 273-296 (Preprint hep-ph/0306219)

[634] Carena M, Heinemeyer S, Wagner C and Weiglein G 1999 Suggestions for improved benchmark scenarios for Higgs-boson searches at LEP2 Preprint hep-ph/9912223

[635] Carena M, Heinemeyer S, Wagner C and Weiglein G 2003 Suggestions for benchmark scenarios for MSSM Higgs boson searches at hadron colliders Eur. Phys. J. C 26 601-607 (Preprint hep-ph/0202167)

[636] ECFA/DESY LC Physics Working Group Collaboration, Aguilar-Saavedra J A et al 2001 TESLA Technical Design Report Part III: Physics at an e+e- Linear Collider Preprint hep-ph/0106315

[637] Bennett C L et al 2003 First year Wilkinson Microwave Anisotropy Probe (WMAP) observations: preliminary maps and basic results Astrophys. J. Suppl. 1481 (Preprint astro-ph/0302207)

[638] WMAP Collaboration, Spergel D N et al 2003 First year Wilkinson Microwave Anisotropy Probe (WMAP) observations: determination of cosmological parameters Astrophys. J. Suppl. 148175 (Preprint astro-ph/0302209)

[639] Goldberg H 1983 Constraint on the photino mass from cosmology Phys. Rev. Lett. 501419

[640] Ellis J R, Hagelin J, Nanopoulos D, Olive K A and Srednicki M 1984 Supersymmetric relics from the big bang Nucl. Phys. B 238 453-476

[641] Heavy Flavor Averaging Group Collaboration, Barberio E et al 2006 Averages of b-hadron properties at the end of 2005 Preprint hep-ex/0603003

[642] Asatrian H M, Hovhannisyan A, Poghosyan V, Greub C and Hurth T 2005 Towards the NNLL precision in anti-B $\rightarrow$ X/s gamma Preprint hep-ph/0512097

[643] Muon Collaboration, Bennett G W 2006 Final report of the muon E821 anomalous magnetic moment measurement at BNL Preprint hep-ex/0602035

[644] Czarnecki A and Marciano W J 2001 The muon anomalous magnetic moment: A harbinger for 'new physics' Phys. Rev. D 64013014 (Preprint hep-ph/0102122)

[645] Carena $\mathrm{M}$ et al 2000 Reconciling the two-loop diagrammatic and effective field theory computations of the mass of the lightest CP-even Higgs boson in the MSSM Nucl. Phys. B 580 29-57 (Preprint hep-ph/0001002)

[646] Randall L and Sundrum R 1999 An alternative to compactification Phys. Rev. Lett. 83 4690-4693 (doi:10.1103/PhysRevLett.83.4690)

[647] Giudice G F, Rattazzi R and Wells J D 2001 Graviscalars from higher-dimensional metrics and curvature- Higgs mixing Nucl. Phys. B 595 250-276 (Preprint hep-ph/0002178)

[648] Chaichian M, Datta A, Huitu K and Yu Z-h 2002 Radion and Higgs mixing at the LHC Phys. Lett. B 524 161-169 (Preprint hep-ph/0110035)

[649] Hewett J L and Rizzo T G 2003 Shifts in the properties of the Higgs boson from radion mixing J. High. Energy Phys. JHEP08(2003)028 (Preprint hep-ph/0202155)

[650] Dominici D, Grzadkowski B, Gunion J F and Toharia M 2003 The scalar sector of the Randall-Sundrum model Nucl. Phys. B 671 243-292 (Preprint hep-ph/0206192)

[651] Battaglia M, De Curtis S, De Roeck A, Dominici D and Gunion J F 2003 On the complementarity of Higgs and radion searches at LHC Phys. Lett. B 568 92-102 (Preprint hep-ph/0304245) 
[652] Azuelos G, Cavalli D, Przysiezniak H and Vacavant L 2002 Search for the radion using the ATLAS detector Eur. Phys. J. direct C 416

[653] Dominici D, Dewhirst G, Nikitenko A, Gennai S and Fanò L 2005 Search for radion decays into Higgs boson pairs in the $\gamma \gamma \mathrm{b} \bar{b}, \tau \tau \mathrm{b} \overline{\mathrm{b}}$ and $\mathrm{b} \overline{\mathrm{b}} \mathrm{b} \overline{\mathrm{b}}$ final states CMS Note $2005 / 007$

[654] Arkani-Hamed N, Cohen A G and Georgi H 2001 (De)constructing dimensions Phys. Rev. Lett. 86 4757-4761 (Preprint hep-th/0104005)

[655] Cheng H-C, Hill C T, Pokorski S and Wang J 2001 The standard model in the latticized bulk Phys. Rev. D 64 065007 (Preprint hep-th/0104179)

[656] Arkani-Hamed N, Cohen A G and Georgi H 2001 Electroweak symmetry breaking from dimensional deconstruction Phys. Lett. B 513 232-240 (Preprint hep-ph/0105239)

[657] Arkani-Hamed N, Cohen A G, Katz E and Nelson A E 2002 The littlest Higgs J. High Energy Phys. JHEP07(2002)034 (Preprint hep-ph/0206021)

[658] Schechter J and Valle J 1980 Neutrino masses in $S U(2) \times U(1)$ Theories Phys. Rev. D 222227 (doi:10.1103/PhysRevD.22.2227)

[659] Ma E and Sarkar U 1998 Neutrino masses and leptogenesis with heavy Higgs triplets Phys. Rev. Lett. 80 5716-5719 (Preprint hep-ph/9802445)

[660] Ma E, Raidal M and Sarkar U 2000 Verifiable model of neutrino masses from large extra dimensions Phys. Rev. Lett. 85 3769-3772 (Preprint hep-ph/0006046)

[661] Ma E, Raidal M and Sarkar U 2001 Phenomenology of the neutrino-mass-giving Higgs triplet and the low-energy seesaw violation of lepton number Nucl. Phys. B 615 313-330 (Preprint hep-ph/0012101)

[662] Ma E and Sarkar U 2006 Connecting dark energy to neutrinos with an observable Higgs triplet Preprint hep-ph/0602116

[663] Marandella G, Schappacher C and Strumia A 2005 Little-Higgs corrections to precision data after LEP2 Phys. Rev. D 72035014 (Preprint hep-ph/0502096)

[664] Huitu K, Maalampi J, Pietila A and Raidal M 1997 Doubly charged Higgs at LHC Nucl. Phys. B 487 27-42 (Preprint hep-ph/9606311)

[665] Han T, Logan H E and Wang L-T 2005 Smoking-gun signatures of little Higgs models Preprint hep-ph/0506313

[666] Azuelos G et al 2005 Exploring little Higgs models with ATLAS at the LHC Eur. Phys. J. C 39S2 13-24 (Preprint hep-ph/0402037)

[667] Gunion J F, Grifols J, Mendez A, Kayser B and Olness F I 1989 Higgs bosons in left-right symmetric models Phys. Rev. D 401546 (doi:10.1103/PhysRevD.40.1546)

[668] Muhlleitner M and Spira M 2003 A note on doubly-charged Higgs pair production at hadron colliders Phys. Rev. D 68117701 (Preprint hep-ph/0305288)

[669] Bityukov S, Krasnikov N and Taperechkina V 2001 Confidence intervals for Poisson distribution parameter Preprint hep-ex/0108020

[670] Martin S P 1997 A supersymmetry primer Perspectives in Supersymmetry ed G. Kane (World Scientific) Preprint hep-ph/9709356

[671] CMS Collaboration Abdullin S et al 2002 Discovery potential for supersymmetry in CMS J. Phys. G 28469 (doi:10.1088/0954-3899/28/3/401)

[672] Paige F E, Protopopescu S D, Baer H and Tata X 2003 ISAJET 7.69: A Monte Carlo Event Generator for $p p$, $\bar{p} p$, and $e^{+} e^{-}$Reactions (Preprint hep-ph/0312045)

[673] De Roeck A et al 2005 Supersymmetric benchmarks with non-universal scalar masses or gravitino dark matter Preprint hep-ph/0508198

[674] de Boer W et al 2004 Excess of EGRET galactic gamma ray data interpreted as dark matter annihilation Preprint astro-ph/0408272

[675] Acosta D et al 2006 Potential to Discover SUSY in Events with Muons Jets and Large Missing Transverse Energy in pp Collisions at $\sqrt{s}=14 \mathrm{TeV}$ CMS Note 2006/134

[676] Acosta D et al 2006 CMS Discovery Potential for mSUGRA in Same Sign Di-muon Events with Jets and Large Missing Transverse Energy in pp collisions at $\sqrt{s}=14$ TeVCMS Note

[677] Chiorboli M, Galanti M and Tricomi A 2006 Leptons + Jets + Missing Energy analysis at LM1 CMS Note 2006/133

[678] Mangeol D and Goerlach U 2006 Search for $\tilde{\tau}$ production in di-tau final states and measurements of SUSY masses in mSUGRA cascade decays CMS Note 2006/096

[679] Bityukov S I 2005 Uncertainty, Systematics, Limits Available at http://cmsdoc.cern.ch/bityukov

[680] Kyriazopoulou S and Markou C 2006 Search for SUSY in Final States with Z Bosons CMS Note 2006/116

[681] Nikitenko A, Quast G and Ciulli V Minimal requirements for significance estimates of the physics resultInfo located at http://cmsdoc.cern.ch/ anikiten/cms-higgs/stat.txt 
[682] Beenakker W, Hopker R and Spira M 1996 PROSPINO: A program for the PROduction of Supersymmetric Particles In Next-to-leading Order QCD Preprint hep-ph/9611232

[683] Allanach B C, Lester C G, Parker M A and Webber B R 2000 Measuring sparticle masses in non-universal string inspired models at the LHC J. High. Energy Phys. JHEP09(2000)004 (Preprint hep-ph/0007009)

[684] Beenakker W et al 1999 The production of charginos/neutralinos and sleptons at hadron colliders Phys. Rev. Lett. 83 3780-3783 (doi:10.1103/PhysRevLett.83.3780)

[685] del Aguila F and Ametller L 1991 On the detectability of sleptons at large hadron colliders Phys. Lett. B 261 326-333 (doi:10.1016/0370-2693(91)90336-O)

[686] Baer H, Chen C-h, Paige F and Tata X 1994 Detecting sleptons at hadron colliders and supercolliders Phys. Rev. D 49 3283-3290 (Preprint hep-ph/9311248)

[687] Denegri D, Rurua L and Stepanov N 1996 Detection of Sleptons in CMS, Mass Reach CMS TN 96-059

[688] Andreev Y M, Bityukov S I and Krasnikov N V 2005 Sleptons at post-WMAP benchmark points at LHC(CMS) Phys. Atom. Nucl. 68 340-347 (Preprint hep-ph/0402229)

[689] Bityukov S I and Krasnikov N V 1999 The search for sleptons and lepton-flavor-number violation at LHC (CMS) Phys. Atom. Nucl. 62 1213-1225 (Preprint hep-ph/9712358)

[690] Krasnikov N 1994 Flavor lepton number violation at LEP-2 Mod. Phys. Lett. A 9 791-794

[691] Arkani-Hamed N, Cheng H-C, Feng J L and Hall L J 1996 Probing lepton flavor violation at future colliders Phys. Rev. Lett. 77 1937-1940 (Preprint hep-ph/9603431) (doi:10.1103/PhysRevLett.77.1937)

[692] Krasnikov N V 1997 Search for flavor lepton number violation in slepton decays at LHC JETP Lett. 65148 (doi:10.1134/1.567315)

[693] Agashe K and Graesser M 2000 Signals of supersymmetric lepton flavor violation at the LHC Phys. Rev. D 61075008 (Preprint hep-ph/9904422)

[694] Hisano J, Kitano R and Nojiri M M 2002 Flavor mixing in slepton production at the large hadron collider Phys. Rev. D 65116002

[695] Baer H, Chen C-h, Paige F and Tata X 1994 Trileptons from chargino - neutralino production at the CERN Large Hadron Collider Phys. Rev. D 50 4508-4516 (Preprint hep-ph/9404212) (doi:10.1103/PhysRevD.50.4508)

[696] Ellis J R, Olive K A and Santoso Y 2002 The MSSM parameter space with non-universal Higgs masses Phys. Lett. B 539 107-118 (Preprint hep-ph/0204192)

[697] Altarelli G, Mele B and Ruiz-Altaba M 1989 Searching for new heavy vector bosons in $p \bar{p}$ colliders Z. Phys. C 45109 (doi:10.1007/BF01556677)

[698] Arkani-Hamed N, Dimopoulos S and Dvali G R 1998 The hierarchy problem and new dimensions at a millimeter Phys. Lett. B 429 263-272 (Preprint hep-ph/9803315)

[699] Lykken J D, Poppitz E and Trivedi S P 1999 Branes with GUTs and supersymmetry breaking Nucl. Phys. B 543 105-121 (Preprint hep-th/9806080)

[700] Antoniadis I 1990 A possible new dimension at a few TeV Phys. Lett. B 246 377-384 (doi:10.1016/0370-2693(90)90617-F)

[701] Lykken J D 1996 Weak scale superstrings Phys. Rev. D 54 3693-3697 (Preprint hep-th/9603133) (doi:10.1103/PhysRevD.54.R3693)

[702] Giudice G F, Rattazzi R and Wells J D 1999 Quantum gravity and extra dimensions at high-energy colliders Nucl. Phys. B 544 3-38 (Preprint hep-ph/9811291)

[703] Han T, Lykken J D and Zhang R-J 1999 On Kaluza-Klein states from large extra dimensions Phys. Rev. D 59 105006 (Preprint hep-ph/9811350) (doi:10.1103/PhysRevD.59.105006)

[704] Cullen S, Perelstein M and Peskin M E $2000 \mathrm{TeV}$ strings and collider probes of large extra dimensions Phys. Rev. D 62055012 (Preprint hep-ph/0001166)

[705] Lykken J D and Randall L 2000 The shape of gravity J. High. Energy Phys. JHEP06(2000)014 (Preprint hep-th/9908076)

[706] Vacavant L and Hinchliffe I 2001 Signals of models with large extra dimensions in ATLAS J. Phys. G 27 $1839-1850$

[707] Ruppert J, Rahmede C and Bleicher M 2005 Determination of the fundamental scale of gravity and the number of space-time dimensions from high energetic particle interactions Phys. Lett. B 608 240-243 (Preprint hep-ph/0501028)

[708] Dudas E and Mourad J 2000 String theory predictions for future accelerators Nucl. Phys. B 575 3-34 (doi:10.1016/S0550-3213(00)00082-1)

[709] Chialva D, Iengo R and Russo J G 2005 Cross sections for production of closed superstrings at high energy colliders in brane world models Phys. Rev. D 71106009 (Preprint hep-ph/0503125)

[710] Bando M, Kugo T, Noguchi T and Yoshioka K 1999 Brane fluctuation and suppression of Kaluza-Klein mode couplings Phys. Rev. Lett. 83 3601-3604 (doi:10.1103/PhysRevLett.83.3601) 
[711] Hewett J L 1999 Indirect collider signals for extra dimensions Phys. Rev. Lett. 82 4765-4768 (doi:10.1103/PhysRevLett.82.4765)

[712] Davoudiasl H, Hewett J L and Rizzo T G 2000 Phenomenology of the Randall-Sundrum gauge hierarchy model Phys. Rev. Lett. 842080 (doi:10.1103/PhysRevLett.84.2080)

[713] Hewett J and Spiropulu M 2002 Particle physics probes of extra spacetime dimensions Ann. Rev. Nucl. Part. Sci. 52 397-424 (doi:10.1146/annurev.nucl.52.050102.090706)

[714] Clerbaux B, Mahmoud T, Collard C and Miné P 2006 Search with the CMS detector for heavy resonances decaying into an electron pair CMS Note 2006/083

[715] Collard C and Lemaire M-C 2004 Search with the CMS Detector for Randall-Sundrum Excitations of Gravitons Decaying Into Electron Pairs CMS Note 2004/024

[716] CMS Collaboration, Acosta D et al 2006 CMS Physics Technical Design Report Volume 1, Section 10 CERN/LHCC, 2006-001

[717] Clerbaux B, Mahmoud T, Collard C, Lemaire M-C and Litvin V 2006 TeV electron and photon saturation studies CMS Note 2006/004

[718] CDF Collaboration, Affolder T et al 2001 Measurement of ds/dM and Forward-Backward charge asymmetry for high-mass Drell-Yan $\mathrm{e}^{+} \mathrm{e}^{-}$pairs from collisions at $\sqrt{s}=1.8 \mathrm{TeV}$ Phys. Rev. Lett. 87131802

[719] CTEQ Collaboration, Lai H L et al 2000 Global QCD analysis of parton structure of the nucleon: CTEQ5 parton distributions Eur. Phys. J. C 12 375-392 (doi:10.1007/s100529900196)

[720] CTEQ Collaboration, Stump D et al 2003 Inclusive jet production, parton distributions, and the search for new physics J. High. Energy Phys. JHEP0310(2003)046 (Preprint hep-ph/0303013)

[721] Davoudiasl H, Hewett J L and Rizzo T G 2001 Experimental probes of localized gravity: on and off the wall Phys. Rev. D 63075004 (Preprint hep-ph/0006041)

[722] Cheung K-m and Landsberg G 2000 Drell-Yan and diphoton production at hadron colliders and low scale gravity model Phys. Rev. D 62076003 (Preprint hep-ph/9909218)

[723] Belotelov I, Golutvin I, Bourilkov D, Lanyov A, Rogalev E, Savina M and Shmatov S 2006 Search for ADD extra dimensional gravity in di-muon channel with the CMS Detector CMS Note 2006/076

[724] Mohapatra R and Pati J 1975 Left-right gauge symmetry and an 'isoconjugate' model of CP violation Phys. Rev. D 11 566-571 (doi:10.1103/PhysRevD.11.566)

[725] Senjanovic G and Mohapatra R 1975 Exact left-right symmetry and spontaneous violation of parity Phys. Rev. D 121502 (doi:10.1103/PhysRevD.12.1502)

[726] Mohapatra R and Senjanovic G 1980 Neutrino mass and spontaneous parity nonconservation Phys. Rev. Lett. 44912 (doi:10.1103/PhysRevLett.44.912)

[727] Pati J and Salam A 1974 Lepton number as the fourth color Phys. Rev. D 10 275-289

[728] CDF Collaboration, Affolder T et al 2001 Search for quark lepton compositeness and a heavy W' boson using the e nu channel in p anti-p collisions at s** $(1 / 2)=1.8-\mathrm{TeV}$ Phys. Rev. Lett. 87231803 (Preprint hep-ex/0107008)

[729] Hof C, Hebbeker T and Hoepfner K 2006 Detection of New Heavy Charged Gauge Bosons in the Muon Plus Neutrino Channel CMS Note 2006/117

[730] Gumus K, Akchurin N, Esen S and Harris R M 2006 CMS sensitivity to dijet resonances CMS Note 2006/070

[731] Baur U, Hinchliffe I and Zeppenfeld D 1987 Excited quark production at Hadron colliders Int. J. Mod. Phys. A 21285

[732] Bagger J, Schmidt C and King S 1988 Axigluon production in hadronic collisions Phys. Rev. D 371188

[733] Chivukula R S, Cohen A G and Simmons E H 1996 New strong interactions at the Tevatron? Phys. Lett. B 380 92-98 (Preprint hep-ph/9603311)

[734] Hewett J L and Rizzo T G 1989 Low-energy phenomenology of superstring inspired E(6) models Phys. Rept. 183193

[735] Lane K and Mrenna S 2003 The collider phenomenology of technihadrons in the technicolor Straw man model Phys. Rev. D 67115011 (doi:10.1103/PhysRevD.67.115011)

[736] Eichten E, Hinchliffe I, Lane K D and Quigg C 1984 Super collider physics Rev. Mod. Phys. 56 579-707

[737] CMS Collaboration, Lemaire M-C, Newman H and Litvin V 2006 Search for Randall-Sundrum excitations of gravitons decaying into two photons for CMS at LHC CMS Note 2006/051

[738] CMS Collaboration, Clerbaux B, Mahmoud T, Collard C, Lemaire M-C and Litvin V 2006 TeV electron and photon saturation studies CMS Note 2006/004

[739] Acosta D et al 2005 Measurement of the Cross Section for Prompt Diphoton Production in $p \bar{p}$ Collisions at $\sqrt{s}=1.96$ TeV Phys. Rev. Lett. 95022003 (doi:10.1103/PhysRevLett.95.022003)

[740] Weng J et al 2006 Search for ADD Direct Graviton Emission in Photon plus Missing Transverse Energy Final States at CMS CMS Note 2006/129

[741] Myers R C and Perry M J 1986 Black holes in higher dimensional space-times Ann. Phys. 172304 
[742] Hawking S W 1975 Particle creation by black holes Commun. Math. Phys. 43 199-220

[743] Argyres P C, Dimopoulos S and March-Russell J 1998 Black holes and sub-millimeter dimensions Phys. Lett. B 441 96-104 (Preprint hep-th/9808138)

[744] Harris C M, Richardson P and Webber B R 2003 CHARYBDIS: A black hole event generator J. High. Energy Phys. JHEP08(2003)033 (Preprint hep-ph/0307305)

[745] Mathews P, Ravindran V and Sridhar K 2005 NLO-QCD corrections to dilepton production in the RandallSundrum model J. High. Energy Phys. JHEP10(2005)031 (Preprint hep-ph/0506158)

[746] Lane K D 2000 Technicolor 2000 Preprint hep-ph/0007304

[747] Kreuzer P 2006 Search for Technicolour at CMS in the $\rho_{\mathrm{TC}} \rightarrow W Z$ channel CMS Note 2006/135

[748] Betev B, Bourilkov D and Mavrodiev S. Shch Structure functions of pion and nucleon determined from high mass muon pair production JINR-E2-85-312

[749] Super-Kamiokande Collaboration, Fukuda Y et al 1998 Evidence for oscillation of atmospheric neutrinos Phys. Rev. Lett. 81 1562-1567 (Preprint hep-ex/9807003)

[750] Esen S and Harris R 2006 CMS sensitivity to quark contact interactions using dijets CMS Note 2006/071

[751] Pati J C and Salam A 1975 Anomalous lepton-hadron interactions and gauge models Phys. Rev. D 11 1137-1154 (doi:10.1103/PhysRevD.11.1137)

[752] CMS Collaboration, Gninenko N, Kirsanov M, Krasnikov N and Matveev N 2006 Detection of Heavy Majorana Neutrinos and Right-Handed Bosons CMS Note 2006/098

[753] Particle Data Group, Hagiwara K et al 2002 Review of Particle Physics Phys. Rev. D 66010001

[754] Barenboim G, Bernabeu J, Prades J and Raidal M 1997 Constraints on the W(R) mass and CP-violation in left-right models Phys. Rev. D 55 4213-4221 (Preprint hep-ph/9611347)

[755] DØ Collaboration, Abachi S et al 1996 Search for right-handed $W$ bosons and heavy $W^{\prime}$ in $p \bar{p}$ collisions at $\sqrt{s}=1.8$ TeV Phys. Rev. Lett. 76 3271-3276 (Preprint hep-ex/9512007)

[756] Datta A, Guchait M and Roy D P 1993 Prospect of heavy right-handed neutrino search at SSC/LHC energies Phys. Rev. D 47 961-966 (Preprint hep-ph/9208228)

[757] CMS Collaboration, Karafasoulis K, Kyriakis A, Petrakou H and Mazumdar K 2006 Little Higgs model and top-like heavy quark at CMS CMS Note 2006/079

[758] D'Hondt J, Lowette S, Hammad G, Heyninck J and Van Mulders P 2006 Observability of same-charge lepton topology in di-leptonic ttbar events CMS Note 2006/065

[759] Larios F and Penunuri F 2004 FCNC production of same sign top quark pairs at the LHC J. Phys. G 30 895-904 (Preprint hep-ph/0311056)

[760] Gouz Y P and Slabospitsky S R 1999 Double top production at hadronic colliders Phys. Lett. B 457 177-185 (doi:10.1016/S0370-2693(99)00516-X)

[761] Yue C-X, Zong Z-J, Xu L-L and Chen J-X 2006 Associated production of the top-pions and single top at hadron colliders Phys. Rev. D 73015006 (Preprint hep-ph/0601058)

[762] Kraml S and Raklev A R 2006 Same-sign top quarks as signature of light stops at the LHC Phys. Rev. D 73 075002 (Preprint hep-ph/0512284)

[763] Bityukov S I and Krasnikov N V 2000 On the observability of a signal above background Nucl. Instrum. and Methods A 452 518-524 (doi:10.1016/S0168-9002(00)00454-X)

[764] Lehmann E L 1957 A theory of some multiple decision problems The Annals of Mathematical Statistics 281

[765] O'Neill R and Wetherill G B 1971 The present state of multiple comparison methods Journal of the Royal Statistical Society, Series B (Methodological) 332

[766] Hauser $\mathbf{J} 2006$ Search for new physics in tails of distributionsPrivate communications, paper in preparation

[767] DØ Collaboration, Abazov V M et al 2005 Measurement of the $t \bar{t}$ production cross section in $p \bar{p}$ collisions at $\sqrt{s}=1.96 \mathrm{TeV}$ using kinematic characteristics of lepton + jets events Phys. Lett. B $62645-54$ (Preprint hep-ex/0504043) (doi:10.1016/j.physletb.2005.08.104)

[768] Abbott B et al 2000 Search for New physics in $e \mu X$ data at DØ Using Sleuth: a quasi-model-independent search strategy for new physics Phys. Rev. D 62 (doi:10.1103/PhysRevD.62.092004)

[769] Abbott B et al 2001 A Quasi-Model-Independent search for new physics at large transverse momentum Phys. Rev. D 64 (Preprint hep-ex/0011067)

[770] Finley C et al 2004 On the evidence for clustering in the arrival directions of AGASA's ultrahigh energy cosmic rays Astroparticle Physics 21

[771] Frixione S, Nason P and Webber B R 2003 Matching NLO QCD and parton showers in heavy flavour production J. High. Energy Phys. JHEP08(2003)007 (Preprint hep-ph/0305252)

[772] Lonnblad L 2002 Correcting the colour-dipole cascade model with fixed order matrix elements J. High. Energy Phys. JHEP05(2002)046 (Preprint hep-ph/0112284)

[773] Frixione S and Webber B R 2002 Matching NLO QCD computations and parton shower simulations J. High. Energy Phys. JHEP06(2002)029 (Preprint hep-ph/0204244) 
[774] Krauss F 2002 Matrix elements and parton showers in hadronic interactions J. High. Energy Phys. JHEP08(2002)015 (Preprint hep-ph/0205283)

[775] Mrenna S and Richardson P 2004 Matching matrix elements and parton showers with HERWIG and PYTHIA J. High. Energy Phys. JHEP05(2004)040 (Preprint hep-ph/0312274)

[776] Butterworth J, Butterworth S, Waugh B, Stirling W and Whalley M 2004 The CEDAR project Preprint hep-ph/0412139

[777] LEP Collaboration, 2004 A combination of preliminary electroweak measurements and constraints on the standard model Preprint hep-ex/0412015

[778] Stump D R 2002 A new generation of CTEQ parton distribution functions with uncertainty analysis Prepared for 31st International Conference on High Energy Physics (ICHEP 2002), (Amsterdam, The Netherlands, 24-31 July 2002 )

[779] Stirling W J, Martin A D, Roberts R G and Thorne R S 2005 MRST parton distributions AIP Conf. Proc. 747 $16-21$

[780] Botje M 2000 A QCD analysis of HERA and fixed target structure function data Eur. Phys. J. C 14 285-297 (Preprint hep-ph/9912439)

[781] Alekhin S I 2001 Global fit to the charged leptons DIS data: alpha(s), parton distributions, and high twists Phys. Rev. D 63094022 (Preprint hep-ph/0011002)

[782] Altarelli G and Parisi G 1977 Asymptotic freedom in parton language Nucl. Phys. B 126298

[783] Lonnblad L 1992 ARIADNE version 4: A program for simulation of QCD cascades implementing the color dipole model Comput. Phys. Commun. 71 15-31

[784] CDF Collaboration, Abe F et al 1994 Evidence for color coherence in $p \bar{p}$ collisions at $\sqrt{s}=1.8 \mathrm{TeV}$ Phys Rev. D 50 5562-5579 (doi:10.1103/PhysRevD.50.5562)

[785] DØ Collaboration, Abbott B et al 1997 Color coherent radiation in multijet events from $p \bar{p}$ collisions at $\sqrt{s}=1.8$ TeV Phys. Lett. B 414 419-427 (doi:10.1016/S0370-2693(97)01190-8)

[786] Knowles I G et al 1995 QCD event generators Preprint hep-ph/9601212

[787] ALEPH Collaboration, Buskulic D et al 1992 Properties of hadronic Z decays and test of QCD generators Z. Phys. C 55 209-234

[788] DELPHI Collaboration, Abreu P et al 1996 Tuning and test of fragmentation models based on identified particles and precision event shape data Z. Phys. C 73 11-60 (doi:10.1007/s002880050295)

[789] OPAL Collaboration, Alexander G et al 1996 A Comparison of b and (u d s) quark jets to gluon jets Z. Phys. C $69543-560$

[790] Andersson B, Gustafson G and Soderberg B 1983 A general model for jet fragmentation Z. Phys. C 20317

[791] ALEPH Collaboration, Heister A et al 2001 Study of the fragmentation of b quarks into B mesons at the Z peak Phys. Lett. B 512 30-48 (Preprint hep-ex/0106051)

[792] OPAL Collaboration, Abbiendi G et al 2003 Inclusive analysis of the b quark fragmentation function in $\mathrm{Z}$ decays at LEP ((B)) Eur. Phys. J. C 29 463-478 (Preprint hep-ex/0210031)

[793] SLD Collaboration, Abe K et al 2002 Measurement of the b-quark fragmentation function in $Z^{0}$ decays Phys. Rev. D 65092006 (doi:10.1103/PhysRevD.65.092006)

[794] Bowler M G 1981 e+ e- Production of heavy quarks in the String Model Zeit. Phys. C 11169

[795] Peterson C, Schlatter D, Schmitt I and Zerwas P M 1983 Scaling violations in inclusive $e^{+} e^{-}$annihilation spectra Phys. Rev. D 27105

[796] Kartvelishvili V, Likhoded A and Petrov V 1978 On the fragmentation functions of heavy quarks into hadrons Phys. Lett. B 78615

[797] Norrbin E and Sjostrand T 2000 Production and hadronization of heavy quarks Eur. Phys. J. C 17 137-161 (Preprint hep-ph/0005110)

[798] Engel R and Ranft J 1999 Color singlet exchange between jets and the PHOJET Monte Carlo Nucl. Phys. Proc. Suppl. A 75 272-274 (doi:10.1016/S0920-5632(99)00263-7)

[799] CDF Collaboration, Affolder T et al 2002 Charged jet evolution and the underlying event in proton anti-proton collisions at 1.8 TeV Phys. Rev. D 65092009 (doi:10.1103/PhysRevD.65.092009)

[800] Nason P et al 1999 Bottom production Preprint hep-ph/0003142

[801] Sjostrand T and Skands P Z 2005 Transverse-momentum-ordered showers and interleaved multiple interactions Eur. Phys. J. C 39 129-154 (Preprint hep-ph/0408302) (doi:10.1140/epjc/s2004-02084-y)

[802] Sapeta S and Golec-Biernat K 2005 Total, elastic and diffractive cross sections at LHC in the Miettinen-Pumplin model Phys. Lett. B 613 154-161 (Preprint hep-ph/0502229)

[803] Lange D J 2001 The EvtGen particle decay simulation package Nucl. Instrum. Methods A 462 152-155 
[804] Bartalini P 2005 Supporting Monte Carlo Generators at the LHC Proceedings of the 10th International Conference on B-Physics at Hadron Machines (Assisi Perugia, Italy June, 2005) ed M Biasini and S Erhan volume 156, Published in Nucl. Phys B - Proceedings Supplements 1 (doi:10.1016/j.nuclphysbps.2006.02.123)

[805] Sullivan Z 2002 Fully differential W production and decay at next-to- leading order in QCD Phys. Rev. D 66 075011 (Preprint hep-ph/0207290)

[806] Dobbs M A et al 2004 Les Houches guidebook to Monte Carlo generators for hadron collider physics Preprint hep-ph/0403045

[807] HIJING Web Site Located at http://www-nsdth.lbl.gov/ xnwang/hijing/index.html

[808] Gleisberg T et al 2005 Event generator for the LHC Preprint hep-ph/0508315

[809] Boos E et al 2001 Generic user process interface for event generators Preprint hep-ph/0109068

[810] Lokhtin I P and Snigirev A M 2000 Nuclear geometry of jet quenching Eur. Phys. J. C 16 527-536 (Preprint hep-ph/0004176) (doi:10.1007/s100520000437)

[811] Lokhtin I P and Snigirev A M 2004 Fast simulation of jet quenching in ultrarelativistic heavy ion collisions SINP MSU-13/752 Preprint hep-ph/0406038

[812] Lokhtin I P and Snigirev A M 2003 Fast simulation of flow effects in central and semi-central heavy ion collisions at LHC Preprint hep-ph/0312204

[813] CDF Collaboration, Field R D 2001 The underlying event in hard scattering processes eConf C 010630 P501 (Preprint hep-ph/0201192)

[814] CompHEP Collaboration, Boos E, Bunichev V, Dubinin M, Dudko L, Edneral V, Ilyin V, Kryukov A, Savrin V, Semenov A and Sherstnev A 2004 Web site at http://theory.sinp.msu.ru/comphep. See also [355]

[815] Lokhtin I P, Sarycheva L I and Snigirev A M 2002 The method for analysing jet azimuthal anisotropy in ultrarelativistic heavy ion collisions Phys. Lett. B 537 261-267 (Preprint hep-ph/0203144) (doi:10.1016/S0370-2693(02)01913-5)

[816] Carena M, Daleo A, Dobrescu B A and Tait T M P $2004 Z^{\prime}$ gauge bosons at the Tevatron Phys. Rev. D 70 093009 (doi:10.1103/PhysRevD.70.093009)

[817] Balazs C, Qiu J-w and Yuan C P 1995 Effects of QCD resummation on distributions of leptons from the decay of electroweak vector bosons Phys. Lett. B 355 548-554 (doi:10.1016/0370-2693(95)00726-2)

[818] Balazs C and Yuan C P 1997 Soft gluon effects on lepton pairs at hadron colliders Phys. Rev. D 56 5558-5583 (doi:10.1103/PhysRevD.56.5558)

[819] Martin A D, Roberts R G, Stirling W J and Thorne R S 2003 Uncertainties of predictions from parton distributions I: Experimental errors Eur. Phys. J. C 28 455-473 (Preprint hep-ph/0211080)

[820] Holland J H 1975 Adaptation in natural and artificial systems (The University of Michigan Press, Ann Arbor)

[821] Goldberg D E 1989 Genetic algorithms in search, optimization and machine learning (Addison: Wesley)

[822] Abdullin S 2003 Genetic algorithm for SUSY trigger optimization in CMS detector at LHC NIM A 502 693-695 (doi:10.1016/S0168-9002(03)00546-1)

[823] TOTEM Collaboration, 2004 TOTEM Technical Design Report CERN/LHCC, 2004-002

[824] TOTEM Collaboration, 2004 Addendum to the TOTEM-TDR CERN/LHCC, 2004-020

[825] Avati V and Österberg K 2005 TOTEM forward measurements: leading proton acceptance Proceedings of the HERA-LHC Workshop (CERN/DESY: January, 2005) Available at http://www.desy.de/ heralhc/proceedings/wg4avati.pdf

[826] Kalliopuska J et al 2005 TOTEM forward measurements: exclusive central diffraction Proceedings of the HERA-LHC Workshop (CERN/DESY: January, 2005) Available at http://www.desy.de/ heralhc/proceedings/wg4pXp_heralhc.pdf

[827] The MAD-X Program, Methodical Accelerator Design Information available at http://www.cern.ch/mad

[828] Arneodo M et al 2005 Diffractive Higgs: CMS/TOTEM Level-1 Trigger Studies Proceedings of the HERA-LHC Workshop (CERN/DESY: January, 2005) Available at http://www.desy.de/ heralhc/proceedings/wg4arneodo.pdf

[829] Croft R 2006 (In preparation) PhD Thesis, University of Bristol

[830] Oljemark F 2006 First level triggering of diffractively produced low-mass Higgs at The Large Hadron Collider, University of Helsinki

[831] Bruni G et al 2005 Leading proton production in ep and pp, experiments: how well do high-energy physics Monte Carlos reproduce the data? Proceedings of the HERA-LHC Workshop (CERN/DESY: January, 2005) Available at http://www.desy.de/ heralhc/proceedings/wg5lps.pdf

[832] Ferro F 2005 Diffractive Higgs in CMS/TOTEM: study of L1 trigger conditions from T1 and T2 TOTEM Note 04-2005

[833] Cox B and Forshaw J 2002 Herwig for diffractive interactions Comput. Phys. Commun. 144 104-110 (doi:10.1016/S0010-4655(01)00467-2) 
[834] Collins J C 1997 Light-cone variables, rapidity and all that Preprint hep-ph/9705393

[835] Albrow M G and Rostovtsev A 2000 Searching for the Higgs at hadron colliders using the missing mass method Preprint hep-ph/0009336

[836] Albrow M G 2005 Double pomeron physics at the LHC AIP Conf. Proc. 792 509-514 (Preprint hep-ex/0507095) 


\section{Colour plates CP1-CP9}

Various figures are in colour throughout the online edition but only plates CP1-CP9 are in colour in both the print and online editions.

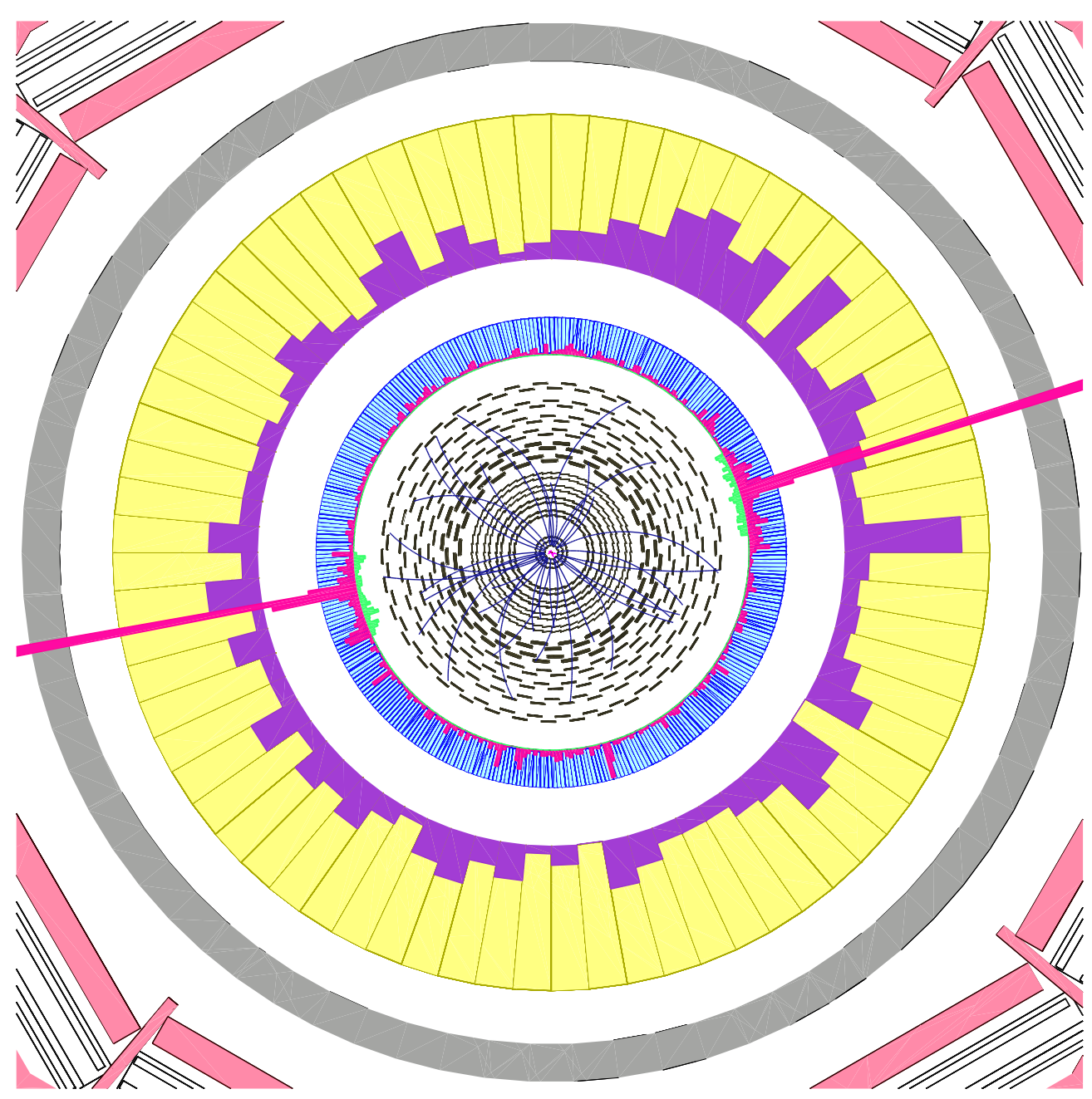

Figure CP1. Example of a $\mathrm{pp} \rightarrow \mathrm{H}+\mathrm{X}$ event with Higgs particle decay $\mathrm{H} \rightarrow \gamma \gamma$. (See section 2.1.) 


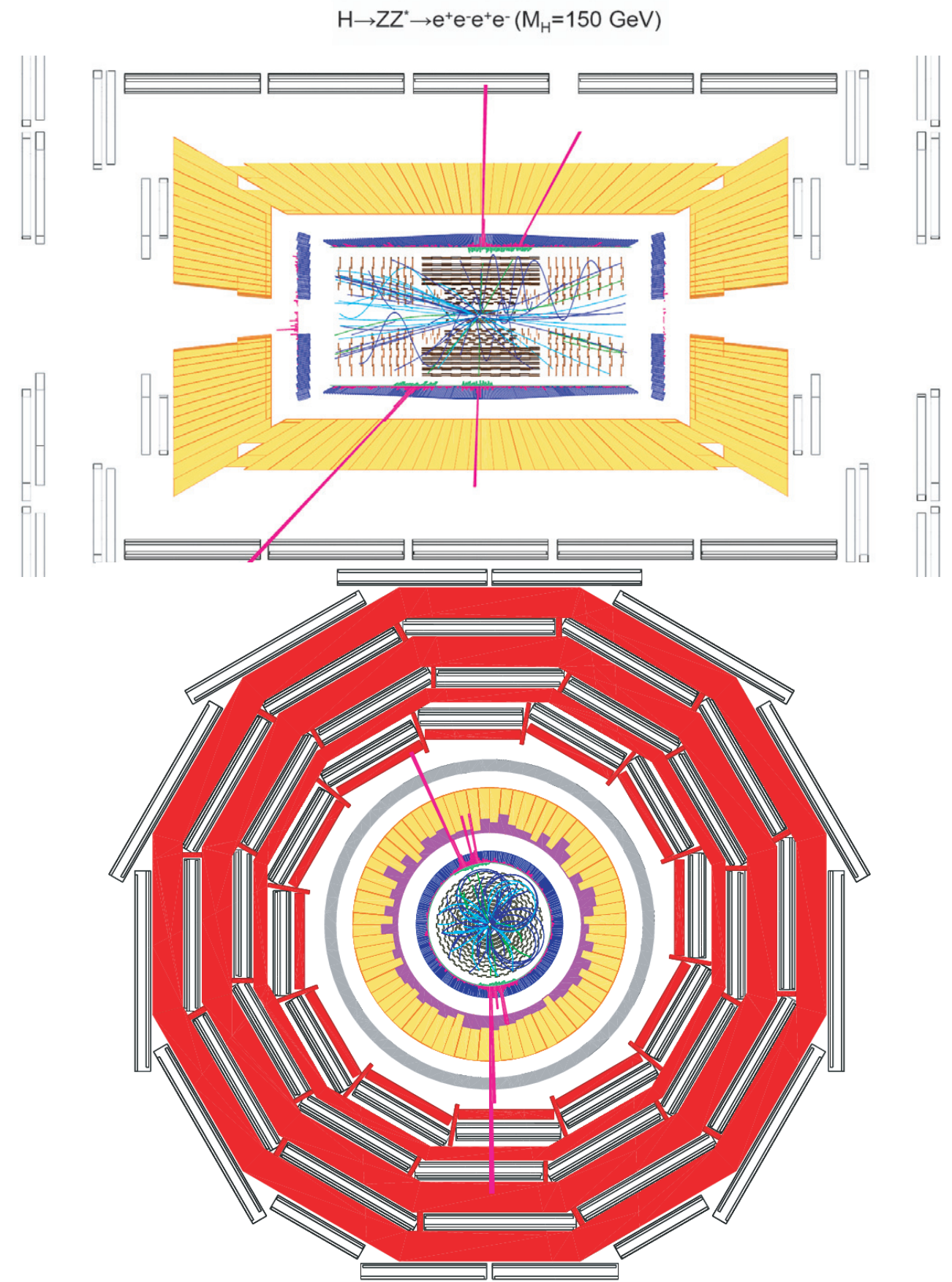

Figure CP2. Display of an event candidate in the CMS detector at the LHC for the Standard Model Higgs boson decay channel $\mathrm{H} \rightarrow \mathrm{ZZ}^{*} \rightarrow 4 \mathrm{e}$. The event is shown in a longitudinal (top) and transversal (bottom) projection of the detector. A mass of $150 \mathrm{GeV} / \mathrm{c}^{2}$ is measured from the reconstructed electrons. (See section 2.2.) 


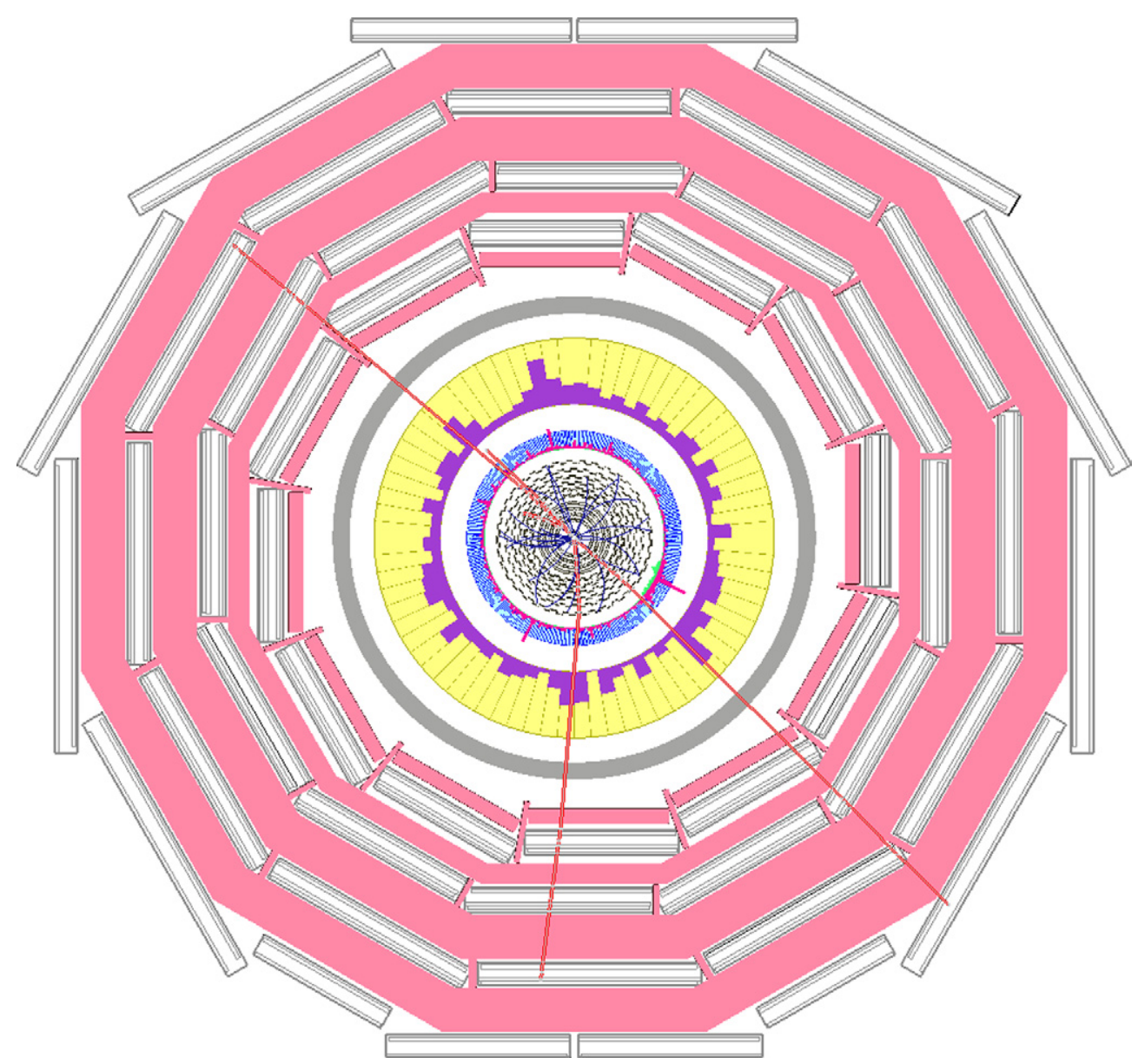

Figure CP3. Example of a $\mathrm{H} \rightarrow \mathrm{ZZ} \rightarrow 4 \mu$ event showing only the reconstructed tracks. One muon goes in the endcap detectors. (See section 3.1.1.) 


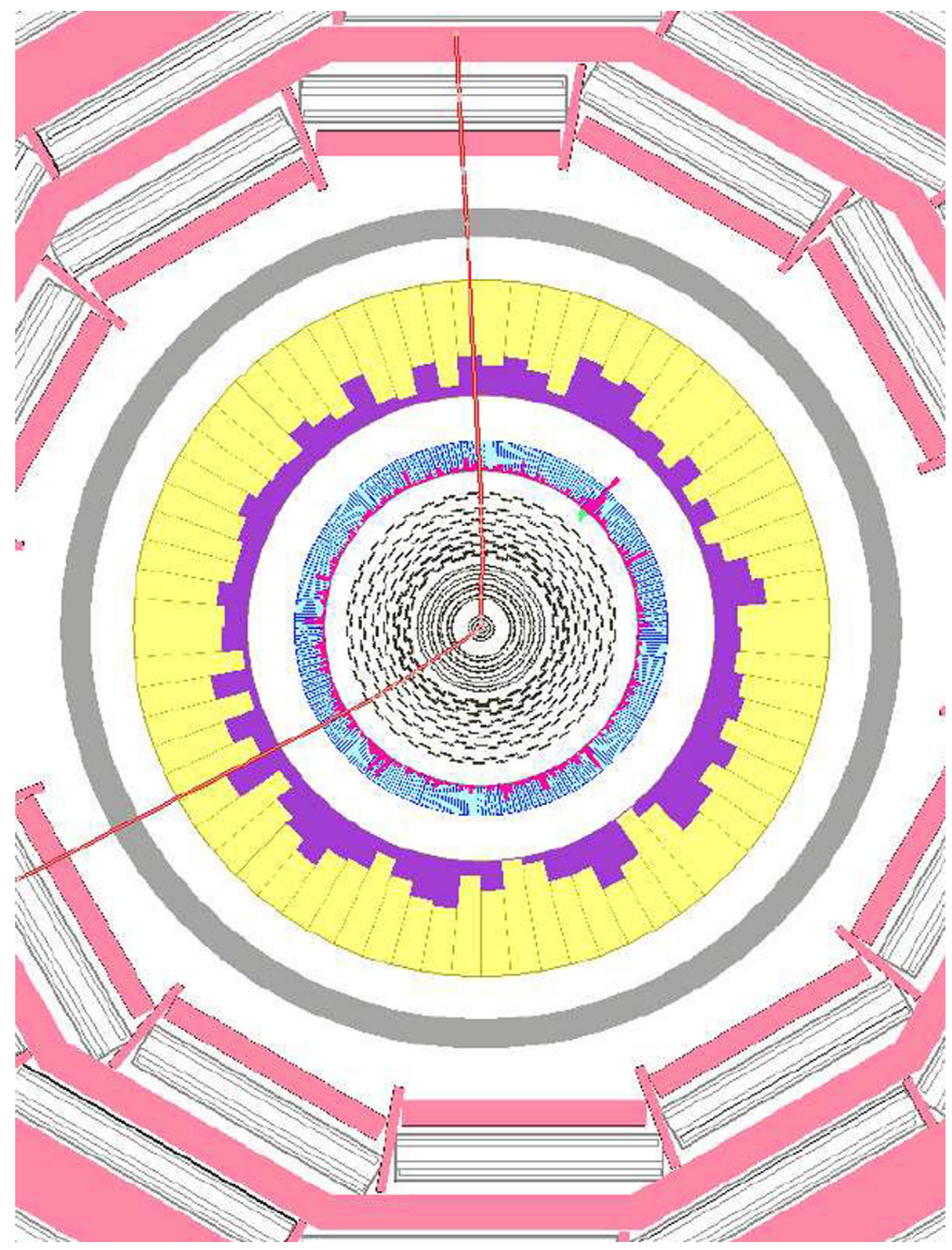

Figure CP4. Example of a $\mathrm{pp} \rightarrow \mathrm{H}+\mathrm{X}$ event with $\mathrm{H} \rightarrow \mathrm{WW} \rightarrow \mu \nu \mu \nu$. (See section 3.2.2.1.) 


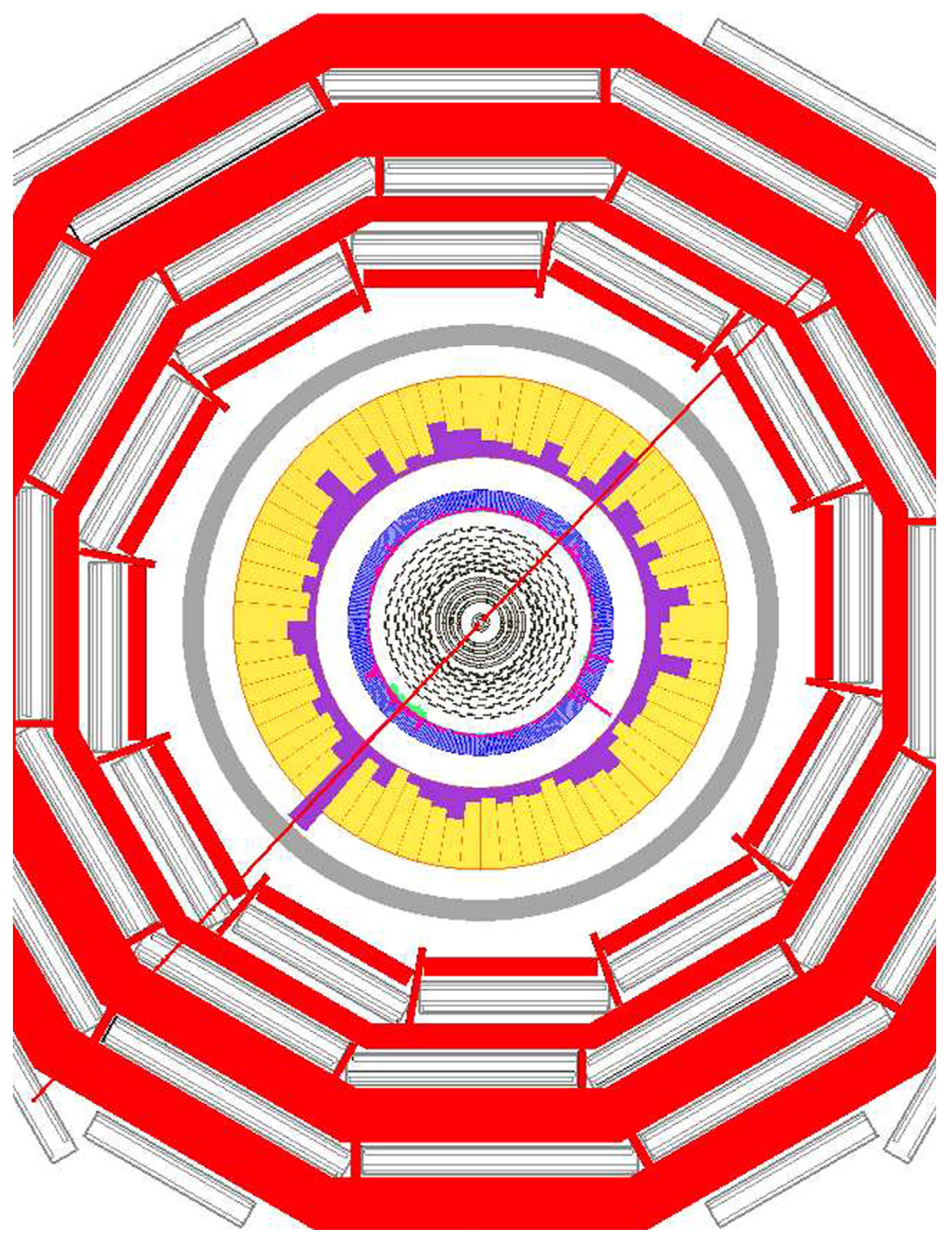

Figure CP5. Typical simulated event of a dimuon decay of $3 \mathrm{TeV} / \mathrm{c}^{2} \mathrm{Z}^{\prime}$ produced at $\mathcal{L}=2 \times 10^{33} \mathrm{~cm}^{-2} \mathrm{~s}^{-1}$, showing the muon tracks only. (See section 3.3.1.) 
CMS Collaboration

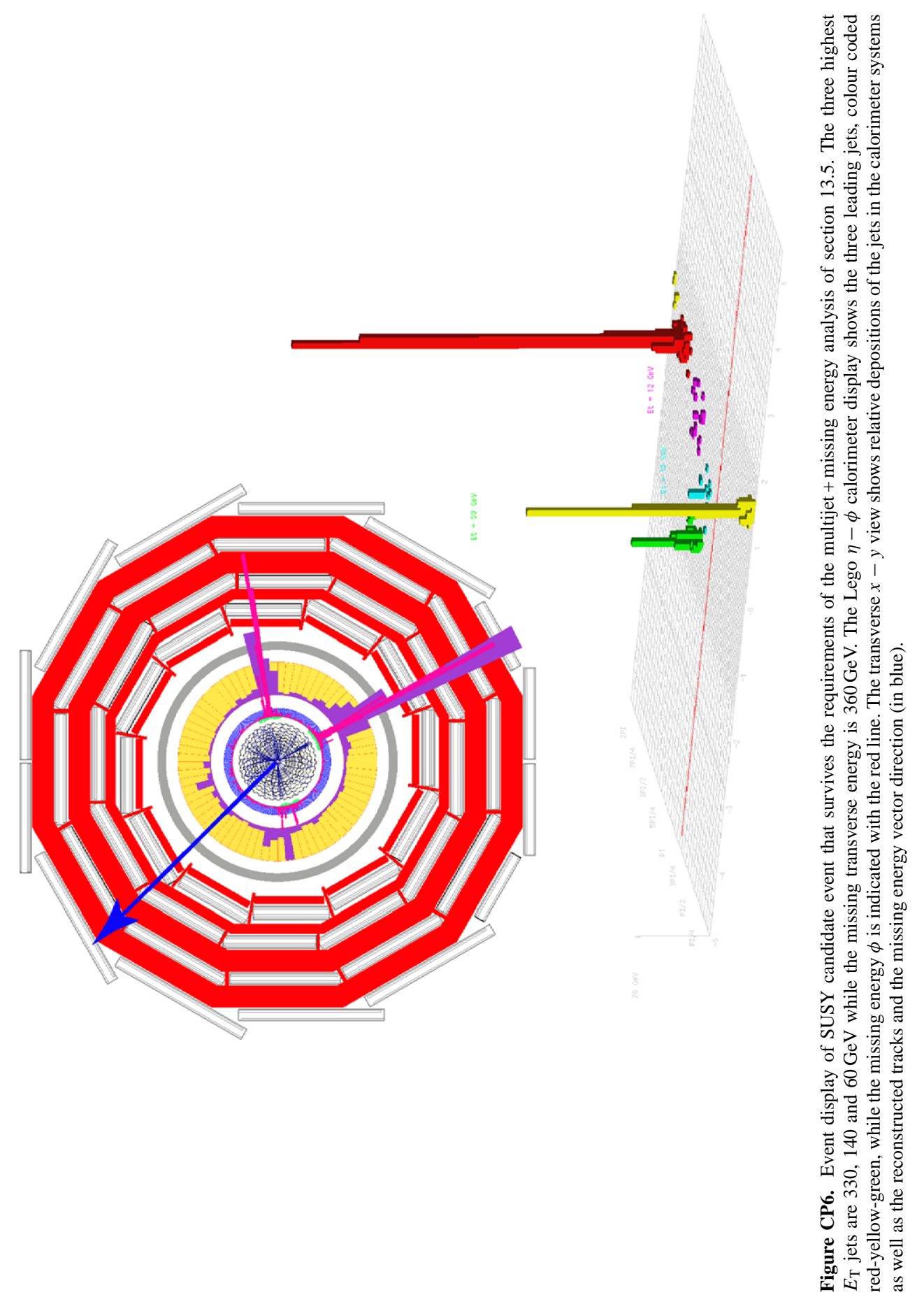



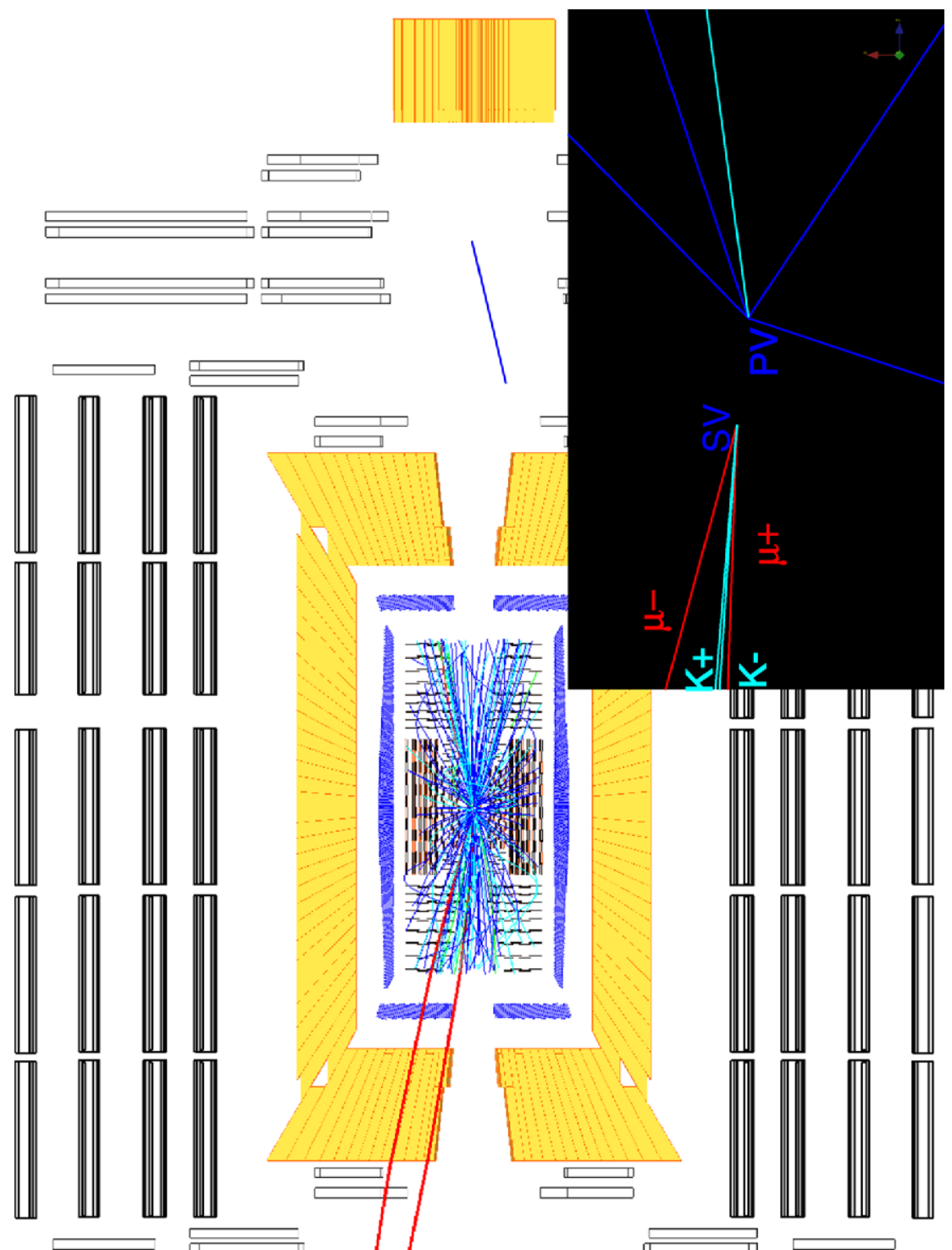


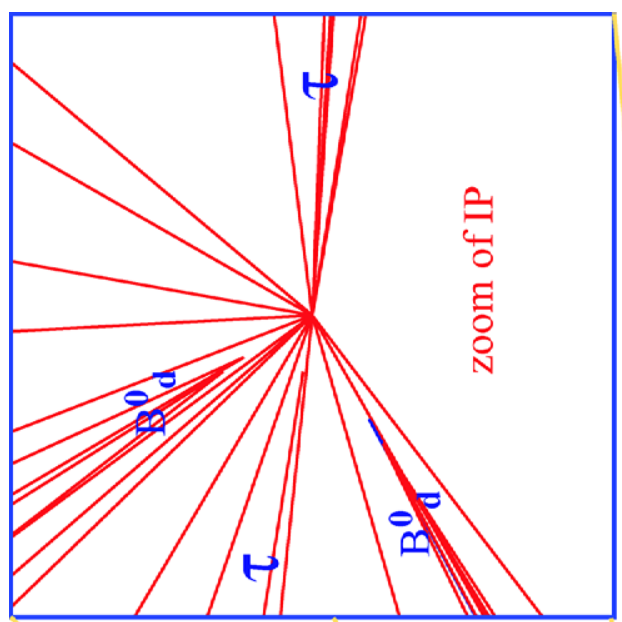

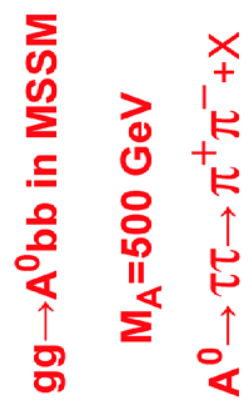

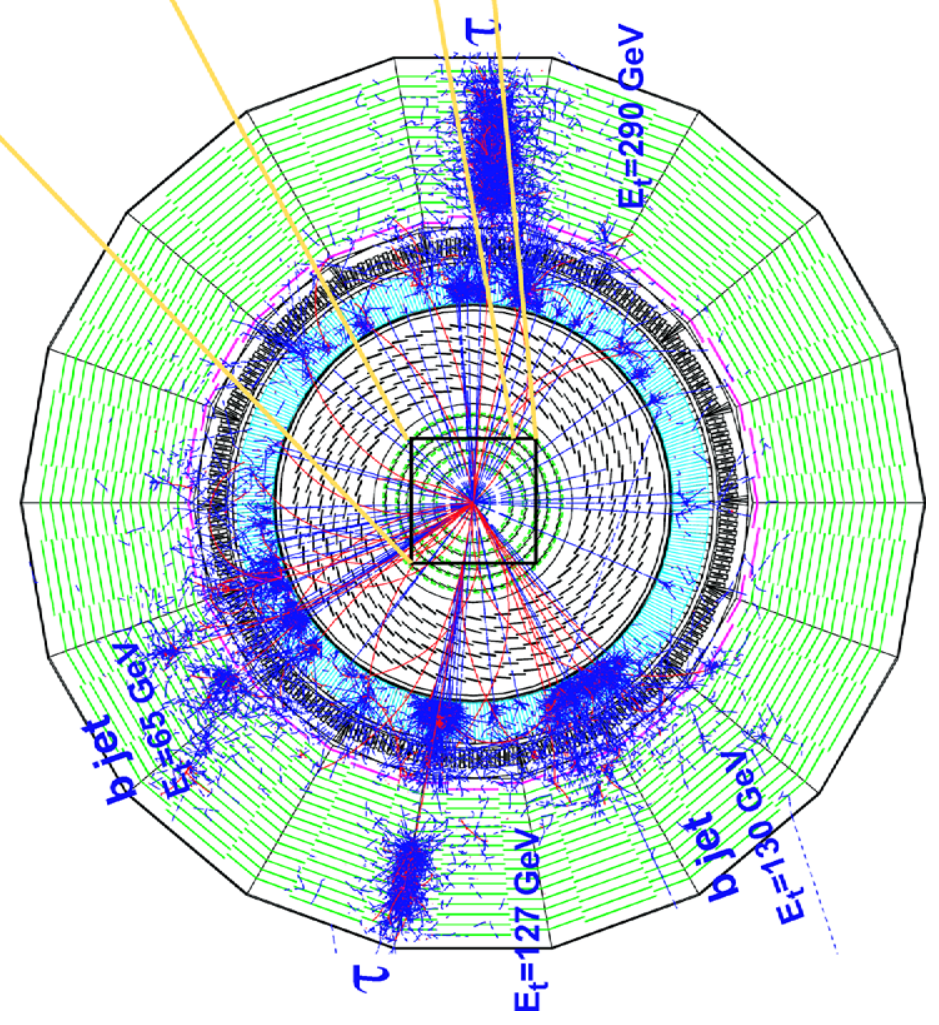

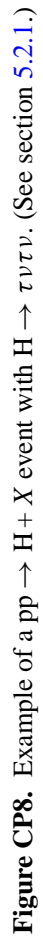




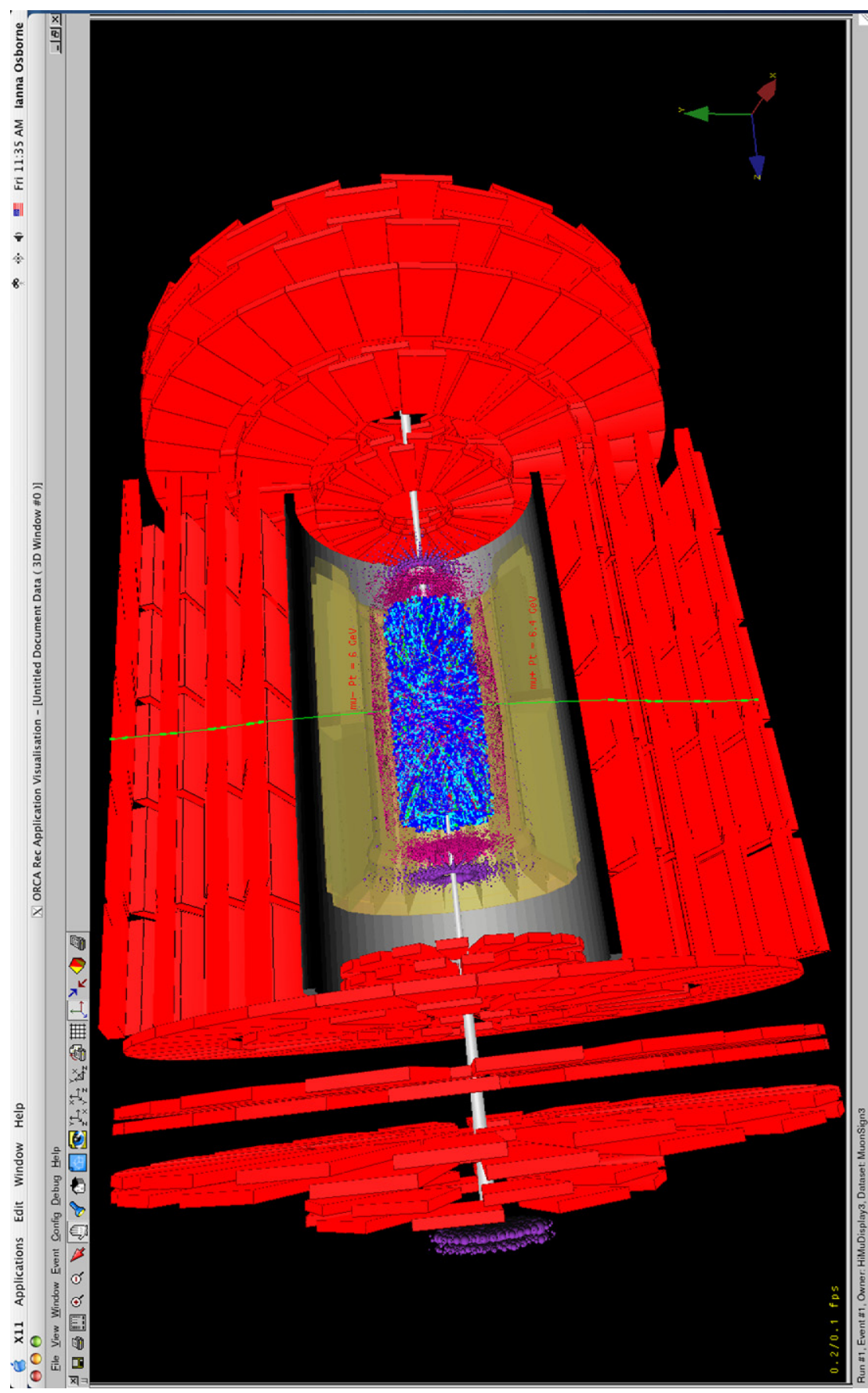

